\title{
World Congress on Osteoporosis, Osteoarthritis and Musculoskeletal Diseases (WCO-IOF-ESCEO 2016): Poster Abstracts
}

(C) International Osteoporosis Foundation and National Osteoporosis Foundation 2016

P100

AXIAL BMD IN SOUTH EAST ASIAN PATIENTS WITH TYPE 2 DM: HOW DO RACE AND BMI MATTER?

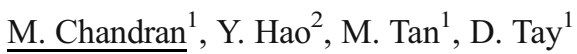

${ }^{1}$ Osteoporosis and Bone Metabolism Unit, Singapore General Hospital, Singapore, Singapore, ${ }^{2}$ Health Services Research and Biostatistics Unit, Singapore General Hospital, Singapore, Singapore

Introduction: Axial BMD is reported to be higher in type 2 Diabetes Mellitus (T2DM). However, it is not entirely clear whether the higher BMD is due to the higher BMI that is commonly seen with T2DM. In Asia, T2DM is not as strongly associated with overweight and obesity as it is in the west and a small but significant number of underweight $(\mathrm{BMI}<18.5)$ Asian type 2 diabetic patients exist. We aimed to explore whether BMD differed at axial sites amongst South East Asian underweight diabetics as compared to their non-diabetic counterparts.

Method: Cross-sectional analysis of 255 diabetic and 148 nondiabetic patients over the age of 50 with new hip fractures.

Results: $84.9 \%$ of the total cohort were Chinese, $9.9 \%$ were Malay and 5.2\% were Indian. There were 37 diabetics and 26 nondiabetics who were underweight. Presence of T2DM was associated with higher absolute BMD $(\mathrm{gm} / \mathrm{cm} 2)$ at the femoral neck $(0.527 \pm 0.103$ vs. $0.491 \pm 0.102, p<0.01)$ and lumbar spine [LS] $(0.798$ \pm 0.147 vs. $0.723 \pm 0.156, p<0.01)$. This association persisted after adjustment for age, BMI, race, gender, renal function, use of statins, proton pump inhibitors, steroids, anticonvulsants and calcium and/or vitamin D supplements. We found statistically significant higher BMD at the LS in underweight diabetics when compared to underweight non-diabetics $(0.733 \pm 0.126$ vs. $0.649 \pm 0.131$ $\mathrm{g} / \mathrm{cm} 2, \mathrm{p}=0.014)$. This difference persisted after correction for age, gender and BMI $(\mathrm{p}=0.005)$. There was no statistically significant difference in radiographically reported osteoarthritic changes at the spine between the diabetics and nondiabetics. There was also a trend towards higher BMD at the NOF in the underweight diabetics compared to underweight nondiabetics.

Conclusion: The finding of higher BMD even in underweight diabetics with new hip fractures compared to their nondiabetic counterparts suggests that type 2 diabetics not only fracture at higher BMD than nondiabetics but also that higher BMD in T2DM is not due to higher BMI.

\section{P101}

ELEVATED BODY IRON STORES AND CIRCULATING OSTEOPROTEGERIN AS INDEPENDENT PREDICTORS OF HIP FRACTURE IN POSTME- NOPAUSAL WOMEN ADMITTED FOR FRAGILITY FRACTURE: TIME FOR NEW SCREENING STRATEGIES?

M. Shargorodsky ${ }^{1}$, Y. Lipovetzky ${ }^{1}$

${ }^{1}$ Wolfson Medical Center, Holon, Israel

Background: Identification of risk factors may help us to understand the pathogenesis of osteoporotic hip fracture as well as to formulate development of better diagnostic, prevention and treatment strategies. The present study was designed to determine the impact of multiple metabolic risk factors such as markers of systemic inflammation (C-reactive protein), body iron stores (ferritin), insulin resistance (HOMA-IR) and bone remodeling (osteoprotegerin), for the prediction of hip fractures in postmenopausal osteoporotic women. 
Methods: The study group consisted of 115 postmenopausal women divided into two groups: Group 1 consisted of 49 subjects hospitalized in the Orthopedic Department, Wolfson Medical Center due to the diagnosis of nontraumatic hip fracture and Group 2 contained 66 postmenopausal osteoporotic women without history of hip fracture. Metabolic parameters were determined.

Results: Circulating OPG was significantly higher in Group 1 than in Group $2(205.2 \pm 177.1$ vs. $60.0 \pm 22.3, p<0.0001)$. While levels of hemoglobin ( $\mathrm{Hb})$ as well as $\mathrm{MCV}$ and $\mathrm{MCH}$ did not differ between groups, circulating ferritin was significantly higher in Group 1 than in Group 2 (217.9 \pm 195.1 vs. $49.7 \pm 31.3, p<0.0001)$. In multiple linear regression analysis, which explains about $40 \%$ of the variability in CRP, $42 \%$ in $\mathrm{OPG}$, and $28 \%$ in ferritin, significant by-group differences in terms of these parameters persisted even after adjustment.

Conclusions: Elevated body iron stores reflected by serum ferritin concentrations and bone remodeling marker, osteoprotegerin, are independent predictors of hip fracture in postmenopausal women hospitalized for fragility fracture.

\section{P102}

SEVERE BONE MARROW EDEMA ON SACROILIAC JOINT MRI INCREASES THE RISK OF LOW BMD IN PATIENTS WITH AXIAL SPONDYLOARTHRITIS

\section{K. Y. Kang ${ }^{1}$, Y. S. Hong ${ }^{1}$}

${ }^{1}$ Internal Medicine/Catholic University of Korea, Seoul, Republic of Korea

Objective: To determine the association between inflammatory and structural lesions on sacroiliac joint (SIJ) MRI and BMD and to identify risk factors for low BMD in patients with axial spondyloarthritis (axSpA).

Methods: Seventy-six patients who fulfilled the ASAS axSpA criteria were enrolled. All underwent SIJ MRI and BMD measurement at the lumbar spine, femoral neck, and total hip. Inflammatory and structural lesions on SIJ MRI were scored. Laboratory tests and assessment of radiographic and disease activity were performed at the time of MRI. The association between SIJ MRI findings and BMD was evaluated.

Results: Among the 76 patients, 14 (18\%) had low BMD. Patients with low BMD showed significantly higher bone marrow edema (BME) and deep BME scores on MRI than those with normal BMD $(p<0.047$ and 0.007 , respectively). Inflammatory lesions on SIJ MRI correlated with BMD at the femoral neck and total hip. Multivariate analysis identified the presence of deep
BME on SIJ MRI, increased CRP, and sacroiliitis on $\mathrm{X}$-ray as risk factors for low BMD (OR: 5.6, 14.6, and 2.5, respectively).

Conclusion: The presence of deep BME on SIJ MRI, increased CRP levels, and severity of sacroiliitis on X-ray were independent risk factors for low BMD.

\section{P103}

TBS REFLECTS TRABECULAR MICROARCHITECTURE IN PREMENOPAUSAL WOMEN AND SIMILARLY AGED MEN WITH LOW TRAUMATIC FRACTURES

C. M. Muschitz ${ }^{1}$, R. Kocijan ${ }^{1}$, J. Haschka ${ }^{1}$, D. P. Pahr ${ }^{2}$, A. K. Kaider $^{3}$, D. H. Hans ${ }^{4}$, A. Fahrleitner-Pammer ${ }^{5}$, H. R. Resch ${ }^{6}$

${ }^{1}$ Medical Department II - St. Vincent Hospital Vienna, Vienna, Austria, ${ }^{2}$ Institute of Lightweight Design and Structural Biomechanics, Vienna University of Technology, Vienna, Austria, ${ }^{3}$ Center for Medical Statistics, Informatics and Intelligent Systems, Medical University of Vienna, Vienna, Austria, ${ }^{4}$ Center of Bone Diseases, Lausanne University Hospital Switzerland, Lausanne, Switzerland, ${ }^{5}$ Department of Internal Medicine, Division of Endocrinology and Metabolism, Medical University of Graz, Graz, Austria, ${ }^{6}$ St. Vincent Hospital - Medical Department II, Vienna, Austria

Purpose: Transiliac bone biopsies are still considered to be the gold standard for the analysis of bone microstructure, but limited to specialized centers. The benefit of Trabecular Bone Score (TBS) in addition to areal BMD (aBMD) for fracture risk assessment has been documented in cross-sectional and prospective studies. The aim of this study was to test if TBS may be useful as a surrogate to histomorphometric trabecular parameters of transiliac bone biopsies.

Methods: Transiliac bone biopsies from 80 female patients (median age 39.9 years - interquartile range, IQR $34.7 ; 44.3$ ) and 43 male patients (median age 42.7 years - IQR 38.9 ; 49.0) with low traumatic fractures and without any comorbidities known to affect bone metabolism were included. Bone volume fraction (BV/TV), trabecular thickness (Tb.Th), trabecular number (Tb.N), trabecular separation (Tb.Sp), structural model index (SMI) as well as serum bone turnover markers (BTMs) sclerostin, intact $\mathrm{N}$-terminal type 1 procollagen propeptide (P1NP) and cross-linked $\mathrm{C}$-telopeptide (CTX) were investigated. Results: Median SMI, Tb.Th and sclerostin levels (45.5 vs. $33.4 \mathrm{pmol} / \mathrm{L}, p<0.0001)$ were higher in males. Multiple regression models including gender, aBMD and BTMs 
revealed TBS as an independent discriminative variable with adjusted R2 values of $69.1 \%$ for SMI, $79.5 \%$ for Tb.N, $68.4 \%$ for $\mathrm{Tb} . \mathrm{Sp}$, and $83.3 \%$ for BV/TV. In univariate regression models BTMs showed statistically significant findings, but in the multiple models only P1NP and CTX were significant for Tb.N.

Conclusions: TBS is a feasible non-invasive surrogate technique for the assessment of cancellous bone microarchitecture and should be implemented as an additional tool for the estimation of trabecular bone properties.

\section{P104}

SCLEROSTIN LEVELS AND CHANGES IN BONE METABOLISM AFTER BARIATRIC SURGERY

C. Muschitz ${ }^{1}$, R. Kocijan ${ }^{1}$, C. Marterer ${ }^{1}$, A. Rhabarnia ${ }^{1}$, G. Muschitz $^{2}$, H. Resch ${ }^{1}$, P. Pietschmann ${ }^{3}$

${ }^{1}$ Medical Department II - St. Vincent Hospital Vienna, Vienna, Austria, ${ }^{2}$ Division of Plastic and Reconstructive Surgery, Department of Surgery, Medical University of Vienna, Vienna, Austria, ${ }^{3}$ Department of Pathophysiology and Allergy Research, Center for Pathophysiology, Infectology and Immunology, Vienna, Austria

Purpose: The role of sclerostin as a key regulator of bone formation remains unknown after Roux-en-Y gastric bypass (RYGB) or laparoscopic sleeve gastrectomy (SG). The objective was the evaluation of sclerostin and Dickkopf-1 (DKK-1) serum levels after surgery and correlations with bone turnover markers (P1NP, CTX), PTH (iPTH) and areal BMD changes at total body, lumbar spine and total hip. This was a prospective observational singlecenter two-arm study in premenopausal women with severe adiposities over 24 months. Prior to surgery and after 1, 3, 6, 9, 12, 18, and 24 months sclerostin, DKK-1, CTX, P1NP and BMD were measured.

Results: 52 premenopausal women ( $40 \pm 8$ years, BMI 43.4 ) after RYGB and 38 premenopausal women ( $41 \pm 7$ years, BMI 45.7) after SG were included. Sclerostin, CTX and to lesser extend P1NP immediately increased after surgery and remained elevated during the entire study period $(p<0.001)$. DKK-1 declined during months 3-9 $(p<0.005)$ and then remained unchanged, serum phosphate continuously increased $(p<0.001)$, iPTH remained in the upper normal limit. Sclerostin increases were significantly positively correlated with CTX and P1NP increases and significantly negatively correlated with BMD loss. BMD independently declined regardless of RYGB and SG. Elevations of sclerostin, CTX, P1NP, and phosphate but not DKK-1 and $\mathrm{PPTH}$, were significant discriminating factors for BMD loss (AUC 0.920).
Conclusions: Rapid and sustained increases of sclerostin, CTX and to lesser extend P1NP causes an increase in bone metabolism and results in BMD loss at all skeletal sites.

\section{P105}

THE IMPACT OF VITAMIN D, CALCIUM, PROTEIN SUPPLEMENTATION, AND PHYSICAL EXERCISE ON BONE METABOLISM AFTER BARIATRIC SURGERY: THE BABS STUDY

C. Muschitz ${ }^{1}$, R. Kocijan ${ }^{1}$, J. Haschka ${ }^{1}$, A. Zendeli ${ }^{1}$, T. Pirker ${ }^{1}$, G. Muschitz $^{2}, \underline{\text { H. Resch }}{ }^{1}$, P. Pietschmann ${ }^{3}$

${ }^{1}$ Medical Department II - St. Vincent Hospital Vienna, Vienna, Austria, ${ }^{2}$ Division of Plastic and Reconstructive Surgery, Department of Surgery, Medical University of Vienna, Vienna, Austria, ${ }^{3}$ Department of Pathophysiology and Allergy Research, Center for Pathophysiology, Infectology and Immunology, Vienna, Austria

Purpose: Bariatric surgery causes ongoing weight loss, an increased bone turnover and loss of areal bone mineral density (aBMD) at different skeletal sites. The hypothesis of this study was to test if a specific postoperative intervention exerts different effects on bone metabolism, aBMD, body lean mass, and Trabecular Bone Score (TBS) compared to no intervention. In this prospective observational single-center two-arm study patients eligible for either laparoscopic Roux-en-Y gastric bypass (RYGB) or sleeve gastrectomy (SG) were randomly assigned into the two groups: The intervention group received preoperative vitamin D loading (28.000 IU/week for 8 weeks) and postoperative supplementation of vitamin D (16.000 IU/week), calcium citrate $(500 \mathrm{mg} / \mathrm{d})$, weight adjusted protein diet (range 50 $110 \mathrm{~g}$ /day including protein supplementation), and physical exercise (Nordic walking $3 \times 45 \mathrm{~min} /$ week). The non-interventional group received regular consultation for food intake behavior. The objectives were the evaluation of changes between the two groups of sclerostin (Scl) and Dickkopf-1 (DKK-1) serum levels, bone turnover markers (P1NP, CTX), intact parathyroid hormone (iPTH), 25-OH vitamin D, areal bone mineral density (BMD) changes at total body, lumbar spine and total hip as well as body lean body mass, and changes of TBS. Measurements were performed prior to surgery and after 1, 3, 6, 9, 12, 18, and 24 months.

Results: In each group 110 female and male patients with a median age of 41 years [IQR 34; 45] and a BMI of $44 \mathrm{~kg} / \mathrm{m} 2$ [IQR 41; 47] participated. Baseline characteristics of both groups did not differ with regard to type of surgery, serum values, and aBMD or body lean mass values. All investigated median values significantly changed within the groups regardless of RYGB or SG. At study endpoint in the intervention 
group median levels were lower for iPTH (51.8 vs $77.6 \mathrm{pg} /$ $\mathrm{mL}), \mathrm{Scl}$ (40.2 vs $53.0 \mathrm{pmol} / \mathrm{L}), \mathrm{CTX}(0.524$ vs $0.640 \mathrm{ng} / \mathrm{mL}$; $p<0.0001$ for all) and P1NP (41.5 vs $51.3 \mu \mathrm{g} / \mathrm{L}, \mathrm{p}=0.004)$. Median levels were higher for $25-\mathrm{OH}$ vitamin (32.5 vs 22.0 $\mathrm{ng} / \mathrm{mL}$ ), body lean mass ( $46.5 \mathrm{vs} 42.6 \mathrm{~kg}$ ), aBMD total body (1.178 vs $1.091 \mathrm{~g} / \mathrm{cm} 2)$, aBMD lumbar spine (1.272 vs 1.130 $\mathrm{g} / \mathrm{cm} 2$ ), aBMD total hip (1.179 vs $1.064 \mathrm{~g} / \mathrm{cm} 2, p<0.0001$ for all), DKK-1 (25.9 vs $22.3 \mathrm{pmol} / \mathrm{L}, p=0.038)$, and TBS (1.226 vs $1.172, p=0.0067)$.

Conclusions: Specific interventions for patients with RYGB and SG reduce high bone turnover, the loss of aBMD and body lean mass, as well as changes in TBS.

\section{P106}

EARLY AND SUSTAINED CHANGES IN BONE METABOLISM AFTER SEVERE BURN INJURY

G. Muschitz $^{1}$, E. Schwabegger ${ }^{2}$, R. Kocijan ${ }^{3}$, A. Baierl ${ }^{4}, \mathrm{H}$. Moussalli $^{3}$, A. Fochtmann ${ }^{1}$, S. Nickl ${ }^{1}$, I. Tinhofer ${ }^{1}$, H. Resch ${ }^{3}$, T. Rath ${ }^{1}$, P. Pietschmann ${ }^{5}$, C. Muschitz ${ }^{3}$

${ }^{1}$ Division of Plastic and Reconstructive Surgery, Department of Surgery, Medical University of Vienna, Vienna, Austria, ${ }^{2}$ Department of Plastic, Reconstructive and Aesthetic Surgery, the Medical University Innsbruck, Innsbruck, Austria, ${ }^{3}$ Medical Department II - St. Vincent Hospital Vienna, Vienna, Austria, ${ }^{4}$ Department of Statistics and Operations Research, the University of Vienna, Vienna, Austria, ${ }^{5}$ Department of Pathophysiology and Allergy Research, Center for Pathophysiology, Infectology and Immunology, Vienna, Austria

Context: Severe burn injury causes a massive stress response, consecutively heightened serum levels of acute phase proteins, glucocorticoids, and catecholamines with accompanying disturbance in calcium metabolism.

Objective: Evaluation of early and prolonged changes of serum bone turnover markers (BTM) and regulators of bone metabolism.

Design: Longitudinal observational design.

Setting: University clinic.

Patients: 32 male patients with a median age of 40.5 years and a median burned total body surface area (TBSA) of $40 \%$ ( $83 \%$ patients with full thickness burn injury).

Interventions: None.

Main outcome measures: Comparison of changes of BTM/ regulators of bone metabolism in the early (days $2-7$ ) and prolonged (days 7-56) phase after trauma.

Results: All investigated BTM/regulators significantly changed. During the early phase pronounced changes were observed for CTX, P1NP, sclerostin, DKK-1, BALP, FGF23, and iPTH levels, whereas albumin and 25-OH vitamin D decreased. Changes of OPG, OCN, and phosphate were less, but remained significant. The levels of serum calcium and CRP remained unaffected in the early stage. In the prolonged phase, changes of P1NP were most pronounced, followed by elevated sclerostin, OCN, BALP, and lesser changes for albumin levels. Calcium and ionized calcium levels tardily increased towards the lower limit of normal. In contrast levels of iPTH, FGF23, CRP, and to a lesser extent CTX and phosphate levels, declined significantly during this phase of investigation.

Conclusions: Ongoing changes of BTM and regulators of bone metabolism suggest alterations in bone metabolism with negative influence on bone quality and structure in male patients with severe burn injuries.

\section{P107}

CLINICAL SIGNIFICANCE AND PATHOGENETIC ROLE OF NICOTINAMIDE PHOSPHORIBOSYLTRANSFERASE IN OSTEOARTHRITIS

$\underline{\text { Y. Polyakova }}^{1}$, B. Zavodovsky ${ }^{1}$, L. Seewordova ${ }^{1}$, Y. Akhverdyan $^{1}$, I. Zborovskaya ${ }^{1}$

${ }^{1}$ Federal State Budgetary Institution «Research Institute of Clinical and Experimental Rheumatology», Volgograd, Russian Federation

Nicotinamide phosphoribosyltransferase (Nampt) regulates the rate of NAD + synthesis in cells. However, the other functions of Nampt remain poorly understood. The role of Nampt in the development of osteoarthritis (OA) is currently widely being studied.

Purpose: To explore pathogenesis of OA by measurement of serum Nampt levels.

Materials and methods: We examined 87patients with different types of OA and 30 healthy donors as control group. We used clinical criteria for the OA classification (Althman, 1991). Serum Nampt levels were measured using and indirect ELISA test system (RaiBiotech, cat No. EIA-VIS-1).

Results and discussion: Median Nampt levels in healthy subjects were $2.29[2.02-2.8] \mathrm{ng} / \mathrm{mL}$, while in patients with OA, it was equal to 3.98 [2.00-9.04] ng/ml, and were significantly higher $(p<0.001)$ than in healthy controls. We revealed significantly higher levels of Nampt in patients who complained of pain at rest in contrast to patients who experienced joint pain exclusively on movement $(\mathrm{p}<0.001)$. We observed the correlation between Nampt level and Lecken's functional in$\operatorname{dex}(r=0.4917, p=0.041)$ and the total WOMAC score $(r=0.589, p=0.049)$ in OA patients. Patients with high levels of Nampt had significantly higher erythrocyte sedimentation rate (ESR) and C-reactive protein (CRP) levels. There are a 
number of patented Nampt inhibitors which downregulate $\mathrm{NF} \kappa \mathrm{B}$. We suppose that Nampt inhibitors may reduce inflammation in $\mathrm{OA}$

Conclusions: We found that $29 \%$ of patients with OA had increased Nampt levels which correlated with secondary synovitis, increased ESR, CRP and joint symptoms severity. These findings support hypothesis of Nampt probable role in the pathogenesis of OA.

References: Polyakova J, et al. Annals Rheum Dis 2015;74(Suppl 2):372.

\section{P108}

DYNAMICS OF BONE MINERAL DENSITY IN ATORVASTATIN-TREATED RHEUMATOID ARTHRITIS PATIENTS COMPLICATED BY OSTEOPOROSIS

Y. Akhverdyan $^{1}$, B. Zavodovsky ${ }^{2}$, J. Polyakova ${ }^{2}$, L. Seewordova $^{1}$, M. Kuznetsova ${ }^{2}$, L. Shilova ${ }^{3}$

${ }^{1}$ Federal State Budgetary Institution «Research Institute of Clinical and Experimental Rheumatology», Volgograd, Russian Federation, ${ }^{2}$ Federal State Budgetary Institution «Research Institute of Clinical and Experimental Rheumatology», Xi'an, China, ${ }^{3}$ Volgograd State Medical University, Volgograd, Russian Federation

Purpose: To improve the quality of treatment of rheumatoid arthritis (RA) patients complicated by osteoporosis (OP) with atorvastatin therapy.

Methods: We examined 170 patients with RA aged 22-75 years (128 females $(75.3 \%)$ and 42 males $(24.7 \%))$. We measured BMD with DXA osteodensitometry (Lunar DPX-Pro, USA). We divided the patients into two groups to study the effectiveness of Atorvastatin in the treatment of patients with RA and osteopenia and those having complicated osteoporosis. Group 1 included 49 individuals with osteopenia and OP. They received atorvastatin for 6 months at a dose of 20-40 $\mu \mathrm{g} /$ day. Group 2 included 35 patients who received calcium supplements and vitamin $\mathrm{D}$ for 6 months at a dose of $1000 \mathrm{mg} /$ day.

Results: We revealed that $84(51.5 \%)$ out of 170 patients with RA had significantly lower Z- and T-scores values. We noted that $17(20.2 \%)$ patients were diagnosed as having osteoporosis, while $67(79.8 \%)$ had osteopenia. There was a significant positive dynamic of clinical and laboratory parameters during 6-month treatment with atorvastatin in patients with $\mathrm{OP}$ and osteopenia. In this patients we observed BMD and muscle strength increasing, reducing of bone and back pain, decreasing of C-reactive protein. Atorvastatin was well tolerated. Side effects were scarce. Symptomatic improvement occurred within 2-3 months after starting Atorvastatin therapy. Second group patients who received calcium monotherapy showed signs of progressive OP: BMD continued to decrease (mean -2.7 $\pm 0.18 \%$ ), while bone pain and biochemical markers of bone metabolism remained unchanged.

Conclusions: We concluded that Atorvastatin used in the treatment of RA patients may help to stabilize BMD and improve the symptoms of OP.

\section{P109}

VITAMIN D AND ITS CORRELATION WITH BONE MINERAL DENSITY AND DISEASE ACTIVITY IN CHILDHOOD SYSTEMIC LUPUS ERYTHEMATOSUS

S. Al-Mayouf $^{1}$, A. AlSaleem ${ }^{2}$

${ }^{1}$ Pediatric Rheumatology, King Faisal Specialist Hospital \& Research Center, Riyadh, Saudi Arabia, ${ }^{2}$ Pediatrics, King Faisal Specialist Hospital \& Research center, Riyadh, Saudi Arabia

Objective: To determine the effect of vitamin D supplement on BMD in children with systemic lupus erythematosus (SLE) and its correlation with disease activity.

Methods: Children with SLE enrolled in a cross-sectional study evaluated for disease activity, which is completed by using the SLE Disease Activity Index (SLEDAI), vitamin D profile, bone markers and BMD of the lumbar spine (LS) and the whole body (WB) using DXA at enrollment and 6 months later. All patients treated with cholecalciferol (vitamin D3) 2000 IU daily and calcium supplement (caltrate 600) mg twice daily.

Results: Twenty-eight patients (26 female) completed the evaluation. The mean age was $9.7+3.2$ years with mean disease duration of $5.4+4.3$ years. The mean baseline of $25-\mathrm{OH}$ vitamin D level was $54.1+30 \mathrm{nmol} / \mathrm{L}$, and SLEDAI score was 5.7+4.7. BMD was subnormal in 23 patients, with mean baseline (Zscore) of LS and WB $(-1.6+1.1,-0.5+1$ respectively). Levels of vitamin D correlated inversely with SLEDAI and positively with bone density but did not reach statistical significance. Bone markers levels were correlated with BMD. After 6 months treatment with cholecalciferol and caltrate, BMD of LS and WB did not show significant improvement $(-1.6+1.2$ and $\mathrm{WB}-0.6+$ 1 respectively). However, there was significant improvement in disease activity correlating with the improvement of $25-\mathrm{OH}$ vitamin D levels.

Conclusion: Our findings indicate that low BMD is common in childhood SLE. Furthermore, daily vitamin D supplementation could improve the serum levels of $25-\mathrm{OH}$ vitamin $\mathrm{D}$ and disease activity. However, there was no improvement in BMD during treatment period with the proposed $25-\mathrm{OH}$ vitamin dose. 


\section{P110}

BONE MINERAL DENSITY, FRACTURE RISK AND ASSOCIATED FACTORS IN ARGENTINIAN TYPE 2 DIABETES MELLITUS PATIENTS AGED OVER 55 YEARS

M. J. Sarli ${ }^{1}$, C. G. Firpo ${ }^{1}$, C. Parisi ${ }^{1}$, J. L. Guerra ${ }^{1}$, N. X. Garcia Basavilbaso $^{1}$, F. Baña ${ }^{1}$, F. H. Subies ${ }^{1}$, P. Guido ${ }^{1}$, M. A. Blanco ${ }^{1}$, F. Geraci ${ }^{1}$

${ }^{1}$ Sanatorio Las Lomas, San Isidro, Argentina

Background and aims: Fragility fractures are associated with higher morbidity and mortality. An association between type 2 Diabetes (DBT2) and hip fractures is being considered. The aim of this study was to compare BMD and fracture risk (FR) by the fracture risk algorithm (FRAX index), between diabetic and nondiabetic patient populations aged 55 years or older and to investigate potential underlying associated factors.

Materials and methods: We conducted a study evaluating BMD and FR in a 102 patients group with T2DM (mean age $64.43 y$ ) compared with a 78 patients control group (mean age 63.01y). All subjects were noninstitutionalized, Caucasian, above 55 years of age. Both sexes were included (65 men and 115 women). Patients who presented any cause of secondary osteoporosis, as those who were receiving drugs which affect BMD were excluded. BMD was assessed by DXA. We obtained anthropometric parameters, habits, personal and family history. FR was evaluated by FRAX index, which was calculated according to interrogatory and femoral neck BMD.

Results: We found that T2DM patients had higher BMD in all areas: femoral neck T-Score $(-0.46$ vs. $-1.39 ; p<0.0001)$ and L1L4 T-Score ( 0.11 vs. $-0.91 ; p<0.02)$. Despite, they had a significant elevated hip FRAX index ( 2.74 vs. $1.43 ; p<0.01)$. In our population the diabetic group had more healthy habits including less tobacco consumption (statistically significant), more physical activity, lower caffeine, soft drinks (primarily cola drinks) and alcohol consumption (not statistically significant). Diet calcium was really low in both groups (256 and $173 \mathrm{mg} /$ day in controls and diabetic group). Diabetic patients had increased abdomen circumference (107.6 vs. 92.32 in the control group), BMI was 31.87 in the diabetic group and 27.09 in the control group, having a positive correlation between BMI and hip T-Score. BMD did not correlate with glucose control, as measured by glycosylated hemoglobin.

Conclusion: Diabetes mellitus is pointed as an independent factor for fragility fractures. Bone fractures in diabetic patients lead to higher morbidity that worsens quality of life and increases mortality, secondary to renal, ophthalmologic, immune and cardiovascular conditions. We found that the diabetic patients group had better healthy habits and their BMD was higher, despite that, their hip fracture risk was elevated. The susceptibility to fractures may be due to worse bone quality and increased risk of fall. We consider it is important to continue working in identifying the causes of this association so as to work on the prevention of hip fractures in these patients.
P111

BMD CHANGE AFTER IBANDRONATE IBANDRONATE TREATMENT IN OSTEOPENIC POSTMENOPAUSAL WOMEN

${ }_{\text {S. Park }}{ }^{1}$, H. Kang ${ }^{1}$

${ }^{1}$ Dongguk University International Hospital, Seoul, Republic of Korea

Background: Ibandronate is effective in the treatment of postmenopausal women with osteoporosis. But, there were few data about ibandronate treatment in Korea. We evaluated the effect of ibandronate therapy on bone mass and compared the effectivity on BMD in 1-year treatment group

Objective: The aim of the study is to assess the effect of 1-year treatment with ibandronate on BMD in postmenopausal women with osteopenia or osteoporosis.

Methods: The BMD was assessed in 118 postmenopausal women with osteopenia or osteoporosis from March 2007 to January 2011, 42 patents who treated with $2.5 \mathrm{mg}$ per day of ibandronate were enrolled to study. BMD of lumbar spine (L2-L4) and femur was assessed by DXA at baseline, 12 months after treatment.

Results: The annual BMD of the lumbar spine showed a $9.11 \%$ increase, while also positive changes were noted in the proximal femur as a $1.89 \%$ increase. The BMD changes were $11 \%(\mathrm{~L}$ : Lumbar spine) and $1.1 \%$ (F: Femur) for the T-scores $<-4.0,6.3 \%$ (L) and $0.9 \%(\mathrm{~F})$ for the T-scores $-3.0 \sim-4.0$, and $3.8 \%(\mathrm{~L})$ and $0.5 \%(\mathrm{~F})$ for the T-scores $>-3.0$ respectively.

Conclusion: This study suggests that ibandronate treatment in postmenopausal women with osteopenia or osteoporosis is effective in terms of improving BMD.

\section{P112}

CLINICAL EPIDEMIOLOGY OF OSTEOPOROSIS AMONG ELDERLY FISHING AND AGRICULTURAL POPULATION IN TAIPEI, TAIWAN C.-Y. Lee ${ }^{1}$, W.-H. Chien ${ }^{2}$, H.-C. Shen ${ }^{3}$, Y.-C. $\mathrm{Hu}^{3}$, Y.-F. Chen $^{4}$, T.-H. Tung ${ }^{5}$

${ }^{1}$ School of Medicine, Fu-Jen Catholic University, New Taipei City, Taiwan, Province of China, ${ }^{2}$ Department of Family and Community Medicine, Cheng-Hsin General Hospital, Taipei, Taiwan, Province of China, ${ }^{3}$ New Taipei City Hospital, New Taipei City, Taiwan, Province of China, ${ }^{4}$ NHI Dispute Mediation Committee, Ministry of Health and Welfare, Taipei, Taiwan, Province of China, ${ }^{5}$ Department of Medical Research and Education, Cheng-Hsin General Hospital, Taipei, Taiwan, Province of China

Purpose: To quantify the prevalence and associated factors for osteoporosis among the elderly fishing and agricultural population in Taipei, Taiwan.

Methods: Subjects $(n=4,360)$ aged 65 years and over voluntarily admitted to a teaching hospital for a physical checkup were 
collected in 2010. Osteoporosis was defined as BMD is 2.5 SD or more below the young adult mean ( $-2.5 \mathrm{SD}$ or lower).

Results: Among these subjects, the over prevalence of osteoporosis was $26.4 \%$ and revealed a statistically significant increase with increasing age $(P<0.001)$. The women exhibited a higher prevalence than the men (48.1 vs. $26.4 \%$; $P<0.001)$. The age-specific prevalence of osteoporosis in 65-74 years, 75-84 years, and $\geq 85$ years was $27.7,40.0$, and $56.7 \%$, respectively. From the multiple logistic regression, age $(O R=1.080,95 \% \mathrm{CI}$ : 1.065-1.095), cholesterol $(O R=1.004,95 \% \mathrm{CI}: 1.002-1.007)$, uric acid $(O R=0.883$, $95 \% \mathrm{CI}: 0.835-0.934)$, body weight $(O R=0.987,95 \% \mathrm{CI}$ : 0.978-0995), regular smoking $(O R=1.293$, $95 \% \mathrm{CI}: 1.061-$ $1.576)$, and regular meat $(O R=1.415,95 \% \mathrm{CI}: 1.151-1.739)$ were statistically significantly related to osteoporosis.

Conclusion: Several clinical factors independently were indicated pertaining to the prevalence of osteoporosis in the elderly among fishing and agricultural population.

\section{P113}

BONE MINERAL DENSITY IN PATIENTS WITH PULMONARY TUBERCULOSIS

V. V. Novikov ${ }^{1}$, S. O. Mazurenko ${ }^{1}$, P. V. Gavrilov ${ }^{2}$, L. I. Archakova $^{2}$

${ }^{1}$ Saint Petersburg State University, Saint Petersburg, Russian Federation, ${ }^{2}$ Saint Petersburg Research Institute Phthisiopulmonology of the Russian Ministry of Health, Saint Petersburg, Russian Federation

Introduction: Low BMD is associated with many chronic diseases, including infections, and usually reflexes the violation of the bone remodeling. Despite considerable prevalence of tuberculosis, the state of BMD in patients with tuberculosis remains unexplored.

Objective: To assess BMD and to identify risk factors for osteoporosis in patients with pulmonary tuberculosis.

Methods: BMD was measured in 40 patients ( 25 male and 17 female) suffering from pulmonary tuberculosis. The mean age was $37.6 \pm 14.4$ years. The mean duration of the disease since diagnosis was 173.2 \pm 9.6 months. BMD was measured in two standard areas (lumbar spine, hip) by DXA with the Lunar Prodigy device. As a control group were used the device database and results of BMD measurement in 75 persons ( 35 men and 40 women) of the same age.

Results: In the study group, 15 patients ( 7 men and 8 women) had a history of fractures. In 3 patients, the fractures were only in childhood and adolescence, 3 patients had recurrent fractures. The mean values of BMD in two standard areas were not significantly different from that of the control group ( $p>0.05$ ). In 9 patients (7 men and 2 women) lumbar spine mineral density Z- index was below -1.0 . BMD negatively correlated with the duration of the disease. The lowest rates were in patients with an indication of the great experience of smoking, menopause and serious comorbidities (diabetes mellitus type 1, hepatitis $\mathrm{C}$, chronic pancreatitis). BMD has positive correlation with the results of lung capacity assessment $(p<0.05)$ and with BMI $(p<0.01)$.

Conclusions: We have identified the negative impact of the duration of pulmonary tuberculosis on BMD. Also we found a positive relationship of BMI as a marker of nutritional status and lung capacity with indicators of BMD in these patients. We plan to continue study the effects of different tuberculosis forms and related diseases on the BMD.

\section{P114}

\section{CHOLECALCIFEROL FOR THE TREATMENT OF LOW LEVELS OF VITAMIN D}

V. Aldasoro Caceres ${ }^{1}$, C. A. Egües Dubuc ${ }^{2}$, N. Errazquin Aguirre $^{2}$, M. Uriarte Ecenarro ${ }^{2}$

${ }^{1}$ Hospital Alto Deba, Mondragon, Spain, ${ }^{2}$ Hospital Universitario Donostia, San Sebastian, Spain

Introduction: With the recent emergence of cholecalciferol (25000 IU, $0.625 \mathrm{mg}, 2.5 \mathrm{ml}$ ) in the market we planned to know which might be the dosing schedule when trying to get vitamin D levels above $30 \mathrm{ng} / \mathrm{mL}$.

Material and methods: The main objective was optimal vitamin D levels $>30 \mathrm{ng} / \mathrm{mL}$. Patients referred for osteoporosis study with low vitamin D to which were administered as follows cholecalciferol were selected:

Vitamin $\mathrm{D}<30-20 \mathrm{ng} / \mathrm{mL}$ : Monthly cholecalciferol (Group 1). Vitamin $\mathrm{D}<20-10 \mathrm{ng} / \mathrm{mL}$ : Biweekly cholecalciferol (Group 2). Vitamin $\mathrm{D}<10 \mathrm{ng} / \mathrm{mL}$ : Weekly cholecalciferol (Group 3).

Patients with PTH, calcium and normal phosphorus were selected. Those patients who performed treatment with calcium and / or vitamin D were excluded. Blood analysis was performed applying calcium, PTH, phosphorus and vitamin D at baseline and at 3 months. All cases had normal renal function.

Results: 50 patients were included in this study. All patients enrolled were women. The mean age was 57.9 years. Pretreatment: 18 patients in Group 1 (mean 25.7), 20 patients in Group 2 (mean 18.3) and 12 patients in Group 3 (mean 9.2) were included. Posttreatment: 9/18 patients achieved optimal levels in Group 1, 12/20 in Group 2 and 5/12 in Group 3. No patient had renal failure. One patient experienced intolerance to cholecalciferol in Group1 as nausea and malaise after the 3rd dose.

Conclusions: The treatment schedule used for the treatment cholecalciferol Vitamin D deficit reaches the therapeutic goal (vitamin $\mathrm{D}>30 \mathrm{ng} / \mathrm{mL}$ ) in about half of them in all groups. It seems that the current treatment scheme would not be enough in half of the cases or perhaps more time is needed to achieve the goal in this paper. A larger number of patients would be required to confirm these results. 


\begin{tabular}{|c|c|c|c|}
\hline & PRETREATMENT (MEAN) & $\begin{array}{l}\text { POSTREATMENT (MEAN): } \\
\text { NUMBER OF NOT } \\
\text { RESPONDERS }\end{array}$ & $\begin{array}{l}\text { PERCENTAGE OF } \\
\text { RESPONDERS (\%) }\end{array}$ \\
\hline Vitamina $\mathrm{D}<30-20 \mathrm{ng} / \mathrm{mL}$ & 18 & 9 & $50 \%$ \\
\hline Cholecalciferol monthly (Grupo1) & (MEAN 25.7) & & \\
\hline Vitamina $\mathrm{D}<20-10 \mathrm{ng} / \mathrm{mL}$ : & 20 & 8 & $60 \%$ \\
\hline Cholecalciferol biweekly (Grupo2) & (MEAN 18.3) & & \\
\hline Vitamina $\mathrm{D}<10 \mathrm{ng} / \mathrm{mL}$ : & 12 & 7 & $41.7 \%$ \\
\hline Cholecalciferol weekly (Grupo3) & (MEAN 9.2) & & \\
\hline
\end{tabular}

\section{P115}

\section{THE SIMULTANEOUS BILATERAL ANTERIOR CRUCIATE LIGAMENT RUPTURE}

M. Milankov ${ }^{1}$, M. Obradovic ${ }^{1}$, M. Vranjes ${ }^{1}$, Z. Budinski ${ }^{1}$

${ }^{1}$ Department of Orthopaedic Surgery and Traumatology, Clinical Center Vojvodina, Medical Faculty of Novi Sad, Novi Sad, Serbia

The anterior cruciate ligament (ACL) injury is frequent in adolescents and young adults and is associated with recreational, competitive and professional athletic activity. The incidence of the anterior cruciate ligament (ACL) injuries reported ranges at $0.01-0.08 \%$ inhabitants/year, with the higher incidence in multidirectional sports. The overall incidence of bilateral ACL rupture at two separate times is $2.4-6 \%$. Simultaneous bilateral ACL rupture is a rare occurrence and only a few cases of this type of injury have been previously reported in the orthopaedic literature. Looking into these case reports, there are a lot of questions about the mechanism of the injury and the most optimal treatment. This study is present a case of simultaneous bilateral ACL tears in an actor who was injured by landing from a jump during his theatrical performance and who was treated surgically with staged bilateral ACL reconstruction using BTB autograft.

The surgery was delayed for 6 weeks during which period he underwent physical therapy. The first surgery involved right ACL reconstruction with BTB autograft fixed using titanium interference screws, and medial meniscectomy of the right knee. 4 months later, ACL reconstruction of the left knee was done. One year after the second surgery, the patient started again playing soccer recreationally. Five years later, the patient has stable knees with full range of motion, good strength of quadriceps femoris muscle, bilaterally, and negative Lachman and pivot shift tests. The patient is still an actor and continues to play recreational soccer without any limitations.

\section{P116}

RELATIONSHIP BETWEEN SERUM FERRITIN AND VITAMIN D IN 240 POSTMENOPAUSAL WOMEN WITH FRAGILITY OF HIP

G.-Y. Zhao ${ }^{1}$

${ }^{1}$ Orthopedics, Affiliated Hospital of Jiangsu University, Zhenjiang, China

Objective: To explore effects of iron accumulation on bone metabolism in postmenopausal women with fragility fractures by analyzing relationship between serum ferritin and vitamin D.

Methods: Serum ferritin, 25(OH)D3, bone turnover and biochemical indicators of 240 postmenopausal women with fragility fractures were detected. All of subjects were divided into groups according to a 10-year division and the level of ferritin respectively. Variance analysis was used to evaluate the change of serum ferritin, 25(OH)D3 and bone turnover indicators with age. $t$-test was used to evaluate the difference of $25(\mathrm{OH}) \mathrm{D} 3$ and bone turnover indicators between ferritin normal and elevation groups. At last, Pearson and partial correlation was used to evaluate associations between ferritin and $25(\mathrm{OH}) \mathrm{D} 3$, bone turnover indicators.

Results: The serum ferritin elevation and 25(OH)D3 insufficiency were prevalent among subjects, and ferritin was increased and $25(\mathrm{OH}) \mathrm{D} 3$ was decreased with the increasing of age $(P<0.05)$. The serum 25(OH)D3 was significantly lower in the group of ferritin elevation than in normal $(P<0.05)$. The serum ferritin was negatively associated with $25(\mathrm{OH}) \mathrm{D} 3$ $(r=-0.22, p=0.001)$. Adjusted age, the serum ferritin also showed negative correlation with 25(OH)D3 $(r=-0.19$, $p=0.004)$. No significant relationship was found between ferritin and bone turnover indicators.

Conclusion: Iron accumulation and vitamin D insufficiency are prevalent in postmenopausal women with fragility fractures. The results provide new clinical evidences for studying relationship of iron accumulation and postmenopausal osteoporosis. 


\section{P117}

TRABECULAR BONE SCORE IS SEVERELY AFFECTED IN MALE PATIENTS WITH CHRONIC OBSTRUCTIVE PULMONARY DISEASE

$\underline{\text { E. Casado }}^{1}$, L. Del Río ${ }^{2}$, M. Gallego ${ }^{3}$, M. Arévalo ${ }^{1}$, M. Larrosa $^{1}$

${ }^{1}$ Rheumatology, University Hospital Parc Taulí Sabadell, Sabadell, Spain, ${ }^{2}$ CETIR Centro Médico, Barcelona, Spain, ${ }^{3}$ Pneumology, University Hospital Parc Taulí Sabadell, Sabadell, Spain

Background: Patients with chronic obstructive pulmonary disease (COPD) have a high risk of osteoporosis and fractures. In a cohort of male patients with COPD we reported $33 \%$ of vertebral fractures, with $27 \%$ of fractures in patients with normal $\mathrm{BMD}^{1}$. Trabecular bone score (TBS) has been described as an index of bone microarchitecture, and could be a new assessment tool to detect bone quality impairment in these patients.

Objectives: Analysis of TBS in a subcohort of male patients with COPD and its possible association with BMD, corticoid use, COPD severity or vertebral fractures.

Methods: Male patients, older than 50 years, with COPD defined according to ATS/ERS classification (FEV1/FVC $<70 \%$ ) were included. Exclusion criteria: Other concomitant pulmonary disease, rheumatologic and/or vertebral disease that may lead to misinterpretation of BMD by DXA. BMD was determined by DXA at lumbar spine and proximal femur. Vertebral fractures were assessed by thoracic and lumbar X-ray. Corticosteroid use in the previous 5 years and number of hospitalizations were recorded in all patients.

Results: We included 98 patients. Mean age $67.8 \pm 7.5$ years. $38 \%$ patients had a mild pulmonary disease, $39 \%$ moderate and $23 \%$ had a severe disease ((FEV1/FVC $<30 \%) .29$ patients $(30 \%)$ had morphometric vertebral fractures. Mean BMD T-score at lumbar spine $-1.67 \pm 1.55$, at femoral neck $-1.81 \pm 1.04$, and al total hip $-1.31 \pm 1.14$. Mean TBS was $1.035 \pm 0,135$ (T-score $-2.85 \pm 1.18$ ). TBS was lower in fractured patients $(\mathrm{T}-2.59 \pm 1.16$ vs. $-2.34 \pm 1.22 ; p=\mathrm{NS})$. According to COPD severity TBS was lower in patients with moderate disease ( $\mathrm{T}-2.57 \pm 1.34)$ and severe disease $(\mathrm{T}-2.54$ \pm 1.34 ) than in patients with mild disease $(\mathrm{T}-2.19 \pm 1.32)$, although without reaching significant differences. We did not find any significant association between TBS and corticosteroid use.

Conclusions: TBS is severely affected in male patients with chronic obstructive pulmonary disease, especially in patients with moderate and severe pulmonary disease. Low TBS seems to be associated with vertebral fractures in this population.

${ }^{1}$ Casado E, et al. JBMR 2007;22 (S1):S202.
P118

VERTEBRAL OSTEOPOROTIC FRACTURESBELATED DIAGNOSE OF OSTEOPOROSIS

$\underline{\text { J. Zvekic-Svorcan }}^{1}$, J. Vasic ${ }^{2}$, T. Jankovic ${ }^{1}$, K. Filipovic ${ }^{1}$, S. Subin-Teodosijevic ${ }^{3}$

${ }^{1}$ Rheumatology, Special Hospital for Rheumatic Diseases, Novi Sad, Serbia, ${ }^{2}$ Rheumatology, Railway Healthcare Center, Belgrade, Serbia, ${ }^{3}$ Rheumatology, General Hospital "Djordje Joanovic", Zrenjanin, Serbia

Objective: To point out that occurrence of vertebral osteoporotic fractures represents the belated diagnose of osteoporosis Material and methods: A retrospective analysis was done on 110 postmenopausal women with diagnosed osteoporosis who had vertebral osteoporotic fractures. Data were retrieved from the National register for osteoporosis of Serbia. Age of subjects was noted at the time of occurrence of nonvertebral small-trauma fractures, vertebral fractures, establishing diagnose by DXA method and by radiography detection of vertebral fracture. After collecting these parameters, we analyzed belatedness in establishing the osteoporosis diagnose. Statistical analyze has been done by $T$-test.

Results: Average age of subjects was $70.01 \pm 8.379$ years. From total number of subjects, $58.3 \%$ also had a nonvertebral smalltrauma fracture. Patients with vertebral fractures were older (statistically significant, $t=6.027, p<0.01$ ) when they were diagnosed with osteoporosis than in time when they suffered nonvertebral fracture. This can be explained only by the fact they were not diagnosed after nonvertebral fracture, but only after vertebral fracture occurred after more than 3 years later (belated diagnosis). There is no statistically significant difference in patients with vertebral fractures between time when they were diagnosed by DXA findings and their age at the time when they were diagnosed using radiological findings $(t=0.099, p>0.05)$ which means that only after detection of vertebral fracture, osteoporosis was diagnosed by referring patients to osteodensitometry.

Conclusion: Osteoporosis diagnose was established when complications had already occurred (vertebral osteoporotic fractures). Therefore it is necessary to establish diagnose in a timely manner and to start treatment in order to prevent osteoporotic fractures carrying increased mortality and morbidity.

\section{P119}

ARTERIAL STIFFNESS IN SUBACUTE STROKE: CHANGING PATTERN AND RELATIONSHIP WITH FUNCTIONAL RECOVERY AFTER REHABILITATION

B. R. Kim ${ }^{1}$, Y. K. Lee ${ }^{1}$

${ }^{1}$ Jeju National University Hospital, Jeju, Republic of Korea

Introduction: Brachial-ankle pulse wave velocity (baPWV) has been proposed as a simple, noninvasive method for 
estimating arterial stiffness, and also has a significant predictive value for cardiovascular mortality and morbidity among stroke patients.

Purpose: To investigate the changing pattern of arterial stiffness and functional outcome at 1 and 3 months after stroke onset, and to determine whether baPWV at 1 month after stroke onset is of predictive value in terms of short-term functional recovery after rehabilitation in patients with subacute stroke.

Method: Sixty subacute stroke patients (43 males and 17 females; average age, 62.7 years) were enrolled for this study. BaPWV was measured as indices of arterial stiffness. Functional assessments included 6-min walk test (6MWT), Fugl-Meyer assessment-hemiparetic upper and lower extremities (FMA-UE, FMA-LE), Functional ambulatory category (FAC), Berg balance scale (BBS), Korean Mini-Mental Status Examination (K-MMSE), and Korean-Modified Barthel Index (K-MBI). Above all measurements were conducted at 1 and 3 months after stroke onset, and all patients received conventional rehabilitation during a mean follow-up period.

Results: BaPWV (1703.8 vs. 1641.8, $p=0.049$ ), 6MWT (187.5 vs. $248.0, p<0.001$ ), FMA-UE (42.6 vs 46.2 , $p=0.001)$, FAC (3.1 vs. $3.8, p<0.001)$, BBS (35.8 vs. 43.9, $p<0.001)$, K-MBI (72.9 vs. $80.6, p<0.001)$ measured at 1 and 3 months after stroke showed statistically significant improvements after rehabilitation. BaPWV, FMA-UE, FMA-LE, K-MMSE, 6MWT, BBS and K-MBI at 1 month after stroke correlated significantly with K-MBI at 3 months after stroke. BaPWV, FMA-UE and K-MBI at 1 month after stroke were significant independent predictors of follow-up K-MBI.

Discussion and Conclusions: These results indicated that arterial stiffness as well as functional outcome showed significant improvements after rehabilitation during the subacute phase of stroke, and the measurement of baPWV at 1 month after stroke might be useful in predicting short-term functional recovery in patients with subacute stroke.

\section{P120}

\section{EFFECTS OF AN EARLY ECCENTRICALLY} BASED REHABILITATION AFTER TOTAL KNEE ARTH- ROPLASTY

Y. K. Lee ${ }^{1}$, B. R. Kim ${ }^{1}$

${ }^{1}$ Jeju National University Hospital, Jeju, Republic of Korea

Objective: This study was undertaken to investigate the effects of early combined eccentric-concentric vs. concentric resistance training on functional performance after total knee arthroplasty

Methods: Thirty-four patients who underwent a primary total knee arthroplasty (TKA) were randomly assigned to 1 of 2 groups: a ECC-CON group that received combined eccentricconcentric exercise $(n=16)$ and a CON group that received concentric exercise $(n=18)$. All randomized patients received early progressive resistance training ( 1 of 2 interventions) with 5 sessions per week for 2 weeks, starting at 2 weeks after TKA. To evaluate self-reported physical function, selfreported disease-specific physical function measured by using the Western Ontario McMaster Universities Osteoarthritis Index (WOMAC) and self-reported quality of life measured by using EuroQOL five dimensions (EQ-5D) questionnaire were used. To evaluate physical performance, the following assessment tools were measured: 6-min walk test (6MWT), timed up and go (TUG) test, timed stair climbing test (SCT), instrumental gait analysis for spatio- temporal parameters, isometric knee flexor and extensor strength of the surgical and nonsurgical knees. All patients underwent these evaluations before surgery and 1 month after surgery (after intervention). Results: Both groups significant improvements in WOMACpain, WOMAC-function, and EQ-5D scores, and the ECCCON group showed statistically significant and clinically meaningful improvements in 6MWT, gait speed, peak torque (PT) extensor of nonsurgical knee while the CON group showed significant improvements in only single support duration. In addition, the $\mathrm{CON}$ group showed a significant increase in the SCT-ascent and H/Q ratio of surgical knee. Although PT extensors of surgical knee in both groups did not reach the preoperative level, the change scores of PT extensor of surgical knee in the ECC-CON group were significant less than those in the $\mathrm{CON}$ group.

Conclusions: This study demonstrated that early combined eccentric-concentric resistance training was effective for not only improving endurance and gait speed but also minimizing a loss of quadriceps strength of surgical knee after TKA.

\section{P121}

PREOPERATIVE PHYSICAL FUNCTION INFLUENCES ON FUNCTIONAL STATUS 1 MONTH AFTER TOTAL KNEE ARTHROPLASTY

Y. K. Lee ${ }^{1}$, B. R. Kim ${ }^{1}$

${ }^{1}$ Jeju National University Hospital, Jeju, Republic of Korea

Objective: This study was undertaken to identify preoperative physical performance factors predictive of self-reported physical function and quality of life for people 1 month following total knee arthroplasty.

Methods: In this prospective cohort study, we assessed a total of 60 patients ( 7 males and 53 females; average age $71.9 \pm 6.1$ years) who underwent a primary total knee arthroplasty (TKA). Before and 1 month after TKA, patients completed self-reported diseasespecific physical function measured by using the Western Ontario McMaster Universities Osteoarthritis Index (WOMAC) and self-reported quality of life measured by using EuroQOL five dimensions (EQ-5D) questionnaire. Physical performance tests included 6-min walk test (6MWT), timed up and go (TUG) test, timed Stair Climbing Test (SCT), instrumental gait 
analysis for spatio-temporal parameters, and isometric knee flexor and extensor strength of the surgical and nonsurgical knees.

Results: In the bivariate analyses, the postoperative WOMAC function score had a significant positive correlation with the preoperative WOMAC function score $(r=0.28, p=0.04)$, WOMAC pain score $(r=0.30, p=0.03)$, WOMAC stiffness score $(r=0.34, p=0.02)$, SCT-ascent $(r=0.39, p=0.01)$, SCTdescent $(r=0.38, p=0.01)$, and a significant negative correlation with preoperative peak torque $(\mathrm{PT})$ extensor $(r=-0.32, p=0.02)$ and PT flexor $(r=-0.32, p=0.02)$ of the surgical knee, PT flexor $(r=-0.42, p=0.01)$ of the nonsurgical knee. The postoperative EQ-5D score had a significant positive correlation with the preoperative EQ-5D score $(r=0.40, p=0.01)$, gait speed $(r=0.31$, $p=0.03)$, and a significant negative correlation with the preoperative SCT-ascent $(r=-0.34, p=0.03)$, SCT-descent $(r=-0.37$, $p=0.01)$. In the linear regression analyses, the preoperative SCT-ascent $(\beta=0.39, p=0.01)$ was a factor predictive of the postoperative WOMAC function score, and the preoperative EQ-5D score $(\beta=0.36, p=0.04)$ and gait speed $(\beta=0.34$, $p=0.04$ ) were factors predictive of the postoperative EQ-5D score.

Conclusions: This study demonstrated that preoperative stair climbing ability and gait speed significantly influenced on postoperative self-reported physical function and quality of life 1 month after TKA. Using variables easily measured before surgery, it may be possible to predict with good accuracy for postoperative functional ability and quality of life. In addition, these results could be of importance in determining variable preoperative rehabilitation strategies, especially focusing on resistance exercise.

\section{P122}

\section{INTENSIVE FAST-TRACK REHABILITATION} FOCUSING ON PROGRESSIVE RESISTANCE T RAINING TO ENHANCE FUNCTIONAL PERFORMANCE EARLY AFTER TOTAL KNEE ARTHROPLASTY

\section{Y. K. Lee ${ }^{1}$, B. R. Kim ${ }^{1}$}

${ }^{1}$ Jeju National University Hospital, Jeju, Republic of Korea

Objective: To demonstrate the effectiveness of intensive fasttrack rehabilitation program to enhance early functional recovery after total knee arthroplasty.

Methods: A total of 60 patients ( 7 males and 53 females; average age $71.9 \pm 6.1$ years) who underwent a primary total knee arthroplasty (TKA) were recruited in this study. All participants received progressive resistive training using incline and leg press, leg extension and curl, hip abduction and adduction with an intensity of $30-60 \%$ of $1 \mathrm{RM}$ for $30 \mathrm{~min}$ with 5 sessions per week for 3 weeks, starting within the first week after TKA. All outcome measures were collected before surgery and at 1 month after surgery.
Primary outcomes included 6-min walk test (6MWT), timed up and go (TUG) test, timed stair climbing test (SCT), instrumental gait analysis for spatio-temporal parameters, isometric knee flexor and extensor strength of the surgical and nonsurgical knees, and range of motion (ROM) of surgical knees. Secondary outcome measures included self-reported diseasespecific physical function measured by using the Western Ontario McMaster Universities Osteoarthritis Index (WOMAC) and self-reported quality of life measured by using EuroQOL five dimensions (EQ-5D) questionnaire.

Results: In the primary outcome, there were statistically significant and clinically meaningful improvements in change scores from before surgery to 1 month after TKA for 6MWT, TUG, instrumental gait analysis for spatio-temporal parameters except single support duration, peak torque (PT) extensor of nonsurgical knee, extension ROM of surgical knee. There were no statistically significant differences in the change scores from before surgery to 1 month after TKA for SCT-descent, single support duration, hamstring/quadriceps $(\mathrm{H} / \mathrm{Q})$ ratio of non-surgical knee, peak torque (PT) flexor of surgical and non- surgical knees, deficit of flexor. Although PT extensor of surgical knee did not reach the preoperative level, the change scores were much smaller compared with previous results in other studies. In the secondary outcome, WOMAC-pain, WOMAC-function, and EQ-5D scores after 1 month after TKA were significant higher compared with those before surgery.

Conclusions: This study demonstrated that early, intensive fast-track rehabilitation program focusing on progressive resistance training was effective for not only improving endurance, balance, gait function, quadriceps strength of nonsurgical knee but also minimizing a loss of quadriceps strength of surgical knee after TKA.

\section{P123 \\ FINITE-ELEMENT ANALYSIS OF THE EFFECT OF BASIC HIP MOVEMENTS ON THE \\ M. P. Diez Garcia ${ }^{1}$, V. M. Dominguez Hernandez ${ }^{1}$, V. M. Araujo Monsalvo $^{2}$, J. F. Tovar Lopez ${ }^{2}$ \\ ${ }^{1}$ Instituto Nacional de Rehabilitacion, Mexico, Mexico, ${ }^{2}$ Biomecanica Instituto Nacional de Rehabilitacion, Mexico, Mexico}

Osteoporosis is a serious and multifactorial disease. The number of people affected with osteoporosis is increasing due to the lengthening of life expectancy. Currently, unlike the genetic, nutritional and hormonal factors that have been the focus of most studies of osteoporosis, mechanical stimuli that potentially can produce an increase in bone strength have not been well studied. Studies suggest that the relationship between the health of the bone and mechanical stimuli occurs through bone adaptive remodeling, which is activated by means of the shear stress transmitted by the interstitial fluid 
flow. The present work consists of a finite element analysis of a femur to simulate the basic movements of the hip (flexion, extension, abduction, and adduction) to compare the shear stresses in a common zone of fracture and in the critical mechanical strength zones of the femoral head. A comparison of the distribution and magnitude of the shear stresses was performed to estimate the movement that could induce a more rapid adaptive bone remodeling. This study is the first step in the development of a physical therapy for a preventive rehabilitation that helps to prevent patients with low BMD to avoid suffering osteoporosis hip fractures. The finite element model was constructed using a free-access three-dimensional standardized femur obtained from the Instituti Ortopedici Rizzoli, Bologna, Italy. The mechanical properties and the muscular forces were obtained from a specialized bibliography. We conclude that the movements that exhibit a higher mean value and a good shear stress distribution in the femoral neck are hip extension and abduction.

\section{P124}

THE EFFECT OF ALFACALCIDOL AND PLAIN VITAMIN D ON OSTEOPOROSIS: A RANDOMIZED CONTROLLED TRIAL

B. P. Shrestha ${ }^{1}$, J. Kathabania ${ }^{1}$

${ }^{1}$ Orthopaedics, B.P. Koirala Institute of Health Sciences, Dharan, Nepal

Objectives: Osteoporosis is a common problem in old age group population leading to fragility fractures. There are multiple drug regimens used for treatment of osteoporosis with a wide range of price variation. This study was conducted to compare the treatment of osteoporosis with either alfacalcidol ( $1 \alpha$-OHD3) or plain vitamin $\mathrm{D}$ (cholecalciferol) combined with alendronate and calcium in terms of efficacy, safety and cost effectiveness.

Materials and Methods: We conducted a randomized controlled trial enrolling 80 cases and randomized into two groups of 40 each, one in which Alphacalcidol was used in combination with Alendronate and Calcium and the second in which plain vitamin D was used in similar combination. Student's test with $95 \%$ confidence interval was used to evaluate the difference between the two treatment groups. Categorical variables were analyzed using chi-square test. A p value of 0.05 was considered significant for all statistical tests.

Results: In the alphacalcidol group the mean percentage change in BMD was $9.3 \%$ and in the plain vitamin D group it was $6.9 \%$, the mean being $8.1 \%$. The mean percentage change in T-score was $15.8 \%$. In the Alphacalcidol group it was $16.9 \%$ and in the other group it was $14.7 \%$.

Conclusion: There is no statistical difference in the results of two treatment groups, though there is increment in BMD and T-score both the groups. The efficacy and safety were similar in both the groups though the treatment cost is much higher in the alphacalcidol group.

\section{P125}

INTRA-ARTICULAR USE OF GO-ON®, A STERILE NONPYROGENIC SOLUTION OF SODIUM HYALURONATE AND TABLETS DONA® (GLUCOSAMINE SULFATE) IN THE TREATMENT OF PATIENTS WITH KNEE OSTEOARTHRITIS

$\underline{Z}_{\text {. Golubovic }}{ }^{1}$, P. Stojiljkovic ${ }^{1}$, Z. Radovanovic ${ }^{1}$, I. Golubovic $^{1}$, I. Micic ${ }^{1}$, S. Stojanovic ${ }^{1}$

${ }^{1}$ Clinic for orthopaedic and traumatology Clinical Center, Nis, Serbia

Degenerative joint diseases were the most common diseases of the locomotor system. The knee osteoarthritis is a degenerative disease of the knee joint, which is accompanied by pain and limited mobility. The knee osteoarthritis is three times more common in women than in men. In the treatment of knee osteoarthritis in addition to antirheumatic drugs and physical therapy is applied and $\mathrm{GO}-\mathrm{ON}^{\circledR}$, sterile nonpyrogenic sodium hyaluronate (intra-articular use). We analyzed 72 patients with knee osteoarthritis who were treated at the Clinic for Orthopedics and Traumatology in Clinical Center Nis Serbia in the period from 1.01.2014 to 1.06 .2015 with intra-articular administration of a syringe of GO-ON ${ }^{\circledR}$ (contains $2.5 \mathrm{ml}$ solution) in the knee joint. GO-ON ${ }^{\circledR}$ was injected into the knee joint a total of 3 times, at weekly. After that, patients were prescribed tablets Dona ${ }^{\circledR}$ (glucosamine sulfate) and $1500 \mathrm{mg}$ per day, for a period of 3 to 6 mounts. In patients accompanied pain and range of motion before and after intraarticular injection of GO ON ${ }^{\circledR}$. In 49 $(68.1 \%)$ of the respondents registered a very good result, in 18 $(25 \%)$ and good in $5(6.94 \%)$ patients, there was a partial improvement. Analyzing the results we found that the GO$\mathrm{ON}^{\circledR}$ in combination with tablets Dona ${ }^{\circledR}$ showed good effect in the treatment of patients with knee osteoarthritis.

\section{P126}

HEPCIDIN MODULATES RUNX2A GENE EXPRESSION TO REGULATE BIOMINERALIZATION OF BONE IN ZEBRAFISH

\section{Y. Jiang $^{1}$}

${ }^{1}$ The Second Affiliated Hospital of Soochow University, Suzhou, China

Iron overload as a risk factor for osteoporosis can result in upregulation of Hepcidin, and Hepcidin knockout mice display bone microarchitecture defects. Molecular and genetic mechanisms underlying Hepcidin deficiency-derived bone loss, however, are still unclear. Here, we showed that knockdown of 
zebrafish hepcidin using Morpholino leads to iron overload. Then, we used CRISPR-Cas9, a versatile genome-editing tool, to generate a zebrafish hepcidin mutant. Iron overload and mineralization loss in early intramembranous bones were observed in hepcidin-/- larvae zebrafish, which can be partially restored with microinjection of hepcidin mRNAs. Quantitative real-time PCR analyses showed down-regulation of osteoblast-specific genes runx2a, runx $2 \mathrm{~b}$, alp, and $\mathrm{sp} 7$ in homozygous hepcidin mutant zebrafish. Luciferase reporter assays showed that Bmp2a enhances runx $2 a$ expression but iron overload represses its expression through bmp2a independent of hjv. Highthroughput transcriptome analysis of hepcidin-/- larvae zebrafish revealed multiple pathways in osteoblast metabolism. Together, these findings showed that iron overload derived from Hepcidin deficiency represses bone formation possibly through the Bmp pathway and affecting runx2 in zebrafish. Iron overload as a risk factor for osteoporosis can result in up-regulation of Hepcidin, and Hepcidin knockout mice display bone microarchitecture defects. Molecular and genetic mechanisms underlying Hepcidin deficiency-derived bone loss, however, are still unclear. Here, we showed that knockdown of zebrafish hepcidin using Morpholino leads to iron overload. Then, we used CRISPR-Cas9, a versatile genome-editing tool, to generate a zebrafish hepcidin mutant. Iron overload and mineralization loss in early intramembranous bones were observed in hepcidin-/- larvae zebrafish, which can be partially restored with microinjection of hepcidin mRNAs. Quantitative realtime PCR analyses showed down-regulation of osteoblastspecific genes runx $2 \mathrm{a}$, runx $2 \mathrm{~b}$, alp, and sp7 in homozygous hepcidin mutant zebrafish. Luciferase reporter assays showed that Bmp2a enhances runx $2 \mathrm{a}$ expression but iron overload represses its expression through bmp2a independent of hjv. Highthroughput transcriptome analysis of hepcidin-/- larvae zebrafish revealed multiple pathways in osteoblast metabolism. Together, these findings showed that iron overload derived from Hepcidin deficiency represses bone formation possibly through the Bmp pathway and affecting runx2 in zebrafish.

\section{P127}

THE EFFECT OF A SACROILIAC BELT ON LUMBOPELVIC MUSCLE ACTIVATION IN DIFFERENT LOADING CONDITION IN PATIENT WITH UNILATERAL SACROILIAC JOINT INSTABILITY

\section{S. Karimi ${ }^{1}$}

${ }^{1}$ Physical Therapy/University of Social Welfare and Rehabilitation Sciences, Tehran, Islamic Republic of Iran

Objective: The sacroiliac joint (SIJ) are one of the potential source of low back pain (LBP), and the prevalence of sacroiliac joint pain (SIJP) is reported to be $13-30 \%$ in patients with nonspecific LBP. The main function of the
SIJs has been often described to transfer the load of the upper body weight to the legs, and transmit ground reaction force from the lower limbs to the trunk. The form closure and force closure mechanisms provide stability of the SIJs. One-leg stance is a necessary sequence for dynamic transitions of body weight loading during walking, and it is often used to assess the capability of the SIJ to maintain lumbopelvic stability during the transmission of load between the lower extremities and the spine. It is often recommended that patients suffering from SIJP wear the SIJ belt while walking and standing, and many clinicians have incorporated the use of the SIJ belt into their routine therapy. The purpose of this study was to compare the effects of the SIJ belt on lumbopelvic muscle activation patterns during different loading condition in subjects with unilateral sacroiliac joint instability.

Methods: Thirteen patients (young women) with unilateral SIJ instability volunteered to participant in this study. Inclusion criteria were positive ASLR and in three of the five provocation tests, exclusion criteria were past or present orthopedic surgeries, severe deformity in spine or lower extremity, history of fracture in trunk and lower limbs and neurological disease. The tasks were done in six different conditions (standing on two legs with same and counter same loading - standing on involved side with same and counter same loading-standing on uninvolved side with same and counter same loading). The six conditions were done with and without belt; loading was equivalent with $\% 10$ of body weight. Loading was in line with the involved and uninvolved side. Surface electromyography (EMG) data (signal amplitude) were collected from latissimus dorsi, gluteus medius, transvers abdominis /oblique internus and biceps femoris in both sides of the body during different loading condition with and without belt.

Results: We found significantly decreased LD [in two conditions: one leg standing on involved side with counter same loading $(P v=0.032)$ - one leg standing on uninvolved side with counter same loading $(P v=0.009)]$, TrA/ IO [in three conditions: one leg standing on involved side with counter same loading $(P v=0.021)$ - one leg standing on uninvolved side with same loading $(P v=0.034)$ - double standing with same loading $(P v=0.036)]$ and $\mathrm{BF}$ [in two conditions: one leg standing on involved side with same loading $(P v=0.011)$ - one leg standing on involved side with counter same loading $(P v=0.006)]$ activities and significantly increased GM [in one condition: one leg standing on involved side with same loading $(P v=0.044)]$ activity during different loading condition in double standing and one leg standing when the SIJ belt was applied.

Conclusion: We investigated the influence of the SIJ belt on lumbopelvic girdle muscles activation patterns during one-leg 
and double standing in subjects with unilateral SIJ instability. Wearing Sacroiliac joint belt leads to increased force closure in lumbopelvic girdle. Stability of the lumbopelvic girdle increase with SIJ belt.

\section{P128}

\section{MONTHS RESULTS OF A MINIMALLY INVASIVE SURGICAL METHOD TO STIFFEN THE SACROILIAC JOINT}

$\underline{\text { R. Bornemann }}^{1}$, T. R. Jansen ${ }^{1}$, Y. Rommelspacher ${ }^{1}$, D. C. Wirtz $^{1}$, R. Pflugmacher ${ }^{1}$

${ }^{1}$ Universitätsklinikum Bonn, Bonn, Germany

Background: Low back pain is often caused by sacroiliac joint degeneration. Most of the affected patients can be treated successfully by conservative treatments, but if conservative treatments fail to improve the symptoms surgical treatments for stabilization are required. The newly developed iFuse implant system (SI-Bone Inc., San Jose, $\mathrm{CA}$ ) is a minimally invasive treatment method for arthrodesis of the sacroiliac joint which enables an individual stabilization by three triangular, titanium plasma spray coated implants.

Purpose: This prospective study evaluates the clinical outcome of minimally invasive arthrodesis with the iFuse system in patients suffering from chronic pain caused by sacroiliac joint degeneration.

Methods: 19 patients (age: $55 \pm 14$ y; gender: 17 female, 2 male) suffering from single-sided sacroiliac joint degeneration were treated with the iFuse implant system. Clinical data were assessed preoperative, 1 month postoperative, 3 months postoperative, 6 months postoperative, and 12 months after surgery. At each visit $\mathrm{x}$-rays were taken to determine possible implant loosening. Furthermore, the patients stated their back pain with the visual analogue scale (VAS), and the functional impairments was assessed with the Oswestry-DisabilityQuestionnaire (ODI). All occurred surgery and device related adverse events were documented.

Results: The mean VAS values were significantly improved from $86 \pm 9 \mathrm{~mm}$ to $41 \pm 9 \mathrm{~mm}$ after surgery $(p \leq 0.0001)$ and ongoing to $27 \pm 5$ at the 12 months evaluation $(p \leq 0.0001)$. The mean ODI was also significantly reduced from 58.1 $\pm 10.5-26 \pm 14.4$ after surgery $(p \leq 0.0001)$ and improved further non-significantly to $33 \pm 6$ at the 12 months examination. None of the patients showed radiographic evidence for implant loosening and neither surgery nor device related adverse events occurred during the study period.

Conclusion: The iFuse system is a safe and effective treatment method for patients suffering from sacroiliac joint degeneration who need to be surgically treated. It leads to significant pain reduction and improved functional impairment.

\section{P129}

EXAMINATION OF VERTEBRAL AND NONVERTEBRAL FRACTURES ACCORDING TO BONE MINERAL DENSITY

${ }_{\text {N. Igic }}{ }^{1}$, J. Zvekic-Svorcan ${ }^{2}$, A. Mikov ${ }^{3}$, K. Filipovic ${ }^{2}$, S. Tomasevic-Todorovic $^{4}$

${ }^{1}$ Medical rehabilitation, Faculty of Medicine, University of Novi Sad, Novi Sad, Serbia, ${ }^{2}$ Rheumatology, Special Hospital for Rheumatic Diseases, Faculty of Medicine, University of Novi Sad, Novi Sad, Serbia, ${ }^{3}$ Institute for Child and Youth Health Care of Vojvodina, Faculty of Medicine, University of Novi Sad, Novi Sad, Serbia, ${ }^{4}$ Rheumatology, Clinic for Medical Rehabilitation, Clinical Center of Vojvodina, Faculty of Medicine, University of Novi Sad, Novi Sad, Serbia

Objective: To examine vertebral and non vertebral fractures according to the BMD and according to the places where osteodensitometric findings occurred.

Material and methods: Retrospective analysis and osteodensitometric findings of 122 postmenopausal women was held from November 2013 to April 2014 in the Special Hospital for Rheumatic Diseases in Novi Sad, Serbia. The occurrence of fractures caused by small trauma and the BMD compared to the fractures was analyzed from medical records. Excluding factors from the examinations were premenopausal women, BMD in the reference values, other metabolic bones diseases. Statistical and data analysis were done in SPSS program ver 20.

Results: The average age of the examined women was $63.85 \pm 8.42$ years and $61 \%$ of them had osteoporosis and $39 \%$ had osteopenia. From the total number of examined women $35.2 \%$ had small trauma fractures, out of which $10.7 \%$ were vertebral and $27 \%$ nonvertebral fractures. Using the $t$-test for large independent samples, it was established that among the women with osteopenia and osteoporosis, there was no statistically relevant difference in the total number of fractures $(p=0.604)$, number of vertebral $(p=0.643)$ and nonvertebral fractures $(p=0.162)$. The T-score of the hip is negatively connected to the number of nonvertebral fractures $(p=0.019)$, and statistically relevant difference was not found compared to the vertebral $(\mathrm{p}=0.596)$ and total number of fractures $(p=0.295)$. For the T-score of spine, there is no statistically relevant difference in the comparison of the total number of fractures $(p=0.499)$, number of vertebral $(p=0.766)$ and nonvertebral $(p=0.467)$ fractures.

Conclusion: Every decrease in BMD is a risk factor for occurrence of low energy fractures. Low BMD values on the hip directly show the possibility of fractures, while low BMD values on the spine does not show, probably because of the false positive results, and these patients should also do a lateral scan of the spine. 


\section{P130}

EFFECTS OF TREATMENT WITH TERIPARATIDE AND QUALITY OF LIFE IN A GROUP OF ELDERLY PEOPLE SUFFERING FROM SEVERE OSTEOPOROSIS

K. Ampatzidis $^{1}$, G. Primavera ${ }^{1}$, D. Ampatzidi ${ }^{2}$, F. Palermo ${ }^{3}$, F. Finocchiaro $^{3}$, R. Sorace ${ }^{1}$, D. Maugeri ${ }^{1}$

${ }^{1}$ Geriatrics Section - University of Catania - A.O. Cannizzaro, Catania, Italy, ${ }^{2}$ Society of Psychosocial Research and Intervention (SPRI - EPSEP in Greek), Ioannina, Greece, ${ }^{3}$ University of Catania, Catania, Italy

Today in Italy live about 5 million people affected by osteoporosis. Observing the increase in life expectancy that has characterized the last decades, we notice the tendency to a higher incidence of this disease. In light of the above, our study was aimed to focus on teriparatide. It assesses the effects of the drug on a group of elderly patients affected by severe osteoporosis, in particular the arrest of bone loss as well as the reduction of new fractures. In addition, the study aims to highlight the influence that the aforementioned therapy can determine the quality of life of these patients. We conducted a study over 24 months on a sample of elderly patients affected by severe osteoporosis, we enrolled 81 subjects, including 73 females (F) and 8 males (M), mean age $69.73 \mathrm{y} \pm 11.05$ SD. The subjects enrolled in the study were examined with DXA of L1-L4 and femur, morphometric analysis and calculation of the S.D.I. on the base of latero-lateral X-ray imaging of the thoracolumbar spine (T4-L4) and questionnaires to assess the presence of peripheral fractures and survey on quality of life QUALEFFO-41, all this at the beginning of treatment $(\mathrm{t} 0)$ and at the end of the 24 months of therapy (t24). In order to compare the results recorded before and after treatment we used the Paired $t$-test and the Wilcoxon matched-pairs signed rank test, after making the test of normality Kolmogorov-Smirnov. After 24 months of treatment with teriparatide, patients showed a significant recovery of BMD at the level of spine and femur in terms of t- score (L1-L4 $p<0.0001$; femoral neck $p<0.0001$; total femur $p=0.024)$, z-score (L1-L4 $p<0.0001$; femoral neck $p<0.0001$; total femur $p<0.0001$ ) and BMD (L1-L4 $p=0.0304$; femoral neck $p<0.0001$; total femur $p=0.0575$ ) with a significant reduction in the incidence of both vertebral and peripheral fractures. It was also shown that this treatment is able to obtain an improvement on the subject of the quality of life in toto $(p<0.0001)$. The findings from our study allow us to confirm the clinical efficacy of the therapy with teriparatide in the treatment of severe osteoporosis in elderly patients. The therapy results effective in protecting against new vertebral and peripheral fractures; it increases both spine and femur bone density, in terms of t-score, $\mathrm{z}$ score and BMD. These advantages appear to be of great importance especially when considering the studied subjects target - elderly and very often suffering from polypathology and at high risk of complications, such as falls and related fractures, which may reduce their life expectancy. Also it should be noted that the positive effects that the teriparatide determines are included pain reduction and improvement of ability to walk and to perform normal activities of daily living. All this argues for greater participation in social life and a smoother socialization that help to preserve the elderly from mood disorders, which are also potentially disabling.

\section{P131}

LOWER BONE TURNOVER AND RELATIVE CORTICAL BONE DEFICITS IN MEN WITH METABOLIC SYNDROME: A MATTER OF INSULIN RESISTANCE? THE EUROPEAN MALE AGEING STUDY

M. R. Laurent $^{1}$, M. J. Cook ${ }^{2}$, E. Gielen ${ }^{1}$, K. A. Ward ${ }^{3}$, J. E. Adams $^{4}$, L. Antonio ${ }^{5}$, B. Decallonne ${ }^{5}$, G. Bartfai ${ }^{6}$, F. F. Casanueva $^{7}$, G. Forti $^{8}$, A. Giwercman ${ }^{9}$, I. T. Huhtaniemi ${ }^{10}$, K. Kula ${ }^{11}$, M. E. J. Lean ${ }^{12}$, D. M. Lee ${ }^{2}$, N. Pendleton ${ }^{13}$, M. Punab $^{14}$, F. Claessens ${ }^{15}$, F. C. W. Wu ${ }^{16}$, D. Vanderschueren ${ }^{5}$, S. R. Pye ${ }^{2}$, T. W. O’Neill ${ }^{2}$

${ }^{1}$ Gerontology and Geriatrics, KU Leuven, Leuven, Belgium, ${ }^{2}$ Arthritis Research United Kingdom Epidemiology Unit, University of Manchester, Manchester, United Kingdom, ${ }^{3}$ Elsie Widdowson Laboratory, Medical Research Council Human Nutrition Research, Cambridge, United Kingdom, ${ }^{4}$ University of Manchester, and the Royal Infirmary, Manchester, United Kingdom, ${ }^{5}$ Clinical and Experimental Endocrinology, KU Leuven, Leuven, Belgium, ${ }^{6}$ Department of Obstetrics, Gynecology and Andrology, Albert SzentGyörgy Medical University, Szeged, Hungary, ${ }^{7}$ Department of Medicine, Santiago de Compostela University, Santiago de Compostela, Spain, ${ }^{8}$ Andrology Unit, Department of Clinical Physiopathology, University of Florence, Florence, Italy, ${ }^{9}$ Department of Urology, Scanian Andrology Centre, Malmö University Hospital, University of Lund, Lund, Sweden, ${ }^{10}$ Department of Reproductive Biology, Imperial College London, London, United Kingdom, ${ }^{11}$ Department of Andrology and Reproductive Endocrinology, Medical University of Lodz, Lodz, Poland, ${ }^{12}$ Department of Human Nutrition, University of Glasgow, Glasgow, United Kingdom, ${ }^{13}$ School of Community Based Medicine, University of Manchester, Manchester, United Kingdom, ${ }^{14}$ Andrology Unit, United Laboratories of Tartu University Clinics, Tarty, Estonia, ${ }^{15}$ Molecular Endocrinology Laboratory, KU Leuven, Leuven, Belgium, ${ }^{16}$ Andrology Research Unit, Manchester Academic Health Science Centre, Manchester Royal Infirmary, University of Manchester, Manchester, United Kingdom

Introduction: Metabolic syndrome (MetS) features relative skeletal deficits i.e. lower bone mass and strength than 
expected for BMI. Whether these relative deficits are related to different MetS components remains unclear.

Participants and methods: We examined cross-sectional associations of MetS, its components and insulin sensitivity (HOMA-S) with bone turnover markers (BTMs) and heel broadband ultrasound attenuation (BUA) in 3129 men aged 40-79 from the multicenter European Male Ageing Study. Two centers additionally measured hip and spine BMD $(n=527)$ and performed radius peripheral quantitative computed tomography (pQCT, $n=595$ ). Associations were examined using linear regression and mediation analysis, adjusted for age, center, smoking, alcohol consumption and BMI.

Results: MetS was present in 975 men (31.2\%). Men with MetS had lower $\beta$-cTX, P1NP and osteocalcin $(P<0.0001)$ and higher hip and spine BMD $(P \leq 0.03)$. Among MetS components, only hypertriglyceridemia and hyperglycemia (components related to insulin sensitivity) were independently associated with P1NP and $\beta$-cTX. Waist circumference was positively associated with heel BUA, hip and spine BMD and radius cross-sectional bone area (CSA), but not independently of BMI. Adjustment for BMI revealed inverse associations of hyperglycemia with BUA, and of hypertriglyceridemia with hip BMD and radius cross-sectional bone area and stress-strain index (a surrogate of bone strength). Impaired insulin sensitivity was similarly associated with lower BTMs and lower heel BUA and radius CSA. In pathway analysis, BMI had both significant beneficial direct effects on $\beta$-cTX, BUA and cortical bone area, as well as negative effects mediated via HOMA-S.

Conclusions: In men with MetS, greater bone mass is dominantly associated with BMI, while lower bone turnover and impaired cortical bone expansion are mainly related to measures of insulin resistance. We hypothesize that insulin resistance may be involved in the failure of cortical bone to adapt to increasing bodily loads in obese middle-aged and elderly men.

\section{P132}

\section{THE INFLUENCE OF VITAMIN D RECEPTOR GENE POLYMORPHISM ON BONE MINERAL DENSITY IN HEMODIALYSIS PATIENTS}

\section{S. O. Mazurenko ${ }^{1}$, A. A. Enkin ${ }^{2}$, M. V. Aseev ${ }^{3}$}

${ }^{1}$ Saint Petersburg State University, Saint Petersburg, Russian Federation, ${ }^{2}$ Dialysis Center of Leningrad Regional Hospital, Saint Petersburg, Russian Federation, ${ }^{3}$ D.O. Ott Research Institute of Obstetrics and Gynecology, Saint Petersburg, Russian Federation

Objective: To examine whether the vitamin D gene TaqI polymorphism is related to the low BMD and the risk of secondary osteoporosis in patients on maintenance hemodialysis.
Materials and methods: To test the relationship between VDR genotype and BMD, parameters of bone metabolism and incidence of osteoporosis, 88 patients ( 43 men and 45 women) aged from 24 to 67 years old (mean $45.5 \pm 12.4$ ), treated on hemodialysis for $6.5 \pm 3.7$ years, have been studied. For molecular-genetic study of VDR gene polymorphism PCR-RFLP analysis was performed. Genotypes TT, Tt and tt have been considered. BMD was measured at the lumbar spine, femoral neck and arm by DXA.

Results: The genotype TT was revealed in $47.7 \%$ patients, генотипы $\mathrm{Tt}$ и $\mathrm{tt}$ соответственно у 29,3 и $23 \%$. There were no remarkable differences in genotype groups in laboratory results, including intact PTH, ionized calcium, phosphates and alkaline phosphatase. BMD parameters were lowest in patients with genotype TT in every studied part of the skeleton, however the difference was statistically significant only in proximal part of the femur assessed by $\mathrm{Z}$ criterion $(p=0.02)$. We did not find statistical difference in BMD in patients with genotypes $\mathrm{Tt}$ and $\mathrm{tt}$. Conclusion: This study suggested the hypothesis that the vitamin $\mathrm{D}$ receptor gene polymorphism could be genetic determinant of bone metabolism in patients with chronic kidney disease on maintenance hemodialysis.

\section{P133}

\section{EFFECT OF MELATONIN ON SOME BONE TURNOVER MARKERS IN VASECTOMIZED} RATS

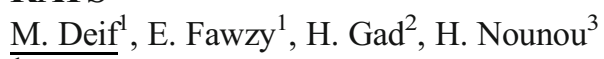

${ }^{1}$ Clinical Physiology - Faculty of Medicine, Alexandria, Egypt, ${ }^{2}$ Biochemistry, Alexandria, Egypt, ${ }^{3}$ College of Science, Al Riyadh, Saudi Arabia

Objective: To evaluate the effect and mechanism of action of melatonin on some bone markers in vasectomized bone loss in rats.

Material and methods: 32 female albino rats underwent either bilateral laparotomy (sham $n=8$ ), or bilateral vasectomy(Ovx, $n=24)$. The ovx rats were divided into 3 groups, each of 8 rats; vehicle treated, estrogen treated(E2) and melatonin treated (Mlt). After 14 weeks treatment, blood and urine were collected. Serum osteoprotegerin (OPG), inhibin, follistatin, and alkaline phosphatase (ALPase) were determined as bone markers. Also, urinary deoxypyridinoline ( $\mathrm{UDPD}$ ) was assayed.

Results: Serum OPG, and follistatin levels significantly decreased upon Ovx. They increased upon either treatment with E2 or Mlt. In addition, serum ALPase and uDPD significantly increased with Ovx and decreased with either therapy with non-significant difference between both.

Conclusion: Administration of Mlt inhibited high bone turnover and prevented calcium loss in Ovx rats. This may be through increasing OPG, inhibin and/or follistatin levels. Mlt could be a candidate for the treatment of postmenopausal osteoporosis. 


\section{P134}

\section{HYPERMOBILITY AND PROPRIOCEPTION IN THE FINGER JOINTS OF FLAUTISTS}

\section{Artigues-Cano ${ }^{1}$}

${ }^{1}$ Institute of Arts Barcelona, Trinity Laban Conservatoire of Music \& Dance, London, United Kingdom

Background: Ergonomically the flute is especially complex amongst wind instruments and flautists may therefore be at particular risk of performance-related musculoskeletal disorders. Yet little is known about injury prevalence amongst flute players, and even less in those flautists who are also hypermobile. Recent research has found hand and wrist pain to be common complaints amongst flautists. Understanding of the predictors of injury and pain is therefore crucial as the presence of pain decreases performance quality and causes unnecessary time loss. There is a strong relationship between hypermobility and impaired proprioception though many musicians may acquire greater proprioception than the average population. We have compared flexibility and proprioception of the hand in a controlled study of flautists.

Methods: Twenty flautists took part in the study. General hypermobility, the passive range of motion of the three specific joints most involved in flute-playing and proprioception acuity were all measured accurately for the first time in this awkward instrument that needs high levels of dexterity.

Results: Flautists' finger joints have a greater range of movement than in the general population. This group of flute players had especially large ranges of movement in the finger joints which take the weight of the instrument. Although flautists have hypermobile finger joints, they are not generally hypermobile elsewhere as measured by the Beighton Scale. Flautists, even with very mobile finger joints, have very accurate proprioception which may be acquired through training.

Conclusions: The study of instrumentalists may provide an ideal model for study of the interaction between localized joint flexibility and joint proprioception, both inherited and acquired.

\section{P135}

ADIPONECTIN AS A USEFUL MARKER FOR DIAGNOSIS OF OSTEOPOROSIS IN RHEUMATOID ARTHRITIS PATIENTS

L. E. Sivordova ${ }^{1}$, B. Zavodovsky ${ }^{2}$, Y. Polyakova ${ }^{2}$, Y. Akhverdyan $^{2}$, M. I. Kuznetsova ${ }^{1}$, A. B. Zborovsky ${ }^{1}$

${ }^{1}$ Federal State Budgetary Institution «Research Institute of Clinical and Experimental Rheumatology», Xi'an, China, ${ }^{2}$ Federal State Budgetary Institution «Research Institute of Clinical and Experimental Rheumatology», Volgograd, Russian Federation
Objective: To study the diagnostic value of serum adiponectin levels for evaluating osteoporosis (OP) in rheumatoid arthritis (RA) patients.

Materials and methods: We examined 88 women with a documented diagnosis of RA and mean disease duration of $6.56 \pm 0.88$ years. Female patients with II degree of disease activity (DAS28), Steinbrocker stage II (erosive), rheumatoid factor- and anti-cyclic-citrullinated peptide antibody-positive were prevalent. A control group of 45 apparently healthy females were included in the study. The groups were adjusted for age $(p>0.05)$ and showed no statistically significant differences. We measured serum Adiponectin levels $(\mu \mathrm{g} / \mathrm{ml})$ using indirect ELISA commercial test systems (BioVendor, Czech Republic, cat № RD195023100). We used a spectrophotometer with wavelength of $450 \mathrm{~nm}$ to detect the test results («Multiskan» immunoenzyme analyzer, Finland). We diagnosed osteoporosis (OP) using DXA with Lunar DPX Pro (GE, USA).

Results: Serum adiponectin levels in the control group were $12.5 \pm 0.9 \mu \mathrm{g} / \mathrm{ml}(\mathrm{M} \pm \mathrm{m})$. Adiponectin levels in healthy subjects measured as $\mathrm{M} \pm 2 \mathrm{~d}$, ranged between 0.44 and $24.56 \mu \mathrm{g} / \mathrm{ml}$. Patients with OP and RA had significantly higher levels of serum Adiponectin $(p<0.001)$ than controls. Mean serum Adiponectin levels in RA patients who had normal bone density and had no OP were $35.21 \pm 0.6 \mu \mathrm{g} / \mathrm{ml}$. Mean serum adiponectin levels in RA/ OP patients with low bone density were 52.42 $\pm 0.69 \mu \mathrm{g} / \mathrm{ml}$. Adiponectin levels of $44 \mu \mathrm{g} / \mathrm{ml}$ and higher were associated with osteoporosis. Adiponectin levels of $43.9 \mu \mathrm{g} / \mathrm{ml}$ and lower were associated with normal BMD. Conclusion: Serum adiponectin levels may be used to evaluate osteoporosis in patients with rheumatoid arthritis. Qualitative diagnostics of OP and RA can help choose the most adequate treatment of the metabolic bone disease. It can reduce the risk of low-energy fractures and to improve the quality of life of OP/RA patients.

\section{P136 \\ NONPHARMACOLOGICAL TREATMENT FOR OSTEOPOROSIS VERTEBRAL FRACTURE. A STUDY OF ECONOMIC IMPACT IN AN PRIVATE HEALTH CARE IN BRAZIL}

E. Tanaka ${ }^{1}$, M. D. Barbosa ${ }^{2}$, C. Saldanha ${ }^{3}$, F. Tanaka ${ }^{1}$

${ }^{1}$ Curitiba Health Institute, Curitiba, Brazil, ${ }^{2} \mathrm{Med}$ Inc Research, Berlin, Germany, ${ }^{3}$ Hospital de Clinicas, Porto Alegre, Brazil

Background: The rising costs of prostheses and orthopedic procedures in pathological fractures due to osteoporosis stimulate the innovation of using drugs, such as bisphosphonates, or nonpharmacological treatment, such as spinomed, intended to minimize skeletal-related events, 
regarding clinical and costs saving. Little is known about the economic impact of these new measures in Brazil.

Objective: Comparing bone-related event treatment costs in a Latin America country private health care provider.

Method: We analyzed the electronic medical records of 100 patients (GROUP A) who received zol at some point during their treatment (2009-2014). Most patients (71 patients) were diagnosed with breast cancer. The other 29 patients were diagnosed with other types of cancer. The predominant age in the sample was over 46 , representing $90 \%$ of the total. We analyzed another group of 88 patients (Group B). Group B patients had osteoporosis vertebral fracture with or without surgery procedure.

Results: The costs for Group A were estimated at approximately R $\$ 876,000.00$ Brazilian reals $(\sim \$ 437,000.00$ USD) with 487 IV applications of zoledronic acid during 5 years considering only bottle/vials spending. Expenses with femur and column prosthesis totaled $\$ 455,000.00$ and $\$ 580,000.00$ USD respectively. For Group B the most important data regarding costs was the calculation based on the pharmacoeconomic model. If all 88 patients were treated with an external prostheses using a non-pharmacological (SPINOMED) treatment for vertebral fracture, the cost would be roughly $€ 35.200$ Euros (88 patients $\times 400$ Euros), that is $\sim \$ 44.000 .00$ USD or $\sim \mathrm{R} \$ 176.000$ Brazilian reals. As secondary end point we still on study for pain drugs and hospitals costs.

Conclusion: The preliminary economic data for nonpharmacological treatment is cost effective due to the results based on the total costs. Further studies seems important to calculate the cost saving for pain drugs and hospitalizations

\section{P137}

OSTEOPOROSIS MANAGEMENT FOLLOWING TERIPARATIDE THERAPY FOR VERTEBRAL FRACTURES: ARE PATIENTS ON CORRECT MAINTENANCE THERAPY?

M. B. O'Connor ${ }^{1}$, D. Gilmartin ${ }^{2}$, S. Scanlon ${ }^{2}$, U. Bond ${ }^{1}$, M. J. Phelan $^{1}$

${ }^{1}$ Department of Rheumatology, South Infirmary Victoria University Hospital, Cork, Ireland, ${ }^{2}$ Department of Medicine, Mercy University Hospital, Cork, Ireland

Background: Teriparatide is used as a daily subcutaneous therapy for severely osteoporotic patients, with therapy duration of 18 to 24 months. It functions as an anabolic agent, and demonstrates increases in cortical thickness and reduces fracture risk. For the benefits of teriparatide to be sustained anti-resorptive therapy, in combination with calcium/vitamin D supplementation, should be initiated/restarted long-term after teriparatide therapy.
Methods: All patients prescribed teriparatide therapy from 2009 to 2012 were identified from departmental prescription records. Contact information was identified from local hospital databases. Patients were sent a pre-study letter outlining the nature of the study and the questions. This was followed by a telephone call, within 2 weeks, from the investigators. Three telephone attempts were made to contact participants after which they were excluded from the study. Participants were asked to list their current medications, background diagnoses and if they sustained a fracture since completing teriparatide therapy.

Results: 113 patients were identified from records. 42 were contacted and consented to participate in the study, 16 were deceased and 55 were uncontactable despite three attempts. Of the 42 enrolled, $45.2 \%(n=19)$ were no longer on a calcium or vitamin D supplementation and $57.1 \%(n=24)$ were no longer on an anti-resorptive, despite it being prescribed at their post-teriparatide Rheumatology assessment prior to discharge to GP care. 3 fractures were experienced post-teriparatide: 2 hip and 1 humerus. All fractures were in those not taking an anti-resorptive.

Conclusions: Despite being prescribed an anti-resorptive osteoporosis medication and calcium/Vitamin D supplementation on completion of teriparatide therapy there was a significant number of patients who no longer took these medications. The reasons for discontinuation are undocumented. This leaves them exposed to a submaximal benefit from therapy and an increased future fracture risk. This care- gap needs to be tackled.

\section{P138}

ASSOCIATION STUDY OF RANKL/RANK/OPG POLYMORPHISMS WITH HEEL QUANTITATIVE ULTRASOUND IN YOUNG ADULTS

M. Correa-Rodriguez $^{1}$, J. Schmidt Rio-Valle ${ }^{1}$, B. RuedaMedina $^{1}$

${ }^{1}$ Department of Nursing, University of Granada, Granada, Spain

Several single-nucleotide polymorphisms of the RANKL/ RANK/OPG signaling pathway have shown association with bone-related phenotypes (BMD at different skeletal sites or risk of fracture) in both, candidate gene and GWAS studies. However, the possible association of RANKL/RANK/OPG genes with heel Quantitative Ultrasound parameters (QUS) has not been analyzed previously. Thus for the first time, we aimed to determine whether genetic markers of this pathway were associated with calcaneal bone properties assessed by QUS. Ten single nucleotide polymorphisms (SNPs) of RANKL (rs9594759, rs12585014, rs7988338, rs2148073), RANK (rs1805034, rs12458117, rs3018362), and OPG 
(rs4355801, rs3102735, rs2073618) were selected as genetic markers and genotyped through the Open Array technology in 575 individuals aged 18 to 25 . Bone mass at the right calcaneus was estimated by QUS using the CUBA clinical ultrasound bone densitometer to obtain broadband ultrasound attenuation (BUA) measurements $(\mathrm{dB} / \mathrm{MHz})$. Linear regression analyses with adjustments for age, sex, weight, height, physical activity and calcium intake were performed to test the possible association between the SNPs and BUA. A trend of association between the rs9594759 RANKL polymorphism and heel QUS was observed ( $p=0.064, \mathrm{~B}=95 \% \mathrm{CI})$. For the rest of the tested SNPs no significant association with BUA parameter was found after adjustments for age, sex, weight, height, physical activity and calcium intake. Our results suggest that polymorphisms in RANKL/RANK/OPG pathway do not contribute significantly as genetic factors of heel ultrasound measurements in a population of Caucasian young adults.

\section{P139}

REPLICATION STUDY OF POLYMORPHISMS ASSOCIATED WITH CALCANEAL QUANTITATIVE ULTRASOUND IN A COHORT OF SPANISH YOUNG ADULTS

$\underline{\text { M. Correa-Rodriguez }}{ }^{1}$, J. Schmidt Rio-Valle ${ }^{1}$, B. RuedaMedina $^{1}$

${ }^{1}$ Department of Nursing, University of Granada, Granada, Spain

Genetic factors are important determinants of calcaneus ultrasound parameters. Family and twin studies have shown a $74 \%$ heritability of QUS parameters. In a recent published meta-analysis by the GENOMOS (Genetic Markers of Osteoporosis) consortium involving European subjects, several susceptibility genes for osteoporosis including SPTBN1, RSPO3, CCDC170, DKK1, GPATCH1 and TMEM135 were confirmed to be associated with heel properties assessed by QUS at a genome-wide significance level. The purpose of the present study was to validate the most significant polymorphisms associations with broadband ultrasound attenuation (BUA) from the meta-analysis of the GENOMOS in an independent population of Caucasian young adults.A crosssectional study was conducted on 575 individuals (mean age $20.41 \pm 2.69)$. Bone mass at the right calcaneus was estimated by QUS using the CUBA clinical ultrasound bone densitometer to obtain BUA measurement (dB/MHz). Six SNPs showing the most significant association at GWAS level in the meta-analysis conducted by GENOMOS consortium in genes SPTBN1 (rs11898505), RSPO3 (rs7741021), CCDC170 (rs4869739), MBL2/DKK1 (rs7902708), TMEM135 (rs597319) and GPATCH1 (rs10416265) were genotyped using an Open Array technology. Association of the tested SNPs with BUA variations was determined using linear regression analysis. The regression analysis revealed a significant association of the rs7741021 SNP in RSPO3 gene with BUA $(p=0.03)$. However, we could not confirm the association of the rest of SNPs with QUS parameter in our population. Our study has provided an independent replication analysis of the SNPs in SPTBN1, RSPO3, CCDC170, MBL2/ DKK1, GPATCH1 and TMEM135 genes and calcaneal QUS in a relatively large population of Spanish young adults. The results only support the association of RSPO3 gene with QUS parameter.

\section{P140}

GENOME-WIDE EPIGENOMIC ELEMENTS ANALYSIS IDENTIFIES NEW SUSCEPTIBILITY GENES INFLUENCING OSTEOPOROSIS Y. Guo $^{1}$, T.-L. Yang ${ }^{1}$

${ }^{1}$ Key Laboratory of Biomedical Information Engineering of Ministry of Education, School of Life Science and Technology, Xi'an Jiaotong University, Xi'an, China

Background: Despite genome-wide association studies (GWASs) have identified many susceptibility genes for osteoporosis, it still leaves a large part of missing heritability to be discovered. Meanwhile, it is difficult to elucidate the functional mechanisms underlying associations only based on these statistical signals.

Methods: We conducted an integrative analysis that combined epigenomic elements and data from previous GWASs to identify common regulatory elements and predict new susceptibility genes that are biologically meaningful to osteoporosis, then followed by a validation at population level using available GWASs datasets, including GEFOS (Genetic Factors for Osteoporosis Consortium) and our three GWASs samples.

Results: We successfully found a set of distinct epigenomic elements as regulatory basis significantly enriched or depleted in the promoters of osteoporosis-associated genes, including 4 transcription factor binding sites (TFBSs), 27 histone marks, and 21 chromatin states segmentation types. Using these significant epigenomic marks, we predicted new candidate genes through reverse analysis. Genes with high priority were further subjected to validation using available GWASs datasets. Three genes, BDNF, PDE4D, and SATB2, were successfully validated for associations with spine BMD. These 3 genes all have close relationship with bone metabolism. Functional enrichment analysis of all the prioritized genes revealed several key osteoporosis related pathways, like Wnt signaling.

Conclusions: Our results demonstrated that epigenomic data could be used to indicate common epigenomic marks to 
discover additional loci with biological functions for osteoporosis, which would offer new targets for research into the etiology of osteoporosis.

Acknowledgement: This work was supported by the National Natural Science Foundation of China (31371278, 31471188, 81573241, 31511140285); and the Fundamental Research Funds for the Central Universities (2012jdgz11).

Disclosure of Interest: None Declared

Keywords: Osteoporosis; Epigenomic element; GWAS; Association

\section{P141}

A FUNCTIONAL SNP IN THE 3"UTR OF THE HUMAN SOX6 GENE INCREASES OSTEOPOROSIS RISK THROUGH MODIFYING MIRNA BINDING

Y. Guo $^{1}$, T.-L. Yang ${ }^{1}$

${ }^{1}$ Key Laboratory of Biomedical Information Engineering of Ministry of Education, School of Life Science and Technology, Xi'an Jiaotong University, Xi'an, China

Background: $\mathrm{SOX} 6$ gene is an essential transcription factor in chondrogenesis and cartilage formation. Previous genomewide association studies (GWASs) have identified SOX6 as a new susceptibility gene for osteoporosis in white populations. Evaluating the genetic associations in different ethnicities, especially in Chinese, is necessary and valuable. Meanwhile, discovering the unknown function of the genetic association is extremely important to offer new targets for research into the etiology of osteoporosis.

Aim: To investigate whether the single nucleotide polymorphisms (SNPs) in SOX6 are associated with osteoporosis in Chinese population, and explore the potential function of the associated SNPs.

Methods: We performed a resequencing and association analysis to investigate the relationship between SNPs in the SOX6 gene and hip BMD in a Chinese population including 100 unrelated Chinese Han subjects (50 high BMD vs. 50 low BMD). Functional studies were conducted to investigate the potential function of associated SNPs.

Results: We identified a SNP rs1065024 (A > G) located in the 3"-untranslated region (3"UTR) of SOX6 associated with hip BMD $(P<0.05)$. Luciferase reporter analysis revealed that gene expression was significantly lower in the presence of the $\mathrm{G}$ allele (mutant type) when compared with the A allele (wild type) in the HEK293T cell and osteoblast cells. Using Target Scan, miRanda and Pictar databases, we predicted that this SNP rs1065024 was the target binding site of miR-190b. After miR-190b co- transfection with the vector carrying the wide type, it showed significant inhibitions of luciferase activity. While, there was no significant difference for the mutant type. Our results suggest that this SNP could increase SOX6 expression through affecting binding affinity of miR-190b.

Conclusions: Our study provide evidence that SNP rs 1065024 in the 3"UTR of SOX6 could be associated with increased risk for osteoporosis in Chinese population, and the SNP-modified posttranscriptional gene regulation by miRNA could be a potentially pathogenetic mechanism of osteoporosis.

Acknowledgement: This work was supported by the National Natural Science Foundation of China (31371278, 31471188, 81573241, 31511140285); China Postdoctoral Science Foundation (2015 M570820); the Natural Science Basic Research Program Shaanxi Province (2015JQ3089), and the Fundamental Research Funds for the Central Universities (2012jdgz11).

\section{P142}

COMPARATIVE STUDY OF BONE MINERAL DENSITOMETRY AND DISCORDANCY BETWEEN WOMEN ON LEVOTHYROXINE FOR HYPOTHYROIDISM AND HEALTHY WOMEN

N. Panahi ${ }^{1}$, A. Hossein-Nezhad ${ }^{2}$, H. R. Aghaei Meybodi ${ }^{3}$, M. $\overline{\text { Qorbani }}^{3}$, A. Soltani ${ }^{3}$, Z. Maghbooli ${ }^{2}$, B. Larijani ${ }^{3}$

${ }^{1}$ Osteoporosis Research Center, Endocrinology and Metabolism Clinical Sciences Institute, Tehran University of Medical Sciences, National Elites Foundation, Tehran, Islamic Republic of Iran, ${ }^{2}$ Osteoporosis Research Center, Endocrinology and Metabolism Clinical Sciences Institute, Tehran University of Medical Sciences, Tehran, Islamic Republic of Iran, ${ }^{3}$ Endocrinology and Metabolism Clinical Sciences Institute, Tehran University of Medical Sciences, Tehran, Islamic Republic of Iran

Objectives: To determine bone mineral densitometry and diagnostic discordance for osteoporosis in a group of women on levothyroxine for treatment of hypothyroidism compared to healthy women.

Material and Methods: This cross-sectional study was conducted on 1133 women with hypothyroidism taking levothyroxine and 483 healthy women undergoing bone mineral densitometry with DXA, for suspected osteoporosis in an outpatient clinic at a referral hospital at Tehran, Iran. Demographic data, anthropometric measurements, and risk factors for osteoporosis were derived. The BMD measures at hip and spine were used to measure T-scores to determine discordance.

Univariate and a multivariate regression model was performed to obtain Factors potentially associated with discordance.

Results: Among women on levothyroxine 947 and among healthy women 153 were postmenopausal. Osteoporotic state was significantly different in all three sites (spine, hip and ward) between women on levothyroxine and healthy women $(p$ value $<0.001)$ with more women on 
levothyroxine being osteoporotic. Major discordance, minor discordance and concordance of T-scores between two sites (spine and Total hip) was observed in 39(3.4\%), $459(40.5 \%)$, and $635(56 \%)$ of women on levothyroxine and $11(2.3 \%), 147(30.4 \%), 325(67.3 \%)$ of healthy women respectively. Discordancy was observed more significantly in women on levothyroxine ( $p$ value $<0.001)$. More discordance was noted in postmenopausal women. Logistic regression analysis shows that in univariate model the association between levothyroxine and discordancy was statistically significant (OR: 1.613(1.290-2.017)); however, in multivariate model this association was not statistically significant (OR: 0.921(0.701-1.210)).

Conclusion: Our study revealed the high prevalence of $\mathrm{T}$ score discordance in women on levothyroxine treatment compared to healthy women.

\section{P143}

EFFECTS OF ALENDRONATE TOPICAL APPLICATION AS A COMPONENT OF A BIOCOMPOSITE MATERIAL ON OSTEOGENESIS IN OSTEOPLASTY

S. Rodionova $^{1}$, A. Torgashin ${ }^{1}$

${ }^{1}$ Federal State Institution « Federal Scientific Research Institute for Traumatology and Orthopedics named after $\mathrm{N}$. N. Priorov », Moscow, Russian Federation

The fact that topical application alendronate as a component of a biocomposite material can decrease the rate of bone synthesis has been prevented their utilization in orthopedic and traumatologic practice.

Objective: To assess effect of topical application alendronate as a component of biocomposite material on osteogenesis.

Methods: Experiment was performed on 20 nonlinear female white mice divided into 2 groups. In the experimental group defect of shin bone was filled with nondemineralized freeze-dried bone implant combined with biocomposite material containing Alendronate $1 \mathrm{mg} / \mathrm{ml}$. In the control group biocomposite material was not containing bisphosphonate. Experiment lasted 90 days. Results evaluation was morphological (light microscope Zeiss Axioskop 40). Samples were stained with hematoxylin-eosin. BMD in experimental area and in the whole segment was assessed with densitometer Hologic and analyzed with software "Performing and Analyzing Small Animal Studies". Rate of osteogenesis and changes in the experimental areas were graded according to the following scale: 1 - poor osteogenesis (area of bone defect is filled with loose connective tissue and fragments of bone implant presented with osteocytes-free bone trabeculae; 2 - moderate osteogenesis (area of bone defect contains foci of newly synthesized mature bone tissue around bone implant or marginal osteogenesis on the cartilage basis with remnants of bone implant); 3 - advanced osteogenesis (area of bone defect is filled with newly synthesized mature bone tissue without remnants of bone implant). Statistical analysis of data was performed on SPSS software, level of statistical significance was set to $p<0,05$.

Results: In the experimental group the mean index of osteogenesis was 1.4 , in the control group - 1.6. Difference in results between groups was not statistically significant.

Conclusion: Topical application of alendronate $1 \mathrm{mg} / \mathrm{ml}$ as a part of biocomposite material does not inhibit osteogenesis.

\section{P144}

IONIZED AND TOTAL CALCIUM LEVELS IN PATIENTS WITH HYPOVITAMINOSIS D : THE PTH CONNECTION

\section{H. M. Al Attia ${ }^{1}$}

${ }^{1}$ Department of Internal Medicine and Rheumatology, Universal Hospital, Abu Dhabi, United Arab Emirates

Methods: Records of 101 patients with hypovitaminosis $\mathrm{D}<30 \mathrm{ng} / \mathrm{ml}$ also underwent assays for ionized $\mathrm{Ca}$, nonionized $\mathrm{Ca}$ and PTH are presented. Data of patients with chronic renal failure or on loop diuretics, corticosteroids, calcium and vitamin D supplementation were not included. They were divided into two groups based on level of ionized Ca.

Results: 26 (25.5\%) had ionized $\mathrm{Ca}<1.16 \mathrm{nmol} / \mathrm{l}$ (group 1) and 75 with normal levels (1.16-1.32 nmol/l) (group 2), $p=0.0001$. The mean of ionized $\mathrm{Ca}$ in group 1 was significantly lower to that in group $2(1.12 \pm 0.116$ vs. $1.23 \pm 0.12) p=0.0001$ respectively. However, the mean of $25(\mathrm{OH}) \mathrm{D}$ in the two groups was not different $(17.7 \pm 5.33 \mathrm{ng} / \mathrm{ml}$ vs. $18.2 \pm 5.89)$ respectively, $p=0.68$. Nonionized $\mathrm{Ca}$ was within normal values in both groups $(9.43$ \pm 0.449 , range $8.9-10.4 \mathrm{mg} / \mathrm{dL}$ vs.9.80 \pm 0.435 , range 8.88 $11.1 \mathrm{mg} / \mathrm{dL}$ ) respectively yet of statistical significance, $p=0.0004$. The $\mathrm{R}$ value was weakly positive in the correlation between the ionized and non ionized $\mathrm{Ca}$ in both groups $(R=0.2327$ and 0.2879$)$ respectively.

10 patients in each group had secondary hyperparathyroidism (SHPTH) with levels above $65 \mathrm{pg} / \mathrm{ml}, 38.5 \mathrm{vs} .13 .5 \%$ $p=0.0095$. The $\mathrm{R}$ value was positive $(0.0147)$ between the patients with subnormal ionized $\mathrm{Ca}$ and SHPTH but negative in nonionized Ca values and SHPT (0.2048).

Conclusions: Subnormal ionized $\mathrm{Ca}$ exhibited higher degree of correlation with secondary hyperparathyroidism due to hypovitaminosis $\mathrm{D}$ than that of the total $\mathrm{Ca}$. Estimating the ionized $\mathrm{Ca}$ therefore should be considered essential in future studies addressing similar or other pertinent issues. 


\section{P145}

\section{UNMET SOCIAL NEEDS OF PATIENT WITH KNEE OSTEOARTHRITIS}

${ }_{\text {V. Sadovici-Bobeica }}{ }^{1}$, V. Salaru ${ }^{1}$, L. Mazur-Nicorici ${ }^{1}$, M. Cebanu $^{1}$, M. Mazur ${ }^{1}$

${ }^{1}$ State University of Medicine and Pharmacy "Nicolae

Testemitanu", Chisinau, Republic of Moldova

Aim: To estimate the level of social support of patients with knee osteoarthritis.

Methods: A cross-sectional study was performed from January 2014 to January 2015. There were included consecutive patients that fulfilled ACR classification criteria for knee OA (1991). We collected data on demographic and socioeconomic characteristics, joint function, use of health and social services, and effect on occupation and living arrangements over the previous 12 months. The social support was assessed by Interpersonal Support Evaluation List (ISEL- 12).

Results: There were 185 patients integrated in the study including 143 females and 42 males, mean age

$62.4 \pm 9.5$ (range 37 to 85 ) years. The disease duration was $8.1 \pm 6.7$ (range 1-51) years. The results of the ISEL 12 evaluation revealed that the social support was scored with 31.7 points, appreciated as satisfying, the higher ISEL score was obtained for appraisal domain -11.98 , meantime, for belonging (9.7 points) and tangible (9.6) was determined a lower result. We established a significant indirect correlation between the patient's age and total ISEL score $r=-0.71$ $(p<0.001)$ and mild correlation between social support and symptoms, knee's functionality and pain $r=0.51-0.54$ $(p<0.05)$.

Conclusion: Patients with knee osteoarthritis were generally positive about their appraisal support; meantime they manifested a high level of dissatisfaction with financial aspects (tangible) and communication (belonging) that demonstrates a poor social support, correlating directly with the age and disease's manifestations.

\section{P146}

\section{AGED-RELATED CHANGES IN THE SYNO-} VIUM USING AN EXPERIMENTAL MODEL

\section{Lopez-Fernandez ${ }^{1}$}

${ }^{1}$ Universidad de Santiago de Compostela, Facultad de Medicina, Santiago de Compostela, Spain

Objective: To determine differences with age in normal synovium.

Material and Methods: The synovium is the soft tissue lining the spaces of diarthrodial joints (except where there is cartilage), tendon sheets and bursae. It is quite important in the joint, not only because it allows movements of adjacent tissues, but also because it lubricates the cartilage, providing the nutrition of chondrocytes and also controlling the synovial fluid volume. Despite the synovium is the central area of pathology in an important number of inflammatory diseases such as rheumatoid arthritis, the architecture of the synovial membrane is not as known as it should be, particularly in normal specimens. Changes to the synovium due aging can adversely affect joint homeostasis. The main changes in synovium with age could be summarize in: decreasing of layers of synovial intima, reduction in the overall population of synovial cells which became atrophic, relative reduction of type B cells that reflects a compromised secretory activity, decreased vascularity and the presence of fibrosis. Forty eight samples of adipose synovium of $12 \mathrm{New}$ Zealand male rabbits have been studied. Animals were divided into 3 groups depending on their age at the time of the euthanasia. Light microscopy using the routine technique with hematoxylin-eosin allowed evaluation of the number of layers in the lining, cellularity in the sublining and vessels. Statistical analysis was performed following Kruskal-Wallis nonparametric test.

Results: The results showed in older specimens decrease in all the countings: cell layers in lining, number of cells found in sublining and vessels found in lining as well as in sublining. There are statistically significant differences.

Conclusion: It seemed worthwhile to study the synovial membrane of young and old synovium in order to quantitate any age-associated changes. Distinguish which differences in synovial tissue are because of aging and which are because of a possible inflammatory disease could lead us to a correct diagnosis and treatment.

\section{P147}

CORRELATIONS BETWEEN CLINICAL PARAMETERS AND HEALTH-RELATED QUALITY OF LIFE IN POSTMENOPAUSAL OSTEOPOROTIC WOMEN

V. Grbovic $^{1}$, A. Jurisic Skevin ${ }^{1}$, K. Parezanovic Ilic ${ }^{1}$, A. Tomic Lucic $^{1}$, D. Djordjevic ${ }^{1}$, D. Pavlovic ${ }^{2}$, J. Nurkovic ${ }^{3}$

${ }^{1}$ Clinical Center Kragujevac, Novi Pazar, Serbia, ${ }^{2}$ Specialized Hospital for Rehabilitation Bukovicka Banja, Arandjelovac, Serbia, ${ }^{3}$ State University of Novi Pazar, Novi Pazar, Serbia

Objectives: The purpose of study was to assess correlation between health related quality of life (HRQoL) of postmenopausal osteoporotic women and clinically relevant osteodensitometric parameters.

Methods: This cross-sectional study was performed within the group of 100 osteoporotic patients ( 56 without and 44 with history of fracture) of the Clinical centre Kragujevac, Serbia. The collection of data lasted was performed from January to December 2012. The study was approved by the Ethical committee of the Clinical centre Kragujevac. BMD and T-score at the lumbar vertebra and femoral neck were assessed in 100 
osteoporotic women (56 without and 44 with previous fracture), using DXA performed on Discovery TM osteodensitometer (Hologic, Bedford, MA). Fracture risk assessment tool (FRAX index) for major osteoporotic and hip fractures was calculated based on demographic data and BMD hip value. HRQoL was assessed using QUALEFFO-41 questionnaire (domains: health perception, pain, physical, social and mental function).

Results: Basic characteristics of participants (age, length of menopause, BMI, smoking habit, hereditary tendency to fractures, fracture history) correlated with some QUALEFFO-41 domains, but coefficients of correlation were low $(r<0.3)$, except in case of pain domain to fracture history relationship $(r=0.638)$. Out of those six chosen predictable variables in the multiple regression model, fracture history was shown to be the most significant predictable variable for three QUALEFFO-41 domains: pain $(b=20.511)$, social function $(b=2.548)$ and health perception $(b=3.185)$. Correlation analysis showed that, after adjustment for basic characteristics of subjects, the strongest correlations were found between BMD and T-score at femoral neck and pain $(r=0.331$ and $r=0.449$, respectively), social function $(r=0.422$ and $r=0.419)$ and health perception domains $(r=0.434$ for Tscore at femoral neck).

Conclusion: The results of our study confirm previously established relationship between BMD at femoral neck and HRQoL in patients with osteoporosis.

\section{P148}

\section{THE WNT-INACTIVATING LIPASE NOTUM AS} A NOVEL ANABOLIC OSTEOPOROSIS DRUG TARGET

$\underline{\text { R. Brommage }}^{1}$, A. Y. Thompson ${ }^{1}$, M. K. Shadoan ${ }^{1}$, J. Liu $^{1}$, S. Jeter-Jones $^{1}$, J. Ciu ${ }^{1}$, D. G. Potter ${ }^{1}$, D. Bright ${ }^{1}$, F. Mseeh ${ }^{1}$, J. P. Bardenhagan $^{1}$, G. M. Hansen ${ }^{1}$, P. Vogel ${ }^{1}$, J. E. Tarver ${ }^{1}$, B. Zambrowicz $^{1}$, Q. Liu ${ }^{1}$, D. R. Powell ${ }^{1}$

${ }^{1}$ Lexicon Pharmaceuticals, The Woodlands, Texas, United States

To identify novel osteoporosis drug targets, Lexicon's gene knockout $(\mathrm{KO})$ phenotyping campaign $(\approx 4,650$ distinct genes) included DXA measurements of body, spine and femur BMD plus microCT analyses of trabecular (LV5) and cortical (midshaft femur) bone. NOTUM inactivates WNTs by removing the essential palmitoleate required for activating Frizzled receptors. Notum KO mice had elevated bone mass and, aside from defects in dentin mineralization, no non-skeletal phenotypes. Elevated cortical bone thickness occurred throughout the skeleton, with trabecular bone unchanged. Treating male mice weekly with NOTUM neutralizing antibody $2.78 .33 \mathrm{a}$ at doses from 0.3 to $10 \mathrm{mg} / \mathrm{kg}$ for 4 weeks increased midshaft femur cortical thickness from 3 to $10 \%$. Treating male mice with the orally active NOTUM inhibitor LP-922056 at $10 \mathrm{mg} / \mathrm{kg}$ for 4 weeks increased $(P<0.001)$ serum ALP $(36 \%)$, serum PINP $(66 \%)$, femoral neck BV/TV (5\%), LV5 vertebral body cortical shell $\mathrm{BV} / \mathrm{TV}(9 \%)$ and both cortical thickness $(12 \%)$ and strength $(29 \%)$ of the midshaft femur. Male mice treated with LP-922056 daily for 12 weeks at doses of 1,3 or $10 \mathrm{mg} / \mathrm{kg}$ showed dose-dependent increases in cortical bone thickness of the humerus ( 5 to $15 \%$ ), femur ( 9 to $17 \%$ ) and rib (12 to $27 \%$ ). Male mice treated with LP922056 twice weekly (Mondays and Thursdays) for 12 weeks at doses of 3,10 or $30 \mathrm{mg} / \mathrm{kg}$ showed dosedependent ( 4 to $11 \%$ ) increases in midshaft femur cortical thickness. Treating both intact and OVX female rats with LP-922056 at $30 \mathrm{mg} / \mathrm{kg}$ for 18 weeks increased $(P<0.002)$ cortical bone parameters in femur, tibia, humerus, radius, ulna, rib, femoral neck and vertebral body cortical shell. Treatment stimulated endocortical BFR (measured between 1 and 9 weeks) at the midshaft femur, distal tibia and rib. As a secreted enzyme inhibitable by neutralizing antibodies and orally active drugs, NOTUM is a potential drug target for stimulating modelingdependent endocortical bone formation and treating osteoporosis.

\section{P149}

EFFICACY OF SHORT TERM TERIPARATIDE FOR UNTREATABLE HIP AND KNEE BONE MARROW EDEMA SYNDROMES

\section{F. Galluccio $^{1}$, E. Bellucci ${ }^{1}$, M. Matucci Cerinic ${ }^{1}$}

${ }^{1}$ Div. of Rheumatology, Department of Clinical and Experimental Medicine, Firenze, Italy

The use of magnetic resonance (MRI) for the diagnosis of hip and knee pain revealed a set of conditions that occur with epiphyseal bone edema ${ }^{1}$. The so-called bone marrow edema syndromes ${ }^{2}$ (BMEs) are challenging for clinicians because it includes a variety of diseases such as transient bone marrow edema and osteoporosis, complex regional pain syndrome I (CRPS type 1), stress fractures and bone bruise, subchondral insufficiency fracture and osteonecrosis. In most cases the medical history facilitate us the correct diagnosis but it is not always true and unfortunately is not always possible to identify a causative factor. The lack of knowledge on the pathological processes between this diseases and the demonstration that some of these can evolve into osteonecrosis, the theory that BMEs are a variable form of an early stage or self-limiting osteonecrosis, further complicates the diagnostic process and the formulation of a correct therapeutic strategy ${ }^{3}$. The goal of conservative treatment is to 
control symptoms, prevent or delay surgery and to protect the joint. The conservative treatments commonly used are AINS, painkillers and opioids, bisphosphonates, iloprost and prostaglandins and hyperbaric therapy all to be combined with rehabilitation, physical therapies and partial or non weight bearing. Therapy with both sysadoas and hyaluronate is useful for secondary osteoarthritis. Despite these treatments have proven to be effective in most of the cases, it is still a subset of patients who are classified as untreatable and are generally intended for prosthetic surgery.

In this patients, the failure of the above mentioned therapy has led us to try a three months therapy with teriparatide when the maximum anabolic effect is present before promoting bone remodeling ${ }^{4}$. Moreover, the up regulation of growth factors like bFGF-2 and IGF- $1^{5}$, $\mathrm{RANKL}^{6}$, the influence on the $\mathrm{Wnt} / \beta$-catenin signaling pathway and the transcriptional suppression of the sclerostin ${ }^{7}$ gene may play an important role on the regulating effect of teriparatide on bone. Teriparatide has also an adrenal effect increasing plasma and urinary cortisol lasting as long as therapy ${ }^{8}$ probably regulating the inflammatory cascade. Lastly, teriparatide has proven to accelerate fracture healing and have a potential effect in increasing bone volume within joints and inhibiting articular cartilage degeneration ${ }^{9,10}$. Until now we have treated nine patients, six of which have exceeded 1 year of follow-up and one over two years. Of these seven patients, two of them with primary hip osteonecrosis, two with post traumatic knee osteonecrosis, two with CRPS type 1 of the knee and one with subchondral insufficiency fracture. Concomitant therapy was with sysadoas, intraarticular hyaluronate and analgesics as needed. All patients experienced a rapid response in terms of pain and progressive recovery of joint function. After three months, teriparatide was discontinued with the only indication to rehabilitation and chondroprotection. MRI at three months showed an almost complete resolution of the initial consensual to the clinical response. Three patients underwent control at 1 year which shows no progression.

We believe that teriparatide is considered as an alternative treatment to refractory BMEs, although further confirmation of efficacy is needed.

\footnotetext{
References:

${ }^{1}$ Schaefer PW, et al. Sem Musculoskeletal Radiol 2006; $10: 177$

${ }^{2}$ Patel S. Rheumatology (Oxford). 2014;53:785.

${ }^{3}$ Korompilias AV, et al. .Skeletal Radiol 2009;38:425.

${ }^{4}$ Dhillon RS, Schwarz EM. Materials 2011;4:1117.
}

${ }^{5}$ Hurley M, et al. Osteoporos Int 2005;16:2080.

${ }^{6}$ Rosen CJ. Horm Res. 2005;64(Suppl 2):81.

${ }^{7}$ Paszty C, et al. J Bone Miner Res 2010;25:1897.

${ }^{8}$ Lasco A, et al. Osteoporosis Int 2011;22:299.

${ }^{9}$ Gomberg SJ, et al. J Clin Endocrinol Metab 2011;96:1627.

${ }^{10}$ Sampson ER, et al. Sci Transl Med 2011;3:101 ra93.

\section{P150}

CENTRAL ADIPOSITY HAS A DETRIMENTAL IMPACT ON TRABECULAR BONE IN OBESE POSTMENOPAUSAL WOMEN: A RETROSPECTIVE STUDY

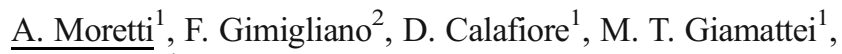
G. Iolascon $^{1}$

${ }^{1}$ Department of Medical and Surgical Specialties and Dentistry, Second University of Naples, Naples, Italy, ${ }^{2}$ Department of Physical and Mental Health and Preventive Medicine, Second University of Naples, Naples, Italy

Objective: To evaluate the effect of visceral adipose tissue (VAT) on bone microarchitecture in postmenopausal obese women.

Materials and Methods: In this retrospective study we reported data from medical records of obese postmenopausal women (BMI $\geq 30 \mathrm{~kg} / \mathrm{m}^{2}$ ) referring to our outpatient rehabilitation service for the prevention and management of osteoporosis over a 3-year period. In these patients we measured BMD at lumbar spine (LS BMD) and at femoral neck (FN BMD), trabecular bone score (TBS), VAT volume and VAT mass. The population was divided into quartiles of VAT volume (VAT $<1.398 \mathrm{~cm}^{3}$; VAT between 1.398 and $1.764 \mathrm{~cm}^{3}$; VAT between 1.765 and $2.371 \mathrm{~cm} 3$; VAT $>2.371 \mathrm{~cm}^{3}$ ). The differences between groups in terms of TBS, according to cutoff proposed by Silva et al. $\left[^{1}\right]$, were assessed using SPSS 21.0 to perform the Kruskal-Wallis test for independent samples.

Results: We analyzed data of 226 women (mean age $64.56 \pm$ SD 8.42, mean BMI $34.18 \pm$ SD 3.32). There were no statistically significant differences for age between groups $(p=0.332)$. In our population, higher VAT volume was associated with a significant worsening of the trabecular bone microarchitecture $(p=0.003)$. In particular, in the first quartile, $40.8 \%$ of patients had a TBS $\leq 1.200$, whereas in the higher quartile $72.2 \%$ of women had degraded microarchitecture.

Conclusions: Nowadays, the relationship between adipose tissue and bone is not well understood. Moreover, the bone involvement in obese patients is still controversial. The amount of visceral adipose tissue (VAT) might be 
one of the pathogenetic factors involved in bone microarchitecture deterioration in obese postmenopausal women.

References: ${ }^{1}$ Silva BC, et al. J Bone Miner Res 2014;29:518.

\section{P151}

\section{VERTEBRAL BONE QUALITY AND INTERVERTEBRAL DISC ALTERATIONS IN PATIENTS WITH BETA-THALASSEMIA: A RETROSPECTIVE STUDY}

A. de Sire $^{1}$, A. Moretti ${ }^{1}$, M. Bianco ${ }^{1}$, F. Gimigliano ${ }^{2}$, G. Iolascon $^{1}$

${ }^{1}$ Department of Medical and Surgical Specialties and Dentistry, Second University of Naples, Naples, Italy, ${ }^{2}$ Department of Physical and Mental Health and Preventive Medicine, Second University of Naples, Naples, Italy

Objective: Bone involvement in patients with $\beta$-thalassemia is well known, but only few studies have analyzed bone microarchitecture and the prevalence of intervertebral disc calcifications (IDCs) in these patients. Objective of our study was to evaluate the vertebral bone involvement in a group of patients with $\beta$-thalassemia in terms of geometry and bone quality; moreover, we evaluated the presence and site of IDCs in these patients.

Material and Methods: Our retrospective case-control study was conducted in adults with $\beta$-thalassemia (aged 1850 years). Patients were divided, according with the ISCD criteria, into 2 groups: subjects with BMD $\mathrm{Zs} \leq-2.0$, below the expected range for age, and subjects with BMD Zs $>-2.0$, within the expected range for age. Assessment of bone quality was performed using the Trabecular Bone Score (TBS), stratifying subjects into 3 groups: with degraded (TBS $\leq 1.200$ ), partially degraded (TBS $>1.200$ and $<1.350$ ), and normal (TBS $\geq 1.350)$ trabecular microarchitecture. Finally, we evaluated the presence of IDCs highlighted by images of vertebral fracture assessment.

Results: We evaluated 49 patients with $\beta$-thalassemia, mean aged 35.2 \pm 9.6 years, divided into two groups: 25 patients with $\mathrm{Zs} \leq-2.0$ and 24 patients with $\mathrm{Zs}>-2.0$. There was a statistically significant difference between groups in number of fragility fractures $(p=0.0339)$. Furthermore, TBS of patients with $\mathrm{Zs} \leq-2.0$ was significantly lower than individuals with $Z_{s}>-2.0$ as mean value $(p=0.0006)$ and as categorized value $(p=0.0061)$. Finally, we evidenced in 7 patients $(14.29 \%)$ the presence of at least one IDC.

Conclusions: Our results showed that $\beta$-thalassemia is characterized not only by a reduction in BMD, but also by a bone geometry and bone microarchitecture involvement, highlighting that TBS should be included in the assessment of these subjects, in order to obtain a proper diagnosis, management and prevention of fragility fractures; furthermore, presence of IDCs should be better investigated.

\section{P152}

ARE DIETARY SUPPLEMENTS AND NUTRACEUTICALS EFFECTIVE FOR MUSCULOSKELETAL HEALTH? A SCOPING REVIEW

A. de Sire $^{1}$, A. Moretti ${ }^{1}$, M. T. Giamattei ${ }^{1}$, F. Gimigliano ${ }^{2}$, G. Iolascon $^{1}$

${ }^{1}$ Department of Medical and Surgical Specialties and Dentistry, Second University of Naples, Naples, Italy, ${ }^{2}$ Department of Physical and Mental Health and Preventive Medicine, Second University of Naples, Naples, Italy

Objective: To analyze the state of the art on micronutrients, available in nutraceuticals or in dietary supplements, in order to identify, according to an evidence-based approach, which of them effectively improve the areas typically involved in functional deterioration of elderly: bone, skeletal muscle and nervous tissues.

Material and Methods: The Group for the Study of Healthy Ageing by Nutraceuticals and Dietary Supplements (HANDS) performed this scoping review through different steps: list of micronutrients available in dietary supplements and nutraceuticals, used in elderly to improve their physical functioning in three systems (bone, muscle and central nervous system); identification of relevant studies on PubMed, using as MeSH terms the selected micronutrients, adding through PubMed Search Builder the terms: "bone", "skeletal muscle" and "central nervous system"; selection of effective micronutrients; identification of effective and safe dosage regimens.

Results: We evaluated 12 relevant studies (1 international society guideline recommendations, 1 systematic review, 7 randomized controlled trials, and 3 prospective cohort studies). Of the 39 micronutrients available in the market, only 16 resulted to have appropriate scientific evidence of their effectiveness in terms of improving musculoskeletal health in older people: beta-alanine, calcium, creatine, fluorides, leucine, magnesium, omega-3 fatty acids, potassium, vitamin B6, vitamin B9, vitamin B12, vitamin C, vitamin D, vitamin E, vitamin $\mathrm{K} 2$, and zinc.

Conclusions: This scoping review shows that selected micronutrients in specific doses might effectively improve the musculoskeletal health and cognitive function in elderly. A precise analysis carried out according to the EBM principles might deliver significant benefits in the field of dietary supplements and nutraceuticals. 


\section{P153}

IMPROVEMENT OF BALANCE IN VITAMIN-D DEFICIENT WOMEN AFTER 6 MONTHS TREATMENT WITH EITHER CHOLECALCIFEROL OR ALPHACALCIDOL

C. Capatina ${ }^{1}$, A. Caragheorgheopol ${ }^{2}$, M. Berteanu ${ }^{1}$, C. Poiana ${ }^{1}$

${ }^{1}$ Carol Davila University of Medicine and Pharmacy, Bucharest, Romania, ${ }^{2 "}$ "C.I. Parhon" National Institute of Endocrinology, Bucharest, Romania

Background: Vitamin D (VD) deficiency is associated with the atrophy of muscle fibers, (predominantly type II), with deleterious consequences on both muscular strength and balance function. These effects are at least partially reversible with normalization of the VD status. We aimed to analyse the differential effects of native VD and alphacalcidol treatment on the balance function of a group of women with VD deficiency.

Material and methods: We analysed 177 women aged 2083 years-old, diagnosed with VD deficiency (by measuring the serum concentration of $25 \mathrm{OHD}$ using an electrochemilu- miniscence method). 72 of the cases were postmenopausal women with low bone mass, 105 were premenopausal women. We randomised the patients to receive colecalciferol 1000 IU daily or alphacalcidol $1 \mu \mathrm{g}$ daily, for 6 months. We administered the Tinetti test both at baseline and after 6 months of treatment and recorded at each evaluation 3 different results (global test score, posture and balance subscores).

Results: The mean baseline 25OHD concentration was 15.38 $\pm 7.55 \mathrm{ng} / \mathrm{ml}$ (mean $\pm \mathrm{SD}$ ). After treatment the serum $25 \mathrm{OHD}$ level was $20.85 \pm 8.88 \mathrm{ng} / \mathrm{ml}$, significantly higher in cases supplemented with cholecalciferol $(22.7 \pm 8.32)$ compared to those treated with alphacalcidol $(13.5 \pm 7.29 ; p=0.000)$. At baseline both the global Tinetti score and the balance specific Tinetti score were directly correlated with the serum 25OHD concentration (correlation coefficients of 0.165 and 0.236 ; $p=0.022$ and 0.001 , respectively). After treatment, the mean results of the Tinetti global and balance-specific items increased slightly but significantly ( $p=0.000$ for both scores). The analysis on age groups revealed significantly more important beneficial effects in both scores in older patients (over 70 years old) ( $p=0.003$ and 0.004 for global and balance item scores, respectively). No differences were noted between the cases treated with alphacalcidol compared to those offered cholecalciferol.

Conclusions: Improvement of VD status is associated with improvement of balance in VD-deficient women, especially in the elderly. Alphacalcidol exerts similar positive effects, despite no apparent increase in serum 25OHD concentration.

\section{P154}

REDUCE ROUTINE BLOOD ORDERING IN DEGENERATIVE LUMBAR ARTHRODESIS SURGERY AT ONE AND TWO LEVELS

M. Angulo Tabernero ${ }^{1}$, D. Peña Jimenez ${ }^{1}$, E. Suñen Sanchez ${ }^{1}$, A. Loste Ramos ${ }^{1}$, A. Tabuenca Sanchez ${ }^{1}$, A. Charlez Marco ${ }^{1}$, J. Rodriguez Vela ${ }^{1}$

${ }^{1}$ Department of Orthopaedic Surgery. H. U. Miguel Servet, Zaragoza, Spain

Introduction: The main surgical activity in the Spinal Surgical Unit at University Hospital Miguel Servet in Zaragoza is based on lumbar arthrodesis regarding the cases of degenerative disease of the lumbar spine with more than 150 cases per year. It is a routine procedure to order blood prior to the operation. However, the necessity of transfusion is uncommon in lumbar arthrodesis surgery which affects one or two levels.

Taking into account this situation, it has been thought the possibility of reducing the routine blood ordering and crossmatch for surgeries of one or two levels, with the consequent economical saving and use of resources involved.

Objective: 1 . To quantify in a prospective way the real necessities of blood transfusion in patients who are going to be operated on lumbar arthrodesis stratifying it according to the levels that could be joined, the quantity of hematocrit and hemoglobin prior to the surgery, the comorbidities and previous treatments. 2. To identify the risk factors in transfusion in these patients. 3 . To estimate the potential saving and reduction in the use of resources that could be obtained by not doing the routine blood ordering in this surgery.

Material and Methods: Data capture of all patients who are going to be operated on lumbar arthrodesis in the Spinal Surgical Unit at the University Hospital Miguel Servet in Zaragoza, classifying them according to the number of levels that could be joined (one level, two levels and three or more levels). The needed information about patients will be: age, sex, hematocrit-hemoglobin preoperative and post-operative, necessity of postoperative transfusion (Yes/No), volume of transfused milliliters, usual medicines that alter coagulation, diseases and physical conditions that may imply a high risk of bleeding and surgical incidents which have produced a higher bleeding.

Results: If the obtained results supported what has been analysed in our clinical experience, new criteria in blood 
reserves policy for the lumbar spinal fusion surgery of one or two levels could be established.

Conclusions: It is expected that our study means a saving for such a frequent process in lumbar spine surgery.

\section{P155}

HOW DOES THE INITIAL SLOPE OF THE VARIOGRAM, THE TEXTURAL BASIS OF THE TRABECULAR BONE SCORE, PREDICT VERTEBRAL STRENGTH?

$\underline{\text { P. K. Zysset }}^{1}$, G. Maquer ${ }^{1}$, Y. Lu ${ }^{2}$, E. Dall'Ara ${ }^{3}$, Y. Chevalier ${ }^{4}$, M. Krause ${ }^{5}$, L. Yang ${ }^{3}$, R. Eastell ${ }^{3}$, K. Lippuner ${ }^{1}$

${ }^{1}$ Institute for Surgical Technology and Biomechanics, University of Bern, Bern, Switzerland, ${ }^{2}$ Hamburg University of Technology, Hamburg, Germany, ${ }^{3}$ University of Sheffield, Sheffield, United Kingdom,

${ }^{4}$ Klinikum Grosshadern, Munich, Germany, ${ }^{5}$ Department of Osteology and Biomechanics, University Medical Center Hamburg-Eppendorf, Hamburg, Germany

The Trabecular Bone Score (TBS) is a 2D textural parameter based on the initial slope of a variogram (ISV) and was reported to be reduced in the DXA images of patients with vertebral fractures. A recent study suggested that bone volume fraction and trabecular anisotropy explain up to $98 \%$ variation of the elastic and yield properties of cubic regions of interest of human trabecular bone and that further textural parameters such as ISV did not bring any further contribution. The aim of this study was therefore to examine the ability of ISV to predict vertebral strength using three different biomechanical experiments. In a first experiment, vertebral bodies $(n=13)$ were loaded in compression to failure between two intervertebral discs. In a second experiment, vertebral bodies $(n=12)$ were freed of their posterior elements, embedded in PMMA and compressed to failure between two parallel plates. In a third experiment, vertebral bodies $(n=37)$ were freed of both posterior elements and endplates and were compressed axially to failure with a ball joint. A synthetic DXA image was projected along the AP direction from the QCT data of all vertebral bodies. Both aBMD and ISV were computed for each DXA projection and combined in a multi-linear model to predict the ultimate force measured in each biomechanical experiment. The correlations between aBMD and ultimate force were moderate for all experiments $\left(0.58<\mathrm{r}^{2}<0.70\right)$, but ISV did not bring any further significant contribution $(p>0.05)$. As a limitation, the initial slope of the variogram used in this study was not the commercial implementation of TBS but was significantly $(p<0.001)$ correlated to it with $\mathrm{R}^{2}=0.75$. To conclude, beyond aBMD, ISV did not contribute to the prediction of vertebral strength and the causal link between TBS and vertebral fractures remains unknown.

\section{P156}

\section{HUNT FOR THE TUMOR OF OSTEOMALACIA} S. Rajoo ${ }^{1}$, M. B. N. Long Bidin ${ }^{1}$, Y. Imanishi ${ }^{2}$

${ }^{1}$ Endocrine Unit, Kuala Lumpur General Hospital, Kuala Lumpur, Malaysia, ${ }^{2}$ Metabolism, Endocrinology and Molecular Medicine, Graduate School Of Medicine, Osaka City University, Osaka, Japan

Oncogenic osteomalacia (OO) is a rare endocrinological paraneoplastic syndrome characterised by defective bone mineralisation from renal phosphate loss. Establishing a diagnosis of OO may be perplexing especially in localising the causal tumour. We report a case of a 39 year old man who presented with progressive weakness over 4 years with severe osteoporosis. He had hypophosphatemia, low total vitamin D and 1,25-dihydroxyvitamin D [(1,25(OH)D3] levels. Serum calcium and intact PTH levels were normal. Phosphate and vitamin D levels failed to improve despite oral supplementation of these minerals. Positron emission tomography with fluoro-D-glucose (PET-FDG) revealed hypermetabolic uptake in the greater trochanter of the right femur which corresponds to magnetic resonance imaging (MRI) of right femur. Initial biopsy of the lesion demonstrated phosphaturic mesenchymal origin tissue which stained positive for fibroblast growth factor-23 (FGF-23). His concurrent serum levels for FGF-23 was $543.4 \mathrm{pg} / \mathrm{ml}$ (normal range (NR), $<40 \mathrm{pg} / \mathrm{ml}$ ). Post tumor resection, patient's phosphate levels normalised and patient regained his muscle bulk and strength. $\mathrm{OO}$ is characterised by hypophosphatemia secondary to an increased activity of novel phosphatonins produced by the neoplasm. One such phosphotonin is FGF-23 which reduces $1,25(\mathrm{OH}) \mathrm{D} 3$ concentrations. Removal of the tumour reverses symptoms and biochemical markers leading to dramatic improvement. However, diagnosis of the oncogenic cause of osteomalacia may be delayed and unrecognised as the tumours are frequently small, asymptomatic and often difficult to find, as in our patient. Early detection and treatment can prevent the progression of the disease to incapacitating disability. 


\section{P157}

\section{TERIPARATIDE: A NEW TOOL FOR ARTHRO- DESIS IN THE AGED SPINE - INITIAL RESULTS IN 17 PATIENTS}

F. A. Lorente Muñoz ${ }^{1}$, S. Cortes Franco ${ }^{1}$

${ }^{1}$ Neurosurgery, University Hospital Miguel Servet, Zaragoza, Spain

Objective: Surgery for lumbar spine arthrodesis in elderly patients has experienced an increased frequency over the past few y; morbidity and mortality in this sense has been proven to be associated to the lack of bone quality and difficulties with fusion. Recombinant human teriparatide [rhPTH(134)], a drug used in the treatment of osteoporosis, has been proposed as a possible co-adjuvant treatment to improve these problems, thanks to its anabolizing effect on bone metabolism. In this study, we analyze the possible beneficial effect of teriparatide as co-adjuvant treatment in elderly patients with poor bone quality who have been operated due to lumbar degenerative disease with a posterolateral arthrosis.

Material and Methods: We present a prospective clinical series of 17 patients of over 70 years of age in whom surgical posterolateral arthrodesis was carried out and who received teriparatide during 18 months as co-adjuvant treatment.

Results: In our series, we found a fusion rate of $94.1 \%$, without observing any vertebral fractures, arthrodesis hardware failure (pull-out) or any other complications associated to bone failure. Moreover, a reduction in pain and in the disability in the VAS and ODI scales was verified after the combined treatment with surgery and teriparatide, which remained stable during follow-up.

Conclusions: In our series, we found promising results with a high rate of fusion, without recording any complications. Teriparatide could be a useful treatment when it comes to favoring lumbar arthrodesis by increasing mineral bone density. However, randomized studies with a greater number of patients are needed to demonstrate the possible beneficial effect.

\section{P158}

SURGICAL FAILURE IN PATIENT WITH CERVICAL FRACTURE AND ANKYLOSING SPONDYLITIS TREATED SUCCESSFULLY WITH TERIPARATIDE ADMINISTRATION

S. Cortes Franco ${ }^{1}$, F. A. Lorente Muñoz ${ }^{1}$

${ }^{1}$ Neurosurgery, University Hospital Miguel Servet, Zaragoza, Spain

Objective: Cervical fractures often require surgical treatment to achieve proper stabilisation and thus prevent neurological damage associated with an unstable cervical spine. Systemic diseases such as ankylosing spondylitis hinder the surgical treatment of fractures, mainly because of the osteoporosis induced by long-term immunosuppressive and glucocorticoid treatments. They also pose a surgical challenge because the approach is limited by stiffness and kyphotic deformity of cervical spine.

Material and Methods: We report a 52-year-old male patient with a long history of ankylosing spondylitis, who suffered a whiplash injury, resulting in a $\mathrm{C} 7$ fracture. We were not able to perform the right approach because of patient's comorbidity, so we started with teriparatide and orthotic treatment.

Results: Two months after teriparatide treatment beginning, the patient was in significantly less pain. Cervical spine CT showed a clear bone consolidation and 12 months later patient remains in the same clinical situation.

Conclusions: The combination of ankylosing spondylitis and vertebral fracture makes it very hard to treat these two conditions. In the event of pseudoarthrosis or when it is impossible to perform appropriate surgical treatment, teriparatide may be an useful treatment because of its bone anabolic action that prevents the complications associated with prolonged orthotic treatment.

P159

ASSESSMENT OF THERAPEUTIC EFFICACY OF WRIST SPLINT IN THE MANAGEMENT OF CARPAL TUNNEL SYNDROME (CTS) LITERATURE REVIEW

S. S. Salih $^{1}$

${ }^{1}$ Bolton GGC, Bolton, United Kingdom

Introduction: "Management of carpal tunnel syndrome should be based on severity. Patients with mild to moderate carpal tunnel syndrome have normal to mildly abnormal nerve conduction study and electromyography findings. Severe disease is suggested by worsening clinical symptoms and clearly abnormal electrodiagnostic studies. Six weeks to three months of conservative treatment is reasonable in pa-

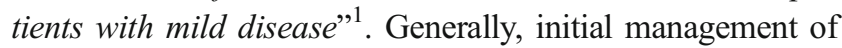
CTS is conservative through holding the wrist in the neutral position overnight using special therapeutic wrist splints, alongside adequate analgesia and considering possible reversible causative factors such as wrist inflammation, obesity, repetitive occupational movements and hormonal factors ${ }^{2}$.

Aim: To evaluate whether wrist splinting is beneficial in the medical management of carpal syndrome. The outcomes of the studies have been measured using a wide range of qualitative and quantitative criteria. See table 1

Methodology: Online databases had been searched, namely, PUBMED, MEDLINE, and Google Scholars for relevant literature published in English in during the last 10 years. Selection criteria: RCT and observational studies comparing wrist splints with no treatment (or a placebo. Also, studies comparing one splint type or regimen vs. another. Excluded 
studies are these comparing splinting with other interventional treatment (steroid injection or surgery).

Results: Initial search yielded 46 articles. 21 articles were duplicates across the utilised databases. The vast majority of articles were either describing therapeutic outcomes of surgical management or comparative studies between surgical and non-surgical management of CTS.

Five relevant articles were eventually identified: (Brininger et al 2007), (M. V. De Angelis et al 2008), (Shingo Nouta et al 2009), and (Forogh, B. et al 2012), (Bo Povlsen et al 2013).

Two randomised control trials were conducted in 2008 and 2013 respectively. The first study compared the efficacy of wrist splint to a soft hand brace recruiting 120 patients over a period of three months. The outcome demonstrated that both devices induced symptomatic and functional benefit in nearly equal number of patients.

The other RCT involved 74 mildly symptomatic patients with CTS. 30 of them used the wrist splint as opposed to a control group of 24. A significant positive impact was recorded on measures of symptom severity and function in the intervention group.

These results have been further reinforced by another RCT completed in 2007, which compared the effects of a neutral wrist and metacarpophalangeal (MCP) splint with a fabricated wrist cock-up splint. 51 patients only completed the study. The results revealed that splints support the wrist and MCP joints in neutral are more effective than the fabricated customized (wrist cock-up) splint.

The above three studies have only evaluated short term outcomes in CTS patients treated with wrist splint. However, another study took place in 2013 on 75 patients with CRT, Which aimed at assessing long-term impact of using wrist splint in CTS. 75 patients treated with overnight wrist splint for 33 months, then referred afterwards to the hand surgeon. 52 of them chose to carry on with wrist splint and refused surgical option. The two groups were followed for further assessment in 33 months period. Patients adherently treated with wrist splint alone were more satisfied compared to their counterparts who failed wrist splint or had surgical decompression.

In 2008, a large case series study was carried out on 214 mildmoderate CTS hands. All cases were treated with mechanical wrist splints only. The follow-up period in this study was 7 months which, compared to the former study (33 months), is relatively shorter. It, however, reasonably examined long-term outcome of treating CTS with wrist splint. The results of this study showed that $74 \%$ of the cohort showed good to excellent outcome. The study has concluded that wrist splinting is effective in mild-moderate severity CTS.

Conclusion: Wrist splinting is an effective measure in managing mild to moderate CTS according to these clinical trials. However, almost all the included studies are vague about the definition of mild-moderate stages of the condition. This is mainly because there is no clear description of the nature of these patients apart from their age and sex. What is mild in an 84 year old patient might be moderate or severe and lifeimpacting in a 30 year old active patient. It is, therefore, important to conduct a study more specific and categorised according to patient's age and their living nature and levels of activates for more robust and reliable outcomes.

Moreover, this literature review is, merely, about assessing the use of wrist splint in CTS patients more or less solely, which is deemed successful according to the results. Yet, other management options might be far more satisfactory and beneficial compared to splinting, such as local steroid injection, and surgical intervention. This potential comparison is essentially a valid base for further researches or extensive literature review on existing evidences.

Table 1 Outcome measures used in the clinical trials

\begin{tabular}{ll} 
Clinical trial & Outcome measured used \\
\hline Brininger et al. & $\begin{array}{l}\text { CTS Symptom Severity Scale (SSS) and the } \\
\text { Functional Status Scale (FSS) }\end{array}$ \\
M. V. De Angelis et al. & $\begin{array}{l}\text { Boston Carpal Tunnel Questionanaire (BCTQ) } \\
\text { and in Visual Analogical Scale (VAS) for } \\
\text { pain and paresthesias } \\
\text { Shingo Nouta et al. }\end{array}$ \\
$\begin{array}{l}\text { Motor nerve conduction measurement was } \\
\text { perfomed in all cases before and } \\
\text { after treatment }\end{array}$ \\
$\begin{array}{l}\text { Semmes-Wein stein monpfilaments, Two Point } \\
\text { discrimination, Visual analog Scale (VAS), } \\
\text { pinch and Grip Strength } \\
\text { Baseline pain and numbness levels were } \\
\text { recorded on a Visual Analogue Scale (VAS) } \\
\text { using a questionnaire upon first presentation }\end{array}$ \\
$\begin{array}{l}\text { A further questionnaire assessing pain and } \\
\text { numbness levels, patients' satisfaction with } \\
\text { the treatment, and whether they had } \\
\text { subsequent surgical decompression }\end{array}$
\end{tabular}

\section{References:}

1. KIM EDWARD Leblanc, MD, Phd,nd WAYNE CESTIA, MD, Louisiana State University Health Sciences Center, New Orleans, Louisiana.. Am Fam Physician. 2011 Apr 15;83(8):952-958 http://www.aafp.org/afp/2011/0415/ p952.html

2. Richard A Watts, Philip Conaghan, Chris Denton, Helen Foster, John Isaacs, Ulf Müller-Ladner. Oxford textbook of Rheumatology, fourth edition Oxford textbook of Rheumatology, fourth edition 2013. https://books.google.co.uk/ books?id=EXGrAAAAQBAJ

3. M. V. De Angelis, F. Pierfelice, P. Di Giovanni, T. Staniscia and A. Uncini.. Article first published online: 13 JUL 2008. DOI: 10.1111/j.1600-0404.2008.01072.x.. http://onlinelibrary. wiley.com/doi/10.1111/j.1600-0404.2008.01072.x/abstract 
4. Baker NA; Moehling KK, Desai AR, Gustafson NP. John Wiley \& Sons Country of Publication: United States NLM ID: 101518086 Publication Model: Print Cited Medium: Internet ISSN:2151-4658 (Electronic) Linking ISSN: 2151464X NLM ISO Abbreviation: Arthritis Care Res (Hoboken) Subsets: MEDLINE.

5. Forogh, B.; Mohammadi, A.; Azad, A. Journal of Gorgan University of Medical Sciences 2009 Vol. 10 No. 4 pp. 18-21, 93. ISSN 1562-4765. http://www. cabdirect.org/abstracts/20093301544.html

6. Brininger TL, Rogers JC, Holm MB, Baker NA, Li ZM, Goitz RJ. Department of Occupational Therapy, School of Health and Rehabilitation Sciences, University of Pittsburgh, Pittsburgh, PA, USA. Arch Phys Med Rehabil. 2007 Nov;88(11):1429-35. http://www.ncbi.nlm.nih.gov/pubmed/ 17964883

7. Bo Povlsen, Muhammad Bashir \& Fabian Wong, Journal of Plastic Surgery and Hand Surgery Volume 48, Issue 3, 2014. pages 175-178. http://www.tandfonline.com/doi/full/10. 3109/2000656X.2013.837392

8. Shingo Nobuta, Katsumi Sato, Tomowaki Nakagawa, Masahito Hatori \& Eiji Itoi. 12 Jul 2009. 10.3109/20001967-228: http://www.tandfonline.com/doi/abs/10.3109/ 2000-1967-228

\section{P160}

INFLUENCE OF PREOPERATIVE BONE MASS DENSITY IN PERIPROSTHETIC BONE REMODELING: A 10-YEAR FOLLOW-UP

A. Aguilar ${ }^{1}$, J. J. Panisello ${ }^{1}$, J. López ${ }^{1}$, L. Gracia ${ }^{2}$

${ }^{1}$ Department of Orthopaedic Surgery. H. U. Miguel Servet, Zaragoza, Spain, ${ }^{2}$ Obispo Polanco Hospital, Teruel, Spain

Introduction: Bone remodeling after total hip arthroplasty is a common event observed in all prosthetic designs. Preoperative Bone Mass Index has proven to be an important factor in periprosthetic bone remodeling in short follow-up studies.

Material and methods: We used DXA to perform a 10-year follow-up study of 39 patients with a unilateral, uncemented THA. BMD measurements were made at 6 months, 1, 3, 5 and 10 years after surgery. Pearson coefficient was used to quantify correlations between preoperative bone mass density and periprosthetic BMD in the seven Gruen zones at 6 months, 1 , 3,5 and 10 years.

Results: Preoperative BMD was a good predictor of periprosthetic BMD one year after surgery in zones 1,2,4,5 and 6 (Pearson index from 0.61 to 0.75 ). Three years after surgery it has good predictive power in zones 1,4 and 5 (0.71-0.61), although in zones 3 and 7 low correlation was observed one year after surgery ( 0.51 and 0.57 respectively). At the end of the follow-up low correlation was observed in the seven Gruen zones. Sex and BMI were found to not have a statistically significant influence on periprosthetic bone remodeling.

Conclusion: Although preoperative BMD seems to be an important factor in periprosthetic remodeling 1 year after THA implantation, it is losing predictive power progressively; not being a major factor in periprosthetic remodeling 10 years after surgery.

\section{P161}

HEALTH-RELATED QUALITY OF LIFE, BACK PAIN AND RISK OF FRACTURE IN OSTEOPOROTIC PATIENTS AFTER TERIPARATIDE TREATMENT

M. Osca Guadalajara ${ }^{1}$

${ }^{1}$ Hospital Obispo Polanco Teruel, Teruel, Spain

Introduction: Osteoporosis is a generalised metabolic disease characterised by loss of bone quantity and quality. In clinical terms, the disease remains silent until a bone fracture occurs, considered to be the most significant consequence of osteoporosis, which has been associated with increased risk of posttreatment fracture, higher direct health care costs, increased morbidity and mortality risk, and worse quality of life and increased pain.

Objectives: The aim of this study is to evaluate back pain, risk of fracture and quality of life in patients with severe osteoporosis treated with subcutaneous teriparatide, a bone induction drug that promotes bone remodeling.

Methods: A prospective observational study with 49 patients treated in the Pain Unit of Hospital Obispo Polanco of Teruel (Spain). They were treated with teriparatide between April 2006 and February 2014. Pain was assessed by the Visual analogue scale (VAS). Health-related quality of life was measured using the European Quality of Life Questionnaire (EuroQol-5D). Pre and post- treatment values were collected respectively, and the number of fractures too. A regression analysis was done to obtain an explicative model of factors.

Results: After the treatment period, improvement in pain was observed in $82 \%$ of the patients. The mean VAS value went from 5.42 to 3.47 , with mean pain improvement measured as 1.76 points. Health-related quality of life increased in $61.22 \%$ of the patients. The mean health status value rose from 0.36 to 0.598 points. VAS-measured pain improvement showed significant pain recovery in the patients with a higher VAS value before treatment. After treatment ended, the number of patients who had a fracture lowered from 30 to 12 . Nonetheless, it was not possible to confirm this fact either statistically or using the duration explanatory variable.

Conclusions: Teriparatide reduces pain, number of fractures and improves quality of life in osteoporotic patients. Studies into the effect of TPTD on reducing pain and improving 
quality of life have been carried out in eight European countries. Nonetheless, no previous studies conducted on this subject in Spain have been found. One major novel aspect that our study offers, unlike other studies, is that patients' initial health status is an important factor for improving VAS and healthrelated quality of life. The worse the baseline situation, the more patients' health improved.

\section{P162}

\section{BONE-ADIPOSE TISSUE RELATIONSHIP}

S. E. Oros ${ }^{1}$, O. Ianas ${ }^{2}$, D. A. Manda ${ }^{1}$, S. Vladoiu ${ }^{1}$, M.

Giurcaneanu $^{1}$, G. Voicu ${ }^{1}$, M. Stoiceanu ${ }^{1}$, R. I. Rosca ${ }^{1}$, D. L. Paun $^{1}$, C. Neamtu ${ }^{1}$, C. Dumitrache ${ }^{1}$

1“C.I. Parhon" National Institute of Endocrinology, Bucharest, Romania, ${ }^{2}$ Romanian Academy-Medical Science, Bucharest, Romania

We previously reported higher adiponectin values in women with osteoporosis compared to age matched women with metabolic syndrome and higher leptin mean values in subjects with metabolic syndrome, positively correlating with hip BMD. We aimed to see the influence of bisphosphonates and vitamin D supplementation on adipokines, cytokines and bone markers. Subjects and methods: 40 subjects were re- evaluated, 6 from the osteoporotic group with metabolic syndrome (group 1), 10 from the osteoporotic group (group 2 ) and 24 from the group with metabolic syndrome without osteoporosis (group 3). Fasting blood samples were used to re-evaluate their haematological, biochemical profile, bone turn-over markers and adipocytokines after one year of antiosteoporotic treatment and vitamin D supplementation. We used R\&D System commercial kits, provided by Cybermed. Control DXA of the spine and the hip (left) was performed also during the second visit. Metsyn was defined using the International Diabetes Federation's criteria. Results were statistically analyzed using SPSS program, version 15. Results are expressed as means \pm standard error and were considered statistically significant if $\mathrm{p} \leq 0.05$.

Results: Pairs sample $t$-test was used to evaluate the influence of osteoporosis treatment and vitamin D supplementation on metabolic, BMD and anthropometric features. The correction of vitamin D led to the improvement of hip BMD $(p=0.008)$, triglycerides $(p=0.024)$ and inflammatory state $(p=0.007)$, although our subjects put on weight $(p=0.045)$, had higher glucose levels $(p=0.05)$ and leptin levels $(p=0.012)$. Spine BMD $(p=0.032)$ improved also in group 2 , with a raise in HDL-cholesterol $(p=0.044)$, insulinemia $(p=0.025), 25-\mathrm{OH}$ vitamin $\mathrm{D}(p=0.014)$ and $\mathrm{TNF} \alpha(p=0.002)$. In group 3 , insulin resistance improved simultaneously with $25-\mathrm{OH}$ vitamin $\mathrm{D}(p=0.004)$. We also noticed a raise in HDL-cholesterol $(p<0.001)$, resistin $(p=0.001)$, leptin $(p=0.004)$ and TNF $\alpha$ $(p<0.001)$.
Conclusion: We may say that leptin plays a key role in adipose-bone tissue relationship and correction of vitamin D insufficiency or deficiency conducts to spine and hip BMD, insulin resistance improvement and a raise in HDLcholesterol.

Acknowledgement: This study is funded by the research project PN II-RU-PD 97/2010

\section{P163}

\section{REVERSE TOTAL SHOULDER ARTHRO- PLASTY IN THE TREATMENT OF OSTEOPO-} ROTIC PROXIMAL HUMERUS FRACTURE

J. López $^{1}$, M. Lillo ${ }^{1}$, N. Perez ${ }^{1}$, V. Roda ${ }^{1}$, A. Aguilar ${ }^{1}$

${ }^{1}$ Department of Orthopaedic Surgery. H. U. Miguel Servet, Zaragoza, Spain

Introduction: The Reverse Total Shoulder Arthroplasty, initially emerged as an alternative for the treatment of massive and irreparable rotator cuff injuries in the elder, arthropathy secondary to chronic rupture of the cuff, and could not benefit from the anatomical glenohumeral arthroplasty. Over time current indications have been extended to other pathologies like osteoporotic proximal humerus fractures.

Objective: The aim of this study was to evaluate clinical and radiographic short-medium term results of the use of the reverse shoulder arthroplasty in the treatment of osteoporotic proximal humerus fractures. Materials and Methods: A retrospective study was performed at the HUMS on 14 patients undergoing reverse total shoulder arthroplasty from 2006 to 2009. The indications for surgical intervention were osteoporotic proximal humerus fracture. Patient-oriented outcomes were obtained using the Constant Score postoperatively at 3, $6,12,24$ months. In addition, functional ranges of motion were measured in forward elevation and internal and external rotation. Preoperative and postoperative Visual Analog Scale (VAS) were recorded.

Results: The mean patient age was 72.6 years (range, 5175 years). The mean follow-up was 5.3 years (range, 6 months -8 years). $87 \%$ of the patients were women. $63 \%$ were right shoulders and $37 \%$ left shoulders. The average preoperative pain score was 7 , with a range from 5 to 9 . Preoperative Constant score averaged 23.9, with a range from 16 to 31 . Postoperatively, all scores improved with a mean pain score noted at 1 , range $0-6$; mean constant 39.2 , with a range $21-51$; mean abduction was 60 , forward elevation was $70^{\circ}$, external rotation of $31^{\circ}$. Internal rotation $33^{\circ} .3$ patients were satisfied with the functional results of surgery while 6 were somewhat dissatisfied and 5 were dissatisfied. The overall incidence of complications was 8 cases, 3 cases of scapular notching, 3 cases of glenohumeral instability, 2 periprosthetic fractures. 
Conclusions: Reverse arthroplasty is an excellent therapeutic option for the treatment of proximal humerus fracture, providing good pain relief, but their long-term functional results are not entirely satisfactory than expected, design improvements are necessary in order to achieve better results.

\section{P164}

\section{TREATMENT OF OSTEOPOROTIC PERIPROS- THETIC HIP FRACTURES WITH DALL MILES} PLATE SYSTEM

M. Lillo ${ }^{1}$, J. López ${ }^{1}$, N. Perez ${ }^{1}$

${ }^{1}$ Department of Orthopaedic Surgery. H. U. Miguel Servet, Zaragoza, Spain

Introduction and objectives: Despite the success of total hip prosthesis is inevitable in many cases the appearance intraand postoperative complications. These complications include: vascular lesions, peripheral nerve injuries, osteoporotic periprosthetic fractures and leg discrepancy. Osteoporotic periprosthetic fracture after a total hip arthroplasty is a common complication due to the increase in hip replacements and the advanced age of the population. Is more common in women with bone comorbidities (osteolysis, osteoporosis) and metabolic diseases (rheumatoid arthritis, corticosteroids, Paget., etc.), after a low-energy trauma or even without it. For the classification of these fractures is used the Vancouver classification, which divides them into types $\mathrm{A}, \mathrm{B}$ and $\mathrm{C}$.

Material and Methods: A retrospective study was performed at the HUMS on 20 patients undergoing Dall Miles plate cable system for the treatment osteoporotic periprosthetic hip fractures, from 2013 to 2014. We analyzed the indications and the complications in the use of this system.

Results: The mean patient age was 76,3. 16 patients were women. In all cases occurred after fall from their own height. 12 cases were classified as B1 Vancouver classification, 6 as B2 and 2 as B3. In cases classified as B1 they were treated by open reduction and osteosynthesis with plate Dall Miles. In cases classified as B2 and B3 the system plate dall miles was associated to the implantation of cemented long stem. The patients was allowed to start walking with partial weight bearing at six weeks after surgery.

There were no complications of the surgical wound. At 12 months after surgery in all cases seen radiographic bone healing. 12 patients refer no subjective sensation of pain and almost rejoined his normally activities. 8 patients had to limit considerably its activity.

Conclusion: Osteoporotic periprosthetic hip fractures incidence is increasing in our health area. For the prevention of these pathology, it is important to educate doctors and patients, monitoring and treating osteoporosis, advising the patient regarding risk activities and caring intraoperative surgical technique.

\section{P165}

MUSCULOSKELETAL INVOLVEMENT IN NEUROMUSCULAR GENETIC DISEASES: THE CASE STUDY OF A FAMILY AFFECTED BY LATE ONSET POMPE DISEASE

D. Calafiore ${ }^{1}$, A. Moretti ${ }^{1}$, A. de Sire ${ }^{1}$, M. T. Giamattei ${ }^{1}$, F. Gimigliano $^{2}$, G. Iolascon ${ }^{1}$

${ }^{1}$ Department of Medical and Surgical Specialties and Dentistry, Second University of Naples, Naples, Italy, ${ }^{2}$ Department of Physical and Mental Health and Preventive Medicine, Second University of Naples, Naples, Italy

Objective: To characterize the bone phenotype, including bone metabolism and skeletal manifestations, in some family members affected by late-onset Pompe disease (LOPD) with the same genotype.

Materials and Methods: In a family of 13 siblings ( 9 males and 4 females) born from nonconsanguineous parents, of which 10 were affected by LOPD, we studied the 7 siblings ( 4 men, 3 women) that presented the same genotype (p.R40X/p.N882fs). The diagnosis of LOPD was confirmed through genetic mutation analysis and measurement of decreased acid alfa-glucosidase gene (GAA) activity in fibroblasts. The BMD at the total body less head (TBLH), at the lumbar spine (LS; L1-L4), and at the femoral neck (FN) was measured by DXA; a standard X-ray lateral projection was performed for each patient. Biochemical monitoring of bone metabolism was also performed. Moreover, muscle strength was assessed by an hand-held Jamar hydraulic dynamometer. All patients started Enzyme Replacement Therapy (ERT) with recombinant human acid $\alpha$ glucosidase (rhGAA) on 2011 and ambulated independently without aids.

Results: The 7 siblings analyzed in the present study had a mean (SD) age of $52(6.2)$ years. Of all patients, 2 had osteoporosis (Ts $<-2.5 \mathrm{SD}), 2$ had a low bone mass (Ts $>-2.5 \mathrm{SD}$ and $<-1 \mathrm{SD}$ ); these 4 patients had also hypovitaminosis $\mathrm{D}$ $(<30 \mathrm{ng} / \mathrm{ml})$. One of them, a female, had also 2 vertebral fragility fractures. Furthermore, all male patients had low grip strength. In all patients there were not alterations of other biochemical parameters.

Conclusions: Results of this study suggested that low BMD, hypovitaminosis $\mathrm{D}$, and reduced muscle strength are common features in our cohort of patients affected by LOPD with p.R40X/p.N882fs genotype. Therefore, assessment of skeletal fragility, muscle function, and 25-OH-D3 serum levels should not be limited to inactive and immobile patients, but it should be also included in an early evaluation of all subjects affected by LOPD. However, further research is needed to fully elucidate the mechanism of bone metabolic alterations in Pompe disease and the effect of ERT on bone involvement in these patients. 


\section{P166}

\section{STRESS FRACTURE OF THE DISTAL TIBIA FOLLOWING TOTAL KNEE ARTHROPLASTY} I. Sancho ${ }^{1}$, A. Echeverria ${ }^{2}$

${ }^{1}$ Department of Trauma and Orthopedics. Hospital Reina Sofia de Tudela, Tudela, Navarra, Spain, ${ }^{2}$ Department of Internal Medicine. Hospital Reina Sofía de Tudela, Tudela, Navarra, Spain

Background: Stress fractures of the lower limb following total knee arthroplasty (TKA) occur primarily in patients in which a considerable limb axis realignment is required. The majority of these fractures are located on the femoral neck and the proximal part of the tibia being typically reported in patients with osteoporosis. To our knowledge, distal tibia stress fractures as an adverse event following TKA have not been described yet.

Material and Methods: In the present study we present a case of TKA in which the patient suffered a stress fracture of the distal tibia six weeks after replacement. Our patient suffered from osteoporosis but she had never received any specific treatment. Preoperative diagnosis was knee varus osteoarthritis with an anatomical lower limb axis of $186^{\circ}$. Surgery was carried out uneventfully and the patient started the ordinary post-TKA rehabilitation program. Six weeks postoperatively, our patient presented acute pain development on the operated extremity, as well as perimalleolar warm and swelling. Radiographs showed a transverse sclerotic line through the metaphyseal region of the distal tibia and fibula. Serum levels of bone metabolism revealed hypovitaminosis $\mathrm{D}$ and the skeletal survey did not show any additional fractures.

Results: The patient was prescribed Calcium and Vitamin D supplementation and Teriparatide. Symptoms subsided in a few weeks with no weight-bearing. Image studies exhibited proper healing of the fracture. The physical therapy program was restarted, and after 1 year of follow-up she has no pain or limitations when carrying out her daily activities.

Conclusions: Distal tibial stress fractures following TKA should be considered as a possible complication during the postoperative period, especially in osteoporotic individuals. A high index of suspicion is needed for an early diagnosis and a proper treatment is requested for a correct final outcome.

\section{P167}

MORTALITY RISK FACTORS FOR HIP FRACTURE IN ELDERLY PATIENTS

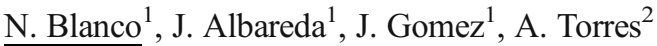

${ }^{1}$ Hcu Lozano Blesa, zaragoza, Spain, ${ }^{2}$ Hospital Obispo

Polanco Teruel, Teruel, Spain

Introduction: The incidence of hip fractures increases with the progressive aging of the population. The hip fracture has more mortality than other types of osteoporotic fractures.
These fractures are a problem that concern three levels: epidemiological, assistance and economical. The aim of the study is to evaluate which factors have more influence in the mortality of the patients with hip fracture.

Material and Methods: A prospective study was performed including patients with hip fractures treated in our department. The data collection period lasted 1 year, from 1 st November 2011 until 31th October 2012. The total number of patients included in the study was 303 . The survival of the study sample and the risk factors were studied using R Core Team Version 3.0.1 statistical software and Kaplan-Meier survival curves. It was used a significance level of $p=0.05$.

Results: The follow-up was at least of 24 months. At the time of admission, the average age of the patients was 84,26 years. The majority of the patients were female $(78,5 \%)$. After the first year following the survival rate was $77,5 \%$. Almost all excess mortality occurred during the first 6 months following. In order of significance, key factors negatively influencing mortality at the end of the following were: age, previous heart disease, poor previous functionality, blood transfusion requirements, waiting time before operation over three days, malnutrition, low hemoglobin level and medical complication during admission.

Discussion: The annual mortality rate after hip fracture is similar to other series. Among the risk factors, waiting time for surgery, blood transfusion requirements, malnutrition and medical complication could be modified. A number of other studies have confirmed the importance of optimizing these factors.

\section{P168}

ESTIMATION OF THRESHOLD SERUM 25-HYDROXYVITAMIN D CONCENTRATIONS FOR MULTIPLE MUSCULOSKELETAL OUTCOMES IN CHINESE OLDER WOMEN

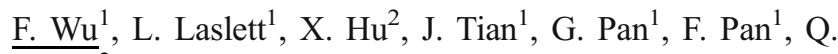
$\overline{\text { Zhang }}^{2}$

${ }^{1}$ Menzies Institute for Medical Research, Hobart, Australia, ${ }^{2}$ National Institute for Nutrition and Health, Chinese Centre for Disease Control and Prevention, Beijing, China

The optimal vitamin D status for musculoskeletal health outcomes remains uncertain, and existing evidence is primarily obtained from Caucasian populations. This study examined thresholds of serum 25-hydroxyvitamin D (25OHD) associated with BMD, muscle strength and balance in older Chinese women. Participants of this cross-sectional study were 441 community-dwelling women aged $60-88$ years (mean age, 68 years). Differences in total body, lumber spine, total hip and femoral neck BMD using DXA, grip strength, lower limb muscle strength (LMS), static (one-leg standing time with eyes closed) and dynamic (timed up and go test) balance tests were assessed across 4 groups of serum 25OHD 
concentrations $(<25,25-37.5,37.5-50$, and $>50 \mathrm{nmol} / \mathrm{L})$ using multivariable linear regression models. Thresholds for associations between 25OHD and all outcomes were estimated using locally weighted smoothing (LOWESS) plots and piecewise regressions. Serum 25OHD was $<50$ and $75 \mathrm{nmol} / \mathrm{L}$ in 78.0 and $95.0 \%$ of the participants, respectively. BMD was greater in the higher 25OHD categories for total body and all sites ( $\mathrm{p}$ for trend $<0.05$ ). Although the shortest one-leg standing time was observed in the $25-37.5 \mathrm{nmol} / \mathrm{L}$ group, it was longer overall in the higher $25 \mathrm{OHD}$ groups $(p=0.002)$. There were no associations of serum $25 \mathrm{OHD}$ with timed up and go test, grip strength or lower limb muscle strength; no thresholds were identified for any outcomes. Vitamin D insufficiency is common in older Chinese women. A serum $25 \mathrm{OHD}$ level of at least $50 \mathrm{nmol} / \mathrm{L}$ and even higher is beneficially associated with bone health and static balance.

\section{P169}

LONG-TERM BONE MINERAL DENSITY FOLLOW-UP AFTER DOUBLE PARATHYROIDECTOMY

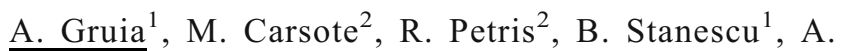
Ghemigian $^{2}$

${ }^{1}$ Medlife, Bucharest, Romania, ${ }^{2}$ Endocrinology Institute CI Parhon, Bucharest, Romania

Background: The correction of PTH levels in primary hyperparathyroidism (PHPT) increases BMD but the response rate depends on the site, on co-morbidities, etc. A part from antiresorptive drugs the remove of the adenoma is needed.

Objective: We aim to present the DXA results during a 5 -year period of time regarding a menopausal female suffering of HPTH.

Material and Methods: This is a case report. DXA, biochemistry and hormonal panel is presented. Results: A 59-year female accused non-specific bone pain for several years. She was admitted in 2010 for an endocrine checkup at Medlife Centre, Bucharest. She suffered of kidney stones and she had 5 previous fragility fractures without prior therapy. Menopause was registered at age of 53. The total calcium ( $\mathrm{tCa}$ ) was $11.66 \mathrm{mg} / \mathrm{dL}$ (normal $<10.2 \mathrm{mg} / \mathrm{dL}$ ), PTH of $266 \mathrm{pg} / \mathrm{mL}(\mathrm{N}<65 \mathrm{pg} /$ $\mathrm{mL}$ ), 25-hydroxyvitamin D (25OHD) of $6.7 \mathrm{ng} / \mathrm{mL}$ $(\mathrm{N}>30 \mathrm{ng} / \mathrm{mL})$. DXA showed: lumbar BMD of $0.624 \mathrm{~g} / \mathrm{cm}^{2}$, T-score of $-3.8 \mathrm{SD}, \mathrm{Z}$-score of $-2.5 \mathrm{SD}$, femoral neck BMD of $0.551 \mathrm{~g} / \mathrm{cm}^{2}$, T-score of $-2.7 \mathrm{SD}$, Z-score of $-1.4 \mathrm{SD}$; hip BMD of $0.75 \mathrm{~g} / \mathrm{cm}^{2}$, T-score of $-1.7 \mathrm{SD}, \mathrm{Z}$-score of $-0.8 \mathrm{DS}$. HPTH together with a secondary component due to vitamin $\mathrm{D}$ deficiency was confirmed. Vitamin D supplements and zolendronic acid (ZA) were started and a parathyroid left superior adenoma was removed by an anterior cervical procedure. After surgery the $\mathrm{tCa}$ was $9.98 \mathrm{mg} / \mathrm{dL}$ but increased to
$11 \mathrm{mg} / \mathrm{dL}$ within 3 months. A left inferior parathyroid adenoma was removed by left latero-cervical approach. TCa and PTH normalised and one year later DXA showed an improvement: lumbar BMD of $0.848 \mathrm{~g} / \mathrm{cm}^{2}$, T-score of $-2.8 \mathrm{SD}, \mathrm{Z}$-score of -2SD. She continued for 2 more years with ZA. In 2013 DXA pointed stationary lumbar BMD and a mild improvement of third distal radius BMD from BMD of $0.513 \mathrm{~g} / \mathrm{cm}^{2}$ (in 2010) to $0.523 \mathrm{~g} / \mathrm{cm}^{2}$, T-score of $-2.7 \mathrm{SD}$. In 2015 (after 1 year of injectable ibandronic acid) the lumbar BMD increased to $0.895 \mathrm{~g} / \mathrm{cm}^{2}$, T- score of $-2.4 \mathrm{SS}$, the neck T-score was $-2.1 \mathrm{SD}$, and the radius $\mathrm{T}$-score was of $-1.1 \mathrm{She}$ continued to have normal $\mathrm{tCa}$, PTH and 25OHD levels. Conclusion: Double synchronic parathyroid adenoma may represent a challenging issue especially if a supplementary secondary mechanism is involved due to vitamin D deficiency, and the BMD improvement reflects the PTH correction with a different pattern depending of the skeleton site.

\section{P170}

LONG-TERM EFFECTS OF GLUCOSAMINE/ CHONDROITIN SULFATE ON THE PROGRESSION OF STRUCTURAL CHANGES IN KNEE OSTEOARTHRITIS: 6-YEAR FOLLOW-UP DATA FROM THE OSTEOARTHRITIS INITIATIVE (OAI)

J. Martel-Pelletier $^{1}$

${ }^{1}$ Osteoarthritis Research Unit, University of Montreal Hospital Research Centre (CRCHUM), Montreal, Quebec, Canada

Objective: This study aimed to examine, for the first time, the long-term (6-year) protective effect of combined glucosamine (Glu) and chondroitin sulfate (CS) treatment on cartilage volume in knee osteoarthritis.

Method: Participants were from the Osteoarthritis Initiative (OAI) Progression and Incidence sub-cohorts who had MRI data on the target knee at baseline and 6 years, joint space width greater than $1 \mathrm{~mm}$, and information on Glu/CS consumption $(n=1593)$. The participants were stratified into two main groups based on whether or not they had medial meniscal extrusion at baseline. The group with meniscal extrusion at baseline $(n=429)$ was further stratified into subgroups based on exposure time to Glu/CS: not exposed, 1 year, 2 to 3 years, and 4 to 6 years. Cartilage volume was assessed using fully automated quantitative MRI technologies ${ }^{1,2}$.

Results: Jonckheere-Terpstra trend test indicated that treatment with Glu/CS significantly reduced the cartilage volume loss in the global knee, associated with the lateral compartment. Multivariate analysis further demonstrated that the extent of the treatment's positive effect was related to exposure time to treatment; the protective 
effect at 6 years was significant in participants exposed to two or more years of treatment.

Conclusion: The findings of this study are in line with previous data showing chondroprotective effects of Glu and CS treatment with preferential effects in the lateral compartment of the knee, and provide future support for the long-term protective structure-modifying effects of such treatment in knee osteoarthritis subjects.

\section{References:}

1. Dodin P, J Biomed Graph Comput 2013;3:51.

\section{P171}

ASSOCIATION OF D IGITAL X-RAY RADIOGRAMMETRY WITH FRAX AND DUAL-ENERGY X-RAY ABSORPTIOMETRY: THE STUDY OF OSTEOPOROTIC FRACTURES J. Kälvesten $^{1}$, L.-Y. Lui ${ }^{2}$, T. B. Brismar ${ }^{3}$, S. R. Cummings ${ }^{2}$

${ }^{1}$ Radiology, Department of Medicine and Health Sciences, Linköping University / Center for Medical Image Science and Visualization (CMIV), Linköping University / Sectra, Linköping, Sweden, ${ }^{2}$ San Francisco Coordinating Center, California Pacific Medical Center, San Francisco, United States, ${ }^{3}$ Karolinska Institutet, Department for Clinical Science, Intervention and Technology, Division of Radiology, Karolinska University Hospital, Stockholm, Sweden
Objectives: The objective of this study was to compare and relate digital X-ray radiogrammetry (DXR) with FRAX ${ }^{\circledR}$ and DXA in discrimination of major osteoporotic fracture (MOF) (hip, clinical spine, forearm or shoulder), hip fracture and femoral neck (FN) osteoporosis.

Material and Methods: The prospective Study of Osteoporotic Fractures (SOF) cohort was used for this study. Baseline hand Xray images of 5,358 women 65 years and older were analyzed and fractures were ascertained during 10 years of follow-up. Methods were compared and related using area under age adjusted receiver operating characteristic curve (AUC) for MOF and hip fracture and for osteoporotic DXA FN BMD.

Results: DXR and FRAX (no BMD) were similar in fracture discrimination; AUC was around 0.65 for MOF and 0.70 for hip fractures for both methods, while femoral neck DXA BMD AUC as expected was higher at 0.68 for MOF and 0.75 for hip fractures, Table 1 . AUC for femoral neck osteoporosis (DXA FN BMD Tscore $<-2.5)$ was higher for DXR-BMD, $0.76(0.74$ $0.77)$, than for FRAX, $0.69(0.67-0.71)$,

$(p<0.0001)$, Table 1.

Conclusions: Incident fractures were discriminated to a similar degree by DXR-BMD as by FRAX in this population based cohort. Femoral neck osteoporosis was predicted to a larger degree by DXR-BMD than by FRAX.

Disclosures: JK was an industrial $\mathrm{PhD}$ student and is an employee by Sectra AB.

Table 1 Area under receiver operating characteristic curve (AUC) for discrimination of major osteoporotic fracture (MOF), hip fracture and femoral neck osteoporosis. Mean and $95 \%$ CI

\begin{tabular}{llll}
\hline & AUC MOF 10 years & AUC hip fracture 10 years & AUC DXA FN BMD T-score $<-2.5$ \\
Age alone & $0.59(0.57,0.61)$ & $0.68(0.65,0.71)$ & $0.64(0.61,0.65)$ \\
Age + DXR & $0.65(0.63,0.67)$ & $0.69(0.66,0.72)$ & $0.76(0.74,0.77)$ \\
Age + DXA FN BMD & $0.68(0.66,0.70)$ & $0.75(0.72,0.77)$ & - \\
Age + FRAX (no BMD) & $0.64(0.61,0.65)$ & $0.71(0.67,0.73)$ & $0.69(0.67,0.71)$ \\
Age + DXA FN BMD + FRAX & $0.69(0.67,0.70)$ & $0.76(0.73,0.78)$ & - \\
\hline
\end{tabular}

\section{P172}

VALIDATION OF FRAGILITY FRACTURES IN PRIMARY CARE ELECTRONIC MEDICAL RECORDS: A POPULATION-BASED STUDY

D. Martinez-Laguna $^{1}$, A. Soria-Castro ${ }^{1}$, C. CarbonellAbella $^{1}$, P. Orozco ${ }^{1}$, P. Estrada-Laza ${ }^{1}$, X. Nogués ${ }^{2}$, A. DiezPerez $^{2}$, D. Prieto-Alhambra ${ }^{3}$

${ }^{1}$ Institut Catala de la Salut and IDIAP Jordi Gol, Barcelona, Spain, ${ }^{2}$ Department of Internal Medicine, Hospital del Mar-IMIM and Autonomous University of Barcelona, Barcelona, Spain, ${ }^{3}$ Oxford NIHR Musculoskeletal Biomedical Research Unit, University of Oxford and IDIAP Jordi Gol, Oxford, United Kingdom
Objective: Electronic medical records databases use validated lists of ICD (or other) codes to identify fractures. These, however, are not specific enough to disentangle traumatic from fragility fractures. We report on the proportion of fragility fractures identified amongst a random sample of coded fractures in SIDIAP, both overall and after stratification by fracture site.

Material and Methods: Retrospective observational study in 6 of the 279 primary care centers includes in the SIDIAP database (www.sidiap.org). SIDIAP contains clinical information from primary care records, hospital admissions, and pharmacy invoice data for $>5$ million patients $(80 \%$ of the population) in Catalonia, Spain. We selected all patients $\geq$ 
50 years old with a clinical fracture registered in 2012 using pre-specified lists of ICD-10 codes. Patients were excluded if they did not respond, had a dementia or a serious psychiatric disease, or died during the study. Data on fracture type (traumatic or fragility), site, as well as on patient characteristics were collected, previous verbal consent.

Results: A total of 491/616 (79.7\%) patients with a registered fracture in 2012 were contacted, and 331 (349 fractures) were included after eligibility screening. Mean age was 69.85 \pm 11.13 and $76.4 \%$ were women. The most prevalent fractures were forearm (82), ribs (38) and humerus (32). 225/349 $(64.5 \%)$ were fragility fractures, with higher proportions for classic osteoporotic sites: $91.7 \%$ for hip, $87.7 \%$ for spine, and $80.5 \%$ for any major fracture (including hip, spine, wrist/ forearm and proximal humerus). Osteoporotic, compared to traumatic, fractures were more frequent in women (85.1 vs. $60.3 \%, p<0.0001$ ), in older patients (mean age $72.23 \pm 10.48$ vs. $65.45 \pm 11.01, p<0.0001)$ and amongst patients with a previously coded diagnosis of osteoporosis (31.6 vs. $15.5 \%$, $p=0,001$ ).

Conclusion: In patients $\geq 50$ years old from the SIDIAP database the majority of hip, vertebral and major fractures are for fragility.

\section{P173}

MANAGEMENT OF SEVERE OSTEOPOROSIS IN A PATIENT WITH THERAPEUTICALLY NEGLECTED ANDROGEN INSENSITIVITY SYNDROME

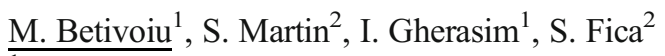

${ }^{1}$ Elias Hospital, Endocrinology Department, Bucharest, Romania, ${ }^{2}$ Elias Hospital, Endocrinology Department, Carol Davila University of Medicine and Pharmacy, Endocrinology Department, Bucharest, Romania

Introduction: Androgen insensitivity syndrome (AIS) is a rare $\mathrm{X}$-linked recessive condition resulting in a failure of normal masculinization of the external genitalia in chromosomally male individuals. Reduced BMD is evident in patients with AIS, mainly when compliance with estrogen replacement therapy is not guaranteed. We report the case of a 46-year-old normal weight phenotypic female patient who presented to our clinic in November 2015 with complaints of bilateral hip pain. She was diagnosed with complete AIS at 18 years old when she was investigated for primary amenorrhea. Surgical resection of the intraabdominal undescended testes and estrogen replacement therapy were recommended but refused at that time. In 2005 surgery was performed for a type A Antoni neurinoma and the atrophic right male gonad. In August 2015 she was hospitalized for suspicion of neurinoma relapse and the left atrophic testicle was resected. She has no bone deformities or history of fragility fractures. She was diagnosed with osteoporosis in 2012 (DXA hip T-score-3.8 SD, Z-score $-3.2 \mathrm{SD})$ but she did not take any medication.

Present laboratory tests: testosterone $=24.28 \mathrm{ng} / \mathrm{dl}$, estradiol $=11.07 \mathrm{pg} / \mathrm{ml}$, iIPTH $=58.82 \mathrm{pg} / \mathrm{ml}$, total calcium $=9.7 \mathrm{mg} / \mathrm{dl}$, phosphorus $=4.4 \mathrm{mg} / \mathrm{dl}, 25 \mathrm{OH}-$ vitaminD $=17.39 \mathrm{ng} / \mathrm{ml}$, alkaline phosphatase $=69 \mathrm{U} / 1$, $\mathrm{TSH}=1.2 \mathrm{mcUi} / \mathrm{ml}$. DXA hip T-score $=-4.4 \mathrm{SD}, \mathrm{Z}$ score $=-3.7 \mathrm{SD}$. Radiography of the spine showed no vertebral fractures. Treatment with iv $5 \mathrm{mg}$ zoledronic acid/ year, calcium $1000 \mathrm{mg} /$ day and cholecalciferol $1000 \mathrm{UI} /$ day was started. Conclusions: In AIS patients, estrogen therapy, calcium and vitamin D are recommended to prevent osteoporosis. DXA scanning should be instituted early with the addition of bisphosphonate therapy when indicated. Bone health remains a crucial aspect in the management of AIS patients, new studies being required to improve their bone health and wellbeing.

\section{P174}

OSTEOPOROTIC HIP REFRACTURE AUGMENTATION PFNA NAIL

N. Pérez Jimeno ${ }^{1}$, J. López ${ }^{1}$, M. Lillo ${ }^{1}$, V. Roda ${ }^{1}$, B. Garcia ${ }^{1}$

${ }^{1}$ Miguel Servet University Hospital, Zaragoza, Spain

Introduction: Hip fractures have social and economic impact due to their high frequency in the elderly.

Objectives: Show our experience with the use of augmentation PFNA nail in an elderly patient who had metafisodiafisaria distal femur fracture with previous screwed.

Methods: 85 year old woman with pain in her left leg after an accidental fall. Operated 8 years before on the same hip for a subcapital fracture (Garden II), performing screwed. The patient had severe pain and functional impotence. Neurovascular exploration unchanged. X-rays showed a distal fracture from the previous screwed. To get more information, we requested a $\mathrm{CT}$, which was informated like isolate external cortical femur fracture in the metafisodiafisaria zone. Based on the patient quality life, the poor bone quality and the bone void expected after removal of osteosynthesis material, we decided implanting a PFNA nail with introduction of PMMA cement in order to prevent mobilization of the proximal nail screw.

Results: After 2 months, the patient presents minimal pain in the operated hip and walks with the aid of a walker. X-rays revealed consolidation fracture and components in their correct position.

Conclusions: In our case, the use of augmentation PFNA nail for greater fixation devices in osteoporotic bone has been satisfactory. Therefore, we recommend these types of nails in osteoporotic proximal femur fractures as long as there is no risk of cement leakage into the joint space or vascular structures. 


\section{P175}

PHILOS® IN OSTEOPOROTIC PROXIMAL HUMERUS FRACTURES

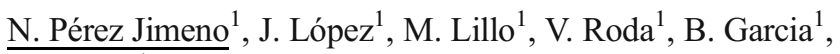
F. Blanco ${ }^{1}$

${ }^{1}$ Miguel Servet University Hospital, Zaragoza, Spain

Introduction: The treatment of proximal humerus fractures remains controversial. They constitute $2-3 \%$ of upper extremity fractures. Higher incidence in patients over 60 years, more common in women than in men.

Objectives: To evaluate clinical, radiological and functional parameters in patients treated with plate fixation for proximal humerus fracture.

Methods: It is a retrospective study in which we have reviewed 36 cases of proximal humerus fractures, undergoing surgery in the last 3 years in our hospital. A minimum of 12 months follow-up was performed including a clinical evaluation, a measurement of radiological parameters and an assessment of the functional state by the Constant-Murlay scale.

Results: The average age of the patients was 66.8 years. The surgical fractures: $52.6 \%$ were in 3 fragments, $42.1 \%$ in 4 fragments and $5.2 \%$ in 2 fragments according to the Neer classification; being displaced fractures $89.4 \%$ of them, with an angle greater than $45^{\circ}$ in $84 \%$ of cases. Up to $94.8 \%$ of fractures had comminution or important osteoporosis; and in $47.3 \%$ of cases, the comminution was in calcar area. In all cases where osteosynthesis treatment was indicated, the Philos ${ }^{\circledR}$ plate was used. The average of epiphyseal screws was 5 . In all short plates were placed 3 metafiso-diaphyseal screws. In the Constant- Murlay scale, the average was 67.2 points out of 100 with variable clinical-radiological correlation. Conclusions: Philos ${ }^{\circledR}$ plate fixation in proximal humerus fractures has a good clinical, radiological and functional outcomes. The results are better in displaced fractures of 2 and 3 fragments than in 4 fragments. The ConstantMurlay scale offers better scores in patients treated with osteosynthesis than in those treated by arthroplasty of the proximal humerus.

\section{P176}

THE STUDY OF RELATIONSHIP ANALYTICAL OF KNEE JOINT PAIN ACCORDING SOME OF BIOMECHANICAL VARIABLES

N. A. Almashaakhi ${ }^{1}$

${ }^{1}$ Sports medicine, Babylon, Iraq

The number of injuries in the failed of sport, it increases, day by day. The cause is giving back to the problem in the component of loading, the many from the players leave the sport in early time.
And through of the last the joint pain of knee with indication of loading mechanics for players. The aim of research to analysis of loading on the knee joint with indication of loading mechanics to identify about the natural of pain for having players. So the hypothesis of research, there is relation between the pain in the knee joint and the mechanics of loading. And after that, the researchers are processing data to the get of result and they are inducing some conclusions and recommendations, its importances are :

1. Sets the drills to development the muscles, which are moving the joint of knee.

2. Sets guidelines to the mechanics of performance in each games.

\section{P177}

\section{LEVEL OF PHYSICAL ACTIVITY IN PATIENTS WITH OSTEOPOROSIS}

$\underline{\text { S. Tomasevic-Todorovic }}^{1}, \mathrm{~K}$. Boskovic ${ }^{1}$, A. Knežević ${ }^{1}, \mathrm{M}$. Eric $^{1}$, S. Pantelinac ${ }^{1}$, F. Hanna ${ }^{2}$

${ }^{1}$ Medical Faculty, University of Novi Sad, Novi Sad, Serbia,

${ }^{2}$ Public Health Program, Department of Health Sciences, College of Arts and Sciences, Qatar University (Doha, Qatar), Doha, Qatar

Objective: To examine the degree and types of physical activity (heavy physical activity, medium heavy and light physical activity) in female patients with lower BMD compared to healthy women.

Materials and Methods: The cross-sectional study included 40 female patients $(65.85 \pm 11.46$ years $)$ with lower BMD and 20 patients $(56.25 \pm 6.6$ years $)$ with no existing abnormality of BMD levels. The research was conducted at the Medical Rehabilitation Clinic, Clinical Center of Vojvodina.

Data were collected using the internationally endorsed questionnaire IPAQ. BMD was measured at lumbar spine (L1-4) and at proximal femur by DXA technique. Osteoporosis was defined as BMD $>-2.5 \mathrm{~T}$-score.

Results: We found that subjects with a normal BMD had the highest level of physical activity (MET $=3154.58)$, followed by subjects with osteopenia (MET $=2311.25)$. Patients suffering from osteoporosis who were physically active had the lowest value of MET (1972.95) ( $p$ 0.01). Sub- analysis based on BMI categories showed $10 \%$ (2 patients) of those with osteopenia were underweight, compared to $40 \%$ (8 patients) in the group with osteoporosis.

Conclusions: Patients with osteoporosis were more obese and less physically active, as compared to healthier females. Our findings support the fact that adapted physical activity seems to be important for the prevention and treatment of patients with osteoporosis. 


\section{P178}

ADIPOSE-DERIVED HUMAN MESENCHYMAL STEM CELLS CAN PRODUCE CARTILAGE

J. Nurkovic $^{1}$, V. Grbovic ${ }^{2}$, S. Nurkovic ${ }^{3}$, S. Vucelj ${ }^{3}$, Z. Dolicanin $^{1}$

${ }^{1}$ Department of Biomedical Sciences, State University of Novi Pazar, Novi Pazar, Serbia, ${ }^{2}$ Clinical Center Kragujevac, Kragujevac, Serbia, ${ }^{3}$ General Hospital Novi Pazar, Novi Pazar, Serbia

Although stem cells have the ability to regenerate new tissue, the theory that depletion of stem cells may be present in degenerative disease has had some evidence in osteoarthritis. We showed that chondrogenesis could be induced by culturing human MSCs (hMSCs) in micromass pellets in the presence of a medium that included 100nM dexamethasone and $10 \mathrm{ng} / \mathrm{ml}$ TGF Beta3. Adipose tissue hMSCs from 10 adult donors aged 1860 years were trypsinized, washed in serum containing medium and suspended in serum-free chondrogenic medium, centrifuged, incubated and left to sediment and form pellet masses at the bottom of the tube. Cell survival was determined by florescence microscopy; and antibodies specific for ECM proteins, collagen I and II used for determining chondrogenic differentiation. The hMSCs yielded positive staining for anionic proteoglycans, as well as type II collagen. hMSCs may have the potential of giving the best repair in terms of the phenotype as well as longevity of the regenerated cartilage. The potential for hMSCs in cartilage repair and regeneration may be tremendous. Much needs to be done in this very exciting area of research. The answers will eventually come from collaborations amongst scientists, engineers and clinicians.

\section{P179}

INSULIN USE AND EXCESS FRACTURE RISK AMONG TYPE 2 DIABETIC PATIENTS: A PROPENSITY-MATCHED COHORT ANALYSIS

E. Losada-Grande ${ }^{1}$, B. Soldevila ${ }^{2}$, S. Hawley ${ }^{3}$, X. Nogués ${ }^{4}$, A. Diez-Perez ${ }^{4}$, D. Martinez-Laguna ${ }^{5}$, M. Puig-Domingo ${ }^{2}$, D. Mauricio $^{2}$, D. Prieto-Alhambra ${ }^{6}$

${ }^{1}$ Medicine Department. Universitat Autonoma de Barcelona. Endocrinology section. Can Misses hospital, Ibiza, Spain, ${ }^{2}$ Department of Endocrinology and Nutrition. University Hospital \& Health Sciences Research institute Germans Trias i Pujol. Institute Research and Hospital Germans Trias i Pujol. CIBER of Diabetes and Associated Metabolic Diseases (CIBERDEM). Instituto de Salud Carlos III (ISCIII), Badalona, Spain, ${ }^{3}$ Oxford NIHR Musculoskeletal Biomedical Research Unit, Nuffield Department of Orthopaedics, Rheumatology and Musculoskeletal Sciences,
University of Oxford, Oxford, United Kingdom, ${ }^{4}$ Department of Internal Medicine, Hospital del Mar-IMIM and Autonomous University of Barcelona, Barcelona, Spain, ${ }^{5}$ Institut Catala de la Salut and IDIAP Jordi Gol, Barcelona, Spain, ${ }^{6} \mathrm{MRC}$ Lifecourse Epidemiology Unit, University of Southampton; MRC Human Nutrition Research, Elsie Widdowson Laboratory Oxford NIHR Musculoskeletal Biomedical Research Unit, University of Oxford, Southampton, United Kingdom

Objectives: Despite higher bone density, type 2 diabetic patients (T2DM) have increased fracture rates. T2DM medications could be involved in this excess risk. We investigated the association between insulin use and risk of bone fractures among T2DM patients.

Design and Population: Population-based matched cohort study.

Setting: Primary care records from the SIDIAP database (Spain).

Participants and Follow-up: All SIDIAP participants newly diagnosed with T2DM in 2006-2013 were eligible $(n=53,383)$. Patients aged $<40$ years, and those with secondary osteoporosis or severe kidney failure were excluded.

Exposure: Patients receiving at least two dispensations of insulin (two months of therapy) were classified as insulin users.

Outcome: Major osteoporotic fractures were ascertained using validated lists of ICD-10 codes.

Statistics: Propensity scores for insulin use were calculated using logistic regression models including the following a priori defined predictors of fracture: $\mathrm{HbAlc}$, comorbidities, anti-osteoporosis and T2DM medications, lifestyle factors, fracture history, and index year. Insulin users were matched 1:5 with comparable non-users using propensity score calliper-matching.

Fine and Gray models were fitted to estimate the relative risk (sub-hazard ratio or SHR) of major fracture according to insulin use.

Results: A total of 2,979 insulin users and 14,895 non-users were observed for a median of 4.45 years. Major fracture rates were 11.2/1,000 person-years while on insulin therapy compared with 8.3/1,000 among non-users. Adjusted models confirmed a significant association, an adjSHR of 1.43 [95\% CI 1.09-1.86] for major fractures. No differences between types of insulin or different regimens were found. Estimated numbers needed to harm were 71 (95\% CI 29 to 227) for major osteoporotic fracture.

Conclusions: Insulin use appears to be associated with a $>40 \%$ excess fracture risk among T2DM patients even in the early stages of the disease. Fracture risk should be included among the risks that are to be taken into account upon the decision to initiate new insulin treatment. 


\section{P180}

\section{THE INFLUENCE OF NEIGHBOURHOOD DEPRIVATION IN ADVANCED ARTHRITIS}

N. Cristall ${ }^{1}$

${ }^{1}$ Social worker (MSW), Concordia Hospital, Winnipeg, Canada

Arthritis is a growing aging and public health concern in Canada and elsewhere. As with many other chronic health conditions, arthritis occurs more often and has a higher impact on functioning for people who have lower incomes or live in an impoverished environment. There is a large body of research that supports a gradient between socioeconomic status and health and between area level poverty and decreased quality of life. Although this relationship is widely acknowledged, less is known about the influence of broader social conditions at the neighbourhood level on health outcomes. I provide an analysis of the direct impact of material and social deprivation on health related quality of life, as well as the impact considering the influence of age, body weight, physical functioning, sex, and co-existing health conditions. I also examine interaction effects between neighbourhood deprivation and individual characteristics. An explanatory three-level multilevel model supported a substantial relationship between individual factors, deprivation at the neighbourhood level, and reduced mental and physical HRQoL. The impact of neighbourhood deprivation was more pronounced for mental health related life quality, with having a history of another health condition making the largest contribution to the model. Physical HRQoL was impacted by sex in interaction with material deprivation and body weight in interaction with social deprivation. I will discuss implications for practice, service delivery, and policy and make suggestions for further research.

\section{P181 \\ UNION OF BIOLOGY AND MATHEMATICS: RA BLOOD BASED DISEASE ACTIVITY SCORE (RABBDAS)}

A. Aggarwal ${ }^{1}$, A. Sharma ${ }^{2}$, A. Bhatnagar ${ }^{3}$

${ }^{1}$ Centre of Excellence in HIV Care, Department of Internal Medicine, PGIMER Chandigarh, Chandigarh, India, ${ }^{2}$ Department of Internal Medicine, PGIMER Chandigarh, Chandigarh, India, ${ }^{3}$ Department of Biochemistry, Panjab University Chandigarh, Chandigarh, India

Background: Improved disease severity prediction markers are need of the hour to aid early prognosis and personalised therapeutic approach. Disease activity score, simplified disease activity index and clinical disease activity index are influenced by intra and inter-assessor variability.
Objectives:

- To elucidate the role of oxidative stress and immunobiology of NK and NKT cells.

- To design a mathematical model for measuring disease severity in RA patients.

Materials and Methods: This prospective study was carried out from January 2011 to December 2012. Following ethical approval, 50 RA patients (39 females, 11 males) were recruited from the rheumatology Clinic of PGIMER Chandigarh. 50 age and sex matched healthy volunteers (41 females, 9 males) were also enrolled. The biochemical parameters investigated in serum samples were Lipid Peroxidation, Reduced Glutathione, Catalase, Superoxide dismutase, Glutathione peroxidase, IL-18 and TNF- $\alpha$. NK and NKT cells related intracellular parameters (DNA damage, caspase-3, perforin, granzyme A and B, IL-4, IFN- $\gamma$ and IL-8 expression) were measured using multicolour flow cytometer. Mathematical model equation was developed for predicting RA blood based disease activity score (RABBDAS) using multiple linear regression analysis.

Results: Oxidative balance was deregulated in RA patients. Granzyme A, perforin and IL-8 were independently associated with RABBDAS as:

RABBDAS $=-1.115+0.054 \mathrm{X} 1+0.032 \mathrm{X} 2$

$$
+0.14 \times 3(\mathrm{r} 2=0.95)
$$

Where X1: NK_GranA; $X 2$ : NKT_Perf; X3: NKT_IL-8

Conclusions: There is a state of profound oxidative stress in RA patients. Immunobiology of NK and NKT cells were severely compromised. This novel severity index may serve as an un-separable tool in quantifying the severity index of RA in patients. a patent application "diagnostic kit for in vitro diagnosis of RA and method of use thereof' has been filed.

\section{P182}

\section{GLYCATED HEMOGLOBIN AS A PREDICTOR OF ARTERIAL OCCLUSION SEVERITY IN DIABETIC FOOT INFECTIONS MANAGED WITH MAJOR AMPUTATION}

J. A. Coruña $^{1}$, T. H. Pacaldo ${ }^{1}$, J. M. Coruña ${ }^{1}$, M. V. L. Guanzon $^{2}$, D. Pedroza ${ }^{3}$

${ }^{1}$ Orthopaedics and Traumatology, Corazon Locsin Montelibano Memorial Regional Hospital, Bacolod, Philippines, ${ }^{2}$ Department of Internal Medicine, Endocrinology, Corazon Locsin Montelibano Memorial Regional Hospital, Bacolod, Philippines, ${ }^{3}$ Laboratory, Corazon Locsin Montelibano Memorial Regional Hospital, Bacolod, Philippines

Objectives: In this retrospective cohort study, we compared $\mathrm{HbAlc}$ levels of all diabetic patients with foot infections, and between those treated with major amputation (below-knee and above-knee) or conservatively. 
Methods: After ethics review board approval, a systematized chart review was performed. Three-hundred two feet from 264 consecutive patient referrals were enrolled in the study from January 1 to December 31, 2014. HbA1c result upon admission and routine histopathology of amputated limb posterior tibial artery were collected and tabulated in a spreadsheet, together with demographic and clinical data.

Results: Majority of the patients were males (58.94 \%). The mean $\mathrm{HbAlc}$ of study participants was $9.81 \%$ (SD: $2.89 \%$ ). Majority $(78.15 \%)$ had uncontrolled blood sugar (HbAlc $>7.0 \%$ ). Forty-five percent underwent major amputation. The mean HbAlc of patients who did not undergo surgery was $7.89 \%$ (SD: $2.41 \%$ ) while patients with major surgery had $11.20 \%$ (SD: $2.68 \%$ ). The mean percentage for posterior tibial artery occlusion among amputated limbs was $31.46 \%$ $n=135$ (95 \% CI: 27.01-35.91\%). On univariate analysis using linear regression analysis, Hbalc correlates with percentage occlusion in posterior tibial artery $(F$ value $=57.17, \mathrm{R}$ square $=0.1601, p$ value $=0.000$ ). Based on the linear regression analysis equation, the lowest $\mathrm{HbA1c}$ level that could predict a $90 \%$ occlusion of the posterior tibial artery is $14.11 \%$. Conclusion: Patients with poor glucose control have higher incidence of undergoing major amputation. Among amputees, poor glucose control is associated with increased occlusion in the posterior tibial artery.

Disclosures: None of the authors or any immediate family member has received anything of value from or owns stock in a commercial company or institution related directly or indirectly to the subject of this research.

\section{P183}

IDENTIFICATION OF SYNOVIAL FLUID MICRORNA SIGNATURE IN KNEE OSTEOARTHRITIS: DIFFERENTIATING EARLY- AND LATE-STAGE KNEE OSTEOARTHRITIS

M. Kapoor ${ }^{1}$, Y. Li ${ }^{2}$, G. Tavallaee ${ }^{2}$, T. Tokar ${ }^{3}$, A. Nakamura ${ }^{2}$, K. Sundararajan ${ }^{4}$, A. Weston ${ }^{4}$, A. Sharma ${ }^{2}$, N. Mahomed ${ }^{4}$, R. Gandhi $^{4}$, I. Jurisica ${ }^{3}$

${ }^{1}$ Surgery, University of Toronto, Toronto, Canada, ${ }^{2}$ Krembil Research Institute, Toronto, Canada, ${ }^{3}$ Princess Margaret Cancer Centre, Toronto, Canada, ${ }^{4}$ Toronto Western Hospital, Toronto, Canada

Objectives: This study aimed to identify circulating microRNA (miRNA) signatures in knee synovial fluid (SF) from early-stage and late-stage knee osteoarthritis (OA) patients.

Methods: miRNAs were screened by miRNA-PCR-arrays and validated by RT-qPCR in SF from patients with earlystage (Kellgren-Lawrence:Grade:I/II) and late-stage OA (Grade:III/IV). OA cartilage or synovial explants were cultured to study the source and release of identified miRNAs.
An integrated computational-approach was utilized to predict gene/pathway targets.

Results: Our screening and validation analysis identified a panel of seven (out of 752) circulating SF miRNAs (23a-3p, 24-3p, 27a-3p, 27b-3p, 29c-3p, 34a-5p and 186-5p) that were significantly differentially expressed in late-stage vs. early-stage OASF, irrespective of age, gender and BMI. miR-378a-5p was mostly detectable in the majority of late-stage OA-SF. Explant cultures revealed that validated miRNAs exhibited greater expression in the synovium compared to cartilage. IL-1 $\beta$-stimulated OA synovial explants exhibited significantly increased expression of miR-34a-5p, 24-3p, 27a-3p, 23a-3p and 27b-39 and release of only $23 a-3 p$ and $27 b-3 p$ in supernatants, suggesting that IL-1 $\beta$ in the synovium likely contributes to the release of $23 a-3 p$ and 27b-3p in the SF. Computational-analysis further identified 2 genes (ROQUIN-1 [RC3H1] and quaking-gene [QKI]) that are targeted by 6 out of 8 miRNAs; miR-27b-3p exhibited greatest association with $R C 3 H 1$ and $Q K I$ genes. Indeed, synovial explants treated with miR-27b-3p-mimic show significant suppression of both RC3HI and QKI genes.

Conclusions: We provide first evidence of the differential expression of circulating miRNAs in early vs. late-stage knee OA-SF. Further, we provide source, release and genes/ pathways regulated by identified miRNAs.

\section{P184}

CARTILAGE-SPECIFIC ABLATION OF UNC-51 LIKE KINASE 1 RESULTS IN AN ACCELERATED OSTEOARTHRITIS PHENOTYPE

M. Kapoor ${ }^{1}$, M. Abou Rjeili ${ }^{2}$, J. Rockel ${ }^{3}$, E. Rossomacha ${ }^{3}$, Y. Zhang $^{3}$, A. Sharma ${ }^{3}$

${ }^{1}$ Surgery, University of Toronto, Toronto, Canada, ${ }^{2}$ Pharmacology, University of Montreal, Montreal, Canada, ${ }^{3}$ Krembil Research Institute, Toronto, Canada

Objective: Unc-51 like kinase-1 (ULK1), a serine/threonine protein kinase, is the most upstream inducer of autophagy. We recently showed that ULK1 expression is suppressed in the articular cartilage during Osteoarthritis (OA); however, the role of ULK1 in OA pathogenesis is not known. We sought to determine the contribution of ULK1 to OA pathogenesis.

Methods: We generated inducible, cartilage-specific ULK1 knockout (KO) mice using Cre-lox technology (ULK1 f/f; Col2-rtTA-cre) and induced experimental OA by surgical destabilization of the medial meniscus (DMM). At 10 weeks post-DMM surgery, the degree of cartilage degeneration, loss of cellularity and expression of cell death and catabolic markers were determined. In vitro, we overexpressed ULK1 in human OA chondrocytes by transfection with a ULK1 expression plasmid to determine the effect of ULK1 on the expression of genes involved in cell death and survival. 
Results: We identified that cartilage-specific ablation of ULK1 resulted in accelerated DMM-induced OA including accelerated cartilage degeneration, proteoglycan loss, chondrocyte cell death, synovial inflammation and increased expression of OA catabolic factors, as compared to control mice. Furthermore, human OA chondrocytes transfected with ULK1 expression plasmid modulated the expression of mTOR, a negative mediator of autophagy, and ADAMTS-5, an OA-associated catabolic factor.

Conclusions: This study provides the first evidence that ULK1 expression is vital to maintaining articular cartilage homeostasis in surgically-induced OA.

\section{P185}

IDENTIFICATION OF METABOLOMIC SIGNATURES IN HIGH-FAT DIET INDUCED ACCELERATION IN AGE-RELATED AND SURGICALLY INDUCED OSTEOARTHRITIS IN MICE

M. Kapoor ${ }^{1}$, P. Datta ${ }^{2}$, Y. Zhang ${ }^{2}$, A. Parousis ${ }^{2}$, E. Rossomacha $^{2}$, A. Sharma ${ }^{2}$, J. Rockel ${ }^{2}$

${ }^{1}$ Surgery, University of Toronto, Toronto, Canada, ${ }^{2}$ Krembil Research Institute, Toronto, Canada

Objectives: We sought to identify the change in metabolomics profile due to distinct diet regimes and their contributions to the pathogenesis of OA.

Methods: 9 weeks (wk) old C57BL6 mice were fed high fat diet (HFD, $60 \%$ ) and lean diet (LD, $10 \%$ fat) for 18 weeks followed by a normal chow diet. Mice were longitudinally evaluated at times 0 (start of diet/ baseline), 18 weeks of diet (end of diet) and at 9 months of age. At each time point BMI, fasting blood glucose level and body weight were measured. At sacrifice, blood plasma was collected to measure leptin levels, metabolite levels and knee joints were collected for histopathological analysis at 9 months of age. Additional mice on HFD and LD were subjected to experimental OA by DMM surgery at the end of diet regime. Mice were maintained on normal chow and knee joints were collected for histopathological analysis at 10 and 20 week post surgery.

Results: We determined that HFD significantly increased fasting blood glucose levels, body weight, BMI and leptin levels as compared to LD fed mice. Histopathological analysis using OARSI scoring clearly showed that HFD fed mice exhibited accelerated spontaneous $\mathrm{OA}$ at 9 months of age as well as acceleration in the surgically induced OA at 10 and 20 week post surgery in comparison to LD fed mice. Of the 170 metabolites analysed in blood plasma at each time point, lysophosphatidyl choline analogues (lysoPCaC20:4, lysoPCaC17:0, lysoPCaC18:0) and one phosphatidyl choline analogue (PCaaC36:2) were increased longitudinally in the HFD fed mice. Our ongoing studies are now evaluating if these LysoPC metabolomics signatures are responsible for initiating and accelerating cartilage degradative process observed in HFD fed mice.

Conclusion: We identified that high fat diet induces and maintains selective metabolic changes and increases OA progression in both spontaneous and surgically induced OA. We anticipate that these identified metabolomics signatures are involved in OA pathogenesis during obesity.

\section{P186}

FACET CARTILAGE FROM PATIENTS WITH LUMBAR SPINE OSTEOARTHRITIS EXHIBIT REDUCED AUTOPHAGY AND ENHANCED EXPRESSION OF CELL DEATH, INFLAMMATORY AND CATABOLIC MEDIATORS

M. Kapoor ${ }^{1}$, A. Nakamura ${ }^{2}$, R. Rampersaud ${ }^{3}$, B. $\mathrm{Wu}^{2}, \mathrm{~K}$. Sundararajan $^{3}$, E. Rossomacha ${ }^{2}$, I. Jurisica ${ }^{4}$

${ }^{1}$ Surgery, University of Toronto, Toronto, Canada, ${ }^{2}$ Krembil Research Institute, Toronto, Canada, ${ }^{3}$ Toronto Western Hospital, Toronto, Canada, ${ }^{4}$ Princess Margaret Cancer Centre, Toronto, Canada

Rationale: Specific mechanisms associated with facet cartilage degeneration during facet joint osteoarthritis (OA) are largely unknown. In this study, we obtained facet joint cartilage from patients undergoing surgery for lumbar spinal canal stenosis (LSCS) and from patients with lumbar intervertebral disc herniation (LDH) (control group) to determine the degree of facet cartilage degeneration and expression of autophagy, apoptosis, inflammatory, catabolic and anabolic markers.

Methods: The severity of degeneration in the facet joint and intervertebral disc was assessed by the MRI grading scores as described by Weishaupt et al. and Pfirrmann et al., respectively. The degree of facet cartilage degeneration was further assessed histologically using the Osteoarthritis Research Society International (OARSI) grading system. Facet joint cartilage (L3 to S1; medial aspect) was collected from patients with LSCS and patients with LDH. Protein and RNA extracted from facet cartilage was subjected to quantitative real time PCR and Western blot analysis.

Results: Our results showed that all patients with LDH exhibited a degenerative score of grade 0 (normal) or grade 1 , whereas all patients with LSCS exhibited scores of grade 2 or 3 for the facet joint. In the lumbar disc, all patients in both groups exhibited moderate to severe disc degeneration. Histological analysis using OARSI grading further revealed a significant degree of facet cartilage degeneration in LSCS patients (OARSI score $=5.5+0.2)$ compared to LDH patients (OARSI score $=0.9 \pm 0.3$ ). Our results further showed that LSCS facet cartilage (in comparison to the LDH control group) exhibited a significant decrease in the expression of chondroprotective autophagy markers (LC3 and ULK1), increase in mammalian target of rapamycin (mTOR, negative regulator of autophagy), increase in cell death (apoptosis) markers 
(PARP-1 and caspase-3), increase in inflammatory factors (IL-1 $\beta$, TNF- $\alpha$, IL-6, COX-2, MCP-1), increase in catabolic factors (MMP-3, MMP-13 and ADAMTS-5) and decrease in the expression anabolic matrix molecules (aggrecan and type II collagen). Conclusions: These results clearly show that the LSCS facet cartilage exhibits an enhanced degree of facet cartilage degeneration associated with reduced expression of autophagy (chondroprotective) and anabolic markers; and enhanced expression of apoptosis, inflammatory and catabolic markers. Our uniqueness of using severely degenerated facet cartilage from patients undergoing LSCS surgery and facet cartilage from patients with LDH (as controls) allows us to understand the endogenous mechanisms associated with facet cartilage degeneration.

\section{P187}

QUALITY IMPROVEMENT PROJECT TO IMPROVE THE TIMELY REVIEW OF ALENDRONATE PRESCRIBED FOR OSTEOPOROSIS IN GENERAL PRACTICE

C. Nye $^{1}$, I. Jarvis ${ }^{2}$

${ }^{1}$ Gloucestershire NHS Foundation Trust, Gloucester, United Kingdom, ${ }^{2}$ London Medical Practice, Gloucester, United Kingdom

Introduction: Current guidelines suggest that patients taking alendronate for osteoporosis should have a review at 5 years to assess their need for ongoing treatment and prevent harm from unnecessarily prolonged treatment. (1) The long time frame means that recognising the appropriate time for review can be particularly challenging in general practice. This project aimed to assess historical and current practice and implement measures to help prompt the 5 year review.

Retrospective audit: A retrospective audit was included to assess the management of alendronate from start to finish of treatment. Vision software was used to identify 50 patients from the practice database who had completed alendronate therapy for osteoporosis within the last 10 years and data was extracted from their records. $42 \%$ of patients had taken alendronate for over 5 years. Only $42.3 \%$ of these patients had had a review of their therapy at an appropriate time (defined as between 4 and 6 years). $19 \%$ of these patients had never had a documented review.

Current practice audit: 49 patients currently taking alendronate were identified using a Vision search. $57 \%$ of those on alendronate for over 5 years had had a review at an appropriate time. $10 \%$ had a clearly documented date of next review.

Interventions: Changes were made to the prescribing tool on the Vision general practice software, such that a first prescription of alendronate automatically requires the practitioner to enter the 5 year review date into the prescription. This is printed on all future repeat prescriptions, to remind doctor, patient and pharmacist of the review date. Patients who were already on alendronate had their repeat prescriptions manually altered to include their review date. Patients who were identified as being overdue for review were invited in for review.

Results: $100 \%$ of patients currently taking alendronate have either a review plan in progress or a clear future date for a review. The intervention was well received by all general practitioners in the practice. This is a simple and practical way to facilitate timely review of prescriptions and could be extended to other medications.

References: Osteoporosis - clinical guideline for prevention and treatment; executive summary. National Osteoporosis Guideline Group. November 2014

\section{P188}

IMPAIRED TRABECULAR BONE SCORE (TBS) IN PATIENTS WITH PRIMARY HYPERPARATHYROIDISM

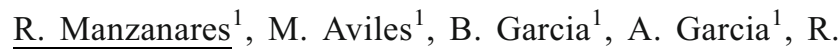

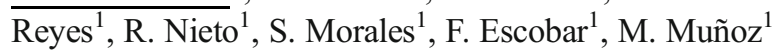

${ }^{1}$ Department of Endocrinology, Bone metabolic Unit, Hospital Universitario San Cecilio de Granada, Granada, Spain

Patients with primary HPT exhibit increased bone fragility which is an indication for surgical treatment. The trabecular bone score (TBS) is a grey-level texture measurement based on the use of experimental variograms acquired during a DXA of lumbar spine (LS). Several studies show that TBS can be an independent predictor of fragility fractures. However, the usefulness of this new technique in patients with PHPT is not well established. Our study was aimed to investigate TBS in patients with PHPT.

Patients and methods: We studied 86 patients with PHPT (mean age $64 \pm 9.9$ years, $82 \%$ females, $85 \%$ postmenopausal. In all patients BMD by DXA and TBS indices derived from LS DXA was assessed (TBS iNsight ${ }^{\circledR}$ software).

Results: TBS in PHPT was low $(1.21 \pm 0.13)$, representing abnormal trabecular microstructure (normal $\geq 1.35$ ). Males showed TBS values significantly lower than the women $(1.15 \pm 0.14$ vs. $1.23 \pm 0.12 ; p=0.028)$. PHPT patients with densitometric criteria of osteoporosis T-score $(\leq 2.5 \mathrm{SD})$ showed lower values of TBS $(1.16 \pm 0.13$ vs. $1.24 \pm 0.13$; $p=0.019$ ). However, we found no significant correlation between the values of TBS and DXA. We did not find any correlation between values of TBS and PTH levels and bone turnover markers. Patients with symptomatic and asymptomatic PHP showed similar values of TBS. We found significant differences when comparing postmenopausal women vs. premenopausal $(1.22 \pm 0.11$ vs. 1.30 $\pm 0.11 ; p=0.028$ ).

Conclusion: TBS measurements show that PHPT patients exhibit a degraded trabecular microstructure. It should be 
investigated if this technique can identify patients with increased bone fragility.

\section{P189}

\section{DOES BISPHOSPHONATE TREATMENT ALTER THE HEALING OF BONE DEFECT WHEN ADMINISTERED AT INITIATION OF THE HEALING PROCESS?}

C. Lavet ${ }^{1}$, R. Rizzoli ${ }^{1}$, P. Ammann ${ }^{1}$

${ }^{1}$ Division of Bone Diseases, Geneva University Hospitals and

Faculty of Medicine, Geneva, Switzerland

Objectives: Whether bisphosphonate treatment alters the healing of bone defect is still debated. We investigated the effects of the bisphosphonate pamidronate (PAM) compared to PTH on rat metaphyseal tibia bone defect (BD) healing.

Material and Methods: From BD creation on, 6-month old female rats received PAM $(0.1 \mathrm{mg} / \mathrm{kg}, 5 \mathrm{~d} /$ week every 4 weeks), PTH $(40 \mu \mathrm{g} / \mathrm{kg}, 5 \mathrm{~d} / \mathrm{w})$, or vehicle for 2,4 and 6 weeks. MicroCT, histomorphometry and nanoindentation test were performed in metaphyseal 2 nd spongiosa (2Spon), within the healing defect (HBD), close to the defect (closeBD) and in cortical bone bridging $\mathrm{BD}$ and at distance of it.

Results: There was no cartilage template in HBD. As expected, PAM progressively reduced active osteoclast surfaces in both trabecular ( $-40 \%$ at week $2,-70 \%$ at week $6, p<0.001)$ and cortical endosteal bone. At week 2, bone formation in HBD trabecular and cortical area was not changed in PAM group as compared to controls: osteoid thickness and surfaces (OS) remained unchanged with a blurry tetracycline labelling indicating a rapid bone formation. In contrast, bone formation rate (BFR) in 2Spon and closeBD was decreased in PAM group ( $-60 \%$ each, $p<0.001)$. At week 4 and 6 , OS and BFR were lower in PAM group independently of the trabecular sites. At all time points, PAM decreased mineral apposition rate in cortical compartment, increased bone mass in all trabecular sites (more than 20 fold in HBD at week 6) and led to a thicker $(+33 \%, p<0.01)$ and more mineralized $(+6.5 \%, p<0.001)$ cortical shell. As compared to PTH treatment, PAM caused a greater trabecular filling of $\mathrm{BD}(+60 \%, p<0.001)$, an important anisotropy as well as a thinner cortical shell $(-25 \%$, $p<0.001)$, with similar mineralization of HBD. By 6 weeks, cortical and trabecular bone hardness and modulus as assessed by nano-indentation were similarly increased in PAM and PTH groups.

Conclusion: Within the healing bone defect, PAM decreased resorption during the early phase of healing while formation was maintained, leading to a positive bone balance. Then, it inhibited both components of bone remodelling. Bone formed under PAM treatment displayed important anisotropy and increased bone material level properties.

\section{P190}

EFFECTIVENESS OF BONE THERAPIES IN ROUTINE CLINICAL PRACTICE: TWO RETROSPECTIVE DATABASE ANALYSES USING US MARKETSCAN AND MEDICARE

A. Yusuf $^{1}$, M. Feudjo Tepie ${ }^{2}$, L. Kalouche-Khalil ${ }^{3}, \mathrm{H}_{\text {. Guo }}{ }^{1}$, Z. Jincheng ${ }^{4}$, R. B. Wagman ${ }^{5}$, J. M. Sprafka ${ }^{5}$, C. Cooper ${ }^{6}$

${ }^{1}$ Chronic Disease Research Group, Minneapolis Medical Research Foundation, Minneapolis, MN, United States,

${ }^{2}$ Amgen Ltd., Uxbridge, United Kingdom, ${ }^{3}$ Amgen (Europe) GmbH, Zug, Switzerland, ${ }^{4}$ Chronic Disease Research Group, Minneapolis Medical Research Foundation Division of Biostatistics, University of Minnesota School of Public Health, Minneapolos, MN, United States, ${ }^{5}$ Amgen Inc., Thousand Oaks, CA, United States, ${ }^{6}$ Unveristy of Southampton, Southampton General Hospital, Southampton; University of Oxford, Oxford, United Kingdom

Objective: Assess the effectiveness of antiresorptive agents for PMO in routine practice using US MarketScan and Medicare databases.

Materials and Methods: Women initiating PMO treatment (index event) between 01Jan2010-31Dec2012 (MarketScan) or 01Jan2009-30Jun2012 (Medicare), with data available for $\geq 12$ months prior to and $\geq 4$ months after index date were included. Primary outcome was fracture (FX). To control for baseline FX risk, patients were their own control and the ratio of the FX rate in the late treatment period (12 months after the first 90 days of treatment) to the rate in the early treatment period (first 90 days of treatment) calculated. No MarketScan data was available for teriparatide(TPTD); Medicare data for oral bisphosphonates were pooled.

Results: Eligible women from MarketScan were younger than those from Medicare (mean [SD], 69.2 [11.1] vs 78.1 [7.4] years). In the early treatment period, FX rates were highest for TPTD, denosumab (DMAb) and zoledronic acid (Table; both databases). In the late treatment period, vertebral FX declined for all therapies, with the greatest reduction observed for TPTD (Medicare). DMAb produced the greatest reduction in non-vertebral FX (MarketScan) and reduced the rate of hip FX by $20 \%$ (Medicare). Results of sensitivity analyses, changing the length of the early/late treatment periods and censoring patients at first FX, were similar.

Conclusions: In these large real-world PMO cohorts, women treated with DMAb or TPTD were at higher FX risk than those treated with other antiresorptives. Despite this higher risk, both therapies were effective in reducing fracture risk.

Acknowledgements: Funded by Amgen 
MarketScan Fx rates per 100-patient years $(N=213,10)^{*}$

\begin{tabular}{|c|c|c|c|c|c|c|}
\hline & & $\begin{array}{l}\text { DMAb } \\
\qquad(n=17,561)\end{array}$ & $\begin{array}{l}\text { IV ZOL } \\
\quad(n=20,223)\end{array}$ & $\begin{array}{c}\text { Oral Ibandronate } \\
\quad(n=40,739)\end{array}$ & $\begin{array}{l}\text { Risedronate } \\
\qquad(n=31,651)\end{array}$ & $\begin{array}{l}\text { Aledronate } \\
\qquad(n=101,698)\end{array}$ \\
\hline \multirow[t]{3}{*}{ Hip FX } & Early & 0.8 & 0.7 & 0.4 & 0.5 & 0.6 \\
\hline & Late & 0.8 & 0.7 & 0.5 & 0.5 & 0.5 \\
\hline & $\operatorname{IRR}[95 \% \mathrm{CI}]$ & $0.99[0.68,1.45]$ & $1.01[0.70,1.47]$ & $1.03[0.74,1.43]$ & $0.88[0.62,1.25]$ & $0.97[0.80,1.17]$ \\
\hline \multirow[t]{3}{*}{ Non-vert FX } & Early & 3.1 & 2.0 & 1.5 & 1.3 & 1.7 \\
\hline & Late & 2.3 & 2.3 & 1.5 & 1.3 & 1.5 \\
\hline & IRR $[95 \% \mathrm{CI}]$ & $0.75[0.61,0.92]$ & $1.13[0.91,1.40]$ & $0.99[0.83,1.19]$ & $0.97[0.78,1.21]$ & $0.89[0.79,0.99]$ \\
\hline \multicolumn{7}{|c|}{ Medicare Fx rates per 100-patient years $(N=1,278,296)^{\dagger}$} \\
\hline & & $\begin{array}{l}\text { DMAb } \\
\quad(n=34,622)\end{array}$ & $\begin{array}{l}\text { IV ZOL } \\
\quad(n=124,857)\end{array}$ & $\begin{array}{l}\text { Other BPs } \\
\qquad(n=997,686)\end{array}$ & $\begin{array}{l}\text { Raloxifene } \\
\qquad(n=100,521)\end{array}$ & $\begin{array}{l}\text { TPTD } \\
\quad(n=20,610)\end{array}$ \\
\hline \multirow[t]{3}{*}{ All FX } & Early & 8.32 & 6.77 & 6.20 & 4.43 & 14.04 \\
\hline & Late & 5.68 & 6.19 & 5.56 & 4.41 & 7.94 \\
\hline & $\operatorname{IRR}[95 \% \mathrm{CI}]$ & $0.68[0.62,0.75]$ & $0.92[0.87,0.96]$ & $0.90[0.88,0.91]$ & $1.00[0.98,1.01]$ & $0.57[0.52,0.62]$ \\
\hline \multirow[t]{3}{*}{ Hip FX } & Early & 1.27 & 1.14 & 1.08 & 1.00 & 1.67 \\
\hline & Late & 1.01 & 1.10 & 1.12 & 1.03 & 1.45 \\
\hline & $\operatorname{IRR}[95 \% \mathrm{CI}]$ & $0.80[0.64,1.00]$ & $0.97[0.86,1.09]$ & $1.04[1.00,1.01]$ & $1.03[0.90,1.19]$ & $0.87[0.68,1.10]$ \\
\hline \multirow[t]{3}{*}{ Vert FX } & Early & 2.82 & 2.07 & 1.76 & 1.01 & 6.27 \\
\hline & Late & 1.41 & 1.50 & 1.29 & 0.89 & 2.26 \\
\hline & IRR $[95 \%$ CI $]$ & $0.50[0.43,0.59]$ & $0.72[0.66,0.79]$ & $0.74[0.71,0.76]$ & $0.88[0.77,1.02]$ & $0.36[0.31,0.42]$ \\
\hline
\end{tabular}

*Hip and non-vertebral fractures analysed for MarketScan; ${ }^{\dagger}$ Hip and vertebral fractures analysed for Medicare. Early, early treatment period, first 90 days of treatment; late, late treatment period, the 12 months following the early treatment period; FX, fracture; IRR, incidence rate ratio, late versus early treatment periods, values $<1$ indicate a reduction in FX risk; $N$, number of eligible women in the database; $n$, number od eligible women receiving the specific treatment; vert, vertebral

\section{P191}

\section{EFFECTS OF POSTURAL AND RESPIRATORY} REHABILITATION IN PATIENTS WITH HYPER- KYPHOSIS BY OSTEOPOROTIC VERTEBRAL FRACTURES: A PILOT STUDY

A. Pompa $^{1}$, G. Lugari ${ }^{1}$, E. Giampà ${ }^{1}$, C. Della Vedova ${ }^{1}$, L.

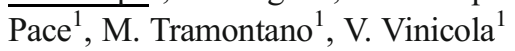

${ }^{1}$ Fondazione IRCCS S. Lucia, Rome, Italy

Objective: There is growing evidence that physiotherapic intervention has an important role in post-vertebral fracture recovery and treatment of dorsal hyperkyphosis. This is an excessive anterior concavity of the thoracic spine, common in osteoporotic patients, that can impair pulmonary function, activities of daily living performance, quality of life, and predict mortality independent of underlying spinal osteoporosis. There is no standard protocol for treating hyperkyphosis, generally the rehabilitative interventions are long lasting and aimed to strength the paravertebral muscles. Our rehabilitation program was short and aimed to restore good mobility of the spine, eliminating paravertebral muscle contractions and secondary reflex dysfunction of the diaphragm, considered also as a an important regulator of lumbar lordosis. The aim of our study was to evaluate the efficacy of a treatment protocol, based on the mobilization in elongation of the paravertebral muscles and respiratory rehabilitation, in improving postural attitude, respiratory compliance, mobility of spine and quality of life in hyperkyphotic patients with postmenopausal osteoporosis.

Patients and Methods: We enrolled women with dorsal hyperkyphosis due to one or more vertebral wedging by postmenopausal osteoporosis (T-score $<-2.5$ ). Necessary criterion was the presence of a kyphosis angle between $35^{\circ}$ and $50^{\circ}$ on Cobb scale. Inclusion criteria were: female, aged between 55 and 65 years, postmenopausal osteoporosis, one or more vertebral fractures. Exclusion criteria were: recent fractures (MRI negative for bone edema); usual program of exercise/ rehabilitation; neurological or orthopedic associated serious diseases. Patients enrolled were randomized into two groups: experimental group, submitted to the rehabilitation program and control group, who did not perform any kind of rehabilitation activity. The rehabilitation protocol included 10 sessions of $1 \mathrm{~h}$ each (with a frequency of one session every 5 days); besides each patient was educated to do some exercises daily. All patients were evaluated at the time T1 (start of training) and at T2 (outcome), after about two months, by the following scales: NUMERICAL RATING SCALE for pain, Distance hands - floor (in flexion and side bending) for evaluation of spinal mobility, Distance tragus- wall and Plumb line 
to $\mathrm{C} 7$ method for the evaluation of the dorsal kyphosis, incentive spirometer Coach II, to estimate lung capacity and QUALEFFO, as an index of quality of life.

Results: We enrolled 14 patients, who were randomly divided into the two groups. Experimental group and control group were homogeneous for age, spine mobility, pain and lung capacity at T1. In the experimental group, at the end of the treatment, we observed: a significant reduction of the kyphosis curve, measured by the method of the plumb line $(p<0.05)$ compared to the control group; greater lung capacity, although not statistically significant; a significant improvement in the mobility of the spine $(p<0.05)$; a significant reduction of pain $(p<0.005)$ than the control group. At the end of the training the patients of the experimental group were very satisfied, referring in most cases a greater feeling of physical well-being, as measured by the QUALEFFO scale, with an improvement of 8.7 points compared to 1 of the control group.

Conclusion: Despite the small number of patients does not allow us to draw final conclusions, preliminary data seem to indicate a good effectiveness of our rehabilitation protocol, in all domains investigated. This result is interesting, because we know that persons who suffer from hyperkyphosis are at increased risk for a variety of adverse health outcomes that include poor physical function, pulmonary compromise, falls, new fractures, and even earlier mortality.

\section{P192}

MUSCULOSKELETAL EXAMINATION IN PATIENTS PRESENTING WITH FALLS AND JUNIOR DOCTORS TRAINING: STILL A CONCERN

\author{
M. Nisar ${ }^{1}$, A. Oomatia ${ }^{1}$ \\ ${ }^{1}$ Luton \& Dunstable University Hospital, Luton, United \\ Kingdom
}

Musculoskeletal (MSK) examination in general medical patients admitted to hospital with locomotor issues is often incomplete or altogether omitted with reported frequency of as low as $20 \%{ }^{1}$. This is despite arthritis known to be the leading cause of non-serious prolonged hospital admission ${ }^{2}$. We audited the frequency of MSK examinations performed in patients admitted to our institution with locomotor issues. A survey of junior doctors was also undertaken to assess their confidence in performing MSK examination. Findings were compared to the same study done in 2011 which had instigated changes in the clerking process and development of a teaching programme for junior doctors ${ }^{3}$. In each audit cycle, 60 patients admitted to our hospital with locomotor complaints such as 'falls', or 'off legs' were identified at random and their admission clerking reviewed. Additionally junior doctors were surveyed to determine any change in their perceived confidence in performing MSK examination. Demographics of the two cohorts (2011 \& 2013) were similar with majority of participants being older ( 80 and $88 \%$ ) women $(63$ and $65 \%$.) 31 (51.7\%) patients in 2011 and 34 (56.7\%) patients in 2013 had no documented MSK examination. Four patients had prolonged hospital stay this year compared to three in 2011 as a result of inadequate MSK examination leading to the delay in diagnosis. This included two patients with vertebral fractures. Comparing junior doctors' confidence in undertaking MSK examination in 2011 $(n=21)$ with $2013(n=23)$, only two and five respectively were fully confident despite 13 vs 15 had received formal MSK education in medical school. However, only 4 doctors in both groups had any postgraduate teaching in locomotor examination. MSK examinations are performed in only $50 \%$ of patients presenting with locomotor system complaints at our institute though still higher than other reports in the literature $^{4-5}$. Inclusion of MSK exam section in the clerking proforma since the last audit failed to improve the outcome leading to delayed diagnosis and a trend towards prolonged hospital stay. An important reason, as our audit demonstrates, is the relative lack of confidence in junior doctors performing MSK examination despite the introduction of postgraduate teaching programme. This might be due to short placements and frequent rotations of junior doctors, hence they may not have had the opportunity to attend the sessions. Focused but flexible training after graduation is required to improve doctors' examination skills, both as formal teaching and during consultant ward rounds to improve patient care and safety.

\section{References:}

${ }^{1 .}$ Lillicrap MS, et al. Rheumatology 2003;42:951.

2. Keough A, Kirwan J. Rheumatology 2003;42:810.

${ }^{3 .}$ Nisar MK, et al. Int J Rheum Dis 2012;15:e43.

4. Doherty M, et al. J Royal Coll Physicians 1990;24:115.

5. Marshall RW, Hull RG. Rheumatology 2004;43:1447.

\section{P193}

DEVELOPMENT AND VALIDATION OF AN OSTEOPOROSIS TREATMENT QUESTIONNAIRE (OSTREQ): EVALUATION OF PHYSICIANS' CRI- TERIA IN THE CHOICE OF TREATMENT P. Makras $^{1}$, A. D. Anastasilakis ${ }^{2}$, A. Galanos ${ }^{3}$, S. Rizou ${ }^{4}$, G. P. Lyritis $^{4}$

${ }^{1}$ Department of Endocrinology and Diabetes, 251 Hellenic Air Force General Hospital, Athens, Greece, ${ }^{2}$ Department of Endocrinology, 424 Military Hospital, Thessaloniki, Greece, ${ }^{3}$ Laboratory for the Research of the Musculoskeletal System, University of Athens, Athens, Greece, ${ }^{4}$ Hellenic Osteoporosis Foundation, Athens, Greece

Objectives: This study describes the development and validation of the Osteoporosis Treatment Questionnaire (OSTREQ), which is a physician-reported outcome tool aiming to evaluate the physicians' criteria in the choice of osteoporosis treatment. 
Materials and Methods: A questionnaire consisted of 17 questions, included in eight sections (health care system, patients' preference in administration routes, usage, cost, severity of disease, treatment efficacy, safety profile, pharmaceutical industry), affecting the decision and overall execution of a therapeutic approach was developed by an expert panel and was later officially translated to English. In the second phase orthopedic surgeons were asked to complete OSTREQ. Six indirect methods to evaluate validity were adopted: exploratory factor analysis, confirmatory factor analysis, subscale validity, known groups validity, floor or ceiling effects, interpretability. To assess the reliability of the questionnaire internal consistency validity, as well as test-retest and parallel forms were calculated.

Results: One hundred seventy two orthopedic surgeons were interviewed with an average experience in clinical practice of 10.5 years ( \pm 8.9 years). Factors "Severity of Disease" and "Treatment efficacy" were the most important ones in the choice of osteoporosis treatment while factor "Pharmaceutical industry" had the least impact. The methodology of validation proved that the questionnaire presents construct validity, discriminant ability, reliability, and sensitivity to change.

Conclusions: OSTREQ represents a comprehensive and focused tool that assesses the physicians' criteria in the choice of osteoporosis treatment. This tool could assist health care systems and pharmaceuticals companies to understand which parameters drive physicians' preferences regarding the treatment of osteoporosis.

\section{P194}

RURAL COMMUNITY EXPERIENCE: OSTEOPOROSIS FIRSTLY PRESENTED BY FRAGILITY FRACTURE

A. M. Ali ${ }^{1}$, H. A. Elazaly ${ }^{1}$

${ }^{1}$ King Faisal Medical Complex, Taif, Saudi Arabia

Objective: To study the incidence of undiagnosed osteoporosis among fractured patient referred from rural communities.

Material and Methods: King Faisal Medical Complex in Taif is a referral hospital for 8 rural communities around Taif city with a collective population of about 800,000 citizen. During the period of 18 months (from April 2014 to September 2015); we conducted a prospective study for referred patients above the age of 50 years who experienced fractures after lowenergy trauma (falling down). We included only patients with osteoporosis; as evident by BMD measurement with a T-score less than or equal to -2.5 . Each fracture was managed accordingly either surgically (mainly for hip and distal radius) or nonsurgically (mainly for spine). Treatment of osteoporosis was started for all patients. We hold in our study a detailed record for osteoporosis previous diagnosis and intake of osteoporosis treatment in the last 5 years. Most of the patients in our series are still following up in outpatient clinics.

Results: 482 patients were included in our study, osteoporosis was undiagnosed in $448(92.94 \%)$ patients prior to the time of fracture, while among the previously-diagnosed (34 patients); only 13 patients were maintained on osteoporosis treatment before the fracture time. The remaining 21 patients were not compliant with the treatment previously prescribed for them. Conclusion: The majority of patients in our study (93\%) had undiagnosed osteoporosis prior to getting fractured, so we conclude that in rural communities osteoporosis is almost always firstly presented by a fragility fracture. This conclusion call for a recommendation to conduct regular screening campaigns for rural communities to discover osteoporosis in population above the age of 50 years. We will progress in our study to collect a larger patient data sample for statistically significant results about site of fracture, re- fracture occurrence, and compliance with osteoporosis treatment.

\section{P195}

COMPARATIVE ANTI-FRACTURE EFFECTIVENESS OF DIFFERENT ANTI-OSTEOPOROSIS MEDICATIONS AMONGST MALE DRUG USERS IN REAL WORLD CLINICAL PRACTICE: A POPULATION-BASED COHORT STUDY

M. Zwart ${ }^{1}$, S. Hawley ${ }^{2}$, T. Van Staa $^{3}$, N. Harvey ${ }^{4}$, A. Judge ${ }^{2}$, M. K. Javaid ${ }^{2}$, C. Cooper ${ }^{4}$, D. Prieto-Alhambra ${ }^{2}$

${ }^{1}$ Catalan Institute of Health (ICS), Barcelona, Spain, ${ }^{2}$ Nuffield Department of Orthopaedics, Rheumatology and Musculoskeletal Sciences, University of Oxford, Oxford, United Kingdom, ${ }^{3}$ Health eResearch Centre, University of Manchester, Manchester, United Kingdom, ${ }^{4}$ MRC Epidemiology Resource Centre, University of Southampton, Southampton General Hospital, Southampton, United Kingdom

Objective: There is a scarcity of data on head-to-head comparison of anti-fracture effectiveness of different antiosteoporosis medications (AOM) for males. We therefore aimed to compare the observed risk of fracture while on treatment amongst male users of different AOMs.

\section{Methods}

Design: Cohort study using primary care data from the United Kingdom CPRD.

Participants, exposures, outcome: All men who received any AOMs [alendronate, other (oral) bisphosphonates (BPs), strontium ranelate, denosumab, or teriparatide] prescribed for at least 6 months in 2000-2012 were eligible and followed from first prescription until occurrence of a fracture (hip, major, or non-hip) or censoring.

Statistical analyses: Multivariable Cox regression models were fitted, adjusting for age, BMI, smoking, alcohol drinking, fracture history, Charlson index, and prior use 
of drugs with an effect on bone metabolism or fracture risk.

Results: A total of 31,846 men were included: 26,124 (82\%) alendronate, 5,244 (16.5\%) other BPs, 470 (1.5\%) strontium, 4 denosumab, and 1 teriparatide users. The two latter groups were excluded due to insufficient power. Compared to alendronate (reference group), adjusted HRs [95\% $\mathrm{CI}]$ for other BP users were 1.26 [0.99-1.60], 1.18 [0.97-1.43], and 0.95 [0.75-1.19] for hip, major and non-hip fracture respectively. Similarly, adjusted HRs for strontium users were 1.26 [0.70-2.2], 1.29 [0.802.08], and 0.87 [0.43-1.77].

Conclusions: Our study shows borderline-significant increases in hip and major fracture risk amongst male users of other oral BP compared to alendronate with no differences in non-hip fracture risk. Similar results are seen for strontium users, although with less significance probably due to lower statistical power. Residual confounding could partially explain these findings, and randomised studies are needed to confirm our results. Acknowledgments: Partial funding from the National Osteoporosis Society UK and the Idiap Jordi Gol (Barcelona).

\section{P196}

REVIEW OF THE EFFICIENCY IN TREATMENT WITH ZOLEDRONIC ACID IN SIX PATIENTS WITH GIOP

T. Jankovic ${ }^{1}$, J. Zvekic-Svorcan ${ }^{2}$, K. Filipovic ${ }^{1}$, S. SubinTeodosijevic $^{3}$, J. Vasic ${ }^{4}$

${ }^{1}$ Rheumatology, Special Hospital for Rheumatic Diseases, Novi Sad, Serbia, ${ }^{2}$ Rheumatology, Special Hospital for Rheumatic Diseases, Faculty of Medicine, University of Novi Sad, Novi Sad, Serbia, ${ }^{3}$ Rheumatology, General Hospital, Zrenjanin, Serbia, ${ }^{4}$ Rheumatology, Railway Healthcare Center, Belgrade, Serbia

Objective: To show the efficiency in treatment with zoledronic acid in 6 patients with GIOP.

Method: The study included 6 patients with GIOP in a 1 year period. They have been diagnosed osteoporosis after DXA findings and vertebral fractures after radiographic examine.

Results: Table BMD and T score before and after treatment with zoledronic acid in 6 patients.

\begin{tabular}{|c|c|c|c|c|c|c|c|c|}
\hline \multirow[t]{2}{*}{ Patient } & \multicolumn{2}{|c|}{ BMD L1-L4 } & \multicolumn{2}{|c|}{ T L1-L4 } & \multicolumn{2}{|c|}{ BMD hip } & \multicolumn{2}{|c|}{ T hip } \\
\hline & begining & after 1 year & begining & after 1 year & begining & after 1 year & begining & after 1 year \\
\hline 1 & 0,767 & 0,834 & $-3,40$ & $-2,80$ & 0,658 & 0,792 & $-2,70$ & $-2,20$ \\
\hline 2 & 0,712 & 0,821 & $-3,70$ & $-3,10$ & 0,562 & 0,715 & $-3,20$ & $-2,40$ \\
\hline 3 & 0,614 & 0,794 & $-4,20$ & $-3,50$ & 0,562 & 0,699 & $-3,10$ & $-2,20$ \\
\hline 4 & 0,823 & 0,911 & $-2,70$ & $-2,20$ & 0,711 & 0,804 & $-2,10$ & $-1,50$ \\
\hline 5 & 0,845 & 0,916 & $-2,20$ & $-2,20$ & 0,751 & 0,798 & $-1,90$ & $-1,20$ \\
\hline 6 & 0,834 & 0,895 & $-3,10$ & $-2,70$ & 0,752 & 0,804 & $-2,10$ & $-1,60$ \\
\hline
\end{tabular}

Conclusion: In all patients, there was an increase in BMD at all measurement points verified with DXA findings and no new fractures.

Reference: Jankovic T, et al. Osteoporos Int 2013;24:S165

\section{P197}

PERINATAL DNA METHYLATION AT THE CDKN2A LOCUS AT BIRTH IS ASSOCIATED WITH CHILDHOOD BONE SIZE AND MINERALISATION: FINDINGS FROM THE SOUTHAMPTON WOMEN'S SURVEY

E. M. Curtis ${ }^{1}$, R. Murray ${ }^{2}$, P. Titcombe ${ }^{1}$, R. Clarke-Harris ${ }^{2}$, P. Costello $^{2}$, E. Garratt ${ }^{2}$, E. Cook ${ }^{2}$, J. Holbrook ${ }^{3}$, S. Barton ${ }^{1}$, H. Inskip $^{1}$, K. Godfrey ${ }^{1}$, C. Cooper ${ }^{1}$, K. A. Lillycrop ${ }^{2}$, N. C. Harvey ${ }^{1}$

${ }^{1}$ MRC Lifecourse Epidemiology Unit, University of Southampton, Southampton, United Kingdom, ${ }^{2}$ Institute of Developmental Sciences, University of Southampton, Southampton, United Kingdom, ${ }^{3}$ Singapore Institute for Clinical Sciences, A*STAR, Singapore, Singapore
Objectives: We used a population-based mother-offspring cohort to explore relationships between DNA methylation at the CDKN2A gene locus (a region involved in cell cycle regulation) in umbilical cord tissue at birth, and bone indices measured by DXA in childhood.

Materials and Methods: We used a genome-scale methylbinding domain capture array (Agilent) to identify potentially informative genomic regions in 19 umbilical cords from infants born in the UK Southampton Women's Survey (SWS). Following adjustment to account for $\mathrm{CpG}$ density via a Bayesian algorithm (BATMAN), we located a differentially methylated region within the CDKN2A gene locus with strong correlations between methylation and childhood bone size and density assessed by DXA. We then used pyrosequencing to carry out in-depth methylation analysis at 9 CpGs within this CDKN2A region in umbilical cords from independent discovery $(n=332)$ and replication $(n=337)$ cohorts of SWS children. Bone mass was assessed by wholebody-minus-head DXA (Hologic Discovery) at 4 and/ or 6 years old. 
Results: Percentage methylation varied greatly across the $9 \mathrm{CpG}$ sites (at $\mathrm{CpG} \mathrm{6,} \mathrm{the} \mathrm{5th} \mathrm{to} \mathrm{95th} \mathrm{percentile}=49.9-82.4 \%$ ). Adjusting for age and sex, there were consistent associations between higher CDKN2A methylation at 6 of $9 \mathrm{CpG}$ sites (CpG 4 9) and lower bone area, bone mineral content (BMC), and areal density in both cohorts at 4 and 6 years, and in further analyses on the pooled dataset (all $p<0.01$ ). For example, for each $10 \%$ increase in methylation at $\mathrm{CpG} 4-9$, BMC decreased by 6-9 $\mathrm{g}$ at age 4 years $(p \leq 0.001)$. Associations remained robust after adjustment for batch effect, maternal height, smoking, walking speed and triceps skinfold thickness in late pregnancy; and offspring lean mass, fat mass or birthweight.

Conclusions: We have demonstrated that perinatal CDKN2A methylation is negatively associated with childhood bone size, mineral content and density. These findings, if replicated in other cohorts, suggest a potential role for CDKN2A in skeletal development and its use as a biomarker for later osteoporosis risk.

\section{P198}

\section{THE ASSOCIATION OF VASCULAR STIFFNESS} PARAMETERS AND SUBCLINICAL ATHEROSCLEROSIS WITH BONE MASS IN POSTMENOPAUSAL WOMEN

I. Skripnikova $^{1}$, N. Alikhanova ${ }^{1}$, O. Tkacheva ${ }^{2}$, E. Abirova $^{1}$, V. Novikov $^{1}$, L. Murashko ${ }^{1}$, N. Sharashkina ${ }^{2}$, O. Isaykina ${ }^{3}$, V. Vygodin $^{4}$

${ }^{1}$ Prophylaxis Osteoporosis Department, Moscow, Russian Federation, ${ }^{2}$ Department of Integrated Non-communicable Disease Rick Reduction, Moscow, Russian Federation, ${ }^{3}$ Laboratory of Outpatient Diagnostic Methods in the Prevention of Chronic Non-Communicable Diseases, Moscow, Russian Federation, ${ }^{4}$ Laboratory of Biostatistics, Moscow, Russian Federation

Objective: To study the relationship between vascular wall condition and bone mass in postmenopausal women.

Materials and Methods: In a cross-sectional study included 107 patients - women at the age range of 45-82 years who were observed outpatient and signed a written informed consent. Exclusion criteria: any clinical signs of atherosclerosis, malignant diseases, diseases causing secondary osteoporosis, taking drugs affecting bone turnover and the indices of vascular stiffness. The intima-media thickness (IMT), the presence and quantity of atherosclerotic plaques (AP), and the degree of carotid stenosis were examined by duplex scanning. The pulse wave velocity (PWV), augmentation index (AI) were measured by applanation tonometry (SphygmoCor). Assessment of BMD was performed in spine and hip using DXA (Delphi W, Hologic, USA). Statistical analysis was performed using the software application Statistical Analysis System (USA).

Results: With the increase in duration of menopause a gradual increase in the stiffness rate (PWV, AI), IMT and decrease of
BMD was noticed in all examined parts of the skeleton. The maximal vascular stiffness and minimal BMD were found in patients with $10+$ years of menopause. Osteopenia and osteoporosis were diagnosed in $61 \%$ of patients. The risk of bone mass loss and osteoporosis increased by 3 times in patients with high values of $\mathrm{PWV} \geq 10 \mathrm{~m} / \mathrm{s}$ [OP-3.1, $95 \% \mathrm{CI}$ 1.13-9.89 $(p<0.05)]$, by more than 4 times in patients with $\mathrm{AI} \geq 20 \%$ [OP-4.35, 95\%CI 1.02-11.0 $(p<0.05)$ ] and the IMT $>9 \mathrm{~mm}$ [OP-4.17 95\%CI $1.21-14.4(p=0.02)]$ and by 2.45 times in patients with presence of AP in the carotid arteries [OP-2.45, 95\% CI 1.07-5.56 $(p=0.03)$ ]. During multivariate regression analysis the negative relationship between IA, IMT and BMD remained highly significant, while in relation to PWV and the AP presence, such a correlation was not confirmed.

Conclusion: Reduced BMD in postmenopausal women is associated with high rates of vascular stiffness. The correlation between duration of menopause and gradual increase in the rates of vascular stiffness (PWV, AI), IMT, as well as loss of bone mass was observed.

P199

EVALUATION OF BONE METABOLISM MARKERS AND QOL IN PATIENTS WITH OSTEOPOROSIS AFTER TREATMENT WITH THE ANTI-RANKL ANTIBODY DENOSUMAB

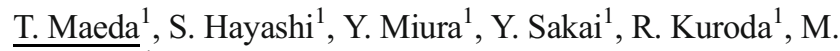
Kurosaka ${ }^{1}$

${ }^{1}$ Department of Orthopaedic Surgery, Kobe University Graduate School of Medicine, Kobe, Japan

Objectives: This study aimed to investigate the effects of denosumab in patients with osteoporosis based on the evaluation of changes in bone density, those in bone metabolism markers detected by blood tests, and those in health-related QOL obtained from an observational study.

Material and Methods: The study included 400 patients with osteoporosis who received denosumab treatment ( $80.4 \mathrm{y}$; including 82 patients switched from the other medications). The following clinical evaluations were performed before the first administration of denosumab and after 6 and 12 months of the treatment: bone mineral assay in the distal end of the radius using DXA; blood test for P1NP and TRACP-5b. A questionnaire survey employing VAS scores for low back pain and patients' QOL using SF-36 was also carried out.

Results: The bone density relative to the YAM ratio was significantly increased at both 6 and 12 months after the treatment with denosumab as compared to that determined before the first administration (51.6\% pre-treatment, $52.1 \%$ $[P<0.05]$ after 6 months, and $52.6 \%[P<0.001]$ after 12 months). The serum levels of P1NP and TRACP-5b were significantly decreased (P1NP: $55.9 \mu \mathrm{g} / \mathrm{L}, 24.5 \mu \mathrm{g} / \mathrm{L}$ $[P<0.001]$ and $26.1 \mu \mathrm{g} / \mathrm{L}[P<0.001]$; TRACP-5b: $545 \mathrm{mU} /$ 
$\mathrm{dL}, 290 \mathrm{mU} / \mathrm{dL}[P<0.001]$ and $288 \mathrm{mU} / \mathrm{dL}[P<0.001]$, respectively). The VAS scores for low back pain showed significant improvement $(41.4 \mathrm{~mm}, 26.8 \mathrm{~mm}[P<0.05]$ and $23.1 \mathrm{~mm}[P<0.001]$, respectively). The SF-36 scores at 6 months after starting denosumab treatment, as compared to those before the treatment, improved in three categories including physical functioning, role physical and bodily pain $(P<0.05)$.

Conclusions: This study demonstrated that denosumab suppressed bone turnover and increased bone strength in patients with osteoporosis. The SF-36 scores showed significant improvement in the physical dimension categories including physical functioning, role physical and bodily pain, suggesting that denosumab can potentially contribute to QOL improvement as well as increasing bone strength.

\section{P200}

INFLUENCE ON BONE MINERAL DENSITY AND PAIN SYNDROME IN PATIENTS WITH RHEUMATOID ARTHRITIS COMBINATION CALCIUM CARBONATE/VITAMIN D ANDOSSEIN HYDROXYAPATITE COMPLEX

A. V. Cherkasova ${ }^{1}$, I. P. Garmish ${ }^{1}$

${ }^{1}$ DSMA Ukraine, Dnipropetrovsk, Ukraine

Background: In patients with rheumatoid arthritis (RA) develop both local and systemic osteoporosis due to inflammation and medications (glucocorticoids) that are used in treatment of RA. Question of treatment of osteopenia among such patients is not still closed. According to the EULAR recommendations in that case as first medication we use combination of calcium and vitamin $\mathrm{D}$, but there are more active compounds.

Objectives: To compare the effect of a standard combination of calcium carbonate $(1000 \mathrm{mg})$ and vitamin D (800ME) and combination of ossein $(291 \mathrm{mg}$ ) and hydroxyapatite (444 mg) (Pierre Fabre, France) on BMD in patients with RA, track the dynamic of pain syndrome.

Methods: We examined 47 patients with RA, 9 men and 38 women, mean age $-51.60 \pm 5.62$ years, midscore of DAS28 is $2.78 \pm 0.35$, randomized into 2 groups. As basic therapy 20 patients $(42.55 \%)$ received methotrexate $7.5-15 \mathrm{mg}$ per week, 23 patients (48.94\%) combined methotrexate with glucocorticoids $5-10 \mathrm{mg}$ per day, 4 patients $(8.51 \%)$ received leflunomide $20 \mathrm{mg}$ per day. Patients of the first group $(n=23)$ along with basic therapy were treated with osteogenon, the second group $(n=24)$ - basic therapy and calcium with vitamin D. Pain syndrome was assessed according to visual analogue scale (VAS) before treatment and after 1,3 and 6 months. BMD was determined by DXA.

Results: Pain decreased by $35.4 \%(p<0.05)$ in the group of patients receiving osteogenon and in the group receiving standard combination of calcium and vitamin D to $9.1 \%$ $(p<0.05)$. BMD increased in the group receiving osteogenon $(+6.9 \%, p<0.001)$ after 6 months of treatment, in contrast to second group, in which was not observed reliable dynamic $(+1.35 \%, p>0.05)$.

Conclusions: More significant improvement of BMD was registered in first group in comparison with a group of patients that received the standard combination of calcium with vitamin $\mathrm{D}$ at recommended doses.

\section{P201}

IMPACT OF PRETRANSFUSION HAEMOGLOBIN AND SERUM FERRITIN LEVELS ON PHYSICAL GROWTH IN PAKISTANI CHILDREN W I T H T RA N S F U S I O N DEPENDEN T B-THALASSAEMIA

M. S. Shaikh ${ }^{1}$, B. Moiz ${ }^{1}$, A. H. Khan ${ }^{1}$, S. Misri ${ }^{1}$, A. Raheem ${ }^{1}$, R. Iqbal ${ }^{1}$

${ }^{1}$ Aga Khan University, Karachi, Pakistan

Objective: To determine association of physical growth with pretransfusion haemoglobin and serum ferritin in Pakistani children with transfusion dependent $\beta$-thalassaemia.

Material and methods: This was an observational descriptive study conducted at Fatimid Foundation Karachi from January 2013 to December 2014. All the patients with diagnosis of transfusion dependent $\beta$ - thalassaemia major were recruited. Demographic and anthropometric profile along with pretransfusion haemoglobin and serum ferritin levels were recorded. BMI was calculated in $\mathrm{kg} /$ height in meters 2 . Zscores were calculated utilizing following formula: $Z=x-\mu /$ $\sigma$. WHO 2007 growth charts for boys and girls were used to assess physical growth. Accordingly, a Z-score of $<-2$ for height was considered as stunted growth and BMI of $<-2$ was considered as underweight or thin for age.

Results: A total of 380 patients were included. Mean age at first evaluation was $10.6 \pm 3.3$ with $\mathrm{M}: \mathrm{F}$ ratio of 1.1:1. Mean \pm SD of different variables were: haemoglobin: 7.6 $\pm 1.3 \mathrm{~g} / \mathrm{dl}$, ferritin $5401 \pm 2650.2 \mathrm{ng} / \mathrm{ml}$, height Z-score: $-2.65 \pm 1.64$, weight Z-score: $-1.82 \pm 1.12$ and BMI Zscore: $-1.77 \pm 1.40$. With increasing age, decrease in pretransfusion $\mathrm{Hb}$ and an increase in ferritin levels along with worsening BMI and height Z-scores were observed. Significant association of higher ferritin levels (5632.75 $\pm 2543.97 \mathrm{ng} / \mathrm{ml}$ ) was found in patients with stunted growth $(n=250,66 \% ; p=0.019)$.

Conclusions: Growth retardation is a common problem in transfusion dependent $\beta$-thalassaemia. Good nutrition, appropriate transfusions and chelation along with height and weight monitoring at regular intervals must be ensured.

Disclosure: This study was supported by institutional research council of Aga Khan University. 


\section{P202}

TOPICAL ALENDRONATE ENHANCE THE HEALING OF MAXILLARY SINUS LIFTING FOR DENTAL IMPLANTATION IN OSTEOPOROTIC WOMEN: CLINICAL STUDY S. Adarnli ${ }^{1}$, G. Bassit ${ }^{1}$, N. Koshaji ${ }^{1}$

${ }^{1}$ International University for Science and Technology, Damascus, Syrian Arab Republic

Introduction \& background: Alveolar ridge resorption is a physiologic process occurs after teeth extraction, it usually complicates the insertion of dental implants as a result of inadequate available alveolar bone; this resorption makes bone augmentation needed in most implantation procedures especially in the posterior maxilla where the maxillary sinus normally expands after tooth extraction creating the least quantity of bone, which is usually the least density (D4) in all oral cavity anatomical locations. This resorption was proved to be more presented in osteoporotic women, moreover the negative effect of osteoporosis on bone graft healing has been previously proved, which will totally affect the quality of life of osteoporotic women.

Aim: To investigate the possible positive effect of topical alendronate $108-\mathrm{M} / \mathrm{L}$ in humans; which we previously showed in experimental animal study, on the healing of maxillary sinus bone grafts prior to dental implants placement, as far as we know this is the first ever histomorphometric study in the field.

Materials \& Methods: This study was performed on 21 osteoporotic female women their ages were between 55 and 73 years, all of them were using alendronate (ALN) $70 \mathrm{mg} /$ 1 time weekly. All the patients in the study were selected with edentulous posterior maxilla with alveolar ridge less than $5 \mathrm{~mm}$ in height confirmed on cone beam computed tomography, patients were divided into two groups; the control group (11 patients) and the ALN group (10 patients). All of patients underwent an external sinus lifting procedure, with Bio-Oss augmentation in the control group, and augmentation with Bio-Oss soaked in 108- M/L Alendronate solution in the ALN group. All the patients were observed for 10 months for optimum graft healing and then a procedure of dental implantation was performed with trephine histologic samples (for histomorphometric study) obtained from the place of implant insertion.

Results: T student analysis for histomorphometric study showed that the ratio of new bone formation in ALN group was $(29.37 \pm 3.67 \%)$ and significantly higher than the Control group (20.65 $\pm 2.27 \%), P<0.01$.

Conclusion: Based on previous results we conclude that topical application of alendronate 10-8 $\mathrm{M} \backslash \mathrm{L}$ enhances bone grafts healing in maxillary sinus of osteoporotic women, which can aid for later more successful rating of dental implantation.

\section{P203}

ECONOMIC STUDY OF NON-PHARMACOLOGICAL AND PHARMACOLOGICAL TREATMENT FOR OSTEOPOROSIS VERTEBRAL FRACTURE AND BONE EVENTS IN ONCOLOGY PATIENTS IN A HEALTH INSURANCE PROVIDER IN A LATIN AMERICA COUNTRY

E. Tanaka ${ }^{1}$, F. Tanaka ${ }^{1}$, M. D. Barbosa ${ }^{2}$

${ }^{1}$ Curitiba Health Institute, Curitiba, Brazil, ${ }^{2}$ Med Inc Research, Berlin, Germany

Objectives: Comparing bone-related event treatment costs in a Latin America country private health care provider. Background: The rising costs of prostheses and orthopedic curative or palliative procedures in oncology patients, and pathological fractures due to osteoporosis stimulate the innovation of using drugs, such as bisphosphonates, intended to minimize skeletal-related events. Little is known about the economic impact of these measures.

Methods: We analyzed the electronic medical records of 100 patients (GROUP A) who received zoledronate (zol) at some point during their treatment (2009-2014). Most (71 patients) were diagnosed with breast cancer. The others 29 were diagnosed with other types of cancer. We analyzed another group of 88 patients (Group B) who had osteoporosis vertebral fracture with or without surgery procedure.

Results: The costs for Group A were estimated at approximately R\$ 876.000,00 Brazilian reals ( USD 437.000, 00 ) with 487 IV applications of zol during five years considering only bottle/vials spending. Expenses with femur and column prosthesis totaled $\$ 455,000.00$ and $\$ 580$, 000.00 USD respectively, if all 100 patients should spent in prosthesis. For both groups the data regarding costs was calculated based on the pharmacoeconomic model. If all 88 patients were treated with an external prostheses using a non-pharmacological (SPINOMED) treatment for vertebral fracture, the cost would be roughly $€ 35.200$ Euros (88 patients $\times 400$ Euros), that is $\sim \$ 70.400 .00$ USD or $\sim R \$ 176.000,00$ Brazilian Reals.

Conclusions: In spite of different clinical aspects of evaluation, due to biologic heterogeneous disease, the use of IV zol in group A patients was favorable and further studies investigating others patterns such as microscopic bone pathology and pharmacologic exposures should be conducted. The non pharmacological treatment is cost effective in group B due to the results based on the total costs. 


\section{P204}

PREVALENCE OF CONCOMITANT BONE AND MUSCLE WASTING IN PATIENTS FROM THE SARCOPHAGE STUDY

M. Locquet ${ }^{1}$, C. Beaudart ${ }^{1}$, J.-Y. Reginster ${ }^{1}$, J. Petermans ${ }^{2}$, S. Gillain $^{2}$, A. Quabron ${ }^{1}$, J. Slomian ${ }^{1}$, F. Buckinx ${ }^{1}$, O. Bruyère ${ }^{1}$

${ }^{1}$ Department of Public Health, University of Liège, Liège, Belgium, ${ }^{2}$ Geriatric Department, CHU of Liège, Liège, Belgium

Background: Recent studies suggest that bone and muscle wasting are closely interconnected. Dysfunctions of the "muscle-bone unit" generate an increased risk of morbid outcomes, i.e. injurious falls and fractures, physical disability, hospitalization, loss of independence and ultimately mortality. However, the relationship between age-related muscle and bone loss is not yet fully elucidated. Our objective is to assess the prevalence of osteoporosis (OP) in a population of individuals diagnosed with sarcopenia ( $\mathrm{Sp}$ ).

Methods: We investigated women, aged 65 years old and above, for whom BMD was available at the time of inclusion in the SarcoPhAge (Sarcopenia and Physical Impairments with advancing Age) study. SarcoPhAge is an ongoing prospective study following community-dwelling elderly subjects to assess health and functional consequences of Sp. For this ancillary study, looking at the prevalence of low BMD in patients with or without Sp, women taking a pharmacological treatment against osteoporosis were excluded. Muscle strength was assessed with a hydraulic hand-dynamometer (Saehan Corporation, MSD Europe Bvba, Belgium), muscle mass (i.e., appendicular lean mass) and BMD by DXA (Hologic Discovery A, USA) and physical performance by the Short Physical Performance Battery (SPPB) test (/12 points). Sp was diagnosed according to the EWGSOP definition, i.e. a low muscle mass $\left(<5.5 \mathrm{~kg} / \mathrm{m}^{2}\right.$ for women and $<$ $7.26 \mathrm{~kg} / \mathrm{m}^{2}$ for men) plus either low muscle strength $(<20 \mathrm{~kg}$ for women and $<30 \mathrm{~kg}$ for men) or low physical performance (SPPB $<8$ points). A BMD T- score equal to or below $-2.5 \mathrm{SD}$ at the lumbar spine or at the hip was used to define osteoporosis (World Health Organization definition).

Results: 106 women aged $73.21 \pm 6.32$ years with a BMD assessment at baseline were included. Among them, 22 where diagnosed with Sp (20.75\%) and 19 (17.92\%) with OP. After adjustment for potential confounders, a significant lower appendicular lean mass was observed in OP women compared to women without OP $\left(5.32 \pm 0.65 \mathrm{~kg} / \mathrm{m}^{2}\right.$ vs. $5.91 \pm 0.78 \mathrm{~kg} / \mathrm{m}^{2}$, $p=0.018$ ). We also observed, in OP subjects, a lower muscle strength $(19.49 \pm 7.23 \mathrm{~kg}$ vs. $22.28 \pm 6.08 \mathrm{~kg}, p=0.021$ after adjustment) and a lower physical performance ( $7.89 \pm 3.23$ points vs. $9.16 \pm 2.67$ points, $p=0.014$ after adjustment). There were more OP women among Sp subjects (36.4\%) than among non-
Sp subjects $(13.1 \%)(p=0.011)$. Numerical values of BMD were lower in the Sp vs. non-Sp populations but the differences did not reach the level of statistical significance: $0.968 \pm 0.224 \mathrm{~g} / \mathrm{cm}^{2} \mathrm{vs}$. $0.998 \pm 0.186 \mathrm{~g} / \mathrm{cm}^{2}$ for the lumbar spine $(p=0.36), 0.766$ $\pm 0.133 \mathrm{~g} / \mathrm{cm}^{2}$ vs. $0.805 \pm 0.120 \mathrm{~g} / \mathrm{cm}^{2}$ for the total hip $(p=.016)$ and $0.667 \pm 0.128 \mathrm{~g} / \mathrm{cm}^{2}$ vs. $0.693 \pm 0.095 \mathrm{~g} / \mathrm{cm}^{2}$ for the femoral neck ( $p=0.38)$. Eventually, appendicular lean mass was positively and significantly correlated with lumbar spine, total hip and femoral neck BMD (respectively, $r=0.29, r=0.33, r=0.29, p<0.05$ ). Conclusion: Muscle mass, muscle strength and physical performance are lower in patients presenting Op, defined by a low BMD. Prospective changes in bone and muscle wasting will be investigated during the follow-up of our cohort.

\section{P205}

SAFETY AND EFFICACY OF SINGLEBALLOON MULTIPLE-PASS TECHNIQUE KYPHOPLASTY IN TREATMENT OF MULTIPLE-LEVEL VERTEBRAL COMPRESSION FRACTURES, SPINAL METASTASES AND SPINAL MULTIPLE MYELOMA

K. C. Mak ${ }^{1}$, G. M. Kuang ${ }^{2}$, Z. M. Zheng ${ }^{3}$

${ }^{1}$ Department of Orthopaedics \& Traumatology, University of Hong Kong, Hong Kong, Hong Kong, ${ }^{2}$ Department of Orthopaedics \& Traumatology, The University of Hong Kong Shenzhen Hospital, Shenzhen, China, ${ }^{3}$ Department of Spine Surgery, The First Affiliated Hospital, Sun Yat-sen University, Guangzhou, China

Objective: To evaluate the safety and efficacy of using a single-balloon for multiple passes PKP in treating multiplelevel vertebral lesions in a single patient.

Patients and Methods: Forty-five consecutive single-balloon multiple pass PKP procedures were performed in 18 patients. The indications included osteoporotic vertebral compression fractures (11 patients), spinal metastases (2 patients) and spinal multiple myeloma (5 patients). The average age was 68.8 $(44 \sim 80)$ years. One kyphoplasty balloon was used for one patient., therefore multiple passes for each balloon. Perioperative variables and bone tamp complications were recorded and analyzed. Preoperative and postoperative radiographs were compared to calculate percentage of height restoration. Outcome data were obtained by comparing preoperative and postoperative Visual Analog Scale (VAS) and Oswestry Disability Index (ODI) data.

Result: All patients underwent the procedure without any major complication, specifically balloon breakage or rupture. The average operative time was $29.3(55 \sim 127)$ min per vertebra. Mean pressure of balloon expansion was 165 (87 210) Psi, and average volume of balloon expansion was $3.3(1.5 \sim 4) \mathrm{ml}$. 
The balloons were expanded an average of $2.9(2 \sim 5)$ times in one operation. The average volume of bone cement injected was $4.0(2 \sim 8.5) \mathrm{ml}$ per vertebrae. Epidural cement leakage was seen in 1 vertebra and paraspinal leakages were seen in 2 vertebrae. The vertebral heights were restored well in both anterior and middle columns. The patients were followed up on average $18.3(12 \sim 24)$ months. At last follow up, VAS was 8.0-3.3 and ODI was $29.5-72.8 \%(p<0.01)$. Both showed significant improvement compared to their pre-operative status.

Conclusion: We have shown that single-balloon multiplepass can be safely and efficiently employed in PKP for multi-level VCFs, metastatic tumor and multiple myeloma in the spine. Thus reducing cost, and would be applicable in developing nations and reducing health costs. We recommend a maximum of 3 passes per balloon at this stage.

\section{P206}

\section{DETERMINING THE EFFECTS OF VITAMIN D} ON OSTEOPOROSIS AND OSTEOPENIA

\section{S. T. Tilgner ${ }^{1}$}

${ }^{1}$ Innovative Health Care, Lake Havasu City, United States

Problem: Vitamin D deficiency is a concern, not only in the United States, but worldwide. Identifying a correlation for osteoporosis and osteopenia in vitamin D deficient patients may reduce the risk of osteoporosis-related fractures. The PICO question for this capstone project was: In osteoporotic or osteopenia patients, what is the relationship between reduced serum levels of vitamin D OH 25 compared to normal serum levels of vitamin D OH 25, in the incidence of osteoporosis or osteopenia? Osteoporosis contributes to fracture risk in patients, which subsequently has been shown to result from an insufficiency in vitamin D.

Purpose: The purpose of the project was to evaluate the relationship between osteoporosis and osteopenia and vitamin D $\mathrm{OH} 25$ levels.

Goals and Objectives: The goal of this study was to determine if a relationship existed between vitamin D deficiency and osteoporosis or osteopenia and note its significance. Identifying a correlation between these factors may help to raise awareness in the investigator's practice and community. Plan: The issue was identified as outlined above, and the project was implemented using PICO analysis at the doctorate level. This quality improvement (QI) project utilized a quantitative retrospective study design involving 91 patients. Outcome data was compared on patients who had previously undergone a bone density test using a DXA scan and also had Vitamin D OH 25 laboratory testing over the previous threeyear period. The main outcome that was measured was the number of patients diagnosed with osteoporosis or osteopenia and the laboratory blood level value of vitamin D OH 25 .
Other variables included DXA scan results, vitamin D OH 25 lab results, gender, age, steroid use history and exercise history. Using one- way ANOVA testing, the study identified four groups; once this was completed, multiple regression was used to analyze the variables involved.

Outcomes and Measures: The study consisted of 12 men and 79 women. $39 \%$ of patients had both osteoporosis or osteopenia and vitamin D deficiency, $36 \%$ of patients had osteoporosis or osteopenia and did not have vitamin D deficiency, $5 \%$ of patients had normal bone and were vitamin $\mathrm{D}$ deficient and $20 \%$ of patients had normal bone and normal vitamin D results. Results of the one-way ANOVA test showed a significance of $p=0.026$ for vitamin $\mathrm{D}$ when compared to the control group diagnosis. The results of this analysis show that vitamin D deficiency is a statistically significant factor in osteoporosis and osteopenia. The factor of vitamin D deficiency had a higher statistical significance than exercise history, steroid use, gender, and age.

\section{P207}

\section{SYNOVIOLOGY: A NEW CHAPTER ENTITLED} TO JOINTS CARE

B. Palmieri ${ }^{1}$, C. Laurino ${ }^{1}$

${ }^{1}$ University of Modena and Reggio Emilia, Modena, Italy

The synovial membrane is the soft tissue between the joint capsule and the joint cavity. The synovial fluid, a clear and viscous fluid secreted by synoviocytes, lubricates this membrane. We have outlined a specific medical branch named "synoviology" which targets the physiopathology of synovial membrane dysfunction with the final aim to identify new therapeutic strategies. In this environment, mesenchymal stem cells (MSCs) interact with extracellular matrix components, leading to the secretion of cytokines and growth factors. Specifically, synovial MSCs are responsible of 1) osteophyte (defined as fibrocartilage-capped bony outgrowth) formation early appearing in the osteoarthritis process that has heavy clinical impact on pain and functional disability; 2) chondrogenesis and cartilage repair that actively involve periosteal cells and can be modulated by growth factors. For the diagnosis and the prognosis of synovium-affected diseases, synoviology employs clinical investigation, based on synovial membrane biopsies by means of proteomic and genetic approaches. The physiological composition of synovial fluid is well known. In fact, some biochemical and cytological parameters of the synovial fluid, such as relative density and viscosity, osmolality, $\mathrm{pH}, \mathrm{pCO} 2, \mathrm{pO} 2$, potassium, sodium, calcium, carbon dioxide, chlorine, iron, copper, zinc, total nitrogen, non-proteic nitrogen, urea, uric acid, total proteins, albumin, $\alpha 1$-antitrypsine, ceruloplasmin, haptoglobins, $\alpha 2$ macroglobuline, lactoferrin, IgG, IgA, IgM, IgE, enzymes, crystals, cytokines, glucose, chondroitin sulphate, hyaluronic 
acid, cholesterol, leucocytes (e.g. monocytes, lymphocytes, macrophages, polymorfonuclear cells) and synoviocytes, are well established. In this context, Synoviology deals with the identification of synovial inflammation markers such as cytokines, adipokines, growth factors and matrix metalloproteinases for diagnosis and prognosis of individual diseases, and the identification of therapeutic approaches tailored to the specific markers identified. Specifically, our commitment is to use the identified markers to design point-of-care instruments for quick and accurate diagnosis leading to selection of the most appropriate intra-articular treatment. In fact, immediately after drained, the synovial exudate offers quickly exhaustive information in order to decide the most effective intra-cavity therapy aiming at restoring at the best the function and reducing or preventing the permanent damage, which is often progressive and invalidating.

Among these therapies, viscosupplementation, with structurally different hyaluronic acid compounds, may be effective for restoring the damaged synovial cartilage. In addition, the contemporary intra-articular multiple drugs delivery, with possible slow release if previously intermixing with a bulk of reticulated hyaluronic acid will be a "holistic" hypothesis based on an appealing multistep therapeutic rationale. Some of these include anaesthetics, corticosteroids, morphine, tramadol, sorbitol, bisphosphonates, somatostatin, magnesium sulfate, vitamin A, anti-TNF $\alpha$ agents, methotrexate, MSCs, nonsteroidal anti-inflammatory drugs, chitosan, collagen, ozone, hypertonic dextrose (prolotherapy), autologous serum injection, lubricin and vitamins. In conclusion, synoviology integrates genomic, proteomic, biochemical, immunological morphological and clinical information for the progress of new diagnostic and taxonomic tools as well as of therapeutic options in osteoarticular pathology.

\section{P208}

THE FIRST ASSESSMENT OF THE PRINCIPLES OF "CO-MANAGED CARE IN ELDERLY PATIENTS" IN MOSCOW CITY HOSPITAL \#13 L. Farba $^{1}$, I. Cypin ${ }^{1}$, I. Spesivtcev ${ }^{1}$

${ }^{1}$ City Hospital № 13, Moscow, Russian Federation

Introduction: In the 21st century, Russia, as well as other European countries, has been facing problems of population aging. According to the UN, the number of persons above 60 years in Russia will increase from 26.5 million (18.6\% of the population) to 39.3 million ( $31.3 \%$ of the population) by the year 2050. Annually, about 200 patients with proximal femur fractures are attended to at Moscow City Hospital No. 13. The principles of "Co-managed care in elderly Patients" with fragility fractures are early «one shot surgery", protocol driven treatment, multiprofessional and interdisciplinary team work, early rehabilitation and secondary fracture prevention.
Aim: To adapt the principles of "co-managed care" in the existing Russian Healthcare System for the treatment of patients with trochanteric hip fractures (31A Fractures AO / OTA Classification).

Materials and Methods: From 2011 to 2014, 714 patients with fracture types $31 \mathrm{~A}$ were treated at Moscow City Hospital No. 13's clinic of traumatology, 535 women (75\%) and 179 men $(25 \%)$. The Average ages of the men and women were 65 years and 80 , respectively. With the introduction of the principles of "Co-managed care" operating activity increased in this group of patients from $72 \%$ in 2011 to $88 \%$ in 2014, and also, in $201486 \%$ of patients were operated within $48 \mathrm{~h}$ after admission to the hospital. Proximal femoral nails were used for osteosynthesis $31 \mathrm{~A} 2$ and $31 \mathrm{~A} 3$ type fractures and dynamic hip screw (DHS) for stable fractures type 31A1. The average preoperative day decreased from 2011's 4.7 to 2.3 in 2014 which ultimately led to a decrease in total hospital stay from 18 days in 2011 to 16 days.

Thus, mortality in this group of patients did not exceed $2.2 \%$. Conclusions: The principles of «co-managed care» must surely be put into practice in respect of the Russian health care of patients with proximal femur fractures. The implementation of «co-managed care» at Moscow City Hospital № 13 has led to a significant improvement in the quality of patient care.

\section{P209}

CALCIUM AND VITAMIN D METABOLISM AMONG PATIENTS WITH EXCESS OF WEIGHT OF A DOCENT CLINIC IN SALVADOR-BA

J. Rodrigues $^{1}$, M. L. Silva ${ }^{2}$, M. Carvalho ${ }^{2}$

${ }^{1}$ Bahiana School of Medicine and Public Health/CNPq, Salvador, Brazil, ${ }^{2}$ Bahiana School of Medicine and Public Health, Salvador, Brazil

Introduction: Hypovitaminosis D is a biochemical change with high prevalence among the population, especially in obese patients. Its function more known relates to bone metabolism, although recently, many functions have been described.

Objectives: Describe the prevalence of vitamin D deficiency and evaluate calcium, phosphorus and PTH metabolism between women with excess of height followed in an obesity clinic of reference in Salvador-BA.

Methods: A descriptive cross-sectional study, which were included women over 18 years old with BMI equal or superior of $25 \mathrm{~kg} / \mathrm{m}^{2}$. Anthropometric measurements were obtained: weight, height, BMI, waist circumference, hip circumference; presence of hypertension and / or metabolic syndrome was obtained by analysing the charts of patients in the study. The criteria for diagnosing hypovitaminosis $\mathrm{D}$ was established according to The Brazilian Society of Endocrinology and Metabology - levels $\leq 20 \mathrm{ng} / \mathrm{dL}$ means deficiency, 21-29 ng/ 
$\mathrm{dL}$ are considered insufficiency and levels between 30 and $100 \mathrm{ng} / \mathrm{dL}$ are normal.

Results: The sample consisted of 121 women with a mean age of $43 \pm 12$ years, BMI of $37 \pm 6.5 \mathrm{~kg} / \mathrm{m}^{2}$, the waist / hip ratio of $0.86 \pm 0.09$ and the abdominal circumference of $109 \pm 13 \mathrm{~cm} \mathrm{~cm}$. The prevalence of obesity was $89.3 \% ; 59 \%$ of the population were hypertensive and $20 \%$ diabetics. The mean of the level of vitamin $\mathrm{D}$ was $23.5 \pm 6 \mathrm{ng} / \mathrm{dL}$. Hypovitaminosis $\mathrm{D}$ was observed in $84.6 \%$ of the population, $25,6 \%$ are vitamin D deficient and $59 \%$ are insufficient. The values of the biochemical in the groups obesity and overweight were respectively: $26.8 \pm 7 \mathrm{ng} /$ $\mathrm{dL}$ and $23.1 \pm 6(p=0,1)$ for vitamin $\mathrm{D}, 41.7[26.4-37.4] \mathrm{pg} / \mathrm{mL}$ and $63.3(p=0.03)$ for PTH, $10.1 \pm 1$ and $9.4 \pm 0.7 \mathrm{mg} / \mathrm{dL}$ $(p=0.052)$ calcium and $3.8 \mathrm{mg} / \mathrm{dL}(p=0.84)$ in both groups for phosphorus. It was observed an inverse correlation between BMI and the levels of vitamin $\mathrm{D}(p=0.04)$.

Conclusion: The prevalence of hypovitaminosis among patients with excess of height is higher than in the general population. There is an inverse correlation between the levels of vitamin D and BMI.

\section{P210}

REAL-WORLD PERSISTENCE TO INJECTABLE OSTEOPOROSIS THERAPY: ANALYSIS OF A MANAGED CARE POPULATION FROM THE US.

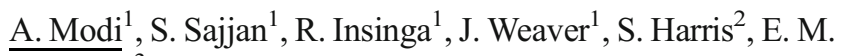
Lewiecki ${ }^{3}$

${ }^{1}$ Merck \& Co., Inc., Kenilworth, NJ, United States, ${ }^{2}$ University of California, San Francisco, San Francisco, CA, United States, ${ }^{3}$ New Mexico Clinical Research \& Osteoporosis Center, Albuquerque, NM, United States

Objectives: This study was designed to evaluate treatment persistence among patients who initiate injectable osteoporosis (OP) therapies and to examine predictors of persistence among OP patients started on these therapies.

Materials and Methods: A claims database from a large US managed care health plan was used to measure persistence to injectable OP therapy at 6 month intervals over 2 years. Women and men included were $\geq 55$ years of age, and had newly initiated injectable OP therapy between January 2008 and June 2012 (index period), and were continuously enrolled for 1 year pre- and 1.5 years post-index. OP treatments included in the analysis were intravenous ibandronate (IBN) and zoledronic acid (ZOL), and subcutaneous teriparatide (TER) and denosumab (DEN). Persistence to treatment was assessed for each drug using Kaplan-Meier survival analysis. A 90 day grace period after the date of the next scheduled refill was allowed for flexibility in scheduling of re-administrations. Sensitivity analyses were conducted using grace periods of 30 and 60 days.
Results: 4,756 patients met the inclusion criteria for the study, with 233 on IBN, 3,128 on ZOL, 778 on TER and 617 on DEN. Depending on treatment type, the mean age of patients at index date ranged from 71.5 years (IBN) to 73.5 years (DEN); history of fracture ranged from $9.4 \%$ (IBN) to $31.2 \%$ (TER); and mean Charlson comorbidity scores ranged from 0.51 (ZOL) to 0.61 (TER). Beyond the 12 month time point, using a 90 day grace period, persistence was greatest among patients using DEN (51.4 \%), followed by ZOL (40.8 \%), TER (33.0 \%) and IBN $(31.8 \%)$. Beyond the 24 month time point, persistence was lower for each treatment, yet remained highest for DEN (35.7 \%) and ZOL (20.2 \%) followed by TER (12.6\%) and IBN (12.5\%). For all therapies, persistence rates were lowest with a 30 day grace period and highest with the 90 day grace period, increasing as the duration of the grace period increased. DEN consistently demonstrated the highest rates of persistence among injectable therapies and TER demonstrated the lowest, regardless of the duration of the grace period or the time point. Conclusion: Over two years, DEN had higher rates of persistence than other injectable OP therapies; however, among those receiving DEN, only one-half remained persistent for more than 1 year after treatment initiation.

\section{P211}

BONE MINERAL DENSITY AND BODY COMPOSITION ANALYSIS IN CHILDREN BORN SMALL FOR GESTATIONAL AGE (SGA) COMPARED TO APPROPRIATE FOR GESTATIONAL AGE (AGA)

D. Fintini ${ }^{1}$, G. Ubertini ${ }^{1}$, R. Fiori ${ }^{1}$, S. Bocchini ${ }^{1}$, S. Cianfarani $^{1}$, M. Cappa ${ }^{1}$

${ }^{1}$ Endocrinology and Diabetology Unit, Bambino Gesù Children's Hospital, Rome, Italy

Background: Conflicting results about influence of birth size (BW) on $\mathrm{BMD}$ and bone mineral content (BMC) are present in the literature. Fetal growth pattern seems to influence bone accrual not only in small for gestational age (SGA) but also when birth weight is maintained in the normal range.

Aim: We analyzed BMD and body composition in children born SGA compared with age and sex matched children born appropriate for gestational age (AGA).

Patients: 53 SGA (26 f. mean age $10.9 \pm 2.5$ years; BW 2.4 $\pm 0.4 \mathrm{~kg}$, percentile $2.9 \pm 0.4$; BMI SDS $1.2 \pm 1.9$; pubertal stage $2.2 \pm 1.4$ ) vs 40 age, BMI matched AGA ( $27 \mathrm{f}$. mean age $11.6 \pm 2.9$ years; BW $3.4 \pm 0.4 \mathrm{~kg}$, percentile $48.8 \pm 16$; BMI SDS 1.3 \pm 1.6 ; pubertal stage $2.6 \pm 1.4$ ) were studied using DXA scan (Hologic) in order to obtain lumbar and whole body BMD, normalized for height BMD (BMDAD and $\mathrm{nWBMD}$ ), lumbar $\mathrm{Z}$ score and fat (FM) and free fat mass (FFM) expressed in gr and percentage of body weight 
$(\% \mathrm{TBW})$. None of the patients were taking medication influencing growth or bone content.

Results (Table): No differences were found in bone parameters between SGA and AGA children, both as a whole group and divided for gender. Statistically higher FM in SGA and FFM/FM ratio in AGA children was found when expressed as row data (gr) but not when we analyzed as \%TBW.

The multivariate analysis showed independent positive correlation between BW SDS and whole body BMD (beta 0.479 ; p0.001) in SGA and in lumbar areal BMD (beta 0.343; p 0.035 ) and $\mathrm{FFM} / \mathrm{FM}(\% \mathrm{TBW})$ only in AGA children (beta $0.330 ; p=0.043$ ).

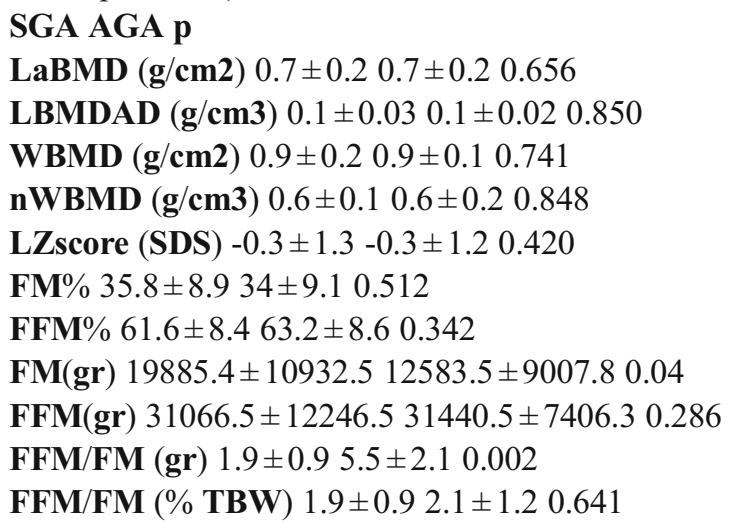
tween birth weight and bone density seems to exist, no statistical difference in bone parameters and body composition is present in peripubertal SGA and AGA children.

\section{P212}

FRACTURE INCIDENCE AMONGST UK CHILDREN IN CPRD 1988-2012: VARIATION BY ETHNICITY AND GEOGRAPHY

N. C. Harvey ${ }^{1}$, R. J. Moon ${ }^{1}$, E. M. Curtis ${ }^{1}$, F. de Vries ${ }^{2}$, T. P. Van $\mathrm{Staa}^{3}, \mathrm{C}$. Cooper ${ }^{1}$

${ }^{1}$ MRC Lifecourse Epidemiology Unit, University of Southampton, Southampton, United Kingdom, ${ }^{2}$ Department of Clinical Pharmacy \& Toxicology, Maastricht University Medical Centre, Maastricht, Netherlands, ${ }^{3}$ Health eResearch Centre, University of Manchester, Manchester, United Kingdom

Objectives: We aimed to document the site-specific incidence of childhood fractures in the United Kingdom (UK), and to describe variation by age-, sex-, ethnicity- and geographic location.

Methods: The Clinical Practice Research Datalink (CPRD) contains anonymised electronic health records for approximately $7 \%$ of the UK population. The occurrence of a fracture 1988-2012 was determined from the CPRD for all individuals $<18$ years of age, and used to calculate site-specific fracture incidence rates by dividing the number of children with a fracture by the total person-years of follow-up within strata of age, sex and ethnicity. Regional fracture incidence rates were also calculated based on general practitioner location within 14 Strategic Health Authorities (SHA) within the UK. Results: Overall, the incidence of any fracture was 137 per 10, 000 person-years (py). Boys had a greater rate (169 per 10,000 py) than girls (103 per 10,000 py). Fracture rates increased with age in both boys and girls, peaking at 14-15 years and 11-12 years respectively, before subsequent decline. In both sexes, the most common site of fracture was at the radius/ulna, followed by the carpal bones and humerus. Black (64 per 10, 000 py) and South Asian (81 per 10,000 py) children had lower rates than white children (150 per 10,000 py). Highest fracture rates were observed in Wales, where boys and girls had 1.82 and 1.97 times greater incidence than those residing in Greater London, respectively.

Conclusion: We have documented notable geographic and ethnic variation in childhood fracture incidence across the UK. Such results are likely to at least partly reflect differences in diet, obesity, physical activity and socioeconomic status and will inform policy makers in the UK and internationally. $\mathrm{NCH}$ and RJM are joint first author. TvS and CC are joint senior author.

\section{P213}

INFLAMMAGING AND BONE HEALTH IN LATER LIFE: THE HERTFORDSHIRE COHORT STUDY

$\underline{\text { A. E. Litwic }}{ }^{1}$, K. A. Jameson ${ }^{1}$, M. Edwards ${ }^{1}$, C. Moss ${ }^{1}$, C. Cooper $^{1}$, E. M. Dennison ${ }^{1}$

${ }^{1}$ MRC Epidemiology Resource Centre, University of Southampton, Southampton General Hospital, Southampton, United Kingdom

Background: Inflammatory burden has been associated with a range of adverse musculoskeletal outcomes in later life, including accelerated osteoarthritis and sarcopenia. Here we report the association between markers of inflammation and bone health among unselected individuals recruited to a population based cohort study.

Methods: The study population was taken from the Hertfordshire Cohort Study who were born in Hertfordshire between 1931 and 1939, and still lived there 70 years later. At baseline, a questionnaire was administered detailing lifestyle and demographic factors, and a detailed comorbidity history was taken. At a clinic visit, fasting blood samples were taken and analysed for Il- 6 and hsCRP. BMD measurements were taken at the lumbar spine and femoral neck using a QDR 4500 instrument. There were 314 men and 288 women for whom we had BMD measurements and IL-6 and/or hsCRP.

Results: The mean SD age was 65.0 (2.6) years in men and 66.2 (2.6) years in women. Geometric mean (SD) BMI was 26.8 (1.1) and 26.9 (1.2) in men and women, respectively. The 
geometric mean (SD) hsCRP was $1.72(2.9) \mathrm{mg} / \mathrm{l}$ in men and $1.85(3.0) \mathrm{mg} / \mathrm{l}$ in women; an Il-6>1.5 pg/1 was observed in $88(28.4 \%)$ men and $58(20.5 \%)$ women. hsCRP z-score was an explanatory variable for lumbar spine BMD in men but not women, after adjustment for age, BMI, comorbidities, social class, smoking status, alcohol consumption, physical activity and dietary calcium intake [regression coefficient -0.14 $(95 \% \mathrm{CI}-0.26,-0.02), p=0.03]$. Relationships at the femoral neck were also apparent but slightly weaker in men only [regression coefficient $-0.11(95 \% \mathrm{CI}-0.23,0.00), p=0.06$ ] after the same adjustments. A baseline Il6 greater than $>1.5 \mathrm{pg} / \mathrm{l}$ was also associated with reduced lumbar spine BMD in men but not women [regression coefficient -0.31 $(95 \% \mathrm{CI}-0.59,-0.04), p=0.03]$ after adjustments.

Conclusions: We have observed associations between inflammatory markers within the normal range and bone health in men but not women in later life. Further studies are now warranted.

\section{P214}

EFFECT OF KINESIO TAPING (KT) ON IMMEDIATE REHABILITATION PROGRAM OF KNEE MEDIAL COMPARTMENT OSTEOARTHRITIS (OA)

A. Alobaidi $^{1}$, Z. Muhammad ${ }^{1}$

${ }^{1}$ College of Health and Medical Technology, Baghdad, Iraq

Objective: To evaluate the role of KT in the immediate rehabilitation management of knee medial compartment OA, through application of Western Ontario and McMaster universities osteoarthritis Index (WOMAC).

Material and Methods: 120 patients (mean \& SD age, $55.16 \& 7.05$ years, body mass $31.23 \& 7.20 \mathrm{Kg} / \mathrm{m}), 80$ females and 40 males diagnosed as candidates of chronic stage medial compartment knee OA regardless the etiology, participated in this randomized controlled clinical trial, they had been referred to the Medical City Teaching Hospital in Baghdad, Iraq from Oct. 2014 till June 2015. The study sample was divided to two groups, Control group ( 60 patients) treated by traditional physical therapy rehabilitation program, the other 60 patients (Experimental group) were treated similarly plus KT application. WOMAC scale was used to evaluate both groups before and after treatment and the responses were scored accordingly and the mean value of the whole group on each scale were calculated and analyzed by using $t$-test.

Results: There was significant $(P<0.05)$ decrease in Pain and Stiffness subscales, and significant $(P<0.05)$ increase in physical function subscale of experimental group compared to control group.
Conclusion: By introducing KT procedure to immediate rehabilitation program for medial compartment knee osteoarthritis, the problem of pain, stiffness and reduced functional activity will not be a concern.

\section{P215}

BONE REABSORPTION IN LONG-TERM TOTAL HIP ARTHROPLASTIES: A MRI STUDY $\underline{\text { I. Pérez-Coto }}^{1}$, D. Hernández-Vaquero ${ }^{1}$, A. Sierra-Pereira ${ }^{1}, \mathrm{~S}$. Roncero-Gonzalez ${ }^{1}$

${ }^{1}$ Hospital San Agustín, Avilés, Spain

Objectives: Since the aseptic loosening due to bone reabsorption after total hip replacement (THR) is considered the most important long-term complication, our aim was to assess, through MRI, the severity and growth pattern of osteolysis, as well as the influence of clinical-radiographic variables.

Material and Method: We analyzed 75 THR with an average evolution time of 13.67 years. The implant was a titanium alloy, non-cemented, multiple-hole model with hydroxyapatite coating. We perform a clinical and radiological follow-up of the cases and a MRI study, where we analyzed the amount, localization and pattern of the bone loss.

Results: Bone resorption was found in 56 of the 75 cases. It had a peripheral pattern in 48 and a central pattern in 6 cases; in 52 cases it was continuous, and in 4, isolated. Out of 118 screws, 20 exhibited lysis. There was a proportional correlation between osteolysis severity and wear rate with age, physical activity and acetabular abduction, as well as an association between said variables and peripheral and continuous patterns.

Conclusions: We found that the bone reabsorption after THR is an important, frequent and underestimated problem with a disastrous clinical consequences. We can affirm that most of the lysis occurred peripherally and continuously and it was incremented by many variables, all relationed with the physical activity of the patient.

Table 1. Frequency of osteolytic presentation in the femoral and pelvic areas.

\begin{tabular}{lllllllllll}
\hline & Total & \multicolumn{3}{c}{ Pelvic areas } & \multicolumn{4}{c}{ Femoral areas } \\
\hline Degree of lesion & & 1 & 2 & 3 & 4 & 5 & 1 & $2-6$ & 7 \\
Osteolysis (NO) & 19 & 48 & 33 & 71 & 59 & 36 & 54 & 75 & 58 \\
Osteolysis (YES) & 56 & 27 & 42 & 4 & 16 & 39 & 21 & 0 & 17 \\
Osteolysis $<4$ areas & 39 & 14 & 25 & 0 & 6 & 24 & 11 & 0 & 6 \\
Osteolysis $\geq 4$ areas & 17 & 13 & 17 & 4 & 10 & 15 & 10 & 0 & 11 \\
\hline
\end{tabular}


P216

BONE MINERAL DENSITY AND PARATHYROID HORMONE AS INDEPENDENT RISK FACTORS FOR MORTALITY IN COMMUNITYDWELLING OLDER ADULTS: A POPULATION-BASED PROSPECTIVE COHORT STUDY IN BRAZIL. THE SÃO PAULO AGEING \& HEALTH (SPAH) STUDY

$\underline{\text { D. Domiciano }}^{1}$, L. Machado ${ }^{1}$, J. Lopes ${ }^{1}$, C. Figueiredo ${ }^{1}$, V. Caparbo $^{1}$, L. Takayama ${ }^{1}$, R. Oliveira ${ }^{2}$, M. Scazufca ${ }^{3}$, M. McClung $^{4}$, R. Pereira ${ }^{3}$

${ }^{1}$ Faculdade de Medicina da Universidade de São Paulo, Rheumatology Division, São Paulo, Brazil, ${ }^{2}$ RDO Diagnósticos Médicos, São Paulo, Brazil, ${ }^{3}$ Faculdade de Medicina da Universidade de São Paulo, Department of Preventive Medicine, São Paulo, Brazil, ${ }^{4}$ Oregon Osteoporosis Center, Portland, OR, United States

Previous studies have shown a relationship between osteoporosis and increased mortality risk. However, none of these studies performed a concomitant evaluation of the PTH-calciumvitamin $\mathrm{D}$ - axis and bone mass to accurately determine the contribution of each of these parameters to survival in older subjects. Thus, we sought to investigate the association between bone parameters and mortality in a longitudinal, prospective, population-based cohort of 839 elderly subjects. Clinical data (including history of fractures and cardiovascular events) were assessed using a specific questionnaire. Laboratory exams, including serum 25OHD and PTH, were also performed. BMD at the lumbar spine and hip were evaluated using DXA. All analyses were performed at baseline (2005-2007). Mortality was recorded during follow-up. Multivariate Cox proportional regression was used to compute hazard ratios for all-cause and cardiovascular mortality. Over a mean $4.06 \pm 1.07$ years, there were $132(15.7 \%)$ deaths. In a multivariate Cox proportional hazards model, age (HR: $1.3295 \% \mathrm{CI}: 1.13-1.55, p=0.001$, for each 5-year increase), male sex (HR: 1.90 95\%CI:1.30-2.79, $p=0.001$ ), recurrent falls ( $\geq 2$ in the previous year; HR: 1.65 95\% CI:1.06-2.56, $p=0.026$ ), diabetes mellitus (HR: 2.17 95\%CI:1.46-3.21, $p<0.001$ ), low physical activity score (HR: $1.7895 \%$ CI:1.14-2.79, $p=0.011$ ), prior cardiovascular event (HR: 1.76 95\%CI:1.18-2.63, $p=0.006$ ), total hip BMD (HR: $1.4195 \% \mathrm{CI}: 1.15-1.72, p=0.001$, per each 1SD-decrease) and iPTH (HR: 1.06 95\%CI:1.04-1.08, $p<0.001$, per each $10 \mathrm{pg} /$ $\mathrm{mL}$-increase) were independently associated with all-cause mortality. The subjects in the highest quartile of PTH $(>49 \mathrm{pg} / \mathrm{mL})$ were at a higher risk of cardiovascular death (HR 3.09, 95\%CI: $1.36-6.99, p=0.007)$ compared with the subjects in the lowest quartile $(<26 \mathrm{pg} / \mathrm{mL})$. Low BMD and higher PTH were significantly associated with mortality in community-dwelling older adults. These findings support the notion that careful screening of these bone parameters might lead to better management of older patients and improve outcomes in this population.

\section{P217}

TEN YEARS OF DENOSUMAB (DMAB) TREATMENT IN POSTMENOPAUSAL WOMEN WITH OSTEOPOROSIS: RESULTS FROM THE FREEDOM EXTENSION TRIAL

H. G. Bone ${ }^{1}$, M. L. Brandi ${ }^{2}$, J. P. Brown ${ }^{3}$, R. Chapurlat ${ }^{4}$, S. R. $\overline{\text { Cummings }}^{5}$, E. Czerwinski ${ }^{6}$, A. Fahrleitner-Pammer ${ }^{7}$, D. L. Kendler $^{8}$, K. Lippuner ${ }^{9}$, J.-Y. Reginster ${ }^{10}$, E. Vittinghoff ${ }^{11}$, N. S. Daizadeh ${ }^{12}$, A. Wang ${ }^{12}$, P. Dakin ${ }^{12}$, R. B. Wagman ${ }^{12}$, S. E. Papapoulos ${ }^{13}$

${ }^{1}$ Michigan Bone and Mineral Clinic, Detroit, MI, United States, ${ }^{2}$ University of Florence, Azienda Ospedaliera Careggi, Florence, Italy, ${ }^{3}$ Laval University and CHU de Québec Research Centre, Quebec City, QC, Canada, ${ }^{4}$ Hôpital Edouard Herriot, Lyon, France, ${ }^{5}$ San Francisco Coordinating Center, California Pacific Medical Center, San Francisco, United States, ${ }^{6}$ Krakow Medical Centre, Krakow, Poland, ${ }^{7}$ Department of Internal Medicine, Division of Endocrinology and Metabolism, Medical University of Graz, Graz, Austria, ${ }^{8}$ University of British Columbia, Vancouver, BC, Canada, ${ }^{9}$ Institute for Surgical Technology and Biomechanics, University of Bern, Bern, Switzerland, ${ }^{10}$ Department of Public Health, University of Liège, Liège, Belgium, ${ }^{11}$ University of California, San Francisco, San Francisco, CA, United States, ${ }^{12}$ Amgen Inc., Thousand Oaks, CA, United States, ${ }^{13}$ Leiden University Medical Center, Leiden, Netherlands

Objective: We report final data from the FREEDOM extension, representing up to 10 years (yrs) of continued DMAb treatment.

Material and Methods: During the extension, subjects were to receive $60 \mathrm{mg}$ DMAb every 6 months and calcium and vitamin D daily (Papapoulos OI 2015). In this analysis, the long-term group received 10 year of DMAb treatment and the cross-over group received 7 years of DMAb treatment.

Results: Of the 4,550 subjects entering the extension, 2, $784(61 \%)$ remained on study at the beginning of yr 10. Of these, $2,212(80 \%)$ had completed their final 10-year visit, 120 (4\%) discontinued, and $452(16 \%)$ were ongoing at the time of this analysis. In the longterm group, further significant increases in BMD occurred with mean cumulative 10-yr gains from FREEDOM baseline of $21.6 \%$ (lumbar spine) and $9.1 \%$ (total hip). The cross over group had mean cumulative 7-year gains of $16.3 \%$ (lumbar spine) and $7.3 \%$ (total hip) from the extension baseline (Figure; 
all $P<0.0001$ vs. FREEDOM baseline, extension baseline, and previous measurement). Similar and sustained reductions in bone turnover markers were observed in both groups. Yearly rates of new vertebral and nonvertebral fractures remained low. Overall incidence rates of adverse events (AEs) and serious AEs were consistent with data reported previously.

Conclusions: DMAb treatment for up to $10 \mathrm{yrs}$ was associated with persistent reduction of bone turnover, continued BMD increases without therapeutic plateau, and low fracture incidence. The benefit/risk profile for DMAb in an aging population of postmenopausal women remains favorable.

Acknowledgements: FREEDOM was funded by Amgen.
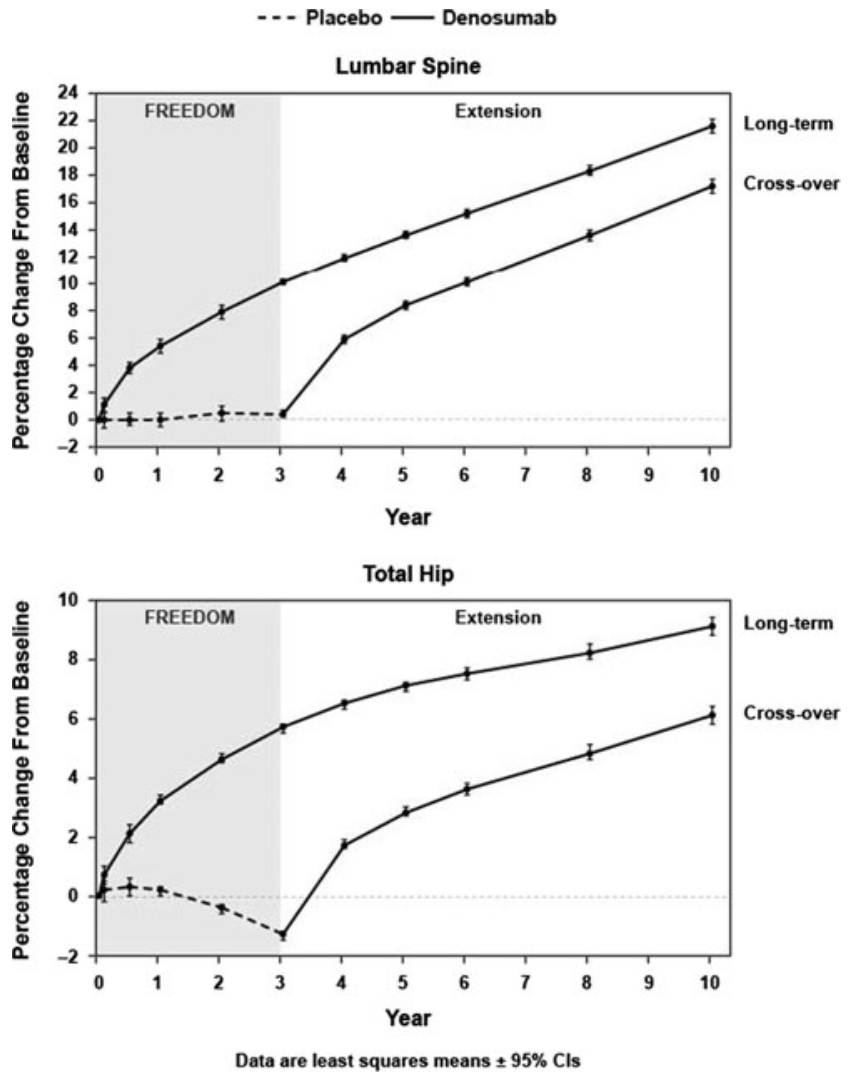

\section{P218}

MEASURING RODS AND PLATES IN TRABECULAR BONE: A 2016 UPDATE

M. Doube $^{1}$, S. J. Shefelbine ${ }^{2}$, C. Ohlsson ${ }^{3}$, P. L. Salmon ${ }^{4}$

${ }^{1}$ The Royal Veterinary College, London, United Kingdom,

${ }^{2}$ Northeastern University, Boston, United States, ${ }^{3}$ University of Gothenburg, Gothenburg, Sweden, ${ }^{4}$ Bruker microCT, Kontich, Belgium
Rods and plates are thought to be important for the mechanical integrity of trabecular bone. Rods are narrow, elongated regions, while plates are flatter and more extensive. Most studies investigating the roles of rods and plates in bone have used Structure Model Index (SMI) or other algorithms that themselves have used SMI as a validation step during development. SMI assumes that the whole bone surface is convex, but we show using X-ray microtomography of diverse bone samples and synthetic images that concave surfaces are abundant in trabecular bone $(20-70 \%$ of the surface) and that SMI is a very unreliable measure of rods and plates. Furthermore, the amount of concave surface increases with increasing bone volume fraction (BV/TV), so conclusions relating rod and plate geometry to $\mathrm{BV} /$ $\mathrm{TV}$, in osteoporotic bone loss for example, are suspicious at best. Studies relating mechanical performance of bone samples to SMI-derived rod and plate geometry are similarly suspect. Doube (2015) developed the Ellipsoid Factor (EF) method by extending to ellipsoid-fitting the Local Thickness sphere-fitting concept used to measure trabecular thickness and spacing. $\mathrm{EF}$ at a point is calculated from the 3 radii $(a \leq b \leq c)$ of the largest ellipsoid that contains the point and that fits within the structure, as $E F=a / b-b / c$. EF ranges from -1 (plate-like, discus shaped ellipsoid) to +1 (rod-like, javelin shaped ellipsoid). In this work we show that EF is nearly independent of $\mathrm{BV} / \mathrm{TV}$, unlike SMI. EF overcomes limitations of techniques such as individual trabecular segmentation (ITS) that split the trabecular continuum into discrete elements, by producing a smooth scale of local geometry that is intuitive to understand and use.

Reference: Doube M. Front Endocrinol 2015;6:15.

Disclosure: PLS is an employee of Bruker microCT, which sells software containing SMI.

\section{P219}

\section{FRACTURE PREDICTION BY TBS AND FRAX} IN FEMALE PATIENTS WITH OSTEOPENIA

M. Kuzma ${ }^{1}$, A. Bednarova ${ }^{1}$, T. Koller ${ }^{1}, \mathrm{H} . \mathrm{Resch}^{2}, \mathrm{Z}$. Killinger $^{1}$, J. Payer ${ }^{1}$

${ }^{1}$ Comenius University, Faculty of Medicine, 5th Department of Internal Medicine, University Hospital, Bratislava, Slovakia, ${ }^{2}$ Medical Department II - St. Vincent Hospital Vienna, Vienna, Austria

Introduction: More than half of osteopenic patients suffer from fracture (Fx), but BMD osteopenia is usually not considered for treatment initiation. To our knowledge there is no 
study which compares risk stratifying methods in Fx prediction of BMD non-therapy group.

Objective: Comparison of three methods, trabecular bone score (TBS), FRAX and FRAX adjusted for TBS in Fx risk prediction of postmenopausal (PM) females.

Methods: Observational cohort study of PM females with BMD osteopenia (defined as T-score $\leq-1 \geq-2,5$ ) during 2/2009-5/2015 was performed. Patients underwent TBS, FRAX and FRAX adjusted for TBS evaluation. Using NOF cutoff values of $20 \%$ for major osteoporotic Fx and $3 \%$ for hip Fx were used to consider patients at high absolute 10 years risk of Fx. With regard to TBS patients were divided to 3 groups: normal, moderate and degraded. According to temporary consensus guidelines patients with BMD osteopenia + very low (degraded) TBS $(<1,1)$ are at high risk of developing Fx. TBS Insight ${ }^{\circledR}$ tool was used to assess TBS derived from L-spine DXA scans. Primary endpoint during follow-up was clinical $\mathrm{Fx} /$ death.

Results: In total, 144 PM females (mean age 66.1 year, BMI $26.7 \mathrm{~kg} / \mathrm{m} 2$, T-score: neck -1.2; L-spine 1.4, TBS 1.24) were included. At baseline, $31.9 \%$; 30.5 and $34 \%$ belonged to high $\mathrm{Fx}$ risk group according to TBS, FRAX and FRAX adjusted for TBS, respectively. Trend to increase Fx risk (RR 2.3; 95\%CI 0.32;12.5) was observed by degraded TBS. Fx/death probability was significantly 4.28-times higher in patients with degraded TBS value (RR 5.28, 95\%CI 1.4;19.1). Mean time to Fx/death was 4.4 years.

Conclusions: Patients with BMD osteopenia with degraded trabecular microarchitecture are at high risk of Fx. This study provides supportive results that TBS is appropriate method to assess high risk of Fx patients with BMD osteopenia.

\section{P220 \\ PILOT STUDY OF BONE MASS IN HEART TRANSPLANT RECIPIENTS}

L. Bautista Aguilar ${ }^{1}$, A. Salmoral Chamizo ${ }^{1}$, I. Gómez Gracia ${ }^{1}$, P. Font ${ }^{1}$, J.-M. Arizon ${ }^{2}$, M. Ruiz Ortiz ${ }^{2}$, E. Collantes Estevez ${ }^{1}$

${ }^{1}$ Department of Rheumatology, Reina Sofía University Hospital, Cordoba, Spain, ${ }^{2}$ Heart Transplant Unit, Reina Sofía University Hospital, Cordoba, Spain

Objective: To describe changes in BMD, incidence of fractures and levels of bone remodeling markers in the first year post-heart transplant (postHTX).

Material and methods: We included patients aged $\geq 18$ years with heart transplant performed in our institution from 01.01.2013 to 31.07.2015, excluding those on antiresorptive or bone forming treatment and pregnant women. Before heart transplant (preHTX) and in the 1 year postHTX visit, the following data were registered: BMI, risk factors for osteoporosis, presence of osteopenia or osteoporosis in the densitometric analysis of lumbar spine and femoral neck, presence of fractures in the X-ray of the spine and echocardiographic left ventricular ejection fraction (LVEF). Biochemical parameters of bone metabolism were registered in the 1 year postHTX.

Results: All 18 patients (age $48 \pm 16$ years, 11 male) included in the study were treated with calcineurin inhibitors and steroids (mean dose $15 \mathrm{mg} /$ day). At the preTX visit, BMI was $26 \pm 5 \mathrm{~kg} / \mathrm{m} 2$, obesity was present in 4 patients, DM in 1, kidney stones in 3 , excessive alcohol consumption in 1, and 11 patients were ex-smokers. Low BMD was present in 12 patients in the preTX visit: 10 osteopenia (6 men and 4 women) and 2 osteoporosis (1 man and 1 woman); and 13 patients in the one year postTX visit: 8 osteopenia ( 5 men and 3 women) and 5 osteoporosis ( 2 men and 3 women). Twelve patients had a vitamin D blood level $<20 \mathrm{ng} / \mathrm{ml}$ at the one year postHTX visit. A man presented a spinal fracture in the follow- up. Mean LVEF was $25 \pm 6 \%$ (preHTX) and 75 $\pm 8 \%$ (postHTX).

Conclusion: In this pilot study, $67 \%$ of heart transplant recipients had a low BMD preHTX and $72 \%$ at the one year postHTX visit. Moreover, $67 \%$ of patients had a vitamin D deficiency at the one year postHTX visit. Larger studies are required to fully evaluate this morbidity and describe clinical predictors.

\section{P221 \\ COST OF HIP FRACTURE IN SPAIN: ANALYSIS BY REGION OF A PROSPECTIVE, OBSER- VATIONAL STUDY (PROA)}

$\underline{\text { I. Etxebarria-Foronda }}^{1}$, A. Bartra ${ }^{2}$, J. R. Caeiro Rey ${ }^{3}$, P.

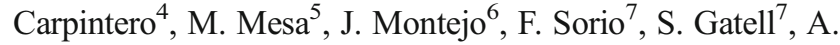
Farre $^{7}$, L. Canals ${ }^{7}$

Superscript $>1$ Department of Orthopaedic Surgery, Alto Deba Hospital, Arrasate, Spain, ${ }^{2}$ Hospital Universitario Mutua de Terrasa, Terrasa, Spain, ${ }^{3}$ Department of Orthopaedic Surgery. Complejo Hospitalario Universitario de Santiago de Compostela, Santiago de Compostela, Spain, ${ }^{4}$ Hospital Reina Sofía de Cordoba, Cordoba, Spain, ${ }^{5}$ Hospital Valle de los Pedroches, Cordoba, Spain, ${ }^{6}$ Hospital Universitario Fundación Alcorcón, Alcorcón, Spain, ${ }^{7}$ Amgen, Barcelona, Spain 
Objectives: To estimate health resource utilization (HRU) and costs during the first year following a first osteoporotic (OP) hip fracture in each of the 6 Spanish regions participating in the PROA study.

Material and Methods: This 12-month prospective observational study included patients (pts) aged $\geq 65$ years hospitalized due to a first OP hip fracture in 6 Autonomous Communities (AC) (Andalusia, Basque Country, Catalonia, Galicia, Madrid, and Valencia). Regions were selected based on population size, geographical spread, and likelihood to request and/or use this data for decision making. Fracture-related HRU was collected during follow-up and total costs calculated using unit costs from the eSalud database (http:// www.oblikue.com/bddcostes) updated to 2012 .

Results: The table presents the main sample characteristics, HRU and costs associated with hip fracture in each region. The gender distribution and mean age was similar across regions, except for a higher percentage of men $(33.7 \%)$ in Valencia (vs. approximately $20 \%$ in the rest). The proportion of pts recruited in large centers was higher in Galicia and Madrid (81.7 and $79.5 \%$ ), compared with Catalonia and Valencia (36.8 and $26.3 \%$ ). Mean hospital stay ranged from 7.9 days (Andalusia) to 16.1 days (Galicia). The mean number of surgical interventions and imaging techniques used during the first hospitalization and outpatient visits were similar across regions. Notable differences were observed in the use of rehabilitation sessions (mean of 17 in Catalonia and Madrid vs. 3 and 2 in Andalusia and Galicia, respectively) and home care (mean of 19 and 29 days in Catalonia and Madrid vs. 2, 0.4 and 0.4 days in Andalusia, Galicia and Valencia, respectively). The mean cost also showed significant differences, being $43 \%$ lower in Andalusia ( $€ 7,031$ in women) compared to the region with the highest cost (Madrid: $€ 12,321$ in women).

Conclusions: OP hip fractures incur a high cost for the Regional Health Systems, mainly due to the first hospitalization, but also to outpatient health care during the following months. Notable differences in HRU and costs are observed between different regions.

Acknowledgements: Financially supported by Amgen S.A.

\begin{tabular}{|c|c|c|c|c|c|c|}
\hline & $\begin{array}{l}\text { Andalusia } \\
(N=105)\end{array}$ & $\begin{array}{l}\text { Basque country } \\
(N=40)\end{array}$ & $\begin{array}{l}\text { Catalonia } \\
(N=114) \\
90(789)\end{array}$ & $\begin{array}{l}\text { Galicia } \\
(N=60) \\
49(817)\end{array}$ & $\begin{array}{l}\text { Madrid } \\
(N=88)\end{array}$ & $\begin{array}{l}\text { Valencia } \\
(N=80)\end{array}$ \\
\hline Gender, women, n (\%) & $82(78.1)$ & & & & & \\
\hline Age, years, mean (SD) & $83.2(6.8)$ & $82.6(5.5)$ & $83.2(6.5)$ & $84.0(7.0)$ & $83.6(7.5)$ & $82.4(6.5)$ \\
\hline $\begin{array}{l}\text { Type of center, large } \\
(>500 \text { beds), } \mathrm{n}(\%)\end{array}$ & $60(57.1)$ & $18(45.0)$ & $42(36.8)$ & $49(81.7)$ & $66(75.0)$ & $21(26.3)$ \\
\hline \multicolumn{7}{|l|}{ HRU, mean or \% pts } \\
\hline Hospital stay & 7.9 days & 12.8 days & 11.6 days & 16.1 days & 15.7 days & 8.7 days \\
\hline Surgical intervention & $99.0 \%$ & $95.0 \%$ & $97.4 \%$ & $91.7 \%$ & $93.2 \%$ & $91.2 \%$ \\
\hline Imaging techniques & 2.7 & 3.9 & 4.7 & 3.6 & 4.8 & 4.3 \\
\hline Outpatient visits & 7.9 & 6.9 & 5.7 & 7.8 & 6.7 & 8.2 \\
\hline Rehabilitation sessions & 3.2 & 10.1 & 16.6 & 2.1 & 17.1 & 8.8 \\
\hline Home care & 2.2 days & 8.2 days & 19.1 days & 0.4 days & 29.2 days & 0.4 days \\
\hline \multicolumn{7}{|l|}{ Total cost, $€(95 \% \mathrm{CI})$} \\
\hline Women & $\begin{array}{l}7031 \\
(6532-7631)\end{array}$ & $\begin{array}{l}9772 \\
(8138-11406)\end{array}$ & $\begin{array}{l}9978 \\
(9071-10884)\end{array}$ & $\begin{array}{l}10940 \\
(9342-12537)\end{array}$ & $\begin{array}{l}12321 \\
(10879-13763)\end{array}$ & $\begin{array}{l}8639 \\
(7299-9980)\end{array}$ \\
\hline Men & $\begin{array}{l}6115 \\
(5168-7063)\end{array}$ & $\begin{array}{l}12905 \\
(7791-18018)\end{array}$ & $\begin{array}{l}10391 \\
(8107-12675)\end{array}$ & $\begin{array}{l}8282 \\
(5506-11057)\end{array}$ & $\begin{array}{l}12297 \\
(9141-15453)\end{array}$ & $\begin{array}{l}7091 \\
(6197-7986)\end{array}$ \\
\hline
\end{tabular}




\section{P223}

OSTEOPOROTIC FRACTURES OF THE PELVIS

J. C. Minarro $^{1}$, R. Quevedo-Reinoso ${ }^{1}$, M. J. López-Pulido ${ }^{1}$, A. González-Fernández ${ }^{1}$

${ }^{1}$ University Hospital Reina Sofía, Córdoba, Spain

Introduction: Osteoporotic fractures of the pelvis are more common than it is thought and its incidence is increasing as population ages. These fractures can occur in patients with severe osteoporosis and no previous trauma. What physicians may not realize is that these injuries do not appear isolated and advance imaging may be needed.

Aim: The aim of this paper is to report our experience in diagnosis and management of this type of fractures which are underestimated despite its high morbidity.

Methods: Between January 2013 and December 2014, ten patients ( 9 women and 1 man) with a mean age of 76,3 (range 59-91) were radiographically diagnosed of an osteoporotic fracture of the pelvis in the ER. A computed tomography (CT) was performed in order to reveal any sacrum fracture associated which appeared in four patients. None of the patients had been previously diagnosed of osteoporosis and thus they were not taking any hypercalcemic medication. Treatment for bone deficiency was applied in all patients, five with calcium, vitamin $\mathrm{D}$ and bisphosphonates and the other five initiated a $\mathrm{rPTH}$ therapy. Patients were followed up for a minimum of 12 months.

Results: Fracture healing was observed in all patients in less than 12 weeks needing less time (2,3 weeks in average) those who had been treated with $\mathrm{rPTH}$. One of the patients suffered a contralateral fragility fracture which is currently under follow.

Discussion: The appearance of an insufficiency fracture can be the first presentation of osteoporosis. Risk factors for an extreme loss of bone mass usually are prolonged immobilization, radiation, long-term intake of corticosteroids, vitamin D deficiency. As a result of loss of autonomy, insufficiency fractures carry a significant morbidity and significant 1 -year mortality risk comparable to hip fractures. Studies have shown that these fractures do not occur in isolation and they appear frequently associated with posterior pelvic ring injuries. Therefore, it is necessary to supplement the radiological study to search sacral injury as pubic rami fractures are often the only initial finding. Because of the general lack of displacement associated, only $20-38 \%$ of sacral insufficiency fractures are identified on plain radiographs so CT exam is helpful in these cases. Pelvic fragility fractures can usually be managed conservatively, but certain unstable patterns may require surgical fixation. Treatment consists on bed rest and analgesics (pain may persist for 6-8 weeks). The relative risk of a second fragility fracture in these patients is $1.8-2.0$, so the bone forming treatment should be used as treatment of choice.

\section{P224}

TRANSIENT OSTEOPOROSIS OF THE HIP. A CASE REPORT OF PATHOLOGIC FRACTURE

${ }_{\text {B. Barriendos }}^{1}$, R. Lumbreras ${ }^{1}$, M. Angulo ${ }^{1}$

${ }^{1}$ Department of Orthopaedic Surgery. H. U. Miguel Servet, Zaragoza, Spain

Introduction: The transient osteoporosis of the hip is transient an unusual, self-limited disorder of unknown etiology that usually lasts for 6-8 months. It is more frequently found in middle aged men but it can also be found in pregnant women during the last three months of pregnancy. It usually appears as an ingrowing pain that enables to patient bear any weight. Complications are rare.

Material and Methods: 39 weeks gravid woman of 37 years old who suffered for severe coxalgia in the last two months treated with paracetamol, just seen by her gynaecologist, that appears with sudden lower limb claudication and fall. Taken to A \& E where X-rays were performed and a nondisplaced subcapital fracture was diagnosed.

Results: An elective section was performed and just afterwards, the fracture was fixed with three canulated screws, not allowing weight bearing for two months but rehabilitation was started two weeks after surgery. She completed weight bearing at three months with some remaining aches and pains but after four months was completed asymptomatic.

Conclusions: Transient osteoporosis is rare but benign even though possible complications have to be taken into account due to its severity. When suspected it has to be studied and until then prevent weight bearing, especially in pregnant women we need to evaluate the risk-benefit of x-ray studies when suspected. 


\section{P225}

\section{SCREW CUT-OUT: MECHANICAL OR BIOLO- GICAL FAILURE?}

B. Limousin Aranzabal ${ }^{1}$, E. Fernandez Tormos ${ }^{1}$, C. Arraiz Diaz $^{1}$, O. Marín Peña ${ }^{1}$, R. Larrainzar Garijo ${ }^{1}$

${ }^{1}$ Hospital Universitario Infanta Leonor, Madrid, Spain

Introduction: The hip fracture is one of the XXI century epidemics. The most common treatment for pertrochanteric hip fractures is the intramedullary nailing, although other types of devices are used also like the nail-plate. The objectives of this work are: to identify the type indecency of screw "cut-out" in our series; analyze the potential mechanical or biological origin; and study the functional significance in those patients.

Method: We analyzed retrospectively a case series of patients with pertrochanteric hip fracture. In our series are 589 cases treated between 2009 and 2013 in our hospital. We selected cases of complications with subsequent reoperation grouped by DRGs. We found 9 cases of "cut out" reoperation. We analyzed demographics data, functional parameters (BARTHEL Index), laboratory parameters (Vit. D), radiological parameters (Tip-Apex Distance (TAD) and Parker Ratio (PR)).

Results: With a comparable groups of cases and controls with respect to demographic data; We found a higher rate of BARTHEL in the series of cases $(10 \%)$. The analytical parameters indicated a clear hypovitaminosis $\mathrm{D}$ in patients with pertrochanteric hip fracture, being more marked in our case series. In our cases the radiological parameters analyzed indicated a $\mathrm{TAD}>25$ in 50 and $50 \% \mathrm{TAD}<25$. In $100 \%$ the screw was in a middle position as PR. The incidence of screw cut-out was $1.35 \%$. We detected a possible mechanical origin in $50 \%$ of cases and biological in the remaining $50 \%$. We detected a reduction in the functional parameters of $18.75 \%$ in the BARTHEL Index.

Conclusion: Even though we have a low incidence of screw cut-out in our series in comparison to the literature, this has not less importance. This phenomenon has a significant impact on the functional parameters of patients. Two important routes of improvement for reducing the incidence are: improve the surgical technique (SBP $<25 \mathrm{~mm}$ and $\mathrm{PR}>66 \%$ ) and improve the biological environment, placing great importance to vitamin $\mathrm{D}$.

\section{P226}

PREVALENCE OF MONOCLONAL GAMMOPATHY IN PATIENTS WITH ACUTE HIP FRACTURE

T. Dukelow ${ }^{1}$, I. Pillay ${ }^{1}$

${ }^{1}$ Department of Geriatric Medicine, South Tipperary General Hospital, Clonmel, County Tipperary, Ireland

Objective: To establish the prevalence of monoclonal gammopathy in a population with acute hip fracture attending an outpatient service in Ireland.

Material and Methods: Secondary osteoporosis screening was carried out on patients attending an outpatient clinic post acute hip fracture. Frequently ordered tests included serum protein electrophoresis (SPEP), thyroid function tests, bone profile, serum Vitamin D levels, immunoglobulins, cortisol, and, where relevant, testosterone. Data pertaining to 100 patients attending over an 18 month period was analysed retrospectively. 8 patients were excluded from data analysis as no information was available regarding basic demographics.

Results: Average patient age was 81.85 years. $35.7 \%$ were men and $64.3 \%$ women. $85.9 \%$ of patients $(79 / 92)$ underwent serum protein electrophoresis (SPEP). Of these individuals, $10.1 \%(8 / 79)$ patients were found to have a monoclonal band on electrophoresis.

Conclusion: Monoclonal gammopathy is a relatively common finding in patients with acute hip fracture, occurring more frequently in this study than previously demonstrated elsewhere. This finding is likely to represent monoclonal gammopathy of undetermined significance (MGUS) in the majority of patients. Multiple myeloma and MGUS remain important considerations for those with suspected or newly diagnosed osteoporosis. Up to 1 in 20 patients with newly diagnosed osteoporosis have multiple myeloma or MGUS ${ }^{1}$. Despite a well documented increase in fracture risk in $\mathrm{MGUS}^{2}$, screening for this condition in populations with fracture may be neglected. Routine SPEP may be advisable in all individuals presenting with hip fracture to assess for MGUS and malignant gammopathies.

References:

${ }^{1}$ Abrahamsen B, et al. BMJ 2005;330:818.

${ }^{2}$ Melton LJ 3rd, et al. J Bone Miner Res 2004;19:25.

\section{P227}

THE EFFECT OF SENSORIMOTOR TRAINING ON HAND AND UPPER EXTRIMITY SENSORY AND MOTOR FUNCTION IN PARKINSON PATIENT

F. Daneshjoo $^{1}$

${ }^{1}$ Iran University of Medical Science, Tehran, Islamic Republic of Iran

Introduction: Parkinson patient suffer from sensorimotor function problems. Their touch sensation, proprioception, discrimination and object weight perception will get affected. This disorder cause bradykinesia, slow reaction time, dis coordination and absence of muscle control. Several studies had considered sensory and motor relation in varies subject and indicated that sensory deficiency specially in hand can be the primary reason of function disorder. Since this matter has not 
been studied, so we decided to do this study as study of hand and upper extremity sensory and motor relation in Parkinson patient to use its results in planning intervention for hand sensorimotor function improvement.

Material and Method: 40 patients (35 male, 5 female) with idiopathic Parkinson recruited in this study (table 1). The study performed in a randomized in this repeated measures design with 2 groups of idiopathic Parkinson between the ages of 22 and 83 years old. The sensory motor training group was made up 20 PD (the mean ages: $61.05 \pm 13.9$ ) participated in a 10- sessions SMT (5-day in 2 weeks) and the control group consisted of 20 PD (The mean ages: $59.15 \pm 11.26$ ).

Result: There were no significant difference between groups at the pretest for all subtest of PPT score and BBT in both dominant and non-dominant hand. A significant group by time interaction was seen for $\mathrm{DH}$, both hand and assembly subtest of PPT. There were no significant difference between the group at the pretest for the sensory tests score in all test points, but there were significant group by time interactions for the sensory tests scores in all test points, indicating the SMT participant improved.

Conclusion: We found that 10 session sensorimotor training improved sensory motor function in hand and upper extremity in Parkinson patient.

\section{P228}

A PROPOSAL FOR THE DEFINITION OF EARLY SYMPTOMATIC KNEE OSTEOARTHRITIS FROM AN INTERNATIONAL CONSENSUS PROMOTED BY THE ITALIAN SOCIETY FOR RHEUMATOLOGY (SIR)

A. Migliore $^{1}$, C. A. Scirè ${ }^{2}$, L. Carmona ${ }^{3}$, G. HerreroBeaumont $^{4}$, J. Branco ${ }^{5}$, E. Bizzi ${ }^{6}$, X. Chevalier ${ }^{7}$, L. Collaku $^{8}$, S. Aslanidis ${ }^{9}$, L. Denisov ${ }^{10}$, L. Di Matteo ${ }^{11}$, G. Bianchi $^{12}$, D. Diracoglu ${ }^{13}$, B. Frediani ${ }^{14}$, E. Maheu ${ }^{15}, \mathrm{~N}$. Martusevich $^{16}$, G. F. Bagnato ${ }^{17}$, M. Scarpellini ${ }^{18}$, G. Minisola $^{19}$, N. Akkoc ${ }^{20}$, R. Ramonda ${ }^{21}$, T. Barskova ${ }^{22}$, D. Babic-Naglic $^{23}$, J. V. Moreno Muelas ${ }^{24}$, R. Ionescu ${ }^{25}$, R. Rashkov $^{26}$, N. Damjanov ${ }^{27}$, M. Matucci-Cerinic ${ }^{22}$

${ }^{1}$ Rheumatology, S. Pietro FBF Hospital, Rome, Italy, ${ }^{2}$ Italian Society for Rheumatology, Epidemiology Unit, Milan, Italy, Milan, Italy, ${ }^{3}$ Instituto de Salud Musculoesquelética, Madrid, Spain, Madrid, Spain, ${ }^{4}$ Bone and Joint Research Unit, Fundación Jiménez Díaz, Universidad Autónoma de Madrid, Spain, Madrid, Spain, ${ }^{5}$ Faculdade de Ciencias Medicas, Universidade Nova de Lisboa, Portugal, Lisboa, Portugal, ${ }^{6}$ Rheumatology, S.Pietro FBF Hospital, Rome, Italy, Rome, Italy, ${ }^{7}$ Department of Rheumatology, Henri Mondor Hospital, Creteil, France, Creteil, France, ${ }^{8}$ Internal Medicine Department, University hospital, Tirana, Albania, Tirana, Albania, ${ }^{9}$ 2nd
Propedeutic Department of Internal Medicine, Hippokration General Hospital, Thessaloniki, Greece., Thessaloniki, Greece, ${ }^{10}$ Institute of Rheumatology, Russian Academy of Medical Science, Moscow, Russia, Moscow, Russian Federation, ${ }^{11}$ Department of Rheumatology, University of Chieti, Chieti, Italy, Chieti, Italy, ${ }^{12}$ Department of Locomotor System Director Division of Rheumatology ASL3-Azienda Sanitaria Genovese, Genoa, Italy, Genova, Italy, ${ }^{13}$ Department of Physical Medicine and Rehabilitation, Division of Pain Medicine, Istanbul University, Faculty of Medicine, Turkey, Istanbul, Turkey, ${ }^{14}$ Rheumatology Unit, University of Siena, Siena, Italy, Siena, Italy, ${ }^{15}$ Rheumatology Department, St-Antoine Hospital, APHP, Paris, France, Paris, France, ${ }^{16}$ Belorussian State Medical University, Department Of Rheumatology, Minsk, Belarus, Minsk, Belarus, ${ }^{17}$ Rheumatology Unit, University of Messina, Italy, Messina, Italy, ${ }^{18}$ Director Rheumatology Unit "Fornaroli Hospital", Magenta (MI) Italy, Milan, Italy, ${ }^{19} \mathrm{OU}$ of Rheumatology, S.Camillo-Forlanini Hospital, Rome, Italy, Rome, Italy, ${ }^{20}$ Professor of Medicine, Division of Rheumatology Immunology, Dokuz Eylul University, School of Medicine, Izmir, Turkey, Izmir, Turkey, ${ }^{21}$ Rheumatology Unit, Department of Medicine DIMED, University of Padova, Padova Italy, Padova, Italy, ${ }^{22}$ Department of Experimental and Clinical Medicine, Division of Rheumatology AOUC, University of Florence, Italy, Florence, Italy, ${ }^{23}$ University of Zagreb School of Medicine, University Hospital Centre, Zagreb, Department of Rheumatology and Rehabilitation, Zagreb, Croatia, Zagreb, Croatia, ${ }^{24}$ Hospital Vall d'Hebron, Barcelona, President of the Spanish Society of Rheumatology., Barcelona, Spain, ${ }^{25} \mathrm{Head}$ of the Department of Internal Medicine and Rheumatology Sf. Maria Hospital, University of Medicine \&Pharmacy "Carol Davila" Bucharest, President of Romanian Society of Rheumatology, Bucharest, Romania, ${ }^{26}$ Clinic of Rheumatology, Medical University, Sofia, Bulgaria, ${ }^{27}$ Institute of Rheumatology, Belgrade University School of Medicine, Belgrade, Serbia, Belgrade, Serbia

Objective: A valid definition of early symptomatic knee osteoarthritis (ESKOA) is still missing. Identifying patients in early stages of disease could improve the management of OA.

Materials and Methods: A set of focus groups composed by patients, basic science researchers and expert clinicians was held to identify signs, symptoms and risk factors of ESKOA. A systematic review of literature was brought out. A modified Delphi survey was then performed by an international panel of experts, to rank proposed items. A face to face meeting was held to achieve a final definition of ESKOA.

Results: Several different constructs of the disease were identified in the literature. A total of 46 items, including 
symptoms, signs and risk factors was identified by focus groups, then it was reduced to 15 by the expert panel. A final definition of criteria of ESKOA (Table 1) was achieved after the modified Delphi survey.

Conclusions: This is the first international consensus for the definition of ESKOA. Knee pain and a set of risk factors represent the core items of this diagnostic algorithm. A validation study is actually ongoing to confirm this definition.

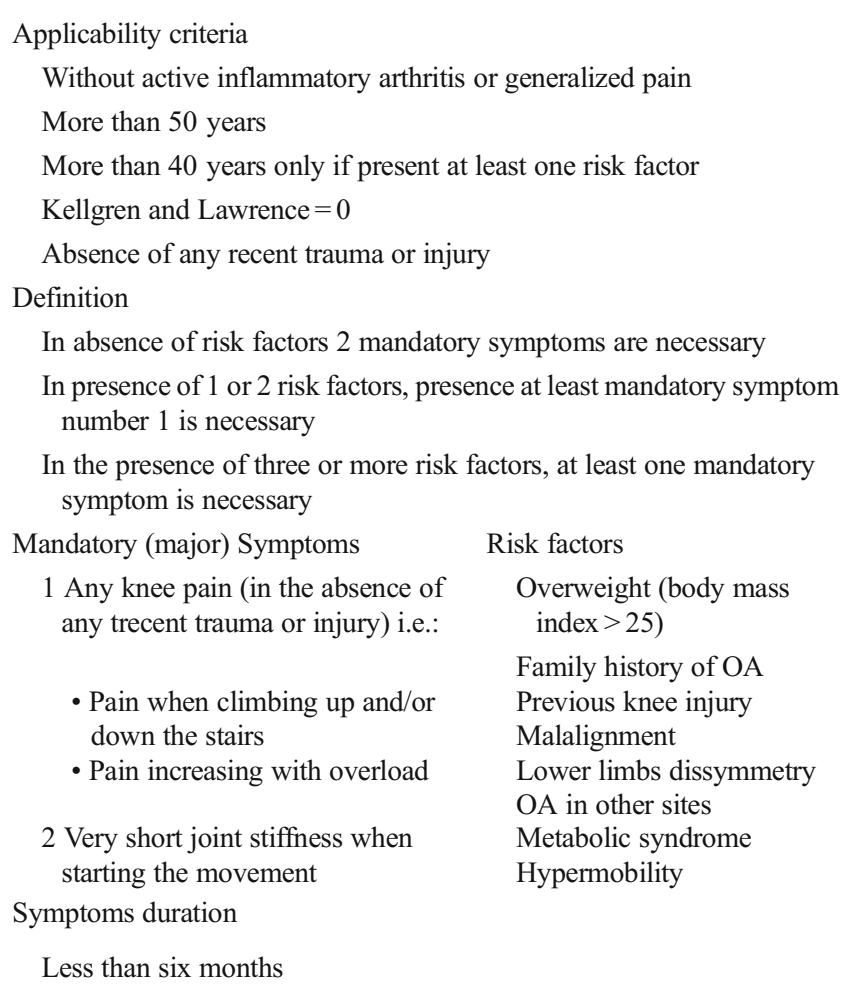

\section{P229}

DIACEREIN RETARDS CELL GROWTH OF CHONDROSARCOMA CELLS AT THE G2/M CELL CYCLE CHECKPOINT VIA CYCLIN B1/CDK1 AND CDK2 DOWNREGULATION

B. Lohberger ${ }^{1}$, A. Leithner ${ }^{1}$, N. Stuendl ${ }^{1}$, H. Kaltenegger ${ }^{1}$, W. Kullich $^{2}$, B. Steinecker-Frohnwieser ${ }^{2}$

${ }^{1}$ Department of Orthopedic Surgery, Medical University Graz, Graz, Austria, ${ }^{2}$ Ludwig Boltzmann Institute for Rehabilitation of Internal Diseases, Saalfelden, Austria

Objectives: Chondrosarcoma is characterized for its lack of response to conventional cytotoxic chemotherapy, propensity for developing lung metastases, and low rates of survival. Research within the field of development and expansion of new treatment options for unresectable or metastatic diseases is of particular priority. Diacerein, a symptomatic slow acting drug in osteoarthritis (SYSADOA), implicates a therapeutic benefit for the treatment of chondrosarcoma by an antitumor activity.
Material and Methods: After treatment with diacerein the growth behaviour of the cells was analyzed with the xCELLigence system and MTS assay. Cell cycle was examined using flow cytometric analysis, RT-PCR, and western blot analysis of specific checkpoint regulators. The status for phosphorylation of mitogen-activated protein kinases (MAPKs) was analyzed with a proteome profiler assay. In addition, the possible impact of diacerein on apoptosis was investigated using cleaved caspase 3 and Annexin V/PI flow cytometric analysis.

Results: Diacerein decreased the cell viability and the cell proliferation in two different chondrosarcoma cell lines in a dose dependent manner. Flow cytometric analysis showed a classical G2/M arrest. mRNA and protein analysis revealed that diacerein induced a downregulation of the cyclin B1-CDK1 complex and a reduction in $\mathrm{CDK} 2$ expression. Furthermore, diacerein treatment increased the phosphorylation of $\mathrm{p} 38 \alpha$ and $\mathrm{p} 38 \beta$ MAPKs, and Akt1, Akt2, and Akt 3 in SW-1353, whereas in Cal-78 the opposite effect has been demonstrated. These observations accordingly to our cell cycle flow cytometric analysis and protein expression data may explain the $\mathrm{G} 2 / \mathrm{M}$ phase arrest. In addition, no apoptotic induction after diacerein treatment, neither in the Cal-78 nor in the SW-1353 cell line was observed.

Conclusion: Our results demonstrate for the first time that diacerein decreased the viability of human chondrosarcoma cells and induces G2/M cell cycle arrest by CDK1/cyclin B1 downregulation. In summary, our findings strongly support diacerein as an interesting target for further investigation and development of novel therapeutics in sarcoma research.

P230

IS CALCIFEDIOL MORE EFFECTIVE THAN CHOLECALCIFEROL IN IMPROVING 25-HYDROXYVITAMIN D3 LEVELS, MUSCLE STRENGTH, AND PHYSICAL PERFORMANCE IN POSTMENOPAUSAL WOMEN?

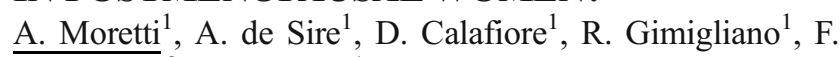
$\overline{\text { Gimigliano }}^{2}$, G. Iolascon ${ }^{1}$

${ }^{1}$ Department of Medical and Surgical Specialties and Dentistry, Second University of Naples, Naples, Italy, ${ }^{2}$ Department of Physical and Mental Health and Preventive Medicine, Second University of Naples, Naples, Italy

Objective: The aim of our study was to evaluate the effects of vitamin $\mathrm{D}$ on $25(\mathrm{OH}) \mathrm{D} 3$ levels, muscle strength, and physical performance in postmenopausal women, comparing calcifediol and cholecalciferol.

Material and Methods: In our real-practice study we included postmenopausal women aged $\geq 50 \mathrm{y}$; they were divided into two groups, according to the prescription: calcifediol and cholecalciferol. We evaluated at the baseline (T0) and after six months (T1): serum levels of 25(OH)D3, appendicular muscle strength, using the hand grip strength test (HGS) and the knee 
extensor strength test (KES), and physical performance, using the short physical performance battery (SPPB).

Results: We evaluated 205 postmenopausal women, mean aged $69.28 \pm 9.16$ years, 103 treated with calcifediol and 102 with cholecalciferol. In Table 1 we showed the results after vitamin D prescription.

Table 1. Outcome measures assessed at the baseline and after 6 months of vitamin D supplementation.

\begin{tabular}{llll}
\hline & $\begin{array}{c}\text { Calcifediol T0 } \\
(n=103)\end{array}$ & $\begin{array}{c}\text { Calcifediol T1 } \\
(n=103)\end{array}$ & Pvalues \\
25(OH)D3 $(\mathrm{ng} / \mathrm{ml})$ & $31.74 \pm 13.03$ & $51.80 \pm 19.85$ & $<0.001$ \\
HGS $(\mathrm{kg})$ & $15.54 \pm 6.65$ & $18.29 \pm 3.85$ & 0.014 \\
KES $(\mathrm{kg})$ & $14.49 \pm 6.92$ & $17.05 \pm 5.62$ & 0.050 \\
SPPB & $8.41 \pm 3.32$ & $9.70 \pm 2.14$ & 0.008 \\
\hline & \multicolumn{1}{c}{$\begin{array}{l}(n=102) \\
\text { Cholecalciferol T0 }\end{array}$} & $\begin{array}{c}\text { Cholecalciferol T1 } \\
(n=102)\end{array}$ & Pvalues \\
25(OH)D3 $(\mathrm{ng} / \mathrm{ml})$ & $35.15 \pm 11.57$ & $40.28 \pm 13.04$ & 0.118 \\
HGS $(\mathrm{kg})$ & $15,36 \pm 6.06$ & $16.38 \pm 5.14$ & 0.257 \\
KES $(\mathrm{kg})$ & $12.12 \pm 5.74$ & $12.58 \pm 5.67$ & 0.627 \\
SPPB & $7.81 \pm 3.66$ & $8.02 \pm 3.41$ & 0.638 \\
\hline
\end{tabular}

Result are expressed as means \pm standard deviations. Within group analysis was performed using a paired $t$-test

Conclusions: Our results showed that postmenopausal women treated with calcifediol had significant improvements in serum levels of $25(\mathrm{OH}) \mathrm{D} 3$, muscle strength, and physical performance.

\section{P231}

\section{ABDOMINAL AORTIC CALCIFICATION IS MORE PREVALENT AND SEVERE IN COM- MUNITY-DWELLING OLDER AUSTRALIANS WITH LOW RELATIVE MUSCLE MASS}

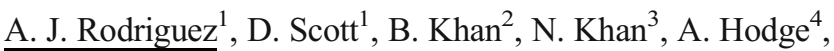
D. R. English ${ }^{4}$, G. G. Giles ${ }^{4}$, P. R. Ebeling ${ }^{1}$

${ }^{1}$ Bone and Muscle Health Research Group, Department of Medicine, Monash Medical Centre, Melbourne, Australia, ${ }^{2}$ Melbourne Medical School (Western Campus), The University of Melbourne, Melbourne, Australia, ${ }^{3}$ Department of Medicine, Diwan Chand Satyapal Aggarwal Diagnostic Imaging Centre, New Delhi, India, ${ }^{4}$ Cancer Epidemiology Centre, The Cancer Council Victoria, Melbourne, Australia

Background: Low muscle mass increases the risk of functional limitation and cardiovascular mortality. Cardiovascular disease burden in the elderly may be related to increased abdominal aortic calcification (AAC) and therefore has prognostic value for cardiovascular risk. This study aimed to determine the association between low muscle mass and AAC in communitydwelling older Australians.
Participants and Methods: Cross-sectional data was extracted from a 2010 sub-study of the Melbourne Collaborative Cohort Study (Australia). Body composition was determined by DXA. Participants were stratified into tertiles of sexspecific BMI-normalised appendicular lean mass (ALM). AAC was determined by radiography. Aortic calcification score (ACS) was determined visually as the extent of aortic wall calcification between L1-L4 vertebrae. Scores could range between $0-3$ per vertebral segment with a maximal cumulative ACS of 24.

Results: Three hundred and twenty-seven participants were included for analysis [mean age $=70.5 \pm 5.5 \mathrm{y}$; mean $\mathrm{BMI}=28.1 \pm 5.2 \mathrm{~kg} / \mathrm{m} 2$; females $n=199$ (61.9\%)]. Prevalence of AAC was highest in the lower ALM/BMI tertile (74.1\%) compared to those in the middle (64.5\%) and upper $(53.2 \%)$ tertiles $(p$-value trend=0.006). Median ACS was highest in the lower tertile $(4$, range $=0-17)$ compared to the middle (3,0-16) and upper (1,0-14) tertiles (p-value trend $=0.001)$. In multivariable logistic regression, the lower tertile had increased odds (Odds $=2.281 ; 95 \%$ confidence interval: $1.133-4.593 ; p=0.021)$ of AAC; and increased odds of more severe AAC $(2.211 ; 1.223-3.999 ; p=0.009)$ independent of traditional AAC risk factors, serum calcium and weekly caloric expenditure.

Conclusion: AAC is more prevalent and severe in community-dwelling older adults with low muscle mass. Prospective studies are required to validate these findings independent of other risk factors and to determine its causality.

\section{P233}

UPDATE ANALYSIS: EVALUATION OF THE STEROID PREMEDICATION FOR CANCER CHEMOTHERAPY ASSOCIATED OSTEOPOROSIS: ESPRESSO-01 STUDY

T. Muranaka $^{1}$, M. Nakamura ${ }^{2}$, A. Ishiguro ${ }^{3}$, S. Yuki ${ }^{4}$, T.

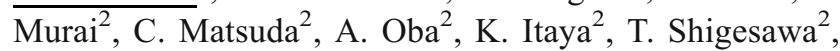
Y. Koike ${ }^{2}$, Y. Fujita ${ }^{2}$, A. Endo ${ }^{2}$, Y. Tsukuda ${ }^{2}$, Y. $\mathrm{Ono}^{2}$, T. $\mathrm{Kudo}^{2}$, A. Nagasaka ${ }^{2}$, S. Nishikawa ${ }^{2}$, Y. Komatsu ${ }^{1}$

${ }^{1}$ Division of Cancer Chemotherapy, Hokkaido University Hospital Cancer Center, Sapporo City, Hokkaido, Japan, ${ }^{2}$ Department of Gastroenterology, Sapporo City General Hospital, Sapporo City, Hokkaido, Japan, ${ }^{3}$ Department of Medical Oncology, Teine Keijinkai Hospital, Sapporo City, Hokkaido, Japan, ${ }^{4}$ Department of Gastroenterology and Hepatology, Hokkaido University Hospital, Sapporo City, Hokkaido, Japan

Objective: We previously reported that glucocorticoid (GC) premedication (GCP) of cancer chemotherapy (CTx) for preventing several side effects such as nausea, vomiting, and infusion reaction caused the reduction of 
bone mineral densities at lumbar spine (BMD-LS) and the increase of bone turnover markers, such as crosslinked N-telopeptide of type I collagen (NTX) and bone alkaline phosphatase (BAP) (ASCO-GI 2016 Abst.523). We now report the updated results of this study (ESPRESSO-01 study).

Material and Methods: The eligibility criteria were as the follows: (i) histologically proven gastrointestinal cancer (GIC).; (ii) The duration of periodical GCP is weekly, biweekly, and triweekly.; (iii) age over 20. The primary endpoint was to investigate the variations of BMD-LS by DXA, NTX, and BAP, between baseline (BL) and 16 weeks after starting CTx (16w). Results: From June 2013 to April 2015, 98 pts were enrolled. Two pts were not proven as GIC histologically. One patient (pt) was not measured on baseline DXA. One pt was taken bisphosphonates already on BL point. Four pts were not administered CTx or GCP, and 16 pts were not measured BMD on $16 \mathrm{w}$ due to several reasons such as pts refusal, discontinuation of CTx, death and so on (74 pts were full analysis set). Not only the levels of BMD-LS but also those of total hip (BMD-TH) and femoral neck BMD (BMD-FN) at 16w were decreased compared with $\mathrm{BL}$ and the average percent changes were $-1.9 \%$ (95\%CI: $-2.7 \%$ to $-1.1 \%, p<0.0001),-2.2 \%$ (95\%CI: $-3.5 \%$ to $-0.9 \%, p=0.002$ ), and $-2.0 \%$ (95\%CI: $-3.0 \%$ to $-1.0 \%, p<0.0001)$, respectively.

Conclusions: We found that there were statistically significant reductions of not only the levels of BMD- LS but also those of BMD-TH and BMD-FN due to periodic GCP in GIC pts. These results indicate that GCP might generate more serious osteoporosis of GIC pts during CTx.

\section{P234}

LOWER LEG ARTERIAL CALCIFICATION ASSESSED BY HIGH-RESOLUTION PERIPHERAL QUANTITATIVE COMPUTED TOMOGRAPHY IS ASSOCIATED WITH BONE MICROSTRUCTURE ABNORMALITIES IN WOMEN

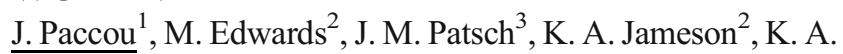

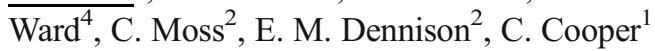

${ }^{1}$ MRC Lifecourse Epidemiology Unit, University of Southampton, Southampton, United Kingdom, ${ }^{2}$ MRC Lifecourse Epidemiology Unit, Southampton, United Kingdom, ${ }^{3}$ Department of Biomedical Imaging and Image-Guided Therapy, Medical University of Vienna, Vienna, Austria, ${ }^{4}$ Elsie Widdowson Laboratory, Medical Research Council Human Nutrition Research, Cambridge, United Kingdom

Purpose: Here we report the relationships of bone geometry, volumetric $\mathrm{BMD}$, and bone microarchitecture with lower leg arterial calcification (LLAC) as assessed by HR-pQCT.
Methods: We utilised the Hertfordshire Cohort Study (HCS), where we were able to study associations between measures obtained from HR-pQCT of the distal radius and distal tibia in 341 participants with or without LLAC. Statistical analyses were performed separately for women and men. We used linear regression models to investigate the cross-sectional relationships between LLAC and bone parameters.

Results: The mean (SD) age of participants was 76.4 (2.6) and 76.1 (2.5) years in women and men, respectively. One hundred and eleven of 341 participants $(32.6 \%)$ had LLAC that were visible and quantifiable by HR-pQCT. The prevalence of LLAC was higher in men than in women $(46.4 \%(n=83)$ vs. $17.3 \%$ $(n=28), p<0.001)$. After adjustment for confounding factors, we found that women with LLAC had substantially lower Ct.area $(\beta=-0.33, p=0.016)$, lower Tb.N $(\beta=-0.54, p=0.013)$ and higher Tb.Sp $(\beta=0.54$, $p=0.012)$ at the distal tibia and lower Tb.Th $(\beta=-0.49$, $p=0.027)$ at the distal radius compared with participants without LLAC. Distal radial or tibial bone parameters analyses in men according to their LLAC status revealed no significant differences with the exception of Tb.N $(\beta=0.27, p=0.035)$ at the distal tibia.

Conclusion: In the HCS, the presence of LLAC assessed by HR-pQCT was associated with relevant bone microstructure abnormalities in women. These findings need to be replicated but may help clinicians and researchers to a better understanding on osteo-vascular interactions.

\section{P235}

\section{NINE LEVELS ELASTOPLASTY}

R. Conti ${ }^{1}$, A. Boschi ${ }^{1}$, G. Marchese ${ }^{1}$, I. Barni ${ }^{1}$

${ }^{1}$ Department of Neurosurgery, University Florence, Florence, Italy

Introduction: Percutaneous kyphoplasty or vetebroplasty with polymethylmethacrylate (PMMA) is successfully used for pain relief in symptomatic neoplastic or osteoporotic fractures. Unfortunately restoration of the hardness of the vertebral body might propagate secondary fracture of adjacent vertebrae. Elastoplasty might prevent these complications. In this report we describe the case of a man affected by Monoclonal gammopathy of undetermined significance (MGUS) with multilevel vertebral fractures. We chose to perform a 9 levels percutaneous elastoplasty. There were no complications during the procedure. After surgery the patient reported complete resolution of symptoms (VAS 2), and he was discharged on the third day post-op. Rx control after six months showed good restoration of the size of the vertebral body and no evidence of secondary fracture of adjacent vertebrae. 
Case report: A 59 years old male presented with thoracic and lumbar pain (visual analogue scale, VAS 8) for 8 weeks arose after an intense effort. In his personal history presence of monoclonal gammopathy of undermined significance (MGUS) and hypertension. There were no urinary or bowel symptoms. Laboratory investigation revealed moderate anemia. Radiological findings showed a T12 and T11 A1.2 fracture and a T5,T6, T8, T10,L1, L2 and L3 A1.1 fracture (Magerl et al. Eur Spine J 1994). All this vertebrae were RMi T2 stir weighted positive. Due to the number and the nature of the fractures we decided to perform a nine level percutaneous elastoplasty with VK100, an elastic silicone biopolymer (BBraun) (BonWrx Inc., member of the Wrx LLC 1507 W Parkside Lane, Phoenix, Arizona). In local anesthesia, with patient positioned prone and under constant radioscopic control VK100 was inserted with a bilateral transpeduncolar approach for T10, T11, T12, L1 L2, L3 and bilateral extrapeduncolar approach for T5,T6,T8. After the procedure the patient referred a complete recovery of the preoperative symptoms. Repeated lumbar and thoracic Rx at the 3 months showed an excellent vertebral augmentation with no sign of leakage or other complications. The 6 month MRI scan showed no evidence of fracture or sufferance of the adjacent vertebrae.

Discussion: VK100 is an elastic silicone biopolymer, made by two dry components combined during the application to become a viscous and fluid mixture injected into the fractured vertebrae to augment the surrounding tissue. It contains barium sulfate to be radiopaque. Thanks to its peculiar chemical/physical structure, VK100 has no documented toxicity, and it has an hard and elastic consistence at the same time and it allows in a successive moment a pedicle screw fixation if needed. Another peculiarity of VK100 is that is possible to add it in an already augmented vertebra in successive procedures. Vertebroplasty and Kyphoplasty using PMMA have been shown to be effective in controlling pain and improving function. PMMA has been demonstrated to be a safe material but some authors recommend that the maximum volume of injected cement should not exceed $30 \mathrm{ml}$ or three levels per session, for a vertebroplasty and three levels for a kyphoplasty due to prevent possible side effects of the toxicity of PMMA (Coumans et al. J Neursurg Spine 2003). Other possible complications presented in literature are fractures of adjacent vertebrae; leakages of cement in spinal canal or foramen, in the disc or in blood flow and related pulmonary embolism (Choe D H et al. A J Roentgenol 2004; Zaryanov AV Neurosurg focus 2014). The two great meta-analysis of Lee et al. and Taylor et al. showed that cement leakages occurred in $14 \%$ of all cases, but only $0.01 \%$ were symptomatic. New vertebral fractures occurred in $17 \%$. Radiculopathy occurred in $0.17 \%$ of cases and the mortality rate was $4.4 \%$ with a perioperative mortality of $0.13 \%$ (Lee MJ et al. Spine 2009; Taylor RS et al. Eur Spine 2007). In the literature there are also rare cases of postoperative aseptic osteonecrosis or delayed bone cement displacement. (Mueller M et al. Eur Spine J 18 Suppl 2009; Wang HS et al. J Korean Neurosurg S0c 43 2008).
Conclusion: From this experience multiple level elastoplasty seems to be an excellent alternative than conventional techniques of vertebral augmentation. In particular for its chemical/physical structure seems to be safer against the risk of fracture adjacent vertebrae. It would be necessary to validate these initial positive impressions, with studies that collect a sufficient number of cases, counting the few cases of elastoplasty reported in the literature.

\section{P236}

A SYSTEMATIC REVIEW AND META-ANALYSIS OF RANDOMISED CONTROLLED TRIALS ON THE EFFECT OF VITAMIN D SUPPLEMENTATION ON MEASURES OF ARTERIAL STIFFNESS

A. J. Rodriguez $^{1}$, D. Scott ${ }^{1}$, V. Srikanth ${ }^{2}$, P. R. Ebeling ${ }^{1}$

${ }^{1}$ Bone and Muscle Health Research Group, Department of Medicine, Monash Medical Centre, Melbourne, Australia, ${ }^{2}$ Stroke and Ageing Research Group, Department of Medicine, Monash Medical Centre, Melbourne, Australia

Background: Low vitamin D has been associated with poor arterial compliance in observational studies. Arterial stiffness has prognostic value for cardiovascular disease risk. The aim of this systematic review and meta-analysis was to clarify the literature surrounding the use of vitamin D to ameliorate arterial stiffness and to quantitatively determine its effect. Data Extraction and Statistical Methods: We conducted a systematic review of the MEDLINE, Scopus and EMBASE databases for randomised controlled clinical trials investigating the effect of vitamin $\mathrm{D}$ supplementation on pulse wave velocity (PWV) and/or augmentation index (AIx) as measures of arterial stiffness. We meta-analysed data and calculated standardised mean difference (SMD) and $95 \%$ confidence intervals (CI) using inverse-variance models on Revman v5.3 software. Study quality was assessed using a modified Jadad scale.

Results: Six hundred and seven unique records were identified, of which 18 studies satisfied our inclusion and exclusion criteria. Study quality was high, ranging from 9 to 12 (out of a maximum 13). Study design in terms of vitamin D dosing protocol (range: 1000-5700 IU/day), follow-up times (range: 1-12 months), sample size (range: $n=29-183$ ) and recruitment strategies varied markedly. Thirteen studies had data included in meta-analysis. Vitamin D supplementation was associated with non-significant reductions in $\mathrm{PWV}$ $[\mathrm{SMD}=-0.10 ; 95 \% \mathrm{CI}:-0.24,0.04 p=0.17 ; n=806$ from ten studies $]$ and AIx $[-0.15 ;-0.32,0.02 ; p=0.08 ; n=551$ from eight studies].

Interpretation: There is inconsistent evidence to suggest that vitamin D supplementation improves measures of arterial stiffness. This may be attributable to the 
heterogeneity in study design. Therefore large, and welldesigned randomised studies are required to determine the casual relationships between vitamin D and arterial stiffness and cardiovascular risk.

\section{P237}

THE WAY OF LIFE MEDIATES THE DIFFERENCES IN THE LEVELS OF VITAMIN D OBSERVED IN THE URBAN AND RURAL POPULATION

A.-M. Borissova ${ }^{1}$, A. D. Shinkov ${ }^{1}$, J. Vlahov $^{1}$, L. Dakovska ${ }^{1}$,

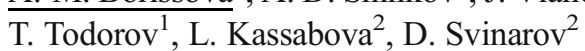

${ }^{1}$ Medical University of Sofia, University Hospital of Endocrinology, Sofia, Bulgaria, ${ }^{2}$ Medical University of Sofia, Central Laboratory for Therapeutic Drug Management, Sofia, Bulgaria

There are many factors, which have an effect on vitamin D daily hours of sun exposure, air pollution, urbanization, sex, age, a way of life (nutrition, physical activity, hours spend in the open, occupation).

Objective: To provide a comparative analysis on the distribution of vitamin D deficiency and insufficiency in Bulgaria depending on the type of residence - town, small town and village.

Material and methods: This cross-sectional study included 2032 subjects - 1076 women (53\%) and 956 men (47\%); mean age $49.3 \pm 14.8$ years ( $\geq 20$ - 80 years) from 12 Bulgarian towns and the adjacent villages. The participants were allocated in three age groups: young (20-44 years) 894 subjects; middle age ( $45-59$ years) 534 subjects; elderly ( $\geq 60$ years) 604 subjects. Venous blood was drawn before 09:00 a.m. measurement of $25(\mathrm{OH}) \mathrm{D}$ by LC-MS/MS.

Results: The prevalence of vitamin $\mathrm{D}$ deficiency $[25(\mathrm{OH}) \mathrm{D}<25.0 \mathrm{nmol} / \mathrm{l}]$ and insufficiency $[25(\mathrm{OH}) \mathrm{D} 25.0$ $\pm 50.0 \mathrm{nmol} / 1]$ was significantly lower in the villages than in the towns/small towns for all three age groups $(66.6 \% \mathrm{vs}$. $77.7 \%$, and $79.9 \%$, both $p<0.05$ for all age groups). Vitamin D insufficiency was less common in the male villagers than the female $(53.9 \%$ vs. $77.2 \%, \mathrm{p}<0.001)$. The elderly male and female rural population had significantly less vitamin $\mathrm{D}$ insufficiency than the town/small town elderly ( $64.5 \%$ vs. $77.1 \%$ and $79.8 \%$, both $p<0.01$ ).

Discussion and conclusions: The regular work in the open and longer sun exposure probably contribute to the lower frequency of vitamin D deficiency and insufficiency among male rural population. The female rural population had the same frequency of vitamin D deficiency and insufficiency as the urban females. The young women from rural areas commute and spend more "urban" hours, while the elderly women in the village spend more time inside, housekeeping, and their traditional clothing leaves less exposed skin to the sun. The rural way of life therefore renders women to less sun exposure and suboptimal synthesis of vitamin $\mathrm{D}$ than the men.

\section{P238}

THERAPEUTIC CONTRIBUTION OF COLLAGEN TYPE II IN TREATMENT OFARTHROSIS AND PSORIATIC ARTHRITIS-CASE REPORT

Z. M. Mirkovic ${ }^{1}$, J. M. Mirkovic ${ }^{1}$, T. N. Novakovic ${ }^{1}$, S. M.

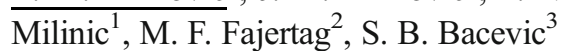

${ }^{1}$ University of Pristina, Faculty of Medicine, Kosovska Mitrovica, Serbia, ${ }^{2}$ Generaly Hospital, Kosovska Mitrovica, Serbia, ${ }^{3}$ Generaly Hospital, Prokuplje, Serbia

Introduction: Today is in the treatment of osteoarthritis and primary arthrosis routinely used collagen type II, with excellent therapeutic effects and good tolerability.

Objective: To contribute to the efficacy and safety of treating patients with psoriatic arthritis and degenerative phenomena.

Material and methods: analysis of the clinical course of the disease and radiography.

Results: The patient MR treated at the Health Center in Kosovska Mitrovica age of 54 years. From 8 years ago she lost her period and immediately afterwards she felt a pain in the shoulder joint, the left ankle was accompanied by swellings, after some time the movements of the knees were followed by crepitations. She has been suffering from psoriatic arthritis for 6 years. She was treated with MTX $7.5 \mathrm{mg}$ per week and occasionally NAIL. Objective findings: patient with skinny constitution, afebrile, eupnoic, psoriatic lesions in the elbows' area. Left ankle slightly swollen and deformed-passive movements accompanied by crepitations. Right shoulder joint accompanied by pain during movement. Other joints moderately painful without objective changes. Radiography of the joints: deformed humeral head of right shoulder and deformed cavitas glenoidalis scapulae. Left ankle: talus deformed and flattened articular surface uneven, joint cracks are hardly visible and are coated with fibrous bone bands. On lateral recording: one-third of crack of talocrural joint is barely visible and lateral on the talus are visible exostoses. The disease has with time, its activity, dictated and enhanced doses of MTX. Because of gastric intolerance, the last 6 months NAIL were replaced by tablets containing highly hydrolyzed collagen type II, due to its content of hydroxyproline and hydroxylysine amino acids that are essential for the construction of the connective tissue of the articular cartilage, there have been improvements, the aforementioned movements of the joints were not followed by pain and crepitations.

Conclusion: This case was seen as arthropathy characterized by arthritic manifestations and simultaneous occurrence of psoriatic arthritis. By combining therapy with collagen type II, benefit was observed in terms of efficacy, tolerability and safety. 


\section{P239}

RELATIONSHIP BETWEEN GASTROINTESTINAL EVENTS AND COMPLIANCE WITH O S T E O P O R O S I S T H E R A P Y : A N ADMINISTRATIVE CLAIMS ANALYSIS OF US MANAGED CARE POPULATION

A. Modi ${ }^{1}$, S. Sajjan ${ }^{1}$, J. Weaver ${ }^{1}$, E. M. Lewiecki ${ }^{2}$, S. Harris ${ }^{3}$

${ }^{1}$ Merck \& Co., Inc., Kenilworth, NJ, United States, ${ }^{2}$ New Mexico Clinical Research \& Osteoporosis Center, Albuquerque, NM, United States, ${ }^{3}$ University of California, San Francisco, San Francisco, CA, United States

Objectives: To estimate the rate of gastrointestinal (GI) events, and association between GI events and compliance with osteoporosis (OP) therapy among osteoporotic women.

Materials and Methods: A retrospective cohort study using a large administrative claims database in the United States from 2007 through 2013 was conducted. We studied women $\geq 55$ years old who were continuously enrolled in a health plan for at least 2 years, assessing a baseline year before and a follow-up year after the date of the first prescription of an oral bisphosphonate (OBP) (index date) as the first OP treatment. OBPs assessed were alendronate, ibandronate, risedronate. Compliance was defined as medication possession ratio (MPR) of $>=0.80$. GI problems were assessed using ICD-9 diagnosis or CPT Procedures. Logistic regression analysis was used to examine the association of post-treatment GI problems and compliance adjusting for baseline covariates. A sensitivity analysis of age was conducted using patients age $\geq 65$ years with a Medicare claim.

Results: A sample consisting of 45,031 women age $\geq 55$ years taking at least one OBP with mean (SD) age of 72 (7.4) years was identified. A total of 17,481 (38.8\%) patients experienced at least one GI event during the follow-up period, of whom 7,074 (40.45\%) had not experienced a GI problem at baseline. 14,071 (31.2 \%) of patients were compliant to BIS. Patients who experienced GI events after initiation of OBP were $24 \%$ less likely to be compliant to OBP as compared to patients who did not experience GI events (OR [95\% CI], 0.76 [0.728-0.796]; $P<.001)$. Patients age $\geq 65$ years old ( 37 , 886 ) had a mean (SD) age of 74 (5.7) years, and 14,732 (38.8 \%) had a GI event in the follow-up period, of whom 5, $946(40.36 \%)$ did not have a GI problem at baseline. 7,942 $(33.83 \%)$ of patients were compliant to OBP. Patients age $\geq 65$ years old who had a GI event after initiation of OBP were also $24 \%$ less likely to be compliant to OBP as compared to patients who did not experience GI events (OR $[95 \% \mathrm{CI}]$, 0.76 [0.721-0.795; $P<.001)$.

Conclusion: Patients experiencing GI events after OBP treatment initiation are less likely to comply with therapy. Patients age $\geq 65$ years do not significantly differ from patients age $\geq 55$ years regarding the relationship between GI events and compliance to OBP treatment.
P240

THE SEASONAL AND METEOROLOGICAL RELATIONSHIPS OF D I A GNOSTIC DISTRIBUTION OF PATIENTS PRESENTING WITH MUSCULOSKELETAL COMPLAINTS ACCORDING TO GENDER

A. Kul $^{1}$, M. Ugur ${ }^{1}$

${ }^{1}$ Physical Medicine and Rehabilitation, Faculty of Medicine, Erzurum, Turkey

Background: The seasonal relationship of incidence of musculoskeletal system (MSS) pathologies according to gender and their meteorological variability has not been investigated satisfactorily.

Objective: To determine diagnostic distribution of patients with MSS-related complaints and their seasonal relationships.

Methods: This study was conducted on 45541 patients having MSS-related complaints. Female/male ratios of eleven diagnosis groups were determined; monthly/ seasonal incidences and relations with climatic factors were evaluated.

Results: While number of patients admitted to polyclinics increased most in winter for both genders, lowest number was in summer. In female patients, knee-related pathologies were most frequent in April (9.4\%) and rheumatologic disorders were most frequent in September $(13.3 \%)$. In male patients, lumbar pathologies were most frequent in December (9.6\%) and rheumatologic disorders were most frequent in September (13.0\%). In all patients, negative correlation was found between average temperatures and knee pathologies $(r=-0.952, p=0.048)$. There were positive correlations between humidity and knee $(r=0.980, p=0.020)$, elbow $(r=0.951, p=0.049)$, hip $(r=0.957, p=0.043)$, hand-wrist $(r=0.963, p=0.037)$ and pathologies necessitating rehabilitation $(r=0.954$, $p=0.046$ ).

Conclusions: The number of admissions increased in both genders during winter, and decreased during summer. Additionally, relationships of some MSS pathologies with seasonal/meteorological changes should be considered.

\section{P241}

LIFESTYLE CORRELATES OF LOW BONE MINERAL DENSITY IN ALBANIAN WOMEN

J. Kollcaku $^{1}$, A. Kollcaku ${ }^{2}$

${ }^{1}$ Polyclinic 3, Tirana, Albania, ${ }^{2}$ University Hospital Center Mother Teresa, Tirana, Albania

Aim: To assess the association of lifestyle/behavioral factors with low BMD in Albanian women. 
Methods: A cross-sectional study was conducted in Tirana city in 2010 including a population-based sample of 549 women aged 35 years and above (response rate: $92 \%$ ). Low BMD (osteopenia and/or osteoporosis defined as a BMD T-score less than -1) was assessed with a bone ultrasound device which is simple and easy to use for screening of BMD in population-based studies. Binary logistic regression was used to determine the relationship of low bone mineral density with behavioral factors in this study population.

Results: The prevalence of low BMD in this study population was $28.4 \%$ (156/549). In multivariable-adjusted logistic regression models, low BMD was positively associated with smoking $(O R=4.1,95 \% \mathrm{CI}=2.2-7.4)$ and coffee consumption $(O R=2.3,95 \% \mathrm{CI}=1.3-4.1)$, but inversely related to overweight and obesity $(O R=0.4,95 \% \mathrm{CI}=0.2-0.7$ and $O R=0.3$, $95 \% \mathrm{CI}=0.2-0.6$, respectively).

Conclusion: This study offers useful evidence about the lifestyle/behavioral determinants of low BMD among Albanian women.

\section{P242}

TOWARDS POINT OF CARE INSTRUMENTATION FOR ACCURATE FRACTURE RISK PREDICTION

A. J. Dicken ${ }^{1}$, J. P. O. Evans ${ }^{1}$, K. Rogers ${ }^{2}$, C. Greenwood ${ }^{2}$, S. $\mathrm{X}_{\text {. Godber }}^{3}$, N. Stone ${ }^{4}$, J. Clement ${ }^{5}$, I. Lyburn ${ }^{6}$, R. Martin ${ }^{7}$, P. Zioupos $^{2}$

${ }^{1}$ Nottingham Trent University, Nottingham, United Kingdom, ${ }^{2}$ CFI, Cranfield University, Swindon, United Kingdom, ${ }^{3}$ Halo X-ray Technologies, Nottingham, United Kingdom, ${ }^{4}$ Physics and Astronomy, Exeter University, Exeter, United Kingdom, ${ }^{5}$ Melbourne Dental School, University of Melbourne, Melbourne, Australia, ${ }^{6}$ Cobalt Health, Cheltenham, Cheltenham, United Kingdom, ${ }^{7}$ Social and Community Medicine, Bristol University, Bristol, United Kingdom

Objective(s): The economic burden of osteoporotic related fractures is large (i.e. $\$ 25.3$ billion anticipated by 2025 ) and therefore improvements in diagnostic performance that enable better informed clinical management would be of significant benefit. Currently at least $30 \%$ of the variation in bone strength is not predicted by BMD thus limiting DXA's predictive ability. This would be improved if bone 'quality' (architecture and physico-chemistry) could be determined simultaneously in vivo. Thus we have been developing new instrumentation to provide quantification of material properties with the potential to extend the approach to microarchitecture.

Material and Methods: Fundamental material properties of bone are measured routinely within the laboratory using coherent X-ray scatter applied to small specimens.
Unfortunately this is a relatively slow process using X-ray energies too low to penetrate even modest bone architectures. However, recent technological advancements in enhanced scatter harvesting have made the implementation of an in vivo diagnostic probe highly achievable. We have exploited this advance ('focal construct technology') in a new benchtop instrument capable of measuring scattering from whole bone samples. The data quality and patient dose have been determined.

Results: We present, for the first time, clinically significant Xray scattering data and show how it can be employed through two methods (parameterisation and discriminant analysis) to discriminate between bone with a range of associated fracture probabilities. Ultrastructure characteristics of bone mineral were determined with classifying precision (e.g. within $2 \mathrm{wt} \% \mathrm{CO}_{3}{ }^{2-}$ substitution) from bone of $1 \mathrm{~cm}$ thickness at $130 \mathrm{kV}$. Exposure times were $<1 \mathrm{~s}$ and absorbed doses $<20$ mGy.

Conclusion(s): This work demonstrates, for the first time, a viable approach for the production of a clinical point of care instrument with the ability to provide simultaneously ultrastructural and conventional (BMD) estimates of fracture risk for individual patients.

Acknowledgments: This programme of work is funded by the UK EPSRC grant number EP/K020196/1 Pointof-Care High Accuracy Fracture Risk Prediction.

\section{P243}

EFFECTS OF DENOSUMAB (DMAB) ON BONE MATRIX MINERALIZATION: RESULTS FROM THE PHASE 3 FREEDOM TRIAL

D. Dempster ${ }^{1}$, J. P. Brown ${ }^{2}$, S. Yue ${ }^{3}$, D. Farlay ${ }^{4}$, S. Rizzo ${ }^{4}$, J. Song $^{3}$, A. Wang ${ }^{3}$, R. B. Wagman ${ }^{3}$, G. Boivin ${ }^{4}$

${ }^{1} 1$ Columbia University, New York, NY, USA and Helen Hayes Hospital, West Haverstraw, NY, United States, ${ }^{2}$ Laval University and CHU de Québec Research Centre, Quebec City, QC, Canada, ${ }^{3}$ Amgen Inc., Thousand Oaks, CA, United States, ${ }^{4}$ INSERM UMR 1033, Université de Lyon, Lyon, France

Objective: Bone biopsy-based assessment of DMAb's effects at the tissue level demonstrated a low remodeling rate consistent with DMAb's mechanism of action (Reid JBMR 2010; Brown JBMR 2014). We report the effects of DMAb on bone matrix mineralization in women who underwent transiliac crest bone biopsy in FREEDOM.

Materials and Methods: Biopsies were performed at year 2 and/or 3 (Reid JBMR 2010). Bone matrix mineralization was assessed in a blinded fashion by digitized quantitative microradiography and analyzed using a Matlab program (Montagner J X-Ray Sci Technol 2015). The mean degree of mineralization of bone (DMB) and heterogeneity index (HI) 
of the distribution of DMB were calculated for cancellous and cortical bone, endocortical and periosteal sub-compartments of cortical bone, and total bone (cancellous and cortical combined).

Results: 72 of 115 biopsies (42 DMAb, 30 Placebo) from the FREEDOM bone biopsy sub-study were evaluated. Demographics for subjects in this sub-study were comparable to FREEDOM. DMAb resulted in a significant increase in mean DMB compared with Placebo (Figure); these findings were consistent across cancellous and cortical compartments $(p<0.01)$. A significant- ly lower HI was observed in total bone and in all compartments assessed in the DMAb-treated group $(p<0.05)$, consistent with reduced bone turnover in response to DMAb.

Conclusions: In women with PMO, DMAb resulted in increased bone matrix mineralization and a lower $\mathrm{HI}$ compared with Placebo. These data are consistent with expected results based on observations with other antiresorptives (Bala Eur J Endocrinol 2011) and with DMAb's mechanism of action.

Conflicts of interest: FREEDOM was funded by Amgen

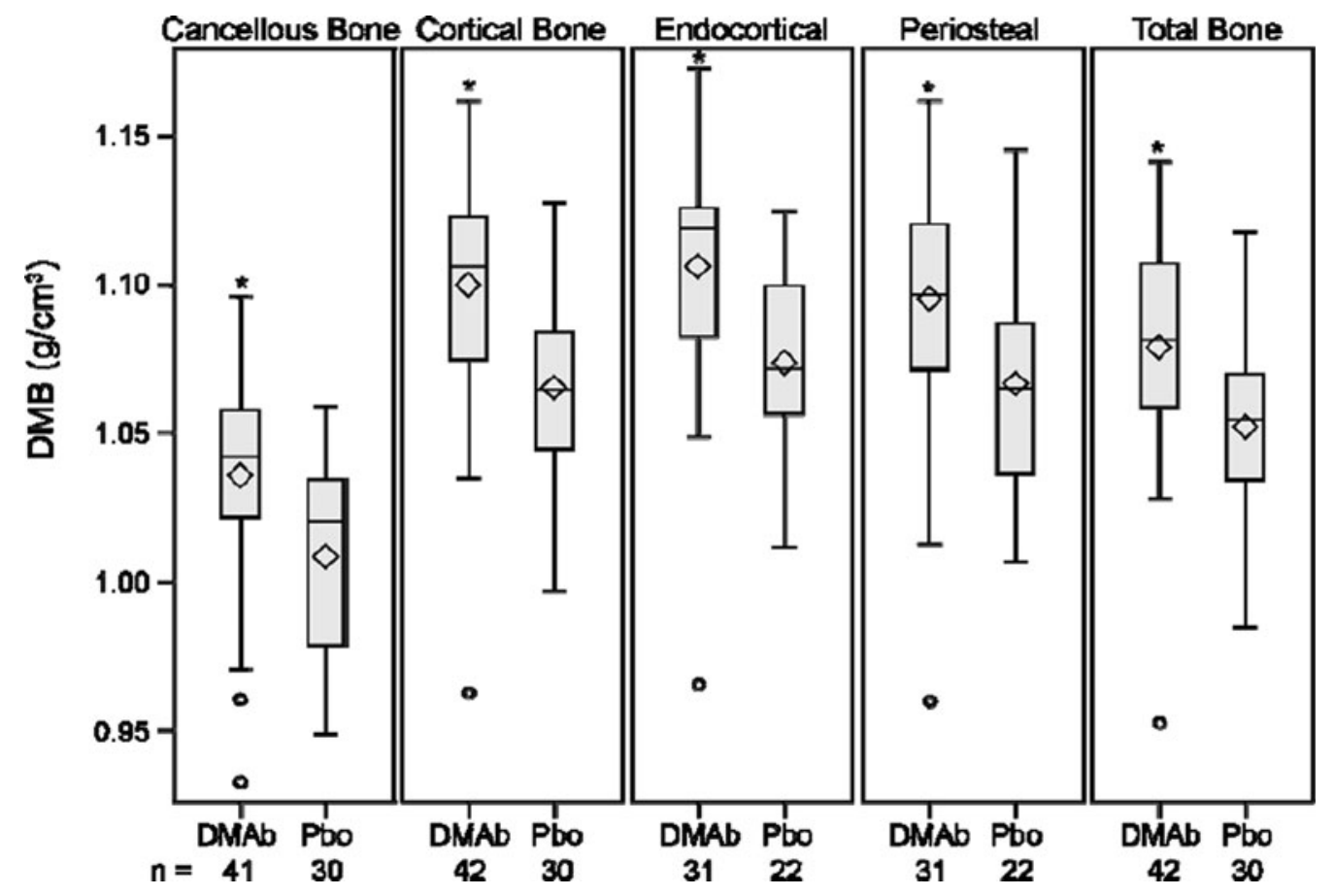

\section{P244}

STRUCTURAL PARAMETERS COMPUTED BY 3D-DXA: ACCURACY EVALUATION AGAINST QUANTITATIVE COMPUTED TOMOGRAPHY

L. Humbert $^{1}$, J. Clotet ${ }^{1}$, Y. Martelli ${ }^{1}$, S. Di Gregorio ${ }^{2}$, L. Del Río ${ }^{2}$

${ }^{1}$ Galgo Medical, Barcelona, Spain, ${ }^{2}$ CETIR Centro Médico, Barcelona, Spain

Objective: To evaluate the accuracy of structural parameters computed by 3D-DXA against quantitative computed tomography (QCT).

Material and Methods: The 3D-DXA software (Galgo Medical, Barcelona, Spain) provides 3D subject-specific models of the proximal femur from DXA projections. The algorithm registers a 3D appearance model of the femoral shape and density onto the DXA image of the hip of the patient, and allows for the quantification of volumetric BMD (vBMD), volume (for trabecular and cortical regions) and cortical thickness distribution. In this study, a software algorithm was developed to compute structural parameters from 3DDXA subject-specific models: cross-sectional area (CSA), cross-sectional moment of inertia (CSMI), section modulus (Z) and buckling ratio (BR). These parameters were computed at the narrow neck, intertrochanteric and lower shaft cross sections. A database of 60 patients with both QCT and DXA acquisitions (CETIR Grup Mèdic, Barcelona, Spain) was used (22 men and 38 women, with a mean age of $60.9 \pm 14.7$ years [34 years - $91 \mathrm{y}]$ ). DXA scans were performed using a Lunar iDXA device (GE Healthcare, Madison, WI). QCT-scans 
were performed using a Philips Gemini GXL 16 (Philips Healthcare, Best, The Netherlands), a Discovery CT750 HD scanner (GE Healthcare) or a GE HiSpeed QX/I scanner (GE Healthcare, Madison, WI). Structural parameters computed by 3D-DXA and QCT were compared to evaluate the accuracy of the proposed method.

Results: Correlation coefficients (R) between CSA computed by 3D-DXA and QCT were 0.95 when computed at the nar-
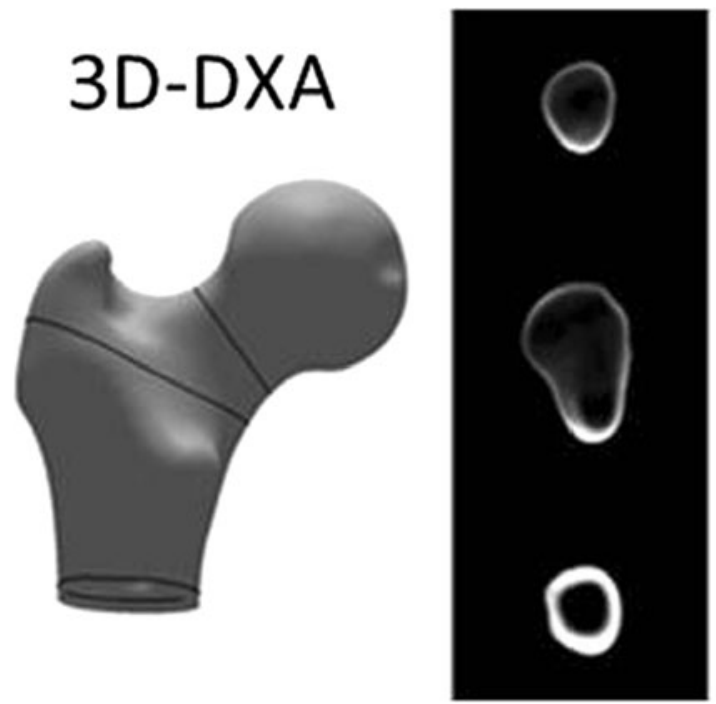

row neck, 0.96 at the intertrochanteric and 0.97 at the lower shaft cross section. Correlation coefficients were of 0.95 , 0.95 and 0.94 for the CSMI, 0.92, 0.94 and 0.94 for the Z, and $0.85,0.96$ and 0.92 for the BR, when computed at the narrow neck, intertrochanteric and lower shaft cross section respectively. A comparison between 3D-DXA and QCT models and cross-sections for one patient of the database can be seen in the figure below.

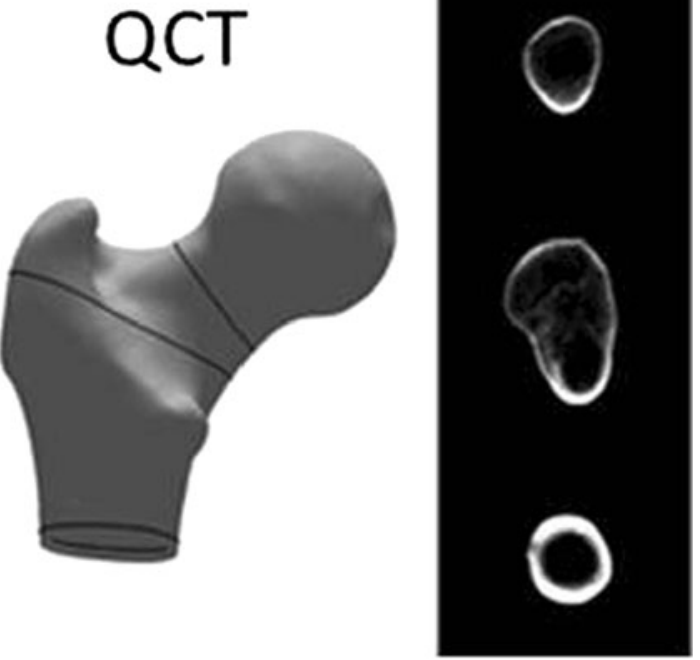

Conclusion: This study demonstrates the ability of 3D-DXA to compute accurate structural parameters and provide a 3D analysis of the proximal femur strength from 2D DXA images.

\section{P245}

THORACIC CORD COMPRESSION BY EPIDURAL MULTIPLE MYELOMA: A RARE PRESENTATION OF MULTIPLE MYELOMA

A.-K. Kul ${ }^{1}$, F.-B. Bagcier ${ }^{1}$, A.-K. Karaman ${ }^{2}$

${ }^{1}$ Physical Therapy and Rehabilitation, Atatürk University Faculty of Medicine, Erzurum, Turkey, ${ }^{2}$ Radiology, Ataturk University Faculty of Medicine, Erzurum, Turkey

45-year-old man presented to our clinic with a -month history of progressive paraparesis. His symptoms began as numbness in the bilateral lower extremity. That progressed over several weeks, followed by progressive weakness affecting the bilateral lower extremity. He denied any trauma to the back, fever, headache, nausea, vomiting or diarrhea or any difficulty in breathing or loss of appetite or weight. On physical examination, T4 sensory level paraplegia was demonstrated with slight motion of the left plantar and dorsal flexors of the foot. There was no sacral sparing. Deep tendon reflexes were absent in lower extremities with no clonus. There was no disturbance of consciousness. An magnetic resonance imaging study revealed a solid mass appearance in soft tissue intensity on 4th - 6th thoracic vertebral levels. The lesion originates from right lateral posterior paravertebral area and extends into spinal canal and making marked pressure on spinal cord. Neurosurgery was called immediately. Patient was taken to the surgery and debulking of the mass was done and sent for pathology study. The biopsy of the mass showed plasma cell malignancy. Immuno-histochemical analysis of the bone marrow showed neoplastic cells of plasma cell origin. These plasma cells expressed monoclonal lambda light chain with absent kappa staining. Post-operative course was uneventful and patient regained the power of $4 / 5$ in both lower legs.

Radiotherapy was started. Slowly patient regained the strength and was able to ambulate with walker. Multiple myeloma is a malignant proliferation of plasma cells usually showing diffuse bony involvement with predilection of the spine. Extramedullary multiple myeloma is very rare, comprising less than $5 \%$ of all plasma cell neoplasms. Spinal cord compression is usually caused by primary involvement of the vertebral body with tumor extension into the adjacent spinal canal ${ }^{1}$. MRI has been 
established as a sensitive method in detecting localized or diffuse bone lesions in multiple myeloma. In our case, no reliable evidence of bone involvement was seen on conventional radiographs, and the vertebral bodies were of normal height and shape. On MRI, the marrow signal in the vertebral bodies was nor-mal; however, MRI demonstrated spinal cord compression over T4-T6 level due to a separate extensive epidural mass in the dorsal spinal canal, which was clearly distinguishable from surrounding bone structures and obviously of extra osseous origin. Treatment options for extra osseous epidural multiple myeloma include surgical resection and local radiation therapy (4000 cGy over 4 weeks) ${ }^{2}$. The response to conventional chemotherapy of extramedullary multiple myeloma is very poor. Although quite rare, extra osseous multiple myeloma without evidence of bone destruction or collapse of vertebral body should be included in the differential diagnosis of epidural mass lesions, causing spinal cord compression.

1. Palmbach M, et al. Eur J Radiol 1996;22:146.

2. Damaj G, et al. Eur J Haematol 2004;73:402.

\section{P246}

GIANT LIMBUS VERTEBRA MIMICKING A VERTEBRAL FRACTURE

F.-B. Bagcier $^{1}$, H.-O. Ogul ${ }^{2}$, A.-K. Kul ${ }^{1}$

${ }^{1}$ Physical Therapy and Rehabilitation, Atatürk University Faculty of Medicine, Erzurum, Turkey, ${ }^{2}$ Radiology, Ataturk University Faculty of Medicine, Erzurum, Turkey

A 58-year-old woman presented to our clinic with a year history of low-back pain radiating to her right and left leg. There was no neuropathic pain. She had no symptoms suggestive of inflammatory back pain. Patient's previous medical history was insignificant. There was no history of trauma, chronic disease, alcohol and substance abuse. Neurological examination revealed a positive straight leg-raising test on the right and left side. Both the upper and lower limb muscle strength and sensory examination was complete. Other system physical examination findings were normal. Lumbosacral magnetic resonance imaging revealed Grade 1-2 spondylolisthesis at L4-L5 and a large bony fragment over the posteriorsuperior portion of L5 lumbar vertebral body. Anti-inflammatory medications, back bracing for stabilisation and physiotherapy treatment regimen(ultrasound, electric stimulation, hot packs) was recommended. Limbus vertebrae results from the marginal disc herniations. The well-shaped triangular bone fragment that is developed by anterior herniation of the nucleus composes the typical form and stands as a separate segment. It has been claimed that the obstructing effect of the replaced disc material from the corpus of the vertebra towards the ring apophysis is responsible for the development of limbus vertebrae. ${ }^{1}$ Limbus vertebra is not an uncommon radiological finding in an adult, it is a rare finding in the child or adolescent.
The most common site for the presence of Limbus vertebra is the mid-lumbar region and less commonly occurs in the mid cervical region. Anterior limbus vertebra is typically asymptomatic, while posterior limbus vertebra has been reported to cause nerve compression and be associated with back pain. The limbus vertebra is generally misdiagnosed as a fracture, which may result in unnecessary invasive procedures. The clue for radiographic differentiation of the limbus vertebra from fracture is the sclerotic margin of the triangular fragment. It can be differentiated from an acute fracture because fractures will not have sclerotic margins, but limbus vertebra will. $^{2}$ Anterior limbus vertebrae are more frequent than posterior limbus vertebrae. A review of literature showed very few reports discussing the limbus vertebrae within the posterior region. $\mathrm{H}$ Baba and friends ${ }^{3}$ report posterior limbus vertebral lesions causing lumbosacral radiculopathy and the cauda equina syndrome. In our case there was posterior limbus vertebrae mimicking the vertebral fracture. As a result fractures, degenerative osteophytes and ring epiphysis should be considered as more common etiologies in addition to the acquired developmental fusion defects of vertebra in differential diagnosis of limbus vertebrae.

\section{References:}

1. Schmorl G, Junghanns H: The Human Spine in Health and Disease, 2d ed, New York, Grune \& Stratton, 1971.

2. Handel SF, et al. Radiology 1979;130:629.

3. Baba H, et al. Spinal Cord 1996;34.7:427.

\section{P247}

\section{A RARE CAUSE OF KNEE FLEXION CONTRA- CTURES: INTRAMUSCULER HEMANGIOMA IN THE QUADRICEPS MUSCLE}

\section{F.-B. Bagcier ${ }^{1}$, R.-S. Sade ${ }^{2}$, A.-K. Kul ${ }^{1}$}

${ }^{1}$ Physical Therapy and Rehabilitation, Atatürk University Faculty of Medicine, Erzurum, Turkey, ${ }^{2}$ Radiology, Ataturk University Faculty of Medicine, Erzurum, Turkey

15 -year-old male presented to our clinic with progressive and painless right knee flexion contracture. The patient noticed a soft tissue mass in the anterior aspect of his right knee a year ago. Throughout this period, swelling gradually increased. The patient had received a diagnosis of presumed congenital cavernosus hemangioma in right ankle and it was operated and the mass removed. There was no history of associated constitutional symptoms, bleeding tendencies, trauma or infection prior to the appearance of the mass. Examination revealed an asymmetric, boggy, noncompressible, and nonpulsatile swelling in the anterolateral aspect of right knee region measuring. The overlying skin was normal with no discoloration and no local raise of temperature. The fossae on either side of the patellar tendon were normal and the patellar tap was negative. There was no distal neurovascular deficit 
or bruit. Limb length was equal to the contralateral limb. MRI scans showed a soft-tissue mass below the anterior thigh muscles, which suggested a hemangioma with no communication with the joint cavity. Patient was planned for surgical excision. A flexion contracture (FC) of the knee is the inability to fully straighten the knee. Normal active range of motion of the knee is $0^{\circ}$ extension and $140^{\circ}$ flexion. In people with a FC one of the motions or both quadriceps muscle are reduced. FC can arise by different causes such as burn scars, intra-articular fractures, septic arthritis, juvenile rheumatoid arthritis, cerebral palsy and many others. Rare reasons such as hemangioma may cause the FC. Hemangioma is a common soft-tissue tumor but intramuscular cavernous haemangiomas represent less than $1 \%$ of all haemangiomas. Most patients present with a mass and/or pain, which often aggravate with activity ${ }^{1}$. The younger age groups are most frequently affected with $85 \%$ of cases having occurred under the age of 30 years, among which $30 \%$ are seen in lower extremities with quadriceps being the most common muscle involved. The majority of tumors are located in the lower extremities. They usually present as slowly enlarging soft tissue masses with few symptoms ${ }^{2}$. There is no agreement about the etiology of these tumors, their appearance is often linked to trauma but much data exists suggesting a congenital origin $^{3}$. Many treatment modalities(percutaneous sclerotherapy, radiotherapy, embolization and freezing) for the symptomatic haemangiomas are available of which surgical excision is the most preferred. The aim of presenting this case is to emphasize that, in cases with chronic, progressive and painless swelling in a muscle or adjacent to a joint with or without an episode of trauma especially in a young individual, the physician should be alert to this early diagnosis.

\section{References:}

1. Wild AT, et al. Arch Orthop Trauma Surg 2000;120:139.

${ }^{2}$. Nagira K, et al. Clin Imag 2001;25:368.

${ }^{3}$. Chaudhary N, et al. J Laryngol Otol 1998;112:1199.

\section{P248}

\section{A RARE COEXISTING OF HIP AND KNEE JOINT'S SYNOVIAL CHONDROMATOSIS}

F.-B. Bagcier ${ }^{1}$, A.-K. Kul ${ }^{1}$, E.-E. Ergül ${ }^{1}$, A.-E. Erdal ${ }^{1}$

${ }^{1}$ Physical Therapy and Rehabilitation, Atatürk University Faculty of Medicine, Erzurum, Turkey

A 29-year-old male patient presented to our clinic with progressively worsening bilateral hip pain, right knee pain and swelling. The hip and knee pain started about 3 months previously and increased over time. Pain of the hip joints were spreading bilaterally to the trochanteric regions. The characteristic of the pain was mechanical. He did not feel pain while sleeping. Prolonged sitting or standing caused the hip to lock. Previously, he had received analgesic medications but there had been no significant improvement. There was no pain in any other joint, and there was no history of disease or trauma associated with the hip and knee. Physical examination revealed an antalgic gait and the patient was seen to have visibly swollen right popliteal fossa. The motion of the right hip joint was limited and painful in all directions, whereas lumbar and left hip joint motions were unrestricted. He could fully extend his knee, but flexion was restricted to only 120 degrees. Radiography of the pelvis and knee indicated a narrowing joint space, and there were erosions on acetabular side of the joint and multiple soft tissue calcifications outside the joint capsule of the right knee. Magnetic resonance imaging of the bilateral hip revealed findings consistent with synovial chondromatosis, which filled the joint space completely and knee magnetic resonance revealed expanded synovium that contained osteochondromas, which were hypointense all sequences. Orthopedic surgical procedures including arthroscopic debridement were planned with symptomatic right hip and right knee joints. Synovial osteochondromatosis is an rare benign condition characterized by the formation of multiple nodules of hyaline cartilage within the sub-synovial connective tissue ${ }^{1}$. The condition is generally monoarticular and involves the knee in more than $50 \%$ of cases ${ }^{2}$. The other common joints involved are hip, elbow, shoulder and ankle. In the literature Kose MM and friends presented with unilateral hip joint synovial osteochondromatosis case, Silvio Giannetti and friends presented with unilateral elbow joint synovial osteochondromatosis case, Heather Shearer and friends presented with bilateral ankle joint synovial osteochondromatosis case ${ }^{3}$. In our case uncommon coexistance of hip and knee involvement was detected. The more common symptoms chronic, progressive pain, swelling, loss of motion, and locking, and the most common physical signs consist of soft-tissue swelling, crepitation, palpable loose bodies, and limited range of motion. As radiotherapy and chemotherapy have no effect on synovial chondromatosis, surgical removal of the affected synovium and the loose bodies is the preferred treatment ${ }^{4}$.

\section{References}

1. Sugimoto K, et al. Skeletal Radiol 2003;32:99.

2. Walling AK, Gasser SI. Clin Sports Med 1994;13:909.

3. Shearer H, et al. Chiropractic Manual Therapies 2007;15.1:18.

4. Mackenzie H, et al. J Med Case Rep 2010;4:113.

\section{P249 \\ PAINLESS COMPLEX REGIONAL PAIN SYN- DROME IN AN OLD MAN}

F.-B. Bagcier $^{1}$, A.-K. Kul ${ }^{1}$, M.-U. Ugur ${ }^{1}$

${ }^{1}$ Physical Therapy and Rehabilitation, Atatürk University

Faculty of Medicine, Erzurum, Turkey

A 62-year-old man presented to outpatient clinic with complaints of swelling and restriction of finger and wrist 
movements of his left hand. He gives history of distal radius fracture on his left hand two months prior to presentation to the our clinic. Following the trauma patient had been treated by immobilization in a cast for six weeks. He had no pain. On examination of the skin of the affected hand; wrist was swollen (non-pitting), hot, was not tender to touch. There was no hyperhidrosis, tremor, allodynia and hyperalgesia. Range of motion was restricted at the wrist and fingers. X-ray of the hand showed demineralization of the bones of the hand. A bone scan performed and there was milder non-specific diffuse increased uptake in the remaining left wrist region. Based on these findings, the patient was diagnosed as complex regional pain syndrome (CRPS) Type I according to Zyluk scoring system.(1) Physical therapy modalities and medical treatment was planned. In the physiotherapy programme; range of motion exercises, contrast baths (four cycles over a 20 minute period), whirpool bath (15 minutes), retrograde massage, pulse ultrasound $\left(1 \mathrm{~W} / \mathrm{cm}^{2}\right)$ was performed. The patient was given intravenous methylprednisolone $30 \mathrm{mg} /$ day posology for a week. After the 20 sessions of physical therapy and medical treatment the patient's signs and symptoms markedly improved since the initial examination. Active and passive wrist flexion was full and he did not report any pain at the control visits.

According to the International Association for the Study of Pain criteria of 1994, pain, evidence of change in blood flow or abnormal sudomotor activity and the absence of conditions that would otherwise account for symptoms are essential diagnostic entities of CRPS type I. (2,5) Eisenberg and Melamed (3) reported a series of five patients with various foot pathologies who had presented with all of the criteria of CRPS except pain. Veldman et al. reported a series of 829 patients with CRPS and among them, $7 \%$ of the patients did not have pain as a symptom. (4) This case presents a patient who, subsequent to trauma, developed full-blown clinical pictures of CRPS, but had neither spontaneous pain nor any hyperalgesia/allodynia whatsoever. The literature suggest that the autonomic, motor, and trophic signs and symptoms of CRPS are not necessarily coupled with either ongoing or evoked pain and point to the existence of a relatively unrecognized syndrome, for which we suggest the name complex regional painless syndrome.

\section{References:}

1. Zyluk A. J Hand Surg 2003;28B:238.

2. Reinders MF, et al. Clin J Pain 2002;18:207.

3. Eisenberg E, Melamed E. Pain 2003;106:263.

4. Veldman PHJM, et al. Lancet 1993;342:1012.

5. Dilek B, et al. (2008) Ağrısız Seyreden Kompleks Bölgesel Ağrı Sendromu: Olgu Sunumu. Turkish Journal of Physical Medicine \& Rehabilitation/Turkiye Fiziksel Tip ve Rehabilitasyon Dergisi, 54 (3).

\section{P250}

A COMPARATIVE STUDY OF THE EFFECTS OF PTH FORMULATIONS ON JAPANESE PATIENTS WITH OSTEOPOROSIS

H. Kikuchi ${ }^{1}$, Y. Itoh $^{2}$, W. Shimada ${ }^{2}$, N. Okada ${ }^{3}$, S. Soen ${ }^{4}$

${ }^{1}$ Department of Orthopaedic Surgery, Sakai Hospital, Kinki University Faculty of Medicine, Sakai-city, Japan, ${ }^{2}$ Orthopaedic Surgery, Sakai Hospital Kinki University Faculty of Medicine, Sakai-city, Japan, ${ }^{3}$ Orthopaedic Surgery, Sumoto-itsuki Hospital, Sumoto-city, Japan, ${ }^{4}$ Orthopaedic Surgery and Rheumatology, Nara Hospital Kinki University Faculty, Nara, Japan

Objective: Daily PTH (D-PTH; teriparatide $20 \mu \mathrm{g}$ /day [Eli Lilly and Company]) stimulates bone formation and is hence commonly used to increase vertebral bone mass. However, it also increases bone resorption, leading to cortical bone porosity. Weekly PTH (W-PTH; teriparatide $56.5 \mu \mathrm{g} /$ week [Asahi Kasei Pharma]) alleviates cortical bone porosity by increasing the dose interval. In this study, we compared the effects of the $2 \mathrm{PTH}$ formulations used in routine clinical practice.

Patients and Methods: Among severe osteoporosis patients receiving outpatient care in the Department of Orthopaedic Surgery at Sakai Hospital, Kinki University Faculty of Medicine, we selected those administered 2 PTH, divided them into 2 groups, and prospectively studied their 1-year clinical outcomes (continuation rates, complications, changes in a bone formation marker level, number of bone fractures during the study, and radius DXA bone density).

Results: Thirty-eight patients in the D-PTH group (2 men and 36 women; mean age, 78 years [range: 55-90 y]) and 41 in the W-PTH group (6 men and 35 women; mean age, 78 years [range: $62-90$ y]) gave consent to participate, except for the times immediately after a fracture or surgery. The continuation rate at week 52 was $76 \%(29 / 38)$ and $56 \%(23 / 41)$ in the DPTH and W-PTH groups, respectively. The rates of discontinuation due to nausea, vomiting, and other side effects were $18 \%(7 / 38)$ and $29 \%(12 / 41)$ in the D-PTH and W-PTH groups, respectively. The D-PTH and W-PTH groups had only 2 and 4 patients, respectively, who dropped out because they either changed the hospital or were lost to follow-up. The median level of the bone formation marker procollagen type $1 \mathrm{~N}$-terminal propeptide increased from 28.7 to $86.7 \mu \mathrm{g} / \mathrm{L}$ in the D-PTH group, but only slightly, from 30.7 to $45.1 \mu \mathrm{g} / \mathrm{L}$, in the W-PTH group. In all, 1 and 5 new fractures occurred during the study period in the D-PTH (clavicle) and W-PTH groups (vertebral body [2 cases], femur, humerus, and rib) groups, respectively. However, the radius DXA bone density decreased from 62.5 to $57.0 \%$ in the D-PTH group, and increased from 61.7 to $63.4 \%$ in the W-PTH group.

Conclusion: Although our findings are for a small sample, no decrease was noted in the mass of the long bones in the WPTH group, indicating that the treatment had achieved its 
intended purpose. However, the clinical outcomes of the WPTH group were no better than those of the D-PTH group.

\section{P251}

DENOSUMAB (DMAB) FREEDOM EXTENSION PSEUDO CONTROL STUDY: A RETROSPECTIVE COHORT STUDY OF COMORBIDITIES IN SWEDISH WOMEN WITH POSTMENOPAUSAL OSTEOPOROSIS (PMO)

M. Feudjo Tepie ${ }^{1}$, J. Banefelt ${ }^{2}$, O. Ström ${ }^{2}$, G. Ortsäter ${ }^{2}$, O. Ljunggren $^{3}$, K. A kesson ${ }^{4}$, J. M. Sprafka ${ }^{5}$, R. B. Wagman ${ }^{5}$

${ }^{1}$ Amgen Ltd., Uxbridge, United Kingdom, ${ }^{2}$ Quantify Research, Stockholm, Sweden, ${ }^{3}$ Uppsala University, Uppsala, Sweden, ${ }^{4}$ Department of Orthopedics, Skane University Hospital and Clinical Sciences Malmö, Lund University, Lund, Sweden, ${ }^{5}$ Amgen Inc., Thousand Oaks, CA, United States

Objective: Provide context for the adverse events (AEs) observed in FREEDOM Extension in which DMAb was administered for up to 10 years with no control group [Papapoulos ASBMR 2015].

Materials and methods: From databases in two Swedish osteoporosis clinics we included women aged 60-90 years with a DXA scan at the hip or spine between 2000-2012 (index event); women prescribed DMAb were excluded. We then selected a PMO cohort using the FREEDOM inclusion/exclusion criteria, e.g. BMD T-score less than -2.5 at lumbar spine or total hip and not less than -4.0 at either site (Cummings NEJM 2009). A general population cohort was selected from a national register, matched to the PMO cohort on demographic factors. Our primary outcome was comorbidities, with exposure-adjusted incidence rates (through Dec2012) calculated for each cohort.

Results: In total, 3,276 women were included in the PMO cohort and 8,569 in the general population cohort; mean (SD) age, 71.9 (7.4) and 70.6 (7.6) years, respectively. Overall, the rates of the comorbidities of interest were comparable to the AE rates observed in years 1-6 of the FREEDOM Extension (Table). From the database analysis, the overall mortality rate was 2.7 per 100 -patient years (comparable across the PMO and general population cohorts). AFF were not recorded in the databases; ONJ were not analysed.

Conclusion: This database analysis indicates that the incidence rates of most AEs of special interest observed in years 1-6 of the FREEDOM extension study are comparable to the rates in the general population and a population of women with PMO unexposed to DMAb. This finding further supports the long term safety of DMAb.

Conflicts of interest: Amgen funded
Subject Incidence Rates per 100-patient years

\begin{tabular}{|c|c|c|c|c|}
\hline & \multicolumn{2}{|c|}{$\begin{array}{l}\text { FREEDOM Extension } \\
\text { Yrs 1-6* }\end{array}$} & \multicolumn{2}{|c|}{ Database analysis } \\
\hline & $\begin{array}{l}\text { Crosss-over } \\
\quad \text { DMAb } \\
(N=2206) \\
\text { Rate }(\mathrm{n})\end{array}$ & $\begin{array}{c}\text { Long-term } \\
\quad \text { DMAb } \\
(N=2343) \\
\text { Rate }(\mathrm{n})\end{array}$ & $\begin{array}{l}\text { PMO cohort } \\
(N=3276) \\
\text { Rate }(\mathrm{n})\end{array}$ & $\begin{array}{l}\begin{array}{l}\text { General } \\
\quad \text { population } \\
(N=8569) \\
\text { Rate }(\mathrm{n})\end{array}\end{array}$ \\
\hline Malignancies & $2.0(206)$ & $2.1(226)$ & $1.88(270)$ & $1.96(661)$ \\
\hline Eczema & $0.9(93)$ & $0.9(102)$ & $0.05(7)$ & $0.06(23)$ \\
\hline Hypocalcemia & $<0.1(10)$ & $<0.1(5)$ & $0(0)$ & $0.02(7)$ \\
\hline Pancreatitis & $<0.1(3)$ & $<0.1(8)$ & $0.10(14)$ & $0.09(32)$ \\
\hline Cellulitis* & $<0.1(6)$ & $<0.1(9)$ & $0.06(8)$ & $0.07(24)$ \\
\hline Fatal AEs & $0.8(78)$ & $0.8(94)$ & \multicolumn{2}{|c|}{ Not recorded in databaes } \\
\hline ONJ & $<0.1(5)$ & $<0.1(5)$ & \multicolumn{2}{|c|}{$\begin{array}{l}\text { Not included in database } \\
\text { analyses }\end{array}$} \\
\hline $\mathrm{AFF}$ & $<0.1$ (1) & $<0.1$ (1) & \multicolumn{2}{|c|}{ Not recorded in databases } \\
\hline
\end{tabular}

${ }^{*}$ Papapoulos ECCEO 2015; ${ }^{\dagger}$ Only serious events of cellulitis summarised for FREEDOM Extension. AE, adverse event; AFF, atypical femoral fracture; DMAb, denosumab; $n$, number of events; ONJ, osteonecrosis of the jaw; yr, year

P252

BONE IMPAIRMENT IN PRIMARY HYPEROXALURIA (PH): AN ULTRASTRUCTURAL BONE ANALYSIS

J. Bacchetta ${ }^{1}$, D. Farlay ${ }^{2}$, P. Cochat ${ }^{1}$, G. Boivin ${ }^{2}$

${ }^{1}$ Service de Néphrologie, Rhumatologie et Dermatologie Pédiatriques, Centre de Référence des Maladies Rénales Rares, Hôpital Femme Mère Enfant, Bron, France, ${ }^{2}$ INSERM, UMR 1033, Université de Lyon, Lyon, France

Background: Deposition of calcium oxalate crystals in the kidney and bone is a hallmark of systemic oxalosis. Patients with PH1 (less frequently PH2) present severe impaired renal function, and thus systemic oxalosis. Since the bone compartment stores massive amounts of oxalate, patients present with recurrent low-trauma fractures, bone deformations, severe bone pains and specific oxalate osteopathy on plain X-ray. The threshold of glomerular filtration rate at which this occurs is debatable and might be as high as 30 to $45 \mathrm{~mL} / \mathrm{min}$ per $1.73 \mathrm{~m}^{2}$. The objective of the study is to present a single-centre experience of bone biopsy and bone ultrastructural analysis in oxalosis.

Methods: We present data obtained in 10 samples from 8 patients with oxalosis (16-68 years) who underwent iliac crest bone biopsy and bone quality analysis using modern methods (microradiography, microindentation, Fourier transform infrared microspectroscopy, transmission electron microscopy) in addition to histomorphometry.

Results: Disseminated calcium oxalate deposits (whewellite) were found in the bone marrow space (with a granulomatous reaction) but not in the bone matrix. Calcium oxalate deposits 
were totally surrounded by macrophages and multinucleated giant cells, and a phagocytosis activity was sometimes observed. Very few calcium oxalate crystals were directly in close contact with the mineral substance of bone. Bone mineralization was not modified by the presence of calcium oxalate even in close vicinity. Bone quality analysis also revealed a harder bone than normal, perhaps in relationship with decreased carbonate content in the mineral.

Discussion: This study reports for the first time in patients with oxalosis the specific location of calcium oxalate deposits (whewellite) in the bone marrow space (with a granulomatous reaction) and not in the bone matrix. Bone hardness is increased, that could explain a more "brittle" bone. The formation and growth of calcium oxalate crystals in bone is independent of apatite. Despite these novel observations, the exact mechanisms leading to nucleation and growth of oxalate deposits are still unclear and deserve further studies.

\section{P253}

MENISCAL EXTRUSION AND BONE MARROW LESIONS ARE INDEPENDENTLY ASSOCIATED WITH INCIDENT AND PROGRESSIVE KNEE OSTEOARTHRITIS

J. Martel-Pelletier $^{1}$, A. J. Teichtahl' ${ }^{2}$, F. M. Cicuttini ${ }^{3}$, F. Abram $^{4}$, Y. Wang ${ }^{3}$, J.-P. Pelletier ${ }^{1}$

${ }^{1}$ Osteoarthritis Research Unit, University of Montreal Hospital Research Centre (CRCHUM), Montreal, Canada, ${ }^{2}$ Department of Epidemiology and Preventive Medicine, School of Public Health and Preventive Medicine, Monash University, Alfred Hospital and Baker IDI Heart and Diabetes Institute, Melbourne, Australia, ${ }^{3}$ Department of Epidemiology and Preventive Medicine, School of Public Health and Preventive Medicine, Monash University, Alfred Hospital, Melbourne, Australia, ${ }^{4}$ Medical Imaging Research \& Development, ArthroLab Inc., Montreal, Canada

Objective: Whether meniscal extrusion and bone marrow lesions (BMLs) independently predict the risk of knee osteoarthritis (OA) remains to be clarified. Yet, while prospective cohort studies have shown that both meniscal extrusion and BMLs increase the risk of progressive knee OA, these studies have focused on the medial rather than the lateral knee joint, have examined people with established knee OA. It remains unclear whether these structural abnormalities predate incident knee OA, and did not distinguish whether meniscal extrusion or BMLs independently predict progressive disease.

Methods: Data was extracted from the Osteoarthritis Initiative (OAI) cohort. Participants were grouped according to the absence (Kellgren-Lawrence (KL) grade $\leq 1, n=2120$ ) or presence ( $\mathrm{KL} \geq 2, n=2249)$ of radiographic OA (ROA). Baseline meniscal extrusion, BMLs and cartilage volume were assessed on the magnetic resonance imaging (MRI). Cartilage volume of the tibial plateau was assessed at baseline and 72 months, while ROA was assessed at baseline and 48 months. Total knee replacement (TKR) was assessed at 72 months.

Results: In those with ROA, the presence of a baseline meniscal extrusion (independent of BMLs) was associated with accelerated cartilage loss (medial tibia: $-2.1 \% /$ annum vs. $-1.5 \%$; lateral tibia: $-2.6 \%$ annum vs. $-1.6 \%$; both $p<0.001$ ), progressive ROA and TKR (OR range 1.4-1.8; $95 \%$ CI range 1.1-2.9, $\mathrm{p} \leq 0.04$ ). The presence of a baseline BML (independent of meniscal extrusion) was associated with accelerated cartilage loss (medial tibia: $-2.1 \%$ /annum vs. $-1.6 \%$; lateral tibia: $-1.9 \%$ / annum vs. $-1.6 \% ; \mathrm{p} \leq 0.02$ ), progressive ROA and TKR (OR range $1.5-2.4 ; 95 \% \mathrm{CI}$ range $1.1-3.4, \mathrm{p} \leq 0.02$ ). In those with no ROA, a baseline medial meniscal extrusion was associated with accelerated cartilage loss (medial tibia: $-2.1 \% /$ annum vs. $-1.2 \%$, $p<0.001$ ) and a baseline medial BML with incident ROA (OR $1.7,95 \%$ CI $1.1-2.9, p=0.03$ ).

Conclusion: The presence of baseline meniscal extrusion and BMLs predict incident and progressive knee OA and represent important and independent structural targets for patients' selection in disease-modifying OA drugs (DMOAD) trials.

P254

IN A TWO-YEAR DOUBLE-BLIND RANDOMIZED CONTROLLED MULTICENTER STUDY, CHONDROITIN SULFATE WAS SIGNIFICANTLY SUPERIOR TO CELECOXIB AT REDUCING CARTILAGE LOSS WITH SIMILAR EFFICACY AT REDUCING DISEASE SYMPTOMS IN KNEE OSTEOARTHRITIS PATIENTS J.-P. Pelletierr $^{1}$, J.-P. Raynauld ${ }^{2}$, A. Beaulieu ${ }^{3}$, L. Bessette ${ }^{4}$, F. Morin $^{5}$, A. J. de Brum-Fernandes ${ }^{6}$, F. Abram ${ }^{7}$, M. Dorais ${ }^{8}$, J. Martel-Pelletier ${ }^{1}$

${ }^{1}$ Osteoarthritis Research Unit, University of Montreal Hospital Research Centre (CRCHUM), Montreal, Canada, ${ }^{2}$ Institut de rhumatologie de Montréal, Montreal, Canada, ${ }^{3}$ Centre de rhumatologie St-Louis, Sainte-Foy, Canada, ${ }^{4}$ Groupe de recherche en Rhumatologie et Maladies Osseuses Inc., SteFoy, Canada, ${ }^{5}$ Centre de recherche musculo-squelettique, Trois-Rivières, Canada, ${ }^{6}$ Rheumatology Division, Sherbrooke University Hospital, Sherbrooke, Canada, ${ }^{7}$ Medical Imaging Research \& Development, ArthroLab Inc., Montreal, Canada, ${ }^{8}$ StatSciences Inc., Notre-Dame de l'Île Perrot, Canada

Objective: Although chondroitin sulfate (CS) was found in a number of studies using X-rays to have a knee osteoarthritis (OA) structure modifying (DMOAD) effect, the question is still under debate. A study using quantitative MRI (qMRI) is thus important.

The present RCT has two main objectives: i) to explore, using qMRI, the DMOAD effect of CS vs celecoxib (CE) on 
cartilage volume loss (CVL) in knee OA, and ii) to compare the effects of the drugs on symptoms.

Methods: Symptomatic primary knee OA patients according to ACR criteria with KL grades 2-3 and synovitis were treated with CS (1200 mg/day) or CE (200 mg/day) for 24 months. Patients at high risk for cardiovascular and/or gastrointestinal disease were excluded. MRI was done at baseline, 12 and 24 months. CVL, bone marrow lesion (BML) size, and synovial membrane thickness (SmT) were evaluated using qMRI. Clinical symptoms were assessed by validated questionnaires. Analyses were done on the intention-to-treat (ITT) $(n=194)$, per protocol $(n=195)$, and according-to-protocol completer $(n=120)$ populations using Student's $t$-test, Wilcoxon Mann-Whitney test, and ANCOVA. Results: In the ITT population, OA patients treated with CS $(n=97)$ had a reduction in CVL at 12 months $(p=0.017)$ and 24 months in the medial tibiofemoral compartment $(p=0.013)$ and global knee at $12(p=0.034)$ and 24 months $(p=0.054)$ compared to CE $(n=97)$. No difference in change in SmT or BML size between the two groups was observed. A marked reduction in incidence of patients with joint swelling plus effusion was observed in both CS (51 \%) and CE (39 \%) groups over 24 months. Both groups had a reduction in VAS and WOMAC pain at 24 months: CS and CE showed $48 \%$ vs $55 \%$, and $43 \%$ vs $54 \%$ respectively. The daily consumption of rescue analgesic (acetaminophen) and incidence of adverse events was similar in both groups.

Conclusion: This is the first study demonstrating the superiority of CS over CE at reducing the long term progression of knee OA structural changes at the same time as effectively reducing OA symptoms. Both drugs were found equally effective at reducing symptoms. These findings have important implications regarding the usefulness of CS for long term management of knee OA.

\section{P255}

ONE-YEAR SURVIVAL AND HEALTHCARE UTILIZATION: IMPACT OF A GERIATRIC FRACTURE PROGRAM

L. McNicoll $^{1}$, N. Mujahid ${ }^{1}$, I. Rich ${ }^{2}$, J. F. G. Monteiro ${ }^{2}$

${ }^{1}$ Medicine, Alpert Medical School of Brown University, Providence, United States, ${ }^{2}$ Medicine, Lifespan, Providence, United States

Background: Hip fracture in older adults is common and is associated with increased morbidity as well as increased 1year mortality as high as $25-40 \%$. In 2011, we implemented a Geriatric Fracture Program (GFP): a co-management program between Geriatrics and Orthopaedics. In this study, we analyzed hip fracture patients 1-year after hospital discharge looking at mortality rates, Emergency Department (ED) visits, and hospital admissions for those admitted under GFP vs. usual care (UC) described as patients admitted under Internal Medicine service with orthopaedics consulting.

Methods: This retrospective study analyzed hip fracture patients 65 years or older admitted from January 2012 to January 2014 who survived the initial hospital stay. Demographic data and outcomes (mortality, ED visits, hospitalizations within the hospital system and respective causes) were collected through the Trauma Registry and chart review. We excluded patients where both geriatrics and internal medicine were consulted or neither, and those who died or have been transferred to hospice after the initial hospitalization.

Results: Of 313 surviving hip fracture patients, 197 were cared for by GFP and 116 by UC. Preliminary analysis shows 1 -year mortality at $0 \%$ for GFP compared to $6.0 \%$ for UC patients. Although the mean age of all patients was 86.2 , the GFP group was much older with $63.7 \%$ of them aged $>85$ years of age. ED visits within 1-year were lower in the GFP group at $16.8 \%$ compared to $20.4 \%$ for UC representing a $17.6 \%$ decrease (similar results for multiple ED visits $2.5 \%$ for GFP and 5.3\% for UC; a $53 \%$ decrease). For 1-year re-hospitalization rates, the GFP patients were $30 \%$ less likely to be hospitalized ( $34.5 \%$ for GFP vs. $49.6 \%$ for UC), with $15.2 \%$ of GFP having $>1$ hospitalization compared to $23.4 \%$ for UC; a $35 \%$ decrease. The most common causes of ED visits and hospitalizations were heart failure and infections.

Conclusions: Our GFP co-management program improved 1year survival compared to UC with no deaths compared to seven in UC. There were also substantially lower ED visits and hospitalizations among patients cared for under GFP. We need further research into co-management programs to better delineate the improvement in outcomes in older hip fracture patients.

P256

PROXIMAL FEMUR GEOMETRY AND BONE MASS IN TROCHANTERIC AND NECK HIP FRACTURES: A RETROSPECTIVE STUDY

M. T. Giamattei ${ }^{1}$, A. Moretti ${ }^{1}$, A. de Sire $^{1}$, F. Gimigliano ${ }^{2}$, G. Iolascon $^{1}$

${ }^{1}$ Department of Medical and Surgical Specialties and Dentistry, Second University of Naples, Naples, Italy, ${ }^{2}$ Department of Physical and Mental Health and Preventive Medicine, Second University of Naples, Naples, Italy

Objective: To evaluate the differences between postmenopausal women with previous medial or lateral hip fracture in terms of BMD and bone geometry.

Material and Methods: We included all consecutive postmenopausal women with a previous proximal femur fracture, aged more than 60 years, referring to our outpatient clinic from September 2015. We divided our population into 2 groups, according to the site of femoral fracture: medial or 
lateral. We performed a hip structural analysis (HSA) from contralateral hip DXA images to measure the following parameters: hip axis length (HAL), femoral strength index (FSI), cross-sectional moment of inertia (CSMI), cross-sectional area (CSA), section modulus (Z), and buckling ratio (BR).

Results: We evaluated 28 postmenopausal women, mean aged $75.57 \pm 6.99$ years, with a mean BMI of 25.49 $\pm 3.05 \mathrm{~kg} / \mathrm{m}^{2}$. Of these, 9 had a previous medial fracture (mean aged $74.67 \pm 6.61)$ and 19 had a previous lateral fracture (mean aged $76.00 \pm 7.30$ ). There were statistically significant differences between groups in lumbar spine BMD $(0.926$ $\pm 0.204 \mathrm{~g} / \mathrm{cm} 2$ vs. $1.107 \pm 0.171 ; p=0.010)$ and femoral neck $\operatorname{BMD}(0.652 \pm 0.066 \mathrm{~g} / \mathrm{cm} 2$ vs. $0.730 \pm 0.097 ; p=0.019)$. However, women with a previous medial hip fracture had a greater HAL than lateral hip fracture group $(109.06 \pm 9.58 \mathrm{vs.}$ $107.07 \pm 6.00 ; p=0.508)$, although there was not a statistically significant difference.

Conclusions: Our results showed that in our population of postmenopausal women the group with a previous lateral hip fracture had a worse BMD and a lower HAL than the group with a previous medial hip fracture.

\section{P257}

FACTORS AFFECTING BONE MINERAL DENSITY AMONG MALE PRIMARY PROGRESSIVE MULTIPLE SCLEROSIS PATIENTS

$\underline{\text { A. Calıskan }}^{1}$, Y. Gokce Kutsal ${ }^{1}$, P. Acar ${ }^{1}$, R. Karabudak ${ }^{1}$, F. Akbiylk $^{1}$, S. Karahan ${ }^{1}$

${ }^{1}$ Hacettepe University Faculty of Medicine, Ankara, Turkey

Objective: It is known that secondary osteoporosis is more common than primary osteoporosis in men. Therefore diagnosis is important to define the prognosis and to choose the appropriate treatment. Multiple sclerosis (MS) is an important of the cause of secondary osteoporosis. There's limited data in Turkey about the effect of MS on male osteoporosis. It's aimed to evaluate the effect of MS on BMD among male primary progressive MS (PPMS) patients excluding the factors such as chronic immobilization and oral glucocorticoid use. Also it's aimed to define the correlations between BMD and bone biomarkers for increasing the use in clinical practice because they can give information about bone metabolism earlier and it is a cheaper, repeatable way and does not have radiation risk.

Material and Methods: 26 male patients who are diagnosed as PPMS according to 2010 McDonald's Criteria for over a year and age matched male healthy volunteers between 25-65 years old are included in the study. Expanded Disability Status Scale (EDSS) scores of the patients, femoral and lumbar BMD measured by DXA, bone turnover markers, biochemical markers, sex hormone binding globulin (SHBG) were evaluated. Demographical data, tobacco and alcohol consumption, presence of any additional systemic diseases, medications especially prior use of steroids, pathological fractures and family histories of MS or osteoporosis were questioned.

Results: Demographical properties, tobacco and alcohol consumption were statistically similar in both groups. Mean age of the patient group were $42.6 \pm 8.9$ (22-60) years, mean disease duration were $3.4 \pm 1.5$ years, mean EDSS scores were $4.4 \pm 1.7$. 25 -hydroxyvitamin D levels were $17.8 \pm 9.2 \mathrm{ng} / \mathrm{ml}$ in patient group and $19.4 \pm 6.3 \mathrm{ng} / \mathrm{ml}$ in control group $(p>0.05)$. Femoral BMD $(p=0.01)$, femoral T-scores $(p=0.04)$, femoral $\mathrm{Z}$ scores $(p=0.05)$ were lower in patient group. There were not any significant correlation between SHBG levels, EDSS, BMD scores and biomarkers (CTX and osteocalcin) and other biochemical variables; but $p$ values were close to the significant value. BMD scores were lower in patient group than in control group.

Conclusion: Osteoporosis should be managed as part of MS patients' treatment protocols.

\section{P258}

INCIDENCE AND PERSISTENCE TO ANTIOSTEOPOROSIS TREATMENTS IN SPANISH PRIMARY CARE SETTINGS: A POPULATIONBASED COHORT STUDY

E. Martín-Merino $^{1}$, A. Álvarez-Gutierrez ${ }^{1}$, C. HuertaÁlvarez ${ }^{1}$, D. Prieto-Alhambra ${ }^{2}$, D. Montero-Corominas ${ }^{3}$

${ }^{1}$ BIFAP (Base de datos para la Investigación Farmacoepidemiológica en Atención Primaria) / AEMPS (Spanish Agency of Medicines and Medical Devices), Madrid, Spain, ${ }^{2}$ MRC Lifecourse Epidemiology Unit, University of Southampton / Oxford NIHR Musculoskeletal Biomedical Research Unit, University of Oxford, Southampton, United Kingdom, ${ }^{3}$ AEMPS (Spanish Agency of Medicines and Medical Devices), Madrid, Spain

Objective: A lack of persistence to antiosteoporosis drugs $(\mathrm{AOM})$ has been reported. In addition, AOM treatments may be compromised by different recent safety alerts. We aimed to estimate the incidence and persistence of AOM use in Spanish primary care.

Methods: A cohort study was performed using anonymized electronic primary care records for 4 million people (BIFAP database). Participants entered the study when aged $\geq 50$ years in 2001-2013 and with $\geq 1$ year of data available. Users of AOM in the previous year were excluded. To identify AOM new users, participants were followed from cohort entry to the earliest of: AOM initiation, or censoring (lost to follow-up, death, or end of 2013). Six cohorts were identified according to first prescribed AOM: alendronate, other bisphosphonates, SERM, strontium ranelate, teriparatide, and denosumab. For the study of persistence, AOM users were followed from initiation to cessation (90 days refill gap), switching, or censoring. Incidence rate (IR) of AOM users per 1,000 person-y 
(py)) was computed dividing number of AOM users by py of follow-up, overall and stratified by sex, assuming Poisson distribution. First-year cessation proportion was estimated using life tables.

Results: Overall, 95,057 new AOM users were identified, resulting in an IR of $14.27 / 1,000$ py (24.90 in women, 2.77 in men). Drug-specific IRs/1,000 py were: 9.35 (women) and 1.21 (men) for alendronate, 9.75 and 1.21 for other bisphosphonates, 3.36 and 0.03 for SERM, 2.08 and 0.25 for strontium, 0.30 and 0.08 for teriparatide, and 0.08 and 0.01 for denosumab. First-year cessation were $51 \%$ overall, highest for strontium (68\%), and lowest for denosumab (28\%).

Conclusions: Bisphosphonates were the most frequently prescribed drugs, followed by SERM in women and strontium in men. Half of the patients initiating AOM therapy ceased treatment during the first year, with strontium being the most commonly stopped drug. Close monitoring of new users of AOM may be needed.

\section{P259}

RISK OF VENOUS THROMBOEMBOLISM AMONGST USERS OF DIFFERENT ANTIOSTEOPOROSIS DRUGS: A MULTINATIONAL POPULATION-BASED COHORT STUDY

$\underline{\text { E. Martín-Merino }}^{1}$, I. Petersen ${ }^{2}$, S. Hawley ${ }^{3}$, A. ÁlvarezGutierrez $^{1}$, A. Delmestri ${ }^{3}$, A. Llorente-Garcia ${ }^{1}$, M. K.

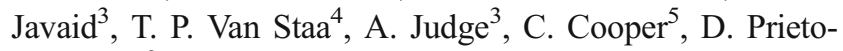
Alhambra $^{3}$

${ }^{1}$ BIFAP (Base de datos para la Investigación Farmacoepidemiológica en Atención Primaria) / AEMPS (Spanish Agency of Medicines and Medical Devices), Madrid, Spain, ${ }^{2}$ Primary Care and Population Health / University College London, London, United Kingdom, ${ }^{3}$ Nuffield Department of Orthopaedics, Rheumatology and Musculoskeletal Sciences, University of Oxford, Oxford, United Kingdom, ${ }^{4}$ Health eResearch Centre, University of Manchester, Manchester, United Kingdom, ${ }^{5} \mathrm{MRC}$ Lifecourse Epidemiology Unit, University of Southampton; MRC Human Nutrition Research, Elsie Widdowson Laboratory Oxford NIHR Musculoskeletal Biomedical Research Unit, University of Oxford, Southampton, United Kingdom

Objective: Most adverse drug reactions for anti-osteoporosis medicines (AO) users have been described comparing AO users to AO-naïve patients. We aimed to compare the risk of venous thromboembolism (VTE) amongst incident users of different AO -and in particular Strontium Ranelate (SR)- in the UK (CPRD) and Spain (SIDIAP and BIFAP).

Methods: Three cohort studies were performed separately using primary care records data from CPRD, SIDIAP and
BIFAP. All patients aged $\geq 50$ years, $>1$ year of data available and a new AO prescription (therapy initiation) in 2001-2013 (BIFAP), 2007-2014 (SIDIAP), or 2000-2014 (CPRD) were included. AO included: 1.alendronate (AL), 2.other (oral) bisphosphonates (OB), 3.SR, 4.denosumab (DE), or 5.teriparatide (TE). SERM users were excluded since VTE was a contraindication, as were TE and DE users in CPRD due to low numbers. Patients were followed to the earliest of: VTE (diagnosis combined with anticoagulant use), AO cessation, switching, drop-out, death, or end of study. Incidence rate (IR) of VTE and Hazard ratios (HR) for VTE were estimated for each cohort vs. AL adjusted for NICE risk factors for VTE. Results: Overall, 2,035/159,209 (1.28\%) in CPRD, 386/148, $564(0.26 \%)$ in SIDIAP, and 401/83,334 (0.48 \%) in BIFAP had VTE. Crude IR per 1,000 py were 4.84, 2.47 and 2.36 for AL; 5.08, 2.43 and 2.21 for OB; and 5.06, 1.60 and 2.89 for SR in CPRD, SIDIAP and BIFAP respectively; 5.08 and 5.16 for DE; and 2.38 and 4.67 for TE in SIDIAP and BIFAP. Adjusted HRs of VTE were 1.05 (0.94-1.18), 1.48 (1.17$1.85)$ and $0.96(0.78-1.18)$ for $\mathrm{OB}$; and 0.90 (0.61-1.34), $1.12(0.67-1.88)$ and $1.19(0.82-1.74)$ for SR in CPRD, SIDIAP and BIFAP respectively; and $1.11(0.55-2.23)$ and $1.77(0.25-12.66)$ for DE; and $1.00(0.37-2.72)$ and 1.27 (0.59-2.71) for TE in SIDIAP and BIFAP.

Conclusions: VTE risk during AO therapy did not differ by drugs, except OB showing higher risk in SIDIAP only. Our data does not support an increased risk of VTE associated with $\mathrm{SR}$ in the UK or Spain.

\section{P260}

\section{CHANGES OF FIBROBLAST GROWTH FACTOR'S BLOOD CONCENTRATION IN DIFFERENT CLINICAL COURSE OF RHEU- MATOID ARTHRITIS}

$\underline{\text { B. A. Rebrov }}^{1}$, E. B. Komarova ${ }^{1}$, A. B. Blagodarenko ${ }^{1}$, I. I. Pokryshka $^{2}$

${ }^{1}$ SI "Lugansk State Medical University", Lugansk, Ukraine, ${ }^{2}$ Lugansk Regional Clinical Hospital, Lugansk, Ukraine

Background: Fibroblast growth factor (FGF) stimulates angiogenesis, proliferation of fibroblasts and endothelial dysfunction. It contributes to suppression of apoptosis reactions of fibroblast-like synoviocytes. This may stimulate hyperplasia of the synovial membrane, pannus growth and cartilagebone destruction in rheumatoid arthritis (RA).

Objectives: To specify the particularize of the FGF blood levels changes, depending on the clinical course of RA.

Methods: Comprehensive clinical and laboratory examination of 194 patients diagnosed with RA (verified according to the criteria of ACR/EULAR 2010): the average age was $47.7 \pm 10.22$ years, mean duration of RA $3.82 \pm 3.43$ years. The control group (CG) consisted of 36 healthy people aged 
$46.8 \pm 7.8$ years. IFA determined the concentration of CRP and TNF- $\alpha$ (Vector-Best, Russia), anti-CCP (Euroimmun, Germany), FGF (BCM Diagnostic, Canada).

Results: The concentration of FGF in all RA patients was 2.5 times higher than in the CG $(p<0,001)$, ANOVA analysis of variance showed that the duration of the RA affects the blood level of FGF $(\mathrm{KW}=21.68, p<0.001)$ with increasing duration of RA FGF blood level increases. In patients with duration of $\mathrm{RA}>5$ years, the FGF was 2-fold higher than in patients with RA duration $<2$ years $(p<0.001)$. Patients with high DAS28 index showed increase in the FGF blood concentration $(p<0.05)$ as compared to low or moderate RA activity. Patients with anti-CCP levels $>40 \mathrm{IU} / \mathrm{ml}$ showed $25 \%$ increase of FGF level as compared to patients with low levels of anti-CCP $(p<0.05)$. ANOVA analysis of variance found that the antiCCP level and the RA activity degree by DAS28 affected the FGF blood level in the examined RA patients $(\mathrm{KW}=4.95$, $p=0.03 ; \mathrm{KW}=8.88, p=0.003$, respectively).

Conclusions: High FGF blood level is defined in the patients with the long RA history, high anti-CCP blood level and high disease activity. Therefore, the FGF blood level upgrowth can serve as a marker of rapid progression of cartilage-bone destruction in these patients.

\section{P261}

INFLUENCE OF SPIRONOLACTONE ON INFLAMMATORY PROCESS MARKERS IN PATIENTS WITH RHEUMATOID ARTHRITIS

E. B. Komarova $^{1}$, B. A. Rebrov ${ }^{1}$, G. A. Belkina ${ }^{1}$, I. I. Pokrushka $^{2}$, R. K. Beshimova ${ }^{2}$

${ }^{1}$ SI "Lugansk State Medical University", Lugansk, Ukraine,

${ }^{2}$ Lugansk Regional Clinical Hospital, Lugansk, Ukraine

Aldosterone blocker - spironolactone has anti-inflammatory, anti-proliferative and anti-oxidative effects (early suppressing effect on some immunoactive and pro-inflammatory cytokines and stimulates effects of apoptosis of different cells, reduced oxidative stress, normalized hypertrophic reconstruction reducing collagen and fibronectin), that is why pathogenetically it is expedient to use it in complex therapy of rheumatoid arthritis (RA).

Objectives: To study influence of spironolactone on inflammatory process markers in patients with RA.

Methods: 93 patients with RA (ACR/EULAR, 2010 criteria) were examined (ESR, CRP, DAS 28 and TNF- $\alpha$ ) and were divided into two groups with the technique of random sampling: I - 47 patients got standard RA therapy according the protocols of rheumatoid diseases treatment, II - 46 patients got complex treatment during 12 months which included standard therapy with adding spironolactone in $25-50 \mathrm{mg}$ per day. Initial dose was $50 \mathrm{mg} /$ day during 2 weeks, and then the dose was corrected depending on the potassium content in blood serum. The patients of both group I and group II statistically did not different in all the characteristics studied before the treatment. Among the patients there were 11 males and 81 females with average age $42.82 \pm 10.2$ years, average duration of the disease is $3.82 \pm 3.43$ years.

Results and their discussions: Level of CRP under the influence of treatment authentically decreased in each of the studied groups concerning an index in this group to treatment, with identical degree of reliability of differences in I $(\mathrm{W}=4.1, \mathrm{p}<0.001)$ and group II $(\mathrm{W}=4.37, \mathrm{p}<0.001)$, led to decreased in ESR in each of the studied groups (both $\mathrm{p}<0.001$ ). In patients of both groups, highly reliable (both $\mathrm{p}<0.001)$ decrease the DAS28 was established. After treatment CRP, ESR, TNF $\alpha$ and DAS28 authentically differed in patients who received complex treatment from those patients who received standard therapy: (according to $Z=3.29$; $\mathrm{Z}=3.15 ; \mathrm{Z}=3.28 ; \mathrm{Z}=2.8$, in total $\mathrm{p}<0.01$ ). The use of spironolactone in complex therapy made reduced DAS28 more than $>0.6$, that according to treatment efficacy estimation EULAR is considered satisfactory.

Conclusions: including of spironolactone into complex RA therapy leads to improvement of inflammatory process markers: contributes to reduce CRP by 1.5 times, ESR by 2 times and increase of blood TNF- $\alpha$ by $17 \%$ comparing to standard therapy; reduces of DAS 28 by $13 \%$ more significant than complex treatment.

\section{P262}

EFFECTIVE TERIPARATIDE TREATMENT OF HYPOPARATHYROIDISM NOT COMPENSATED ON HIGH DOSES OF CALCIUM AND ALFACALCIDOL DUE TO ACQUIRED COLON PSEUDOMELANOSIS

J. A. Krupinova $^{1}$, E. A. Pigarova ${ }^{1}$, L. Y. Rozhinskaya ${ }^{1}$, Z. E. Belaya $^{1}$

${ }^{1}$ Neuroendocrinology and Bone Diseases, Moscow, Russian Federation

Objective(s): Hypoparathyroidism (HypoP) is a common complication after thyroidectomy. In some cases, its effective compensation is challenging.

Material and Methods: A 47-yo female was admitted to our hospital due to uncompensated HypoP after subtotal thyroidectomy for diffuse toxic goiter performed 2 years ago. Since this surgery, she was experiencing 1-2 tetany attacks per day despite taking $3 \mu \mathrm{g}$ of alfacalcidol (Alf) and $5 \mathrm{~g}$ of calcium carbonate $(\mathrm{CaC})$. High severity of these attacks made iv access hard to perform and required priming with im diazepam or im injections of 20-30 $\mathrm{ml} 10 \%$ calcium gluconate (CaGlu). Nevertheless, regular iv injection of CaGlu had a temporary effect.

Results: At admission the patient presented with tetanic convulsions of the whole body (Ca ion level was $0.71 \mathrm{mmol} / \mathrm{l}$ 
(range 1.03-1.30)) that required the abovementioned priming measures and iv administration of $40 \mathrm{ml}$ bolus followed by slow infusion of $120 \mathrm{ml} 10 \%$ CaGlu. The daily dose of Alf had been gradually increased to $6 \mu \mathrm{g}$ and $\mathrm{CaC}$ to $4 \mathrm{~g}$ per day, but tetany attacks still occurred every day (Ca ion $0.75-0.96$ ). Her colonoscopy identified a colon pseudomelanosis as a side effect of laxatives used to overcome spastic and $\mathrm{CaC}$ induced constipation. Given that the conventional therapy of HypoP was proved ineffective, it was decided to give the patient $20 \mu \mathrm{g}$ of teriparatide twice a day. This reduced the required daily dose of Alf down to $3 \mu \mathrm{g}$, and $\mathrm{CaC}$ to $2 \mathrm{~g}$. During the teriparatide treatment, the patient greatly improved clinically: no tetany attacks; Chvostek and Trousseau signs completely disappeared with the induction of mild hypercalcemia (Ca ion - 1.42) due to bone metabolism activation that required teriparatide dose reduction down to $20 \mu \mathrm{g}$ a day.

Conclusion: In case of severe HypoP with compromised gut $\mathrm{Ca}$ absorption treatment with teriparatide showed to be a promising option for restoration of $\mathrm{Ca}$ levels and clinical well being.

\section{P263}

ARTERIOVENOUS MALFORMATIONS PRESENTING WITH BACK PAIN AND LEG WEAKNESS

A.-K. Kul $^{1}$, F.-B. Bagcier ${ }^{1}$, A.-K. Karaman ${ }^{2}$

${ }^{1}$ Physical Therapy and Rehabilitation, Atatürk University

Faculty of Medicine, Erzurum, Turkey, ${ }^{2}$ Radiology, Ataturk University Faculty of Medicine, Erzurum, Turkey

50-year-old male presented with progressive paraparesis together with low back pain. There was no neuropathic pain. He had no symptoms suggestive of inflammatory back pain. Patient's previous medical history was insignificant. There was no history of trauma, chronic disease, alcohol and substance abuse. Physical examination revealed wasting of the lower extremities, with weakness involving extension(3/5) and flexion(3/5) of the bilateral ankle. Deep tendon reflexes were normoactive. Neurological examination revealed a negative straight leg-raising test and femoral nerve stretch test on the right and left side. A myelogram revealed serpiginous filling defects compatible with a midline dilated vessel extending from L1 to L5 (Fig. 1). Spinal arteriovenous malformation (AVM) was diagnosed at the L4 vertebral level. (Fig. 2). Embolization of the AVM was performed with $0.2 \mathrm{ml}$ of bucrylate diluted with an equal amount of Pantopaque contrast medium injected through a 3-French polyethylene catheter under fluoroscopic control. The malformation was successfully occluded (Fig. 3) There was progressive improvement in the patient's ambulation up to 6 months postembolization. Spinal epidural AVMs were first discussed in a 1914 case report by Dr. Charles Elsberg, who described a patient with severe spastic paraparesis with a T9 sensory level ${ }^{1}$. Spinal AMVs are divided angiographically into retromedullary and intramedullary lesions supplied by posterior and anterior spinal arteries, respectively. Retromedullary malformations typically present as progressive neurological deficits in elderly men, while intramedullary lesions are characteristically seen in young patients who often have subarachnoid hemorrhage. On MRI, the AVM type cannot always be differentiated. The typical appearance of spinal cord AVMs is a conglomerate of dilated, peri- and intra-medullary located vessels that are demonstrated on T2-weighted sequences as flow voids, whilst they appear on T1-weighted sequences depending on their flow velocity and direction as mixed hyper- or hypo-intense tubular structures. Contrast enhancement may vary ${ }^{2}$. The therapeutic approach of asymptomatic AVM is difficult since data concerning the spontaneous prognosis are not available; however, in symptomatic AVMs, therapy ameliorates the prognosis of the patient. The therapy of choice for all spinal cord AVMs is the endovascular embolisation with coils, glue or particles after careful analysis of the selective spinal angiography with the embolising agent being dependent on the specific angioarchitecture ${ }^{3}$.

References:

1. Elsberg CA. Ann Surg 1914;60:454.

2. Grunwald I, et al. Radiologe 2001;41:961.

3. Van Dijk JM, et al. Stroke 2002;33:1578.

\section{P264}

INFLUENCE OF GLUCOSE-LOWERING STRATEGIES ON FRACTURE RISK IN TYPE 2 DIABETES

A. A. Gusova $^{1}$, M. G. Pavlova ${ }^{2}$, G. A. Melnitchenko ${ }^{2}$

${ }^{1}$ Outpatient Department, Scientific Research Institute of Neurosurgery named after N.N. Burdenko, Moscow, Russian Federation, ${ }^{2}$ Endocrinology Department, I.M. Sechenov First Moscow State Medical University, Moscow, Russian Federation

Objective: To examine an impact of diabetes therapy regimens on risk of any fracture in type 2 diabetic (T2DM) population.

Material and Methods: We analyzed local trauma clinic data for 3 sequential years and compared it with data from The National Register of diabetes mellitus patients.

Results: In The National Register of diabetes mellitus patients there were 1995 patients with T2DM, 72 of them developed fractures in observation period. Most common therapy was insulin secretagogues (IS group, $48.2 \%$ ) followed by insulinotherapy (IT) and metformin (M), few people received thiazolidinediones. The youngest group was M, and the eldest - IS and ND groups. Diabetes duration was the longest in IT group followed by IS. The level of $\mathrm{HbAlc}$ and rate of diabetic microangiopathy was the highest in IT group. There was $1.8 \%$ of patients who did not receive no anti-diabetic drugs (ND group).

There were registered 80 fractures in 72 persons. Most fractures developed in ND group, in this group was also registered highest rate of diabetic complications other than microangiopathy. 
Multiply fracture were observed only in IT and IS groups. In IT group incidence of fractures was significantly higher than in $\mathrm{M}$ group ( $p=0,036)$. Patients in IT grope were prone to hip fractures (compared to IS group, $\mathrm{p}=0,061$ ).

Conclusion: In our study higher incidence of fracture in "NoDrugs" group was observed, but only the trend to it in insulinotherapy group. We believe that it was explained rather with higher rate of diabetic complications in this groups than with the influence of therapy itself.

\section{P265}

MOLECULAR INVESTIGATIONS OF BONE QUALITY FROM OSTEOPOROTIC WOMEN TREATED WITH ALENDRONATE OR STRONTIUM RANELATE AFTER 12 MONTHS

G. Falgayrac $^{1}$, B. Cortet $^{2}$, C. Olejnik ${ }^{1}$, G. Penel ${ }^{1}$

${ }^{1}$ PMOI EA4490, Faculty of Dentistry, Lille, France,

${ }^{2}$ Rheumatology Department, Roger Salengro Hospital, Lille, France

Objective: This study compares the effects of alendronate (ALN) and strontium ranelate (SrRan) on bone quality at molecular level for 12 months.

Material and methods: Paired iliac crest biopsies were harvested on postmenopausal osteoporotic women (50-84 years) at baseline (M0, $n=10)$ and after 12 months (M12, $n=10)$ for each treatment (ALN/SrRan). This study was a sub- study from a larger double blind study which compared the effect of ALN and SrRan on bone by histomorphometry. New bone, old bone and Sr were located by Scanning Electron Microscopy. Raman microspectroscopy was done on new and old bone. The bone quality was assessed from Raman spectra through the parameters: mineralization ratio, type-B carbonization, crystallinity, collagen quality (hydroxyproline/proline and crosslinks ratio) and relative non-collagenous content (PG/AmideIII). Multivariate analyses (MVA) were used to find specific Raman bands due to ALN and SrRan treatments. The results were interpreted as function of the tissue age, the treatment and the nature of bone (cortical (ct)/trabecular (tb)).

Results: As the tissue age increases, the mineralization, the type-B carbonization and the crystallinity increases in ct and tb bone. MVA revealed organic bands characteristics of new bone and mineral bands characteristics of old bone. As function of treatment, the mineralization increased $(+9.3 \%$, $p=0.0026)$ in tb and PG/Amide III increased $(+8.5 \%$, $p=0.0030)$ in ct and tb between ALN-M0 and ALN-M12 in new bone. The type-B carbonization $(-9.6 \%, p<0.0001)$ and the crystallinity $(-1.8 \%, p=0.017)$ decreased in ct and tb new bone between SrRan-M0 and SrRan-M12. The PG/AmideIII $(-8.3 \%, p=0.0047)$ decreased in tb new bone between SrRan-M0 and SrRan-M12. No difference was observed between M0 and M12 in old bone for both drugs.
Conclusions: ALN suppresses the bone remodeling and enhances the secondary mineralization process which explains the increase of mineralization. A different mechanism may occur at molecular level for SrRan, which could explain the decrease of carbonate and crystallinity. Both drugs may have an opposite effect on the mineralization mechanism of collagen matrix according to PG/AmideIII. These findings indicate that the effects of ALN and SrRan at molecular level are different. Acknowledgement: The authors acknowledge SERVIER Company for their financial support.

\section{P266}

COMPARISON OF QUALITY OF LIFE OUTCOMES BETWEEN BILATERAL SIMULTANEOUS AND BILATERAL STAGED TOTAL KNEE REPLACEMENTS 2 YEARS POST OPERATION

A. A. Sayampanathan ${ }^{1}$, H. C. Chong ${ }^{2}$, S. J. $\mathrm{Yeo}^{2}$, S. L. Chia ${ }^{2}$, D. K. J. Tay ${ }^{2}$, H. N. Pang ${ }^{2}$, N. N. Lo ${ }^{2}$

${ }^{1}$ Yong Loo Lin School of Medicine, National University of Singapore, Singapore, Singapore, ${ }^{2}$ Department of Orthopaedic Surgery, Singapore General Hospital, Singapore, Singapore

Introduction: The aim of this study was to compare the difference in quality of life (QoL) outcomes of individuals who undergo bilateral staged and bilateral simultaneous TKRs.

Method: We prospectively followed up 300 patients who underwent bilateral TKRs over a period of two years. Of these, $150(50.0 \%)$ underwent bilateral staged TKRs within 6 months and 150 (50.0\%) underwent bilateral simultaneous TKRs. Demographic information, preoperative mean Range of Movement (ROM), Knee Society Score (KSS) (Function Score), KSS (Knee Score), Oxford Knee Score (OKS) and SF36 scores were matched for staged and simultaneous groups. SF-36 and patient satisfaction scores were assessed at 6 months and 2 years post-operation. Statistical analysis was performed using SPSS Version 21.0.

Results: 6 months postoperatively, no significant difference was noted in SF-36 scores for both the staged and simultaneous groups. Similarly, 2 years postoperatively, there was no significant difference in SF-36 scores between the staged and simultaneous groups. There was also no significant difference in patient satisfaction scores 6 months post-operatively between both groups $(p=0.086)$. However, patients who underwent simultaneous TKRs mentioned higher patient satisfaction scores 2 years post-operatively ( $p=0.016$ ).

Conclusion: There was no significant difference in QoL outcomes between patients who underwent bilateral staged or simultaneous TKRs for bilateral osteoarthritis 2 years postoperatively. Nevertheless, patients who underwent bilateral simultaneous TKRs were more satisfied with the outcomes from their operation 2 years post-operatively. 


\section{P267}

\section{COMPARISON OF FUNCTIONAL OUTCOMES} BETWEEN BILATERAL SIMULTANEOUS AND BILATERAL STAGED TOTAL KNEE REPLACEMENTS 2 YEARS POST OPERATION

A. A. Sayampanathan ${ }^{1}$, H. C. Chong ${ }^{2}$, S. J. $\mathrm{Yeo}^{2}$, S. L. Chia ${ }^{2}$, D. K. J. Tay ${ }^{2}$, H. N. Pang ${ }^{2}$, N. N. Lo $^{2}$

${ }^{1}$ Yong Loo Lin School of Medicine, National University of Singapore, Singapore, Singapore, ${ }^{2}$ Department of Orthopaedic Surgery, Singapore General Hospital, Singapore, Singapore

Introduction: To compare the difference in functional outcomes of individuals who undergo bilateral staged and bilateral simultaneous TKRs.

Methodology: We prospectively followed up 315 patients who underwent bilateral TKRs over a period of two years. Of these, $150(47.6 \%)$ underwent bilateral staged TKRs within 6 months and 165 (52.4\%) underwent bilateral simultaneous TKRs. Demographic data, preoperative mean range of movement (ROM), Knee Society Score (KSS) (Function Score), KSS (Knee Score) and Oxford Knee Score (OKS) were matched for staged and simultaneous groups. Functional scores were reassessed 6 months and 2 years post-operation. Statistical analysis was performed with SPSS Version 21.0.

Results: 6 months postoperatively, patients in the simultaneous group did better than patients in the staged group based on KSS (Function Score) (76.4 (SD: 16.8) vs. 64.8 (SD: 18.4) $(p<0.001))$ and OKS (18.6 (SD: 5.12) vs. 19.6 (SD: 4.95) $(p=0.01))$. There was no significant difference in KSS (Knee Score) (85.1 (SD: 11.3) vs. 85.3 (SD: 9.72) $(p=0.782)$ ). Similarly, 2 years postoperatively, patients in the simultaneous group did better than patients in the staged group based on KSS (Function Score) (81.7 (SD: 17.2) vs. 76.4 (SD: 17.5) $(p<0.001))$ and OKS (17.1 (SD: 4.79) vs. 18.0 (SD: 4.32$)(p=0.017))$. There was no significant difference in KSS (Knee Score) (85.8 (SD: 12.1) vs. 85.7 (SD: 11.2) $(p=0.942)$.

Conclusion: Bilateral simultaneous TKRs results in better functional outcomes 2 years postoperatively compared to patients who undergo bilateral staged TKRs within 6 months of each operation.

\section{P268}

PREVALENCE OF LOW BMD AMONG PATIENTS WITH NEWLY DIAGNOSED RHEUMATOID ARTHRITIS

$\underline{\text { S. Arain }}^{1}$, A. Riaz ${ }^{1}$, L. Nazir ${ }^{1}$, T. Perveen Umer ${ }^{1}$

${ }^{1}$ Liaquat National Hospital and Medical College, Karachi, Pakistan
Objective: Reduced BMD is one of the most common complications seen in patients with rheumatoid arthritis (RA) and dramatically affects quality of life. The aim of this study was twofold; to quantify the magnitude of low BMD among patients with newly diagnosed RA and to compare the BMD in newly diagnosed patients with seronegative and seropositive RA.

Method: The cross-sectional study was conducted at the Rheumatology Department of a tertiary care hospital in Karachi from July, 2013 to January, 2015. The data was prospectively collected from all patients with age $>15$ years presented with seronegative or seropositive RA with $<6$ months duration were included whose BMD of lumbar spine and hip was measured using DXA scan. Variables like age, gender, BMI, menstrual status, duration of disease, erythrocyte sedimentation rate, vitamin D level, CDAI and seropositivity were measured along with outcome variables. SPSS 21 was used for statistical analysis.

Results: Of the 76 patients in this study, 68 (89.50\%) were female, with mean age of the patients with low BMD was $50.95 \pm 7.87$ years while mean age in normal patients was $39.04 \pm 10.33$ years (p-value $<0.001) .19(25 \%)$ patients had low BMD, $68.52 \%$ had low BMD at spine, while $10.52 \%$ at hip and 21.05 at both spine and hip. Low BMD was found higher in patients with seronegative $7(50 \%)$ as compared to seropositive patients $12(19.4 \%)$ (p-value 0.017$)$, whereas low BMD was found higher $12(70.6 \%)$ among patients with postmenopausal status as compared to the patients with premenopausal status $5(9.8 \%)$ (p-value $<0.001)$.

Conclusion: Low BMD was found in $25 \%$ patients at earlier stage of the RA with seropositivity, age and menopausal status as significant risk factors.

\section{P269}

DIAGNOSTIC ACCURACY OF ACHILLES TENDON PATHOLOGY BY ULTRASONOGRAPHY AND PLAIN X-RAY

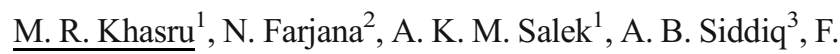
Haseen $^{4}$, M. S. A. $\operatorname{Azad}^{5}$, N. Anwar ${ }^{6}$, M. A. Karim ${ }^{7}$, S. M. Rahman $^{5}$

${ }^{1}$ Physical Medicine and Rehabilitation, Faculty of Medicine, Bangabandhu Sheikh Mujib Medical University, Dhaka, Bangladesh, ${ }^{2}$ Radiology and Imaging, Bangabandhu Sheikh Muijb Medical University, Dhaka, Bangladesh, ${ }^{3}$ Physical Medicine and Rehabilitation, Feni Diabetic Hospital, Feni, Bangladesh, ${ }^{4}$ Public Health and Informatics, Bangabandhu Sheikh Mujib Medical University, Dhaka, Bangladesh, ${ }^{5}$ Radiology and Imaging, Bangabandhu Sheikh Mujib Medical University, Dhaka, Bangladesh, ${ }^{6}$ Neurology, Bangabandhu Sheikh Mujib Medical University, Dhaka, Bangladesh, ${ }^{7}$ Radiology and Imaging, Bangabndhu Sheikh Mujib Medical University, Dhaka, Bangladesh 
Background: Objective of this study is to establish diagnostic usefulness of Ultrasonography in Achilles tendon pathology.

Methods: This cross sectional study was carried out on purposively selected 61 patients having clinical suspicion of Achilles tendon pathology, attended in the Department of Physical Medicine \& Rehabilitation, Bangabandhu Sheikh Mujib Medical University, Dhaka. All cases were evaluated by history, clinical examination, X-ray and ultrasonography. Four were refused to do USG, and no X-ray reports were available from 7 patients and thus 50 respondents are considered as study sample.

Results: Among all respondent maximum $38.0 \%$ were belonged to $36-45$ years age group, $68.0 \%$ were male, maximum $(32.0 \%)$ were service holder, $76.0 \%$ had been suffering from present illness up to 1 year and $26.0 \%$ had systemic diseases of them $76.9 \%$ had DM, $7.7 \%$ had dyslipidaemia, $7.7 \%$ had SLE, and $7.7 \%$ had AS. Among all responders, $62.0 \%$ Achilles tendinitis, $14.0 \%$ rupture, $6.0 \%$ retrocalcaneal bursitis, $2.0 \%$ tendonxanthoma and $16.0 \%$ others were diagnosed sonographically. Sensitivity of USG to diagnose Achilles tendon pathology was $95.7 .0 \%$, specificity $50.0 \%$, positive predictive value $95.7 \%$, negative predictive value $50.0 \%$ and accuracy $92.0 \%$. Sensitivity of USG to diagnose Achilles tendonitis was $86.7 \%$, specificity $75.0 \%$, positive predictive value $83.9 \%$, negative predictive value $78.9 \%$ and accuracy $82.0 \%$. Sensitivity of USG to diagnose Achilles tendon rupture was $87.5 \%$ specificity $100 \%$ positive predictive value $100 \%$ negative predictive value $97.7 \%$ and accuracy $98 \%$. Sensitivity of USG to diagnose retrocalcaneal bursitis was $50.0 \%$, specificity $100.0 \%$, positive predictive value $100.0 \%$, negative predictive value $93.6 \%$ and accuracy $94.0 \%$. Sensitivity of X-ray to diagnose Achilles tendon pathology was $39.0 \%$, specificity $75.0 \%$, positive predictive value $94.7 \%$, negative predictive value $9.7 \%$ and accuracy $42.0 \%$. Diagnostic accuracy was determined as receiver operating characteristic (ROC) curve, suggesting that the area under the curve (AUC) of USG and X-ray was 0.728 and 0.571 , respectively.

Limitation: Clinical diagnosis itself is not a gold standard, for that reason using clinical diagnosis as a test of reference we mere compare the validity tests of USG and X-ray for the diagnosis of Achilles tendon pathology.

Conclusion: MRI could be added as an additional imaging modality in solvent patients and comparing its diagnostic accuracy with clinical findings and ultrasonography and further research work could be done on individual pathology of Achilles tendon using large sample in the same procedure.

\section{P270}

DENOSUMAB REDUCES BACK PAIN RELATED DISABILITY AND IMPROVES HEALTH RELATED QUALITY OF LIFE IN WOMEN WITH VERTEBRAL FRAGILITY FRACTURES: A PROSPECTIVE COHORT STUDY

$\underline{\text { G. Iolascon }^{1}}{ }^{\text {, A. Moretti }}{ }^{1}$, A. de Sire ${ }^{1}$, G. Di Pietro ${ }^{1}$, F. Gimigliano $^{2}$

${ }^{1}$ Department of Medical and Surgical Specialties and Dentistry, Second University of Naples, Naples, Italy, ${ }^{2}$ Department of Physical and Mental Health and Preventive Medicine, Second University of Naples, Naples, Italy

Objective: To evaluate the effectiveness of denosumab in reducing back pain related disability and improving Health Related Quality of Life (HRQoL) in women with postmenopausal osteoporosis and vertebral fractures.

Material and Methods: We included women aged $\geq 50$ years with a diagnosis of postmenopausal osteoporosis that had already experienced at least one vertebral fragility fracture. They were treated with subcutaneous denosumab (60 mg/every 6 months) and a supplementation of calcium carbonate $(500-1000 \mathrm{mg} /$ day) and cholecalciferol (800 IU/day) for 1 year. We evaluated at the baseline (T0), after 6 months (T1), and after 12 months (T2) of treatment the following outcomes: back pain related disability, assessed by Spine Pain Index (SPI), and HRQoL, using the 12-Item Short Form Health Survey (SF-12), with Physical (PCS) and Mental Health Composite Scores (MCS) and the European Quality of Life - 5 Dimensions - 3 Levels (EuroQol-5D-3 L) index (EQ5D-3 L index) and the EuroQol-Visual analogue scale scores (EQ VAS).

Results: We enrolled 140 women, mean aged 74.89 \pm 8.80 years, with a mean BMI of $26.13 \pm 3.93 \mathrm{~kg} / \mathrm{m}^{2}$. Results are reported in Table 1.

Table 1. Outcome measures at the baseline (T0), after 6 months (T1), and after 1 year (T2) of subcutaneous denosumab therapy

\begin{tabular}{|c|c|c|c|c|}
\hline & T0 & $\mathrm{T} 1$ & $\mathrm{~T} 2$ & $P$ values \\
\hline SPI & $58.49 \pm 19.94$ & $45.03 \pm 21.97$ & $40.38 \pm 21.21$ & $\mathrm{P}<0.001^{*}$ \\
\hline SF-12PCS & $\begin{array}{l}29.5(24.6- \\
34.1)\end{array}$ & $\begin{array}{c}35.4(29- \\
40.9)\end{array}$ & $\begin{array}{c}35.7(29.3- \\
42.95)\end{array}$ & $\mathrm{P}<0.001 * *$ \\
\hline SF-12MCS & $\begin{array}{c}37.0(29.55- \\
44.72)\end{array}$ & $\begin{array}{l}44.6(35.5- \\
53.85)\end{array}$ & $\begin{array}{l}45.9(36.92- \\
55.17)\end{array}$ & $\mathrm{P}<0.001^{*}$ \\
\hline $\begin{array}{l}\text { EQ-5D-3 L } \\
\text { index }\end{array}$ & $\begin{array}{l}0.60(0.32- \\
0.76)\end{array}$ & $\begin{array}{c}0.69(0.6- \\
0.81)\end{array}$ & $\begin{array}{c}0.69(0.6- \\
0.82\end{array}$ & $\mathrm{P}<0.001^{*}$ \\
\hline EQ VAS & $4.67 \pm 1.86$ & $5.68 \pm 1.50$ & $6.20 \pm 1.63$ & $\mathrm{P}<0.001 *$ \\
\hline
\end{tabular}

Continuous variables are expressed as means \pm standards deviations. Categorical variables are expressed as median values (interquartile interval). $*=$ Friedman Test; $* *=$ ANOVA for repeated measures 
Conclusions: Our results demonstrated that denosumab is significantly effective in reducing back pain related disability and in improving HRQoL in women with vertebral osteoporotic fractures already after 6 months, and these findings are confirmed even at one year of treatment.

\section{P271}

SURVEY RESULTS OF TRAUMATOLOGISTSORTHOPAEDISTS ABOUT OSTEOPOROSIS DIAGNOSTICS AND TREATMENT, SECONDARY PREVENTION OF OSTEOPOROTIC FRACTIONS IN RUSSIA

${ }_{\text {A. U. Kochish }}{ }^{1}$, S. N. Ivanov ${ }^{1}$, E. V. Sannikova ${ }^{1}$

${ }^{1}$ Russian Research Institute of Traumatology and Orthopedics named after RR Vreden, St. Petersburg, Russian Federation

Nowadays, an attitude of traumatologists-orthopaedists to the osteoporosis diagnostics and treatment in Russian Federation is not investigated and following scientific publications are absent. That is why a voluntary writing specialists survey was held aiming to achieve their awareness degree and opinion record about osteoporosis diagnostics and treatment, secondary prevention of osteoporotic fractions and registration of their real participation in solving specified problems.

Materials and methods: This survey was held among traumatologists and orthopaedists in the largest in Russia annual conference of experts of this profile «Vreden reading» from 8th to 10th October, 2015. The conference was attended by 802 traumatologists-orthopaedists. The survey involved 153 doctors from all the regions of Russia, $92.8 \%$ of surveyed doctors work in hospitals. The questionnaire contained 4 main sections: the first - general info, the second - the organizational aspects of treatment and registration of patients with osteoporosis, the third - osteoporosis treatment and its effectiveness control and the fourth - secondary prevention of osteoporotic bone fractions.

Results: Osteoporosis has its own cipher as an independent nosologic unit with an own ICS code only in $23.5 \%$ of medical facilities. $90.8 \%$ of doctors have an opportunity to lead the patients towards X-ray densitometry, but $34.3 \%$ of respondents refer patients with low energy fractions to densitometry. By the way, traumatologists use a simple X-ray radiography more often than X-ray densitometry $-73.5 \%$ and $65.4 \%$ cases respectively. FRAX technique is used for the following problem barely in $22.2 \%$ cases. The main drugs for medical treatment of osteoporosis due to the trauma and orthopaedics experts opinion are complex drugs of calcium and vitamin $\mathrm{D}(63.9 \%)$. The other drugs are prescribed in less than $50 \%$ cases. Bisphosphonates are likely prescribed intravenously. Only $25.2 \%$ of doctors prescribe an antiosteoporotic therapy lasting more than a year. The effectiveness therapy control is made only by $55.5 \%$ respondents.
The effectiveness control is made in different ways: $59.7 \%$ of doctors use an X-ray densitometry, $26.1 \%$ take into consideration the repetitive bone fractions, $24.4 \%$ use laboratory results and barely $10.1 \%$ make FRAX calculations. Actions for secondary prevention of osteoporotic bone fractions are made only in $29 \%$ of medical facilities.

Conclusion: This survey has revealed the problems of diagnostic and treatment nature in case of providing care to patients with osteoporotic bone fractions in Russian Federation. The survey has explored necessary materials for planning and solving these problems.

P272

ORGANIZATION EXPERIENCE OF SECONDARY PREVENTION OF OSTEOPOROTIC FRACTURES SERVICE IN THE SCIENTIFIC RESEARCH INSTITUTE OF TRAUMATOLOGY AND ORTHOPAEDICS NAMED AFTER R.R.VREDEN, ST. PETERSBURG, RUSSIA

$\underline{\text { S. N. Ivanov }}^{1}$, A. U. Kochish ${ }^{1}$

${ }^{1}$ Russian Research Institute of Traumatology and Orthopedics named after RR Vreden, St. Petersburg, Russian Federation

The Russian Osteoporosis Association takes an active participation in secondary prevention of osteoporotic fractures according to the special program "Capture the Fracture". The Scientific Research Institute of Traumatology and Orthopaedics named after R.R.Vreden with 760 beds is the largest federal hospital of trauma and orthopaedics profile in Russia. A special program of secondary prevention of osteoporotic bone fractures was designed and started in this medical facility in 2015.

Aim: Organize the work in the institute matching the International Osteoporosis Foundation program "Capture the Fracture" standards. Targets: 1 . Work out the routing of patients to osteoporosis specialists. 2. Diagnose osteoporosis and count the repetitive fractions risks. 3. Organize the choice and the registration of patients requiring the secondary prevention of osteoporotic fractures. 4. Follow the matching indications and prescribe an adequate antiosteoporotic therapy. 5 . Organize the system of dispensary care of the following patients during the year after trauma. 6. Estimate the adherence and effectiveness of an antiosteoporotic therapy.

Materials and methods: Patients over 50 years with low energy trauma bone fractions that arrived in the emergency department of the Institute were chosen as the objects of the program. A routing scheme for patients and activity algorithm was designed to achieve the aims of the study. The FRAX calculation, $\mathrm{X}$-ray densitometry were made, drug therapy was prescribed, therapy adherence and results treatment were studied.

Results: Patients with forearm (37,3\%) and shoulder (27\%) bone fractures were leading among the incoming injured in the 
Institute. $66 \%$ patients had had previous fractures before. 73, $6 \%$ were diagnosed osteoporosis by densitometry. According to FRAX all the patients required an antiosteoporotic therapy. Due to this aim alendronate acid drug was prescribed as the most frequent $(35,8 \%)$. However, $20,8 \%$ did not take the prescribed drugs in 6 months.

Conclusion: The first experience of establishing the service for secondary prevention of fractures in patients with osteoporosis in the largest trauma and orthopaedics hospital revealed high medical and social significance of this program.

\section{P273}

\section{VERTEBRAL FRACTURES IN PATIENTS WITH END-STAGE KIDNEY DISEASE UNDERGOING} DIALYSIS

J. Przedlacki $^{1}$, P. Żebrowski ${ }^{1}$, E. Wojtaszek ${ }^{1}$, M. Mieczkowski $^{1}$, A. Grzejszczak ${ }^{1}$, P. Kulicki ${ }^{1}$, M. Kaszyńska ${ }^{1}$, J. Matuszkiewicz-Rowińska ${ }^{1}$

${ }^{1}$ Chair and Department of Nephrology, Dialysis and Internal Diseases, Warsaw Medical University, Warsaw, Poland

Objective: Reports on the real frequency of vertebral fractures in patients with end-stage renal failure treated with dialysis are limited. The aim of the study was to assess the frequency of the low-energy vertebral fractures in dialysis patients.

Material and Methods: One hundred eight patients from our one dialysis center (62 males and 46 females; 75 hemodialysis and 34 peritoneal dialysis patients) aged $59.4 \pm 13.8$ years were examined during 2010-2013. Densitometric vertebral fracture assessment (VFA) was done with the use of Discovery A, Hologic machine. Genant method of vertebral fracture was used. In the case of the new fracture diagnosis routine X-ray was done to confirm it. In 2010, 74 patients were examined and in the next three years 34 new patients were examined also. The control VFA examinations were done every 1-2 years in most of patients.

Results: Altogether, 15 vertebral fractures (5 thoracic and 10 lumbar) were recognized in 9 patients $(8.3 \%$; $8 \mathrm{HD}$ and 1 CAPD patients; 6 males and 3 females). In 2 patients ( 1 male and 1 female) the diagnosis was known previously (clinically overt fracture) from X-ray. There were wedge deformity in 4 thoracic vertebras ( 2 of grade 1 and 2 of grade 2 ) and in 2 lumbar vertebras (grade 1) and biconcave deformity in 1 thoracic vertebra (grade 1) and in 8 lumbar vertebras (4 of grade 1 and 4 of grade 2). There was no statistically significant difference between fractured and not-fractured patients depending on the form of dialysis, gender, age, duration of dialysis treatment and intact-PTH. There were only 3 fractured patients with DXA spine and 2 with DXA hip result of osteoporosis (in accordance to WHO criterion, T- score $\leq-2.5$ ).

Conclusion: Densitometric VFA was useful in diagnosis of vertebral fractures in dialysis patients. They were clinically silent in most cases. Because the information on the new vertebral fractures informs on the increased further fracture risk it seems reasonable to perform VFA assessment in dialysis patients as the routine procedure in the case of availability of this method.

\section{P274}

INCREASED SERUM URIC ACID (SUA) LEVELS ARE A COMMON FINDING IN PATIENTS WITH HIGH BLOOD PRESSURE, INSULIN RESISTANCE, OBESITY AND CARDIOVASCULAR (CV) DISEASE

I. Tasic ${ }^{1}$, S. Kostic ${ }^{1}$, V. Skakic ${ }^{1}$, A. Djordjevic ${ }^{1}$, D. Djordjevic $^{1}$, J. Radicovic ${ }^{2}$, M. Nikolic ${ }^{1}$, S. Andonov ${ }^{3}$, M. Karadzic $^{3}$, I. Krstic ${ }^{3}$

${ }^{1}$ Institute for Therapy \& Rehabilitation, Nis, Serbia, ${ }^{2}$ University of Nis, Medical Faculty, Institute for Therapy and Rehabilitation, Nis, Serbia, ${ }^{3}$ Institute for Therapy \& Rehabilitation, Niska Banja, Nis, Serbia

Objective: To evaluate the connection between SUA and severity of coronary heart disease (CHD) assessed by angiography in patients with CHD.

Design, Setting, and Participants: 198 patients $(75.8 \%$ men) undergoing cardiovascular rehabilitation at the Institute "Niska Banja" from October 1, 2014, to January 31, 2015, were evaluated. Mean (SD) patient age $(62.1 \pm 10.4)$, prevalence of patients with 3 -vessel coronary artery disease with stenosis $>70 \%(31.3 \%)$, coronary artery bypass grafting (CABG) $(37.4 \%)$, percutaneous coronary intervention (PCI) $(38.9 \%)$, primary PCI $(32.8 \%)$, mean ejection fraction $(50 \% \pm 11)$. Prevalence of hyperuricemia $(>386 \mu \mathrm{mol} / 1) 33.3 \%$, hypertension $85.9 \%$, hyperlipoproteinemia $81.8 \%$, diabetes mellitus $24 \%$, family history of CHD (47\%) and mean number risk factors 2.65. Patients were separated into a normal SUA group $(n=132$, with SUA $<386 \mu \mathrm{mol} / \mathrm{L})$ and a high SUA group $(n=66$, with $\mathrm{SUA} \geq 386 \mu \mathrm{mol} / \mathrm{L})$.

Results: Patients with high SUA had significantly higher 3 -vessel coronary artery disease with stenosis $>70 \%$ (40.9 \% vs. $26.5 \% ; P=0.04)$, low ejection fraction $(\mathrm{EF}<34 \%)(19.7 \%$ vs. $8.3 \% ; p=0.021)$, obesity (27\% vs. 14.4; $P=0.03$ ), significantly higher triglycerides $(2.38 \pm 1.7$ vs. $1.88 \pm 1.44 ; P=0.028)$ and serum creatinine $(111.5 \pm 46$ vs. $90 \pm 21 ; p<0.001)$. The SUA level was positively correlated with creatinine $(r=0,379$, $p<0.001)$, obesity $(r=0.214 ; p=0.002), 3$-vessel coronary artery disease with stenosis $>70 \%(r=0.129$, $p=0.071)$ and negative correlated with ejection fraction $(r=-.321 ; p<0.001)$. After multiple linear regression analysis, SUA levels were identified to be independently correlated with ejection fraction $(\mathrm{B}=-0.33 ; p<0.001)$ and creatinine $(\mathrm{B}=0.131 ; p<0.001)$. 
Conclusions: SUA levels were independently associated with $\mathrm{EF}$ in patients with CHD. Hyperuricemia is associated with severity of CAD- 3-vessel coronary artery disease with stenosis $>70 \%$.

\section{P275}

\section{VITAMIN D DEFICIENCY AND BONE DENSITY IN PATIENTS AFTER STROKE}

S. Tomasevic-Todorovic ${ }^{1}$, M. Savic ${ }^{2}$, K. Boskovic ${ }^{2}, \mathrm{~K}$.

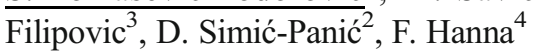

${ }^{1}$ Rheumatology, Clinic for Medical Rehabilitation, Clinical Center of Vojvodina, Faculty of Medicine, University of Novi Sad, Novi Sad, Serbia, ${ }^{2}$ Medical Faculty, University of Novi Sad, Novi Sad, Serbia, ${ }^{3}$ Rheumatology, Special Hospital for Rheumatic Diseases, Novi Sad, Serbia, ${ }^{4}$ Public Health Program, Department of Health Sciences, College of Arts and Sciences, Qatar University (Doha, Qatar), Doha, Qatar

Introduction: Vitamin D deficiency and osteoporosis are highly prevalent among stroke patients. The adverse effects of vitamin D deficiency on bone metabolism, seem to be aggravated by immobilization of paretic extremities.

Objectives: The purpose of this study was to determine the level of vitamin D in patients with osteoporosis following a stroke.

Material and methods: In this cross-sectional study participants consisted of 40 patients ( 33 women, 7 men) with mean age of $66.5 \pm 9.8$ years. The inclusion criteria were patients after a first stroke ( $\leq 1.5$ years from a stroke), who were treated at the Medical Rehabilitation Clinic, Clinical Center of Vojvodina in Novi Sad, Serbia. Lunar Prodigy Primo densitometer was used to determine the BMD in patients after stroke. DXA measurement was performed at the lumbar spine L2-L4 segment in antero-posterior (AP) position. Vitamin D was determined as follow; reference values: $25(\mathrm{OH})$ vitD $30 \mathrm{ng} / \mathrm{dl}$, total calcium $2.1-2.60 \mathrm{mmol} / \mathrm{l}$, phosphorus 3.0$4.5 \mathrm{mg} / \mathrm{dl}$, PTH (PTH) $14.0-72.0 \mathrm{pg} / \mathrm{ml}$.

Results: Seventeen $(42.50 \%)$ patients had osteoporosis of the lumbar spine, while $6(15 \%)$ had osteoporosis of the femur. Our study showed that ten patients $(25 \%)$ had levels of serum $25(\mathrm{OH})$ vitamin D lower than $30 \mathrm{ng} / \mathrm{dl}$, while 30 patients $(75 \%)$ had serum levels of $25(\mathrm{OH})$ vitamin $\mathrm{D} \geq 30 \mathrm{ng} / \mathrm{dl}$. The average values of PTH were $48,71 \pm 36 \mathrm{pg} / \mathrm{ml}$, and phosphorus $3.51 \pm 0.72 \mathrm{mg} / \mathrm{dl}$. Only $2.5 \%$ of the patients were receiving antiresorptive bone therapy and vitamin D supplementation at the moment of initial evaluation.

Conclusion: Low BMD and low levels of serum 25(OH) vitamin D are common amongst patients after stroke. Vitamin D supplementation and antiresorptive therapy prevent further bone loss, falls and fracture in poststroke patients, but further research need to confirm this hypothesis.
P276

MOTION CHARACTERISTICS OF THE VERTEBRAL SEGMENTS WITH LUMBAR DEGENERATIVE SPONDYLOLISTHESIS IN ELDERLY PATIENTS

H. El-Nemr ${ }^{1}$, H. Ibrahim ${ }^{1}$

${ }^{1}$ Department of Neurosurgery, Benha University, Banha, Egypt

Background: Spondylolisthesis refers to the forward displacement of one vertebra relative to another. Objectives: to compare the clinical results of lumbar decompression vs. lumbar decompression with bilateral pedicle screw fixation and postero-lateral fusion in elderly patients with lumbar degenerative spondylolisthesis (DS). Patients and Methods: Thirty patients (age more than 55 years) with lumbar DS and failed conservative measures for at least three months before surgical treatment were included. Vertebral kinematics obtained using dynamic plain X-ray; also MRI and/or $\mathrm{CT}$ scan of lumbosacral spine were done. During functional postures, vertebral instability was studied. All cases operated upon from May 2013 to December 2013 in the Neurosurgery Department of Benha University hospital and followed up 6 months for at least. Patients were grouped into 2 groups according to vertebral instability: Group A (fifteen cases) and Group B (fifteen cases) chosen without privilege to sex, or weight, but suffering from midline low back pain and/or claudication that were proven to be attributed to spondylolisthesis.

Results: Adequate lumbar decompression alone in cases of Group A (with no evident range of motion) showed good outcome with less complications when compared with cases of Group B (showing instability) treated with decompression with postero-lateral instrumented fusion using bilateral pedicle screw fixation.

Conclusion: Lumbar DS is a degenerative disease of lumbar spine results in neural compression but does not result necessarily in vertebral instability, some cases of lumbar DS may need only neural decompression as re-stabilization process may have occurred but other cases show instability which required decompression with instrumented fusion.

\section{P277 \\ CORRELATIONS BETWEEN CLINICAL AND HISTOPATHOLOGICAL ASPECTS IN ASEPTIC OSTEONECROSIS OF THE FEMORAL HEAD \\ D. Kamal ${ }^{1}$, R. M. Trăistaru ${ }^{1}$, K. C. Kamal ${ }^{1}$, D. O. Alexandru ${ }^{1}$, O. Rogoveanu ${ }^{1}$ \\ ${ }^{1}$ University of Medicine and Pharmacy, Craiova, Romania}

Objectives: Aseptic osteonecrosis of the femoral head is a condition whose pathogenesis remains unclear despite many theories developed so far, and the discovery of numerous risk factors. The objective of this study was to evaluate the histopathological and clinical features and to correlate them, in order to more accurately stage the disease. 
Material and methods: This retrospective study was performed on a total of 73 patients with clinical and radiological suspicion of unilateral or bilateral osteonecrosis. For the diagnosis criteria we used clinical information, pelvic X-ray images, MRI or CT. For the inclusion of patients in a disease stage we used the ARCO classification system. From patients diagnosed at an advanced stage, who underwent hip arthroplasty, we harvested biological material necessary for the histopathological study.

Results: We included a total of 73 patients in the study, aged between 26 and 64 years, with a mean age of 47.32 years. $42.04 \%$ of patients were from rural areas and $57.96 \%$ from urban areas. Out of the 73 patients, 59 were men and 14 women. The study results, according to stage of disease, showed that most patients $(90.41 \%)$ were diagnosed in the last four stages. Although patients in stages III and IV presented alterations on the imaging scans such as flattening of the femoral head or decrease in bone density, the precise extension of the necrotic and perilesional areas was much wider on the histopathological examination. In patients from stage $\mathrm{V}$ we noticed, besides changes in the shape and contour of the femoral head, changes in the internal architecture and overlying cartilage.

Conclusions: There were differences in the appearance and extent of the lesion on the histological samples compared to macroscopic examination and even those obtained through imaging methods, particularly for patients in evolutionary stage III. Even though the clinical features are poor, the extension of the lesion can be important. Before classifying patients in a certain stage, after correlating clinical and imaging data, histopathological aspects have to be considered, particularly in patients in stages III and IV, in which total hip arthroplasty could be delayed.

\section{P278}

\section{EVALUATION OF THE LESION EXTENSION IN PATIENTS WITH POSTTRAUMATIC ASEPTIC OSTEONECROSIS OF THE FEMORAL HEAD}

D. Kamal $^{1}$, R. M. Trăistaru ${ }^{1}$, K. C. Kamal ${ }^{1}$, M. D. MacoveiMoraru $^{1}$, L. D. Marinescu

${ }^{1}$ University of Medicine and Pharmacy, Craiova, Romania

Objective: Trauma is the most common cause of developing secondary aseptic osteonecrosis of the femoral head. The first step in diagnosing the disease is the early use, after the trauma occurs, of imaging techniques such as X-rays, MRI, CT, SPECT. The objective of this paper is to emphasize the role of imaging techniques and the correlation of histopathological methods in order to more accurately stage the disease, and to apply the optimal therapeutic methods.

Material and methods: This retrospective study was performed on a total of 32 patients with clinical and radiological suspicion of unilateral or bilateral posttraumatic aseptic osteonecrosis of the femoral head. Patients were examined using X-rays, magnetic resonance and CT. We used the ARCO classification system for staging patients. From the patients in an advanced stage of the disease, who underwent hip replacements surgery, we harvested biological material from the lesion area. We compared the extension of the actual injuries through histopathological techniques, and checked the accuracy of the imaging methods used to diagnose the disease, on which the staging and treatment methods are based on.

Results: Microscopic appearance varied according to the stage of the condition and was similar for patients in the same stage, regardless of their age or time passed since injury. We found changes in their blood circulation, both in the area of necrosis, as well as the under and superjacent areas, especially for patients in stage IV. Macrophages exhibited a particularly intense lysosomal activity especially in patients at stages III and IV. In these patients we highlighted at and endosteum level, numerous macrophages with foamy cytoplasm, indicating an intense activity of these cells. We noted the presence of macrophages on slides from patients in the last two evolutionary stages, but they were small in number and had an erratic disposition.

Conclusions: Aspects such as the extension of the area of fibrosis, remodelling of bone tissue, neo-formation vascular network density and degree of impairment of the cartilage were determined more accurately using histopathological methods than imaging techniques, especially in patients in stages III and IV.

\section{P279 \\ DEPRESSION IN PATIENTS WITH CHRONIC LOW BACK PAIN \\ M. Vukovic $^{1}$, S. Nejkov ${ }^{1}$, Z. Skaric-Karanikic ${ }^{1}$, V. Bokan- Mirkovic $^{1}$ \\ ${ }^{1}$ Center of Physical Medicine and Rehabilitation, Clinical Center of Montenegro, Podgorica, Serbia}

Objectives: The aims of this study were to assess the prevalence and degree of depression in patients with chronic low back pain, than compare it with prevalence of depression in group of patients who did not have chronic pain of any etiology. The last objective was to estimate the correlation between the severity of depression and intensity of pain in patients with chronic low back pain.

Material and Method: This study included 78 patients who were divided into two groups. The first group (G1) consisted of 41 patients (average ages $49.61 \pm 11.9$ ) with chronic back pain. The second group included 37 patients who did not refer any chronic pain (patients on rehabilitation program after non complicate fractures of arms and legs) average ages 50.78 \pm 14.16 . The presence and severity of the depressive syndrome was assessed by Patient Health Questionnaire (PHQ9), scored by depression severity score (DSS) and graded as none/minimal (0-4), mild (5-9), moderate (10-14), 
moderately severe (15-19) and severe (20-27) (1). Intensity of pain was evaluated using the Visual Analog Scale (VAS) rated $0-10$. In the statistical analysis we used Mann-Whitney test and Spearman's rank correlation coefficient.

Results: Distribution of patients in G1 by DSS was: none/minimal 8 , mild 16 , moderate 14 , moderately severe 2 and severe depression 1; in G2 none/minimal 19, mild 10, moderate 7 , moderately severe 0 , and severe depression 0 . Statistical analysis of the median DSS values of both groups was found statistically significant difference in severity of depression in these two groups $(\mathrm{W}=426.5$, $\mathrm{p}$-value $<0.001)$. Mean of VAS value in G1 was $6.93 \pm 2.0$ and there was a statistically significant strong positive correlation between the DSS and VAS in the group G1 (Spearman's rank correlation coefficient $0.705 \mathrm{p}$ value $<0.001$ ).

Conclusion: We found that patients with chronic low back pain have a tendency to depression, most often to mild and moderate depression. It has shown a strong positive correlation between the intensity of pain and severity of depression, which is consistent with previous studies $(2,3)$. Patients with chronic low back pain have a severe degree of depression than patients without chronic problems.

\section{References:}

${ }^{1}$ Kroenke K, Spitzer RL. Psychiatric Annals 2002;32:509.

${ }^{2}$ Bair MJ, et al. Archiv Int Med 2003;163:2433.

${ }^{3}$ Arnow BA, et al. Psychosomatic Med 2006;68:262.

\section{P280}

SERUM URIC ACID AND RADIOGRAPHIC KNEE OSTEOARTHRITIS

V. Skakic ${ }^{1}$, I. Tasic ${ }^{1}$, A. Skakic ${ }^{2}$

${ }^{1}$ Institute for Therapy \& Rehabilitation, Niska Banja, Serbia,

${ }^{2}$ Urology Clinic, Clinical Center Nis, Nis, Serbia

Objectives: To study the association between serum uric acid levels and ultrasonographic knee osteoarthritis (KOA).

Methods: Hundred patients (45 males, 55 females) prospectively examined at the Ultrasound Cabinet of the Institute Niska Banja, were included in this study. Their serum uric acid results and ultrasonography of knees were recorded during routine examination. The presence of KOA was analysed in relation to serum uric acid values.

Results: Their mean age was $63.14 \pm 9.8$ (female $63.8 \pm 9.2$, males $62.2 \pm 10.3)$. The KOA was present in 48 patients (48 \%), 23 (23.6\%) females and 63 (26.1\%) males. After adjusting for age, sex, BMI, serum cholesterol and triglycerides, there was an association between knee OA, generalized $\mathrm{OA}$ and the highest tertile of serum uric acid [adjusted odds ratio (OR) 2.03, $95 \% \mathrm{CI}, 1.58-2.71$ and $1.81,95 \% \mathrm{CI}, 1.33-$ 2.57 respectively).

Conclusion: It is concluded that KOA is associated with hyperuricemia.

\section{P281}

OSTEOGENIC LOADING PHYSICAL INTERVENTION, FUNCTIONAL BONE PERFORMANCE INCREASES AND BONE HEALTH RESTORATION

J. Jaquish $^{1}$, L. Freeman ${ }^{2}$

${ }^{1}$ Jaquish Industrial Research, Nevada City, California, United States, ${ }^{2}$ PATH- Research Institute, Sammamish, Washington, United States

Objective: To ascertain the effectiveness, compliance and patient satisfaction of osteogenic loading (OL) type therapy device application with a community dwelling frailelderly population with chronic conditions including osteoporosis.

Materials and Methods: 100 frail-elderly $\mathrm{m} / \mathrm{f}$ subjects, age $72.4( \pm 7.2 \mathrm{SD})$ were randomly selected for a 9-week trial using $\mathrm{OL}$ in order to isolate impact ranges of motion and allow for comfort/biofeedback mediated, axial compression of bone at multiples of subject bodyweight (MOB). Loading when using the OL modality are higher than those seen in conventional exercise: (1.26 to $1.54 \mathrm{MOB}$ in the hip ${ }^{1}$ ).

Results: The population was able to show adaptation via increases in functional bone performance (FBP) greater than age matched norms over the intervention, exceeding 8.5 MOB and 2.8 MOB in the hip and spine, respectively $(p=0.006)^{2}$. Hip loading exceeded the previously identified threshold for osteogenesis $(4.2 \mathrm{MOB})^{3}$. Loading measured via MOB was also congruent with existing OL literature showing BMD gain ${ }^{4}$. Post intervention survey results showed subjects: 1) strongly agree that the OL influenced their decision to continue after the study; 2) agree that OL influenced satisfaction; 3) strongly agree that OL therapy impacted confidence/functional mobility; 4) had total compliance with subjects. No adverse effects were reported.

Conclusion: These data suggest that adding OL therapy to standard rehabilitation can have positive outcomes with frail-elderly populations suffering from multiple chronic conditions, as indicated by BMD increase, satisfaction, and overall confidence in functional mobility.

\section{References:}

${ }^{1}$ American College of Sports Medicine (2009). ACSM's Guidelines for Exercise Testing and Prescription, 8th ed. LWW, Philadelphia, PA.

${ }^{2}$ Huck C \& Jaquish J. Osteoporosis Int 2015;26(1):s391-s392, NS12.

${ }^{3}$ Tobias J, et al. Front in Endo. 2014;5:1.

${ }^{4}$ Hunte B, et al. JOPA 2015;3:2.

Disclosures: Second author is a paid consultant for PHS, a US-based company that manufactures OL therapy devices. 


\section{P282}

CLINICAL EVIDENCE OF THE ATTENUATION EFFECT OF MAGNESIUM SULPHATE ON THE TERTIARY HYPERALGESIA IN STAGED BILATERAL TOTAL KNEE ARTHROPLASTY: A DOUBLE-BLIND RANDOMIZED CONTROLLED TRIAL

H.-J. Shin ${ }^{1}$, H.-S. Na ${ }^{1}$, S.-H. Do ${ }^{1}$, T.-K. Kim ${ }^{2}$

${ }^{1}$ Department Anesthesiology and Pain Medicine/Seoul National University Bundang Hospital, Seongnamsi, Republic of Korea, ${ }^{2}$ Department of Orthopedic Surgery, Seoul National Bundang Hospital, Seongnamsi, Republic of Korea

Objective: Tertiary hyperalgesia may occur in staged bilateral total knee arthroplasty (TKA) due to repeated surgical injury, possibly via central sensitization. Magnesium attenuates the central sensitization due to its antagonistic effect on N-methyl-d-aspartate receptors. We evaluated the effects of magnesium on tertiary hyperalgesia in patients undergoing staged TKA.

Materials and Methods: Forty-four patients undergoing staged bilateral TKA were enrolled in this study. The magnesium group $(n=22)$ received magnesium sulfate at $50 \mathrm{mg} / \mathrm{kg}$ for 15 minutes followed by $15 \mathrm{mg} / \mathrm{kg} / \mathrm{h}$ by continuous i.v. infusion until the end of surgery. The control group $(n=22)$ received the same volume of isotonic saline over the same period. Postoperative pain (numerical rating scale, NRS) at rest, the amount of patient-controlled analgesic (PCA, i.v. fentanyl), and rescue analgesic (i.v. ketoprofen) administered during the 48-h period after the operation were compared between the two groups and the first and second periods within groups.

Results: NRS scores were greater in the control group at 24 and 48 hours postoperatively in the first TKA $(P=0.001$ and $P=0.001$, respectively) and in the second TKA $(P<0.001$ and $P<0.001$, respectively) than in the magnesium group. The amount of rescue analgesics used during the 48-h postoperative period in the second TKA was greater in the control group than the magnesium group $(P=0.001)$. Patients received more fentanyl via PCA in the first $(P=0.014)$ and second $(P=0.001)$ TKA over $48 \mathrm{~h}$ postoperatively in the control group than the magnesium group. In the control group, at 24 and $48 \mathrm{~h}$ postoperatively, NRS scores $(P<0.001$ and $P=0.006$, respectively) and the amount of rescue analgesics $(P=0.011$ and $P=0.004$, respectively) were greater for the second than the first operated knee. The cumulative consumption of PCA during the first $48 \mathrm{~h}$ postoperatively was greater after the second than the first TKA $(P<0.001)$. In the magnesium group, there were no significant differences in NRS score at postoperative 24 or $48 \mathrm{~h}$ between the first and second operated knee. The amount of rescue analgesics used during $24 \mathrm{~h}(P=0.021)$ and the cumulative amounts of PCA during first $48 \mathrm{~h}(P=0.004)$ were greater in the second operated knee.

Conclusions: Administration of magnesium significantly reduced postoperative pain and the difference in pain intensity between the first and second operated knee in staged bilateral TKA. These observations suggest that magnesium attenuates tertiary hyperalgesia induced by surgical injury.

\section{P283}

MAINTENANCE VITAMIN D3 SUPPLEMENTATION REQUIRED TO MAINTAIN SUFFICIENCY IN INDIAN WOMEN WITH POSTMENOPAUSAL OSTEOPOROSIS LIVING IN THE TROPICS

S. F. Wan Muhamad Hatta ${ }^{1}$, G. K. Goh ${ }^{2}$, S. P. Chan ${ }^{2}$, S. R. Vethakkan $^{2}$

${ }^{1}$ Department of Endocrinology, University Teknologi MARA, Selangor, Malaysia, ${ }^{2}$ Department of Endocrinology, University of Malaya, Wilayah Persekutuan Kuala Lumpur, Malaysia

Background: Vitamin D supplementation is a mainstay of osteoporosis-therapy. Serum vitamin D levels in unsupplemented subjects are mainly dependent on sun exposure and may thus vary with geography and ethnicity (differing skin pigmentation/cultural clothing practices). Various guidelines have proposed different thresholds for sufficiency and maintenance doses ranging from 600 to 2000 IU daily. ${ }^{1,2,3}$

Objective: To determine the dose of vitamin D required to maintain sufficiency in postmenopausal osteoporosis(PMO) women of South-Indian descent living in Kuala Lumpur $\left(3.1333^{\circ} \mathrm{N}\right.$, $101.6833^{\circ} \mathrm{E}$ ) who may have different requirements given their tropical climate exposure and darker skin pigmentation.

Design: In this randomized controlled parallel arm trial, 33 PMO Indian women with baseline serum 25(OH)D $>20 \mathrm{ng} / \mathrm{ml}$ were randomly allocated to 3 different dosingregimens for 6 months i.e. Group(a) 25,000 IU/monthly $(\approx 900 \mathrm{IU} /$ day $)$, Group(b) 50,000 IU/monthly $(\approx 1800 \mathrm{IU} /$ day) and Group(c) 50,000 IU/fortnightly ( $\approx 3300 \mathrm{IU} /$ day). Serum 25(OH)D, calcium, phosphate, iPTH, and 24-h urine calcium were measured at baseline and 6 months. Vitamin D sufficiency was defined as $\mathrm{Sr} .25(\mathrm{OH}) \mathrm{D}>30 \mathrm{ng} / \mathrm{ml}$. Results: Mean age and BMI in the entire population were 67.4 \pm 6.2 years and $26.8 \pm 4.9 \mathrm{~kg} / \mathrm{m} 2$. At baseline, $66.7 \%$ had serum 25(OH)D levels of 20.1-29 ng/ml (insufficiency) and $33.3 \%$ levels $\geq 30 \mathrm{ng} / \mathrm{ml}$ (sufficiency). 8,10 and 15 women were randomized to group (a),(b) and (c), respectively. Baseline characteristics were similar between groups (age, BMI, waist circumference, skin colour, sun exposure index, dietary vitamin $\mathrm{D} /$ calcium intake, supplementary calcium intake, Sr. 25(OH)D and iPTH). After 6 months, regardless of dosing-regimen, 21 out of 22 (95\%) of individuals who were insufficient at baseline became sufficient and $100 \%$ 
$(n=12)$ of those with baseline sufficiency remained sufficient. There were no significant differences in mean incremental rise of 25(OH)D from baseline-to-6-months of 13.37 $\pm 14.67,18.38 \pm 15.76$ and $21.82 \pm 11.43 \mathrm{ng} / \mathrm{ml}$ in group (a), (b) and (c), respectively $(p=0.467)$. Mean serum vitamin $\mathrm{D}$ was not significantly different between the 3 dosing regimens at the 2, 4 and 6-month time points.

Those on the highest dose of vitamin D attained higher mean serum $25(\mathrm{OH}) \mathrm{D}$ but this difference was not statistically significant(group (a) 44.6 \pm 7.7 , (b) $42.9 \pm 12.9$ and (c) 48.8 $\pm 11.9 \mathrm{ng} / \mathrm{ml}(p=0.531))$. None of the dosing regimens resulted in hypercalcemia, hypercalciuria or vitamin $\mathrm{D}$ intoxication. Conclusions: All three vitamin D3 doses of 25,000 IU/month ( $\sim 00 \mathrm{IU} /$ day), 50,000 IU/month ( 1800 IU/day) and 100, $000 /$ month ( $\sim 330 \mathrm{IU} /$ day) safely correct insufficiency and maintain sufficiency ( $\geq 30 \mathrm{ng} / \mathrm{ml}$ ) in dark skinned IndianMalaysian PMO women living in the tropics. It may not be necessary for these women (with baseline levels $>20 \mathrm{ng} / \mathrm{ml}$ ) to ingest $>900 \mathrm{IU} /$ day to maintain sufficiency.

References:

${ }^{1}$ Holick MF, et al. J Clin Endocrinol Metab 2011;96:1911.

${ }^{2}$ Ross A, et al. (2011) Dietary reference intakes for calcium and vitamin D. Institute of Medicine report. Washington DC: The National Academies Press.

3.2014 National Osteoporosis Malaysian Guidelines.

\section{P284}

PORE NET WORK ARCHITECTURE DETERMINES CORTICAL BONE ELASTICITY DURING GROWTH AND AGEING

$\underline{\text { Y. Bala }}^{1}$, E. Lefevre ${ }^{2}$, J.-P. Roux ${ }^{1}$, C. Baron ${ }^{2}$, P. Lasaygues ${ }^{3}$, M. Pithioux ${ }^{2}$, V. Kaftandjian ${ }^{4}$, H. Follet ${ }^{1}$

${ }^{1}$ INSERM UMR 1033, Université de Lyon, Lyon, France, ${ }^{2}$ Aphm, Marseille, France, ${ }^{3}$ Laboratory of mechanics and acoustics, Marseille, France, ${ }^{4}$ Lva Insa, Villeurbanne, France

Cortical porosity originating from a dynamic network of interconnected channels in 3D is a determinant of bone strength. Given the changes in bone remodeling across life, we hypothesized that the $3 \mathrm{D}$ architecture of the pore network is agedependent and influences its stiffness. Two-mm cubes of fibula cortex of 13 children $(9 \mathrm{M}, 4 \mathrm{~F}, 12 \pm 4 \mathrm{yrs})$ and 16 adults $(7 \mathrm{M}$, $9 \mathrm{~F}, 74 \pm 12 \mathrm{yrs}$ ) were imaged at $8 \mu \mathrm{m}$ using microCT. Pores were segmented to assess porosity (Ct.Po\%), pore number (Po.N $1 / \mathrm{mm}$ ), diameter (Po.Dm $\mu \mathrm{m}$ ), separation (Po.Sp $\mu \mathrm{m})$ and connectivity (ConnD 1/mm3). Compression (C33) and shear (C66) elastic coefficients were derived by assessing waves velocity using ultrasonic measurements. Spearman correlation coefficients ( $\left.\mathrm{r}^{\prime}\right)$ are reported when $p<0.05$. Ct.Po did not differ between growing children and adults groups but originated from different architectural pattern. Relative to children, adults had
$46 \%$ higher Po.N, $17 \%$ lower Po.Sp, $250 \%$ higher ConnD, $14 \%$ and $13 \%$ higher compressive (C33) and shear (C66) stiffness (All $p<0.02$ ). During growth, age correlated with a decrease in Ct.Po $\left(\mathrm{r}^{\prime}=-0.6\right)$ due to decreasing Po.N $\left(\mathrm{r}^{\prime}=-0.5\right)$, Po.Dm $\left(r^{\prime}=-0.4\right)$ and increasing Po.Sp $\left(r^{\prime}=0.6\right) . C 33$ and C66 were inversely correlated with Ct.Po, Po.N, Po.Dm and ConnD ( $\mathrm{r}$ ' ranging from -0.56 to -0.82 ), Correlation between ConnD and c33 remained significant after adjustment for Ct.Po. During ageing, age correlated with higher Ct.Po $\left(r^{\prime}=0.7\right)$ originating from an increase in Po.N $\left(\mathrm{r}^{\prime}=0.8\right), \operatorname{Po} . \mathrm{Dm}\left(\mathrm{r}^{\prime}=0.5\right)$ and a decrease in Po.Sp $\left(r^{\prime}=-0.8\right) . \mathrm{C}^{\prime} 3$ was inversely correlated with Ct.Po $\left(r^{\prime}=-0.6\right)$, Po.N $\left(r^{\prime}=-0.6\right)$ and positively with Po.Sp $\left(r^{\prime}=0.7\right)$. Po.Sp remained correlated with $\mathrm{C} 33$ after accounting for Ct.Po contribution. Changes in intracortical remodeling across life alter the distribution, size and connectedness of the channels forming cortical porosity. These alterations in pore network architecture influence age-related changes in compressive stiffness during growth and ageing independently of changes in porosity.

\section{P285}

SHOULDER PAIN ACCORDING TO TIME OF ONSET OF SYMPTOMS: A RETROSPECTIVE ULTRAS ONOGRAPHIC FEATURES COMPARISON.

$\underline{\text { C. A. Guillén-Astete }}^{1}$, M. Villarejo-Botija ${ }^{1}$, A. Boteanu ${ }^{1}, M$. A. Blazquez-Cañamero ${ }^{2}$

${ }^{1}$ Ramon y Cajal University Hospital, Madrid, Spain, ${ }^{2}$ Leganes Hospital, Madrid, Spain

Background: Ultrasonographic features in shoulder pain have been extensively described in many series. Most epidemiological studies have focused on ultrasonographic findings without considering time of onset of symptoms. In our experience with patients with shoulder pain of recent onset (less than a week) we find ultrasonographic features which proportions are discordant with global epidemiological data especially in terms of prevalence of subacromial bursitis and supraspinatus tendinosis.

Objective: To describe and compare ultrasonographic findings on patients with shoulder pain who consulted to our emergency department and underwent an ultrasonographic shoulder assessment.

Methods: The registries of the musculoskeletal and rheumatologic urgencies unit of our A\&E department, from 2013 to 2015 were consulted. The known ratio of ultrasonographic assessment of our unit is close to $95 \%$ of all shoulder pain cases. Two rheumatologists with full ultrasonographic training and at least 3 years of experience performed the studies following EULAR shoulder ultrasonographic definitions. Findings were grouped according to time of onset of symptoms using an arbitrary definition of hyperacute ( $<7$ days), acute ( 1 to 6 weeks) and chronic $(>6$ weeks) of shoulder pain. 
Results: 474 registries of shoulder ultrasonographic assessments were reviewed. Average age of patients was 59 SD 17 years old. Male proportion was $67 \%$. Global findings showed that supraspinatus tendon was affected in $86.7 \%$ of all records, while subescapularis tendon in $26.8 \%$ and biceps tendon in $11.8 \%$. 67, 47 and 20 registries were classified as hyperacute, acute and chronic shoulder pain, respectively. $38.6 \%$ of all supraspinatus tendinosis was present in hyperacute, $25.1 \%$ in acute and $36.3 \%$ in chronic shoulder pain. $50 \%$ of all subacromial bursitis detected were present in hyperacute shoulders, $35.1 \%$ in acute and $14.9 \%$ in chronic shoulder pain. $34.2 \%$ of all supraespinatus tears were present in hyperacute shoulders, $47.4 \%$ in acute and $18.4 \%$ in chronic shoulder pain. $70 \%$ of all subscapularis tendinosis were present in hyperacute and $30 \%$ in acute shoulders. $12.7 \%$ of all subscapularis calcifications were present in hyperacute shoulders, $31.7 \%$ in acute and $55.6 \%$ in chronic shoulders. All of these distributions had differences statistically significant. Supraspinatus calcification distribution was not statistically different in the three groups.

Discussion: Subacromial bursitis and subscapular tendinosis seems to be the most frequent finding in patients with hyperacute shoulder pain. Our results, in patients with less than a week of symptoms, do not agree with most epidemiological ultrasonographic studies of the shoulder. It is possible that differences could be explained by the fact that most studies have not been performed in patients with hyperacute shoulder pain. In our opinion, a better comprehension of shoulder ultrasonographic features in shoulder pain should be linked to the time of onset of symptoms.

\section{P286 \\ HOW TO ASSESS DISABILITY AFTER A SECOND CONTRALATERAL HIP FRACTURE? \\ E. Dubljanin Raspopovic $^{1}$, S. Tomanovic Vujadinovic ${ }^{1}, \mathrm{U}$. Nedeljkovic $^{1}$, N. Ilic ${ }^{1}$, S. Stoicic ${ }^{2}$ \\ ${ }^{1}$ Clinic for Physical Medicine and Rehabilitation, Clinical Center Serbia, School of Medicine, University of Belgrade, Belgrade, Serbia, ${ }^{2}$ Clinic for Physical Medicine and Rehabilitation, Clinical Center Serbia, Belgrade, Serbia}

The aim of this study was to determine the outcome for patients who sustain a second hip fracture compared with those who sustain a first fracture. We included 343 patients who presented with acute hip fractures to an university-associated orthopedic hospital during a 12 month period. Patients with a first ( 318 patients, $78.10 \pm 7.53$ years) and second ( 25 patients, $78.96 \pm 6.02)$ hip fracture were compared regarding all baseline variables. Regression analysis was also performed to assess the independent relationship between the presence of a second hip fracture and observed outcome variables at discharge (physical disability, complications, length of stay, mortality) and 1-year after surgery (physical disability, mortality). Disability when performing instrumentalized activities of daily living (IADL) at 1-year follow-up is independently related to the presence of a second hip fracture. There were no other statistically significant relationships between the presence of a second hip fracture and other observed outcome variables. IADL is a good tool to assess disability after a second hip fracture. and could be thus a more reliable outcome measure when investigating differences in functional recovery in patients with a second hip fracture compared to conventionally used ADL scales.

\section{P287}

E F F E T IVENESS OF THE USE OF TRANSDERMAL BUPRENORPHINE THERAPY IN AXIAL PAIN RELATED TO NON-ACUTE VERTEBRAL FRACTURE IN COMPARISON TO OTHER PAIN PHARMACOLOGICAL MANAGEMENTS: A RETROSPECTIVE ROUTINE CLINICAL PRACTICE-BASED STUDY IN TERMS OF REDUCTION OF NEED OF URGENT RE-ASSESSMENT

C. A. Guillén-Astete $^{1}$, C. Fernández-Pérez ${ }^{2}$, A. Boteanu $^{1}, \mathrm{M}$. Luque-Alarcón $^{3}$, C. Carballo-Cardona ${ }^{1}$, F. Roldán-Moll ${ }^{1}$

${ }^{1}$ Ramon y Cajal University Hospital, Madrid, Spain, ${ }^{2}$ San Carlos, Clinical Hospital, Madrid, Spain, ${ }^{3}$ El Tajo Hospital, Aranjuez, Spain

Background: Pain control is the most relevant issue clinicians must face in patients who demands medical attention. Vertebral fracture, with independence of its cause it a major cause of axial pain not only in the acute phase but as a chronic contributor to the instability of the spine and ultimate cause of many alterations of the axial biomechanics. Previous studies demonstrated that lumbar and dorsal pain episodes are two to three times more frequent in patients of the same age when a vertebral fracture is present. Many treatments have been tested in musculoskeletal pain with different results. There are no studies conducted to compare different therapeutic strategies in back pain treatment related to nonacute vertebral fractures. Methods: A retrospective observational study was conducted. Data was retrieved from the database of registries of A\&E department of our hospital and the follow-up registries of the rheumatologic and musculoskeletal urgencies unit. No identity data was gathered. Registries included had the following criteria: (1) Al least 60 years old, (2) Radiological demonstration of a previous known vertebral fracture/collapse since at least 3 months, and (3) chief complain related to dorsal or back pain. Patients were grouped according to the treatment at discharge: 
First scale of the WHO pain treatment ladder (Group I), second scale (Group II) and Transdermal buprenorphine (Group III). Main outcome variable was the time until the need of a further urgent assessment (Kaplan-Meier curve). Results: 180 registries were analyzed. Group I, II and III contained 39, 74 and 67 cases. No demographic statistically significant differences were founded between groups. Group I consisted by patients who underwent treatment with metamizol, paracetamol or both (24), NSAID alone or associated with metamizol or paracetamol (15). Group II consisted by patients who underwent treatment with an average dose of $155 \mathrm{mg} /$ day of tramadol (range 37.5-300 mg/day) and patients treated with codein (2). Group III consisted by patients treated with $35 \mu \mathrm{g} / 72 \mathrm{~h}$ of transdermal buprenorphine, $50 \mu \mathrm{g} / 72 \mathrm{~h}$ (2) and $70 \mu \mathrm{g} / 72 \mathrm{~h}$ (1). $50 \%$ of patients of group III had to be assessed after the 50th day since the first urgent visit (ICR 4160 ), while same proportion of patients from group I and II consulted at the 28th (ICR 21-53) and 19th (ICR 10-37) day, respectively. Patients from group I drugs increase their re-assessment rate 4.19 fold (CI95\% 2.57-6.80; $P<0.001)$ while patients from group II have a 1.91 fold re-assessment rate (CI95\% 1.22-2.99, $P=0.005)$ compared with group III patients. 3/39, 10/74 and 1/67 patient from groups I, II and III, respectively requested a new urgent assessment due to what was finally interpreted as a prescription side effect. Tramadol users (Group II) had a significantly higher proportion of side effects than transdermal buprenorphine by the first month $(P=0.01)$. There were no statistically significant differences between proportions of side effects in patients from group I compared to group III $(P=0.14)$.

Conclusions: Our results suggest that pharmacological treatment with transdermal buprenorphine in patients with nonacute vertebral fracture reduces the ratio of further need of urgent reassessments compared to tramadol or first scale drugs of the WHO pain treatment ladder. Considering that need of reassessment is the result of an insufficient pain control and presence of side effects, our study could support the statement that transdermal buprenorphine treatment is a better choice in these patients.

\section{P288}

SYMPTOMS MODIFYING EFFECT OF ORAL AVOCADO/SOYBEAN UNSAPONIFIABLES (ASU) IN A ROUTINE TREATMENT OF KNEE OSTEOARTHRITIS (OA) IN POLAND: AN OPEN, OBSERVATIONAL STUDYOF OUTPATIENTS ADHERENT TO A 6-MONTH TREATMENT

P. Gluszko ${ }^{1}$

${ }^{1}$ Department of Rheumatology, NIGRR, Warsaw, Poland
Objectives: To investigate the effects of ASU capsules on pain relief and functional ability during 6 months of the routine treatment of knee OA.

Methods: A prospective, observational 6- months study was conducted in a group of 4822 patients with symptomatic knee OA treated with $300 \mathrm{mg}$ ASU capsules / day, as a routine medication in 99 centers in Poland. The patients did not suffer from any other rheumatic diseases and were not treated with other SYSADOA and/or other procedures. Data collection on OA symptoms and therapy was performed starting from the initiation of ASU treatment (visit 0 ) and during 3 consecutive control visits performed every 2 months (visits I-III). Functional Lequesne index, severity of joint pain of one symptomatic knee (Laitinen index and VAS), use of analgesics and NSAIDs, adherence to treatment and adverse events were evaluated and recorded using electronic case report forms.

Results: 4186 patients $(86.8 \%)$ attended all 4 visits. $94.2 \%$ of patients (mean age 60.7 years. $\pm 11.6 \mathrm{SD}, 73.4 \%$ female) had at least one OA risk factor. There was a significant improvement of functional ability between the last and baseline visits: median Lequesne index decreased from 8 to 4 points $(p<0.001)$. Pain diminished significantly $(p<0.001)$ throughout the study: median Laitinen score decreased from 6 to $3 p$, median pain at rest VAS - from 1.8 to $0 \mathrm{~cm}$ and median pain during walking VAS - from 5.6 to $1.9 \mathrm{~cm}$. The significant differences were also noted between consecutive visits. The number of patients using analgesics and NSAIDs fell down from $58.8 \%$ at baseline visit to $24.9 \%$ at last visit III $(p<0.001)$. Defined daily dose of NSAIDs significantly lowered throughout the study from 1 at baseline visit to 0.67 at visit III. Conclusions: It was the first investigation in Poland evaluating the effects of the routine knee $\mathrm{OA}$ treatment with ASU. A small group of patients (13.2 \%) treated with ASU dropped out from the study.

Almost all patients adherent to the ASU treatment for 6 months showed gradual alleviation of joint pain, functional ability improvement and reduction in NSAIDs intake.

Acknowledgements: The study got grant/research support from Angelini, Poland.

P289

POLYMORPHISMS VDR, ESR1,LRP5,OPG,SOST AND ROR2 IN PATIENTS WITH OSTEOPENIA AND OSTEOPOROSIS AND THEIR RELATIONS WITH OSTEOLOGICAL PARAMETERS AND FRACTURES.

$\underline{\text { P. Novosad }}^{1}$, P. Hrdý ${ }^{1}$, P. Fojtík ${ }^{2}$, M. Běhal ${ }^{3}$, M. Zemánek ${ }^{3}$, M. Janura ${ }^{4}, K$. Janurová ${ }^{5}$

${ }^{1}$ Mediekos Amb. Ltd, Zlín, Czech Republic, ${ }^{2}$ AGEL Research and Training Institute-Ostrava Vítkovice Branch, Ostrava- 
Vítkovice Hospital Centre of Care for the Gastrointenstinal Tract, Ostrava-Vítkovice, Czech Republic, ${ }^{3}$ Imalab Lab. Ltd, Zlín, Czech Republic, ${ }^{4}$ Department of Nature Science in Kinanthropology, Faculty of Physical Culture, Palacky University, Olomouc, Olomouc, Czech Republic, ${ }^{5}$ IT4 Innovations National Supercomputing Centre VŠB-Technical University, Ostrava, Czech Republic

Aim: Individual polymorphism frequencies are examined within two defined groups of probands. We examine a relation of individual polymorphisms towards defined osteological parameters, including fractures.

Examined polymorphisms: In the examined cohort for osteopenia and osteoporosis we examined the following polymorphisms: VDR(vitamin D receptor)-BsmI, FokI, ApaI, TaqI, ESR1 (estrogen receptor 1) - PvuII and XbaI, OPG (osteoprotegerin) $-1181 \mathrm{G}>\mathrm{C}$, SOST (sclerosteosis) - 10565ins GGA, LRP5 (lipoprotein receptor-related protein5)-Val667 Met and Ala 1330 Val, and ROR-2-rs7048756. In the first cohort we examine only VDR (BmsI) and do not examine ROR2.

Examined osteological parameters: BMD of lumbar spine and femoral neck (iDXA GE Lunar) markers for bone metabolism P1NP(procollagen type I N-terminal propeptide) and CTx(beta C-terminal crosslinked telopeptide of type I collagen), vitamin $25(\mathrm{OH}) \mathrm{D} 3$ and $1.25(\mathrm{OH}) 2 \mathrm{D} 3, \mathrm{PTH}, \mathrm{Ca}$ (total calcium). Clinical characteristics of the cohort 1 and 2: These cohorts are with primary osteoporosis or osteopenia. Probands with secondary osteoporosis or with skeleton damaging medications were excluded. Male population is not evaluated. The first cohort was composed of female population from 45 to 79 years of age. The total number of probands in this first cohort was 213 . The control cohort had 63 probands included with no symptoms of osteoporosis or any other heterogeneous or mutated disorders. The second cohort counted 231 probands from 43 to 84 years of age.

Types of fractures: No fracture -0 , distal forearm -1 , vertebra -2 , femoral neck -3 , humeral neck -4 , multiple major osteoporotic fractures -5 , non-vertebral fractures with inappropriate injury history -6 , traumatological fractures with appropriate injury history -7 .

Laboratory methods: DNA was isolated by kit MagNA Pure Compact Nucleic Acid Isolation Kit I using automated isolator MagNA Pure MagNA) from $400 \mu$ noncoagulable blood samples. Detection of all polymorphisms was carried out by a method of real time PCR with the use of hydrolysation and FRET probes on LC $480 \mathrm{II}($ Roche).). Correctness of the molecular examination results was randomly checked by sequencing on a sequenator ABI 3130 AVANT (Applied Biosystem).

Statistical processing of cohort 1: Basic descriptive characteristics (mean, standard deviation, median) were calculated from the measured data (STATISTICA Version
10.0, Stat-Soft, Inc., Tulsa, Oklahoma, USA).Normality of data distribution was verified using the KolmogorovSmirnov test. The data were analyzed using a one-way ANOVA. Fisher's least significant difference (LSD) procedure was used for all post-hoc pair-wise comparisons. Kruskal-Wallis test with Turkey-Kramer post-hoc test was used for parameters that did not show a normal data distribution.

Statistical processing of cohort 2: We carried out a statistic data processing with the use of Statgraphics Plus 5.1 software and R software 3.2.0. Normality of data distribution was verified with the use of Shapiro-Wilk test. Homoscedasticity was tested with Barlette's test. The data were analyzed using a one-way ANOVA.. Bonferroni posthoc test was used for all pairwise comparisons. KruskalWallis test with Turkey-Kramer post-hoc test was used for parameters that did not show a normal data distribution. To determine the dependence of vitamin $\mathrm{D}$ and complex diagnosis we used Pearson's chi-square test with Yates's correction.

Results: Statistic data relation: In the cohort 1 we have found statistically significant difference $(p<0.05)$ with the following: BMD femoral neck for homozygote ESR1, OPG, SOST and VDR(BmsI), next with BMD-L of spine for OPG and SOST, also with PTH for SOST. We find fractures to have a lower statistic relation with both polymorphisms LRP5 (Val 667 Met) where $p=0.023$, respectively LRP5 (Ala330Val) $p=0.052$ for fractures of types 5 and 6 . In the cohort 2 we do not find statistically relevant data, only those relations that are at lower levels, with homozygote for CTx in VDR (ApaI) $p=0.021$, in P1NP in OPG $p=0.007$ and CTX $p=0.037$ in OPG. In case of femoral neck fractures for VDR (TaqI) $p=0.018$ and VDR BsmI $p=0.021$ for fractures types 4,5 , and 6.

Conclusion: Our work aims to search for genetic parameters useful in clinical diagnostics and further treatment in personalised approaches. From the above declared data results we can utilize, with some limits, OPG, SOST and VDR (BmsI). We propose more potential in a way of evaluating combined genetic disorders. This may be the aim of further study.

\section{P290}

SALIVARY TESTOSTERONE AND 17-BETA ESTRADIOL IN SYSTEMIC LUPUS ERYTHEMATOSUS

A. Dima $^{1}$, B. Calenic ${ }^{2}$, E. Balanescu ${ }^{1}$, P. Balanescu ${ }^{1}$, M. Greabu $^{2}$, C. Baicus ${ }^{1}$

${ }^{1}$ Colentina Clinical Hospital, Colentina Research Center, Bucharest, Romania, ${ }^{2} \mathrm{UMF}$ Carol Davila, Bucharest, Romania 
Background: Imbalanced expression of sexual hormones, low androgen vs. high estrogen levels, might be involved in systemic lupus erythematosus (SLE) development and course. Methods: 33 female patients fulfilling the 2012 Systemic Lupus Collaborating Clinics (SLICC) SLE's criteria were enrolled. Furthermore, 5 male SLE patients, 5 female controls, and 5 male controls were included. SLE diseases activity was assessed by the Systemic Lupus Erythematosus Disease Activity Index (SLEDAI) score. Saliva collection was done around 8 am before eating or drinking in all subjects. Salivary testosterone (T2) and 17-beta estradiol (E2) were than determined using ELISA method (IBL International GMBH, Hamburg, Germany).

Results: The median (med), quartile 1 and 3 (q1; q3) female SLE patients' age of inclusion and disease duration were 51.0 $(39.0 ; 58.5)$, respectively $10.0(4.5 ; 14.5)$. Overall, salivary T2 and E2 levels were correlated ( $\mathrm{rs}=0.362 ; p=0.012)$. Also, salivary E2 (but not T2) correlated with patients' BMI ( $\mathrm{rs}=-0.004 ; p=0.004)$. Salivary E2 levels were not different in SLE females $[5.9(4.2 ; 29.9) \mathrm{pg} / \mathrm{mL}$ ] when compared with SLE male patients or respective female or male controls $(p>0.05)$. In regard with salivary T2, significant lower levels $[15.0(10.8 ; 63.1) \mathrm{pg} / \mathrm{mL}]$ were found in SLE female patients when compared with female controls $[78.3(56.2 ; 230.0 \mathrm{pg} /$ $\mathrm{mL}), p=0.015]$, but no difference was noted when compared with SLE male patients $[67.1(9.4 ; 182.1) \mathrm{pg} / \mathrm{mL}, p=0.271]$ or male control subjects [93.2 $(9.8 ; 269.4) \mathrm{pg} / \mathrm{mL}, p=0.897]$. Salivary T2 presented also lower levels in female patients known with renal involvement when compared with those without this SLE clinical feature $[13.3(8.0 ; 41.3)$ vs. 15.2 $(14.2 ; 91.1) \mathrm{pg} / \mathrm{mL}, p=0.036]$. Moreover, patients with low salivary T2 levels had higher daily corticosteroids doses at inclusion $[10.0(5.0 ; 20.0)$ vs. $5.0(0.0 ; 8.8)$ equivalence $\mathrm{mg}$ Prednisone, $p=0.031]$ and those with high salivary E2 levels presented with higher SLEDAI scores $[5.5(1.5 ; 13.5)$ vs. 0.0 $(0.0 ; 4.0)$ points, $p=0.040]$.

Conclusion: Significant lower salivary T2 levels are found in female SLE patients and this hormonal imbalance might be related also to renal involvement. Also, more active disease was observed in patients with high salivary E2.

\section{P291}

IMPACT OF NON-ACUTE VERTEBRAL FRACTURE IN CLINICAL AND EPIDEMIOLOGICAL FEATURES OF DORSAL AND BACK PAIN IN AN URGENCY ENVIRONMENT OF A TEACHING HOSPITAL IN MADRID.

C. A. Guillén-Astete ${ }^{1}$, M. A. Terán-Tinedo ${ }^{1}$

${ }^{1}$ Ramon y Cajal University Hospital, Madrid, Spain
Background: Lumbar and dorsal musculoskeletal pain is a major chief complain in A\&E and primary healthcare units worldwide. In people over 65 years old, many circumstances increase the frequency and intensity of back pain including osteoarthritis, less muscle mass and kyphosis. Acute vertebral fracture (VF) is also a cause of intense spine pain and a relevant cause of consultation due to its locomotive impairment and need of potent pharmacological analgesia. After treatment of the acute pain axial syndrome, those patients seem to complain more frequently and demands more intense pharmacological treatment, however this impression have not been sustained statistically yet.

Objective: To compare the frequency of healthcare demand and intensity of analgesic treatment of patients with lumbar or dorsal pain according to the existence or not of a vertebral fracture excluding the acute event.

Methods: A retrospective comparative study was conducted. Registries of patients who consulted in our A\&E department by lumbar or dorsal pain, older than 65 years old from November 2013 to November 2015 were identified using the electronic database of our institution. Those records were grouped according to the presence or absence of a known vertebral fracture. Records of patients who were diagnosed by the first time of vertebral fracture during the medical attention were excluded. No identifying data was retrieved from the database. Epidemiological and clinical information were gathered. Main outcome variables were number of consultations/year and type of treatment according to the WHO pain ladder.

Results: The inclusion criteria were achieved in 1528 registries. Among them, 76 registries were incomplete or unavailable. Of the remaining 1452 registries, 168 (11.5\%) presented a previously documented vertebral fracture (PDVF) and the remaining did not (nVF). Average age in the PDVF and $\mathrm{nVF}$ group was 75.9 SD 3.3 and 73.6 SD 4.2 years old, respectively $(P=0.0001)$. Proportion of women was higher than men in both groups $(85.9 \%$ and $60.9 \%$, respectively; $P=0.0001$ ). Distribution of time of onset of symptoms were $36.8 \%$ and $27.2 \%$ for less than a week $(P=0.0106), 34 \%$ and $26.8 \%$ for more than a week and less than two weeks $(P=0.0663)$, and $29.1 \%$ and $45.9 \%$ for more than a week $(P=0.0001)$ in both groups, respectively (Fisher's exact test). Proportion of patients previously on treatment with a third scale WHO-ladder drug was $11.9 \%$ in PDVF group and $3.04 \%$ in $\mathrm{nVF}$ group $(P=0.0001)$. Average number of consultations was $1.58 \mathrm{SD} 0.23$ consultations/year in PDVF group and $1.02 \mathrm{SD} 0.11$ consultations/year in nVF group (95\%CI of difference 0.5391-0.5809; 
$P=0.0001 ;$ T Student's test). The odds ratio of require a further consultation within a month of first assessment was 2.24 when PDVF group was compared with nVF group (CI95\% 1.54-3.26; $P<0.0001)$. At discharge, 42 $(25.0 \%)$ patients of PDVF group and $652(50.7 \%)$ of nVF group were sent to primary care units and 98 $(58.3 \%)$ patients of PDVF group and $210(16.3 \%)$ of nVF group were sent to secondary care units $(P=0.0001$ for both comparison, Fisher's exact test). The odds ratio of be discharged without need of further follow-up was 0.40 when compared PDVF and nVF group (CI95\% 0.26$0.62 ; P<0.0001) .53 .5 \%$ of PDVF patients and $23.4 \%$ of $\mathrm{nVF}$ patients were treated at discharge with $2^{\circ}$ scale WHO-pain ladder drugs $(P=0.0001) .29 .1 \%$ of PDVF group and $23.4 \%$ of nVF group were treated with $3^{\circ}$ scale WHO-pain ladder drugs $(P=0.0001)$. The odds ratio of be treated with major opioids at discharge was 78.02 when compared PDVF with nVF group (CI95\% 38.57161.86; $P<0.0001)$.

Conclusions: Patients with nonacute vertebral fractures have a higher index of urgent consultations per year, are more probably to be treated with major opioids and generates a higher burden of primary or secondary care appointments. As far as we know, this is the very first study which measures the impact of VF in an urgency environment. It seems that its relevance could justify the development and diffusion of protocols of lumbar or dorsal pain in patients with VF.

\section{P292}

EFFECT OF ALLOPURINOL AND FEBUXOSTAT ON FLARE-FREE SURVIVAL IN GOUTY PATIENTS ON OR AFTER PROPHYLACTIC TREATMENT WITH COLCHICINE: A RETROSPECTIVE KAPLAN-MEIER ANALYSIS C. A. Guillén-Astete ${ }^{1}$, M. Villarejo-Botija ${ }^{1}$, G. Silvestre-Egea ${ }^{1}$

${ }^{1}$ Ramon y Cajal University Hospital, Madrid, Spain

Background: Among inflammatory joint diseases, gout is a main cause of recidivism in urgency departments due to insufficient pain control or development of arthritis flares. This burden produces time and resources consumption and time of work off. New and old hypouricemic treatments are not only intended to control uric acid level below $6 \mathrm{mg} / \mathrm{dL}$ but to reduce the number of flares per year. It is well known that their activity removing uric deposits could trigger new flares so prophylactic treatment is always recommended when those treatments are initiated. There are many studies conducted to compare hypouricemic effectiveness with those treatments, however, their impact in flare-free survival is a topic not previously studied.

Objective: To compare the effectiveness of Allopurinol and febuxostat in terms of recidivism in patients with gout.

Methods: A descriptive retrospective study was performed. Electronic registries of patients diagnosed by gout and treated with allopurinol $(100-200 \mathrm{mg} / \mathrm{d}$ and $300-600 \mathrm{mg} / \mathrm{d})$ and febuxostat $(80 \mathrm{mg} / \mathrm{d}$ and $120 \mathrm{mg} /$ d) were included. Prophylaxis treatment was considered as positive in patients on treatment with colchicine 0.5 $1 \mathrm{mg}$ /día or NSAIDs according to the 2012 ACR guidelines for management of gout (Khanna et al. Arthritis Care Res 2012:64:1447). Follow-up period was 20132015 over a maximum of 6 months since hypouricemic treatment was started. Data recovered from electronic registries were: demographic and clinical data, number of consultations and work off time where appropriated. Comparisons were made according to hypouricemic treatment and colchicine prophylaxis treatment.

Results: 90 registries of different patients were identified: 18 were under treatment with febuxostat $(14 \mathrm{on} 80 \mathrm{mg} /$ day and 3 on $120 \mathrm{mg} /$ day) and 72 under treatment with allopurinol (22 on $100-200 \mathrm{mg} /$ day, 50 on $300-600 \mathrm{mg} /$ day). Difference on average of age, proportion of males and proportion of tophus were not statistically significant between both groups. Mean of time until the first flare after starting treatment with allopurinol was 132.4 SD 6.8 days (CI95\% 119.0-145.8) and 142.6 SD 11.63 (CI95\% 119.8-165.4) with febuxostat (Chi-squared 0.304; $P=0.581$, Hazard ratio 0.806 ; $95 \% \mathrm{CI} 0.3935-1.654)$. Excluding patients without prophylaxis treatment ( 2 on febuxostat and 26 on allopurinol), the mean of time until the first flare was 116.4 SD 8.0 days (CI95\% 100.7-132.1) in the group on allopurinol and 153.8 SD 9.9 days (CI95\% 134.3-173.3) in the group on febuxostat (chi-squared 5.984; $P=0.0144$, hazard ratio 0.356 (CI95\% 0.183-0.692). No differences statistically significant were observed when Kaplan Meier curves were built groping patients according to the doses of allopurinol or febuxostat.

Conclusion: Although our retrospective cohort is not large, our observations point that there is a tendency to higher surveillance without in gouty patients on febuxostat than on allopurinol. This tendency becomes statistically significant when only patients on colchicine treatment were compared, so it seems that prophylaxis treatment benefits specially this group of patients. However, larger observational studies should be conducted in order to establish a general recommendation on prolongation of colchicine prophylactic/concomitant therapy. 


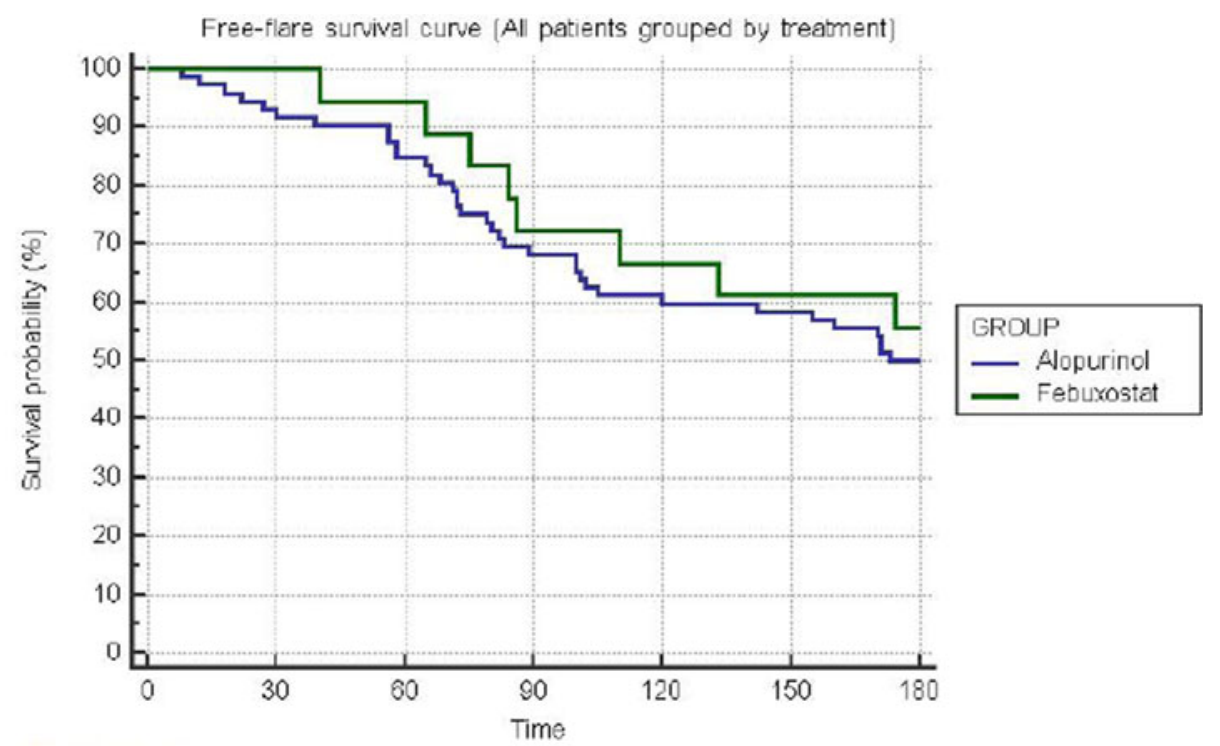

Number at risk

Group: Alopurino

$\begin{array}{lllllll}72 & 66 & 61 & 49 & 43 & 42 & 0\end{array}$

Group: Febuxostat

18

$17 \quad 13$

12

$11 \quad 0$

Free-flare survival curve (Only patients on prohylaxis treatment, both groups)

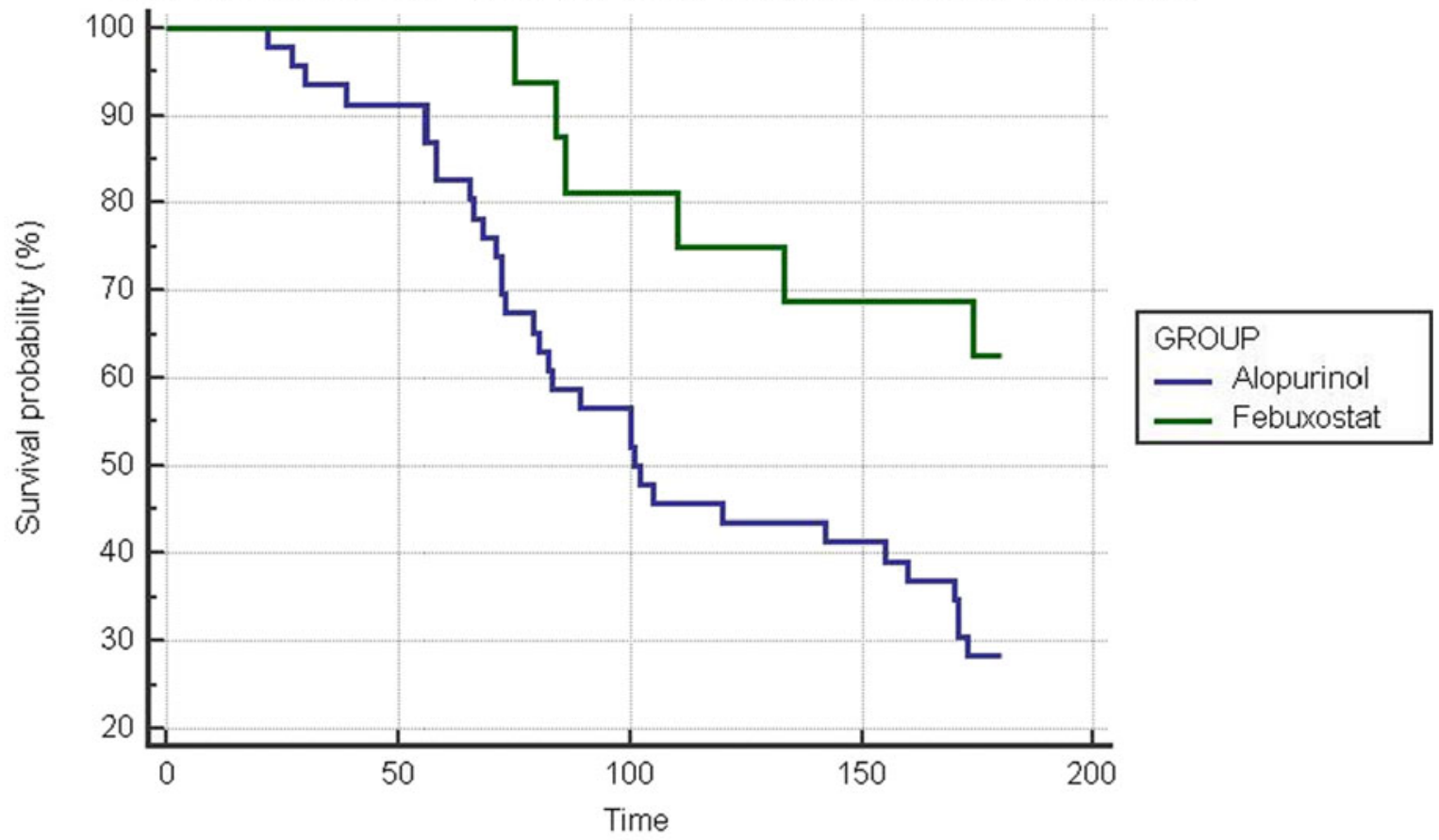

Number at risk

Group: Alopurinol

46

Group: Febuxostat

16

42

24

19

0

13

11

0 


\section{P293}

TREATMENT OF IBANDRONIC ACID ASSOCIATED WITH VITAMIN K2 IN OSTEOPOROSIS FROM DELAYED PUBERTY S. A. Preda ${ }^{1}$, M. Bistriceanu ${ }^{2}$, I. Bistriceanu ${ }^{3}$, D. M. Albulescu ${ }^{4}$, C. Constantin $^{4}$, O. M. Marioara ${ }^{5}$, A. Turculeanu ${ }^{6}$, A. Covei ${ }^{7}$, A. Camen ${ }^{1}$ ${ }^{1}$ Faculty of Dentistry, University of Medicine and Pharmacy, Craiova, Romania, ${ }^{2}$ Department of Endocrinology, University of Medicine and Pharmacy, Craiova, Romania, ${ }^{3}$ Department of Endocrinology, Emergency Hospital, Craiova, Romania, ${ }^{4}$ Department of Radiology, University of Medicine and Pharmacy, Craiova, Romania, ${ }^{5}$ University of Medicine and Pharmacy, Craiova, Romania, ${ }^{6}$ Biochemical Department, University of Medicine and Pharmacy, Craiova, Romania, ${ }^{7}$ Endocrinology Department, Philanthropy Hospital, Craiova, Romania

Discovering frequently feminine cases with delayed puberty (hypogonadotropic or hypergonadotropic hypogonadism), is motivating the therapeutically broaching of hypogonadism osteoporosis that is installed more precocious compared with menopause. Objectives: Gonadal insufficiency diagnosis and its etiology, the BMD evaluation, initiating differentiated therapeutically measures by rapport with the evolutive stage of bone mass deficit (osteoporosis/osteopenia) and with hypogonadism etiology.

Material and Methods: Were included in the study 18 cases with delayed feminine puberty with ages 17 to 24 .At all cases BMD was evaluated by DXA. The therapeutically options were aiming to nonpharmacological undertake: a diet with a positive level of calcium and vitamin $\mathrm{D}$, modifying the lifestyle and easy physical exercises. Pharmacological therapy was applied at cases with osteoporosis which administrated antiresorptive agent ibandronic acid in $150 \mathrm{mg}$ doses at 30 days) in association with $45 \mathrm{mg}$ vitamin $\mathrm{K} 2$ per day.

Results: Osteoporosis was confirmed at 8 cases. The efficiency of the treatment with ibandronic acid in association with vitamin $\mathrm{K} 2$, after 12 months of therapy, was superior then the separately bisphosphonates therapy. It was remarked an increase of BMD with $4.4 \%$ at lumbar spine level and with $3.6 \%$ at femoral cervix level compared to 4.2 and $2.1 \%$ reported for ibandronic acid.

Conclusions: The study is confirming the efficiency association of ibandronic acid with vitamin $\mathrm{K} 2$ in delayed feminine puberty.

\section{P294}

STUDY OF BIOCHEMICAL MARKERS OF BONE TURNOVER AND BONE MINERAL DENSITY IN TURNER SYNDROME

S. A. Preda ${ }^{1}$, M. Bistriceanu ${ }^{2}$, I. Bistriceanu ${ }^{3}$, D. M. Albulescu ${ }^{4}$, C. Constantin $^{4}$, O. M. Marioara ${ }^{5}$, A. Turculeanu ${ }^{6}$, A. Covei ${ }^{7}$, A. Camen ${ }^{8}$

${ }^{1}$ Student Faculty of Dentistry, University of Medicine and Pharmacy, Craiova, Romania, ${ }^{2}$ Department of Endocrinology, University of Medicine and Pharmacy, Craiova, Romania, ${ }^{3}$ Department of Endocrinology, Emergency Hospital, Craiova,
Romania, ${ }^{4}$ Department of Radiology, University of Medicine and Pharmacy, Craiova, Romania, ${ }^{5}$ University of Medicine and Pharmacy, Craiova, Romania, ${ }^{6}$ Biochemical Department, University of Medicine and Pharmacy, Craiova, Romania, ${ }^{7}$ Endocrinology Department, Philanthropy Hospital, Craiova, Romania, ${ }^{8}$ Faculty of Dentistry, University of Medicine and Pharmacy, Craiova, Romania

The gonadal dysgenesis is the definition of all formation failings of embryonic gonad. Sexual chromosomes have an important role in the etiology of gonadal dysgenesis because is conditioning the development of the gonad and implicit of enzymatic battery which is ensuring a biosynthesis of sexual hormones. Transformation of gonadogenesis process (the morphological dysgenesis) has negative impacts: perturbation of hormonal biosynthesis process, alteration of structure reactivity on gonadic hormones and, under clinical aspect, the perturbing of sexualisation process. Sexual hormones exclusion from the organism economy is seriously influencing the bone structure, being the major cause of osteoporosis.

Objectives: Identification of cases with Turner syndrome with feminine phenotype, hormonal evaluation on gonadotropic axis and the study of BMD and biochemical markers of bone turnover. Material and Methods: The study were performed on 17 cases with Turner syndrome with ages between 12-18 years. In the same time with karyotype study of ovarian (estradiol, progesterone) and gonadotropic hormones (LH, FSH) were evaluated the biochemical markers of bone turnover (serum osteocalcin and CrossLaps) and BMD were appreciated by DXA.

Results: It was highlighted osteoporosis in 9 cases, osteopenia in 4 cases ( $T$-score $=-2.5 \mathrm{DS}$ ), and for the remnants patients (4) biochemical markers and mineral bone density were in normal limits. Conclusions:

1. The study of biochemical markers of bone turnover and mineral bone density is mandatory for all cases with Turner syndrome cytogenetic confirmed.

2. The precocious diagnosis of osteoporosis/osteopenia, is claiming the estro-progestative hormonal substitution which represent the therapeutically attitude from the main intention.

3. Hormonal substitution associated with therapeutically means specific to bone remineralisation is preventing the apparition of fragility fractures.

\section{P295}

EVALUATION OF BIOCHEMICAL MARKERS OF BONE TURNOVER IN PREMATURE OVARIAN INSUFFICIENCY

S. A. Preda ${ }^{1}$, M. Bistriceanu ${ }^{2}$, I. Bistriceanu ${ }^{3}$, D. M. Albulescu ${ }^{4}$, C. Constantin $^{4}$, O. M. Marioara ${ }^{5}$, A. Turculeanu ${ }^{6}$, A. Covei ${ }^{7}$, A. Camen ${ }^{1}$

${ }^{1}$ Faculty of Dentistry, University of Medicine and Pharmacy, Craiova, Romania, ${ }^{2}$ Department of Endocrinology, University of Medicine and Pharmacy, Craiova, Romania, ${ }^{3}$ Department of Endocrinology, Emergency Hospital, Craiova, Romania, 
${ }^{4}$ Department of Radiology, University of Medicine and Pharmacy, Craiova, Romania, ${ }^{5}$ University of Medicine and Pharmacy, Craiova, Romania, ${ }^{6}$ Biochemical Department, University of Medicine and Pharmacy, Craiova, Romania, ${ }^{7}$ Endocrinology Department, Philanthropy Hospital, Craiova, Romania

The follicular dower is much reduced in premature ovarian insufficiency or the syndrome of pauper ovaries, resulting the deficiency of ovarian hormonogenesis. The lower number of ovarian follicles is inducing the ovary's life span, making the clinical spectrum to be characterized through the absence of puberty sexuality total or partial until the precocious installation of climacterium.

Objectives: The identification of ovarian failure secondary amenorrhea cases caused by premature ovarian insufficiency. Is demonstrated that the perturbation of hormonal secretion which controls the bone homeostasis, ratio bone formation-resorption is damaged and thus, bone mass decreases and causes osteoporosis. Material and Methods: The study was performed on 68 patients whose ages ranged from 19 to 44 years. Hormonal investigations focused on the study of FSH, LH, PRL, estradiol, progesterone and also on the biochemical markers of bone turnover (osteocalcin and CrossLaps). BMD was measured by DXA.

Results: Hormonal doses showed low levels of estradiol and progesterone, instead of gonadotropic that hormones were above the normal upper limit between $210-390 \mathrm{mUI} / \mathrm{ml}$. There were also showed low levels for biochemical markers of bone turnover at half of the studied cases. BMD measurements revealed the presence of osteoporosis at 29 cases which represents $42.6 \%$ and 5 cases with osteopenia. BMD values correlate with biochemical markers of bone turnover.

\section{Conclusions:}

1. Evaluation of BMD and biochemical markers of bone turnover in premature ovarian failure must be done regularly after amenorrhea appearance, to identify patients who rapidly lose bone mass and are at increased risk of osteoporosis/osteopenia.

2. Estrogen-progesterone substitution is the main and first treatment in premature ovarian failure to prevent osteoporosis/osteopenia, metabolic and visceral complications.

3. Patients with osteoporosis will receive antiresorptive agents or proformative medication to prevent fragility fractures.

\section{P296}

COLLAGEN INJECTIONS GUNA MDS FOR TREATING UPPER BACK PAIN DUE TO OSTEOPOROTIC VERTEBRAL FRACTURES

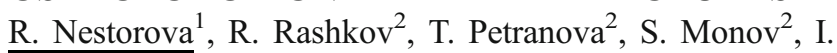
Sheytanov $^{2}$

${ }^{1}$ Rheumatology Centre "St. Irina", Sofia, Bulgaria, ${ }^{2}$ Clinic of Rheumatology, Medical University, Sofia, Bulgaria

Aim: To evaluate the effectiveness of collagen injections GUNA MDs regarding back pain in patients with osteoporosis vertebral fractures in the thoracic region of the spine.

Methods: We studied 15 patients with osteoporosis and compression fractures on regular antiosteoporosis treatment, started it at least 1 year before the screening visit. All patients were with back pain, proved osteoporosis (lumbar BMD) and X-ray of the thoracic spine with vertebral fractures. Clinical assessment included demographic and clinical data, neurological exam, a visual analog scale (VAS) for pain (0-100) and Quality of life Questionnaire (IOF) on the baseline, 60th day and 180th day. Evaluations of the efficacy according to the patient and the physician were performed. We applied collagen injections "GUNA MDs-Thoracic" into the most painful points of paravertebral muscles in a total course of treatment 24 weeks, following the scheme: first 2 weeks -2 applications per week, 6 weeks -1 application per week, and last 16 weeks with a single injection every 2 weeks. Results: Pain was significantly reduced and quality of life improved. $70 \%$ of patients gave a very good and good assessment of efficacy, which coincided with the opinion of the physician. Conclusions: The collagen injections GUNA MDs are an innovative and effective approach with analgesic effect in the treatment of back pain due to osteoporosis fractures, thereby increasing the quality of life. The easy application and total absence of side effects makes them a modern device of choice in daily practice of the physician.

\section{P297}

RESEARCH OF POPULATION KNOWLEDGE ON OSTEOPOROSIS AS THE BASIS OF FORMATION OF EDUCATIONAL PROGRAMS

M. A. Dobritsyna ${ }^{1}$, L. A. Marchenkova ${ }^{1}$, M. Y. Gerasimenko ${ }^{1}$ ${ }^{1}$ Federal State Budgetary Institution Russian Scientific Center of Medical Rehabilitation and Balneology of the Russian Ministry of Health, Moscow, Russian Federation

Aim: To study of the awareness of the broad masses population about the importance, clinical presentation and prevention of osteoporosis (OP) and fractures.

Materials and methods: A questionnaire survey of a random sample of 2018 (1712 women and 306 men) respondents without OP aged 20-93 years. The questionnaire included 10 points with answers «Yes» or «No» on general issues, OP prevention and diagnosis. In addition to the "knowledge level test" everyone answer a question "From where you receive information on osteoporosis and methods of its prevention?"

Results: Among the respondents, the level of knowledge on OP in women was higher than in men, speaking of the questionnaire as a whole, as well as of all 10 items separately $(p<0.001)$. There was found a significant non-monotone dependence of knowledge level in OP on age with the peak in the age group of 40-59 $\mathrm{y}: \mathrm{H}(6$, $n=2013)=120.57, p<0.0001)$. Awareness in the field of OP was significantly affected by the level of education - the ratio of gamma 
correlation $\mathrm{g}=0.039(\mathrm{Z}=-15.7), p<0.000001$. There was found a statistically significant dependence of knowledge level on OP on employment: $\mathrm{H}(4, n=1711)=45.97, p=0.0001$. The main share of respondents $(38.9 \%)$ called television as used information source in OP, $31 \%$ of ones noted that receive information on OP from the attending physician. Most respondents less often for replenishment of the knowledge of OP used the internet in $9.6 \%$ of cases or any other sources of information in $9.3 \%$ of cases.

Conclusions: Results of the research can be used as the basis of for formation of educational programs on OP for the population and indicate the need for broad implementation them in Russian Federation.

\section{P298}

IMMEDIATE EFFECTIVENESS OF SCAPULAR REPOSITIONING WITH ACTIVE CERVICAL ROTATION IN ACUTE SPASMODIC TORTICOLLIS S.-K. Khatri ${ }^{1}$

${ }^{1}$ APJ Abdul Kalam College of Physiotherapy, Ahmednagar, India

Objectives: To investigate the immediate effects on pain and pressure pain threshold (PPT) of a scapular repositioning technique in patients with acute spasmodic torticollis.

Methods: A randomized, single blind pilot study was conducted. The subjects were 23 individuals (age 20-40 years) with a clinical diagnosis of spasmodic torticollis. Visual analog scale pain score, cervical active ranges of motion, and PPT were assessed before and after the intervention. The comparison group was treated with only conventional physiotherapy (microwave diathermy, submaximal isometrics, and ergonomic advice). The intervention group was given scapular repositioning, active cervical rotation technique, in addition to conventional physiotherapy treatment.

Results: There were significant improvements in intensity of pain ( $\mathrm{P} \mathrm{b} .01)$, cervical rotation to the ipsilateral side $(\mathrm{P} \mathrm{b} .01)$, cervical side flexion to the contralateral side (P b.01), and PPT (P b.01) immediately after the treatment of the scapular repositioning and conventional therapy compared with the conventional therapy alone.

Conclusion: The present pilot study demonstrated that scapular repositioning may have an immediate hypoalgesic effect on individuals with spasmodic torticollis in terms pain severity, PPT, and cervical range of motion. Therefore, further controlled trials are warranted.

\section{P299}

EFFECTIVENESS OF HYALURONIC ACID "OSTENIL TENDON" IN PARTIAL THICKNESS ROTATOR CUFF TEARS OF THE SHOULDER

R. Nestorova ${ }^{1}$, P. Todorov ${ }^{2}$, T. Petranova ${ }^{3}$, Z. Kolarov ${ }^{3}$

${ }^{1}$ Rheumatology Centre, Sofia, Bulgaria, ${ }^{2}$ Clinic of Rheumatology, Medical University, Plovdiv, Bulgaria, ${ }^{3}$ Clinic of Rheumatology, Medical University, Sofia, Bulgaria
Objective: To ascertain the level and duration of the effectiveness of hyaluronic acid (HA) injections "Ostenil Tendon" regarding pain, functional activity and tendon repair in patients with partial thickness tears (PTT) of the rotator cuff (RC) of the shoulder. The accuracy of musculoskeletal ultrasound (US) for diagnosis of PTT of the RC, benefit of performance of USguided procedures and monitoring of therapy have been proved. Material and Methods: 31 patients with painful shoulder(VAS $>40 \mathrm{~mm}$ and duration of symptoms up to 7 days) and sonographically proved PTT of the RC were included. A Pain Diary with a ten point visual analogue scale (VAS), Shoulder function assessment (SFA) scale (0-70) and questionnaires of the efficacy according to the patient and the physician were evaluated. US examination was provided by Mindray M5 scanner with multi-frequency linear transducer (7.5-12.0 MHz). HA “Ostenil Tendon" $40 \mathrm{mg} / 2.0 \mathrm{ml}$ was injected around the affected tendon once a week for a total of two injections. All applications were performed under US control. Two and six months after the baseline were performed VAS, SFA and US of the RC.

Results: Pain was significantly reduced after the first injection and this effect was maintained until the end of the observational period of six months. SFA Index was significantly improved. $74.2 \%$ of patients gave a very good and good assessment of the efficacy, which coincided with the opinion of the physician. $77.4 \%$ of patients had a complete recovery or improved structure of the SSP which was US demonstrated by two independent sonographers.

Conclusions: HA "Ostenil Tendon" resulted in a relatively rapid and sustained relief of pain and improvement in the functional capacity of the shoulder. Thanks to lubricating and viscoelastic properties of HA, RC tendons with incomplete lesions showed improved or restored structure, which was sonographically proved. No adverse events were observed. Injections under MSUS control were received favorably by patients.

\section{P300 \\ RELATIONSHIP BETWEEN LEPTIN AND BONE MINERAL DENSITY IN OBESE SUBJECTS IN THE HOSPITAL SAN JOSÉ OF BOGOTÁ}

A. S. Sierra ${ }^{1}$, A. M. Medina ${ }^{1}$, R. R. Rosero ${ }^{1}$, W. R. Rojas ${ }^{1}$, C. C. Castro ${ }^{1}$, A. E. Ely ${ }^{1}$

${ }^{1}$ Hospital San José, Bogotá, Colombia

Background and Objective: To establish the correlation between leptin levels and BMD in obese patients in the Obesity Program, of the Department of Endocrinology, Hospital San José of Bogotá.

Methods: A cross-sectional study was conducted, in patients aged $18-75$ years with $\mathrm{BMI} \geq 30 \mathrm{~kg} / \mathrm{m} 2$. Anthropometric and 
laboratory parameters (leptin and bone densitometry) were determined. A descriptive analysis of the information and correlation between variables through the Pearson correlation coefficient was performed.

Results: 90 patients with an average age of 48 years to leptin $45 \mathrm{ng} / \mathrm{ml}$ and BMI $36.8 \mathrm{~kg} / \mathrm{m} 2$ were analyzed. The Pearson correlation coefficients between leptin and BMD were for lumbar spine (LS) of -0.03 for femoral neck (FN) of -0.18 and total hip (TH) of -0.25 . Anova statistical test was applied to the of obesity and BMD in CL, CF, CT, with significant results in FN $(p=0.01)$ and TH $(p=0.0009)$. In the multiple linear regression model statistically significant associations between BMD and leptin TH, with a coefficient of $-0.002(p=0.017)$ were found. Conclusion: Our study found a weak relationship between leptin levels in obese patients and its effect on BMD in $\mathrm{TH}$ adjusted by degree of obesity.

\section{P301}

\section{SERUR MYOKINES LEVELS IN PATIENTS} WITH ENDOGENOUS CUSHING'S SYNDROME AND ACROMEGALY

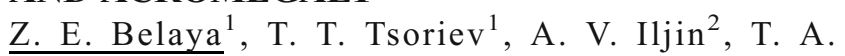
${\text { Grebennikova }^{1}, \text { L. Y. Rozhinskaya }}^{1}$, G. A. Melnitchenko ${ }^{3}$

${ }^{1}$ Neuroendocrinology and Bone Diseases, Moscow, Russian Federation, ${ }^{2}$ Biochemistry and Hormonal Assays, Endocrinology Research Centre, Moscow, Russian Federation, ${ }^{3}$ Neuroendocrinology and Bone Diseases, Endocrinology Research Centre, Moscow, Russian Federation Myokines are produced and released by muscle cells in response to muscular contractions. Endogenous Cushing's syndrome (CS) and acromegaly cause significant changes in muscle tissue leading to atrophy or hypertrophy. However, there is no data whether these endocrine abnormalities influence myokine secretion.

Objective: To evaluate serum levels of myostatin, IL-6 and irisin in patients with CS and acromegaly.

Materials and Methods: fasting serum samples were taken and stored in aliquot at $\leq-20{ }^{\circ} \mathrm{C}$ from consecutive subjects with clinically evident and biochemically confirmed active CS, acromegaly and healthy volunteers matched by age, sex and BMI. None of the participants attended any specific physical training. Myostatin ELISA kit Immunodiagnostic AG, IL6 (eBioscience BMS 213HS) and irisin E_ENG.001.B ELISA kit were used to assay serum myokine levels. Grip strength was measured by dynamometer. IGF1 was measured by immunochemiluminescence assay (Liaison) reference range (60-280 ng/ml). $24 \mathrm{~h}$ urine free cortisol (24hUFC) was measured by an immunochemiluminescence assay on a Vitros ECi (60-413 nmol/24 h). One-way ANOVA was utilized to assess the difference between groups.

Results: We enrolled 88 subjects (30 patients suffered from CS (group 1), 28 - acromegaly (2) and 30 matched healthy controlled (3)) in total 51 females and 37 males ( $p=0.492$ among groups), the mean age was 38 years ( $95 \%$ confident interval $(\mathrm{CI})$ $35-41$ ) with no difference among the groups $p=0.062$; BMI 27 (95\%CI 26-28) kg/m2 $p=0.174$. Mean 24hUFC in subjects with CS - 2887 (1285-4489) nmol/24 h, mean IGF1 in subjects with acromegaly $803(688-917) \mathrm{ng} / \mathrm{ml}$ were significantly higher as compared to other groups $(p<0.001)$. Right hand grip strength was lower in patients with CS - 32 (27-36) daN as compared to both patients with acromegaly - $40(34-45) \mathrm{daN}$ and healthy subjects $-40(32-38)$ daN $(p=0.04)$.

However, among these young adults we did not find statistically significant differences in measured myokines levels: myostatin: (1) 22.7 (19.3-26.1); (2) 20.1 (17.2-23.0); (3) 22.5 (18.5-26.6) $\mathrm{ng} / \mathrm{ml} p=0.50$; IL6: (1) 1.7 (0.7-2.7), (2) 0.89 (0.54-1.24), (3) $1.2(0.3-2.0) \mathrm{pg} / \mathrm{ml} p=0.34$; irisin: (1) 3.0 (2.6-3.4), (2) 2.5 (1.9-3.1), (3) $3.4(2.6-4.1) \mu \mathrm{g} / \mathrm{ml} p=0.15$.

Conclusion: Hypercortisolism or supraphysiological IGF1 levels do not significantly influence serum levels of myostatin, IL6 and irisin in young adults.

Acknowledgements: МД-3332.2015.7

\section{P302}

THE IMPACT OF FUNCTIONAL DISABILITY TO FATIGUE IN PATIENTS WITH RHEUMATOID ARTHRITIS

J. Jovanovic $^{1}$, V. Jovanovic ${ }^{2}$, M. Karadzic ${ }^{3}$, S. Milenkovic ${ }^{3}$, V. Skakic ${ }^{3}$, I. Aleksic ${ }^{3}$

${ }^{1}$ Institute for Treatment and Rehabilitation Niska Banja, Nis, Serbia, ${ }^{2}$ Clinic for Orthopedics, Clinical Centre, Nis, Serbia

Introduction: Rheumatoid arthritis (RA) is an autoimmune disease characterized by chronic symmetric polyarthritis causing progressive joint destruction and disability. Major patients complaints are pain, disability and fatigue.

Objective: Investigate impact of functional disability to fatigue in patients with RA.

Material and Method: A prospective study included 109 patients with random sample method for patients treated in in Niška Banja Institute. The average age of patients was $58.86 \pm 9.54$ and the average disease duration was $10.74 \pm 7.43$ godine. Functional disability is represented with HAQ questionnaire filled in by patients themselves. Based on the obtained values they are classified in three groups: I group with HAQ value score of $0.125-1.000$ indicate modest disability, II group with HAQ value score of 1.125-2.000 indicate more severe disability and III group with HAQ value score of 2.125-3.000 indicate a complete functional disability. Fatigue was measured by questionnaires for fatigue evaluation: Fatigue Facit Scala (FFS) and visual analog scale of fatigue (VAS of Fatigue). Comparison of numerical variables classified according to the type of normality was performed by ANOVA test. Statistical significance was at the level of $<0.05$. 
Results: Average value of FFS, in the group of patients with total functional disability was $13.02 \pm 5.65$, that was statistically significantly worse than in the group of patients with more severe disease and $21.01 \pm 7.62$, $(p<0.001)$ and in the group with moderate functional disability $32.29 \pm 7.27,(p<0.001)$. FFS was statistically significantly better with the patients with moderate functional disability compared to the patients with more severe disability $(p<0.001)$. Average value fatigue by estimated VAS of Fatigue, in the group of patients with complete functional disability accounted for 75.38 \pm 13.26 , that was statistically more significantly worse than in the group of patients with more severe disability $65.42 \pm 18.59,(p<0.001)$ and in the group of patients that had moderate functional disability $40.23 \pm 18.87$. FFS was statistically much better with the patients with moderate functional disability in regard to patients with more severe disability $(p<0.001)$.

Discussion: Fatigue is a common symptom of RA, but it is not included in the disease activity core set measures and indices in RA. Fatigue is a subjective phenomenon and assessed by individual self-report. Various selfreport measures have been developed to evaluate fatigue in chronic diseases. It was suggested as a useful instrument for assessment of fatigue.

Conclusion: Our results have confirmed the hypothesis that functional disability has adverse impact on fatigue. Fatigue should be included in clinical practice and clinical trials as a RA outcome measure.

\section{P303}

BONE MICROARCHITECTURE AND BONE MINERAL DENSITY IN PATIENTS WITH CHRONIC RENAL FAILURE

D. Monova $^{1}$, S. Monov ${ }^{2}$, D. Mluchkova ${ }^{3}$, M. Stambolova ${ }^{1}$, E. Peneva ${ }^{1}$

${ }^{1}$ Department of Internal Diseases, Medical Institute, Sofia, Bulgaria, ${ }^{2}$ Department of Rheumatology, Medical University, University Hospital “St. J. Rilski”, Sofia, Bulgaria, ${ }^{3}$ Department of Radiology, Medical Institute, Sofia, Bulgaria

Objective: Fractures are common in patients with chronic kidney disease (CKD) and associated with substantially high morbidity, mortality and economic costs. Bone mass measurements are commonly used to assess fracture risk in the general population, but the utility of these measurements in patients with CKD, and specifically among those on hemodialysis, is unclear. CKD affects bone quality through changes in bone turnover, microarchitecture, and mineralization. The aim of this study was to assess if volumetric BMD (vBMD) and microarchitecture could be impaired early in the course of
CKD. We compare BMD and microarchitectural parameters in patients II-IV CKD stages and controls.

Material and Methods: We examined 47 patients with CKD II-IV stage (21 men and 26 women; mean age $65.6 \pm 9.2$ years, mean glomerular filtration rate $36 \pm 11 \mathrm{~mL} / \mathrm{min}$ per $1.73 \mathrm{~m} 2$, mean serum PTH level $84 \pm 54 \mathrm{pg} / \mathrm{mL}$ ) and 43 age- and sexmatched controls with estimated glomerular filtration rate (eGFR) $\geq 60 \mathrm{ml} / \mathrm{min} / 1.73 \mathrm{~m} 2$. We used DXA at the spine, hip and radius to measure BMD. Bone microarchitecture was examined with a noninvasive $3 \mathrm{D}$ imaging technique (HR-pQCT) at the tibia and radius.

Results: We found that CKD patients had lower BMD measured by both techniques compared to controls, but by DXA this was only significant at the hip. At the tibia, total $\mathrm{VBMD}$ was significantly lower in CKD patients compared to controls and there was a trend towards lower cortical and trabecular vBMD, but this was not significant. At the distal radius, total $\mathrm{VBMD}$ was lower in CKD patients, but trabecular (tb) vBMD was significantly lower compared to controls $(169 \pm 38$ vs. $189 \pm 35 \mathrm{mg} \mathrm{HA} / \mathrm{cm})$. Tb number was reduced $(1.71 \pm 0.24$ vs. $1.92 \pm 0.27 \mathrm{~mm})$, and $\mathrm{Tb}$ separation was increased $(514 \pm 89$ vs. $458 \pm 81 \mu \mathrm{m})$. Meanwhile, cortical vBMD in CKD at this site was similar to controls.

Conclusion: Our data suggests that CKD is associated with different effects in trabecular and cortical bone, but DXA is unable to effectively differentiate these bone compartments. HR-pQCT can overcome this limitation. Decreased bone mass and disruption of microarchitecture occur early in the course of $\mathrm{CKD}$ and worsens with the progressive decline in renal function. This study, using a noninvasive bone-imaging device, shows an early impairment of trabecular microarchitecture in stage II-IV CKD patients. HR-pQCT is a useful research tool in detecting bone changes in CKD although this needs to be verified in larger longitudinal studies and with bone histomorphometry.

\section{P304 \\ COMPARISON OF CONVENTIONAL PHYSICAL THERAPY MODALITIES AND KINESIOTAPING THERAPIES' EFFECTS ON ACTIVE RANGE OF MOTION IN THE SHOULDER IMPINGEMENT SYNDROME}

A. Kul $^{1}$, K. Yildirim ${ }^{1}$

${ }^{1}$ Physical Medicine and Rehabilitation, Faculty of Medicine, Erzurum, Turkey

Background: Shoulder impingement syndrome is one of the most common shoulder disorders which characterized by shoulder pain that is exacerbated with arm elevation or overhead activities, in adults, with a high socioeconomic impact on working ability. The aim is to stop the inflammatory process and prevent the progressive pathological variations with treatment. Otherwise, surgical treatment would be unavoidable. 
Objective: The study assessed the effects of conventional physical therapy modalities (PM) and of kinesiotaping (KT) therapy on the shoulder active range of motion (ROM) in the patients with impingement syndrome.

Material and Method: Diagnosed clinically and radiologically with impingement syndrome but with no total rupture, a total of 40 patients were randomly grouped into two equal parts and included in the study (According to Neer Staging, stage 2-tendinitis- 15 patients and stage 3-partial rupture- 25 patients). While KT during the course of 15 days ( 3 times in total every 5 days) was applied to supraspinatus muscle with inhibition technique and to deltoid muscle with stimulation and mechanical correction techniques and physical therapy modalities of fifteen séances, Hot-Pack, USG, TENS, were applied to the second group (once a day). Both groups were given home-based exercise programs. Prior to the treatment (PT), after the treatment (AT), and one month after the treatment (T3), Shoulder active ROMs were measured with a standard goniometry. Freidman and Wilcoxon test was used in intragroup measures, and Mann Whitney $U$ test in intergroup comparisons.

Results: A statistically meaningful difference was established between intra-group measure scores in both groups $(p<0.05)$. In both groups, after the treatment, a meaningful improvement was observed in active ROM values $(p<0.001)$. After the treatment, In the intergroup comparison, except for the T2T3 active extension value $(p<0.05)$, in period of PT-AT, AT$\mathrm{T} 3$ and PT-T3, there was no significant between the active ROM values $(p>0.05)$.

Conclusion: The study has shown that KT exercise treatment is as effective as FM+ exercise treatment in terms of the improvement of shoulder active rom. Therefore, we believe that KT application provides a remarkable benefit in the treatment. It should be examined whether KT alone is effective in the treatment of IS, adding KT group alone.

\section{P305}

VISCERAL FAT MEASURED BY DXA IS ASSOCIATED WITH INCREASED RISK OF NONSPINE FRACTURES IN NONOBESE ELDERLY WOMEN: A POPULATION-BASED PROSPECTIVE COHORT ANALYSIS FROM THE SÃO PAULO AGEING \& HEATHY (SPAH) STUDY

L. Machado ${ }^{1}$, R. M. Pereira ${ }^{1}$, D. Domiciano ${ }^{1}$, C. Figueiredo ${ }^{1}$, J. Lopes ${ }^{1}$, V. Caparbo $^{1}$, L. Takayama ${ }^{1}$, R. Oliveira ${ }^{2}$, P. Menezes $^{3}$

${ }^{1}$ Faculdade de Medicina da Universidade de São Paulo, Rheumatology Division, São Paulo, Brazil, ${ }^{2}$ RDO Diagnósticos Médicos, São Paulo, Brazil, ${ }^{3}$ Faculdade de Medicina da Universidade de São Paulo, Department of Preventive Medicine, São Paulo, Brazil
Objective: The aim of this study was to investigate the association of visceral fat measured by DXA with the incidence of nonspine fractures in community-dwelling elderly women.

Material and Methods: This is a longitudinal prospective population-based cohort study evaluating 433 communitydwelling women aged 65 years or older. A specific clinical questionnaire, including personal history of fragility fracture in non-spine osteoporotic sites, was performed at baseline and after an average of 4.3 years. All incidences of fragility fractures during the study period were confirmed by affected site radiography. Visceral adipose tissue (VAT) was measured in the android region of a whole body DXA scan.

Results: The mean age was $72.8 \pm 4.7$ years, and 28 incident nonspine osteoporotic fractures were identified after a mean follow-up time of $4.3 \pm 0.8$ years. According to the Lipschitz classification for nutritional status in the elderly, $38.6 \%$ of women were nonobese $\left(\mathrm{BMI} \leq 27 \mathrm{~kg} / \mathrm{m}^{2}\right)$ and $61.4 \%$ were obese/overweight. Logistic regression models were used to estimate the relationship between VAT and nonspine fractures in elderly women. After adjusting for age, previous fracture and BMD (significant parameters at univariate analysis), VAT (mass, area, volume) had a significant association with the incidence of non-spine fractures in nonobese elderly women (VAT mass: RR:1.49 [95\%CI, 1.22-1.81; $p=0.008]$ ), (VAT area: RR:1.19 [95\%CI, 1.04-1.37; $p=0.007]$ ), (VAT volume: RR:1.34 [95\%CI, $1.10-1.64 ; p=0.007]$ ).

Conclusion: This study suggests a potential negative effect of visceral adiposity on bone health in nonobese women.

\section{P306}

BONE TURNOVER MARKERS AND SERUM SCLEROSTIN LEVELS IN PATIENTS WITH ACROMEGALY

T. T. Tsoriev $^{1}$, T. A. Grebennikova ${ }^{1}$, Z. E. Belaya ${ }^{1}$, A. V. Iljin $^{2}$, L. Y. Rozhinskaya ${ }^{1}$, G. A. Melnichenko ${ }^{3}$

${ }^{1}$ Neuroendocrinology and Bone Diseases, Moscow, Russian Federation, ${ }^{2}$ Biochemistry and Hormonal Assays, Endocrinology Research Centre, Moscow, Russian Federation, ${ }^{3}$ Neuroendocrinology and Bone Diseases, Endocrinology Research Centre, Moscow, Russian Federation

Although growth hormone and IGF1 are considered to be anabolic hormones for bone, patients with acromegaly seems to have skeletal fragility.

Objective: To evaluate serum levels of sclerostin and bone turnover markers in patients with acromegaly. Materials and Methods: Fasting serum samples were taken from consecutive subjects with biochemically confirmed active acromegaly and healthy volunteers matched by age, sex and BMI.

IGF1 was measured by immunochemiluminescence assay (Liaison) reference range (60-280 $\mathrm{ng} / \mathrm{ml})$. Osteocalcin (OC) 
and carboxyterminal crosslinked telopeptide of type I collagen (CTx) were assayed by electrochemiluminescence assay (ECLIA) Cobas e601 Roche. Serum sclerostin was measured by commercially available immunoassay (Biomedica Medizinprodukte GmbH \& Co KG, Vienna, Austria). Results: 28 subjects with acromegaly mean age 43 (95\%CI 35-51) years old, 15 females and 13 males, BMI - 28 (25-30) kg/m2 were enrolled along with 30 healthy control subjects (16 females, 14 males) matched by age $p=0.57$ and BMI $p=0.17$. Mean IGF1 in subjects with acromegaly - 803 (95\%CI 688-917) ng/ml was higher as compared to healthy control $-176(150-202) \mathrm{ng} / \mathrm{ml}$ $p<0.001$. Low-traumatic fractures were registered in 9 cases out of 28 patients with acromegaly including vertebral body compression in 3 cases and 8 nonvertebral fractures. We did not find statistically significant difference in serum sclerostin levels in subjects with acromegaly 37 (28-47)pmol/1 vs. healthy control 40 (32-47) pmol/1 $p=0.46$. Nevertheless, both markers of bone turnover were higher in subjects with acromegaly OC 44 (27-61) ng/ml vs. 24 (17-31) ng/ml $(p=0.001)$; CTx 1.1 $(0.62-1.5)$ vs. $0.52(0.37-0.66) \mathrm{ng} / \mathrm{ml}(p=0.001)$.Patients with acromegaly did not differ in BMD Z-score L1-L4 $0.48(-0.51-$ $1,48)$ or neck Z- score $0.63(-0.05-1.32)$ as compared to healthy subjects L1-L4 $-0.02(-0.61-0.57)$, neck $0.25(-0.18-0.69)$ $p=0.21$ or serum calcium levels $p=0.39$, but their serum phosphorus was significantly higher $1.41(1.34-1.47) \mathrm{mmol} / \mathrm{l}$ vs. 1.19 (1.11-1.26) $\mathrm{mmol} / \mathrm{l} p<0.001$.

Conclusion: Patients with acromegaly have increased rate of bone turnover without any difference in serum sclerostin levels or BMD, but with a high prevalence (up to $32 \%$ ) of low-traumatic fractures.

Acknowledgements: РНФ 15-15-30032

\section{P307 \\ PREVALENCE OF POSTOPERATIVE DELI- RIUM ACCORDING TO THE USE OF DEXME- DETOMIDINE OR PROPOFOL IN ELDERLY PATIENTS UNDERGOING ORTHOPAEDIC SURGERY: RETROSPECTIVE STUDY}

H.-J. Shin ${ }^{1}$, H.-S. Na ${ }^{1}$, B.-W. Koo ${ }^{1}$

${ }^{1}$ Department of Anesthesiology and Pain Medicine, Seoul National University Bundang Hospital, Seongnamsi, Republic of Korea

Objective: With advancements in health care and increasing life expectancy, orthopaedic surgery in older patients is being performed in increasing numbers. Delirium is a common postoperative complication of surgery in elderly adults, and postoperative delirium (PD) is associated with adverse surgical outcomes, including prolonged hospital stays, delayed functional recovery, future institutionalization and higher mortality rate. Intraoperative sedation can be provided to the patients undergoing surgery via regional anaesthesia to reduce anxiety or stress response of patients. We evaluated the incidence of PD in elderly patients undergoing orthopaedic surgery under regional anaesthesia according to the sedative agents.

Materials and Methods: A total 855 adults patients, who underwent orthopedic surgery under regional anesthesia between July 2012 and September 2015, were surveyed to evaluate the incidence of PD: 592 using propofol and 263 using dexmedetomidine for sedative agents. Patient, surgery, and anesthesia- related factors were investigated from all patients. Results: The incidence of PD was higher in the propofol group compared with the dexmedetomidine group [40 (6.5\%) vs. 6 $(2.3 \%), P=0.008]$. Postoperative creatinine was higher in the propofol group than the dexmedetomidine group $(0.8 \pm 0.5 \mathrm{vs}$. $0.7 \pm 0.2, P=0.022$ ), and postoperative glomerular filtration rate was lower in the propofol group than the dexmedetomidine group ( $94.2 \pm 32.6$ vs. $100.5 \pm 33.7, P=0.020)$.

Conclusions: The results of this study suggest that dexmedetomidine may have beneficial effect for reducing PD in elderly patients undergoing orthopedic surgery compared propofol.

\section{P308}

\section{PHYSICAL THERAPY AND OSTEOPOROSIS}

K. Miladinovic ${ }^{1}$, V. Zejnilagic ${ }^{1}$

${ }^{1}$ Clinic for Physiatry and Rehabilitation, University Clinical Centre Sarajevo, Sarajevo, Bosnia and Herzegovina

Background: Certain physical modalities have osteogenic effect. These are: pulsed electromagnetic field, low intensity pulsed ultrasound, and, of course, exercises.

Objective: To analyse treatment of primary postmenopausal osteoporosis with physical modalities among patients on outpatient rehabilitation in one year period.

Material and methods: Analytic, retrospective study included 16 women with primary postmenopausal osteoporosis, selected from the total number of 487 outpatients who were rehabilitated in the period from January 2015. to December 2015. at the Clinic for Physiatry and Rehabilitation. Their cards with a prescribed program of physical therapy were analyzed.

Results: Annual prevalence of primary postmenopausal osteoporosis at outpatient rehabilitation was $3.3 \%$. The average age of patients was 65.7 years ranging from 48 to 82 .

Out of total 62 prescribed physical modalities, the most widely used was electrotherapy $(33.87 \%)$, including galvanic current, transcutaneous electrical stimulation and interference currents. Then followed pulsed electromagnetic field $(16.13 \%)$ and thermotherapy (16.13\%), which included hot and cold procedures. Manual massage was presented with $12.9 \%$. Exercises with osteogenic effect were presented with $11.29 \%$ and low intensity pulsed ultrasound with $8.06 \%$. 
Conclusion: Results of the study showed that physical therapy in patients with primary postmenopausal osteoporosis still being used for its analgesic effects, but because of its osteogenic effect.

\section{P309}

\section{ASSESSMENT OF BONE-RELATED MARKERS AND BONE MINERAL DENSITY IN RECENT} KIDNEY TRANSPLANTATION PATIENTS

E. Tomatsu $^{1}$, Y. Yoshino ${ }^{1}$, I. Hiratsuka ${ }^{1}$, S. Sekiguchi-Ueda ${ }^{1}$, M. Shibata ${ }^{1}$, T. Itoh $^{2}$, H. Sasaki ${ }^{3}$, M. Hasegawa ${ }^{4}$, M. Kusaka ${ }^{3}$, R. Shiroki ${ }^{3}$, T. Kenmochi ${ }^{2}$, Y. Yuzawa ${ }^{4}$, K. Hoshinaga ${ }^{3}$, A. Suzuki ${ }^{1}$

${ }^{1}$ Division of Endocrinology and metabolism, Fujita Health University, Toyoake, Japan, ${ }^{2}$ Division of Organ Transplant Surgery, Fujita Health University, Toyoake, Japan, ${ }^{3}$ Division of Urology, Fujita Health University, Toyoake, Japan, ${ }^{4}$ Division of Nephrology, Fujita Health University, Toyoake, Japan

Background: In kidney transplantation (KTx) recipients, preexisting chronic kidney disease-mineral and bone disease (CKD-MBD) and other clinical risk factors such as hyperparathyroidism and glucocorticoid therapy would make osteoporosis as a serious problem. With progress of transplantation procedure and its safety, the number of preemptive KTx from living-donor has been increasing in Japan. In order to improve bone health after KTx, more precise assessment of CKDMBD at KTx under current situation should be needed. In the present study, we estimated bone-related markers and BMD in short- term after KTx.

Subjects and Methods: This observational study was done in Fujita Health University Hospital, and post- KTx recipients in 2014 and 2015 were recruited $(n=33, \mathrm{M} / \mathrm{F}=20 / 13$, age 51 \pm 14 years old). Serum samples were collected and lumbar and femoral neck BMD was measured before initial discharge after KTx (mean duration $22 \pm 12$ days). Single KTx was done in 24 cases, while other 9 cases with type 1 diabetes were performed simultaneous pancreas-kidney transplantation. Maintenance dialysis before KTx was done in 23 cases, whose median hemodialysis duration was 22 months (9-67, interquartile range).

Their mean serum creatinine level after KTx was 1.31 $\pm 0.46 \mathrm{mg} / \mathrm{dL}$.

Result: Median intact PTH (iPTH), bone-specific alkaline phosphatase, tartrate-resistant acid phosphatase form $5 \mathrm{~b}$ (TRACP 5b), fibroblast growth factor-23 and 25 hydroxyvitamin D levels were $100 \mathrm{pg} / \mathrm{mL}, 12.3 \mu \mathrm{g} / \mathrm{L}$, $301 \mathrm{mU} / \mathrm{dL}, 128 \mathrm{pg} / \mathrm{mL}$, and $13.0 \mathrm{ng} / \mathrm{mL}$, respectively. Mean BMD of lumber spine evaluated by DXA was 0.94 $\pm 0.16 \mathrm{~g} / \mathrm{cm}^{2}$ (Z-score, $0.12 \pm 1.48$ ), and their mean femoral neck BMD was $0.64 \pm 0.12 \mathrm{~g} / \mathrm{cm}^{2}$ (Z-score, $-0.91 \pm 1.02$ ). Serum iPTH was positively associated with hip Z score, but not with other BMD data. Other bone-related markers were not related to BMD or Z-score at both sites.

Conclusion: We found that low iPTH level seems to be related to the decrease of hip BMD in KTx recipients just after their transplantation. Although pre-existent hyperparathyroidism has been reported to be a risk factor for future fracture after KTx, modest elevation of iPTH does not seem harmful for bone in post-KTx patients.

\section{P310}

MONTHLY ORAL IBANDRONATE (IBN) 100MG IS AS EFFECTIVE AS MONTHLY INTRAVENOUS (IV) IBN 1MG: SUBGROUP ANALYSES OF THE MOVEST STUDY

H. Hagino $^{1}$, T. Nakamura ${ }^{2}$, M. Ito $^{3}$, J. Hashimoto ${ }^{4}$, Y. Asao ${ }^{4}$, M. Yamamoto ${ }^{4}$, K. Endo ${ }^{4}$, K. Katsumata ${ }^{4}$, R. Matsumoto ${ }^{5}$, T. Nakano $^{6}$, H. Mizunuma ${ }^{7}$

${ }^{1}$ School of Health Science \& Rehabilitation Division, Tottori University Faculty of Medicine, Yonago, Japan, ${ }^{2}$ National Center for Global Health and Medicine, Tokyo, Japan, ${ }^{3}$ Center for Diversity and Inclusion, Nagasaki University, Nagasaki, Japan, ${ }^{4}$ Chugai Pharmaceutical Co. Ltd., Tokyo, Japan, ${ }^{5}$ Taisho Pharmaceutical Co. Ltd., Tokyo, Japan, ${ }^{6}$ Tamana Central Hospital, Kumamoto, Japan, ${ }^{7}$ Department of Obstetrics and Gynecology, Hirosaki University School of Medicine, Hirosaki, Japan

Objective: We present subgroup analyses of the randomized double-blind MOVEST study that compared the efficacy and safety of monthly oral IBN with monthly IV IBN in Japanese pts with primary osteoporosis.

Methods: Ambulatory pts aged $\geq 55$ yrs with primary osteoporosis were randomized to receive monthly oral IBN $100 \mathrm{mg}+$ monthly IV placebo, or monthly IV IBN $1 \mathrm{mg}+$ monthly oral placebo. The primary endpoint was noninferiority of oral vs. IV IBN with respect to BMD gains at the lumbar spine (LS) after 12 months. BMD gains were compared in subgroups classified by the number of prevalent baseline vertebral fractures, LS BMD T-score $(\geq-3$ or $<-3)$ at screening, and bisphosphonate (BP) pretreatment.

Results: 422 pts were randomized. The per-protocol set comprised 183 and 189 pts in the oral and IV IBN groups, respectively. Baseline characteristics were balanced. Mean relative changes from baseline in LS BMD at 12 months were $5.22 \%$ (95 \%CI 4.65-5.80) with oral IBN and 5.34 \% (95\%CI 4.785.90 ) with IV IBN, showing non-inferiority of oral to IV IBN. BMD gains at the total hip and femoral neck were evident. In pts with LS BMD T-score $\geq-3$ or $<-3$ at screening, BMD gains were $4.42 \%$ and $5.79 \%$, respectively, with oral IBN and $4.60 \%$ and $5.83 \%$ with IV IBN. In pts with or without BP pretreatment, BMD gains were $4.33 \%$ and $5.47 \%$, respectively, with oral IBN and $4.22 \%$ and $5.70 \%$ with IV IBN. In 
pts with or without prevalent vertebral fractures, BMD gains were 5.21 and $5.23 \%$, respectively, with oral IBN and 5.01 and $5.49 \%$ with IV IBN. There were no apparent differences in the safety profiles of the two treatment groups.

Conclusions: Monthly oral IBN $100 \mathrm{mg}$ demonstrated the same BMD gains as monthly IV IBN $1 \mathrm{mg}$ in Japanese osteoporotic pts. IV IBN and oral IBN increased BMD levels to the same extent in subgroups defined by LS BMD T-score at screening, BP pretreatment, and prevalent vertebral fractures. IBN is expected to show beneficial efficacy in osteoporotic pts.

\section{P311}

CALCIUM AND VITAMIN D SUPPLEMENTATION ARE NOT ASSOCIATED WITH INCREASED RISK OF ISCHAEMIC CARDIAC EVENTS: RESULTS FROM UK BIOBANK

N. C. Harvey ${ }^{1}$, S. D’Angelo ${ }^{1}$, J. Paccou ${ }^{1}$, M. Edwards ${ }^{1}$, S. E. Petersen $^{2}$, C. Cooper ${ }^{1}$

${ }^{1}$ MRC Lifecourse Epidemiology Unit, University of Southampton, Southampton, United Kingdom, ${ }^{2}$ NIHR Cardiovascular Biomedical Research Unit at Barts, William Harvey Research Institute, Queen Mary University of London, London, United Kingdom

Objective: Supplementation with calcium is widely used, both alone and as an adjunct to therapy for osteoporosis. However, recent meta-analyses have suggested a possible association with increased risk of myocardial infarction. We aimed to investigate such relationships longitudinally in a UK population-based cohort.

Methods: UK Biobank is a large prospective cohort comprising 502,664 men and women aged 40-69 years, with detailed assessment at baseline. Supplementation with calcium and/ or vitamin $\mathrm{D}$ was self-reported, and we obtained information on incident hospital admission (ICD-10) for ischaemic heart disease (IHD: I20-I25), any cardiovascular event (I63/I64 or I20I25) and death following these events, through linkage to Hospital Episode Statistics with up to 7 years follow-up. We used Cox Proportional Hazards models to investigate the prospective relationships between use of calcium and/or vitamin D supplementation and hospital admission for men and women, controlling for age, BMI, smoking, alcohol, educational level, physical activity, and in a further model, HRT use (in women). Results: All 502,664 participants (median age of 58 years, $54.5 \%$ women) had complete data on calcium and vitamin D supplementation. 34,890 participants reported taking calcium supplements, 20,004 taking vitamin D supplements, and 10,406 taking both $(2.1 \%)$. In both crude and adjusted analyses, there were no associations between use of calcium supplements and risk of incident hospital admission with ischaemic heart disease, any cardiovascular event, or death following either admission category. Thus, for example, in adjusted models, the hazard ratio for IHD admission was 1.06 (95\%CI: $0.85,1.31 ; p=0.62$ ) amongst women taking calcium supplementation. The corresponding HR for men was 1.02 (95\%CI: $0.80,1.30 ; p=0.87)$. The adjusted HRs for death from IHD were 0.71 (95\%CI: $0.32,1.61 ; p=0.42)$ in women, and 0.92 (95\%CI: $0.52,1.62 ; p=0.76)$ in men. Results were similar for vitamin D and combination supplementation, and in women, further adjustment for HRT use did not alter the associations. Conclusions: In this very large prospective cohort, use of calcium supplementation was not associated with increased risk of hospital admission or death following ischaemic or non-ischaemic cardiovascular events. These findings thus support the cardiovascular safety of calcium and vitamin D supplementation, at least within the age range studied. This research has been conducted using the UK Biobank Resource.

\section{P312}

FERRI ION: A POTENTIAL PATHOGENIC TOXICITY FACTOR INHIBITING BONE FORMATION OF POSTMENOPAUSAL OSTEOPOROSIS

W. Xiao ${ }^{1}, \mathrm{X}$. Youjia ${ }^{1}$

${ }^{1}$ Department of Orthopaedics, The Second Affiliated Hospital of Soochow University, Suzhou, China

Primary type I osteoporosis is a bone metabolic disease induced by estrogen deficiency. Our previous research have revealed a negative correlation between ferritin level and bone mass. We asked whether ferri ion may further aggravate the effect of estrogen deficiency on bone status. Ferric ammonium citrate, a most commonly used chalybeate, was injected to set up an iron overload mouse model. We examined and compared the BMD among three groups of mice: E+F -, E - F and $\mathrm{E}-\mathrm{F}+$. Our comprehensive in vivo time-course experiments directly demonstrated that bone mass was declined to a certain degree by ovariectomy and maximally by iron excess. These effects were accompanied by fluctuations in the level of bone formation markers and reactive oxygen species. We extracted cDNA from every mice femur and measured osteogenic reactive related gene expression. These differences were associated with bone status. To further study whether these effects were related to bone formation, mouse primary osteoblasts were used $\mathrm{f}$ or in vitro experiments. We collected the serum from three groups of mice and cultured osteoblasts with th ese serum. Using the cell count assay, alkaline phosphatase staining and von Kossa staining, we found that ferri ion did inhibit not only the viability but also the osteogenic ability of osteoblasts obviously through the production of oxygen species (ROS). Our data provided direct evidence that ferri ion inhibited postmenopausal bone formation particularly via stimulating oxidative stress level or other yet unknown mechanisms. 


\section{P313}

\section{PLASTIN 3: A NEW REGULATOR OF BONE} STRUCTURE IN ZEBRAFISH, MICE AND HUMAN

J. Milbradt ${ }^{1}$, N. Hamann ${ }^{2}$, M. Peters ${ }^{1}$, K. Hupperich ${ }^{1}$, A. $\overline{\text { Hoffmann }}^{1}$, J. Heilig ${ }^{3}$, D. Micha ${ }^{4}$, F. Van Dijk ${ }^{4}$, N. Mendoza-Ferreira $^{1}$, M. Hammerschmidt ${ }^{5}$, G. Pals ${ }^{4}$, F. Zaucke $^{3}$, A. Niehoff ${ }^{2}$, B. Wirth ${ }^{1}$

${ }^{1}$ Human Genetics, Cologne, Germany, ${ }^{2}$ German Sport University, Cologne, Germany, ${ }^{3}$ Biochemistry II, Cologne, Germany, ${ }^{4}$ Clinical Genetics, Amsterdam, Netherlands, ${ }^{5}$ Developmental Biology, Cologne, Germany

Objective: Elucidation of the disease-modifying characteristics of actin-bundling protein Plastin 3 (PLS3) relevant for the development of osteoporosis.

Material and Methods: This study used morpholino-based knock-down of pls3 in five day old (5 dpf) OsxNTRChr $x$ CollaleGFP transgenic zebrafish to analyse craniofacial muscular-skeletal malformations and rescue effects of coinjected PLS3 mRNA as well as other actin-bundling proteins. Moreover, examination of 5-day and 3-month-old femora of mice overexpressing human PLS3 was performed through micro-computed tomography $(\mu \mathrm{CT}), 3$-point-bending-test (3PBT) as well as histological experiments and protein analysis. Furthermore, primary cell culture of bone marrow, obtained from WT and PLS3 overexpressing animals, differentiated into osteoclasts was achieved and analysed via TRAP staining, immunofluorescence staining and bone resorption assay.

Results: We have demonstrated that PLS3 mRNA co-injection dose dependently rescued malformations of the craniofacial muscular-skeletal system induced upon pls3 morpholino injection in $5 \mathrm{dpf}$ transgenic zebrafish. Additionally, body axis and tail phenotype were also rescued upon PLS3 mRNA co-injection. The same findings were confirmed for the actin-bundling proteins, alpha-actinin 1 and alpha-actinin 4. Analysis of femora by $\mu \mathrm{CT}$ in 3-month-old PLS3 overexpressing animals showed a significantly increased thickness of cortical as well as trabecular bone. Results obtained with the 3-point-bendingtest (3PBT) showed an increased breaking force, stiffness and E-module in femora of PLS3 overexpressing animals. Histological as well as protein expression based experiments suggested a lower expression of Tartrate-resistant-alkalinephosphatase (TRAP) in PLS3 overexpressing animals. Osteoclasts differentiated from bone marrow of PLS3 overexpressing animals showed a significantly reduced resorption compared to WT. Additionally, sealing zone formation showed disrupted formation in PLS3 overexpressing osteoclasts, certainly explaining the reduction in resorption and the obtained murine bone phenotype.

Conclusion: These results strongly indicate an essential function of PLS3 as a new regulator of bone development and maintenance most likely involved in common, multifactorial osteoporosis.

Acknowledgements: This work has been funded by the DFG WI945/16-1 and CMMC C1 to BW.

\section{P314}

AN ORAL NONSTEROIDAL SELECTIVE ANDROGEN RECEPTOR MODULATOR, TEI-SARM2, PREVENTS DISUSE MUSCLE ATROPHY AND CANCER CACHEXIA

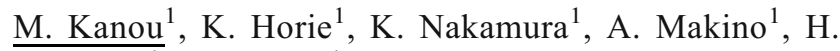
$\overline{\text { Sugiyama }}^{1}$, K. Yamana ${ }^{1}$

${ }^{1}$ Teijin Pharma Ltd, Tokyo, Japan

Muscle wasting is a frequent concomitant condition with hip fracture, cancer cachexia and aging. TEI- SARM2 is an oral nonsteroidal selective androgen receptor modulator (SARM) selected as a pharmaceutical candidate for treatment of muscle wasting diseases by its potent muscle anabolic activity, favorable pharmacological and pharmacokinetic properties in nonclinical studies. TEI-SARM2 has a non- steroidal scaffold structure, highly selective to AR among nuclear receptors, bound as a novel binding mode to AR and efficacious to increase muscle mass in normal and orchiectomized rats. In this study, we evaluated the efficacy of TEI-SARM2 during progressive disuse muscle atrophy. Female Wistar rats were hindlimb-unloaded for 14 days and received a daily oral administration of TEISARM2 ranging from 0.1 to $3 \mathrm{mg} / \mathrm{kg}$ or single subcutaneous administration of nandrolone decanoate (ND) ranging from 1 to $30 \mathrm{mg} / \mathrm{kg}$. TEI-SARM2 could reduce unloadinginduced muscle atrophy without any effects on ovary and uterus weight while ND also inhibited muscle atrophy with significant effects on ovary and uterus weight. TEI-SARM2 and ND dose dependently increased the local IGF-1 expression and decreased IGF binding protein 3 expression in muscle, suggesting that the amount of free IGF-1 was increased in TEI-SARM2-treated muscle. These results indicate that TEI-SARM2 and ND also prevents unloadinginduced decrease of muscle mass at least in part by influencing the local IGF-1 availability. Furthermore, we evaluated the effect of TEI-SARM2 in a mice model of cancer cachexia. BALB/c nu/nu mice were transplanted with HMV-II human malignant melanoma and received a daily oral administration of TEI- SARM2 at $3 \mathrm{mg} / \mathrm{kg}$ or subcutaneous administration of ND at $10 \mathrm{mg} / \mathrm{kg}$ biweekly. TEI-SARM2 and ND prevented muscle wasting without any effects on tumor growth. Interestingly, food intake was increased by TEISARM2 and ND, while no effect on fat mass was observed. Taken together, our results indicate that TEI-SARM2 is a promising drug candidate for muscle wasting diseases such as hip fracture, cancer cachexia and sarcopenia. 


\section{P315}

ONE SIZE FITS ALL? COMPARING EFFICACY OF STANDARD CHOLECALCIFEROL LOADING FOR VITAMIN-D DEFICIENCY IN MALAYSIAN WOMEN OF DIFFERENT ETHNICITIES WITH POSTMENOPAUSAL OSTEOPOROSIS

K. G. Goh ${ }^{1}$, S. F. Wan Muhamad Hatta ${ }^{2}$, S. R. Vethakkan ${ }^{1}$, S. P. Chan ${ }^{1}$, L. L. Lim ${ }^{1}$, A. T. Tan ${ }^{1}$, L. Ibrahim ${ }^{1}$, S. S. Paramasivam $^{1}$, J. Ratnasingam ${ }^{1}$

${ }^{1}$ Department of Endocrinology, University of Malaya, Wilayah Persekutuan Kuala Lumpur, Malaysia, ${ }^{2}$ Department of Endocrinology, University Teknologi MARA, Selangor, Malaysia

Introduction: Vitamin D supplementation is vital to osteoporosis management: reducing fractures/falls. Deficiency is prevalent in Southeast Asia despite year long sunlight exposure. Factors modulating serum $25(\mathrm{OH}) \mathrm{D}$ include those that limit sun exposure-induced cutaneous synthesis (latitude, conservative dressing, darker skin pigmentation) and cholecalciferol dietary intake. This study evaluated efficacy of standard loading dose regimen advocated by Endocrine Society 2011 guidelines for treatment of deficiency, among Malay(conservative dress) and Indian(darker skin pigmentation) women in Malaysia. Methods: In this prospective trial, community-dwelling postmenopausal osteoporosis(PMO) $\operatorname{Indian}(n=36)$ and Malay $(n=20)$ women living in Kuala Lumpur $\left(3.1333^{\circ} \mathrm{N}, 101.6833^{\circ} \mathrm{E}\right)$, Malaysia with vitamin $\mathrm{D}$ deficiency $(25(\mathrm{OH}) \mathrm{D}<20 \mathrm{ng} / \mathrm{mL})$ had serum $25(\mathrm{OH}) \mathrm{D}$ measured at baseline and after 8 weeks oral cholecalciferol 50000 IU weekly. Sufficiency was defined as $>30 \mathrm{ng} / \mathrm{ml}$.

Results: Mean baseline vitamin-D was not different between the Malay and Indian group $(14.79 \mathrm{ng} / \mathrm{mL}$ vs. $15.13 \mathrm{ng} / \mathrm{mL}$, respectively, $p=\mathrm{NS})$. At study end final median $\mathrm{Sr} 25(\mathrm{OH}) \mathrm{D}$ was higher in Malays compared with Indians $(37.2 \mathrm{ng} / \mathrm{mL}$ vs. $35.28 \mathrm{ng} / \mathrm{mL}$, respectively, $p<0.05$ ). There was no significant difference in delta serum $25(\mathrm{OH}) \mathrm{D}(8$ weeks-baseline) between groups(Malay $21.7 \mathrm{ng} / \mathrm{mL}$ vs. Indian $18.7 \mathrm{ng} / \mathrm{mL}, p=0.06$ ). However, whilst $90 \%(n=18)$ of the Malay population achieved vitamin D sufficiency $(>30 \mathrm{ng} / \mathrm{ml})$ after eight weeks; only $63.9 \%(n=23)$ of the Indian population achieved sufficiency(Fisher exact test, $p=0.072$ ). Only one Indian subject remained vitamin D deficient after 8 weeks. All patients selfreported good compliance with the treatment-regimen. At baseline, the only significant difference in clinical characteristics between the two groups was a lighter skin tone in Malay compared with Indian women(mean von Luschan score 26.28 vs. 29.32, $p<0.05$ ). There were no significant differences in BMI, sun exposure index, pretrial vitamin D supplementation dose or dietary vitamin D intake. Subgroup analysis exploring potential factors contributing to persistent vitamin D deficiency/ insufficiency in Indians revealed that while BMI was higher and pretrial cholecalciferol supplementation dose/dietary cholecalciferol intake lower in the deficient/insufficient group in comparison with the sufficient group, these differences were not statistically significant. Baseline vitamin $\mathrm{D}$, skin pigmentation and sun-index were similar between subgroups.

Conclusions: One-third of Indian PMO women failed to achieve sufficiency despite adherence to an 8-week loading dose regimen, whereas $90 \%$ of Malays attained sufficiency. We postulate darker Indian skin pigmentation necessitates higher dose/longer duration loading compared to lighter skinned Malay despite similar sun exposure. Alternatively, Indians may have genetically derived differences in vitamin $\mathrm{D}$ binding protein as has been hypothesized with African-Americans. Our findings indicate the need for loading regimens tailored to ethnicity/skin pigmentation and serum 25(OH)D assessment post loading to confirm attainment of sufficiency.

\section{P316}

INCREASED CADMIUM LEVELS DO NOT PREDICT FRACTURE RISK, BUT INCREASED MORTALITY IN WOMEN: THE MALMÖ DIET AND CANCER STUDY

$\underline{\text { L. Moberg }}^{1}$, P. M. Nilsson ${ }^{2}$, G. Samsioe ${ }^{1}$, G. Sällsten ${ }^{3}$, L. Barregård $^{3}$, G. Engström ${ }^{4}$, C. Borgfeldt ${ }^{1}$

${ }^{1}$ Department of Obstetrics and Gynaecology, Clinical Sciences, Lund University, Skåne University Hospital, Lund, Sweden, ${ }^{2}$ Department of Internal Medicine, Clinical Sciences, Lund University, Skåne University Hospital, Malmö, Sweden, ${ }^{3}$ Department of Occupational and Environmental Medicine, Institute of Medicine, Sahlgrenska Academy, University of Gothenburg, and Sahlgrenska University Hospital, Gothenburg, Sweden, ${ }^{4}$ Cardiovascular Epidemiology Research Group, Department of Clinical Sciences Malmö, Lund University, Malmö, Sweden

Background: Cadmium exposure at high levels has been found to negatively affect BMD and increase fracture risk, but the effect of low level cadmium exposure needs to be further explored.

Objectives: To investigate the association between baseline blood cadmium (B-Cd) levels and risk of fracture or mortality during follow-up in Swedish women.

Material and methods: The Malmö Diet and Cancer Study Cardiovascular cohort is population-based and includes 2927 middle-aged women with background questionnaire and B$\mathrm{Cd}$ measurements. Women were divided into quartiles $(\mathrm{Q})$ according to their cadmium levels $(\mathrm{Q} 1:<0.18$; Q2: 0.18-0.28; Q3: 0.28-0.51; and Q4: $>0.51 \mu \mathrm{g} / \mathrm{L}$ ). National registries were analysed for prospective risk of fractures occurring after individual inclusion in the study. Time to study endpoint was calculated from inclusion to fracture, emigration, death or end of follow-up. 
Results: In total, 604 first incident fractures occurred in women during follow-up for $20.6 \mathrm{y}$ (14.4-21.3 years) (median (25th-75th percentile)). The number of fractures during follow-up were similar for women in Q1 and Q4: $20.6 \%$ vs. $19.8 \% ; p=0.71$. More women in Q4 were current smokers than in Q1: $78.4 \%$ vs. $3.4 \%(p<0.001)$ and the number of smoked cigarettes/day correlated with B-Cd: correlation coefficient $0.49 ; p<0.001$. Cox-regression analysis for time to endpoint and B-Cd in Q4 vs. Q1 did not show significantly increased fracture risk for Q4 hazard ratio (HR) 1.27; $95 \%$ confidence interval (CI): 0.89-1.82)) when adjusting for age, BMI and smoking status (yes/no), nor when adjusting for age, BMI, smoking status (yes/no), age at menopause, physical activity, death during follow- up (yes/no) and self-reported treatment (yes/no) for diabetes mellitus, rheumatoid arthritis and gastric ulcer. Cox-regression analysis for time to endpoint and Q4 vs. Q1 found increased risk of death for Q4: HR 2.04 (95 \% CI: 1.64-2.54) compared to women in Q1.

Conclusion: Higher levels of cadmium in blood at baseline did not increase fracture risk in middle-aged women. Women with high levels of B-Cd show shorter overall survival.

\section{P317}

ALUMINUM AND BONE: NEW CLINICAL CIRCUMSTANCES ASSOCIATED WITH AL3+ DEPOSITION IN BONE, CO-LOCALIZATION WITH IRON

D. Chappard $^{1}$, L. Hubert ${ }^{1}$

${ }^{1}$ GEROM, University of Angers, Angers, France

Several decades ago, aluminum encephalopathy associated with osteomalacia has been recognized as the major complication of chronic renal failure in dialyzed patients. Removal of aluminum from the dialysate has led to a disappearance of the disease. However, aluminum deposit can occur in the hydroxyapatite crystals of the bone matrix in some other clinical circumstances. We have encountered aluminum in bone in patients with an increased intestinal permeability (coeliac disease) or in case of prolonged administration of aluminum anti-acid drugs. A co-localisation of aluminum with iron was also noted in cases of hemochromatosis and sickle cell anemia. Aluminum was also identified in a series of patients with exostosis, a frequent benign bone tumor. Corrosion of prosthetic implants composed of grade $\mathrm{V}$ titanium (TA6V is an alloy containing $6 \%$ aluminum and $4 \%$ vanadium) was also observed in peri-prosthetic bone retrieved in a series of hip or knee revisions. Aluminum can be identified in undecalcified bone sections stained by solochrome azurine, a highly specific stain allowing the detection of 0.03 atomic $\%$. Iron $\left(\mathrm{Fe}^{3+}\right)$ can be identified by Perls' Prussian blue technic allowing the detection of 0.035 atomic $\%$. Histochemical methods are by far superior to spectroscopic methods used in conjunction with scanning electron microscopy (EDS and WDS). $\mathrm{Al}^{3+}$ can bind to the phosphate groups of HA of the calcified bone matrix and $\mathrm{Fe}^{3+}$ can replace calcium in the hydroxyapatite crystals. Colocalization of aluminum and iron in bone does not seems to be the fruit of chance but the cellular and molecular mechanisms are still poorly understood. Much efforts are still needed to improve our knowledge on the toxic activity of metals on mineralization and bone cells. Histochemistry performed on undecalcified bone sections is the gold standard for the identification and localization of these metals in bone laboratories.

\section{P318 \\ BONE GRAFT WITH BETA TRICALCIUM PHOSPHATE (B-TCP) IN EDENTULOUS PATIENTS: A NANOTOMOGRAPHIC AND RAMAN STUDY}

D. Chappard $^{1}$, F. PascarettiI-Grizon ${ }^{1}$, B. Guillaume ${ }^{1}$, L. Terranova $^{1}, \mathrm{H}$. Libouban ${ }^{1}$

${ }^{1}$ GEROM, University of Angers, Angers, France

Edentulism leads to a decreased bone mass at the maxilla or/and the mandible. At the maxilla, the bone mass can be so dramatically reduced that dental implants cannot be placed to support the dental prostheses. Sinus lift elevation restores bone mass at the maxilla in patients with edentulism. The method consists in opening the lateral side of the sinus and grafting a granular biomaterial ( $\beta$-TCP is presently the most recommended) under the olfactory membrane. In this way, several millimeters in thickness of bone are obtained after healing of the maxillary bone in 6-9 months. Bone biopsies were obtained in the grafted area in five patients at that time. They were studied undecalcified after embedding in pMMA; blocks were analyzed by nanocomputed tomography and specific areas studied by Raman microspectroscopy. Histologically, remnants of the $\beta$ TCP granules were found osseointegrated and covered with mineralized bone; however, some areas containing only osteoid tissue were observed inside the granules. Macrophages having engulfed numerous $\beta$-TCP grains were observed in the marrow spaces. NanoCT identified the biomaterial (due to its high calcium content) when it is osseointegrated and in the macrophages. Raman microscopy permitted to compare the spectra of crude $\beta$ TCP, $\beta$-TCP embedded in pMMA, osseointegrated $\beta$-TCP and finally intracytoplasmic $\beta$-TCP grains. The ratio of the $820 \mathrm{~cm}^{-1}$ band of pMMA (-CH2 groups) on the $960 \mathrm{~cm}^{-1}$ of the $v 1$ phosphate band is an indicator of tissue hydration. In the bone matrix, the ratio of the $960 \mathrm{~cm}^{-1}$ to the $1675 \mathrm{~cm}^{-1}$ amide- 1 band reflects the mineralization degree. Specific bands of $\beta$-TCP were found in the osseointegrated $\beta$-TCP and also in the macrophages. The hydration degree was maximal for the biomaterial phagocytosed by these cells. Raman microspectroscopy associated with nanoCT is a powerful tool in the analysis of the biomaterial degradation and osseointegration in osteoporotic patients. 


\section{P319}

\section{COMPREHENSIVE ASSESSMENT OF MENO-} PAUSAL DISORDERS IN WOMEN WITH DIFFERENT LEVELS OF BONE MINERAL DENSITY

Postmenopausal osteoporosis is a pathology that is not an isolated lesion of the skeleton, and systemic violations of the metabolic processes associated with menopause. An important role in the mechanism of formation of these disorders play a decline in estrogen, leading to various disorders of the female body, leading to the formation of the climacteric syndrome. Considering the general features of postmenopausal osteoporotic changes and other disorders associated with menopause, conducted a comprehensive analysis of menopausal disorders in patients with various degrees of BMD.

The study included 96 women in the early postmenopausal period. Clinical evaluation of disorders associated with the menopause carried out based on the Menopause Rating Scale (MRS). In assessing the reliability of tests conducted by Cronbach's alpha coefficient was 0.80 ; retest correlation 0.98 . Women with more severe manifestations of menopausal disorders in early postmenopausal women have lower values in lumbar spine BMD $(r=-0.57 ; p=0.001)$. Score of menopausal disorders was significantly higher in women with T-score -2.5 or lower $(p=0.04)$. The process of bone resorption is dependent on osteoclast activity. Given the importance of reactive oxygen species in stimulating resorptive processes were evaluated correlation between the level of malondialdehyde plasma and urine deoxypyridinoline concentrations. There was a positive correlation between the concentration of MDA, a marker of bone resorption - urine deoxypyridinoline $(r=0.36 ; p=0.001)$ and negative correlation between MDA and lumbar spine BMD $(r=-0.46 ; p=0.001)$. In addition, a statistically significant feedback SOD activity and intensity of menopausal symptoms $(r=-0.62 ; p=0.001)$, as well as a direct link between the activity of SOD and the level of lumbar spine BMD $(r=-0.64 ; p=0.001)$.

Thus, the results of the study may be an additional prognostic factors in assessing the risk of reducing BMD in early postmenopausal women.

\section{P320}

A CASE SERIES OF PREGNANCY-AND LACTATION-ASSOCIATED OSTEOPOROSIS

\section{A. Kornete ${ }^{1}$, M. Mukane ${ }^{2}$, I. Rasa ${ }^{3}$}

${ }^{1}$ Riga Stradins University, Riga, Latvia, ${ }^{2}$ Riga Stradins University, Riga East Clinical University Hospital, Latvian Osteoporosis and Metabolic Bone Diseases Association, Riga, Latvia, ${ }^{3}$ Riga East Clinical University Hospital, Riga Stradins University, Latvian Osteoporosis and Metabolic Bone Diseases Association, Riga, Latvia
Objective: Pregnancy- and lactation-associated osteoporosis (PLO) is a rare syndrome characterized by significant changes in calcium and bone homeostasis during pregnancy and the early postpartum period. At this time, calcium demands are increased to meet the needs of the growing fetus and the losses that occur during lactation. PLO leads to fragility fractures, most commonly in the vertebral bodies. We present the clinical findings of two cases of PLO during peripregnancy period.

Material and Methods: From patients treated at Riga East Clinical University Hospital with multiple peripartum vertebral fractures, two were selected for the study. The findings are discussed in the view of current literature.

Results: We came across a case of a 34-year-old primiparous woman who had multiple vertebral fractures on her thoracic (Th11, Th12) and lumbar (L1, L2) spine and severe lower back pain that worsened two months after a Cesarean delivery. We also encountered a 29 -year-old patient with vertebral compressions of the thoracic (Th11, Th12) and lumbar (L1) spine, osteoporotic fracture of the L2 vertebra, where musculoskeletal pain syndrome began in the third trimester of pregnancy and deteriorated immediately postpartum. The first patient had the serum 25-hydroxyvitamin D (25[OH]D) deficiency $(11.5 \mathrm{ng} /$ $\mathrm{mL}$ ) while the second patient had $25(\mathrm{OH}) \mathrm{D}$ insufficiency $(21.6 \mathrm{ng} / \mathrm{mL})$. Peripheral blood examination revealed calcium $2.53 \mathrm{mmol} / \mathrm{L}$ in case 1 and $2.45 \mathrm{mmol} / \mathrm{L}$ in case 2 . Neither patient had any disease or other osteoporosis risk factors.

Conclusions: PLO, although a rare disorder and often confused with other causes of low back pain associated with pregnancy and lactation, should be kept in mind when an expectant or new mother develops persistent back pain. PLO can result in devastating physical, psychosocial and economic consequences, thus monitoring the patients with risk factors or secondary causes of osteoporosis, early diagnosis and management is essential.

\section{P321}

DISTINGUISHING CHARACTERISTICS OF INJURIOUS VERSUS NON-INJURIOUS FALLS

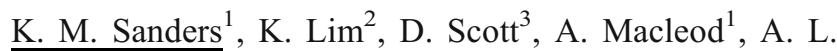
Stuart $^{4}$, G. C. Nicholson ${ }^{1}$, L. Busija ${ }^{1}$

${ }^{1}$ Institute for Health and Ageing, Australian Catholic University, Melbourne, Australia, ${ }^{2}$ Institute for Health \& Ageing, Australian Catholic University, Melbourne, Australia, ${ }^{3}$ Bone and Muscle Health Research Group, Department of Medicine, Monash Medical Centre, Melbourne, Australia, ${ }^{4}$ IMPACT, Deakin University, Geelong, Australia

As many studies investigate only falls resulting in a hospital presentation, limited information exists to compare non-injurious vs. injurious falls. We investigated 
differences in fall outcomes using data from the placebo group of our RCT where falls/fracture was the primary outcome.

Invitation letters were sent to all community-dwelling women aged $70+$ years residing in our region (Australia; latitude $\left.38^{\circ} \mathrm{S}\right)$. Falls and fractures (Fx) were prospectively ascertained by monthly calendars and confirmed by phone interview. Hospital admissions and fractures were confirmed but other injuries were self-reported. Fall characteristics were analysed according to injury status ( 1 ; none), (2; injury without $\mathrm{Fx}$, e.g. abrasion, bruising), ( $3 ; \mathrm{Fx})$ using Chi square tests and logistic regression.

Of 888 women, $64 \%(n=566)$ reported $\geq 1$ fall between 2006-2008 and detailed fall event information was available for 1,211 of 1,215 falls. $32 \%(n=384)$ of falls resulted in no injury, $63 \%(n=764)$ resulted in non- Fx injury and $5 \%(n=63)$ resulted in $66 \mathrm{Fx}$. Ascertainment time $(1.6 \pm 0.3 \mathrm{yrs})$, BMI $(26.2 \pm 5.2)$ and age (79.1 \pm 4.9 yrs $)$ did not vary between injury groups $(p>0.05)$. Compared to groups $1 \& 2$, Fx falls were more likely to involve slipping on a hazard (40\% vs. $20 \& 23 \%$, $p<0.017$ ); being away from home (52\% vs. $31 \&$ $40 \%, p<0.017)$ and outdoors $(67 \%$ vs. $49 \& 58 \%$, $p<0.017)$. Fewer 'trip' falls resulted in Fx $(10 \%$ vs. 22 $\& 24 \%$, respectively, $p<0.019$ ). Injury status was not related to loss of balance or feeling dizziness ( $32 \%)$ and falling during night-time $(12 \%)(p>0.1)$. However despite no differences in the likelihood of injury, night fallers were nevertheless four times more likely to be hospitalised (OR; 95 \% CI: 4.0; 2.1, 7.7; adjusted for age $\&$ injury group).

These findings facilitate cost-effective programs of falls prevention and injury management by identifying value in targeting specific risk factors for Fx falls in older women and a need to reduce hospitalisations for night fallers.

\section{P322}

DIFFERENCES IN PEAK BONE MINERAL STATUS BETWEEN URBAN AND RURAL MEN AND WOMEN: BANGLADESH PERSPECTIVE

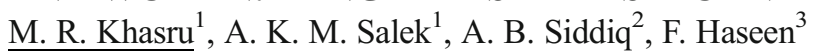

${ }^{1}$ Physical Medicine and Rehabilitation, Faculty of Medicine, Bangabandhu Sheikh Mujib Medical University, Dhaka, Bangladesh, ${ }^{2}$ Physical Medicine and Rehabilitation, Feni Diabetic Hospital, Feni, Bangladesh, ${ }^{3}$ Public Health and Informatics, Bangabandhu Sheikh Mujib Medical University, Dhaka, Bangladesh

Background: Osteoporosis is a silent killer. Several studies of western populations have shown a lower prevalence of fractures in rural populations compared with their urban counterparts. Higher bone mineral density (BMD) or bone mass content (BMC) in distal forearm and central bones were also reported in rural populations from studies in different western countries. More than $65 \%$ of total populations of Bangladesh are rural residents, who are dissimilar to their urban peers in terms of socioeconomic status, diet and lifestyle. Similar study was not found among Bangladeshi population.

Objective: The objective of this study is to primarily determine whether urban-rural differences in peak bone mineral status of aged 25-35 years of both gender and secondly to determine whether these differences can be explained by differences in body size or certain lifestyle factors.

Methodology: This Cross sectional survey will be carried out at the department of Physical Medicine \& Rehabilitation of Bangabandhu Sheikh Mujib Medical University, Dhaka, Bangladesh in the year 2016. Sample will be collected from population of both urban rural setting. Total one thousand subjects of which 500 from urban and 500 from rural setting will be enrolled. A multistage stratified cluster sampling method will be used to select the study participants.

Selection criteria: Eligible subjects should be stable healthy residents (lived $\geq 10$ years), aged $25-25$ of both genders.

Measurements: Information on demographics, socioeconomic status, medical history, smoking and drinking habits will be collected using a standardized questionnaire from each participant during an in-house face-to-face interview. Standardized measurements of height and weight, Body mass index (BMI) waist circumference will be measured. Bone mineral content (BMC, g) and bone area (BA, cm2) of the anteroposterior (AP) spine L1-4 will be measured using DEXA. Bone mineral density (BMD, $\mathrm{g} / \mathrm{cm} 2$ ) will be calculated as BMC divided by BA.

Statistical analysis: Student's $t$-test, Chi-square test, linear regression analysis will be performed using SPSS V21.0. A $p$ value $<0.05$ will be considered as statistical significant.

Conclusion: Information will be disseminated to all stakeholders.

\section{P323}

TRENDS IN FRACTURE HOSPITALIZATION AMONG OLDER ADULTS IN ECUADOR

C. H. Orces ${ }^{1}, \mathrm{~K}$. Weisson ${ }^{2}$

${ }^{1}$ Medicine/Laredo Medical Center, Laredo, United States,

${ }^{2}$ Baptist Hospital, San Antonio, United States

Objective: To examine trends in fracture hospitalization among adults aged 65 years and older in Ecuador between 1999-2014. 
Material and Methods: The National Hospital Discharge Surveillance system database was used to identify older adults hospitalized with a principal diagnosis of hip (S72.0-1), femur (S72.2-9), lower leg and ankle (S82.09), foot, except ankle (S92.0-9), lumbar spine and pelvis (S32.0-8), ribs (S22.1-4), shoulder and upper arm (S42.09), forearm (S53.0-9), wrist and hand (S62.0-8) fractures. Hospitalization rates per 100,000 persons were ageadjusted using the 2010 Census Ecuadorian population as the standard. The joinpoint regression program was used to examine the average annual percent change (AAPC) in rates between 1999 and 2014 and the annual percent change (APC) in fracture rates where a statistically significant change occurred in the linear slope of rates.

Results: A total of 55,621 older adults were hospitalized for fractures in Ecuador between 1999 and 2014. Women represented $64.6 \%$ of the hospital admissions and the majority of subjects $(66.9 \%)$ with these injuries were admitted to public hospitals. Overall, hip and femur fractures were the leading admitting diagnoses in both genders. Fracture hospitalization rates among women increased on average by $4.6 \%(95 \% \mathrm{CI} ; 3.4,5.7 \%)$ per year between 1999 and 2014. This upward trend in fracture hospitalizations was predominantly attributed to a significant annual increase in non-hip fracture rates by $8.7 \%$ (95\% CI, 7.1, $10.3 \%$ ) between 2006 and 2014. Moreover, hip fracture rates among women increased on average by $4.8 \%$ (95\% CI, 3.7, $5.9 \%)$ per year during the study period. In men, the increase in fracture hospitalizations was less accentuated at an AAPC in rates of $2.9 \%(95 \%$ CI $, 1.7,4.1 \%)$ during the study period. Nevertheless, upward trends in hospitalization rates were seen since 2004 onwards at an annual rate of $5.5 \%$ (95\% CI, 4.3, $6.7 \%$ ). Of interest, hip fracture rates among men also increased on average by $3.8 \%(95 \%$ CI, 2.6, $4.9 \%$ ) per year during the study period. Table 1 shows the AAPC and APC change in fracture hospitalization rates by gender and affected body area. In general, it appears that the increased femoral and forearm fracture hospitalization rates among women since 2006 and femoral fractures in men since 2005 may have accounted for the marked upward trend in no-hip fracture hospitalizations in women and men seen after these years, respectively.

Conclusions: Hip and non-hip fracture hospitalization rates significantly increased among older adults in Ecuador during the study period. The present findings may assist public health authorities to implement osteoporosis and fall prevention awareness programs among subjects at risk for these injuries.

Table 1. Fracture hospitalization rates among older adults in Ecuador, 1999-2014

\begin{tabular}{|c|c|c|c|c|c|}
\hline Fractures & Number (\%) & Rates $^{\mathrm{a}}$ & AACP & $\mathrm{ACP}$ & $\mathrm{ACP}$ \\
\hline \multicolumn{6}{|l|}{ Women } \\
\hline Hip & $11,238(31.3)$ & 155.3 & $4.8 \%$ & - & - \\
\hline Femur & $9,310(25.9)$ & 129.5 & $1.8 \%$ & $1999-2006 \mathrm{APC}=-5.2 \%{ }^{*}$ & 2006-2014 APC $=8.4 \%{ }^{*}$ \\
\hline Lower leg and ankle & $4,606(12.8)$ & 66.1 & $4.5 \%$ & - & - \\
\hline Foot & $299(0.8)$ & 4.3 & $6.3 \%$ & - & - \\
\hline Lumbar spine and pelvis & $1,303(3.6)$ & 18.4 & $5.4 \%$ & - & - \\
\hline Ribs & $357(1.7)$ & 6.5 & $1.6 \%$ & - & - \\
\hline Shoulder and upper arm & $3.135(8.7)$ & 44.4 & $6.1 \%$ & $1999-2003 \mathrm{APC}=-0.0 \%$ & 2003-2014 APC $8.4 \%{ }^{*}$ \\
\hline Forearm & $4,224(11.4)$ & 60.2 & $8.3 \%$ & $1999-2006 \mathrm{APC}=2.5 \%$ & 2006-2014 APC $=13.7 \%{ }^{*}$ \\
\hline Wrist and hand & $1,483(4.1)$ & 21.1 & $9.4 \%$ & - & - \\
\hline \multicolumn{6}{|l|}{ Men } \\
\hline Hip & $4,983(25.3)$ & 85.9 & $3.8 \%{ }^{*}$ & - & - \\
\hline Femur & $4,698(23.9)$ & 80.5 & $0.4 \%$ & $1999-2005$ APC $-7.0 \%{ }^{*}$ & $2005-2014 \mathrm{APC}=5.6 \%^{*}$ \\
\hline Lower leg and ankle & $3,990(20.3)$ & 66.4 & $3.5 \%{ }^{*}$ & - & - \\
\hline Foot & $404(2.1)$ & 6.7 & $4.6 \%^{*}$ & - & - \\
\hline Lumbar spine and pelvis & $678(3.4)$ & 11.4 & $0.4 \%$ & - & - \\
\hline Ribs & $506(2.6)$ & 8.5 & $0.4 \%$ & - & - \\
\hline Shoulder and upper arm & $1,696(8.6)$ & 28.4 & $5.6 \%^{*}$ & - & - \\
\hline Forearm & $1,864(9.5)$ & 31.1 & $7.2 \%{ }^{*}$ & $1999-2009 \mathrm{APC}=3.1 \%$ & 2009-2014 APC $16.1 \%{ }^{*}$ \\
\hline Wrist and hand & 847 & 14.1 & $7.1 \%{ }^{*}$ & - & - \\
\hline
\end{tabular}

${ }^{a}$ Age-adjusted hospitalization rates per 100,000 persons

${ }^{*}$ The AAPC and APC is significantly different from zero at alpha $=0.05$ 


\section{P324}

DOES ADIPONECTIN IN SERUM OR SYNOVIAL FLUID PREDICT ARTHROSCOPY ASSESSED CARTILAGE DAMAGE SEVERITY IN PATIENTS WITH SYMPTOMATIC KNEE OSTEOARTHRITIS?

M. Shargorodsky ${ }^{1}$, O. Nugzar ${ }^{1}$

${ }^{1}$ Wolfson Medical Center, Holon, Israel

Background: Osteoarthritis (OA), the prevalent joint-affecting disease characterized by cartilage damage, is the leading cause of disability in adults and contributes to the excess of morbidity and healthcare costs. We performed the current trial to evaluate biomarkers, specifically adiponectin in serum and synovial fluid, associated with cartilage damage severity assessed by arthroscopy, in patients with symptomatic knee OA.

Methods: The 40 subjects (mean age 51.8, $35 \%$ female, mean BMI 28.8) were divided into two groups according to arthroscopy assessed cartilage damage, using Outerbridge (OB) grading: Group 1 included 20 patients without cartilages surface defects (OB grade 0, I), Group 2 included 20 patients with cartilages surface defects (OB grade II, III). Metabolic parameters, insulin resistance markers and serum adiponectin levels were determined. Results: Both groups were similar in terms of serum adiponectin levels $(p=0.806)$. Synovial fluid adiponectin levels tended to be lower (not statistically significant) in subjects with cartilage damage (1718.6 vs. 2738.1). Knee Society Score was significantly lower in subjects with cartilage damage $(113.0 \pm 24.9$ vs. $142.7 \pm 25.1, p<0.001)$. In multiple linear regression analysis BMI was a significant independent determinant of cartilage damage in non-obese patients with knee osteoarthritis, such that each 1-unit increase in BMI was associated with a $21.7 \%$ increase in risk of cartilage damage (OR1.217, $95 \%$ CI 0.998-1.483, $p=0.05$ ).

Conclusions: We did not find an association between serum adiponectin as well as adiponectin in synovial fluid, and arthroscopy assessed cartilage damage severity. BMI was a significant independent determinant of cartilage damage in nonobese patients with knee OA.

\section{P325}

MUSCLE MASS PREDICTS INCIDENT FRACTURE IN POSTMENOPAUSAL WOMEN : THE OFELY STUDY

$\underline{\text { E. Sornay-Rendu }}^{1}$, F. Duboeuf ${ }^{1}$, S. Boutroy ${ }^{1}$, R. D. Chapurlat $^{1}$

${ }^{1}$ INSERM UMR 1033, Université de Lyon, Lyon, France

The relationships between body composition and BMD are well established but the independent contribution of body composition to the risk of fracture (Fx) has rarely been evaluated prospectively. The aim of this study was to prospectively investigate the prediction of fragility Fx by fat, lean and muscle mass in postmenopausal women.

We measured body composition at the 9th annual follow-up of the OFELY study in 595 postmenopausal women, mean age $66 \pm 8$ yrs. Whole body DXA (Hologic ${ }^{\circledR}$, QDR4500) exams were analyzed using the APEX version 4.0.2 to get visceral (VFAT) and subcutaneous fat mass (SFAT) in an abdominal region of interest along with measurement of total body fat mass (FM), lean mass (LM), appendicular skeletal muscle mass (ASM) and Femoral Neck BMD (FNBMD). The LM (LMI) and ASM indexes (ASMI) were obtained by dividing by height squared.

During a median[IQ] 13.1[1.9] yrs of follow-up, 138 women sustained a first incident fragility Fx, including 85 women with a major osteoporotic Fx (MOP Fx: hip, clinical spine, shoulder or wrist). After adjustment for age, women who sustained Fx had lower BMI $(-4 \%, p=0.01)$, VFAT $(-4 \%$, $p=0.07)$, SFAT $(-6 \%, p=0.06)$, LMI $(-6 \%, p=0.002)$ and ASMI $(-3 \%, p=0.003)$, compared with women without Fx $(n=457)$. The differences were more pronounced for women who sustained MOP Fx ( $p<0.01$ for all). After adjustment for age, prevalent Fx, physical activity, incident falls (during the whole follow-up in women without Fx and until the first incident Fx in fractured women) and FNBMD, each SD increase of baseline values of LMI and ASMI were associated with significant decreased fracture risk with adjusted hazard ratios $[\mathrm{HR}(95 \% \mathrm{CI})]$ of $0.76(0.61-0.95)$ and $0.76(0.62-0.94)$, $\mathrm{p} \leq 0.02$. Those associations remained significant after adding body weight in the multivariate model $(p=0.02)$. For all Fx, FM, VFAT and SFAT were not independently associated with fracture risk in the multivariate model with or without adding body weight. For MOP Fx, FM, VFAT and SFAT, were associated with fracture risk in the multivariate model without $(p<0.05)$, but not with body weight in the model.

We conclude that both visceral and subcutaneous fat mass are associated with fracture risk but not independently of BMD and body weight. In contrast, lean mass and appendicular muscle mass indexes predict the risk of fracture in postmenopausal women independently of BMD and clinical risk factors.

P326

REVISIONAL STUDY OF 123 OSTEOPOROTIC PATIENTS COMPARING SERUM LEVELS OF THE BONE RESORPTION MARKER CTX-I, IN A SYSTEMATIC WAY BEFORE AND AFTER THE ANTIRESORPTIVE MEDICATION

A. F.França $^{1}$, A. P.F. Filho ${ }^{1}$, A. F.F. Filho ${ }^{2}$, P. D. Zardetto ${ }^{2}$, G. F. França ${ }^{3}$

${ }^{1}$ Clinica Santa Helena of Orthopedics and Traumatology, Catanduva, Brazil, ${ }^{2}$ Araraquara University (UNIARA), Araraquara, Brazil, ${ }^{3}$ Barão de Mauá University, Ribeirão Preto, Brazil 
Objective: The purpose of this osteoporotic individuals medical record review was to compare the serum levels of the bone resorption biochemical marker CTX-I [1] collected before and after 25 days of antiresorptive drug administration, furthermore verify the possible precocious reduction in the serum concentration of this marker. This way, we could be able to demonstrate if the systematic sample method of collection can support the early decrease of bone resorption activity.

Material and Methods: We reviewed the medical records of 123 osteoporotic patients with average age of 65 years which 112 were female and 11 male. The blood samples were obtained after $8 \mathrm{~h}$ of fasting during the morning period, one before the antiresorptive medication (initial CTX-I) and the other (final CTX-I) 25 days after endovenous drug administration (zoledronic acid $5 \mathrm{mg}$ ) or the subcutaneous (denosumab $60 \mathrm{mg}$ ). We compared the sample serum levels before and after the medication, calculating this way the variation (delta) of the final CTX-I - initial CTX-I related to the initial CTX-I. Another measure was the relation of the final CTX-I divided by the initial CTX-I. The following step was the statistical analysis applied by the Tstudent test two-tails paired samples. The research was made between January and December of 2015.

Results: The average concentration in 123 patients of the initial CTX-I was $0.281 \mathrm{ng} / \mathrm{ml}$ and the average concentration 25 days after medication was $0.122 \mathrm{ng} / \mathrm{ml}$, therefore we obtained an average reduction of $56.62 \%$ on the final CTX-I. When the delta was used an average decrease of $39.13 \%$ was noticed. $p=1,2 \times 10-12$.

Conclusion: We conclude that exists a significant statistical difference when comparing the initial CTX-I of the osteoporotic patients with the same person 25 days after the antiresorptive medication confirmed by the $56.62 \%$ biochemical marker reduction and the $39.13 \%$ delta average reduction. The serum dosage of the biochemical marker of bone destruction CTX-I, made in a systematic way showed us to be efficient when used after the antiresorptive medication, decreasing the bone turnover. References:

Szulc P. Bone turnover biochemical markers on osteoporosis. In: Rosen $\mathrm{C} \mathrm{J}$ et al., editors. Primer on the metabolic bone diseases and disorders of mineral. Rio de Janeiro: Grupo editorial nacional; 2014. P.241-246.

\section{P327 \\ COMPARISON OF CLINICAL AND MAGNETIC RESONANCE IMAGING DIAGNOSIS OF DEGENERATIVE CHANGES OF TEMPORO- MANDIBULAR JOINT}

A. Knežević ${ }^{1}$, M. Jeremic Knezevic ${ }^{1}$, S. TomasevicTodorovic $^{1}$, D. Simić-Panić ${ }^{1}$, J. Ivacic ${ }^{2}$, K. Boskovic ${ }^{1}$

${ }^{1}$ Medical Faculty, University of Novi Sad, Novi Sad, Serbia,

${ }^{2}$ Physical Medicine and Rehabilitation, General Hospital, Senta, Serbia
Objective: To compare temporomandibular joint (TMJ) clinical findings according to the Research Diagnostic Criteria for Temporomandibular Disorders (RDC/TMD) and findings based on MRI.

Material and Methods: The prospective study was conducted in the period from 2011 to 2012 at The Center for Imaging Diagnostic, Institute for Oncology of Vojvodina. It included 60 randomly selected patients (aged 18-65) who were scheduled for MRI of the head. The patients were examined and a diagnosis of temporomandibular degenerative disease was established according to RDC/TMD criteria. Later on, the same day MRI was performed and examined by experienced radiologist.

Results: After clinical examination we established a diagnosis of osteoarthritis (OA) in $5(4.2 \%)$ TMJs according to the RDC/TMD. MRI showed degenerative changes in 44 (36.7 \%) TMJs, fossa was affected in 17 (14.2\%), and condyles were affected in 34 (28.3\%) TMJs. Approximately the equal number of TMJs had unilateral and bilateral mandibular fossa affection (5 (4.2\%) unilateral vs. $6(5 \%)$ bilateral) and condyles affection (12 (10\%) unilateral vs. 11 (9.2) bilateral). Solely mandibular fossa or condyle was affected by OA in the majority of affected TMJs (37 (84.1\%)), while degenerative changes both on the mandibular fossa and condyle was found in only 7 (15.9 \%) TMJs. Cohen's kappa statistics showed poor agreement between clinical and MRI findings (kappa $=-0.037 ; p=0.430$ ).

Conclusion: Our data showed that clinical diagnostic criteria were unreliable predictor of MRI diagnoses of TMJ osteoarthritis.

\section{P328 \\ THE ANALYSIS OF OSTEODENSITOMETRY FINDINGS ON THE HIP IN PATIENTS WITH OSTEOPOROTIC FRACTURES}

Z. G. Grujic ${ }^{1}$, T. T. Tomic ${ }^{1}$, L. J. Josovic ${ }^{1}$, J. D.-R. DzagicRistic $^{1}$, A. J. Jokic ${ }^{1}$

${ }^{1}$ Specialized Rehabilitation Hospital Banja Koviljaca, Banja Koviljaca, Serbia

Objective: To study and analyze all parameters acquired by measuring BMD of the neck of the femur in patients with fractures of osteoporotic etiology, and to discover their significance as a predictor of unfavourable prognosis.

Material and methods: BMD was measured using the device Lunar DPX on the neck of the femur, according to the standard propositions for central DXA. The following values were analyzed - BMD values (absolute, expressed in $\mathrm{g} / \mathrm{cm} 2$ and deviations from normal values expressed in percentages), T-score and Z-score values on the hip and FRAX index values (hip fracture). Demographic data and fracture information were collected from 
a survey which all patients fill out, and body weight and height measurements were performed using standard methods. This study encompasses 1499 patients, of both sexes, and older than 50 years. Acquired data were statistically processed using chisquare test, $T$-test and ROC analysis.

Results: Average age of the examinees was 63.72 years. 235 of them had one or more fractures, of osteoporotic etiology. 44 examinees had a hip fracture, and 45 had two or more fractures. Based on the T-score values on the hip in patients with osteoporosis $-36.4 \%$ of them had a fracture and $8.9 \%$ of patients with normal findings had fractures $\left(X^{2}=24.281\right.$; $p<0.001)$. In the group with fractures, average T-score value was $-1.596 \mathrm{SD}$ and without fractures $-0.850 \mathrm{SD}(\mathrm{t}=-8.209$, $p<0.001)$. Average $\mathrm{Z}$-score value in examinees with fractures was $-0.292 \mathrm{SD}$, and $-0.139 \mathrm{SD}(\mathrm{t}=-4.374, p<0.001)$ without fractures. Surface beneath the curve for hip fractures is 0.739 , cutoff $2.05, p=0.000$ ).

Conclusion: Measuring bone density on the neck of femur is highly reliable method for the estimation of the density of the entire bone system and can predict with great certainty osteoporotic fractures, since it greatly correlates with their frequency. It will significantly add to the proper methods of treating osteoporosis.

\section{P329}

\section{CLINICAL RESULTS IN APPLICATION OF} AUTOLOGOUS PLATELET-RICH PLASMA (PRP) IN OSTEOARTHRITIS OF THE KNEE

L. Smolina $^{1}$, L. Khimion ${ }^{1}$

${ }^{1}$ Department of Family Medicine, Institute of Family Medicine, P.L. Shupyk Medical Academy of Postgraduate Education, Kiyv, Ukraine

Summary: Obtain information about improving the performance of complex treatment with PRP compared to standard therapy of OA for 12 months.

Introduction: Osteoarthritis( $\mathrm{OA})$ is a chronic degenerative progressive pathology of hyaline cartilage. OA accounts for profound morbidity, pain and health care expenses. There are few validated interventions that can improve the functional state of patients once the degenerative process becomes symptomatic. Taking into account the lack of response of the body's healing mechanisms to degenerative conditions generally, local use of the stimulating and growth factors to activate cartilage regeneration is sensible. At the moment the steam cells therapy, platelet rich plasma (PRP) and mixed techniques are intensively investigating for OA treatment. PRP is a novel therapeutic tool of autologous nature that has emerged in recent years. The application of PRP in different tissues has given promising results in different pathologies such as acute and chronic injuries of bone and cartilage. PRP treatment has demonstrated a quite prominent potential in rheumatology, orthopedics and sport medicine. Its therapeutic target eminently comprises chronic processes, although the range of indications is constantly expanding; PRP has been successfully used in many ailments, including knee osteoarthritis. Its low cost, ease of use, usefulness in pathological processes and high safety put it at the center of the researchers interest. PRP owes its therapeutic interest to the crucial role of platelets in the wound healing and tissue regeneration. This role is not related to the repairing properties of the platelets themselves but, rather, to growth factors released by its $\alpha$-granules, which possess multiple regenerative properties. Also platelet quantification and the growth factor content definition must be defined in order to understand molecular mechanisms behind PRP regenerative strength. Standardization of PRP preparations is thus urgently needed.

Objective: To study the efficacy and safety of the PRP use in knee OA (I-IIst.) treatment.

Methods: The study was conducted at the Department of Family Medicine of the National Medical Academy of Postgraduate Education (Kyiv, Ukraine). We have examined 127 patients. In the study we included 68 patients with diagnosed knee OA (I-II radiological stage), according to the required diagnostic criteria. The patients with severe co-morbidities, trauma or after knee surgery were not included at the study. 68 patients $(27$ men $(39.7 \%)$ and 41 women (60.3\%), aged $35-55$ years (mean age $41.3 \pm 1.2 \mathrm{y}$ ) were divided on 2 groups. Group 1 included 34 patients who consented to receive standard OA treatment (nonsteroidal anti-inflammatory drugs (NSAID), physiotherapy, massage, exercises) and 3 intra- articular injections of PRP (total volume-24-30 ml, mean platelets number $869.24 \pm 32.1 \times 109 / \mu \mathrm{l})$ weekly, a second course of treatment 3 intra-articular injection we performed 6 months after first use PRP; group 2 consisted of 34 patients of comparable age with the same diagnosis who received only standard OA treatment. The CRP-level, WOMAC scale and Lequesne index was analyzed before treatment and 1, 3, 6 and 12 months after course of treatment in both groups.

Results: CRP level was modestly abnormal in the $43.5 \%$ patients of group 1 and $38.7 \%$ patients of group 2; during the treatment period CRP got to normal in all patients, but during the follow-up period after 6 and 12 months of treatment in the study group it was significantly lower $(6.44 \pm 0.52)$ and $(5.34 \pm 0.22) \mathrm{mg} / \mathrm{L}$, than in the control group $(7.96 \pm 0.60)$ and $(6.72 \pm 0.51) \mathrm{mg} / \mathrm{L}(p<0.005)$; in addition in 3 patients of group 2 CRP level had increased again after 6 months which was the sign of OA exacerbation; in group 1 there were no OA exacerbations during 6 months of follow-up. WOMAC and Lequesne index had positive dynamic during treatment in both patients groups. At month 12 functional status of the patients in group 1 improved by $33 \%$ in stiffness (at the beginning of the study it was $(4.8 \pm 1.0)$ which had significant difference with patients of control group $(4.6 \pm 0.1)$ - at the beginning of 
the study and $(3.4 \pm 0.1)$ in 12 month after treatment. Pain during movement and after passing the distance decreased after treatment by $38.5 \%$ in group 1; pain and discomfort when climbing and descending the stairs decreased by $38.9 \%$ (these indicators in group 1 at the beginning of the study were $(10.9 \pm 1.3)$ in group $2-(10.2 \pm 0.9)$ and in 12 month after treatment $-(2.1 \pm 0.3)$ in group 1 and (3.9 $\pm 0.3)$ in group $2,(p<0.05)$. At the end of follow-up period patients functional activity increased by $47.4 \%$ in group 1 which was better than positive changes in group 2 and was accompanied by a significant reduction of restrictions in daily activities. There were no adverse events due to use of PRP injections in group 1.

Conclusion: The course of 6 intra-articular injections added to the standard treatment of knee OA improves functional activity, reduces pain and probably can prolong remission in patients with the early stages of disease. The further long-term studies are needed with the use of ultrasound and MRI monitoring of the articular cartilage to obtain more accurate information and determine the most effective methods of PRP use in $\mathrm{OA}$ treatment.

\section{References:}

1. Rodeo SA, et al. Clin J Sport Med 2013;23: 238.

2. Jordan KM, et al. Ann Rheum Dis 2013;62:1145.

3. Wang-Saegusa A, et al. Arch Orthop Trauma Surg 2010;131:311.

4. Sampson S, et al. Am J of Physical Med \& Rehab 2010;89:961.

5. Beizel K, et al. Muscles ligaments tendons J 2014;4:38.

6. Kon et al. Knee Surg Sports Traumatol Arthrosc 2010;18:472.

7. http://www.hindawi.com/journals/mi/2010/174341/fig

\section{P330}

CYTOKINE PROFILES IN OSTEOPOROSIS: A PRO-INFLAMMATORY BIAS?

F. Azizieh $^{1}$, R. Raghupathy ${ }^{2}$, D. Shehab ${ }^{2}$, K. Al-Jarallah ${ }^{2}$, R. Gupta $^{2}$

${ }^{1}$ Gulf University for Science and Technology, Mubarak AlAbdullah Area, Kuwait, ${ }^{2}$ Kuwait University, Faculty of Medicine, Jabriya, Kuwait

Objective: The immune system is suggested to contribute to the increased incidence of osteoporosis seen in menopause, but these interactions are poorly understood. This study was aimed at measuring osteoclastogenesis stimulator cytokines TNF $\alpha$, IL-6, IL-17, IL-20 and inhibitor cytokines IFN $\gamma$, IL4, IL-10, IL-12, IL-13, IL-23 produced by peripheral blood mononuclear cells (PBMC) from postmenopausal women with normal, osteopenic and osteoporotic bone density.

Methods: The study population included 48 postmenopausal women of whom 19 had normal BMD, 19 had osteopenia and
10 had osteoporosis. PBMC were stimulated with a mitogen and cultured for 4 days; cytokine levels in culture supernatants were measured using the Multiplex system (Millipore) and read on the Magpex ELISA platform.

Results: PBMC from osteoporosis women produced significantly lower levels of the osteoclastogenesis inhibitor cytokines IL-4, IL-13 and IL-23 compared to women with normal BMD $(p=0.038,0.047,0.016)$ or with osteopenia $(p=0.049,0.03$, $0.016)$. Women with osteopenia produced lower levels of the anti- inflammatory IL-10 and IL-13 compared to women with normal BMD ( $p=0.032,0.032)$. Osteopenic women produced significantly higher levels of the osteoclastogenesis stimulator cytokines IL-6 and IL-17 compared to women with normal $\operatorname{BMD}(p=0.044,0.042)$. Osteoporotic women produced significantly higher levels of the inflammatory TNF $\alpha$ compared to osteopenic women $(p=0.021)$. This suggests a decreased anti-inflammatory or increased pro-inflammatory cytokine bias in osteopenic and osteoporotic women compared to those with normal BMD. Ratios of osteoclastogenesis stimulator cytokines to osteoclastogenesis inhibitor cytokines suggest a dominance of osteoclastogenesis stimulator cytokines in postmenopausal women with lower BMD.

Conclusion: These data provide insights into the possible influence of proinflammatory cytokines on the BMD of postmenopausal women.

Acknowledgment: This study is supported by Kuwait Foundation of Advancement of Science (KFAS) project no. 2013-1302-02.

\section{P331}

REAL-WORLD HIP AND OTHER FRAGILITY FRACTURE INCIDENCE IN TERIPARATIDETREATED PATIENTS IN THE UNITED STATES R. T. Burge ${ }^{1}$, D. P. Disch ${ }^{1}$, S. Gelwicks ${ }^{1}$, X. Zhang ${ }^{1}$, J. H. Krege ${ }^{1}$

${ }^{1}$ Eli Lilly and Company, Indianapolis, United States

Objective: To examine the relationship of treatment adherence and persistence with hip and non-hip fracture incidence rate among teriparatide (TPTD) patients in a U.S. claims database.

Materials and Methods: The Truven MarketScan ${ }^{\circledR}$ Research Databases, 2005-2011, were used to identify new TPTD users $\geq 18$ years old with continuous medical and pharmacy coverage 12-months pre- and 24-months post-first TPTD prescription. Adherence (medication possession ratio, MPR) groups were defined as high (MPR $\geq 0.80)$, medium $(0.5 \leq \mathrm{MPR}<0.8)$, and low (MPR $<0.5)$. TPTD persistence allowed for $<90$ day gaps between prescriptions. Persistence groups were defined by periods of $1-6,7-12,13-18$, and 1924 months. Patient baseline characteristics and concomitant 
medications were compared across MPR or persistence groups. Fracture incidence per 1,000 patient years (PYs) was summarized for any fractures (AF), hip fractures (HF), wrist fractures (WF), clinical vertebral fractures (VF), and non-vertebral fractures (NVF) separately. Fracture incidence rates by MPR and persistence groups were compared to the lowest MPR and persistence group, respectively, using ANOVA.

Results: Among 14,284 new TPTD subjects, baseline mean age was 68.4 years, $89.8 \%$ were female, and $29.6 \%$ had a previous fracture. Baseline characteristics were similar across MPR and persistence groups; though use [mean; range] of glucocorticoids [62.1; 58.3-67.3\%], anticonvulsants [25.5; 22.2-29.3\%], and bisphosphonates [58.3; 53.9-62.1\%] were statistically different $(p<0.05)$. Unadjusted incidence rates per 1000 PYs by fracture types (for low, medium, and high MPR) were: AF 82.9, 76.4, and 55.6**; HF 12.1, 9.4, and 5.8**; WF 13.0, 14.7, and 11.3; VF 33.8, 30.1, and 22.2**; and NVF 49.1, 46.3, and 33.4**. For treatment persistence, incidence rates per $1000 \mathrm{PYs}$ by fracture types (for 1-6, 7-12, 13-18, and 19-24 months persistence) were: AF 91.2, 74.2**, 69.2**, and 55.5**; HF 13.3, 10.4, 8.1*, and 5.9**; WF 13.9, 13.2, 13.0, and 11.4; VF 38.4, 28.0**, 28.0**, and 21.8**; and NVF 52.7, 46.2, 41.3*, and $33.7^{* *}(* * p<0.01$; * $p<0.05$ vs. lowest MPR or persistence group).

Conclusions: Among TPTD patients in a U.S. claims database, fracture incidence rates significantly decreased as MPR and persistence increased for vertebral, non-vertebral, hip, and any clinical fractures.

\section{P332}

RISK FACTORS FOR POSTMENOPAUSAL OSTEOPOROSIS DO NOT CHARACTERIZE PREDICTORS FOR SECOND HIP FRACTURE

$\underline{\text { E. Fernandez Tormos }}{ }^{1}$, B. Limousin Aranzabal ${ }^{1}$, C. Arraiz

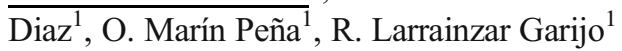

${ }^{1}$ Hospital Universitario Infanta Leonor, Madrid, Spain

Introduction: About $5-10 \%$ of the patients admitted for a hip fracture have in the past sustained a fracture of the contralateral hip. The second fracture is associated with high morbidity and mortality. Despite its importance, they are not described predictors that identify this risk, as standard practice to equate risk factors for postmenopausal osteoporosis.

Objective: Establish the incidence of second contralateral hip fracture in our historical data and analyze predictors.

Materials and Methods: Of a total of 1392 hip fractures treated in our hospital we identified 34 patients with second hip fracture, with an average age of 83.91 years, BMI of 25 and a predominance of women $(75.75 \%)$. The first half FRAX fracture was 7.49. The pattern followed of both hip fractures and the elapsed time between the two pathological events are collected. Risk factors analyzed include levels of vitamin D, FRAX, caffeine, alcohol, tobacco, thinness, drugs (steroids, benzodiazepines, immunosuppressants, etc.), maternal history of hip fracture and personal antecedent of vertebral compression. We also analyzed the osteoporosis treatment at the first hip fracture and at the time of the diagnosis of the second one.

Results: The $64.70 \%$ of cases of second fracture repeat first pattern presentation, averaging 31 months. When the patient is affected by the second fracture, the $60.61 \%$ of them were not taking any treatment for osteoporosis, despite being prescribed at high in the first fracture. Mean levels of vitamin D are $19.51 \mathrm{ng} / \mathrm{mL}$. As for risk factors for osteoporosis include smoking 6.06; $3.03 \%$ alcohol; coffee consumption of $3.03 \%$, history of prior fracture of $33.33 \%$, and maternal history of hip fracture in $9.09 \%$.

Conclusions: The high repetition rate of the fracture pattern, in accordance with some previous studies, suggests a structural alteration or biomechanics of both femurs. In our study the second hip fracture is an early event (31 months on average), and also a large number of patients are not taking medication at the time of the second fracture, indicating that treatment program strategy should be refined so as to improve the medical care after the first hip fracture.

Maternal history of hip fracture seems to be predictive for developing a second hip fracture. While there is a low percentage of traditional risk factors associated with postmenopausal osteoporosis, indicating that elderly hip fracture could be an independent entity, with their own risk factors.

References:

1. Dretakis E, et al. Acta Orthop Scand 1981;52:227.

2. Yamanashi A, et al. Osteoporos Int 2005;16:1239.

${ }^{3}$ Mitani S, et al. J Orthop Sci 2010;15:192.

4. Zhu Y, et al. Geriatr Gerontol Int 2015;15:11.

5. Shen SH, et al. J Am Med Dir Assoc 2014;15:725.

P333

OVARIECTOMY EXACERBATES EXPRESSION OF PROINFLAMMATORY CYTOKINES AND MATRIX METALLOPROTEINASES IN APICAL PERIO- DONTITIS IN RATS

P. C. Romualdo $^{1}$, M. P. Lucisano ${ }^{1}$, F. W. G. Paula-Silva ${ }^{1}$, R. A. B. Silva ${ }^{1}$, L. A. B. Silva ${ }^{1}$, A. M. Queiroz ${ }^{1}$, P. Nelson-Filho ${ }^{1}$ ${ }^{1}$ Department of Pediatric Dentistry, School of Dentistry of Ribeirão Preto, University of São Paulo, Ribeirão Preto, SP, Brazil

Objective: To evaluate the expression of mRNA of proinflammatory cytokines and matrix metalloproteinases in apical periodontitis (AP) of ovariectomized (OVX) rats compared with sham-OVX (sham) rats. 
Material and Methods: Twenty 12-week-old female Wistar rats were submitted to ovariectomy or sham surgery and followed by 9 weeks. Then, the dental pulp of upper first molars was exposed to the oral environment for induction of AP. The groups were as follows: sham, OVX, sham-AP and OVXAP. After 21 days of AP induction, the animals were euthanized and the blocks containing teeth and bone were collected to be analyzed by real time polymerase chain reaction (RT-PCR) for quantification of proinflammatory cytokines and matrix metalloproteinases genes (Il1b, Tnfa, Il6, Mmp8 and Mmp13).

Results: AP in OVX rats showed increased expression of Illb, Tnfa, Il6, Mmp8 and Mmp13 compared with AP in sham rats $(p<0.05)$. The ovariectomy alone (without AP induction) did not interfere with the expression of the genes evaluated. These findings indicate that infection associated with ovariectomy play an important role in the regulation of cytokines during the development of AP.

Conclusion: AP in OVX rats have increased expression of proinflammatory cytokines and matrix metalloproteinases compared with AP in sham rats. Based on that, we speculate that the hypoestrogenic condition aggravates inflammation and degradation of extracellular matrix components in AP which may offer insight into understanding the development of AP in female postmenopausal patients.

Acknowledgments: This work was supported by the São Paulo Research Foundation (FAPESP) grant 2013/18231-9 and 2014/13238-8.

\section{P334}

DLAT STUDY (PART 2): ANALYSIS OF SELFREPORTED PHYSICAL ACTIVITY AND HEALTH-RELATED QUALITY OF LIFE IN YOUNG LATVIAN ADULTS WITH VITAMIN D DEFICIENCY

M. Mukane $^{1}$, I. Rasa ${ }^{2}$, M. Mukans ${ }^{3}$, M. Orlova ${ }^{4}$

${ }^{1}$ Riga Stradins University, Riga East Clinical University Hospital, Latvian Osteoporosis and Metabolic Bone Diseases Association, Riga, Latvia, ${ }^{2}$ Riga East Clinical University Hospital, Riga Stradins University, Latvian Osteoporosis and Metabolic Bone Diseases Association, Riga, Latvia, ${ }^{3}$ Riga Stradins University, Riga East Clinical University Hospital, Riga, Latvia, ${ }^{4}$ Riga Stradins University, Riga, Latvia

Vitamin D (vit.D) deficiency is associated with higher morbidity and mortality rates, it also affects both physical and mental health. Participants of most vit.D studies were elderly or had various diseases. The aim of this cross-sectional study was to compare self-reported physical activity and healthrelated quality of life of young Latvian adults with vit.D insufficiency $(25(\mathrm{OH}) \mathrm{D}$ 20.0-29.9 $\mathrm{ng} / \mathrm{ml})$ and deficiency $(25(\mathrm{OH}) \mathrm{D}<19.9 \mathrm{ng} / \mathrm{ml})$.
The study was made from 4.02.14-20.11.14. Respondents (rsp.) of this study were 228 young adults that participated in DLAT study and had vit.D level $<30.0 \mathrm{ng} / \mathrm{ml}$. Each of them filled Likert scale-based form that involved questions about physical activity and health-related quality of life.

Prevalent group of rsp. were females $79 \%(n=180)$, with median age $23(26-22)$ y; $39 \%(n=89)$ of rsp. had vit.D insufficiency and $61 \%(n=139)$ rsp. had vit.D deficiency. The median 25(OH)D level was $17.8(23-14) \mathrm{ng} / \mathrm{ml}$.

The capability of rsp. physical activity, according to their opinion, was excellent in $81 \%(n=185)$ cases (with no difference in vit.D levels, $p=0.334), 92 \%(n=210)$ or rsp. reported their health as good or worse than good (with no difference in vit.D level, $p=0.624)$. According to data about behavior and cognitive functions, $22 \%(n=50)$ of rsp. evaluated their mental health as fair or worse than fair (with no difference in vit.D levels, $p=0.839$ ). Pain influenced daily activities (such as work and housework) of $37 \%(n=85)$ cases (with no difference in vit.D levels, $p=0.251$ ). Physical health or emotional problems had a negative impact on social activities in $37 \%(n=85)$ of cases (with no difference in vit.D levels, $p=0.251$ ).

Conclusion: There was no significant difference in physical, mental and social health conditions between young Latvian adults with vit.D insufficiency and deficiency. The main part of rsp. reported their physical activity as excellent and general health as good or worse than good, and some part of the rsp. mentioned having fair or worse than fair mental health and problems in social life.

\section{P335 \\ NEUROGENIC OSTEOPOROSIS IN PATIENTS WITH POST POLIOMYELITIS SYNDROME M. A. Lissens ${ }^{1}$ \\ ${ }^{1}$ Mechanical Engineering Sciences, KU Leuven University, Geel, Belgium}

Muscle mass, muscle strength, muscle density, sarcopenia, exercise and physical activity are well known factors to be correlated with bone mass, bone density and osteoporotic fractures. Both bone and muscle decay and dysfunction are seen in ageing. However, in neurological disorders resulting in muscular atrophy, bone loss may be seen also at younger ages. In this study BMD is measured both in the femoral neck and in the lumbar spine using DXA in 30 patients with so called post poliomyelitis syndrome, 18 women and 12 men, with a mean age of 56.3 years (ranging from 37 to 70). Mean age in men was 56 and in women 56.5 years. Eight women were postmenopausal. Femoral neck BMD was $-22.76 \%$ or -1.86 SD and lumbar BMD was $-13.21 \%$ or -1.25 SD. Low femoral bone mass was found in $80 \%$ of the patients $(12$ men and 12 
women, of whom 5 were postmenopausal) and low lumbar bone mass was seen in $63 \%$ (9 men and 10 women, of whom 8 were postmenopausal). Six patients (all women) had normal femoral BMD and 11 had normal lumbar BMD, whereas 6 (3 male and 3 female) had osteoporotic femoral BMD-scores $(<-2.5 \mathrm{SD})$ and 3 (1 man and 2 women) had osteoporotic lumbar BMD. The remaining patients had osteopenia: 18 femoral osteopenia $(<-1 \mathrm{SD})$ and 16 lumbar osteopenia. A significant difference in femoral (cortical) BMD between men and women was found: $-27.72 \%$ or $-2.24 \mathrm{SD}$ in men and $-19.46 \%$ or $-1.62 \mathrm{SD}$ in women. Only small sex differences were seen in lumbar (trabecular) BMD: $-13.85 \%$ or $-1.37 \mathrm{SD}$ in men and $-12.78 \%$ or $-1.17 \mathrm{SD}$ in women. The lowest (mainly cortical) BMD scores were present in (mainly male) patients with visible and clear lower limb muscle atrophy. These results showing a correlation between low muscle mass and low cortical BMD can be explained by neurogenic bone loss, both due to muscle atrophy and disturbed regulation of bone by the central nervous system (e.g., poliomyelitis and spinal cord injury).

\section{P336}

CHANGES IN THE UTILIZATION OF OSTEOPOROSIS DRUGS AFTER THE 2010 FDA BISPHOSPHONATE DRUG SAFETY COMMUNICATION: AN EVALUATION OF THE US MEDICAID PROGRAM

$\underline{\text { B. Balkhi }}{ }^{1}$, E. Seoane-Vazquez ${ }^{2}$, R. Rodriguez-Monguio ${ }^{3}, \mathrm{~K}$. $\overline{\text { Seoyoung }}^{4}$

${ }^{1}$ Dept. of Clinical Pharmacy, College of Pharmacy, King Saud University, Riyadh, Saudi Arabia, ${ }^{2}$ International Center for Pharmaceutical Economics and Policy, Massachusetts College of Pharmacy and Health Sciences, Boston, United States, ${ }^{3}$ School of Public Health and Health Sciences, University of Massachusetts, Amherst, MA, United States, ${ }^{4}$ Division of Pharmacoepidemiology and Pharmacoeconomics, Department of Medicine, Brigham and Women's Hospital and Harvard Medical School, Boston, United States

Introduction: In October 2010, the U.S. Food and Drug Administration (FDA) issued a safety communication regarding the risks of atypical fractures of the femur, for bisphosphonates drugs. This study evaluated the impact of the bisphosphonates FDA safety communication on the utilization of osteoporosis medications in Medicaid programs.

Methods: Osteoporosis drugs utilization data from the July 2006 to June 2014 were extracted from the National Summary Files from the Medicaid State Drug Utilization Data maintained by the Centers for Medicare \& Medicaid Services. We performed interrupted time series analyses to evaluate trends in utilization of osteoporosis drugs before and after the 2010 FDA Drug Safety Communication (DSC). Results: Time-series analyses of osteoporosis drug utilization in Medicaid program revealed a significant downward trend associated with the 2010 FDA bisphosphonates safety communication. Before the DSC was issued, the utilization rate slightly decreased between 2006 and 2010. In the year following the FDA safety communication the bisphosphonate DDDs per 1000 beneficiaries fell $22 \%$ yearly until the end of the study period.

Conclusions: The 2010 FDA bisphosphonates safety communication appeared to have influenced osteoporosis utilization in Medicaid recipients. The 2010 FDA bisphosphonates safety communication was associated with a significant reduction in the utilization of bisphosphonates in the Medicaid program.

\section{P337}

\section{ROLE IN THERAPY AND SAFETY OF FDA AP- PROVED OSTEOPOROSIS DRUGS}

B. Balkhi ${ }^{1}$

${ }^{1}$ Dept. of Clinical Pharmacy, College of Pharmacy, King Saud University, Riyadh, Saudi Arabia

Purpose: Clinical management of osteoporosis prevention and treatment changed substantially over the past two decades. This study assessed trends in approvals, safety profile, and place in therapy of osteoporosis drug in the US in the period 1984-2014.

Methods: Drug regulatory data for all FDA approved osteoporosis drugs including new molecular entities (NME) and therapeutic biologic license applications (BLA) were derived from the FDA website. Anatomical Therapeutic Chemical (ATC) classes and daily defined dosages (DDD) were extracted from the World health Organization Collaborating Centre for Drug Statistics Methodology. Safety data were derived from the FDA website and FDA drug approved labels. Information regarding the role of each drug in therapy was extracted from clinical guidelines and published literature. Descriptive statistics were performed.

Results: The FDA approved 23 drugs for the prevention and treatment of osteoporosis between 1984 and 2014; 3 in the 1980 s, 8 in 1990s, 8 in 2000s, and 4 in the period 2010 2014. The FDA approved 8 NME, 1 BLA, 5 new combinations, 7 new dosage forms and 1 new formulation of osteoporosis drugs. FDA issued a boxed warning for 11 osteoporosis drugs, including 8 for hormone replacement therapy products that were approved before 1999 .

Conclusions: Prior to 1995, two hormonal therapies were available for the prevention and treatment of osteoporosis. In 2014, 23 treatments were available with different mechanisms of action, dosing regimens and routes of administration. The FDA issued boxed warnings and REMS for several osteoporosis drugs. Long term effects of osteoporosis drugs remain a safety concern. 


\section{P338}

AGE-RELATED REFERENCE CURVES ADJUSTED TO WEIGHT AND HEIGHT OF VOLUMETRIC BONE DENSITY, STRUCTURE, AND BIOMECHANICAL PARAMETERS IN A POPULATION OF HEALTHY WOMEN: A HR-PQCT STUDY

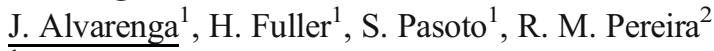

${ }^{1}$ Faculdade de Medicina da Universidade de São Paulo, Rheumatology Division, São Paulo, Brazil, São Paulo, Brazil

Introduction: The aimed of this study is evaluate age-related changes regarding volumetric BMD (vBMD), structure and biomechanical parameters in cortical and trabecular compartments in a healthy female population using HR-pQCT.

Methods: A cross-sectional cohort of 450 Brazilian women aged 20-85 years, reference curves age-related adjusted to weight and height of vBMD, structure, and biomechanical parameters at distal radius(DR) and distal tibia(DT) were constructed and the comparison of different decades was done.

Results: A significant trabecular and cortical bone loss were found at fifth decade compared to second decade. After $65 \mathrm{y}$ old, a decrease of the trabecular (vBMD:-10.8 \%, number:$5.3 \%$, separation:8.1 \%) was found at DR, and a cortical decrease (vBMD:-8.4\%, thickness:-13.2 \%) was observed at DT, compared to fifth decade $(p<0.05)$. An increase of cortical porosity (Ct.Po) was observed at fifth decade (DR:84.6\%, DT:107.2 \%; $\leq \leq 0.001)$ compared to second decade. Furthermore, after $65 \mathrm{y}$-old a further increase of more than $50 \%$ of Ct.Po was demonstrated in both sites compared to fifth decade $(p<0.001)$. Change of stiffness (S) became more prominent after age of 65 (DR:-12.1\%, DT:-9.7\%; $p<0.001$ ). Pearson correlations between stiffness and HR-pQCT parameters revealed a significant correlation with densities and structure in trabecular and cortical compartments. A weak correlation was observed between $\mathrm{S}$ and Ct.Po (r:-0.162 at DR, r:0.273 at DT; $p<0.05$ ).

Conclusions: This study highlight important site and compartment differences in patterns of age-related bone loss on a healthy population of women and showed the importance of trabecular and cortical parameters to predict bone strength.

\section{P339 \\ COMPLEX THERAPEUTIC APPROACH FOR PATIENTS WITH RHEUMATOID ARTHRITIS}

N. Nikolov $^{1}$, M. Krastanova ${ }^{1}$, M. Panchovska ${ }^{2}$

${ }^{1}$ UMHAT G. Stranski, Pleven, Bulgaria, ${ }^{2}$ Medical University, Plovdiv, Bulgaria

Introduction: Rheumatoid arthritis (RA) is a chronic, systematic and autoimmune disease. Around $40 \%$ of the patients become handicapped and incapable of work to a various degree after the 10th year with the disease. Currently there is no scientifically proven treatment for patients with RA. For some of the patients the leading symptoms are pain and stiffness in the joints, while others develop heavy joint deformations and permanent disabilities. Applying a complex approach with rheumatologic aid and a physio- rehabilitation program significantly improves the condition of the patients with RA, the strength of their symptoms diminishes and becoming handicapped is avoided or delayed.

Aim: To measure the effect of conducting a complex drug treatment combined with a physio-rehabilitation program in patients with RA.

Methods and Materials: Between 2012 and 2015, 57 patients with rheumatoid arthritis were hospitalized and treated at the rheumatology ward at UMBAL "Dr. Georgi Stranski" Pleven. 23 of them were male and 34 female, aged between 23 and 69 years old, with moderate activity (SUE $<25 \mathrm{~mm}$, fibrinogen and CRP at relevant values, AST and RF (-). In addition to the drug therapy with analgesics, NSAIDs and chondroprotectors, DMARD the patients also undergo a complex physical and rehabilitation program consisting of: electrotherapy, therapeutic massages, cryotherapy, active methods of kinesitherapy and occupational therapy. Functional tests and measurements were made for the purpose of the study: VAS for the pain, a daily activities life test (DAL) and complex functional assessment test (CFA).

Results and analysis: An individual file was created for every patient where the results from the tests and measurements are stored in order to track the patient's condition over the course of treatment (which consists of 3 stages: a period in the rheumatology ward, treatment in the medical rehabilitation ward and ambulatory physio-rehabilitation courses). The Wilcoxon rank test (a statistical method for analysis and spreading of nonparametric data) was applied to interpret the complex functional assessment test results. The Wilcoxon curve shifts to the right, which indicates an improvement of the patients' self-sufficiency and their quality of life. When applying the VAS test for pain and daily activities life test, the average values at the beginning and at the end of the observed period were measured and compared. The results show a pain decrease by around $35 \%$, and an increase in the joint function. The ANOVA statistical analysis method was applied to the results of the both tests (VAS and DAL).

Results and discussion: The collaboration between rheumatologists and physiotherapists for treatment of patients with RA widens the range of daily activities that the patients can perform without experiencing pain, slows down the progression of the deterioration process and makes the daily life of patients more complete and self-sufficient. For patients who are still at working age, the duration of their temporary inability to work is decreased, and they can resume working activities sooner, or they can acquire new professional skills with a lower degree of physical stress which suits their physical 
condition and abilities. The advent of permanent disability for patients is delayed.

Conclusion: The complex physio-rehabilitational and rheumatological treatment for patients with RA is effective and leads to a significant improvement in the quality of life of patients.

\section{P340}

ULTRASONOGRAPHIC FEATURES OF ACUTE ANSERINE SYNDROME AND ITS CLINICAL AND EPIDEMIOLOGICAL RELATIONSHIPS.

C. A. Guillén-Astete ${ }^{1}$, G. Silvestre-Egea ${ }^{1}$

${ }^{1}$ Ramon y Cajal University Hospital, Madrid, Spain

Background: Anserine syndrome includes two different pathological features: The anserine bursitis and the anserine tendinitis. Last one could be properly named as anserine enthensytis. Although is considered a minor complain, anserine syndrome is a relative frequent cause of pain among athletes and even sedentary people. Most diagnostics of anserine syndrome are made considering topographic semiotics. It is logical to assume that treatment of different physiopathological features are associated with different clinical history but not necessary with differences in the clinical exam.

Objective: To find relationships between ultrasonographic findings and epidemiological data in patients with anserine syndrome.

Methods: All the images of ultrasonographic studies of patients from 2013 to 2014 who were clinically assessed due to anserine syndrome were reviewed. For that purpose we use the electronic database of the A\&E department. Registries with the terms "anserine bursitis", "anserine tendinitis", "anserine enthesitis" and "anserine syndrome" in the field of diagnostic were collected. No identity information was captured. Gender, age, history of hypothyroidism and diabetes, and time from onset of symptoms were used to find correlation with ultrasonographic findings. Cases with more than month within the onset of symptoms were excluded. Ultrasonographic assessments were performed by two trained rheumatologist using standard EULAR definitions as part of common clinical practice during the period chosen. Findings considered positive were: bursitis and enthesitis.

Results: 113 registries which fulfilled the clinical diagnostic criteria were analyzed with their corresponding ultrasonographic images. 38 cases were unrelated to any particular ultrasonographic finding, 20 were compatible with bursitis and 56 with enthesitis. Average age in the three groups were 55 SD 9, 46 SD 7 and 69 SD 8 years old (ANOVA, $P<0.0001$ ). Patients with bursitis where significantly younger than patients with enthesitis (Student's $t$-test, $P=0.0001$ ). Also, patients without findings were significantly older than both other groups (Student's $t$-test, $P<0.0001$ for both comparisons).
Proportion of female gender was 58.9, 65,0 and 53.6\%, in enthesitis, bursitis and no-findings group, respectively (chisquare test, $P=0.647$ ). Only two diabetic and two hypothyroid patients were identified in the bursitis group (No comparisons were made). According to time since onset of symptoms, patients with less than a week presented bursitis and enthesitis in 6.67 and $44.67 \%$, respectively. Patients with symptoms within 7 to 14 days presented bursitis and enthesitis in a proportion of 55.17 and $34.48 \%$, respectively. Finally patients with symptoms within 15 days and less than a month presented bursitis in $2.56 \%$ and enthesitis in $64.10 \%$. Bursitis was significantly higher in patients with acute symptoms compared to the other two groups $(P<0.001)$. In the other hand, most patients with enthesitis were classified into the groups with a longer time since onset of symptoms $(P<0.05)$.

Conclusions: Our population of study is mainly composed by patients with less than a month of disease. Comparing to other series, we can consider ours as an acute symptomatic one. The older age founded in patients without ultrasonographic findings could be explained by the presence of disease related with the middle collateral ligament as many other series has previously found as one of the main differential diagnostic in anserine syndrome. Our results points to state that bursitis is a finding more prevalent in acute scenarios while bursitis is more frequently seen in patients with more time of evolution. To perform this differential diagnosis could be relevant when choosing a therapeutic approach (infiltration, physiotherapy or local NSAID) so we consider the ultrasonography a very useful tool when assessing an anserine syndrome. As our study is based on a retrospective analysis of ultrasonographic studies, the presence of concomitant bursitis and enthesitis could be underestimated, so we recognize that prospective larger studies should be conducted in order to support our observations.

\section{P341 \\ THICKER AND DENSER CORTICES BUT LESS INTACT TRABECULAE ARE ASSOCIATED WITH ATYPICAL FEMUR FRACTURES: A CROSS-SECTIONAL CASE-COHORT ANALYSIS}

A. K. Wong ${ }^{1}$, K. S. Davison ${ }^{2}$, J. D. Adachi ${ }^{3}$, J. P. Brown ${ }^{4}$, R. G. Josse ${ }^{5}$, A. Khan ${ }^{6}$, A. M. Cheung ${ }^{1}$

${ }^{1}$ Osteoporosis Program, University Health Network, Toronto, Canada, ${ }^{2}$ University of Victoria, Victoria, Canada, ${ }^{3}$ Department of Medicine, St. Joseph's Hospital, McMaster University, Hamilton, Ontario, Canada, ${ }^{4}$ Laval University and CHU de Québec Research Centre, Quebec City, QC, Canada, ${ }^{5}$ St. Michael's Hospital, Toronto, Canada, ${ }^{6}$ Medicine, McMaster University, Hamilton, Canada

Objectives: To determine bone quality parameters associated with AFFs and to evaluate potential diagnostic thresholds. 
Methods: A cross-sectional analysis combined women with AFFs with controls from three clinical studies. AFFs were ascertained by ASBMR task force criteria. HR-pQCT distal radius and tibia scans were obtained. Fracture risk categories were determined by 2010 Osteoporosis Clinical Guidelines. Covariates: age, height, weight, history of fragility fractures, parental hip fractures, glucocorticoids use, and antiresorptive therapy duration were obtained.

Data analysis: Fixed effects models determined differences in HR-pQCT radius and tibia parameters between fracture risk groups and the AFF cohort, adjusting for covariates. Diagnostic thresholds were evaluated on 1000 bootstrapped samples summarizing diagnostic parameters and odds ratios for AFFs when HR-pQCT parameters were beyond the diagnostic thresholds.

Results: Among 1151 women $(68.4 \pm 9.5 y$ old $)$, those with AFFs $(N=122)$ used antiresorptives for longer (10.4y vs. $2.3 y)$ and $33.6 \%$ were Asian (7.9 \% for non-AFFs). Ct.Th and vBMD were larger at both sites in the AFF group compared to moderate and high fracture risk groups $(p<0.001)$. $\mathrm{Tb} . \mathrm{Sp}$ was larger in the AFF group at the tibia compared to other fracture risk groups $(p<0.001)$. Duration antiresorptive therapy $>4.6$ years showed $88.4 \%$ sensitivity, $78.2 \%$ specificity and $97.6 \%$ negative predictive value but only $12.2 \%$ positive predictive value for AFFs. Smaller Tb.Th and larger $\mathrm{Tb} . \mathrm{Sp}$ at the tibia and higher Ct.Th and cortical vBMD at the radius all showed sensitivity and negative predictive values $>60 \%$. Among Asians, positive predictive value was larger for $\mathrm{Tb} . \mathrm{Sp}$ in the fully adjusted model (42.5-47.3\%) compared to duration antiresorptive therapy alone (40.4$40.5 \%$ ). Odds for an AFF were significant for Ct.Th $(1.78(1.00,3.19))$ and cortical vBMD $(2.09(1.20,3.66))$ at the radius and for $\mathrm{Tb} . \mathrm{Sp}(2.00(1.14,3.54))$ and trabecular vBMD $(2.25(1.26,4.02))$ at the tibia. Similar patterns were observed among Asians but with larger effect sizes and confidence intervals.

Conclusions: Thicker, denser cortices and less intact trabeculae are risk factors for AFFs. These parameters remain poor diagnostic candidates; although within Asians, there may be stronger diagnostic value for AFFs using antiresorptive therapy duration, with a mild added effect by HR-pQCT parameters.

\section{P342}

EFFECT OF DENOSUMAB ON BONE MINERAL DENSITY AND TRABECULAR BONE SCORE IN POSTMENOPAUSAL OSTEOPOROSIS: THREE -YEAR RESULTS

T. Petranova $^{1}$, I. Sheytanov ${ }^{1}$, S. Monov ${ }^{1}$, R. Nestorova ${ }^{2}$, R. Rashkov

${ }^{1}$ Clinic of Rheumatology, Medical University, Sofia, Bulgaria,

${ }^{2}$ Rheumatology Centre "St. Irina", Sofia, Bulgaria
Aim: To evaluate the three year efficacy and safety of Denosumab on BMD and bone microarchitecture, measured by trabecular bone score (TBS), in women with postmenopausal osteoporosis.

Patients and Methods: Study included 71 postmenopausal women mean age 62.1 (7.6), diagnosed with osteoporosis with T-score lower than $-2.5 \mathrm{SD}$ at lumbar spine and/or total hip. Patients with history of endocrine, renal and severe GI tract diseases, such as those receiving other bone-active drugs, were excluded from the study. Participants received Denosumab $60 \mathrm{mg}$ once per 6 months for 3 years and daily calcium/vitamin D. DXA BMD and T-score, TBS and presence of low energy fractures (anamnesis, medical history, $\mathrm{X}$ ray of thoracic and lumbar spine) were assessed at baseline and Month 12, 24 and 36. BMD was measured on lumbar spine and hip using DXA (Lunar Prodigy ${ }^{\circledR}$ Primo, GE Healthcare). The software program (TBS iNsight ${ }^{\circledR}$ v1.9, v 2.1 Med-Imaps, Pessac, France) was applied to the lumbar spine DXA scans to determine their TBS indexes.

Statistical analysis: Statistical analysis was performed by Kolmogorov- Smirnov test, paired samples test or Wilcoxon signed ranks test using the SPSS 13.0 for Windows.

Results: The three-yr treatment with denosumab was associated with a continuous improvement in BMD and trabecular bone score. The values of the examined parameters changed as follows - lumbar spine BMD increased from 3.7 \% at M12 to $8.9 \%$ at M36 $(\mathrm{p}<0.05)$, total hip - from $1.5-5.6 \%$ $(\mathrm{p}<0.05)$ and TBS - from $1.7-4.3 \%(\mathrm{p}<0.05)$. The T-score of lumbar spine and total hip changed in corresponding proportion to the increase of BMD. We did not observe clinically manifested fractures during the evaluation period. Serious adverse reactions were not registered.

Conclusions: Denosumab was highly effective in the 36month treatment of postmenopausal women with OP leading in a significant increase of BMD (lumbar spine and hip) and TBS. These results clearly demonstrate that denosumab is a valuable therapeutic option in the present day treatment of OP.

\section{P343 \\ RELATION AMONG BONE PHENOTYPES AND ESTROGEN RECEPTOR ALPHA GENE POLY- MORPHISM XBAI IN THE CENTRAL MORAVIA REGION \\ P. Hrdý $^{1}$, P. Novosad ${ }^{1}$, P. Fojtík ${ }^{2}$, M. Běhal ${ }^{3}$, M. Zemánek ${ }^{3}$, P. Horák $^{4}$ \\ ${ }^{1}$ Mediekos Amb. Ltd, Zlín, Czech Republic, ${ }^{2}$ AGEL Research and Training Institute-Ostrava Vítkovice Branch, Ostrava-Vítkovice Hospital Centre of Care for the Gastrointenstinal Tract, Ostrava-Vítkovice, Czech Republic, ${ }^{3}$ Imalab Lab. Ltd, Zlín, Czech Republic, ${ }^{4}$ University Hospital Olomouc, Olomouc, Czech Republic}


Aims: Osteoporosis is a systemic disease of a skeleton which is characterized by a lowered amount of BMD and an increased risk for fractures. Characteristics of a bone mineral are described in bone phenotypes: BMD, bone quality, bone turnover markers (BTM). A level of clinical expression of bone phenotypes is significantly influenced by genes for osteoporosis. Individual genotypes are determined by combinations of single nucleotide polymorphisms (SNP) of these genes. In our work we studied the polymorphism XbaI (rs9340799, 156751A $>$ G) of gene for estrogen receptor alpha (ESR1).

Method: DNA was isolated by kit MagNA Pure Compact Nucleic Acid Isolation Kit I (using automated isolator MagNA Pure MagNA) from $400 \mu$ l noncoagulable blood samples. Detection of polymorphism was carried out by using a method of real time PCR using hydrolysation and FRET probes on LC 480 II (Roche). Correctness of the molecular examination results was randomly checked by sequencing on a sequenator ABI 3130 AVANT (Applied Biosystem). Osteological parameters were then compared with frequency of genotype occurrence as wild type, SNP heterozygote or SNP homozygote. BTM as osteocalcin (OC) and beta CrossLaps (CTX) were measured using standard biochemical methods. BMD was measured in lumbar spinal column, total hip and femoral neck using densitometer (DXA) Lunar iDXA (GE Healthcare).

Results: We studied a group of 596 female patients with postmenopausal osteoporosis. Above stated polymorphism were compared with a level of expression of bone phenotypes in the given individuals. Studied bone phenotypes are: BMD measured by DXA, laboratory parameters of calcium phosphate metabolism (Ca, P), BTM (OC, CTX, bone ALP). From anamnesis we followed mainly typical osteoporotic fractures. We managed to prove a significantly lower BMD in the lumbar spine area for genotype GG SNP XbaI of gene for ESR1: $0.852 \mathrm{~g} / \mathrm{cm}^{2}$ vs. $0.891 \mathrm{~g} / \mathrm{cm}^{2}(p=0.05)$.

Conclusion: Our observations show a slight but statistically significant difference in an amount of bone phenotype BMD expression in observed polymorphism XbaI.

\section{P344 \\ FREQUENCY AND RELATION OF BONE PHENOTYPES AND VITAMIN D RECEPTOR GENE POLYMORPHISMS BSMI, TAQI, APAI AND FOKI IN THE CENTRAL MORAVIA REGION}

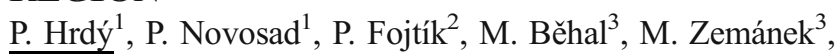
M. Janura ${ }^{4}, K$. Janurová ${ }^{5}$, P. Horák ${ }^{6}$

${ }^{1}$ Mediekos Amb. Ltd, Zlín, Czech Republic, ${ }^{2}$ AGEL Research and Training Institute-Ostrava Vítkovice Branch, Ostrava-Vítkovice Hospital Centre of Care for the Gastrointenstinal Tract, Ostrava-Vítkovice, Czech Republic,

${ }^{3}$ Imalab Lab. Ltd, Zlín, Czech Republic, ${ }^{4}$ Department of
Nature Science in Kinanthropology, Faculty of Physical Culture, Palacky University, Olomouc, Olomouc, Czech Republic, ${ }^{5}$ IT4 Innovations National Supercomputing Centre VŠB-Technical University, Ostrava, Czech Republic, ${ }^{6}$ University Hospital Olomouc, Olomouc, Czech Republic

Aims: Osteoporosis is a systemic disease of a skeleton which is characterized by a lowered amount of BMD and an increased risk for fractures. Characteristics of a bone mineral are described in bone phenotypes: $\mathrm{BMD}$, bone quality, bone turnover markers (BTM). A level of clinical expression of bone phenotypes is significantly influenced by genes for osteoporosis. Individual genotypes are determined by combinations of single nucleotide polymorphisms (SNP) of these genes. In our work we studied the polymorphisms BsmI, TaqI, ApaI and FokI of gene for vitamin D receptor.

Method: DNA was isolated by kit MagNA Pure Compact Nucleic Acid Isolation Kit I (using automated isolator MagNA Pure MagNA) from $400 \mu 1$ noncoagulable blood samples. Detection of all polymorphisms was carried out by using a method of real time PCR using hydrolysation and FRET probes on LC $480 \mathrm{II}$ (Roche). Correctness of the molecular examination results was randomly checked by sequencing on a sequenator ABI 3130 AVANT (Applied Biosystem). Osteological parameters were then compared with frequency of genotype occurrence as wild type, SNP heterozygote or SNP homozygote.

BTM as osteocalcin (OC) and C-terminal peptide collagen I, beta CrossLaps (CTX) were measured using standard biochemical methods. BMD was measured in lumbar spinal column, total hip and femoral neck using densitometer (DXA) Lunar iDXA (GE Healthcare). We carried out a statistic data processing with the use of Statgraphics Plus 5.1 software and $\mathrm{R}$ software 3.2.0. Normality of data distribution was verified with the use of Shapiro-Wilk test. Homoscedasticity was tested with Barlette's test. With such parameters that did not show a normal data distribution we used Kruskal-Wallis test and Turkey-Kramer post hoc test.

Results: We studied a group of 230 female patients with postmenopausal osteoporosis. Above stated polymorphism were compared with a level of expression of bone phenotypes in the given individuals. Studied bone phenotypes are: BMD measured by DXA, laboratory parameters of calcium phosphate metabolism $(\mathrm{Ca}, \mathrm{P})$, bone turnover markers (OC, CTX, bone ALP). From anamnesis we followed mainly typical osteoporotic fractures. We managed to prove a significantly higher frequency of fractures for genotype CC SNP TaqI $(p=0.018)$ and for genotype AA SNP BsmI $(p=0.011)$. Next we managed to prove a significantly higher level of CTX for genotype AA SNP ApaI $(p=0.021)$.

Conclusion: Our observations show a slight but statistically significant difference in an amount of bone phenotypes (fractures and CTX) expression in observed polymorphisms TaqI, BsmI and ApaI. 


\section{P345}

\section{MULTIPLE MYELOMA PRESENTING AS CER- VICAL SPINE COMPRESSION}

\section{A.-K. Kul ${ }^{1}$, F.-B. Bagcier ${ }^{1}$, U.-B. Bayraktutan ${ }^{2}$}

${ }^{1}$ Radiology, Ataturk University Faculty of Medicine, Erzurum, Turkey, ${ }^{2}$ Physical Therapy and Rehabilitation, Atatürk University Faculty of Medicine, Erzurum, Turkey

Multiple myeloma (MM) is a malignant monoclonal proliferation of plasma cells usually showing bone involvement with predilection of the spine ${ }^{1}$. The cervical spine is the least common site of disease involvement. We report the case of 86-year-old man presenting with progressively increased cervical pain, associated with disturb walking. The patient was diagnosed as having MM with cervical vertebra involvement and he has started chemo - therapy. A 86-year-old female patient was referred to our department with a-week history of neck pain, lower and upper extremities weakness. Physical examination found a stiff cervical spine. His neurological examination revealed quadripyramidal syndrome, superficial sensory disturbance at the level of the fifth cervical vertebra (C5) and urinary troubles. Biological abnormalities consisted of: sedimentation rate $100 \mathrm{~mm} / \mathrm{h}$, C-reactive protein $60 \mathrm{mg} /$ $\mathrm{dl}$, haemoglobin $9.2 \mathrm{~g} / \mathrm{dl}$. White cell count, platelet count, serum creatinine, calcaemia were within the normal range. The X-ray examination of the cervical spine showed lytic lesions of C5. Spinal MRI showed an epidural mass compressing the spinal cord at the level of C5 with osteolysis of the posterior arch. The patient underwent an open surgery consisting of C4-C5 laminectomy, resection of the tumor, decompression of the spinal cord reconstruction and stabilisation of the vertebral column. Postoperatively, the examination showed the improvement of neurological deficit and of sensory disturbance. Microscopic examination and immunohistochemical studies confirmed the diagnosis of multiple myeloma of kappa subtype. The protein electrophoresis of the blood and urine, showed the presence of a paraprotein (monoclonal protein type $\operatorname{IgG}$ ) band, with reduction of the other (normal) immunoglobulins. Serum immunoglobulin (Ig) levels were as follows: Ig G: $50 \mathrm{~g} / \mathrm{L}$; $\operatorname{IgA}: 0,67 \mathrm{~g} / \mathrm{L}$ and IgM: $0,4 \mathrm{~g} / \mathrm{L}$. The Bence Jones protein was positive (type kappa light chains). The bone marrow aspiration showed infiltration with $45 \%$ of plasma cells. The diagnosis of IgG MM with kappa light chains, stage IIIB according to the Durie- Salmon staging system was made. The patient started systemic vincristine, melphalan, cyclophosphamide and prednisone combination chemotherapy. Currently, he received 18 cycles of chemotherapeutic regimen and showed a complete response. The bisphosphonate zoledronic acid was also administrated at the same time. During the follow-up the general condition of our patient was well and he had complete relief of pain. The clinical examination was normal and he showed no residual neurological deficit. Cervical spine cord compression can be the first presentation of MM and should bear in mind the rare possibility of the disease ${ }^{2}$. Treatment should include surgical resection and local radiation therapy, as the response to conventional chemotherapy of extramedullary multiple myeloma is considered very poor ${ }^{3}$.

${ }^{1}$ Angtuaco EJ, et al. Radiology 2004;231:11.

${ }^{2}$ Chakraborti C, Miller K. JMCR 2010;4:251.

${ }^{3}$ Rao G, et al. J Neurosurg Spine 2006;5:140.

\section{P346}

FASTING PLASMA GLUCOSE AND GLYCATED HEMOGLOBIN A1C LEVELS WERE ASSOCIATED POSITIVELY WITH BONE DENSITY AND INVERSELY WITH TRABECULAR BONE SCORE IN JAPANESE ELDERLY MEN: FUJIWARA-KYO OSTEOPOROSIS RISK IN MEN (FORMEN) STUDY

M. Iki $^{1}$, Y. Fujita ${ }^{1}$, K. Kouda ${ }^{1}$, A. Yura ${ }^{1}$, Y. Sato ${ }^{2}$, J.-S. $\overline{\text { Moon }}^{3}$, J. Tamaki ${ }^{4}$, R. Winzenrieth ${ }^{5}$, N. Okamoto ${ }^{6}$, N. Kurumatani $^{6}$

${ }^{1}$ Department of Public Health, Kindai University Faculty of Medicine, Osaka-Sayama, Japan, ${ }^{2}$ Department of Human Life, Jin-ai University, Echizen, Japan, ${ }^{3}$ Department of Nursing and Medical Care, Kio University, Koryo-cho, Japan, ${ }^{4}$ Department of Hygiene and Public Health, Osaka Medical College, Takatsuki, Japan, ${ }^{5}$ Med-Imaps, Bordeaux, France, ${ }^{6}$ Department of Community Health and Epidemiology, Nara Medical University School of Medicine, Kashihara, Japan

Objectives: Fracture risk in patients with type 2 diabetes mellitus (T2DM) is elevated even though they have quite high areal BMD (aBMD). Mechanism for this increase in fracture risk is not completely understood. We aimed to clarify the association between T2DM and glucose metabolism indices and bone microarchitecture represented by trabecular bone score (TBS).

Materials and Methods: 2012 community-dwelling men aged 65 years and older completed the baseline study of the Fujiwara-kyo Osteoporosis Risk in Men (FORMEN) Study comprising measurements of aBMD at the spine (QDR4500A, Hologic, USA), fasting plasma glucose (FPG), glycated hemoglobin A1c (A1c), height and weight, and interviews covering past history of diseases including diabetes mellitus.

T2DM was defined as middle-age or elderly onset of physician-diagnosed T2DM or diabetes mellitus without specification of type 1 or 2, or biochemical test results obtained in the present study: FPG level $\geq 126 \mathrm{mg} / \mathrm{dl}$ or A $1 \mathrm{c}$ levels $\geq 6.5 \%$. TBS was calculated in the same vertebrae for aBMD using TBS iNsight software (v. 2.1, Med-Imaps, France). 
Results: (1) After exclusion of participants with history of diseases or medications affecting bone metabolism, 1684 men ( $72.9 \pm 5.2$ years) were analyzed. (2) aBMD of men with T2DM was significantly higher $(p<0.0001)$ than those without while significant difference was not found in TBS. (3) FPG and A1c levels were significantly positively correlated with aBMD after adjusting for age, BMI and TBS $(p<0.005)$, and inversely with TBS after adjusting for age, BMI and aBMD $(p<0.05)$. (4) The least square mean TBS adjusted for age, BMI and aBMD was significantly lower in the highest quartile of FPG or A1c than in the lowest quartile $(p<0.05)$ with a significant decreasing trend over the quartile groups $(p<0.005)$ while the mean aBMD adjusted for age, BMI and TBS was significantly higher in the highest quartile than in all the other groups $(p<0.05)$.

Conclusions: Trabecular bone microarchitecture may be deteriorated in T2DM. This may account for the elevated risk of fracture in patients with T2DM.

Disclosure: R. Winzenrieth is a senior scientist at Med-Imaps.

\section{P347}

INCIDENCE OF FALL AND STABILITY FOLLOWING TOTAL KNEE REPLACEMENT

\section{Khanna ${ }^{1}$}

${ }^{1}$ Department of Orthopaedics, National Institute of Medical Sciences, Jaipur, India

\begin{abstract}
Aim: To analyse the incidence of fall following total knee replacement and comparison with a similar age group of patients.
\end{abstract}

Material And Methods: A total of 200 patients, aged between 60 to 65 years, were included in this study. Out of these 100 patients(50 males and 50 females) were 6 months postoperative patients of total knee replacement and 100 were normal population. All the patients were selected randomly. A detailed history was taken and the patients were assessed for the presence of any varus or valgus instability. The patients were asked if they had suffered from a fall in the past 3 months or not. If yes then how many falls and what treatment was taken by them.

Results: Out of 100 postoperative patients 29 suffered from a fall within the past 3 months as compared to the to 11 patients in the control group. This was found to be statistically significant. Out of the 29 patients who fell in the postoperative period 19 were found to be unstable whereas there was no instability seen in the patients who did not fall.

Conclusion: Patients undergoing total knee replacement were more prone to fall down as compared to healthy individuals of the same age group of the same race and ethnicity. This incidence was higher in patients with postoperative instability of knee. Hence, this study indicates that the instability acts as an important prognostic factor for the outcome of total knee replacement.

\section{P348}

ASSOCIATIONS OF BODY COMPOSITION AND MUSCLE FUNCTION WITH PQCTDETERMINED BONE DENSITY AND GEOMETRY IN OVERWEIGHT AND OBESE OLDER ADULTS

D. Scott ${ }^{1}$, L. McMillan ${ }^{1}$, C. A. Shore-Lorenti ${ }^{2}$, K. M. Sanders $^{3}$, P. R. Ebeling ${ }^{1}$

${ }^{1}$ Bone and Muscle Health Research Group, Department of Medicine, Monash Medical Centre, Melbourne, Australia,

${ }^{2}$ Melbourne Medical School, The University of Melbourne, Melbourne, Australia, ${ }^{3}$ Institute for Health and Ageing, Australian Catholic University, Melbourne, Australia

Objective: To determine associations of body fat and components of sarcopenia (low muscle mass and function) with bone density and geometry in overweight and obese communitydwelling older adults.

Materials and Methods: 67 overweight or obese (mean \pm SD BMI: $33.0 \pm 6.4)$ volunteers aged $\geq 50$ years $(62.8 \pm 8.0$ y; $66 \%$ female) underwent whole-body DXA (for assessment of appendicular lean mass [ALM] and total body fat), hand grip strength (HGS) and gait speed assessments. Bone density and geometry were assessed by pQCT at the distal $(4 \%)$ and proximal $(66 \%)$ radius and tibia. Generalised linear models examined associations of ALM, HGS, gait speed and total body fat with bone density and geometry indices after mutual adjustment, and further adjustment for age, sex and selfreported physical activity.

Results: There were no associations of total body fat with bone density or geometry indices at radius or tibia (all $P>0.05$ ). Conversely, ALM was positively associated with distal tibial total bone density $(\beta=3.00 ; p=0.011)$ and area $(\beta=15.99$; $p<0.001)$; proximal tibial cortical area $(\beta=5.79 ; p<0.001)$, stress-strain index (SSI) $(\beta=73.34 ; p<0.001)$ and periosteal circumference $(\beta=0.65 ; p=0.010)$; and proximal radial cortical area $(\beta=1.45 ; p<0.001)$, SSI $(\beta=7.02 ; p<0.001)$, periosteal $(\beta=0.57 ; p=0.001)$ and endosteal $(\beta=0.48 ; p=0.030)$ circumference. HGS was positively associated with distal tibial total area $(\beta=6.45 ; p=0.009)$ and proximal radial periosteal $(\beta=0.23 ; p=0.019)$ and endosteal $(\beta=0.28 ; p=0.031)$ circumference. Gait speed was positively associated with proximal tibial cortical area $(\beta=76.66 ; p=0.004)$ and thickness $(\beta=1.12 ; p=0.009)$, and distal radial total density $(\beta=77.99$; $p=0.037)$. At proximal radius, gait speed was positively associated with cortical thickness $(\beta=0.64 ; p=0.011)$, but negatively associated with periosteal $(\beta=-8.10 ; p=0.032)$ and endosteal $(\beta=-12.12 ; p=0.013)$ circumference.

Conclusions: Despite a perception that higher fat mass is beneficial for bone health, this does not appear to be case in overweight and obese older adults. Loss of body fat during weight loss interventions is unlikely to 
negatively affect bone health if exercise targeting maintenance of muscle mass and function is incorporated.

\section{P349}

LONGITUDINAL STUDY INVESTIGATING ASSOCIATIONS OF SARCOPENIC OBESITY WITH BONE MINERAL DENSITY AND INCIDENT FALLS AND FRACTURES AMONG COMMUNITY-DWELLING OLDER MEN: THE CONCORD HEALTH AND AGEING IN MEN PROJECT (CHAMP)

D. Scott ${ }^{1}$, V. Hirani ${ }^{2}$, V. Naganathan ${ }^{2}$, F. Blyth ${ }^{2}$, D. G. Le

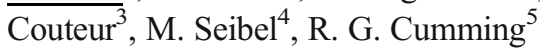

${ }^{1}$ Bone and Muscle Health Research Group, Department of Medicine, Monash Medical Centre, Melbourne, Australia, ${ }^{2}$ Centre for Education and Research on Ageing, Ageing and Alzheimer's Institute, Concord Hospital, University of Sydney, Sydney, Australia, ${ }^{3}$ ANZAC Research Institute \& Charles Perkins Centre, University of Sydney, Sydney, Australia, ${ }^{4}$ Bone Research Program, ANZAC Research Institute, and Dept of Endocrinology \& Metabolism, Concord Hospital, The University of Sydney, Sydney, Australia, ${ }^{5}$ School of Public Health, University of Sydney, Sydney, Australia

Objective: To investigate associations of sarcopenic obesity with five-year changes in BMD, and six-year incident falls and fractures, among Australian community-dwelling older men.

Materials and Methods: 1529 CHAMP participants with complete baseline data for hip, spine and total body DXA (BMD, appendicular lean mass (ALM) and body fat), hand grip strength (HGS) and gait speed were included. 1367 and 958 had DXA at two and five years, respectively. Participants were contacted every 4 months for $6.0 \pm 2.2$ years to ascertain incident falls and fractures (confirmed by radiographic reports). Sarcopenia was defined by European Working Group on Sarcopenia (EWGSOP) and Foundation for the National Institutes of Health (FNIH) algorithms. Obesity was defined as body fat $\geq 30 \%$. Generalised estimating equations and logistic regression determined associations of sarcopenia and obesity with changes in BMD, falls and fracture adjusting for age, physical activity, smoking, living alone, comorbidity, vitamin $\mathrm{D}$, albumin, haemoglobin, and psychotropic, bisphosphonate and corticosteroid use.

Results: After multivariate adjustment, ALM was associated with BMD at all sites $(\beta=0.08 ; p=0.004)$. Body fat percentage was associated with hip BMD only $(\beta=0.01 ; p<0.001)$. During follow-up, $51 \%$ of participants reported at least one fall, $26 \%$ had multiple falls, and $10 \%$ had at least one fracture. The likelihood of at least one $(\beta=-0.87 ; p=0.004)$ and multiple $(\beta=-1.42 ; p<0.001)$ falls was reduced with higher gait speed but multiple falls increased with higher body fat percentage $(\beta=0.03 ; p=0.03)$. Higher hip BMD $(\beta=-4.07 ; p<0.001)$, but not body fat or sarcopenia components, was associated with lower likelihood of incident fracture. According to EWGSOP and FNIH definitions, 106 (7\%) and 63 (4\%) participants were sarcopenic obese, respectively. Compared to non-sarcopenic non-obese, EWGSOP- defined sarcopenic obese men had increased likelihood of at least one (odds ratio: $2.04,95 \% \mathrm{CI}$ : $1.24-3.34)$ and multiple $(2.49,1.53-4.06)$ falls.

Conclusions: Interventions which target increases in muscle mass and function, and also reductions in body fat, are likely to be most beneficial for prevention of incident osteoporosis and falls in community- dwelling older men.

\section{P350}

\section{SEROTONIN TRANSPORTERS COULD} AFFECT BMD IN OLDER WOMEN

$\underline{\text { S. Patru }}^{1}$, A. C. Bighea ${ }^{1}$ I. R. Marcu ${ }^{1}$, D. Matei ${ }^{1}$, A.-M. $\overline{\text { Bumbea }}^{1}$, D. V. Caimac ${ }^{1}$

${ }^{1}$ University of Medicine and Pharmacy, Craiova, Romania

Background: Antidepressants are often prescribed for depressive symptoms, and depression itself has been associated with a lower BMD. But many clinical studies raise the possibility that serotonin transporters may affect bone metabolism.

Aim: To determine if selective serotonin reuptake inhibitors (SSRI) use among older women is associated with increased rates of hip bone loss.

Material and Methods: Were included 52 patients (women, mean age, 72.4 years): 27 nonusers (defined as not taking an SSRI or any other type of antidepressant) and 25 users (taking only SSRI). Total hip BMD was measured at baseline and at the end of the study, after one year using the same instruments as used for the initial measurements.

Results: On average, women taking SSRI experienced a higher rate of bone loss at the total hip than nonusers $(-0.67 \%$ vs. $-0.42 \%$ per year; $P=.005)$.

Conclusions: In this cohort of older women use of SSRI was associated with an increased rate of bone loss at the total hip. References:

${ }^{1}$. Hubbard R, et al. Am J Epidemiol 2003;15877.

2. Diem SJ, et al. Arch Intern Med 2007;167:1240.

\section{P351}

TUBERCULOSIS PSOAS ABSCESS PRESENTING WITH CHRONIC LOW BACK PAIN

\section{A.-K. Kul ${ }^{1}$, F.-B. Bagcier ${ }^{1}$, U.-B. Bayraktutan ${ }^{2}$}

${ }^{1}$ Radiology, Atatürk University Faculty of Medicine, Erzurum, Turkey, ${ }^{2}$ Radiology, Ataturk University Faculty of Medicine, Erzurum, Turkey 
Tuberculosis (TB) is a common infectious disease, caused by various strains of mycobacteria, usually Mycobacterium tuberculosis in human. Vertebral TB (Pott's disease) is the most common form of skeletal TB which may give rise to psaoas abscess because of close vicinity. We present a case of a woman who presented to our university hospital with a history of acute back pain. She was subsequently diagnosed with tuberculosis spondylitis (Pott's disease) and psoas abscess. Surgical drainage with adjuvant anti- tuberculosis therapy was carried out. A 50-yearold woman with complains of fatigue, low back pain for 2 years presented our university hospital. There was no neuropathic pain. He had no symptoms suggestive of inflammatory back pain. Patient's previous medical history was insignificant. There was no history of trauma, chronic disease, alcohol and substance abuse. She had subjective weakness in both legs but denied changes in urinary or bowel habits. Naproksen partially relieved the back pain. In her physical examination, She had painful abdomen at palpation at the right iliac fossa. During her follow-up, the patient was investigated for the etiology. Ultrasound of the abdomen demonstrated a hypoechoic mass suggestive of psoas abscess. Computed tomography of abdomen revealed a hypodense lesion of the right psoas muscle suggesting abscess with muscular edema and lytic lesions at L3 and L4 vertebral bodies. A needle biopsy of L3 was performed, and pathology revealed necrotizing granulomatous inflammation. Biopsy specimen was sent for polymerase chain reaction (PCR) for M. tuberculosis, the result for M. TB was positive subsequently surgical drainage of the abscess with a great quantity of pus and necrotic tissue was performed and the patient was placed on pharmacological therapy (isoniazid, rifampin, pyrazinamide, and ethambutol). At the moment, the patient is in the third month of the treatment and she is in a very good condition after surgery without symptoms, undergoing anti-tTB treatment. TB is still common in our country. The diagnosis of Pott's disease should be considered in patients who live in endemic TB regions have chronic back pain ${ }^{1}$. Microscopy and culture are recommended to confirm the diagnosis. Antimicrobial therapy, usually in multidrug combinations, is the primary spinal TB treatment; however, adjuvant surgical treatment may be warranted in cases of neurologic involvement or medical treatment failure ${ }^{2}$.

1Sternbach G, Pott P. J Emerg Med 1996;14:79

2Maron R, et al. Spine 2006;31:E561

$<$ ptyle= "margin-left:18.0 pt">

\section{P352}

\section{A RARE CAUSE OF ANKLE PAIN: PROXIMAL TIBIOFIBULAR SYNOSTOSIS}

A.-K. Kul $^{1}$, F.-B. Bagcier ${ }^{1}$, U.-B. Bayraktutan ${ }^{2}$

${ }^{1}$ Physical Therapy and Rehabilitation, Atatürk University Faculty of Medicine, Erzurum, Turkey, ${ }^{2}$ Radiology, Atatürk University Faculty of Medicine, Erzurum, Turkey
Tibiofibular synostosis or heterotopic ossification within the interosseous membrane of ankle is an uncommon cause of ankle disability. We report the case of a 30 -year-old man who presented with ankle pain of unknown etiology. A fulllength tibia film revealed a proximal tibiofibular synostosis. He successfully underwent a fibular osteotomy with complete symptomatic relief. A 30-year-old man presented with complaints of left ankle pain for five years that was worse after walking a few hundred yards and was completely relieved by rest. He had broken his tibia 6 years prior to presentation and the fracture had healed uneventfully with conservative treatment. For almost a year after the fracture had healed, the patient was totally asymptomatic, but then his ankle started to ache on exertion. He was seen twice, in 2013 and 2014, at his local hospital where ankle radiographs did not reveal any abnormalities. Therefore, he was referred to the author for a second opinion. Clinical examination was entirely normal except for pain and discomfort in his ankle during the push-off phase of walking. A full-length tibia radiograph taken at that time showed a proximal tibiofibular synostosis with a normal ankle joint, which was further confirmed on MRI scan. Following a literature search to find the various treatment modalities for this condition. He successfully underwent fibular osteotomy. The patient remained symptom free at one year follow-up. Although there are a few reports of distal tibiofibular synostosis in the literature, an isolated posttraumatic proximal synostosis presenting with ankle pain has never been reported ${ }^{1}$. If a synostosis is present from birth or occurs before closure of the physis, it is always associated with at least one deformity, such as distal positioning of the proximal tibiofibular joint, leg length discrepancy, bowing of the fibula, or valgus deformity of the knee. The absence of all of these anomalies in our case, along with a history of trauma, supports a post-traumatic etiology, and suggests that the synostosis occurred after physeal plate closure 2 .

${ }^{1}$ Frick SL, et al. J Bone Joint Surg 2001;83-A:247.

${ }^{2}$ O'Dwyer KJ. Acta Orthop Belg 1991;57:204.

\section{P353 \\ OSTEOPOROSIS AND FRACTURES IN PATI- ENTS WITH RHEUMATIC INFLAMMATORY DISEASES}

E. G. Gladkova ${ }^{1}$, E. K. Kozhemyakina ${ }^{2}$, O. M. Lesnyak ${ }^{3}$

${ }^{1}$ Ural State Medical University, Ekaterinburg, Russian Federation, ${ }^{2}$ Regional Hospital \#1, Yekaterinburg, Russian Federation, ${ }^{3}$ North-Western State Medical University named after I.I. Mechnikov, Saint-Petersburg, Russian Federation

Objectives: To estimate the burden of fractures in patients with rheumatic inflammatory diseases.

Materials and Methods: A specially designed questionnaire was introduced to patient of rheumatic department 
Sverdlovsk regional hospital №1. The study included 242 patients aged 50 to 79 years (mean age $58.4 \pm 6,5$ years, 194 women and 48 men).

Results: High incidence of osteoporosis $(35,5 \%)$ was observed in patient with rheumatic inflammatory diseases. Intervention threshold (FRAX) was identified in $46.8 \%$ of patients. Low-energy fractures of the skeleton were identified in $33,9 \%$ of patient. Oral glucocorticoid therapy increased the risk (odds ratio $(95 \% \mathrm{CI})$ ) of osteoporotic fractures by 2.68 fold $(95 \%$ CI $1.55-4.63, p=0.004)$.

Conclusion: High incidence of osteoporosis and osteoporotic fractures was observed in patient with rheumatic inflammatory diseases.

\section{P354}

ELISA MEASUREMENT OF CIRCULATING PERIOSTIN IN ANIMAL MODELS OF BONE LOSS OR BONE FORMATION AND IDENTIFICATION OF CIRCULATING AND TISSUE-SPECIFIC ASSOCIATED FORMS OF PERIOSTIN

E. Gineyts ${ }^{1}$, N. Bonnet ${ }^{2}$, C. Bertholon ${ }^{1}$, A. Pagnon-Minot ${ }^{3}$, O. Borel $^{1}$, D. Hartmann ${ }^{4}$, R. D. Chapurlat ${ }^{5}$, S. Ferrari ${ }^{2}$, P. Garnero $^{1}$, P. Clezardin ${ }^{1}$, J.-C. Rousseau ${ }^{1}$

${ }^{1}$ INSERM 1033 Hôp E. Herriot, Lyon, France, ${ }^{2}$ Geneva University Hospital, Geneva, Switzerland, ${ }^{3}$ Novotec, Lyon, France, ${ }^{4}$ CNRS 5510 / Mateis, Lyon, France, ${ }^{5}$ INSERM UMR 1033, Université de Lyon, Lyon, France

Purpose: Matricellular protein periostin (PN) mediates the bone anabolic effect of PTH and is associated with metastasis from solid tumors. The aim of this study was to develop an enzyme-linked immunosorbent assay (ELISA) to measure PN levels in the serum of animals treated with intermittent PTH or inoculated with $4 \mathrm{~T} 1$ breast cancer cells. Circulating- and tissue-associated forms of PN recognized by the antibodies used for the ELISA were also characterized.

Methods: We developed a sandwich ELISA using polyclonal antibodies raised against the recombinant mouse PN (Ab1) or the last C-Ter 22 amino acids (Ab2). In the "PTH model", mice (12 weeks old) were treated with intermittent PTH $(40 \mathrm{mg} / \mathrm{kg} /$ day $)$ or vehicle for 5 weeks. In the "metastasis model", mice (5 weeks old) were inoculated iv with $4 \mathrm{~T} 1$ cells and sacrificed at day 14 after tumor cell inoculation. Mice developed bone and lung metastases. Osteolytic lesions were monitored at day 13 by radiography. Lung metastases were assessed by histology and immunohistochemistry. At sacrifice, serum and tissues were collected and stored at $-80{ }^{\circ} \mathrm{C}$. Using 2-D gel electrophoresis and western blotting, $\mathrm{PN}$ forms in the serum and bone and soft tissues of wild type mice were characterized and PN spot specificity was controlled using a model of Postn deficient mice. The ability of recombinant human cathepsin $\mathrm{K}$ to cleave the recombinant mouse PN (R\&D) was also tested.

Results: In the "PTH model", serum PN levels were significantly increased compare to untreated animals, $(+44 \%$, mean \pm SD: $2178 \pm 198$ vs. $3139 \pm 213 \mathrm{ng} / \mathrm{ml}, p=0.02)$. In contrast, in the "metastasis model", a significant decrease of serum PN levels was observed, compared to age-matched control mice $(-26 \%$, mean \pm SD: $560 \pm 100$ vs. $756 \pm 64 \mathrm{ng} / \mathrm{ml}, p<0.002)$. In serum and tissues, the two antibodies recognized several PN spots differing according to their isoelectric points and molecular weights (MW). We found a "bone signature" corresponding to a $50 \mathrm{kDa}$ PN fragment not recognized by Ab2. Additionally, PN was a substrate for cathepsin $\mathrm{K}$, leading to the generation of fragments with a range of MWs similar to that found in the bone signature.

Conclusion: ELISA measurement of serum PN levels reflects variations of the bone metabolism that are associated with an anabolic treatment or cancer-induced bone disease. Additionally, we found that PN was cleaved by cathepsin K, enabling the identification of a "bone signature" in the animals.

\section{P355}

INVERSE ASSOCIATION BETWEEN BODY FAT MASS WITH 25(OH)D LEVELS IN INDIVIDUALS WITH OSTEOPENIA AND OSTEOPOROSIS

$\underline{\text { B. S. E. Peters }}^{1}$, N. A. G. França ${ }^{1}$, E. B. Curatella ${ }^{1}$, M. M. S. Lima $^{1}$, P. C. Santos ${ }^{1}$, L. A. Martini ${ }^{1}$

${ }^{1}$ Nutrition Department, School of Public Health, University of São Paulo, São Paulo, Brazil

Objective: It is controversial if the vitamin D deficiency is a consequence or a factor predisposing to obesity. The fact is that previous studies have demonstrated positive association between vitamin $\mathrm{D}$ deficiency and obesity. Therefore, the aim of this study was to assess the association between fat mass and serum levels of $25(\mathrm{OH}) \mathrm{D}$ in individuals with osteopenia and osteoporosis.

Material and Methods: A subsample of a cross-sectional study of a representative sample of individuals living in Sao Paulo, Brazil, aged older than 40 years of both genders, diagnosed with osteoporosis and osteopenia according to the criteria of WHO, was evaluated $(n=65)$. Body weight (BW), height, BMI, waist circumference (WC), hip circumference $(\mathrm{HC})$, fat mass percentage $(\% \mathrm{FM})$, fat mass index (FMI), were evaluated. Body composition was analyzed by iDXA (GE Healthcare, Madison, WI). Serum levels of $25(\mathrm{OH}) \mathrm{D}$ were measured by chemiluminescence assay (Advia Centaur XP-Siemens). Spearman correlation, Student's $t$-test and One-way ANOVA were performed. 
Results: 81.5 and $18.5 \%$ of the sample presented osteopenia and osteoporosis, respectively. Underweight was observed in $15.4 \%$ of subjects, normal weight in $43.1 \%$, overweight in 16.9 and $24.6 \%$ presented obesity. The mean of $\% \mathrm{FM}$ was 37 , $7 \%$. Excess of FMI was present in $69.2 \%$ of subjects. The mean serum levels of 25(OH)D was $16.2(7.0) \mathrm{ng} / \mathrm{ml}$. Insufficiency of vitamin D [serum 25(OHD): $29.99-20 \mathrm{ng} / \mathrm{ml}$ ] was observed in $29.2 \%$ of subjects, and deficiency of vitamin $\mathrm{D}$ [serum 25(OHD): $<20 \mathrm{ng} / \mathrm{ml}$ ] in $55.8 \%$. There was an inverse correlation between serum levels of $25(\mathrm{OH}) \mathrm{D}$ with $\mathrm{BW}$ $(r=-0.309 ; P=.012)$, BMI $(r=-0.311 ; P=0.012)$, WC $(r=-0.348 ; P<0.01), \mathrm{HC}(r=-0.301 ; P=0.02), \% \mathrm{FM}$ $(r=-0.344 ; P<0.01)$ and FMI $(r=-0.351 ; P<0.01)$. Serum levels of $25(\mathrm{OH}) \mathrm{D}$ was significantly higher among individuals with normal weight and underweight compared with obese individuals ( $P=0.017$ and $P=0.30$, respectively).

Conclusion: There were a high prevalence of excess of body weight (overweight + obesity), fat mass and vitamin D deficiency in our population. It was observed an inverse association between fat mass and serum levels of $25(\mathrm{OH}) \mathrm{D}$ in the present study.

\section{P356}

CAN CT IMAGE DEBLURRING IMPROVE FINITE ELEMENT PREDICTIONS AT THE PROXIMAL FEMUR?

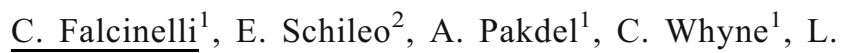
Cristofolini $^{3}$, F. Taddei ${ }^{2}$

${ }^{1}$ Orthopaedic Biomechanics Laboratory, Sunnybrook Health Sciences Centre, Toronto, Canada, ${ }^{2}$ Laboratorio di Tecnologia Medica, Istituto Ortopedico Rizzoli, Bologna, Italy, ${ }^{3}$ Dipartimento di Ingegneria Industriale, Universita' di Bologna, Bologna, Italy

Objective: Clinical application of computed tomography (CT) based finite element (FE) estimates of bone strength recently showed encouraging results [1]. However, model performance may be improved. In fact, in-vitro validation studies showed systematic errors in strain prediction, especially where bone cortex is thin (i.e. the femoral neck) [2].

This work seeks to verify if, and to what extent, a CT deblurring algorithm restoring both geometry and intensity data in thin bone structures [3], can improve strain and failure load prediction accuracy of CT-based FE models of the proximal femur.

Material and Methods: CT scans were acquired of 14 fresh-frozen human cadaveric femora. An estimate of the 3D Point Spread Function for each CT scan was used within a deconvolution solver to perform deblurring. Using the restored images, FE models of the proximal femur were generated [4]. Each femur was tested nondestructively in both stance and fall loading configurations to measure surface strains, and then loaded to failure in stance or fall.

Deblurred FE predictions of strains and failure load were compared to experimental measurements, and FE predictions obtained from the original CT data (no deblurring).

Results: An enhancement in strain prediction accuracy was obtained using deblurred FE models, with the Standard Error of Estimate reduced by $11 \%$ with respect to reference FE models. Marked improvements at the femoral neck were achieved (e.g. peak error reduced by $38 \%$ ). Using deblurred models, the regression equation between FE-predicted and measured failure loads was characterized by a slope not significantly different from one, with $\mathrm{R}^{2}=0.89$, unchanged with respect to reference models. Absolute differences between estimated and measured failure loads were consistently reduced by deblurring in stance (mean error 10 vs. $15 \%$ ) but not in fall (32 vs. $17 \%$ ).

Conclusions: The proposed $\mathrm{CT}$ deblurring technique yielded moderate but significant improvements in femoral FE predictions, and can thus be seen as a first and worthwhile step in the improvement of CT-based FE models of the human femur.

References:

1. Falcinelli et al. Bone 2014;67:71

2. Grassi et al. J Biomech 2012;45:394

3. Pakdel et al. Phys Med Biol 2012;57:8099

4. Schileo et al. J Biomech 2014;47:3531

\section{P357}

BONE STATUS IN A PATIENT WITH THYMOMA- ASSOCIATED MYASTHENIA GRAVIS, ADRENAL TUMOR, AND LONG-TIME CORTICOTHERAPY FOR RHEUMATOID ARTHRITIS M. Carsote $^{1}$, A. Ghemigian ${ }^{1}$, A. Valea ${ }^{2}$, S. E. Albu ${ }^{3}$, C. Vasiliu $^{3}$

${ }^{1}$ C.Davila University of Medicine and Pharmacy \& C.I.Parhon National Institute of Endocrinology, Bucharest, Romania, Bucharest, Romania, ${ }^{2}$ I.Hatieganu University of Medicine and Pharmacy \& Clinical County Hospital, Cluj-Napoca, Romania, Clu-Napoca, Romania, ${ }^{3}$ C.Davila University of Medicine and Pharmacy \& University Emergency Hospital, Bucharest, Romania, Bucharest, Romania

Introduction: Women with regular menses may associate bone loss due to various rheumatologic and/or endocrine causes like autoimmune conditions and associated therapy or tumor disorders (of adrenals or hypophysis).

Material and Method: This is a case report of an adult subject who was investigated in different medical centers from 
Romania for almost 3 decades of life. Bone evaluation included: central DXA (GE Lunar); 25-hydroxyvitamin D (25-OH D) assay (chemiluminescence); and endocrine panel for adrenals. Case data: A 49-old nonsmoking female was operated at age of 28 for a thymoma the myasthenia gravis was diagnosed. 2 years later rheumatoid arthritis was diagnosed and corticotherapy was continued for 22 years. At age of 47 she had persistent uterine bleeding so total hysterectomy with ovaries preservation was done and no hormonal replacement therapy was recommended. A few months later she suffered a gastric hemorrhage because of a gastric ulcer. Recently the patient accused persistent lumbar pain. The investigations lead to the discovery of a left adrenal tumor of 2.15 by $2.2 \mathrm{~cm}$. The endocrine phenotype was not suggestive for Cushing's syndrome and the glucocorticoid axes were not evaluated because the patient did not tolerate the stop of corticoids. The aldosterone/rennin ratio was normal like plasma metanephrines (10 pg/mL, normal: 10-90 pg/mL), and normetanephrines (of $20 \mathrm{pg} / \mathrm{mL}$, normal: 15-180 ng/mL). Chromogranin A was mildly increased at $147 \mathrm{ng} / \mathrm{mL}$ (normal: 20-125 ng/mL). FSH levels revealed persistent ovarian activity (of $8 \mathrm{mUI} / \mathrm{mL}$ ). The assays showed vitamin D deficiency (25OHD of $18.1 \mathrm{ng} / \mathrm{mL}$, normal $>30 \mathrm{ng} / \mathrm{mL}$ ); low BMD of $0.728 \mathrm{~g} /$ $\mathrm{cm} 2$, Z-score of -2.6 SD (femoral neck), of $0.762 \mathrm{~g} / \mathrm{cm} 2, \mathrm{Z}$ score of $-2.6 \mathrm{SD}$ (total hip). No vertebral fractures were found. Intravenous ibandronate every 3 months was given together with vitamin D and calcium supplements. Close adrenal and bone follow-up is recommended a part from previous comorbidities.

Conclusion: In pre-menopausal women despite the estrogen protection a complex panel of causes may lead to bone loss. Early screening and intervention might prevent the skeleton impairment. The adrenal tumors affect BMD in cases with persistent hypercortisolemia and adrenal incidentalomas with exogenous Cushing's syndrome.

\section{P358}

EFFICACY OF CFA IN EARLY AND LATE KNEE OSTEOARTHRITIS: A MULTICENTER STUDY

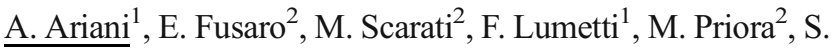
Parisi $^{2}$

${ }^{1}$ UO Medicina Interna e Reumatologia - Azienda Ospedaliero Universitaria di Parma, Parma, Italy, ${ }^{2}$ Struttura Complessa Reumatologia - Azienda Ospedaliero Universitaria Città della Salute e della Scienza di Torino, Torino, Italy

Introduction: Knee osteoarthritis (OA) is a chronic disease characterized by pain, limited gait and patients' autonomy reduction. These symptoms often implicate the use of analgesic or oral non steroid anti- inflammatory drugs (NSAIDs) with the well-known side effects. Cetylated fatty acids (CFA) are emerging as a viable alternative to topical NSAIDs. The aim of this work is to verify if a short-time
CFA treatment is effective in patients with early vs. late radiological knee OA.

Materials and methods: Sixty patients with knee OA according to ACR classification criteria were enrolled. Each of them underwent to knees' X-rays and, afterwards, to a CFA topical treatment (two applications per day for one week). Before the treatment beginning and three days after its conclusion, all patients completed a WOMAC (Western Ontario and McMaster Universities) questionnaire. All knees' X- rays were classified according to Kellgren-Lawrence scale. Nonparametric tests were applied; $p$-value $<0.05$ was considered statistically significant.

Results: After a week of treatment the median WOMAC questionnaire increased from 54.0 (95\% confidence interval: $50.4-57.5)$ to 61.6 (95\%CI: 58.1-65.1) $(p<0.0001)$. In particular we observed statistically significant increase of the scores in all four WOMAC domains increased $(p<0.0001)$. The improvement in the overall score of the WOMAC is slightly higher in those patients with X-rays knees in stage 2 according to the scale of Kellgren-Lawrence $(p=0.05)$.

Conclusions: This study's results suggest that topical CFA treatment in patients with knee OA may represent a therapeutic option available to the rheumatologist. CFA improve all the knee OA aspects investigated by the WOMAC questionnaire. Our findings leads one to suppose that CFA are effective in all patients notwithstanding the radiological stage of knee X-rays.

P359

MULTIPLE MECHANISMS OF LOW BLOOD CALCIUM LEVELS IN A PATIENT WITH PROSTATE CANCER-ASSOCIATED HYPOGONADISM AND THYROIDECTOMY FOR SUSPECTED THYROID MALIGNANCY

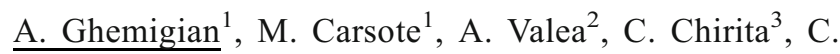

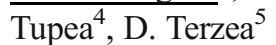

${ }^{1}$ C.I. Parhon National Institute of Endocrinology, Bucharest, Romania, ${ }^{2}$ I. Hatieganu University of Medicine and Pharmacy \& Clinical County Hospital, Cluj-Napoca, Romania, ${ }^{3}$ Medicover, Bucharest, Romania, ${ }^{4}$ C.I.Parhon National Institute of Endocrinology, Bucharest, Romania, ${ }^{5}$ C.I.Parhon National Institute of Endocrinology \& Monza Oncoteam, Bucharest, Romania

Introduction: Men with prostate cancer might associate bone status damage and not only because of bone metastases (for instance hypogonadism-related bone loss or vitamin $\mathrm{D}$ deficiency). Material and Method: An adult male case is reported during follow-up at different medical centers from Romania. Bone assays included: central DXA (GE Lunar) providing BMD; circulating 25-hydroxyvitamin D (25-OH D) assay (chemiluminescence); and endocrine panel for thyroid masses. 
Case presentation: A 66-old smoking male was diagnosed in 2013 with prostate adenocarcinoma (a nodule of $13 \mathrm{~mm}$ at MRI) based on biopsy: Gleason grade of $7(3+4)$; $64 \%$ infiltration on the surface; with extra-capsular invasion; and treated with local radiotherapy and GnRH analogues for the following 2 years. Later on, the $\mathrm{CT}$ scan (to check the disease extension) incidentally showed a thyroid nodule of $48 \mathrm{~mm}$ at left thyroid lobe with right deviation of the trachea. He intermittently accused mild breathing problems.

On admission, normal thyroid function, calcitonin levels and thyroid antibodies were found. Low levels of total testosterone were detected as well as low-normal levels of total serum calcium (of $8.9 \mathrm{mg} / \mathrm{dl}$ ) according to decreased 25-OHD (of $12.69 \mathrm{ng} / \mathrm{mL}$, normal: $>30 \mathrm{ng} / \mathrm{mL}$ ). A suspected second malignancy needed total thyroidectomy. The pathological report confirmed a follicular adenoma with hyperplasic siderophage epithelium and nodules of aniso-follicular adenomatous aspects, with reactive lymph nodes. After surgery low levels of ionic calcium (of $3.6 \mathrm{mg} / \mathrm{dl}$, normal: $3.9-4.9 \mathrm{mg} / \mathrm{dl}$ ) were consistent for post- operative tetany. The patient was given vitamin $\mathrm{D}$ and calcium supplements without the correction of hypogonadism because of hormone-dependent gonad neoplasia. BMD was normal: of $1.297 \mathrm{~g} / \mathrm{cm} 2$ at lumbar level (T-score of $-0.6 \mathrm{SD}$, T-score of $-0.8 \mathrm{SD}$ ), $0.963 \mathrm{~g} / \mathrm{cm} 2$ at femoral neck (T-score of $-0.8 \mathrm{SD}, \mathrm{Z}$ - score of $0.1 \mathrm{SD}$ ), $1.142 \mathrm{~g} / \mathrm{cmv}$ at total hip (T-score of $0.3 \mathrm{SD}, \mathrm{Z}$-score of $0.7 \mathrm{SD}$ ). Close follow-up of bone status is considered useful as well as oncologic periodic checkup.

Conclusion: Hypocalcemia in a male patient already diagnosed and treated for a testosterone- dependent cancer may be related to hypovitaminosis $\mathrm{D}$ because of pelvic-abdominal radiotherapy, low appetite and consecutive nutrient deficiencies and, as in our case, a second tumor (which we not malign) caused thyroidectomy-related hypoparathyroidism.

\section{P360 \\ OBESITY-RELATED FRAGILITY FRACTURE: THE NEED FOR PERSISTENT HYPERCOR- TISOLEMIA ASSAY}

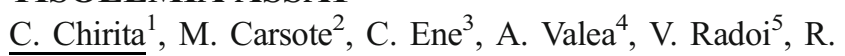
Petrescu $^{6}$

${ }^{1}$ Medicover, Bucharest, Romania, ${ }^{2}$ C. Davila University of Medicine and Pharmacy \& C.I.Parhon National Institute of Endocrinology, Bucharest, Romania, ${ }^{3}$ Babes Medical Centre, Bucharest, Romania, ${ }^{4}$ I. Hatieganu University of Medicine and Pharmacy \& Clinical County Hospital, ClujNapoca, Romania, ${ }^{5}$ Romanian Association of Psychiatry and Psychotherapy, Bucharest, Romania, ${ }^{6}$ Military Central Hospital, Bucharest, Romania

Introduction: Despite old theories, obesity does not protect against fractures as ankle, humerus, and distal forearm. The inflammation, the weight- related physical forces involved in the fall might contribute to these but persistent mild high cortisol may play a role, especially in cases without a classic phenotype of Cushing's syndrome and thus associating a delayed diagnosis.

Material and Method: This is a case presentation of a female who suffered of long-term obesity and associated osteoporosis. She was evaluated in a few Romanian medical centers. Endocrine assessment was done by central DXA (GE Lunar Prodigy), bone turnover markers, 25-hydroxyvitamin D (25$\mathrm{OH}$ D; normal levels of $>30 \mathrm{ng} / \mathrm{mL}$ ); dexamethasone (DXM) suppression tests, etc.

Case presentation: A 58-old nonsmoking female suffered of obesity since childhood. Multiple methods of diet and psychological approaches were tried without an adequate response. Progressively, she associated high blood pressure, cardiac insufficiency, hyperlypemia, impaired glucose tolerance and a left humerus fragility fracture. Bariatric surgery was recommended and a prior endocrine evaluation. Normal TSH, freeT4 levels were found. High baseline plasma cortisol (of $31 \mu \mathrm{g} / \mathrm{dL}$, normal $<21 \mu \mathrm{g} / \mathrm{dL}$ ) and suppressed ACTH (of $2.57 \mathrm{pg} / \mathrm{ml}$, normal between 3-66 pg/mL) were found. The DXM 2 days $\times 2$ mgs test also confirmed the Cushing's syndrome based on plasma morning cortisol of $4.44 \mu \mathrm{g} / \mathrm{dL}$. CT scan found a right adrenal tumor of $5.4 \mathrm{~cm}$ and laparoscopic adrenalectomy was performed first. Adrenal insufficiency was registered and the patient lost 15 kilos within the first three months. Bariatric surgery is postponed for the moment depending on clinical outcome. Before surgery the bone indices were more consistent with osteoporotic obesity than glucocorticoid osteoporosis: nondominant forearm BMD of $0.655 \mathrm{~g} /$ $\mathrm{cm}^{2}$, T-score of $-0.8 \mathrm{SD}, \mathrm{Z}$-score of $-0.1 \mathrm{SD}$; normal circulating levels of P1NP, CrossLaps, osteocalcin, alkaline phosphatase, and PT; low 25-OHD of $9.7 \mathrm{ng} / \mathrm{mL}$.

Conclusion: BMD is inadequately high in patients with high BMI. However fragility fractures might be seen. Vitamin D has been correlated with metabolic components without a clearly established cause-effect relationship. Based on our observations we encourage the adrenal axes evaluation in obese patients especially if complications as osteoporosis are diagnosed since tumor-related persistent mild hypercortisolemia is one of the mechanisms that can easily be corrected by adequate nonbariatric surgery.

\section{P361 \\ CHANGES IN OSTEOPOROTIC HIP FRAC- TURES WITHIN A 25-YEAR PERIOD}

M. Angulo Tabernero ${ }^{1}$, A. Aguilar Ezquerra ${ }^{1}$, J. Ungria Murillo $^{2}$, M. C. Cassinello Ojea ${ }^{1}$, J. Sanz Pascual ${ }^{3}$, J. Cuenca Espierrez ${ }^{1}$, C. Martín Hernández ${ }^{1}$

${ }^{1}$ Hospital Universitario Miguel Servet, Zaragoza, Spain,

${ }^{2}$ Hospital de Barbastro, Barbastro, Spain, ${ }^{3}$ Hospital Rio Carrión, Palencia, Spain 
Introduction: Hip fractures are a major cause of public health burden because of the morbidity, mortality and high health care costs. It is known that there has been an important change in the age distribution of the population, with an increase in the population aged more than 80 years. Recent reports have shown that the increasing age of the population and the longer survival of the oldest people resulted in a greater incidence of hip fractures especially unstable and comminuted fractures.

Objectives: To investigate whether hip fracture morphology and the mean age and sex of patients with various types of fracture had changed over the 25-year period (during 1990, 2000, 2010 and 2015) in our province.

Methods: The study included all the cervical, trochanteric and sub-trochanteric fractures in patients aged 65 years or more who were admitted to the Department of Orthopaedic Surgery at the Miguel Servet University Hospital, Zaragoza, during four years 1990, 2000, 2010 and 2015. Fractures related to bone tumors and metastases were excluded. The etiology was a low-energy trauma caused by a simple fall. For each patient we registered the age, sex and type of fracture. The population of this catchment area has increased from 522661 inhabitants in 1989, 559621 in 2009.

Results: Because of the timeliness of the 2015 patients we are still analyzing the results of the last 5 years of the study. The preliminary results of the first 20 years shows a significant increase of the incidence (per 100000 population) from 98 to $159(p<0.001)$. The mean age increased during the three periods from 80.3 in 1990 to 82 years in $2010(p<0.041)$. The proportion of the different types of hip fractures did not differ significantly between the three periods, despite the trend of increasing proportion of trochanteric fractures and the decrease of cervical fractures between 1989 and 2010. Significant changes were observed in the incidences of the different types of cervical fracture. Nondisplaced cervical fractures were significantly decreased and the incidence of displaced cervical fractures was significantly increased. We observed an increasing trend of conmminuted and unstable trochanteric fractures.

Conclusions: In the first 20 years of the study It supports other international studies by showing changes in the incidence of hip fractures. We observed an increase in the incidence, in the mean age and a change in the pattern of hip fracture with an increase of displaced cervical fractures and comminuted and unstable trochanteric hip fractures. These changes modify the preoperative management and functional recovery of the patient. The increasing numbers of cervical fractures that have to be treated by hemiarthroplasties increases the cost of surgical treatment. Older people have greater morbidity and they are more likely to require long-term institutionalization. These factors can lead to an increase in the total economic cost of the healthcare system.

\section{P362}

LOW DOSE COMPARISON OF SYSTEMIC CORTICOSTEROIDS THERAPY IN DIABETIC PATIENTS WITH OLIGOARTICULAR OR POLYARTICULAR GOUT FLARES: AN OBSERVATIONAL RETROSPECTIVE STUDY

C. A. Guillén-Astete ${ }^{1}$

${ }^{1}$ Ramon y Cajal University Hospital, Madrid, Spain

Background: ACR and EULAR recommendations in management of gout flares with oligoarticular involvement points that systemic corticosteroids should be strongly considered in patients with oligoarticular gout flares. However, some considerations must be taking into account before been used, especially in diabetic patients with an unstable glycemic control of with history of hyperglycaemic states after corticoids administration. Instead of avoiding its use, some clinicians use doses lesser than recommended in such patients with different results.

Objective: To compare the effectiveness of different doses of corticoids in diabetic patients with acute oligoarticular gout flares fixed to weight and colchicine doses.

Methods: An observational retrospective study was performed. Electronic charts of diabetic patients who consulted to our A\&E department from 2013-2015 and were diagnosed by oligoarticular gout flare were identified and revised. Registries included patients with oligoarticular gout flares who were not treated with parenteral corticoids or joint infiltrations. They were grouped according to the corticoids dose received fixed by weight and colchicine treatment. Main outcome variables were: need of further consultation before 14th day, VAS-pain score at 7th and 14th day, hyperglycemic states and time off work, where applied. Treatments with systemic corticoids were classified in two arbitrary groups: up to $0.30 \mathrm{mg} / \mathrm{kg} /$ day (Group I) and 0.31 to $0.49 \mathrm{mg} / \mathrm{kg} /$ day (Group II). Patients who were treated with higher doses were considered regular doses and were excluded.

Results: During the period of observation, 81 registries of diabetic patients diagnosed by gout flares with more than a joint involved were detected. Among them, only 62 had complete registries of weight (diabetic control at primary care facility) and complete follow-up assessment after gout flare. 29 and 32 patients were included in groups I and II, respectively.

Conclusions: In diabetic patients with oligoarticular or polyarticular gout flares the therapeutic approach with prednisone doses higher than $0.3 \mathrm{mg} / \mathrm{Kg} /$ day seems to be associated with a lower tendency of need of new clinical assessment, and a better pain control without a higher frequency of hyperglycemic states. As a retrospective observational study, its most relevant limitation is the loss of information about follow-up. However, considering that diabetes is a relatively frequent comorbidity of gout, prospective studies should be conducted 
in order to establish a evidence-based recommendation about corticoid systemic therapy in oligoarticular flares in gouty and diabetic patients.

\begin{tabular}{|c|c|c|c|}
\hline Variable & $\begin{array}{l}\text { Group I } \\
<0.31 \mathrm{mg} / \\
\mathrm{Kg} / \text { day }\end{array}$ & $\begin{array}{l}\text { Group II } \\
0.31-0.49 \mathrm{mg} / \\
\mathrm{Kg} / \text { day }\end{array}$ & Statistics \\
\hline Age and SD & $55.0 \mathrm{SD} 8.1$ & $57 \mathrm{SD} 6.4$ & $P=0.286$ \\
\hline Male proportion & $\begin{array}{l}27 / 29 \\
\quad(93.1 \%)\end{array}$ & $\begin{array}{l}31 / 32 \\
\quad(96.9 \%)\end{array}$ & $P=0.600$ \\
\hline \multicolumn{4}{|l|}{ Time after tappering } \\
\hline $\begin{array}{l}\cdot<7 \text { days } \\
\cdot 7-30 \text { days } \\
\cdot>30 \text { days }\end{array}$ & $\begin{array}{l}2 \\
22 \\
5\end{array}$ & $\begin{array}{l}2 \\
24 \\
6\end{array}$ & $P=1.000$ \\
\hline \multicolumn{4}{|l|}{ Colchicine therapy } \\
\hline $\begin{array}{l}\cdot \text { None } \\
\cdot 0.5 \mathrm{mg} / \text { day } \\
\cdot 1 \mathrm{mg} / \text { day } \\
\cdot 2 \mathrm{mg} / \text { day }\end{array}$ & $\begin{array}{l}1 \\
9 \\
11 \\
8\end{array}$ & $\begin{array}{l}2 \\
8 \\
13 \\
9\end{array}$ & $P=1.000$ \\
\hline $\begin{array}{l}\text { Proportion of patient } \\
\text { who asked for medical } \\
\text { assessment before } \\
\text { 14th day }\end{array}$ & 13 (44.8\%) & $8(25.0 \%)$ & $P=0.068$ \\
\hline $\begin{array}{l}\text { VAS pain score and SD } \\
\text { at 7th day }\end{array}$ & $4,3 \mathrm{SD} 2,2$ & $3,1 \mathrm{SD} 1,9$ & $P=0.025$ \\
\hline $\begin{array}{l}\text { VAS pain score and SD } \\
\text { at } 14 \text { th day }\end{array}$ & $4,1 \mathrm{SD} 2,5$ & $2,0 \mathrm{SD} 0,5$ & $P=0.0001$ \\
\hline $\begin{array}{l}\text { Hyperglucemic state } \\
\text { during treat ment }\end{array}$ & $\begin{array}{l}1(3.45 \%) \\
6.3 \operatorname{SD} 2.6\end{array}$ & $1(3.13 \%)$ & N.A \\
\hline $\begin{array}{l}\text { Time off work } \\
\text { (where corresponds) }\end{array}$ & $\mathrm{d} /$ patient & $\begin{array}{r}\text { 5.1 SD } 1.9 \\
\text { d/patient }\end{array}$ & $P=0.051$ \\
\hline
\end{tabular}

\section{P363}

SENSITIVITY OF 3D KNEE KINEMATIC BIOMARKERS DURING A SINGLE-LIMB TASK IN A KNEE OSTEOARTHRITIS COHORT WITH A MILD TO SEVERE RADIOLOGICAL SEVERITY

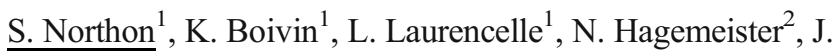
de Guise $^{2}$

${ }^{1}$ Département des sciences de l'activité physique, Université du Québec à Trois-Rivières, Trois-rivieres, Canada, ${ }^{2}$ École de Technologie Supérieure, Département de génie de la production automatisée, Montréal, Canada

Objectives: Study the effects of knee osteoarthritis (KOA) and its severity on 3D knee kinematics during a single-limb task (SLT).

Material and Methods: Knee kinematic data of six SLT trials obtained from 15 asymptomatic individuals (AS) and $31 \mathrm{KOA}$ patients categorized with $\mathrm{Rx}$ as mild (KOAM) or severe (KOAS). SLT data were divided as the loading (LP) and stance (SP) phase using a $2 \%$ body weight (BW) cutoff, measured by two force plates. A median $1 \mathrm{~s}$ period was used as a time window for SP. Participants wore an exoskeleton ${ }^{1}$ equipped with two rigid marker bodies (femoral and tibial levels), tracked by six VICON ${ }^{\mathrm{TM}}$ cameras. Knee joint coordinate system was determined using the FP method ${ }^{2}$. Biomarkers for the three knee rotations and translations were proposed for LP (values at $2 \%$ and $100 \% \mathrm{BW}$, ROM, deltas) and SP (mean, ROM, trajectory). Averages computed on the three most similar trials and compared using ANOVA with contrasts 'KOA vs. AS' and 'KOAS vs. KOAM'.

Results: 29/31 patients successfully performed SLT trials. At LP, KOA and severity effects were a greater varus-valgus ROM, varus thrust motion, and an anteriorly positioned femur. At SP, linear anteroposterior axis parameters were sensitive to KOA and, along with varus-valgus angles, to its severity.

Conclusions: KOA patients exhibit instability in varus-valgus during LP, a finding that increases with severity. The anterior femoral shift and varus angle suggest abnormal knee patterns. Further validity of these biomarkers (e.g. inter-session reliability, sensitivity to change) needs to be studied.

Acknowledgments: We thank Gerald Parent from the Laboratoire de recherche en imagerie et orthopédie (LIO) for his technical support.

References:

1. Ganjikia S, et al. (2000). Knee 2000;7:221.

2. Hagemeister N, et al. J Biomech 2005;38:1926.

Grants: Canadian Institutes of Health Research, Natural Sciences and Engineering Research Council of Canada, Chaire Marie-Lou et Yves Cotrel en orthopédie du CHUM et de l'Université de Montréal, Chaire de recherche du Canada en imagerie 3D et ingénierie biomédical

\section{P364}

RESULTS OF ANKLE ARTHROPLASTY IN CASES OF OSTEOARTHRITIS AND TUMORS OF THE DISTAL TIBIA

V. Protsenko $^{1}$, V. Chorneyi ${ }^{1}$

${ }^{1}$ Institute of Traumatology and Orthopedics of NAMS of Ukraine, Kiev, Ukraine

Objectives: Osteoarthritis of the ankle and tumors of the distal tibia are rarely met. Earlier in cases of osteoarthritis arthrodesis of the ankle was carried out for reconstruction purposes. In cases of tumors of the distal tibia an amputation or disarticulation of limbs was carried out. With new design of orthopaedic and oncological implants, improved surgical techniques, development of new chemotherapy regimens, the individual arthroplasty is applied to replace bone defects. Osteoarthritic joint replacement is necessary at the stage $3-4$ due to severe pain. For tumors of the distal tibia arthroplasty is the method 
of choice because of the difficulties to preserve biomechanical joint activity and soft tissue closure of the implant.

Materials and methods: From 2009-2015, joint replacement for osteoarthritis of the ankle was performed in 6 patients, for distal tibia tumor -8 patients. Women amounted 9 , men -5 . The average age of the patients was $42,4 \pm 0,8$ years. Morphologically, 6 patients had osteoarthritis, 3 - giant cell tumor of bone, 2 - osteosarcoma, 2 - chondrosarcoma, 1 - bone angiosarcoma. All 6 patients with osteoarthritis in the preoperative period received conservative treatment, 2 patients with osteosarcoma, and 1 patient with angiosarcoma of bone in the schemes of complex treatment courses received chemotherapy. In 6 patients with osteoarthritis "Implantkast" orthopedic implants were applied, in 8 patients with tumors of the distal tibia "Inmed" individual oncological implants of the ankle were applied.

Results: Complications associated with arthroplasty were not observed in the postoperative period. In 1 patient with osteosarcoma three months after hip replacement and in 1 patient with chondrosarcoma 16 months after were found local recurrences of the tumor, therefore the patients underwent amputation of a limb. Overall disease-free survival of 6 patients with tumors of the distal tibia for the period of 72 months was 73,4 $\pm 0,5 \%$. The functional outcome of the ankle joint replacement for osteoarthritis was $92,4 \%$, and functional outcome after joint replacement for tumors (system MSTS) averaged 72,4 \%.

Conclusions: To achieve good clinical and functional results in patients with osteoarthritis of the ankle and tumors of the distal tibia must be observed clear indications for ankle joint replacement, careful selection of patients, taking into account the effect of preoperative treatment.

\section{P365}

SERUM IGF-1, OSTEOCALCIN, PTH, AND VITAMIN D LEVELS, IN POSTMENOPAUSAL WOMEN WITH LOW BONE MASS

E. López Gavilanez ${ }^{1}$, K. Guerrero Franco ${ }^{1}$, M. Navarro Chavez $^{2}$, A. Segale Bajana ${ }^{3}$, N. Bautista Litardo ${ }^{4}$ J. Nicola Celleri $^{5}$

${ }^{1}$ Servicio de Endocrinologia Hospital Docente Policia Nacional Guayaquil \#2, Guayaquil, Ecuador, ${ }^{2}$ Servicio Medicina Interna Hospital Luis Vernaza; Guayaquil, Ecuador, ${ }^{3}$ Servicio Medicina Interna. Hospital Docente Policía Nacional Guayaquil $\mathrm{N}^{\circ} 2$, Guayaquil- Ecuador, Guayaquil, Ecuador, ${ }^{4}$ Servicio de Endocrinología Hospital de SOLCA Guayaquil, Guayaquil, Ecuador, ${ }^{5}$ Departamento de Pediatria Hospital Docente Policia Nacional Guayaquil \#2, Guayaquil, Ecuador

Objectives: To assess whether there are correlation between serum levels of insulin-like growth factor 1(IGF-1), osteocalcin (OSC), PTH, and total vitamin D (VD) with BMD in postmenopausal women with low bone mass (LBM). Participants, materials, and methods: In 96 women who attended at routine control in Endocrinology service of HDPN, we determined the BMD at the lumbar spine and neck of femur by DXA. We separated the patients in 2 groups according to the levels of BMD T-score $\geq 0,9$ and $\leq-1$. We excluded patients with impaired renal function, with leading causes of secondary osteoporosis or treatments that could alter the bone mass. We realize an analysis of linear correlation and multiple regression between age, IGF-1 and BMD. The results are expressed as mean $\pm \mathrm{SD}$.

Results: We found a significant reduction in serum levels of IGF-1 in patients with LBM. There was no statistically significant difference between groups in relation of PTH, VD and OSC levels. Positive correlation in BMD and IGF-1 $(r=0.35$, $\mathrm{p}:<0.05)$ was found, but not between BMD, PTH, VD neither OSC.

Table 1.-

\begin{tabular}{llll}
\hline & $\begin{array}{l}\text { Normal Bone Mass } \\
(n=36)\end{array}$ & $\begin{array}{l}\text { Low Bone Mass } \\
(n=61)\end{array}$ & $p$ \\
\hline Age & $56.72 \pm 10.47$ & $68.22 \pm 8.57$ & $<0,0001$ \\
T Score BMD & $0.15 \pm 0.88$ & $-1.99 \pm 0.66$ & $<0,0001$ \\
IGF-1 & $121.06 \pm 43.78$ & $96.49 \pm 40.58$ & $<0,01$ \\
PTH & $34.91 \pm 17.38$ & $37.23 \pm 19.00$ & $(\mathrm{~ns})$ \\
ionic calcium & $4.86 \pm 0.36$ & $4.91 \pm 0.23$ & $(\mathrm{~ns})$ \\
VD & $23.18 \pm 7.58$ & $21.25 \pm 7.75$ & $(\mathrm{~ns})$ \\
OSC & $19.87 \pm 14.65$ & $16.43 \pm 7.17$ & $(\mathrm{~ns})$ \\
\hline
\end{tabular}

Conclusion: In this selected group of postmenopausal women with LBM, there is a significant decrease in serum levels of IGF-1. We found a positive correlation between BMD and IGF-1, while OSC, PTH and VD seem to be independent.

\section{P366 \\ EFFECTS OF HIGH-FAT DIET ON BONE MINERAL DENSITY AND MECHANICAL PROPERTIES IN GROWTH RATS}

G. R. Yanagihara ${ }^{1}$, R. C. Shimano ${ }^{1}$, J. A. Tida ${ }^{1}$, B. B. Brancaleon $^{1}$, A. P. Macedo ${ }^{1}$, A. C. Shimano ${ }^{1}$

${ }^{1}$ Department of Biomechanics, Medicine and Rehabilitation of the Locomotor System, Ribeirão Preto Medical School, University of São Paulo, Ribeirão Preto, Brazil

Objective: To evaluate experimentally the effects of a high-fat diet on BMD and mechanical properties of bone in growth rats. Materials and Methods: This study was approved by the Ethics Committee of our Institution. We used 20 rats Wistar 
requested with 3 weeks old, immediately after weaning. The animals were divided equally into 2 groups: PAD group, which received standard diet for rats AIN-93, and HIP group, which received high-fat diet (AIN-93 diet with $60 \% \mathrm{kcal}$ in the form of saturated fat). The experiment period was 5 weeks and was performed weekly body mass control. After the experiment was carried out the killing of animals for extreme anesthesia and femurs were removed. We used DXA to assess BMD of the femoral neck. A universal testing machine was used to test the Maximal Load (ML) and Stiffness (ST) of femurs through low-speed mechanical tests (flexo-compression of the femoral neck). The Shipiro-Wilk test was used to test the normality of the data and chosen the T-Student or Mann- Whitney tests to compare groups. We considered significant when $\mathrm{p} \leq 0.05$.

Results: At the beginning of the experiment, the rats had similar body mass $(p=0.520)$, but at the end of the experiment the rats HIP group acquired greater body mass $(p=0.022)$. The results showed that BMD of femoral neck of HIP group was $28.45 \%$ higher than the PAD group $(p=0.031)$. Data of ML and ST were statistically similar between groups.

Conclusion: A high-fat diet can increase the BMD of the femoral neck in growth rats, but does not alter its mechanical properties.

\section{P367}

SARCOPENIA AND HIGH RISK OF NONVERTEBRAL FRACTURE IN OLDER MEN: THE STRAMBO STUDY

P. S. Szulc ${ }^{1}$, R. D. Chapurlat ${ }^{1}$

${ }^{1}$ INSERM UMR 1033, Université de Lyon, Lyon, France

In older men sarcopenia is associated with higher fracture risk. It is not clear whether sarcopenia predicts both vertebral and nonvertebral (NV) fracture. Our aim was to study the association of sarcopenia with the risk of vertebral and NV fracture in older men. The cohort of 815 men aged 60-87 yrs was followed up prospectively. Hip BMD and body composition were assessed using a HOLOGIC Discovery A device. Relative appendicular skeletal muscle mass (RASM) was calculated as the sum of lean mass of four limbs divided by (height)2. Self-reported incident non-vertebral fractures were confirmed by inspection of medical records and X- rays. Incident vertebral fractures (assessed on the lateral DXA spine scan) were defined by $>20 \%$ decrease in any vertebral height. Over the median follow-up of 8 yrs, 67 men sustained NV fractures. After adjustment for age, weight and prior falls and fractures, both lower RASM and lower femoral neck BMD were associated with higher fracture risk $(H R=1.43$ per SD decrease, 95\%CI: 1.09-1.98, $p<0.05$ and
$H R=1.90$ per SD decrease, $95 \% \mathrm{CI}: 1.34-2.70 ; p<0.001$, respectively). After adjustment for confounders the risk of NV fracture was higher in the lowest RASM quartile $(<7.65 \mathrm{~kg} / \mathrm{m} 2)$ vs. three upper quartiles combined $(H R=1.95,95 \% \mathrm{CI}: 1.09-3.51 ; p<0.05)$. The NV fracture risk was higher in men who had both low femoral neck BMD (T- score $<-2.0)$ and low RASM $(<7.65 \mathrm{~kg} / \mathrm{m} 2)$ vs. men who had normal BMD and normal RASM $(H R=3.76$, 95\%CI: $1.40-10.12 ; p<0.01)$. Interestingly, the lowest RASM quartile was associated with higher risk of nonvertebral fracture in men with femoral neck BMD Tscore $\geq-2.0(H R=2.11,95 \% \mathrm{CI}: 1.04-4.28, p<0.05)$. Among the 658 men who had follow-up spine scans, 47 men had incident vertebral fractures. RASM was not associated with the risk of vertebral fracture regardless of statistical approach. In conclusion, in older men, low RASM is associated with higher $\mathrm{NV}$ fracture risk independently of potential confounders.

\section{P368}

SARCOPENIA IS PREDICTIVE OF MAJOR CARDIOVASCULAR EVENTS IN OLDER MEN: PROSPECTIVE STRAMBO STUDY

${ }^{\text {P. S. Szulc }}{ }^{1}$, R. D. Chapurlat ${ }^{1}$

${ }^{1}$ INSERM UMR 1033, Université de Lyon, Lyon, France

Data on the association between sarcopenia and cardiovascular risk in men are scanty. Our aim was to assess the association between muscle mass and risk of major cardiovascular event in a cohort of 795 home-dwelling older men. Body composition was assessed by DXA (Hologic Discovery A). Relative appendicular skeletal muscle mass (RASM) is the sum of lean mass of four limbs divided by (height) 2 . Incident major cardiovascular event was defined as acute coronary syndrome (myocardial infarction, coronary insufficiency with or without ST-T elevation confirmed by angiography), stroke or sudden death. During the median follow-up of 8 years, 82 men sustained major cardiovascular events. We used a Cox proportional hazards model adjusted for age, central fat, physical activity, current smoking, co-morbidities, aortic calcification, blood pressure, treatments, and serum levels of osteoprotegerin (OPG), C-reactive protein, FGF23 and 25OHD. The incidence of major cardiovascular events increased from $6 \%$ in the upper RASM quartile $(>8.81 \mathrm{~kg} /$ $\mathrm{m} 2)$ to $13 \%$ in the lowest quartile $(<7.65 \mathrm{~kg} / \mathrm{m} 2)$ (trend $p<0.05$ ). After adjustment for confounders, the incidence of cardiovascular events increased with decreasing RASM $(H R=1.52$ per SD, 95\%CI: 1.17-1.96, $p<0.005)$. Their incidence was higher in the second quartile $(H R=2.38, p<0.05)$ and in the lowest quartile $(H R=4.07, p<0.001)$ vs. the upper 
quartile. Hypertensive men in the lowest RASM quartile had higher cardiovascular risk $(H R=5.26,95 \% \mathrm{CI}$ : $1.65-16.82$, $p<0.01$ ) vs. normotensive men with high RASM (upper quartile). Diabetic men with low RASM had higher cardiovascular risk $(H R=9.25,95 \% \mathrm{CI}: 2.06-41.48, p<0.005)$ vs. nondiabetic men with high RASM. Men who had low RASM and high OPG level (highest quartile, $>4.9 \mathrm{pmol} / \mathrm{L}$ ) had higher cardiovascular risk $(H R=6.94,95 \% \mathrm{CI}: 1.28-37.74 ; p<0.05)$ vs. men with high RASM and normal OPG level. Our data suggest that sarcopenia is an independent indicator of cardiovascular risk in older home-dwelling men.

\section{P369}

ACTIVITY RESTRICTIONS AFTER TJA: ARE THEY ESSENTIAL?

K. M. Ganapathy ${ }^{1}$, S. Nandkumar Sundaram ${ }^{1}$

${ }^{1}$ Helios Hospitals, Chennai, India

\section{Background:}

- Early post-operative dislocation.

- Concerns vary depending upon approach.

- Restrictions designed to prevent excessive hip flexion, adduction and internal rotation.

- $\quad 0.3 \%$ to $10 \%$ of primary THA patients.

- $10 \%$ to $28 \%$ following revision THR.

- Majority occur within 3 months of surgery.

Causes of repeated dislocation:

- Component malposition.

- Soft tissue tension insufficiency.

- Impingement.

- Abductor muscle insufficiency.

General hip precautions:

- Do not flex beyond 90 degrees.

- Do not cross the legs.

- Do not internally rotate beyond the neutral.

- Do not excessively externally rotate.

Additional restrictions:

- Abduction pillow.

- Use elevated toilet seat/ chair.

- Avoid sleeping on the sides.

- Do not travel in/ drive a car.

Aim:

- The aim of this study was to evaluate the functional activities allowed following TJA. The patients are allowed to return to sports and daily activities involving squatting and sitting cross legged. Expectations of patients requiring total Joint replacement have become higher than in the past and are often well beyond pain relief and improved mobility. Return to work and sporting activity are important factors to be considered when advising patients preoperatively. With the trend toward demand for higher performance, exercise, and athletic activity, clinicians have been increasingly forced to question how much athletic activity a patient can participate in after TJA and what kind of athletic activities are deemed acceptable. Defining "reasonable" levels of activity with predictable outcomes has proven to be a difficult tasks.

- To study the effect of dispensing with activity restrictions after THA on the early dislocation rate and patient functional recovery and satisfaction

\section{Methods:}

- Prospective randomized study.

- Anterolateral approach.

- 'Restricted' group or 'Unrestricted' group.

- General hip precautions in all patients.

- Additional restrictions in 'Restricted' group for first 6 weeks.

Results:

- 203 hips in 196 patients.

- 1998-2005.

- 189 unilateral and 7 bilateral THAs.

- 112 right, 77 left and 7 bilateral THAs.

- 107 women and 89 men.

- Age: 22 to 78 years.

- Anterolateral approach.

Indications:

- Osteoarthritis: 79

- Rheumatoid arthritis: 60

- AVN: 26

- Post-traumatic arthritis: 15

- Fracture NOF: 10

- SLE: 02

- DDH: 10

Results (1 year):

- No dislocations in either group.

- Mean HHS improved from 40.1 pre-op to 96.4 post-op in Unrestricted group and from 42.3 pre-op to 90.1 post-op in the Restricted group.

- Shorter duration of hospital stay in unrestricted group (7 days) compared to restricted group (12 days).

- Lesser utilization of physiotherapy services in unrestricted group (20 days) than in restricted group (53 days).

- Faster return to normal activities in the unrestricted group.

- Greater patient satisfaction in the unrestricted group.

\section{Conclusion:}

- Dispensing with activity restrictions did not increase the incidence of early post- operative dislocation following primary THA.

- It did, in fact, improve patient independence and satisfaction and significantly reduced healthcare utilization and costs. 


\section{P370}

EFFECT OF TERIPARATIDE IN CHINESE AND CAUCASIAN POSTMENOPAUSAL WOMEN WITH OSTEOPOROSIS: BRIDGING FINDINGS ON EFFICACY

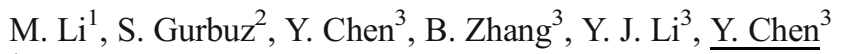

${ }^{1}$ Department of Endocrinology, Key Laboratory of Endocrinology of Ministry of Health, Peking Union Medical College Hospital, Chinese Academy of Medical Sciences, Beijing, China, ${ }^{2}$ Eli Lilly and Company, Indianapolis, United States, ${ }^{3}$ Lilly Suzhou Pharmaceutical Co. Ltd, Medical department, Shanghai, China

Objective: Currently there lacks data of response to teriparatide (TPD) between Chinese and Caucasian. We compare the efficacy and safety of TPD in matched patients from a Chinese trial (CNT) and Global Fracture Prevention Trial (FPT) to estimate if response of Chinese and Caucasian to TPD is similar and bridging strategy can be adapted.

Material and Methods: CNT and FPT were phase III, multicenter and randomized. CNT was a 24-week, open-label, active comparator trial and FPT was a 19-month, global, doubleblind, placebo-controlled study. Postmenopausal women with osteoporosis and similar baseline characteristics except for ethnicity (98\% Caucasian for FPT) from both studies were paired based on propensity score matching method. The percentage change in mean lumbar spine (LS) BMD and fracture incidence within 19-month in matched patients in FPT were measured as efficacy. Safety was assessed by adverse events during 24-week treatment.

Results: There are total of 456 patients paired from 2 studies. CNT included 228 patients randomized to TPD $(n=148)$ and calcitonin $(n=80)$; FPT included 228 patients randomized to TPD $(n=107)$ and placebo $(n=121)$. TPD produced a significant increase in LS-BMD from baseline to week 24 in both studies (Least squares mean of difference $[95 \% \mathrm{CI}]: 5.0[3.2,6.7] \%$ for $\mathrm{CNT}$ and $5.4[2.5,8.2] \%$ for FPT $(\mathrm{Ps}<0.001)$. In matched patients from FPT, after 19 months of TPD treatment, percentage change in LS-BMD was $11.5[9.2,13.8] \%(P<0.001)$ and the incidence of fractures was numerically decreased (relative risk: 0.587 , $P=0.07)$ compared with placebo. The adverse events were consistent between two studies.

Conclusions: The analysis of matched patients from both trials showed similar increases in LS-BMD and safety profile during 24 weeks of TPD treatment. This analysis is in accordance to ICH E-5 guideline and suggests that it might be reasonable to extrapolate the long term LS-BMD improvement and fracture result of FPT to Chinese patient.
P371

PREVALENCE OF FRAILTY AMONG NURSING HOME, ACCORDING TO DIFFERENT OPERATIONAL DEFINITIONS

F. Buckinx $^{1}$, J.-Y. Reginster ${ }^{1}$, S. Gillain ${ }^{2}$, J. Petermans ${ }^{2}$, T. Brunois $^{1}, \mathrm{O}$. Bruyère ${ }^{1}$

${ }^{1}$ Department of Public Health, University of Liège, Liège, Belgium, ${ }^{2}$ Geriatric Department, CHU of Liège, Liège, Belgium

Background: Data on prevalence of frailty vary from one study to another, which is probably due to the method used to evaluate this geriatric syndrome. The aim of this study is to compare the prevalence of frailty according to different operational definitions, among elderly nursing home resident in Belgium.

Methods: : This is an analysis of data collected at baseline in the SENIOR (Sample of Nursing home Elderly Individuals: an Observational Research) cohort. Subjects are defined frail, pre-frail or robust according to 10 different operational definitions: Clinical Frailty Scale, Groningen Indicator, Edmonton scale, Frail Scale Status, Frailty Index, Fried definition, Sega gird, Share Frailty Index, Strawbridge questionnaire and Tilburg indicator. The percentage of frail and prefrail subject is calculated for each definition. A large number of demographic and clinical characteristics are also collected : age, sex, BMI, walking support, number of drugs consumed, number of comorbidities (CIRS-G), cognitive status (MMSE), energy expenditure (Minnesota questionnaire), quality of life (EQ-D and SF-36 questionnaires), level of autonomy (Katz), functional and motors skills (Tinetti test, SPPB test, Timed Up and Go test and gait speed). Relationship between the different operational definitions of frailty and subjects' characteristics is evaluated by means of a multiple or logistic regression, when appropriate.

Results: A total of 662 volunteers subjects from 28 nursing homes are included in this analysis. Among them, the mean age is $83.2 \pm 8.99$ years and $484(73.1 \%)$ of them are women. Prevalence of frailty varies from $1.7 \%$ (Frailty Index) to $76.3 \%$ (Groningen Frailty Indicator) according to the tool. Prevalence of pre-frailty varies from 28\% (Clinical Frailty Scale) to $60.8 \%$ (Fried definition). After adjustment on age, sex and BMI, some demographic and clinical characteristics are significantly different between frail subjects diagnosed by the different definitions. This is true for walking support, number of drugs consumed, cognitive status, energy expenditure, nutritional status, quality of life, level of autonomy, Tinetti test, SPPB test and gait speed.

Conclusion: Prevalence of frailty is highly dependent of the operational definition used. It is therefore necessary to reach a consensus on the operational definition of frailty to use, in 
order to make data comparable between epidemiological studies but also from a clinical point of view, to optimize the management of subjects suffering from frailty.

\section{P372}

\section{PHYSICAL AND MUSCLE PERFORMANCES AMONG ELDERLY NURSING HOME RESIDENTS}

F. Buckinx $^{1}$, J.-Y. Reginster ${ }^{1}$, J.-L. Croisier ${ }^{2}$, J. Petermans ${ }^{3}$, O. Bruyère ${ }^{1}$

${ }^{1}$ Department of Public Health, University of Liège, Liège, Belgium, ${ }^{2}$ Department of Motricity Sciences, University of Liège, Liège, Belgium, ${ }^{3}$ Geriatric Department, CHU of Liège, Liège, Belgium

Background: Aging is generally characterized by a progressive loss of muscle function (i.e. muscle strength and muscle performance). This change is often associated with a decrease in mobility that in turn could lead to disability, frailty, falls, and even death. The aim of this study is to assess muscle function among frail, pre-frail and robust elderly nursing home residents, in Belgium.

Methods: All subjects from the SENIOR (Sample of Elderly Individuals: an Observational Research) cohort were included in this analysis. The SENIOR cohort is a prospective follow-up of nursing home residents aimed to identify prognostic factors of frailty. Physical and muscular performances have been evaluated. Grip strength is measured using an hydraulic dynamometer. Maximal isometric strength of 8 different muscle groups (knee flexors and extensors, ankle flexors and extensors, hip abductors and extensors, elbow flexors and extensors) is evaluated with a portable dynamometer (i.e. the MicroFET2). Functional and motors skills are evaluated by means of the Tinetti test, the Timed Up and Go test, the SPPB test and the gait speed. The diagnosis of frailty is based on Fried's definition. Physical and muscle performances are then compared between frail, pre-frail and robust subjects by means of an Anova analysis.

Results: The SENIOR cohort included 662 nursing home subjects aged 83.2 \pm 8.99 years on average, among which $484(73.1 \%)$ are women. These subjects are relatively oriented (MMSE $=24.1 \pm 4.52$ points) and able to walk. The mean grip strength of the subjects is $18.6 \pm 10.9 \mathrm{~kg}$ and their isometric muscle strength measured at 8 different muscle groups ranged from $63.2 \pm 29.1 \mathrm{~N}$ (elbow extensors) to 101.6 $\pm 52.4 \mathrm{~N}$ (knee extensors). These subjects have a mean Tinetti score of $22.4 \pm 6.23$ points and a mean SPPB score of $5.56 \pm 3.23$ points. Their average time to perform the timed up and go test is $25.6 \pm 19.3$ seconds whereas their gait speed is
$0.89 \pm 4.05$ seconds. All these data of strength and muscle performances are significantly different $(p<.0001)$ between frail, pre-frail and robust.

Conclusion: The decline of mobility is common among elderly, especially in nursing homes. The frailest subjects have muscle and physical performances reduced. These results provide important public health perspectives, to identify subjects requiring intervention.

\section{P373}

REBOUND-ASSOCIATED VERTEBRAL FRACTURES AFTER DENOSUMAB DISCONTINUATION: A SERIES OF 8 WOMEN WITH 35 SPONTANEOUS VERTEBRAL FRACTURES.

O. Lamy ${ }^{1}$, D. Stoll ${ }^{1}$, E. Gonzalez Rodriguez ${ }^{1}$, D. H. Hans ${ }^{1}$, B. Aubry-Rozier ${ }^{1}$

${ }^{1}$ Center of Bone Diseases, Lausanne University Hospital Switzerland, Lausanne, Switzerland

Introduction: Osteoporosis (OP) treatments are given for a limited period of time because of a risk / benefice balance. Reversibility of OP treatment is observed by the measurements of bone markers turnover (BMTs) and BMD. The effect on vertebral fracture (VFx) is difficult to evaluate. The OP treatment discontinuation is associated with an increase of BMTs and a more or less rapid decrease of BMD. Denosumab (Dmab) discontinuation is associated with a severe rebound effect on BTMs and BMD for near 24 months. A recent publication suggests an increase of VFx (Osteoporos Int 2015 Oct 28).

Method: We report the cases of 8 postmenopausal women. They received Dmab $60 \mathrm{mg}$ every 6 months for 2 to 8 doses. The 8 women were on calcium and vitamin D. A wide biological assessment excluded a secondary cause of OP. VFx were documented by MRI.

Results: Five OP women without any prior fragility fracture were treated every 6 months with 4 to 6 Dmab doses. Dmab was stopped because there was no more OP on BMD (3 women 55, 56 and 59 years old), the aromatase inhibitors were stopped (77y old) and according to the wish of the patient $(77 \mathrm{y}$ old). 9 to 16 months after Dmab discontinuation, they presented respectively 5 (D11, D12, L2-L4), 7 (D9, D12-L5), 2 (D11 and D12), 3 (D12-L2) and 9 (D5-D9 and D11-L2) symptomatic spontaneous (SS) VFx. A 65 years old woman with osteoporosis and 3 prevalent VFx was treated every 6 months with 8 Dmab doses. Ten months after Dmab discontinuation she presented 2 SSVFx (D12, L3). These 62 years old woman (osteopenia, treated with aromatase inhibitors) received 2 Dmab doses every 6 months. The subsequent 
Dmab dose was forgotten. Twelve months after the last Dmab dose she presented a D10 SSFx. A 71 years old woman (one prevalent VFx and one hip fracture) received 2 Dmab doses with a delay of 11 months because of a lack of compliance. Eleven months after the last Dmab dose she presented 5 SSVFx (D12, L2-L5).

Conclusion: These 8 cases show a severe increased risk of vertebral fractures in the 9 to 16 months after the last injection of Dmab. The occurrence of these fractures can be explained by the severe rebound effect observed after denosumab discontinuation. It is urgent to: 1) inform the health authorities and patients of this risk; 2) determine treatment regimens before or at the time of denosumab discontinuation.

\section{P374}

THE SPANISH REGISTRY OF HIP AND PROXIMAL FEMUR FRACTURES (SPARE-HIP): BASELINE CHARACTERISTICS OF THE FIRST PATIENTS AND PARTICIPATING CENTRES.

D. Prieto-Alhambra $^{1}$, I. Aguado-Maestro ${ }^{2}$, I. Andrés Cano ${ }^{3}$, M. Asenjo Cambra ${ }^{4}$, A. Balfagon Ferrer ${ }^{5}$, F. Brañas Baztán 6 , M. F. Bravo Bardají ${ }^{7}$, V. Climent-Peris ${ }^{8}$, J. C. Díaz Miñarro ${ }^{9}$, A. Díez Rodríguez ${ }^{10}$, E. Escudero Martinez ${ }^{11}$, I. EtxebarriaForonda $^{12}$, L. Evangelista-Cabrera ${ }^{13}$, L. Ezquerra Herrando ${ }^{14}$, J. Fernandez-Lombardia ${ }^{15}$, A. Freire Romero ${ }^{16}$, M. Martínez $\operatorname{Ros}^{17}$, D. Mifsut Miedes ${ }^{18}$, S. Mills Gañan ${ }^{19}$, J. Olmos Martínez $^{20}$, M. Osca Guadalajara ${ }^{21}$, M. Salomó Doménech $^{22}$, N. Sánchez Hernández ${ }^{23}, \mathrm{M}$. Sanz Sainz ${ }^{24}, \mathrm{~J}$. J. Sierra Serrano ${ }^{25}$, J. Teixidor ${ }^{26}$, O. Tendero $\mathrm{Gómez}^{27}$, O. Torregrosa Suau ${ }^{28}$, A. Herrera ${ }^{29}$, A. Diez-Perez ${ }^{30}$

${ }^{1}$ Oxford NIHR Musculoskeletal Biomedical Research Unit, University of Oxford, MRC Lifecourse Epidemiology Centre, University of Southampton, IDIAP Jordi Gol, Oxford, United Kingdom, ${ }^{2}$ Department of Orthopaedic Surgery, Hospital Universitario del Río Hortega, Valladolid, Spain, ${ }^{3}$ Department of Orthopaedic Surgery, Traumatology and Rheumatology, Hospital Universitario Puerta del Mar, Cádiz, Spain, ${ }^{4}$ Geriatric Department, Hospital Universitario de Getafe, Getafe, Spain, ${ }^{5}$ Trauma Unit, Unit of Orthopaedic Surgery and Traumatology, Hospital Universitario y Politécnico La Fe de Valencia, Valencia, Spain, ${ }^{6}$ Geriatric and Internal Medicine Department, Infanta Leonor University Hospital, Madrid, Spain, ${ }^{7}$ Department of Orthopaedic Surgery, Hospital Regional Universitario Carlos Haya, Málaga, Spain, ${ }^{8}$ Orthopaedics and trauma Departament, Hospital Lluís Alcanyís, Xàtiva, Spain, ${ }^{9}$ Hospital Universitario Reina Sofía, Córdoba, Spain, ${ }^{10}$ Orthopaedic Surgery and Traumatology Unit, Hospital Virgen del Puerto, Plasencia, Spain, ${ }^{11}$ Department of
Orthopaedic Surgery, Complejo Hospitalario Universitario de Pontevedra, Pontevedra, Spain, ${ }^{12}$ Department of Orthopaedic Surgery, Alto Deba Hospital, Arrasate, Spain, ${ }^{13}$ Geriatric Department, Hospital Universitario San Carlos, Madrid, Spain, ${ }^{14}$ Orthopaedic Surgery and Traumatology Unit, Hospital Clínico Universitario de Zaragoza, Zaragoza, Spain, ${ }^{15}$ Department of Orthopaedic Surgery, Hospital Universitario San Agustin, Avilés, Spain, ${ }^{16}$ Department of Orthopaedic Surgery, Complejo Hospitalario Universitario de Santiago de Compostela, Santiago de Compostela, Spain, ${ }^{17}$ Orthogeriatrics Unit, Hospital Virgen de la Arrixaca, Murcia, Spain, ${ }^{18}$ Hospital Clínico de Valencia, Valencia, Spain, ${ }^{19}$ Department of Orthopaedic Surgery, Hospital Universitario La Paz, Madrid, Spain, ${ }^{20}$ Department of Internal Medicine, RETICEF, IDIVAL, Universidad de Cantanbria, Santander, Spain, ${ }^{21}$ Hospital Obispo Polanco Teruel, Teruel, Spain, ${ }^{22}$ Department of Orthopaedic Surgery and Traumatology, Hospital Universitario Parc Tauli, Savadell, Spain, ${ }^{23}$ Department of Orthopaedic Surgery, Hospital Ntra. Sra. de Sonsoles, Ávila, Spain, ${ }^{24}$ Orthopaedic Surgery and Traumatology Unit, Hospital Universitario Miguel Servet, Zaragoza, Spain, ${ }^{25}$ Orthopaedic Surgery and Traumatology Unit, Hospital San Pedro, Logroño, Spain, ${ }^{26}$ Trauma Unit, Hospital Vall d Hebron, Universitat Autónoma de Barcelona, Barcelona, Spain, ${ }^{27}$ Department of Orthopaedic Surgery, Hospital Universitario Son Espases, Palma de Mallorca, Spain, ${ }^{28}$ Metabolic Bone Unit, Department of Internal Medicine, Hospital General Universitario, Elche, Spain, ${ }^{29}$ Department of Surgery, Medicine School, University of Zaragoza, Zaragoza, Spain, ${ }^{30}$ Department of Internal Medicine, Hospital del Mar-IMIM and Autonomous University of Barcelona, Barcelona, Spain

Objective: We report on the first patients and contributing centres to the Spanish Registry of Hip and Proximal Femur Fractures (SPARE-HIP).

Methods: SPARE-HIP is a prospective cohort study aiming to recruit 1,000 consecutive patients. Participant centres were selected to maximise geographical and hospital volume representativeness. We here report on those patients and hospitals recruited/participating between April/2014 and October/2015. Socio-demographics, clinical, and bone health patient features are described. Hip fracture care pathways or units existing in participating hospitals are also characterised. Mean (SD) or $\mathrm{N}(\%)$ are reported.

Results: A consecutive sample of 557 patients, recruited from 22 hospitals, were included. Age was 84.0 (8.5) years, 435 (78.1\%) were women, and BMI was $24.9(4.4) \mathrm{kg} / \mathrm{m}^{2}$; all but 4 participants were Caucasian. Most $(80.4 \%)$ were community-dwelling before admission. ASA grade 3 was most common (51.3\%), and pre-fracture Barthel index scored 
77.9/100 (24.1). In terms of bone health, 212 (36.6\%) had a previous fracture history ( $11.9 \%$ contralateral hip), 63 (11.6\%) osteoporosis, 80/545 (14.7\%) no previous DXA scan, and $242(44.0 \%)$ a history of fall/s in the previous year (144>2 falls). 25 (4.6\%) were on systemic steroids, and prefracture FRAX risks were $15.6 \%(8.8 \%)$ and $8.7 \%(7.0 \%)$ for hip and major fracture respectively. As for centre characteristics, 10/22 (45.5\%) referred hip fracture patients to other wards (not trauma) for admission, 18/22 (81.8\%) shared care with a medical department, 12/22 (54.6\%) had a liaison nurse/ doctor in place, $7 / 22(31.8 \%)$ had a specific hip fracture unit, and 17/22 (77.3\%) followed specific procedures/protocols. Hip fracture volume per hospital ranged between 150 and 700 , and the number of dedicated physicians were 3 to 34 .

Conclusions: The first 557 hip and/or proximal femur fracture patients in the SPARE-HIP registry have similar characteristics to those recruited in similar national audits/registries. Despite the believe that these patients have poor health before fracturing, most of the participants enrolled were communitydwelling and independent. We also report on great heterogeneity in the care processes for these patients in different hospitals across the Spanish geography. This, together with recent evidence on the overall benefits of such audits, supports the need for a Spanish Registry of Hip and Proximal Femur fractures to include all public hospitals.

Acknowledgments: Amgen provided an unrestricted research grant for the SPARE-HIP study. ADP and DPA receive partial support from RETICEF (Instituto de Salud Carlos III, Spain) and NIHR (United Kingdom).

\section{P375}

PERIPHERAL BONE RESORPTION IS SUPPRESSED WHEN SYSTEMIC INFLAMMATION IS IMPROVED IN RA PATIENTS AFTER B CELL DEPLETION TREATMENT

M.-J. Lim ${ }^{1}$, W. Park ${ }^{1}$, S.-R. Kwon ${ }^{1}$, K.-Y. Jung ${ }^{1}$, H.-A. Kim ${ }^{2}$ ${ }^{1}$ Inha University Hospital, Incheon, Republic of Korea, ${ }^{2} \mathrm{Aju}$ University Hospital, Suwon, Republic of Korea

Objectives: We investigated peripheral bone turnover before, 6 and 12 months after rituximab therapy in seropositive RA patients.

Materials and Methods: Ten seropositive RA patients were enrolled. They had been refractory to antirheumatic drugs and anti TNF- $\alpha$ therapy, thus they were preparing for rituximab, monoclonal anti-CD 20 antibody, treatment. Peripheral blood mononuclear cells were collected before, 6 and 12 months after rituximab treatment. They were cultured and histochemical staining for tartrate resistant acid phosphatase (TRAP) was carried out. TRAP positive giant cells with more than 3 nuclei were regarded as osteoclasts. In addition, CD 19 cells along with ESR, CRP and RA disease activity such as DAS28 were compared before, 6 and 12 months after anti rituximab therapy. Bone turnover markers including c-terminal telopeptide (CTX), osteocalcin, bone specific alkaline phosphatase (BSALP) were also measured using enzyme linked immunosorbent assay.

Results: After 6 months of rituximab therapy, number of cultured osteoclasts from peripheral blood and serum level of CTX were both decreased (Table 1) while RA disease activity was improving in accordance with depletion of B-cells (Table 2). BSALP increased after the treatment as well.

Table 1. Peripheral osteoclastogenesis and bone turnover

\begin{tabular}{lllll}
\hline markers & & At baseline & At 6 months & At 12 months \\
\hline Ex vivo & $\begin{array}{c}\text { Number of } \\
\text { osteoclasts } \\
\text { Bone resorption } \\
\text { pit by } \\
\text { oteoclasts (\%) }\end{array}$ & $524 \pm 301.1$ & $299 \pm 270.8^{*}$ & $174 \pm 131$ \\
In vivo & $\begin{array}{l}\text { CTX (ng/mL) } \\
\text { Cathepsin K } \\
\text { (pmol/L) }\end{array}$ & $0.86 \pm 0.45$ & $0.5 \pm 0.211^{*}$ & $0.54 \pm 0.19$ \\
& $\begin{array}{l}\text { BSALP (U/L) } \\
\text { Osteocalcin } \\
(\mathrm{ng} / \mathrm{mL})\end{array}$ & $7.42 \pm 2.51$ & $10.81 \pm 5.2^{*}$ & $8.58 \pm 3.12$ \\
& $28.11 \pm 8.07$ & $40.71 \pm 18.19$ & $30.6 \pm 7.96$ \\
& & & \\
\hline
\end{tabular}

Table 2. CD 19 cells, systemic inflammation and RA disease activity

\begin{tabular}{llll}
\hline & At baseline & At 6 months & At 12 months \\
\hline CD 19 cells $(/ \mu \ell)$ & $660 \pm 345.5$ & $42 \pm 103.5^{*}$ & $298 \pm 563^{+* *}$ \\
ESR $(\mathrm{mm} / \mathrm{hr})$ & $56 \pm 21.1$ & $23 \pm 13^{*}$ & $31 \pm 23.4^{\dagger * *}$ \\
CRP $(\mathrm{mg} / \mathrm{dL})$ & $3.72 \pm 2.72$ & $0.82 \pm 1.46^{*}$ & $1.16 \pm 1.11^{\dagger}$ \\
DAS28-ESR & $7 \pm 0.9$ & $4.04 \pm 0.86^{*}$ & $5.55 \pm 1.89^{* *}$ \\
DAS28-CRP & $6.39 \pm 0.98$ & $3.52 \pm 0.69^{*}$ & $5.04 \pm 1.77^{* *}$ \\
\hline
\end{tabular}

*denotes $p \leq 0.25$, at Baseline vs. at 6 months

${ }^{\dagger}$ Denotes $p=0.008$, at Baseline vs. at 12 months

${ }^{* *}$ denotes $p<0.05$, at 6 months vs. at 12 months

Conclusion: Peripheral bone resorption is suppressed when systemic inflammation is improved in RA patients 6 months after B cell depletion treatment. This was accompanied by an increase in bone formation marker reflected by BSALP. However, one year after rituximab treatment, ESR and CRP began to increase along with peripheral B cell counts, resulting in worsened RA disease activity. The area of bone resorption pit by cultured osteoclasts from peripheral blood increased as well. Therefore, rituximab therapy decreases bone resorption and increases bone formation in RA patients when B cells were depleted and RA activity was improved. 


\section{P376}

PREVALENCE AND DETERMINANTS FOR NEUROPATHIC PAIN IN KNEE OSTEOARTHRITIS. AN ALGERIAN MULTICENTER STUDY

S. Slimani ${ }^{1}$, K. Testas ${ }^{2}$, A. Abbas ${ }^{3}$, D. Kebaili ${ }^{4}$, R. Chiheub ${ }^{5}$

${ }^{1}$ Department of Medicine, University of Batna, Batna, Algeria, ${ }^{2}$ Department of Medicine, Khroub Public Hospital, Constantine, Algeria, ${ }^{3}$ Private Practice, Msila, Algeria, ${ }^{4}$ Department of Medicine, Oum El Bouaghi Hospital, Oum El Bouaghi, Algeria, ${ }^{5}$ Private Practice, Constantine, Algeria

Background: Pain in osteoarthritis (OA) is typically described as being nociceptive; however, the partial efficacy of peripheral analgesics and the demonstration of the efficacy of central analgesics such as duloxetine, suggest a complex mechanism for pain in OA. Very few reports in the world have assessed the prevalence of neuropathic pain in knee OA, no work in Africa and the Arab world has been conducted in that purpose. The aim of our study was to evaluate the prevalence of neuropathic pain in Algerian knee OA, and to look for possible associated demographic, clinical and radiographic factors.

Methods: A cross sectional multicenter study of knee OA patients (ACR criteria), aged 20 years and more, with the exclusion of patients with any neurological condition. Demographic and clinical data were collected, as well as the radiographic assessment using the Kellgren and Lawrence (KL) grading system. Severity of pain was evaluated using a numerical scale. The detection of neuropathic pain was performed separately on each knee using the DN4 score, with a positive cutoff of 4 . Descriptive data were expressed as means $\pm \mathrm{SD}$, and proportions. Determinants of neuropathic pain were evaluated by a univariate followed by a multivariate regression analysis. Statistical analyses were carried out using SPSS version 17.0.

Results: We have included 205 OA knees in 105 patients with a mean age of $62.1 \pm 11.1$ years, $88 \%$ were females, and the mean BMI was $32.3 \pm 6.1$. The main comorbidities were hypertension (39.5\%) and diabetes $(16.1 \%)$. Neuropathic pain $(\mathrm{DNA} \geq 4)$ was found on $39 \%$ of knees. The univariate analysis revealed a significant association with age $(p<0.001)$ and with a longer disease duration $(p<0, .001)$. Also, there was a tendency towards a more prevalent neuropathic pain in more severe K-L grades $(p=0.098)$. No significant association was found with the presence of diabetes and other comorbidities. The multivariate regression found an independent association with the disease duration $(b=0.376 ; p<0.001)$.

Conclusion: A high proportion (39\%) of knee OA presented with neuropathic pain, in an Algerian rheumatology outpatient setting. This reinforces the idea of the intricacy of nociceptive and neuropathic pain in OA and may explain the efficacy of some neurotropic medications in knee OA.

\section{P377}

\section{YEARS EXPERIENCE OF USING TERIPA- RATIDE IN SEVERE OSTEOPOROSIS}

M. Siddiqi ${ }^{1}$, A. Murray ${ }^{1}$, A. Gatignol ${ }^{1}$, J. Ohare $^{1}$

${ }^{1}$ Aintree University Hospital, Liverpool, United Kingdom

Objective: Teriparatide, was authorised for use in UK in 2003. Here we report on our experience of using it in severe osteoporosis for last 10 years (2005-2015) in our Metabolic Bone Disease Clinic.

Material and Methods: We retrospectively collected the information through patient electronic case notes. The duration of treatment was 18 months till June 2008 and is 24 months since.

Results: 307 patients were included in the study, 265 were females (age 50-92, avg-75.13) and 42 males (age 27-85, avg-66.31). All suffered with severe osteoporosis (T-score -3.5 or below and at least 1 fracture). Only 20 patients were treatment naïve, rest were previously treated with bisphosphonates (254), denosumab (18) or strontium(15). Major indications were treatment failure (fracture despite treatment $>12$ months or deteriorating BMD despite 5 years of treatment) 258 , intolerance or contraindication to other treatments 23 , severe steroid induced osteoporosis 16 , after atypical femoral fracture 7 and after ONJ 3. 21 patients experienced side effects including headache (12), aches and pains(7), nausea and vomiting (5) and dizziness(3). Only 11 had to stop the treatment due to SEs or inability to inject. There was a significant increase in BMD at spine both in 18 months (49 pts, $14.65 \%, p=0.0106)$ and 24 months (167 pts, $16.12 \%, p=0.0051)$ treated patients but the increase at femoral neck did not reach statistical significance $(8.11 \%$, $p=0.251$ in 18 months and $11.47 \%, p=0.0569$ in 24 month group). 156 patients had over 12 months follow-up (avg51.5 months). 128 ( $82 \%$ ) were still on some treatment for osteoporosis $(110,70.5 \%$ on a bisphosphonate and 18 , $12 \%$ on denosumab). 18 patients had fractures during follow-up ( 5 vertebral, 8 NOF and 5 NVNF) despite being on anti-resorptive treatment. The average time to fracture after completion of teriparatide treatment was 36.5 months. Our usage of teriparatide has increased in recent years (12 patients per year from 2005-2010 and 43.5 patients per year since then $p=0.0027$ ) possibly because of concerns with long term usage of bisphosphonate.

Conclusions: Teriparatide is safe, well tolerated and increases the BMD at spine significantly. 


\section{P378}

PARATHORMONE STABILITY IN HEMODIALYZED PATIENTS AND HEALTHY SUBJECTS: COMPARISON ON NON-CENTRIFUGED EDTA AND SERUM SAMPLES WITH 2 ND AND 3RD GENERATION ASSAYS

E. Cavalier ${ }^{1}$, M.-L. Schleck ${ }^{1}$, A.-C. Bekaert ${ }^{1}$, N. Ferrante ${ }^{1}$, O. Rousselle $^{1}$, A. Carlisi ${ }^{1}$

${ }^{1}$ Clinical Chemistry, University of Liège, CHU de Liège, Liège, Belgium

Introduction: PTH stability is of importance. Many studies have shown divergent results between EDTA and serum, mainly linked to differences in protocols or cutoffs used to decipher whether PTH remained stable or not. No studies have yet compared PTH as measured by 2 nd and 3rd generation assays on the same samples in hemodialyzed (HD) patients and healthy subjects.

Methods: Five pairs of samples (EDTA and gel tubes) were obtained in $10 \mathrm{HD}$ patients before session and 10 healthy subjects. One pair was centrifuged and run immediately to establish the "T0". Two pairs were kept at $+4{ }^{\circ} \mathrm{C}$ and $+25^{\circ} \mathrm{C}$. They were centrifuged after 4 and 18 hours to mimic real laboratory storage conditions of none directly treated samples (external laboratory delivery, etc.). Supernatant was kept at $-80^{\circ} \mathrm{C}$ for one week. Then, all samples were measured in a single batch, on Roche Cobas and DiaSorin XL 2nd and 3rd generation PTH assays. Samples were considered as stable if $90 \%$ of subjects had a decrease lower than a total change limit that takes both analytical $(\mathrm{CVa})$ and biological variability $(\mathrm{CVb})$ into consideration $\left(\mathrm{TCL}=\mathrm{SQR}\left((1.96 * \mathrm{sqr} 2 * \mathrm{CVa})^{\wedge} 2+\right.\right.$ $\left.\left.\left(0.5^{*} \mathrm{CVb}\right)^{\wedge} 2\right)\right)$. All determinations were run in duplicates.

Results: At T0, no difference was observed between plasma and serum for any of the methods. PTH decreased in all samples and degradation was the same with 2 nd or 3rd generation assays, whatever the method used and in both groups studied. Percentages of decrease were systematically lower in EDTA plasma compared to serum and none of our subjects presented a decrease higher than the TCL with EDTA plasma. In serum, PTH was considered as unstable only when kept at $25^{\circ} \mathrm{C}$ for 18 hours. There was no significant difference in PTH stability between HD patients and healthy subjects.

Conclusion: PTH stability is of paramount importance for correct interpretation of the results. Many studies have tried to evaluate this stability but are not free from criticisms, mainly due to a poorly defined $\mathrm{T} 0$ and questionable acceptation limits. In this study, we used an unfrozen $\mathrm{T} 0$ and decision limits based on analytical and biological variations. Moreover, we tested 2nd and 3rd generation PTH on two automated platforms and in 2 different groups of patients. Our results show that, if PTH is basically more stable in EDTA plasma than in serum, this advantage is only clinically significant when samples are stored for a long period
(18 h) at $+25^{\circ} \mathrm{C}$. However, if PTH analysis is performed directly, it can be done using EDTA plasma or serum. Finally, we found that PTH was as stable in healthy than in HD patients.

\section{P379}

ENGLISH TRANSLATION AND CROSS-CULTURAL ADAPTATION OF THE SARQOL ${ }^{\circledR}$ QUESTIONNAIRE

C. Beaudart ${ }^{1}$, M. Edwards ${ }^{2}$, E. M. Dennison ${ }^{2}$, C. Moss $^{2}$, O. Bruyère $^{1}$, J.-Y. Reginster ${ }^{1}$, R. J. Moon ${ }^{3}$, C. Cooper ${ }^{4}$, C. Demoulin $^{5}$

${ }^{1}$ Department of Public Health, University of Liège, Liège, Belgium, ${ }^{2}$ MRC Lifecourse Epidemiology Unit, Southampton, United Kingdom, ${ }^{3}$ MRC Lifecourse Epidemiology Unit, University of Southampton, Southampton, United Kingdom, ${ }^{4}$ MRC Epidemiology Resource Centre, University of Southampton, Southampton General Hospital, Southampton, United Kingdom, ${ }^{5}$ Department of Sport and Rehabilitation Sciences, University of Liege, Liege, Liège, Belgium

Background: Recently, the SarQoL ${ }^{\circledR}$ (Sarcopenia and Quality of Life), a quality of life questionnaire specific to sarcopenia, has been developed and validated in French ${ }^{1}$. To extend the availability and utilisation of this questionnaire, its translation and validation in other languages is necessary. The purpose of this study was therefore to translate the SarQoL ${ }^{\circledR}$ questionnaire into English.

Methods: The translation was performed according to translation guidelines ${ }^{2}$. Five different phases were followed: 1 . The initial translation from French to English by two independent bilingual translators which were English native speakers; 2 . The synthesis of the first two translations to provide a single "version 1" of the translated questionnaire; 3. The backward translation by two independent bilingual blinded to the original French version and having French as their first language; 4. An Expert Committee review to compare the backward translations with the original questionnaire and consent on a "version 2" of the translated questionnaire; 5. The pre-test of the "version 2" of the SarQoL ${ }^{\circledR}$ to ensure good comprehension of each question of the questionnaire and conclude with the "version 3", final version of the English SarQoL ${ }^{\circledR}$.

Results: The 22 questions of the SarQoL ${ }^{\circledR}$ questionnaire were translated without any major difficulties. Some complications have however been encountered regarding the choice of responses displayed for the 4-likert scale. Cross-cultural adaptation have been made on one question and one example used in this question has consequently been deleted. A pre-test was performed on 10 different subjects. Minor changes have consequently been made to the questionnaire "version 2". These changes, which did not modify the meaning of the sentences, 
were mainly related to choice of words used for the 4-Likert scale choices. The rigorous translation and adaptation processes provide equivalence between the French and the English version of the SarQoL ${ }^{\circledR}$.

Conclusion: The English version of the SarQoL ${ }^{\circledR}$ questionnaire is now available and has been showed to be comprehensible. Investigations are now required to verify the psychometric properties (validity, reliability and responsiveness) of the English version of the SarQoL ${ }^{\circledR}$ questionnaire.

${ }^{1}$ Beaudart C, et al. Age Ageing 2015;Oct 3. pii: afv133. [Epub ahead of print]

${ }^{2}$ Beaton DE, et al. Spine 2000;25:3186

\section{P380}

MIR-214-3P TARGETS TRAF3 TO PROMOTE OSTEOCLASTIC BONE RESORPTION DURING THE DEVELOPMENT OF POSTMENOPAUSAL OSTEOPOROSIS

J. Liu ${ }^{1}$, D. F. Li ${ }^{1}$, L. Dang ${ }^{1}$, A. P. Lu ${ }^{1}$, G. Zhang ${ }^{1}$

${ }^{1}$ Hong Kong Baptist University, Hong Kong, Hong Kong

Introduction: The over-activation of osteoclast could lead to skeletal diseases with excessive bone resorption, such as postmenopausal osteoporosis. Emerging evidences indicate that microRNAs (miRNAs) participate in regulating osteoclastic bone resorption. However, there still lack of miRNAs directly identified from human bone specimens to contribute to the pathophysiological regulation of PMO. Recently, our preliminary study found that miR-214-3p was significantly upregulated and positively correlated with bone resorption marker genes in bone specimens from osteoporotic patients in the early stage (less than 5 years) of menopause. In addition, the bioinformatics analysis of miRNA target prediction by miRBase showed that Traf3, a previously identified negative regulator for osteoclastogenesis (Xiu et al. J Clin Invest 2014), has a miR-214-3p binding site in its 3'UTR. Thus, we hypothesis that the elevated miR-214-3p could be involved in the regulatory mechanism on the excessive bone resorption under pathological condition to contribute to postmenopausal osteoporosis.

Methods: First, we applied genetic approach to examine the bone phenotype in vivo when miR-214-3p was overexpressed within osteoclast in the osteoclast-specific miR-214-3p knock-in (OC-miR-214) mice.

The OC-miR-214 mice were obtained by crossing the mice containing the miR-214-3p knock-in allele with the Ctsk-cre transgenic mice. Thereafter, we performed luciferase reporter assay and gain- and loss-of- function experiments in vitro to investigate whether miR-214-3p could directly target TRAF3 to regulate osteoclast activity. Finally, we studied the therapeutic effect of osteoclast-targeted inhibition of miR-214-3p on the estrogen depletion-induced osteoporosis in ovariectomized (OVX) mice.
Results: The OC-miR-214 mice showed a remarkably higher number of osteoclasts and bone resorption surfaces as well as downregulated TRAF3 protein expression, lower bone mass and deteriorated trabecular architecture when compared to the wildtype (WT) controls. Moreover, the above bone phenotype could be markedly rescued after osteoclast-targeted inhibition of miR-214-3p in OC-miR-214 mice by pharmacological approach. In the in vitro experiments, we observed a gradual increase in the miR-214-3p level in company with a decrease in TRAF3 protein level in RAW 264.7 cells during RANKLinduced osteoclastogenesis. The luciferase reporter assay and the gain- and loss-of-function studies showed that miR-214$3 p$ could directly target Traf3 to promote osteoclast activity. Furthermore, therapeutic inhibition of miR-214-3p through administration of antagomir-214-3p delivered by our previously developed osteoclast-targeting delivery system in OVX mice significantly upregulated the intraosseous TRAF3 protein expression, suppressed bone resorption and attenuated the development of estrogen depletion-induced osteoporosis.

Conclusion: Collectively, our study demonstrated that miR214-3p could target TRAF3 to promote osteoclastic bone resorption during the development of postmenopausal osteoporosis. Targeting osteoclastic miR-214-3p may be a potential therapeutic strategy for postmenopausal osteoporosis.

\section{P381 \\ PREMATURE MENOPAUSE AND RISK OF OSTEOPOROSIS}

A. Mirica $^{1}$, I. Cotofana ${ }^{1}$, R. Petris ${ }^{1}$, M. Stoiceanu ${ }^{1}$, C.

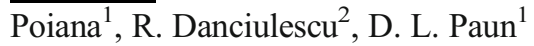

1“C.I. Parhon" National Institute of Endocrinology, Bucharest, Romania, ${ }^{2}$ N.C. Paulescu National Institute of Diabetes, Bucharest, Romania

Introduction: Premature menopause is related to menopause that occurs before age of 40 years, under the median age of natural menopause of 51 years old. Premature menopause can be spontaneous or induced by surgical interventions or cancer treatments. Regardless of cause, women who experience premature menopause are now recognized to be at increased risk for premature morbidity and mortality.

Objectives: The aim of the present study was to assess the prevalence of osteoporosis in a sample of 40 patients with premature menopause in comparison to control group of 38 natural postmenopausal women.

Material and Methods: We conducted a retrospective study over a year, collecting data in patients hospitalized in Department of Gonads and Bone pathology of Institute CI Parhon. We made comparisons between a group of patients with premature menopause $(n=40)$ and a control group of women with natural menopause $(n=38)$. Hip (femoral) and 
spinal L1-L4 BMD measurements were performed by DXA in the two groups.

Results: The median \pm SD age of premature menopause patients at the time of diagnosis was $36.8 \pm 1.6$ years, with a median age of menarche of $12.5 \pm 1.41$ years. The mean \pm SD age of natural menopausal women was $52.2 \pm 1.6$ years, with a median age of menarche of $13.1 \pm 1.32$ years. At the time of BMD measurement, the mean \pm SD age of premature menopause patients $(45.26 \pm 8.26$ years $)$ was lower than that of natural menopause patients. Femur BMD was significantly lower in the study group $\left(0.802 \pm 0.16 \mathrm{~g} / \mathrm{cm}^{2}\right)$ compared to the reference group $\left(1.024 \pm 0.14 \mathrm{~g} / \mathrm{cm}^{2} ; P=0.02\right)$. Spinal L1-L4 BMD in the study group was $0.931 \pm 0.12 \mathrm{~g} / \mathrm{cm}^{2}$, similar to spinal BMD of $0.980 \pm 0.17 \mathrm{~g} / \mathrm{cm}^{2}$ in the control group. In addition, $57.5 \%(n=23)$ of patients in the study group had osteoporosis (T-scores $\geq-2.5 \mathrm{SD}$ ) comparative with $21.05 \%$ $(n=8)$ of the control group $(P=0.04)$.

Conclusions: Femoral BMD is significantly lower in patients with premature menopause than in age-matched control group. Osteoporosis is more frequent in women with premature menopause compared with women with natural menopause.

\section{P382}

IS OSTEOPOROSIS ASSOCIATED WITH THE OCCURRENCE OF SARCOPENIA? A FOUR-YEAR FOLLOW-UP TO THE ROAD STUDY

$\underline{\text { N. Yoshimura }}^{1}$, S. Muraki ${ }^{1}$, H. Oka ${ }^{2}$, S. Tanaka ${ }^{3}$, H. Kawaguchi $^{4}$, K. Nakamura ${ }^{5}$, T. Akune ${ }^{5}$

${ }^{1}$ Department of Joint Disease Research, Tokyo University, Tokyo, Japan, ${ }^{2}$ Department of Medical Research and Management for Musculoskeletal Pain, Tokyo University, Tokyo, Japan, ${ }^{3}$ Department of Orthopaedic Surgery, Tokyo University, Tokyo, Japan, ${ }^{4} \mathrm{JCHO}$ Tokyo Shinjuku Medical Center, Tokyo, Japan, ${ }^{5}$ National Rehabilitation Center for Persons with Disabilities, Saitama, Japan

Objective: The present 4-year follow-up study was performed to clarify the contribution of osteoporosis (OP) to sarcopenia (SP) in the general population.

Material and Methods: The second survey of the Research on Osteoarthritis/Osteoporosis Against Disability (ROAD) study - a large-scale population-based cohort study-was conducted between 2008 and 2010. We enrolled 1099 participants (aged $\geq 60$ years, 377 men, 722 women) from the second survey of the ROAD study who had complete assessments of handgrip strength, gait speed, skeletal muscle mass measured by bioimpedance analysis, and BMD measured by DXA. The third survey was conducted between 2012 and 2013; 767 of the 1,099 individuals who were enrolled from the second trial $(69.8 \%, 253$ men, 514 women) had completed assessments identical to those in the second trial. SP was defined as per the algorithm of the Asian Working Group for Sarcopenia, whereas OP was defined based on the World Health Organization criteria.

Results: The prevalence of SP in the second survey was found to be $8.2 \%$ (men, $8.5 \%$; women, $8.0 \%$ ). The cumulative incidence of SP during the 4-year period between the second and third visits was $2.0 \% / \mathrm{yr}$ (men, $2.2 \% / \mathrm{yr}$; women, $1.9 \% /$ $\mathrm{yr}$ ). After adjustment for age, gender, regional differences, and emaciation (body mass index $<18.5 \mathrm{~kg} / \mathrm{m}^{2}$ ), a logistic regression analysis using the occurrence of SP as the objective variable and the presence of $\mathrm{OP}$ as the explanatory factor indicated that the presence of OP was significantly associated with the occurrence of SP in the near future (odds ratio, 2.86; $95 \%$ confidence interval, $1.41-5.79 ; p=0.003$ ).

Conclusions: This prospective study suggests that the presence of OP might predict the incidence of SP in the near future. The treatment of the OP might reduce the risk not only of osteoporotic fracture but also that of SP.

\section{P383}

POTENTIAL EXTENSION OF FRAX ALGORITHM AND PROBABILITY OF BONE FRACTURE

$\underline{\text { A. Andreadi }}{ }^{1}$, B. Capuani ${ }^{2}$, M. Romano ${ }^{3}$, A. Galli ${ }^{3}$, M. P. Caputo $^{3}$, M. Cerilli ${ }^{3}$, F. Pozzi ${ }^{3}$, A. Bellia ${ }^{1}$, D. Della Morte ${ }^{1}$, D. Lauro ${ }^{1}$

${ }^{1}$ Department of System's Medicine, University of Tor Vergata, UOC of Endocrinology, Diabetology and Metabolic Diseases, University Hospital Foundation PTV, Rome, Italy, ${ }^{2}$ Department of System's Medicine, University of Tor Vergata, Rome, Italy, ${ }^{3}$ UOC of Endocrinology, Diabetology and Metabolic Diseases, University Hospital Foundation PTV, Rome, Italy

Osteoporosis is a pandemic chronic noncommunicable disease, with worldwide expansion. This condition and the resulting bone fractures are a major cause of disability and mortality for millions of people worldwide. FRAX is an algorithm an algorithm that relies on a questionnaire for the prediction of bone fracture. This algorithm was recognized and approved by World Health Organization (WHO) on 2008.

FRAX estimates the probability in $\%$ to have a fracture in the following 10 years. The aim of this study was to evaluate whether patients lifestyle and dietary habits may be considered as additional risk factors for new bone fractures and evaluate how it correlates to the degree of osteoporosis. Furthermore, we analyzed if obesity can be associated with reduced levels of BMD. Between March-October 2015, 1132 patients have been enrolled, at the Unit of Endocrinology, Diabetes and Metabolic Diseases, University Hospital of Tor Vergata, Rome Italy. All subjects underwent to evaluation of anthropometric data (weight, height, BMI), bone densitometry with DXA Hologic QDR 4500 Delphi series, Food frequency 
questionnaire (1) to assess dietary habits during a week and FRAX questionnaire. From the 1132 subjects, the media of the age was $63.3( \pm 9.5 \mathrm{SD})$; from which 1086 were women $(63.3 \mathrm{y} \pm 9.4 \mathrm{SD})$ and $45 \mathrm{men}(63.8 \mathrm{y} \pm 11.6 \mathrm{SD})$. The food questionnaire data when divided considering the T- score in three groups, 210 subjects normal $(-0.29 \mathrm{SD} \pm 0.636 /$ $0.818 \mathrm{~g} / \mathrm{cm} 2 / \pm 0.07), 712$ with osteopenia $(-1.74 \mathrm{SD} \pm 0.42 /$ $0.649 \mathrm{~g} / \mathrm{cm} 2 \pm 0.05)$ and 210 with osteoporosis $(-2.86 \mathrm{DS}$ $\pm 0.38 / 0.515 \mathrm{~g} / \mathrm{cm} 2 \pm 0.04$ ), confirming that patients with osteoporosis consume less foods with calcium and vitamin $\mathrm{D}(\mathrm{p}$ value 0.01). Patients were also divided according the BMI. From 1.132 people, the $0.79 \%$ were underweight ( 9 subjects), $34.8 \%$ (395 subjects) normal weight (22.6 \pm 1.59$), 36.7 \%$ (416 subjects), overweight (27.2 \pm 1.4$)$ and $27.5 \%$ (312 subjects) obese (33.7 \pm 3.7$)$. It is important to consider that the prevalence of overweight and obesity in our population was $64.2 \%$. If then we analyze the patients that are overweight and obese, the majority of the sample (80\%) presents osteopenia or osteoporosis; this result is controversy from the supposed protective role of adipose tissue on BMD.

\section{P384}

THE USABILITY OF FRAX INDEX IN EVERYDAY DOCTOR'S PRACTICE FOR DECISIONMAKING REGARDING THE NECESSITY OF TREATMENT OF OSTEOPOROSIS

T. T. Tomic ${ }^{1}$, Z. G. Grujic ${ }^{1}$, A. J. Jokic ${ }^{1}$, V. T. Tomic ${ }^{2}$

${ }^{1}$ Specialized Rehabilitation Hospital Banja Koviljaca, Banja Koviljaca, Serbia, ${ }^{2}$ Faculty of Medicine, Novi Sad, Novi Sad, Serbia

Objective: To test the rationality of application of FRAX index in every-day practice for decision-making when treating postmenopausal osteoporosis.

Material and methods: This study encompasses 310 postmenopause women that have performed bone densitometry analysis using the DXA LUNAR machine at the Specialized Rehabilitation Hospital in Banja Koviljaca, Serbia. The criteria for the inclusion in the study were: that the women have entered menopause and that they have been neither diagnosed with nor treated for osteoporosis. For the estimation of treatment necessity for examinees, FRAX index for the population of UK was used, which is according to the risk factors similar to Serbia. In the statistical analysis, chi-square test and analysis of agreement (Kappa) were used.

Results: Average age of the examinees was approximately 63 years. All 63 of them suffered a previous fractures caused by a small force. According to the FRAX index the treatment is advised for $58.7 \%$ of examinees with previous fractures and for $14.2 \%$ of examinees without previous fractures. Based on the Hi-square test results, it has been determined that there is a high statistically significant difference between patients with and without previous fractures according to the FRAX proposition
$\left(X^{2}=55.896 ; p<0.001\right)$. Based on the DXA findings, 60 female examinees had normal findings, 94 of them had osteoporosis and 156 of them had osteopenia. 131 of them had osteopenia without any previous fracture. By analyzing FRAX proposition for that group of women, FRAX advised treatment for 11 of them. Using analysis of agreement (Kappa) was determined that there is a high statistically significant correlation between the decision regarding the manner of treatment based on the DXA findings and FRAX index (Kappa $=0.444 ; p<0.001)$.

Conclusion: Based on the research results, it has proven that there is a high correlation of FRAX proposition regarding the treatment necessity with the proposition based on the DXA findings and previous fractures. It is also proven that FRAX is very useful for decision-making regarding the treatment of persons with osteopenia.

\section{P385}

ASSOCIATION BETWEEN MYOCYTE QUALITY CONTROL SIGNALING AND SARCOPENIA IN OLDER HIP-FRACTURED PATIENTS

$\underline{\text { E. Marzetti }^{1}}$, M. Lorenzi ${ }^{2}$, E. D'Angelo ${ }^{2}$, A. Collamati ${ }^{2}$, G. Savera $^{2}$, A. Menghi ${ }^{1}$, R. Bernabei ${ }^{1}$, F. Landi ${ }^{1}$, R. Calvani ${ }^{1}$

${ }^{1}$ Department of Geriatrics, Neurosciences and Orthopedics, Teaching Hospital “Agostino Gemelli”, Rome, Italy, ${ }^{2}$ Department of Geriatrics, Neurosciences and Orthopedics, Catholic University of the Sacred Heart, Rome, Italy

Objective(s): Sarcopenia may impact the clinical outcome of hip fractured elderly. A better understanding of the signaling pathways responsible for age-related muscle decline is necessary to design targeted interventions. The present study was undertaken to explore the association between alterations in myocyte quality control signaling and sarcopenia in elderly hip-fractured patients. Materials and Methods: Twenty-five older hip-fractured patients ( 20 women and 5 men; mean age $84.9 \pm 1.65$ years) were enrolled in the study. Intraoperatory biopsies from the vastus lateralis muscle were obtained and assayed for the expression of a set of quality control signaling proteins. The presence of sarcopenia was established according to the European Working Group on Sarcopenia in Older People (EWGSOP) criteria. Whole-body fat-free mass was estimated via bioelectrical impedance analysis (BIA). Muscle strength was assessed by means of handgrip strength testing. Differences in the expression of myocyte quality control signaling proteins between sarcopenic and non-sarcopenic patients were assessed by the independent samples $t$-test or the Newman-Keuls test, as appropriate.

Results: Sarcopenia was identified in 12 subjects (48 \%). The expression of mitochondrial fusion protein mitofusin 2 (Mfn2) and the autophagic mediators, lysosomal associated membrane protein-2 (LAMP-2) and microtubule-associated protein 1 light chain 3 beta (LC3B), was lower in older subjects with sarcopenia relative to non-sarcopenic patients (all $\mathrm{p}$ values $<0.05$ ). No 
differences between groups were observed for the other mediators investigated (fusion proteins Mfn1 and Opa1, fission protein Fis1, the regulator of mitochondrial biogenesis PGC- $1 \alpha$ and the pleiotropic transcription factor FoxO3a).

Conclusions: Data from this exploratory study show that reduced mitochondrial fusion and disrupted autophagic signaling are associated with sarcopenia in older hip-fractured patients. Future larger-scale studies are needed to corroborate these preliminary findings and determine whether myocyte quality control pathways may be targeted to improve muscle tropism and function recovery in older patients with hip fracture.

\section{P386}

ANIMAL-DERIVED DIETARY PROTEIN, MUSCLE MASS AND FUNCTION IN COMMUNITY-DWELLERS VISITING MILAN EXPO: RESULTS FROM THE VIP STUDY

R. Calvani ${ }^{1}$, E. Marzetti ${ }^{2}$, M. Tosato ${ }^{3}$, G. Savera ${ }^{1}$, A. Collamati $^{1}$, A. Sisto ${ }^{1}$, E. Ortolani ${ }^{3}$, A. M. Martone ${ }^{3}$, R. Bernabei $^{2}$, F. Landi ${ }^{2}$

${ }^{1}$ Catholic University of the Sacred Heart, Rome, Italy, ${ }^{2}$ Department of Geriatrics, Neurosciences and Orthopedics, Teaching Hospital, Rome, Italy, ${ }^{3}$ Department of Geriatrics, Neurosciences and Orthopedics, Teaching Hospital “Agostino Gemelli”, Rome, Italy

Objective(s): The loss of muscle mass and function with aging (sarcopenia) is associated with numerous adverse health events. Behavioral factors, including protein intake, influence the quantity and quality of skeletal muscle.

Material and Methods: The VIP (Very Important Protein) Study, conducted during EXPO 2015 in Milan, consisted of a population survey aimed at assessing the prevalence of health metrics in a population outside of a research setting with a special focus on the relation between animal protein intake and muscle mass and function. A brief questionnaire exploring lifestyle habits, dietary preferences and the consumption of selected foods was administered. Muscle mass was estimated by midarm muscle circumference (MAMC) and calf circumference of the dominant side. Muscle strength and function was assessed through handgrip strength testing and repeated chair stand test.

Results: 2854 adult persons (53\% women, $47 \%$ men, mean age 51 years) were enrolled. Both muscle strength and function declined significantly with age $(p<0.0001)$. Muscle mass assessed either by MAMC or calf circumference was similar across age groups. The average daily consumption of animal derived protein in the study population was $21.9 \mathrm{~g}$. The nadir of animal protein consumption was found in the 50-60 years age group. Persons in the highest tertile of protein consumption (over $30 \mathrm{~g} /$ day) showed better performance both at the handgrip strength $(p<0.0001)$ and chair stand tests $(p<0.005)$ than those in the lowest tertile. The same results were found for MAMC $(p<0.0001)$ and calf circumference $(p<0.001)$
Conclusions: The results of the VIP survey suggest that in the oldest age group, protein consumption from animal sources, albeit not lower than in younger participants, may not be sufficient in supporting muscle health. This observation corroborates the paradigm of muscle anabolic resistance in old age and suggests that an increased amount of animal derived high-quality protein may be required to preserve muscle tropism in late life.

\section{P387}

AGE-RELATED TRAJECTORIES OF MUSCLE MASS AND FUNCTION IN ITALIAN COMMUNITY-DWELLERS VISITING THE MILAN EXPO: RESULTS FROM THE VIP STUDY

M. Tosato ${ }^{1}$, R. Calvani ${ }^{2}$, E. Marzetti ${ }^{1}$, G. Savera ${ }^{2}$, A. Collamati $^{2}$, A. Sisto ${ }^{2}$, E. Ortolani ${ }^{1}$, A. M. Martone ${ }^{1}$, R. Bernabei ${ }^{1}$, F. Landi ${ }^{1}$

${ }^{1}$ Department of Geriatrics, Neurosciences and Orthopedics, Teaching Hospital, Rome, Italy, ${ }^{2}$ Catholic University of the Sacred Heart, Rome, Italy

Objective(s): Declining muscle mass and function is a hallmark of the aging process. The preservation of muscle tropism may protect against various negative health outcomes. Age- and sexspecific curves of muscle mass and strength using data from a large sample of "true" free-living people have yet to be established.

Material and Methods: The VIP (Very Important Protein) Study, conducted during EXPO 2015 in Milan, consisted in a population survey aimed at assessing the prevalence of health metrics in a population outside of a research setting, with a special focus on the relation between animal protein intake and muscle mass and function. A brief questionnaire exploring lifestyle habits, dietary preferences and the consumption of selected foods was administered. Muscle mass was estimated by mid-arm muscle circumference (MAMC) and calf circumference of the dominant side. Muscle strength and function was assessed through handgrip strength testing and repeated chair stand test.

Results: 2800 adult persons (53\% women, $47 \%$ men, mean age 51 years) were enrolled. Both muscle strength and function declined significantly across decades ( $p$ for trend $<0.0001$ ) irrespective of sex and BMI. This pattern was more evident in females (33\% reduction in handgrip strength for females vs. $25 \%$ in males). Muscle mass assessed either by MAMC or calf circumference was similar across age groups. Muscle quality of the upper extremities, defined as handgrip strength divided by MAMC, declined significantly with aging $(p<0.0001)$.

Conclusions: Muscle mass and strength curves may be used to extract reference values for subsequent use in research as well as in the clinical setting. In particular, the analyses of trajectories of muscle parameters may help identify cutoffs for the estimation of risk of adverse events. 


\section{P388}

\section{THE INCIDENCE OF SARCOPENIA AMONG OLDER IN-PATIENTS: RESULTS FROM THE GLISTEN STUDY}

A. M. Martone ${ }^{1}$, M. Tosato ${ }^{1}$, S. Volpato ${ }^{2}$, E. Marzetti ${ }^{1}$, R. Calvani $^{3}$, A. Sisto ${ }^{3}$, E. Ortolani ${ }^{1}$, R. Bernabei ${ }^{1}$, F. Landi ${ }^{1}$

${ }^{1}$ Department of Geriatrics, Neurosciences and Orthopedics, Teaching Hospital, Rome, Italy, ${ }^{2}$ Department of Medical Science, University of Ferrara, Italy, Ferrara, Italy, ${ }^{3}$ Catholic University of the Sacred Heart, Rome, Italy

Objective(s): Lean body mass is an important determinant of illness and recovery during hospital stay. The objective of the study was to evaluate the incidence of sarcopenia in hospitalized older patients.

Material and Methods: Data are from the Italian Study of the Gruppo Lavoro Italiano Sarcopenia - Trattamento e Nutrizione (GLISTEN), conducted in nine acute care wards across Italy. Sarcopenia was assessed and diagnosed according to the EWGSOP criteria, with bioelectrical impedance analysis (BIA) used for muscle mass estimation.

Results: 453 inpatients (48\% women; mean age 80.9 \pm 6.4 years) were enrolled. At hospital admission, sarcopenia was detected in 148 patients (32.7\%), with no differences in gender. Among non-sarcopenic patients at admission, $14.3 \%$ developed sarcopenia during their hospital stay. Patients who developed sarcopenia were significantly older than those with no sarcopenia at discharge $(81.5 \pm 6.9$ vs. $79.3 \pm 5.9$ years, $p<0.01$ ). The development of sarcopenia during hospital stays was significantly associated with the number of days spent without mobilization, while it was unrelated to the length of stay. Patients who developed this condition had a significantly lower BMI and muscle mass at admission relative to those who did not develop sarcopenia ( $p<0.01$ for both comparisons).

Conclusions: Our findings show that hospital admission is associated with a high risk of developing sarcopenia. This phenomenon is primarily related to the length of time spent in bed. Given the relevance of lean body mass for patient recovery, strategies should be developed to preserve muscle mass and function during hospital stays (e.g., early mobilization, nutritional support).

\section{P389}

GROWING TREND OF OSTEOPOROSIS TREATMENT AFTER HIP FRACTURE IN THAILAND K. K. Klunklin ${ }^{1}$, T. L. Leerapun ${ }^{1}$

${ }^{1}$ Department of Orthopedics, Faculty of Medicine, Chiangmai University, Chiangmai, Thailand

Objective: Hip fracture is the common consequence of osteoporosis and generally the most severe form of osteoporotic fracture. After the first hip fracture, the rate of further fracture increases 2.5 fold for vertebral fracture and 2.3-fold for second hip fracture. Osteoporosis treatment is required to prevent the new fracture. The purpose of the present study was to assess the proportion of osteoporosis patients who were treated with antiresorptive or anabolic medication after hip fracture.

Material and Methods: A retrospective database study of all low energy osteoporotic hip fracture patients at the age of 50 years old or more was performed at Chiangmai university hospital, between 2012-2015. The National Osteoporosis Foundation guideline was used to identify the adequacy of treatment of osteoporosis in this group of patients. Age, sex, admitted diagnosis, mechanism of injury, treatment procedures and discharge medication were extracted and analysed. Results: A total of 769 patients who had sustained hip fracture were identified. Of these patients, from 2012 to $2015,10.5 \%$, $12.3 \%, 18.7 \%$, and $20.7 \%$, respectively, were receiving antiresorptive or anabolic medication. The percentage of calcium supplementation for the discharged patients was $48.3 \%$, $54.5 \%, 60.8 \%$ and $66.9 \%$, respectively, and the percentage of combination of calcium and vitamin D supplementation for the discharged patients was $45.4 \%, 51.8 \%, 57.3 \%$ and $63.3 \%$, respectively. All three types of medication treatment were increased significantly by a trended chi-square test $(p<0.05)$.

Conclusion: Along the 4 years of our study, we found improvement in the treatment of osteoporosis after hip fracture by increasing of antiresorptive or anabolic medication prescription. However, there are still some patients who have not been treated adequately so we need to encourage physicians about the importance of osteoporosis treatment.

\section{P390 \\ PREGNANCY ASSOCIATED OSTEOPOROSIS WITH VERTEBRAL FRACTURES IN YOUNG FEMALE}

$\underline{\text { T. Bajraktarova Prosheva }}{ }^{1}$, S. Markovik Temelkova ${ }^{2}$, S. Josifovska Tofiloska ${ }^{1}$

${ }^{1}$ Department of Internal Medicine, Clinical Hospital Shtip, Shtip, Former Yugoslav Republic of Macedonia, ${ }^{2}$ University Clinic of Endocrinology, Diabetes and Metabolic Disorders, Skopje, Former Yugoslav Republic of Macedonia

Objective: To present the rare case of postpartum osteoporosis (PPO) with thoracolumbar compression fractures in previous healthy young woman and improving in clinical manifestation and BMD after one year treatment.

Material and methods: We report 31-y-old female with severe lumbar back pain, started the last month of the pregnancy. One week after delivery she felt severe pain when she bent forward, after what she became immobile in a wheelchair. On the physical examination, the movement in the lumbar region was limited, painful and with paravertebral muscle spasm.

Results: X-ray and computer tomography (CT) of the thoraco-lumbar spine was performed. Osteoporotic changes 
of the vertebral bodies and reduced cranio-caudal diameter with compressive fractures of Th12, L3 and L5 were found. DXA showed significantly decreased bone density, with osteoporosis on all lumbar vertebrae: Z-score: L1 -3.7; L2 -3.6; L3 -2.7; L4 -3.7; (T-score L1-L4 -4.4). The patient was underweight with BMI $17.5 \mathrm{~kg} / \mathrm{m} 2$. The family anamnesis and the anamnesis for medications intake was negative. The laboratory tests showed increased bone turnover markers (osteocalcin $42 \mathrm{ng} / \mathrm{ml}$, $\beta$ CrossLaps $0.704 \mathrm{ng} / \mathrm{ml}$ ), normal level of PTH, vitamin $\mathrm{D}$ and calcium. The thyroid function was normal, the protein electrophoresis was normal with negative findings for multiple myeloma. An antiresorptive treatment with i.v. bisphosphonate, calcium and vitamin D was started. The condition of the patient and the DXA results were significantly improved after one year of the treatment.

Conclusion: Although pregnancy is not known as a risk factor for osteoporosis, we should think about it in women with low back pain during and after pregnancy. Early diagnosis is important in the prevention of fractures and the disability of the patients aiming an increased life quality. The treatment of PPO with bisphosphonates is recommended and effective.

\section{P391}

\section{A RANDOMIZED CONTROLLED TRIAL OF TIDAL IRRIGATION VERSUS INTRA-ARTI- CULAR CORTICOSTEROID INJECTION ON EFFUSION IN KNEE OSTEOARTHRITIS}

S. Mostefai $^{1}$, D. Hannoune ${ }^{2}$, H. Djoudi ${ }^{3}$, S. Lefkir Tafiani ${ }^{4}$

${ }^{1}$ Rheumatology, University Hospital Centre, Beni-Messous, Algiers University Medicine Faculty, Algiers, Algeria, ${ }^{2}$ National Institute of Public Health (INSP), Algiers, Algeria, ${ }^{3}$ Rheumatology Service, EHS Douéra, Algiers, Algeria, ${ }^{4}$ Rheumatology, University Hospital Centre, Beni-Messous, Algiers, Algeria

Introduction: Tidal irrigation consists in cleaning the joint from debris and inflammatory mediators. Its place in the treatment of knee osteoarthritis is variously appreciated. The objective of the study is to evaluate the efficacy of the tidal irrigation associated with the corticosteroid injection vs. the intra articular corticosteroid injection alone, on the effusion in advanced knee osteoarthritis of III and IV stages of the Kellgren-Lawrence classification.

Material and Methods: This is a prospective clinical trial, which is open, controlled and randomized over a period of six months. This study included subjects aged 50 or over, suffering from advanced knee osteoarthritis requiring a surgical treatment that cannot be done for various reasons such as the advanced age, the existence of other serious diseases that can be life threatening. Knee osteoarthritis is characterized by an effusion, refractory to a medical treatment. A standard X-ray shows an advanced knee osteoarthritis of III and IV stages of the Kellgren-Lawrence classification. Patients are randomized into two groups:
- The joint puncture + tidal irrigation + corticosteroid injection - The joint puncture + intra articular corticosteroid injection The assessment of patients focused on the recurrence of the joint effusion which is analyzed by measuring the circumference of the knee and the recidivism period of the effusion during the monitoring in both groups. The evaluation was made at the $1 \mathrm{st}, 4 \mathrm{th}, 12$ th and 24 th weeks.

Results: Our study involves 140 patients whose average age is 69.5 years. It allowed to demonstrate that the joint puncture followed by irrigation and corticosteroid injection has a better effect on effusion and prevents its recurrence in time in comparison to the puncture + injection whose effect does not exceed 4 weeks. At the 6 th month, $88.7 \%$ of patients who underwent tidal irrigation have no more effusion. While in the injection group, $50 \%$ of patients continue to have effusion despite the treatment given. The difference is statistically significant between the two groups at the 6th month $(p<10-6)$.

Conclusion: The risk of the recurrence of effusion is lower in the irrigation group compared to injection group over a period of six months. The effect of injection does not exceed 4 weeks. Tidal irrigation can be a therapeutic alternative in the treatment of advanced knee osteoarthritis.

P392

THE ROLE OF IL-6 IN THE ACCELERATION OF SARCOPENIA IN FEMALE PATIENTS WITH RHEUMATOID ARTHRITIS

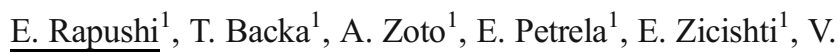
Salko $^{1}$, L. Nuhaj ${ }^{1}$

${ }^{1}$ Service of Rheumatology, University Hospital Center "Mother Theresa", Tirana, Albania

Background: Rheumatoid arthritis (RA) is a chronic inflammatory disease, in its fispathology participate various number of inflammatory cytokines (TNF $\alpha$, IL-1 $\beta$, IL-6, etc.), which increase muscle mass loss leading or accelerating sarcopenia. Objective: Evaluation of the role of IL-6 in causing sarcopenia in female patients with RA.

Material and Methods: 42 female patients diagnosed with RA according to ACR/EULAR 2010 criteria were involved in the study. Sarcopenia status in patients with RA was evaluated by assessing body composition using DXA. Sarcopenia was defined as a relative skeletal mass index (SMI) of $\leq 5.75 \mathrm{~kg} / \mathrm{m}^{2}$ in women. In all patients was evaluated seric level of IL- $6(<4.0 \mathrm{pg} / \mathrm{ml})$. Were excluded from this study patients in menopause (to exclude the hormonal effect on immunologic alteration and their effect in sarcopenia), and those with other chronic disease such as diabetes mellitus or other connective tissue disease.

Results: From all the data collected was found that SMI was diminished $4.93 \pm 0.96 \mathrm{~kg} / \mathrm{m}^{2}$ in patients with RA. Seric level of IL- 6 was found increased in 20 patients $19 \pm 3.5 \mathrm{pg} / \mathrm{ml}$ and normal levels in 22 patients $2.11 \pm 1.3 \mathrm{pg} / \mathrm{ml}$. Patients with 
diminished levels of SMI $3.03 \pm 0.4 \mathrm{~kg} / \mathrm{m}^{2}$ presents increased seric levels of IL-6 $(19 \pm 3.5 \mathrm{pg} / \mathrm{ml})(p<0.05)$, meanwhile patients with SMI levels close to normal range $4.02 \pm 0.9 \mathrm{~kg} / \mathrm{m}^{2}$ presents lower levels of IL-6 $(2.11 \pm 1.3 \mathrm{pg} / \mathrm{ml}(p<0.05)$.

Conclusion: IL-6 has an important role in decreasing muscle mass in patients with RA. Its noted a very strong relation between sarcopenia and increased seric level of IL- 6 in patients with RA, leading to the infliction and acceleration of sarcopenia in these patients.

\section{P393}

\section{FEMALE PATIENTS SUFFERING FROM} SECONDARY HYPERPARATHYROIDISM WHO UNDERGO TOTAL KNEE ARTHROPLASTY DO NOT FACE INCREASED DANGER TO DEVELOP ASEPTIC LOOSENING OF THEIR IMPLANTS

$\underline{\text { K. Papavasiliou }}^{1}$, T. Michael ${ }^{1}$, D. Tsitouras ${ }^{1}$, M. Karanassos ${ }^{1}$, J. Kirkos ${ }^{1}$

${ }^{1}$ 3rd Orthopaedic Dept. Aristotle University of Thessaloniki, Papageorgiou General Hospital, Thessaloniki, Greece

Objectives: Aim of this prospective case-controlled study was to assess whether the incidence of aseptic loosening of implants in patients undergoing total knee arthroplasty (TKA) is higher among those with secondary hyperparathyroidism (SP) at the time of the primary operation.

Material and Methods: Caucasian female patients with knee osteoarthritis who underwent TKA between November 2004 and March 2007 were enrolled. Those with endocrine disorder, rheumatoid or other secondary arthritis, end-stage renal disease and who had sustained a fracture or had undergone orthopaedic surgery 24 months before the TKA, were excluded. Patients with osteopenia/osteoporosis were not excluded. The serum levels of intact PTH (I-PTH), calcium, phosphorus, creatinine, and the clearance of creatinine were determined pre-operatively. The case-notes were reviewed and any revision operation was registered.

Results: 283 women aged 49-81 (mean, 70) were enrolled; 100 had abnormally elevated serum I-PTH levels. Two had primary hyperparathyroidism and therefore excluded from further analysis. The overall incidence of $\mathrm{SH}$ was $35 \%$. The serum I-PTH level correlated positively with patient age $(r=0.158, p=0.008)$ and serum creatinine level $(r=0.138$, $p=0.021)$ and negatively with the clearance of creatinine $(r=-0.169, p=0.004)$. In multiple regression analysis, $7.3 \%$ of the variance in serum I-PTH values $(\mathrm{R} 2=0.073$, $p<0.001$ ) was significant; serum creatinine level was the largest contributor (standardised beta $=0.275, p=0.08$ ). 265 patients were available for re-evaluation at an average follow-up period of 115 (105-132) months. Three patients with SH and 7 with normal I-PTH values (at the initial operation) were re- operated due to aseptic loosening at an average period of 51.3 (26-90) and 49.9 (25-94) months respectively. The difference between the number of re-operated patients with pre-operative $\mathrm{SH}$ and normal I-PTH values, was statistically non-significant $(p>0.005)$. The mean time to re-operation was also non- significant $(p>0.005)$.

Conclusion(s): The initial hypothesis that $\mathrm{SH}$ enhances the early aseptic loosening of implants in TKA due to excessive activation of osteoclasts is not supported by the results of this study. Further studies are needed to assess the potentially negative impact of SH in the orthopaedic implants' incorporation.

\section{P394}

PREVENTION AND MANAGEMENT OF OSTEOPOROSIS AMONG CANADIAN ADULTS

\section{S. O'Donnell ${ }^{1}$}

${ }^{1}$ Surveillance and Epidemiology Division, Center for Chronic Disease Prevention, Health Promotion and Chronic Disease Prevention Branch, Public Health Agency of Canada, Ottawa, Canada

Objectives: To describe the use of osteoporosis (OP) prevention and management strategies among Canadians age $>40$ years.

Methods: Respondents to the 2009 Canadian Community Health Survey's Osteoporosis Rapid Response, a nationally representative sample of 5,849 Canadians age $>40$ years, were asked about OP prevention and management strategies. We describe these according to four risk groups based on OP diagnosis and fracture history i.e., fracture after age 40 of the wrist, upper arm, spine, pelvis or hip.

Results: Approximately 1.5 million Canadians 40 years of age or older $(10 \%)$ reported having been diagnosed with OP of which, one in five (21\%) reported having had a fracture. The majority of those diagnosed with OP reported having been prescribed OP medication(s) (59\%) and taking vitamin $\mathrm{D}$ and calcium supplements. Less than $40 \%$ reported regular physical activity. Among those who did not report having been diagnosed with OP, $7 \%$ reported having had a fracture of which, one-third reported having had a bone density test of the spine or hip, less than half reported taking vitamin D or calcium supplements and engaging in regular physical activity.

Conclusions: OP is common among Canadian adults. A large proportion at risk for OP are not being screened and many (especially those without an OP diagnosis) are not engaging in lifestyle approaches recommended to help maintain healthy bones.

Notes: Proportions (\%) and $95 \%$ Confidence Intervals (CIs) are based weighted data; ${ }^{\dagger}$ Fracture after age 40 of the wrist, upper arm, spine, pelvis or hip; ${ }^{*}$ Age-standardized proportions significantly different from the group with OP diagnosis, fracture; ${ }^{\in}$ Interpret with caution as coefficient of variation between $16.6-33.3 \%$. 


\begin{tabular}{|c|c|c|c|c|}
\hline & $\begin{array}{l}\text { OP diagnosis, fracture }{ }^{\dagger} \\
n=153(2.1 \%)\end{array}$ & $\begin{array}{l}\text { OP diagnosis, no fracture } \\
n=519(7.9 \%)\end{array}$ & $\begin{array}{l}\text { No OP diagnosis fracture } \\
n=393(6.3 \%)\end{array}$ & $\begin{array}{l}\text { No OP diagnosis, no fracture } \\
n=4,639(83.6 \%)\end{array}$ \\
\hline & $\%(95 \% \mathrm{CI})$ & $\%(95 \% \mathrm{CI})$ & $\%(95 \% \mathrm{CI})$ & $\%(95 \% \mathrm{CI})$ \\
\hline Bone density test & $88.9(82.2-95.7)$ & $86.0(81.3-90.7)$ & $33.0(26.1-39.8)^{*}$ & $20.3(18.6-21.9)^{*}$ \\
\hline Prescribed OP medication & $69.5(58.5-81.6)$ & $56.6(49.3-63.9)^{*}$ & $\mathrm{n} / \mathrm{a}$ & $\mathrm{n} / \mathrm{a}$ \\
\hline Vitamin D & $89.1(83.2-95.0)$ & $68.6(60.3-76.9)$ & $44.9(36.9-52.6)^{*}$ & $34.0(32.1-36.0)^{*}$ \\
\hline Calcium & $90.7(85.1-96.4)$ & $81.8(76.6-87.0)$ & $40.5(32.8-48.2)^{*}$ & $34.0(32.1-36.0)^{*}$ \\
\hline Regular Physical Activity & $37.6^{€}(23.6-52.6)$ & $29.3(23.2-35.4)$ & $45.1(37.2-53.1)$ & $43.1(40.7-45.4)$ \\
\hline
\end{tabular}

P395

EFFECTS OF WEEKLY VITAMIN D SUPPLEMENTATION ON BONE TURNOVER MARKERS IN FEMALE ADOLESCENTS WITH VITAMIN D INSUFFICIENCY/DEFICIENCY

$\underline{\text { R. Sulimani }^{1}}{ }^{1}$ A. $\operatorname{Gad}^{1}$, S. Alshehri ${ }^{2}$, A. Alfadda ${ }^{1}$, A. Alothman $^{1}$, N. Aldaghri ${ }^{1}$

${ }^{1}$ King Saud University, Riyadh, Saudi Arabia, ${ }^{2}$ Ministry of health, Riyadh, Saudi Arabia

Vitamin D deficiency is considered a major public health problem in Saudi Arabia. Different regimens for supplementation have been advocated. This study examines the efficacy of weekly loading vitamin D supplementation in Saudi adolescent females and its effects on bone turnover markers (BTMs).

Methods: 68 adolescent girls with vitamin D insufficiency $(25(\mathrm{OH}) \mathrm{D}<50 \mathrm{nmol} / 1$ and deficiency $(25(\mathrm{OH}) \mathrm{D}<25 \mathrm{nmol} / \mathrm{L}$ were recruited from high schools. Their age ranged from 13-19 years with a mean of $15.1 .7 \pm 1.7$ years. Fasting morning blood samples were taken for assay of $25(\mathrm{OH}) \mathrm{D}$, calcium, phosphorus, PTH, osteocalcin and carboxyterminal collagen crosslinks (CTX). Girls were supplemented with weekly 35,000 units of vitamin D3 drops for 4 weeks. Blood samples were taken at baseline and one day after completion of the 4 week supplementation course.

Results: Mean baseline 25(OH)D level was 27.60 $\pm 14.56 \mathrm{nmol} / 1$ and $55.96 \pm 23.03$ after 4 weeks of treatment $(p<001)$, PTH $6.32 \pm 5.55 \mathrm{pmol} / \mathrm{L}$ and post $3.51 \pm 1.33 \mathrm{pmol} / 1$, CTX $0.52 \pm 0.41 \mu \mathrm{g} / \mathrm{ml}$ pretreatment and $0.32 \pm 0.18 \mu \mathrm{g} / \mathrm{ml}$ post treatment and osteocalcin $52.75 \pm 41.80 \mathrm{ng} / \mathrm{ml}$ pretreatment and $34.54 \pm \mathrm{ng} / \mathrm{ml}$ post treatment.

Conclusions: weekly $35,000 \mathrm{u}$ vitamin D3 is effective in restoring vitamin $\mathrm{D}$ levels to acceptable values and is accompanied with reduction in PTH and bone turnover markers.
P396

VISCERAL FAT MASS AND BONE MINERAL DENSITY IN A GROUP OF OBESE CHILDREN

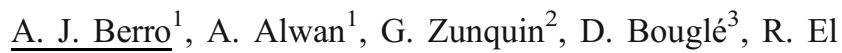
Hage $^{1}$

${ }^{1}$ Department of Physical Education, Fass, University of Balamand, Kelhat El Kurah, Lebanon, ${ }^{2}$ Staps, Ulco, Dunkerque, France, ${ }^{3}$ Service de Pédiatrie, CHU de Caen, Caen, France

Introduction: We have previously shown that obesity is associated with higher BMD values in children [1]. However, the relation between visceral fat mass and BMD in obese children remains unclear. The aim of this study was to investigate the relationship between visceral fat mass and BMD in a group of obese children.

Methods: 35 obese girls and 31 obese boys whose ages range between 7-17 years participated in this study [2,3]. Weight and height were measured, and BMI was calculated [3]. Body composition, bone mineral content and BMD were measured at whole body, lumbar spine (L1-L4), total hip (TH) and femoral neck (FN). Trunk fat mass was measured and percent trunk fat was calculated as previously described [3]. Percent trunk fat was used as a surrogate for visceral fat mass [4].

Results: Age, weight, height, BMI, lean mass, fat mass and trunk fat mass were positively correlated to bone variables (BMC and BMD) in both sexes. Visceral fat mass was not significantly correlated to BMC and BMD in girls while it was negatively correlated to these bone variables in boys. After controlling for lean mass and age using multiple linear regression models, visceral fat mass was negatively correlated to WB BMD in girls while it was not significantly correlated to bone variables in boys.

Conclusion: This study suggests that, in obese children, visceral fat mass is not beneficial to BMD.

\section{References:}

1. Rocher E, et al. J Clin Densitom 2013;16:244.

2. Bouglé D, et al. Int J Pediatr 2010;2010:580897.

3. Cole TJ, et al. BMJ 2000;320:12403. 
4. Ackerman KE, et al. J Pediatr Endocrinol Metab $2011 ; 24: 497$

\section{P397}

\section{BONE DENSITY IN SAMPLE OF IRAQI DIABETIC PATIENTS}

A. Abdulbari ${ }^{1}$, A. Rahmah ${ }^{2}$, H. Fadhil ${ }^{2}$

${ }^{1}$ Al Yarmouk Teaching Hospital, Baghdad, Iraq, ${ }^{2}$ National

Diabetic Center, Baghdad, Iraq

Background: Diabetes as a common metabolic disorder is not only a disorder of glucose metabolism but it has an effect on the metabolism of almost all other materials in the body. Accordingly, diabetics might have different bone density parameters.

Objectives: To estimate the frequency of bone density in Iraqi diabetic patients and the effects of different demographic and metabolic factors on the distribution of bone marrow density in this group of patients.

Methods: This is a cross-sectional study the enrolled 41 diabetic patients who attended the outpatient unit in the National Center of Diabetes in Baghdad, Iraq during the period between January 2012 and December 2013. After taking detailed history and doing thorough physical examination, all patients did bone density.

Results: In general, there was no statistically significant difference between the mean of bone density in diabetic from that in the control (nondiabetic) groups. But there was statistically significant difference in the mean bone density in obese diabetics $(1.15 \pm 0.2)$ compared to non-obese diabetics $(0.83 \pm 0.15)$. This significant difference was observed in different age groups, but it is increased with increasing age in obese diabetics $(1.31 \pm 0.21$ vs. $1.19 \pm 0.3$ in nonobese and obese diabetics in their forties, respectively) and (1.12 \pm 0.2 vs. $0.82 \pm 0.1$ in nonobese and obese diabetics in their sixties, respectively). In addition, it had been found that diabetics who were on insulin therapy had bone density of $0.92 \pm 0.4$ while those on oral antidiabetic agents had a bone density of $1.03 \pm 0.19$, but it had been found that this difference is not statistically significant.

Conclusions: This study showed that obesity is a major risk factor for lower bone density and its effect is increased with increasing age and using of insulin therapy.

\section{P398}

A POPULATION-BASED ANALYSIS OF THE POST-FRACTURE CARE GAP IN HONG KONG: THE SITUATION IS NOT IMPROVING

A. W. H. Ho ${ }^{1}$, S. H. Wong ${ }^{1}$

${ }^{1}$ Caritas Medical Centre, Hong Kong, Hong Kong

Introduction: Patients who sustain an osteoporotic fracture are at increased risk of sustaining further osteoporotic fracture.
There are international guidelines to close this post-fracture care gap but not in Hong Kong. The practice varies between different hospitals and departments. We report the current situation and practice of DXA assessment after osteoporotic hip fracture in all public hospitals.

Methods: We retrieved patients with new osteoporotic hip fractures age 65 or above with DXA performed within one year after fracture, admitted from 1st Jan 2012 to 30th June 2014 using Hospital Authority Clinical Data Analysis Reporting System.

Results: 11397 osteoporotic hip fracture patients were included. Only 398 patients (3.49\%) received DXA within one year after fracture. Among the group with DXA performed, $69 \%$ were from two Hospitals. The other 11 hospitals contribute the remaining $31 \%$.

Discussion and conclusion: The waiting time for DXA in public hospital of Hong Kong varies from 1-4 years. There is a huge post-fracture care gap in secondary prevention for osteoporotic hip fracture patient in Hong Kong. Patients presenting with lowenergy fractures should be offered assessment for osteoporosis by axial bone densitometry. However majority of these patients in Hong Kong are neither diagnosed nor being tested for osteoporosis and remained untreated. The government needs to allocate more resources to implement the best practices framework to those high risk post-hip fracture patients before they go on to break another bone. By reducing the number of subsequent osteoporotic fractures, the government can get significant cost savings that can be utilized in other valuable healthcare program.

\section{P399}

DAYTIME GERIATRIC HIP FRACTURE SURGERY REDUCES EARLY AND LATE MORTALITY AND IMPROVE LONG-TERM SURVIVAL

A. W. H. Ho $^{1}$, S. H. Wong ${ }^{1}$

${ }^{1}$ Caritas Medical Centre, Hong Kong, Hong Kong

Introduction: Geriatric hip fracture and their care are a major burden and challenge to our health care system. The trend is ever increasing due to our ageing population. Although it is recommended in many international guidelines for hip fracture management, no major study has been performed to examine the relationship of daytime surgery and mortality in Chinese population.

Material and Methods: We undertook a retrospective review of data collected from the Clinical Data Analysis and Reporting System (CDARS) of Hospital Authority of Hong Kong on all patients aged 65 or above who presented to all acute public hospitals between 2000-2014 with hip fracture treated surgically. Patients were divided into two groups according to timing of surgery: daytime group (surgery performed from 8 am to $8 \mathrm{pm}$ ) and nighttime group (from $8 \mathrm{pm}$ to $8 \mathrm{am}$ ). Between the two groups, the 30 -day, one-year and 
five-year mortality were compared using chi-square test. The result was considered statistically significant if $p$ value is less than 0.05. All analysis was performed using SPSS software.

Results: There were 42590 patients (13817 men and 32160 women) included in the study. The patient age ranged from 65-112 (mean: 82). $92.6 \%$ of patients had day time surgery while $7.4 \%$ of patients had nighttime surgery. For each age group the percentage of daytime surgery or nighttime surgery was similar and no correlation between the timing of surgery and sex was found. There was no statistically significant difference of survival in 30-day, 1-year and 5-year mortality between daytime and nighttime surgery. By breaking down into sex and different age group, there were decreases in 30-day, 1-year and 5-year mortality in daytime surgery group among different age group, and the reduction in mortality in female aged 75-84 was statistically significant. The male and female mortality are depicted in Tables $1 \& 2$.

Table 1: Age- and Sex-specific Mortality in Male patients operated in daytime or nighttime

\begin{tabular}{|c|c|c|c|c|c|c|c|c|c|c|}
\hline \multirow[t]{2}{*}{ Male/ age } & \multicolumn{2}{|c|}{$65-69$} & \multicolumn{2}{|c|}{$70-74$} & \multicolumn{2}{|c|}{$75-79$} & \multicolumn{2}{|c|}{$80-84$} & \multicolumn{2}{|c|}{$85+$} \\
\hline & Night & Day & Night & Day & Night & Day & Night & Day & Night & Day \\
\hline $\begin{array}{l}\text { Mean } \\
\text { survival } \\
\text { (days) }\end{array}$ & 2629 & 2674 & 2197 & 2309 & 1829 & 1886 & 1623 & 1454 & 1146 & 1056 \\
\hline $\begin{array}{l}30 \text { days } \\
\text { Mortality } \\
\text { (\%) }\end{array}$ & 1.0 & 2.1 & 5.4 & 2.7 & 6.4 & 4.0 & 3.1 & 4.6 & 6.0 & 7.1 \\
\hline $\begin{array}{l}1 \text { year } \\
\text { mortality } \\
\text { (\%) }\end{array}$ & 9.7 & 14.6 & 19.6 & 18.0 & 25.5 & 20.3 & 28.7 & 26.2 & 36.1 & 35.5 \\
\hline $\begin{array}{l}5 \text { years } \\
\text { mortality } \\
(\%)\end{array}$ & 41.3 & 43.0 & 50.4 & 50.0 & 57.9 & 55.8 & 62.5 & 66.0 & 74.5 & 77.7 \\
\hline Population & 94 & 1135 & 146 & 1806 & 217 & 2933 & 251 & 3251 & 298 & 3634 \\
\hline $\mathrm{p}$ & \multicolumn{2}{|c|}{0.349} & \multicolumn{2}{|c|}{0.863} & \multicolumn{2}{|c|}{0.126} & \multicolumn{2}{|c|}{0.719} & \multicolumn{2}{|c|}{0.721} \\
\hline
\end{tabular}

Night: Night time; Day: Day time

day time: 12811 night time: 1206

Table 2: Age-and sex-specific mortality in female patients operated in daytime and nigh time

\begin{tabular}{|c|c|c|c|c|c|c|c|c|c|c|}
\hline \multirow{2}{*}{$\begin{array}{c}\text { Female/ } \\
\text { age }\end{array}$} & \multicolumn{2}{|c|}{$65-69$} & \multicolumn{2}{|c|}{$70-74$} & \multicolumn{2}{|c|}{$75-79$} & \multicolumn{2}{|c|}{$80-84$} & \multicolumn{2}{|c|}{$85+$} \\
\hline & Night & Day & Night & Day & Night & Day & Night & Day & Night & Day \\
\hline $\begin{array}{l}\text { Mean } \\
\text { survival } \\
\text { (days) }\end{array}$ & 4072 & 3840 & 3402 & 3326 & 2539 & 2804 & 2036 & 2348 & 1685 & 1687 \\
\hline $\begin{array}{l}30 \text { days } \\
\text { Mortality } \\
(\%)\end{array}$ & 0.6 & 0.47 & 0.45 & 0.12 & 0.22 & 0.15 & 0.23 & 0.20 & 0.30 & 0.30 \\
\hline $\begin{array}{l}1 \text { year } \\
\text { mortality } \\
\text { (\%) }\end{array}$ & 0.82 & 0.56 & 0.78 & 0.73 & 10.5 & 0.98 & 15.1 & 12.5 & 17.9 & 18.4 \\
\hline $\begin{array}{l}5 \text { years } \\
\text { mortality } \\
(\%)\end{array}$ & 18.1 & 22.1 & 26.8 & 28.1 & 42.0 & 34.8 & 49.1 & 44.0 & 56.8 & 59.4 \\
\hline Population & 168 & 1503 & 223 & 3048 & 400 & 5856 & 586 & 7757 & 1002 & 11604 \\
\hline$p$ & \multicolumn{2}{|c|}{0.352} & \multicolumn{2}{|c|}{0.573} & \multicolumn{2}{|c|}{0.014} & \multicolumn{2}{|c|}{0.003} & \multicolumn{2}{|c|}{0.678} \\
\hline
\end{tabular}


Discussion and Conclusion: This is a large observational study of geriatric hip fracture in Chinese population, with complete follow-up for mortality statistics. The result showed that female aged 75-84 benefit the most from daytime surgery with statistically significant decreases in short-term and long-term mortality. This group of patient comprise of $32 \%$ of all geriatric hip fracture population. In view of the significant survival benefit, daytime geriatric hip fracture surgery should be recommended.

\section{P400}

ORTHOGERIATRIC COLLABORATIVE INTERVENTION PROGRAM FOR HIP FRACTURE SURGERY: A REVIEW ON SOUTHERN CHINESE POPULATION

A. W. H. Ho ${ }^{1}$, S. H. Wong ${ }^{1}$

${ }^{1}$ Caritas Medical Centre, Hong Kong, Hong Kong

Introduction: Geriatric hip fractures and their care are a major burden and challenge to our health care system. It is not uncommon to see these patients with multiple comorbidities, which complicates the perioperative course. Although it is recommended in many international guidelines for hip fracture management, no major study has been performed to examine the effect of orthogeriatric collaborative intervention program on mortality in the Chinese population.

Material and Methods: We undertook a retrospective review of data collected from the Clinical Data Analysis and Reporting System (CDARS) of Hospital Authority of Hong
Kong from 2005-2014, of all patients aged 65 or above who presented to five acute public hospitals between 2000-2014 with hip fracture treated surgically. Patients were divided into two groups according to availability of orthogeriatric collaborative intervention program. Those without these programs were classified as the control group. Among these two groups, the 30-day, one-year and five-year mortality were compared using chi-square test. Survival was calculated using survival analysis. The result was considered statistically significant if $p$ value is less than 0.05 . All analysis was performed using SPSS software.

Results: There were 15584 patients (4862 men and 10722 women) included in our study. $52.4 \%$ of patients belonged to orthogeriatric collaborative intervention program while $47.6 \%$ of patients had not. Patients under orthogeriatric collaborative intervention program had longer survival (mean survival 1907 days) while for the control group had mean survival was 1856 days $(p=0.05)$. Those under orthogeriatric intervention program also had lower 30-day, 1-year and 5-year mortality compared with the control group. It was statistically significant (table 1). By breaking down into sex and different age group, there were decrease in 30-day 1-year and 5-year mortality in patient under orthogeriatric intervention program among different age group, and the reduction in mortality in males aged 70-74 was statistically significant (table 2).

Table 1: Cumulative survival curve for hip fracture patient under orthogeriatric collaborative intervention program (With Geri) vs. control (Without Geri)

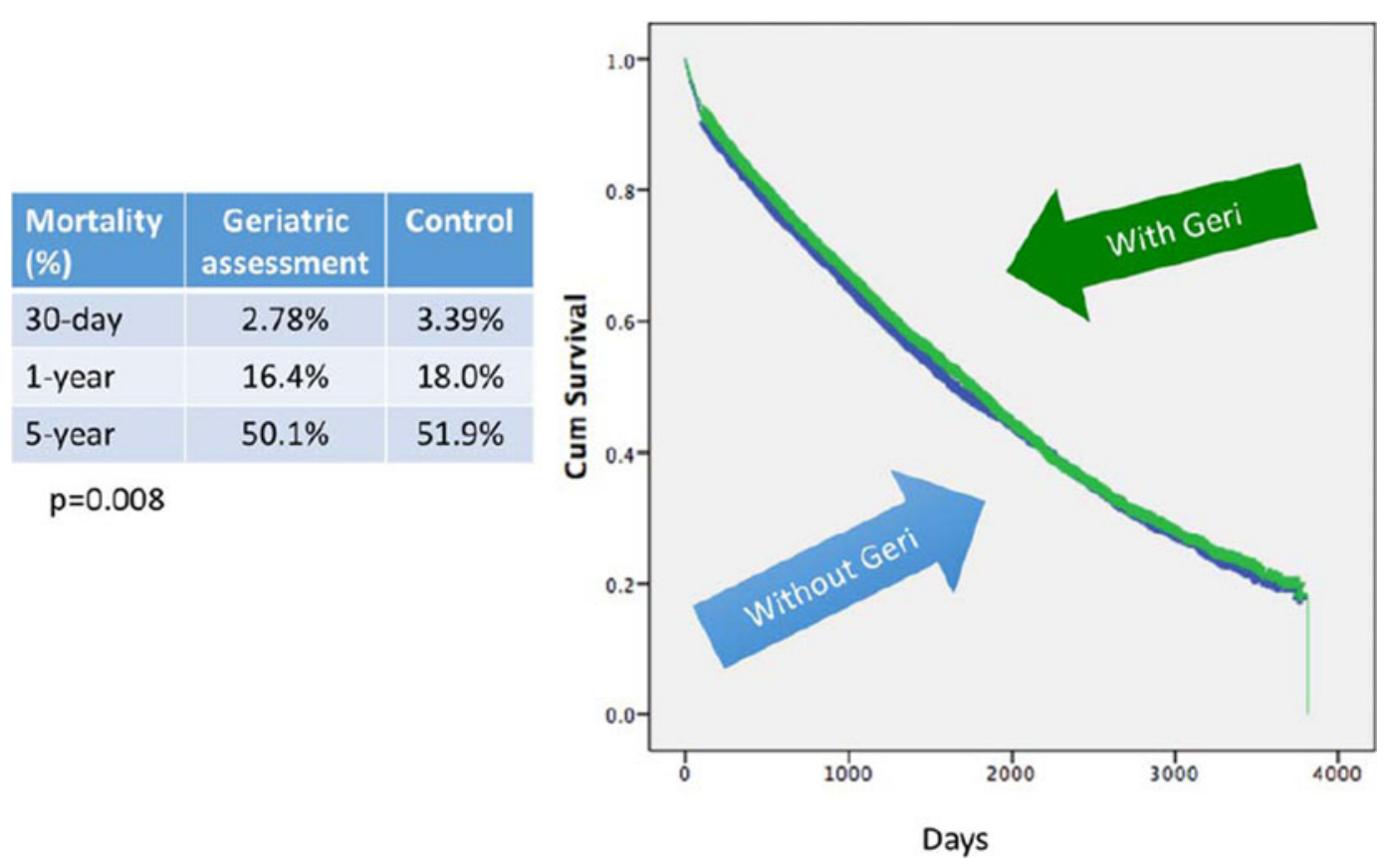




\begin{tabular}{|l|c|c|c|c|c|c|c|c|c|c|}
\hline Male/ age & \multicolumn{2}{|c|}{$65-69$} & \multicolumn{2}{|c|}{$70-74$} & \multicolumn{2}{c|}{$75-79$} & \multicolumn{2}{|c|}{$80-84$} & \multicolumn{2}{|c|}{$85+$} \\
\hline & $\begin{array}{l}\text { With } \\
\text { out } \\
\text { geri }\end{array}$ & $\begin{array}{l}\text { With } \\
\text { geri }\end{array}$ & $\begin{array}{l}\text { Witho } \\
\text { ut } \\
\text { geri }\end{array}$ & $\begin{array}{l}\text { With } \\
\text { geri }\end{array}$ & $\begin{array}{l}\text { With } \\
\text { out } \\
\text { geri }\end{array}$ & $\begin{array}{l}\text { With } \\
\text { geri }\end{array}$ & $\begin{array}{l}\text { Witho } \\
\text { ut } \\
\text { geri }\end{array}$ & $\begin{array}{l}\text { With } \\
\text { geri }\end{array}$ & $\begin{array}{l}\text { Witho } \\
\text { ut } \\
\text { geri }\end{array}$ & $\begin{array}{l}\text { With } \\
\text { geri }\end{array}$ \\
\hline $\begin{array}{l}30 \text { days } \\
\text { Mortality } \\
(\%)\end{array}$ & 2.2 & 1.9 & 3.5 & 3.4 & 5.0 & 3.6 & 4.8 & 3.4 & 7.8 & 6.4 \\
\hline $\begin{array}{l}1 \text { year } \\
\text { mortality } \\
(\%)\end{array}$ & 12.6 & 14.3 & 21.0 & 15.5 & 18.9 & 18.7 & 27.4 & 24.9 & 37.1 & 36.6 \\
\hline $\begin{array}{l}5 \text { years } \\
\text { mortality } \\
(\%)\end{array}$ & 41.2 & 47.4 & 54.1 & 47.1 & 53.7 & 57.2 & 66.9 & 63.9 & 78.7 & 75.0 \\
\hline Population & 227 & 207 & 344 & 354 & 536 & 554 & 609 & 653 & 679 & 699 \\
\hline p & 0.263 & \multicolumn{2}{|c|}{0.049} & \multicolumn{2}{|c|}{0.95} & 0.369 & 0.317 \\
\hline
\end{tabular}

Discussion and Conclusion: This is a large observational study of geriatric hip fracture in southern Chinese population, with a complete follow up for mortality statistics. The result showed that orthogeriatric collaborative intervention program improves survival and decreases early and late moralities, especially in male patients age $70-74$. The result is promising and warrants the implementation of orthogeriatric collaborative intervention program in hip fracture patient care in Hong Kong.

\section{P401}

\section{CHALLENGES IN FIXING THE OSTEOPORO- TIC INTERTROCHANTERIC HIP FRACTURE} $\underline{\text { R. K. Kumar }}^{1}$, P. C. Chawla ${ }^{1}$

${ }^{1}$ Indian Spinal Injuries Centre, New Delhi, India

The principle aim of urgent surgical fixation of the elderly hip fractures is pain relief to enable mobilization out of bed.

Objectives: To analyze the failures of fixation of the intertrochanteric femur fractures.

Material and methods: 100 elderly (above 60 years) patients with intertrochanteric fractures were prospectively followed up after fixation till union or till complications complained of. The protocol for post operative visits was 02 weeks, 03 months, 06 months and at 1 year.

Results: Failure was seen in 13 cases; backing out of the hip screw/blade, superior cut-out of the hip device, insufficiency fracture of the femur head and fracture through the femur head after removal of lag screw.

Conclusions: Attention to detail in fracture reduction is the first essential step. Establishing bone contact across the fracture and maintaining it till union is crucial. Implant selection is important. Augmenting purchase of hip device in femur head cancellous bone must be anticipated. Antiosteoporotic medical management must be continued or initiated before discharge from hospital.

\section{P402}

TOWARDS A STRATEGY FOR SECONDARY FRACTURE PREVENTION IN QUEBEC

S. N. Morin $^{1}$, S. Jean ${ }^{2}$, J. P. Brown ${ }^{3}$, L.-G. Ste-Marie ${ }^{4}$, P. Dubois $^{5}$, G. Boire ${ }^{6}$, I. Gaboury ${ }^{7}$, F. Cabana ${ }^{8}$, J. Fernandes ${ }^{9}$, J. Delisle ${ }^{9}$, M. Wall ${ }^{10}$, D. Theriault ${ }^{11}$, M.-C. Beaulieu ${ }^{12}$

${ }^{1}$ Medicine/McGill University/McGill University Health Center, Montreal, Canada, ${ }^{2}$ Institut National de Sante Publique du Quebec, Quebec, Canada, ${ }^{3}$ Laval University and CHU de Québec Research Centre, Quebec City, QC, Canada, ${ }^{4}$ Medicine/Université de Montreal/Centre de Recherche du Centre Hospitalier Universitaire de Montreal, Montreal, Canada, ${ }^{5}$ Medicine, Hôpital de Verdun, Montreal, Canada, ${ }^{6}$ Medecine/Université de Sherbrooke, Sherbrooke, Canada, ${ }^{7}$ Médecine Familiale/Université de Sherbrooke, Sherbrooke, Canada, ${ }^{8}$ Orthopedie/Université de Sherbrooke, Sherbrooke, Canada, ${ }^{9}$ Orthopedie/Université de Montreal, Montreal, Canada, ${ }^{10}$ Research Institute of the McGill University Health Center, Montreal, Canada, ${ }^{11}$ Osteoporosis Canada, Halifax, Canada, ${ }^{12}$ Médecine Familiale/ Université de Sherbrooke, Sherbrooke, Canada

Objective: We aim to share the Province of Quebec (Canada) collaborative experience in post-fracture management programs (fracture liaison services) and to propose an approach towards setting the foundations for a coordinated provincial evidence-based strategy in secondary fracture prevention.

Methods: We obtained peer-reviewed funding to hold a one-day meeting (February 12th 2016) where we will bring together primary care and specialist clinicians and trainees, patient representatives and Osteoporosis Canada Fracture Liaison Service Chief Medical Officer as well as key experts and policy makers from the Institut national de santé publique du Québec (INSPQ), Institut national d'excellence en santé et en services sociaux (INESSS) and the Ministry of Health (MSSS) to review post fracture interventions currently in place in Quebec and develop a minimum common dataset.

Results: We will characterize and compare the organizational structures, processes of care and data collection systems in the 4 largest secondary fracture prevention management programs in Quebec: a) OPTIMUS- OPTI-FRAC, Sherbrooke b) Lucky Bone ${ }^{\mathrm{TM}}$, Hôpitaux Sacré- Coeur and Jean Talon, Montreal c) Programme régional de soins post-fracture de fragilisation CSSS Sud-Ouest Verdun, Montreal and d) One Day One Site Post-Fracture Integrated Service, Quebec City. We will present consensus on a minimum set of data to be collected as part of secondary fracture prevention programs and of quality of care indicators to evaluate the performance of these programs and discuss next steps in a provincial strategy. We will discuss the launch of a working group whose overarching mandate would 
be to elaborate a provincial health services delivery strategy, to close the "know-do" care gap in secondary fracture prevention. Conclusion: A provincial comprehensive evidence-based approach to secondary fracture prevention that includes not only investigation and anti-osteoporosis treatment but also fall prevention programs, will hopefully lead to patient empowerment to improve adherence to effective interventions, improved primary care knowledge about osteoporosis and fracture prevention, reduced rates of falls and fractures and be the foundation for the delivery of appropriate, accessible, efficient and cost-effective care.

\section{P403}

\section{ANTI-OSTEOPOROTIC CONSTITUENTS IN TRADITIONAL ROMANIAN MEDICINAL PLANTS}

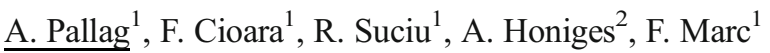

${ }^{1}$ University of Oradea, Faculty of Medicine and Pharmacy, Oradea, Romania, ${ }^{2}$ Vasile Goldis Western University of Arad, Arad, Romania

Objective: The phytotherapy in osteoporosis treatment focuses on the use of plants with estrogenic properties that compensate for the loss of estrogen and plants to helps provide more calcium to the bones or help the body to produce the principles that help calcium absorption. Through the traditional Romanian medicinal plants there are many species which has proven anti-osteoporotic action.

Material and methods: We made a study concerning the knowledge of people about the anti-osteoporotic and osteoporosis preventive effects of some traditional Romanian medicinal plants. The study was made in 2014 and 2015, with 364 people involved, who agreed to complete a questionnaire. The distribution of the respondents;

- Gender: Male -169, Female -195

- Location: urban -188 , rural-176

- Age: 24-68

The questions of the questionnaire made reference to the following traditional Romanian medicinal plants: Urtica dioica (stinging nettle), Juglans regia (walnut), Equisetum arvense (horsentail), Achillea millefolium (milfoil), Petroselinum crispum (parsley), Medicago sativa (alfalfa), Hippophäe rhamnoides (sea-buckthom).

Results: Our results show that the majority of the respondents don't know about the anti-osteoporotic and osteoporosis preventive effects of these medicinal plants. The most known and used plants are: Achillea millefolium (milfoil) (46\%), Equisetum arvense (horsentail) (41\%), Urtica dioica (stinging nettle) (33\%) si Hippophäe rhamnoides (sea-buckthorn) (31\%). Just a small percent of the respondents use: Petroselinum crispum (parsley) (11\%), Medicago sativa (alfalfa) (10\%), Juglans regia (walnut) (7\%). More women than men know about these plants and their effects on human organism, but there is no difference between rural or urban provenience of the participants

Conclusions: It turned out that many traditional medicinal plants have anti-osteoporotic effect.

Phytotherapy in osteoporosis treatment is very important due to the absence of secondary effects and can be used simultaneously with drug therapy.

\section{P404}

THE IMPACT OF THE BALANCE DISORDERS ON THE COMPLEX RECOVERY FOR OSTEOPOROSIS

L. Vicas ${ }^{1}$, F. Cioara ${ }^{1}$, C. Nistor Cseppento ${ }^{1}$

${ }^{1}$ University of Oradea, Faculty of Medicine and Pharmacy, Oradea, Romania

Introduction: Osteoporosis is a major disease characterized by reduced bone mass and microarchitecture destruction leading to decreased bone strength and increased risk of fracture. In this condition there are some required physical exercises which determine pressure load bone structures and rhythmic muscle contractions. The association of several diseases requires adapting the kinetotherapy. Elder predisposition to falls is increased by adding more factors such as decreased visual acuity, sarcopenia, vestibular syndrome, neurological disorders, orthopedic etc.

Objective: To identify patients at risk for falling, and the impact of associated disorders, particularly those manifested by balance disorder on the recovery of patients with osteoporosis. In this work we also evaluated the effectiveness of physical therapy program tailored to the clinical aspects mentioned and tried to define the difficulties in achieving physical therapy programs.

Material: We used a lot of 56 female patients diagnosed with primary osteoporosis, type I. Age was between 57-67 years. The pathology associated with osteoporosis was marked by peripheral vestibular syndrome, sensory ataxia, vertebrobasilar circulatory failure etc. The monitoring was conducted over a period of 4 years.

Methods: Patients were treated based on specific balneokinetotherapy means kinetic and Felix spa resort in Romania, alongside with drug therapy based on bisphosphonates, vitamin D3, calcium and the specific treatments for the associated 
conditions. The treatment options are aimed at reducing the risk of falls and fractures, through action on health-related and environmental factors that can be influenced. The program aimed at lifestyle changes and to adopt a personalized physical therapy program. Balance and coordination exercises aimed at improving gait and balance, reducing vertigo and enhancing physical activity in general. We evaluated the quality of life by applying Qualeffo-41 standardized questionnaire and by applying a satisfaction questionnaire to the patients. We also test the lower limb functionality (Tandem Standing, Up \& Go, Chair Rising Test). We evaluated the stability, balance and coordination (Berg test) and pain. For quantitative comparison we used the Student $t$-test for comparing the average values of variables and Pearson correlation coefficient for calculating correlations between the rows of values of quantitative variables.

Results: The results evaluated before and after monitoring showed improvement that reach the limit of statistical significance $(p<=0.05)$ at physical performance in patients with high compliance to the program, but without balance disorders. Patients with balance disorders had only temporary relief. Assessment of quality of life is different during monitoring developments with or without improvement is related directly to practicing rhythmic exercise. There is a greater responsiveness and efficiency and for the elderly, proving the undeniable value of complex functional recovery as the possibility of maintaining, strengthening or developing the lack of functionality. Conclusions: Adherence to treatment depends on a prophylaxis and therapy professionals with a full staffed team. Exercises, in particular loading and resistance exercises have a beneficial effect on quality of life. It contributes along with other factors in aggravation prevention of osteoporosis and fall prevention, particularly in elderly women. Marked physical limitations require physical therapy programs different from traditional ones. Oligo-mineral spa external treatment influences thermal reactivity and body condition and the patient perceive a positive well-being due to natural healing factors and contribute to reaching the goals of the proposed therapeutic schema.

\section{P405}

\section{ASSESSMENT OF THE FUNCTIONAL STATUS OF THE KNEE JOINT OF A PATIENT WITH OSTEOARTHRITIS}

\section{S. Kevic $^{1}$, K. Boskovic ${ }^{2}$, S. Todorovic-Tomasevic ${ }^{1}$}

${ }^{1}$ Clinic for Medical Rehabilitation and Physical Medicine, Clinical Center of Vojvodina, Serbia, Novi Sad, Serbia, ${ }^{2}$ Clinic for Medical Rehabilitation and Physical Medicine, Clinical Center of Vojvodina, Novi Sad, Serbia
Introduction: Chronic degenerative disease that is characterized by pain, swelling, limitation of movement of knee and audible crepitus. Activities of daily living with the limitations and difficulty walking. The specific structure of the cartilage allows the knee "depreciation".

Aim: To assess the functional status of patients with knee osteoarthritis to a before and after therapy.

Material and Methods: The study included 120 patients with knee osteoarthritis, whose established diagnosis was based on ACR criteria. All patients were evaluated by orthopedist and physiatrist / rheumatologist, and all were handled by a single criterion. The average age was 74 years, $70 \%$ were women. The degree of radiological change is estimated to Kellgren-Lawrence classification. $70 \%$ of patients had radiological changes of the third degree and $30 \%$ of second degree. Treatment of patients included i.a. application of hyaluronic acid and then conducting physical procedures. The degree of pain was evaluated with VAS. Functional status was evaluated according to the Oxford Knee Score and the T.Lysholm Knee Scoring Scale before and after therapy.

Results: The mean value for pain before treatment was 8 and 4 after treatment. The mean value of the functional status according to the Oxford score was 22 before treatment and after treatment 38 . The mean value of the functional status according T.Lysholm score was 43 before treatment and after treatment 76.

Conclusion: The significant increase in functional status knee can be expected after application i.a. hyaluronic acid and physiotherapy.

\section{P406 \\ HEIGHT LOSS STARTING IN MIDDLE AGE PREDICTS INCREASED FRACTURE RISK IN ELDERLY MEN AND WOMEN: HIRIOSHIMA COHORT STUDY}

S. Fujiwara $^{1}$, M. Masunari ${ }^{2}$, I. Takahashi ${ }^{3}$, W. Ohishi ${ }^{3}$

${ }^{1}$ Hiroshima Atomic Bomb Casualty Council, Hiroshima, Japan, ${ }^{2}$ Department Public Health, Yamaguchi Prefectural University, Yamaguchi, Japan, ${ }^{3}$ Department Clinical Studies, Radiation Effects Research Foundation (RERF), Hiroshima, Japan

Objective: Our previous reports demonstrated that height loss significantly affected quality of life (QOL) (Osteoporos Int 
2007;18:1493) and mortality independent of vertebral fracture (VF) in the elderly (JBMR 2013;28:179). The objective of this study was to determine fracture risk among Japanese elderly men and women with height loss starting in middle age, taking into account BMD and prevalent VF.

Methods: From among a population-based community cohort, 2498 subjects (755 men and 1743 women) aged 47-91 years old were enrolled in a prospective and longitudinal cohort study for osteoporosis in Hiroshima, Japan. Since 1958, the cohort members have been followed through biennial health examinations including physical examination, measurements of height and weight, and chest X-ray exam. These individuals underwent spinal X-ray and BMD measurement in addition to usual physical exam during the period 1994-95, and were followed for incident fracture through 2007. Incident VFs were identified from lateral chest radiographs, and incident nonvertebral fragility fractures were collected using interviews by trained nurses and physicians during the health exams. Cox hazard model was used for analyses.

Results: There were 146 incident VFs, 29 hip fractures, and 142 non-spine fractures during the follow-up period. Height loss starting in middle age predicted VFs, and the hazard ration of height loss per $1 \mathrm{~cm}$ was 1.10 (95\%CI 1.04-1.15). Height loss was not significantly associated with hip fracture risk and nonspine fracture risk. Those with at least $3 \mathrm{~cm}$ of height loss had twice as high risk of VFs $(H R=2.17,95 \%$ CI 1.54-3.06), after adjustment was made for age, sex, atomic-bomb radiation exposure, and BMD. Height loss at least $3 \mathrm{~cm}$ was also significantly associated with VF risk $(H R=1,720(95 \% \mathrm{CI} 1.21-2.45$, even after adjusting for prevalent $\mathrm{VF}$.

Conclusions: Height loss starting in middle age predicted incident vertebral fracture, even after adjusting for prevalent vertebral fracture. Height loss is a good predictor for future vertebral fracture.

Acknowledgments: This study was supported by the research funding for Longevity Sciences from the Ministry of Health and Welfare (LOCOMO Study. H25-Longevity SciencesGeneral-007).

\section{P407}

THE HISTOLOGICAL EFFECTS OF TERIPARATIDE, DENOSUMAB AND SWITCHING FROM TERIPARATIDE TO DENOSUMAB ON BONE METABOLISM

T. Omiya ${ }^{1}$, J. Hirose ${ }^{1}$, S. Tanaka ${ }^{1}$

${ }^{1}$ Department of Orthopaedic Surgery, The University of Tokyo, Tokyo, Japan

Objective: We examined the effects of teriparatide and switching from teriparatide to anti-RANKL monoclonal antibody in ovariectomized mice to compare the differences of $\mathrm{BMD}$ and histological findings.
Material and Methods: Twelve-week-old female C57BL/6 mice were ovariectomized or sham operated (SHAM group). Four weeks after the surgeries, the ovariectomized mice were subjected to one of the following four treatment options; PBS for 8 weeks (OVX group), PTH for 4 weeks followed by PBS for the next 4 weeks (PTH4W group), PTH for 8 weeks (PTH8W group) and PTH 4 weeks followed by anti-RANKL antibody (single injection of $5 \mathrm{mg} / \mathrm{kg}$ ) (SWITCH group). All mice were sacrificed 12 weeks after the operation. BMD was measured at the tibia, femur and lumbar spine. Hind limbs and lumbar spines were subjected to histological and histomorphometric analysis.

Results: BMD increased similarly in PTH8W and SWITCH groups, while BMD in PTH4W group was markedly decreased after discontinuation of the treatment. Histomorphometric analysis demonstrated that both bone formation and resorption increased extremely in PTH8W group and were suppressed in SWITCH group in cancellous bone. In contrast, bone formation increased on the periosteal surface in PTH8W group and SWITCH group, while that in the endocortical surface was suppressed in SWITCH group.

Conclusion: Anti-RANKL antibody is a good therapeutic option after discontinuation of teriparatide therapy, in particular for cortical bones.

\section{P408}

ASSOCIATION BETWEEN BONE MINERAL DENSITY AND URINARY INCONTINENCE IN ELDERLY KOREAN WOMEN

J.-S. Huh $^{1}$, J.-H. Moon ${ }^{2}$, H.-J. Kim ${ }^{3}$

${ }^{1}$ Department of Urology, Jeju National University \& Hosipital, Jeju, Republic of Korea, ${ }^{2}$ Jeju National University Hospital, Jeju, Republic of Korea, ${ }^{3}$ Family Medicine, Jeju National Universitiy \& Hospital, Jeju, Republic of Korea

Objective: Urinary incontinence and impaired bone health are important senior health problems. In this study, we evaluated the impact of urinary incontinence on BMD in elderly Korean women.

Material and Methods: Our study used data from the Korea National Health and Nutrition Examination Survey from 2008-2009. Total 901 women aged 65 yr or older were included. BMD was measured by DXA. Lumbar spine (L 1-4) and proximal femur BMD were obtained. The presence of impaired bone health was defined by the calculation of a lumbar spine, femoral neck or total femur T-score $<-1.0 \mathrm{SD}$. Each participant's incontinence status was collected using constructed questionnaires. 
Results: The prevalence of self-reported urinary incontinence in individuals aged over 65 women was $9.7 \%$. After adjusting for age, sex, height, weight, smoking status, alcohol consumption, frequency of exercise, activity limitation, experience of hormone therapy and marital status, the odds ratios for impaired bone health of total femur, femoral neck and lumbar spine were $1.27(0.70-2.29), 1.64(0.51-5.28), 1.69$ (0.654.37), respectively.

Conclusion: This study found no association between urinary incontinence and impaired bone health in elderly Korean women.

\section{P409}

RELATIONSHIP OF LIPID PARAMETERS WITH BONE MINERAL DENSITY IN POSTMENOPAUSAL WOMEN.

K. Baccouche $^{1}$, S. Belghali ${ }^{1}$, N. Elamri ${ }^{1}$, Z. Alaya ${ }^{1}, \mathrm{H}$. Zeglaoui $^{1}$, E. Bouajina ${ }^{1}$

${ }^{1}$ Rheumatology, Farhat Hached Hospital, Sousse, Tunisia

Introduction: Cardiovascular disease and osteoporosis share common risk factors including dyslipidemia. There are conflicting reports of differential relation of various lipid parameters on BMD in postmenopausal women. Hence, we studied the correlation between lipid parameters and BMD in postmenopausal women.

Methods: A total of 113 postmenopausal women included in this cross-sectional study were divided into two groups: control group (BMD: normal) and osteoporosis group. Fasting blood samples were drawn for biochemical parameters: total cholesterol (TC), low-density lipoprotein cholesterol (LDL-C), high- density lipoprotein cholesterol (HDL-C), triglyceride (TG), apoprotein B and lipoprotein (a). BMD at lumbar spine and femur were measured by DXA.

Results: The presence of dyslipidemia was significantly higher in the group with osteoporosis than normal BMD $(p=0.004)$. After adjustment for age, multivariate regression analysis revealed that the risk of osteoporosis persists and even increases with cases of dyslipidemia. Indeed, $p$ decreases from 0.009 to 0.006 and the relative risk increases from 1.7 to 2.3. However, student's $t$-test revealed no significant difference between the two groups, osteoporosis and normal BMD, in serum concentrations of the TC, TG, HDL, LDL, lipoprotein or for the atherogenic index. However, the atherogenic index, and the average concentrations of LDL -C, apoprotein B and lipoprotein (a) appear to be higher in the group of osteoporotic women, but the difference is not significant.

Conclusion: Our study may exhibit an atherogenic profile associated with osteoporosis but other larger studies are needed to confirm this hypothesis.

\section{P410}

WHOLE BODY AND REGIONAL AREA, BONE MINERAL CONTENT AND DENSITY IN BULGARIAN WOMEN OF DIFFERENT AGES

M. Boyanov ${ }^{1}$

${ }^{1}$ Department Internal Medicine, Medical University Sofia, Alexandrovska University Hospital, Endocrinology Clinic, Sofia, Bulgaria

Up-to-date DXA offers an insight into bone mineral content (BMC) and density (BMD) in different body regions.

Objectives: To measure the regional BMC and BMD in women of different age and to describe age-related changes.

Material and Methods: 120 women referred for DXA participated in the whole body study - age range was $20-75$ yrs. They were subdivided in three age groups: $20-44$ (20 premenopausal women), 45-59 (80 postmenopausal women), and 60-75 (20 women). Whole body (WB) DXA was performed on a Hologic QDR 4500 A bone densitometer (Hologic Inc., Bedford MA). Total body area, BMC and BMD were measured. Regional analysis included arm, rib, legs and pelvis area and BMC (as the sum of both left and right), as well as BMD (BMC divided by the area). Descriptive and regression analyses with age as independent variable were performed on an IBM SPSS Statistics 19.0 for Windows platform. The data were first analyzed as a whole and then separately for the different age groups.

Results: WB area decreased slightly with age while BMC and BMD showed more pronounced changes. WB BMC was $2163 \pm 393 \mathrm{~g}$ in the $20-44 \mathrm{yr}$ group; $2023 \pm 325 \mathrm{~g}$ in the $45-$ $59 \mathrm{yr}$ group and $1819 \pm 335 \mathrm{~g}$ in the $60-75 \mathrm{yr}$ group. Arms BMC was by approximately $15 \%$ lower in age group $60-75$ compared to $20-44 \mathrm{yr}$; while ribs BMC - by $14 \%$, leg BMC by $12.5 \%$. As both BMC and area decreased with age, BMD changes were more subtle. Best fitting regression models with age as independent variable are shown.

Conclusion: Aging has the most pronounced effect on WB and regional $\mathrm{BMC}$, followed by regional area and BMD. This type of data could be useful in the study of sarcopenia.

\section{P411}

\section{USE OF TERIPARATIDE IN NONUNION HEALING: BRIEF REPORT}

J. Palencia $^{1}$, R. Zahid ${ }^{1}$, F. Serro ${ }^{1}$, J. Fakehaa ${ }^{1}$

${ }^{1}$ Orthopedic Department, King Saud Medical City, Riyadh, Saudi Arabia

Introduction: 5-10\% of bone fractures does not heal spontaneously and need treatment. The nonhealing $(27-34 \%)$ of segmental fractures leads to high rates of disability (40-50\%). Allograft healing procedures for fractures are associated with high complications rate namely late graft fracture (24\%- 
$27 \%$ ), and infection (9\%-16\%). Human PTH stimulates osteoblasts and reduces osteoblast apoptosis. Intermittent administration of human PTH increases callus formation and improves mechanical strength. It has proven to increase skeletal bone mass in osteoporotic patients.

Literature review: Research on human PTH is conducted mainly on animal models. A recent study from US has reported that delayed short treatment with teriparatide in rats enhances intramembranous bone formation at the graft-host junction and therefore improves femoral allograft healing. There are several case reports among humans. A study from Japan has reported 2 cases of ulnar fracture in smokers who had nonunion after ulnar shortening osteotomy. Both were treated with Teriparatide in addition to low-intensity pulsed ultrasound. At follow-up after 10 and 6 months of treatment with Teriparatide, both patients had successful bone healing without additional surgical interventions. Teriparatide and low-intensity pulsed ultrasound are presented as a possible alternative to surgical intervention particularly among smokers who have a higher incidence of nonunion (30\%). Another report from UK in 21-year-old female with extended oligoarticular JIA diagnosed at age of 8 years who also sustained a low-impact short oblique closed fracture of her right lower tibia. She was treated with Teriparatide and later zoledronic acid. She started showing signs of healing within 12 months period. But authors have advised to interpret the results with caution. A case report from Italy reports successful callus formation and healing of traumatic fractures of lower limb with teriparatide in 4 patients who had undergone open fixation.

Summary: The effect of human PTH on callus formation and its potential role in nonunion of fractures cannot be doubted. But we have to interpret data from animal models with great caution. We need RCT comparing gold standard procedures before we can replace surgical options in the treatment of fracture with teriparatide.

\section{P412}

\section{DISTAL FEMORAL OSTEONECROSIS TREATMENT WITH ALLOGRAFT}

J. Palencia ${ }^{1}$, R. Zahid ${ }^{1}$, F. Serro ${ }^{1}$, J. Fakehaa ${ }^{1}$

${ }^{1}$ Orthopedic Department, King Saud Medical City, Riyadh, Saudi Arabia

Introduction and Objectives: In this study we show the treatment of distal femoral osteonecrosis with allograft, its evolution and the indications of this technique. Distal femoral osteonecrosis has a low prevalence. Its origin is not well defined. The most accepted hypothesis is traumatic causes, though a known traumatic episode is only referred in half of the cases. It affects mainly teenagers and young people, more frequently males, 2 or 4 times more than females, and 2 over 3 cases are on internal condyle.
Materials and Methods: 37 distal femoral osteonecrosis have been treated surgically along the last 10 years in our Hospital. We present 3 cases of distal femoral osteonecrosis treated with allograft. All patients were males, and the affected side was right. They complained from low pain of mechanic characteristics, affecting mainly the internal compartment, hydrarthrose and pseudoblocking. Classical signs of Axhausen and Troel, as well as Wilson sign were positive. X-ray and MRI status corresponded to Bedouelle IV. Operation took place under intradural anesthesia and limb ischemia. The operative technique was arthrotomy, cleaning and refreshment of affected surfaces, and insertion of the graft, fixed with resorbable material. The remaining 34 cases were treated with autograft and are not the objective of our present study. Patients used after operation a flexo-extension controlled orthosis, and started early recovering exercises. Limb weight bearing was allowed after 3 months. Follow-up was 2 years, with X-ray controls at 4,8 , and 12 weeks, and X-ray and magnetic resonance every year.

Results:

- Evolution was satisfactory, with full integration of the graft in X-ray and MRI.

- Patients improved clinically and achieved total passive and active movement of the limb.

- We did not found significant differences with the evolution of patients treated with autograph during these two years of follow up.

\section{Discussion and Conclusions:}

1. This technique must be used in young people with advanced and extended affectation in Bedouelle IV status, in which total knee replacement is not indicated.

2. This is a simple technique to perform.

3. X-ray and magnetic resonance studies show good integration of the allograft, though two years may not be enough follow up time to evaluate total integration of the graft.

4. Other authors report around $75 \%$ of good results with this technique.

\section{P413}

\section{AMBIENT AIR POLLUTION AS A MAJOR BONE HEALTH THREAT}

A. Hossein-Nezhad $^{1}$, Z. Maghbooli ${ }^{1}$, H. Adibi ${ }^{1}$, M. Ramezani $^{1}$, F. Zare ${ }^{1}$, C. Gorgani ${ }^{1}$, B. Larijani ${ }^{1}$

${ }^{1}$ Osteoporosis Research Center, Endocrinology and Metabolism Clinical Sciences Institute, Tehran University of Medical Sciences, Tehran, Islamic Republic of Iran

This population-based retrospective cohort study conducted to evaluate the association between ambient air pollution and BMD as well as risk of osteoporosis in Tehran residents exposed to air pollution. DXA results of the total hip and lumbar spine and clinical data were obtained from 
participants that referred to an outpatient bone clinic affiliated to osteoporosis research center of Tehran University. The study subjects were 6308 subjects (514 men) aged 40-100 years. Any chronic illness like auto immune disorders, cancers, neurological diseases and any conditions that under treatment with corticosteroids were excluded. Detailed daily data on air pollution were obtained from archive data that collected by Tehran Air Quality Control Company (AQCC) from 2014 to 2015. The yearly average concentrations of air pollutants were categorized into quartiles, and BMD, T-score, Z-score and the risks of osteoporosis were evaluated among 4 stages of air pollutants (Q1-Q4). In women, AQI and air pollutants exposure were positively and significantly associated with risk of osteoporosis. After adjustment for BMI and menopause status in women, BMD and related $\mathrm{T}$ and $\mathrm{Z}$ scores in $\mathrm{Q} 4$ were compared with Q1. There were significant negative association between Air Quality Index (AQI), carbon monoxide (CO), PM2.5 and PM10 with lumbar spine BMD, T-score and Z-sore. Similar association detected between nitrogen dioxide (NO2) with total hip BMD, T-score and Z-sore. Ozone (O3) adversely affects both lumbar spine and hip BMD. Air pollution is one of the most serious health hazard and our findings show that ambient air pollution exposure is associated with an increased risk of osteoporosis in the Tehran population.

\section{P414}

BONE MINERAL DENSITY IN ADOLESCENT AND YOUNG ADULTS WITH CHRONIC KIDNEY DISEASE TREATED WITH HEMODIALYSIS

$\underline{\text { S. O. Mazurenko }}^{1}$, R. K. Kuanshkaliev², A. A. Enkin ${ }^{3}$, O. Mazurenko $^{4}$

${ }^{1}$ Saint Petersburg State University, Saint Petersburg, Russian Federation, ${ }^{2}$ Saint Petersburg Pediatric Hospital \# 1, Saint Petersburg, Russian Federation, ${ }^{3}$ Dialysis Center of Leningrad Regional Hospital, Saint Petersburg, Russian Federation, ${ }^{4}$ Saint Petersburg Hospital named after L.G. Sokolov, Saint Petersburg, Russian Federation

Osteoporosis, pathological fractures, growth retardation, and skeletal deformities occur occasionally in children with end stage renal disease. This study was performed to assess the prevalence and severity of low BMD and growth retardation in 42 young adults and adolescent dialysis patients ( 23 females and 19 males) aged 14-25 years (mean age 19.9 \pm 3.85$)$ with duration of hemodialysis from 1-12 years (mean $4.9 \pm 3.5$ ). BMD of the lumbar spine (L1 - L4) was measured using DXA by Hologic Discovery W. Height and weight were recorded, and BMI calculated. Bone age was assessed by analysis of wrist $\mathrm{x}$-rays films.
Laboratory investigations included intact PTH, serum total and ionized calcium, serum phosphate, osteocalcin, alkaline phosphatase. Mean BMD corrected for gender and age (Z-score) of the lumbar spine was $-2.01 \pm 1.6 .59 .5 \% \mathrm{had}$ osteopenia or osteoporosis. $45.2 \%$ had growth retardation. Growth retardation and a low lumbar BMD correlated significantly $(p<0.001)$ with age at start of hemodialysis and pubertal status, duration of hemodialysis and weight of the patients $(p<0.001)$.

Laboratory results did not correlate significantly with BMD and skeletal status. Fracture risk was not influenced significantly by bone age and BMD. Our results indicated that end stage renal disease and hemodialysis are associated with serious skeletal abnormalities in adolescent and young adults.

\section{P415}

\section{SARCOPENIA AND OSTEOPOROSIS IN HONG KONG CHINESE GERIATRIC HIP FRACTURE PATIENT}

A. W. H. Ho $^{1}$, M. L. Lee ${ }^{1}$, W. C. Chan ${ }^{1}$, M. Y. $\mathrm{Ng}^{1}$, C. W.

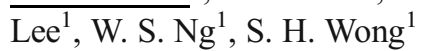

${ }^{1}$ Caritas Medical Centre, Hong Kong, Hong Kong

Introduction: Sarcopenia and osteoporosis are age-related declines in the quantity of muscle and bone respectively. Both contribute in disability, fall and hip fracture in elderly.

Methods: We included all geriatric primary hip fracture patients admitted to Kowloon West Cluster Orthopaedic Rehabilitation Centre. Hand grip strength and body composition measurement using DXA were performed.

Results: There were 356 patients with mean age 81.7. By stratifying in male and female, the mean hand grip strength were 20.8 and $13.7 \mathrm{~kg}$, the mean Relative Skeletal Muscle Mass Index (RASM) were 5.79 and $4.90 \mathrm{~kg} / \mathrm{m}^{2}$, the hip BMD were 0.699 and $0.992 \mathrm{~g} / \mathrm{cm}^{2}$. The prevalence of sarcopenia based on RASM according to the Asian Working Group for Sarcopenia (AWGS) definition were $89.5 \%$ in male and $76 \%$ in female. The prevalence of femoral neck osteoporosis based on hip T-score less than -2.5 were $43.4 \%$ in male and $63.6 \%$ in female. RASM was positively correlated with hand grip strength, body weight, femur T-score, hip BMD, BMI and total fat mass in both male and female. All were statistically significant.

Discussion and Conclusion: The prevalence of sarcopenia was very high in geriatric hip fracture patients, much higher than community dwelling elderly. Apart from treatment of osteoporosis, sarcopenia screening and treatment should be addressed, which is essential to reduce subsequent fall, subsequent fracture and the fracture-related complications and economic burden to Hong Kong. 


\section{P416}

PRIMARY HYPOTHYROIDISM IN YOUNG PATIENTS WITH ALBRIGHT'S HEREDITARY OSTEODYSTROPHY

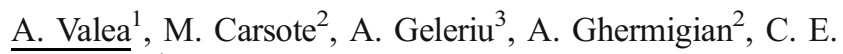
Georgescu $^{1}$

${ }^{1}$ I. Hatieganu University of Medicine and Pharmacy \& Clinical County Hospital, Cluj-Napoca, Romania, ${ }^{2}$ C.I. Parhon National Institute of Endocrinology, Bucharest, Romania, ${ }^{3}$ Queen Mary Center, Bucharest, Romania

Introduction: Albright's hereditary osteodystrophy (AHO) is caused by resistance to PTH action due to a genetic epigenetic imprinting which affects Gs alpha protein. Despite the inherited autosomal dominant pattern the diagnosis is sometimes delayed and complications as hypocalcemia-related seizures or teeth anomalies are registered. Primary hypothyroidism $(\mathrm{PH})$ might be included in some phenotypes.

Material and Methods: We introduce two cases of AHO associating PH who were first recognized around pubertal years and followed-up in different medical centers. The bone metabolism was evaluated as well as dental examination. The informed consent was obtained.

Case 1: A 20-year female was admitted for short stature (of $1.36 \mathrm{~m}$ ). The phenotype revealed rounded face, bilateral brachydactylic, abnormal implant of left premolars, positive tetany tests. Lab assays confirmed mild PH in association with low calcium levels and high PTH. AHO was diagnosed and therapy with calcium and vitamin D was started as well as daily morning levothyroxine (LT4). She was periodically checked-up and normal levels of calcium were obtained due to daily supplements with persistent mildly elevated PTH values.

Case 2: A 12-year male is admitted for repeated seizures since early childhood with persistent hypocalcemia. The endocrine exam revealed chronic asthenia, dry skin, lateral incisors disturbances. Blood tests confirmed high PTH and TSH levels establishing the diagnosis of OHD and PH. LT4 and calcium/ $\mathrm{D}$ vitamin supplements were started as well as anti-epileptic drugs. He was followed-up but both endocrinologist and pediatrician but he proved a relatively low compliance at therapy without the normalization of calcium levels, neither PTH (of $324.7 \mathrm{pg} / \mathrm{mL}$ at age of $15 \mathrm{yrs} ; \mathrm{N}: 15-65 \mathrm{pg} / \mathrm{dL}$ ). Thyroid assessment showed adequate LT4 substitution (free T4 of $16.73 \mathrm{pmol} / \mathrm{L}$; N: $12-22 \mathrm{pmol} / \mathrm{L}$, TSH of $7.1 \mu \mathrm{UI} / \mathrm{mL} ; \mathrm{N}$ : $0.28-4.3 \mu \mathrm{UI} / \mathrm{mL}$, at age of 15 ).

Conclusions: Albright's hereditary osteodystrophy represents a rare condition with a delayed diagnosis in some situations despite highly suggestive phenotype based on the association between seizures, nanism, and symptomatic tetany. Primary hypothyroidism, although not severe in most cases, may complicate the general picture by influencing the stature and the mental development.

\section{P417}

BEHIND LUMBAR PAIN: THE ROLE OF BONE SCINTIGRAM IN FOLLOWING-UP SEVERE IDIOPATHIC MALE OSTEOPOROSIS WITH PERSISTENT THROMBOCYTOPENIA

A. Ghemigian $^{1}$, M. Carsote ${ }^{1}$, D. Terzea ${ }^{2}$, A. Mazilu ${ }^{3}$, A. Goldstein $^{4}$, A. Dumitrascu ${ }^{4}$, A. Valea ${ }^{5}$

${ }^{1}$ C.I. Parhon National Institute of Endocrinology, Bucharest, Romania, ${ }^{2}$ C.I.Parhon National Institute of Endocrinology \& Monza Oncoteam, Bucharest, Romania, ${ }^{3}$ Military Central Hospital, Bucharest, Romania, ${ }^{4}$ C.I.Parhon National Institute of Endocrinology, Bucharest, Romania, ${ }^{5}$ I. Hatieganu University of Medicine and Pharmacy \& Clinical County Hospital, Cluj-Napoca, Romania

Introduction: Male osteoporosis, although less frequent than menopause-related bone loss, is caused by many known mechanisms; yet, the idiopathic type needs to be considered in some situations. A recent lumbar pain of an already treated patient may be associated with a trauma, a new vertebral fracture, chronic spondylo-arthritis or even a bone metastasis of a prior unknown cancer.

Material and Method: We introduce a male case with a history of idiopathic osteoporosis currently under anabolic medication. We performed central DXA (GE Lunar Prodigy) to have the BMD providing Z-score. The informed consent was signed by the patient.

Case data: A 64-year nonsmoking male was diagnosed with severe lumbar fractures without an evident trigger and neurosurgery was performed. Normal gonad and adrenal axes were found. As risk factor we mention potential alcohol consumption (although the patient denied it) based on persistent high hepatic enzymes with negative liver antibodies and viral tests. Low normal levels of circulating plates had no obvious cause at the moment. A local bone tumor was suspected but the pathological report after surgery excluded it. L3-4 DXA assay showed a Z-score of $-3.8 \mathrm{SD}$. The patient was offered for a year weekly risendronate and vitamin $\mathrm{D} /$ calcium supplements. Despite the L3-4 Z-score increase to -3SD a new thoracic vertebra fracture was registered and the therapy was switched to $20 \mu \mathrm{g}$ SC daily teriparatide. A hepatic and hematological assessment was also done since liver enzymes were twice as normal and the number of plates decreased to $124.000 / \mathrm{mmc}$ (normal $>150.000$ ) but no diagnosis was ruled. 6 months later the patient accused again intense lumbar pain without evidence of a trauma. The whole body bone scintigram (99mTC-HDP, $20 \mathrm{mCi}$ ) showed no new fractures (moderate uptake of the tracer at the level of different articulations consistent with a degenerative aspect, and a T7 osteoporotic fracture which was already registered 6 months before). No malignancy was suspected after performing a computed tomography scan and idiopathic thrombocytopenia was considered after gastroenterological examination. 
Conclusion: Idiopathic severe male osteoporosis in association with persistent thrombocytopenia might raise the question of a secondary cause (of liver or oncologic origin). However, once the safety profile of the patient is achieved, the decision of anabolic anti-osteoporosis therapy may be done.

\section{P418}

\section{DEFICIT OF ESTROGEN IN MENOPAUSE AND OSTEOPOROSIS}

T. Petrusic $^{1}$

${ }^{1}$ Health Centre, Nis, Serbia

Background: Postmenopausal osteoporosis develops in women due to a decrease in the hormone estrogen and the loss of its protective functions in the bones. In Serbia suffers from osteoporosis 375000 women, and only $30 \%$ of that number are curing. Every fifth woman after $50 \mathrm{y}$ of life gain one or more fractures. One third of menopausal women in the world suffer from osteoporosis, and the optimal time to prevent osteoporosis is the first control a year since the start of menopause.

Aim: To display the percentage of representation of osteoporosis due to prolonged lack of the hormone estrogen in postmenopausal women.

Methods: Measurements of BMD in 516 postmenopausal women (mean age 67) in the period from 15.9.2015 to 31.12.2015god, in the department of Physical Medicine and Rehabilitation Health Center in Nis. The apparatus sonost 3000 osteodensitometer.

Results: The parameter measurements of BMD at the calcaneus of the left foot of the patients with risk factors estrogen deficit T-score, for statistical processing of the results was determined by Fisher test (Fisher's exact probability). Osteoporosis is diagnosed in $121(23.45 \%)$ patients with T-score of over $-2.5 \mathrm{SD}$. Osteopenia Tscore of -1 to $-2.5 \mathrm{SD}$ had 168 (32.56\%) women. Normal finding with the measured T-score minor revamp of -1 SD had 227 (43.99 \%) women. Number of patients with osteoporosis compared to the total number of respondents statistically significant $(p<0.005)$ and statistically significant $(p<0.001)$ for those with osteopenia.

Conclusion: Osteoporosis is a disease of old people who frequent in women than men. For it was largely to blame the deficit of the female sex hormone estrogen at menopause. Appears 5 to 15 years after the last menstrual period. It is estimated that about $25 \%$ of menopausal women have osteoporosis, and $15 \%$ of them experience a dangerous fractures of the femoral neck which is the main cause of high morbidity and mortality assembly osteoporosis. The pronounced increase in bone resorption in women is directly related to the lack of estrogen, but not with age women. Fact that women 50 and 70 years have the same bone density when they postmenopausal equal length, convincingly shows that menopause determines the structure of the skeleton woman.

\section{P419}

\section{CAUSES OF SENILE OSTEOPOROSIS} T. Petrusic ${ }^{1}$

${ }^{1}$ Health Centre, Nis, Serbia

Introduction: Osteoporosis is a global health problem, and from this disease today falling ill about one-fifth of men and one of women around the raceway world. Her importance is growing as more and more extends the average life span of a man and how to increase the population of older people.

Objective: The diagnosis of osteoporosis is important for mass illness and to prevent more serious consequences and complications such as fractures of the vertebrae, femur, disability.

Methods: Measurements of BMD was performed ultrasound densitometer in the department of physical medicine and rehabilitation at the Health centre Nis. This research was performed as a screening test, and included 612 respondents older than 70 prominently in the period of three years. Results of the work of the total number of respondents was 318 (51.96\%) females and 294 (48.04\%) men Normal finding of the patients of the female population was $101(16.50 \%)$, at the male population 159 (25.98\%). Osteopenia had $150(24.51 \%)$ of the female population and $99(16.18 \%)$ male population Osteoporosis was diagnosed in 67 (10.95\%) women and 36 (5.88\%) men.

Conclusion: Senile osteoporosis is a disease that faces, population of the third age, or older person. According latest research the number of people who had been in osteoporosis will double in the next 50 years, due to the aging of the world population and the impact of various risk factors among which an important place occupied way of life. Age-related osteoporosis occurs in both sexes after age 70, presents the hip fractures and is caused by aging. The most common causes of secondary osteoporosis (caused by disease or drugs) are the treatment of steroids and anticonvulsants (medications to treat epilepsy). Osteoporosis long time remain clinically inconspicuous - it does not have characteristic symptoms and most importantly, it does not hurt. Therefore, it is rightly called "the silent thief" because bone mass impoverishes years until it falls below the so called "threshold for fracture" when they appear clinically dramatic complications of the disease - fractures of the vertebrae. Vertebral fracture usually occurs with a sudden movement, bending, lifting, and sometimes for no apparent reason. Fracture of the vertebrae leading to hunched posture characteristic of osteoporosis. Body height decreases with each fracture about 2-4 cm. 


\section{P420}

HORMONAL THERAPY-RELATED OSTEOPOROSIS IN A PATIENT WITH PROSTATE ADENOCARCINOMA AND ADRENAL INCIDENTALOMA

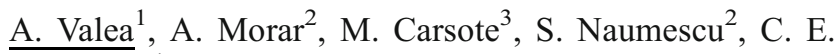
Georgescu $^{1}$

${ }^{1}$ I. Hatieganu University of Medicine and Pharmacy \& Clinical County Hospital, Cluj-Napoca, Romania, ${ }^{2}$ Clinical County Hospital, Cluj-Napoca, Romania, ${ }^{3}$ C.I. Parhon National Institute of Endocrinology, Bucharest, Romania

Introduction: Hormone therapy for prostate cancer induces hypogonadism which is a leading cause of decreased BMD and associated fragility fractures in men.

Materials and methods: We report the case of an adult male with complex medical conditions which were periodically checked-up in several medical centers from Romania. Bone profile evaluation included: central dual X-ray energy assay measuring BMD; intact PTH, serum 25-hydroxyvitamin D (25-OH D); whole body MRI.

Case presentation: A 84 year-old male was diagnosed with prostate adenocarcinoma with extracapsular invasion (T2N0M6, G5) in 2012, and was treated with local radiotherapy and gonadotropic analogues $(\mathrm{GnRH})$ for 3 years. Vitamin D supplements were offered to the subject. During therapy the whole body MRI was done to check the disease extension and a solid pulmonary nodule (measuring maximum $9 \mathrm{~mm}$ ) was revealed in the right upper lobe and a cortical tumor (apparently suggesting benign features) in the left adrenal gland, measuring $23 \mathrm{~mm}$ at maximum diameter. Hormonal profile was assessed, although the patient showed no clinical signs of hormone excess, except for high blood pressure controlled by current medications. The diagnosis of adrenal incidentaloma was confirmed by normal levels of plasma cortisol and aldosterone and urinary fractionated metanephines. At the moment, no association was established between prostate cancer, lung nodule and adrenal incidentaloma. Recently, the subject complained of persistent back pain. The laboratory investigations showed: vitamin D deficiency (25-OHD of $20.2 \mathrm{ng} / \mathrm{mL}$, normal value above $30 \mathrm{ng} / \mathrm{mL}$ ), high PTH level (of $76.6 \mathrm{pg} / \mathrm{mL}$, normal ranges between 15-66 pg/ $\mathrm{mL}$ ), while 24 -h urinary calcium levels were slightly above the upper limit of normal $(307.8 \mathrm{mg} / 24-\mathrm{h}$, normal ranges between $100-300 \mathrm{mg} / 24-\mathrm{h}$ ), with normal serum total calcium levels $(9.21 \mathrm{mg} / \mathrm{dl}$, normal ranges between 8.8 $10.2 \mathrm{mg} / \mathrm{dl}$ ). Low BMD at DXA was consistent with a Tscore of -3 SD (femoral neck), of - 2.1 SD (total hip), -2.5 SD (lumbar). No vertebral fractures were detected. Weekly oral acidum alendronicum (5600 mg/week) was recommended together with vitamin $\mathrm{D}$ and calcium. Also, the patient continued oral antiandrogen therapy.
Conclusions: Hormone therapy significantly decreases BMD in men with prostate cancer especially in elderly population. High rates of bone loss persist despite concurrent administration of vitamin D supplements and after careful exclusion of secondary causes of osteoporosis as bone metastasis.

\section{P421}

DUAL ENERGY X-RAY ABSORPTIOMETRY AND QUANTITATIVE ULTRASOUND MEASUREMENTS OF BONE MINERAL DENSITY OF TRABECULAR BONE PHANTOMS IN THE PRESENCE OF STRONTIUM

A. M. Pejović-Milić ${ }^{1}$, D. Hyun Jang ${ }^{1}$, E. Da Silva ${ }^{1}$, L. Slatkovska $^{2}$, A. M. Cheung ${ }^{2}$, J. Tavakkoli ${ }^{1}$

${ }^{1}$ Department of Physics, Ryerson University, Toronto, Canada, ${ }^{2}$ Osteoporosis Program, University Health Network, Toronto, Canada

Objective(s): The aim of this study was to investigate the effect of bone strontium concentration on determined BMD when measured by DXA and quantitative ultrasound (QUS) techniques.

Materials and Methods: Bone mineral phantoms with clinically relevant molar percentages of strontium $(\% \mathrm{Sr} /[\mathrm{Sr}+\mathrm{Ca}])$ were prepared as previously described [1] by substituting calcium for strontium. The strontium-substituted apatitic material was then mixed homogenously with gelatin to produce trabecular bone-mimicking phantoms, compatible with both DXA and QUS. Seven phantoms with a constant volumetric BMD of $200 \mathrm{mg} / \mathrm{cm}^{3}$ and different molar percentage of strontium ranging from $0 \mathrm{~mol} \%$ to $2.0 \mathrm{~mol} \%$ were produced. The phantoms were assessed using a Hologic Discovery DXA and a Hologic Sahara QUS systems.

Result: A linear relationship $\left(r^{2}>0.99\right)$ was observed between BMD and the molar percentage of strontium when measured by DXA. Upon the analysis of the linear regression, the increase in the measured BMD for every molar percentage of strontium was observed to be $(13.5 \pm 0.5) \%$, which is comparable with the correction reported [2]. In contrast, stiffness index, measured by QUS to derive BMD, did not exhibit a correlation with the strontium content $\left(r^{2}>0.22\right)$.

Conclusions: The results of this study suggest that, unlike DXA, QUS is capable of estimating BMD independently of the bone strontium content. Therefore, it is recommended that the assessment of BMD in patients undergoing treatment with strontium should be done using QUS rather than DXA. This would eliminate overestimation of patients' BMD due to strontium content in their bone, and potentially provide better understanding of strontium based treatments.

\section{Reference:}

1. Da Silva E, et al. Analytical Chemistry 2013;85:9189.

2. Blake GM, Fogelman I. J Clin Densit 2007;10:34. 


\section{P422}

VITAMIN D AND ITS ASSOCIATION WITH PHYSICAL PERFORMANCE IN PRIMARY KNEE OSTEOARTHRITIS PATIENTS

P. Yuktanandana ${ }^{1}$, P. Manoy ${ }^{2}$, A. Tanavalee ${ }^{1}$, S. Ngarmukos $^{1}$, T. Tanpowpong ${ }^{1}$, W. Anomasiri ${ }^{2}$, S. Honsawek $^{2}$

${ }^{1}$ Department of Orthopedics, Faculty of Medicine, Chulalongkorn University, Bangkok, Thailand, ${ }^{2}$ Department of Biochemistry, Faculty of Medicine, Chulalongkorn University, Bangkok, Thailand

Background: The implication of vitamin D in physical performance among patients with knee osteoarthritis $(\mathrm{OA})$ is still controversial. We postulated that among individuals with $\mathrm{OA}$, lower levels of serum 25-hydroxyvitamin $\mathrm{D}$ would be associated with poorer physical performance. The present study aims to investigate the association between 25-hydroxyvitamin D and physical performance in primary knee OA patients.

Methods: In this cross-sectional study, 150 elderly patients with clinical and radiological findings of primary knee OA were recruited. Knee OA grading was assessed using the Kellgren-Lawrence grading system. Serum levels of 25-hydroxyvitamin D (vitamin D) were analyzed using an automated chemiluminescent immunoassay. Intact PTH, creatinine, calcium, phosphate, albumin, and lipid profile levels were also measured. We defined vitamin $\mathrm{D}$ insufficiency as $\leq 75 \mathrm{nmol} / 1$ and deficiency as $\leq 50 \mathrm{nmol} / \mathrm{l}$. Each patient was evaluated for gait speed, five-time-sit-to-stand, time-up-and-go, and six-minute walk tests.

Results: Twenty-six percent of knee OA patients had normal serum vitamin D. Serum vitamin D insufficiency was observed in $48 \%$ of patients with knee OA, whereas vitamin D deficiency was found in $26 \%$ of knee OA patients. Physical performance measures were significantly reduced in OA patients with low serum vitamin D compared to those with normal serum vitamin D $(P<0.01)$. We found positive significant associations of lower levels of 25 -hydroxyvitamin $\mathrm{D}$ with poorer physical performance measures $(P<0.05)$.

Conclusion: These findings indicate that there were significantly positive associations between serum 25hydroxyvitamin D levels and all physical performance measures in primary knee $\mathrm{OA}$ and suggest assessment of serum 25-hydroxyvitamin D in any patients with symptoms suggestive of knee OA and low physical performance. Treatment with vitamin D supplements may provide beneficial effects to OA patients and further prospective studies are in progress to elucidate the role of vitamin D supplements in physical performance of primary knee osteoarthritis patients.
P423

OSTEOARTHRITIS INCREASES THE RISK OF CARDIOVASCULAR DISEASES IN THE ELDERLY: THE PROGETTO VENETO ANZIANO STUDY

N. Veronese $^{1}$, C. Trevisan ${ }^{1}$, G. Bano ${ }^{1}$, G. Sergi ${ }^{1}$, S. Maggi ${ }^{2}$

${ }^{1}$ University of Padova, Department of Medicine, Geriatrics Section, Padova, Italy, ${ }^{2}$ National Research Council, Padova, Padova, Italy

Objective: The possible relevance of osteoarthritis (OA) as a cardiovascular disease (CVD) risk factor is still debated. We aimed to investigate the association between $\mathrm{OA}$ and the onset of CVD in older people.

Material and Methods: Among a sample of 3099 elderly subjects, we considered 2158 who had no CVD at the baseline and were followed for $4.4 \pm 1.2$ years. OA was defined using a standardized algorithm investigating disease history, medical documentation (including X-ray reports), symptoms, and physical examination of the joints. Incident CVD was defined as the onset of coronary artery disease, heart failure, stroke/ transient ischemic attack, peripheral artery disease, and CVDrelated hospitalization or mortality.

Results: At the baseline, 1336 participants $(=61.9 \%)$ suffered from OA. Participants with OA had more potential CVD risk factors, including obesity, hypertension, high LDL and inflammation levels, and a worse renal function than those without. During the follow-up, $47.8 \%$ of the participants with OA at the baseline developed a new CVD vs. $41.3 \%$ of those without. Using an adjusted Cox's regression analysis, the presence of OA significantly increased the risk of CVD $(H R=1.22,95 \% \mathrm{CI}: 1.02-1.49, p=0.04)$. The association between OA and CVD was stronger when the hip or knee were affected, in women, and when more than two joints were involved. Considering single CVD outcomes, the presence of OA significantly increased the risk of new coronary artery disease, heart failure, and hospitalization for CVD.

Conclusions: OA significantly predicted the onset of CVD in elderly people, particularly when it involved lower limbs, women, and more than two joints.

\section{P424}

OSTEOARTHRITIS AND MORTALITY: A PROSPECTIVE COHORT STUDY AND SYSTEMATIC REVIEW WITH META-ANALYSIS

N. Veronese $^{1}$, E. Cereda ${ }^{2}$, B. Stubbs ${ }^{3}$, M. Solmi ${ }^{4}$, E. Manzato $^{1}$, S. Maggi ${ }^{5}$

${ }^{1}$ University of Padova, Department of Medicine, Geriatrics Section, Padova, Italy, ${ }^{2}$ Fondazione IRCCS Policlinico San Matteo, Pavia, Italy, ${ }^{3}$ King's College London, London, United Kingdom, ${ }^{4}$ University of Padova, Department of Neuroscience, Padova, Italy, ${ }^{5}$ National Research Council, Padova, Padova, Italy 
Background: Osteoarthritis (OA) is a leading cause of disability, but the relationship with premature mortality remains uncertain. In this work, we aimed to investigate the relationship between OA and mortality from any cause and from cardiovascular disease (CVD).

Materials and Methods: In addition to the Progetto Veneto Anziani (PRO.V.A.) study, other prospective studies comparing mortality in a sample of people with and without OA were searched by two independent authors in PubMed, EMBASE, CINAHL and SCOPUS until 17th November 2015 and without language restrictions.. Data were summarized using hazard ratios (HRs) for death together with $95 \% \mathrm{CI}$, adjusted for the maximum number of potential confounders. Data about single joints (hand, hip and knee) and about CVD mortality were extracted were available.

Results: From the PRO.V.A. study $(N=2,927)$, there was no significant increase in mortality risk for participants with anyjoint OA $(N=1,858)$ compared to non-OA (all-cause, $H R=0.95$ [95\%CI, 0.78-1.16], $p=0.59$; CVD, $H R=1.12$ [95\%CI, 0.82-1.54], $p=0.52$ ). On meta-analysis, seven studies (OA: 10,018; non-OA: 18,541) with a median follow-up of 12 years, reported no increased risk of premature mortality in those with any-joint $\mathrm{OA}(H R=1.10$ [95\%CI, 0.97-1.25], $p=0.12 ; \mathrm{I}^{2}=67 \%$ ). In the following study strata (European studies; death ascertainment acquired with death certificates; multiple joints affected; radiological diagnosis of $\mathrm{OA}$ ), we observed a significantly higher risk of all-cause mortality and a reduction of between-study heterogeneity. OA was associated with significantly higher CVD attributed mortality without heterogeneity $(H R=1.21$ [95\% CI, 1.10-1.34], $p<0.001 ; \mathrm{I}^{2}=0 \%$ ).

Conclusions: People with OA are at increased risk of premature death due to CVD, but not all cause mortality.

\section{P425}

OSTEOARTHRITIS AS A RISK FACTOR FOR FRAILTY IN THE ELDERLY: THE PROGETTO VENETO ANZIANI STUDY

N. Veronese $^{1}$, S. Carraro ${ }^{1}$, L. Berton ${ }^{1}$, F. Gessoni ${ }^{1}$, S. Maggi ${ }^{2}$ ${ }^{1}$ University of Padova, Department of Medicine, Geriatrics Section, Padova, Italy, ${ }^{2}$ National Research Council, Padova, Padova, Italy

Objective: While osteoarthritis (OA) increases the risk of disability and other negative outcomes in elderly populations, there is a paucity of data concerning its link with frailty. This study aimed to investigate the association between OA and OA-related pain with frailty in a cohort of older subjects coming from the Progetto Veneto Anziani (PRO.V.A.) study. Material and Methods: OA and OA-related pain were defined using a standardized algorithm taking into consideration disease history, medical records, symptoms/signs, pain/use of analgesics, and a physical examination of the joints. Participants were considered frail if they met 3 out of 5 (unintentional weight loss, exhaustion, weakness, low physical activity, slow walking speed for 4 meters) criteria of Fried's Index.

Results: Among the 3099 initially involved, 2830 were included for cross-sectional analyses. After adjusting for potential confounders, OA was found to be associated with significantly higher odds of frailty (hand $\mathrm{OA}, O R=1.72$, $95 \% \mathrm{CI}=1.55-1.90$; hip OA, $O R=2.50,95 \% \mathrm{CI}=2.26-2.77$; knee $\mathrm{OA}, \mathrm{OR}=1.81,95 \% \mathrm{CI}=1.62-2.02$ ). Of the $1951 \mathrm{sub}-$ jects who were not considered frail at baseline, $302(=15.5 \%)$ became so after a mean of 4.4 years. A fully-adjusted logistic regression analysis showed that OA increases the risk of frailty independently from the site that is affected (hand OA, $O R=1.22,95 \% \mathrm{CI}=1.11-1.34$; hip $\mathrm{OA}, O R=1.90$, $95 \% \mathrm{CI}=1.73-2.10$; knee $\mathrm{OA}, O R=1.24,95 \% \mathrm{CI}=1.13$ 1.37). Similar patterns were observed with regard to OArelated pain.

Conclusion: Our results suggest that OA and OA related-pain were found to be associated with an increased risk of frailty in older men and women.

P426

OLDER PEOPLE WITH OSTEOARTHRITIS ARE AT INCREASED RISK OF DEVELOPING COGNITIVE IMPAIRMENT: AN ANALYSIS OF THE PROGETTO VENETO ANZIANI COHORT N. Veronese ${ }^{1}$, S. Facchin ${ }^{1}$, G. Girotti ${ }^{1}$, E. Valmorbida ${ }^{1}$, S. Maggi $^{2}$

${ }^{1}$ University of Padova, Department of Medicine, Geriatrics Section, Padova, Italy, ${ }^{2}$ National Research Council, Padova, Padova, Italy

Objective: Osteoarthritis is associated with a number of medical morbidities in the elderly. However, its relationship to cognitive impairment is still unknown. This study sought to determine whether osteoarthritis can increase the risk of developing cognitive impairment in older people.

Material and Methods: Data was gathered from the Progetto Veneto Anziani (Pro.V.A) dataset, a cohort study of community-dwelling older people in Italy. Osteoarthritis was defined using a standardized algorithm investigating disease history, medical documentation, symptoms, and physical examination. Cognitive impairment was assessed through the mini-mental state examination (MMSE) defined as a score of less 24/30.

Results: In total 1418 people were analyzed ( 817 with osteoarthritis compared to 601 without). On adjusted logistic regression analysis, people with osteoarthritis had a significant increased probability of experiencing cognitive impairment compared to those without (odds ratio (OR): 1.18 ; 95\% 
1.08 to 1.30 ). When assessed by anatomical location, hand osteoarthritis had a $62 \%$ greater chance of cognitive impairment (OR: 1.62 ; 95\%CI: 1.44 to 1.82 ). Mono-articular (OR: 1.14; $95 \% \mathrm{CI}$ : 1.01 to 1.27 ) and poly- articular osteoarthritis (OR: $1.26 ; 95 \% \mathrm{CI}: 1.13$ to 1.41 ) also demonstrated higher probability of cognitive impairment compared to nonosteoarthritis cohorts. There was a greater association of cognitive impairment in males compared to females with osteoarthritis.

Conclusions: Osteoarthritis is significantly associated with an increased risk of developing cognitive impairment in older people. Clinicians managing this population should therefore be aware of this association when assessing the global healthcare needs of this population at potential higher risk of poor cognitive status.

\section{P427}

OLDER WOMEN WITH OSTEOARTHRITIS ARE AT INCREASED RISK OF INCIDENT DEPRESSION: A PROSPECTIVE ANALYSIS OF THE PROGETTO VENETO ANZIANI COHORT N. Veronese ${ }^{1}$, B. Stubbs ${ }^{2}$, S. Pizzato ${ }^{1}$, B. Zanforlin ${ }^{1}$, F. Bolzetta $^{1}$, S. Maggi ${ }^{3}$

${ }^{1}$ University of Padova, Department of Medicine, Geriatrics Section, Padova, Italy, ${ }^{2}$ King's College London, London, United Kingdom, ${ }^{3}$ National Research Council, Padova, Padova, Italy

Objective: To provide meta-analytical evidence of BMD, fractures, and osteoporosis rates in eating disorders (ED) vs. healthy controls (HCs).

Material and Methods: Three independent authors searched major electronic databases from inception till 08/2015 for cross-sectional studies reporting BMD in people with ED (anorexia nervosa, (AN); bulimia nervosa, $(\mathrm{BN})$; eating disorders not otherwise specified, (EDNOS)) vs. HCs. Standardized mean differences (SMDs) $\pm 95 \% \mathrm{CI}$ were calculated for BMD; and odds ratios (ORs) for osteopenia, osteoporosis and fractures.

Results: Overall, 57 studies were eligible, including 21,607 participants $(\mathrm{ED}=6,485, \mathrm{HCs}=15,122)$. Compared to $\mathrm{HC}$, AN subjects had significantly lower BMD values at lumbar spine $(\mathrm{SMD}=-1.51,95 \% \mathrm{CI}=-1.75,-1.27$, studies $=41)$, total hip $(\mathrm{SMD}=-1.56,95 \% \mathrm{CI}=-1.84,-1.28$, studies $=23)$, intertrochanteric region $(\mathrm{SMD}=-1.80,95 \% \mathrm{CI}=-2.46$, -1.14 ,studies $=7)$, trochanteric region $(\mathrm{SMD}=-1.05$, $95 \% \mathrm{CI}=-1.44,-0.66$, studies $=7$ ) and femoral neck $(\mathrm{SMD}=-0.98,95 \% \mathrm{CI}=-1.12,-0.77$, studies $=20)$. Reduced BMD was moderated by ED illness duration and amenorrhea $(p<0.05)$. AN was associated with an increased likelihood of osteoporosis $(O R=12.59,95 \% \mathrm{CI}=3.30-47.9$, $p<0.001$, studies $=4)$ and fractures $(O R=1.84$,
$95 \% \mathrm{CI}=1.17-2.89, \mathrm{I}^{2}=56$, studies $=6$ ). No difference in BMD was found between BN or EDNOS and HC.

Conclusion: People with AN have consistently reduced BMD resulting in a markedly increased risk of fractures. Proactive monitoring and interventions are required to ameliorate bone loss in AN.

\section{P428}

BONE MINERAL DENSITY, OSTEOPOROSIS, AND FRACTURES AMONG PEOPLE WITH EATING DISORDERS: A SYSTEMATIC REVIEW AND META-ANALYSIS

N. Veronese ${ }^{1}$, M. Solmi ${ }^{2}$, C. Correll ${ }^{3}$, A. Favaro ${ }^{2}$, P. $\overline{\text { Santonastaso }}^{2}$, L. Caregaro ${ }^{4}$, D. Vancampfort ${ }^{5}$, C. Luchini ${ }^{6}$, B. Stubbs ${ }^{7}$

${ }^{1}$ University of Padova, Department of Medicine, Geriatrics Section, Padova, Italy, ${ }^{2}$ University of Padova, Department of Neuroscience, Padova, Italy, ${ }^{3}$ Department of Psychiatry, North Shore-Long Island Jewish Health System, Glen Oaks, New York, USA, New York, United States, ${ }^{4}$ University of Padova, Department of Medicine, Padova, Italy, ${ }^{5}$ University of Leuven Department of Rehabilitation Sciences, Leuven, Belgium, ${ }^{6}$ University of Verona, Verona, Italy, ${ }^{7}$ King's College London, London, United Kingdom

Objective: To provide meta-analytical evidence of bone mineral density (BMD), fractures, and osteoporosis rates in Eating Disorders (ED) vs healthy controls (HCs).

Material and Methods: Three independent authors searched major electronic databases from inception till 08/2015 for cross-sectional studies reporting BMD in people with ED (anorexia nervosa, (AN); bulimia nervosa, (BN); eating disorders not otherwise specified, (EDNOS)) versus HCs. Standardized mean differences (SMDs) $\pm 95 \%$ and confidence intervals (CIs) were calculated for BMD; and odds ratios (ORs) for osteopenia, osteoporosis and fractures.

Results: Overall, 57 studies were eligible, including 21,607 participants $(\mathrm{ED}=6,485, \mathrm{HCs}=15,122)$. Compared to $\mathrm{HC}$, AN subjects had significantly lower BMD values at lumbar spine $(\mathrm{SMD}=-1.51,95 \% \mathrm{CI}=-1.75,-1.27$, studies $=41)$, total hip $(\mathrm{SMD}=-1.56,95 \% \mathrm{CI}=-1.84,-1.28$, studies $=23)$, intertrochanteric region $(\mathrm{SMD}=-1.80,95 \% \mathrm{CI}=-2.46$, -1.14 , studies $=7)$, trochanteric region $(\mathrm{SMD}=-1.05$, $95 \% \mathrm{CI}=-1.44,-0.66$, studies $=7)$ and femoral neck $(\mathrm{SMD}=-0.98,95 \% \mathrm{CI}=-1.12,-0.77$, studies $=20)$. Reduced BMD was moderated by ED illness duration and amenorrhea $(\mathrm{p}<0.05)$. AN was associated with an increased likelihood of osteoporosis $(O R=12.59,95 \% \mathrm{CI}=3.30-47.9$, $p<0.001$, studies $=4)$ and fractures $(O R=1.84$, $95 \% \mathrm{CI}=1.17-2.89, \mathrm{I}^{2}=56$, studies $=6$ ). No difference in BMD was found between BN or EDNOS and HC. 
Conclusion: People with AN have consistently reduced BMD resulting in a markedly increased risk of fractures. Proactive monitoring and interventions are required to ameliorate bone loss in AN.

\section{P429}

DEVELOPMENT AND VALIDATION OF THE OSTEOPOROSIS RISK SCORE (ORS) TOOL TO ENHANCE TARGETING WOMEN WITH LOW BONE MINERAL DENSITY

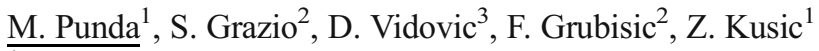

${ }^{1}$ Department of Oncology and Nuclear Medicine, UHC Sisters of Mercy, Zagreb, Croatia, ${ }^{2}$ Department of Rheumatology, Physical Medicine and Rehabilitation, UHC Sisters of Mercy, Zagreb, Croatia, ${ }^{3}$ Department of Trauma and Orthopedic Reconstructive Surgery, UHC Sisters of Mercy, Zagreb, Croatia

Objectives: Clinical osteoporosis prescreening tools are intended to select individuals most likely to benefit from BMD testing. This study aims to develop and validate Osteoporosis Risk Score (ORS), a new prescreening tool intended to be used in women aged 45 years and older.

Materials and Methods: Two cohorts of women aged 45-87 years without previous diagnosis of osteoporosis participated in the development $(n=200)$ and the validation $(n=1000)$ of the ORS tool. Data on risk factors for osteoporosis were assessed by questionnaire and BMD by DXA (Hologic Delphi C, Bedford, USA). Risk factors that best predicted Tscore $\leq-2.5$ at the femoral neck region were selected for inclusion into the ORS. Logistic regression analysis was used to select significant predictors of BMD, whereas receiveroperating characteristic (ROC) analysis was used to assess the discriminatory performance of the ORS tool. Validation of the ORS tool and comparison with other validated osteoporosis tools were evaluated in terms of sensitivity and specificity using the original developer's cutoffs and densitometric target outcomes.

Results: The ORS tool was developed based on four variables: age $>65$ years ( 3 points), years post- menopause of $>14$ years ( 3 points), height $<160 \mathrm{~cm}$ ( 2 points) and weight $<73 \mathrm{~kg}$ ( 1 point). Cut-off value of $>6$ points (out of 9 possible) was associated with the $80.0 \%$ sensitivity and $81.6 \%$ specificity to identify women with low bone mass. Applying the ORS tool among the study population (validation cohort) showed lower sensitivity $(58.9 \%)$ and higher specificity $(82.1 \%$ ) compared to ORAI, ABONE, OST tools (sensitivity of $86.3 \%, 83.6 \%, 80.8 \%$ and specificity of $52.5 \%, 55.9 \%, 58.6 \%$ ), respectively. ORS tool showed better prediction of low BMD among women above 65 years compared to group of women aged 45-64 years.
Conclusions: The Osteoporosis Risk Score tool is a simple scoring system based on four risk factors for osteoporosis. Due to its higher specificity and somewhat lower sensitivity ORS tool showed better performance in discrimination of women in whom BMD testing is unnecessary. Further investigation is needed to determine prescreening osteoporosis scoring system among women aged 45-64 years.

\section{P430}

\section{HEALTH WITHOUT PILLS}

E. Maftei $^{1}$, A. Mandrut ${ }^{2}$, C. Nistor Cseppento ${ }^{3}$

${ }^{1}$ Faculty of Medicine of Oradea, Oradea, Romania, ${ }^{2}$ Medical rehabilitation, Faculty of Medicine, University of Oradea, Oradea, Romania, ${ }^{3}$ University of Oradea, Faculty of Medicine and Pharmacy, Oradea, Romania

As we look around us it is impossible not to notice people that are dealing with pain because of their bones, muscles or articulation and we often see that in the long run these aches do not only produce discomfort but they also determine some issues regarding body coordination, standing, balance or worst case scenarios when they get to spasticity or paralysis that thoroughly severe the patient's ability to move. The emergence of these sorts of pain related to bones, muscles or articulations depend on many factors and people that suffer because of them think that by using pain killers they will get better without any additional effort. In doing so they forget that these medication only calms the effect without directly dealing with the cause of it, that is why in this article we shall examine why medical recovery/therapy is the best solution in maintaining health as it is using exercises which conform to mechanical laws that imply disciplines such as: physical therapy, physiotherapy, electrotherapy, hydrotherapy, thermotherapy, etc. The purpose of these exercises is: pain removal, muscle relaxation, the improvement of joint mobility, blood circulation, tissue trophicity and cell metabolism. Recovery programs are perfectly adapted to the needs of every patient alike and they take in account the diagnostic and the evolutionary stage of disease. These is no standard recovery program for all patients as they are all adapted to their current pathology either: neurologically, cardiac, respiratory, traumatic, orthopedic. By using the experience of Felix station we shall help you see how the picture in which a sad patient arrives at his therapy with sayings like: 'It's hurting really bad", "I can't do it" changes to: "Today really feels a lot better than yesterday" or "Your hands have made me well! Thank you" and you could how he shines of gladness due to real progress. We shall also try to describe this little place, so rich in mineral waters, which regardless of season satisfies with its warm waters any person, either sick or healthy. 


\section{P431}

THE INFLUENCE OF BISPHOSPHONATE THERAPY ON THE INCREASE OF BONE MINERAL DENSITY IN WOMEN WITH DIFFERENT MENOPAUSE DURATION

I. Aleksic $^{1}$, S. Milenkovic ${ }^{1}$, B. Stamenkovic ${ }^{1}$, S. Stojanovic ${ }^{1}$, V. Zivkovic $^{1}$, J. Jovanovic ${ }^{1}$

${ }^{1}$ Rheumatology department Institute for Treatment and Rehabilitation Niska Banja, Niska Banja, Nis, Serbia

Introduction: A key factor of the pathogenesis of postmenopausal osteoporosis is estrogen deficiency. The estrogens protect bone against osteoporosis indirectly, stimulating the synthesis of calcitonin than inhibiting bone resorption and directly, by estrogen (E2) receptors activity as well as increasing the synthesis of osteoprotegerin (OPG) in osteoblasts and stimulating the osteoblastic activity. Osteoclastogenesis is related to menopause as well as the increase secretion of PTH. Objectives: To determine the effect of bisphosphonate therapy on osteoporosis in postmenopausal women with different duration of menopause.

Material and method: Our investigation consist of 106 women with newly diagnosed postmenopausal osteoporosis without previous vitamin $\mathrm{D}$ treatment. Bone density was measured by DXA Hologic densitometer at L1-L4 spine and proximal femur in all patients before and after one year of treatment with bisphosphonates (Alendronate $70 \mathrm{mg}$ per week, vitamin D 800 IU per day). OP is defined as the mean bone density expressed as a T-score $<-2.5$.

Results: According to the duration of menopause examinees were divided into 3 groups. The first group consisted of women with menopausal duration of 5 years (36 examinees); in the second group the duration was 6 to 10 years (32 examinees) and the third group with menopausal duration 11 years and over (38 examinees). The mean value of the T-score and BMD before the therapy was highest in women with the duration of menopause 5 years $\left(-2.78 \pm 0.28 ; 0.734 \pm 0.033 \mathrm{~g} / \mathrm{cm}^{2}\right)$, then in women with duration of menopause 6-10 years $(-2.99$ $\left.\pm 0.61 ; 0.722 \pm 0.064 \mathrm{~g} / \mathrm{cm}^{2}\right)$, and the lowest T-score and BMD value was found in women with duration of menopause 11 years and more $\left(-3.09 \pm 0.55 ; 0.708 \pm 0.064 \mathrm{~g} / \mathrm{cm}^{2}\right)$. ANOVA and Danet's test did not show statistically significant. differences between these values. The mean value of T-score and BMD after 12 months of treatment was highest in women with duration of menopause 5 years $(-2.51 \pm 0.350 ; 0.768$ $\left.\pm 0.038 \mathrm{~g} / \mathrm{cm}^{2}\right)$, then in women with duration of menopause $6-10$ years $\left(-2.73 \pm 0.61 ; 0.748 \pm 0.065 \mathrm{~g} / \mathrm{cm}^{2}\right)$, and the lowest in women with duration of menopause 11 years and more $\left(-2.85 \pm 0.54 ; 0.733 \pm 0.056 \mathrm{~g} / \mathrm{cm}^{2}\right)$, but Danet's and ANOVA test did not show statistically significant differences among these values. We found the most significant increasing in T- score values after one year of bisphosphonates therapy in group with menopause duration of 5 years $(0.270 \pm 0.205)$;
( -2.78 vs. $-2.51, p<0.01)$, then in second group with duration of menopause $6-10$ years $(0.260 \pm 0.218)$; $(-2.99$ vs. -2.73 , $p<0,01)$. The lowest increasing of T-score was in third group $(0.240 \pm 0.223) ;(-3.09$ vs. $-2.85, p<0.01)$. With ANOVA and Danet's test we found statistically significant differences between these values. According to the BMD values after one year of the therapy, we found highest increasing in the first group $\left(0.034 \pm 0.024 \mathrm{~g} / \mathrm{cm}^{2}\right) ;(0.734$ vs. $0.768, p<0,01)$, then in the second group $\left(0.026 \pm 0.027 \mathrm{~g} / \mathrm{cm}^{2}\right) ;(0.722 \mathrm{vs} .0 .748$, $p<0.01)$, and the lowest increasing was in the third group of examinees $\left(0.025 \pm 0.022 \mathrm{~g} / \mathrm{cm}^{2}\right) ;(0.708$ vs. $0.733, p<0.01)$. With ANOVA and Danet's test we found statistically significant differences between these values.

Conclusion: After 12 months of bisphosphonate treatment we found statistically significant increasing in BMD in all three groups of our examinees with different menopausal duration. The effect of bisphosphonate therapy was highest in women with shortest menopausal duration (5 years), but without statistical significance as compared to the other groups.

\section{P432}

SERUM RANKL AND FRACTURE RISK USING FRAX TOOL IN POSTMENOPAUSAL RHEUMATOID ARTHRITIS PATIENTS

S. I. Nasef ${ }^{1}$, H. H. Omar ${ }^{2}$, H. M. Abdalla ${ }^{3}$

${ }^{1}$ Rheumatology Department, Faculty of Medicine, Suez Canal University, Ismailia, Egypt, ${ }^{2}$ Clinical Pathology Department, Faculty of Medicine, Suez Canal University, Ismailia, Egypt, ${ }^{3}$ Radiology Department, Faculty of Medicine, Suez Canal University, Ismailia, Egypt

Objectives: To assess the level of serum receptor activator of nuclear factor kappa beta ligand (RANKL) in postmenopausal rheumatoid arthritis (RA) patients and its correlation with the fracture risk using fracture risk assessment tool (FRAX).

Materials and Methods: We recruited 116 individuals (78 postmenopausal RA patients and 38 age- matched postmenopausal control subjects). All patients and controls were not receiving osteoporosis treatment. We measured serum RANKL, C reactive protein (CRP) and rheumatoid factor (RF). Disease activity score (DAS)-28 was used to assess RA activity. We measured BMD at lumbar spine (L1- L4), right femur neck and right forearm radius with DXA. We have calculated the absolute 10-year major osteoporotic fracture and hip fracture risks with FRAX tool.

Results: Mean age was $54.83 \pm 6.6$ years in RA patients vs. $55.05 \pm 6.2$ years in controls. Mean disease duration was $10.53 \pm 6.6$ years. Serum RANKL was significantly higher in RA patients vs. controls $(971.0$ pmol/L vs. 177.85 $\mathrm{pmol} / \mathrm{L}, \mathrm{P}$ value $<0.0001)$. Mean DAS was $3.94 \pm 1.3$ and $76.9 \%$ of patients were on steroids. Mean BMD of RA patients at lumbar spine was $0.990 \pm 0.2 \mathrm{~g} / \mathrm{cm}^{2}$, at femur neck 
was $0.870 \pm 0.19 \mathrm{~g} / \mathrm{cm}^{2}$ and at forearm was $0.568 \pm 0.14 \mathrm{~g} /$ $\mathrm{cm}^{2}$. Prevalence of osteoporosis in RA patients at lumbar spine was $34.6 \%$, at femur neck was $23.1 \%$ and at forearm was $42.3 \%$. Risk of major osteoporotic fracture in RA patients was $8.62 \%$ and the risk of hip fracture was $3.3 \%$. There was a highly significant positive correlation between serum RANKL and the risk of major osteoporotic fracture $(r=0.736$, P value $<0.0001)$. There was a highly significant positive correlation between serum RANKL and the risk of hip fracture $(r=0.859, \mathrm{P}$ value $<0.0001)$. Serum RANKL was negatively correlated with femur neck BMD $(r=-0.529$, P value $<0.001)$ and negatively correlated with forearm BMD $(r=-0.289$, P value $<0.01)$. No significant correlation was found between serum RANKL and CRP, DAS- 28 or BMD at lumbar spine.

Conclusion: In postmenopausal RA patients, increased serum RANKL level is associated with an increased risk of major osteoporotic and hip fractures. Therefore, antiRANKL therapy could have a role in prevention of RArelated fracture risks in postmenopausal RA patients.

\section{P433}

CORTICAL AND TRABECULAR BONE ANALYSIS WITH 3D-DXA IN PATIENTS WITH TYPE 2 DIABETES, LATENT AUTOIMMUNE DIABETES IN ADULTS (LADA) AND HEALTHY CONTROLS: A PRELIMINARY REPORT

M. A. Guagnelli ${ }^{1}$, R. A. Gomez-Diaz ${ }^{2}$, N. Wacher ${ }^{2}$, L. H. Humbert $^{3}$, Y. Martelli ${ }^{3}$, G. Gonzalez-Castelan ${ }^{1}$, P. Clark ${ }^{1}$

${ }^{1}$ Clinical Epidemiology Unit, Hospital Infantil Federico Gómez-Facultad de Medicina UNAM, Mexico City, Mexico, ${ }^{2}$ Unidad de Investigación Médica en Epidemiología Clínica, Centro Medico Nacional Siglo XXI, Instituto Mexicano del Seguro Social, Mexico City, Mexico, ${ }^{3}$ Galgo Medical, Barcelona, Spain

Objective: 3D-DXA technology provides a threedimensional analysis of femoral shape and bone density distribution from a conventional DXA scan, allowing a separate assessment of cortical and trabecular bone. Differences between BMD and fracture risk have been described for diabetic patients. The objective of the present study was to analyze BMD and 3D-DXA structural characteristics in a group of patients with type 2 diabetes (T2D), latent autoimmune diabetes in adults (LADA), and healthy controls.

Methods: Patients diagnosed with T2D according to ADA criteria and LADA patients (aged 30-70 years, under treatment with diet and/or oral hypoglycemic agents for at least the first 6 months, and positive for at least 1 autoantibody: GAD and/or IA-2) were compared with healthy controls. Patients with any other immune disease associated with diabetes or a fragility fracture were excluded. Anthropometric measurements, C-peptide, serum vitD, lipid profile, insulin, glucose, glycosylated hemoglobin $(\mathrm{HbA} 1 \mathrm{c})$ and conventional DXA scans for body composition, lumbar and femoral BMD with iDXA GE Lunar and 3D-DXA analysis of left femur were obtained with Galgo Medical software. The 3D-DXA software was used to analyze the cortical and trabecular bone in all cases, and to obtain a 3D subject-specific model of the femur of the patient and quantify the volumetric BMD (vBMD), volume (for trabecular and cortical regions) and cortical thickness distribution.

Results: Healthy controls $(n=51$, mean age $53.9 \pm 11.3)$, T2D $(n=74,53.6 \pm 13.6)$ and LADA $(n=26,51.2 \pm 9.7)$ were included. Lower Z-score BMD for lumbar region compared to controls were found in LADA patients and higher in T2D $(p=0.008)$, total body BMD showed significant differences $(p=0.028)$ and no significant differences were found in the femur BMD. 3D-DXA analysis showed the trabecular content in neck and greater trochanter diminished in T2D patients compared to controls and LADA patients $(p=0.005)$. Total cortical thickness showed no difference between groups, but was lower in the neck region in LADA patients compared to controls and DM2 $(p=0.044)$.

Conclusion: This preliminary report indicates that structural differences between patients with T2D and LADA are found with 3D-DXA. Further studies are needed.

\section{P434 \\ UNDIAGNOSED OCHRONOSIS PRESENTED WITH SEVER OSTEOARTHRITIS WITH CONCOMITANT OSTEOPOROSIS}

M. Zabihiyeganeh $^{1}$, H. Chehregosha ${ }^{2}$

${ }^{1} 1$ Bone and Joint Reconstruction Research Center, Shafa Orthopedic Hospital, Iran University of Medical Sciences, Tehran, Islamic Republic of Iran, ${ }^{2} 2$ Department of Internal Medicine, Rasole Akram Hospital, Iran University of Medical Sciences, Iran, Islamic Republic of Iran

Alkaptonuria (AKU) or ochronosis is a rare autosomal recessive disorder of the tyrosine metabolism. We present the case of a middle-aged female patient who had been referred to the clinic to evaluate chronic lumbosacral and peripheral joint pain. Her findings were in accordance with the diagnosis of ochronosis. Our case was a 56year-old woman presented with progressive inflammatory low back pain and pain in both knees and hips since 
3 years ago. That was associated with limitation of motion and reduced daily activity. During the 4 months before admission, right hip arthroplasty was performed due to pain and severe osteoarthritis but the pain and stiffness of lumbar spine increased and she did not able to sleep supine. Physical examination and imaging were compatible with the diagnosis of ochronosis. Also, Characteristic features of ochronosis as urine darkening, subcutaneous pigmentation and degenerative ochronoticarthropathy resembling osteoarthritis were found in our patient. BMD of the femoral neck showed osteoporosis and the lumbar spine was osteopenic with increased risk of fracture. So, treatment with zoledronic acid started for her. The mean age of presentation in previous literature evaluated. In a study of 58 patients, one-half of the patients had replacement of one knee, hip, or shoulder before 55 years of age and in most patients, the mean age at diagnosis was 29 years. In one study reported by Azami et al., seven patients with ochronotic arthropathy with mean age of 46.1 y presented. The age of presentation and delayed diagnosis were notable in our patient. However, there was not enough studies about the mean age of presentation. We found five relevant articles in the literature review in Iran. They concluded alkaptonuria must be considered in the evaluation of low back pain of patients especially with having a positive family history and bluish discoloration of cartilage tissues. One of these articles suggested more widespread screening in order to assess the true incidence of the disorder. But none of these articles discussed about the relationship of ochronosis with osteoporosis and the etiology of it. We evaluated the relationship between osteoarthritis (OA) and osteoporosis (OP). Previous studies suggest that OA is inversely related to OP. However when analyzed in individual bones, the BMD of the appendicular skeleton in OA-affected joints may be decreased, particularly in the upper extremities. Patients with OA should not be considered to be at a lower risk of fracture than the general population and physicians should be aware that a high BMD in patients with OA may be falsely reassuring. So, as noted above, low BMD in spine and femur were not be expected in this patient due to severe osteoarthritis and calcifications. On the other hand, studies suggested ochronosis may be associated with increased bone resorption rate leading to an accelerated bone loss. They hypothesized a role of the homogentisic acid polymer deposit in bone matrix and cells, possibly with osteocyte damage and interference in collagen metabolism. The same authors showed the ineffectiveness of alendronate for reverting the process of bone mass loss in a considerable number of patients with ochronosis and osteoporosis. This suggests that the existence of a specific mechanism (microlesions of the matrix, viability of the osteocytes and deterioration of the collagen network) could be responsible. But treatment with bisphosphonates is well tolerated and can improve BMD in some cases. So, this treatment could be useful in patients with ochronosis and osteoporosis. However osteoarthritis is associated with increase of BMD but treatment with Alendronate may be considered. We suggested other treatments such as teriparatide, calcium and vitamin $\mathrm{D}$, as a trial study, for the management of osteoporosis in these

\section{P435}

\section{PATTERN OF BONE MINERAL DENSITY AMONG ELDERLY HOUSEWIVES AT LOW RESOURCE SETTING IN BANGLADESH}

\section{S. Rahman ${ }^{1}$}

${ }^{1}$ Physical Medicine and Rehabilitation, Faculty of Medicine, Bangabandhu Sheikh Mujib Medical University, Dhaka, Bangladesh

Objectives: Active lifestyles, weight bearing exercises, exposure to sunlight and diet rich in calcium and vitamin D are essential component to maintain bone health. Elderly housewives of lower middle class family in Bangladesh mostly remain in the house and get less exposure to sunlight. Postmenopausal conditions are also important. Their average diet does not contain adequate calcium and vitamin. This small laboratory data based survey was done to see the pattern of BMD among elderly housewives in Bangladesh.

Materials and methods: We have randomly selected 75 women of more than 50 years old from principal author's private chamber, who were housewives of lower middle class family. They stay in the house most of the time and get less exposure to sunlight. Secondary causes were excluded. Tscores of spines and neck of femurs were recorded from all patients. Serum vitamin D level was not measured. Collected data were analyzed to see the pattern of BMD among the study population.

Results: This was a laboratory based collection of data. Age ranges of the patients were 53-82 years. Weight range was 52 to 90 kilograms. $60(80 \%)$ of the patients had T-score of the spine below -2.5 and $45(60 \%)$ had Tscore of hip below -2.5 . Only $15(20 \%)$ of patients had Tscore of both spine and hip below -3.5 which indicates severe osteoporosis.

Conclusion: Sedentary lifestyles, lack of exposure to sunlight, nutritional deficiencies of calcium and vitamin $\mathrm{D}$ and postmenopausal conditions are reasonable cause 
of decreased BMD in these elderly housewives at low resource setting. Serum vitamin D measurement could have better reflect the cause. This study suggests the need of anti-osteoporotic treatment in all elderly housewives irrespective of FRAX calculations or BMD measurement. Larger multicenter study can better delineate the prevalence of osteoporosis and fracture risk at low resource setting in Bangladesh.

\section{P436}

AN OPEN-LABEL, SINGLE CENTER STUDY ASSESSING THE EFFICACY OF AMORPHOUS CALCIUM CARBONATE (ACC) SUPPLEMENT IN THE MANAGEMENT OF PRIMARY HYPOPARATHYROIDISM

E. Segal $^{1}$, S. Ish-Shalom ${ }^{2}$

${ }^{1}$ Rambam Health Care Campus, Endocrine Institute, Haifa, Israel, ${ }^{2}$ Elisha Hospital, Haifa, Israel

Hypoparathyroidism is characterized by the absence or inactivity of PTH, leading to decreased intestinal calcium absorption, reduced bone turnover, hypocalcemia and hyperphosphatemia. Standard treatment in hypoparathyroidism consists of calcium and active vitamin D metabolites. High doses of oral calcium are frequently required.

Aim: To assess the effect of ACC, a soluble highly bioavailable form of calcium carbonate, on calcemia, calciuria in hypoparathyroid patients(HPP), previously treated with crystalline calcium carbonate supplement (CCS).

Patients and Methods: 10 consecutive HPP, aged 50.9 \pm 17.17 years, stable on CCS for at least one year, were enrolled in 28 days study. Patients with renal impairment and conditions influencing calcium absorption were excluded. The total daily dose of elemental calcium supplementation was gradually decreased while replacing CCS by ACC: on day $1,10 \%$ of CCS were replaced by $5 \%$ of elemental calcium from ACC; by day 14, $50 \%$ of CCS were replaced by $25 \%$ ACC; by day $21100 \%$ CCS were replaced by $50 \% \pm 10 \%$ ACC. Five patients consumed ACC before meals; 5 - after meals, the groups were similar in in BMI, age and ethnicity ( $p=0.17 ; 0.54 ; 1.0$ respectively). Serum albumin adjusted calcium (SAACa) and serum phosphorus (SP) were evaluated weekly; calciuria - at baseline and at day 28 .

Results: Initial elemental CCS doses ranged 1000$10800 \mathrm{mg} / \mathrm{d}$, alfacalcidol doses ranged $0.25-1.75 \mu \mathrm{g} / \mathrm{d}$ (remained unchanged during the study period). SAACa and SP were $8.1 \pm 0.15$ and $4.39 \pm 0.186($ mean \pm SEM) and $8.19 \pm 0.14$ and $4.4 \pm 0.12(p=0.6 ; 0.9)$ on day 1 and 28 respectively, while elemental ACC doses ranged 500$5400 \mathrm{mg} / \mathrm{d}$. During the trial SAACa was maintained between $7.2-9.2 \mathrm{mg} / \mathrm{dl}$. One patient had asymptomatic hypocalcemia during physical activity. Two hypercalciuric episodes were observed and treated.

Conclusion: Initial CCS dose was replaced in all subjects with ACC dose of $50 \% \pm 10 \%$ while SAACa remained stable. The study was sponsored by Amorphical Ltd., NesTziona, Israel.

\section{P437}

COMMON BONE PARAMETERS DO NOT CORRELATE WITH CORONARY CALCIFICATION IN TYPE 2 DIABETES PATIENTS

M. Cokolic ${ }^{1}$, M. Krajnc ${ }^{1}$

${ }^{1}$ Department of Endocrinology and Diabetology, University Medical Centre, Maribor, Slovenia

Objectives: To assess the impact of common bone parameters: serum calcium $(\mathrm{Ca})$, phosphate $(\mathrm{P})$, alkaline phosphatase (AP), 25-hydroxyvitamin D (25-OHD), intact PTH and BMD on the coronary calcification and its progression in asymptomatic type 2 diabetes patients.

Materials and methods: We included 45 type 2 diabetes patients without previously known cardiovascular disease. They were managed as out-patients, according to the newest national and European guidelines. We measured serum $\mathrm{Ca}, \mathrm{P}, \mathrm{AP}, 25-\mathrm{OHD}$ at baseline and after 18 months, PTH (EIA) at baseline. Femoral neck BMD was determined at baseline with DXA. Coronary calcification was measured as calcium score (CS) with 64-slice CT according to the Agatston's method at baseline and after 18 months. CS progression was defined as absolute and relative (in\%). Univariate analysis was performed with Spearman correlation coefficient.

Results: Patients were $51 \%$ males, age (average \pm SD) 59 \pm 8 years, diabetes duration $10 \pm 8$ years, $\mathrm{HbA} 1 \mathrm{c} 7.2$ $\pm 0.9 \%$. Median CS (interquartile range) at baseline was 63 (6.384), after 18 months 100 (13.532). Baseline average Ca $2.38 \pm 0.11 \mathrm{mmol} / 1$, P $1.15 \pm 0.17 \mathrm{mmol} / \mathrm{l}$, AF 1.21 $\pm 0.30 \mu \mathrm{kat} / 1,25$-OHD $58.0 \pm 35.1 \mathrm{nmol} / 1$, PTH 36.2 $\pm 17.3 \mathrm{pg} / \mathrm{ml}$, BMD $0.82 \pm 0.16 \mathrm{~g} /$. After 18 months average Ca $2.34 \pm 0.09$, P $1.11 \pm 0.16$, AP $1.21 \pm 0.27,25$-OHD $40.6 \pm 11.9$. There was no significant univariate correlation (p 0.05) of any of the studied bone parameters with baseline CS or absolute/relative change in CS. Due to the absence of univariate correlation, we did not perform multivariate analysis.

Conclusions: In asymptomatic type 2 diabetes patients, serum $\mathrm{Ca}, \mathrm{P}, \mathrm{AP}, 25-\mathrm{OHD}, \mathrm{PTH}$ and femoral neck BMD do not correlate with baseline coronary calcification or its absolute/ relative progression in 18 months. Our data do not support the impact of common bone parameters on subclinical vascular injury (coronary calcification). 


\section{P438}

PRIMARY CULTURES OF HUMAN SKELETAL MUSCLE SATELLITE CELLS: A NOVEL MODEL TO ASSESS SKELETAL MUSCLE CELL DIFFERENTIATION

L. Cianferotti ${ }^{1}$, C. Romagnoli ${ }^{1}$, M. Vigna ${ }^{1}$, R. Zonefrati ${ }^{1}$, C. Mavilia $^{1}$, G. Galli ${ }^{1}$, M. Innocenti ${ }^{2}$, G. Marcucci ${ }^{1}$, A. Tanini ${ }^{1}$, M. L. Brandi ${ }^{1}$

${ }^{1}$ Department of Surgery and Translational Medicine, Unit of Bone and Mineral Diseases, University of Florence, University Hospital of Florence, Florence, Italy, ${ }^{2}$ Department of Surgery and Translational Medicine, Plastic Surgery and Reconstructive Microsurgery Unit, University of Florence, University Hospital of Florence, Florence, Italy

Objectives: Myosatellite cells (MSCs) constitute small subpopulation (2-10\%) of mononuclear stem and progenitor cells residing in mature skeletal muscle. These cells lay in a niche between the plasma membrane of the myofiber and the basal laminal membrane. In adult skeletal muscle, MSCs are generally quiescent, but ready to be activated, self-renew and differentiate for muscle growth and regeneration. Aim of the study has been to isolate MSCs from human skeletal muscle and test their capacity to generate mature myofibers in vitro, to develop a useful model for studies of muscle differentiation and for the screening of new molecules to be employed in the treatment of sarcopenia and skeletal muscle diseases.

Materials and methods: Human MSCs have been isolated from biopsies of skeletal muscles (pectoralis major, recto abdominal muscles) in healthy young adult volunteers undergoing plastic surgery. The minced specimens have been digested with collagenase at $37{ }^{\circ} \mathrm{C}$ for $3 \mathrm{~h}$. Primary cells have been cultured in a specific growth medium (PromoCell cod.C39360) and differentiated for 14 days with appropriate differentiation medium (PromoCell cod.C-39366) for 14 days to evaluate the expression of main markers of myogenic differentiation by real-time-qPCR and immunocytochemistry.

Results: MSCs express PAX-7, the main early marker of myogenic differentiation. After 4 days in culture, elongated cells resembling myotubes have appeared in culture. After 10 days in culture a significant increase in the expression of markers of myogenic differentiation has been observed $(p<0.005$ with respect to baseline levels). The expression of the protein myosin heavy chain, characterizing mature striated muscle fibers, was confirmed.

Conclusions: MSCs derived from human skeletal muscle biopsies constitute a good model to study mechanisms of skeletal muscle differentiation and can serve to test in the future therapeutical agents for skeletal muscle diseases in a preclinical setting.

\section{P439}

INCIDENCE OF FOREARM AND ANKLE FRACTURES IN THE POPULATION OF EAST SIBERIA, RUSSIA

L. Menshikova $^{1}$, O. Dvoeglazova ${ }^{1}$

${ }^{1}$ Irkutsk State Medical Academy of Postgraduate Education, Irkutsk, Russian Federation

Objectives: the incidence of distal forearm fractures and ankle fractures in persons aged more than 40 years was carried out in Angarsk - city of Irkutsk region (East Siberia, Russia).

Material and Methods: Medical documentation of traumatological departments and stations for 2011-2012 years was analyzed. The information on the population number in the town, sex and age distribution were obtained in the regional statistical department. The osteoporotic fracture frequency was calculated for 100000 person/year in each age group (4044, 45-49, etc.).

Results: Among 111478 (2011) and 111729 (2012) citizens were registered 1264 distal forearm fractures and 309 ankle fractures. Distal forearm fractures incidence was 565.8/ $100000 \mathrm{p} / \mathrm{y}$ among females 735.3/100000 p/y and in males $300 / 100000 \mathrm{p} / \mathrm{y}$ (ratio female/male $-2.5: 1$ ). The highest fractures frequency was occurred at the age group of 65-69 years, amounting to $978 / 100000 \mathrm{p} / \mathrm{y}$ in women and 366/100000 p/y in men with decrease after 70 years. These fractures dominated in women of all age group. Ankle fractures incidence was $138.4 / 100000 \mathrm{p} / \mathrm{y}$ (in males - 126.5/100000 and among females $-146 / 100000 \mathrm{p} / \mathrm{y}$, ratio female/male $-1,2: 1)$. An increased of fractures was marked at the age 50-54 years in females $-207.8 / 100000 \mathrm{p} / \mathrm{y}$, in male at the age group 45-49 and after 70 years (145.1 and 141.5/100000 p/y correspondingly).

Conclusions: The incidence of distal forearm fractures were in 4 times higher than that of ankle fractures with reliable domination in women in all the age groups. The frequency ankle fractures is approximately equal in men and women. The data obtained lay the for developing the regional program of prophylaxis and treatment of osteoporosis.

\section{P440}

COMPARATIVE BETWEEN SURGICAL TREATMENT VS. MEDICAL TREATMENT IN DUPUYTREN'S DISEASE

M. Perez Santamaria ${ }^{1}$, M. J. Dominguez Arevalo ${ }^{1}$

${ }^{1}$ Hospital de Laredo, Laredo, Spain

Objective: The aim of the study was to compare the results between surgical (aponeurectomy) vs. medical treatment (injectable collagenase of Clostridium histolyticum $)^{1}$ in Dupuytren's disease. 
Material and methods: 62 patients were attended for this disease in our centre in the last two years. As inclusion criteria we used a single axis affected palmar and digital, discarding bleeding disorder, anticoagulant therapy, recurrent cases and only palmar affectation. Total patients studied was 27, 12 with surgery and 15 with injections. The treatment received was determined by the surgeon responsible of the patient. Age range: $49-81$ years. Distribution by sex: 25 males-2 females. Minimum 6 months follow-up. The treatment consisted in double injection of collagenase within a one month interval compared with classic surgical treatment (partial aponeurectomy) $)^{2}$.

Results: In the group treated with collagenase we observed objectively, on average, a $141^{\circ}$ to $21^{\circ}$ improvement of contracture $\left(5^{\circ}\right.$ metacarpal level; $16^{\circ}$ interphalangeal) in opposition to $143^{\circ}$ to $32^{\circ}$ of contracture in those who were treated surgically $\left(8^{\circ} \mathrm{MTC} ; 24^{\circ} \mathrm{IFP}\right)$. Lower rates of complications such as neurovascular injuries, infection or skin disorders were registered in collagenase group. Recovery time: 2 months in collagenase group vs. 6 months postsurgery. No recurrences were registered in both groups.

Conclusions: Injection of collagenase is a reasonable alternative in the treatment of Dupuytren's disease because it improves deformity effectively with a lower rate of complications $^{3}$, higher patients subjective acceptance and lower economic costs ${ }^{4}$.

References:

${ }^{1}$ Badalamente MA, et al. NEJM 2009;361:968.

2 McGrouter AD: Dupuytren's Contracture. Green's Hand Surgery. 5th Edition. Ed: Green DP, Hotchkiss RN, Pederson WC, Wolfe SW, New York 2007. Pp 159-169.

${ }^{3}$ Witthaut J, et al. J Hand Surg Am 2013;38:2.

${ }^{4}$ Chen NC, et al. J Hand Surg Am 2011;36:1826.

\section{P441}

\section{EFFICACY OF DENOSUMAB ON BONE MINERAL DENSITY IN PATIENTS WITH POSTMENOPAUSAL OSTEOPOROSIS}

M. Tsagareli ${ }^{1}$, E. Giorgadze $^{1}$, N. Dolidze ${ }^{1}$, T. Sulikashvili ${ }^{1}$, N. Jeiranashvili ${ }^{1}$

${ }^{1}$ National Institute of Endocrinology, Tbilisi, Georgia

Objective: To assess the efficacy of Denosumab on BMD in patients with postmenopausal osteoporosis.

Material and methods: 67 postmenopausal women from age 49-74 with osteoporosis and prior fragility fracture at vertebrae, hip or other sites were enrolled in the study. We measured ionized calcium $(\mathrm{Ca}++), 25(\mathrm{OH}) \mathrm{D}$ values, lumbar spine (LS) and proximal femur (PF) BMD values were obtained using DXA. $70 \%$ of patients revealed $25(\mathrm{OH}) \mathrm{D}$ values $<30 \mathrm{ng} / \mathrm{ml}$. All patients received $60 \mathrm{mg}$ Denosumab injections + elemental calcium $500-1000 \mathrm{mg} /$ day (calcium carbonate)+ Vit D 800-1000 IU/day for 12 months. Denosumab therapy was initiated after achieving 25(OH)D levels $>30 \mathrm{ng} / \mathrm{ml}$. Bone density was measured at baseline and after 12 months.

Results: At the baseline the mean value of T-score was -2.8 $\mathrm{SD}$ at LS and $-2.7 \mathrm{SD}$ at PF. After a year of commencing treatment majority of patients ( $80 \%$ ) revealed significant increase of BMD with mean value of $5.6 \%$ at LS and $3.9 \%$ at PF.

Conclusion: Administration of $60 \mathrm{mg}$ denosumab injections every 6 months effectively increases BMD at LS and $\mathrm{PF}$.

\section{P442}

\section{SHOULDER PAIN AFTER SUBACROMIAL} INJECTION

M. J. Dominguez Arevalo $^{1}$, M. Perez Santamaria ${ }^{1}$

${ }^{1}$ Hospital de Laredo, Laredo, Spain

Objective: To describe an unusual cause of humeral head necrosis not mentioned until now in medical literature.

Material and Methods: a 78 years old woman was referred with shoulder pain lasting several months without improvement with conservative measures. Her clinical history was irrelevant,. We decided to order a X-ray study which was consistent with a rotator cuff arthropathy (Fig. 1). She was suffering intense pain, so we decided to offer her a subacromial injection with corticosteroids. At the follow-up four weeks later, her clinical status become deteriorated with a worsening of pain control. New $\mathrm{X}$-ray study was made and showed a massive destruction of humeral head (Fig. 2). Complementary studies were made in order to prove a septic or tumoral etiology that could explain the quickness of bone necrosis evolution. All studies were consistent with a humeral head necrosis without a clearly defined etiology.

Results: in view of pathological changes (massive rotator cuff tear plus humeral head destruction) we performed an inverted shoulder arthroplasty. Microbiologic tests were negative and pathology examination showed only bone necrosis. Currently the patient shoulder remains painless and she is capable to do activities of daily leaving without limitations.

Conclusion: humeral head necrosis is the second site most frequent of osteonecrosis. It is often associated with corticosteroids use, usually at high doses and over a long time period. The physiopathology is not well understood. It is believed that some changes of fat metabolism lead to ischemic events within the bone, mostly at subchondral level. The most relevant event of this case was that subacromial injection was the only risk factor that could be linked with this 
quick progression to bone necrosis, not mentioned before in medical literature.

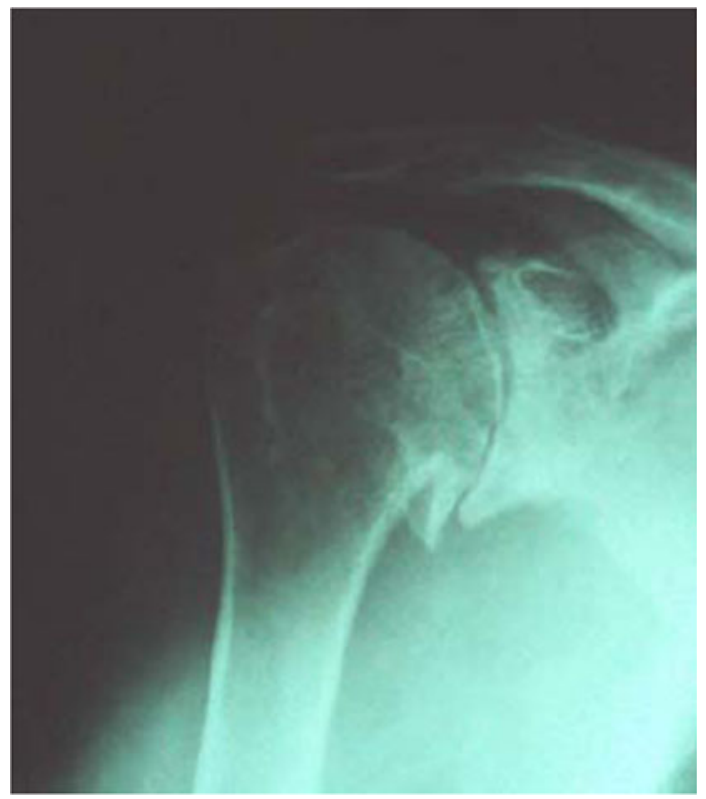

Fig. 1

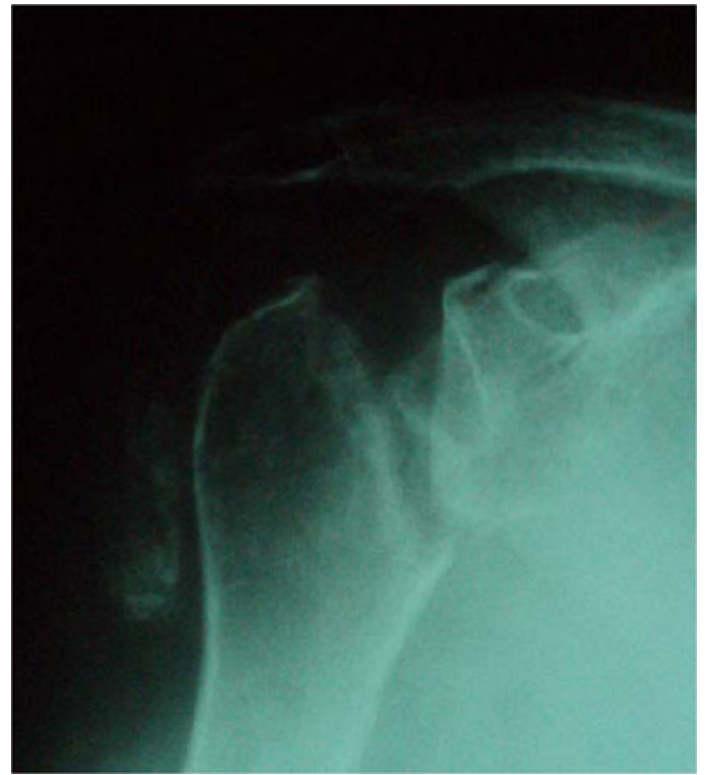

Fig. 2

\section{P443 \\ DEVELOPMENT OF BONE MINERAL DENSITY IN ADOLESCENT: A PROSPECTIVE 3- YEAR FOLLOW-UP STUDY}

L. Menshikova $^{1}$, A. Menshikov ${ }^{2}$, T. Maksikova ${ }^{3}$, L. Dats ${ }^{1}$

${ }^{1}$ Irkutsk State Medical Academy of Postgraduate Education, Irkutsk, Russian Federation, ${ }^{2}$ Region Clinical Diagnostic Center, Irkutsk, Russian Federation, ${ }^{3}$ Irkutsk State Medical University, Irkutsk, Russian Federation
Objectives: To assess BMD and bone mineral content (BMC) in Irkutsk (East Siberia) adolescent in 3-year follow-up study, the frequency and the risk factors of low level BMD for chronological age.

Material and Methods: 217 adolescents (132 girls and 85 boys) aged 14-17 years were included. BMD was evaluated by DXA (Lunar, USA) with reference pediatric base in lumbar spine (L2-L4) were defined twice in dynamics. At 29 adolescents with low values of BMD (Z-score $\leq-2 \mathrm{SD}$, ISCD) the densitometry parameters were measured in the third time. Anthropometrical parameters, questionnaire study considering risk factors were performed.

Results: The positive dynamics of densitometry parameters was observed at the second stage of the study in the whole group $(n=217)$. At primary inspection the values of BMD and BMC were $1.049 \pm 0.155 \mathrm{~g} / \mathrm{cm}$ and $34.94 \mathrm{~g}$, at the second stage $-1.059 \pm 0.125 \mathrm{~g} / \mathrm{cm}$ and $41.98 \pm 8.36 \mathrm{~g}$. Frequency of low BMD (Z-score $\leq-2)$ for chronological age at adolescents was $10.6 \%$ at the first stage of the study and $16.1 \%$ on the second. Both at boys and girls at the second age of the study BMC and BMD positively correlated with height, weight, arms span and muscle strength $(p<0.001)$. Besides that absorptiometry parameters in girls negatively correlated with age of menses onset. Statistically significant distinctions $(p<0.001)$ were defined between adolescents with that score $\leq-2$ and Z-score $>-2$. The boys in those groups differed in height, weight, arms span and muscle strength, the girls - in height, weight, age of menarche, morbidity at backbone pressing $(p<0.001)$.

Conclusions: The estimation of BMD and BMC among adolescents, studding of changes densitometry parameters and risk factors of low BMD for chronological age at adolescents allows to reveal in due time children with low parameters BMD and BMC and to warn development of osteoporosis at later age.

\section{P444}

EVALUATION OF DENTAL HEALTH IN PATIENTS WITH OSTEOPOROSIS

I. Mazur $^{1}$, I. Bilozetskyi ${ }^{2}$

${ }^{1}$ Department of Dentistry PL Shupyk National Medical Academy of Postgraduate Education, Kyiv, Ukraine, ${ }^{2}$ Department of Surgical Dentistry I.Ya.Horbachevsky Ternopil State Medical University, Ternopil, Ukraine

The etiology of periodontitis is multifactorial, but one particular focus of interest has been whether osteoporosis is a contributory factor. Studies have shown different results on the impact of osteoporosis on periodontal status in the general population, and in case of rheumatoid arthritis (RA) such data is very limited. Aim: To determine the dental condition of patients with osteoporosis. 
Materials and methods: The study involved 60 patients with RA who were divided into three groups: normal $\operatorname{BMD}(n=17)$; osteopenia ( $n=15)$ and osteoporosis (OP) $(n=24)$. All patients conducted X-ray examination - diagnostics state BMD using dual-energy $\mathrm{x}$-ray by densitometry (DPX-A «Lunar»). Clinically, was studied on the following indicators - the CFE (caries, filling, extracted), number of teeth; the periodontal health: OHI-s, papillary marginal attached (PMA) index, periodontal index (PI), community periodontal index of treatment needs (CPITN) indexes; panoramic radiography (PMX) allows studies of mandibular cortical index (MCI).

Results: The results stated that all indexes are highly significant. Established that overall dental condition indicators CFE $(p=0.0003)$ and number of teeth $(\mathrm{r} 0-1=0.029112 ; \mathrm{r} 0$ $2=0.000017$; r1 $-2=0.004026$ ) was better in patients with a presence of normal $\mathrm{BMD}$, whereas in its reduction observed their deterioration. Patients with OP demonstrated significant periodontal destruction compared with those who had normal BMD OHI-s $(p=0.0001)$, PI $(p=0.0001)$, PMA $(=0.000771$ r0-1; r0-2 $=0.000009)$ and CPITN $(1-\mathrm{r} 0-1=0.0398$; r0$2=0.0057 ; \mathrm{r} 0-22=0.0036$ ). Dentistry study showed the deterioration of dental health using $\mathrm{MCI}$ index, as a marker of systemic osteoporosis $(\mathrm{C} 1-\mathrm{r} 0-2=0.0006 ; \mathrm{r} 1-2=0,0047$; $\mathrm{C} 3$ $\mathrm{r} 0-2=0.0004)$, the level of resorption $\mathrm{C} 1$ and $\mathrm{C} 3$ were significantly higher in patients with OP compared with osteopenia. Conclusion: Our results show that BMD is an important indicator that affects severity of periodontitis and dental health, as well as bone density of the jaws.

\section{P445}

\section{EFFECTS OF DAILY PHYSICAL ACTIVITY} AND BODY COMPOSITION ON BONE TURNOVER MARKERS IN YOUNG ADULT MEN

$\underline{\text { C. Verroken }}{ }^{1}$, B. Lapauw ${ }^{1}$, H. Zmierczak ${ }^{1}$, S. Goemaere ${ }^{1}$, J.M. Kaufman ${ }^{1}$

${ }^{1}$ Unit for Osteoporosis and Metabolic Bone Diseases, Ghent University Hospital, Ghent, Belgium

Objective: Biochemical markers of bone turnover are higher in young adult men than in middle-aged men or young adult women, but little is known about the determinants hereof. We aimed to investigate associations of body composition, daily physical activity (PA), and estimates of mechanical loading with bone turnover in healthy young men.

Material and Methods: A cross-sectional, population-based sibling study was conducted in 1001 healthy men aged 25-45 years. Bone turnover markers including osteocalcin (OC), procollagen type 1 amino-terminal propeptide (P1NP), and C-terminal telopeptide of type 1 collagen (CTX) were measured from fasting serum samples. Body composition was assessed using DXA. PA levels at work, during sports activities, and during leisure time excluding sport were scored using a questionnaire as proposed by Baecke et $\mathrm{al}^{1}$. Estimates of mechanical loading include pQCT-derived muscle crosssectional area (CSA) at the forearm and lower leg, grip strength, and biceps and quadriceps peak torque.

Results: Median OC, P1NP and CTX levels were 21.7 (25th75th percentile 18.5-26.3) $\mu \mathrm{g} / \mathrm{L}, 50.8$ (41.7-63.7) $\mu \mathrm{g} / \mathrm{L}$ and 0.41 $(0.31-0.52) \mu \mathrm{g} / \mathrm{L}$. In age and height-adjusted analyses, all bone turnover markers correlated inversely with fat mass $(\beta=-0.21$, $\beta=-0.13$ and $\beta=-0.19, p<0.001)$, and $\mathrm{OC}$ and CTX correlated inversely with lean mass $(\beta=-0.15$ and $\beta=-0.09, \mathrm{p} \leq 0.009)$. When fat and lean mass were both included in the regression models, only fat mass remained an independent determinant of the bone turnover markers $(\beta=-0.20, \beta=-0.16$ and $\beta=-0.21$, all $p<0.001)$. Sport index but not total PA correlated positively with the bone turnover markers after adjustment for age, height and body composition $(\beta=0.09, \beta=0.11$ and $\beta=0.07$, $\mathrm{p} \leq 0.020$ ). No associations were found with muscle CSA, grip strength, or biceps or quadriceps peak torque.

Conclusion: Whereas bone turnover in healthy young men correlates inversely with the amount of fat mass and positively with PA levels during sports activities, it is not associated with the amount of lean mass or other estimates of mechanical loading. These findings suggest that the relatively high levels of biochemical markers of bone turnover in young adult men may only for a minor part be explained by higher physical activity.

Reference: ${ }^{1}$ Baecke et al., Am J Clin Nutr 1982;36:936.

\section{P446}

ASSOCIATIONS OF INSULIN SENSITIVITY WITH CORTICAL BONE GEOMETRY IN HEALTHY ADULT MEN

$\underline{\text { C. Verroken }}^{1}$, B. Lapauw ${ }^{1}$, H. Zmierczak ${ }^{1}$, S. Goemaere ${ }^{1}$, J.M. Kaufman ${ }^{1}$

${ }^{1}$ Unit for Osteoporosis and Metabolic Bone Diseases, Ghent University Hospital, Ghent, Belgium

Objective: In type 2 diabetes mellitus, fracture risk is increased despite preserved BMD. This might in part be due to insulin resistance affecting bone geometry; however, studies investigating the associations of insulin sensitivity with bone geometry are lacking. We explored this relationship in a cohort of healthy, young adult men.

Material and Methods: A cross-sectional, population-based sibling study was conducted in 1001 healthy men aged 25-45 years. Cortical bone geometry at the radius and the tibia $(66 \%$ from distal end point) was assessed by pQCT. Insulin and glucose were measured from fasting serum samples; insulin resistance was evaluated using the homeostasis model assessment of insulin resistance (HOMA-IR).

Results: In age and BMI-adjusted analyses, HOMA-IR and insulin levels correlated inversely with periosteal circumference (PC), endosteal circumference (EC) and polar strength- 
strain index (SSIp) at both the radius (PC: both $\beta=-0.13$, $p<0.001$; EC: both $\beta=-0.08, \mathrm{p} \leq 0.031$; SSIp: $\beta=-0.14$ and $\beta=-0.16, p<0.001$ ) and tibia (PC: both $\beta=-0.12, p<0.001$; EC: $\beta=-0.10$ and $\beta=-0.09, p \leq 0.007$; SSIp: both $\beta=-0.10$, $\mathrm{p} \leq 0.003$ ). Moreover, insulin levels but not HOMA-IR correlated inversely with cortical thickness at the radius $(\beta=-0.07$, $p=0.048)$. After adjustment for DXA-derived body composition (total fat and lean body mass) instead of BMI, the associations of HOMA-IR and insulin with PC and SSIp at both the radius and the tibia and with $\mathrm{EC}$ at the tibia remained significant (B-coefficients from -0.07 to -0.11 ), whereas the associations of HOMA-IR and insulin with EC and the association of insulin with cortical thickness at the radius lost significance.

Conclusion: In this cohort of healthy young men, insulin sensitivity correlates with cortical bone size at least in part independently of body composition. Given the important contribution of bone geometry to overall bone strength, this might contribute to the paradoxically increased fracture risk in patients with type 2 diabetes mellitus. Whether this association is a direct consequence of impaired insulin signaling in bone tissue or reflects indirect effects through modulation of the muscle-bone relationship remains to be established.

\section{P447}

\section{INITIATION OF ANTI-OSTEOPOROTIC} TREATMENT AND MEDICAL RESOURCE UTILIZATION IN FRANCE BETWEEN 2007-2013

M. Belhassen ${ }^{1}$, C. B. Confavreux ${ }^{2}$, B. Cortet ${ }^{3}$, L. Lamezec ${ }^{4}$, M. Ginoux ${ }^{1}$ E. Van Ganse ${ }^{1}$

${ }^{1}$ Lyon Pharmaco-Epidemiology Unit- UMR 5558 CNRS-Claude Bernard Lyon 1 University, Lyon, France, ${ }^{2}$ INSERM UMR 1033 - University of Lyon, Rheumatology Department, Hôpital Edouard Herriot, Hospices Civils de Lyon, Lyon, France, ${ }^{3}$ Department of Rheumatology, Lille University Hospital, Lille, France, ${ }^{4}$ Merck Sharp \& Dohme, Courbevoie, France

Objective: Using the French epidemiological cohort "Permanent Beneficiaries Sample" database (EGB), we conducted a study to describe anti-osteoporotic strategies and medical resources utilization (MRU) in patients treated for osteoporosis between 2007 and 2013.

Material and methods: EGB is a 1/97th representative sample of French health insurance beneficiaries indicating that in the cohort, an observation for 1000 individuals reflects 97 , 000 patients in the whole French health insurance. We included all patients aged $\geq 50$ years who received a first antiosteoporosis treatment in 2007 and who completed 6 years of follow-up. We collected data on all anti-osteoporotic drugs, hormone replacement therapy, consumption of osteoporosisrelated therapy and medical cares. Data were collected at entry and during a follow-up period of 6 years.
Results: We identified 2,219 patients. Mean age was $68.5 \mathrm{y}$ $( \pm 10.2)$. There were 1,994 women $(89.9 \%) .1,539$ patients $(69.4 \%)$ received oral bisphosphonate (BP) as first line therapy and $12(0.5 \%)$ received zoledronic acid. Respectively 454 patients $(20.5 \%)$ and 6 patients $(0.3 \%)$ started strontium ranelate and teriparatide. At baseline, 212 patients (9.6\%) underwent bone densitometry testing, 407 (18.3\%) have visited a rheumatologist, $653(29.4 \%)$ a nurse, and $644(29.0 \%)$ a physiotherapist. $1,328(78.9 \%)$ patients treated by alendronic acid had no switch during the follow-up, as 872 patients (78.1\%), 85 patients $(84.2 \%)$, and 489 (77.3\%) treated by risedronic acid, zoledronic acid and raloxifene, respectively. Only $58.8 \%$ of patients treated by teriparatide had a switch. Among patients who switch after teriparatide, $90 \%$ went on with a BP and $10 \%$ with strontium ranelate. During the baseline period, 426 patients (19.2\%) presented a hospitalization for a related osteoporosis. By contrast, only between $1.3 \%$ and $2.6 \%$ of patients were hospitalized during follow-up.

Conclusion: This study allowed us to describe the MRU of a cohort of ambulatory osteoporotic patients, over a 6-year period. As expected, the most frequently prescribed treatment class were oral weekly bisphosphonates. Initiation of antiosteoporotic treatment reduced MRU during the follow-up. Medical education is needed to develop the use of the bone densitometry testing.

\section{P448 \\ PREVALENCE AND RISK FACTORS OF OSTEOPOROSIS IN PATIENTS WITH ANKYLOSING SPONDYLITIS: A 7-YEAR RETROSPECTIVE STUDY OF 133 CASES}

K. Baccouche $^{1}$, D. Amri ${ }^{1}$, S. Belghali ${ }^{1}$, Z. Alaya ${ }^{1}, \mathrm{H}$. Zeglaoui $^{1}$, E. Bouajina ${ }^{1}$

${ }^{1}$ Rheumatology, Farhat Hached Hospital, Sousse, Tunisia

Objectives: To assess the prevalence and risk factors of osteoporosis (OP) in patients with ankylosing spondylitis (AS).

Methods: Demographic and clinical data of 133 AS patients were collected. BMD measurements of the lumbar spine and proximal femur were performed by DXA.

Results: Osteoporosis was found in $66.7 \%$ of patients. The average age of onset of the disease was 27 years. The average development time was 12.24 years. The BMD was significantly lower in patients with elevated erythrocyte sedimentation rate (ESR) or C-reactive protein (CRP) than patients with normal ESR and CRP. Juvenile onset, morning stiffness lasting over 0.5 hours, spine involvement and elevated ESR levels were risk factors for bone loss at the lumbar spine; female gender, older age, hip involvement and lack of regular treatment were risk factors for bone loss at the femur. Hip and/or spine involvement and persistent elevated ESR levels, 
but not GCs treatment, were associated with decreased BMD at both the lumbar spine and the femur.

Conclusion: High disease activity, juvenile onset, spine and hip involvement are risk factors of bone loss in patients with AS.

\section{P449}

SYMPTOMATIC SHORT TERM EFFECT OF CRYSTALLINE GLUCOSAMINE SULFATE IN OSTEOARTHRITIS OF THE KNEE

K. Filipovic ${ }^{1}$, J. Zvekic-Svorcan ${ }^{1}$, T. Jankovic ${ }^{1}$, S. Tomasevic-Todorovic $^{2}$, C. Demesi Drljan ${ }^{3}$, G. Galetic ${ }^{3}$

${ }^{1}$ Rheumatology, Special Hospital for Rheumatic Diseases, Novi Sad, Serbia, ${ }^{2}$ Rheumatology, Clinic for Medical Rehabilitation, Clinical Center of Vojvodina, Novi Sad, Serbia, ${ }^{3}$ Institute for Child and Adolescent Healt Care of Vojvodina, Medical Faculty Novi Sad, University of Novi Sad, Novi Sad, Serbia

Aim: Research and establish symptomatic effect of crystalline glucosamine sulfate (CGS) (1.5 g/day) in knee osteoarthritis. CGS treatment was compared to nonsteroid antiinflammatory drugs (NSAID) (ibuprofen 1200-1600 mg/ day; diclofenac sodium $75 \mathrm{mg}$ /day).

Material and method: The open, prospective study with one month included 111 outpatients of both genders, 55 years of age or more. Study was carried out in the Special hospital for rheumatic diseases in Novi Sad. Assessment of therapy effect has been done using clinical (WOMAC index and Lequesne index). Results: After one month, a statistically significant reduction of pain and stiffness with improvement of joint function $(p<0.05)$ was noted in patients from NSAID group. In this period, both groups had lower Lequesne index, without statistical significance $(p>0.05)$.

Conclusion: Although is not shown clinical efficacy CGS (WOMAC pain, stiffness and function) patients who taking CGS had less difficulty in activities of daily life and walking (measured by Lequesne index).

\section{P450}

VANISHING TESTES SYNDROME WITH UNANTICIPATED NORMAL BONE MINERAL DENSITY

A.-T. erban ${ }^{1}$, D.-S. Cristofor ${ }^{2}$

${ }^{1}$ Medlife, Bucharest, Romania, ${ }^{2}$ Hexi Med, Bucharest, Romania

Objective: We present a case of a young hypogonadal patient with vanishing testes syndrome (also referred as testicular regression syndrome) and normal BMD, despite inconsistent androgen replacement therapy given till presentation.

Material and Methods: A 19 years old male was diagnosed at birth with bilateral anorchidism and underwent inconstant oral androgen supplementation after the age of 14 for less than 2 years. A total of two MRI's and two surgical explorations excluded the presence of testicular tissue remnants; his karyotype was $46, \mathrm{XY}$. The following clinical data were noted at presentation: height $182 \mathrm{~cm}$, weight $81 \mathrm{~kg}$, BMI $28 \mathrm{~kg} / \mathrm{m} 2$, eunucoidal habitus (arm span $193 \mathrm{~cm}$, upper segment/lower segment ratio $82 / 100 \mathrm{~cm}$ ), incomplete virilisation, no palpable testes in the scrotum but normal penis development, pubic hair Tanner stage P5, adipomastia without significant gynecomastia. Blood tests showed low haemoglobin, low testosterone levels with high FSH and LH, low levels of inhibin B, undetectable antimullerian hormone, normal thyroid and adrenal function. Subsequently, we initiated androgen therapy with testosterone undecanoate given via intramuscular injection.

Results: Considering the open growth plates in our patient, we performed whole body DXA for the measurement of BMD, which proved unexpectedly normal. Normal testosterone and LH levels were achieved after 6 months of treatment but his bone age remained delayed (approximately 17 years old). During the first year of treatment he continued to grow $4 \mathrm{~cm}$.

Conclusion: Hypogonadism in adults is an indication for testing BMD because it can be associated with low bone mass despite androgen replacement therapy. We intend to reassess the BMD in our patient because the data show inconstant response of bone mass to testosterone replacement.

\section{P451 \\ LOW BONE MINERAL DENSITY IN PATIENTS AT DIAGNOSIS OF CELIAC DISEASE}

K. Baccouche ${ }^{1}$, D. Amri ${ }^{1}$, M. Bouzaoueche ${ }^{1}, \mathrm{~S}$. Belghali $^{1}, \mathrm{H}$. Zeglaoui $^{1}$, E. Bouajina ${ }^{1}$

${ }^{1}$ Rheumatology, Farhat Hached Hospital, Sousse, Tunisia

Background: Low BMD is considered an extra-intestinal manifestation of celiac disease with reduced bone mass, increased bone fragility, and risk of fractures. Celiac disease is considered a condition at high risk for secondary osteoporosis and the evaluation of bone density is very important in the clinical management of these patients.

Objective: The present study aimed to investigate bone alterations in celiac patients at diagnosis, correlating the findings with age and gender.

Methods: Patients who were included have consulted our department of rheumatology from January 2002 to 
December 2015. The diagnosis of celiac disease was done through clinical, serological and histological findings. All data were collected from the medical charts of the patients. After the diagnosis of celiac disease, evaluation for low BMD was requested by DXA. DXA bone densitometer was used to estimate low BMD at the lumbar spine and femur.

Results: A total of 8 patients, $100 \%$ female, with mean age of 35.7 years (16-60) were included.

At celiac disease diagnosis, 4 were younger than 30 years, 3 were between $31-50$ years, and 1 was older than 50 years. All patients presented low BMD, being 1 with osteopenia and 7 with osteoporosis. The means vertebral T- score and femoral T-score were respectively, $-3.5 \mathrm{SD}(-2.5$ to -5.7$)$ and $-3 \mathrm{SD}$ $(-2.5$ to -4.1$)$. It was osteomalacia in 3 cases. The low BMD was indicative of celiac disease in 3 cases. All patients were treated with a gluten-free diet associated with calcium and vitamin D substitution. we have not checked the BMD.

Conclusion: This study demonstrated that $100 \%$ of our patients with celiac disease at diagnosis had low BMD. Hence the interest of systematic practice of the BMD in patients with celiac disease.

\section{P452}

FRAX AND MANDIBULAR SPARSE TRABECULATION AS FRACTURE PREDICTORS: A LONGITUDINAL STUDY 1980-2002

V. Sundh ${ }^{1}$, G. Jonasson ${ }^{2}$

${ }^{1}$ Dept. of Public health and Community Medicine, Institute of Medicine, Sahlgrenska Academy, University of Gothenburg, Gothenburg, Sweden, ${ }^{2}$ Department of Behavioral and Community Dentistry, Institute of Odontology at the Sahlgrenska Academy, University of Gothenburg, Gothenburg, Sweden

Objectives: The purpose of this study was to analyze which cutoff value for FRAX would provide the best fracture risk prediction in middle-aged and elderly women and to examine if visual assessments of mandibular trabecular bone may improve the FRAX tool prediction.

Materials and Methods: From the Prospective Population Study of Women in Gothenburg data two baseline examinations in 1981 and 1992 with follow-up information on fracture events during approximately ten years were available for 499 of the women 1981-1992 and for 413 women 1992-2002. FRAX was calculated and mandibular trabeculation assessed as dense, mixed dense plus sparse, and sparse.

Results: Both sparse trabeculation and FRAX $>15 \%$ were in a common model associated with a more than 2 -fold increase in age-adjusted risk for future fracture in the younger group, and a 3 to 4 -fold increase in the elder group. In individuals with neither of these two factors, the fracture risk was $8.5 \%$ and $1.5 \%$ during the two follow-up periods. In individuals with one of the two factors it was $18 \%$ and $16 \%$, and among those with both factors it was $50 \%$ and $34 \%$. Considered as two independent risk factors, sparse trabeculation and FRAX $>15$ had approximately the same predictive value, with a clear advantage to FRAX $>15$ in the elder group, but both gave a significant improvement to the prediction model when added as covariate. The improvement in predictive power, as measured by AUC (Area Under Curve), by adding sparse trabeculation to the model after FRAX $>15$ was an increase from $0.59(C I=0.54-0.64)$ to $0.66(C I=0.60-0.73)$ for the younger group, and from $0.69(C I=0.63-0.74)$ to $0.75(C I=0.70-0.81)$ in the elder group. Of the separate items in FRAX, previous fracture, BMI $(\mathrm{BMI}<19)$ and body height $(>160 \mathrm{~cm})$ showed (a more than marginal) significant association with higher fracture risk for the younger group, but none of them were significant as separate effects in the elder group.

Conclusion: Both FRAX $>15 \%$ and sparse trabeculation predicts fracture independently of each other, and their predictive power is better at a higher age. FRAX is a well working tool in the current sample with cut-off value set to $>15$, and mandibular sparse trabeculation has an additive effect.

\section{P453}

IMPACT OF ADHERENCE TO ANTI-OSTEOPOROTIC DRUGS ON THE OCCURRENCE OF FRACTURES

M. Belhassen $^{1}$, B. Cortet $^{2}$, C. B. Confavreux ${ }^{3}$, L. Lamezec ${ }^{4}$, M. Ginoux ${ }^{1}$, E. Van Ganse ${ }^{1}$

${ }^{1}$ Lyon Pharmaco-Epidemiology Unit- UMR 5558 CNRSClaude Bernard Lyon 1 University, Lyon, France, ${ }^{2}$ Department of Rheumatology, Lille University Hospital, Lille, France, ${ }^{3}$ INSERM UMR 1033 - University of Lyon, Rheumatology Department, Hôpital Edouard Herriot, Hospices Civils de Lyon, Lyon, France, ${ }^{4}$ Merck Sharp \& Dohme, Courbevoie, France

Objective: Using the French epidemiological cohort "Permanent Beneficiaries Sample" database (EGB), we conducted a nested-case control study to assess the impact of adherence to anti-osteoporotic treatment on the occurrence of fractures, between 2007-2013.

Material and methods: EGB is a 1/97th representative sample of French health insurance beneficiaries indicating that in the cohort, an observation for 1000 individuals reflects 97 000 patients in the whole French health insurance. We identified all patients aged $\geq 50$ years who received an anti- 
osteoporotic treatment between 2007-2013. Cases were defined as patients presenting an osteoporosis (OP)-related fracture leading to hospitalization between January 1, 2008 ( $\geq 2$ year history required) and December 31, 2013. Controls were hospitalized patients at the time where the matched case presented the event studied but without OP-related fracture. Cases and controls were matched on age ( \pm 5 years), gender, duration of their participation in the cohort, and main comorbidities. Cases and controls were compared for their respective exposure to bisphosphonate (BP) during the 24 months before index date. Co-variates of the models were antiosteoporotic drugs (except BP), oral corticosteroids, diuretics, NSAIDs, cardiovascular drugs, anti-hypertensives and vasodilators.

Results: Among the 9859 OP patients included in the cohort, we found $434(4.4 \%)$ cases and 1,123 controls. In the cases group, $311(71.7 \%)$ patients received at least one time a BP in the last 24 months compared to the controls (827 patients, $73.6 \%)(p=0.29)$. Over the 24 months before index date, patients with a Cumulative Medication Adherence (CMA) of $\leq 20 \%,>80 \%$ and $>90 \%$ were $162(37.3 \%$ ), $154(35.5 \%)$ and $112(25.8 \%)$, in comparison to controls (384 (34.2\%), 441 (39.3\%), 299 (26.6\%); $p=0.29)$. A CMA of $80 \%$ does not significantly reduce the risk of fractures related to osteoporosis $(O R=0.851$, IC95\% [0.668-1.084]). Nevertheless, patients with high coverage $(>90 \%)$ had a $25 \%$ decreased risk of fracture in comparison with low $(<10 \%)$ covered patients $(O R=0.741$, IC95\% [0.608-0.903], $p=0.003)$.

Conclusion: In this study, adherence to anti-osteoporotic treatment affected the occurrence of fractures when comparisons were performed between extreme patients with a very high coverage with a $25 \%$ decreased risk of fracture.

\section{P454}

RISK FACTORS FOR POSTMENOPAUSAL OSTEOPOROSIS IN BOSNIAN WOMEN: CASE SERIES STUDY

S. Sokolovic $^{1}$, R. Alimanovic-Alagic ${ }^{2}$

${ }^{1}$ University Clinical Center \& Medical Faculty, Sarajevo, Bosnia and Herzegovina, ${ }^{2}$ University Clinical Center Sarajevo, Sarajevo, Bosnia and Herzegovina

Introduction: Osteoporosis affects every third postmenopausal women in general. Primary prevention include the risk management, lifestyle changes, medication and physical therapy. It is necessary to obtain all risk factors for osteoporosis in order to provide the comprehensive programme including patient education, adherence and compliance. The measurement of the BMD should be done in all women, especially with a known risk factors.

Objective: To evaluate postmenopausal osteoporosis risk factors in Bosnian women.

Material and Methods: This is a retrospective clinical analysis of total 117 Bosnian postmenopausal women. The Inclusion criteria was the postmenopausal osteoporotic women detected by BMD. Exclusion criteria was other related osteoporosis including. The statistical analysis included a composite score.

Results: Average age of total 117 postmenopausal women was 65 age. BMI in average was 23.5. BMD was average -3.1 for the hip and -3.4 for the lumbar spine. The following risk factors were observed: Sedentary lifestyle in 26 women, coffee consumption in 94 women $>4$ cups/day, smoking in 46 patients, family history of fracture $=4$ and vitamin $\mathrm{D}$ level was in average 16.9 in $82 \%$ of patients.

Discussion: This study of 117 subjects showed the following postmenopausal risk factors among case series Bosnian women: coffee consumption, deficient and insufficient vitamin D, smoking, decreased BMI and sedentary lifestyle.

\section{P455}

AUTOMATED DIRECT MEASUREMENT OF FREE 25OH VITAMIN D

N. Heureux $^{1}$, M. Geurts ${ }^{2}$, L. M. Swinkels ${ }^{2}$, E. Lindhout ${ }^{2}$, G. Mayer $^{2}$, M. Martens ${ }^{2}$

${ }^{1}$ DIAsource Immunoassays, Louvain-la-Neuve, Belgium, ${ }^{2}$ Future Diagnostics Solutions, Wijchen, Netherlands

The objective of this project was to implement and validate an ELISA assay for the direct measurement of free 25-hydroxyvitamin D (25OH Vit D) on the Dynex DS2 platform. Recent studies suggest that the concentration and genotype of Vitamin D binding protein (DBP) are important factors that determine the bioavailability of $25 \mathrm{OH}$ Vit $\mathrm{D}$ in blood. It has been suggested that measurement of free, non-protein bound $25 \mathrm{OH}$ Vit D in serum, may provide more relevant diagnostic information than total $25 \mathrm{OH}$ Vit $\mathrm{D}$, for instance in chronic kidney disease, bladder cancer and pancreatic cancer, or in hemodialysis patients. After adaptation of the assay protocol on the instrument, manual and automated results were compared in terms of dose response curve, accuracy, precision, sensitivity and drift. An assay that reproducibly determines the level of free $25 \mathrm{OH}$ Vit D in serum was implemented and validated on an open ELISA platform. This can be used as a valuable tool in studies to establish the clinical relevance of free $25 \mathrm{OH}$ Vit D. 


\section{P456}

\section{THE THERAPEUTIC EFFECT OF DENOSUMAB IN OSTEOPOROSIS PATIENTS}

T. Fujito ${ }^{1}$, H. Kanda ${ }^{1}$, S. Sato ${ }^{1}$, M. Maeda ${ }^{1}$, K. Mori ${ }^{1}$, Y. Hattori $^{1}$, A. Kaneko ${ }^{1}$, N. Takahashi ${ }^{1}$, D. Kida ${ }^{1}$

${ }^{1}$ National Hospital Organization Nagoya Medical Center, Aichi, Japan

Purpose: Denosumab is a fully human monoclonal antibody against RANKL which was released as a therapeutic drug of the osteoporosis in Japan. We examined the usefulness of it, based on a use experiences.

Patients and Methods: In 148 osteoporosis patients who started denosumab from July 2013 to July 2014 in our hospital, we investigated a difference of BMD with lumbar vertebrae and trans-cervical of femur, bone metabolism markers (TRACP-5b, urinary NTx/Cre, BAP), for before and after one year denosumab administration. We also observed a cancellation reason and a continuation rate. Baseline demographics were shown in Table 1. Then, patients were divided into two groups: rheumatoid arthritis (RA) group and non-RA group. Furthermore, statistical analysis was performed between two groups.

Results: Although lumbar vertebrae BMD were significantly increased, but the transcervical BMD were not significantly increased (Table 2). All bone metabolism markers were significantly decreased.

\begin{tabular}{lll}
\hline Table 1 & $N=148$ \\
\hline Age (year) & & 73.4 \\
Sex (\% female) & 95.9 \\
Previous fracture (\%) & 43.9 \\
Previous medication (\%) & 91.2 \\
\hline & & \\
\hline Table 2 & control & $1 \mathrm{Y}$ \\
\hline lumbar vertebrae BMD $\left(\mathrm{g} / \mathrm{cm}^{2}\right)$ & 0.817 & 0.864 \\
trans-cervical BMD $\left(\mathrm{g} / \mathrm{cm}^{2}\right)$ & 0.537 & 0.548 \\
TRACP-5b $(\mathrm{mU} / \mathrm{dL})$ & 456.8 & 263.7 \\
Urinary NTx/Cre $(\mathrm{nmoIBCE} / \mathrm{mmol}$ • Cre) & 44.6 & 20.4 \\
BAP $(\mu \mathrm{g} / \mathrm{L})$ & 29.25 & 9.37 \\
\hline
\end{tabular}

Discussion: Because of previous teriparatide (TPD) use in $84 \%$ of patients, it was considered that higher effect could be expected in previous TPD users, compared with non-TPD users.

Conclusion: The curative effect of denosumab for the osteoporosis was shown in increasing of BMD and reducing of bone turnover, significantly in TPD users compared with non-TPD users, regardless of RA or non-RA patients.
P457

EPIDEMIOLOGICAL STUDY OF FOREARM FRACTURE IN POSTMENOPAUSAL WOMEN

K. Baccouche ${ }^{1}$, N. Elamri ${ }^{1}$, S. Belghali ${ }^{1}$, M. Bouzaoueche ${ }^{1}$, Z. Alaya $^{1}$, H. Zeglaoui ${ }^{1}$, E. Bouajina ${ }^{1}$

${ }^{1}$ Rheumatology, Farhat Hached Hospital, Sousse, Tunisia

Introduction: The forearm fracture is frequent. It can be the expression of a serious pathology, osteoporosis. The aim of this study is to determine the epidemiological characteristics of this fracture in a Tunisian population of postmenopausal women.

Methods: This is a cross-sectional study of 86 postmenopausal women with forearm fracture, for which we practiced BMD. We excluded from our study any patient with a predisposing factor for secondary osteoporosis.

Results: The mean age of the occurrence of the fracture is 60.5 years, on average 14 years after the onset of menopause. A Conservative orthopedic treatment is indicated in $60 \%$ of cases. In $16 \%$ of cases, there is a history of fracture in the mother. The majority ( $70 \%$ ) of our women are multifarious. A fracture of the proximal femur, associated, is found in two patients. Latent vertebral fractures are identified in $33 \%$ cases and are multiple in $64 \%$ of cases. The lumbar spine is most affected. Bone densitometry performed, objective osteoporosis in $69.8 \%$, osteopenia in $20.6 \%$ and normal BMD in $9.6 \%$ of patients.

Conclusion: The forearm fracture is strongly related to osteoporosis. The detection of this pathology in the year following the occurrence of the fracture must be systematic in order to prevent the occurrence of other fracture events.

\section{P458}

\section{CELLULAR COUPLING OF ANGIOGENESIS} AND OSTEOGENESIS IN BONE

A. P. Kusumbe $^{1}$, S. K. Ramasamy ${ }^{1}$, R. H. Adams ${ }^{1}$

${ }^{1}$ Max Planck Institute for Molecular Biomedicine, Muenster, Germany

Osteogenesis is indispensable for the homeostatic renewal of bone as well as regenerative fracture healing, but these processes frequently decline in ageing organisms leading to loss of bone mass and increased fracture incidence. Decline or impairment of blood vessel growth is a characteristic feature of osteoporosis, osteonecrosis and delayed fracture healing. There is evidence indicating that the growth of blood vessels in bone and osteogenesis are coupled, but relatively little is known about the underlying cellular and molecular mechanisms. Here we identify a new capillary subtype in the murine skeletal system with distinct morphological, molecular and functional 
properties. These vessels are found in specific locations, mediate growth of the bone vasculature; maintain perivascular osteoprogenitors, and couple angiogenesis to osteogenesis. Combination of inducible, cell typespecific mouse genetics and pharmacological approaches, showed that endothelial hypoxia-inducible factor 1 $(\mathrm{H} 1 \mathrm{~F} 1-\alpha)$ promotes angiogenesis and thereby bone formation in the postnatal skeletal system. The abundance of these vessels and associated osteoprogenitors was strongly reduced in bone from aged animals, which was pharmacologically reversible to restore bone mass. Thus, the skeletal vasculature is a potential therapeutic target that could be modulated for treating several bone diseases.

\section{P459}

GLUCOCORTICOID-INDUCED OSTEOPOROSIS IN AMBULATORY PRACTICE

M. Menshikov $^{1}$, L. Menshikova ${ }^{1}$, E. Belikh ${ }^{2}$, O. Grudinina ${ }^{3}$ ${ }^{1}$ Irkutsk State Medical Academy of Postgraduate Education, Irkutsk, Russian Federation, ${ }^{2}$ Regional Clinical Diagnostic Center, Irkutsk, Russian Federation, ${ }^{3}$ Region Clinical Diagnostic Center, Irkutsk, Russian Federation

Objectives: The arm of this work is to define the frequency of secondary glucocorticoid-induced osteoporosis (GIP) and the risk factors in the women with bronchial asthma (BA) and rheumatoid arthritis (RA) in East Siberia, Russia.

Material and Methods: There have been investigated 296 women with BA (mean age $52.9 \pm 12.8 \mathrm{y}$ ) and 178 women with RA (mean age 53.1 $\pm 11.7 \mathrm{y}$ ). The diagnosis was verified according to the criteria GINA and ARA. The BMD was measured by DXA ("DPX-IQ", "Lunar", USA) at the lumbar spine (L2-L4) and at the hip. Were excluded diseases and conditions which may affect the bone metabolism. Risk factors for OP calculated as odds ratio (OR) in logistic regression analysis models and multivariate analysis in 244 women with BA and 146 women.

Results: Osteoporosis was observed in $88(29.7 \%)$ and osteopenia had 120(40.5\%) women with BA and in $80(44.9 \%)$ and 69(38.8 \%) with RA. In control group (896 women) were $18.1 \%$ and $26 \%$ correspondingly. Low trauma fractures were in significantly higher among pts with BA and RA. The independent risk factors $(95 \% \mathrm{CL})$ were found to be duration of taking $\mathrm{GC}>5$ years $(O R=2.5)$ in pts with BA and $>2$ years $(O R=4.7)$ with $\mathrm{RA}$; the dose of $\mathrm{GC}>7.5 \mathrm{mg} /$ day $(\mathrm{BA}-\mathrm{OR}=2.2 ; \mathrm{RA}-O R=4.8)$ and cumulative dose of $\mathrm{GC}>10000 \mathrm{mg}(O R=2.8)$ in pts with BA and $>2000 \mathrm{mg}$ in RA $(O R=4.6)$. Other risk factors for $\mathrm{OP}$ in pts with $\mathrm{BA}$ and RA were: age $>60$ years, early menopause, duration of menopause $>10$ years, history of fractures, weight $<60 \mathrm{~kg}$, lowering of height $<1.6 \mathrm{~m}$, low BMI, low physical activity, duration of diseases $>10$ years, high severity and activity of diseases, functional capacity. There was no relation between seropositive or seronegative variants of RA, dietary calcium intake, smoking and alcohol.

Conclusions: Prevalence of OP and fractures were high among patients with BA and RA, were found different dose and duration of taking oral GC in women with $\mathrm{BA}$ and RA and knowledge other risk factors of the development of OP will allow to conduct the preventive measures.

\section{P460}

GENDER SIMILARITIES AND DIFFERENCES IN CROSS-SECTIONAL CORTICAL AND TRABECULAR BONE OF FEMORAL NECK IN ELDERLY CHINESE POPULATION

L. Wang ${ }^{1}$, X. G. Cheng ${ }^{1}$, A. H. Yu ${ }^{1}$, Y.-B. $\mathrm{Su}^{1}$

${ }^{1}$ Department of Radiology, Beijing Jishuitan Hospital, Beijing, China

Objective: To investigate gender similarities and differences in femoral neck (FN) structure of elderly population by bone investigational toolkit (BIT) of quantitative computed tomography (QCT).

Material and Methods: This cross-sectional study was part of China Action on Spine and Hip Status (CASH) study, including 207 males (from 55 to 87 years old) and 400 females (from 55 to 96 years old). QCT scans were performed in the hip for the subjects, and we used BIT software which directed automatically the lowest area of FN cross-section perpendicular to FN axis to measure cortical and trabecular bone in anatomic quadrants of FN. The measurements of cortical thickness (Cor.T), cortical vBMD (Cor.vBMD), trabecular vBMD (Tra.vBMD) and integral vBMD (Int.vBMD) at FN were determined in BIT sectors. This resulted in four anatomical quadrants, Quadrant 1(Supero-anterior, SA), Quadrant 2 (Infero-anterior, IA), Quadrant 3 (Infero-posterior, IP), Quadrant 4 (Supero-posterior, SP). The study was approved by the ethics committee of Beijing Jishuitan Hospital.

Results: The greatest difference between genders after adjustment was in Cor.T of Quadrant SA, $27.3 \%$ lower in women $(p=0.000)$. The estimated Cor.T of Quadrant IA was $14.8 \%$ lower in men than women $(p=0.027)$. The differences in Cor.T of Quadrant IA and IP between the genders did not get statistical significance. There was no gender-related difference in Int.vBMD of Quadrant IA, IP and SP. However it was detected that elderly men had a higher vBMD in the trabecular bone compartment than elderly women in same regions. With aging, Cor.T of superior FN declined significantly in both sexes, in contrast Cor.T of inferior region maintained in men different from that in women. 
Conclusions: Our results indicate that women have thinner cortical thickness in superior quadrants. With aging, cortical thickness of cross-sectional FN declines significantly in women, in contrast cortical thickness of Inferior region maintains in men. Thinner cortical thickness in the superior region of the femoral neck may be a stronger predictor for hip fracture, implying the mechanism causing a higher fracture incidence in women.
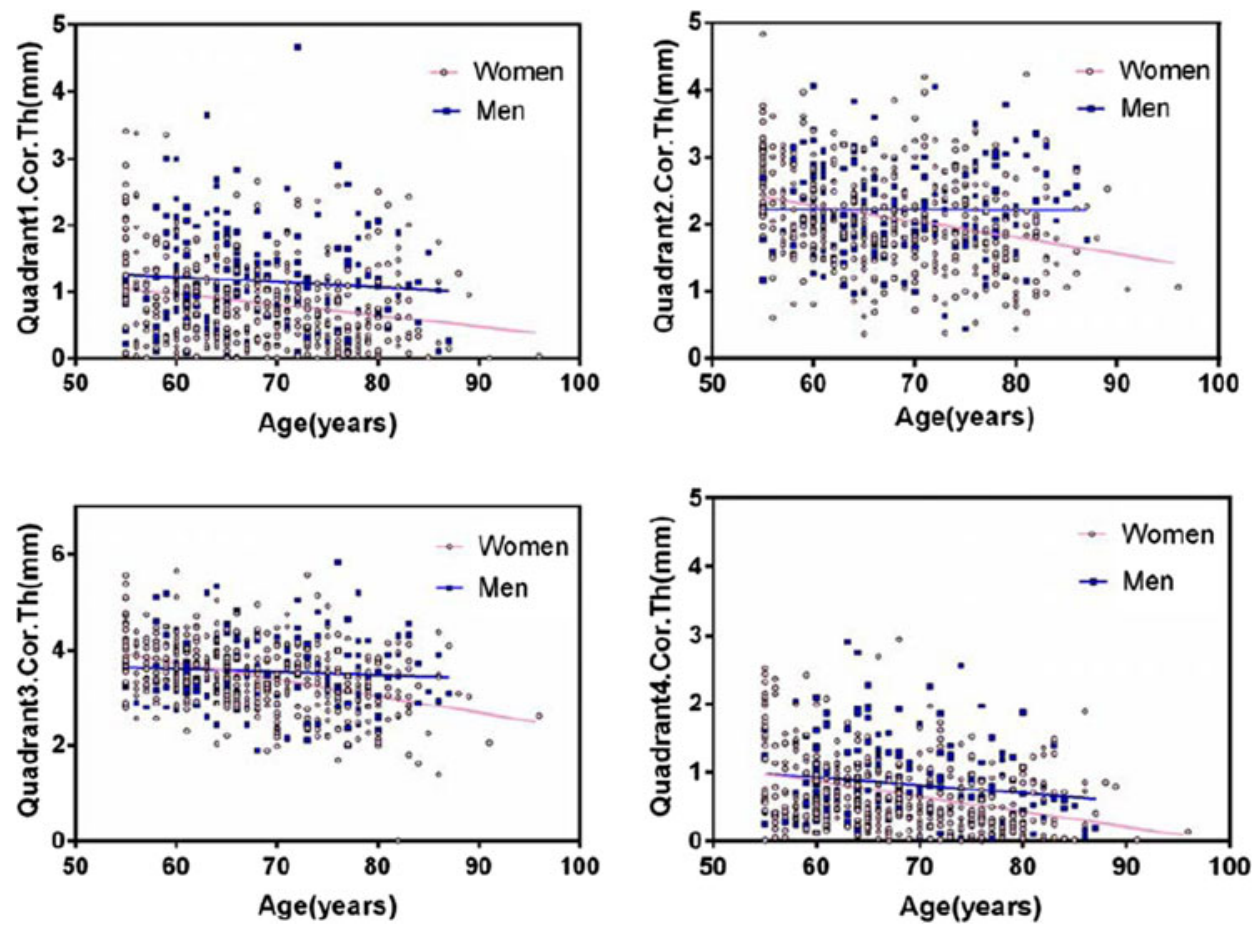

\section{P461}

\section{EFFECTS OF HIGH IMPACT TRAINING ON} BONE MINERAL DENSITY AND MECHANICAL PROPERTIES OF BONE OF RATS

B. B. Brancaleon $^{1}$, G. R. Yanagihara ${ }^{1}$, J. A. Tida ${ }^{1}$, A. P. Macedo $^{1}$, R. Carminati Shimano ${ }^{1}$, A. C. Oliveira Penoni ${ }^{1}$, A. C. Shimano ${ }^{1}$

${ }^{1}$ University of São Paulo, Biomechanics, Medicine and Rehabilitation of Locomotor System/Ribeirão Preto Medical School, Ribeirão Preto, Brazil

The aim of this work study was to evaluate the effects of different phases of the jump on BMD and mechanical properties of bone of healthy rats. 32 female Wistar rats were used. Rats had body mass ranging between 200 and $250 \mathrm{~g}$. The animals received standard rat chow and water ad libitum and were equally divided into 4 groups $(n=8)$ : Eccentric Group (EG): animals that have been subjected to just eccentric phase of jump (landing/braking); Concentric Group (CG): animals that have been subjected to just concentric phase of jump (pulse); Combined Group (COMBG): animals that have been subjected to concentric and eccentric phases of jump and

Control group (CONTG): animals that have remained in the cage for 8 weeks and have not undergone any training. The trained groups made the training protocol of 10 jumps/day, 5 days per week, during i 8 weeks. After the training period, all the animals were killed with an overdose of anesthetic and the tibiae were dissected. We used a DXA to evaluate the BMD of the proximal metaphysis of the tibiae. Static mechanical tests of flexion in three points were used to evaluate the mechanical properties of maximum load (MaxL) and Stiffness (St). The statistical test of Kolmogorov-Smirnov was used to test the normality of the data. The comparison between the groups was made by testing One-way ANOVA or KruskalWallis and was adopted the significance level of $5 \%$. No statistically significant differences were observed in relation to BMD of the tibiae. About MaxL, we observed statistically significant differences between the groups $(p=0.031)$, being the COMBG $>$ EXCG $(p=0.021)$. Regarding the St, we observed statistically significant differences between the groups ( $p=0.05)$, being the COMBG $>$ EXCG $(p=0.003)$ and the COMBG $>$ CONTG $(p=0.048)$. Our data indicate that the high-impact exercise with two phases of jumps (COMB) is more beneficial than exercise with one of the phases alone. 


\section{P462}

A NON-RESOLVED CARE GAP: SECONDARY FRACTURE PREVENTION AFTER A FRAGILITY HIP FRACTURE IN SPAIN. A NATIONWIDE, PROSPECTIVE COHORT STUDY.

${ }_{\text {D. Prieto-Alhambra }}{ }^{1}$, I. Aguado-Maestro ${ }^{2}$, N. Alonso García $^{3}$, I. Andrés Cano ${ }^{4}$, J. D. Avilés Hernández ${ }^{5}$, M. Barrés Carsi ${ }^{6}$, F. Brañas Baztán ${ }^{7}$, M. F. Bravo Bardaji ${ }^{8}$, J. R. Caeiro Rey ${ }^{9}$, P. Carpintero ${ }^{10}$, V. Climent-Peris ${ }^{11}$, A. Díez Rodríguez $^{12}$, I. Etxebarria-Foronda ${ }^{13}$, L. Ezquerra Herrando $^{14}$, N. Fernández González ${ }^{15}$, P. Gonzalez García ${ }^{16}$, C. Martín Hernández ${ }^{17}$, D. Mifsut Miedes ${ }^{18}$, S. Mills Gañan $^{19}$, J. Mora-Fernández ${ }^{20}$, J. Olmos Martínez ${ }^{21}$, I. Pérez-Coto $^{22}$, N. Quiroga Veiga ${ }^{23}$, M. Salomó Doménech ${ }^{24}$, J. Teixidor ${ }^{25}, \mathrm{C}$. Tejedor Carreño ${ }^{26}, \mathrm{O}$. Tendero $\mathrm{Gómez}^{27}, \mathrm{O}$. Torregrosa $\mathrm{Suau}^{28}$, A. Herrera ${ }^{29}$, A. Diez-Perez ${ }^{30}$

${ }^{1}$ Oxford NIHR Musculoskeletal Biomedical Research Unit, University of Oxford, MRC Lifecourse Epidemiology Centre, University of Southampton, IDIAP Jordi Gol, Oxford, United Kingdom, ${ }^{2}$ Department of Orthopaedic Surgery, Hospital Universitario del Río Hortega, Valladolid, Spain, ${ }^{3}$ Department of Orthopaedic Surgery, Hospital Ntra. Sra. de Sonsoles, Avila, Spain, ${ }^{4}$ Department of Orthopaedic Surgery, Traumatology and Rheumatology, Hospital Universitario Puerta del Mar, Cádiz, Spain, ${ }^{5}$ Orthogeriatrics Unit, Hospital Virgen de la Arrixaca, Murcia, Spain, ${ }^{6}$ Trauma Unit, Unit of Orthopaedic Surgery and Traumatology, Hospital Universitario y Politécnico La Fe de Valencia, Valencia, Spain, ${ }^{7}$ Geriatric and Internal Medicine Department, Infanta Leonor University Hospital, Madrid, Spain, ${ }^{8}$ Department of Orthopaedic Surgery, Hospital Regional Universitario Carlos Haya, Málaga, Spain, ${ }^{9}$ Department of Orthopaedic Surgery, Complejo Hospitalario Universitario de Santiago de Compostela, Santiago de Compostela, Spain, ${ }^{10}$ Hospital Reina Sofía de Cordoba, Cordoba, Spain, ${ }^{11}$ Orthopaedics and trauma Departament, Hospital Lluís Alcanyís, Xàtiva, Spain, ${ }^{12}$ Orthopaedic Surgery and Traumatology Unit, Hospital Virgen del Puerto, Plasencia, Spain, ${ }^{13}$ Department of Orthopaedic Surgery, Alto Deba Hospital, Arrasate, Spain, ${ }^{14}$ Orthopaedic Surgery and Traumatology Unit, Hospital Clínico Universitario de Zaragoza, Zaragoza, Spain, ${ }^{15}$ Geriatric Department, Hospital Universitario de Getafe, Getafe, Spain, ${ }^{16}$ Department of Geriatrics, Orthogeriatric Unit, Hospital Obispo Polanco, Teruel, Teruel, Spain, ${ }^{17}$ Orthopaedic Surgery and Traumatology Unit, Hospital Universitario Miguel Servet, Zaragoza, Spain, ${ }^{18}$ Hospital Clínico de Valencia, Valencia, Spain, ${ }^{19}$ Department of Orthopaedic Surgery, Hospital Universitario La Paz, Madrid, Spain, ${ }^{20}$ Geriatric Department, Hospital Universitario San Carlos, Madrid, Spain, ${ }^{21}$ Department of Internal Medicine, RETICEF, IDIVAL, Universidad de Cantanbria, Santander, Spain, ${ }^{22}$ Hospital San Agustín, Avilés, Spain, ${ }^{23}$ Department of Orthopaedic Surgery,
Complejo Hospitalario Universitario de Pontevedra, Pontevedra, Spain, ${ }^{24}$ Department of Orthopaedic Surgery and Traumatology, Hospital Universitario Parc Tauli, Savadell, Spain, ${ }^{25}$ Trauma Unit, Hospital Vall d Hebron, Universitat Autónoma de Barcelona, Barcelona, Spain, ${ }^{26}$ Orthopaedic Surgery and Traumatology Unit, Hospital San Pedro, Logroño, Spain, ${ }^{27}$ Department of Orthopaedic Surgery, Hospital Universitario Son Espases, Palma de Mallorca, Spain, ${ }^{28}$ Metabolic Bone Unit, Department of Internal Medicine, Hospital General Universitario, Elche, Spain,

${ }^{29}$ Department of Surgery, Medicine School, University of Zaragoza, Zaragoza, Spain, ${ }^{30}$ Department of Internal Medicine, Hospital del Mar-IMIM and Autonomous University of Barcelona, Barcelona, Spain

Objective: Despite compelling evidence, clinical guidance (including NICE TA/161), and scientific initiatives such as the IOF Capture The Fracture, the care gap for secondary fracture prevention is unresolved. We studied the first 557 patients recruited in the Spanish Registry of Hip and Proximal Femur Fractures (SPARE-HIP) to describe and compare the proportion of inpatients prescribed 1.thromboprophylaxis, 2.antibiotic prophylaxis, and 3.active anti-osteoporosis drugs (excluding calcium/vitamin D supplements).

\section{Methods}

Design: Cohort study using data from the SPARE-HIP cohort. Participants: A consecutive sample of hip/proximal femur fractures recruited from Sept/2014 to Oct/2015 in a representative 22 hospitals from the different Spanish regions.

Statistical analyses: Patient baseline characteristics are reported in detail elsewhere, and summarized here as mean(standard deviation) or $\mathrm{N}(\%)$. The $\mathrm{N}(\%)$ starting thrombo-prophylaxis, antibiotic prophylaxis, and antiosteoporosis therapy whilst inpatient, and $95 \% \mathrm{CI}$ (calculated assuming a binomial distribution) are presented.

Results: A total of 557 patients were included, aged 84.0 (8.5) years, $78.1 \%$ women. Among them 541/549 (98.5\% [95CI 97.1-99.4\%]) started antibiotics, and 549/550 (99.8\% [99.0 $99.9 \%]$ ) drug thrombo- prophylaxis. Conversely, only 143/ $548(26.1 \%$ [22.5-30.0\%]) initiated anti-osteoporosis therapies (whilst inpatient (35 (6.4\%) were previous users of such drugs), whilst 20 (3.7\%) and 43 (7.9\%) were awaiting DXA scan or bone specialist opinion respectively. According to recruiting physicians, 133 (24.3\% [20.7-28.1\%]) were not assessed for anti-osteoporosis treatment, and 135 (24.6\% [21.1-28.5\%]) were considered not to need such therapies.

Conclusions: In our data, only 1 in 4 patients suffering an incident hip fracture is started on anti-osteoporosis therapies, whilst almost $50 \%$ are either not evaluated or not considered to need such treatment. Interestingly, other preventative strategies such as thrombo- and antibiotic prophylaxis are well implemented, with uptake close to $100 \%$. There is an urgent 
need for a better understanding on the reasons for this care gap, and on research on strategies for a better secondary fracture prevention in Spain.

Acknowledgments: Amgen provided an unrestricted research grant for the SPARE-HIP study. ADP and DPA receive partial support from RETICEF (Instituto de Salud Carlos III, Spain) and NIHR (United Kingdom).

\section{P463}

\section{A PRELIMINARY STUDY TO COMPARE EFFECTS OF TWO DIFFERENT HYALURONIC ACID COMPOUNDS WITH AND WITHOUT LIDOCAINE CONTENT FOR TREATMENT OF KNEE AND SHOULDER OSTEOARTHRITIS} B. Palmieri ${ }^{1}$, C. Laurino ${ }^{1}$

${ }^{1}$ University of Modena and Reggio Emilia, Modena, Italy

Objectives: We investigated the clinical effectiveness of viscosupplementation comparing a cross-linked hyaluronic acid (CL-HA) without lidocaine (control formulation, CF) and CL-HA containing $0,3 \%$ lidocaine (experimental formulation, EF) in patients affected by knee osteoarthritis (KOA) and shoulder osteoarthritis (SOA).

Materials and Methods: 24 patients (13 males and 11 females, $57.5 \pm 1.09$ ) affected by symptomatic arthritis with $\operatorname{VAS}^{3} 30$, dissatisfaction with conservative methods of treatment (including NSAIDs therapy) and without any hypersensitivity to HA and lidocaine participated in this preliminary clinical study. Patient affected by bilateral KOA $(n=4)$ and SOA $(n=2)$ received parallel injection of control or experimental formulation in each limb. Patients with monolateral OA $(n=18)$ received a single injections $(2 \mathrm{ml})$ of the $\mathrm{CF}$, and 3 to 6 months later, when relapsed, received a single injections $(2 \mathrm{ml})$ of EF containing $0.3 \%$ lidocaine. The following assessments between two treatments were compared: (1) pain and functional disability by VAS and WOMAC scale; (2) intolerance symptoms; (3) pedal rehab exercise and (4) patients' satisfaction after 6 months.

Results: VAS pain was reduced 78,0 \% after use of EF and 52, $8 \%$ after use of CF injection. WOMAC pain, stiffness and physical activity were reduced $41,0 \%, 48,0 \%$ and 53,9 \% after use of EF and 20,2 \%, 22,3\%, 29,6\% after CF injection, respectively. After injections, pedal cycling exercise was used to assess pain symptoms. $29 \%$ patients didn't show any pain symptoms during exercise time ${ }^{3} 300 \mathrm{sec}$ after use of EF, while for $\mathrm{CF}$ the average time was $60 \mathrm{sec} \pm 52$ and all patients had pain. Pain during injection of EF was: absent (a) in $71 \%$ (17/ 24) patients, mild in $29 \%(7 / 24)$ patients, and there was no medium or strong event. Pain after injection was absent in $83 \%(20 / 24)$ patients, mild in $17 \%(4 / 24)$ patients, and there was no medium or strong event. Redness and swelling was absent in $92 \%(22 / 24)$ patients and mild in $8 \%(2 / 24)$ patients. Itching, discoloration and hematoma were not observed in any patients.

Conclusion: Treatment with the EF resulted in a higher percentage improvement in VAS pain, WOMAC score pain, stiffness and physical activity, when compared to the CF. Lidocaine even at very low concentration $(0.3 \%)$ had a significant effect on pain assessments and joint motility in OA patients. As to the literature claims of cartilage toxicity by local anesthetics, our opinion is that the low concentration and quick absorption of single small molecules had not produced any damage and helped restore the normal function of joint. Spontaneous motion due to absent pain helped spread the viscosupplement.

\section{P464}

PREVALENCE OF VERTEBRAL FRACTURES, LOW BONE MINERAL DENSITY AND HYPOVITAMINOSIS D IN AN HIV-INFECTED COHORT

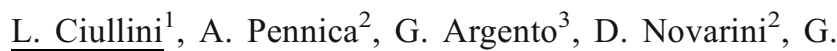
Pugliese $^{1}$, F. Conti ${ }^{1}$

${ }^{1}$ Department of Clinical and Molecular Medicine, Sapienza University of Rome, S. Andrea Hospital - Osteoporosis and Other Metabolic Bone Diseases Unit - Diabetes Unit, Rome, Italy, ${ }^{2}$ Clinical Infectious Diseases, Sapienza University of Rome, S. Andrea Hospital, Rome, Italy, ${ }^{3}$ Radiology, Sapienza University of Rome, S. Andrea Hospital, Rome, Italy

Objective: To investigate bone alterations in a cohort of HIVinfected subjects.

Material and Methods: 149 HIV-infected patients on combined-antiretroviral therapy (cART) with undetectable viral load (88\% males, median age 44 years) underwent viroimmunological investigations and bone metabolism biochemical analysis. BMD was measured with DXA. Thoracic and lumbar spine X-ray examination was performed. Pearson's chi-squared test was used to compare frequencies.

Results: Low 25(OH)vitamin D levels $(<30 \mathrm{ng} / \mathrm{ml})$ were found in $139(93 \%)$ and 105 patients $(74 \%)$, before and after seasonal sun exposure, respectively. PTH was increased in $29 \%$ of the individuals, urinary deoxypyridinoline/ creatinine ratio in $62 \%$ and bone-specific alkaline phosphatase in $17 \%$. Other parameters did not show remarkable alterations. BMD was normal in 54 patients $(36.2 \%)$; 82 patients $(55 \%)$ showed osteopenia and 13 patients $(8.8 \%)$ had osteoporosis (lumbar spine and/or femoral T- score $\leq-2.5 \mathrm{SD}$ ). Vertebral fractures (VFs) (vertebral deformity $>20 \%)$ were found in 19 patients $(13.5 \%)$. HIV-related parameters (duration of HIV infection, cART duration and classes) and bone biochemical parameters did not show any association with VFs. No statistically significant differences were found comparing VFs 
rate with BMD status: VFs were detected in $18 \%$ of subjects with normal BMD whereas patients with reduced BMD did not show a higher rate of VFs as expected (12\% of patients with osteopenia and $8 \%$ of patients with osteoporosis).

Conclusions: Bone diseases have a high prevalence among HIV-infected subjects under cART. Hypovitaminosis D was found to be very frequent in our cohort regardless of sun exposure. Reduced BMD and sub-clinical VFs are common findings despite young median age. Bone density seem not to be associated to VFs among HIV-positive patients.

\section{P465}

\section{IDIOPATHIC MALE OSTEOPOROSIS WITH} CASCADE FRACTURES AT YOUNG AGE

A. Valea ${ }^{1}$, A. Morar ${ }^{2}$, M. Carsote ${ }^{3}$, A. Patronea ${ }^{2}$, C. Ghervan ${ }^{1}$, C. E. Georgescu ${ }^{1}$

${ }^{1}$ I. Hatieganu University of Medicine and Pharmacy \& Clinical County Hospital, Cluj-Napoca, Romania, ${ }^{2}$ Clinical County Hospital, Cluj-Napoca, Romania, ${ }^{3}$ C.I. Parhon National Institute of Endocrinology, Bucharest, Romania

Introduction: Idiopathic osteoporosis of young men may progressively associate multiple fractures despite adequate therapy. However, the therapeutically resources are challenging because of large epidemiological studies and lack of mechanisms understanding which does not allow an etiopathogenic approach.

Material and Method: We report the case of a young adult osteoporotic providing the data based on central DXA (BMD, and associated Z-score); the vitamin D status revealed by circulating 25-hydroxyvitamin D (25-OH D) assay and PTH (iPTH); the blood bone turnover markers evaluation: $\beta$ CrossLaps (ß-CTx), osteocalcin, as well as the endocrine panel necessary to exclude a potential thyroid, adrenal, and gonad pathology as cause of secondary osteoporosis.

Case presentation: A 31-old nonsmoking male with irrelevant personal and family medical history is admitted for persistent lumbar pain especially if he needed to stand up for a few hours. He denies current alcohol consumption. Four months ago a posterior arch fracture of the 7th right rib was diagnosed correlated to a mild coughing effort and also a fracture at the level of the 5th right metacarpal bone was presented after a small effort. Moderate weight gain (around 5$6 \mathrm{~kg}$ in the last 2 years) was described by the patient. The endocrine phenotype was not suggestive for Cushing's disease and the endocrine testes confirmed the intact glucocorticoid axes. Normal levels of TSH, free T4, and testosterone were found. The assays showed a medium vitamin D deficiency: $25-\mathrm{OHD}$ of $21.3 \mathrm{ng} / \mathrm{m}$ (normal values between $30-100 \mathrm{ng} /$ $\mathrm{mL}$ ) B-CTx of $0.36 \mathrm{ng} / \mathrm{mL}$ (normal levels $\leq 0.584 \mathrm{ng} / \mathrm{mL}$ ), and osteocalcin (of $20.9 \mathrm{ng} / \mathrm{mL}$, normal between 14-22 ng/
$\mathrm{mL}$ ). DXA showed L1-4 BMD of $0.815 \mathrm{~g} / \mathrm{cm}^{2}$, Z-score of -3.8 SD. X-ray confirmed a T12 vertebral fractures. No other rheumatologic, neither oncologic cause of bone loss was found. Idiopathic osteoporosis was diagnosed and intravenous ibandronate every 3 months was given together with vitamin $\mathrm{D}$ and calcium supplements. Close follow-up is considered essential in this situation.

Conclusions: Idiopathic osteoporosis should be included in differential diagnosis of young patients with repeated fragility fractures. Although no specific therapy has been proven to be very effective in idiopathic male osteoporosis, an individualized approach is necessary as well as long term serial check up to evaluate the therapy effect or/and the decision of changing/ stopping the medication.

\section{P466}

SKELETAL HEALTH ANOMALIES IN OVARIAN CANCER SURVIVOR NEWLY DIAGNOSED WITH ADRENAL CUSHING'S SYNDROME

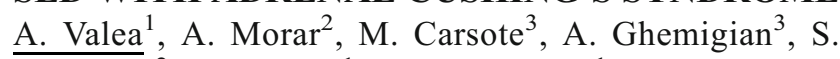
Naumescu $^{2}$, C. Ghervan ${ }^{1}$, C. E. Georgescu ${ }^{1}$

${ }^{1}$ I. Hatieganu University of Medicine and Pharmacy \& Clinical County Hospital, Cluj-Napoca, Romania, ${ }^{2}$ Clinical County Hospital, Cluj-Napoca, Romania, ${ }^{3}$ C.I. Parhon National Institute of Endocrinology, Bucharest, Romania

Introduction: The associations between osteoporosis and ovarian cancer largely reflect the consequences of antiestrogen therapy on BMD. Cushing's syndrome is further responsible for worsening skeleton status because of excessive glucocorticoid levels negatively acting on the bone. If these maladies are registered in menopause an age-related mechanism of bone loss might also be incidental.

Patient and Methods: A menopausal woman was followed in different endocrine and oncologic departments for multiple medical conditions. Endocrine assessment was focused on adrenal profile based on baseline morning ACTH levels, glucocorticoid axes before and after dexamethasone (DXM) suppression test and skeleton evaluation consistent with 25hydroxyvitamin D (25-OH D), PTH (iPTH) assays and DXA calculating BMD.

Case report: A 59 year-old nonsmoking woman underwent surgery for a left breast fibroadenoma at the age of 36 . Four years later she was diagnosed with ovarian adeno-carcinoma and bilateral oophorectomy with total hysterectomy was performed, followed by anti-estrogen therapy. Intermittent vitamin D and calcium supplements were taken. The cancer was considered remitted and the patient was further followed- up. At age of 58 she complained of persistent lumbar pain, central obesity (a BMI of $33 \mathrm{~kg} / \mathrm{m}^{2}$ ), abdominal striae and high blood pressure. Hormonal profile revealed high baseline plasma cortisol of $29 \mu \mathrm{g} / \mathrm{dl}$, with normal values less than $21 \mu \mathrm{g} / \mathrm{dl}$ and 
suppressed baseline ACTH levels of $6.08 \mathrm{pg} / \mathrm{ml}$ with normal ranges between 7.2 and $63.3 \mathrm{pg} / \mathrm{ml}$. The standard 2-days low dose DXM suppression test confirmed the diagnosis of Cushing's syndrome. Abdominal computed tomography exam revealed a $29 \mathrm{~mm}$ left adrenal tumor and laparoscopic adrenalectomy was performed with confirmation of benign features. Bone profile included vitamin D deficiency (25-OHD of $24.2 \mathrm{ng} / \mathrm{mL}$ with normal levels $\geq 30 \mathrm{ng} / \mathrm{mL}$ without secondary hyperparathyroidism (iPTH of $58.6 \mathrm{pg} / \mathrm{mL}$ with normal ranges between 15 and $66 \mathrm{pg} / \mathrm{mL}$ ); and osteoporosis based on low L13 BMD of $0.785 \mathrm{~g} / \mathrm{cm}^{2}$, T-score of $-3.2 \mathrm{SD}, \mathrm{Z}$-score of 2.5SD; femoral neck BMD of $0.884 \mathrm{~g} / \mathrm{cm}^{2}$, T-score of -1.3 $\mathrm{SD}, \mathrm{Z}$-score of $-0.2 \mathrm{SD}$. No vertebral fractures were found at profile X-ray. Thyroid tests showed normal TSH and free T4 levels. Therapy with weekly oral alendronic acid was prescribed, together with vitamin D and calcium supplements. The patient will be further followed-up by a multidisciplinary team regarding the adrenal, bone and oncologic profile.

Conclusion: In young women, surgical menopausal for gonad cancer and anti-estrogen therapy increase the risk of osteoporosis. However, multiple mechanisms of bone loss might be incidental as a second non- malign tumor causing persistent hypercortisolemia and associated skeleton damage. The risk of fall is elevated by vitamin D deficiency.

\section{P467}

THE EFFECT OF MANGANESE ENHANCED HYDROXYAPATITE IMPLANT INTO THE TIBIA ON CHEMICAL COMPOSITION OF THE LOWER INCISOR DENTIN IN RATS

$\underline{\text { D. A. Astrakhantsev }}^{1}$, V. I. Luzin ${ }^{1}$, E. P. Berezhnoy ${ }^{1}$, L. V. Stklyanina

${ }^{1}$ SE Lugansk State Medical University, Lugansk, Ukraine

Objectives: The study is aimed at analysis of chemical composition of the lower incisor dentin (LDI) after implantation of manganese (Mn) enhanced hydroxyapatite into the tibia.

Methods: The study involved 252 male rats. The 1st group comprised intact animals, the 2 nd group - animals with $2.2 \mathrm{~mm}$ defect in the tibia, and the groups 3 through 6 - the animals with the defects filled with hydroxyapatite enhanced with $0.1 \%, 0.25 \%$, and $0.5 \%$ share of Mn. LDI were prepared for chemical analysis.

Results: A plain defect in tibia resulted in instability of chemical composition of the LDI up to the 180th day of observation. In the 3 rd group, alterations in comparison with the 2 nd group continued manifesting as instability of $\mathrm{Ca}$ levels and microelemental exhaust up to the 60th day. Later on, $\mathrm{F}$ and $\mathrm{Zn}$ levels by the 90th and the 180th days were higher than those of the 2nd group by $9.25 \%$ and $5.42 \%, 7.35 \%$ and $6.98 \%$. Implants with $0.1 \%$ of Mn share had nearly the same effect and few significant differences were revealed.
With Mn concentration increase up to $0.25 \%$ restoration rate of chemical composition of LDI appeared to be higher beginning from the 30th day. Ca level from the 30th to the 60th day and $\mathrm{Ca} / \mathrm{P}$ ratio from the 30 th to the 90th day were higher than those of the 3rd group by $6.16-7.35 \%$ and $5.91-15.36 \%$ respectively and $\mathrm{Mn}$ level from the 15th to the 180th day was higher by $5.36-14.29 \%$. Mn share of $0.5 \%$ did not have positive effect on chemical composition of the LDI. What is more, from the 90th to the 180 th day $\mathrm{Ca}$ level and $\mathrm{Ca} / \mathrm{P}$ ratio in this group were lower by $5.12 \%$ and $5.18 \%$, and by $8.84 \%$ and $8.72 \%$ respectively and Mn level from the 15 th to the 180th day was higher by $8.13-15.63 \%$.

Conclusions: Application of Mn enhanced implants significantly reduces negative effects of bone fracture on chemical composition of the LDI. Implants with $0.25 \%$ share of $\mathrm{Mn}$ proved to be the most effective while implants with $0.5 \%$ share of Mn produced signs of Mn intoxication.

\section{P468}

ZOLEDRONIC ACID UTILIZATION AND FACTORS ASSOCIATED WITH RECEIPT OF FOLLOW-UP INFUSION: A SURVEY OF PATIENTS WITH POSTMENOPAUSAL OSTEOPOROSIS

D. T. Gold ${ }^{1}$, L. Cheng ${ }^{2}$, B. J. Chastek ${ }^{3}$, J. C. White ${ }^{3}, \underline{\text { S. L. }}$ Silverman $^{4}$

${ }^{1}$ Duke University Medical Center, Durham, United States, ${ }^{2}$ Amgen Inc., Thousand Oaks, United States, ${ }^{3}$ Optum Life Sciences, Eden Prairie, United States, ${ }^{4}$ Cedars-Sinai Medical Center, UCLA School of Medicine, and OMC Clinical Research Center, Los Angeles, United States

Objectives: To estimate the proportion of patients who received a follow-up zoledronic acid (ZOL) infusion after the index infusion for the treatment of postmenopausal osteoporosis (OP) and to assess factors associated with receipt of follow-up infusion.

Material and Methods: A cross-sectional study of patients affiliated with the Optum Research Database (ORD) in the US was conducted via a mailed survey. Female patients aged $\geq 50$ years with an OP diagnosis who received a ZOL infusion (index date) 13-19 months prior to survey administration were included. The survey (collected from November 2013 to July 2014) assessed clinical and demographic characteristics, knowledge of OP and ZOL therapy, attitudes toward medication, and availability of support for disease management.

Results: Of the 616 surveys sent, 175 evaluable surveys were returned (response rate $=31.3 \%$ ). The mean $(\mathrm{SD})$ age of the respondents was 62.4 (7.0). Based on the survey, $49.7 \%$ of the respondents reported receiving the follow-up infusion; however, only $21.1 \%$ had evidence of the infusion in the ORD claims data. Differences in patient characteristics were not 
statistically significant between those with vs. without a claim for a follow-up infusion. Patients with a claim for a follow-up infusion were more likely to report having had a discussion with the physician about the need to return for additional infusions compared with those who did not return (100.0 \% vs. $89.1 \% ; p=0.043)$. Although not statistically significant $(p=0.061)$, there was a trend indicating that more patients with a claim for a follow-up infusion (66.7 \%) reported having been contacted by the physicians about scheduling their next infusion than those without (48.2\%).

Conclusions: The proportion of patients who received followup ZOL infusion was low. This study suggests that increased patient-physician interactions may improve treatment continuation for less frequent injectable OP medications.

\section{P469}

PRIMARY AUTOIMMUNE HYPOTHYROIDISM AND MAMMARY CANCER: WHAT ABOUT THE BONE?

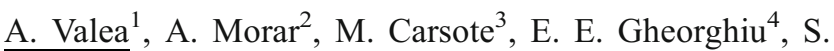
Naumescu $^{5}$, C. E. Georgescu ${ }^{1}$

${ }^{1}$ I. Hatieganu University of Medicine and Pharmacy \& Clinical County Hospital, Cluj-Napoca, Romania, ${ }^{2}$ Clinical County Hospital, Bucharest, Romania, ${ }^{3}$ C.I. Parhon National Institute of Endocrinology, Bucharest, Romania, ${ }^{4}$ C.I.Parhon National Institute of Endocrinology, Bucharest, Romania, ${ }^{5}$ Clinical County Hospital, Cluj-Napoca, Romania

Introduction: Breast cancer damages the bone status by inhibition of ovarian function as potential source of estrogens, by aromatase inhibitors which additionally block the estrogens production, by chemotherapy with potential direct negative effect on bone, and by mammary malignant cells itself that may stimulate the osteoclasts production and function.

Materials and Methods: For this case report we tested central DXA, 25-hydroxyvitamin D (25-OH D), PTH, thyroid function based on TSH and freeT4 levels and thyroid autoimmunity was evaluated based on circulating thyroid antibodies.

Results: A 59 year-old nonsmoking female had a radical surgery performed at the age of 46 for a right breast carcinoma, followed by radiotherapy and long-term chemotherapy (for almost 10 years with different regimes). For the last 3 years the patient accused diffuse back pain. Whole body bone scintigraphy revealed no metastasis, neither visible fracture. Vitamin D was deficient according to 25-OHD levels of $22.3 \mathrm{ng} / \mathrm{mL}$ (with normal ranges between 30 and $100 \mathrm{ng} / \mathrm{mL}$ ) in association with high- normal PTH value of $65.8 \mathrm{pg} / \mathrm{mL}$ (normal values between considered between 15 and $66 \mathrm{pg} / \mathrm{mL}$ ). DXA showed decreased BMD of $0.713 \mathrm{~g} / \mathrm{cm}^{2}$, T-score of $-4.1 \mathrm{SD}$ and Zscore of -3SD, femoral neck BMD of $0.687 \mathrm{~g} / \mathrm{cm}^{2}$, T-score of $-2.5 \mathrm{SD}, \mathrm{Z}$-score of $-1.4 \mathrm{SD}$, total hip BMD of $0.696 \mathrm{~g} / \mathrm{cm}^{2}, \mathrm{~T}-$ score of $-2.6 \mathrm{SD}, \mathrm{Z}$-score of $-1.7 \mathrm{SD}$. Thyroid tests found low free T4 levels (FT4 of $10.2 \mathrm{pmol} / \mathrm{L}$, normal range: 11-22 $\mathrm{pmol} / \mathrm{L}$ ), elevated TSH (TSH of $7.9 \mu \mathrm{UI} / \mathrm{mL}$, normal between 0.5-4.5 $\mu \mathrm{UI} / \mathrm{mL}$ ) and positive thyroid antibodies (antithyreoperoxidase antibodies of $398 \mathrm{UI} / \mathrm{mL}$, normal less than $34 \mathrm{UI} / \mathrm{mL}$ ). Thyroid hormone replacement therapy was prescribed (daily $50 \mu \mathrm{g}$ of levothyroxine) in addition to vitamin $\mathrm{D}$ and calcium supplements. We found significant BMD improvement after 2 years of injectable ibandronate (every 3 months) without any incidental fragility fracture.

Conclusions: Given the rising incidence of breast cancer and the improvement of long-term survival rates, bone health and fracture prevention have become important health issues. IV bisphosphonates have demonstrated their utility in this type of bone loss. Active skeleton evaluation and case finding strategies are essential in this matter. Whether autoimmune thyroid condition has a potential role in bone anomalies is still difficult to establish.

\section{P470}

WHOLE BODY ELECTROMYOSTIMULATION AND PROTEIN TO FIGHT SARCOPENIC OBESITY IN WOMEN 70 YEARS AND OLDER: PRELIMINARY DATA OF THE FORMOSASTUDY

W. Kemmler $^{1}$, K. Engelke ${ }^{2}$, S. von Stengel ${ }^{1}$, F. Jakob ${ }^{3}$, C. Sieber $^{4}$

${ }^{1}$ Institute of Medical Physics, University of ErlangenNürnberg, Erlangen, Germany, ${ }^{2}$ Institute of Medical Physics, University of Erlangen-Nürnberg and Bioclinica-Synarc Germany, Hamburg, Germany, ${ }^{3}$ Muskuloskelettales Zentrum Würzburg, University Würzburg, Würzburg, Germany, ${ }^{4}$ Institute of Biomedicine of Aging, University ErlangenNürnberg, Nürnberg, Germany

Aim: To determine the effect of whole-body electromyostimulation on sarcopenic obesity in sedentary community dwelling women, 70 years and older, with sarcopenic obesity (SO).

Methods: 75 women $(77 \pm 4$ years) with sarcopenic obesity (i.e. $<5.99 \mathrm{~kg} / \mathrm{m}^{2}$ skeletal muscle mass index (SMI) and $>35 \%$ total body-fat) were randomly assigned to a wholebody electromyostimulation- (WB- EMS), a WB-EMS + Protein- (WB-EMS\&P) or a non-training control group (CG). WB-EMS training consisted of one session/week with 20 min of intermitted WB-EMS application (i.e. $6 \mathrm{~s}$ impulse phase $-4 \mathrm{~s}$ of rest, $85 \mathrm{~Hz}, 350 \mu \mathrm{s}$ ) in a sitting/lying supine position over 6 months. Participants performed slight movements during the impulse phase. In the WB-EMS\&P-group protein supplementation averaged $0.33 \mathrm{~g} / \mathrm{kg}$ bodyweight per day, in order to ensure a minimum daily intake of at least $1.25 \mathrm{~g} / \mathrm{kg} / \mathrm{d}$. All participants were supplemented with $800 \mathrm{IE} / \mathrm{d}$ of cholecalciferol. Primary endpoint was the sarcopenia Z-Score, generated by SMI, gait speed and hand 
grip strength according to the EWGSOP sarcopenia definition ${ }^{1}$.

Results: Sarcopenia Z-Score significantly comparably improved in both exercise groups $(p<.029)$, and significantly worsened $(p=.008)$ in the CG. Differences between the exercise groups and the CG were significant $(p<.001)$. SMI showed the most profound difference between WB-EMSgroups and CG $(p<.001)$, while the discrepancy in grip strength and gait speed was lower. Body-fat significantly decreased in the WB-EMS groups $(p<.047)$ and nonsignificantly decreased in the CG $(p=.270)$, however, differences between the groups were not significant.

Conclusion: WB-EMS with and without additional protein supplementation significantly affects Sarcopenia, however, the effect on body-fat was not satisfying at least in the group of sedentary females with SO. However, due to the fact that many people are unwilling or unable to start an intensive exercise protocol at higher age, the time-efficient and less exhaustive WB-EMS may be an option for this cohort. Acknowledgement: We gratefully acknowledge the support of the Bayerische Forschungsstiftung (Munich, Germany), miha-bodytec (Gersthofen, Germany), Nutricia (Erlangen, Germany) and physiomed (Laipersdorf, Germany).

Reference: ${ }^{1}$ Cruz-Jentoft AJ, et al. Age Ageing 2010;39:412.

P471

EFFECT OF PARITY ON BONE MINERAL DENSITY AMONG POSTMENOPAUSAL TUNISIAN WOMEN

$\underline{\text { K. Baccouche }}^{1}$, M. Bouzaoueche ${ }^{1}$, S. Belghali ${ }^{1}$, N. Elamri ${ }^{1}$, Z. Alaya ${ }^{1}$, H. Zeglaoui $^{1}$, E. Bouajina ${ }^{1}$

${ }^{1}$ Rheumatology, Farhat Hached Hospital, Sousse, Tunisia

Objective: Osteoporosis and osteopenia among postmenopausal Tunisian women are common. Pregnancy, multiparity and prolonged lactation are suggested as factors modifying negatively in the development of osteoporosis. We conducted this study to measure the effect of parity on BMD measurement of lumbar spine and the upper femur.

Methods: We conducted this cross-sectional study at department of rheumatology. This study analyzed 177 patients. The data gathered was age, duration of menopause, number of children borne and the duration of breastfeeding. We excluded women with secondary osteoporosis from the study. BMD measurements of the lumbar spine and proximal femur were performed by DXA. We analyzed the data using SPSS package with significance at $p<0.05$ and confidence interval of $95 \%$.

Results: We analyzed the available data of 177 patients. We divided the patients into 2 groups; group A with $>6$ children and group B with women of $<5$ children. In group A, there were 116 women and 61 in group $B$. The mean age of patients in group A was 58.21 (49-70) years and in group B the mean age was 62.45 years (45-78). The T-score of the lumbar spine of group A was $-2.15 \mathrm{SD}( \pm 1.3)$ compared to group B of -1 . $69 \mathrm{SD}( \pm 1.41), p<0.05$. The T-score of the hip region of group A was $-1.67 \mathrm{SD}( \pm 1.21)$ and that of group B patients was $-1.16 \mathrm{SD}( \pm 1.14)(p>0.05)$. In women with $<5$ children, $21.7 \%$ had normal BMD as compared to $10.4 \%$ in women with $>6$ children, $58 \%$ were osteoporotic in group $\mathrm{A}$ and in group B $37 \%(p=0.007)$. As per the World Health Organization classification $70 \%$ in group A had an increased risk of fracture as compared to $53 \%$ in group B women. A negative correlation of lumbar and femoral T-score is objectified respectively, with the number of pregnancies, the number of children breastfed and duration of breastfeeding.

Conclusion: Our results agree with the hypothesis to classify increasing number of pregnancies beyond 6 and the duration of breastfeeding among the risk factors for osteoporosis in postmenopausal women.

Further larger studies are needed to confirm this finding.

P472

THE EFFECT OF 60-DAY EXPOSURE TO EPICHLORHYDRINE VAPOR ON CHEMICAL COMPOSITION OF SKELETAL BONES IN RATS OF VARIOUS AGES

$\underline{\text { A. N. Skorobogatov }}^{1}$, V. I. Luzin ${ }^{2}$, M. G. Hryshchuk ${ }^{2}$, E. Y. Shutov $^{2}$

${ }^{1}$ National University of Physical Education and Sports of Ukraine, Kiev, Ukraine, ${ }^{2}$ SE Lugansk State Medical University, Lugansk, Ukraine

Objectives: This study is aimed at investigating of chemical composition of skeletal bones in rats after 60-day exposure of epichlorohydrin vapors (EV) and administration of thiotriazoline (Th) and Echinaceae tinctura (ET) as medication. Methods: The experiment involved 420 male rats (young, mature and old), separated into: control, animals that received daily EV as a single 5-h exposure to $10 \mathrm{MPC}$ for 60 days and the groups 3 and 4 received $2.5 \%$ intraperitoneal $\mathrm{Th}$ in dosage $117.4 \mathrm{mg}$ per $\mathrm{kg}$ and per os ET in dosage of $0.1 \mathrm{mg}$ of active component per $100 \mathrm{~g}$. The hipbone, the tibia, and the third lumbar vertebra were prepared for chemical analysis.

Results: By the 1st day upon EV discontinue, share of minerals in the tibia, the hipbone, and the vertebra was lower than that of the control by 5.24-6.90\% and Ca share decreased by 12.46$13.16 \%$. In adult animals the same values changed in the same way by $5.97-6.59 \%$, and $10.33-11.07 \%$ and in old animals by $5.93-6.65 \%$ and $7.61-8.05 \%$. In readaptation period in young animals exhibited restoration of deranged features by the 60th day of observation, in adult - alterations persisted up to the 30th day of observation and old - did not exhibit marked restoration. After Th administration, deranged features of the 
chemical composition of bones restored in young animals by the 1th day to 30th day of observation, and in adult and old animals - by the 15 th to the 60th day. After ET administration, in young animals restoration of the chemical composition was observed by the 1th to the 30th day of observation, and in adult and old animals - by the 30th to the 60th day.

Conclusion: 60-day inhalation of EV results in destabilization of the chemical composition of bones. Deviations degree and recovery rate depend on age of animals. Faster recovery rate was observed in young animals while old animals exhibited few signs of recovery. Application of Th or ET reduces negative effects of EV on the chemical composition of bones. We proved Th to be more effective than ET.

\section{P473}

THE EFFECT OF DEFECT IN TIBIA AFTER 60-DAY INTAKE OF TARTRAZINE ON PHASE CONTENTS OF BIOMINERAL OF THE HIP BONE IN RATS

G. V. Lukyantseva ${ }^{1}$, V. I. Luzin ${ }^{2}$, V. V. Bybyk ${ }^{2}$, V. N. Morozov $^{3}$

${ }^{1}$ National University of Physical Education and Sports of Ukraine, Kiev, Ukraine, ${ }^{2}$ SE Lugansk State Medical University, Lugansk, Ukraine, ${ }^{3}$ Belgorod National Research University, Belgorod, Russian Federation

Objectives: To test biomineral phases of the hipbones in adult rats with modeled fracture of tibia after 60-day application tartrazine (Tr) in various concentrations.

Material and methods: The experiment involved 280 male rats with initial body weight of 200-210 g. The K group control animals, the $\mathrm{T} 1$ and the $\mathrm{T} 2$ groups received per os $1 \mathrm{ml}$ of $\mathrm{Tr}$ in dosage of 750 or $1500 \mathrm{mg} / \mathrm{kg}$ of body weight, the D group - animals with defect in both tibiae made when in groups 2 and 3 Tr was discontinued. The T1D and the T2D groups comprised the animals who received $\mathrm{Tr}$ and had defects in tibiae also made after Tr discontinue and the T1DM and T2DM groups also received mexidol (M) in dosage of $50 \mathrm{mg} / \mathrm{kg}$. Hipbone biomineral analyzed for presence whitlockite (Wh), calcite (Cal), and hydroxyapatite (HA) using internal check method.

Results: In the T1D group as compared to D group HA share was smaller by $3.61 \%, 1.13 \%$ и $1.08 \%$ (the $3 \mathrm{rd}$, the 24 th, and the 45th days) and $\mathrm{Cal}$ share increased by $9.07 \%$ and $5.95 \%$ by the 3rd and 10th days. In the T2D group HA share was lower by $1.27-3.94 \%$ in all observation terms. Cal share was higher by $10.83 \%, 10.32 \%$ and $3.65 \%$ by the $3 \mathrm{rd}$, the 10 th, and the 24th day. In the group T1DM HA share was higher than that of T1D group by $3.41 \%$ and $4.27 \%$ by the 3 rd and the 10th day, and Wh and Cal share was smaller by $5.77 \%$ and $7.45 \%$ by the 3 rd day respectively. In T2DM group HA share was higher than that of T2D group by $2.69 \%$ only by the 3 rd day and Cal share was smaller by $6.40 \%$ in the same period. In T1DM group as compared toT1D group alterations were observed from the 3rd to the 10th day and in T2DM group as compared to T2D group - only by the 3rd day.

Conclusions: Fracture modeling after Tr discontinue results in increase of amorphous minerals share in bone mineral and depended directly on Tr dosage. Same fracture modeling after combined action of $\operatorname{Tr}$ and $\mathrm{M}$ has less expressed effects on phase contents of bone mineral.

\section{P474}

THE EFFECT OF 6 O-DAY SOD I UM GLUTAMATE INTAKE AND EXPOSURE TO IONIZING RADIATION ON ULTRASTRUCTURE OF DENTIN MINERAL OF THE LOWER INCISOR IN RATS

$\underline{\text { K. T. Simrok }}{ }^{1}$, V. I. Luzin ${ }^{2}$, G. V. Miakotkyna ${ }^{2}$, E. N. Skryabina $^{2}$

${ }^{1}$ Clinics of Therapeutic Dentistry, LLC Medeurobud, Kiev, Ukraine, ${ }^{2}$ SE Lugansk State Medical University, Lugansk, Ukraine

Objectives: To analyze ultrastructure of dentin mineral of the lower incisor in rats after 60-day sodium glutamate (SG) intake and exposure to ionizing radiation (IR) and to test correction properties of Spirulina $(\mathrm{Sp})$.

Material and methods: The experiment involved 240 rats with body weight of 180-200 g. The animals were distributed: intact animals, animals that received SG in dosage of $30 \mathrm{mg}$ per $\mathrm{kg}$ daily for 60 days, animals exposed to IR (total 4 Grey in 4 sessions), received $\mathrm{Sp}$ in dosage of $250 \mathrm{mg}$ per kg, combined SG and IR, SG and Sp, Sp and IR, and all three agents simultaneously. Burned and powdered dentin were taken to Xray scatter analysis and calculated crystallographic parameters of the dentine mineral.

Results: By the 1st day after SG discontinue, dimensions of elementary cells along $a$ and $c$ axes were larger than controls by $0.13 \%$ and $0.17 \%$ and microtexture coefficient (MC) was lower by $6.02 \%$ (from here and on, all numeric values are significant with $p<0.05)$. After SG discontinue, dentin mineral ultrastructure restores by the 15th day of observation. Combined action of SG and IR affected crystal lattice to a greater extent: by the 1st day elementary cells were larger than controls by $0.22 \%$ and $0.28 \%$ and crystallites were larger by $7.96 \%$, MC was lower than controls by $8.84 \%$. Restoration of crystal lattice was not observed. Application of Sp in animals under combined influence of SG and IR resulted in diminishing of elementary cells along $a$-axis by $0.12 \%$ and increase of $\mathrm{MC}$ by $4.89 \%$ by the 1 st day of observation.

Conclusions: Daily 60-day per os intake of SG in dosage of $30 \mathrm{mg}$ per $\mathrm{kg}$ of body weight results in instability of crystal lattice of dentin mineral of lower incisor 
in rats. Combined action of SG and IR has more expressed adverse effects on ultrastructure of crystal lattice of dentin mineral. Administration of $\mathrm{Sp}$ together with SG and IR reduces effects of experimental conditions on crystal lattice of dentin mineral.

\section{P475}

\section{CHEMICAL COMPOSITION OF DENTIN OF THE LOWER INCISOR AND STRUCTURE OF RENAL PROXIMAL CONVOLUTED TUBULES IN RATS AFTER 60-DAY INTAKE OF TOLUENE VAPORS}

V. A. Gavrilov ${ }^{1}$, V. I. Luzin ${ }^{1}$, O. N. Fastova ${ }^{1}$

${ }^{1}$ SE Lugansk State Medical University, Lugansk, Ukraine

Objectives: Aim of the study is to analyze chemical composition of dentin of the lower incisor and structure of renal proximal convoluted tubules in rats after 60-day exposure to toluene (Tol) and application of thiotriazoline (Th) and Echinaceae tinctura (ET) as medication.

Material and Methods: The experiment involved 420 male rats (young, adult, and old): the first control group; the second group - animals that received inhalations of Tol; the third group received Tol and Th; the fourth group - animals that received Tol and $E T$.

Results: In young rats shares of mineral content, $\mathrm{Ca}, \mathrm{F}$ and $\mathrm{Ca} / \mathrm{P}$ ratio were lower than those of the control group by $8.90 \%, 9.82,6 ., 55 \%$ and $11.94 \%$; in adult animals - by $6.43 \%, 8.71 \%, 8.54 \%$ and $13.05 \%$; in senile animals by $6.18 \%, 7.78 \%, 7.85 \%$ and $9.65 \%$ respectively. Thus, diameters of proximal tubules and tubular lumen were higher than control by $9.53 \%$ and $17.66 \%, 8.28 \%$ and $15.47 \%, 7.26 \%$ and $18.84 \%$ respectively. The height of the epithelium in immature rats was higher than control data by $3.39 \%$, in senile animals - by $5.97 \%$. Application of Th or ET reduced negative effects of Tol. After Th application, deranged features of the crystal lattice restored in young animals by the 7th day, adult animals exhibited restoration signs throughout the whole observation period and in old animals signs of restoration were observed by the 7th and to the 60th days. After ET application, in young animals restoration of the crystal lattice was observed by the 7 th day, in adult by the 15 th day, and in old animals - by the 30th and the 60th days of observation.

Conclusions: 60-day inhalation of Tol results in instability of chemical composition of dentin and failure of reabsorption in proximal convoluted tubules. Deviations degree and recovery rate depend on age of animals. Faster recovery rate was observed in young animals while old animals exhibited few signs of recovery. Application of T or ET reduces negative effects of toluene.

\section{P476}

ILIAC ANEURYSMAL BONE CYST INVOLVING THE SACROILIAC JOINT

A.-K. Kul $^{1}$, F.-B. Bagcier ${ }^{1}$, U.-B. Bayraktutan ${ }^{2}$

${ }^{1}$ Physical Therapy and Rehabilitation, Atatürk University Faculty of Medicine, Erzurum, Turkey, ${ }^{2}$ Radiology, Atatürk University Faculty of Medicine, Erzurum, Turkey

Aneurysmal bone cysts are destructive, expansile bone lesions characterized by a reactive proliferation of connective tissue containing multiple blood filled cavities. Aneurysmal bone cysts most commonly occur in the distal femur or proximal tibia. We are reporting a case of iliac aneurysmal bone cyst involving the sacroiliac joint. A 60-year-old male patient was admitted to our hospital with tenderness over the right sacroiliac region and a history of unsuccessful conservative treatment for lower back pain. The patient had no neurologic deficit. She underwent routine radiographic evaluation. Anteroposterior pelvic radiograph showed lytic lesion of the sacrum adjacent to the right sacroiliac joint. On computed tomography septated lytic lesion of the sacrum and right iliac bone involving the right sacroiliac joint was observed. An open biopsy confirmed this. After preoperative embolization the patient underwent a thorough curettage of this lesion. There was resolution of the symptoms following surgery and no evidence of recurrence after years. Many different clinical cases were identified for the definitive diagnosis of sacroiliac jointrelated paint. Selim cystic bone lesions are among these diagnoses. Bone tumours which show pelvic involvement are mostly malign tumours. ${ }^{1}$ Selim cystic tumour-like lesions of the bone mainly hold the lengthy bones of the lower and higher extremities. Selim cystic tumour-like lesions should be taken into consideration in the definitive diagnosis of the sacroiliac joint-related pain. Such lesions could be treated safely and effectively with expanded intralesional curettage, local adjuvant cures and grafting. ${ }^{2}$

${ }^{1}$ Bloem JL, Reidsma II. Eur J Radiol 2012;81:3793.

${ }^{2}$ Unni KK. Dahlin's bone tumors: general aspects and data on 11,087 cases. 5th ed. Philadelphia: Lippincott-Raven; 1996. P. 382-90.

\section{P477 \\ BONE STATUS IN ADULT PATIENTS WITH TURNER SYNDROME}

C. Di Somma ${ }^{1}$, V. V. Zhukouskaya ${ }^{1}$, E. Scarano ${ }^{1}$, C. Savanelli ${ }^{2}$, A. Renzullo ${ }^{1}$, A. Colao ${ }^{1}$

${ }^{1}$ Dipartimento di Medicina Clinica e Chirurgia, Divisione Endocrinologia, University of Naples Federico II, Naples, Italy, ${ }^{2}$ IOS \& Coleman Srl, Naples, Italy

Background and aim: Osteoporosis is a common feature of Turner Syndrome, the aetiology of which is unclear. The histology of osteoporosis in Turner patients resembles to that observed in postmenopausal osteoporosis. On the other hand, it has been 
demonstrated that the addition of low dose estrogen to a regimen of growth hormone (GH) improves bone deposition and calcium metabolism in girls with Turner Syndrome. Estrogen seems to be facultative for the GH effects on the bone. However, most studies on bone mineral status in Turner syndrome were carried out in pre-pubertal or pubertal age. Therefore, the aim of the present study was to determine whether osteoporosis is a feature of adult patients with Turner syndrome.

Patients and methods: We evaluated bone mass and bone parameters in adult patients after the closure of the epiphyseal plates. Ten patients with Turner syndrome (28-35 yr aged) and 10 age-, height-, weight-, and BMI-matched healthy subjects entered the study. The patients were treated in pre-pubertal and or pubertal age with rhGH until the closure of the epiphyseal plates. All patients were treated with estrogen and progesterone using standard protocol before and throughout the study. In all patients and controls lumbar spine (LS) and femoral neck (FN) BMD, using Hologic QDR 1000, serum osteocalcin and urinary N-terminal crosslinked telopeptide of type I collagen (NTx) levels have been evaluated.

Results: No significant differences were found in BMD values at both sites (LS: $0.97 \pm 0.02$ vs. $0.99 \pm 0.01 \mathrm{~g} / \mathrm{cm}^{2}$, $p<0.05$; FN: $0.89 \pm 0.01$ vs. $0.91 \pm 0.01 \mathrm{~g} / \mathrm{cm}^{2}, p<0.05$ ), in osteocalcin $(8.5 \pm 2.7$ vs. $9.2 \pm 2.1 \mathrm{mg} / \mathrm{L}, p<0.05)$ and urinary NTx levels $(77.4 \pm 2.5$ vs. $76.3 \pm 2.8 \mathrm{nmol} \mathrm{BCE} / \mathrm{mmol} \mathrm{Cr}$, $p<0.05$ ) between patients and controls, respectively.

Conclusion: This study shows that adult patients with Turner syndrome, treated with rhGH until the closure of the epiphyseal plates and estrogen, show BMD values at lumbar spine / femoral neck and parameters of bone metabolism comparable to healthy subjects. These findings could be likely due to a direct effect of rhGH and estrogen therapy on bone mineral status, although further studies are mandatory to confirm these results.

\section{P478}

CORTICAL AND TRABECULAR BONE ANALYSIS OF PATIENTS WITH EARLY BREAST CANCER UNDER AROMATASE INHIBITORS, B-ABLE COHORT, USING 3D-DXA: A LONGITUDINAL STUDY

$\underline{X . ~ N o g u e ́ s ~}^{1}$, L. H. Humbert ${ }^{2}$, M. Rodriguez-Sanz ${ }^{1}$, S. Servitja ${ }^{3}$, N. Garcia-Giralt ${ }^{1}$, L. Garrigos ${ }^{3}$, J. Rodriguez-Morera ${ }^{1}$, L. Mellibovsky $^{1}$, M. Martinez-Garcia ${ }^{3}$, R. F. Fonolla ${ }^{2}$, Y. Martelli $^{2}$, J. Romera ${ }^{2}$, A. Diez-Perez ${ }^{1}$, I. Tusquets ${ }^{3}$

${ }^{1}$ Internal Medicine Department, Parc de Salut Mar, Universitat Autònoma de Barcelona, IMIM (Hospital del Mar Research Institute), Red Temática de Investigación Cooperativa en Envejecimiento y Fragilidad (RETICEF), Instituto de Salud Carlos III FEDER, Barcelona, Spain, ${ }^{2}$ Musculoskeletal Unit, Galgo Medical S.L., Barcelona, Spain, ${ }^{3}$ IMIM (Hospital del Mar Research Institute), Medical
Oncology Department, Parc de Salut Mar, Universitat Autònoma de Barcelona, Barcelona, Spain., Barcelona, Spain Objective: To analyze the cortical and trabecular bone changes after treatment with aromatase inhibitors (AI) in patients with early breast cancer.

Material and Methods: A subgroup of 40 women with early breast cancer from B-ABLE cohort was included in this study. DXA scans (QDR 4500 SL, Hologic, Waltham, MA, USA) were acquired at baseline and after a 5-y of treatment with letrozole. The 3D-DXA software (Galgo Medical, Barcelona, Spain) was used to analyze the cortical and trabecular bone of patients and control group. 3D-DXA registers a 3D appearance model of the femoral shape and density onto the DXA projection to obtain a 3D subject-specific model of the femur of the patient and quantify the volumetric BMD (vBMD), volume (for trabecular and cortical regions) and cortical thickness distribution. The 3D-DXA measurements for both groups were compared using paired samples Student's $t$-test.

Results: Mean age at baseline was $60.1 \pm 6.9$ years. A statistically significant decrease of the mean cortical thickness of $-0.1 \mathrm{~mm}(-2.7 \%, p=0.003)$ and of the cortical density of $-4.5 \mathrm{mg} / \mathrm{cm}^{3}(-0.43 \%, p=0.005)$ was observed at 5-y follow-up at the total femur region of interest. No statistical difference was observed in terms of trabecular density $(p=0.813)$. An increase of the trabecular volume of $+1.2 \mathrm{~cm}^{3}(+2.2 \%$, $p=0.003)$ and an increase of the integral volume of $+0.8 \mathrm{~cm}^{3}$ $(+1.2 \%, p=0.050)$ were observed, together with a decrease of the volume of the cortex $(-0.3 \%, p=0.050)$.

Conclusion: The cortical thinning and a decrease in density observed after 5-y follow-up of women with early breast cancer treated with AI was accompanied of an outward cortical displacement and an endosteal resorption. Those findings should be compared with results obtained from a similar study including a control population.

\section{P479}

SECULAR TRENDS IN FRACTURE INCIDENCE IN THE UNITED KINGDOM BETWEEN 1990-2012 IN SUBJECTS 50 YEARS OR OLDER

R. Y. van der Velde ${ }^{1}$, C. E. Wyers ${ }^{1}$, E. M. Curtis ${ }^{2}$, P. Geusens ${ }^{3}$, J. P. van den Bergh ${ }^{1}$, F. de Vries ${ }^{4}$, C. Cooper ${ }^{2}$, T.-P. van de $\mathrm{Staa}^{5}$, N. C. Harvey ${ }^{2}$

${ }^{1}$ Department of Internal Medicine, VieCuri Medical Center, Venlo, Netherlands, ${ }^{2}$ MRC Lifecourse Epidemiology Unit, University of Southampton, Southampton, United Kingdom, ${ }^{3}$ Department of Internal Medicine, Subdivision of Rheumatology, Maastricht University Medical Centre, Maastricht, Netherlands, ${ }^{4}$ Department of Clinical Pharmacy \& Toxicology, Maastricht University Medical Centre, Maastricht, Netherlands, ${ }^{5}$ Health eResearch Centre, University of Manchester, Manchester, United Kingdom 
Objectives: There is increasing evidence of secular changes in age and sex adjusted fracture incidence globally. Since altered fracture rates have major implications for healthcare provision and planning, we investigated secular trends in age and sex adjusted fracture incidence amongst the UK population aged 50 years or above from 1990 till 2012.

Materials and methods: We undertook a retrospective observational study using the Clinical Practice Research Data link (CPRD), representative for the UK population. Site specific fracture incidence was calculated by calendar year for men and women separately, with fracture type categorized according to ICD-9 classification. Linear regression analyses were used to calculate mean annualized change in absolute fracture incidence and to test for trend by calendar year. For presentational purposes, mean rates in the first 5 years (1990-1994) and last 5 years (2008-2012) were calculated.

Results: Overall fracture incidence was unchanged in both women (168.2 to 170.0 per 10,000 py) and men ( 74.8 to 78.9 per 10,000 py) from 1990 to 2012 . The incidence of hip fracture remained stable in women (1990-1994: 33.8 per 10,000 person years (py) vs. 2008-2012: 33.5 per 10,000 py; $\mathrm{p}$ trend annualized change in incidence $=0.80$ ), but increased in men (from 10.8 to 13.4 per $10,000 \mathrm{py} ; p=0.002$ ). Incidence of clinical vertebral fractures increased in women (8.9 to 11.8 per $10,000 \mathrm{py} ; p=0.005)$ but remained stable in men (4.6 to 5.9 per 10,000 py; $p=0.72$ ). Similarly incidence of radius/ ulna fractures did not change in men (9.6 per 10,000 py; $p=0.25$ ), and decreased in women ( 50.4 to 41.2 per 10,000 py; $p=0.001)$. Secular trends amongst fractures of the carpus, scapula, humerus, foot, pelvis, skull, clavicle, ankle, patella and ribs varied according to fracture site and sex.

Conclusion: Although, overall sex-specific fracture incidence in the UK population aged 50 years or over appears to have remained stable over the last two decades, there have been noticeable changes in incidences of individual fracture types. Given that the impact of a fracture on morbidity, mortality and health economy varies according to fracture site, these data clearly inform the provision of healthcare services in the UK.

\section{P480 \\ CHANGES IN BONE STATUS AND EXPRESSION OF MECHANO-GROWTH FACTOR BY SKELE- TAL MUSCLE AND BONE IN RESPONSE TO CHIROPRACTIC MANIPULATION IN AN OSTEOPOROTIC MODEL}

A. López-Herradón ${ }^{1}$, R. Fujikawa ${ }^{1}$, M. Gómez-Marín ${ }^{1}$, J.-P. Stedile-Lovatel $^{1}$, F. Mulero ${ }^{2}$, I. Muñoz-García ${ }^{1}$, P. RuizCarretero $^{1}$, P. Esbrit ${ }^{3}$, A. Ortega-De Mues ${ }^{1}$

${ }^{1}$ Madrid College of Chiropractic-Real Centro Universitario Escorial-María Cristina, San Lorenzo de El Escorial, Spain,
${ }^{2}$ de Imagen Molecular, Centro Nacional de Investigaciones Oncológicas (CNIO), Madrid, Spain, ${ }^{3}$ Laboratorio de Metabolismo Mineral y Óseo, Instituto de Investigación Sanitaria (IIS)-Fundación Jiménez Díaz-UAM, Madrid, Spain

Chiropractic aims to alleviate the morbidity of musculoskeletal disorders. Current data suggest that some pathological situations such as osteoporosis can be improved by chiropractic manipulation (CM). However, the molecular mechanisms involved in this positive effect of $\mathrm{CM}$ remain to be understood. Muscle strength changes occurring during $\mathrm{CM}$ could account in part for its beneficial skeletal effects. The aim of the present study was to evaluate the possible changes elicited by $\mathrm{CM}$ on bone structure and skeletal muscle in ovariectomized rats. Shamoperated $(\mathrm{Sh} ; n=10)$ and ovariectomized (OVX; $n=15)$ Sprague Dawley rats (6 months-old) were submitted to CM (M group) or not (NM group) using the chiropractic adjusting instrument Activator $\mathrm{V}^{\circledR}$ (ACTV) as follows: Force 1 setting was applied onto the tibial tubercle of the right hind limb of Mrats (true $\mathrm{CM}$; tM), at an angle of approximately $90^{\circ}$, from medial to lateral side; the corresponding left hind limb received a false CM (fM) consisting on firing the ACTV in the air and touching the tibial tubercle slightly. These procedures were repeated 3 times a week for 6 weeks. BMD and bone mineral content (BMC) were determined in the long bones and L3-L4 vertebrae in all rats in vivo. After euthanasia, both femora and tibiae were removed for $\mu \mathrm{CT}$ analysis and osteocyte apoptosis determination by terminal deoxynucleotidyltransferase dUTP nick end labeling (TUNEL) assay. Mechano-growth factor (MGF), a splice variant of IGF-1 that is expressed in myocytes and bone cells, was detected by immunohistochemistry in both femora and tibiae. Moreover, MGF protein expression in soleus, quadriceps and tibial muscles was analyzed by Western blot. A significant decrease of BMD and BMC as well as trabecular bone impairment in the long bones was observed in OVX rats vs. Sh controls. We found no differences in parameters related to bone mass or bone structure between NM and fM conditions in both Sh and OVX groups. However, a significant improvement of bone parameters occurred in the OVX-tM group vs. OVX-NM or fM. In contrast, bone mass values of vertebrae were similar in all OVX groups, independently of $\mathrm{CM}$. These changes were associated with higher values of MGF protein expression in the quadriceps and the anterior tibial muscle, but a decreased expression of this factor in the cortical femora and tibiae, in the tM-OVX group, compared to those in NM-OVX rats.

Finally, we found a significant decrease in the number of apoptotic osteocytes in the former group when compared to NM-OVX rats. These results indicate that $\mathrm{CM}$ can improve deteriorated bone, and suggest the involvement of MGF as a putative mechanotransduction signal in bone and muscle triggered by CM in a rat model of primary osteoporosis. 


\section{P481}

VERTEBRAL OSTEOPOROTIC FRACTURES AS THE ONLY SYMPTOM OF NONSECRETORY MULTIPLE MYELOMA: CASE REPORT

S. Subin-Teodosijevic ${ }^{1}$, J. Zvekic-Svorcan ${ }^{2}$, T. Jankovic ${ }^{2}$, K. Filipovic $^{2}$

${ }^{1}$ Rheumatology, Internal Medicine, General hospital Dr Dj.Joanovic, Zrenjanin, Serbia, ${ }^{2}$ Rheumatology, Special Hospital for Rheumatic Diseases, Novi Sad, Serbia

Objective: Multiple myeloma is a malignant proliferation of plasma cells within the bone marrow. Standard for diagnosis, prognosis and management is measurement of circulating monoclonal immunoglobulin. In about 1-5 \% of multiple myeloma cases no protein can be detected ${ }^{1}$ and these patients are known to have a nonsecretory type of myeloma. These patients are often a diagnostic dilemma, especially in a patient with osteolytic lesions ${ }^{2}$.

Material and Methods: We present a case of a 58-year-old male who came to our clinic with complaints of intensive low back pain of 6 months duration.

Results: Examination revealed tenderness on thoracic and lumbosacral spine and kyphosis.

An x-ray of the spine showed multiple vertebral fractures of vTh 8,9,10,12 and v L1,2 vertebra which are confirmed on MRI, and osteoporosis was described in radiological findings. Bone scintigraphy revealed areas of increased activity suspicious for osteolytic lesions in spine end ribs. DXA vL1-4 Tscore $-3,0$ and total hip T- score $-2,0$. Lab investigations ESR 18 Hgb 130 g/l Hct 0.357 Plt 357 WBC 7,2, Creatinine 70 urea 6,0, calcium 2.32,P 1,21, ALP 164 LDH 363 TP 72 g/1 alb 36,2 PSA 2,1. Immunoglobulins were in normal ranges, serum and urine protein immunoelectrophoresis did not show any abnormalities. Trauma and significant medical co-morbid conditions were excluded - we consulted orthopedic surgeon, endocrinologist, gastroenterologist, oncologist (normal findings of chest RTG, echosonography of abdomen and urinary tract, gastroscopy, colonoscopy, tumour markers, etc.) and finally hematologist who performed bone marrow biopsy which showed significant percent of plasmocytes $(20 \%)$ and established diagnosis.

Conclusion: Diagnosis in these cases depends on bone marrow biopsy. Absence of paraprotein in the blood does not exclude multiple myeloma. Consultation with hematologist is required when diagnosis of MM is strongly suspected, and other predisposing conditions for secondary osteoporosis excluded, in spite of absence of any abnormal protein, to avoid delay in diagnosis. Uncommon presentation may lead to delay in diagnosis if presented to rheumatologist or orthopedic surgeons.
References:

${ }^{1}$ Smith DB, et al. Haematol Oncol 1986;4:307.

${ }^{2}$ Middela S, Kanse P. Indian J Orthop 2009;43:408.

\section{P482}

THE BLADE AUGMENTATION TECHNIQUE IN OSTEOPOROTIC PERTROCHANTERIC HIP FRACTURES

A. Bañuelos Diaz ${ }^{1}$, F. J. Nistal Rodríguez ${ }^{1}$, R. Escudero Marcos $^{1}$, E. Cebrian Rodriguez ${ }^{1}$, I. Aguado Maestro ${ }^{1}$, A. Quintanilla Garcia $^{1}$, J. Lopez Sanchez ${ }^{1}$, M. F. Garcia Alonso ${ }^{1}$ ${ }^{1}$ Hospital Universitario Rio Hortega, Valladolid, Spain

Objective: Evaluate the technical performance, clinical outcomes and mid-long term results of augmentation technique associated to proximal femoral nail antirotation system (PFNA).

Material and Methods: We performed a retrospective analysis including patients treated with this technique between January 2010 and February 2014. 48 patients were treated; 44 pertrochanteric fragility fractures and 4 surgical revisions due to mechanical complications of a previous implant. Data collected and evaluated included complications associated with surgical technique, distribution of the cement around the helical blade, functional results and radiological outcomes such as fracture reduction (gap, CCD angle) and blade position.

Results: A total number of 48 patients ( 38 women and 10 men) were included in the analysis. Average follow-up was 14.5 months (12-24 months). Fracture reduction was good in $33(68.8 \%)$ patients, acceptable in $13(27 \%)$ and poor in 2 $(4.2 \%)$ cases. Blade position was in centre-centre position in $39(81.2 \%)$ patients and centre-inferior in $9(18.8 \%)$ cases. The mean volume of cement used was $4.2 \mathrm{ml}(3-5)$. The mean operative time was 52.7 minutes (25-120). Cement distribution did not show a clear pattern. There was an intraoperative complication: minimal leakage of cement into the hip joint. This complication appeared in one of the fragility fractures and had no further repercussions. No complications associated with augmentation occurred during follow-up; no signs of femoral head necrosis were observed. Two fractures collapsed without mobilization of the implant. $57.5 \%$ of patients achieved their pre-fracture functional status regarding capability to walk. 8 (16.7\%) patients died within the first 2 months for reasons no related to the surgical technique.

Conclusions: The PFNA-augmentation technique is safe and reproducible in osteoporotic pertrochanteric fractures, with low complication rate when adequate surgical technique is performed. It is of utmost importance to inject contrast into 
the helical blade before the introduction of the cement to prevent leakage. Furthermore, it could be a good alternative to increase implant anchorage on osteoporotic bone and decrease the rate of failures.

\section{P483}

FERMENTATION POTENTIATES THE PREVENTIVE EFFECT OF SOYBEAN FOR SENILE OSTEOPOROSIS IN SAMP6 MICE

$\mathrm{J}^{\mathrm{J} \text { A. Ahn }}{ }^{1}$, H. L. Lee ${ }^{1}$, C. J. Jung ${ }^{1}$, T. H. Ha ${ }^{1}$

${ }^{1}$ Korea Food Research Institute, Seongnam-si, Republic of Korea

Osteoporosis increases fragility fractures and is a major health problem in the elderly. The SAMP6 mouse is a substrain of senescence-accelerated mice which spontaneously develops osteoporosis early in life. The protective effects of soybean or fermented soybean for bone loss were reported. However, how fermentation process influences the physiological effect of soybean against osteoporosis has not been studied yet. Therefore, this study compares the effect of raw soybean and its fermented product, Cheonggukjang (CJ) for age-related bone loss in SAMP6 mice. Feedings of both soybean and CJ improve aging-associated decrease of trabecular bone mineral density (BMP). But the improvement of BMP was higher when the mice were fed with CJ. This was resulted from increase of bone formation and decrease of bone resorption as confirmed by bone-specific ALP and CTx-1 levels, respectively. CJ was more effective for lowering urine DPD level compare to soybean. Moreover, soybean and CJ upregulated BMP signalingrelated genes and RANK/OPG ratio. These results indicate that fermentation potentiates the effect of soybean for amelioration of senile osteoporosis and can suggest evidence for the health beneficial effect of fermentation.

\section{P484}

\section{ASSOCIATION BETWEEN BODY COMPO- SITION AND BONE MASS DENSITY}

E.-H. Kim ${ }^{1}$, H.-A. Seo ${ }^{1}$

${ }^{1}$ Departments of Internal Medicine, Daegu Fatima Hospital, Daegu, Republic of Korea

Introduction: Due to its high prevalence, osteoporosis is considered as a serious public health concern. Overweight is known to have a beneficial effect on BMD. But, there are not many studies about the independent effect of fat mass and lean mass on BMD. In this study, we investigated the association between the body composition and BMD.
Method: A total of 760 Korean (446 male, 314 female), who had undergone comprehensive routine health examinations at the Daegu Fatima Hospital, were included in this study. The BMD of lumbar spines (L1 to L4) was measured by DXA. Bioelectrical impedance analysis was used to measure the body composition by composition analyzer (Inbody 3.0, Biospace, Seoul, Korea).

Result: The mean age of total subjects was $47.29 \pm 11.60$. There was significantly positive association between muscle mass and BMD of lumbar spines in both males and females. Fat mass was significantly related with BMD of lumbar spines in male subjects, but this association was not shown in females. After adjustments for age and height, the significant positive correlation between muscle mass and BMD of lumbar spines was retained in both males and females. In males, the positive association between fat mass and BMD of lumbar spines was only shown in L1 and L2. In females, all sites of lumbar spine were related with fat mass.

Conclusion: In this study, muscle mass was highly related with BMD of lumbar spines in males and females. Association between fat mass and BMD was inconsistent. Muscle mass is more important that fat mass for BMD.

\section{P485}

OPTICAL COHERENCE TOMOGRAPHY FOR BONE MINERAL DENSITY QUANTITY

S. Khijmatgar $^{1}$

${ }^{1}$ Queen Mary, University of London, London, United Kingdom

\section{Objectives:}

- To determine whether the depth of light penetration into demineralized bone matrix is further than the depth of light penetration into mineralized bone matrix.

- To compare depth of light penetration into bone matrix in different mineralized bones of rats

Materials and Methods: Two pairs of carpel bones $(2 \mathrm{~cm}$ length) of the porcine forelimb and rats of age were cleared of attendant soft tissues and fixed overnight in $3.7 \%$ buffered formal saline. Following fixation, one bone was decalcified in 1:1 mixture of $8 \%$ hydrochloric acid and $8 \%$ formic acid solution for three days, with solution changes each day. Acid was neutralised, rinsed in water and bones were cut lengthwise to provide access to the endosteal surfaces and stored in $70 \%$ ethanol. Similarly rat of age 12 weeks and 8 weeks; calvaria, mandible and ulnar bones were imaged after cleared soft tissues. Samples were imaged using an OCT microscope (EX1301, Michelson Diagnostics Ltd, UK) operating at a central wavelength of $1300 \mathrm{~nm}$ with axial and lateral resolutions 
of approximately 10 and $9 \mu \mathrm{m}$, respectively. OCT image volumes $(3 \times 3 \times 1.5 \mathrm{~mm})$ were obtained from the interior, exterior and cross-sectional surfaces of each bone sample. The light penetration depth in each sample was measured for mineral content using Image $\mathrm{J}$ (National Institutes of Health, USA) and OCT images obtained is processed in scattering attenuation microscopy software (SAM) to get SAM images.

Results: The percentage change in the depth of penetration of light varies between $7 \%-64 \%(p<0.001)$. The penetration of light is more in demineralized than in mineralized bone samples but for mineralized interface cross-section, the percentage change in the penetration of light is more in mineralized than in demineralized bone. When seen in rat samples the penetration of light is further in the 12 week aged rat compared to 8 weeks rat.

Conclusion: The optical coherence tomography and SAM software is sensitive enough to detect the changes in association with the levels of BMD.

\section{P486 \\ PREVALENCE OF VITAMIN D DEFICIENCY AND ITS GENETIC AND NONGENETIC CORRELATES}

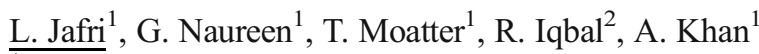

${ }^{1}$ Department of Pathology and Laboratory Medicine, Aga

Khan University, Karachi, Pakistan, ${ }^{2}$ Department of Community Health Sciences, Aga Khan University Hospital, Karachi, Pakistan

\begin{abstract}
Aim: Despite recent concerns about high prevalence of vitamin D deficiency (VDD) in adolescents, few studies have investigated the underlying reason. The study objective was to determine 25-hydroxyvitamin D (25OHD) status in young adults and its relation with socioeconomic factors, dietary intake, physical activity and single nucleotide polymorphisms linked to vitamin D binding protein (GC) gene (rs4588 and rs7041).
\end{abstract}

Material and Methods: A cross-sectional study on 211 medical students was conducted from July 2014-Nov 2015. Sociodemographic factors of healthy medical students were assessed; validated food frequency and physical activity questionnaires were filled. Quantitative heel ultrasound was done using Osteosys Sonost-3000. Total 25OHD was measured on ADVIA-Centaur; Siemens. Genomic DNA was extracted from whole blood samples of 86 subjects by Wizard Genomic DNA Purification Kit (Promega) and genotyped using polymerase chain reaction-based restriction fragment length polymorphism assay.

Results: Mean age of study group was $20.0 \pm 0.9$ years, $58.4 \%$ being females. Overall mean 25OHD levels were $17.5 \pm 15.2 \mathrm{ng} / \mathrm{ml}, 79.1 \%$ were $25 \mathrm{OHD}$ deficient, $10.4 \%$ insufficient and $10.4 \%$ sufficient. Out of the total students, $11.4 \%$ had BMD z-scores 2SD below the age and gender matched mean. Mean 25OHD in students with low z score were $14.8 \pm 7.7 \mathrm{ng} / \mathrm{ml}$ as compared to those with normal $\mathrm{z}$ scores, $17.8 \pm 15.9 \mathrm{ng} / \mathrm{ml}$ ( $\mathrm{p}$ value $>0.05$ ). Mean 25OHD $(15.9 \pm 10.9 \mathrm{ng} / \mathrm{ml})$ was comparatively high in individuals with the AC genotype followed by CC $(14.1 \pm 6.5 \mathrm{ng} / \mathrm{ml})$ and AA $(12.8 \pm 5.7 \mathrm{ng} / \mathrm{ml})$ amongst rs 4588 genotypes (p-value $>0.05$ ). As for rs7041, the mean 25OHD (15.6 $\pm 10.7 \mathrm{ng} / \mathrm{ml}$ ) was raised in individuals with GT compared with GG genotype $(14.5 \pm 6.7 \mathrm{ng} / \mathrm{ml})$ and TT genotype carrying individuals $(13.3 \pm 5.0 \mathrm{ng} / \mathrm{ml})(\mathrm{p}$-value $>0.05)$. Linear regression analysis was carried out to assess the association between 25OHD and other variables. No significant association was found between BMI, waist circumference, physical activity, protein/fat/calories intake, z-scores and 25OHD, while a poor positive association between $25 \mathrm{OHHD}$ and daily calcium intake was noted; $r=0.2, p<0.05$. Students living in apartments/small houses were significantly associated with VDD and lower z-scores.

Conclusion: Pakistani adolescents have high prevalence of VDD and insufficiency. With prevalent VDD in our population, it is vital to correct this deficiency and health care providers should tackle predictors of vitamin D status, favoring a healthier lifestyle and promoting supplementation in the groups at higher risk of VDD.

\section{P487 \\ BONE IN BONE IMAGE AFTER TREATMENT WITH INTRAVENOUS INFUSIONS OF DISO- DIUM PAMIDRONATE IN A CHILD SUFFE- RING FROM OSTEOGENESIS IMPERFECTA} J.-P. Devogelaer $^{1}$, F. Lecouvet ${ }^{2}$, G. Depresseux ${ }^{1}$, A. Durnez ${ }^{1}$ ${ }^{1}$ Rheumatology Department/Cliniques Universitaires SaintLuc, B-1200 Bruxelles, Belgium, ${ }^{2}$ Radiology Department/ Cliniques Universitaires Saint-Luc, B-1200 Bruxelles, Belgium

A boy aged 12 suffering from osteogenesis imperfecta, complicated by several peripheral and thoracic vertebral fractures was treated by intravenous infusions of disodium pamidronate ( $1 \mathrm{mg} / \mathrm{kg}$ per infusion), 3 consecutive days every 3 months for 2 years. His BMD measured by DXA increased from 0.416 to $0.600 \mathrm{~g} / \mathrm{cm}^{2}$ at the (L1-L4) lumbar spine and from 0.590 to $0.804 \mathrm{~g} / \mathrm{cm}^{2}$ at the total hip after 4 years. In the same time, he grew $16 \mathrm{~cm}$. The view of the postero-anterior DXA scan of the lumbar spine obtained 2 years after the last course of therapy demonstrated the presence of "arrest lines" close to the plateaus of the vertebral bodies. X-ray films confirmed that this aspect was attributable to a "bone in bone image" due to bisphosphonate therapy, a well-known radiological aspect in growing children 
treated by cyclical intermittent bisphosphonates ${ }^{1}$. This DXA aspect corresponding to the radiological image should be recognized, because much of the bone gain can be explained by it.

${ }^{1}$ Devogelaer J-P. Curr Opin Pharmacol 2002;2:748.

\section{P488}

CHARACTERISTICS OF WOMEN WITH POSTMENOPAUSAL OSTEOPOROSIS (PMO) RECEIVING DENOSUMAB (DMAB) OR MONTHLY ORAL BISPHOSPHONATES (OBPS) IN BULGARIAN CLINICAL PRACTICE

T. Petranova ${ }^{1}$, M. Boyanov ${ }^{2}$, A. Shinkov ${ }^{3}$, R. Petkova ${ }^{4}$, M. Intorcia $^{5}$

${ }^{1}$ Clinic of Rheumatology, Medical University, Sofia, Bulgaria,

${ }^{2}$ University Hospital Alexandrovska, Sofia, Bulgaria,

${ }^{3}$ University Hospital of Endocrinology "Acad. Iv. Penchev", Sofia, Bulgaria, ${ }^{4}$ Amgen Bulgaria, Sofia, Bulgaria, ${ }^{5}$ Amgen (Europe) $\mathrm{GmbH}$, Zug, Switzerland

Objective: Describe characteristics of women with PMO receiving DMAb or monthly oBPs in Bulgaria.

Material and Methods: This national retrospective chart review included women $\geq 50$ years old with a PMO diagnosis, initiating DMAb or monthly oBPs from 1Oct2011 to 30Sep2012, followed for up to 24 months. BMD T-scores and oBP discontinuation date (including switch to another treatment/regimen) were taken from medical records; if no discontinuation date was recorded, treatment was assumed to have continued. DMAb persistence was defined as receiving the relevant injection within 6 months +60 days of the previous injection.

Results: 224 women initiating DMAb and 217 initiating monthly oBPs met the inclusion criteria. Of these, 57 [25\%] and 38 [18\%] women $\mathrm{had} \geq 1$ prior OP fracture, respectively; $3(1.3 \%)$ and $8(3.7 \%)$ experienced $\geq 1 \mathrm{OP}$ fracture during follow-up. Women initiating DMAb had lower BMD T-scores and experienced greater BMD increases during follow-up (Fig). $4.5 \%$ of women initiating $\mathrm{DMAb}$ and $56 \%$ initiating monthly oBPs discontinued treatment; where recorded, reasons were: $\mathrm{DMAb}$, financial [1 (10\%) patient]; oBPs, poor adherence (41 [34\%]), lack of efficacy (12 [10\%]), adverse event (9 [7\%]), hospitalisation $(1[<1 \%))$. Using a 60 -day permissible gap, $\mathrm{DMAb}$ persistence at 12,18 and 24 months was $100 \%$, $99.1 \%$ and $98.7 \%$, respectively.

Conclusions: In our study of clinical practice, women initiating DMAb had more severe disease and greater BMD increases than those initiating monthly oBPs. Only $4.5 \%$ of women initiating DMAb discontinued treatment within 24 months. Such high persistence could lead to improved clinical outcomes, including fracture risk reduction.
Acknowledgements: Funded by Amgen

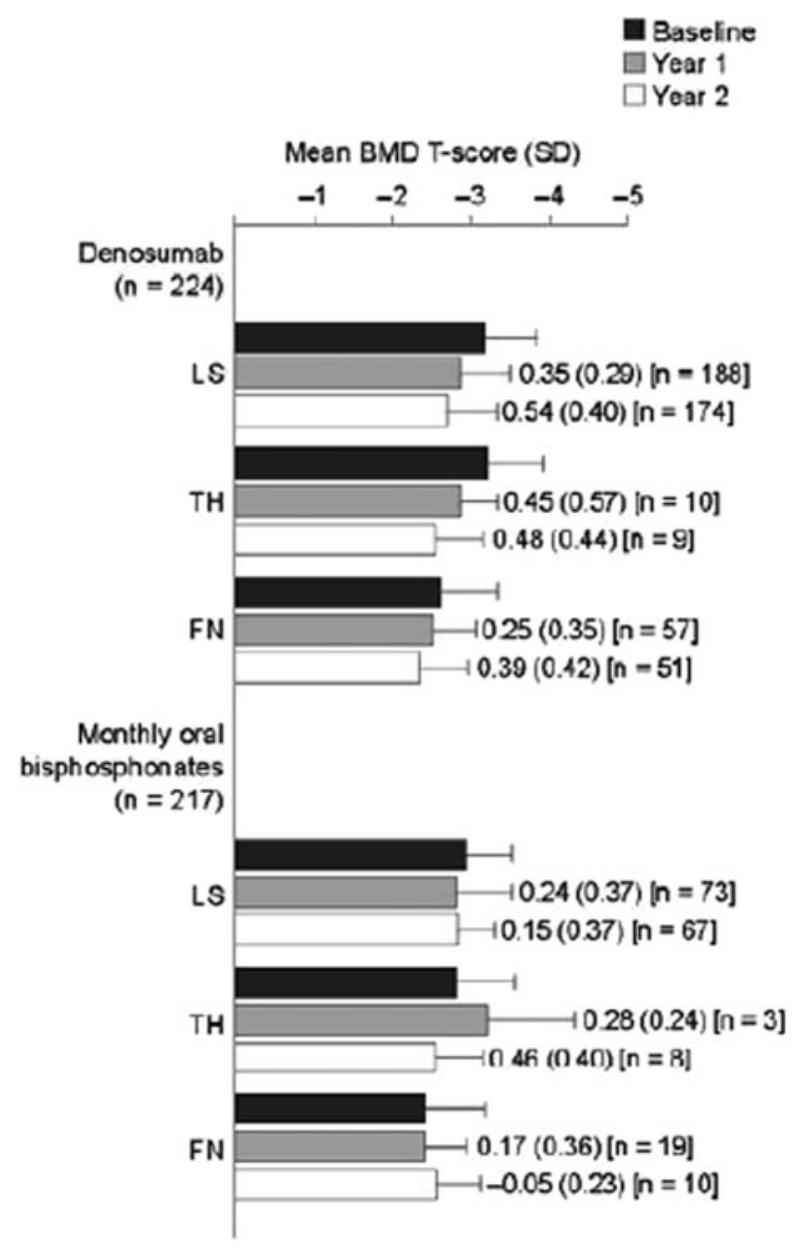

Bars represent mean BMD T-sccre. Error bars represent the SD of the mean; data labels show the mean (SD) change from besoline calculated from the number of patients with a BMD T-score at both time paints.

\section{P489}

THE QUALITY OF BONE OF OVARIECTOMIZED RATS TREATED WITH SULFUROUS WATER

G. Stefanovski ${ }^{1}$, T. Popovic ${ }^{2}$, M. Ceko ${ }^{2}$, M. Stefanovski ${ }^{2}$ ${ }^{1}$ IPRM "Dr M. Zotovic"-Rheumatology, Banja Luka, Bosnia and Herzegovina, ${ }^{2}$ Mljecanica Spa-Balneology, K. Dubica, Bosnia and Herzegovina

Introduction: The most common type of osteoporosis is associated with ovarian hormone deficiency at menopause. Sulfurous mineral water is used widely in the treatment of skeletal disorders. Aim: To evaluate the influence of sulfurous mineral water "Mljecanica" on bone quality of ovariectomized rats.

Material and method: The 14-weeks old female Wistar rats $(N=14)$ were ovariectomized and randomized to experimental (OVX SW) and control (OVX) group, seven in each, and seven 
with sham surgery served as intact controls (INT). The accommodation conditions and feeding were the same for all groups. After six weeks the OVX SW rats were supplied with sulfurous mineral water ad libitum, during four weeks, five days per week, while the OVX and INT rats were drinking taped water. At the end of four weeks period biochemical analyzes of osteocalcin (OC), alkaline phosphatase (AP), calcium (Ca) and phosphorus (P) were evaluated. The histological analyzes of left tibia were studied by routine microscopy. Biomechanical properties were assessed on TOMI 2001.

Results: Statistically significant increase of OC, Ap and P was obtained in OVX SW compared to INT group $(p<0.05)$. The increase of same parameters was not significant in comparison with OVX group. Histological analyses showed the growth of young chondrocytes in the central zone of bone and their migration to peripheral parts. The trabecules were enlarged, sill disconnected, with bone marrow in between. Bending and torsion of left femur showed better quality in OVX SW group. Conclusion: This study shows that sulfurous water, used in experimental osteoporosis, induces the repairing mechanism and has positive impact on mechanical properties of osteoporotic bone. Clinical research follows our experimental study. Acknowledgements: This study is supported by Grant N 06/ 06-020/961-49/05 Ministry of Science and Technology, Republic of Srpska.

\section{P490}

A NOVEL INJECTABLE VISCO-ANTALGIC (JTA- 004) WITH PROLONGED LUBRICATION EFFECTS AND ANTI-INFLAMMATORY PROPERTIES FOR KNEE OSTEOARTHRITIS

J. Winand $^{1}$, S. Pietri ${ }^{1}$, S. Ena ${ }^{1}$, N. Stricwant ${ }^{1}$, E. Bastianelli ${ }^{1}$

${ }^{1}$ Bone Therapeutics S.A., Gosselies, Belgium

Bone Therapeutics, a bone and joint regenerative company, develops innovative medicinal products for the treatment of osteoarthritis (OA). The company has developed a novel injectable visco-antalgic (JTA-004 ${ }^{\circledR}$ ) intended to restore the natural composition and the viscous properties of synovial fluid of osteoarthritic knees. The specific composition of JTA- $004{ }^{\circledR}$ confers a prolonged stability to the product once injected and reduces local inflammation and pain. The purpose of the study was to demonstrate (i) in vitro the anti-inflammatory effects of JTA-004 ${ }^{\circledR}$ and (ii) in vivo the prolonged lubrication effects of JTA-004, compared to commercially available viscosupplements.

Methods: The anti-inflammatory properties of JTA- $004^{\circledR}$ were assessed in an activated peripheral blood mononuclear cells model (PBMC; from healthy donors) where activated PBMC were contacted with JTA- $004^{\circledR}$, a commercially available viscosupplement (Ostenil ${ }^{\circledR}$ Plus) or positive controls (methotrexate and dexamethasone) $(n=2)$. After 3 days of culture, cell counting, cell characterization and levels of TNF- $\alpha$ secretion were assessed. In vivo, the lubrication efficacy of JTA-004 ${ }^{\circledR}$ and of the marketed viscosupplement (Synvisc-One ${ }^{\circledR}$ ) was assessed by measuring the viscosity of synovial fluid in a model of knee OA induced by complete resection of the medial meniscus in rat. Synovial fluid viscosities were assessed 2, 4, 6 and 10 days after injection ( $n=5$ per group).

Results: After 3 days of culture, JTA-004 ${ }^{\circledR}$ decreased the proliferation of activated PBMCs by $52 \pm 2 \%$ (relative to activated PBMCs alone), a level close to the positive control methotrexate $(73 \pm 8 \%)$. The secretion of TNF- $\alpha$ by activated PBMCs was decreased by $41 \pm 14 \%$ in presence of JTA- $004^{\circledR}$ as compared to activated PBMCs, a level close to the positive control dexamethasone $(54 \pm 6 \%)$. Ostenil ${ }^{\circledR}$ Plus did not affect the proliferation and the levels of cytokine secretion. In vivo at each study time point, the synovial fluid viscosity of meniscectomized knees treated with JTA- $004^{\circledR}$ was higher than that of healthy knees. Ten days after injection, the synovial fluid viscosity of meniscectomized knees injected with JTA-004 ${ }^{\circledR}$ was still $67 \%$ higher than the viscosity of healthy knees against only $11 \%$ with Synvisc-One ${ }^{\circledR}$; the difference between JTA- $004^{\circledR}$ and SynviscOne ${ }^{\circledR}$ was statistically significant.

Conclusion: these studies demonstrate that as compared to commercially available viscosupplements, JTA-004 ${ }^{\circledR}$ displays potent anti-inflammatory properties in vitro by reducing the proliferation of activated mononuclear cells as well as the secretion levels of TNF- $\alpha$, a major player in OA pathophysiology and in vivo a prolonged lubrication effect in joints submitted to mechanical and/or biological stresses. Altogether these data suggest a superiority of JTA- $004^{\circledR}$ as compared with commercially viscosupplements.

\section{P491 \\ IDENTIFICATION OF NOVEL REGULATORS OF OSTEOBLAST DIFFERENTIATION IN PRIMARY CALVARIAL OSTEOBLASTS USING A HIGH- THROUGHPUT SIRNA SCREEN

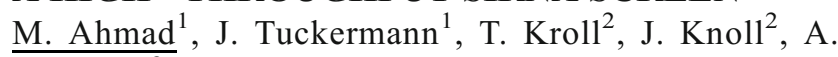 Ploubidou $^{2}$ \\ ${ }^{1}$ Institute for Comparative Molecular Endocrinology (CME), University of Ulm, Ulm, Germany, ${ }^{2}$ Leibniz Institute for Age Research - Fritz Lipmann Institute (FLI), Jena, Germany}

Osteoblasts are specialized, differentiated descendants of mesenchymal stem cells, responsible for the formation and 
mineralization of the skeleton. To date, several osteoblastdifferentiating genes have been identified, whose dysregulation leads to several debilitating disorders including osteoporosis and genetic diseases affecting bone. Besides, glucocorticoids are widely used to treat acute and chronic inflammatory diseases, and lead to multiple side effects including glucocorticoid induced osteoporosis (GIO), the most common form of secondary osteoporosis. We have previously shown that GIO depends on glucocorticoid receptor mediated reduction of osteoblast differentiation (Cell Metabolism 2010 11:517). Although, important advances in the molecular basis of osteoblast differentiation have been achieved, a complete understanding of its mechanistic underpinnings is still relatively unclear. In order to identify novel regulators of osteoblast differentiation with respect to osteoporosis and GIO, and to gain further insights into the mechanistic role of these factors, we carried out an unbiased large-scale siRNA screen in primary calvarial osteoblasts. Using a cell-based functional assay for alkaline phosphatase activity using high content analysis microscopy. We identified novel gene networks in an siRNA screen that have previously unknown roles in bone development. Validation of selected candidates using targeted drug inhibitors confirmed the screening results and, most importantly, abrogated the deleterious effects of glucocorticoids on osteoblast differentiation. We will present data suggesting that these novel drug targets are promising candidates for pharmaceutical intervention in preventing GIO and improving bone strength in osteoporosis.

\section{P492 \\ POST-FRACTURE CARE: DO WE NEED TO EDUCATE THE PATIENTS RATHER THAN THE DOCTORS? THE PREVOST RANDO- MIZED CONTROL TRIAL}

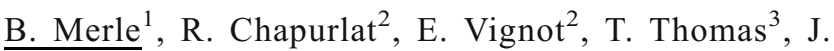
Haesebaert ${ }^{4}$, A.-M. Schott ${ }^{4}$

${ }^{1}$ INSERM UMR 1033, Université de Lyon, Lyon, France, ${ }^{2}$ INSERM 1033 Hôp E. Herriot, Lyon, France, ${ }^{3}$ INSERM U1059, Hôpital Bellevue, St Etienne, France, ${ }^{4}$ Unité Epidémiologie, Hospices Civils de Lyon, Lyon, France

Osteoporotic fractures predispose to future fractures and related morbi-mortality, but so far only a minority of patients with recent osteoporotic fracture receives adequate therapy. Therefore, we implemented a patient-centered post-fracture care program, Prevost (PREVention of OSTeoporosis) and evaluated its impact on BMD prescription and/or anti-osteoporotic treatment prescription. We enrolled 436 women aged 50 to 85 years in a multicenter, randomized controlled trial, while visiting emergency and orthopaedic departments of 21 hospitals from Region Rhône-Alpes, France, for a low-energy fracture of the radius/ulna or humerus, between March 2012 and December 2013. The randomization was stratified on age, hospital department type and site of fracture. The intervention was performed by a centralized coordinator who provided information solely to the patient about fragility fractures and osteoporosis, and encouraged her to contact her primary care physician for a follow-up concerning prevention of fragility fractures and osteoporosis. Patients from control group received usual care. All patients completed a standard questionnaire at baseline and 6 months later. Data were analyzed in intention to treat. At 6 months of follow-up, we found a significant increase in the primary endpoint, i.e. the proportion of women who initiated a post-fracture care, with $53 \%$ in the intervention group vs. $33 \%$ for usual care (OR:2.3, $95 \%$ CI [1.54-3.36], $p<0.001)$. Post-fracture management was more frequent after wrist than humerus fracture (46\% vs. $31 \%, p=0.009)$, and for women aged 50 to 69 than for those aged 70 and older, in both arms (48\% vs. $36 \%$, $p=0.01)$. The intervention resulted in $50 \%$ of BMD prescription vs. $33 \%$ in usual care (OR:2.0, 95\% CI [1,352.94], $p<0.001$ ), and $41 \%$ of BMD test performed vs. $25 \%$ for usual care (OR:2.1, 95\% CI [1.37-3.10], $p<0.0005)$. BMD results showed that $87 \%$ of women had a low BMD: $28 \%$ had a t-score $<-2.5 \mathrm{SD}, 59 \%$ had a $-2.5<\mathrm{t}$-score $<-1 \mathrm{SD}$. Having performed a BMD test significantly increased the set-up of a pharmacological treatment: $+22 \%$ (OR:8.3, 95\%CI [4.08-16.8], $p<0.001)$ and Calcium/vitamin D supplementation: $+36 \%$ (OR:5.1, 95\%CI [3.26-8.12], $p<0.001$ ). However, only $7 \%$ of women with humerus fracture were treated at 6-months post- fracture. In conclusion, our trial showed that a patient-centered care program with a dedicated coordinator can significantly improve post-fracture BMD testing and treatment initiation.

This study was supported by a grant from the French Ministry of Health: PREVOST, PREPS 2012- $n^{\circ}$ : 12-027-0088

\section{P493}

USING ONLINE PROGRAM OF GOOD NUTRITION AND RIGHT DIET MAY REDUCE THE RISK OF FRACTURES IN THE FUTURE.

\author{
V. Krylov ${ }^{1}$ \\ ${ }^{1}$ University Clinical Hospital \#1, Moscow, Russian \\ Federation
}


Introduction: It is too much easier and cheaper to prevent a decrease a bone density and fractures, than in the future to treat osteoporosis and its complications.

Materials and methods: we used an online system for patient education www.rightdiet.ru based on the video lessons, full of humor, pictures, and cartoons to convey the necessary information on good nutrition, necessary to do exercises and the need for exposure to the sun to our patients.

Study results: Watching the short movies, the patients formed the habits of good nutrition during the first month already, which includes a diet with restriction of fat, digestible carbohydrates and daily consumption of low-fat dairy products, slow carbohydrates, protein and fiber. Were also presented recommendations for compliance with the physical activity, as well as vitamin D consumption. A patient was in touch with a doctor-endocrinologist, if he has any additional questions. We examined data from a survey of 50 patients registered in the online system www.rightdiet.ru and 20 patients control group who were given the same recommendations on the appointment. Surprisingly, the consumption of milk and dairy products increased by 2.6 times, compared with patients in the control group. The exposure to the sun was observed 15 to 30 minutes daily, compared with the control group 5-10 minutes. Regular physical activity were the main group of 260 minutes per week, in control group 80 minutes per week.

Conclusions: Very important how we can make delivery of the material. We live in a world of high technologies and lack of time. Often the patient has no opportunity to go to the doctor for an appointment, and during reception it is not always possible to discuss all aspects, and even if it was possible, some information is forgotten by patients. So, we need to improve the quality of information material, including using online technologies to improve the quality and duration of life of our patients.

\section{P494}

\section{ASSOCIATION BETWEEN ADJUVANT THERAPIES AND RISK OF OSTEOPOROSIS IN YOUNG BREAST CANCER PATIENTS: A POPULATION-BASED STUDY}

C. H. Chang ${ }^{1}$, S. J. Chen ${ }^{2}$, C. Y. Liu ${ }^{3}$

${ }^{1}$ China Medical University Hospital, Taichung, Taiwan, Province of China, ${ }^{2}$ Mackay Memorial Hospital, Taitung, Taiwan, Province of China, ${ }^{3}$ National Taipei University of Nursing and Health Sciences, Taipei, Taiwan, Province of China
Background: Breast cancer survivors have an increased risk of osteoporosis. But the risk among young patients with adjuvant therapies remain conflicting. This population-based study is aimed to assess the incidence and risk of osteoporosis among young (age of 20-39 years) breast cancer patients who received adjuvant therapies including selective estrogen receptor modulators, third-generation aromatase inhibitors (AIs), monoclonal antibody, chemotherapy, and radiotherapy. Methods: From January 2001 to December 2007, 5,301 newly diagnosed breast cancer patients were enrolled from the National Health Insurance Research Database (NHIRD) in Taiwan. Patients were observed for a maximum of 6 years to determine the incidence of newly onset osteoporosis. Kaplan Meier and Cox regression analyses were used to evaluate the risk of osteoporosis in young breast cancer patients who received adjuvant treatments.

Results: Of the total 5301 young (age of 20-39 years) newly diagnosed breast cancer patients, the Cox multivariate proportional hazards analysis showed that AIs (adjusted HR $[\mathrm{aHR}]=2.974,95 \% \mathrm{CI}=1.876-4.715, p<0.001)$, trastuzumab $(a H R=1.960,95 \% \mathrm{CI}=1.067-3.603, p=0.03)$, and radiotherapy $(a H R=1.737,95 \% \mathrm{CI}=1.140-2.646, p=0.010)$ were significantly associated with a higher risk of osteoporosis after age and comorbidities were controlled for. Moreover, patients who received AIs for more than 180 days had a high hazard ratio (HR) of $1.414(95 \% \mathrm{CI}=0.7071-2.8279)$, and patients who received more than four radiotherapy visits had a high HR of $3.5486(95 \% \mathrm{CI}=1.9617-6.4192)$. Besides, young breast cancer patients with AIs treatment had the highest risk of hip fracture $(H R=8.520,95 \% \mathrm{CI}=1.711-42.432, p=0.04)$, while patients with radiotherapy treatment had the highest risk of vertebral fracture $(H R=5.512,95 \% \mathrm{CI}=1.847-16.451, p<0.01)$.

Conclusion: Young breast cancer patients who are receiving AIs, radiotherapy or monoclonal antibody need to be more careful for preventing osteoporosis and fracture events. Treatment plans are suggested to incorporate osteoporosis prevention interventions for young breast cancer patients.

\section{P495 \\ ASSOCIATION STUDY OF POLYMORPHISM IN THE LRP5 GENE REGION AND CALCANEAL ULTRASOUND PARAMETER IN YOUNG ADULTS \\ M. Correa-Rodriguez ${ }^{1}$, J. Schmidt Rio-Valle ${ }^{1}$, E. González- Jiménez $^{1}$, B. Rueda-Medina ${ }^{1}$ \\ ${ }^{1}$ Department of Nursing, University of Granada, Granada, Spain}


Introduction: Wnt signaling through low-density lipoprotein receptor-related protein 5 (LRP5) and sclerostin (SOST) plays an important role in the regulation of bone formation and resorption. Quantitative ultrasound (QUS) is a relatively recent and non-invasive method providing structural information on microstructure, bone elasticity and connectivity. For the first time we aimed to investigate the possible implication of these genes as genetic factors influencing calcaneal QUS in a population of Caucasian young adults.

Methods: The study population comprised a total of 575 individuals (mean age 20.41; SD 2.36) whose bone mass was assessed through QUS to determine broadband Ultrasound Attenuation (BUA, dB/MHz). Six single nucleotide polymorphisms (SNPs) of LRP5 (rs2306862, rs556442 and rs3736228) and SOST (rs4792909, rs851054 and rs2023794) were selected as genetic markers based on their association with BMD in previous genetic association studies and genotyped through the Open Array technology.

Results: Linear regression analysis revealed that the rs3736228 SNP of LPR5 was significantly associated with BUA parameter after adjustments for age, sex, weight, height, physical activity and calcium intake $(p=0,028, \beta(95 \% \mathrm{CI})=0,089(0,099$, 1,691). For the rest of SNPs no significant association with QUS measurements was observed.

Conclusion: We reported, for the first time, that LRP5 genetic polymorphism influences calcaneal QUS parameter in a population of Caucasian young adults. This finding suggests that LRP5 might be an important genetic marker contributing to bone mass accrual early in life.

\section{P496 \\ THE EFFECT OF ANTI-INFLAMMATORY THE- RAPY ON BONE MINERAL DENSITY IN PATIENTS WITH ANKYLOSING SPONDYLITIS ACCORDING TO THE RESULTS OF A THREE-YEAR OB- SERVATION}

T. Raskina ${ }^{1}$, O. Pirogova ${ }^{1}$, O. Malyshenko ${ }^{1}$, V. Fanaskov ${ }^{2}$

${ }^{1}$ Kemerovo State Medical Academy, Kemerovo, Russian Federation, ${ }^{2}$ Regional Clinical Hospital for War Veterans, Kemerovo, Russian Federation

Purpose: To evaluate BMD in the dynamics in patients with ankylosing spondylitis (AS) depending on the therapy threeyear prospective study.

Materials and methods: Under supervision there were 72 male patients with reliable diagnosis of AS. To study the effect of anti-inflammatory therapy on the indices of the BMD and Z-test, patients were divided into 2 groups: 1st group - 29 patients with the ineffectiveness of systematic long-term administration of NSAIDs, which were administered infliximab in the standard scheme at the rate of $5 \mathrm{mg} / \mathrm{kg}$ of body weight of the patient: in 1 day, then 2 weeks and 6 weeks after the first injection and then every 8 weeks; 2nd group-43 patients, the AS, which were scheduled NSAIDs: diclofenac $150 \mathrm{mg} /$ day $(n=22)$, nimesulide $-200 \mathrm{mg}$ / day $(n=19)$, meloxicam $15 \mathrm{mg}$ /day $(n=2)$. When poor tolerability of the initially selected drug was replaced with another NSAID in the equivalent dose. The BMD was determined by using a stationary DXA densitometer Excell XR-46 (Norland, USA) every 12 months (for 36 months).

Results: When including patients in the study in both groups of patients, the decrease in BMD and Z- scores in the neck of the 1174 femur. In group 1 of the BMD amounted $0.862 \pm 0.14 \mathrm{~g} / \mathrm{cm} 2$, Z-criterion $-1.51 \pm \mathrm{SD}$ of 0.11 , in group $2-0.852 \pm 0.16 \mathrm{~g} / \mathrm{cm} 2$ and $-1.61 \pm \mathrm{SD}$ of 0.12 ( $p>0.05$ and $p>0.05$, respectively). After 36 months of therapy no statistically significant differences at the level of BMD and Z-criterion in the neck of the femur relative to the original data is not revealed in both groups: group 1 - IPC hip amounted to 0.936 $\pm 0.12 \mathrm{~g} / \mathrm{cm} 2$, Z-criterion $-1.30 \pm 0.04 \mathrm{SD}$, in group 2 IPC $-0.836 \pm 0.17 \mathrm{~g} / \mathrm{cm} 2, Z$ - criterion $-1.68 \pm \mathrm{SD}$ of 0.06 ( $p>0.05$ and $p>0.05$, respectively). Comparison of the densitometric indices in the neck of the thigh of two groups after three years of therapy found that in group 1 the BMD and the Z- criterion was statistically significantly higher than the corresponding indicators 2 group (BMD group $1-0.936 \pm 0.12 \mathrm{~g} / \mathrm{cm} 2$ and Z-criterion $-1.30 \pm 0.04 \mathrm{SD}, \mathrm{BMD}$ group $2-0.836$ $\pm 0.17 \mathrm{~g} / \mathrm{cm} 2$, Z-criterion $-1.68 \pm \mathrm{SD}$ of $0.06, p<0.05$ and $p<0.05$, respectively). When measuring BMD in the lumbar spine marked an increase and stabilization of the BMD indices and Z-scores in both groups of patients. In group 1 indicators of the BMD before the start of treatment was $0.820 \pm 0.14 \mathrm{~g} / \mathrm{cm} 2,36$ months $1.003 \pm 0.10 \mathrm{~g} / \mathrm{cm} 2(p<0.05)$, the Z-criterion $-1.41 \pm \mathrm{SD}$ of 0.11 and $-0.98 \pm 0.04 \mathrm{SD}$, respectively $(p<0.05)$. In group 2 the performance of the BMD before the start of treatment was $0.869 \pm 0.16 \mathrm{~g} / \mathrm{cm} 2,36$ months - 1.103 $\pm 0.10 \mathrm{~g} / \mathrm{cm} 2 \quad(p<0.05)$, the Z-criterion $-1.51 \pm \mathrm{SD}$ and $-1.010 .12 \pm 0.04 \mathrm{SD}$, respectively $(p<0.05)$. Significant differences in the studied indicators in comparison of the two groups is not obtained. 
Conclusions: These results indicate that a lack of adverse effects of infliximab therapy on the BMD and the authentic improvement of indicators of the BMD as in the neck of the femur and in the lumbar spine.

\section{P497}

DELAYED EFFECTS OF SHORT TERM ACUTE AEROBIC EXERCISE ON MICROALBUMINURIA AND INSULIN SENSITIVITY

K. Frames $^{1}$

${ }^{1}$ Department of Biokinetics and Sports Science, University of Zululand, Richards Bay, South Africa

Objectives: The prevalence of obesity has increased worldwide and represents a major public health concern. Obesity is often associated with an increase in urinary albumin excretion and impaired insulin sensitivity. Whilst it is clear that exercise is beneficial in terms of improving insulin sensitivity, the optimal exercise prescription in different cohorts is still unknown, hence the recent increase in studies investigating high vs. moderate intensity interventions. It is also unclear what effect acute bouts of exercise may have on microalbuminuria, a marker which is more commonly being utilized for screening and prognosis of diabetes and cardiovascular disease.

Aim: To investigate the effects of an acute bout of aerobic exercise on insulin sensitivity and microalbuminuria in obese, and normal weight sedentary females.

Methods: Eighteen female participants $(24.78 \pm 5.17 \mathrm{y}$; BMI $34.55 \pm 6.22 \mathrm{~kg} / \mathrm{m}^{2}$ ) and ten normal weight participants (24 $\pm 3.74 \mathrm{y}$; BMI $\left.22.98 \pm 1.48 \mathrm{~g} / \mathrm{m}^{2}\right)$ leading sedentary lifestyles, participated in a single, 30 minute bout of moderate $(65 \%$ $75 \%$ Heart Rate Reserve [HRR]; 12-13 Rate of Perceived Exertion $[\mathrm{RPE}])$ and high intensity exercise (75\%-85\% HRR;14-15 RPE), over a two week period. Participants provided blood (GLUCOSE/INSULIN RATIO, HOMA INDEX, QUICKI INDEX, plasma insulin, plasma glucose) and urine samples (ALBUMIN/CREAT, creatinine, microalbumin) prior to each exercise bout and at $24 \mathrm{~h}, 48 \mathrm{~h}$ and $72 \mathrm{~h}$ postexercise.

Results: Fasting pre-exercise plasma glucose, HOMA Index and QUICKI Index were significantly $(p<0.05)$ different between the obese and control groups. No change in microalbuminuria was observed following the two bouts of exercise. A significant $(p=0.026)$ time effect was observed for albumin- creatinine ratio (ACR) with it being reduced at $48 \mathrm{~h}$ and $72 \mathrm{~h}$ post exercise. No interaction effects were observed between groups or exercise intensities. U-creatinine was significantly $(p<0.05)$ elevated in the normal weight group at $48 \mathrm{~h}$ post exercise. Both the obese and normal weight groups showed significant $(p=0.001)$ reductions in the glucose/insulin ratio at $24 \mathrm{~h}$ and $48 \mathrm{~h}$ post exercise with no differences observed between the groups or exercise intensities.

Conclusion: Acute bouts of different aerobic exercise intensities (moderate and high) failed to induce significant changes in overall microalbuminuria and insulin sensitivity in obese and normal weight females.

\section{P498}

EFFECTS OF CYCLIC YOGA ON BONE HEALTH IN POSTMENOPAUSAL WOMEN

Z. Maghbooli $^{1}$, A. Bakhshizadeh ${ }^{2}$, M. Machawe ${ }^{3}$, A. Hossein-Nezhad $^{1}$, B. Yarjoo ${ }^{2}$, S. Ahmadi ${ }^{3}$, M. A. Azarbayjani ${ }^{4}$, S. Shirazi ${ }^{1}$, S. M. Eshaghi ${ }^{1}$

${ }^{1}$ Osteoporosis Research Center, Endocrinology and Metabolism Clinical Sciences Institute, Tehran University of Medical Sciences, Tehran, Islamic Republic of Iran, ${ }^{2}$ Islamic Azad University, Tehran, Islamic Republic of Iran, ${ }^{3}$ Payam-e-Mehr International Yoga Center, Tehran, Islamic Republic of Iran, ${ }^{4}$ Department of Exercise Physiology, Islamic Azad University, Central Tehran Branch, Tehran, Islamic Republic of Iran

Aims: The purpose of this study was to investigate the effect of $>5$ years continuous practice with cyclic yoga training on BMD in postmenopausal women.

Methods: 21 trained women were randomly assigned as yoga group (YE) and compared with age matched control group $(\mathrm{CON})$. The YE participated in cyclic yoga training classes for $>5$ years, 60 min per week; the control group did not participate in any regular exercise programs. Any chronic illness like auto immune disorders, cancers, neurological diseases and any conditions that under treatment with corticosteroids were excluded. BMD was measured using DXA and also clinical data was obtained. 
Results: The results showed that regular long-term cyclic yoga program had a small positive effect on BMD of all sites. Also, $\mathrm{BMI}$ in YE was significantly lower than CON. While BMD did not vary significantly between $\mathrm{YE}$ and $\mathrm{CON}$, total hip and thoracolumbar spine BMD In YE were higher than CON after adjustment for BMI. Z-score discordance between spine and total hip measurement sites in YE was lower than CON.

Conclusion: Our results suggest that regular long-term cyclic yoga had a positive effect on BMD in postmenopausal women. Future yoga interventions should focus on greater sample size to elicit improvements in BMD.

\section{P499}

HOW MAJOR THALASSEMIA AFFECTS ENDOCRINOLOGICAL, BMD AND BONE METABOLISM PARAMETERS: A CROSS SECTIONAL STUDY

Z. Hamidi ${ }^{1}$, A. A. Hamidieh ${ }^{2}$, M. R. Mohajeri-Tehrani ${ }^{1}$, A. Naghghash $^{3}$, M. Behfar ${ }^{2}$, F. Mohseni ${ }^{1}$, K. Alimoghaddam ${ }^{2}$, H. Rashidian ${ }^{1}$, A. Ghavamzadeh ${ }^{2}$, S. Shirazi ${ }^{4}$, M. Pajouhi ${ }^{1}$, B. Larijani $^{1}$

${ }^{1}$ Endocrinology and Metabolism Research Center, Endocrinology and Metabolism Clinical Sciences Institute, Tehran University of Medical Sciences, Tehran, Islamic Republic of Iran, ${ }^{2}$ Hematology-Oncology and Stem Cell Transplantation Research Center of Tehran University of Medical Sciences, Tehran, Islamic Republic of Iran, ${ }^{3}$ Shahid Beheshti University, Torfeh Hospital, Tehran, Islamic Republic of Iran, ${ }^{4}$ Osteoporosis Research Center, Endocrinology and Metabolism Clinical Sciences Institute, Tehran University of Medical Sciences, Tehran, Islamic Republic of Iran

Background: $\beta$-thalassemia major patients frequently have low BMD and increased fracture risk. We tried to determine how thalassemia affects endocrinological, BMD and bone metabolism parameters.

Methods: Thirty $\beta$-thalassemia major patients with mean age of $18.2 \pm 11(2-45 y / 0)$ entered the study. Female to male ratio was $8 / 22$. 18 of patients aged less than 20 years (pediatric patients). Physicians collected demographic; anthropometric; menstrual; transfusion and treatments histories; and serum levels of ferritin, prolactin, LH,FSH,T4,T3, TSH, IGF-1, testosterone (in males) or estradiol (in females) ACTH, cortisol and bone-specific alkaline phosphatase and osteocalcin (bone formation markers), NTX (bone resorption marker), Ca, P, Alk ph, PTH and vit-D determined. Study participants underwent a measurement of BMD by Hologic Discovery QDR model.

Results: Low IGF1, short stature, subclinical hypothyroidism, hypogonadotropic hypogonadism, high prolactin level were found in $63 \%, 40 \%, 10 \%, 13 \%$ and $6.6 \%$ of our patients, respectively. Low vit-D and Zscore $\leq-2$ in spinal and femoral regions (neck and total) found in $76 \%, 43 \%, 20 \%$ and $16 \%$ of our patients. Correlation of age, sex and ferritin with anthropometric, laboratory and BMD parameters examined. Women showed significantly higher FSH $(\mathrm{P}$ value $=0.031)$. Ferritin correlated only with prolactin and LH (negatively) and positively with phosphorus ( $\mathrm{P}$ values, $0.027,0.049$ and 0.016 , respectively). Age correlated positively to height, weight, BMD of femoral (neck and total) and spinal regions ( $\mathrm{P}$ values, $<0.001,<0.001$, $0.005,0.013$ and $<0.001$, respectively). T3, total Alk ph, bone specific alkaline phosphatase, NTX and Zscores of total femoral and spinal regions correlated negatively with age $(\mathrm{P}$ values, $0.006,0.041,0.010$, $0.008,0.015$ and $<0.001$, respectively). Mean age of short stature patients and who had Z-score less than -2 in spinal region were significantly higher than those who were normal (P values, 0.024 and 0.002 , respectively).

Conclusion: Low prevalence of hypogonadotropic hypogonadism may be due to HRT in our adult patients (a routine treatment in them). However it seems replacement of sex hormones do not reduce the prevalence of short stature in them. This may be due treatment onset that begins only after delayed puberty clears. The main factor related with short stature and lower Z-scores of the femur and spine was age. So, we recommend early monitoring of thalassemia patients (in their childhood and before adolescence) for these complications.

Acknowledgments: We thank Dr. M. Qorbani for his assistance in data analysis and Mrs Eskandari, Miss Habibi, Mrs Pouladian, Mrs Mohamad-Ali, Miss Vossogh, Mrs. Sarkisian, Miss Dadashi, Mrs. Aref and Mrs. Javadi for their valuable assistance in data collection. 


\section{P500}

\section{REPETITIVE FRACTURES DURING CHILDHOOD} AND LOW BONE MINERAL DENSITY FOR PUBERTAL AGE: WHERE TO?

A. Ghemigian $^{1}$, M. Carsote ${ }^{2}$, A. Valea ${ }^{3}$, S. E. Albu ${ }^{4}$, I. Oprisor $^{5}$, M. Paulic ${ }^{5}$, C. Procopiuc ${ }^{1}$, C. Vasiliu ${ }^{4}$, C. E. Georgescu $^{3}$

${ }^{1}$ C.I. Parhon National Institute of Endocrinology, Bucharest, Romania, ${ }^{2}$ C. Davila University of Medicine and Pharmacy \& C.I.Parhon National Institute of Endocrinology, Bucharest, Romania, ${ }^{3}$ I. Hatieganu University of Medicine and Pharmacy \& Clinical County Hospital, Cluj-Napoca, Romania, ${ }^{4}$ C.Davila University of Medicine and Pharmacy \& University Emergency Hospital, Bucharest, Romania, ${ }^{5}$ Clinical County Hospital, Cluj-Napoca, Romania

Introduction: Osteoporosis is a condition of low bone mass and architectural disruption and associated fractures which are triggered by minimal trauma. In teenagers and young adults low BMD for age without an evident cause is very rare, most probably related to the polygenic variation of BMD. Another genetic form is osteogenesis imperfecta (OI). In any case the condition impairs the quality of life and lifelong follow-up.

Material and Method: This is the presentation of two cases with multiple fragility fractures since a very young age followed in two Romanian Tertiary Centers of Endocrinology. Bone was evaluated by central DXA providing BMD; phosphocalcic metabolism assay (including the bone turnover markers). The informed consent was obtained.

Case 1: A 21-yr female has been diagnosed with OI (mother with OI) and she suffered several fractures during childhood. At age of 16 the parameters showed: alkaline phosphatase of $143 \mathrm{U} / \mathrm{L}(\mathrm{N}: 35-129)$, 25hydroxyvitamin D of $8.37 \mathrm{ng} / \mathrm{mL}(\mathrm{N}>30)$, PTH of $53 \mathrm{pg} / \mathrm{mL}(\mathrm{N}: 15-65)$, normal CrossLaps and osteocalcin; lumbar (L1-4) of $0.797 \mathrm{~g} / \mathrm{cm}^{2}$, Z-score of $-3 \mathrm{SD}$. IV ibandronate (despite recommendations) was given every 3 months (with vitamin D/calcium supplements) since the age of 19 and currently the (L1-L4) BMD became of $0.918 \mathrm{~g} / \mathrm{cm}^{2}, Z$-score of $-2.2 \mathrm{SD}$. Further follow-up is necessary.

Case 2: An 18-yr nonsmoking male has a history of lowtrauma fractures since childhood: right clavicle at age of 13 and both forearms at age of 14 . The medical family history includes the mother and grandmother diagnosis of osteoporosis. He was given vitamin D and calcium supplements with a poor compliance. At age of 17 he was diagnosed with primary hypothyroidism and levothyroxine (a daily dose of $75 \mu \mathrm{g}$ ) was started. On admission, he complained of persistent lumbar pain for several months. The clinical phenotype was not specific. The biochemical parameters revealed: alkaline phosphatase of $52 \mathrm{U} / \mathrm{L}$ (normal: $40-150 \mathrm{U} / \mathrm{L}$ ), circulating osteocalcin of $2.1 \mathrm{ng} / \mathrm{ml}$ (normal $24-70 \mathrm{ng} / \mathrm{ml}$ ), with decreased levels of ionized calcium (of $3.92 \mathrm{mg} / \mathrm{dl}$, normal: 4.2-5.4 mg/dl) and normal blood phosphorus (of $3.75 \mathrm{mg} / \mathrm{dl}$, normal: $2.5-4.5 \mathrm{mg} / \mathrm{dl}$ ). DXA found (L1-4) BMD of $0.839 \mathrm{~g} /$ $\mathrm{cm}^{2}, \mathrm{Z}$-score of $-2.8 \mathrm{SD}$. No genetic test of OI was done. IV ibandronate with vitamin $\mathrm{D} /$ calcium supplements were started.

Conclusion: Juvenile and youth osteoporosis represents challenging diagnosis but also therapies since long- term antiosteoporotic drugs are useful.

\section{P501}

\section{DIAGNOSIS ALGORITHM OF LOW LEAN MASS IN} ADULT MEN WITH TYPE 1 DIABETES

Y. Dydyshka ${ }^{1}$, A. Shepelkevich ${ }^{1}$

${ }^{1}$ Belarusian State Medical University, Minsk, Belarus

Background: According to modern concepts, diabetic myopathy is also a common clinical condition characterized by a lower muscle mass, weakness, and an overall reduced physical capacity.

Aim: To determine the criteria for reducing the content of appendicular muscle mass (AMM) in type 1 diabetes mellitus (T1DM) men.

Materials and methods: 35 men with (mean age: 32.4 (24.4 37.2)yrs, duration of DM: $13(7-20)$ yrs, age of manifestation: 17(13-21)yrs, BMI: 23.67(20.88-25.37), HbA1c: 8 , $2(7.5-9.1) \%)$ and 24 men controls. In patients with T1DM and in control group revealed comparable $(\mathrm{U}=130 ; p=0.423)$ levels of free testosterone 15.1(9-24) and 19.7(14.75-23.05) $\mathrm{nmol} / \mathrm{L}$, respectively $(\mathrm{U}=130 ; p=0.423)$, indicating the presence of normagonadal status of the surveyed men. An appendicular skeletal muscle mass index (ALMI) was measured as AMM (lean mass arms + lean mass legs)/height2.

Results: There were significant differences in T1DM males and control: Lean Arms: $6573(5915$ - 7454) vs. 7540 (6658 - 8729)g; $(\mathrm{U}=248 ; p=0.017)$; Lean Legs: $18054(16361-19794)$ vs. $20455(18803-22761) \mathrm{g}$; $(\mathrm{U}=208 ; p=0.002)$; Lean (Arms + Legs) - 25241 (22102-27784) vs. 28370 (25541-31367)g; $(\mathrm{U}=219$; $p=0.004)$, Total Body Lean (54082 (48999-60653) vs. 60969 (54273-65082)g; $\mathrm{U}=259 ; p=0.027)$, Lean Gynoid Lean (7711 (7155-8843) vs. 8423 (80009891)g; $\mathrm{U}=241 ; p=0.013$. Using ROC analysis determined the cutoff point of the ALMI for patients with T1DM $(p=0.008)-8.44 \mathrm{~kg} / \mathrm{m}^{2}$.Considering ORs and $95 \%$ CIs determined probability of decrease of AMM in young men with T1DM age: $O R=5.59(2.88-10.84$; $\mathrm{c} 2=9.16 ; p=0.025$ ).

Conclusions: Thus, it was determined the significant $(p=0.008)$ threshold ALMI $-8.44 \mathrm{~kg} / \mathrm{m}^{2}$, allowing to 
allocate a group of men with a reduction in the AMM $\left(\right.$ ALMI $\left.\leq 8.44 \mathrm{~kg} / \mathrm{m}^{2}\right)$ and people with a normal amount of AMM $\left(\right.$ ALMI $\left.>8.44 \mathrm{~kg} / \mathrm{m}^{2}\right)$.

\section{P502}

\section{OSTEOPOROSIS PREVENTION: WHERE ARE THE BARRIERS TO IMPROVEMENT IN PATIENTS AND DOCTORS?}

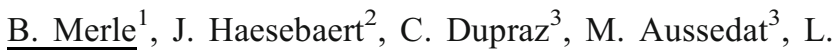
Barraud $^{3}$, A. Bedouet ${ }^{3}$, C. Motteau ${ }^{3}$, V. Simon ${ }^{3}$, A.-M. Schott ${ }^{2}$, M. Flori ${ }^{3}$

${ }^{1}$ INSERM UMR 1033, Université de Lyon, Lyon, France, ${ }^{2}$ Unité Epidémiologie, Hospices Civils de Lyon, Lyon, France, ${ }^{3}$ Collège Universitaire de Médecine Générale, Université Lyon 1, Lyon, France

Despite numerous programs aiming at improving osteoporosis care, prevention, diagnostic and treatment remain suboptimal. It is important to understand barriers to preventive care initiation to improve awareness and management of osteoporosis. We implemented a qualitative study to explore the knowledge and representations of general practitioners in Region RhôneAlpes, France regarding osteoporosis and its prevention in women and men. Four types of focus groups were conducted until data saturation, with women aged 50 to 85 years and men aged 60 to 85 years, with or without a history of fragility fracture or an osteoporosis diagnostic (referred to as "aware" or "unaware"). We involved 57 men, 23 aware and 34 unaware, in 7 focus-groups; and 45 women, 23 aware and 22 unaware in 9 focus groups. In parallel, semi-directed face-to face interviews were conducted with 16 general practitioners. A thematic analysis of transcripts was made with Nvivo 10 software.

Results showed that osteoporosis is not considered as an illness but rather associated to old age by women and men who usually do not feel concerned, even if they had suffered a fragility fracture. No differences could be observed between aware and unaware patients. If osteoporosis was associated with bone fragility and fractures by women, for most of the men it was a women pathology which they deny. Fragility fracture was usually not linked to osteoporosis but to the fall. Men and women agreed with a healthy lifestyle although the benefits of dairy products, sun exposure and sport practicing were discussed. Pharmacological treatments were only mentioned by women, and always with suspicion due to their side effects. Women and men thought that their general practitioner is the right person to inform them about OP although it is rarely done, but women were more likely to be active in their health. For general practitioners, osteoporosis was associated with fragility fractures, women, menopause, and old age but rarely with men. BMD measurement was regarded as the referent diagnostic test, but sometimes with difficulties to interpret the results, and questions as to when to prescribe. Bisphosphonates were the reference treatment. Above all, they were all waiting for clear and pragmatic guidelines to better manage this disease.

In conclusion, understanding barriers to osteoporosis care in patients and general practitioners will help to set up effective strategies to improve prevention and treatment.

\section{P503}

CYCLIC YOGA FOR THE PREVENTION OF AGERELATED SARCOPENIA

A. Hossein-Nezhad ${ }^{1}$, B. Yarjoo ${ }^{2}$, G. Niketeghad ${ }^{1}$, A. Bakhshizadeh $^{2}$, M. Machawe ${ }^{3}$, S. M. Eshaghi ${ }^{1}$, S. Shirazi ${ }^{1}$, Z. Maghbooli ${ }^{1}$, M. A. Azarbayjani ${ }^{2}$

${ }^{1}$ Osteoporosis Research Center, Endocrinology and Metabolism Clinical Sciences Institute, Tehran University of Medical Sciences, Tehran, Islamic Republic of Iran, ${ }^{2}$ Department of Exercise Physiology, Islamic Azad University, Central Tehran Branch, Tehran, Islamic Republic of Iran, ${ }^{3}$ Payam-e-Mehr International Yoga Center, Tehran, Islamic Republic of Iran

Aims: To evaluate the effect of continuous cyclic yoga training on muscle strength and body composition in postmenopausal women.

Methods: Cyclic yoga is a new style in Hatta yoga that has been providing harmony of streaming cycle of poses to fortify the positive effects of each asana on muscle strange and flexibility. DXA was used to assess whole body and regional soft tissue mass, fat mass and lean body mass composition. Muscle strength was assessed by handgrip dynamometry tests. Any chronic illness like auto immune disorders, cancers, neurological diseases and any conditions that under treatment with corticosteroids were excluded. Forty-six postmenopausal women were recruited as yoga group (YE, $n=21$; age 54.5 years) and control group (CON, $n=25$; age 55.6 years). Participants in YE group performed 60 min of a cyclic-based yoga series one time/week for $>5$ years.

Results: There was not significant group difference in age. The results showed that BMI in YE was significantly lower than CON (25.7 vs. 28.2) as well as trunk and total fat percent (41\% vs. $43 \%)$. Also, total lean body mass in YE was higher than CON. In YE hand dynamometer test result of right hand was stranger than $\mathrm{CON}$ $(23.6 \mathrm{~kg}$ vs. $20.3 \mathrm{~kg}$ ). After adjustment for age and social economic status, years of continuous practice in cyclic yoga was directly associated with muscle mass and strange and inversely associated with fat mass.

Conclusion: The findings of this study suggest that regular long-term cyclic yoga had a positive effect on muscle mass, muscle strange and body fitness in postmenopausal women. Cyclic yoga may recommend for the prevention of age-related sarcopenia in postmenopausal women. 


\section{P504}

\section{ROLE OF SMOKING AS RISK FACTOR FOR OSTEO- POROSIS}

\author{
V. Duraj ${ }^{1}$, A. Kollcaku ${ }^{1}$, E. Memlika ${ }^{1}$, A. Tafaj ${ }^{1}$ \\ ${ }^{1}$ University Hospital Center Mother Teresa, Tirana, Albania
}

Background: Smoking is an important factor of risking for osteoporosis because the components that it releases directly affect in these hormones metabolism: adrenocortical, sexual hormones, also in calcium metabolism and vitamin D3.

Methods: This study is based in measuring BMD by DXA on patients infected by arthritis rheumatoid disease, hospitalized in rheumatology ward at Hospital Center "Mother Teresa" Albania, where values are taken into account of L1-L4 and femoral neck BMD, values of calcium, vitamin D3 and estradiol.

Results: In this study are included 282 females infected by arthritis rheumatoid that varies between ages $30-40.85 \%$ of these females don't smoke and $15 \%$ of them were smoking. While measuring the BMD by DXA the results were:

1. In nonsmoking females the L1-L4 T-score results were between -2.2 and -1.6 and the femoral neck T-score varies between -1.9 and -0.8 . Calcium levels where between $8-8.4 \mathrm{mg} / \mathrm{dl}$. Vitamin D3 levels were $50-80 \mathrm{mg} / \mathrm{dl}$ and estradiol levels were between 50-700 pg/dl.

2. In smoking females the L1-L4 T-score varies were between -3.1 and -2.5 and the femoral neck T- score varies between -2.8 and -2.1 . Calcium levels where between $6-$ $7.2 \mathrm{mg} / \mathrm{dl}$. Vitamin D3 levels were $30-48 \mathrm{mg} / \mathrm{dl}$ and estradiol levels were up to $70 \mathrm{pg} / \mathrm{dl}$.

Conclusion: Smoking is an important factor of risking for osteoporosis because the components that it releases directly affect in these hormones metabolism: adrenocortical, sexual hormones also in calcium metabolism and vitamin D3. Its components directly affect estradiol levels and metabolism by producing a component with estrogenic minimum effects.

\section{P505}

DIETARY SOURCES OF CALCIUM AMONG ADOLESCENTS AND ITS EFFECT ON BONE TURNOVER Z. Maghbooli ${ }^{1}$, E. Feizabad ${ }^{1}$, G. Niketeghad ${ }^{1}$, A. HosseinNezhad $^{1}$, E. Taheri ${ }^{1}$, B. Larijani ${ }^{1}$

${ }^{1}$ Osteoporosis Research Center, Endocrinology and Metabolism Clinical Sciences Institute, Tehran University of Medical Sciences, Tehran, Islamic Republic of Iran

Dietary calcium sources may differ by race/ethnicity and dietary acculturation. Our aim was to study dietary calcium intake and sources of calcium in adolescents as well as evaluating its effect on bone turnover. This cross-sectional population-based study is conducted on
444 middle and high school students (both girls and boys) in Tehran. A short food frequency questionnaire designed to estimate dietary calcium and vitamin D consumption. Serum levels of calcium, phosphorus, PTH, bone specific alkaline phosphates, $25(\mathrm{OH}) \mathrm{D}$, osteocalcin, crosslinked C-telopeptide (CTX), total protein, albumin and creatinine were determined. Average calcium intake was significantly lower in girls. Popular dietary sources of calcium among adolescents in both sexes were plain yogurt, milk, cheese, dough (yogurt drink) and ice cream. Fluid milk and plain yogurt provided the largest mean percentage of calcium intake for both girls and boys. Several dietary sources of calcium were used in Persian dietary pattern like dough and Kashk (drained yogurt or sour milk). There was significant association between calcium intake and improving bone turnover in adolescents. The results alert to an insufficient calcium intake and suggest that certain subgroups of adolescents need specific strategies to increase the intake of this nutrient. Understanding calcium food sources and changes in dietary patterns that affect calcium intake among adolescents is important to better promote adequate intake.

\section{P506}

DENOSUMAB: ADHERENCE TO THERAPY AND SAFETY IN WOMEN FOLLOWED IN A VERTEBRAL FRACTURE OUTPATIENT UNIT

M. Mazzantini ${ }^{1}$, V. Bottai ${ }^{2}$, A. Figliomeni ${ }^{1}$, G. Raffaetà ${ }^{2}$, M. Mosca $^{1}$, O. Di Munno ${ }^{1}$, G. Guido ${ }^{2}$

${ }^{1}$ Division of Rheumatology, Azienda UniversitarioOspedaliera di Pisa, Pisa, Italy, ${ }^{2}$ Division of Orthopedics, Azienda Universitario-Ospedaliera di Pisa, Pisa, Italy

Objective: To assess adherence to denosumab (D) and adverse events (AEs) in women with severe osteoporosis followed in a vertebral fracture outpatient unit.

Study design: Prospective observational.

Patients and Methods: 307 patients (P), baseline mean age $75 \pm 9$ years (range 40-100). 292 had previous osteoporotic fractures (33 hip, 227 vertebral, 32 both), 15 had not, but they had DXA T-score $\leq-4$. The mean $\mathrm{n}$. of fractured vertebrae was $3.4 \pm 2.3$ (range 1-13). The mean DXA T-scores were: lumbar $-3.0 \pm 1.3$, femoral neck $-2.6 \pm 0.8$, and total hip $-2.5 \pm 0.9$. At each 6months visit, clinical fractures and AEs were assessed; $\mathrm{P}$ who did not return to visit were investigated by telephone interview and asked about reasons for discontinuation. The mean duration of follow-up was 12 \pm 10 months (range 1-50), as on July 31, 2015.

Results: 13 of 307 P (4.2\%) were lost to follow-up: they did not return to a second visit, and we were 
unable to contact them. Furthermore, $9 \mathrm{P}$ did not take D due to fear of AEs; $35 \mathrm{P}$ withdrew $\mathrm{D}$ after the first dose for personal reasons not related to AEs; 6 stopped D as they had diseases which made them impossible to return to visit; 4 died for reasons unrelated to D; 3 stopped D for AEs. As a result, over a mean 12-month follow-up, adherence to $\mathrm{D}$ was $77.2 \% ; 44 \mathrm{P}$ were intentionally non-adherent (14.9\%), and 13 (4.2\%) were nonintentionally nonadherent. $285 \mathrm{P}$ took at least one dose of D. AEs probably related to $\mathrm{D}$ were observed in $6 \mathrm{P}(2 \%)$, which caused the interruption of therapy in 3: osteonecrosis of the jaw, marked elevation of transaminases in course of $\mathrm{HCV}$ infection, and diffuse skin erythema and subcutaneous edema with arthritis; further $6 \mathrm{P}$ had AEs possibly related to $\mathrm{D}(2 \%)$. Twenty-four $\mathrm{P}(8.4 \%)$ had a new clinical fracture.

Conclusion: In a tertiary care unit, adherence to D was high (77.2 \%, or $81.6 \%$ after exclusion of non intentional nonadherence) after a mean 12-months follow-up; nonadherence due to AEs was very low $(1 \%)$. One case of osteonecrosis of the jaw was recorded.

\section{P507}

THE DATA OF CENTRAL AORTIC PRESSURE AND PULSE WAVE VELOCITY IN WOMEN WITH OSTEOPOROSIS

$\underline{\text { S. Tsarenok }}^{1}$, V. Gorbunov ${ }^{1}$, S. Verhoturova ${ }^{1}$, T. Aksenova ${ }^{1}$

${ }^{1}$ Chita State Medical Academy, Chita, Russian Federation

Aim: To evaluate the data of central aortic pressure and pulse wave velocity (PWV) and their relationship with osteoporosis. Materials and Methods: 120 women aged between 60 and 77 (mean age 68.7 \pm 8.8 ) were examined. The patients were divided into 2 groups: group 1, $n=74$, patients with osteoporosis; group 2, $n=46$, control group. BMD was studied in 2 areas: the lumbar vertebrae and proximal hip by DXA densitometry (Challenger, France). Indicators of central aortic pressure and PWV were determined by applanation tonometry by SphygmoCor, Australia. For statistical analysis we used Wald-Wolfowitz criteria, multiple regression model. The study was based on GCP principles.

Results: Increased levels of central diastolic pressure (78.7 $\pm 9.575 .4 \pm 10.4 \mathrm{p}=0.01)$, pulse pressure $(48.5 \pm 20.145 .5$ $\pm 15.5 \mathrm{p}=0,0000)$ and mean pressure $(96.9 \pm 15.493 .7$ $\pm 12.7 \mathrm{p}=0.0008)$ were determined in patients with osteoporosis. The level of central systolic blood pressure was similar in the examined groups. PWV for carotid-femoral segment in the first group was higher than in the control group $(9.7 \pm 2.39 .0 \pm 2.1 \mathrm{p}=0.0000)$. The increase of the augmentation index was revealed in women with osteoporosis, no significant differences of augmentation pressure between the examined groups were found out $(15.9 \pm 9.5$ $14.9 \pm 8.0 \mathrm{p}=0.1)$. The decrease of BMD mean values in L1-L5 was established in women of group $1(0.974 \pm 0.230$ $1.031 \pm 0.200 p=0.00006) . Z(0.59 \pm 1.750 .95 \pm 1.27$ $p=0.0000)$ and T-score $(-0.8 \pm 1.6-0.3 \pm 1.2 p=0.0004)$ indices were lower in the group of patients with osteoporosis. BMD (Neck 0.62 $\pm 0.160 .72 \pm 0.18 \mathrm{p}=0.00005$; Ward's area $0.47 \pm 0.290 .56 \pm 0.21 \mathrm{p}=0.0003$; Total hip $0.80 \pm 0.160 .86 \pm 0.15 \mathrm{p}=0.0000), \mathrm{Z}($ Neck $0.67 \pm 1.31$ $0.91 \pm 1.41 \mathrm{p}=0.0000 ;$ Ward's area $-0.67 \pm 1.92-0.14$ $\pm 1.54 \mathrm{p}=0.00009$; Total hip $2.7 \pm 1.43 .3 \pm 1.7$ $\mathrm{p}=0.0002)$ and T-score (Neck $-0.78 \pm 1.59-0.74 \pm 1.78$, $\mathrm{p}=0.0000$; Ward's area $-2.1 \pm 1.9-1.6 \pm 1.5, \mathrm{p}=0.001$; Total hip $0.94 \pm 1.661 .19 \pm 1.45, \mathrm{p}=0.0000)$ proved to be significantly lower in women with osteoporosis than in women of the control group. Multiple regression analysis, which included 32 parameters was conducted to identify factors affecting PWV. It was ascertained that age factor ( $\beta=0.3$ 95CI $0.08-0.11 p=0.027)$, Z-score of the proximal femur $(\beta=5.02$ 95CI 8.4-10.6 $p=0.0059)$ and T-score of the Ward area $(\beta=5.07$ 95CI $6.0-8.1 p=0.005)$ were the independent determinants of arterial stiffness.

Conclusion: Increasing performance of arterial stiffness, PWV, augmentation index, pulse aortic pressure and decreasing BMD of L1-L5 and proximal hip were determined in women with osteoporosis. Age and BMD were independent factors affecting arterial stiffness.

\section{P508}

GLUCOCORTICOID THERAPY AND FRACTURE: OCCURRENCE OF DOSE, DURATION AND TIMING, THE NEED FOR A NEW APPROACH

D. E. Robinson $^{1}$, E. M. Dennison ${ }^{2}$, C. Cooper ${ }^{2}$, T. P. Van

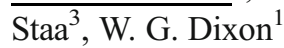

${ }^{1}$ Arthritis Research UK Centre for Epidemiology, University of Manchester, Manchester, United Kingdom, ${ }^{2} \mathrm{MRC}$ Lifecourse Epidemiology Unit, University of Southampton, Southampton, United Kingdom, ${ }^{3}$ Health eResearch Centre, Farr Institute for Health Informatics Research, University of Manchester, Manchester, United Kingdom

Objective: To investigate which methods are currently used to attribute fractures to glucocorticoid exposure in patients with rheumatoid arthritis (RA) and investigate whether such methods can consider the dose, duration and timing of this time-varying exposure.

Material and Methods: A literature search was carried out using Medline and Embase. Search terms for glucocorticoids, fractures, and RA where used to identify papers of interest. Papers were restricted to adults, English language and humans. Following exclusions, abstracts were screened to ensure the risk of fracture due to glucocorticoids in RA patients 
was measured with at least 1 fracture in the exposed and unexposed cohorts.

Results: Thirty-eight studies used five common definitions of risk attribution to glucocorticoid exposure: "current use", "ever use", "daily dose", "cumulative dose" and "time variant". One study attempted to combine multiple definitions where "cumulative dose" was nested within "daily dose", covering the effects of dose and duration but not timing. The majority of results demonstrated an equivocal or increased risk of fracture with increased exposure, although there was wide variation, with odds ratios, hazard ratios and relative risks ranging from 0.16 to 8.16. Within definitions there was also variability in the results with the smallest range for "time variant", 1.072.80 , and the largest for "cumulative dose", ranging from risk estimates of $0.88-8.12$.

Conclusion: Many studies have looked into the effect of glucocorticoids on fracture risk in patients with RA. Despite this, there is no clear consensus about the magnitude of risk. This is partly a consequence of the varied analysis models and their different assumptions. Moreover, no current analysis method allows consideration of dose, duration and timing of glucocorticoid therapy, preventing a clear understanding of fracture risk for patients and their individual treatment patterns.

\section{P509}

VITAMIN D STATUS IN WOMEN LIVING IN REPUBLIC OF BELARUS

A. Rudenka $^{1}$, E. Rudenka $^{2}$, T. Tyabut ${ }^{1}$

${ }^{1}$ Belarusian Medical Academy of Postgraduate Training, Minsk, Belarus, ${ }^{2}$ Belarusian State Medical University, Minsk, Belarus

Background: Hypovitaminosis D is a frequent phenomenon that has a significant impact on health in individuals of any age but particularly in the elderly.

Aim: To determine the levels of vitamin D in Belarusian women according to age and season of year.

Materials and methods: We analyzed data of the laboratory results of the serum content of total vitamin D $(25(\mathrm{OH}) \mathrm{D})$ which were performed in our republic in women aged over 20 years in 2015 . Serum content of total vitamin D was determined by electrochemiluminescence protein binding assay technology using Cobas e411 immunoassay analyzer (Roche Diagnostic, Germany) and original reactants Roche Diagnostics GmbH. 2561 results obtained from 4 laboratories situated in different regions of the country, were analyzed. Statistical analysis was performed using the software package Statistica 6.0.

Results: Obtained data were divided to 3 age groups: group I consisted of women younger than 40 years old (n 198), group II - of women at age $40-60$ years (n 1264) and group III - of those older than 60 years (n 536). Mean levels of $25(\mathrm{OH}) \mathrm{D}$ in studied groups in spring and summer was $26.6 ; 11.0 \mathrm{ng} / \mathrm{ml}$ in group I (n 438), 25.9; $9.9 \mathrm{ng} / \mathrm{ml}$ in group II ( $\mathrm{n} \mathrm{721),} \mathrm{23.9;}$ $8.5 \mathrm{ng} / \mathrm{ml}$ in group III (n 293). In autumn and winter this figures were $26.3 ; 10.4$ (n 323), 24.4; 9.2 (n 543) and 23.4; 9,1 (n 243) ng/ml, respectively. There were no statistically significant differences of seasonal serum vitamin D levels in groups I ( $p$ 0.69) and III ( $p$ 0.43), while such differences were found in women of the second group: showings of $25(\mathrm{OH}) \mathrm{D}$ in spring and summer were higher than those in autumn and winter $(p<0.05)$.

Conclusion: The average level of vitamin D in studied sample demonstrates lack of its content in all age groups regardless of the season with increasing in the spring and summer months in women aged 40$60 \mathrm{y}$.

\section{P510}

LOW BONE MINERAL DENSITY AND OSTEOMALACIA DUE TO RESTRICTED DIET: A CASE REPORT

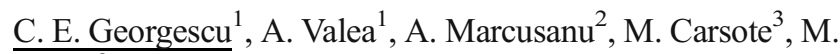
Paulic $^{2}$

${ }^{1}$ I. Hatieganu University of Medicine and Pharmacy \& Clinical County Hospital, Cluj-Napoca, Romania, ${ }^{2}$ Clinical County Hospital, Cluj-Napoca, Romania, ${ }^{3} \mathrm{C}$. Davila University of Medicine and Pharmacy \& C.I.Parhon National Institute of Endocrinology, Bucharest, Romania

Introduction: Osteomalacia is a metabolic bone disease, characterized by decreased mineralization, occurring after the epiphysis plates have closed. The most frequent cause of osteomalacia is vitamin D (VD) deficiency. Malnutrition, different restriction diets, malabsorption impair VD status. Prompt therapeutically intervention with VD and calcium supplements usually is followed by a good response rate but the underlying cause is necessary to be treated or corrected, as well. The differential diagnosis with osteoporosis is established by different tests as tetracycline test but this is not available in many medical centers.

Material and Method: A case report is introduced regarding a young patient with osteomalacia. Bone evaluation included: central DXA providing BMD and calculated Z-score; 25hydroxyvitamin D (25-OH D) assay (chemiluminescence); the calcium/phosphorus assessment as well as circulating bone turnover markers.

Case data: A 25-year old vegetarian Caucasian female, diagnosed with lactose intolerance, was admitted for 
diffuse bone pain, especially in lumbar and sacral spine, ribs, coxofemoral joint. She also had marked astenia and motor deficiency in the right proximal lower limb. The symptoms were presented since 2013, but became persistent and intense in the last few months. At that moment, a skeletal scintigraphy was performed and demonstrated increased uptake with foci of radiotracer accumulation over the cranium, spine, ribs, coxofemoral joints and long bones, who indicated a metabolic disease or a malign condition. The last one was excluded based on an oncologic evaluation. VD supplements were intermittently taken by the patient. On admission, the biochemical parameters revealed markedly increased alkaline phosphatase (of $1201 \mathrm{U} / \mathrm{L}$, normal levels between 50-150 U/L), severe VD deficiency (circulating 25-OH D of $17.2 \mathrm{ng} / \mathrm{mL}$, normal 30-100 ng/mL), normocalcemia, and hypophosphatemia (of $2.1 \mathrm{mg} / \mathrm{dl}$, normal $2.3-4.7 \mathrm{mg} / \mathrm{dl}$ ). DXA showed very low lumbar BMD for age of $0.408 \mathrm{~g} /$ $\mathrm{cm}^{2}, \mathrm{Z}$-score of - $5.3 \mathrm{SD}$; femoral neck BMD of $0.408 \mathrm{~g} /$ $\mathrm{cm}^{2}, \mathrm{Z}$-score of $-4.9 \mathrm{SD}$; total hip BMD of $0.460 \mathrm{~g} / \mathrm{cm}^{2}$, $\mathrm{Z}$-score of $-4.2 \mathrm{SD}$. A high level of PTH was found. The thyroid function was normal. The patient was treated with calcium and vitamin D (parenterally and then orally) with a clinical improvement within weeks.

Conclusion: In the presented case, the severe deficiency of vitamin $\mathrm{D}$ was determined by the strict vegetarian diet and the lactose intolerance since no other obvious cause was detected. The severe decreased of BMD for age with high alkaline phosphatase (about 8 times above the normal upper limit) might suggest a rare bone condition as Paget's disease. Since no bone biopsy was done, the therapeutical test with high vitamin $\mathrm{D} /$ calcium was the argument for osteomalacia. Overall, nutrition-related osteomalacia has a good prognostic, with significant clinical improvement and increment in bone mass after adequate treatment.

\section{P511}

PREVALENCE OF FRAILTY IN A PROSPECTIVE COHORT OF OLDER WORKERS: THE HEALTH AND EMPLOYMENT AFTER FIFTY (HEAF) STUDY S. D'Angelo ${ }^{1}$, K. Walker-Bone ${ }^{1}$, E. C. Harris ${ }^{1}$, C. Linaker ${ }^{1}$, H. Syddall $^{1}$, A. A. Sayer ${ }^{1}$, M. Evandrou ${ }^{2}$, C. Gale ${ }^{1}$, C. Cooper $^{1}$, D. Coggon ${ }^{1}$, K. T. Palmer ${ }^{1}$

${ }^{1}$ MRC Lifecourse Epidemiology Unit, University of Southampton, Southampton, United Kingdom, ${ }^{2}$ Centre for Research on Aging, University of Southampton, Southampton, United Kingdom

Background: Frailty is a multidimensional syndrome, defined in older people and associated with a higher risk of falls, fractures, hospitalisation and death. Studies on its prevalence in relatively younger populations are limited. The aim of this analysis was to assess the prevalence of frailty and its determinants among a cohort of 50-64 year-olds from 24 English general practices.

Methods: Data $(N=8134)$ came from the baseline questionnaire of the HEAF study. A revised version of the Fried criteria was used to define frailty as presence of at least three of the following: unintentional weight loss, poor grip strength, exhaustion, slow walking speed and low physical activity. Data were also collected on some sociodemographic characteristics and health variables. Associations with frailty were assessed by Poisson regression with robust standard error, adjusted for age and sex.

Results: The prevalence of frailty was $3 \%$ among men and $6 \%$ among women. The majority of people classified as frail reported poor grip strength and low physical activity. Older age, obesity, low level of education, not owning/mortgaging a house, living alone and finding it very difficult to manage financially, were all associated with a higher risk of being frail. Even in this age band, frailty was associated with a significantly greater risk of falls (RR 18.8; 95\%CI $(14.9,23.7)$ ), report of musculoskeletal pain (RR 4.8; 95\%CI $(4.4,5.2)$ ) and fair/poor self-rated health (RR $8.8 ; 95 \% \mathrm{CI}(8.0,9.6)$ ).

Conclusions: Frailty is not uncommon among middle-aged people. As in older people, it is associated with social disadvantage and comorbidities.

\section{P512}

PREVALENCE AND RISK FACTORS OF OSTEOPOROSIS AND VERTEBRAL FRACTURES IN PATIENTS WITH ANKYLOSING SPONDYLOARTHRITIS

M.-D. Djennane ${ }^{1}$

${ }^{1}$ Department Medicine Mouloud Mammeri, Tizi Ouzou, Algeria

IBackground: Ankylosing spondylitis (AS) is a chronic inflammatory joint disease that leads over time to bone formations on entheses and para vertebral structures. Osteoporosis is now regarded as a common feature of the AS.

Objectives: Estimate the Prevalence and risk factors for osteoporosis and vertebral fractures in patients with ankylosing spondyloarthritis.

Methods: 104 patients with AS were included in the study. The clinico-biological and radiological characteristics were assessed by the Bath AS Disease Activity Index (BASDAI), Bath Functional Index (BASFI), the erythrocyte sedimentation rate, $\mathrm{C}$ reactive protein (PRC), the 25OHD and the Bath AS Total Index radiology (BASRI). BMD was measured by DXA (Hologic) at the spine and femoral neck. VFA (vertebral fracture assessment), T4-L4. The evaluation was done qualitatively and semiquantitatively as classified by Genant.

Results: The average age is $34.6 \pm 9.4$ years. The mean disease duration was $8.6 \pm 4.2$ years. The average BMI was 21.6 
$\pm 4.5 \mathrm{~kg} / \mathrm{m}^{2}$. Osteoporosis was found in $20 \%$. Vertebral fractures were present in $14.7 \%$ of patients. Risk factors associated with osteoporosis were low weight, duration of disease, elevated ESR and CRP and a high index of Badai and BASFI. Vertebral fractures were associated with older age, high disease duration, high BASRI index and a low BMD at the femoral neck.

Conclusions: Osteoporosis is common in patients with AS and appears to be related to disease activity while vertebral fractures seem to be related to the duration and severity of structural disease rather than BMD.

\section{P513}

MANDIBULAR CORTICAL INDEX IS INDICATOR OF OSTEOPOROSIS IN POSTMENOPAUSAL WOMEN

M. I. Majumder ${ }^{1}$, M. A. S. I. Harun ${ }^{2}$, N. Sakib ${ }^{3}$

${ }^{1}$ Comilla Medical College, Comilla, Bangladesh, ${ }^{2}$ Chattgram International Dental College, Chittagong, Bangladesh, ${ }^{3}$ BRAC University, Dhaka, Bangladesh

The consequences of aging often involves the risk of osteoporosis, leading to an impaired quality of life of the elderly patients specially postmenopausal women. Osteoporosis accounts $0.83 \%$ of noncommunicable disease globally having significant health and economic impact. Aim of this study was to evaluate and correlate the changes of mandibular cortical bone with BMD in postmenopausal osteoporotic patient. 300 postmenopausal osteoporotic patients included in these study. All patients was evaluated by DXA for BMD, and orthopantomograph(OPG). Mandibular cortical index (MCI) was seen from OPG categorized into $\mathrm{C} 1, \mathrm{C} 2$ and $\mathrm{C} 3$ as appearance of the mandibular inferior cortex distal to the mental foramen. The criteria of $\mathrm{C} 1$ - endosteal margin of the cortex is even sharp on both sides of the mandible, C2- endosteal margin has semilunar defects (resorptive cavities) with cortical residues one to three layers thick on one or both sides, C3endosteal margin consists of thick cortical residues and is clearly porous. The result of this study was showed that mean femoral neck T-score in $\mathrm{C} 1$ group and $\mathrm{C} 2$ were $2.26( \pm 0.81)$ vs. $2.88( \pm 0.73)$ respectively $(p<0.05)$ that was statistically significant, lumbar spine $\mathrm{T}$-score in $\mathrm{C} 1$ group and $\mathrm{C} 2$ were $2.49( \pm 0.96)$ and $2.62( \pm 0.72)$, respectively, $(p>0.05)$ was not statistically significant and mean femoral neck T-score in $\mathrm{C} 2$ group and $\mathrm{C} 3$ were $2.88( \pm 0.73)$ vs. $2.49( \pm 0.96)$, respectively, $(p>0.05)$ that was not statistically significant, lumbar spine Tscore in $\mathrm{C} 2$ group and $\mathrm{C} 3$ were $2.62( \pm 0.72)$ and $3.21( \pm 1.18)$, respectively, $(p<0.05)$ was statistically significant. MCI- C3 is almost perfect indicator of osteoporosis. Changes of MCI are correlate significantly well with osteoporosis variable. Simple, low cost investigation OPG determining mandibular cortical index may be used as diagnostic tool for osteoporosis.

\section{P514}

PREVALENCE AND RISK FACTORS OF VERTEBRAL FRACTURES IN WOMEN WITH RHEUMATOID ARTHRITIS WITH THE VERTEBRAL FRACTURE ASSESSMENT

M.-D. Djennane ${ }^{1}$

${ }^{1}$ Department Medicine Mouloud Mammeri, Tizi Ouzou, Algeria

Background: Rheumatoid arthritis (RA) is the most common inflammatory joint disease, affects $0.5-1 \%$ of the world's general population. Osteoporosis and vertebral fractures are well known complications of PR. Vertebral fractures (VF) are the most common type of fragility fracture. They are associated with chronic back pain, height loss, kyphosis, reduced lung function, abdominal pain, disability and death. Objectives: the main objective is to evaluate the prevalence of vertebral fractures in patients with rheumatoid arthritis with vertebral fracture assessment (VFA).

Methods: We recruited 173 women with RA, none of it was taking medication against osteoporosis. A bone BMD at the hip and spine and VFA were performed in all patients. They answered a questionnaire. Vitamin D status was assessed by measurement of the total 25OHD. The VFA was ranked using the Genant semiquantitative morphometric classification.

Results: Patients had a mean disease duration of 10.3 (7.2) years. Vertebral fractures were found in $23 \%$ of cases $(39 / 173)$. Osteoporosis is found in $43 \%$ of cases. The vitamin D deficiency was found in $87 \%$ of patients. Patients with fractures had a lower BMI, a BMD at the lower hip. They also had a duration of the longest disease and greater use of corticosteroids. The multivaried analysis showed that the presence of VF was independently associated with osteoporosis of the hip, a low level of total $25 \mathrm{OHD}$, high consumption of corticosteroids, high disease activity and an average length of the longest disease.

Conclusions: RA is a risk factor for development of osteoporosis and vertebral fractures. This risk increases with low weight, duration and severity of the disease. These results should suggest a vertebral fracture occurrence prevention program treating patients quickly and optimizing vitamin D status in patients with RA.

\section{P515}

REVISIONAL STUDY OF 119 OSTEOPOROTIC PATIENTS COMPARING THE BIOCHEMICAL BONE DESTRUCTION MARKER CTX-I AVERAGE REDUCTION AFTER ANTIRESORPTIVE MEDICATION (ZOLEDRONIC ACID OR DENOSUMAB)

G. F. França $^{1}$, A. P.F. Filho ${ }^{2}$, A. F.F. Filho ${ }^{3}$, P. D. Zardetto ${ }^{3}$, A. F.França $^{2}$

${ }^{1}$ Barão de Mauá University, Ribeirão Preto, Brazil, ${ }^{2}$ Clinica Santa Helena of Orthopedics and Traumatology, Catanduva, Brazil, ${ }^{3}$ Araraquara University (UNIARA), Araraquara, Brazil 
Objective: The intent of this osteoporotic individuals medical record review was to compare the serum levels of the biochemical bone resorption marker CTX-I [1] collected before and after 25 days of antiresorptive drug administration such as zoledronic acid $5 \mathrm{mg}$ or denosumab $60 \mathrm{mg}$, therefore verify which medication can promote the most precocious and intense decrease of the CTX-I serum concentration, demonstrating if the systematic sample method of collection can support the early reduction of bone resorption activity.

Material and methods: We reviewed the medical records of 119 osteoporotic patients divided in two groups: one containing 45 patients which received the zoledronic acid $5 \mathrm{mg}$ and another of 74 individuals who received denosumab $60 \mathrm{mg}$. The blood samples were obtained after $8 \mathrm{~h}$ of fasting during the morning period [1], one before the antiresorptive medication (initial CTX-I) and the other (final CTX-I) 25 days after endovenous drug administration (zoledronic acid $5 \mathrm{mg}$ ) or the subcutaneous (denosumab $60 \mathrm{mg}$ ). We compared the serum levels of the samples before and after the medication, calculating the initial CTX-I and final CTX-I average, the following step was the relation between final CTX-I divided by the final CTX-I on percentage. At last, the T-student two-tail paired samples were applied to each group. The research was made between January and December of 2015.

Results:

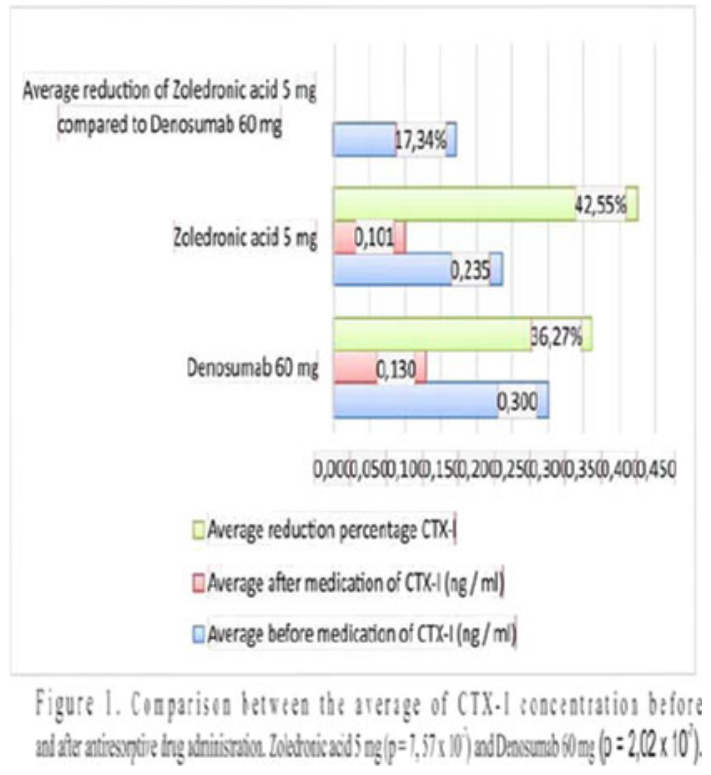

Conclusion: We conclude that there is a significant statistical difference when we compare the initial CTX-I with the final CTX-I 25 days after the antiresorptive treatment such as zoledronic acid $5 \mathrm{mg}$ (average reduction 42,55\%) or denosumab $60 \mathrm{mg}$ (average reduction 36,27\%) in the same osteoporotic patients. Zoledronic acid $5 \mathrm{mg}$ reduced 17, $34 \%$ over denosumab $60 \mathrm{mg}$. The serum dosage of the biochemical marker of bone destruction CTX-I, made in a systematic way showed us to be efficient when used to monitor the early reduction on patients treated with antiresorptive drugs.

\section{References:}

${ }^{1}$ Szulc P. Bone turnover biochemical markers on osteoporosis. In: Rosen C J et al., editors. Primer on the metabolic bone diseases and disorders of mineral. Rio de Janeiro: Grupo editorial nacional; 2014. p.241-246.

\section{P516}

THE RELATIONSHIP BETWEEN 25-HYDROXYVITAMIN D, VITAMIN D-BINDING PROTEIN, FREE 25-HYDROXYVITAMIN D AND BONE MINERAL DENSITY IN MALAYSIAN RHEUMATOID ARTHRITIS PATIENTS AND CONTROLS

$\underline{\text { T. H. Wong }}^{1}$, S. S. Yeap ${ }^{2}$, A. K. Radhakrishnan ${ }^{1}$, S. C. Gun ${ }^{3}$, C. Gandhi ${ }^{3}$, E. Das Gupta ${ }^{4}$

${ }^{1}$ Pathology Division, School of Medicine, International Medical University, Kuala Lumpur, Malaysia, ${ }^{2}$ Department of Medicine, Subang Jaya Medical Centre, Subang Jaya, Malaysia, ${ }^{3}$ Internal Medicine Department, Hospital Tuanku Jaafar, Seremban, Malaysia, ${ }^{4}$ Internal Medicine Department, School of Medicine, International Medical University, Seremban, Malaysia

Background: 25-hydroxyvitamin D (25(OH)D) circulates bound to vitamin D-binding protein (VDBP). Levels of 25(OH)D and VDBP have not been consistently shown to be related to $\mathrm{BMD}$. It has been suggested that (calculated) free $25(\mathrm{OH}) \mathrm{D}$ has a stronger correlation with $\mathrm{BMD}^{1}$.

Objective: The objective of the study was to explore the correlations between $25(\mathrm{OH}) \mathrm{D}$, VDBP, free $25(\mathrm{OH}) \mathrm{D}$ and $\mathrm{BMD}$ in a group of female rheumatoid arthritis (RA) patients and healthy controls.

Methods: 77 RA patients and 30 controls were studied. BMD was measured using DXA. 25(OH)D was measured using electrochemiluminescence immunoassay using a Cobas e411 immunoassay analyzer (Roche Diagnostics Limited, Basel, Switzerland). VDBP levels were measured using Human Vitamin D BP Quantikine ELISA kit by R\&D Systems, USA. Free 25(OH)D was calculated as previously published 1 .

Results: The average age of the RA patients and controls were 54.03 and 52.64 years $(p=\mathrm{NS})$. BMD at the lumber spine (LS), femoral neck (FN) and total hip (TH) was not significantly different (Mann-Whitney U $p=0.16,0.15$ and 0.59 , respectively) between the RA patients and controls. There was a significant correlation between 25(OH)D and FN BMD (Spearman's rho $p=0.042)$ but not at the $\operatorname{LS}(p=0.24)$ or $\mathrm{TH}$ $(p=0.27)$. There was no significant correlations between 
BMD at any site and VDBP or BMD and calculated free $25(\mathrm{OH}) \mathrm{D}$. This lack of correlation remained when the RA patient and healthy control groups were analysed separately.

Conclusions: In this population of Malaysian subjects, there was a significant correlation between 25(OH)D and FN BMD. However, VDBP and calculated free $25(\mathrm{OH}) \mathrm{D}$ were not positively correlated with BMD.

\section{Reference:}

${ }^{1}$ Powe CE, et al. J Bone Miner Res 2011;26:1609

\section{P517}

POSSIBLE ALTERNATIVE OF BONE MINERAL DENSITY IN DIAGNOSIS OF OSTEOPOROSIS

M. I. Majumder ${ }^{1}$, M. A. S. I. Harun ${ }^{2}$, N. Sakib ${ }^{3}$

${ }^{1}$ Comilla Medical College, Comilla, Bangladesh, ${ }^{2}$ Chattagram International Dental College, Chittagong, Bangladesh, ${ }^{3} \mathrm{BRAC}$ University, Dhaka, Bangladesh

Osteoporosis is characterised by progressive decrease in bone mass that results in increased bone fragility and higher fracture risk. Osteoporosis is considered a serious public health concern and accounts $0.83 \%$ of global burden of non communicable diseases. Among multiple modalities of osteoporosis investigations BMD is considered in gold standard by WHO. Aim of this study was to evaluate simple and cost effective estimation of pixel intensity of mandibular alveolar bone determining mandibular alveolar bone mass(MABM) by radiovisiography (RVG) as alternative procedure for diagnosis of osteoporosis with BMD correlation. 1751 patients with osteopenia and osteoporosis was included in this study. All patients were evaluated by DXA for BMD and RVG MABM. Statistical analysis were performed by Stata SE version 12.0 (Stata Corp LP). Mean age of osteopenic patients were 56.13 years with SD 10.68 years $(n=475)$, mean BMD was -1.67 with SD 0.64 $(n=475)$. Linear regression (simple/ unadjusted) shows significant $(p<0.05)$ relationship between MABM \& BMD. Here, estimated BMD $=-2.015+$ $\left(0.0035^{*} \mathrm{MABM}\right)$. Mean age of osteoporotic patients with and without fracture was $62.03 \mathrm{y}$ with SD 10.96 years $(n=1276)$. Mean BMD was -3.78 with SD 0.967. Linear regression (simple/unadjusted) shows relationship between MABM \& BMD $(n=1276)$ which is not statistically significant $(p>0.05)$. Here, estimated $\mathrm{BMD}=-3.848+(0.0008 * \mathrm{MABM})$. Linear regression (adjusted for fracture) shows relationship between BMD and MABM in fracture patients which is not statistically significant $(p>0.05)$. Here, estimated $\mathrm{BMD}=-3.673+$ $(0.0011 * \mathrm{MABM})+(-0.452 *$ fracture $)[$ fracture $=1$, no fracture $=0$ ]. It means, BMD will be estimated -0.452 units more in fracture patients, compared to their nonfractured counterparts, keeping other variables constant. Estimation of MABM is simple, less expensive and can be alternative diagnostic tool for osteoporosis or can be predictor for osteoporosis and BMD can be calculated with MABM value. A large sample evaluation may give more statistically significant value correlating BMD and MABM.

\section{P518}

VITAMIN D STATUS AND ITS DETERMINANTS AMONG PREMENOPAUSAL AND POSTMENOPAUSAL WOMEN IN DHAKA CITY SLUMS

F. R. Bhuiyan ${ }^{1}$, R. J. Janny ${ }^{2}$, H. A. Chowdhury ${ }^{3}$, M. A. Zubair $^{2}$ L. Ali ${ }^{4}$

${ }^{1}$ Human Nutrition Unit, Dept of Biochemistry \& Cell Biology, Bangladesh University of Health Sciences (BUHS), Dhaka, Bangladesh, ${ }^{2}$ Department of food technology \& nutritional science, Mawlana Bhashani Science \& Technology University (MBSTU), Dhaka, Bangladesh, ${ }^{3}$ Department of Biostatistics, Bangladesh University of Health Sciences (BUHS), Dhaka, Bangladesh, ${ }^{4}$ Department of Biochemistry \& Cell Biology, Bangladesh University of Health Sciences (BUHS), Dhaka, Bangladesh

Background and aims: Vitamin D is a fat soluble pro hormone which plays an important role in bone metabolism through regulation of calcium and phosphate homeostasis. It has been estimated that 1 billion people worldwide have vitamin D adequacy or inadequacy. An observational analytical study with a group comparison design was conducted among the Dhaka city slums to measure the vitamin D status in Bangladeshi pre and postmenopausal women.

Materials and methods: Total 88 women were included in each group. Sociodemographic information was taken by semistructured questionnaire, anthropometric and clinical information were taken from the subjects. Serum glucose fasting was analyzed by glucose oxidase-method (Randox, UK). HbA1c was measured by HPLC method, Serum lipid profile (Total cholesterol, TG, and HDL) and clinical enzyme like liver enzyme (SGPT), uric acid, creatinine was measured by enzymatic-colorimetric methods (Randox, UK). Serum $\mathrm{Ca}$ was measured by calcium-colorimetric method. Vitamin D was measured by chemical autoanalyzer.

Results: Among the study subjects age (y, mean $\pm \mathrm{SD})$ was $31 \pm 8$ in premenopausal and $54 \pm 6$ in postmenopausal women. Vitamin D adequacy was observed among $57 \%$ of premenopausal and $43 \%$ of the postmenopausal women and inadequacy was observed among $42 \%$ of premenopausal and $58 \%$ of the postmenopausal women. Out of 
total in inadequacy, moderate deficiency was found about $92 \%$ of each groups. However, using cutoff point $<15 \mathrm{ng} / \mathrm{ml}$ as hypovitaminosis D defined was showed significant association with sun exposure, $\mathrm{S}$ creatinine and total fat intake per day in postmenopausal women ( $p=0.009, p=0.009$ and 0.005 , respectively). Major confounder of vitamin D inadequacy, the sun exposure ( $\mathrm{min} /$ day) in the postmenopausal women was significantly lower compare to the premenopausal women $(p=0.011)$. On Pearson's correlation analysis physical activity showed positive correlation with vitamin $\mathrm{D}$ in both premenopausal $(r=0.266, p=0.012)$ and postmenopausal $(r=0.286$, $p=0.007)$ women. Sun exposure showed positive correlation with vitamin $\mathrm{D}$ in both premenopausal $(r=0.217$, $p=0.042)$ and postmenopausal $(r=0.378, p=<0.001)$ women. On the other hand $\mathrm{HbA} 1 \mathrm{C}$ and $\mathrm{S} \mathrm{Cr}$ showed negative correlation with vitamin $\mathrm{D}$ in postmenopausal ( $r=-0.228, p=0.033$ and $r=-0.191, p=0.075)$ women. But S Ca showed positive correlation with vitamin D in premenopausal $(r=0.280, p=0.008)$ women. On binary regression analysis of vitamin $\mathrm{D}$ according to the confounding independent variables (Age, Sun exposure, S $\mathrm{Cr}$, Fat intake, $\mathrm{Ca}$ intake). There was a positive association was found of Sun exposure $(b=0.006$ [95\%CI: $1.002-1.010] ; p=0.005)$ and Fat intake $(b=0.432$ [95\%CI: $1.133-2.095] ; p=0.006)$. In $\mathrm{S} \mathrm{Cr}$ was a negative association was found $(b=-3.643[95 \% \mathrm{CI}:<0.001-$ $1.756] ; \mathrm{p}=0.090$ ) with vitamin $\mathrm{D}$ in postmenopausal women. But this association was disappeared after adjusting the same confounder in premenopausal women. Conclusion: To concluded that, insufficient exposure to sunlight, deficient fat intake and compromised renal function are the major determinants of hypovitaminosis D among postmenopausal women. For premenopausal women the determinants of vitamin D inadequacy are less clear; however, sun exposure and outdoor physical activity may have some assumption with vitamin D status in this group. Further studies needs to be elucidated by considering a follow-up study with large sample size.

\section{P519}

\section{THE INFLUENCE OF OSTEOPOROSIS ON WELL-BEING IN MEN}

D. M. Farcas ${ }^{1}$, L. Sachelarie 2 , A. I. Gasparik ${ }^{3}$

${ }^{1}$ Physical Rehabilitation Department, University of Oradea, Faculty of Medicine and Pharmacy, Oradea, Romania, ${ }^{2}$ Apollonia University, Iasi, Romania, ${ }^{3}$ University of Medicine and Pharmacy, Tirgu Mures, Romania

Aim of the study: To assess self-esteem, anxiety and wellbeing in men with osteoporosis.
Material and method: Quality of life was lower in the group of men with osteoporosis than in the control group. Also the mean self-esteem value was lower in the group of men with osteoporosis than in the control group. The mean anxiety value was higher in the group of men with osteoporosis than in the group of men without osteoporosis. We also found correlations between quality of life and the psychological parameters investigated.

Results: The relative expressions of catabolic genes (IL1B, iNOS, MMP13, and IL8) in OA patients were significantly higher than in NOF patients and, this was correlated with either hypomethylated $\mathrm{CpG}$ sites in the proximal promoters or in a specific enhancer element for $i N O S$ gene. In contrast, the relative expression level of the chondrocyte anabolic gene, COL9A1, was abolished in OA chondrocytes compared with healthy controls. This observation was correlated with hypermethylated $\mathrm{CpG}$ sites in its proximal promoter. Critically, we observed, for the first time, demethylation of an NF-kB enhancer element orchestrates $i N O S$ induction in $\mathrm{OA}$ via cell cycle regulation.

Conclusions: It seems that osteoporosis has an impact on men's quality of life and on their psychological wellbeing. We tried to underline that there's a correlation between the physical and psychological aspects in osteoporosis. Also these correlations point out the need of developing future strategies for coping with psychological disturbances in patients with chronic illnesses such osteoporosis.

\section{P520}

IMPACT OF AIR POLLUTION ON VITAMIN D DEFICIENCY AND BONE HEALTH IN ADOLESCENTS

E. Feizabad $^{1}$, B. Larijani ${ }^{1}$, A. Hossein-Nezhad ${ }^{1}$, Z. Maghbooli $^{1}$, M. Ramezani ${ }^{1}$, H. Adibi ${ }^{1}$

${ }^{1}$ Osteoporosis Research Center, Endocrinology and Metabolism Clinical Sciences Institute, Tehran University of Medical Sciences, Tehran, Islamic Republic of Iran

The aim of this study was to evaluate the association between ambient air pollution and bone turnover in adolescents and also, to compare the prevalence of vitamin $\mathrm{D}$ deficiency in polluted and non-polluted regions of Tehran. This cross-sectional population-based study is conducted on 444 middle and high school students (both girls and boys) in Tehran. Detailed daily data on air pollution were obtained from archive data that collected by Tehran Air Quality Control Company (AQCC). Serum levels of calcium, phosphorus, PTH, bone specific alkaline phosphates, 25(OH)D, osteo- 
calcin, crosslinked C-telopeptide, total protein, albumin and creatinine were determined. Vitamin D deficiency was more prevalent in polluted area. Air Quality Index (AQI) and PM2.5 exposure were significantly associated with risk of vitamin D deficiency in both sexes. After adjustment for age and sex there was significant association between AQI and PM2.5 with bone turnover. Interestingly, greater calcium intake $(>5000 \mathrm{mg} /$ week) in girls had protective effect against air pollution on bone turnover. The results indicated that air pollution adversely affects on bone health. Air pollution is a chief factor determining the extent of solar UVB that reaches earth surface thus atmospheric pollution may play a significant independent role in the development of vitamin D deficiency.

\section{P521}

\section{A RARE CASE OF CRANIOFACIAL DEFORMITY IN} A PATIENT WITH UNTREATED SECONDARY HYPERPARATHYROIDISM AND CHRONIC RENAL FAILURE: CASE REPORT

T. A. Grebennikova ${ }^{1}$, T. S. Zenkova ${ }^{1}$, J. A. Krupinova ${ }^{1}$, L. Y. Rozhinskaya $^{1}$, Z. E. Belaya ${ }^{1}$

${ }^{1}$ Neuroendocrinology and Bone Diseases, Moscow, Russian Federation

Sagliker et.al described 25 patients with chronic renal failure (CRF), secondary hyperparathyroidism (SH), maxillary and mandibular bone changes, teeth/dental abnormalities disfiguring the face. We describe a similar case with a severe involvement of the skull. A 29-yearold female with end-stage CRF (peritoneal dialysis started at the age of 20 y.o.) with $\mathrm{SH}$ at the time of admission: hyperplasia of three parathyroid glands, PTH - $4327 \mathrm{pg} / \mathrm{ml}$ (15.0-65), serum calcium - $2.29 \mathrm{mmol} / \mathrm{l}$ (2.10-2.55); phosphorus - $2.06 \mathrm{mmol} / \mathrm{l}$ (0.74-1.52), alkaline phosphatase $1819 \mathrm{u} / 1$ (50-150), height loss and significant craniofacial changes with first signs up to 2 years ago. Examination at the time of admission revealed maxillary and mandibular hyperplasia, enlarged cheekbones, sphenoid bone and bones of the cranial vault (Fig. 1), fingertip changes, chest deformity with coexisting vertebral body compression (Th5-Th8, L3L4), total hip Z-score $-2,7$, neck Z-score $-2,6$, radius $33 \% \mathrm{Z}$-score $-4,9$. carboxyterminal crosslinked telopeptide of type I collagen - $4.99 \mathrm{ng} / \mathrm{ml}$ (0.01-0.6), osteocalcin $300 \mathrm{ng} / \mathrm{ml}$ (11-43), vitamin D - $11.9 \mathrm{ng} / \mathrm{ml}$. Conclusion: This rare deformity of the craniofacial complex, coexisting with expected vertebral body compression and bone loss, demonstrates a rare probably genetically determined consequence of $\mathrm{SH}$ requiring further investigation and early parathyroidectomy.

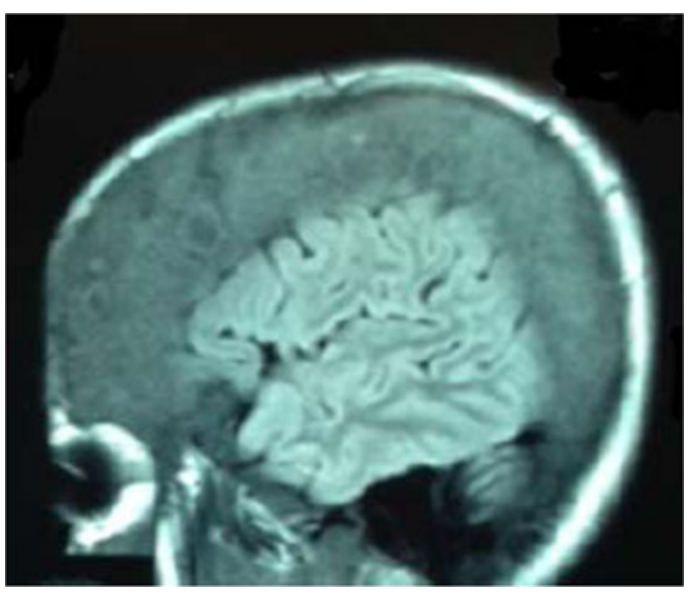

P522

TURNER SYNDROME-ASSOCIATED LOW BONE MINERAL DENSITY IN YOUNG ADULTS

A. A. Galateanu-Gheorghisan $1^{1}$, M. Carsote ${ }^{1}$, D. Peretianu ${ }^{2}$, O. M. Popa ${ }^{3}$, A. Valea ${ }^{4}$, A. Ghemigian ${ }^{1}$

${ }^{1}$ C. Davila University of Medicine and Pharmacy \& C.I.Parhon National Institute of Endocrinology, Bucharest, Romania, ${ }^{2}$ S.C.M. Povernei, Bucharest, Romania, ${ }^{3}$ C.I. Parhon National Institute of Endocrinology, Bucharest, Romania, ${ }^{4}$ I. Hatieganu University of Medicine and Pharmacy \& Clinical County Hospital, Cluj-Napoca, Romania

Introduction: Turner syndrome (TS) is complex monogenetic condition (45, XO) involving multiples comorbidities as short stature, kidney and heart anomalies, autoimmune hypothyroidism, hearing loss, glucose metabolism impairment, etc. The bone is affected by deficiency of ovarian estrogen production (and associated lack of spontaneous puberty), inefficient or unused hormonal replacement therapy, celiac disease-associated vitamin D deficiency celiac and potential effect of growth hormone $(\mathrm{GH})$ therapy. The technique aspects in skeleton assessment based on central DXA is related to dwarfism, the discrepancies between bone age and chronological age during childhood and puberty. The transition to adult life makes necessary a different approach regarding the complications and follow-up.

Material and Method: The case we present was evaluated based on karyotype (metaphase cytogenetic diagnosis from peripheral blood, Leica CW4000 Karyo kit), contrast computed tomography scan, DXA (GE Lunar Prodigy device; an age matched database providing Z-score); bone, thyroid and ovarian indices. 
Case data: A 21-old nonsmoking Caucasian female was diagnosed with TS since the age of 11 (aneuploidy by lack of X chromosome in $100 \%$ of the metaphases, negative Barr test). She associates short stature (a final height of $135 \mathrm{~cm}$, without prior GH therapy), horseshoe kidney, and mild mental retardation. Puberty was induced by estro-progestative (E-P) therapy since the age of 12. Oral contraceptives were prescribed during puberty which was intermittently taken by the patient. On admission, the subject presents obesity (a BMI of $35 \mathrm{~kg} / \mathrm{m} 2$ ), and persistent back pain.

Hashimoto's thyroiditis is suggested by intense hypoechoic thyroid pattern and anti-thyroglobulin antibodies of $86 \mathrm{UI} / \mathrm{L}$ (normal $<70$ ), in association with normal thyroid function was found (TSH of $2.3 \mu \mathrm{UI} / \mathrm{mL}$, normal: $0.5-4.5 \mu \mathrm{UI} / \mathrm{mL}$ ). Cardiac and eye exam is within normal limits. High FSH levels of $68 \mathrm{mUI} / \mathrm{mL}$ suggest no adherence to E-P therapy but growth cartilages are closed. 25-hydroxyvitamin D is low of $12 \mathrm{ng} / \mathrm{mL}$ with normal recommended levels of $\geq 30 \mathrm{ng} / \mathrm{ml}$, and negative blood tests for celiac disease. Intact PTH is within normal ranges (of $46.81 \mathrm{pg} / \mathrm{mL}, 15-65 \mathrm{pg} / \mathrm{mL}$ ). Total serum calcium and phosphorus is normal (of $0.6 \mathrm{mg} / \mathrm{dL}, \mathrm{N}: 8.5-$ $10.2 \mathrm{mg} / \mathrm{dL}$, respective $4.2 \mathrm{mg} / \mathrm{dL}, \mathrm{N}: 2.5-5.5 \mathrm{mg} / \mathrm{dL}$ ) with high-normal total alkaline phosphatase (of 106 U/L, N: 38-105 U/L). Lumbar DXA shows a BMD of $0.785 \mathrm{~g} / \mathrm{cm} 2$, Z-score of $-2.8 \mathrm{SD}$. Weekly oral alendronate with vitamin $\mathrm{D} /$ calcium supplement is recommended with E-P therapy.

Conclusion: Low BMD for adult age or for transition to adult age in females with TS needs an early recognition before complication as fractures are detected. However, the adequate DXA devices and BMD measurements are still challenging because of the final height. The vitamin D status also should be periodically checked up. The skeleton health, as well as others organs like heart, ear, kidney, thyroid, etc. is assessed lifelong.

\section{P523}

VITAMIN D DEFICIENCY, BONE TURNOVER MARKERS AND CAUSATIVE FACTORS AMONG ADOLESCENTS OF TEHRAN

B. Larijani ${ }^{1}$, A. Hossein-Nezhad ${ }^{1}$, E. Feizabad ${ }^{1}$, Z. Maghbooli $^{1}$, H. Adibi ${ }^{1}$, M. Ramezani ${ }^{1}$, E. Taheri ${ }^{1}$

${ }^{1}$ Osteoporosis Research Center, Endocrinology and Metabolism Clinical Sciences Institute, Tehran University of Medical Sciences, Tehran, Islamic Republic of Iran

This cross-sectional population-based study is conducted to elucidate the prevalence of vitamin D deficiency, bone turnover markers variation and its influencing factors among adolescents of Tehran. Totally 444 middle and high school (53\% in high school) students (both girls and boys) were recruited. A short food frequency questionnaire designed to estimate dietary calcium and vitamin D consumption. Serum levels of calcium, phosphorus, PTH, bone specific alkaline phosphates, $25(\mathrm{OH}) \mathrm{D}$, osteocalcin, cross-linked C-telopeptide (CTX), total protein, albumin and creatinine were determined. Vitamin D deficiency was prevalent in adolescents and serum vitamin D in only $22.7 \%$ of participants was normal. Results revealed that vitamin D insufficiency reported in $34 \%$ of participants and vitamin D deficiency was prevalent in $43.3 \%$ of them. Serum vitamin D, osteocalcin, CTX and bone alkaline phosphates were significantly higher in boys in all different ages. Serum levels of $25(\mathrm{OH}) \mathrm{D}$ had positive influences on bone turnover markers and had negative correlation with PTH. Vitamin D deficiency and insufficiency is common among healthy adolescents of Tehran. There is a pressing need to improve vitamin D status among adolescents. Increasing vitamin $\mathrm{D}$ fortification of dairy products can be considered as a population-wide public health strategy in Iran.

\section{P524}

MONITORING OPTIMIZATION OF TREATMENT WITH VITAMIN D3

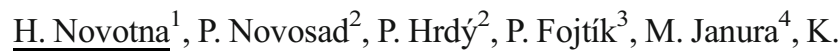
Janurová $^{5}$

${ }^{1}$ Mediekos Amb. Ltd., Osteological Centre, Zlin, Czech Republic, ${ }^{2}$ Mediekos Amb. Ltd, Zlín, Czech Republic,

${ }^{3}$ Agel Research and Training Institute, Ostrava-Vítkovice, Czech Republic, ${ }^{4}$ Department of Nature Science in Kinanthropology, Faculty of Physical Culture, Palacky University, Olomouc, Czech Republic, ${ }^{5}$ IT4 Innovations National Supercomputing Centre VŠB-Technical University, Ostrava, Czech Republic

Objective: To evaluate the status and effectiveness of treatment with vitamin $\mathrm{D}$ in patients with osteoporosis, we measure serum level of vitamin $25(\mathrm{OH})$ vitamin D3 test. In case we use plain vitamin D - cholecalciferol for supplementary treatment, it is possible to monitor the treatment with $25(\mathrm{OH}) \mathrm{D} 3$. However, when selecting active metabolite alfacalcidiol for the treatment, the relation with the level of $25(\mathrm{OH}) \mathrm{D} 3$ is not as direct. In this research we want to find a relation among therapeutically administered vitamin D3, cholecalciferol as well as alfacalcidiol, and levels of $25(\mathrm{OH}) \mathrm{D} 3$ and 1 , 25(OH)2D3 towards markers of bone turnover (P1NP a CTX) with the aim to find how to best monitor the treatment with alfacalcidiol.

Method: This is a randomly selected cohort (n-266) of postmenopausal women from our clinic, average age 
67.7 years, with normal renal functions (eGF CKD EPI 1, $248 \mathrm{ml} / \mathrm{s}$ ). The patients were examined during the last week of March and the first week of June 2015. All were diagnosed with levels for 25(OH)D3, 1,25(OH)2D3, markers of bone metabolism P1NP (procollagen type I $\mathrm{N}$-terminal propeptide) and $\operatorname{CTX}(\beta \mathrm{C}$-terminal crosslinked telopeptide of type I collagen), PTH, Ca (total calcium), P (phosphorus). This group was sorted according to the basic diagnostics (osteoporosis $n=126$, osteopenia $n=106$, with a monitoring group with normal bone density $n=34$. Next we sorted according to vitamin D supplementation. Group 1 of patients $(n=89)$ with no vitamin D treatment, Group $2(n=26)$ with cholecalciferol up to $800 \mathrm{IU}$ and Group $3(n=94)$ with cholecalciferol at $800 \mathrm{IU}$ and above. Group 4 received combined therapy $(n=57)$ with $1 \mu \mathrm{g}$ alfacalcidol and cholecalciferol of average dosage 459 IU. Group 5 received only alfacalcidol $1 \mu \mathrm{g}$.

Statistical data processing: We carried out a statistical data processing with the use of Statgraphics Plus 5.1 software and R software 3.2.0. Normality of data distribution was verified with the use of Shapiro- Wilk test. Homoscedasticity was tested with Barlette's test. To compare the groups we used a single factor (the amount of vitamin D) analysis of variance (ANOVA) and also the Bonferroni's post hoc test. With such parameters that did not show a normal data distribution we used Kruskal-Wallis test and Turkey-Kramer post hoc test. To determine the dependence of vitamin $\mathrm{D}$ and complex diagnosis we used Pearson's chi-square test and Yates' correction.

Results: A Groups according to dosage and type of vitamin D: As expected, the statistically relevant values $(p<0.01)$ in monitored vitamin $25(\mathrm{OH}) \mathrm{D} 3$ were found in the group which was not treated with vitamin $\mathrm{D}(0)$ and the groups receiving cholecalciferol in any dosage (Groups 1 and 2), and also in the group with combined treatment (Group 3) but not in the group with individually administered alfacalcidiol. When evaluating the level of $1.25 \mathrm{OH} 2 \mathrm{D} 3$ there was no strong relation with the type of the chosen treatment (single factor ANOCA $p=0.028$ ) and there was only a tendency towards significance among groups 1 and 2,2 and 3 . On the contrary, the groups treated only with $1 \mu \mathrm{g}$ alfacalcidiol show significant changes $(p<0.05)$ in the sense of lowering values P1NP and CTx when compared with a group with no treatment. B Groups according to diagnosis: According to given calculations, we can find only values with medium dependence $(0.282)$ which is not clinically significant.

Conclusion: When monitoring treatment with vitamin D, the basis is $25(\mathrm{OH}) \mathrm{D} 3$. We cannot rely on this value in more complex clinical studies where treatment with alfacalcidiol is required, also the level of 1,25 (OH)2
D3 has little validity, and it is better to extend the evaluation with osteomarkers evaluating bone turnover.

\section{P525 \\ HOMOCYSTEINE, ABDOMINAL AORTIC CAL- CIFICATION AND VERTEBRAL FRACTURES IN POSTMENOPAUSAL WOMEN.}

I. Ghozlani ${ }^{1}$, A. Mounach ${ }^{2}$, A. Rezqi ${ }^{2}$, A. Nouijai ${ }^{2}$, L. Achemlal $^{2}$, A. Bezza ${ }^{2}$, Z. Ouzzif ${ }^{3}$, A. El Maghraoui ${ }^{2}$

${ }^{1}$ Rheumatology, Military Hospital, Agadir, Morocco, ${ }^{2}$ Rheumatology, Military Hospital Mohammed V, Rabat, Morocco, ${ }^{3}$ Biochemistry, Military Hospital Mohammed V, Rabat, Morocco

The main of this study was to examine the relationship between plasma homocysteine (Hcy), abdominal aortic calcification (AAC) and prevalence of asymptomatic osteoporotic vertebral fractures (VFs) using vertebral fracture assessment (VFA) in Moroccan postmenopausal women. The study cohort consisted of 188 consecutive postmenopausal women with no prior known diagnosis of osteoporosis or taking medication interfering with bone metabolism. Mean age, weight, and BMI were measured. Lateral VFA images and scans of the lumbar spine and proximal femur were obtained using a Lunar Prodigy Vision densitometer (GE Healthcare Inc., Waukesha, WI). VFs were defined using a combination of Genant's semiquantitative approach and morphometry. VFA images were also scored for AAC using a validated 24 point scale. Fifty-eight (30.9\%) patients had densitometric osteoporosis. VFs were identified using VFA in $76(40.4 \%)$ patients: 61 women had grade 1 VFs and 15 had grade 2 or 3 VFs. One hundred twenty nine women $(68.6 \%)$ did not have any detectable AAC, whereas the prevalence of significant atherosclerotic burden defined as AAC score of 5 or higher, was $13.8 \%$. A significant positive correlation between AAC score and homocysteine was observed. Women with extended AAC, were older, had a lower weight, BMI and BMD, higher homocysteine levels and more prevalent VFs than women without extended AAC. No statistical difference was shown between the 3 groups (absence of VFs, VFs grade 1 , and VFs grade $2 / 3$ ) and homocysteine. The group of women with $2 / 3$ VFs was more aged, had a statistically significant higher AAC score and higher proportion of subjects with extended AAC, and lower lumbar spine and hip BMD and T-scores than those without VFA-identified VFs. Multiple regression analysis showed that the presence of extended AAC was significantly associated with Age and grade $2 / 3 \mathrm{VFs}$ and not independently associated with homocysteine levels. This study did not confirm 
that homocysteine is important determinant of prevalent asymptomatic VFs in postmenopausal women although a significant association between Hcy and AAC was showed. However, prevalent VFs are independently associated with extended aortic calcifications regardless of age.

\section{P526}

SHORT-TERM RADIOGRAPHIC PROGRESSION IN PATIENTS WITH NONTRAUMATIC CLINICAL VERTEBRAL FRACTURE

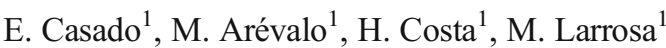

${ }^{1}$ Rheumatology, University Hospital Parc Taulí Sabadell, Sabadell, Spain

Background: Clinical vertebral fracture can be a serious and disabling complication in patients with osteoporosis (OP). Although spine X-ray can easily confirm the diagnosis, it is very important to know the radiological evolution of the fracture in the following weeks in order to establish a suitable therapeutic approach.

Objective: To assess the radiological outcome in patients with acute symptomatic osteoporotic vertebral fracture in the first 8 weeks of symptoms. To analyze what clinical variables are associated with radiological progression, defined as an increase in the severity of the fracture or the presence of new vertebral fractures.

Patients and methods: All patients with acute osteoporotic vertebral fracture attended in a quickly specific OP clinic, from January 2013 to October 2014 were included. Patients who have undergone vertebroplasty, and patients with previous diagnosis or receiving treatment for OP (including calcium and vitamin D) were excluded. All patients gave their consent to participate in the study. Clinical evaluation of vertebral pain (VAS), lateral thoracic and lumbar spine X-ray (to establish the number and severity of vertebral fractures according to the Genant semiquantitative scale), DXA (lumbar spine, femoral neck and total hip BMD) and phospho-calcium metabolism parameters (Ca, P, 25OHD, PTH) were assessed in all patients at baseline. A new clinical assessment of vertebral pain and spine X-ray were performed at 8 weeks.

Results: 31 patients ( 24 women and 7 men) were included. Mean age $74 \pm 9$ years. Sixteen patients had postmenopausal osteoporosis and 15 secondary osteoporosis (6 early menopause, 5 , corticosteroids, 4 other causes). 10-year fracture risk (FRAX) was $17.1 \pm 11.0$ for major fracture and $8.7 \pm 8.4$ for hip fracture. All patients were early assessed (less than 1 month after the onset of symptoms of vertebral pain). At baseline VAS for pain was $8.8 \pm 1.3$. Lateral thoracic and lumbar spine X-ray showed 53 vertebral fractures in 31 patients
$(1.7 \pm 0.9$ fractures per patient): 17 patients had 1 vertebral fracture, 7 patients had 2 vertebral fractures and other 7 patients had 3 or more vertebral fractures. Five patients also had some previous non vertebral fractures. The mean vertebral fracture severity was $1.6 \pm 0.6$ (13 patients grade $0-1,18$ patients grade $>1)$. Baseline T-score at lumbar spine and femoral neck was $-3.0 \pm 1.3$ and $-2.4 \pm 0.6$, respectively. Mean $25(\mathrm{OH}) \mathrm{D}$ and $\mathrm{PTH}$ was $22.9 \pm 12.9 \mathrm{ng} / \mathrm{ml}$ and 66.6 $\pm 31.6 \mathrm{pg} / \mathrm{ml}$ respectively. At 8 weeks, despite clinical improvement $(4.6 \pm 2.7$ VAS pain; $p<0.00001)$, a radiological progression was observed in $15 / 31$ patients (48 \%). An increase in the fracture severity $(2.0 \pm 0.6$; $p=0.0014)$ and 10 new vertebral fractures $(2.1 \pm 1.3$ fracturesper patient; $p=0.07)$ were observed. No differences were found in age, FRAX, BMD, 25(OH) D and PTH between patients with radiological progression and patients with stable fracture. Patients with radiological progression had fewer vertebral fractures ( $1.4 \pm 0.8$ vs. $2.0 \pm 1.0)$, lower severity $(1.3 \pm 0.6$ vs. $2.4 \pm 2.8)$ and worse pain control $(5.5 \pm 2.8$ vs. $4.1 \pm 2.5)$, although differences were not significant.

Conclusions: Half acute clinical vertebral fracture patients will have a radiological progression in the following weeks. Patients with a single, mild-moderate vertebral fracture, without clinical improvement would have to be assessed for radiological progression.

\section{P527}

THE IMPACT OF THE NATIONAL BONE HEALTH ALLIANCE (NBHA) DIAGNOSTIC CRITERIA ON THE PREVALENCE OF OSTEOPOROSIS IN THE UNITED STATES

N. C. Wright $^{1}$, K. G. Saag ${ }^{2}$, B. Dawson-Hughes ${ }^{3}$, S. Khosla ${ }^{4}$, E. S. Siris ${ }^{5}$

${ }^{1}$ Department of Epidemiology, University of Alabama at Birmingham, Birmingham, United States, ${ }^{2}$ Division of Clinical Immunology \& Rheumatology, University of Alabama at Birmingham, Birmingham, United States, ${ }^{3}$ USDA Nutrition Research Center at Tufts University, Boston, United States, ${ }^{4}$ Division of Endocrinology, Metabolism, Diabetes, Nutrition, and Internal Medicine, Mayo Clinic, Rochester, United States, ${ }^{5}$ Department of Medicine, College of Physicians and Surgeons of Columbia University, New York, United States

Objectives: To evaluate the prevalence of osteoporosis using the new NBHA osteoporosis diagnostic criteria in adults' $\geq 50$ years in the United States (US), and to compare the prevalence of osteoporosis based on the NBHA criteria with estimated proportion of adults meeting National Osteoporosis Foundation (NOF) treatment thresholds. 
Material and Methods: We included participants' $\geq 50$ years of age from the 2005-2008 data of the National Health and Nutrition Examination Survey (NHANES). We identified those with osteoporosis if they had any one of the following: 1) BMD based Tscore $\leq-2.5$; 2) qualifying low trauma hip fracture irrespective of BMD, or clinical vertebral, proximal humerus, pelvis, or distal forearm fracture with low BMD; or 3) FRAX score at the NOF intervention thresholds ( $\geq 3 \%$ for hip fracture risk or $\geq 20 \%$ for major osteoporotic fracture risk). We estimated the prevalence (standard error) of osteoporosis according to the NBHA criteria overall, by gender and age, and used test of proportions to compare with the estimated proportion of adults meeting NOF treatment thresholds.

Results: The population included 1,948 (54.3 \%) men and 1,639 (45.7\%) women. Approximately a third of participants were 50-59 years (30.6\%), $12 \%$ were $80+$ years, and $21 \%$ were from racial/ethnic minority groups. We estimated that $16.0 \%(0.8)$ of men and $29.9 \%$ (1.0) of women $50+$ years have osteoporosis according to the NBHA criteria (Table). The prevalence increases with age, with an estimated of $46.3 \%$ of men and $77.1 \%$ of women $80+$ years meeting the criteria. In men, overall and in the two youngest age groups, the NBHA criteria identified a significantly smaller proportion than that indicated for treatment using the NOF guidelines; whereas there were no statistical differences between the two proportions in women.

Conclusions: In a representative sample of US adults age $50+$ years, the inclusion of qualifying fractures and FRAX increases the prevalence of osteoporosis compared to BMD alone based definitions; however the proportions of women eligible for treatment are similar by the NHBA and the NOF clinical guidelines criteria, but less men overall, particularly those aged 50-69 years, qualify for treatment by the NHBA criteria than by the NOF clinical guidelines.

\section{P528}

QUANTITY AND QUALITY OF BONE IN PATIENTS WITH OSTEOGENESIS IMPERFECTA MEASURED BY DXA (BMD AND TBS): CORRELATION WITH GENOTYPE AND FRACTURE RISK.

B. Aubry-Rozier ${ }^{1}$, A. Safran ${ }^{2}$, A. Bregou ${ }^{3}$, S. Unger $^{4}$, D.

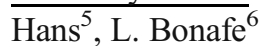

${ }^{1}$ Center of Bone Diseases, Lausanne University Hospital Switzerland, Lausanne, Switzerland, ${ }^{2}$ Rheumatology Unit Lausanne University Hospital, Lausanne, Switzerland, ${ }^{3}$ Orthopedic Unit Lausanne University Hospital, Lausanne, Switzerland, ${ }^{4}$ Genetic Unit Lausanne University Hospital, Lausanne, Switzerland, ${ }^{5}$ Center of Bone Diseases Lausanne University Hospital, Lausanne, Switzerland, ${ }^{6}$ Centre for Molecular Diseases, Lausanne, Switzerland

Osteogenesis imperfecta (OI) is a rare genetic disease caused by a defect in type I collagen or in proteins that interact with it. Recent knowledge regarding the genetics of OI allows an accurate diagnosis of the specific type of OI and its molecular mechanism, with an alteration in quantity and/or quality of the bone. If the fracture risk is high, medications against osteoporosis are usually proposed. Some are more efficient on bone quantity and others on bone quality or both. In this study we evaluated the bone quantity by DXA and the bone quality by trabecular bone score (TBS) and analyzed their relation with fracture risk, phenotype and genotype for 31 OI adult patients.

Method: retrospective study of 31 adult patients with OI. BMD was measured at the femoral neck (FN), the total hip (TH) and the spine (LS). TBS was analyzed by the means of DXA images of the LS. BMD and TBS results were expressed in T-score and means respectively. They were calculated for the entire study population for comparison with the normal population and separately for the genotype and phenotypes subgroups.

Results: 31 patients, 21 women, 10 men, mean age 40 \pm 14 years. Phenotypes: 21 type I, 7 type III, 3 type V.

Table A. Comparison of the Number of US Adults 50 Years and Older Meeting NOF Osteoporosis Treatment Guidelines with NBHA Osteoporosis Diagnostic Criteria

\begin{tabular}{|c|c|c|c|c|c|c|}
\hline & \multicolumn{3}{|l|}{ Men } & \multicolumn{3}{|l|}{ Women } \\
\hline & $\begin{array}{l}\text { NOF Guidelines* } \\
\% \text { (SE) }\end{array}$ & $\begin{array}{l}\text { NBHA } \\
\% \text { (SE) }\end{array}$ & $\mathrm{p}$-value & $\begin{array}{l}\text { NOF Guidelines } \\
\% \text { (SE) }\end{array}$ & $\begin{array}{l}\text { NBHA } \\
\%(\text { SE) }\end{array}$ & p-value \\
\hline $50+(\text { age adjusted })^{\mathrm{a}}$ & $19.3(1.2)$ & $16.0(0.8)$ & 0.007 & $30.8(1.0)$ & $29.9(1.0)$ & 0.575 \\
\hline $50-59$ & $9.0(1.6)$ & $5.5(1.1)$ & 0.015 & $12.3(1.9)$ & $10.9(1.7)$ & 0.510 \\
\hline $60-69$ & $14.5(1.9)$ & $10.1(1.5)$ & 0.016 & $19.8(2.1)$ & $19.9(2.1)$ & 0.965 \\
\hline $70-79$ & $29.6(2.9)$ & $25.9(2.9)$ & 0.304 & $52.3(1.6)$ & $51.3(1.6)$ & 0.782 \\
\hline $80+$ & $47.1(3.2)$ & $46.3(3.0)$ & 0.867 & $78.7(2.9)$ & $77.1(3.0)$ & 0.698 \\
\hline
\end{tabular}

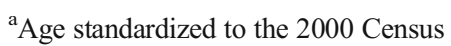

*Dawson-Hughes et al. Osteoporos Int. 2012; 23(3): 811-20 
Genotypes: 9 COL1A1, 6 COL1A2, 3 IFITM5 mutations. Mean age of the first fracture was $4 \pm 2$ years. 26 patients had multiple fractures. Mean BMD T-score were: FN -1.83 $\pm 1.19, \mathrm{TH}-1.5 \pm 1.11, \mathrm{LS}-2.77 \pm 1.30$. Mean TBS was 1.25 \pm 0.13 . For this young population, all results were lower than the normal population $(1.48 \pm 0.09$, normal TBS). Comparison between the phenotypes: no difference between type I and III regarding the LS BMD ( -2.8 vs. $-3.0, p=0.6)$ but TBS was significantly lower for type III compared to type I ( 1.16 vs. $1.27, p=0.03)$. Comparison between type III and V: same results. Comparison between the genotypes: LS BMD and TBS were lower in COL1A2 patients compared to COL1A1 patients $(-4.15 \pm 0.44$ vs. $-2.8 \pm 0.5, p=0.04$, and $1.15 \pm 0.03$ vs. $1.29 \pm 0.14, p=0.02)$. No difference was found for $\mathrm{TH}$ and $\mathrm{FN}$.

Conclusion: in our group of $31 \mathrm{OI}$ adult patients, we found that all measurements of bone health (BMD and TBS) are lower than in the normal population. We can distinguish OI types I, III and $\mathrm{V}$ based on the TBS measurement with the lower values for the type III and the higher values for the OI type V. Patients with COL1A2 mutation had the lower TBS and LS BMD. Based on these data, we can confirm that some OI patients had a predominantly bad quality of bone. This knowledge could help the clinician in the choice of the best treatment.

\section{P529}

\section{ASSESSMENT OF PHYSICAL PERFORMANCE IN SARCOPENIA}

M.-L. Cevei ${ }^{1}$, D.-L. Stoicanescu ${ }^{2}$, R.-N. Suciu ${ }^{1}$

${ }^{1}$ University of Oradea, Faculty of Medicine and Pharmacy, Oradea, Romania, ${ }^{2}$ University of Medicine and Pharmacy "Victor Babes", Timisoara, Romania

Sarcopenia is an age-related disease, defined as progressive loss of skeletal muscle mass and muscle strength with a risk of adverse outcomes such as physical disability, poor quality of life and death. The consequences of muscle decline often contribute to fractures, loss of independence and increased health care costs. Sarcopenia was associated with a loss of skeletal muscle fiber number and a change in the cross- sectional area of the remaining fibers. Different mechanisms were taken into account to explain the gradual loss of muscle tissue.

Objective: Our objective was to identify patients in whom occupational performance is affected by decreased muscle strength due to sarcopenia.

Material and Methods: We evaluated 77 patients with average age $67 \pm 7.6$ years, hospitalized at the Medical Rehabilitation Clinical Hospital Baile Felix, Romania, who fulfilled the inclusion criteria for the diagnosis of sarcopenia. Exclusion criteria were severe mobility disorders of the weight-bearing joints (legs) and cases with neurological conditions, cases with secondary sarcopenia, associated with organ failure in advanced stages, inflammatory diseases, endocrine diseases, malignancies, malabsorption, gastrointestinal disorders or use of medications that cause anorexia were also excluded. Assessment of physical performance in our patients was performed with gait speed, short physical performance battery test (SPPB test), sit to stand 5 times, self-reported physical function, timed up and go test, stair climb, standing balance.

Results: Gait speed value vas $0.83 \pm 0.14 \mathrm{~m} / \mathrm{s}$. Regarding SPPB: Total SPPB was $8.2 \pm 1.63$, SPPB gait speed was 2.31 \pm 0.76 , SPPB balance $3.58 \pm 0.84$, SPPB standing from a chair $2.47 \pm 0.92$. Timed Up and Go test was $18.7 \mathrm{~s} \pm 4.2 \mathrm{~s}$, Sit to stand 5 times $21.3 \pm 4.4$ s. Stair climb: $17.43 \pm 8.35$. Standing balance without movement: standing with one leg $1.4 \pm 0.86 \mathrm{~s}$.

Conclusion: Once these cases are diagnosed the main intervention is regular physical exercise, which restores muscle strength.

\section{P530}

\section{PAIN MANAGEMENT IN OSTEOPOROSIS}

M. I. Majumder ${ }^{1}$

${ }^{1}$ Comilla Medical College, Comilla, Bangladesh

Osteoporosis is characterised by progressive decrease in bony mass that results in increased bone fragility and higher fracture risk and accounts for $0.83 \%$ of global burden of non communicable disease. It is often called a 'silent' disease because it has no discernible symptoms until there is a bone fracture, usually compressive vertebral and other fracture. About $2 / 3$ of vertebral fractures causes no symptoms and remaining typically present with acute back pain. Pain is not universal feature in osteoporosis $50 \%$ of women and $20 \%$ of men above 50 will have an osteoporosis related fracture in their lifetime which increases dramatically with ageing and more in women than men, predominantly in white race, recurrent falls, tobacco use and excessive alcohol intake. Most osteoporotic vertebral fractures are not the result of external trauma and their diagnosis is delayed. Almost all nonspine related fractures occur in context of trauma and present with acute pain. Clinical consequences of hip, vertebral and wrist fractures are acute or chronic pain, excess mortality, increase fracture risks, further decline in psychosocial deterioration and decrease quality of life. Chronic discomfort is due to poorly healed fracture or side effects of osteoporotic medications. Pain evaluation can be performed daily by visual analog scale - 0 for no pain and 10 for agonising pain and also by NRS (numerical rating scale).The management of patients with acute vertebral fractures or other fractures should include measures to reduce pain and improve mobility, as well as starting treatment for osteoporosis. Traditional management has concentrated on analgesia, rest with physical support with brace or corset, and subsequent gradual mobilisation within the limit of pain. Calcitonin has proven efficacy in acute pain in osteoporosis 
related vertebral fracture. The acute pain normally subsides by the end of the healing and rehabilitation period (6-8 weeks). As proposed by the World Health Organisation (WHO), according to the intensity of chronic pain, analgesic treatment can be established. For mild pain (NRS $\leq 3$ ) is recommend the use of NSAIDs or acetaminophen with the possibility of adding the adjuvants drugs(antiresorptive-bisphosphonates, antidepressants, benzodiazepines) for moderate pain (NRS comprised equal to 4-6), is suggested a treatment with weak opioids integrated with or without NSAIDs or acetaminophen, and with the possibility of adding adjuvants. In case of severe pain $(\mathrm{NRS}>6$ ), the WHO plans to undertake a more integrated opioid treatment with NSAIDs or paracetamol, with the possibility of adding adjuvant. Despite published guidelines and WHO's pain ladder to the point of view for the management of chronic pain, the treatment of this condition remains suboptimal. The complexity of the problems of chronic osteoporotic pain needs a multimodal and multidisciplinary approach to diagnose and treat the disease. Other treatment modalities with relaxation techniques, meditation, gentle massage, light stretching/strengthening exercises, applications of heat and ice, acupuncture, transcutaneous electrical nerve stimulation, ultrasound, pain medication, a brace or support (only as a temporary measure). Physical therapy helps the patient to build strength and flexibility with 3-5 sessions per week weight bearing exercise 45-60 min per session. If acute pain not subsided, options for painful vertebral compression fractures are vertebroplasty and kyphoplasty. Other osteoporotic fracture require pain management with orthopaedic care.

\section{P531}

\section{MANAGING A PATIENT WITH VERTEBROGENIC CHRONIC LOW BACK PAIN AND MULTIPLE COMORBIDITIES}

D.-L. Stoicanescu ${ }^{1}$, M.-L. Cevei ${ }^{2}$

${ }^{1}$ University of Medicine and Pharmacy "Victor Babes", Timisoara, Romania, ${ }^{2}$ University of Oradea, Faculty of Medicine and Pharmacy, Oradea, Romania

We present the case of a man aged 59 years, who presented in the Medical Rehabilitation Clinical Hospital with 10 months history of cervical, thoracic and lumbar pain of moderate intensity, VAS 6 . His medical history was significant for many comorbidities: Hepatitis B virus-related cirrhosis, stage A, hypertriglyceridemia controlled by medication, type 2 diabetes mellitus needing insulin complicated by diabetic polyneuropathy and diabetic retinopathy, grade II hypertension, very high added risk, coronary artery disease without chest pain, right aortofemoral bypass graft surgery, residual hypothyroidism after right lobe thyroidectomy for thyroid adenoma, obesity grade 2, bilateral knee osteoarthritis. The case was managed by medication and medical rehabilitation. Sparing digestive hygienic-dietary regime was recommended, with salt restriction, hypolipidemic, hypoglucidic diet, with 1600 calories/day. Drug treatment included antidiabetic, lipid-lowering, hepatoprotective, antihypertensive, antiplatelet medication, coronary vasodilators drugs. For the rheumatic disease, as NSAIDs cannot be given because of the liver disease and antiplatelet medication, adequate chondroprotective agents and local topicals containing capsaicin were chosen for therapy. Physical-kinetic therapy used different procedures with painkiller purpose (TENS), to fight against knee joint and paravertebral lumbar contracture (ultrasound with gel), daily kinetotherapy (posture, stretching) without charge, occupational therapy that teaches the patient to spare the spine and knees during daily activities, evaluating and recommending to avoid different postures and activities that involve heavy lifting or twisting of the vertebral column in everyday activities, walking assist devices. The improvements were seen after 2 weeks of treatment.

Conclusion: Individualized daily exercise therapy was very effective for improving pain and function, but also for ameliorating parameters of the other comorbidities in our patient.

\section{P532}

TREATMENT PARTICULARITIES OF OSTEOPOROSIS IN A CASE WITH MULTIPLE SCLEROSIS M.-L. Cevei ${ }^{1}$, D.-L. Stoicanescu ${ }^{2}$, A.-C. Oprea ${ }^{3}$, I.-R. Cevei ${ }^{2}$ ${ }^{1}$ University of Oradea, Faculty of Medicine and Pharmacy, Clinical Medical Rehabilitation Hospital Felix Spa, Oradea, Romania, ${ }^{2}$ University of Medicine and Pharmacy "Victor Babes", Timisoara, Romania, ${ }^{3}$ University of Oradea, Faculty of Medicine and Pharmacy, Oradea, Romania

A 56 y-old Caucasian female patient was diagnosed with osteoporosis type 1 in 2014. She presented in the Medical Rehabilitation Clinical Hospital Baile Felix, Romania with paraparetic type motor deficit, impossible orthostatism and walking, self-care deficit, neuropathic pain in legs, fatigue. Her medical history revealed surgery for herniated disc L4-L5 in 2002 and multiple sclerosis diagnosed in 2003. Upon admission, her physical examination revealed dorsal kyphosis in a patient in wheelchair with impossible orthostatism and walking, who does not actively mobilize lower limbs, marked spasticity in the lower limbs, poor coordination of upper limbs, present pyramidal syndrome, neurogenic bladder, bowel control but cannot perform the act of defecation in the toilet because the inability to walk. She was able to perform transfers with assistance from another person. DXA investigation showed decreased BMD, lumbar spine $\mathrm{T}$ score was -2.6 and hip $\mathrm{T}$ score was -2.4 . Vertebral radiography revealed vertebral compression involving four osteoporotic vertebrae. Treatment targets aimed at improving BMD, increase stability in orthostatism and sitting, increase voluntary motor control of the lower limbs, daily activities training, 
preventing and combating complications, management of fatigue, increase overall resistance to effort, prevent falls and injuries, improving quality of life. Therapeutic means: balanced diet with high calcium content; vitamin D $1000 \mathrm{mg} /$ day, physical therapy that will avoid fatigue, hydrokinetotherapy with water temperature not exceeding $34{ }^{\circ} \mathrm{C}$, occupational therapy, psychotherapy. Daily physical exercise remains the long-lasting therapeutic tool, which meets the objectives, as anti-osteoporotic medication was not administered to this patient because it cannot fulfill the condition of lack of immobilization in bed, crucial for the prevention of adverse effects. Occupational therapy will improve functional independence through environment necessary changes and application of assistive devices: wheelchair, orthotics, modified kitchen utensils.

Conclusion: Early diagnosis and intervention can decrease fracture risk in cases with multiple sclerosis and osteoporosis. Treatment should be individualized according to the complexity of the case.

\section{P533}

RECOVERY TREATMENT INFLUENCE ON THE PERIMETERS OF THE LOWER LIMBS IN REFLEX SYMPATHETIC DYSTROPHY SYNDROME

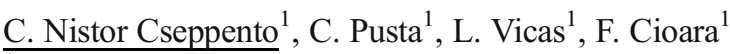
${ }^{1}$ University of Oradea, Faculty of Medicine and Pharmacy, Oradea, Romania
Introduction: The algoneurodistrophy, according to the International Association of pain, defines a regional complex syndrome, painful, chronic, without dermathomeric topography. Diagnosis supports triad vegetative symptomatology, motor and sensory.

Material and methods: We included in the study a group of 8 patients with the following configuration: 2 of them with AND knees, tibial plateau fracture, 6 patients with knee sprain,

To assess the benefits therapeutic I made three comparative measurements in the two legs, both the affected and unaffected at, used as controls, with centimeter. Measurements were made when initiating therapy recovery at 14 days and 3 months.

Results: The mean value obtained by the difference between the two states, the first review is $1.15 \mathrm{~cm}$, $0.73 \mathrm{~cm}$ second determination. The value of $0.1 \mathrm{~cm}$ 3 months.

\section{Conclusion:}

1. $27 \%$ of patients included in the study come from rural areas, the rest in urban areas.

2. Lower limb edema occurs rapidly decrease with $36.5 \%$ at 10 days of treatment and three months practically reaches normal values.

3.This is explained by the fact that patients were affected leg were in stage I, therefore treatment response is very good in that the swelling.

\title{
evalution of the average values in $\mathrm{cm}$
}

\author{
Mb. inf
}




\section{P534}

\section{MANAGEMENT APPROACH OF PAIN IN OSTEO- ?POROSIS}

M. I. Majumder ${ }^{1}$

${ }^{1}$ Comilla Medical College Talk, Comilla, Bangladesh

The term 'pain' is currently defined as an unpleasant sensory and emotional experience associated with actual or potential tissue damage. It is a complex multidimensional experience encompassing biological, emotional and cognitive factors and reflecting a psychological state. Osteoporosis is characterized by progressive decrease in bony mass that results in increased bone fragility and higher fracture risk and accounts for $0.83 \%$ of global burden of non communicable disease. About $2 / 3$ of vertebral fractures causes no symptoms and remaining typically present with acute back pain. Chronic discomfort is due to poorly healed fracture or side effects of osteoporotic medications. Almost all nonspine related fractures occur in context of trauma and present with acute pain. A series of compression fractures in the spine, rubbing of bone against bone, muscle spasms and microscopic fractures result in pain. Pain evaluation can be performed daily by VAS(visual analog scale)- 0 for no pain and 10 for agonizing pain and also by NRS (numerical rating scale). The management of patients with acute vertebral fractures or other fractures should include measures to reduce pain and improve mobility, as well as starting treatment for osteoporosis. As proposed by the World Health Organisation (WHO), according to the intensity of chronic pain, analgesic treatment can be established. For mild pain $(\mathrm{NRS} \leq 3)$ is recommend the use of NSAIDs or acetaminophen with the possibility of adding the adjuvants (Antiresorptive drugs as Bisphosphonates, steroids, antidepressants, Benzodiazepines) for moderate pain (NRS comprised equal to 4-6 and), is suggested a treatment with weak opioids integrated with or without NSAIDs or acetaminophen, and with the possibility of adding adjuvants. In case of severe pain (NRS >6), the WHO plans to undertake a more integrated opioid treatment with NSAIDs or paracetamol, with the possibility of adding adjuvant. Surgery as vertebroplasty or kyphoplasty in vertebral fracture is option in patients not relieved with medical treatment. Pain medication with orthopaedic care is necessary in nonvertebral fracture. Physical therapy and exercise helps the patient to regain muscle strength and mobility. Thus a broad, comprehensive approach is key in management of pain with biopsychosocial approach. The presentation will address management approach built upon biopsychosocial framework of osteoporosis pain.
P535

ASSOCIATION BETWEEN PREOPERATIVE SARCOPENIA AND FUNCTIONAL OUTCOME OF THE PATIENTS FOLLOWING TOTAL KNEE REPLACEMENT

V. Khanna ${ }^{1}$

${ }^{1}$ Department of Orthopaedics, National Institute of Medical Sciences, Jaipur, India

Objectives: To find any association if present between the preoperative sarcopenic status and the postoperative functional outcome in Total Knee Replacement patients. Material And Methods: One hundred male patients between the age group of 60-65 years coming to the hospital for total knee replacement were explained about the study. All the total knee replacements were done in grade 4 osteoarthritis. The anthropometric measurement was done in which the thigh muscle bulk was measured at a point $15 \mathrm{~cm}$ proximal to the lateral joint line in all the patients. In the postoperative period the functional tests (using the stair climbing and the six minute walk test) of the patient was assessed with the help of range of motion, pain and the quality of life assessment. Both these values were correlated and the results were computed.

Results: The mean age of the patient was found to be 62.93 and there was no significant variation in the age of the patient. Only male patients were included in this study. The mean diameter of the thigh was found to be $36.32 \mathrm{~cm}$. On calculating the correlation between the muscle bulk and the functional status of the patient, there was a weak positive correlation seen between the muscle bulk and the functional tests.

There was no correlation seen between the pain, range of motion and the muscle bulk.

Conclusion: In conclusion it was seen by this study that it was seen that functional outcome was better with the increase in the preoperative muscle bulk and hence, sarcopenia might be taken as a predicting factor for the functional outcome of total knee replacement; whereas, there was no effect of the muscle bulk on the functional outcome of the patient.

\section{P536}

THE EVOLUTION OF EDEMA AT THE UPPER LIMB IN SYMPATHETIC REFLEX DYSTROPHY SYNDROME

$\underline{\text { C. Nistor Cseppento }}^{1}$, F. Cioara ${ }^{1}$, C. Pusta ${ }^{1}$, R. Suciu ${ }^{1}$, D. Iovanovici $^{2}$

${ }^{1}$ University of Oradea, Faculty of Medicine and Pharmacy, Oradea, Romania, ${ }^{2}$ UMFT Victor Babes Timisoara, Timisoara, Romania 
Introduction: The etiology of algoneurodystrophy is manifold. Remembering trauma, poliomyelitis, polymyalgia rheumatica, carpal tunnel syndrome, prolonged immobilization, prolonged bed rest, stroke, ALS, cardiovascular disease, surgery. In $25 \%$ of cases is unknown etiology. Clinically diagnosed in 4 of the 5 signs: pain, swelling, skin color changes in temperature and local decreased range of motion.

Material and methods: We included in the study a group of patients with the following configuration: 2 patients with upper limb and post stroke, 5 patients with upper limb post and humerus-humeral head fracture and cervical surgery. During the study we conducted comparative measurements of the perimeters of three, of both upper limbs, at treatment initiation complex recovery, after 10 days of treatment and at 3 months.

Results: Differences averages from three measurements were $1.74 \mathrm{~cm}$, at the first measurement, the second measurement 1.25 and $0.5 \mathrm{~cm}$ at 3 months.

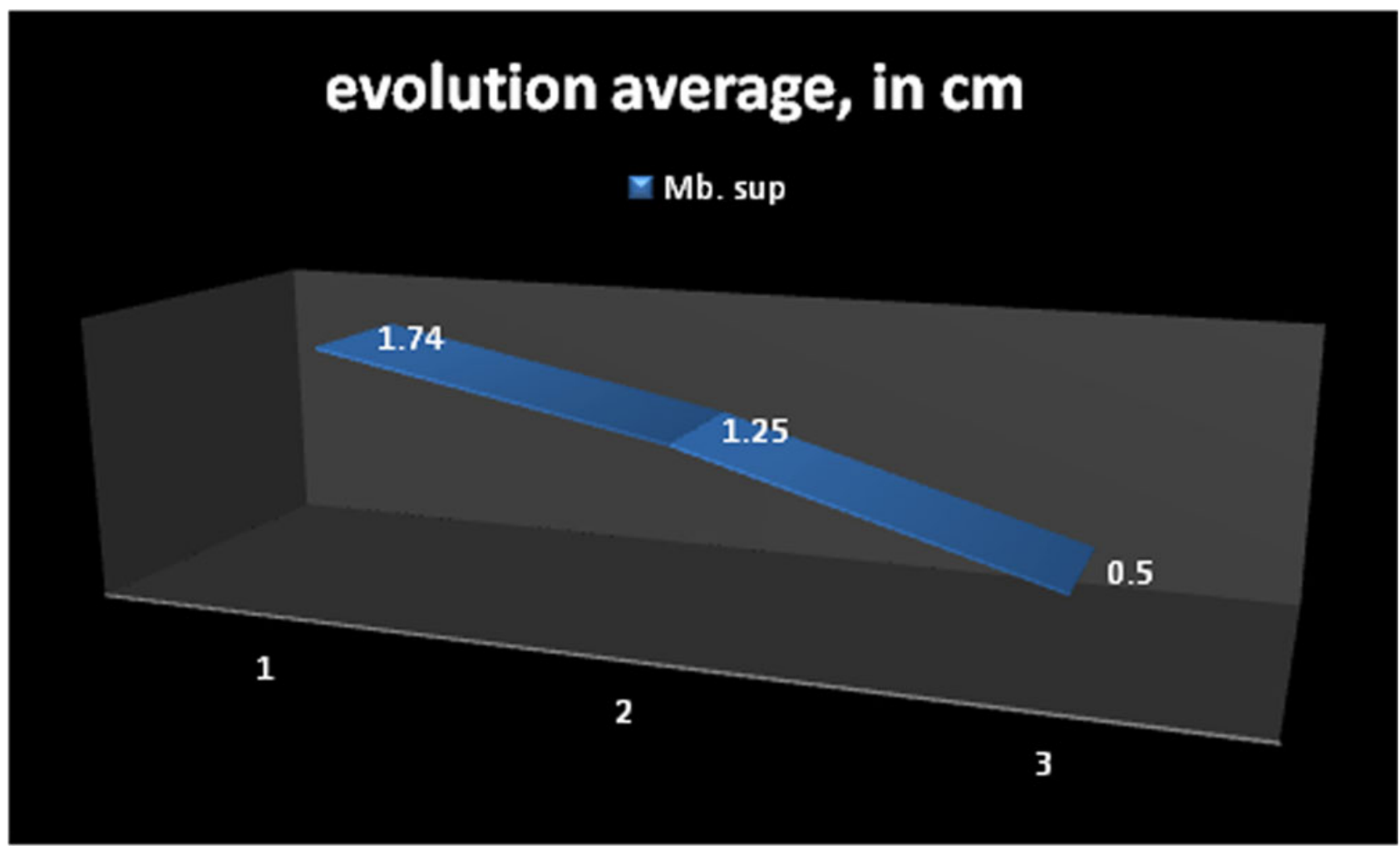

\section{Conclusions:}

1. Comparative measurements between of the both limbs, showing initial average $1.74 \mathrm{~cm}$, the second determination decreases to $1,25 \mathrm{~cm}$, following the end of the study to reach to $0.5 \mathrm{~cm}$.

2. The calculations show that the upper limb edema decreases in the first 10 days of treatment with $28 \%$, and after 3 months reaches at $60 \%$ from the second evaluation values and $71.26 \%$ compared to the first assessment.

3. The results of this study demonstrates the importance of our recovery treatment regardless of stage of disease, the more since initiating the earliest.
Reference: Algoneurodistrofia, sindromul umăr - mână, Filed in Recuperare si FizioKinetoterapie on Oct.17, 2013

\section{P537}

RECRUITMENT OF RNA TARGETS BY CONNEXIN 43 RNA-BINDING MOTIFS: NOVEL MECHANISMS OF CELLULAR COMMUNICATION

M. Varela-Eirin ${ }^{1}$, A. Varela-Vazquez ${ }^{1}$, M. Rodriguez-Candela Mateos $^{1}$, A. Vila-Sanjurjo ${ }^{2}$, B. Acea ${ }^{1}$, E. Fonseca ${ }^{1}$, M. D. Mayan ${ }^{1}$ ${ }^{1}$ CellCOM Research Group, Institute for Biomedical Research of A Coruña (INIBIC), A Coruña, Spain, ${ }^{2}$ GIBE Research Group, Facultade de Ciencias, Universidade de A Coruña, A Coruña, Spain 
Background: Connexins (Cxs) are integral membrane proteins that form plasma membrane channels, allowing cellmatrix and cell to cell communication. Initially described in joining excitable cells (nerve and muscle), gap junctions (GJs) are found joining virtually all cells in solid tissues and are essential for the functional co-ordination of organs by enabling direct transfer of small signalling molecules, metabolites, ions, and electrical signals. Several studies have revealed diverse channel-independent functions of Cxs, including control of cell growth and tumorigenicity. Chondrocytes from osteoarthritic cartilage have increased expression of connexin 43 (Cx43). The myriad roles of $\mathrm{Cx} 43$ and its implication in the development of diseases such as cancer, osteoarthritis or Alzheimer have risen to many novel questions.

Objective: The aim of this study was to investigate possible RNA binding domains in the $\mathrm{Cx} 43$ and $\mathrm{Cx} 26$ sequences in order to study if cells are able to exchange molecules of RNA anchored to $\mathrm{Cx} 43 / \mathrm{Cx} 26$ sequences present in exosomes or vesicles.

Methods: Amino acids sequences from $\mathrm{Cx} 43$ and Connexin 26 (Cx26) were obtained from the Protein Database (NCBI) and the $\mathrm{Cx} 26$ structure from the Protein Data Bank (RCSB). Protein sequences from both Cxs were aligned and compared with Protein Blast and Lalign servers. Analysis of RNAbinding propensity of $\mathrm{Cx} 43$ and $\mathrm{Cx} 26$ sequences was evaluated with three computational methods with the high predicting accuracy aaRNA, Bind + and Pprint.

Results: Protein sequences were highly conserved between $\mathrm{Cx} 43$ and $\mathrm{Cx} 26$ with the exception of the C-terminal end (CTD). The combination of the scores obtained from the three predictors showed four domains that potentially interact with RNA sequences. Two of these domains include the intracellular loop (ICL) and the CTD.

Conclusions: Our results suggest that $\mathrm{Cx} 43$ and $\mathrm{Cx} 26$ are able to interact with RNA targets including siRNA and mRNA. These interactions would have a range of potential implications for cellular communication and control of signalling pathways.

\section{P538}

THE ROLE OF THE BODY MASS INDEX IN EVALUATION OF THE OPERATIVE VASCULAR RISK IN PATIENTS WITH HIGHLY ACTIVE RHEUMATOID ARTHRITIS

V. Popova ${ }^{1}$, A. Batalov ${ }^{1}$, Z. Vazhev ${ }^{1}$, Y. Ronchev ${ }^{1}$, M. Geneva-Popova $^{1,2}$, A. Ivanov ${ }^{2}$, J. Peeva ${ }^{3}$, L. Stoyanova ${ }^{3}$, K. $\mathrm{Kraev}^{3}$, S. Terziiska ${ }^{3}$, I. Yakov ${ }^{3}$, E. Chonova ${ }^{3}$

${ }^{1}$ Department of Rheumatology, Medical university, UMHAT "Kaspela", Plovdiv, Bulgaria, ${ }^{2}$ Clinic of Cardiosurgery, Medical university, UMHAT "Sv. Georgi", Plovdiv, Bulgaria, ${ }^{3}$ Department of Public Health, Medical university, Plovdiv, Bulgaria
Introduction: The vascular operative risk in patients with rheumatoid arthritis is 1.5-2 times higher than the general population. The role of the risk factors amplify this risk proportionally and leads to more severe and prolonged postoperative period. Materials and methods: defining the role of the score of the BMI; evaluation of the operative vascular risk was made with the EuroSCORE calculator in according to the inflammatory activity of the Rheumatoid arthritis- patients from cardiac surgery were divided into three groups - with normal BMI 18.5$29.9 \mathrm{~kg} / \mathrm{m}^{2}$, overweight- BMI- $30-34.9 \mathrm{~kg} / \mathrm{m}^{2}$ and obese -BMI above $35 \mathrm{~kg} / \mathrm{m}^{2}$. The evaluation of the inflammatory activity of RA was made with DAS28 and CDAI. The accompanying risk factors were taken into account.

Results: 67 patients with rheumatoid arthritis with high activity of the disease DAS28 $>6.02$ and CDAI $>22$ were studied. From them $26 / 0.34, p<0.005 /$ had normal BMI and high operative risk EuroSCORE $>27.89 \%, 21 / 0.31, p, 0.005 /$ patients were overweight and with moderate risk - EuroScore $<10.89 \%$, the rest$10 / 15 \%, p<0.005 /$ were overweight and with high vascular risk EuroSCORE $>29.0 \%, 10$ patients $/ 15 \%, p<0.05 /$ were with normal BMI and high vascular risk- EuroSCORE $>27.89 \%$.

Conclusion: The BMI score is important for the evaluation of the operative cardiac risk in patients with inflammatory joint diseases but the risk factors, mediating the vascular risk and lethality should also be taken in account.

References:

1. Avina-Zubieta J, et al. Arthritis Rheum 2008;59:1690

2. Cavagna L, et al. Mediators Inflamm 2012;2012:147354

\section{P539}

CONTENTS OF SERUM MYOSTATIN IN TYPE 1 DIABETIC PATIENTS ASSOCIATED WITH AMOUNT OF MUSCLE MASS

A. Shepelkevich $^{1}$, Y. Dydyshka ${ }^{1}$

${ }^{1}$ Belarusian State Medical University, Minsk, Belarus

Background and aims: Myostatin primarily synthesized by skeletal muscle and a negative regulator of muscle growth. Reducing the amount of muscle mass in patients with type 1 diabetes (T1DM) may be associated with changes of serum myostatin. Therefore, the aim of study was to assess the possible association between serum myostatin with a reduction in muscle mass in T1DM.

Materials and methods: 95 patients with T1DM (60 women, 35 males) (mean age: $31.61 \pm 7.98 \mathrm{yrs,} \mathrm{duration} \mathrm{of}$ DM: $13(7-20)$ yrs, age of manifestation: $17(12-23)$ yrs, BMI: $23.41 \pm 3.04$, HbA1c: $8.3 \pm 1.014 \%$ ) and 55 (31 women, 24 men) controls. The research involved anthropometry of patients (height, weight, BMI, waist circumference), general clinic examination, glycated hemoglobin test, serum myostatin, DXA using a program "body composition". 
Results: There were revealed comparable levels of serum myostatin in patients with T1DM and control groups, respectively $589(457.26-826)$ and $675.38(491.94-750.34) \mathrm{pg} / \mathrm{mL}$, $\mathrm{U}=838 ; p=0.98)$. Men with T1DM showed a significant and high levels of myostatin (792.64 (557.03 - 972.83529 .23$)$ vs. (443.55-625.86) $\mathrm{pg} / \mathrm{mL}, \mathrm{U}=232.5 ; p=0.006$ ) compared to women that confirm the need for clinical interpretation of this indicator taking into account gender. Men and women with T1DM have a comparable content of serum myostatin with control groups: women 529.23 (455.65-625.86) vs. 604.54 (391.53-745.53)pg/ml, $\mathrm{U}=309 ; p=0.585$; men 792.64 (557.025-972.82) vs. $704.95(593.54-790.23) \mathrm{pg} / \mathrm{mL}$, $\mathrm{U}=90 ; p=0.48$, respectively. T1DM men with normal and reduced content have comparable values of serum myostatin (826 (638-905.65) vs. 750.34 (499.19-1040) pg/mL, U=53, $p=0.703)$, regardless of the content of the appendicular muscle mass.

Conclusions: The lack of significant differences in the levels of myostatin in patients with T1DM may be due to the preservation of the secretory activity of muscle mass considering younger patients.

\section{P540 \\ OLIVE-DERIVED OLEUROPEIN AS A POTENTIAL TREATMENT FOR BONE AND CARTILAGE AGE- RELATED DISORDERS}

M. Varela-Eirin ${ }^{1}$, A. Casado-Díaz ${ }^{2}$, J. M. Quesada-Gómez ${ }^{2}$, M. D. Mayan $^{1}$

${ }^{1}$ CellCOM Research Group, Institute for Biomedical Research of A Coruña (INIBIC), A Coruña, Spain, ${ }^{2}$ Clinical Management Unit of Endocrinology and Nutrition, Maimónides Biomedical Research Institute of Córdoba (IMIBIC), Hospital Universitario Reina Sofía - RETICEF, Universidad de Córdoba, Córdoba, Spain

Background: Oleuropein, a glycoside from olive, is well known to have several beneficial effects on human health. However, the mechanisms associated with its potential pharmacological properties are not clear yet. Human bone marrow mesenchymal stem cells (hMSCs) exhibit an agedependent reduction in osteogenesis and an increased propensity toward adipocyte differentiation. This switch has been associated with different bone disorders characterized by reduced bone formation and increased bone marrow fat accumulation. Connexin $43(\mathrm{Cx} 43)$ is an integral membrane protein that forms gap junction channels (GJs) and it is implicated in multiple cellular functions including cellular differentiation and control of bone and cartilage remodelling.

Objective: To study if oleuropein could act as an adipogenic suppressor in order to promote cartilage and bone regeneration through a Cx43-dependent mechanism.
Methods: hMSCs differentiation was performed in the presence of oleuropein. Cellular differentiation was evaluated using histological stains: Oil Red O, Alizarin Red, Toluidine Blue and Safranin O/ Fast Green. Scrape loading assays were used to study the cellular communication through GJs and hemichannels formed by $\mathrm{Cx} 43$. Western blot and immunohistochemistry assays were performed to study the levels of Cx43 protein.

Results: hMSCs treated with oleuropein showed a twofolded decrease in adipogenic differentiation, while osteogenesis was significantly increased. Micromasses cultured in chondrogenic medium using $10 \mu \mathrm{M}$ of oleuropein showed a more intense toluidine blue and Safranin O stain, suggesting an increase in chondrogenesis. RealTime qPCR, western blot and scrape loading assays showed changes in the levels of $\mathrm{Cx} 43$ and dye transference through GJs channels when the hMSCs were grown in the presence of oleuropein.

Conclusions: Our results suggest that oleuropein via $\mathrm{Cx} 43$ and GJs intercellular communication increases the propensity towards osteogenesis and chondrogenesis, reducing adipocyte differentiation. Our preliminary assay indicates that oleuropein may represent a potential therapeutic target for cartilage and bone age-related disorders in order to promote cartilage and bone regeneration.

\section{P541}

\section{TARGETING SIALIC ACID-MODIFIED RECEPTORS AS A POTENTIAL THERAPY FOR OSTEOAR- THRITIS}

$\underline{\text { P. Carpintero-Fernandez }}{ }^{1}$, R. Gago-Fuentes ${ }^{1}$, A. Alessandra-

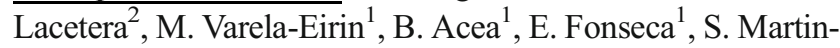
Santamaria $^{2}$, M. D. Mayan ${ }^{1}$

${ }^{1}$ CellCOM Research Group, Institute for Biomedical Research of A Coruña (INIBIC), A Coruña, Spain, ${ }^{2}$ Chemical and Physical Biology, Centro de Investigaciones Biológicas, CIB-CSIC, C/ Ramiro de Maeztu, Madrid, Spain

Background: Osteoarthritis (OA) is one of the most common diseases worldwide. Its prevalence and severity increase with age, but treatments only provide symptomatic relief. The articular cartilage consists of a collagenproteoglycan matrix containing highly glycosylated proteins synthesized by chondrocytes. A notable shift from glycoproteins containing a-2,6-linked sialic acids to those containing a-2,3-linked sialic acids has been associated with progressive cartilage degeneration and with the onset of disorders such as rheumatoid arthritis (RA) and OA. However, the pathophysiology of a-2,3-sialylation in cartilage has not yet been elucidated.

Objective: Lectins recognize specific terminal aspects of glycan chains and the Maackia amurensis seed lectin (MASL) is 
a plant lectin that can bind to sialylated glycoproteins. Here, we study the effects of the lectin MASL on chondrocytes and cartilage integrity from healthy donors, OA patients and animal models of arthritis.

Methods: Cell viability, cell adhesion and growth were performed using commercial kits. Reactive oxygen species (ROS) levels were measured by DCFH-DA and by flow cytometry. Gene expression was analyzed by qPCR. Cartilage integrity was studied by staining methods. Oligomycin and LPS were used to induce cartilage degeneration in vitro and in vivo (human cartilage and mouse model, Mus musculus $\mathrm{BALC} / \mathrm{c})$.

Results: The expression of the a-2,3-sialylated transmembrane mucin receptor podoplanin (PDPN) is induced in cartilage from osteoarthritic patients. Nanomolar concentrations of MASL protected primary chondrocytes and prevented cartilage breakdown in human tissue from OA patients ex vivo and in an animal model of OA initiated by ROS, inflammatory cytokines, and metalloproteinases. Besides, the increased levels of the $\alpha-2,3$ sialyltransferase isoforms and the corresponding increase in the levels of a-2,3-sialylated glycoproteins in osteoarthritic chondrocytes may shed mechanistic light on the pathophysiology of OA. We also provide a three-dimensional molecular model for such an interaction.

Conclusions: The ability of MASL to target a-2,3-sialylated glycoproteins such as PDPN, which is also induced during $\mathrm{OA}$ and RA, and to protect chondrocytes from insults leading to cartilage degradation might offer further possibilities for therapeutic interventions and novel arthritis treatments that may include the regulation of sialylation during acute disease stages.

\section{P542}

RHIZARTHROSIS: TREATMENT WITH GLUCOSAMINE, CHONDROITIN OR COMBINATION REGIMENS

M. G. Geneva-Popova $^{1}$, V. S. Popova ${ }^{2}$, A. Z. Batalov ${ }^{2}$, S. D. Popova $^{1}$, P. T. Selimov ${ }^{1}$

${ }^{1}$ Medical University of Plovdiv, Plovdiv, Bulgaria,

${ }^{2}$ Department of Rheumatology, Medical university, UMHAT, Plovdiv, Bulgaria

Objective: In a normal joint, cartilage covers the end of the bones and serves as a shock absorber to allow smooth, painfree movement. The thumb basal joint is a specialized saddleshaped joint that is formed by a small bone of the wrist and the first bone of the thumb. The aim of the study is to determine if the dietary supplements, glucosamine and/or chondroitin, result in reduced joint space narrowing and pain among people with rhizatrosis.

Methods: A clinical study was with 18-months follow-up. 114 participants, aged $49-84$ years, with rhizartosis were treatment to once daily: glucosamine sulfate (DONA pulv, NaturProduct) $1500 \mathrm{mg}(n=30)$, chondroitin sulfate (Biovea,UK) $800 \mathrm{mg}(n=34)$, both dietary supplements (Chondrosave, Ecopharm) $(n=36)$ and 14 patients weren't treated. Joint space narrowing $(\mathrm{mm})$ was measured from radiographs. Joint pain $(0-10)$ was self-reported in a patients.

Results: After adjusting for factors associated with structural disease progression (gender, baseline structural disease severity and Heberden's nodes), allocation to the dietary supplement combination resulted in a statistically significant ( $p=0.051)$ reduction of 18 months compared to placebo. All groups demonstrated reduced hand pain over the first year, but no significant between-group differences $(p=0.71)$ were detected.

Conclusions: Allocation to the glucosamine-chondroitin combination resulted in a statistically significant reduction in joint space narrowing at 18 months in patients with rhizarthrosis. While all groups demonstrated reduced hand pain over the study period, none of the treatment groups demonstrated significant symptomatic benefit above nontreatment patients.

\section{References:}

1. Felson DT. Arthritis Res Therapy 2009;11(1, article 203)

2. Hochberg MC, et al. Arthritis Care Res 2012;64:465.

3. Gabay C, et al. Arthritis Rheum 2011;63:3383.

\section{P543}

THE POSITIVE EFFECT OF HORMONE REPLACEMENT THERAPY ON BONE MINERAL DENSITY AND TRABECULAR BONE SCORE PERSISTS AFTER ITS WITHDRAWAL: THE OSTEOLAUS COHORT

G. Papadakis ${ }^{1}$, D. H. Hans ${ }^{2}$, P. Vollenweider ${ }^{3}$, G. Waeber ${ }^{3}$, M. Preisig $^{4}$, P. Marques-Vidal ${ }^{3}$, O. Lamy ${ }^{2}$

${ }^{1}$ Department of Medicine, Division of Endocrinology, Lausanne University Hospital Switzerland, Lausanne, Switzerland, ${ }^{2}$ Center of Bone Diseases, Lausanne University Hospital Switzerland, Lausanne, Switzerland, ${ }^{3}$ Department of Medicine, Division of Internal Medicine, Lausanne University Hospital Switzerland, Lausanne, Switzerland, ${ }^{4}$ Department of Psychiatry, Lausanne University Hospital Switzerland, Lausanne, Switzerland

Objectives: We aimed to explore the effect of hormone replacement therapy (HRT) on the main surrogate markers of bone strength: BMD and bone texture, assessed by TBS. It is well established that HRT increases BMD but controversy exists regarding residual effect after withdrawal. No trial has explored the effect of HRT on TBS.

Material and Methods: The Osteolaus cross-sectional population-based study includes 1500 women aged 50 to 80 years 
(Lausanne, Switzerland). Current or past HRT use was assessed by questionnaire along with other factors affecting bone health. BMD at lumbar spine (LS), femoral neck (FN) and total hip (TH), and TBS were measured. Exclusion criteria included current or past anti-osteoporotic treatments other than HRT and history of hormone modifying treatments. The remaining 1279 women were divided in 3 groups based on HRT status: Never (NU, $n=617$ ), Current (CU, $n=282)$ and Past (PU, $n=380$ ) Users.

Results: The 3 groups differed in age: $67.4 \pm 6.8,64.0$ \pm 6.8 and $62.1 \pm 8.0$ years for PU, CU and NU respectively $(p<0.001)$ but not in BMI $(p=0.084)$ nor in dietary calcium intake $(p=0.102)$. Age and BMI-adjusted FRAX $^{\circledR}$ for major osteoporotic fractures was $12.3 \%$ in NU, $11.5 \%$ in PU and $10.5 \%$ in CU (CU vs. NU, $p<0.001$.). After adjustment for age and BMI, there was a significant decrease for all BMD and TBS values according to HRT status ( $\mathrm{CU}>\mathrm{PU}>\mathrm{NU}, p<0.01)$. The between-groups difference was significant for all BMD LS and TH values. The between-groups difference was significant for BMD FN and TBS when CU were compared to PU or NU (PU vs NU for TBS: $p=0.066$ ). After adjusting for BMI, TBS was negatively associated with age: slopes and (95\% confidence interval) for a 10-year increment were $-0.051(-0.060 ;-0.041),-0.032(-0.048$; $-0.017)$ and $-0.022(-0.038 ;-0.005)$ in NU, PU and CU respectively, with differing slopes between groups (CU vs. NU, $p=0.003$. PU vs. NU, $p=0.048$ ). Adjusted slopes for association of BMD with age were all significantly lower according to HRT status ( $p<0.015, \mathrm{CU}<\mathrm{PU}<\mathrm{NU})$, except for the comparison for FN between PU and NU (ns).

Conclusion: Our results show for the first time that current HRT use is associated with a significantly better preservation of the bone microarchitecture assessed by TBS compared to NU or PU, in addition to higher BMD values. The benefits of HRT use for the TBS and the BMD at LS and TH, seem to persist in PU.

\section{P544 \\ SEVERE OSTEOPOROSIS AND MULTIPLE FRACTURES IN AN ICHTHYOSIS CASE}

D.-L. Stoicanescu ${ }^{1}$, M.-L. Cevei ${ }^{2}$

${ }^{1}$ University of Medicine and Pharmacy "Victor Babes", Timisoara, Romania, ${ }^{2}$ University of Oradea, Faculty of Medicine and Pharmacy, Oradea, Romania

Ichthyosis comprises a very heterogeneous group of skin disorders, characterized by excessive amounts of dry scales. Inherited ichthyosis occur due to mutations in different genes and are classified in five types with different inheritance patterns. The disease may be complicated by low levels of vitamin D. We present the case of a Caucasian man aged 59 years, height $1.68 \mathrm{~m}$, weight $68 \mathrm{~kg}$, diagnosed with inherited ichthyosis, X-linked form. His past medical history revealed left humeral neck fracture with displacement operated with osteosynthesis material in 2013, comminuted fracture of the right distal radial epiphysis operated in 2014. He was diagnosed with duodenal ulcer in 2012, with osteoporosis (hip T-score -2.6, lumbar spine T-score -3.3) in 2014 and with hip osteoarthritis in 2015. His family history was negative for ichthyosis, but positive for osteoporosis, as he had a maternal grandmother with osteoporosis. He did not use vitamin D supplements. His physical examination revealed the following abnormal data: brownish scales over the whole body, also present in the flexor areas, sparing the palms and soles; mild dorsal kyphosis limiting the mobility of the cervical and lumbar spine in all axes, right fist with limited mobility; limited coxofemoral joint mobility, right gluteus medius muscle with $3+$ value at muscle testing. VAS value was 7. Radiological examination revealed arthritic changes of both coxofemoral joints and four dorsal vertebrae compression. Laboratory tests at presentation revealed vitamin D deficiency, thyroid and PTH were within normal ranges. Conclusions: For several reasons ichthyosis patients avoid sun exposure, which causes vitamin D deficit. Our patient is young with severe osteoporosis and multiple fragility fractures. Correct treatment and follow-up of the patient is very important to prevent other complications.

\section{P545 \\ SARCOPENIA PARAMETERS AND INCIDENCE OF RECURRENT FALLS AND FRACTURES}

L. A. Schaap $^{1}$, N. M. van Schoor ${ }^{2}$, P. Lips ${ }^{2}$, M. Visser ${ }^{1}$

${ }^{1}$ Department of Health Sciences, Faculty of Earth and Life Sciences, Vrije Universiteit, Amsterdam, Netherlands, ${ }^{2}$ Department of Epidemiology and Biostatistics, VU University Medical Center, Amsterdam, Netherlands

The aim of our study was to examine and the association between individual sarcopenia parameters, according to the EWGSO and the FNIH criteria, and incidence of recurrent falls and fractures in older persons. We investigated the associations of individual sarcopenia parameters (low muscle mass (DXA), low grip strength and slow gait speed) with incidence of recurrent falls (at least two falls within six months, 3-year follow-up) and fractures (10-year follow-up) in 498 older men and women (mean age 75.2 (SD 6.4)) from the Longitudinal Aging Study Amsterdam (LASA). Different cutoff values for the parameters based on the EWGSOP and FNIH were compared. One hundred and thirty persons were considered a recurrent faller and 60 persons experienced a fracture during follow-up. Cox regression analyses, adjusting for age, sex and total body fat, using the EWGSOP definition, revealed that low grip strength 
was associated with recurrent falls (Hazard Ratio (HR) 1.60 (95\% CI 1.09-2.37)). Low muscle mass and slow walking speed were not associated with recurrent falls. None of the parameters were associated with incidence of fractures. When using the FNIH definition, both low grip strength and slow gait speed were associated with recurrent falls (HR 1.60 (1.092.37) and HR 1.47 (1.00-2.17), respectively). Low muscle mass was not associated with falls. None of the parameters were associated with incidence of fractures.

Additional analyses combining all parameters in one model revealed that low grip strength is associated with recurrent falls, independent of muscle mass or gait speed. The results suggest that differences in operationalization of sarcopenia parameters result in different predictive validity regarding recurrent falls and fractures. Moreover, only low grip strength seems to be clinically useful in terms of risk identification. More studies are needed to demonstrate the clinical usefulness of sarcopenia definitions in terms of risk identification and subsequent strategies for prevention and/or treatment of important health outcomes in geriatric medicine.

\section{P546}

DEVELOPING A COMPLEX INTERVENTION FOR PATIENTS WITH OSTEOARTHRITIS WHO HAVE LONG-TERM PAIN AFTER TOTAL KNEE REPLACEMENT

$\underline{\text { R. Gooberman-Hill }}^{1}$, V. Wylde ${ }^{1}$, N. Howells ${ }^{2}$, F. MacKichan ${ }^{3}$,

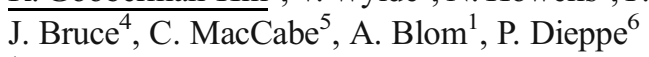

${ }^{1}$ Musculoskeletal Research Unit, School of Clinical Sciences, University of Bristol, Bristol, United Kingdom, ${ }^{2}$ North Bristol NHS Trust, Bristol, United Kingdom, ${ }^{3}$ School of Social and Community Medicine, University of Bristol, Bristol, United Kingdom, ${ }^{4}$ Warwick University, Coventry, United Kingdom, ${ }^{5}$ University of West of England, Bristol, United Kingdom, ${ }^{6}$ University of Exeter, Exeter, United Kingdom

Objective: To refine an assessment protocol and care pathway for patients with osteoarthritis who have long-term pain after knee replacement.

Methods: Healthcare professionals with experience of caring for patients with long-term pain after knee replacement completed a questionnaire to rate the appropriateness of different aspects of the care pathway from 1-9 (not appropriate to very appropriate). Data were collated and anonymised mean appropriateness ratings and summaries of free-text comments discussed in 4 facilitated meetings with professionals in the future trial centres (Bristol, Cardiff, Exeter and Oxford). The final stage of this study will involve the summary report and revised care pathway being sent to participants for further comments.
Results: 28 professionals completed the questionnaire and/ or attended a meeting. Participants included surgeons, physiotherapists, nurses, pain specialists and rheumatologists. Mean appropriateness scores ranged from 6.9 to 8.4. Taking a score of 7-9 as agreement, consensus was achieved that the assessment should be performed at 3 months post-operative by an extended scope practitioner/nurse, treatment be guided by a standardised assessment of pain, and treatment individualised. There was also agreement that referrals in the care pathway to surgical review, GP and pain clinics were appropriate. Nurseled/self-monitoring was rated lower (6.9) because of concerns over ensuring that patients receive appropriate support and are followed-up and referred to other services if needed.

Conclusion: The next stage of this intervention development work will be to assess the acceptability and reliability of the assessment process, and the usability of the intervention standard operating procedures. The intervention will then be evaluated in a multi-centre randomised controlled trial, beginning in Autumn 2016.

Acknowledgement: This abstract summarises independent research funded by the National Institute for Health Research (NIHR) under its Programme Grants for Applied Research programme (RP-PG-0613-20001). The views expressed in this abstract are those of the authors and not necessarily those of the NHS, the NIHR or the Department of Health. The STAR research team acknowledge the support of the NIHR, through the Clinical Research Network.

\section{P547}

INTERVENTIONS FOR THE MANAGEMENT OF LONG-TERM POST-SURGICAL PAIN AFTER TOTAL KNEE REPLACEMENT: A SYSTEMATIC REVIEW OF RANDOMISED CONTROLLED TRIALS $\underline{\text { R. Gooberman-Hill }}^{1}$, A. Beswick ${ }^{1}$, V. Wylde ${ }^{1}$, J. Dennis ${ }^{1}$, N. Howells $^{2}$

${ }^{1}$ Musculoskeletal Research Unit, School of Clinical Sciences, University of Bristol, Bristol, United Kingdom, ${ }^{2}$ North Bristol NHS Trust, Bristol, United Kingdom

Objective: Total knee replacement (TKR) is usually conducted to relieve pain and improve function in osteoarthritis. However, around $10-34 \%$ of people who have TKR report moderate to severe long-term post-surgical pain. We synthesised evidence on the effectiveness of pain management strategies for patients with long-term pain after TKR. Also, as pain occurs after other types of surgery, we identified evaluations of interventions for longterm pain after other surgeries. 
Material and Methods: We conducted a systematic review of randomised controlled trials (RCT) that evaluated interventions in patients with long-term pain after TKR. MEDLINE, Embase and Cochrane library were searched to September 2015. We also conducted a scoping search in other types of surgery.

Results: Searches identified one RCT evaluating an intervention in patients with long-term pain after TKR. Botulinum toxin A injection provided about 40 days of meaningful pain relief. The authors described the need for a further large trial of repeated injections. Regarding other surgeries, over 13,000 articles were screened and 36 evaluations of interventions were identified for pain management after back surgery ( 9 studies), amputation (12 studies), sternotomy (1 study), thoracotomy (1 study), cancer surgery (6 studies), hernia repair (3 studies), and spinal surgery (4 studies). Interventions focused on oral drug therapy (16 studies), injected drugs (3 studies), topical drugs (2 studies), acupuncture (1 study), exercise (1 study), epidural or spinal blocks (12 studies), or magnetic stimulation (1 study).

Conclusions: Our review highlights the lack of robust evidence about management strategies for long-term pain after TKR. While treatments identified in other surgeries may have relevance to long-term pain after TKR, no trials of multidisciplinary interventions or individualised treatments were identified. Future interventions should consider the multifactorial nature of pain after TKR.

Acknowledgement: This abstract summarises independent research funded by the National Institute for Health Research (NIHR) under its Programme Grants for Applied Research programme (RP-PG-0613-20001). The views expressed in this abstract are those of the authors and not necessarily those of the NHS, the NIHR or the Department of Health.

\section{P548}

HYDROXYCHLOROQUINE (RESOCHINE) TREATMENT IN PATIENTS WITH HAND OSTEOARTHRITIS

M. G. Geneva-Popova ${ }^{1}$, A. Z. Batalov ${ }^{2}$, V. S. Popova ${ }^{2}$, S. D.

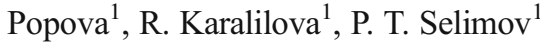

${ }^{1}$ Medical University of Plovdiv, Plovdiv, Bulgaria,

${ }^{2}$ Department of Rheumatology, Medical university, UMHAT, Plovdiv, Bulgaria

Objective: Osteoarthritis is the most common type of degenerative arthritis, causing significant joint pain and disability. It is already a major cause of healthcare expenditure and its incidence will further increase with the ageing population. Current treatments for OA have major limitations and new analgesic treatments are needed.
$\mathrm{OA}$ is associated with severe pain. Hydroxychloroquine is used in routine practice for treating synovitis in patients with rheumatoid arthritis or other rheumatoid diseases.

Aim: To determine if hydroxychloroquine can be used for treatment in patients with hand osteoarthritis.

Methods: A clinical study was with 12-months follow-up. 32 participants, aged 54-83 years, with hand osteoarthritis were treatment once daily with hydroxychloroquine (Resochin $250 \mathrm{mg}$ ) and the control group included 15 patients without treatment. All patients were with symptomatic, radiographic hand osteoarthritis and inadequate response or toxicity to the existing medication (to include paracetamol, oral NSAID or opioid). Vital signs, height and weight will be performed at the beginning. Physical examination included an examination of both hands to document whether any finger joints are painful, swollen or tender.

Results: The primary outcome was a decrease in hand pain rated on a visual analogue scale for pain. Secondary outcomes included change in score on the 10point Arthritis Impact Measurement Scales 2 (AIMS2-SF) (Table 1).

Table. Mean Difference in Measures from beginning to 12 months

$\begin{array}{llll}\text { Outcome Measure } & \begin{array}{c}\text { Hydroxychloroquine } \\ \text { (Resochin 250 mg) } \\ \text { Group }(n=\mathbf{3 2})\end{array} & \begin{array}{c}\text { Control } \\ \text { group } \\ (n=15)\end{array} & \text { P Value } \\ \text { VAS - Pain, mm } & -22 \mathrm{~mm} & -5 \mathrm{~mm} & \mathrm{P}<0.05 \\ \text { Total AIMS2-SF } & -0.3986 & -0.026 & \mathrm{P}<0.01\end{array}$

Conclusions: There were a significant differences in pain scores and AIMS-SF2 scores between the two groups at any time point. Hydroxychloroquine is an effective analgesic treatment for patients with hand osteoarthritis. The time course for symptomatic relief with hydroxychloroquine is at 12 months in patients with hand osteoarthritis. Hydroxychloroquine has an excellent safety profile, with toxicity generally associated with sustained periods of use.

\section{References:}

1. Pereira D, et al. Osteoarthritis Cartilage 2011;19:1270.

2. Hill S, et al. Disabil Rehabil 2011;33:1866.

3. Myers HL, et al. BMC Musculoskelet Disord 2011;12:3.

4. Sellam J, Berenbaum F. Nat Rev Rheumatol 2010;6:625.

5. Sellam JH-BG, Berenbaum F. In: EULAR Compendium on Rheumatic Diseases. Bijlsma JWJ BG, de Silva JA P, Faarvang KL, Hachulla E, Mariette X, editor. London: BMJ Publishing Group; 2009. Osteoarthritis: pathogenesis, clinical aspects and diagnosis; pp. 444-463. 


\section{P549}

PATHOPHYSIOLOGICAL CONSEQUENCES AND EFFECTS OF 25-HYDROXYVITAMIN D DEFICIENCY

R. Amir $^{1}$

${ }^{1}$ Centre de Santé des Fagnes, Chimay, Belgium

Aims: To synthesize many effects in different areas of significant 25-hydroxyvitamin D [25(OH)D] deficiency by reviewing different articles. Knowledge about vitamin D has greatly improved. Its role in the prevention of some osteoporotic fractures in the elderly with calcium nutrition is now well recognized and many studies argue for a role in the prevention of several diseases or anomalies (cancer, auto-immunes diseases, cardiovascular events, sarcopenia, etc.). While in some areas, a consensus seems to emerge, other issues still require a complementary research in order to have an impact on practice. Recent studies ask many questions concerning the reference values of serum $25(\mathrm{OH}) \mathrm{D}$ level. It seems that the best representative reference value below witch vitamin D insufficiency can be present, is between 30 $44 \mathrm{ng} / \mathrm{ml}$, with a clear tendency to target values above $30 \mathrm{ng} / \mathrm{ml}$ (75 pmol/l).

Methods: The analysis of data reported by several studies may be expressed by these preliminary acquisitions. Most pre and postmenopausal women had low vitamin D status with increased risk of musculoskeletal effects ${ }^{(1)}$. A low level of $25(\mathrm{OH}) \mathrm{D}$ is an independent risk factor for cardiovascular events and for sudden cardiac death ${ }^{(2)}$. Despite this epidemiological evidence, whether vitamin D screening and supplementation reduce cardiovascular risk is still matter of debate ${ }^{(3)}$. It has been suggested that in patients without coronary artery disease, the coronary flow reserve was strictly and directly related to the circulating levels of the active form of vitamin D.

Results: Several recent studies have established that a significant $25(\mathrm{OH}) \mathrm{D}$ deficiency is often associated to the diagnosis of breast and prostate cancer. A recent Saudi study where the breast cancer has a higher incidence by comparison to several European countries, linked to the absence of sun exposition in many women, had clearly confirm the presence of most aggressive breast cancer, by comparison to women not concerned by vitamin $\mathrm{D}$ deficiency, as in other countries. But as in many other situations, the effect of preventive action is not yet clearly demonstrated. Vitamin D deficiency has been associated or implicated with the pathophysiology of the gastrointestinal conditions inflammatory bowel disease and colorectal cancer as well as with depression and irritable bowel syndrome ${ }^{(4)}$.

\section{References:}

1. Amir R. Osteoporos Int 2013;24(suppl 4):170.
2. Lavie CJ, et al. J Am Coll Cardiol 2011;58:1547.

3. Elamin MB, et al. J Clin Endocrinol Metab 2001;96:1931

4. Tazzyman S, et al. BMJ Gast 2015;2:000052.

\section{P550}

OSTEOPOROSIS IN PRE- AND POSTMENOPAUSAL RHEUMATOID ARTHRITIS PATIENTS

O. Garmish $^{1}$, A. Romanovskiy ${ }^{1}$, V. Levchenko ${ }^{1}, \mathrm{~T}$.

Gavrilenko ${ }^{1}$

${ }^{1}$ Institute of Cardiology named by M.D. Strazhesko, Kiev, Ukraine

Objective: To compare BMD and bone turnover markers in pre- and postmenopausal rheumatoid arthritis patients. Material and methods: The study was performed on 106 women with RA (ACR criteria 1987), mean age was $43.6 \pm 1.7$ years and mean disease duration was 8.2 \pm 0.8 years. $91.6 \%$ have moderate/high disease activities by DAS 28. $63.3 \%$ received GC more than 3 month. 43 (40.2\%) patients were in menopause.Osteoporosis was assessed measuring BMD in 3 part of the skeleton: hip, lumbar spine, distal part of forearm. Serum $25(\mathrm{OH})$ vitamin D, PTH, osteoprotegerin (OPG), RANKL was analysed.

Results: Patients were divided in two groups by menopause (premenopausal, mean age $57.1 \pm 0.9$ and postmenopausal, mean age $36.7 \pm 1.5)$. Osteoporosis was present in $22(51.2 \%)$ of postmenopausal (PsM) RA patients, osteopenia in $15(23.8 \%)$ and normal in 5 $(11.6 \%)$. In premenopausal (PrM) group in $6(9.5 \%)$, $15(23.8 \%), 42(66.7 \%)$ patients respectively. Vitamin D deficiency was observed only in patient with osteoporosis in both group $(p<0.001)$. The mean level of PTH was normal in all subgroups. In PsM group serum OPG level was significantly higher in osteoporosis (5.9 vs. 3.6, $p<0.001)$. In PrM group all patients have high level of OPG (5.2). It was found negative correlation between BMD in distal part of forearm and PTH level $(r=-0.3, p=0.005)$, and OPG level $(r=-0.61, p<0.01)$; and also between BMD in lumbar spine and RANKL $(r=-0.35, p<0.01)$ and $\mathrm{OPG}(r=-0.32, p<0.01)$. There are no differences was found in BMD in PsM group according to glucocorticoids intake. In PrM group BMD in the hip was found to be significantly lower in patients with glucocorticoid intake $(-0.87$ vs. 0.2$)$, 3 of them have low energy hip fracture.

Conclusion: Osteoporosis is much more frequently in postmenopausal vs. premenopausal RA woman. Vitamin D deficiency has strong predictive value for the osteoporosis risk in both groups. GC intake has predictive value only in premenopausal patients in the hip. Osteoporosis risk was strong 
associated with disease duration and no association with GC intake in dose $<10 \mathrm{mg} /$ day.

\section{P551}

NEED TO INTEGRATE ORAL HEALTH ASSESSMENT WITH COMPREHENSIVE GERIATRIC ASESSMENT

S. Gupta $^{1}$, A. Gupta ${ }^{2}$

${ }^{1}$ Morriston Hospital, Swansea, United Kingdom, ${ }^{2}$ Glangwili Hospital, Carmarthen, United Kingdom

Objective: Good oral health is important for well-being especially in the elderly. Osteonecrosis of Jaw is rare side effect of bisphosphonates and Denosumab. Patients need adequate dental precautions and appropriate advice from doctors and dentists to reduce the chance of this condition. Aim of the study was to assess the dental health of patients with osteoporotic fractures receiving bisphosphonate or denosumab treatments.

Methods: Single person administered Questionnaire study. Subjects- Patients who had suffered fragility fracture and were receiving Bisphosphonate or Denosumab treatment. Setting- Teaching hospital in United Kingdom. Analysis- Responses were summarised and percentages calculated.

Results: Age range 65-94 years Females- $74 \%$, Number of patients studied- $100.66 \%$ patients were registered with a dentist. Reasons mentioned for those not registered were: not found necessary (30\%), cost (35\%) availability $(35 \%) .68 \%$ visited their dentist twice or more within last 2 years. $64 \%$ brushed their teeth daily. $18 \%$ had full or partial dentures. $74 \%$ reported loose or sensitive teeth. Self-reported dental health rating was: food $32 \%$, average $25 \%$ and bad $43 \%$. $23 \%$ had some dental procedure done within last 2 years. $55 \%$ reported dental/gum infections in last 2 years. $54 \%$ reported medical/dental health professional informing side effects and precautions associated with osteoporosis drugs. $10 \%$ reported having discussion about BRONJ. $52 \%$ had dental checkup done prior to initiating osteoporosis treatment.

Conclusions: This large study shows poor dental health is common amongst patients receiving osteoporosis treatments. There is a need to monitor, improve and maintain good oral health both by medical and dental professionals. All patients need information, education and advice about risks and benefits associated with osteoporosis therapies. Further large studies are needed to develop and validate a useful and practical Oral health assessment tool. We recommend that 'oral health risk assessment' should be integrated with 'comprehensive Geriatric assessment tools' for elderly patients.

\section{P552}

\section{DENTAL HEALTH RISK ASSESSMENT AMONGST BISPHOSPHONATE USERS}

S. Gupta ${ }^{1}$, A. Gupta ${ }^{2}$

${ }^{1}$ Morriston Hospital, Swansea, United Kingdom, ${ }^{2}$ Glangwili Hospital, Carmarthen, United Kingdom

Objective: With increasing usage of bisphosphonates and increasing longevity, many elderly patients are on long term bisphosphonates and may require some dental procedure. We wanted to determine the dental risk status based on Scottish guidance for Dental practitioners 2011.

Methods: An interviewer recorded information needed to classify risk status amongst a randomly selected database of bisphosphonate users. Setting- Teaching hospital in United Kingdom.

Results: age range 65-94 years, total number studied $=100.74 \%$ were females. $78 \%$ users were at low risk (taking bisphosphonates for the prevention/ management of osteoporosis. $22 \%$ patients were at higher risk(previous diagnosis of BRONJ, taking a bisphosphonate as part of the management of a malignant condition, Paget's disease, osteogenesis imperfecta, systemic corticosteroids, immunosuppressants, coagulopathy, chemotherapy or radiotherapy) for development of rare condition osteonecrosis of jaw.

Conclusions: This study shows that amongst Bisphosphonate users, a significant proportion of elderly are at high risk of developing dental complications following invasive dental procedure. Risk assessment should be performed and documented as a routine in these patients. If any extraction or invasive dental procedure is deemed necessary then especially amongst high risk patients it should be carried out before commencement of bisphosphonate therapy.

\section{P553}

DEMENTIA AND HIP FRACTURES- IS THERE A CARE GAP?

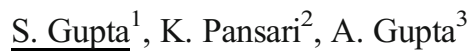

${ }^{1}$ Psychiatry, Birmingham, United Kingdom, ${ }^{2}$ Psychiatry, Hywel Dda, Carmarthen, United Kingdom, ${ }^{3}$ Medicine, Hywel dda, Carmarthen, United Kingdom

Objective: From April 2012, pre- and post-operative cognitive assessment amongst hip fracture patients is one of the criteria for enhanced payment to hospitals in England in UK. Aim of the study was to assess the prevalence of dementia amongst hip fractures and assessments from specialist mental health services. 
Methods: Prospective study. Setting - acute hip unit at a UK hospital in Wales(not England) Subjects- patients admitted following hip fractures. A single researcher collected data on the prevalence of dementia and mild cognitive impairment (MCI) amongst hip fracture patients after their surgery just before discharge using MMSE and 6CIT cognitive assessments scales.

Results: Age range 65 to 94 years $70 \%$ females, total number of study population $=80$. The prevalence of dementia amongst older hip fracture patients was $26 \%$. The prevalence of mild cognitive impairment was $42 \%$. No significant differences in the prevalence of dementia or cognitive impairment was noted between the sexes. Patients from long-term care setting were significantly more likely to have dementia ( $49 \%$ ) compared to those admitted from the community $(18 \%) .40 \%$ of patients had a documented past history of dementia and $25 \%$ of these were on dementia medications. Despite access to specialist mental health services, only $15 \%$ patients were referred/assessed by specialist Mental Liaison team.

Conclusions: A significant proportion of elderly hip fracture patients have cognitive impairment and dementia, many are previously undiagnosed. These patients would benefit from further detailed assessment from LIAISON Psychiatry team following initial diagnosis. Our findings suggest need for improved staff awareness and training to detect dementia early, to ensure that hospitals have a care pathway in place for dementia that fits with existing acute care pathways. Improved diagnosis and management for this high risk group has potential implications in reducing length of stay not only in acute hospital but also the overall burden of dementia after discharge.

\section{P554}

TOWARDS AN INTEGRATED PROACTIVE APPROACH FOR DEMENTIA AND HIP FRACTURE CARE

S. Gupta ${ }^{1}$, K. Pansari ${ }^{2}$, A. Gupta ${ }^{3}$

${ }^{1}$ Psychiatry, Birmingham, United Kingdom, ${ }^{2}$ Psychiatry, Hywel Dda, Carmarthen, United Kingdom, ${ }^{3}$ Medicine, Carmarthen, United Kingdom

Objective: From April 2012, pre- and post-operative cognitive assessment amongst hip fracture patients is one of the criteria for enhanced payment to hospitals in England in UK. Aim of the study was to assess the association between dementia and hip fractures.

Methods: Setting- Acute hip unit at a UK hospital in Wales (not England) Subjects- patients admitted following hip fractures. A single researcher collected data on the prevalence of dementia based on AMTS amongst hip fracture patients from medical records.

Results: Age range 65 to 94 years $70 \%$ females, total number of study population $=130.20$ patients did not have any mental assessments done. The prevalence of dementia amongst 110 hip fracture patients was $26 \%$. Patients from long-term care setting were significantly more likely to have dementia (49\%) compared to those admitted from the community (18\%). $40 \%$ of dementia patients had a documented past history of dementia. Conclusions: Fracture patients have a high prevalence of dementia and cognitive impairment. Many are previously undiagnosed, do not routinely receive cognitive assessment. This is a missed opportunity for the early diagnosis and providing integrated care for patients suffering with dementia. There is a need for goodquality early diagnosis and intervention for all suspected dementia patients- through specialist assessment that delivers rapid assessment, accurate diagnosis and treatment, care and support.

\section{P555}

PSYCHIATRIC ILLNESS IN HIP FRACTURE PATIENTS

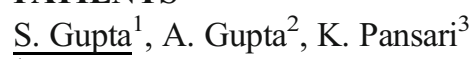

${ }^{1}$ Psychiatry, Birmingham, United Kingdom, ${ }^{2}$ Medicine, Glangwili, Carmarthen, United Kingdom, ${ }^{3}$ Psychiatry, Hywel Dda, Carmarthen, United Kingdom

Hip fracture occurs in elderly and many patients have comorbid psychiatric conditions. We assessed the prevalence of psychiatric comorbidity such as Dementia depression or other psychiatric illnesses in hip fracture patients following their surgery and their impact on outcome.

Method: Prospective data collection Setting- acute hip unit in UK hospital Subjects- patients with hip fracture. Measurement scales used were for assessment of dementia (6CIT) delirium (4AT) and depression (short GDS). Outcomes studied were hospital length of stay.

Results: Age range 65-94 years. $70 \%$ females, total number of study population $=130$ The prevalence of conditions as assessed 5 days following surgery was- dementia $20 \%$ depression $35 \%$ delirium $54 \%$.only $10 \%$ patients had known diagnosis of dementia or depression in the past. As compared with patients without these conditions (number $=67$ ), patients with psychiatric comorbidities stayed in acute hospital 8 days more.

Conclusions: Psychiatric illness including dementia, delirium and depression is common after hip fracture, and has significant impact on duration of hospital stay. Our study suggests a need for routine screening for psychiatric illness after hip fracture, followed by the investigation /treatment/ psychiatric referral to improve the quality and outcomes of this high risk group. 


\section{P556}

\section{DETERMINANTS OF PERFORMANCE-BASED FUNCTION IN WOMEN WITH KNEE OSTEOARTHRITIS}

H. Nur ${ }^{1}$, B. Sertkaya ${ }^{1}$, T. Tuncer ${ }^{1}$

${ }^{1}$ Department of Physical Medicine and Rehabilitation, Akdeniz University, Faculty of Medicine, Antalya, Turkey

Objective: In this study we aimed to explore the impacts of a wide variety of demographic, clinical, neuromuscular, and psychological factors on physical function in women with knee OA

Material and Methods: One hundred and ten women with knee OA participated in this study. Physical performance tests included a 10 meter walk test, timed up and go (TUG) test, and a stair test. Muscle strength was evaluated using an isokinetic dynamometer. Knee pain was measured with the visual analog scale. Age, disease duration, BMI, comorbities were recorded. Hospital anxiety and depression (HAD) scale was used to assess anxious and depressive symptoms. The knee flexion range of motion (ROM) was measured. Radiographs were analyzed according to Kellgren-Lawrence grading scale. Regression analyses were used to assess the associations.

Results: Muscle weakness, diminished knee flexion ROM, pain intensity, and older age were significantly associated with the duration of time performing the 10 meter walk test, TUG test, and the stair test. BMI, disease duration, comorbidities, radiologic severity, anxiety and depression scores did not affect physical performance tests in regression models.

Conclusion: In women with knee OA muscle weakness, lower knee flexion ROM, pain intensity, and older age are related to decreased performance-based function. Rehabilitative approaches maintaining improvement in muscle strength, diminished ROM, and pain intensity must be applied to prevent disability in the management of women with knee OA.

\section{P557 \\ DOUBLE ROW SUTURE ANCHOR FIXATION OF POSTERIOR CRUCIATE BONY AVULSION: A NEW TECHNIQUE WITH REPORT OF TWO CASES \\ K. K. V. Acharya ${ }^{1}$, V. Pandey ${ }^{1}$ \\ ${ }^{1}$ Arthroscopy \& Sports Injuries, Manipal University, Manipal, India}

Posterior cruciate ligament (PCL) injury is frequent in road traffic accidents, most common being fall from a vehicle. A fall over the knee or posteriorly directed blow over the proximal tibia often results in PCL bony avulsion. Often, the PCL bony avulsion is quite displaced and requires fixation. Fixation can be achieved by arthroscopic or open technique using sutures or cannulated cancellous screw. Often, the fragment is too comminuted to allow screw fixation. In such cases, fixation of fragment by suture anchor is a plausible option. However, while fixation of fragment with a single anchor in the crater of avulsed fragment may lead to lifted margins of fragment. This results in not-so-secure fixation of fragment. In such conditions, addition of footprint anchor to further secure the fixation adds on to the strength of fixation. We report a series of two cases which were fixed by such technique by direct open posterior approach which resulted in excellent outcome. The fracture united in three months and patients resumed their original activity status. Double row PCL fixation technique could be opted whenever open fixation is required in a comminuted fragment. It is also a good option in patient who have multiple implant in proximal tibia wherein insertion of one or two cannulated screw is difficult due to space crunch.

\section{P558}

VITAMIN D SUPPLEMENTATION AND VITAMIN D INSUFFICIENCY IN INSTITUTIONALIZED SENIORS

S. B. Ang ${ }^{1}$, S. J. Cheng ${ }^{1}$, M. T. Chua ${ }^{1}$

${ }^{1}$ Family Medicine Service, KK Women's and Children's Hospital, Singapore, Singapore

Objectives: This study aims to investigate the relationship between vitamin D supplementation and serum 25-hydroxyvitamin D levels in institutionalized seniors.

Method: Convenient sampling was done and the initial population of 30 were selected for osteoporosis screening in a nursing home. Osteoporosis screening was done on site with a mobile BMD bus to do BMD screening using the Hologic DXA machine. 19 residents with osteoporosis had their 25hydroxyvitamin D levels screened.

Results: Vitamin D levels were done for 19 residents. Residents who were screened for vitamin D level were between the ages 58-92 years old. 9 (56\%) were males and 10 (44\%) were females. $16(84 \%)$ were Chinese and $3(16 \%)$ were Malays. Vitamin D level showed 3 (16\%) residents were $<20 \mu \mathrm{g} / \mathrm{L}, 13$ (68 \%) were between 20.1-30 $\mu \mathrm{g} / \mathrm{L}, 3$ $(16 \%)$ were $>30.1 \mu \mathrm{g} / \mathrm{L} .4$ residents were not on any vitamin D supplement, 1 was on $200 \mathrm{IU} /$ day, 8 were on $800 \mathrm{IU} /$ day and 6 were on $800 \mathrm{IU}$. Vitamin D levels were analyzed using multivariable Logistic Regression to assess the effect of supplementation after adjustment for age and gender. Residents who received Vitamin D supplement of at least 400 IU were 9.5 (1.44-62.83) times more likely to have a level of above $20 \mu \mathrm{g} / \mathrm{L}$ as compared to those that did not.

Conclusion: esults show that in osteoporotic institutionalized seniors supplemented with less than 400 IU of vitamin D3 daily were more likely to have insufficient 25 - 
hydroxyvitamin D levels $(<20 \mu \mathrm{g} / \mathrm{L})$. This could impact on the efficacy and safety of treatment of osteoporosis in this group of patient. However due to low sample population, the effect of resident's race on vitamin D levels could not be assessed. Further studies could be conducted by racial breakdown to assess the beneficial effect of Vitamin D dosage among various races and determination of the minimum vitamin D supplementation. The beneficial effects of varying dosages could also be accessed over varying timelines to look at the outcomes of resident's hospitalization rate, mortality, fall rate as well as other quality of life indicators.

\section{P559}

DISCRIMINATIVE VALUE OF DXA CHARACTERISTICS AND FRAX ${ }^{\circledR}$ FOR NONVERTEBRAL FRACTURE IN PATIENTS WITH RHEUMATOID ARTHRITIS

T. V. Gracheva ${ }^{1}$, E. V. Kozhemyakina ${ }^{1}$, O. M. Lesnyak ${ }^{2}$

${ }^{1}$ Regional Hospital \#1, Yekaterinburg, Russian Federation, Yekaterinburg, Russian Federation, ${ }^{2}$ North-Western State Medical University named after I.I. Mechnikov, SaintPetersburg, Russian Federation

Objective: To evaluate impact of BMD, trabecular bone score(TBS), FRAX ${ }^{\circledR}$ on nonvertebral fracture risk assessment in patients with rheumatoid arthritis (RA).

Materials and methods: Participants of this were 152 patients who were admitted to the Rheumatology Department of Regional Hospital\#1 of Yekaterinburg from January 12, 2012 to July 31, 2013 with confirmed diagnosis of RA. Vertebral fracture score from $\mathrm{T} 4$ to L4 were evaluated using vertebral fracture assessment software on DXA device and X-ray of spine for excluding patients with vertebral fractures $(n=11) .141 \mathrm{pa}-$ tients were included in our cross-sectional study aged $53.64 \pm 11.41$ with duration of RA $9.59 \pm 8.89$ year group 1 with nonvertebral fracture $(n=15)$ and group 2 without history of any fracture $(n=126)$ with similar demographic and disease related data in both groups. TBS was calculated from anteroposterior image of lumbar spine BMD. For all patients the $10-\mathrm{y}$ probability of major osteoporotic fracture (MOF) (clinical spine, forearm, hip or shoulder fracture) and 10-year probability of hip fracture (HF) were calculated with FRAX algorithm. The discriminative value of BMD at all bone sites, TBS (L2-L4), FRAX MOF facture risk, FRAX HF were assessed by determining the area under the receiving operator characteristic curve.

Results: Number of patients with osteoporosis on base of $\mathrm{T}$ and $\mathrm{Z}$ criteria were 6 from $15(40.00 \%)$ in group with fracture and 34 from $126(26.68 \%)$ without fracture $(p=0.221)$. We have found $4 / 15(26.66 \%)$ patients with osteopenia in group with fractures and 40/126
$(31.74 \%)$ patients without fractures $(p=0.470)$. The incidence rate of nonvertebral fracture was 2.69 per 100 patients/year. The areas under the curves (AUC) has shown the better discriminative value for FRAX HF $(\mathrm{AUC}=0.701)$, FRAX MOF $(\mathrm{AUC}=0.693)$, and $\mathrm{T}$ criteria hip (AUC $=0.668)$, meanwhile T-criteria in lumbar spine, BMD hip, and TBS had no significant value $(\mathrm{AUC}=0.565,0,573$ and 0.549 respectively).

Conclusions: Our data shows that T-criteria hip, FRAX MOF, FRAXHF have own discriminative value, whereas T-criteria in lumbar spine, BMD lumbar spine, TBS in our measurements have not get significant level for nonvertebral fractures in patients with RA.

\section{P560}

PREVALENCE OF VITAMIN D DEFICIENCY IN BELARUSIAN WOMEN

A. Rudenka $^{1}$, E. Rudenka $^{2}$, T. Tyabut ${ }^{1}$

${ }^{1}$ Belarusian Medical Academy of Postgraduate Training, Minsk, Belarus, ${ }^{2}$ Belarusian State Medical University, Minsk, Belarus

Objective: Because of the high prevalence of hypovitaminosis $\mathrm{D}$ and its negative impact on human health identification of persons with its deficiency is one among the priorities of health care. The aim of our study was to determine the prevalence of vitamin D deficiency in Belarusian women.

Materials and methods: We analyzed the results of the laboratory testing of serum levels of total vitamin D (25(OH)D) performed in republic of Belarus in women aged over 20 years in 2015. In general 2561 results, obtained from 4 laboratories situated in different regions of the country, were analyzed. Serum content of total vitamin D was determined by electrochemiluminescence protein binding assay technology using Cobas e411 immunoassay analyzer (Roche Diagnostic, Germany) and original reactants Roche Diagnostics $\mathrm{GmbH}$. According to international recommendations vitamin $\mathrm{D}$ levels considered to be normal when values of $25(\mathrm{OH}) \mathrm{D}$ exceeded the meaning $30 \mathrm{ng} / \mathrm{ml}$, showings from $20-29 \mathrm{ng} / \mathrm{ml}$ were determined as insufficiency, less than $20 \mathrm{ng} / \mathrm{ml}$ - as deficiency of vitamin D. Statistical analysis was performed using the software package Statistica 6.0.

Results: Received data were divided into 3 groups according to age: group I consisted of women younger than 40 years old, group II - of women at age $40-60$ years old and group III - of those older than 60 years. The number of examined individuals in group I was 198, in group II - 1264, in group III - 536. The prevalence of vitamin D deficiency and insufficiency were studied in each age group according to the season: spring-summer and autumn-winter and presented in Figures I and II. 
Fig.I Prevalence of hypovitaminosis D in studied sample in autumn and winter.

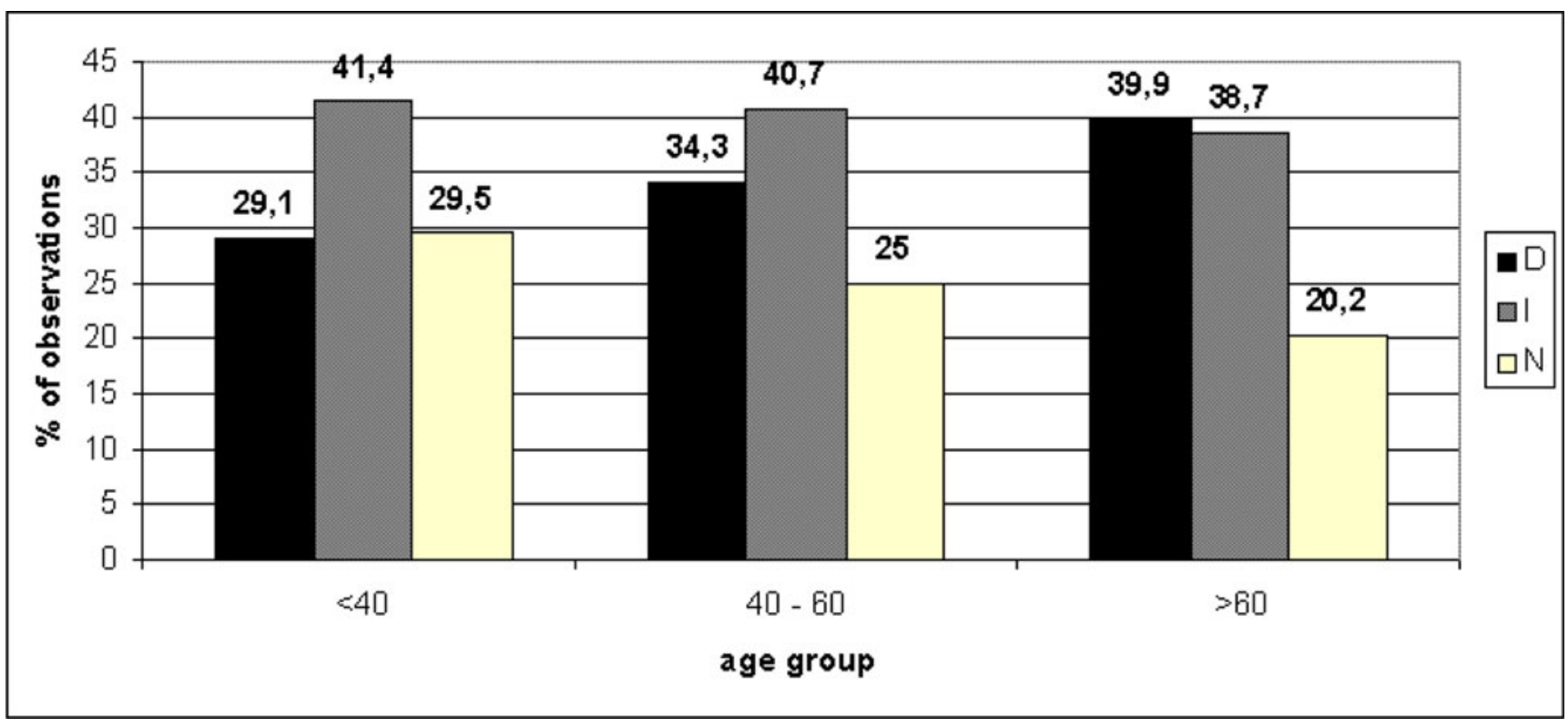

Fig. II. Prevalence of hypovitaminosis D in studied sample in spring and summer.

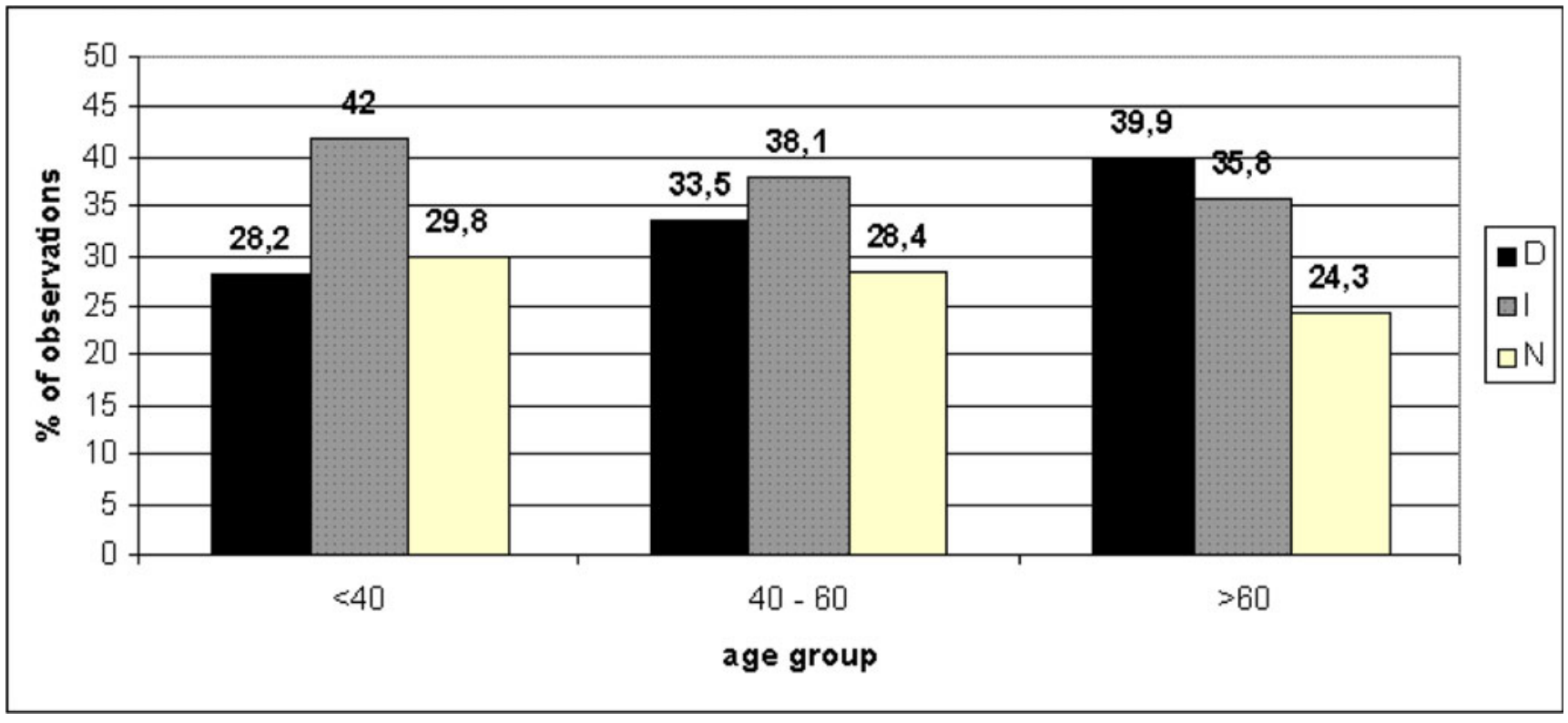


Conclusion: The prevalence of vitamin D deficiency and insufficiency in Belarusian women for the surveyed period is high and exceeds $50 \%$ irrespective of age and season.

\section{P561}

\section{REFERENCE POINT MICROINDENTATION FOR} SUPPLEMENTING FRACTURE RISK ASSESSMENT AT THE FEMORAL NECK

\section{P. J. Thurner ${ }^{1}$, T. Jenkins ${ }^{2}$, L. V. Coutts ${ }^{2}$, S. D'Angelo ${ }^{3}$, D. G.}

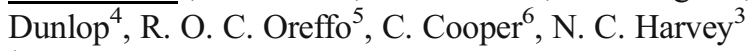

${ }^{1}$ Institute of Lightweight Design and Structural Biomechanics, TU Wien, Vienna, Austria, ${ }^{2}$ Faculty of Engineering and the Environment, University of Southampton, Southampton, United Kingdom, ${ }^{3} \mathrm{MRC}$ Lifecourse Epidemiology Unit, University of Southampton, Southampton, United Kingdom, ${ }^{4}$ University Hospital Southampton NHS Trust, Southampton, United Kingdom, ${ }^{5}$ Bone and Joint Research Group, Centre for Human Development, Stem Cells and Regeneration, Institute of Developmental Sciences, Southampton, United Kingdom, ${ }^{6}$ MRC Epidemiology Resource Centre, University of Southampton, Southampton General Hospital, Southampton, United Kingdom

In the clinical setting, fracture risk is assessed by combining BMD and risk factors e.g. FRAX ${ }^{\circledR}$. Yet such approaches do not incorporate any direct measure of bone biomechanical properties. A recent technique, Reference Point microindentation (RPI), which permits a direct assessment of bone mechanics, appears to discriminate patients with fragility fracture from controls through in vivo measurements at the tibia [1]. However, it is unclear how this relates to testing at the site of most clinically relevant fracture, the femoral neck. Further, RPI derived measurement parameters are not defined on purely mechanical or physical grounds, but rather trying to exploit all the information that can be obtained from such measurements.

Therefore, the actual meaning of RPI measures in a mechanical or physical sense is unclear. Femoral neck samples were collected at surgery following low trauma hip fracture ( $n=46 ; 17$ male, 83 (IQR 77-87) years), and compared with 16 cadaveric femoral neck samples, free from bone disease, obtained from a tissue bank (7 male; 65 (IQR 61-74) years). RPI measures (Biodent $\mathrm{Hfc}^{\mathrm{TM}}$, ActiveLife Scientific) were compared between fracture patients and controls using Mann-Whitney Utests, and discriminative ability using ROC analysis. A subset of fracture patients returned for DXA assessment (Hologic Discovery), and BMD values for cadaveric samples were retrieved using a phantom-calibrated micro-computed tomography device ( $\mu \mathrm{CT})$ (HMX, Nikon) in radiography mode. A subset of these samples was then used to machine fracture toughness samples from the inferomedial femoral neck. These samples were tested in a 3-point bending geometry with concomitant videography [2], and subsequently subjected to RPI. Additional samples were machined for RPI with subsequent imaging of residual indents from osteoporotic and control samples in the transverse direction, approximately perpendicular to the long axis of the osteons. Residual RPI imprints were imaged, using $\mu \mathrm{CT}$ ( 3 osteoporotic donors and 4 control donors), serial sectioning with fluorescence microscopy after basic fuchsin staining (FLM, 5 osteoporotic donors and 5 control donors), as well as atomic force microscopy (AFM) and polarised light microscopy (PLM) in one osteoporotic sample. This gave a total of 16 samples from 14 donors, 8 osteoporotic (4 female, $54-88$ years, mean $72.0 \pm 10.3$ years) and 6 control ( 5 female, $58-92$ years, mean $67.7 \pm 11.9$ years). The RPI measured Total Indentation Depth (TID) was higher in femoral neck samples from osteoporotic fracture patients $(131.3 \mu \mathrm{m})$ than that of cadaveric controls $(105.9 \mu \mathrm{m})$ $p<0.001$. This difference persisted with adjustment for age, sex, BMI and height $(p<0.001)$. TID demonstrated good discrimination between fracture and controls using ROC analysis $(A U C=0.89)$. RPI appeared to measure a property distinct from existing clinical factors with low correlation between TID and each of FRAX probability $(r=0.31, p=0.026, n=53)$, and $\operatorname{BMD}(r=-0.32, p=0.063, n=35)$. The clinical thresholds for FRAX and BMD did not identify the majority of individuals in the fracture group (60-86\%), and yet, in this subset the ROC values are still high for TID (AUC $=0.88$ to 0.93 ) showing potential to supplement current techniques, together having a sensitivity up to $100 \%$ (for an $80 \%$ specificity). Alternatively, summation of normalised TID to normalised BMD or FRAX probability also resulted in an improved AUC (0.95 To 0.99). Despite these encouraging findings, the bulk material properties of the bone samples in the subgroups in terms of fracture resistance $\left(\mathrm{K}_{\text {slope }}\right.$ and $\left.\mathrm{J}_{\text {slope }}\right)$, fracture toughness $\left(\mathrm{K}_{\max }\right.$ and $\left.\mathrm{J}_{\max }\right)$, elastic modulus $\left(\mathrm{E}_{\mathrm{mod}}\right)$ and indentation properties (TID, IDI and CID) are generally not significantly different across groups. Overall, fracture toughness exhibited a clear correlation with donor / patient age. Importantly, stress-whitening, an indicator of diffuse damage during mechanical testing [3], was significantly increased in the healthy bone samples. 2D and 3D indent imaging further revealed that RPI is largely influenced by proximity to larger pores. Therefore, it is possible that the material properties of osteoporotic bone at the inferomedial neck do indeed change, in addition to age- related deterioration. In this study, it appears however, that this is countered by increased porosity in the healthy control samples. In conclusion, the RPI technique at the femoral neck discriminated fracture cases from controls independent of BMD and traditional risk factors. The approach, in its clinical form, may therefore usefully add to risk assessment, and requires testing in prospective cohorts. To 
exploit the full potential of such findings and provide mechanistic understanding further efforts are necessary to provide insights in to changes of structure, composition and porosity of cortical bone at the osteoporotic femoral neck.

1. Diez-Perez et al., JBMR 25(8), 2010

2. Katsamenis et al., PLoS ONE 8, 2013

3. Thurner et al., Eng Frac Mech 74, 2007

We gratefully acknowledge funding from EPSRC (EP/ J008192/1) and University of Southampton alumnus, Mike Russell.

\section{P562}

FACTORS INFLUENCING BMD AND TRABECULAR BONE SCORE IN PATIENTS WITH RHEUMATOID ARTHRITIS

E. V. Kozhemyakina ${ }^{1}$, O. Lesnyak ${ }^{1}$

${ }^{1}$ Regional Hospital \#1, Yekaterinburg, Russian Federation, Yekaterinburg, Russian Federation

Objective: To Evaluate the factors that affect BMD and the index of trabecular bone score in patients with rheumatoid arthritis(RA).

Materials and methods: A specially designed questionnaire was introduced to patient of rheumatic department Sverdlovsk regional hospital №1. The study included 152 patients with diagnosis of RA (ARA criteria, 1987) is $78 \%$ women and $22 \%$ men (mean age $53.01 \pm 12.3$ years). The average duration of RA of $9.46 \pm 8.6$ years. The patients received glucocorticosteroids (GCS) was $44 \%$ (67 people) of them $33(83 \%)$ women and $12(17 \%)$ men. The cumulative dose of GCS of $10 \mathrm{mg}$. All patients on the unit Hologic QDR Discovery were assessed BMD and TBS iNsight.

Results: Patients BMD decreased significantly with age $(O R=3.00(95 \% \mathrm{CI} 1.14-7.84))$. The decline in BMD was significantly associated with duration of RA $(O R=2.52(95 \% \mathrm{CI}$ 1.19-of 5.36)) and prolonged administration of corticosteroids $(O R=4.49(95 \% \mathrm{CI}$ of $2.09-9.62))$. We found a significant decrease in the quality of trabecular bone with age of the patient with RA $(O R=\mathrm{OF} 8.56(95 \%$ CI 1.10-66.12)). The disease duration of RA also significantly influenced the decline in the quality of trabecular bone tissue $(O R=1.71(95 \%$ CI $0.83-$ 3.50)). Intake of GCS for more than three months significantly reduces the quality of the trabecular bone score $(\mathrm{OR}=1.61$ (95\%CI 0.77-3.36)). In patients with RA with normal BMD values, it was found a significant decrease in the quality of trabecular bone score $(O R=3.90(95 \%$ CI $1.41-10.75))$.

Conclusions: The decrease in BMD and quality of trabecular bone in patients with RA significantly influenced by patient age, duration of RA, receipt of GCS for more than three months. In patients with normal BMD values had a statistically significant decrease in the quality of trabecular bone score.

\section{P563}

THE RELATIONSHIP BETWEEN BONE MINERAL DENSITY AND DIABETIC WOMEN

H. Hamdy ${ }^{1}$

${ }^{1}$ Al-Helal Hospital, Cairo-Nasr City, Egypt

Aim: The aim was to compare bone mineral density of preand postmenopausal diabetics.

Methodspine: A cross sectional study of 25 premenopausal diabetics and postmenopausal diabetics was carried out where, BMD at forearm, hip and spine were measured and compared with reference values obtained from measurments of normal healthy pre-postmenopausal women.

Results: The DEXA measures in postmenopausal diabetic group at forearm, lumbar spine, hip showed normal results in $56 \%$ at forearm, $32 \%$ at lumbar spine, $28 \%$ at hip,while osteopenia was found in $36 \%$ at forearm, $60 \%$ at spine, $40 \%$ at hip and osteoporosis was found in $8 \%$ at forearm, $8 \%$ at spine, $32 \%$ at hip.While the DEXA measures in premenopausal diabetic group at forearm, lumbar spine, hip showed normal results in $88 \%$ at forearm, $60 \%$ at lumbar spine, $56 \%$ at hip, while osteopenia was found in $12 \%$ at forearm, $40 \%$ at spine, $36 \%$ at hip, and osteoporosis was found in $0 \%$ at forearm, $0 \%$ at spine, $8 \%$ at hip.

Conclusion: Both postmenopausal \& premenopausal diabetic women had lower BMD values than normal women in same age.

Keywords: Bone mineral density, IDDM.

\section{P564}

ANGIOTENSIN (1-7)/MAS RECEPTOR AXIS MODULATE LOSARTAN AND CAPTOPRIL EFFECTS ON TRABECULAR AND CORTICAL BONE MICROARCHITECTURE AND METABOLISM $\underline{\text { H. M. Abuohahsih }}{ }^{1}$, M. M. Ahmed ${ }^{2}$, M. M. Khattab ${ }^{3}$, S. S. Al-Rejaie $^{2}$

${ }^{1}$ Department of Biomedical Dental Sciences, College of Dentistry, University of Dammam, Dammam, Saudi Arabia. Department of Pharmacology and Toxicology, Faculty of Pharmacy, Cairo University, Cairo, Egypt, ${ }^{2}$ Department of Pharmacology and Toxicology, College of Pharmacy, King Saud University, Riyadh, Saudi Arabia, ${ }^{3}$ Department of Pharmacology and Toxicology, Faculty of Pharmacy, Cairo University, Cairo, Egypt

Background and Objective: Recent data has linked the renin angiotensin system (RAS) and osteoporosis associated impaired bone micro-architecture, structure and metabolism. Studies showed that angiotensin II (Ang II) may prompt bone resorption whereas RAS suppressive medications including angiotensin receptors blockers (e.g., losartan) and angiotensin converting enzyme inhibitors (e.g., captopril) were found to 
ameliorate such effect. Angiotensin-(1-7) (Ang 1-7) is a bioactive heptapeptide in RAS with potent opposing effects against many actions of Ang II maybe as a Mas receptor ligand. In the present project, effects of Ang (1-7) on bone impairments were studied and allied to losartan and captopril osteo-protective properties.

Material and Methods: Ovariectomized (OVX) Wistar albino rats were allocated into eight groups as follow; (A) Sham, (B) OVX, (C) OVX+Ang (1-7), (D) OVX+A-779, (E) OVX+Losartan, (F) OVX + Losartan + A-779, (G) OVX + Captopril, and $(\mathrm{H}) \mathrm{OVX}+$ Captopril $+\mathrm{A}-779$. Animals in (C), (D), (F) and (H) groups were implanted with subcutaneous osmotic pumps (model 2006, Alzet, CA) that delivered Ang-(1-7) and the specific Mas receptor blocker (A-779) (200 and $400 \mathrm{ng} . \mathrm{kg}^{-1} \mathrm{~min}^{-1}$; respectively). Peptides infusion and oral treatments started 8 weeks after OVX for 6 consecutive weeks. Bone turnover biomarkers were measured in serum using ELISA analysis. Trabecular and cortical bone mass and microarchitecture at the distal left femora were evaluated by $\mu-\mathrm{CT}$.

Results: Estrogen deficiency by OVX procedure elevated the serum levels of osteocalcin, bone specific alkaline phosphatase, tartrate resistant acid phosphatase and collagen type I Ctelopeptide, while altered cortical and trabecular microarchitecture of the distal femoral bones. The raised bone turnover biomarkers were markedly ameliorated following 6 weeks of Ang (1-7), losartan and captopril treatments. In addition, bone $3 \mathrm{D}$ scanning and analysis using $\mu$-CT showed that the OVX-induced trabecular and cortical morphometric structure impairments were alleviated by Ang (1-7), losartan and captopril. However, these protective effects were inhibited after blocking of Mas receptor by A-779 infusion.

Conclusion: Findings of the current study suggested a beneficial function of the endogenous peptide Ang (1-7). Moreover, the reported attenuation of bone loss following angiotensin receptors blocking or angiotensin converting enzyme inhibition might be via Ang-(1-7)/Mas dependent pathway.

\section{P565}

EFFECT OF DENOSUMAB ON BONE MINERAL DENSITY IN PATIENTS WITH PRIOR BISPHOSPHONATE EXPOSURE

Y. C. Woo ${ }^{1}$, C. H. N. Loong ${ }^{1}$, V. W. K. Chau ${ }^{2}$, J. K. Y. Lam ${ }^{1}$, M. M. A. Yuen ${ }^{1}$, C. H. Lee ${ }^{2}$, A. C. H. Lee' ${ }^{1}$ K. C. B. Tan ${ }^{2}$

${ }^{1}$ Department of Medicine, Queen Mary Hospital, Hong Kong, Hong Kong, ${ }^{2}$ Department of Medicine, The University of Hong Kong, Hong Kong, Hong Kong

Objectives: It has been demonstrate that denosumab can effectively improve the BMD of patients with short-term (6 months) exposure of oral bisphosphonate. The aim of this study is to evaluate the change in BMD after switching to denosumab for one year in patients with prior oral bisphosphonate use for a longer duration.

Material and Methods: Patients with osteoporosis were followed according to a standard protocol. Patients using denosumab for more than one year, with history of bisphosphonate exposure for at least one year, were recruited for analysis. Patients were monitored regularly in nurse-led clinic to ensure good drug adherence. Subcutaneous denosumab injection was administered by nurses as outpatient. BMD was measured by DXA scan. All subjects had adequate calcium and vitamin D supplementation. The BMD of the total spine, neck of femur and total hip before and after commencement of denosumab was compared by dependent $T$-test.

Results: 104 female subjects with a mean age of 73.7 \pm 10.5 years were included in this report. All of the subjects had history of fragility fracture. Duration of oral bisphosphonate exposure before switching to denosumab was 4.5 \pm 2.7 years. The oral bisphosphonate used was alendronate $70 \mathrm{mg}$ once weekly. There was no statistically significant difference observed in BMD of the total spine $(0.655 \pm 0.105 \mathrm{vs}$. $\left.0.662 \pm 0.107 \mathrm{~g} / \mathrm{cm}^{2}, p=0.24\right)$, neck of femur $(0.485 \pm 0.080$ vs. $\left.0.480 \pm 0.080 \mathrm{~g} / \mathrm{cm}^{2}, P=0.30\right)$ and total hip $(0.589 \pm 0.109$ vs. $0.598 \pm 0.112 \mathrm{~g} / \mathrm{cm}^{2}, p=0.09$ ) before and 1 year after initiation of denosumab. Conclusions: Patients with prevalent fragility fracture, despite having relatively long history of bisphosphonate exposure, should be benefited by continuation of anti-osteoporosis treatment because of high fracture risk. However, switching this group of patients to denosumab may not reproduce the similar BMD improvement observed in patients who had taken bisphosphonate for short term. However, the efficacy of denosumab in fracture risk reduction in this group of patients needs further evaluation.

\section{P566 \\ BONE HEALTH OF MEN AND WOMEN AGED 50 YEARS OLD AND ABOVE IN KUALA LUMPUR MALAYSIA}

K. Y. Chin ${ }^{1}$, N. Y. Low ${ }^{2}$, K. Alia Annessa ${ }^{2}$, S. Ima-Nirwana ${ }^{1}$ ${ }^{1}$ Department of Pharmacology, University Kebangsaan Malaysia Medical Centre, Kuala Lumpur, Malaysia, ${ }^{2}$ ASASIpintar Foundation, PERMATApintar National Gifted Centre, University Kebangsaan Malaysia, Bangi, Malaysia

Objective: Osteoporosis is a major health problem in the aging society due to the healthcare burden caused by fragility fracture. Early detection is the best way to prevent osteoporosis. Quantitative ultrasound (QUS) offers a convenient approach to detect osteoporosis in countries where access to DXA device is limited. This study aimed to determine the bone health status of men and women aged 50 years old and above in Kuala Lumpur using a QUS device. 
Materials and Methods: A total of 233 men and 300 women aged 50 years and above from three major ethnic groups (Chinese, Malay and Indian) were recruited in a crosssectional study conducted from December 2014 to December 2015 at University Kebangsaan Malaysia Medical Centre. They completed a demographic questionnaire, anthropometric and bone health measurements during the screening session.

Results: A significant age-related decline in bone health indicated by calcaneal speed of sound (SOS), broadband attenuation of sound (BUA), stiffness index (SI) and T-score based on SI was observed in women $(p<0.05)$ but not in men $(p>0.05)$. It was revealed that $10.7 \%$ of the elderly men were osteoporotic and $26.6 \%$ were osteopenic. For women, $8 \%$ were osteoporotic and $23.3 \%$ were osteopenic. There were no significant differences in SOS, BUA, SI and T-score among the three ethnic groups studied $(p>0.05)$.

There was no significant ethnic difference in the prevalence of osteoporosis and osteopenia in men and women $(p>0.05)$.

Conclusion: Age-related decline in bone health is more significant in elderly women compared to elderly men. A significant proportion of the elderly population has suboptimal bone health but ethnic difference in bone health status is not apparent.

Acknowledgement: We thank University Kebangsaan Malaysia for funding this study via grants FF-2015-412 and FF-2015-396.

\section{P567}

CURING OSTEOPOROSIS CARE: NEW STRATEGIES FOR QUALITY IMPROVEMENT

P. Khashayar $^{1}$, M. Ebrahimi ${ }^{2}$, H. Ebrahimi ${ }^{3}$, M. Raji ${ }^{2}$, Z.

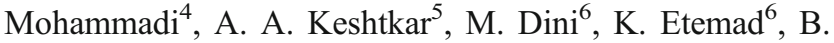
Larijani $^{7}$

${ }^{1}$ Orc/EMRI/TUMS-CMST/UGENT, Tehran, Islamic Republic of Iran, ${ }^{2} \mathrm{Orc} / \mathrm{EMRI} / \mathrm{TUMS}$, Tehran, Islamic Republic of Iran, ${ }^{3}$ Orc/EMRI/TUMS, Shahroud, Islamic Republic of Iran, ${ }^{4}$ Ddri/ TUMS, Tehran, Islamic Republic of Iran, ${ }^{5}$ Tums, Tehran, Islamic Republic of Iran, ${ }^{6} \mathrm{MoHME}$, Tehran, Islamic Republic of Iran, ${ }^{7}$ Endocrinology and Metabolism Research Center, Dr Shariati Hospital, Tehran University of Medical Sciences, Tehran, Islamic Republic of Iran

While the prevention, diagnosis and treatment of osteoporosis has gained more importance in many parts of the world, recent studies report under-treatment in many cases, showing that as high as one fifth of the sufferers remain untreated. Therefore, the focus is now shifted to studies addressing barriers to appropriate management of the disease as well as the implementation of effective strategies to close the osteoporosis care gap. The present study was conducted to identify the osteoporosis care gap and develop a model to improve the condition in Iran.

Methods: Six specialists active in the field of osteoporosis were asked about the care gap in osteoporosis management. They were requested to prioritize the barriers and then provide possible solutions to improve the condition at national level. The list provided by each expert was then gathered and merged to deliver the final results.

Results: From the specialists' point of view, the main barriers to high-quality osteoporosis care were:

1) Health system-related barriers

- Poor/no access to BMD

- Poor/no access to specialists working in the field of osteoporosis

- Lack of osteoporosis care management programs in national healthcare guidelines

- Lack of bone health coordinator-based clinics

2) Healthcare provider-related barriers

- Lack/poor knowledge of osteoporosis management

- Underestimation of the prevalence of osteoporosis and not evaluating the patients regarding the disease

- Poor knowledge of BMD criteria and inappropriate use of BMD

3) Patient-related barriers

- Low socioeconomic status

- Lack/poor knowledge, attitude and practice of osteoporosis

- Low Ca-vit D dietary intake and/or supplementation

- Poor insurance coverage

Possible solutions to improve osteoporosis care in the specialists' point of view were as following:

- Development and implementation of a national guideline for the diagnosis and treatment of osteoporosis and its complications

- Supervision of the implementation of the guidelines by the health system and insurance companies

- Holding CMEs for different groups of healthcare providers active in the field of osteoporosis

- Implementation of a multidisciplinary team-based model of care through coordinator-based osteoporosis clinics

- Public education about osteoporosis through seminars, talk-shows in mass media, booklets and...

Conclusion: In the present study we defined osteoporosis care as a system-based model composed of health structure (Healthcare system-related) and two processes of care (healthcare provider-, and patient-related).Similar to many other studies, poor knowledge of osteoporosis among both patients and healthcare providers (process of care) and lack of a national guideline for osteoporosis management (health structure) were the main barriers to quality osteoporosis care. Developing a guideline, implementing it and supervising its implementation along with educating both the healthcare 
providers and patients can help improve the condition. The effectiveness of these strategies should be assessed through studying the reduction in the number of osteoporotic fractures and the burden the disease and its complications impose on the society. It should be added that the guideline has already been developed by the Osteoporosis Research Center (ORC) of the Endocrinology and Metabolism Research Institute affiliated with Tehran University of Medical Sciences in collaboration with the Center for Non-Communicable Disease Management (CNCDM) of the Iranian Ministry of Health and Medical Education and the Iranian Osteoporosis Research Network (IORN), and its implementation would soon be secured.

\section{P568}

CORTICAL AND TRABECULAR BONE ANALYSIS OF PATIENTS WITH HIGH BONE MASS FROM THE BARCOS COHORT USING 3D-DXA: A CASECONTROL STUDY

J. Rodriguez-Morera $^{1}$, L. H. Humbert ${ }^{2}$, R. F. Fonolla ${ }^{2}$, J. Romera $^{2}$, M. Oanea ${ }^{1}$, A. Gonzalez-Lizaran ${ }^{1}$, A. Diez-Perez ${ }^{1}$, X. Nogués ${ }^{1}$, L. Mellibovsky ${ }^{1}$

${ }^{1}$ Internal Medicine Department, Parc de Salut Mar, Universitat Autònoma de Barcelona, IMIM (Hospital del Mar Research Institute), Red Temática de Investigación Cooperativa en Envejecimiento y Fragilidad (RETICEF), Instituto de Salud Carlos III FEDER, Barcelona, Spain, ${ }^{2}$ Galgo Medical, Barcelona, Spain

Objective: To analyze the cortical and trabecular bone of patients with high bone mass (HBM) and control group using 3D-DXA.

Material and Methods: 8 women with HBM from the BARCOS cohort and 8 age-matched women (control group) collected at Hospital del Mar (Barcelona, Spain) were included in this study. DXA scans (QDR 4500 SL, Hologic, Waltham, MA, USA) were performed for all the subjects. One of the inclusion criteria for HBM cases was a sum of hip and lumbar spine Z-scores equal to or greater than four. Age- matched controls not having HBM were randomly selected from patients of the densitometry department of Hospital del Mar, with no particular exclusion criteria in terms of Z-score or T-score. The 3D-DXA software (Galgo Medical, Barcelona, Spain) was used to analyze the cortical and trabecular bone of patients and control group. 3D-DXA registers a 3D appearance model of the femoral shape and density onto the DXA projection to obtain a 3D subject-specific model of the femur of the patient and quantify the volumetric BMD (vBMD), volume (for trabecular and cortical regions) and cortical thickness distribution. The 3D-DXA measurements for both groups were compared using Student's $t$-test.

Results: No statistically significant differences were found in terms of age (64.3 \pm 8.0 years for cases, $61.5 \pm 9.4$ years for controls, $p=0.54$ ) and height. The weight of HBM cases $(80.6 \pm 13.4 \mathrm{~kg})$ was found to be significantly higher than the weight of controls $(63.6 \pm 7.4 \mathrm{~kg}, p=0.01)$. vBMD of HBM patients was on average higher in comparison with controls: $+27 \%\left(+90 \mathrm{mg} / \mathrm{cm}^{3}\right)$ for integral bone $(p=0.008)$, $+46 \%$ for trabecular bone $(p=0.029)$ and $+3 \%$ for cortical bone $(p=0.005)$. The mean cortical thickness was also higher: $+0.38 \mathrm{~mm}(+21 \%, p=0.002)$. No statistically significant difference was found in terms of volume of the whole proximal femur $(p=0.918)$ and trabecular compartment $(p=0.526)$. However, the volume of the cortex was found to be significantly higher $(+3.4 \mathrm{~cm} 3$ or $+21 \%)$ for HBM patients.

Conclusion: HMB patients were found to have a thicker cortex and a higher vBMD for both trabecular and cortical compartment, while having femurs with similar size than controls.

\section{P569}

PROLIFERATION AND MINERALIZATION OF HUMAN MESENCHYMAL STEM CELLS DERIVED FROM ADIPOSE TISSUE ON POLY( $\varepsilon$-CAPROLACTONE) 3D SCAFFOLD FOR BONE TISSUE ENGINEERING APPLICATION

C. Romagnoli ${ }^{1}$, R. Zonefrati ${ }^{1}$, G. Galli ${ }^{1}$, D. Puppi ${ }^{2}$, A. Pirosa $^{2}$, F. Chiellini ${ }^{2}$, F. S. Martelli ${ }^{3}$, A. Tanini ${ }^{1}$, M. L. Brandi ${ }^{1}$ ${ }^{1}$ Department of Surgery and Translational Medicine, Unit of Bone and Mineral Diseases, University of Florence, University Hospital of Florence, Florence, Italy, ${ }^{2}$ BIOLab Research Group, Department of Chemistry and Industrial Chemistry, University of Pisa, UdR INSTM, Pisa, Italy, ${ }^{3}$ Microdentistry Institute, Florence, Italy

Objective: The need to restore and maintain the function of human bone tissue in skeletal defects makes bone tissue engineering a valuable strategy for tissue regeneration, thanks to the combination between appropriate materials and mesenchymal stem cells. Aim of this study was to evaluate the proliferation and mineralization of human adipose-derived stem cells (hADSCs) in association with poli( -caprolactone) 3D scaffold (3D-PCL) for future application in regenerative medicine.

Material and Methods: hADSCs were cultured in growth medium (10\% FBS, $1 \mathrm{ng} / \mathrm{ml} \mathrm{bFGF}$ and $1 \%$ antibiotics) at $37{ }^{\circ} \mathrm{C}$ and $5 \% \mathrm{CO}_{2}$. hADSCs were plated on 3D-PCL to evaluate fibronectin adhesion protein and cytoskeleton by immunocytochemistry. Proliferation was analyzed by MTT assay after cell seeding on 3D-PCL at appropriate density. Alkaline phospatase (ALP) activity and mineralization were quantified, during osteoinduction, by spectrofluorometric assays. Statistical analysis was performed by linearity test and Student's $t$-test, comparing the values obtained on $3 \mathrm{D}$-PCL with those obtained on PCL film, used as control ${ }^{1}$. 
Results: No qualitative differences were found in cell adhesion and cytoskeleton fiber morphology between hADSCs grown on 3D-PCL and hADSCs grown on PCL film. MTT assay has measured a consistent growth of absorbance during all the studied period (from 2 to 16 days) for hADSCs seeded on 3D-PCL (linear regression $r^{2}=0,94$ ), suggesting that good cell proliferation and cytocompatibility were achieved on the scaffold. Osteogenic differentiation of hADSCs on 3D-PCL was assessed from 0-35 days and has shown not only a significant increase on ALP activity, after 21 days of induction ( $+4579 \%$ vs. time 0$)$, but also on $\mathrm{Ca}^{2+}$ deposition, after 35 days of induction $(+6859 \%$ vs. time 0$)$. The same and not significantly different behavior was obtained with hADSCs induced on PCL film, as control.

Conclusion: This work has shown encouraging results in terms of proliferation and mineralization of hAMSCs on 3D-PCL scaffold prepared by wet spinning, suggesting and promoting the research of new strategies to improve this model for future applications in bone tissue regeneration.

Reference: ${ }^{1}$ Romagnoli C, et al. Biomed Res Int. 2015.

Acknowledgments: Regione Toscana-POR CREO FESR 2007-2013. Progetto REOSS.

\section{P570}

\section{EVALUATION OF CALCIUM INTAKE IN MALE WORKERS IN A RECYCLING FACTORY}

$\underline{\text { M. Sarıdogan }}^{1}$, S. Tuzun ${ }^{1}$, N. Eskiyurt ${ }^{2}$, M. Ahmetoglu ${ }^{1}$, U. Akarirmak $^{1}$

${ }^{1}$ Istanbul University, Cerrahpasa Medical Faculty, Physical Medicine and Rehabilitation Department, Istanbul, Turkey, ${ }^{2}$ Istanbul University, Istanbul Medical Faculty, Physical Medicine and Rehabilitation Department, Istanbul, Turkey

Objective: The Turkish Osteoporosis Society and Osteoporosis Patient Society of Turkey were invited to Marzinc Recycling Factory in Karabük located in Middle Anatolia region of Turkey. Aim: Educational project, in collaboration with 2 societies and Marzinc was to evaluate calcium uptake - nutrition and provide education and awareness about male osteoporosis and prevention. This was the 2 nd year of the educational project.

Results: A total of 55 healthy male laborers were evaluated in terms of calcium intake by the IOF Calcium Calculator. A specific work-shop program was performed in three interactive groups in the factory. Instructions were given on how to fill the Turkish version of the Calcium Calculator, accepted by IOF in 2015. Mean calcium intake was found to be $706.388 \mathrm{mg}$. Daily calcium intake below $500 \mathrm{mg}$ was detected in 14 persons $(25.45 \%)$. Daily calcium intake between 500 $1000 \mathrm{mg}$ was detected in 30 persons $(54.55 \%$ ). Daily calcium intake over $1000 \mathrm{mg}$ was detected in 11 persons $(20 \%)$. Approximately half of the included subjects consumed 500
$1000 \mathrm{mg}$ daily calcium. Approximately $80 \%$ had a daily calcium intake below $1000 \mathrm{mg}$ (Table 1).

Conclusion: Preventive strategies for male patients as well as healthy male population is of great importance. The primary aim is to establish a lifelong approach, address balanced nutrition, vitamin $\mathrm{D}$, healthy level of physical activity and avoid negative lifestyle in males.

Table 1 Calcium intake in different age groups

\begin{tabular}{lllll}
\hline $\begin{array}{l}\text { Level of Calcium } \\
\text { intake }\end{array}$ & $<500 \mathrm{mg}$ & $500 \mathrm{mg}$ & $>1000 \mathrm{mg}$ & Total \\
$\mathrm{n} / \%$ & $14(25.45 \%)$ & $30(54.55 \%)$ & $11(20 \%)$ & 55 \\
Mean age & 34,78571429 & 29 & 28.1 & 33.1 \\
Minimum age & 26 & 19 & 22 & 19 \\
Maximum age & 54 & 49 & 35 & 54 \\
Mean Calcium intake & 706.388 & & & \\
Global average age & 33.1 & & & \\
\hline
\end{tabular}

P571

BONE MINERAL DENSITY AND TRABECULAR BONE SCORE (TBS) IN TYPE 2 DIABETIC WOMEN AND OSTEOPOROTIC POSTMENOPAUSAL ONES A. Wawrzyniak $^{1}$, J. Dytfeld $^{1}$

${ }^{1}$ Department of Family Medicine University of Medical Science Poznan, Poznan, Poland

Introduction: It has been recently proven that type 2 diabetes predisposes to low-energy fractures, despite high or normal BMD values. Therefore it has been believed DXA does not reflect the complexity of bone pathology in diabetes. Trabecular bone score (TBS) is a new, noninvasive method that might shed some new light on pathology of bone in diabetes.

Aim: To compare BMD L1-L4 and L1-L4 TBS between postmenopausal women with type 2 diabetes and osteoporotic women without diabetes.

Materials and methods: In a retrospective, cross-sectional study, we studied a small group of 58 Caucasian, noninstitutionalized, postmenopausal female patients: 29 with type 2 diabetes (mean age $73.0 \pm 10.7$ years) and 29 diagnosed with osteoporosis without diabetes (mean age $73.6 \pm 9.5$ years). All of them were patients of Osteoporosis Outpatient Clinic. All subject underwent clinical examination, L1-L4 DXA (Lunar, GE) and TBS analysis (iNsight software, Medimaps Group). Results: Women with diabetes had greater body weight $(69.1$ vs. $59.6 \mathrm{~kg} p<0.05)$ and BMI $\left(27.6\right.$ vs. $\left.24.4 \mathrm{~kg} / \mathrm{m}^{2}, p<0.05\right)$ when compared to osteoporotic ones. L1-L4 BMD in diabetic females was higher, although not statistically different from the BMD of osteoporotic women ( 0.939 vs. 0.876 , respectively), neither was L1-L4 T-score (-2.0 vs. -2.52). The adjusted 
and unadjusted TBS values were also comparable between diabetics and non-diabetics (unadjusted 1.100 vs. 1.120, NS; age, BMI and BMD adjusted TBS - 1.109 vs. 1.130 , NS). Prevalent fractures were more frequent in diabetics $(72.4 \%)$ than in nondiabetics $(65 \%)$.

Conclusions: Despite the same DXA and TBS parameters, low-energy fractures were more frequent in diabetic women than in osteoporotic ones.

\section{P572}

ESTABLISHMENT AND CHARACTERIZATION OF A CANCER STEM CELL LINE FROM A RARE BONE SARCOMA

G. Palmini ${ }^{1}$, R. Zonefrati ${ }^{1}$, A. Franchi ${ }^{2}$, A. Simoni $^{2}$, A. Aldinucci $^{3}$, C. Ballerini ${ }^{3}$, C. Mavilia ${ }^{1}$, R. Capanna ${ }^{4}$, D. Campanacci $^{4}$, P. Cuomo ${ }^{4}$, F. Marini ${ }^{1}$, E. Luzi ${ }^{1}$, A. Tanini ${ }^{1}$, M. L. Brandi ${ }^{1}$

${ }^{1}$ Department of Surgery and Translational Medicine, Unit of Bone and Mineral Diseases, University of Florence, University Hospital of Florence, Florence, Italy, ${ }^{2}$ Department of Surgery and Translational Medicine, Unit of Anatomical Pathology, University of Florence, Florence, Italy, ${ }^{3}$ Department of Neurofarba, University of Florence, Florence, Italy, ${ }^{4}$ Unit of Ortopedia Oncologica e Ricostruttiva, AOU Careggi, University Hospital of Florence, Florence, Italy

Objective: To establish of a bone cancer stem cell (CSC) line from a rare human bone disease, the high grade osteoblastic osteosarcoma (OS).

Material and Methods: Central high grade osteoblastic OS samples were collected at the "Unit Ortopedia Oncologica e Ricostruttiva", AOUC Careggi, Florence, with informed consent approved by the local Ethical Committee. First, primary human cancer cell cultures of osteoblastic OS have been established. After that, the subpopulations of CSCs have been isolated from these, with the sphere-formation assay ${ }^{1}$. Hence, the cancer stem cell phenotype has been evaluated by several cellular assays/ stainings, by flow cytometry analysis and by analyzing the profiles expressions of a few genes related to CSCs.

Results: We have established primary cell cultures of high grade osteoblastic OS from each samples collected. Consequently, from these we have started to isolate CSCs. Nowadays we have established a OS-CSC line, called as OSA5-CSC. The cancer stem cell phenotype from OSA5CSC line was confirmed by observing the capacity of the OSA5-CSCs to differentiate into osteoblasts and into adipocytes, by showing the positive presence of the MSCs and of the ESCs markers into the cell line, and by evaluating a good rate $(14 \%)$ as clonogenic capacity. The presence of the ESC markers was confirmed also by their gene expression together with the expression of CD133. On the other hand, the malignant phenotype was verify by demonstrating the presence of the expression of genes involved in invasion/ migration process (i.e., AXL and EZR) and in the pluripotency of CSC (i.e., MYC), by the agar soft and by the ALDH1A1 assays.

Conclusions: In conclusion, we have collected a few biopsies of this rare bone disease. We have established primary cell cultures from these. We have completely characterized and studied a OS-CSC line at cellular and molecular level. Nowadays we are concluding the characterization of the OSCSCs isolated from the relative cell cultures and we are starting the study of micro-RNAs related to their phenotype. References: ${ }^{1}$ Gibbs CP et al., Neoplasia.2005;7:967.

Acknowledgements: Supported by the Istituto Toscano Tumori (ITT)_Regione Toscana_Grant Proposal 2010.

\section{P573}

THE VALUE OF ULTRASOUND IN THE DIAGNOSIS OF FIRST CARPOMETACARPAL JOINT OSTEOARTHRITIS

L. Giovannini ${ }^{1}$, A. Calabro ${ }^{1}$, L. Tofani ${ }^{1}$, M. Bustamante ${ }^{1}$, C. Mariana $^{1}$, F. Holzmeister ${ }^{1}$, C. Zorzanelli ${ }^{1}$, R. Ranza ${ }^{1}$, M. Matucci Cerinic ${ }^{1}$

${ }^{1}$ Università di Firenze, Firenze, Italy

Objective: First carpometacarpal (CMC) joint osteoarthritis (OA) (or rhizoarthrosis) is a degenerative disease of cartilage and bone that affects women more frequently than men, whose incidence increases with aging. Several factors, including joint biomechanics, are thought to be implicated in its pathogenesis. This condition can be very disabling, causing significant pain and leading to limitations in functionality of the hand and reduced grip strength. The ultrasonography (US) generated an increasing popularity among rheumatologists due to excellent potentiality and numerous applications in rheumatology (1). Although the application of ultrasonography to inflammatory diseases has been common and widespread, it has been applied to osteoarthritis less frequently (2). The aim of our study was to evaluate first CMC joint osteoarthritis sonographic features in order to assess an ultrasound cutoff value.

Materials and methods: We enrolled 45 symptomatic patients with primary first CMC joint osteoarthritis developed within 24 months and 81 healthy controls and we performed ultrasound of the I CMC joint measuring the articular space and misalignment between the trapezium and first metacarpus. Results: The mean articular space in CMC osteoarthritis patients was $1.01 \mathrm{~mm}$ (SD 0.58) vs. 1.89 (SD 0.55) in the control group, indeed the medium misalignment was $3.06 \mathrm{~mm}$ (SD 1.93 ) in the patient group and $1.50 \mathrm{~mm}$ (SD 1.15) in controls, respectively. We confirmed that articular space discriminates 
healthy controls between rhizoarthrosis patients [area under the curve -AUC- 0.9696 (CI 0.9485-0.9907)]. Furthermore we observed that a CMC articular space inferior to $1.2 \mathrm{~mm}$ allows distinction between patients and controls. In our population, this cut off value has a sensibility of $95.5 \%$ and a specificity of $89.3 \%$ ( -value $<0.0001$; odds ratio $<0.001$ ). Indeed we could not identify differences in bone misalignment, because of the high heterogeneity in the obtained data. Conclusion: With this study we demonstrated that ultrasound allows early detection of I CMC osteoarthritis alterations and we identified, for the articular space, a cut off value with remarkable diagnostic accuracy that discriminate rhizoarthrosis patients. However these data need external validation.

\section{References:}

1. Keen HI, Wakefield RJ. Ann Rheum Dis 2009;68:611

2. D’Agostino MA, Conaghan PG. J Rheumatol 2009;36:1829

\section{P574}

THE EFFECTIVENESS OF DYNAMIC ELECTRONEUROSTIMULATION (DENS) IN THE PAIN MANAGEMENT IN KNEE OSTEOARTHRITIS: RESULTS OF THE MULTICENTER RANDOMIZED TRIAL

O. M. Lesnyak ${ }^{1}$, E. Y. Kadochnikova ${ }^{1}$, A. A. Vlasov ${ }^{2}$, L. I. Alekseeva $^{3}$, I. G. Didikina ${ }^{4}$, O. B. Ershova ${ }^{4}$, E. M. Zaitseva ${ }^{3}$, T. A. Popova ${ }^{5}$, E. A. Taskina ${ }^{3}$, V. N. Khodyrev ${ }^{1}$, E. P. Sharapova $^{3}$, A. G. Solodovnikov ${ }^{1}$, T. A. Korotkova ${ }^{3}$, M. L. Sukhareva ${ }^{3}$

${ }^{1}$ Ural State Medical University, Ekaterinburg, Russian Federation, ${ }^{2}$ Denas MS Corporation, Ekaterinburg, Russian Federation, ${ }^{3}$ Research Institute of Rheumatology, Moscow, Russian Federation, ${ }^{4}$ Yaroslavl State Medical Academy, Yaroslavl, Russian Federation, ${ }^{5}$ GKB№40 Rheumatology center, Ekaterinburg, Russian Federation

Dynamic electroneurostimulation (DENS) is a type of transcutaneous electrical neurostimulation (Tens), based on the use of weak (200-400mkA), low frequency (10-200 Hz) pulse currents. A feature of this type of physiotherapy is the pulse shape, which changes following the change of total electrical resistance of under electrode skin area, which enables prolonged use without "addiction".

Objective: was to investigate the effectiveness and safety of the DENS therapy in the pain management in knee osteoarthritis.
Subjects and methods: It was a multicenter randomized double blind placebo-controlled trial. 132 subjects with knee osteoarthritis were recruited. Inclusion criteria were: the pain severity more than $40 \mathrm{~mm}$ by the visual analogue scale, the Lequesne index between 4 and 12. The DENS group (66 subjects) received the DENS treatment on the target knee, by the applicator, connected to DiaDENS PC device, with the stimulation parameters $-77 \mathrm{~Hz}$ frequency and $15 \mathrm{~mA}$ intensity of the current. Patients in the placebo group received the placebo procedure. The treatment duration was $30 \mathrm{~min}$ at the 10 sessions. The outcome measure was a knee pain severity measured by the visual analogue scale (VAS). All participants were evaluated before and after the treatment procedure at the 1, 3, 6, 10 treatment days and after 2 and 4 weeks of the followup. Both groups were comparable at baseline for the evaluated indicators, except for difference in the patients age: patients in the placebo group were older than the DENS group by an average 3.2 years. In the DENS group twice more patients received nonsteroidal antiinflammatory drugs (NSAIDs) in comparison with placebo group, although statistically insignificant $(p=0.101)$.

Results: A distinct analgesic effect associated directly with the treatment procedure was found in the DENS group: after each DENS procedure, 5-8 mm VAS pain reduction was noted. The largest immediate analgesic effect of therapy in the DENS group was found after the first $(8.73 \mathrm{~mm} \mathrm{VAS})$ and the sixth procedure (6.28 mm VAS). In the placebo group, immediate analgesic effect was not observed. A statistically significant difference between two groups was noted after the first $(p=0.014)$, third $(p=0.018)$, and sixth $(\mathrm{p}=0.047)$ treatment procedures. In both groups there was a statistically significant reduction in pain during the treatment $(p<0.0001)$. We did not register statistically significant difference in groups in NSAIDs use during the whole trial. Although, even during the follow-up the difference in pain intensity between two groups was still statistically significant $(p=0.003)$. Both the physicians $(p<0.0001)$ and the patients $(p=0.004)$ positively evaluated the effectiveness of the DiaDENS PC treatment in the DENS group as compared to the placebo group. The side effects frequency did not differ significantly in the DENS group from placebo and the DENS therapy was well tolerated by patients $(p=0.999)$. 
Conclusion: Dynamic electroneurostimulation is an effective and safe method of pain relief in patients with knee osteoarthritis.

\section{P575}

\section{THE LEVEL OF ADHERENCE TO CALCIUM AND VITAMIN D IN RELATION TO DEMOGRAPHIC VARIABLES IN POSTMENOPAUSAL WOMEN WITH LOW BONE MINERAL DENSITY}

B. Erdeljan $^{1}$, J. Zvekic-Svorcan ${ }^{1}$, M. Maksimovic ${ }^{1}$, B. Lazarevic $^{1}$, M. Lazarevic ${ }^{1}$

${ }^{1}$ Rheumatology, Special Hospital for Rheumatic Diseases, Novi Sad, Serbia

Objective: To show the level of adherence to calcium and vitamin $\mathrm{D}$ in relation to demographic variables in postmenopausal women with reduced BMD.

Material and Methods: The prospective analysis is performed from 20 November to 20 December 2015 at the Special Hospital for Rheumatic Diseases Novi Sad, Serbia. The study involved 80 postmenopausal women who were diagnosed with reduced BMD by DXA and all patients filled in the same questionnaire. Morisky scale was used to analyze adherence to calcium and vitamin D intake. We also monitored correlation of adherence according to age, site of residence and level of formal education. The central tendency measures, ANOVA test and $\chi^{2}$ test were used for statistical analysis.

Results: The average age of participants was 65.52 \pm 8.29 years. $65 \%$ live in the city and $35 \%$ in the countryside. $28.8 \%$ had basic, $55 \%$ medium and $16.2 \%$ high level of education. $67.5 \%$ had osteoporosis (duration $M 4.29 \pm 3.36$ years) and $32.5 \%$ osteopenia (duration M 3.54 \pm 2.42 years). Most patients showed a low level of adherence to calcium $(81.3 \%)$ and vitamin D (82.8 \%). Looking at the age there is no statistically significant difference in relation to the medium and low adherence to calcium $(\mathrm{F}=1.92$, $p=0.172, p>0.05)$ and vitamin $\mathrm{D}(\mathrm{F}=0.399$, $p=0.530, p>0$. 05). Considering urban and rural population and the level of education there is no statistically significant difference related to adherence to calcium $\left(\chi^{2}=0.093, \mathrm{df}=1, p=0.760, p>0.05 ; \chi^{2}=0.111\right.$, $\mathrm{df}=2, p=0.946, p>0.05)$ and vitamin $\mathrm{D}\left(\chi^{2}=0.001\right.$, $\mathrm{df}=1, p=0.974, p>0.05 ; \chi^{2}=1.22, \mathrm{df}=2, p=0.542$, $p>0.05)$.
Conclusion: In all observed variables there are critically low adherence to calcium and vitamin D, which may cause complications in patients with reduced BMD. We should actively seek causes of the low adherence and ways of its increase in order to better treatment and prevention of complications.

\section{P576}

DETERMINANTS OF VITAMIN D DEFICIENCY AMONG SAUDI MALES AND FEMALES IN RIYADH, SAUDI ARABIA

A. M. AlQuaiz ${ }^{1}$, M. Fouda Neel ${ }^{1}$, A. Kazi ${ }^{1}$

${ }^{1}$ Research chairs program, Princess Nora Chair for Women's Health Research, College of Medicine, King Saud University, Riyadh, Saudi Arabia

Introduction: Internationally as well as nationally, Vitamin D deficiency has become a recognized public health issue. The objective of this study was to measure the prevalence and correlates of vitamin D deficiency in men and women in Riyadh, Saudi Arabia.

Methodology: A cross-sectional survey was designed and conducted in all five areas of Riyadh. We randomly selected 18 Primary health care centers, 14 schools and 8 Government offices from all over Riyadh. A total of 2997 Saudi male and females aged between 30-75 years participated in the study. Four female and two male phlebotomists conducted the interviews, took the anthropometric measurements and blood samples for vitamin D assessment. Interviews were based on structured questionnaire comprising of common factors associated with vitamin D deficiency. Blood samples were analyzed for vitamin D status.

Results: In total 2,997 people participated in the study; 2029 females and 968 males. The mean $( \pm$ SD) age was $43.6( \pm 11.2)$ years; females $43.9( \pm 10.9)$ and males 41( \pm 11.7$)$. Results for vitamin $\mathrm{D}$ found that around $90 \%$ males (866) and $82 \%$ (1660) females are suffering from vitamin D insufficiency or deficiency. Mean vitamin D levels were significantly lower in males as compared to females $(42.7( \pm 24.1$ vs $48.0( \pm 30.1)$; $\mathrm{p}<0.001)$. Multiple logistic regression analysis for males found that odds for belonging to age group 41-50 years and 30-40 years were associated with 5.0 (95\% CI 1.8,13.3) and $3.9(95 \%$ CI $1.7,9.2)$ times of having vitamin D deficiency as compared to 61-75 years old. Similarly, participants not taking vitamin D supplements were 3.8 
(95\% CI1.9, 7.4) times more atrisk of vitamin D deficiency as compared to those who were taking supplements. For females, we found that in addition to younger age $(30-40$ years OR $2.9(95 \%$ CI $1.7,4.8)$ and not taking vitamin D supplements (OR 3.4, 95\%CI 2.6,4.5), low income (OR 1.8, 95\% CI 1.1, 3.0), less fruit intake (OR 1.7, 95\% CI 1.2,2.5), and increased waisthip ratio (OR 1.4, 95\% CI 1.1, 1.9) were associated with increased risk for vitamin D deficiency.

Discussion: It is commonly believed that vitamin D is a problem of the elderly population, hence various effective measures are being taken to prevent this problem in the elderly, however, it is high time that similar measures are taken to prevent this problem in the young population as well, among both males and females. Healthy life style can help in preventing vitamin D deficiency among all age groups.

\section{P577}

\section{FRACTURE DISCRIMINATION USING A NOVEL PULSE-ECHO ULTRASOUND DEVICE} J. P. Karjalainen $^{1}$, O. Riekkinen ${ }^{1}$, J. T. Schousboe ${ }^{2}$

${ }^{1}$ Bone Index Finland Ltd., Kuopio, Finland, ${ }^{2}$ HealthPartners Institute, Minneapolis, United States While DXA remains the primary method of diagnosing osteoporosis, it has the disadvantages of being nonportable and exposing those having the test to some ionizing radiation. Although the cost of DXA is modest, this is still sufficient to limit access. The aim of this study was to estimate whether or not pulse-echo ultrasonometry (US) can discriminate those who had from those who did not have fractures. In total 555
Caucasian female subjects were enrolled between ages 50 and 89 stratified by age decade. Subjects were examined using US measurements of cortical bone thickness (radius and tibia) and Density Index (DI) 1 and DXA measurement of BMD of the femoral neck and total hip. Fractures within the prior five years before the study date were confirmed by review of all radiology reports by the author (JTS). Logistic regression models were run to estimate the associations of multisite and single-site DI, cortical thickness each with confirmed clinical fractures. Ninety-five individuals $(17.0 \%)$ had 102 fractures within the five years prior to the study date. The majority of these were in the distal radius/wrist, lumbar spine, or thoracic spine. One standard deviation decrease in femoral neck BMD was associated with fractures with an odds ratio of 1.47 (95\% C.I. 1.11 to 1.95). Decreases of multi-site and single-site DI and cortical thickness at the proximal and distal tibia were similarly modestly associated with a higher age-adjusted odds ratio of a fracture (table 1). Measures of cortical thickness of the tibia were as strongly associated with radiographically confirmed fractures as was femoral neck BMD, and our results compare favorably to the discrimination of prior fractures that have been shown with other ultrasound and peripheral bone mass measurement devices. Pulse-echo US shows promise as a tool for fracture risk assessment, and future prospective studies on prediction of incident fractures are warranted.

\section{References:}

[1] Karjalainen, Osteoporos Int., 2015

Disclosures:Karjalainen, Riekkinen, stock ownership, Bone Index Finland Ltd.

Table 1. Associations* of Bone Ultrasound Parameters with clinical Fracture (Confirmed on Clinical Radiographs) within the Prior 5 years

\begin{tabular}{|c|c|c|c|}
\hline \multirow[t]{2}{*}{ Parameter } & \multicolumn{3}{|c|}{ Odds Ratio per SD decrease (95\%C.I.) } \\
\hline & $\begin{array}{l}\text { Ultrasound Parameter, } \\
\text { Age-adjusted only }\end{array}$ & $\begin{array}{l}\text { Ultrasound Parameter Adjusted for Age } \\
\text { \& Femoral Neck BMD }\end{array}$ & $\begin{array}{r}\text { Femoral Neck BMD (Adjusted for } \\
\text { Age and Ultrasound Parameter) }\end{array}$ \\
\hline Multi-Site Density Index & $1.36(1.05$ to 1.76$)$ & $1.18(0.88$ to 1.58$)$ & 1.37 (1.01 to 1.86$)$ \\
\hline Single-Site Density Index & 1.37 (1.04 to 1.82$)$ & $1.20(0.89$ to 1.61$)$ & $1.38(1.03$ to 1.85$)$ \\
\hline Proximal Tibia Thickness & $1.56(1.18$ to 2.06$)$ & $1.43(1.08$ to 1.91$)$ & $1.34(1.01$ to 1.78$)$ \\
\hline Distal Tibia Thickness & $1.38(1.09$ to 1.76$)$ & $1.29(1.00$ to 1.65$)$ & $1.38(1.05$ to 1.82$)$ \\
\hline Distal Radius Thickness & $1.09(0.83$ to 1.43$)$ & $0.98(0.74$ to 1.29$)$ & $1.47(1.11$ to 1.95$)$ \\
\hline
\end{tabular}

*Association with p-values $<0.05$ shown in bold italics 


\section{P578}

\section{VITAMIN D STATUS AND BODY COMPOSITION IN ATHLETES}

T. Karonova ${ }^{1}$, P. Globa ${ }^{2}$, M. Budanova ${ }^{1}$, S. Shishkin ${ }^{2}$, E. Grineva $^{1}$

${ }^{1}$ Federal Almazov North-West Medical Research Centre, St. Petersburg, Russian Federation, ${ }^{2}$ First St. Petersburg State Medical University, St. Petersburg, Russian Federation

Objectives: Recent studies have suggested high prevalence of vitamin D deficiency in general population. However, data from athletes are still contradictive. We examined athletes from North-West region of Russia (St. Petersburg) and analyzed serum 25(OH)D level to assess potential interlinks between vitamin D status and kind of sport and body composition.

Material and Methods: A total of 120 athletes aged from 15 to 25 years (mean $18.0 \pm 1.8$ ), 64 male and 56 female, were examined. DXA (Lunar Prodigy, USA) was performed in 50 subjects. Serum levels of $25(\mathrm{OH}) \mathrm{D}$ (AbbottArchitect 8000) was performed using lab kits for AbbottArchitect 8000, intra-assay CV ranged from 1.60 to $5.92 \%$ whereas the inter-assay $\mathrm{CV}$ ranged from 2.15 to $2.63 \%$.

Results: The study results showed that serum $25(\mathrm{OH}) \mathrm{D}$ level was between 9.9 and $80.2 \mathrm{ng} / \mathrm{ml}$ and was lower in males than in females $(19.8 \pm 0.9 \& 23.2 \pm 1.3 \mathrm{ng} / \mathrm{ml}$ accordingly; $p=0.07$ ). We found negative correlation between 25(OH)D level and weigh $(r=-0.21, p=0.013)$ and BMI $(r=-0.20, p=0.016)$. Only 17 subjects $(14.2 \%)$ had $25(\mathrm{OH}) \mathrm{D}$ level more than $30 \mathrm{ng} / \mathrm{ml}, 22$ subjects $(35 \%)$ were insufficient and 61 subjects $(50.8 \%)$ - deficient. This data was similar to the previous results we had seen in the general population of the North-West region of Russia. Lower 25(OH)D level was found in sportsmen involved is such sport as basketball, volleyball and handball than in swimmers, gymnasts and racing cyclists $(16.8 \pm 1.1 \& 21.7 \pm 1.6 \mathrm{ng} / \mathrm{ml}$ accordingly; $p<0.05)$. Analysis of vitamin $\mathrm{D}$ status in athletes who have 2 hours indoor training session per day in comparison to subjects who only have indoor training sessions did not show any difference (21.9 $\pm 1.2 \& 21.1 \pm 0.9 \mathrm{ng} / \mathrm{ml})$. BMD correlated with height and was higher in team-ball players $(4044.9 \pm 145.6 \mathrm{~g})$ than others $(3239.2 \pm 83.8 \mathrm{~g}, p<0.05)$. Though we did not find an interlink between BMD, lean quantity and $25(\mathrm{OH}) \mathrm{D}$ level in athletes.

Conclusion: Our results showed that healthy sportsmen have low 25(OH)D level and high prevalence of vitamin D insufficiency regardless of the quantity of indoor training sessions. We found that body composition did not depend on vitamin D status in athletes.
P579

CONTINUOUS IMPROVEMENT OF IMPAIRED TRABECULAR BONE MICROARCHITECTURE AFTER 3 YEARS ON GLUTEN-FREE DIET: A PROSPECTIVE LONGITUDINAL HR-PQCT STUDY IN WOMEN WITH CELIAC DISEASE

M. B. Zanchetta ${ }^{1}$, V. Longobardi ${ }^{1}$, F. Costa $^{2}$, C. Bogado ${ }^{1}$, J. $\mathrm{Bai}^{2}$, J. R. Zanchetta ${ }^{1}$

${ }^{1}$ Idim, Universidad del Salvador, Ciudad Autónoma de Buenos Aires, Argentina, ${ }^{2}$ Hospital de Gastroenterología Dr. Bonorino Udaondo, Ciudad Autónoma de Buenos Aires, Argentina

Previously we identified a significant deterioration of trabecular and cortical microarchitecture in peripheral bones of patients with undiagnosed celiac disease (CD) using HR-pQCT. Such affectation was mainly produced in the trabecular bone. Up to now, the effect of the gluten-free diet (GFD) on microstructural parameters of peripheral bones has not been assessed.

Aim: To describe three-year changes of bone microstructure produced by GFD in a prospective cohort of premenopausal women with newly diagnosed $\mathrm{CD}$. We prospectively enrolled 31 consecutive females with newly diagnosed $\mathrm{CD}$. Up to now, 19 patients were reassessed at a mean time of 35.1 \pm 3.6 months after diagnosis. Clinical and biochemical status, $\mathrm{CD}$ specific serology, bone densitometry and microstructural determinations (HR-pQCT) were performed at both time points.

Results: Patients mean age was $34 \pm 8.8$ years and BMI $25.4 \pm 7.4$ at three years' time (previous value $24.1 \pm 6.5$ $p=0.06$ ). PTH and vitamin D were the laboratory parameters that improved significantly over three-y compared to baseline (PTH $44.5 \pm 17.3$ vs. $59.3 \pm 23$, $p=0.02$ and vitamin $\mathrm{D} 23.5 \pm 5$ vs. $17.6 \pm 5.7, p<0.01$ ), without significant change in serum calcium and cross laps. The absolute DXA values were significantly increased in lumbar spine, total hip and ultradistal radius (Table 1). The microstructure of the trabecular compartment in the distal radius was significantly improved (BV/TV, trabecular density and trabecular thickness, $p<0.01)$ as well as the total volumetric density $(p=0.03)$. At the level of tibia, treatment was associated with significant increase of the total volumetric density, cortical density, trabecular density, BV/TV and trabecular thickness $(p<0.01)$.

Conclusions: Our study shows that trabecular parameters impaired at the time of diagnosis continue to improve significantly over time with GFD in this group of young premenopausal women. We postulate that trabecular bone microarchitecture improvement might be the substrate associated with the decreased risk of fractures observed after GFD in former studies. 
Table 1.

\begin{tabular}{|c|c|c|c|c|}
\hline & & Basal Assessment & 3 years GFD & $\mathrm{P}$ \\
\hline \multirow[t]{3}{*}{ Anthropometric data } & Height (m) & $1.59 \pm 0.05$ & $1.58 \pm 0.04$ & 0.89 \\
\hline & Weight (kg) & $60.4 \pm 14.8$ & $63.6 \pm 18.4$ & 0.13 \\
\hline & BMI $(\mathrm{kg} / \mathrm{m} 2)$ & $24.1 \pm 6.5$ & $25.4 \pm 7.4$ & 0.06 \\
\hline \multirow[t]{3}{*}{ Lab test } & Crosslapps & $396.7 \pm 206.5$ & $338 \pm 169.8$ & 0.36 \\
\hline & PTHi (65) & $59.3 \pm 23$ & $44.5 \pm 17.3$ & 0.02 \\
\hline & Vitamin D & $17.6 \pm 5.7$ & $23.5 \pm 5$ & $<0.01$ \\
\hline \multirow[t]{2}{*}{ 0DXA L1-L4 } & BMD & $1.129 \pm 0.153$ & $1.170 \pm 0.150$ & $<0.01$ \\
\hline & Z-score & $-0.3 \pm 1.1$ & $-0.1 \pm 1.1$ & 0.03 \\
\hline \multirow[t]{2}{*}{ DXA Femoral Neck } & $\mathrm{BMD}$ & $0.929 \pm 0.098$ & $0.943 \pm 0.107$ & 0.10 \\
\hline & Z-score & $-0.2 \pm 0.8$ & $-0.2 \pm 0.9$ & 0.38 \\
\hline \multirow[t]{2}{*}{ DXA Total Hip } & $\mathrm{BMD}$ & $0.940 \pm 0.157$ & $0.970 \pm 0.155$ & $<0.01$ \\
\hline & Z-score & $-0.4 \pm 1.1$ & $-0.2 \pm 1.1$ & 0.05 \\
\hline \multirow[t]{2}{*}{ DXA Radio UD } & DMO & $0.398 \pm 0.048$ & $0.427 \pm 0.038$ & $<0.01$ \\
\hline & Z-score & $-1.5 \pm 1.1$ & $-0.8 \pm 0.9$ & $<0.01$ \\
\hline \multirow[t]{6}{*}{ Xtreme-CT Distal Radius } & Total Density (mg HA/cm³) & $290 \pm 47.6$ & $302.7 \pm 45.4$ & 0.03 \\
\hline & Trabecular Density $\left(\mathrm{mg} \mathrm{HA} / \mathrm{cm}^{3}\right)$ & $125.3 \pm 25.1$ & $141 \pm 24.4(+12.5 \%)$ & $<0.01$ \\
\hline & $\mathrm{BV} / \mathrm{TV}(\%)$ & $10.4 \pm 2.1$ & $11.7 \pm 2(+12.5 \%)$ & $<0.01$ \\
\hline & Trabecular number $(1 / \mathrm{mm})$ & $1.75 \pm 0.19$ & $1.78 \pm 0.26$ & 0.53 \\
\hline & Trabecular Thickness (mm) & $0.059 \pm 0.009$ & $0.066 \pm 0.009(+9 \%)$ & $<0.01$ \\
\hline & Cortical Density (mg HA/ $\mathrm{cm}^{3}$ ) & $882.8 \pm 39.1$ & $890 \pm 33.5$ & 0.19 \\
\hline \multirow[t]{6}{*}{ Xtreme-CT Distal Tibia } & Total density $\left(\mathrm{mg} \mathrm{HA} / \mathrm{cm}^{3}\right)$ & $273.9 \pm 37.6$ & $289.4 \pm 43.9$ & $<0.01$ \\
\hline & Trabecular Density $\left(\mathrm{mg} \mathrm{HA} / \mathrm{cm}^{3}\right)$ & $131.6 \pm 30$ & $145.9 \pm 32.4$ & $<0.01$ \\
\hline & $\mathrm{BV} / \mathrm{TV}(\%)$ & $11 \pm 2.5$ & $12.2 \pm 2.7$ & $<0.01$ \\
\hline & Trabecular number $(1 / \mathrm{mm})$ & $1.63 \pm 0.32$ & $1.64 \pm 0.34$ & 0.85 \\
\hline & Trabecular Thickness (mm) & $0.068 \pm 0.011$ & $0.075 \pm 0.014$ & $<0.01$ \\
\hline & Cortical Density (mg HA/cm³) & $911.1 \pm 37.9$ & $929 \pm 36.3$ & $<0.01$ \\
\hline
\end{tabular}

\section{P580}

\section{THE RUDY STUDY: A NOVEL APPROACH TO PATIENT DRIVEN RESEARCH IN RARE MUSCULOSKELETAL DISEASES}

L. Watts ${ }^{1}$, L. Zhang ${ }^{1}$, A. Turner ${ }^{1}$, H. Teare ${ }^{2}$, D. Gray ${ }^{1}$, N. Gray $^{1}$, R. Popert ${ }^{1}$, J. Hogg ${ }^{1}$, J. Barrett ${ }^{1}$, R. Luqmani ${ }^{1}$, P. Wordsworth $^{1}$, J. Kaye ${ }^{2}$, M. K. Javaid ${ }^{1}$

${ }^{1}$ Nuffield Department of Orthopaedics, Rheumatology and Musculoskeletal Sciences, University of Oxford, Oxford, United Kingdom, ${ }^{2}$ Centre for Health, Law and Emerging Technologies (HeLEX), University of Oxford, Oxford, United Kingdom

Objectives: Rare disease research is more challenging as patients are often geographically dispersed, may not be able to travel long distances for traditional research studies and recruitment via tertiary centres would result in a sample biased towards more severe disease. We here describe the initial results from the RUDY study (rare and undiagnosed diseases study), a prospective cohort study of rare musculoskeletal diseases which uses a web based platform and patient involvement to maximise engagement and satisfaction.

Materials and Methods: Participants are eligible if they are aged $0-100$ with a rare disease (defined as a prevalence of less than 1 in 2000) or are related to somebody with a rare disease. Recruitment occurs via registration on a secure website, followed by a telephone call from the research team and a consent form. Consent is dynamic, with participants able to alter their consent options online at any time. Data is collected via the secure website, with a timeline of significant clinical events and six monthly validated questionnaires addressing quality of life, pain, sleep, function, fatigue and mood.

Results: To date, 371 participants with rare musculoskeletal conditions have been recruited, including 83 with osteogenesis imperfecta, 63 with fibrous dysplasia, 34 with X-linked hypophosphatemia and 50 with granulomatosis with polyangiitis. Baseline questionnaire completion is in excess of $50 \%$ for all conditions. One individual has withdrawn and two have amended their consent options. Participant involvement has been essential, with substantial changes to study design 
resulting from participant suggestions via the RUDY patient forum.

Conclusions: RUDY has successfully addressed many of these issues through a web-based platform and high participant engagement in study design and potential for use in other diseases and nations.

\section{P581}

WHY IS IMPORTANT TO EMPHASIZE THE RISK FACTORS FOR OLDER FEMALES WITH HIP OSTEOARTHRITIS: OBSERVATIONAL STUDY

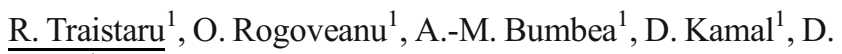
Matei $^{1}$

${ }^{1}$ University of Medicine and Pharmacy, Craiova, Romania

Background and Purpose: Several risk factors have been linked to hip osteoarthritis (HOA); malalignment of hip (coxa valga, dislocation of hip) represents one of the mechanical factor that influences on cartilage loss and osteoarthritis progression. C-telopeptide of type II collagen (CTX-II), a specific marker reflecting degradation of articular cartilage, is one of the most important biochemical biomarkers of collagen degradation and potential indicator for early articular cartilage degeneration. In our cross-sectional study we investigated the correlations and association between various factors (clinical, hip alignment, functional status and value of serum measurement of CTX-II) in older females with disabling HOA.

Materials and Methods: We studied 42 females aged between 69-76 years, with painful HOA diagnosed by ACR criteria. In all patients, clinical, functional and radiographic examination was performed; we used VAS scale for pain and the WOMAC index for functional status. Hip radiographs were evaluated according to the Kellgren and Lawrence (KL) classification. Serum CTX-II levels were analyzed with an enzyme-linked immunosorbent assay (ELISA) kit. We followed the correlations between hip malalignment, CTX-II level and the scale scores that defined painful disabling status. We used Pearson's correlation and linear regression model.

Results: According to the KL grading scale, 13 patients were KL grade 2, 15 patients were KL grade 3 and 14 patients were KL grade 4 . We found significant correlations between KL grade, pain and disabling status (R 0.735) and the CTX - II levels (R 0.721 ). The values of correlation and predictivity were significant in women with previous dislocation of hip (R 0.73).

Conclusions: A complex relationship exists between loss of cartilage and hip malalignment. The serum CTX-II level could really reflects the cartilage degradation. The mechanical disturbances and biochemical biomarkers of collagen degradation are important to take into account for older patients with HOA, focus mainly in the early stage of this multifactorial disease.

\section{P582}

THE ROLE OF REHABILITATION IN ACTIVE PATIENTS WITH ATRAUMATIC OSTEONECROSIS OF THE FEMUR HEAD

R. Traistaru ${ }^{1}$, A.-M. Bumbea ${ }^{1}$, O. Rogoveanu ${ }^{1}$, D. Kamal ${ }^{1}$

${ }^{1}$ University of Medicine and Pharmacy, Craiova, Romania

Background and Aim: Atraumatic osteonecrosis of the femoral head (AONFH) is a pathologic process with multifactorial etiology that results from disruption of the osseous arterial inflow or the venous outflow. The risk factors include corticosteroid administration, heavy alcohol intake, sickle cell disease, and other systemic diseases. The successful treatment is related directly to the stage of disease at diagnosis. Early detection and treatment with physiotherapy and kinetic program is the key to nonsurgical management. The objective of our study (a single blind, randomized controlled trial) was to evaluate the effect of rehabilitation program on clinical and functional parameters and to assess the short-term effects of this program on quality of life of patients with AVNFH.

Materials and Methods: 28 patients aged between 34-43 years with AONFH stage I and II, were randomized into two groups: Group 1-16 patients (G1) was treated by complex therapy (medication, stretching exercises and pedaling of the bike, physical therapy - ultrasound and interferential current, modification of activity - the use of a walking aid), and group 2-12 patients (G2) control receiving only medication. The duration of rehabilitation program was 4 weeks. All patients were evaluated initial and finally. The outcome assessments at the beginning and at the end of the rehabilitation were pain intensity (with dolorimeter), six minute walk test and WOMAC Index.

Results: In both groups, improvements were found in pain intensity ( $55 \%$ in G1 and $23 \%$ in G 2, respectively) and this reduction was significantly higher in G1. The mean value of 6MWD and WOMAC Index showed significant improvements in G1 group.

Conclusions: Our study confirms the literature data that rehabilitation program can be very effective in treating AVNFN if it is detected early. Conservative treatment with physical therapy, modification of activity, and analgesics are best treatment measures for precollapse stages I and II. Decreasing the load that the hip joint bears, proper protection from vigorous activities, maintaining range of motion and muscle strength are crucial to avoid further femoral head collapse and managing pain. 


\section{P583}

\section{BONE DENSITY IN PATIENTS WITH PRIMARY HYPOTHYROIDISM: EFFECTS OF THYROID HORMONE THERAPY}

I. Pankiv $^{1}$

${ }^{1}$ Endocrinology, Bukovinian State Medical University, Chernivtsi, Ukraine

Objective: Previous studies on BMD abnormalities associated with hypothyroidism are scarce and not conclusive.

Material and Methods: This cross-sectional descriptive study was performed on 95 women aged over 55 years with primary hypothyroidism. They were assigned to one of three study groups: Group I consisted 30 patients who had been recently diagnosed with primary hypothyroidism. The second groups of 45 patients diagnosed with primary hypothyroidism for at least 3 years and were treated with levothyroxine. The third group of 20 healthy individuals was selected as a control group. The bone density based on the T-score of femoral neck and lumbar vertebrae were compared between three groups. For densitometry, DXA was used to measure the bone density in lumbar vertebrae and femoral neck. The measurement was performed by an expert technician using the Lunar Prodigy Primo bone densitometer manufactured in USA (General Electric). Data of 95 individuals was analyzed by SPSS version 10 (SPSS, Inc., Chicago, IL, USA) using the regression analysis. $P<0.05$ was considered statistically significant.

Results: This study showed that the T-score at lumbar spine L2-L4 regions were significantly different. Prevalence of osteoporosis was $51.1 \%$ in second group that was higher than other groups. The mean T- score at lumbar spine L2-L4 region between second group and control group and between first and second groups were significantly different. Regression analysis has shown correlation between serum TSH levels and T-score at femoral neck and lumbar spine.

Conclusion: The treatment of primary hypothyroidism with thyroid hormones reduces bone density.

\section{P584}

COMPARISON AMONG ANTHROPOMETRIC INDICATORS THAT BEST PREDICT THE VISCERAL ADIPOSE TISSUE: PRELIMINARY RESULTS OF A SUBSAMPLE WITH OSTEOPOROSIS AND OSTEOPENIA FROM ISA-CAPITAL 2014/2105 STUDY

B. S. E. Peters ${ }^{1}$, M. M. S. Lima ${ }^{1}$, N. A. G. França ${ }^{1}$, E. B. Curatella $^{1}$, P. C. Santos ${ }^{1}$, L. A. Martini ${ }^{1}$

${ }^{1}$ Nutrition Department, School of Public Health, University of São Paulo, São Paulo, Brazil
Objective: The objective of this study was to evaluate which anthropometric indicator of low cost best predicts the visceral adipose tissue (VAT) in a subsample with osteoporosis and osteopenia.

Material and Methods: Were evaluated 206 people of both genders, $35.9 \%$ male and $64.1 \%$ female, with a mean age of 57.6 (14.09) years. Were evaluated such anthropometric indicators: Waist Circumference (WC), BMI and hip circumference, to be calculated waist to hip ratio (WHR). The first indicator was classified according to the cutoff point of the International Diabetes Federation (IDF), and the other two, according to the World Health Organization (WHO). The VAT was assessed by total body densitometry - DXA. Descriptive analyzes and Spearman correlation test were performed using SPSS 20.0. The significance level was $5 \%$. Results are expressed as mean (SD).

Results: The average BMI of the participants was 27.8 (5.3) $\mathrm{kg} / \mathrm{m}^{2}$. It was observed that $53.4 \%$ of individuals present overweight (obesity and overweight according to $\mathrm{BMI})$, and most of them had inadequate WC (81.9\%) and WHR (62.7\%) measurements. Considering the total group, BMI, WC and WHR were significantly correlated with VAT, however the strongest correlation was with the WC $(r=0.797 ; p<0.001)$ and the weakest with WHR $(r=0.614 ; p<0.001)$. When evaluated separately by gender, anthropometric indicator that presented stronger correlation with VAT in males was WC $(r=0.849$; $p<0.001)$, followed by WHR $(r=0.762 ; p<0.001)$, and women's group were the WC $(r=0.791 ; p<0.001)$ followed by the BMI $(r=0.769 ; p<0.001)$. In women WHR did not show significant correlation with VAT $(r=0.422 ; p<0.001)$, and in men the correlation between BMI and VAT was weaker compared to WC and WHR $(r=0.760 ; p<0.001)$.

Conclusions: In this population, WHR showed not be the best predictor of VAT, particularly in women. The WC was the indicator that showed the best correlation with the VAT in both genders, and in the total sample. Therefore, WC, as low cost and easy measurement showed to be the best predictor of visceral obesity.

\section{P585}

MATERNAL CALCIUM SUPPLEMENTATION AND OFFSPRING GROWTH

K. A. Ward ${ }^{1}$, L. Jarjou ${ }^{2}$, A. Prentice ${ }^{3}$

${ }^{1}$ MRC Lifecourse Epidemiology Unit, University of Southampton, Southampton, United Kingdom, ${ }^{2}$ MRC The Gambia Unit, Keneba, Gambia, ${ }^{3} \mathrm{MRC}$ Human Nutrition Research, MRC The Gambia Unit, Cambridge, Keneba, United Kingdom 
Objectives: To determine whether previously reported ${ }^{1}$, sex-specific effects of maternal calcium supplementation on offspring bone were robust to correction for bone and body size, and whether there were effects on body composition.

Materials and methods: In rural Gambia (West Africa) 447 children (216 males; 231 female), mean (SD) age 9.3(0.1)y, 9.2(0.1)y, respectively were measured. Their mothers were randomized to receive $1500 \mathrm{mg} / \mathrm{d}$ calcium carbonate

$(\mathrm{Ca})$, or placebo $(\mathrm{P})$, from 20wk gestation to term (ISCRTN96502494) ${ }^{2}$; children were not supplemented. At age 8-12 years, DXA (whole body) and pQCT (tibia) scans were performed; outcomes were DXA- bone mineral content (BMC), bone area, lean (LM) and fat mass (FM); pQCT- BMC, bone cross-sectional area and cortical area. Differences were tested for a sex-supplement effect using linear regression; length at 52 weeks, current age, weight (bone outcomes), height, DXA-BMC bone area were included in the model. Data are given as percent difference between children borne to the Ca vs. P groups (mean[SE]).

Results: There were no significant differences in bone outcomes between the $\mathrm{Ca}$ and $\mathrm{P}$ groups nor a sex- supplement interaction after size correction for weight, height and where applicable DXA bone area. There was a sexsupplement interaction for LM and FM, before (LM $p=0.08, \mathrm{FM} p=0.02$ ) and after adjustment for body size (LM $p=0.08$, FM $p=0.07$ ). Ca-boys had greater FM than P-boys (10 [4.9], $p=0.04)$ and Ca-girls less LM (-2.3 [0.08], $p=0.08)$ than P-girls.

Conclusions: Maternal Ca supplementation has affected longitudinal and somatic growth in this population and differences were divergent in boys and girls. Growth in Ca-boys was faster than P-boys, whereas in Ca-girl's growth was slower than P-girls. These data highlight the importance of not assuming more $\mathrm{Ca}$ is necessarily better for growth in populations accustomed to lower calcium intakes than international recommendations.

References:

${ }^{1}$ Ward et al. J Bone Miner Res 30(S1),

${ }^{2}$ Jarjou et al. Am J Clin Nutr 2006;83:

${ }^{3}$ Goldberg et al. Am J Clin Nutr 2013;98.

Acknowledgements: This research is jointly funded by the MRC and DFID under the MRC/DFID Concordat agreement U105960371, U123261351, MR/J004839/1

\section{P586 \\ POST TRAUMA GONARTHROSES: FORENSIC CONNOTATIONS}

C. Pusta $^{1}$, C. Nistor Cseppento ${ }^{1}$, A. Bodea ${ }^{1}$

${ }^{1}$ University of Oradea, Faculty of Medicine and Pharmacy, Oradea, Romania
Abstract: Post trauma injuries located to the knee is a frequently pathological in forensic practice. These lesions are most often the result of the mechanical traumatic actions (in $85 \%$ of cases). The purpose of this paper is to highlight the importance of knowledge about the development of the traumatic injuries and its complications, to an accurate forensic evaluation.

Material and Methods: We conducted a clinical trial and statistics within Bihor County Forensic Service, to a group of 50 people with traumatic injuries of the knee, aged between 25-69 years. Parameters analyzed: context and the production mechanism of injury, the initial diagnosis, the evolution and complications of injuries, age, sex, profession, personal history and family history of pathological collateral.

Results and Discussion: In the study group, the most common mechanism was the passive kicking (within some falls) (47\% of cases), followed by a complex mechanism with flexion / extension of the leg on the thigh and a forced knee rotation ( $35 \%$ of cases), and active hard hitting with blunt objects ( $18 \%$ of cases). Identified injuries were: injuries to soft tissue (bruising, hematoma, wound) (54\% of cases), joint damage (broken ligament, damage to the meniscus, sprains) (29\% of cases) and bone injuries, the most serious (fractured kneecap, broken upper epiphysis of the tibia / fibula and of the lower femur epiphysis) (17\% of cases). In $38 \%$ of cases, into the evolution of lesions, occurred complications; out of which the most common was osteoarthritis (48\%). The favoring factors of the osteoarthritis recorded to the group study were associated with: metabolic diseases, physically demanding professions, females, older aged, obesity, and the heredity. The occurrence of these complications required a forensic reassessment to these cases.

Conclusions: The occurrence of osteoarthritis into the development of a posttraumatic knee injury requires forensic revaluation of the case and increase the number of care days of medical care required granted healing.

Key words: knee, trauma, osteoarthritis, forensic revaluation.

\section{P587}

EFFECTIVENESS OF A MULTICOMPONENT PHYSICAL TRAINING INTEGRATED INTO A MULTIMODAL PRIMARY CARE PROGRAM IN HYPERFRAILTY OLDER: A RANDOMIZED CLINICAL TRIAL - STUDY POWER_AGING

A. Cuesta-Vargas $^{1}$, J. Bedoya-Belmonte ${ }^{2}$, E. Bueno-Caro ${ }^{2}$, E. Díaz-Caro $^{2}, \mathrm{M}$. González-Sánchez ${ }^{3}$, G. Ortega-Núñez ${ }^{2}, \mathrm{M}$. Rodríguez-González ${ }^{2}$, A. Galan-Mercant ${ }^{3}$

${ }^{1}$ Physiotherapy Department at Universidad de Malaga, Malaga, Spain, ${ }^{2}$ Tiro Pichón Primary Care Center, National Health Service., Malaga, Spain, ${ }^{3}$ Physiotherapy Department at Universidad de Jaen, Jaen, Spain 
Objective: To compare short-term and medium-term effectiveness of a multimodal program that integrates a program of therapeutic exercise, medication review, adequacy of nutrition and health education, compared with standard medical practice in improving neuromuscular and physiological status. Another aim is to analyze the maintenance of these effects by monitoring long-term.

Design: Randomized control trial.

Study subjects: Frailty older recruited in clinical unit Tiro Pichon, basic health area of Malaga into last tercil of Fried phenotype.

Methods: The subjects to be included in the study will be randomized into two groups: control group whose intervention will be to review adequacy of medication + food + health education (regular physical activity recommendation) and an experimental group whose intervention will consist of a multicomponent physical training integrated into a multimodal primary care program. Also tracking variables at baseline will be collected at 2 and 6 months after starting the intervention and at 3 and 6 months after the intervention (monitoring). The tracking measured variables are: anthropometric and sociodemographic, health services (visit, medications, etc.); neuro-cognitive-motor (attention-concentrationmemory, memory motor), spatial orientation, overall health, fatigue, neuromotor control (grip strength, balance, gait speed) and metabolomic variables.

\section{P588}

BONE DENSITY IN ALGERIAN PATIENTS WITH ANKYLOSING SPONDYLITIS

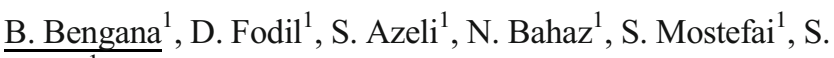
Lefkir $^{1}$

${ }^{1}$ Department of Rheumatology Beni-Messous Hospital, Algiers University Medicine Faculty, Algiers, Algeria

Background: Ankylosing spondylitis (AS) is a chronic inflammatory disease of the axial skeleton and the joints, characterized by osteoproliferation, leading to painful rigidity of the spine and disability.

Osteoporosis can be a complication of AS and can be an early event in this disease. The aims of our study is to explore prevalence of bone loss in a group of AS and to deduct risk factors for reduced BMD in this population.

Methods: A monocentric prospective study were conducted, including forty eight AS patients, naive for all biotherapy. A demographic checklist was completed and the disease activity was evaluated using BASDAI and ASDAS indexes. Serum levels of vitamin D, calcium, phosphorus were measured in all participants. DXA was done to evaluate the bone density.
Results: For the 48 patients, the mean age of patients were $28.62( \pm 9.28)$ years. Nineteen $(39.6 \%)$ of them were normal, $9(18.8 \%)$ were osteopenic, 20 (41.6\%) were osteoporotic. There was no relationship between the different groups (AS patients with osteopenia or osteoporosis and those with normal BMD) and the use of steroids. Statistically significant differences among the three groups were found for BMI, age and disease duration $(P=0.001, P<0.0001$ and $P=0.02$ respectively). Multivariate analysis revealed that the most significant factors associated with BMD were age and BMI $(P<0.0001)$. A slight but statistically significant correlation was also found for disease duration $(P=0.03)$.

Conclusion: In conclusion, our study showed that the prevalence of osteopenia and osteoporosis is high in a Algerian population of AS patients. Major risk factors for low BMD values were low BMI, age and disease duration.

P589

THE INFECTIOUS PROFILES OF BIOTHERAPEUTIC AGENTS IN AN ALGERIAN POPULATION

$\underline{\text { B. Bengana }}^{1}$, D. Fodil ${ }^{1}$, S. Azeli ${ }^{1}$, N. Bahaz ${ }^{1}$, S. Mostefai $^{1}$, S. Lefkir $^{1}$

${ }^{1}$ Department of Rheumatology Beni-Messous Hospital, Algiers University Medicine Faculty, Algiers, Algeria

Background: The management of inflammatory arthritis has been revolutionized by the use of biologic therapy. However, infections represent the first cause of morbidity in people treated for chronic inflammatory diseases (CID) with biotherapy. The main objective of the study is to describe the incidence and risk-factors for infections in people treated with biotherapeutic agents (BA) for CID.

Methods: For a period of nine years, from January 2006 to December 2015, a prospective and descriptive study recruited all patients treated by biologics for CID. We reported all infections encountered during the treatment.

Results: We have identified 107 patients; indications for BA were mainly rheumatoid arthritis in 45 cases, ankylosing spondylitis in 34 cases, 20 for enteropathic arthritis and 8 for psoriatic arthritis. The products used were adalimumab (33.6 \%) etanercept (29\%) rituximab (13\%) tocilizumab (13\%) and infliximab (11.2\%).There were 33 incidents of infections described. The majority of them were under corticosteroids and/or immunosuppressive drugs. Nine of them were diabetics, half of whom have a corticosteroidinduced diabetes. We have noted 23 viral infections, 10 bacterial infections, the most infections $(N=19)$ were 
under anti-tumor necrosis factor alpha (anti-TNF, 3 under tocilizumab, 1 under rituximab. The overall infection rates were significantly increased after anti $-\mathrm{TNF} \alpha$ treatment $(P<0.0001)$. All patients recovered and there were no instances of opportunistic infections, death or stopping treatment.

Conclusions: Infections events are more common in antiTNF $\alpha$ treated patients than those on rituximab or tocilizumab. The co-prescription of steroids and immunosuppressive drugs is an additive risk factor. This underlines the importance of adherence to guidelines for their prevention and management.

\section{P590}

INCIDENCE OF BISPHOSPHONATE ASSOCIATED

ATYPICAL FEMORAL FRACTURES IN A DISTRICT GENERAL HOSPITAL 2007-2014

O. Eseonu $^{1}$, M. Brown ${ }^{1}$, K. Cheng ${ }^{1}$

${ }^{1}$ Department of Trauma \& Orthopaedics, University Hospital Ayr, Ayr, United Kingdom

Introduction: Bisphosphonates are the mainstay of treat- ment for osteoporosis. Their use in Western Countries increased rapidly from the early $1990 \mathrm{~s}$, peaking between 2005 and 2008, and subsequently declining following reports, in literature and in the media, of adverse side effects from long-term use, including atypical femoral fractures. We sought to identify the trend in the presentation of atypical femoral fractures (AFFs), which are associated with bisphosphonate use, in the patient population of a District general hospital in South Ayrshire, United Kingdom, to see if this bore any correlation to the trends in bisphosphonate use.

Methods: We identified patients who had been implanted with proximal femoral nails - the primary management option for subtrochanteric and diaphyseal femoral fractures in our practice - between 2007, when operative records were computerized in our hospital, and 2014.

We subsequently examined the radiographs of these patients to identify atypical femoral fractures, using the American Society of Bone and Mineral Research (ASBMR) criteria $^{(1)}$. We also reviewed their medical notes to identify patients that had been on bisphosphonate therapy.

Results:

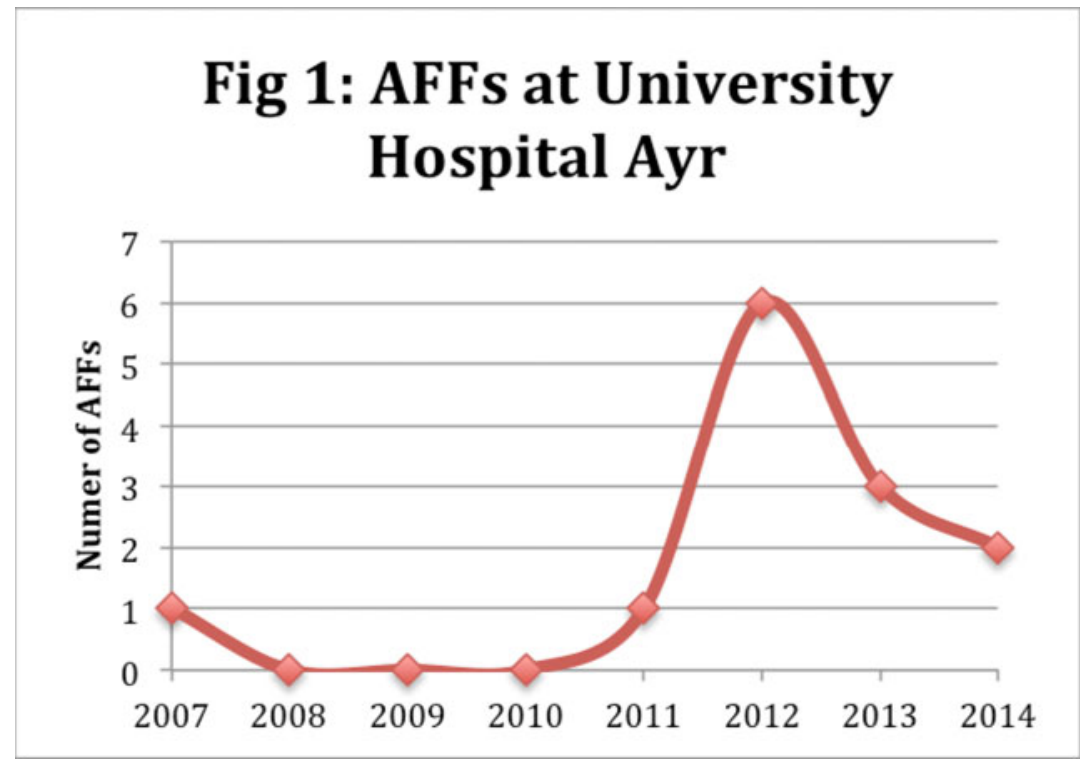

AFFs presenting at University Hospital Ayr peaked in 2012, and have subsequently shown a decreasing trend (Fig. 1).
11 of the 14 patients who presented with AFFs had been on long-term bisphosphonates for an average of 47.7 months (Range 26-84 months). 


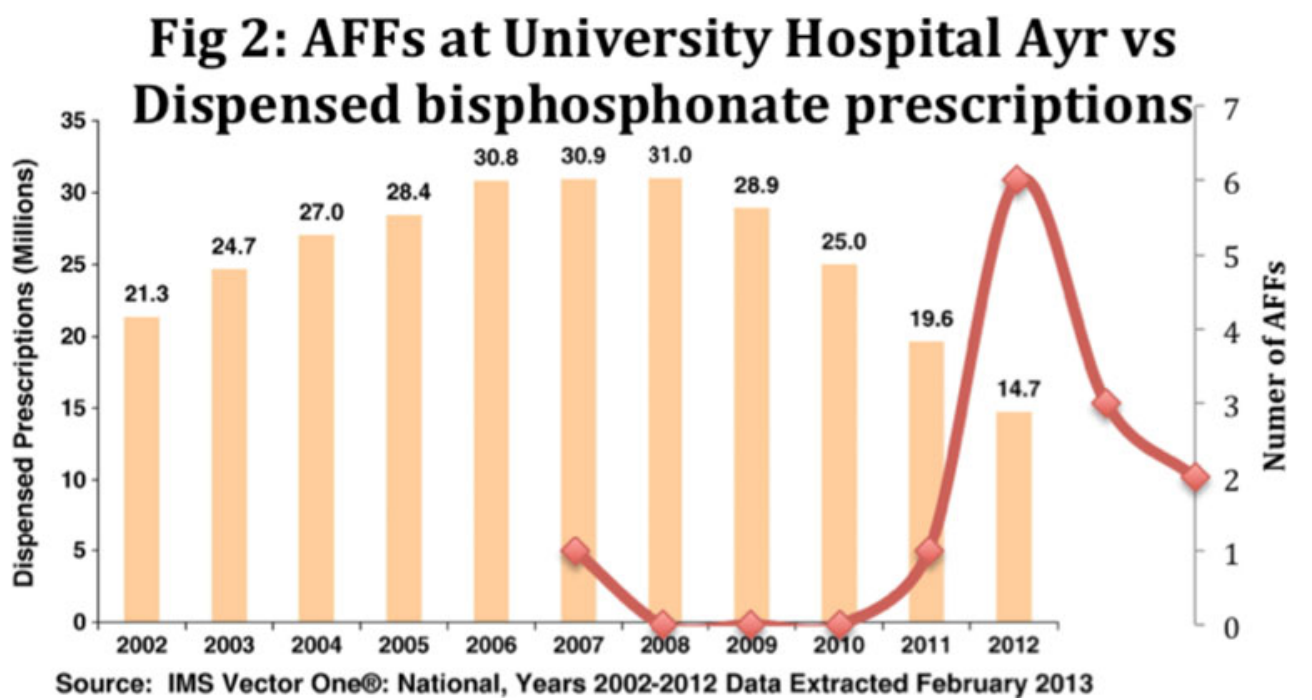

When the graph of AFF incidence at our District hospital is superimposed on the graph showing dispensed bisphosphonate prescriptions in the U.S.A (U.K trends in bisphosphonate use mirror that in the U.K $\left.{ }^{(2,3)}\right)$, there is a similarity in trends of bisphosphonate use and AFF presentations, with a lag of about 5 years.

Conclusions: The trend of AFF presentations at our district hospital seem to mirror trends in the use of long-term bisphosphonate therapy. References:

${ }^{1}$ Shane E, et al. J Bone Miner Res 2014;29:1.

2 Jha S, et al. J Bone Miner Res 2015.

${ }^{3}$ Watson J, et al. Eur J Clin Pharmacol 2007;63:843.

\section{P591}

IMPAIRED QUALITY OF LIFE ON AN ADULT MALE WITH IDIOPATHIC HYPOPARATHYROIDISM AND HASHIMOTO'S THYROIDITIS

\section{$\underline{\text { R. Baloescu }}^{1}$, M. Carsote $^{2}$, A. Valea ${ }^{3}$, A. Dumitrascu $^{1}$}

${ }^{1}$ C.I.Parhon National Institute of Endocrinology, Bucharest, Romania, ${ }^{2}$ C. Davila University of Medicine and Pharmacy \& C.I.Parhon National Institute of Endocrinology, Bucharest, Romania, ${ }^{3}$ I. Hatieganu University of Medicine and Pharmacy \& Clinical County Hospital, Cluj-Napoca, Romania

Introduction: Hypoparathyroidism (HypoPT) represents an orphan condition with both decreased PTH and calcium levels. The most frequent adult cause is thyroid surgery but idiopathic cases are described, potentially associated with an autoimmune environment or metabolic maladies as hemochromatosis, etc. The antibodies against parathyroids do not represent a routine assay so the context of a secondary endocrine or non-endocrine autoimmune disease (for instance, Hashimoto's thyroiditis or HT, adult onset hypogonadism, chronic adrenal insufficiency) may indirectly indicate this type of etiology. However, most of the autoimmune forms are seen in children with type 1 not in adults with type 2 autoimmune polyendocrine syndromes.

Material and Method: The bone metabolism is assessed performing biochemistry panel (total/ionic calcium levels or $\mathrm{T} / \mathrm{ICa}$, serum phosphorus or $\mathrm{Ph}$, serum magnesium or $\mathrm{Mg}$ ), bone turnover markers: alkaline phosphatase (AP), CrossLaps (CL), osteocalcin (OC); hormones: 25-hydroxyvitamin D (25-OHD), PTH. Thyroid, adrenal and testicular profile was also evaluated. Case data: A 43-year old Caucasian smoking male has suffered for several years of chronic asthenia, and fatigue, mild depression, muscle cramps, with acute episodes of tetany. He was evaluated in different Romanian medical centers and normal imunogram, renal function and sideraemia were found as well as negative $B$ and $C$ hepatitis markers. Persistent levels of calcium were not corrected despite different regimes of activated vitamin D analogues and calcium supplementation. On admission, the parameters showed: TCa of $5.7 \mathrm{mg} / \mathrm{dL}$ (8.4$10.3 \mathrm{mg} / \mathrm{dL}$ ), $\mathrm{Mg}$ of $1.5 \mathrm{mg} / \mathrm{dL}$ (N; $1.6-2.4 \mathrm{mg} / \mathrm{dL}$ ), 25-OHD of $38 \mathrm{ng} / \mathrm{mL}(\mathrm{N}:>30 \mathrm{ng} / \mathrm{mL})$, PTH of $2.15 \mathrm{pg} / \mathrm{mL}(\mathrm{N}: 15-$ $65 \mathrm{pg} / \mathrm{mL}$ ). Thyroid profile revealed: intense hypo- echoic pattern at ultrasound suggestive for an autoimmune condition with normal function (TSH of $1.7 \mu \mathrm{UI} / \mathrm{mL}, \mathrm{N}: 0.35-4.94 \mu \mathrm{UI} / \mathrm{mL}$ ). Total testosterone and morning plasma cortisol after stimulation test showed an adequate response of $48 \mu \mathrm{g} / \mathrm{dL}$. Cerebral, lung and cervical computer tomography detected no abnormal calcifications or masses. Electrocardiogram showed sinusal rhythm of 75 beats/ minute without ST-T wave's anomalies. Magnesium supplements were added to prior therapy. The subject was followed for 1 more year and despite therapy he continued to accuse the same symptoms. The endocrine assessment showed: T/I Ca of 5.2/2.1 mg/dL (N ICa: 3.9-4.9 mg/ 
dL), Ph of $5.64 \mathrm{mg} / \mathrm{dL}(\mathrm{N}: 2.5-4.5 \mathrm{mg} / \mathrm{dL}), \mathrm{Mg}$ of $1.85 \mathrm{mg} / \mathrm{dL}$ $(1.6-2.55 \mathrm{mg} / \mathrm{dL})$, PTH of $4.55 \mathrm{pg} / \mathrm{mL}, \mathrm{CL}$ of $0.14 \mathrm{ng} / \mathrm{mL}$ (0.142-0.584 ng/mL), OC of $6.44 \mathrm{ng} / \mathrm{mL}(\mathrm{N}: 14-41 \mathrm{ng} / \mathrm{mL})$, P1NP of $16.1 \mathrm{ng} / \mathrm{mL}(\mathrm{N}: 15-74 \mathrm{ng} / \mathrm{mL}), 25-\mathrm{OHD}$ of $48 \mathrm{ng} /$ $\mathrm{mL}$. No kidney stone was detected at ultrasound. TSH continued to be within the normal limits (of $3.28 \mu \mathrm{Ui} / \mathrm{mL}$ ) with positive antibodies (anti-thyreoperoxidase antibodies of $417 \mathrm{UI} /$ $\mathrm{ml}, \mathrm{N}: 0-35 \mathrm{UI} / \mathrm{mL}$ ). Further follow-up is necessary.

Conclusion: Despite different regimes of supplements we could not correct the blood levels of calcium only the low magnesium deficiency. 25-hydroxyvitamin $\mathrm{D}$ continued to be normal with persistent almost detectable levels of intact PTH. General data of how to treat best HypoPT vary thus general recommendations regarding vitamin $\mathrm{D}$ and calcium supplements may not entirely help to adequately correct the levels of calcium, finally impairing the quality of life.

\section{P592}

\section{TUMOR-INDUCED OSTEOMALACIA (TIO):}

\section{DIFFERENT ASPECTS OF THE SAME SYNDROME} G. Fabbriciani $^{1}$, M. Massarotti ${ }^{2}$, L. Callarelli ${ }^{3}$, R. Radicchi ${ }^{4}$, P. Filipponi ${ }^{5}$

${ }^{1}$ IRCCS Istituto Ortopedico Galeazzi, Rheumatology Unit, Milano, Italy, ${ }^{2}$ Humanitas Research Hospital, Rheumatology Unit, Rozzano, Milano, Italy, ${ }^{3}$ Ospedale di Assisi, Emergency Medicine, Perugia, Italy, ${ }^{4}$ University of Perugia, Institute of Geriatrics, Perugia, Italy, ${ }^{5}$ Associazione Regionale Umbria Osteoporosi (ARUO), Perugia, Italy

Objectives: TIO is a rare acquired syndrome characterized by severe hypophosphatemia due to abnormal production of fibroblast growth factor 23 (FGF 23) and we hereby report four cases of TIO with diagnostics and therapeutic challenges.

Material and Methods: All patients have similar clinical and biochemical features with long history of diffused pain, progressive fatigue and fragility fractures, low serum phosphorus with reduced tubular resorption, increased serum alkaline phosphatase, normal serum calcium, iPTH and 25-OH-D, calcitriol inappropriately low and elevated FGF 23 levels.

Results: The 1st patient is 40-year old man with primary TIO, had FGF 23 value of $690 \mathrm{RU} / \mathrm{mL}($ n.r. $<100)$ due to hemangiopericytoma in the right maxillary sinus. The 2 nd patient is 54 years old woman with neurofibromatosis causing TIO, the FGF 23 was $237 \mathrm{RU} / \mathrm{mL}$. The $3 \mathrm{rd}$ and the 4th patients were diagnosed with TIO-like syndrome, they are women of 50 and 61 years old, FGF 23 levels of 560 and 440 RU/mL respectively, neoplasm production FGF 23 was not detected and genetic causes of hypophosphatemia were excluded. The 1st case showed normalization of FGF 23 levels after tumour removal and after 6 months he recovered completely. The 2nd and the 3rd were treated with phosphate supplements and calcitriol with partial response. For the 4th patient the standard medical therapy of TIO was failed, it was decided to treat her with cinacalcet resulting increased serum phosphorus and significant bone healing.

Conclusions: Patients with acquired form of hypophosphatemic osteomalacia driven by FGF-23 where the source of ectopic FGF 23 is not detected, are classifiable as TIO-like syndrome. It is not known whether this is due to inability to find the tumor or they represent separate forms of the same syndrome. Cincalcetinduced hypoparathyroidism reduces phosphaturic effect of FGF-23, therefore it can be promising option for TIO-like syndromes resistant to the therapy with phosphate and calcitriol. To our knowledge this is the 2nd report in the international literature reporting the efficacy of cincalcet in TIO syndrome1.

References: 1 Geller JL, et al. J Bone Miner Res 2007;22:931

P593

THE ASSOCIATION BETWEEN SERUM

SCLEROSTIN OR FGF-23 LEVELS AND

FRACTURES DIFFERS IN RELATION TO MAJOR COMORBIDITIES IN ELDERLY HOSPITALIZED INDIVIDUALS

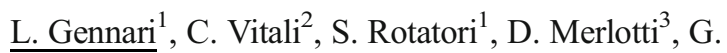
Gussoni $^{4}$, D. Diacinti ${ }^{5}$, L. Sinigaglia ${ }^{6}$, A. Valerio ${ }^{5}$, M. B. Franci $^{1}$, B. Lucani ${ }^{1}$, S. Gonnelli ${ }^{1}$, R. Nuti ${ }^{1}$

${ }^{1}$ Department of Medicine, Surgery and Neurosciences, University of Siena, Siena, Italy, ${ }^{2}$ Piombino Hospital, Livorno, Italy, ${ }^{3}$ Division of Genetics and Cell Biology, San Raffaele Scientific Institute, Milan, Italy, ${ }^{4}$ FADOI Foundation, Research Department, Milan, Italy, ${ }^{5}$ Section of Osteoporosis and Musculoskeletal Diseases, Department of Radiological Oncological and Anatomical-Pathological Sciences, University "La Sapienza", Rome, Italy, ${ }^{6}$ Rheumatology, Gaetano Pini Institute, Milan, Italy

Objective: Sclerostin (SCL) and FGF23 are osteocytesecreted factors with a major role in bone and mineral homeostasis. However the determinants of circulating SCL and FGF23 fractions as well as their association with fracture risk remain to be established.

Material and Methods: We assessed SCL and FGF23 levels in peripheral blood samples from the POINT (Prevalence of Osteoporosis in INTernal medicine) study cohort $(n=1199$ patients, mean age $75 \pm 8 \mathrm{yrs}$ ), a cross-sectional multicenter sample that was specifically designed to assess the prevalence of morphometric vertebral fractures in patients hospitalized in Internal Medicine Units. For each patient, the index of coexistent disease (ICED, which assesses the severity and the index of physical impairment for each disease category according to 5 levels of severity) was calculated.

Results: SCL levels were positively correlated with CTX $(p<0.05)$, creatinine $(p<0.0001)$ and inversely with physical activity score $(p<0.05)$, while FGF23 levels were positively correlated with PTH $(p<0.05)$ and creatinine $(p<0.05)$. In the 
overall sample, SCL did not significantly differ between patients with or without prevalent fractures or morphometric vertebral fractures. A significant association was observed between FGF23 and prevalent nonvertebral fractures $(p<0.005)$ but not morphometric vertebral fractures. Either SCL or FGF23 levels progressively increased with the increase of comorbid conditions, particularly in subjects with the highest ICED levels of severity for cardiovascular disorders, cerebrovascular diseases, diabetes and CRF. After exclusion of subjects with all those comorbid conditions, the association between FGF23 levels and fractures became nonsignificant. Conversely, a statistically significant association was observed between SCL and morphometric vertebral fractures $(p<0.01)$ when patients with cardiovascular disorders, diabetes and/or CRF were considered.

Conclusions: The relationship between circulating SCL or FGF23 levels and fractures is complex and differ in patients with or without major cardiovascular and/or metabolic conditions. Despite their measurement remains of limited value in clinical practice, a better understanding of the mechanisms underlying their increase in the presence of extraskeletal disorders is necessary.

\section{P594}

PREVALENCE OF LOW LEAN MASS AND ITS ASSOCIATION WITH OSTEOPOROSIS DRAMATICALLY DEPEND ON THE DEFINITION USED: A CROSS-SECTIONAL STUDY OF 653 WOMEN WITH HIP FRACTURE

M. Di Monaco $^{1}$, C. Castiglioni ${ }^{1}$, R. Tappero ${ }^{2}$

${ }^{1}$ Osteoporosis Research Center, Presidio San Camillo, Fondazione Opera San Camillo, Torino, Italy, ${ }^{2}$ Division of Physical Medicine and Rehabilitation, Presidio San Camillo, Fondazione Opera San Camillo, Torino, Italy
Objective: To investigate the prevalence of low appendicular lean mass (aLM) according to different definitions and its association with low BMD in women with hip fracture.

Methods: We studied 653 of 720 women with a fragility fracture of the hip. DXA was used to measure body composition. Low aLM was identified according to two different definitions. Baumgartner's criteria identified the women whose aLM/height 2 was less than two standard deviations below the mean of the young reference group. FNIH criteria identified the women whose aLM was lower than $15.02 \mathrm{~kg}$, or whose aLM adjusted for BMI was $<0.512$. Low BMD was diagnosed with a T-score $<$ -2.5 at unfractured femoral neck.

Results: We found a significant positive correlation between aLM and BMD: $r=0.34 ; p<0.001$. Using Baumgartner's definition, 432 of the 653 women ( $66 \%$; $95 \%$ CI $63 \%-70 \%$ ) had low aLM/height ${ }^{2}$ whereas using the definition from the FNIH the women with low aLM were $548(84 \%, 95 \% \mathrm{CI} 81 \%-87 \%)$, but they dropped to 224 (34 \%, 95\%CI $31 \%$-38 \%) with the FNIH definition based on aLM/BMI ratio. Low BMD was diagnosed in 432 of the 653 women $(66 \%$; 95\%CI $63 \%-70 \%)$. After categorization according to Baumgartner's criteria, the association between low aLM/height 2 and low BMD was significant: $\operatorname{chi}^{2}(1, n=653)=8.52(p=0.004)$, but it was erased by adjustments for age and fat mass. After categorization according to the FNIH threshold for aLM, the association between low aLM and low BMD was significant $\operatorname{chi}^{2}(1, n=653)=42.5(p<0.001)$, and it was confirmed after adjustment for age and fat mass. Conversely, the categorization according to the FNIH threshold for aLM/BMI ratio did not result in a significant association between low aLM/BMI ratio and low BMD: $\operatorname{chi}^{2}(1$, $n=653)=0.003(p=0.957)$.

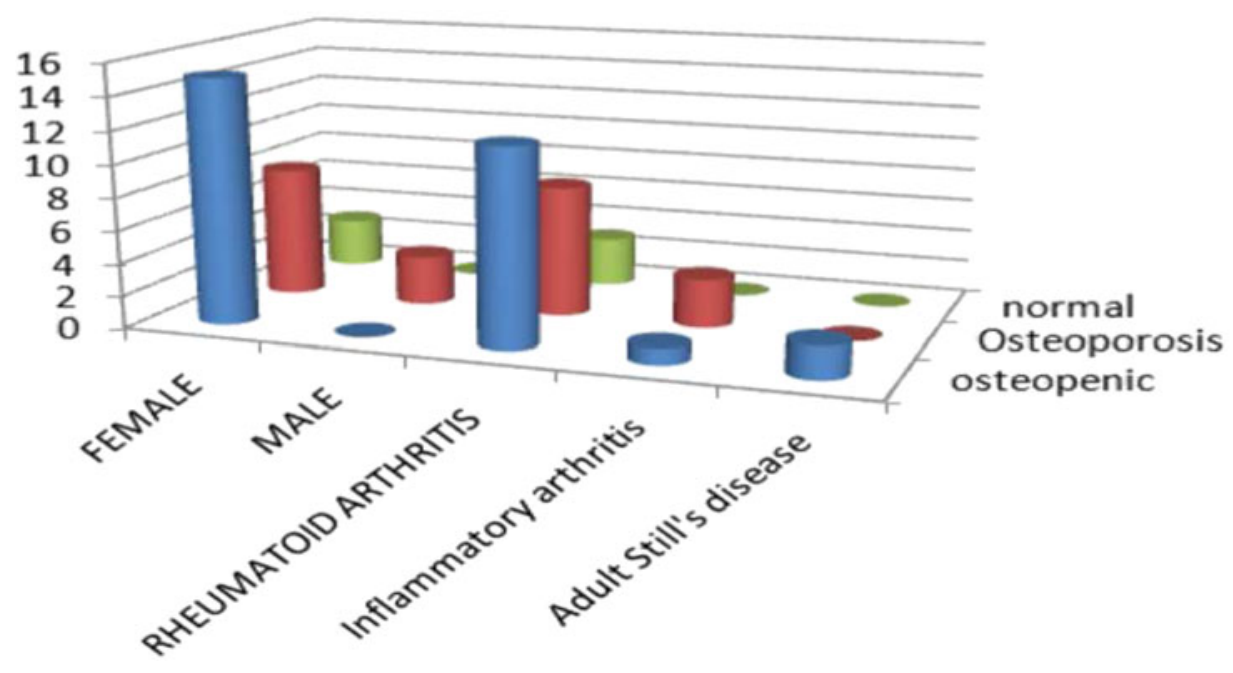

- osteopenic

- Osteoporosis

normal 
Conclusions: In women with hip fracture, both prevalence of low aLM and its association with low BMD dramatically depend on the adopted definition.

\section{P595 \\ BONE MINERAL DENSITY IN ADULT PATIENTS WITH INFLAMMATORY ARTHROPATHY}

A. Taysir ${ }^{1}$, K. Khawaja ${ }^{1}$, M. AlMaini ${ }^{1}$

${ }^{1}$ Rheumatology, Immunology and Allergy, Abu Dhabi, United Arab Emirates

Objectives: Abu Dhabi has a diverse multinational population that is reflected in our cohort of inflammatory arthropathy patients. Osteoporosis and low BMD is one of the comorbidities associated with inflammatory arthropathy. The primary objective of this audit is to look at the percentage of patients with inflammatory arthropathy referred for BMD. The frequency of osteopenia and osteoporosis in these patients referred. The secondary objective is to look at gender differences, age and weight in patients with osteopenia and osteoporosis.

Methods: Cases with inflammatory arthropathy identified through hospital electronic coding system from January 2014 to end of August 2015 (20 months). ICD 9 codes (714.00, 714.04, 714.89, 714.9, and 714.95).Demographic and clinical data was retrospectively collected from the hospital electronic medical records for all patients. We looked at the patients referred for BMD. Measures of BMD assessed by DXA. T-scores of hips and lumbar spines used. Patients who underwent DXA assessment identified and results described. Results: Total of 58 patients with inflammatory arthropathy identified. 29 (50\%) patients had DXA performed. Out of these $29(85.7 \%)$ had abnormal results; 11 (37.9\%) osteoporotic (BMD T-score of $<-2.5)$ and $15(51.7 \%)$ osteopenic (BMD T-score $<1$ and $>-2.5$ ). 3 normal DXA (BMD T-score $>1$ ). Of the 11 osteoporotic patients: 8 female and 3 male. Age range 24 to $79 \mathrm{yrs}$. For osteopenic patients all were female and age range 24-77 yrs of age. 20 patients had the diagnosis of rheumatoid arthritis, four patients had diagnosis of Inflammatory arthritis and two patient with osteopenia had stills disease.

Conclusions: Numbers are small and we only looked at 20 months data. We should look investigate BMD and refer patients with rheumatoid arthritis more often for DXA scan.

\section{P596}

\section{VITAMIN D STATUS IN MEN LIVING IN THE RE-} PUBLIC OF BELARUS

E. Rudenka $^{1}$, A. Rudenka ${ }^{2}$, N. Serduchenko ${ }^{3}$

${ }^{1}$ Belarusian State Medical University, Minsk, Belarus,

${ }^{2}$ Belarusian Medical Academy of Postgraduate Training, Minsk, Belarus, ${ }^{3}$ National Academy of Sciences of Belarus, Minsk, Belarus
Objective: Although much attention is paid to the problem of vitamin $\mathrm{D}$ deficiency, frequency of this condition in men remains understudied. The aim of our study was to determine the levels of vitamin D and prevalence of its deficiency in Belarusian men in accordance with age and season of year.

Materials and methods: 400 results of the laboratory testing of the serum levels of total vitamin $\mathrm{D}(25(\mathrm{OH}) \mathrm{D})$, performed in republic of Belarus in men aged over 20 years in 2015, were analyzed. Serum content of total vitamin D was determined by electrochemiluminescence protein binding assay technology using Cobas e411 immunoassay analyzer (Roche Diagnostic, Germany) and original reactants Roche Diagnostics $\mathrm{GmbH}$. According to international recommendations vitamin $\mathrm{D}$ levels considered to be normal when values of $25(\mathrm{OH}) \mathrm{D}$ exceeded the meaning $30 \mathrm{ng} / \mathrm{ml}$, showings from $20-29 \mathrm{ng} / \mathrm{ml}$ were determined as insufficiency, less than $20 \mathrm{ng} / \mathrm{ml}$ - as deficiency of vitamin D. Statistical analysis was performed using the software package Statistica 6.0.

Results: Received data were divided into 3 groups according to age: group I consisted of men younger than 40 years old ( $\mathrm{n}$ 198), group II - of men at age $40-60$ years old (n 157) and group III - of those older than 60 years (n 45). The prevalence of vitamin D deficiency and insufficiency were studied in each age group according to the season: spring-summer and autumn-winter. Results of determination of seasonal differences in vitamin D levels in each age group with the use of Student's $t$-test are presented in Table 1.

Table 1. Seasonal differences in vitamin $\mathrm{D}$ levels in each age group

\begin{tabular}{lllll}
\hline Age group & autum-winter & spring-summer & $\mathrm{t}$ & $\mathrm{p}$ \\
\hline$<40$ & $\begin{array}{l}26,1 ; 10,6 \\
(\mathrm{n} 79)\end{array}$ & $\begin{array}{l}25,1 ; 9,3 \\
(\mathrm{n} 119)\end{array}$ & 0,467 & 0,46 \\
$40-60$ & $\begin{array}{l}26,1 ; 10,1 \\
(\mathrm{n} 70)\end{array}$ & $\begin{array}{l}30,6 ; 14,8 \\
(\mathrm{n} 87)\end{array}$ & $-2,179$ & 0,03 \\
$>60$ & $\begin{array}{l}27,0 ; 7,7 \\
(\mathrm{n} 19)\end{array}$ & $\begin{array}{l}28,7 ; 9,4 \\
(\mathrm{n} 26)\end{array}$ & $-0,631$ & 0,53 \\
& & & & \\
\hline
\end{tabular}

Statistically significant differences of seasonal serum vitamin D levels were found in individuals of group II. Prevalence of vitamin D deficiency, insufficiency and normal values of $25(\mathrm{OH}) \mathrm{D}$ in the studied sample during different seasons is shown in table 2.

Table 2. Incidence of vitamin D deficiency, insufficiency and normal values of 25(OH)D in the studied sample

\begin{tabular}{llllllll}
\hline \multirow{2}{*}{ Age group } & \multicolumn{2}{l}{ autumn-winter } & & & \multicolumn{2}{c}{ spring-summer } \\
\cline { 2 - 3 } \cline { 7 - 8 } & $\mathrm{D}$ & $\mathrm{I}$ & $\mathrm{N}$ & & $\mathrm{D}$ & $\mathrm{I}$ & $\mathrm{N}$ \\
\hline$<40$ & 30,3 & 44,3 & 25,4 & & 26,8 & 51,3 & 21,9 \\
$40-60$ & 28,5 & 47,2 & 24,3 & & 25,3 & 27,6 & 47,1 \\
$>60$ & 15,8 & 57,9 & 26,3 & & 17,2 & 38,5 & 42,3 \\
\hline
\end{tabular}

Conclusion: The prevalence of vitamin D deficiency in men living in Belarus for the surveyed period is greater than $50 \%$ irrespective of age and season, the highest frequency of 
normal values of $25(\mathrm{OH}) \mathrm{D}$ in the studied sample was found in spring and summer in men aged from $40-60$ and over 60 years old.

\section{P597}

\section{A RETROSPECTIVE STUDY OF VITAMIN D STATUS IN 1599 INPATIENTS WITH HIP FRACTURE: TIME TREND 2000-2013}

M. Di Monaco $^{1}$, C. Castiglioni ${ }^{1}$, E. Lamarmora ${ }^{1}$, I. Filipovich $^{1}$, G. Massazza ${ }^{2}$, R. Tappero ${ }^{3}$

${ }^{1}$ Osteoporosis Research Center, Presidio San Camillo, Fondazione Opera San Camillo, Torino, Italy, ${ }^{2}$ Physical Medicine and Rehabilitation Unit, University Hospital Città della Salute e della Scienza, Torino, Italy, ${ }^{3}$ Division of Physical Medicine and Rehabilitation, Presidio San Camillo, Fondazione Opera San Camillo, Torino, Italy

Objective: To assess time trend of calcifediol serum levels in the hip fracture patients admitted to our rehabilitation ward.

Methods: We retrospectively evaluated 1599 inpatients with a hip fracture admitted between January 1, 2000 and December 31, 2013. A blood sample was collected in the morning following an overnight fasting 14.4 \pm 4.4 (mean $\pm \mathrm{SD}$ ) days after surgery. We assessed 25hydroxyvitamin D levels by an immunoenzymatic assay (coefficient of variation intra-assay $<8 \%$; interassay $<10 \%$ ). For statistical analyses, the 14-year period of observation was divided into seven two-year periods (2000-2001, 2002-2003, 2004-2005, etc.).

Results: We found a significant difference in calcifediol levels across the seven periods of two y: $\operatorname{chi}^{2}(6, N=1599)=194.1$, $p<0.001$. Calcifediol median level was very low in the first period of observation: $5.4 \mathrm{ng} / \mathrm{ml}$ (interquartile range 3.5$9 \mathrm{ng} / \mathrm{ml})$. It significantly increased till 2006-2007 $(13.1 \mathrm{ng} / \mathrm{ml}$, interquartile range $7.9-25 \mathrm{ng} / \mathrm{ml} ; p<0.001)$, but significantly decreased afterward. In the last two-year period (2012-2013) calcifediol median level was $7 \mathrm{ng} / \mathrm{ml}$ (interquartile range 5$14 \mathrm{ng} / \mathrm{ml})$. It was still slightly higher than the one found in the first period $(p<0.001)$, but significantly lower than the one found in 2006-2007 $(p<0.001)$. The association between periods of observation and 25-hydroxyvitamin $\mathrm{D}$ levels persisted after adjustment for age, BMI, sex, albumin, comorbidity, pressure ulcers, and cognitive impairment $(p<0.001)$.

Conclusions: A significant increase in calcifediol concentrations was seen till 2006-2007, but a significant decrease was observed afterward. Calcifediol levels were only slightly higher in the last two years of observation than in the first two years and severe vitamin D deficiency was common during the whole 14-year study period. Heightened awareness is needed to prevent and treat vitamin $\mathrm{D}$ deficiency in hip fracture patients.
P598

TO STUDY THE CORRELATION BETWEEN ABNORMALITIES IN LIPID PROFILE AND SEVERITY AND DISEASE ACTIVITY IN RHEUMATOID ARTHRITIS

A. Mittal ${ }^{1}$

${ }^{1}$ University of Delhi, New Delhi, India

Objective: Rheumatoidarthritis (RA) is a common disease which affects all populations of the world though prevalence varies in different populations. Recent studies have shown that cardiovascular disease plays an important role in increased mortality associated with RA. The excess cardiovascular disease encountered is believed to be due to traditional risk factors (e.g., dyslipidemia, hypertension) as well as due to novel risk factors (e.g., systemic inflammation).The present study was conducted as there is paucity of data on prevalence of lipid abnormalities in patients of RA. Material and Methods: Thirty patients of RA satisfying The 2010 American College of Rheumatology-European League Against Rheumatism (ACR/EULAR) classification criteria were enrolled in the study. The patients were subjected to detailed clinical history and examination with special emphasis on evaluation for swollen and tender joint count. Correlation between variables i.e. lipid profile indices and DAS 28 score, CRP and ESR was examined using the Pearson's correlation coefficient (r) and ANCOVA test. To find out the significance of Pearson's coefficient value, student- $t$ test was used, and $p$ value of less than 0.05 was considered statistically significant.

Results: The mean age of patients was $44.6 \pm 12.6$ years. DAS score was statistically significantly correlated with serum HDL cholesterol. Pearson's correlation coefficient value ranged from -0.19 (serum total cholesterol) to 0.26 (serum VLDL and triglyceride) for different lipid indices but none of the values was statistically significant.

Conclusions: Rheumatoid arthritis is a dyslipidemic state. There is significant inverse correlation between serum HDL cholesterol level and disease activity. There is no significant relationship between disease activity and levels of serum cholesterol, serum LDL cholesterol, serum VLDL cholesterol, serum triglyceride level and atherogenic index.

\section{P599}

BONE PROTECTION IN PATIENTS SUSTAINING PROXIMAL FEMUR FRAGILITY FRACTURES AND ASSOCIATED FRAGILITY INJURIES PRIOR AND AFTER ADMISSION TIME

I. Aguado-Maestro ${ }^{1}$, A. Bañuelos Diaz ${ }^{2}$, M. Panteli ${ }^{3}$, M. F. Garcia Alonso $^{2}$, F. J. Nistal Rodríguez ${ }^{2}$, A. Quintanilla Garcia $^{2}$, J. Lopez Sanchez 2 , P. V. Giannoudis ${ }^{3}$

${ }^{1}$ Department of Orthopaedic Surgery, Hospital Universitario del Río Hortega, Valladolid, Spain, ${ }^{2}$ Hospital Universitario Rio Hortega, Valladolid, Spain, ${ }^{3}$ Department of Traumatology and Orthopaedics, Leeds General Infirmary, Leeds, United Kingdom 
Objectives: To investigate whether patients presenting with fragility fractures of the proximal femur are receiving osteoporosis treatment and to assess the number of other fragility fractures they have sustained prior to admission.

Methods: +All patients presenting to our institution with fragility fractures of the proximal femur within an 18-month period (January 2012 - August 2013) were included. Patient demographics; classification (AO/OTA); American Society of Anesthesiologists (ASA) grade; Abbreviated Mental Test Score (AMTS) on admission; type/time to operation; perioperative complications; length of hospital stay (LOS); walking status; osteoporotic medication; DXA results; additional fragility fractures; and mortality were collected and analysed. Results: A total of 1004 patients (278 male) met the inclusion criteria and were included into the study. The mean age was 82.01 years and mean LOS was 19.54 days. Fifty four per cent of the patients were admitted from their own homes whereas $43 \%$ were capable to walk indoors without any aids before their injury. Mean time to surgery was 2.06 days (0-26 days). Three hundred and six patients $(30.48 \%)$ had at least another fragility fracture before the index episode (mean 1.40 fractures; SD: 0.71 fractures; range: $1-6$ fractures). Only $16.4 \%$ were under complete osteoporosis treatment on admission, defined as receiving calcium with vitamin $\mathrm{D}$ and a bisphosphonate or an alternative agent. When we compared patients without a history of a previous fragility fracture (Group A) and patients with at least another previous fragility fracture (Group B), we found that patients in Group B had a significantly lower AMTS score, lower BMD as evident on the DXA scan, an inferior mobility before admission and a higher incidence of extracapsular fractures $(p<0.05)$. On discharge, patients in Group B had a higher chance of receiving complete bone protection compared to group A (27.9 \% vs. $41.7 \% ; p<0.01)$. Following discharge, $11.2 \%$ of the patients sustained an additional fragility fracture. The mean time from the index episode to the additional fracture was 0.65 years, whilst these injuries were more frequent in Group B $(R R=1.46 ; p<0.05)$.

Conclusion: Patients sustaining a hip fracture are generally under-treated for osteoporosis. Assessment by a designated geriatrician and use of a standardised protocol is of paramount importance for reducing the risk of additional fragility fractures.

\section{P600 \\ MICRO-RNA EXPRESSION PROFILE IN PAGET'S DISEASE OF BONE AND OSTEOPOROSIS}

D. Merlotti ${ }^{1}$, S. Bianciardi ${ }^{2}$, G. Sebastiani ${ }^{2}$, M. Valentini ${ }^{2}$, S. Gonnelli $^{2}$, C. Caffarelli ${ }^{2}$, I. A. Evangelista ${ }^{2}$, S. Cenci ${ }^{1}$, R.

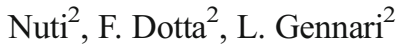

${ }^{1}$ Division of Genetics and Cell Biology, San Raffaele Scientific Institute, Milan, Italy, ${ }^{2}$ Department of Medicine, Surgery and Neurosciences, University of Siena, Siena, Italy
Objective: Since their initial discovery, microRNAs (miRNAs) have emerged as critical post-transcriptional regulators of gene expression that are able to modulate bone remodeling. Nonetheless, despite the peculiar and aggressive phenotype of Pagetic osteoclasts, whether deregulation of miRNAs is involved in Paget's disease of bone (PDB) remains unknown.

Material and Methods: we performed a miRNA expression of 6 major miRNAs previously associated with osteoclast activity (miR21, miR34, miR146a, miR148a, miR155, miR223) in peripheral blood mononuclear cells from 44 patients with active Paget's disease of bone (PDB, of whom 14 with SQSTM1 mutation) and 38 controls (CT, of whom 15 with osteoporosis and fractures). Quantitative analysis of miRNAs was performed using specific stem-loop primers followed by real-time polymerase chain reaction. All values were normalized to endogenous control U6.

Results: among the tested miRNAs associated with osteoclast activity miR146a and miR148a were significantly upregulated in either PDB patients and osteoporotic subjects than in nonosteoporotic controls, while miR21, and miR34a were overexpressed in PDB cases only. Moreover, a positive correlation between bone turnover markers and miR148 was observed in controls, but not in patients with PDB. While the increase in miR21, miR146a and miR148a is consistent with their pro-osteoclastogenic effects observed in vitro by previous studies, and might account for the increase in bone resorption of either osteoporosis and PDB, the increase in miR34a in patients with PDB was unexpected, given its role as a suppressor of osteoclastogenesis and since in mice models its overexpression attenuated ovariectomy-induced bone loss as well as the development of osteolytic bone metastases.

Conclusions: Taken together these results confirms the overexpression of miR148a in osteoporosis and show for the first time an increase in expression levels of most osteoclast-related microRNAs in PDB.

\section{P601 \\ CORRELATION BETWEEN LEPTIN AND RADIO- LOGICAL CHANGES IN OSTEOARTHRITIS OF COXOFEMORAL JOINT IN OBESE WOMEN}

T. Backa ${ }^{1}$, E. Rapushi ${ }^{1}$, L. Nuhaj ${ }^{1}$, V. Salko ${ }^{1}$, K. Skrame ${ }^{1}$, A. Zoto $^{1}$, E. Zicishti ${ }^{1}$, A. Ylli ${ }^{2}$

${ }^{1}$ Service of Rheumatology, University Hospital Center, Tirana, Albania, ${ }^{2}$ Service of Immunology, University Hospital Center, Tirana, Albania

Background: Osteoarthritis (OA) is a multifactorial disease is a multifactorial disease of the joints characterized by progressive loss of articular cartilage. Obesity is considered as a risk factor for $\mathrm{OA}$ of the coxofemoral joint. 
BMI represents its influence in OA of the coxofemoral joint in obese women.

Metabolic disorders associated with obesity are some of the causes of OA. Leptin is a protein that is secreted by adipocytes but also by chondrocytes, it regulates food intake and energy costs, it correlates with BMI in female sex. Leptin is considered an proinflammatory adipocytokine and as such it has a role in OA of coxofemoral joint.

Purpose: Correlation between leptin and radiological changes in the osteoarthritis of coxofemoral joint in obese women.

Method: Were involved in the study 62 obese women of an average age of $39 \pm 2.5$ y.o. All underwent the anteroposterior radiographic examination of sacral region for determining the OA changes: narrowing of articular space, sclerosis, osteophytes on acetabular margins. Leptin seric level was determined to all the women included in the study and also was made the evaluation of adipose mass through DXA and BMI. Results: The average leptin seric level of the group involved in the study resulted $29 \pm 18.5 \mathrm{ng} / \mathrm{ml}$, the average BMI value of the group resulted $23 \pm 4.45$. Radiological founds of the group included in this study were narrowing and sclerosis of articular margins in 37 females(59.67\%) and osteophytes in 25 females $(40.32 \%)$. There is a positive correlation between leptin seric level, BMI and adipose mass measured with $\operatorname{DXA}(r=0.48$ dhe $P<0.001)$. Obese women with the highest leptin seric level presents more significant changes for OA of coxofemoral joint $(P<0.005)$.

Conclusion: This study shows that leptin has an important role in leading to OA of coxofemoral joint in relatively young obese women.

\section{P602 \\ PROXIMAL FEMUR FRACTURE PATTERNS AND THEIR ASSOCIATION WITH HIP OSTEOAR- THRITIS}

I. Aguado Maestro $^{1}$, A. Bañuelos Diaz ${ }^{1}$, M. Panteli ${ }^{2}$, M. F. Garcia Alonso $^{1}$, F. J. Nistal Rodríguez ${ }^{1}$, A. Quintanilla Garcia $^{1}$, J. Lopez Sanchez ${ }^{1}$, P. V. Giannoudis ${ }^{2}$

${ }^{1}$ Hospital Universitario Rio Hortega, Valladolid, Spain, ${ }^{2}$ Department of Traumatology and Orthopaedics, Leeds General Infirmary, Leeds, United Kingdom

Introduction: Hip osteoarthritis has been thought to prevent proximal femur fractures, as intracapsular fractures arthroplasty demonstrated in most of cases an undamaged femoral head. Newer studies haven't been able to demonstrate this asseveration. However, it has been shown a tendency to intracapsular fractures in patients with lower grades of hip osteoarthritis.

The aim of our study is to demonstrate the relationship between the grade of hip osteoarthritis and fracture pattern shown in proximal femur fractures in the elderly.
Materials and methods: We retrospectively included all proximal femur fragility fractures admitted in our teaching hospitals between 2011 and 2013 (an 18 months period). Pathological fractures, high-energy injuries and patients with less than one year of follow-up were excluded. Admission xrays were assessed recording radiological features of osteoarthritis according to Kellgren and Lawrence scale (minimal: grades 1-2 and severe: grades 3 and 4). Fracture pattern was classified according to AO/OTA fracture classification system (31A: intertrochanteric; 31B: intracapsular).

Results: A total of 1003 patients ( 725 female; 1003 fractures) met the inclusion criterio, having a mean age of 82.0 (46-106 years). With regards to fracture classification, $417(41.5 \%)$ fractures were classified as intertrochanteric and $586(58.4 \%)$ as intracapsular. $939(93.6 \%)$ patients had minimal hip osteoarthritis and $61(6.1 \%)$ severe osteoarthritis. 61 patients presenting with severe osteoarthritis had 42 (68.9\%) 31Aintertrochanteric fractures and 19 (31.1\%) 31B-intracapsular fractures. 939 patients presenting with minimal osteoarthritis had 375 (39.9\%) 31A-intertrochanteric and 567 (60.4\%) 31B-intracapsular fractures. $(p<0.01)$. The relative risk for patients with advance hip osteoarthritis for sustaining an extracapsular fracture is 1.72 .

Conclusions: Higher grades of hip osteoarthritis are significantly related to extracapsular hip fractures, whereas lower grades of hip osteoarthritis are related to intracapsular patterns.

\section{P603}

DIFFERENT TREATMENT STRATEGIES FOR CHRONIC NONBACTERIAL OSTEOMYELITIS

M. Kostik ${ }^{1}$, O. L. Kopchak ${ }^{2}$, I. A. Chikova ${ }^{1}$, V. V. Masalova ${ }^{1}$, M. F. Dubko ${ }^{1}$, L. S. Snegireva ${ }^{1}$, E. A. Isupova ${ }^{1}$, O. V. Kalashnikova $^{1}$, V. G. Chasnyk ${ }^{1}$, A. Y. Mushkin ${ }^{3}$

${ }^{1}$ Saint-Petersburg State Pediatric Medical University, SaintPetersburg, Russian Federation, ${ }^{2}$ Kirov's regional children's hospital, Kirov, Russian Federation, ${ }^{3}$ Science research Institute of Phthisiopulmonology, Saint-Petersburg, Russian Federation

Objectives: Chronic nonbacterial osteomyelitis (CNO) is a heterogeneous group of immune mediated inflammatory bone diseases, often coexist with other rheumatic diseases. There are no approved treatments for CNO except nonsteroidal anti-inflammatory drugs (NSAID). The efficacies of methotrexate (MTX), sulfasalazine, pamidronate (PAM), anti-IL1 and TNF $\alpha$-inhibitors (TNF $\alpha$-inh) were shown in different reports.

Aim: To compare the efficacy of nonrandomized different treatment approaches in pediatric patient $\mathrm{CNO}$ cohort.

Material and methods: 152 children $(25 \mathrm{M}$ and $27 \mathrm{~F})$ with $\mathrm{CNO}$ has average age at the onset of disease 8.4 years $(5.4$ $\pm 11.0)$, the number of foci - $3.0(2.0 \pm 6.0$, incl. multifocal 
cases in $80.8 \%$ ), fever at the onset $38.5 \%$, spine involvement $-34.6 \%$, positive family autoimmune diseases (AID) history $7.7 \%$, concomitant AID $-67.3 \%$. NSAID was the first-line treatment for nonvertebral cases, as well as PAM for vertebral involvement. Second-line treatment includes MTX, PAM and $\mathrm{TNF} \alpha$-inh. Dynamics of pain, patient's (PVAS) and physician's (MDVAS) assessment and ability to each medication to achieve remission of $\mathrm{CNO}$ activity we evaluated.

Results: According to the NSAID, MTX, SSZ, PAM and TNF $\alpha$-inh groups next data were registered: PVAS: $-14.2 \%$ $(p=0.05),-50.0 \%(p=0.04),-23.1 \quad(p=0.89),-83.3 \%$ $(p=0.0001),-73.6 \%(p=0.0007)$; pain: $-21.9 \%(p=0.01)$, $-18.6 \%(p=0.13),+36,4(p=0.89),-79.7 \%(p=0.00016)$, $-74.1 \%,(p=0.0015)$; MDVAS: $-13.8 \%(p=0.13) ;-56.4 \%$ $(p=0.09),+30.8 \%(p=0.89),-74.7 \%,(p=0.0001),-82.1$ $(p=0.0015)$, respectively. The ability of each treatment strategy to achieve the CNO remission was $52.6 \%, 44.4 \%, 57,1 \%$, $88.8 \%$ and $73.3 \%$, respectively (log-rank test, $p=0,001$ ). $\mathrm{TNF} \alpha$-inh usually used as second-third line treatment in cases where other options, especially PAM were fall.

Conclusions: The most effective treatment approaches for $\mathrm{CNO}$ were PAM and TNF $\alpha$-inh. The randomized controlled trials for assessment efficacy and safety of these medications is mandatory to confirm these results.

\section{P604}

SERUM PHOSPHATE LEVELS ARE ASSOCIATED WITH FRACTURE RISK: THE ROTTERDAM STUDY M. C. Zillikens $^{1}$, N. Campos-Obando ${ }^{1}$, W. N. Koek ${ }^{1}$, B. C. van der Eerden $^{1}$, J. P. van Leeuwen ${ }^{1}$, A. G. Uitterlinden ${ }^{1}$

${ }^{1}$ Department of Internal Medicine, Erasmus MC, Rotterdam, Netherlands

Objectives: Although abnormal serum phosphate levels (P) have been associated with specific BMD phenotypes, such as rickets/osteomalacia and tumoral calcinosis the nature of an association of phosphate levels with BMD and fracture risk in the general population is unknown. We studied these relations in elderly Dutch participants from the Rotterdam Study (RS).

Material and methods: Serum phosphate was measured in the fasting state. BMD at the femoral neck (FN-BMD) and lumbar spine (LS-BMD) was assessed by DXA. The association between $\mathrm{P}$ and BMD was tested through linear models using three cohorts (RS-I, RS-II and RS- III). We applied competing risk regression models to assess the relation between $\mathrm{P}$ and prospective all-type fracture risk in two RS cohorts (RS-I and RS-II). Basic adjustments included age, BMI and smoking. Betas $(\beta(95 \% \mathrm{CI}))$ and hazard ratios $(\mathrm{HR}(95 \% \mathrm{CI}))$ were meta-analyzed applying fixed-effect model. Results are expressed per $1 \mathrm{mg} / \mathrm{dL}$ increase in $\mathrm{P}$.
Results: $\mathrm{n}$ women, there was no association between $\mathrm{P}$ and BMD. In men, $\mathrm{P}$ was inversely related only to LS-BMD $(-0.13(-0.23$ to- 0.02$))$ and this association was not substantially modified after further adjustments for calcium and 25hydroxyvitamin $\mathrm{D}$ levels $(-0.11(-0.22$ to -0.001$))$. There was a positive association between $P$ and all-type fracture risk in both sexes (men: 1.54(1.20-1.97); women: 1.23(1.01-1.49); sex-combined: 1.35 (1.15-1.58)). Results were similar after further adjustments for levels of calcium, 25-hydroxyvitamin $\mathrm{D}$, kidney function (defined as creatinine-based estimated glomerular filtration rate) and BMD (men: 1.57(1.11-2.22); women: $1.30(1.04-1.64)$ ), or after restricting analyses with subjects with normal kidney function (men 1.81(1.28-2.55); women: 1.25(1.01-1.55)).

Conclusions: Serum P was negatively related to LS-BMD in men but not women, and no association with FN-BMD was found in either sex. $P$ was positively related to all-type fracture risk in both sexes. Our findings suggest that increased $\mathrm{P}$ even within normal range might be deleterious for bone health in the general population.

\section{P605}

PREDICTORS OF IMMINENT FRACTURE RISK IN WOMEN AGED $\geq 65$ YEARS WITH OSTEOPOROSIS $\underline{\text { D. Weycker }}^{1}$, A. Kartashov ${ }^{1}$, J. Edelsberg ${ }^{1}$, A. Grauer ${ }^{2}$, B. Crittenden $^{3}$, R. Barron ${ }^{3}$

${ }^{1}$ Policy Analysis Inc., Brookline, United States, ${ }^{2}$ Amgen Inc., Thousand Oaks, United States, ${ }^{3}$ Amgen Inc., Thousand Oaks, CA, United States

Background: Fractures are the major source of morbidity among women with osteoporosis. However, evidence on factors leading to imminent risk for hip or other non-vertebral fracture within the next 12 months among women aged $\geq 65$ years with osteoporosis is limited.

Methods: A retrospective cohort design and data from the Study of Osteoporotic Fractures (SOF) - which includes 20 years of prospectively collected data on osteoporosis care and outcomes - were employed. The study population comprised all women aged $\geq 65$ years in the Caucasian cohort with osteoporosis (T-score $\leq-2.5$ at total hip). Hip and other non-vertebral fractures were ascertained over a 1-year follow-up period. Potential predictors of fracture observed 12 months prior to fracture were evaluated using multivariate regression models and included anthropometric measures, BMD, cognitive function, comorbidities, drug use, fracture/fall history, lifestyle variables, medical symptoms, physical function/performance, quality of life, and vision status.

Results: The study population included 2.499 women with osteoporosis who contributed 6.811 observations. During the 1-year follow-up, $2.2 \%$ had a hip fracture and $6.6 \%$ 
had any non-vertebral fracture (including hip). Independent predictors of hip and/or non-vertebral fracture included impaired cognitive function, total hip Tscore, falls history, fracture history, and lower physical performance (Table).

Table. Independent predictors of 1-year hip and non-vertebral fracture in $\underline{\text { osteoporotic women aged } \geq 65 \text { years from multivariate regression models }}$

\begin{tabular}{|c|c|c|}
\hline \multirow[b]{2}{*}{ Risk Factors } & \multicolumn{2}{|c|}{ Hazard Ratios* $(95 \%$ CI) } \\
\hline & Hip Fracture & $\begin{array}{c}\text { Non-Vertebral } \\
\text { Fracture** }\end{array}$ \\
\hline \multicolumn{3}{|l|}{ Age (vs. referent: 65-74) } \\
\hline$\geq 75$ to 79 & - & $0.9(0.6-1.3)$ \\
\hline$\geq 80$ to 84 & - & $1.0(0.7-1.4)$ \\
\hline$\geq 85$ & - & $1.4(0.9-1.9)$ \\
\hline \multicolumn{3}{|l|}{$\begin{array}{l}\text { Total Hip T-Score } \\
\text { (vs. referent: }>-3.0 \text { too }-2.5 \text { ) }\end{array}$} \\
\hline$\leq-3.5$ & $2.3(1.5-3.7)$ & $1.9(1.5-2.5)$ \\
\hline$>-3.5$ to -3.0 & $1.6(1.6-2.6)$ & $1.6(1.2-2.0)$ \\
\hline \multicolumn{3}{|l|}{$\begin{array}{l}\text { No. of Falls in Last } 12 \text { Months } \\
\text { (vs. referent: 0) }\end{array}$} \\
\hline 1 & - & $1.2(0.9-1.5)$ \\
\hline$\geq 2$ & - & $1.7(1.3-2.2)$ \\
\hline \multicolumn{3}{|l|}{$\begin{array}{l}\text { History of Fracture } \\
\quad \text { (vs. referent: no history) }\end{array}$} \\
\hline Non-Vertebral Fracture & $1.6(1.0-2.6)$ & - \\
\hline Any Fracture & - & $1.4(1.1-1.7)$ \\
\hline \multicolumn{3}{|l|}{$\begin{array}{l}\text { Walking Speed }(\mathrm{m} / \mathrm{s}) \\
\quad(\text { vs. referent: }>1.0)\end{array}$} \\
\hline$\leq 0.70$ & $3.0(1.5-5.9)$ & $1.5(1.1-2.1)$ \\
\hline 0.70 to 1.0 & $2.6(1.4-5.0)$ & $1.4(1.1-1.9)$ \\
\hline $\begin{array}{l}\text { Short MMSE } \leq 23 \\
\quad(\text { vs. referent: }>23)\end{array}$ & $1.7(1.2-2.4)$ & - \\
\hline $\begin{array}{l}\text { Use of Arms for Chair } \\
\text { Stands or Poor/Very } \\
\text { Poor Tandem Stand } \\
\text { (vs. referent: no use of arms) }\end{array}$ & $1.7(1.1-2.6)$ & - \\
\hline $\begin{array}{l}\text { Parkinson's or Stroke } \\
\text { (vs. referent: without } \\
\text { conditions) }\end{array}$ & - & $1.3(1.0-1.8)$ \\
\hline $\begin{array}{l}\text { Smoker, Pack Years } \\
\quad \text { (continuous measure) }\end{array}$ & - & $1.0(1.0-1.0)$ \\
\hline $\begin{array}{l}\text { Bisphosohonate Use } \\
\quad \text { (vs. referent: non-users) }\end{array}$ & $0.3(0.1-0.9)$ & - \\
\hline $\mathrm{C}$ statistic $(95 \% \mathrm{CI})$ & $0.71(0.67-0.76)$ & $\begin{array}{l}0.62(0.59- \\
0.065)\end{array}$ \\
\hline
\end{tabular}

MMSE: Mini-Mental State Examination

*Only hazard ratios for variables retained in the final model (i.e., those with $p$-values $<0.10$ ) are reported; grouped dichotomous variables were retained if any of the grouped variables had a p-value $<0.10$

**Includes fracture of any non-vertebral site, defined-by SOF in a composite measure-as an incident, non-traumatic fracture of ankle, clavicle, elbow, face, foot, finger, hand, hip, humerus, knee, lower leg, pelvis, rib, toe, upper leg, or wrist

**After age 50 years
Conclusions: Imminent fracture risk within 12 months among osteoporotic women is higher among those with a history of fracture, history of falls, lower BMD, physical dysfunction, and/or cognitive dysfunction. Careful consideration should be given to identifying this population so that those at imminent risk may be targeted for the appropriate therapy.

\section{P606}

THE COMPARATIVE STUDY OF CHRONIC NONBACTERIAL OSTEOMYELITIS, ACUTE NONSPECIFIC OSTEOMYELITIS AND TUBERCULOSIS OSTEITIS

O. L. Kopchak ${ }^{1}$, M. Kostik ${ }^{2}$, A. A. Denisov ${ }^{2}$, V. V. Avramenko $^{2}$, D. M. Vorypin ${ }^{2}$, D. V. Philippov ${ }^{2}$, S. S. Peredereev $^{2}$, Y. D. Vorypin ${ }^{2}$, A. S. Maletin ${ }^{3}$, Y. A. Goryshina $^{2}$, A. Y. Mushkin ${ }^{3}$

${ }^{1}$ Kirov's regional children's hospital, Kirov, Russian Federation, ${ }^{2}$ Saint-Petersburg State Pediatric Medical University, Saint-Petersburg, Russian Federation, ${ }^{3}$ Science research Institute of Phthisiopulmonology, Saint-Petersburg, Russian Federation

Objectives: Chronic nonbacterial osteomyelitis (CNO) is an immune mediated inflammatory bone affect often coexisting with other autoimmune and auto-inflammatory rheumatic condition. CNO diagnosis is required the exclusion of the broad spectrum of infection, malignant and metabolic diseases. The aim of study was to find special clinical features which can help to better distinguish $\mathrm{CNO}$ with similar infection bone diseases.

Material and Methods: 142 children with bone inflammatory diseases were included: $\mathrm{CNO}$ - 52, acute osteomyelitis (AO) - 47, tuberculosis osteomyelitis (TBO) - 43. X-ray, MRI, CT-scan, bone scintigraphy, bone biopsy with cultural analysis and histology examination and routine blood tests were performed in all patients.

Results: Significant differences were found in gender distribution (more females in CNO, $p=0.03$ ), onset age (CNO-8.4, AO11.0 , TBO-2.2 years, $p=0.00001$ ), delay in diagnosis CNO-6.3, AO-0.1, TBO-2.9 months, $p=0.0001$ ), symptomatic arthritis (CNO-69.2 \%, AO-23.4 \%, TBO-48.8 \%, $p=0.00003$ ), X-ray findings, such as bone destruction with perifocal sclerotic zone (CNO-100 \%, $p=0,000001)$, multifocal lesions (CNO- $80.8 \%$, AO-0 \%, TBO- $18.6 \%, p=0.0000001$ ) and number of lesions per patient (CNO-3, AO-1, TBO-1, years, $p=0.00001)$, presence of fever at onset (CNO-38.5\%, AO-91.5\%, TBO$34.9 \%$ years, $p=0.0000001$ ), positive bone culture (CNO$0 \%$, AO- $67.4 \%$, TBO- $60.5 \%$ years, $p=0.0000001)$ and inflammatory activity between groups: $\mathrm{Hb}(p=0.006), \mathrm{WBC}$ $(p=0.0001), \operatorname{PLT}(p=0.003), \operatorname{ESR}(p=0.01), \operatorname{CRP}(p=0.001)$. There were differences in bone involvement, incl. spine (CNO$34.6 \%$, AO- $4.3 \%$, TBO-7.0 \%, $p=0.00003$ ), femur (CNO- 
$34.6 \%$, AO- $14.9 \%$, TBO-44.2 \%, $p=0.009$ ), tibia (CNO$44.2 \%$, AO-25.5\%, TBO-23.2 \%, $p=0.049$ ), foot (CNO$23.1 \%$, AO- $6.4 \%$, TBO- $11.6 \%, p=0.00001$ ), pelvis (CNO$23.1 \%$, AO- $6.4 \%$, TBO-7.0 \%, $p=0.02)$ and clavicular (CNO$11.5 \%$, AO-2. $1 \%$, TBO- $0 \%, p=0.02$ ).

Conclusion: there were differences and similarities between nonbacterial and infectious bone inflammatory diseases. $\mathrm{CNO}$ more similar to TBO, and both diseases differ from AO. The crucial signs were radiological findings, bone pathology and positive bone culture which help to distinguish these diseases.

\section{P607}

SERUM PHOSPHATE IS RELATED TO ALL-CAUSE, CARDIOVASCULAR AND COPD MORTALITY IN MEN: THE ROTTERDAM STUDY

M. C. Zillikens ${ }^{1}$, N. Campos-Obando ${ }^{1}$, L. Lahousse $^{2}$, G. G. Brusselle $^{2}$, B. H. Stricker ${ }^{3}$, O. H. Franco ${ }^{3}$, A. G. Uitterlinden ${ }^{1}$ ${ }^{1}$ Department of Internal Medicine, Erasmus MC, Rotterdam, Netherlands, ${ }^{2}$ Department of Respiratory Medicine, Ghent University Hospital, Ghent, Belgium, ${ }^{3}$ Department of Epidemiology, Erasmus MC, Rotterdam, Netherlands

Objectives: High serum phosphate (P) levels have been associated with increased mortality risk in chronic kidney disease but the nature of such a relation in the general population is unclear.

Material and methods: In two cohorts from the Rotterdam Study (RS-I and RS-II) we assessed the relation between (P) levels and all-cause and cause-specific mortality divided into 7 groups, using ICD-10: cardiovascular diseases (CVD), cancer, external causes, infectious diseases, dementia, chronic lung diseases and other causes.In 3732 elderly participants from RS-I and 2494 from RS-II serum P was measured in the fasting state. Cox and competing risk regression models were applied, adjusted for age, BMI and smoking; results from both cohorts were meta-analyzed applying fixed-effect model. Pooled HRs (95\% CI) are expressed for each $1 \mathrm{mg} / \mathrm{dL}$ increase in serum P. Due to interaction by gender, we performed sex-stratified analyses. Average follow-up was 14.5 y (RS-I) and 10.9 y (RS-II).

Results: There was a significant positive association between $\mathrm{P}$ and all-cause mortality in men $1.46(1.27-1.69)$ but not women $(0.90(0.77-1.04))$. In men, higher phosphate increased the risk for CVD mortality (1.66 (1.29-2.14)), other causes (1.67 (1.16-2.41)) and chronic lung disease mortality (1.96 (1.02-3.76)), the latter driven by mortality due to Chronic Obstructive Pulmonary Disease (COPD) (4.39 (2.12-9.07)). No significant relations were found for mortality due to infections, cancer, dementia or external causes. Analyses restricted to male subjects with P levels within normal range yielded essentially same results. Findings were not essentially modified after adjustments for calcium and 25- hydroxyvitamin D levels; further adjustment for eGFR produced only a slight attenuation We observed no associations between P and cause-specific mortality in women.

Conclusion: Even within normal range serum $P$ levels are associated with increased all-cause, cardiovascular and COPD mortality in men but not women not explained by calcium, 25-hydroxyvitamin D levels or kidney function. Our results suggest that the concept of phosphotoxicity beyond CKD population deserves further attention. The novel finding of an association of phosphate levels with COPD mortality needs further research on underlying mechanisms.

\section{P608 \\ BONE FIBROUS DYSPLASIA: A RETROSPECTIVE REVIEW OF 34 CASES}

G. Marcucci ${ }^{1}$, L. Masi ${ }^{1}$, L. Cianferotti ${ }^{1}$, M. L. Brandi ${ }^{1}$

${ }^{1}$ Department of Surgery and Translational Medicine, Unit of Bone and Mineral Diseases, University of Florence, University Hospital of Florence, Florence, Italy

Objectives: Fibrous dysplasia (FD) is a rare bone disease, caused by missense mutations of GNAS gene. It is a bone developmental anomaly characterized by replacement of normal bone and marrow bone by fibrous tissue, and can present in monostotic or polyostotic forms. About $3 \%$ of lesions are associated with skin pigmentation and hyperfunctioning endocrine disorders known as the McCune Albright syndrome (MAS). Because FD is an uncommon disease, there are limited data in the literature. The aim of the study has been to describe the clinical characteristics of disease and response treatment of observed cases.

Material and Methods: The subjects with FD were evaluated between 2010 and 2015 at the Unit of Bone and Mineral Metabolism Diseases, Florence University Hospital. Main outcome measures included diagnostic evaluation, disease presentation, management strategy, and outcome.

Results: Thirty-four patients with FD were identified (16 monostotic forms, 18 polyostotic forms, and of these 3 MAS). The mean age at diagnosis was 29 years. The monostotic forms affected the craniofacial bones in 6 cases, long bones in 9 cases, and vertebra in 1 case. The polyostotic forms involved the cranial facial bones, long bones, pelvis, ribs and vertebrae. The levels of markers for bone formation and resorption were higher in polyostotic than monostic forms. The clinical manifestations were heterogeneous, characterized by bone pain, fractures, bone deformities, gait abnormality, loss of balance, hearing loss, visual impairment, and headache. Ten fractures have been reported in 8 patients. The average age of the first or single fracture was 23 years. Seventeen patients had bone pain, of these 6 patients reported a marked improvement in pain after therapy with bisphosphonates (BPs). The patients have been treated with 
calcium and vitamin D supplements, pamidronate or zoledronate, except one case with MAS, BPs nonresponder, treated with denosumab.

Conclusions: The clinical presentation of FD is highly variable and dependent upon lesion location and size. BPs have been shown to reduce bone pain in uncontrolled studies, but their influence on bone strength remains to be fully clarified. Large controlled clinical trials, and evaluations of new therapy are absolutely necessary.

\section{P609}

\section{OSTEOPOROSIS ASSOCIATED WITH SECONDARY HYPOGONADISM}

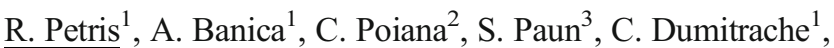
D. L. Paun ${ }^{4}$

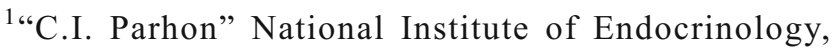
Bucharest, Romania, ${ }^{2}$ Carol Davila University of Medicine and Pharmacy, Bucharest, Romania, ${ }^{3}$ Emergengcy Hospital Floreasca Bucharest, Bucharest, Romania, ${ }^{4}$ U.M.F "Carol Davila” Bucharest, Bucharest, Romania

We have presented the case of a 42 years old man with craniopharyngioma operated by transfrontal approach with secondary hypogonadism in treatment with testosterone undecanoate who was admitted in National Institute of Endocrinology C.I.Parhon Bucharest for reevaluation. Two years ago the patient has presented in an ophthalmologic department for visual field defect. He had low visual acuity and the visual field performed at that time revealed bitemporal hemianopsia. Brain MRI revealed a pituitary tumor with the radiologic appearance of craniopharyngioma and associated optic nerve atrophy. He was sent in a neurosurgical department where they have evacuated $7-8 \mathrm{ml}$ of liquid by transfrontal approach. After evacuation of the liquid visual acuity has improved but he still had bitemporal hemianopsia. Endocrinologic evaluation showed secondary hypogonadism and all other pituitary hormones were normal. IGF 1 was low normal and he had mixed dyslipidemia. He started therapy with testosterone undecanoate and statins and he was sent to the neurosurgical department for surgery but he declined surgery. One year and a half later (on intermittent treatment with testosterone undecanoate), he had tibial plateau fracture. Endocrinologic evaluation showed secondary hypogonadism and hypothyroidism and DXA revealed a Z-score $-2.7 \mathrm{SD}$ at lumbar spine and -3 DS at the left hip and a T-score $-2.1 \mathrm{SD}$ at lumbar spine and -2.9 at the left hip. IGF 1 was low normal and he had mixed dyslipidemia. Echocardiography was normal. 25(OH)VitD was deficient, PTH, serum calcium, osteocalcin and CrossLaps were normal. Visual field showed bitemporal hemianopsia and visual acuity has decreased (mainly on the right eye) compared with the previously evaluation. Cerebral CT showed pituitary tumor $3 / 2.7 \mathrm{~cm}$. We started treatment with alendronate, vitamin d, calcium, 1-thyroxine, continued treatment with testosterone undecanoate, higher dose of statins and sent the patient to the neurosurgical department.

In conclusion: We have presented the case of a young man with secondary hypogonadism and associated osteoporosis. Also IGF 1 was normal, a deficiency of GH is possible but GH treatment in this patient is not an option at this moment because of the large pituitary tumor.

P610

BMD CHANGE AFTER ONE YEAR OF TREATMENT WITH DENOSUMAB OR BISPHOSPHONATES IN POSTMENOPAUSAL WOMEN WITH SEVERE OSTEOPOROSIS PRETREATED WITH TERIPARATIDE

$\underline{\text { T. Kocjan }}{ }^{1}$, A. Sabati Rajic ${ }^{1}$, M. Jensterle Sever ${ }^{1}$, B. Ostanek ${ }^{2}$, $\overline{\text { N. Orehek }}^{2}$, J. Marc ${ }^{2}$, M. Pfeifer ${ }^{1}$, J. Prezelj ${ }^{1}$

${ }^{1}$ University Medical Center Ljubljana, Ljubljana, Slovenia, ${ }^{2}$ Faculty of Pharmacy, University of Ljubljana, Ljubljana, Slovenia

Objective: Sequential therapy with bisphosphonates after teriparatide treatment for osteoporosis is well established while data on denosumab for this purpose is limited. Our aim was to compare BMD changes after one year of therapy between denosumab and bisphosphonates in osteoporotic patients who had completed treatment with teriparatide.

Patients and Methods: We retrospectively analyzed 98 postmenopausal women (aged $73.5 \pm 6.9$ years, $25.3 \pm 7.6$ years from menopause, BMI $26.6 \pm 4.7 \mathrm{~kg} / \mathrm{m}^{2}$ ) with severe primary osteoporosis who had been treated between 2006 and 2012 at our outpatient clinic with teriparatide for 18-24 months. After stopping teriparatide, treatment had been continued with a bisphosphonate (alendronate $70 \mathrm{mg}$ once weekly, risedronate $35 \mathrm{mg}$ once weekly, ibandronate $150 \mathrm{mg}$ once monthly or zoledronic acid $5 \mathrm{mg}$ i.v. once yearly) or with denosumab $60 \mathrm{mg}$ s.c. every 6 months according to the physician's and/ or patient's preference. All patients were prescribed with vitamin D3 1000 IU daily and were instructed to ingest $1200 \mathrm{mg}$ of calcium daily. BMD was measured at lumbar spine (LS), total hip (TH) and femoral neck (FN) by DXA when teriparatide was stopped and after 12 months of further treatment. Differences were statistically tested with independentsamples $t$-test and Mann-Whitney test.

Results: 50 patients continued treatment with a bisphosphonate and 48 patients received denosumab. The two groups did not differ significantly in any baseline characteristic (P 0.1360.577). After 12 months, the bisphosphonate group showed stable BMD values (nonsignificant decrease in LS and $\mathrm{FN}$ BMD $(-0.4 \pm 5.5 \%$ and $-0.1 \pm 5.7 \%$, respectively) and increase in TH BMD $(1 \pm 4.3 \%))$, whereas the denosumab 
group showed significant increase in LS, FN and TH BMD $(3.4 \pm 4.8 \%, P<0.001,1.9 \pm 4.6 \%, P<0.001$, and $2 \pm 3.5 \%$, $P=0.004$, respectively). When comparing the two groups, only the difference in LS BMD change after 12 months reached statistical significance $(P=0.001)$.

Conclusion: Twelve months after stopping teriparatide, sequential osteoporosis treatment with a bisphosphonate preserves previous BMD gain whereas with denosumab one might additionally increase BMD. This effect seems more pronounced at trabecular bone.

\section{P611}

\section{MUSCULOSKELETAL SIGNS IN PRIMARY HYPER-} PARATHYROIDISM

S. Lefkir ${ }^{1}$, D. Fodil ${ }^{1}$, M. Benchennaf ${ }^{2}$, L. Charabi ${ }^{2}$, N. Brahimi $^{3}$, A. Ladjouz-Rezig ${ }^{3}$

${ }^{1}$ Department of Rheumatology Beni-Messous Hospital, Algiers University Medicine Faculty, Algiers, Algeria, ${ }^{2}$ Rheumatology, University Hospital Centre, Beni-Messous, Algiers University Medicine Faculty, Algiers, Algeria, ${ }^{3}$ Rheumatology department, Benaknoun Hospital, Algiers University Medicine Faculty, Algiers, Algeria

Primary hyperparathyroidism (PHPT) is an endocrine disease characterized by excessive secretion of PTH resulting in practice by elevated levels of PTH (PTH) and hypercalcaemia. It is a predominantly female disease. The etiology is parathyroid adenoma in $90 \%$ of cases; hyperplasia and carcinoma are very rare. Secondary hyperparathyroidism (HPS) is often associated with chronic kidney disease (CKD) or chronic hypocalcaemia by calcium and/or vitamin D deficiency. In our country PHPT pattern has not changed as we continue to observe severe bone disease. We provide a descriptive study, longitudinal, mono centric cases of PHPT hospitalized in Rheumatology department between 1995 and 2010 because of: functional impairment, gait disturbance, bone tumors and fractures. Our aim is to describe the different clinical characteristics, radiographic images, biological assessments and additional tests contributing to diagnosis, complications, treatment and monitoring of these patients. Our series consists of 55 patients predominantly female (Sex ratio: $12 \mathrm{~F} / 1 \mathrm{H}$ ) with average age 43 years (19-82) years at diagnosis. Diagnosis delay was 4 years in our study. The HPT was primitive in $56 \%$, secondary and tertiary $44 \%$ by vitamin $\mathrm{D}$ deficiency in the majority of cases (12 by insufficient intake by celiac disease and renal failure was observed in $8 \%$ of PHPT. Clinical manifestations were predominantly diffuse bone pain $(86.5 \%)$, gait disorders $(71 \%)$, bone deformities $(28 \%)$, pathological fractures $(39 \%)$ of the femoral neck, distal radius, clavicle and maxilla. Arthralgias were observed in $35 \%$ and chondrocalcinosis joint damage in two cases. These signs were associated with asthenia (25\%), neuromuscular signs (42\%), renal colic crisis (19\%), gut symptoms (15\%), cardiovascular (5\%), renal failure (8\%). The biological signs focused on calcium and phosphate objectified hypercalcaemia in $48 \%$ cases, normocalcaemia ( $8 \%$ ), hypocalcaemia (32.6\%), hypophosphatemia (54\%). High PTH level was noted in $92.3 \%$, elevation of alkaline phosphatases in $75 \%$ and vitamin $\mathrm{D}$ deficiency in $14 \%$. The $\mathrm{x}$ ray radiographs objectified a diffuse bone loss with vertebral fractures in (44\%), peripheral fractures (38\%), worm-eaten skull (33\%), pelvic deformation (17\%), looser milkman striation (32\%), phalangeal tufts resorption (17\%), osteolytic images (15\%) and calcic edging in 2 patients. Cervical ultrasonography showed parathyroid adenoma (56\%), confirmed by scintigraphy in (30\% cases). Surgery was conducted in 35 patients $(67 \%)$ objectifying adenoma in 23 cases and hyperplasia in 12 cases. $33 \%$ of patients received vitamin and calcium treatment and gluten-free diet in 8 patients, intravenous rehydration associated with bisphosphonates in 5 patients for serious hypocalcaemia. Evolution was excellent in 36 patients whereas paresthesia or tetany crisis were observed in 8 patients leading to calcium addition in post-surgery. One patient presented papillary thyroid carcinoma referred for radiotherapy died 3 days after surgery. PHPT is a common bone disease that must be diagnosed early by blood calcium measurement, because of severe signs that could occur and compromise functional prognosis. Curative treatment of symptomatic forms is essentially surgical.

\section{P612 \\ OSTEOARTHRITIS AND PRIMARY AND SECON- DARY PREVENTION OF FRACTURES}

B. Stolnicki ${ }^{1}$, A. Libman ${ }^{1}$, H. Malleh ${ }^{1}$

${ }^{1}$ Creb - Prevrefrat, Rio de Janeiro, Brazil

During 18 months (May 2014 to November 2015) were evaluated 187 patients (67 men and 120 women) over 65 years of age referred for evaluation of fracture risk. 34 (20.3\%) had a fragility fracture history. Most patients had pain complaint in at least one joint or spine. Has been evaluated clinical risk factors and associated diseases. All patients were subjected to X-rays of the dorsal and lumbar spine and painful joints (in cases of synovitis ultrasound test was done), DXA scans, blood tests, balance, gait and postural analysis. After discarded secondary causes of osteoporosis and rheumatic conditions, all patients underwent supplementation of calcium and vitamin D, drugs for osteoporosis when necessary (DXA was less than 2.0 SD and associated risk factor). When needed NSAID, SYSADOA or DMADOA was prescribed. The patients underwent physical therapy in order to relieving joint pain, improve balance to prevent falls, muscle tone and posture. Resources were used as electrotherapy, acupuncture, therapeutic exercise (in soil and pool), neurological rehabilitation, Pilates and RPG. Patients were assessed periodically in relation to metabolic bone disease and changes in symptoms of osteoarthritis. 
Within the study period there were no new fractures. In our opinion the attention given to the rehabilitation of osteoarthritis (pain, range of motion and muscle tone) in combination with other measures to reduce the risk of new fractures was of great importance to the successful outcome. We believe that other studies with longer duration, will be necessary to include up osteoarthritis protocols to osteoporosis treatment.

\section{P613}

\section{THE ROLE OF MRI IN THE DIAGNOSIS OF} ASEPTIC OSTEONECROSIS OF FEMORAL HEAD A. Venter ${ }^{1}$, A. Pirte ${ }^{1}$, F. Cioara ${ }^{1}$, O. Straciuc ${ }^{1}$, I. Oswald ${ }^{1}$

${ }^{1}$ University of Oradea, Faculty of Medicine and Pharmacy, Oradea, Romania

Aseptic osteonecrosis of the femoral head is an increasingly common cause of musculoskeletal disability. Although patients are initially asymptomatic, disease usually progresses to joint destruction, requiring total hip replacement, usually before the fifth decade. Avascular necrosis is characterized by osseous cell death due to vascular compromise.

Material and Methods: The study included 42 patients, investigated during 2013-2015 in Department of Medical Imaging of Emergency Hospital Oradea. Patients were examined by standard X-ray and MR exam of the hip. Sequences used were $\mathrm{T} 1$ and $\mathrm{T} 2$ ponderation, $\mathrm{T} 2$ with fat suppression(at field strength of $1.5 \mathrm{~T}$ ), contrast medium injected to detect early ischemia. Sequences were used in coronary plan - to compare changes in left and right, in the sagittal plan- to highlight the early changes of the femoral head shape and the axial plane- to balance the extension of necrosis. Double-line sign is definitively a point of no return.

Results: Of the cases studies, 11 were in stage I(normal aspect of radiographs of the pelvis), 15 stage II and 16 in stage III and IV, as classified by Mitchell.

Conclusions: MRI is currently the most sensitive technique in diagnosis and prognosis of aseptic osteonecrosis of femoral head; we found aseptic osteonecrosis of femoral head with no clinical sign, neither radiographic, by positive MRI. Moreover, it enables the simultaneous study of both hips. It gives with precision the age and extent of the necrosed fragment and enables an absolute differential diagnosis with hip algodystrophy.

\section{P614 \\ PHALANGEAL QUANTITATIVE ULTRASOUND IN POSTMENOPAUSAL WOMEN RECEIVING AROMATASE INHIBITORS}

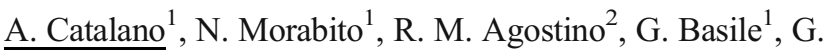
Natale $^{3}$, E. Morini ${ }^{1}$, A. Lasco ${ }^{1}$

${ }^{1}$ Department of Clinical and Experimental Medicine, University Hospital of Messina, Messina, Italy, ${ }^{2}$ Department of Human Pathology, University of Messina, Messina, Italy, ${ }^{3}$ Mineral Metabolism and Nephrology Clinic of Vibo Valentia Hospital, Vibo Valentia, Italy

Objective: Bone loss is a frequent occurrence in breast cancer (BC) women receiving aromatase inhibitors (AIs). Our aim was to assess bone status by phalangeal quantitative ultrasound (QUS) and by DXA, and to evaluate bone turnover in women treated with AIs.

Materials and methods: 60 postmenopausal $\mathrm{BC}$ women and 42 matched control were recruited (mean age 61.64 \pm 8.33 yr.). AD-SoS, UBPI, BTT as QUS parameters, L1-L4 and femoral neck BMD by DXA, vertebral fractures ( $\mathrm{Vfx}$ ) by $\mathrm{X}$-ray were assessed at baseline and after 18 months; serum levels of bone-specific alkaline phosphatase (BSAP) and CTX, as bone formation and resorption markers respectively, were measured every 9 months.

Results: FRAX derived 10-y probability of major fractures and hip fractures were significantly associated to AD-SoS ( $r=-0.381, p=<0.001$ and $r=-0.370, p<0.001$, respectively), UBPI $(r=-0.434, p=<0.001$ and $r=-0.409, p=<0.001$, respectively), BTT ( $r=-0.309, p=0.002$ and $r=-0.340, p=0.001$, respectively). The median percent changes of AD-SoS $[-3.71$ $(-5.38-0.11)$ vs. $-0.7(-4.15-0.83), p=0.02$, respectively] and BTT $[-8.4(-14.91--3.53)$ vs. $-1(-5.72-3.75)$, $p<0.001$, respectively] were significantly different between AI users and controls, and the same was observed for percent changes of lumbar spine BMD $[-2.94(-5.67--0.43)$ vs. $-0.69(-1.21$ to 0.11$), p=0.001$, respectively], and femoral neck BMD [ -2.5 $(-6.39-0.83)$ vs. $-0.3942(-1.49-1.29), p=0.01$, respectively]. In AI users, increased bone turnover rate was observed, the percent changes of BSAP and CTX being significantly different to controls $(p<0.01)$. AD-SoS modification was associated with change of BMD at lumbar spine ( $\beta$ 0.16, SE 0.08, $p=0.04)$ and change of BSAP $(\beta-0.04$, SE $0.02, p=0.04)$.

Conclusion: Phalangeal QUS appeared a reliable tool to evaluate bone quality also in $\mathrm{BC}$ women on AIs.

\section{P615}

\section{THE IMPACT OF LOW-DOSE DMARDS ON BONE MINERAL DENSITY IN EARLY RHEUMATOID ARTHRITIS}

S. Rexhepi ${ }^{1}$, V. Sahatçiu-Meka ${ }^{2}$, M. Rexhepi ${ }^{1}$, B. Rexhepi ${ }^{1}$, V. Mahmutaj ${ }^{3}$, G. Rexhepi ${ }^{4}$, S. Boshnjaku ${ }^{4}$

${ }^{1}$ Rheumatology Clinic, University Clinical Centre of Kosovo, Prishtina, Albania, ${ }^{2}$ University of Prishtina, Prishtina, Albania, ${ }^{3}$ University Clinical Centre of Kosovo, Prishtina, Albania, ${ }^{4}$ Private Clinic "Rheuma", Prishtina, Albania

The aim of this study was to assess the impact of low dose of methotrexate on BMD in patients with early rheumatoid arthritis (RA). This paper follows a retrospective study, which 
involves 60 female patients with early onset RA diagnosed according to the American Rheumatism Association Criteria (ACR/EULAR 2010). Inclusion criteria included premenopausal women with range of age 32-47 years, with disease duration $<12$ months and no previous disease modifying antirheumatic drugs (DMARDs) or corticosteroids. Exclusion criteria included: drug hypersensitivity, hepatic, renal, hematologic, pulmonary, cardiovascular diseases, as well as active peptic ulcer medications that affect bone turnover. The patients were divided into two groups: group I was composed of thirty patients treated with dose of $7.5 \mathrm{mg} /$ weekly methotrexate (MTX), while group II included thirty patients treated with dose of $2 \mathrm{~g}$ /daily salazopyrine (SSZ). The disease activity was measured by a combination of erythrocyte sedimentation rate (ESR) and disease activity score (DAS-28). BMD of the lumbar spine (L2-4), and femoral neck, was measured by DXA (Stratos 800). Laboratory findings that were done at baseline and in the end of the study included: serum calcium, phosphorus and alkaline phosphatase. From total number of patients included in the study, four patients were excluded from the group I with MTX, due to side effects. In this study, we found no negative effect on BMD in RA patients treated with low dose MTX in comparison to patients treated with SSZ. At 12 months, there were no significant differences in BMD of the lumbar spine and femoral neck. At the baseline BMD, results at the lumbar spine in group I $0.95 \pm 0.14$ vs. group II $0.97 \pm 0.12$ compared at 12 months, where group I $0.95 \pm 0.12$ vs. group II $0.93 \pm 0.19$, showed no significant difference ( $P=0.954$ vs. $P=0.073$ ). Also, BMD in femoral neck region at the results in group I $0.88 \pm 0.10$ vs. group II 0.87 \pm 0.11 compared at 12 months in group I $0.88 \pm 0.09$ vs. group II $0.86 \pm 0.11$, showed no significant difference ( $P=0.708$ vs. $P=0.222$ ). Statistical analysis was followed through SPSS. The probability level was expressed by $p$-value of $<0.05$. As conclusion, based on the results of our study, low dose of methotrexate has no negative effect on BMD in premenopausal RA patients. We believe that these results might provide new insights and that further longitudinal studies with larger groups of premenopausal RA patients are required.

\section{P616 \\ MULTIDISCIPLINARY TREATMENT OF FRAGILITY FRACTURE IN THE ELDERLY: CREATION OF A NEW PROTOCOL}

O. Fernandez-Berrizbeitia $^{1}$, A. Hernaiz-Alzamora ${ }^{2}$

${ }^{1}$ Rheumatology Department, Basurto University Hospital, Bilbao, Spain, ${ }^{2}$ Traumatology Department, Basurto University Hospital, Bilbao, Spain

Introduction: Fragility fracture is defined as those fractures originated from a low energy impact in people older than 50 years, without any other secondary reason for such fracture.
There are about 3 million people with osteoporosis in Spain. The expenses resulting from an elderly hip fracture seem to be one of the most important type of fracture in Europe, with 9936 $€$ per hospitalization. The total expenses must count as well the expenses originated from the patient's environment (about 15.000 millions of euros the first year) and the non-quantified ones due to changes in the lifestyle either in the patients or their relatives. Nevertheless, the real problem appears when the real impact in the morbimortality is measured. The correct approach and treatment of the patients that have a fragility fracture reduces, up to a $50 \%$, the posterior risk of undergoing a new fracture. The reality is that only $25 \%$ of the people who suffers a fragility fracture in our community receives some kind of treatment or recommendation after the fracture.

Methods: We have revised medical records of patients who were hospitalized and treated in Traumatology Department at Basurto U. Hospital from fragility fractures in 2015. We included 408 hip fractures, 107 vertebral fractures and 100 shoulder fractures. We recorded epidemiological data (mean age, sex), previous medical history (diseases, previous fractures), received treatment (surgical or nonsurgical), complications during the admission, and recommendations given in the patient's discharge. The presence of new fractures at the time of the review was also reviewed.

Objective: To describe risk factors for fragility fractures and common medical mistakes (the absence of osteoporosis diagnosis or treatment prescription in those patients) for a better disease management and reduction of such mistakes through the development of a new protocol.

Results: $90 \%$ of the patients hospitalized were discharged without any treatment or recommendation for the management of the diagnosed bone fragility. $85 \%$ of the rest were not admitted in the orthopedic surgery department but in other departments. The high incidence of medical complications during the hospitalization requires the collaboration of different specialists to the correct management of OS patient.

Conclusions: The prevention of fragility fractures must be ground, in the first place, in the medical treatment for osteoporosis. In addition, correction of the potentially modifiable risk factors, and prevention of falls must be promoted. We can improve the compliance of these recommendations from $25 \%$ to $61 \%$ with the collaboration of different medical specialists. we have just started a new multidisciplinary protocol for the management and treatment of the fragility fracture patients in Basurto Hospital, which will be carried out by Orthopedic Surgery, Rheumatology, Internal Medicine, Rehabilitation and Social Assistance departments. The real impact of this project will need to be evaluated over the next few years.

\section{References:}

- Herrera A y Grupo de Expertos en Osteoporosis de la SECOT. REEMO 2003;12:125

- Jódar Gimeno E. Rev Osteoporos Metab Miner 2010;2:79

- Bouxsein ML, et al. J Am Acad Orthop Surg 2004;12:385

- Herrera A, et al. Int Orthop 2006;30:11. 


\section{P617}

\section{ONLINE USAGE OF FRAX FRACTURE RISK} ASSESSMENT AND NATIONAL OSTEOPOROSIS GUIDELINE GROUP GUIDANCE BY UK CLINICAL PRACTITIONERS

$\underline{\text { E. McCloskey }}{ }^{1}$, H. Johansson ${ }^{1}$, N. C. Harvey ${ }^{2}$, J. E. Compston $^{3}$, J. A. Kanis ${ }^{1}$

${ }^{1}$ Centre for Metabolic Bone Diseases, University of Sheffield Medical School, Sheffield, United Kingdom, ${ }^{2}$ MRC Lifecourse Epidemiology Unit, University of Southampton, Southampton, United Kingdom, ${ }^{3}$ Department of Medicine, University of Cambridge School of Clinical Medicine, Cambridge, United Kingdom

In several countries, the online output of FRAX is linked to independent country-specific guidelines (e.g. UK, Lebanon, and Finland) which facilitates treatment decisions according to local assessment guidelines. In the UK, guidance provided by the National Osteoporosis Guideline Group (www.shef.ac. uk/NOGG) was made available in 2008. The aim of this study was to determine the uptake of this facility by exploring website activity using Google Analytics software. We have undertaken an analysis of FRAX and NOGG website usage for the year between 1st July 2013 and 30th June 2014 (Google Analytic reports 8th August 2014). During this period, there was a total of $1,774,812$ sessions (a user interaction with the website) on the FRAX website with 348,964 of these from UK-based users. Over the same time, 253,530 sessions were recorded on the NOGG website. Of the latter, two-thirds were returning users, one-third new users and the vast majority $(208,766,82 \%)$ arose from users in the UK. The remainder of users were from other countries with the US comprising approximately half of the non-UK use, demonstrating that some users of FRAX in other countries make use of the NOGG guidance. Of the UK-sourced sessions, the majority were from England, but the session rate (adjusted for population) was highest for Scotland (Table). Almost all (95. $7 \%$ ) of the UK sessions arose from calculations being passed through from the FRAX tool (www.shef.ac.uk/FRAX) to the NOGG website, comprising FRAX calculations in patients without a BMD measurement $(155 \mathrm{~K}, 74.5 \%)$ or FRAX calculations with a BMD result (44 K, $21.2 \%)$. A minority of sessions were conducted for other reasons (manual calculations, document downloads, FAQs etc.). National Health Service (NHS) sites were identified as the major source of visits to the NOGG website, comprising $64 \%$ of the identifiable visiting locations, but this is an underestimate as many sites from within the NHS are not classified as such. The study shows that the facilitated interaction between web based fracture risk assessment and clinical guidelines is widely accepted by clinical users. The approach could usefully be adopted in all countries for which a FRAX model is available.
Table. Population adjusted rates (for those aged 50 years and over) of usage of the FRAX and NOGG websites in the four nations of the United Kingdom.

\begin{tabular}{lccccc}
\hline Country & $\begin{array}{c}\text { FRAX } \\
\text { ses- } \\
\text { sions }\end{array}$ & $\begin{array}{c}\text { NOGG } \\
\text { ses- } \\
\text { sions }\end{array}$ & $\begin{array}{c}\text { Population } \\
\text { aged 50 and } \\
\text { over }\end{array}$ & $\begin{array}{c}\text { FRAX } \\
\text { session } \\
\text { rate }\end{array}$ & $\begin{array}{c}\text { NOGG } \\
\text { session } \\
\text { rate }\end{array}$ \\
\hline England & 270,748 & 163,749 & $14,829,804$ & 18,257 & 11,042 \\
Scotland & 55,999 & 32,740 & $1,936,000$ & 28,925 & 16,911 \\
Wales & 14,384 & 7,677 & $1,155,951$ & 12,443 & 6,641 \\
$\begin{array}{c}\text { Northern } \\
\text { Ireland }\end{array}$ & 7,784 & 4,586 & 574,215 & 13,556 & 7,986 \\
\hline
\end{tabular}

\section{P618}

\section{COMPLEX REGIONAL PAIN SYNDROME SECONDARY RECOVERY OF UPPER LIMB} INJURIES

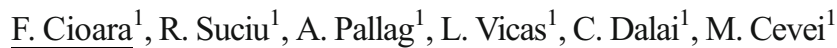
${ }^{1}$ University of Oradea, Faculty of Medicine and Pharmacy, Oradea, Romania

Objectives: Assessment of the effects of physical therapy recovery in patients according to diagnosis complex regional pain syndrome (CRPS).

Materials and methods: We studied a number of 18 patients with upper limb secondary algoneurodystrophic trauma, who conducted the rehabilitation treatment between February 2015-June 2015, at the Medical Rehabilitation Clinical Hospital Baile Felix. Patients were assessed through anamnesis, clinically and laboratory. Questionnaires were applied to the functional and psychological assessment. Evaluation tests of motor function and pain that we applied were: MPQ-SF scale, Balance of functional independence in the event of damage to the train's superior. For the evaluation of psychoaffective status we used Hamilton Scale. We finally appreciated the patient satisfaction using the Likert scale.

Results: Through recovery treatment applied to our patients, have obtained good results over pain (Pain Scale scale McGill), functional independence (according to the balance sheet of the functional independence in the event of damage to the train's superior) and on the psycho-emotional status. Subjective symptoms of the patients have been pain, upper limb joint dysfunction, anxiety and the altered pattern of sleep. The most common objective claims have been caused pain, altered prehension, as well as joint mobility. In the presence of CRPS, psychoemotional sphere is negative influenced, in all patients, most of them presenting moderate and severe anxiety.

Conclusion: CRPS patient needs are complex and must be addressed to the pain, motor function and psycho-emotional status. The role of interdisciplinary team was important in the recovery treatment, thus bringing great results over boosting patient (Likert scale). 10 sessions of rehabilitation treatment 
are insufficient to the CRPS patients, relating to the complexity of somatic type and psycho-affective becoming evident the need of long-term recovery for this type of pathology.

\section{P619}

\section{UNDERSTANDING HIP FRACTURE BY} TWO-LEVEL BIOMECHANICAL MODELING OF SIDEWAYS FALL

Y. Luo ${ }^{1}$, M. Nasiri Sarvi ${ }^{2}$, W. D. Leslie ${ }^{3,4}$

${ }^{1}$ Department of Mechanical Engineering, Faculty of Engineering, University of Manitoba, Winnipeg, Canada, ${ }^{2}$ Department of Biomedical Engineering, Faculty of Engineering, University of Manitoba, Winnipeg, Canada, ${ }^{3}$ Department of Internal Medicine, Faculty of Health Science, University of Manitoba, Winnipeg, Canada, ${ }^{4}$ Department of Radiology, Faculty of Health Science, University of Manitoba, Winnipeg, Canada

Objectives: To understand how fall-related hip fracture risk (HFR) is affected by biomechanical factors including BMI, hip soft-tissue thickness (HSTT) and BMD, comprising factors that affect HFR at different musculoskeletal levels.

Material and Methods: A two-level biomechanical model, consisting of a musculoskeletal dynamic model and a proximal femur finite element (FE) model, were constructed from a subject's whole body and hip DXA images, respectively, which were used to determine impact force in sideways fall ${ }^{1,2}$ and to compute stresses in the proximal femur induced by the impact force $^{3,4}$. HFR was measured as the ratio of actual to yield stress. The biomechanical model was applied to an independent convenience sample of 130 subjects ( 80 women with mean age 53.7 years and BMI $27.1 \mathrm{~kg} / \mathrm{m} 2 ; 50$ men with mean age 39.8 years and BMI $\mathrm{kg} / \mathrm{m}^{2}$ ). HFR was predicted for each subject, and associations with biomechanical factors were studied. Results: Sex-stratified Pearson correlations and testing for sex interaction are provided in the table.

\begin{tabular}{llllllll}
\hline \multirow{2}{*}{$\begin{array}{l}\text { Correlation } \\
\text { between }\end{array}$} & HFR & & & Impact & \multicolumn{2}{l}{ BMI } \\
\cline { 2 - 4 } \cline { 6 - 8 } & FMI & HSTT & BMD & HSTT & HSTT & BMD \\
\hline Women & $-0.30^{* *}$ & $-0.43^{* *}$ & $-0.71^{* *}$ & $-0.27^{*}$ & $0.78^{* *}$ & $0.55^{* *}$ \\
Men & $-0.13^{*}$ & 0.22 & $-0.82^{* *}$ & -0.17 & 0.72 & $0.62^{* *}$ \\
p-interaction & 0.332 & $<0.001$ & 0.144 & 0.569 & 0.459 & 0.562 \\
\hline
\end{tabular}

$* p<0.05, * * p<0.01$

Greater BMI was predicted to be protective against hip fracture (lower HFR) due to associations with impact force (thicker HSTT) and bone strength (higher BMD) seen in both women and men. BMI protection was slightly stronger in women $(r=-0.30)$ than in men $(r=-0.13)$, but the sex difference was not significant. On the other hand, HSTT was predicted to be protective against hip fracture in women but not men $(r=-0.43$ vs $r=0.22$, p-interaction $<0.001)$. Women may have thicker HST (mean HSTT $54.3 \pm 23.3 \mathrm{~mm}$ for women vs. $49.9 \pm 23.1 \mathrm{~mm}, p=0.3$ ).

Conclusions: Image-based two-level biomechanical modeling may provide insights into the pathophysiology of hip fracture.

Acknowledgements: The first author gratefully acknowledges NSERC grant (37098).

Disclosures: No conflict of interest.

References:

1. Luo y, et al. Int Biomech 2014;1:1

2. Nasiri Sarvi M, et al. J Biomed Sci Engin 2014;7:405

3. Luo Y, et al. J Engin Med 2011;225:1188

4. Naylor KE, et al. J Bone Miner Res 2013;28:1014

\section{P620}

IS FEMUR CROSS-SECTIONAL STIFFNESS A BETTER SURROGATE OF BONE STRENGTH THAN GEOMETRY?

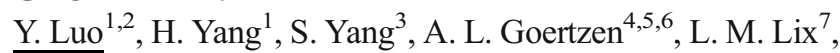
W. D. Leslie ${ }^{3,4}$

${ }^{1}$ Department of Mechanical Engineering, Faculty of Engineering, University of Manitoba, Winnipeg, Canada, ${ }^{2}$ Department of Biomedical Engineering, Faculty of Engineering, University of Manitoba, Winnipeg, Canada, ${ }^{3}$ Department of Internal Medicine, Faculty of Health Sciences, University of Manitoba, Winnipeg, Canada, ${ }^{4}$ Department of Radiology, Faculty of Health Sciences, University of Manitoba, Winnipeg, Canada, ${ }^{5}$ Department of Physics \& Astronomy, Faculty of Science, University of Manitoba, Winnipeg, Canada, ${ }^{6}$ Section of Nuclear Medicine, Health Sciences Centre, Winnipeg, Canada, ${ }^{7}$ Department of Community Health Sciences, Faculty of Health Sciences, University of Manitoba, Winnipeg, Canada

Objective: Geometric parameters of femur cross-section, such as cross-sectional area (CSA) and cross- section moment of inertia (CSMI) ${ }^{1}$, have limited accuracy for discriminating hip fractures as they cannot accurately represent bone strength. The objective of this study was to investigate whether femur cross-sectional stiffness, which integrates both bone material properties and cross-section geometry, is a better surrogate of bone strength using prior hip fracture as a proxy for proximal femur strength.

Material and Methods: We compared femur cross-sectional stiffness and geometric parameters ${ }^{1}$ for discriminating 99 women with prior hip fracture (mean age 78.1y) from 294 women without fracture (mean age $74.2 \mathrm{y}$ ) identified in the Manitoba BMD Database (excluding women with normal hip T-scores or on anti-osteoporosis therapy). Femur axial/ shear (AS) and bending stiffness (BS) were calculated from hip DXA (dual energy X-ray absorptiometry) using elasticity- 
BMD relations ${ }^{2}$. Hip fracture discrimination was assessed from the area under the receiver operating characteristic curve (AUC) and age-adjusted odds ratio (OR per SD decrease) in logistic regression models.

Results: All femur cross-sectional stiffness (AS and BS) and geometry measures (CSA, CSMI and ZZ) showed significant hip fracture discrimination. BS had greater AUC than CSMI $(P=0.03)$. The AUC for AS was slightly greater than for CSA, but the difference was not significant $(p>0.2)$.

\begin{tabular}{lcc}
\hline & AUC $(95 \% \mathrm{CI})$ & $\begin{array}{c}\text { Age-adjusted OR } \\
(95 \% \mathrm{CI})\end{array}$ \\
\hline Cross-Section Area (CSA) & $0.723(0.662-0.784)$ & $2.34(1.75-3.15)$ \\
Axial/Shear Stiffness (AS) & $0.734(0.672-0.795)$ & $2.42(1.80-3.26)$ \\
Cross-Section Moment of & $0.734(0.529-0.674)$ & $1.42(1.08-1.85)$ \\
$\quad$ Inertia (CSMI) & & \\
Cross-Section Modulus (ZZ) & $0.632(0.560-0.703)$ & $1.63(1.24-2.15)$ \\
Bending Stiffness (BS) & $0.632(0.560-0.704)$ & $1.63(1.24-2.15)$ \\
\hline
\end{tabular}

Conclusions: BS showed greater hip fracture discrimination than CSMI, suggesting that femur cross- sectional stiffness may be better than geometry as a surrogate measure for proximal femur strength.

Acknowledgements: The first author gratefully acknowledges funding from the Natural Sciences and Engineering Research Council (\#37098).

\section{References:}

1. Luo Y, et al. Applied Mechanics Materials, 2015;775:415

2. Beck TJ, et al. Invest Radiol 1990;25:6

\section{P621}

\section{REGULATION OF ENDOTHELIAL NOTCH SIGNALLING IN AGE-RELATED BONE LOSS}

S. K. Ramasamy $^{1}$, A. P. Kusumbe ${ }^{1}$, R. H. Adams ${ }^{1}$

${ }^{1}$ Max Planck Institute for Molecular Biomedicine, Muenster, Germany

Angiogenesis and osteogenesis are coupled coherent processes occurring during the development of skeletal system. A distinct capillary subtype called type $\mathrm{H}$ vessels has been identified to play an important role in coupling of angiogenesis and osteogenesis. These blood vessels show presence of specialized structures like loops and bulges, but their involvement in mediating non-sprouting angiogenesis is not known. Here, using a novel intra vital imaging technique, we describe the mechanism of blood vessel growth in the mice long bones, which involves the extension, and anastomotic fusion of endothelial buds. The formation of these structures is severely impaired in aged mice bones due to the progressive loss of endothelial Notch signalling. This leads to decline in angiogenesis and coupled osteogenesis. However reactivation of endothelial Notch signalling in aged bone leads to the generation of angiogenic structures to promote vessel growth and type $\mathrm{H}$ vessels, which ultimately triggers osteogenesis. Thus modulation of Notch activity in blood vessels could be a potential therapeutic strategy to prevent and manage age-related bone loss.

\section{P622}

\section{DIFFERENT APPROACHES IN THE MANAGEMENT OF BONE DENSITOMETRY IN CLINICAL PRACTICE: OLD CERTAINTIES AND ROOM FOR} IMPROVEMENT

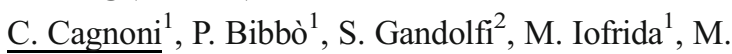
Occhipinti $^{1}$

${ }^{1}$ Department of Internal Medicine, Azienda USL di Piacenza, Piacenza, Italy, ${ }^{2}$ Department of Staff, Azienda USL di Piacenza, Piacenza, Italy

Introduction: DXA is currently the best technique available to evaluate bone mass, enabling the diagnosis of osteoporosis, the prediction of fracture risk and monitoring. Clinical good practices consider as preferred measuring sites the lumbar (L1-L4) and proximal femur (neck and total). The vertebral morphometry is a quantitative method for the diagnosis of vertebral fractures based on measuring vertebral heights. There is an uneven supply of diagnostic densitometric that historically represents a significant problem for the consequences that entails, in terms of money expenditure (duplication of incorrect examinations), of credibility of the diagnostic method and, in particular, of the accuracy of the subsequent clinical management.

Objective: Contrary to the prevailing standard in our area that includes the performance of a single scan (lumbar at age $<65$ years and unilateral femoral at age $>65$ years), the benchmark operating standard at our Center, designated to the diagnosis and treatment of osteoporosis, involves running contextual lumbar scans, bilateral femoral and lateral to evaluate morphometric vertebral (VFA). We conducted a prospective data collection aimed at detecting whether there are significant differences in the two approaches considering a strictly diagnostic and clinical.

Material and Methods: 311 consecutive unselected patients underwent DXA examination according to the extensive method (lumbar and femoral bilateral, VFA); in each case, we have also detected what would be the diagnostic conclusion if it had applied a restrictive method (DXA lumbar age $<65$ years or femoral age $>65$ years); we also researched the impact resulting from the bilateral femoral scan, compared to unilateral; finally, we measured the impact of the VFA, in addition to DXA lumbar and femoral bilateral. 
Results: In our experience, the contextual execution of the DXA in two standard sites (lumbar and femoral) produces an increase in diagnostic sensitivity of $40.5 \%$. The simultaneous execution of DXA in the lumbar and femoral bilateral, plus vertebral morphometry, to the execution of conventional test (vertebral or femoral) induces an increase in the percentage of pathologic findings of $47.17 \%$. There is a particularly significant increase in the evidence of severe osteoporosis (which is tripled, with the ability to document unrecognized vertebral deformities). The impact of the two different approaches on clinical decisions resulted to be significant: we record a $34.4 \%$ increase of prescriptions of first level antiresorptive therapy (alendronate, raloxifene) and more than doubled ( $130 \%$ increase) recourse to a second level treatment (denosumab, zoledronic acid, teriparatide).

Conclusions: The availability of suitable equipment and the investment of resources (time and clinical skills) fully compatible and sustainable with the current practice, allow us to apply operating standards in diagnostic densitometry that significantly increase the accuracy of the examination and its usefulness in practice, as well as the credibility of the diagnostic path.

\section{P623}

\section{INCIDENCE OF PNEUMONIA IN HIP FRACTURE PATIENTS OVER A 12-MONTH PERIOD IN A} MAJOR TRAUMA CENTRE IN UK

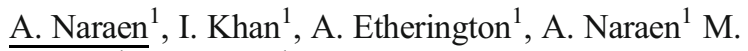
Memon $^{1}$, S. Naraen ${ }^{1}$

${ }^{1}$ Orthogeriatrics, liverpool, United Kingdom

Background: Pneumonia is a significant cause of morbidity in the UK. Community acquired pneumonia affects from $0.5 \%$ $1 \%$ of the adult population. In those adults presenting with lower respiratory tract infections, $5 \%-12 \%$ will be diagnosed with pneumonia, amongst these $22-42 \%$ admitted to hospital where the mortality is $5 \%-14 \%$. Hospital acquired pneumonia affects $1.5 \%$ of inpatients at any time. It is reported to increase stay by around 8 days and has a mortality rate ranging 30-70\%.

Aim: In a teaching hospital in the UK it was noted that there was an increased death rate due to pneumonia in patients staying in the hip fracture ward. This study aimed to look at the incidence of pneumonia in neck of femur fracture patients and their outcomes following treatment.

Method: This is a retrospective study involving the review of hospital records of 381 hip fracture patients over a period of 12 months. The primary outcome was looking at mortality with pneumonia in hip fracture patients. The following outcomes were also looked at in hip fracture patients in relation to; age, gender, type of surgery and comorbidities.

Results: Looking at the primary outcome: $15 \%$ (54 of 381) of hip fracture patients were confirmed to be afflicted with chest infection. $76 \%(41)$ of these were confirmed to have pneumonia and $20 \%$ died as a complication of pneumonia. The highest incidence of pneumonia was noted in ages above 75 with $74 \%$ (40 out of 54 patients). The highest frequency age group was $81-85$ with $24 \%$ (13 out of 54). With respect to the secondary outcome; $74 \%$ had cardiac comorbidities, $43 \%$ had hypertension, $53 \%$ of patients had at least one respiratory co-morbidity and $39 \%$ had COPD, $41 \%$ of patients had one CNS comorbidity and $17 \%$ had dementia, $30 \%$ of the patients were gastro/renal patients, and $28 \%$ had at least one endocrine comorbidity.

Conclusion: There is a rise in the incidence of mortality due to pneumonia in ward patients with hip fracture, the consequences of which are far reaching for both the hospital and the relatives. Pneumonia needs to be actively prevented. Once diagnosed, it must be treated promptly and aggressively.

\section{P624}

\section{CLINICAL APPLICABILITY OF 3D-DXA IN INDIVIDUALS WITH SMALL BONE SIZE: A CASE REPORT}

B. M. Camargos ${ }^{1}$, B. C. Silva ${ }^{2}$, L. H. Humbert ${ }^{3}$, L. Del Río ${ }^{4}$ ${ }^{1}$ Densimater - Rede Mater Dei de Saúde, Belo Horizonte, Brazil, ${ }^{2}$ Santa Casa de Belo Horizonte, Belo Horizonte, Brazil, ${ }^{3}$ Musculoskeletal Unit, Galgo Medical S.L., Barcelona, Spain, ${ }^{4}$ CETIR Centro Médico, Barcelona, Spain

Introduction: Small bone size underestimates areal BMD (aBMD). 3D-DXA algorithm builds a volumetric model from DXA images.

Objective: Explore clinical applicability of 3D-DXA on a small bone-sized subject.

Material and Methods: A 50 y.o., $128 \mathrm{~cm}$ height man, evaluated by DXA (GE Lunar Prodigy Advanced+), was compared to a control group (26 men, 45-55 years, randomly selected). On the patient, QCT (QCT Pro 5.1, Mindways) showed normal volumetric BMD (vBMD) at lumbar spine $\left(148 \mathrm{mg} / \mathrm{cm}^{3}\right)$, low vBMD at total femur $\left(112 \mathrm{mg} / \mathrm{cm}^{3}\right)$ and normal TBS (TBS iNsight 2.1, Medimaps) value (1.392).

Control group DXA was performed on GE Lunar iDXA $(n=17)$ and DMS Stratos dR $(n=9)$. The estimated vBMD (3D-DXA software 1.2, Galgo Medical) evaluated proximal femur vBMD, trabecular and cortical bone compartments in subject and controls. Student's $t$-test compared the results.

Results: Mean age at control group was $49.8 \pm 2.7$ years. Short stature patient total hip aBMD Z-sore $=-2.6, \mathrm{~T}$-score $=-2.9$. Estimated proximal femur volume was $32.2 \mathrm{~cm} 3$ for the shortsized individual showing a -5.1 SD difference from control group $\left(100.6 \pm 13.5 \mathrm{~cm}^{3}\right)$. Proximal femur cortical thickness was $1.2 \mathrm{~mm}$ on the short stature individual, showing a $-2.1 \mathrm{SD}$ difference from controls $(1.8 \pm 0.2 \mathrm{~mm})$. 3D-DXA volume difference was more pronounced at the cortex $\left(961 \mathrm{mg} / \mathrm{cm}^{3}\right.$ vs. 1044 
$\pm 20 \mathrm{mg} / \mathrm{cm}^{3} ;-4.1 \mathrm{SD}$ ) than at trabecular compartment (78 mg/ $\mathrm{cm}^{3}$ vs. $\left.140 \pm 41 \mathrm{mg} / \mathrm{cm} 3 ;-1.5 \mathrm{SD}\right)$.

Conclusion: 3D-DXA volume was lower on the short-stature case than in controls. Differences were more evident at the cortex than trabecular compartment. For short-stature individuals, 3D-DXA might be associated to bone fragility related to cortex thinning.

\section{P625}

\section{INCIDENCE AND RISK OF FRACTURE IN} DIABETIC PATIENTS AGED 50 AND ABOVE A. A. Gusova ${ }^{1}$, M. G. Pavlova ${ }^{2}$, G. A. Melnitchenko ${ }^{2}$ ${ }^{1}$ Outpatient Department, Scientific Research Institute of Neurosurgery named after N.N. Burdenko, Moscow, Russian Federation, ${ }^{2}$ Endocrinology Department, I.M. Sechenov First Moscow State Medical University, Moscow, Russian Federation

Objective: To study if elder people with type 2 diabetes mellitus (T2DM) have an increased risk of fracture.

Methods: The records for two-year period were analyzed in the only local trauma clinic in the territory with elder population of 29,512 people. It was compared with data of The National Register of diabetes mellitus patients (1981 persons over 50 years with T2DM).

Results: There was not an elevation in total fracture risk as well as risk of particular localisations in diabetic population in our study. Moreover, there was observed lower rate of shin and proximal humerus fracture. Women with DM had lower total fracture risk than women without DM (relative risk 0.43 ; 95\%confidence interval 0.30-0.61). In men fracture risk was similar in diabetic and non-diabetic groups (RR 0.97; 95\%CI 0.591.60). We also haven't found an increased hip fracture rate in diabetic people compared to non-diabetic ones. There was a substantial elevation in the incidence of multiply or repeated fractures in diabetic population (1.5\%o vs. $0.3 \%$ ), which in 5-fold higher risk of these fractures for elder people with T2DM. There was no significant influence on fracture risk in elder T2DM population of age, BMI and diabetes duration. The only significant fracture risk factor was the presence of diabetic vascular complications (both microangiopathy and macroangiopathy).

Conclusion: In our study we found that elder diabetic patients did not have an increased total fracture risk. T2DM in women was associated with a lower frequency of all fractures. This could be explained by decreased physical activity of these patients. There was an elevation of multiply/repeated fracture rate which could indicates poor quality and/or higher risk of traumatic falling in elder diabetic patients.

\section{P626}

\section{PREVALENCE OF VITAMIN D DEFICIENCY AND BONE MINERAL DENSITY STATUS AMONG U.S.} ADULTS

C. H. Orces ${ }^{1}$, K. Weisson ${ }^{2}$

${ }^{1}$ Medicine/Laredo Medical Center, Laredo, United States,

${ }^{2}$ Baptist Hospital, San Antonio, United States

Objectives: To examine the prevalance of $25(\mathrm{OH}) \mathrm{D}$ deficiency among adults aged 50 years and older according to their bone mineral density (BMD) status in the United States (U.S.).

Material and Methods: The present study was based on data from the National Health and Nutrition Examination Survey 2007-2010. Participants underwent an in-person 24-hour recall dietary and supplement component interview. Since 2007, the vitamin D values in this dataset reflect the sum of ergocalciferol (vitamin D2) and cholecalciferol (vitamin D3) content of foods reported by survey participants. Moreover, sociodemographic variables previously reported to be associated with 25(OH)D deficiency were included in this analysis. According to the WHO, the specific NHANES III cutoff values to define osteopenia were 0.56 to $0.74 \mathrm{~g} / \mathrm{cm}^{2}$ and $0.56 \mathrm{~g} / \mathrm{cm}^{2}$ or less to define osteoporosis of the femur neck. Serum $25(\mathrm{OH}) \mathrm{D}$ was measured from a standardized liquid chromatography-tandem mass spectrometry (LC-MS/MS) method and reported in $\mathrm{ng} / \mathrm{ml}$. 25(OH)D concentrations $<20 \mathrm{ng} / \mathrm{ml}$ were defined as $25(\mathrm{OH}) \mathrm{D}$ deficiency. The crude prevalence of $25(\mathrm{OH}) \mathrm{D}$ deficiency was estimated according to participants' BMD status. Sex-specific logistic regression models were used to examine the independent association between BMD status and $25(\mathrm{OH}) \mathrm{D}$ deficiency.

Results: A total of 4,310 participants had complete data on BMD, 25(OH)D concentrations, and total vitamin D intake, representing 139 million adults in the U.S. Of these, the prevalence of osteoporosis was $7.0 \%$ (95\% CI, $5.9 \%, 8.3 \%)$ and $1.2 \%$ (95\% CI, $0.8 \%, 1.9 \%$ ) among women and men, respectively. Moreover, osteopenia was present in $51.4 \%$ (95\% CI, $49.2 \%, 53.5 \%)$ of women and $29.2 \%(95 \% \mathrm{CI}$, $27.0 \%, 31.5 \%$ ) of men. The mean vitamin D intake was 385 (SD 18.8) IU and vitamin D supplements were reported by only $29.6 \%$ (95\% CI, $27.1 \%, 32.3 \%$ ) of participants. Overall, 25(OH)D deficiency was prevalent in $19.0 \%(95 \%$ CI, $16.5 \%, 21.9 \%$ ) of men and $21.4 \%$ (95\% CI, $18.9 \%$, $24.2 \%$ ) of women in the U.S. As shown in Table 1, the prevalence of $25(\mathrm{OH}) \mathrm{D}$ deficiency among U.S. adults stratified by BMD status differs according to participants characteristics. In fact, higher $25(\mathrm{OH}) \mathrm{D}$ deficiency prevalence rates were seen among women, non-Hispanics whites, subjects examined between May 1 and Oct 31, those with lower education, current smokers, and obese subjects with osteoporosis as compared with participants with normal BMD. Moreover, participants with osteoporosis who self-reported their health 
status as fair to poor and those who did not participate in moderate work or recreational activities had higher $25(\mathrm{OH}) \mathrm{D}$ deficiency prevalence rates. Of relevance, a marked higher prevalence of $25(\mathrm{OH}) \mathrm{D}$ deficiency was seen among participants with lower calcium and vitamin D intake as compared with subjects who reported adequate nutrients intake. After adjustment for potential confounding variables, men and women with osteoporosis had 1.1- and 2.0-fold higher rates of $25(\mathrm{OH}) \mathrm{D}$ deficiency than those with normal BMD, respectively. Similarly, among U.S. adults with osteopenia, the odds of $25(\mathrm{OH}) \mathrm{D}$ deficiency were $1.4(95 \% \mathrm{CI}, 1.0 \%, 1.9 \%)$ among men and 1.0 (95\% CI, $0.7 \%, 1.5 \%$ ) among women. Conclusions: U.S. adults with osteoporosis had considerably higher $25(\mathrm{OH}) \mathrm{D}$ deficiency prevalence rates. Adequate vitamin $\mathrm{D}$ intake and lifestyle modification may improve $25(\mathrm{OH}) \mathrm{D}$ concentrations among U.S. adults with low BMD.

Table 1. Prevalence of 25(OH)D deficiency according to BMD status, NHANES 20072010

\begin{tabular}{|c|c|c|c|}
\hline & $\begin{array}{l}\text { Normal BMD } \\
\qquad(n=2,378) \\
\quad \%(\mathrm{SE})\end{array}$ & $\begin{array}{l}\text { Osteopenia } \\
\qquad(n=1,729) \\
\quad \%(\mathrm{SE})\end{array}$ & $\begin{array}{c}\text { Osteoporosis } \\
(n=203) \\
\%(\mathrm{SE})\end{array}$ \\
\hline \multicolumn{4}{|l|}{ Six month time period } \\
\hline Nov 1 to April 30 & $27.7(2.2)$ & $28.6(2.2)$ & $22.7(5.2)$ \\
\hline May 1 to Oct 31 & $15.4(1.0)$ & $15.8(1.3)$ & $31.9(6.0)$ \\
\hline \multicolumn{4}{|l|}{ Gender } \\
\hline Male & $18.1(1.3)$ & $21.2(2.5)$ & $20.9(6.5)$ \\
\hline Female & $22.2(2.2)$ & $19.8(1.2)$ & $29.8(4.7)$ \\
\hline \multicolumn{4}{|l|}{ Age groups } \\
\hline $50-64$ years & $20.9(1.4)$ & $20.7(2.0)$ & $(29.2(8.0))$ \\
\hline$\geq 65$ years & $16.6(1.6)$ & $19.9(1.3)$ & $28.4(4.0)$ \\
\hline \multicolumn{4}{|l|}{ Race/Ethnicity } \\
\hline Mexican Americans & $37.1(3.1)$ & $37.3(3.3)$ & $38.9(14.1)^{*}$ \\
\hline Non-Hispanic Whites & $12.4(1.3)$ & $16.3(1.2)$ & $28.0(5.2)$ \\
\hline Non-Hispanic Blacks & $54.9(3.7)$ & $57.3(5.2)$ & $40.6(12.9)^{*}$ \\
\hline Other Hispanics & $27.7(5.0)$ & $23.4(3.7)$ & $39.9(8.4)$ \\
\hline Other race & $26.8(8.1)^{*}$ & $35.8(8.8)$ & $18.7(10.7)^{*}$ \\
\hline \multicolumn{4}{|l|}{ Education } \\
\hline 11th grade and below & $29.5(2.9)$ & $26.8(2.2)$ & $34.1(5.0)$ \\
\hline $\begin{array}{l}\text { High school graduate } \\
\text { and above }\end{array}$ & 17.4 & $18.5(1.4)$ & $26.1(5.3)$ \\
\hline \multicolumn{4}{|l|}{ Smoking status } \\
\hline Never & $21.0(2.0)$ & $19.5(1.4)$ & $19.5(3.4)$ \\
\hline Former & $14.3(1.4)$ & $16.4(2.1)$ & $33.4(7.1)$ \\
\hline Current & $28.4(2.7)$ & $33.2(3.1)$ & $47.6(11.1)$ \\
\hline \multicolumn{4}{|l|}{ Obesity } \\
\hline Yes & $25.6(1.7)$ & $29.3(3.0)$ & $49.0(12.1)$ \\
\hline No & $14.5(1.4)$ & $17.5(1.1)$ & $26.5(3.8)$ \\
\hline \multicolumn{4}{|l|}{ Moderate work activity } \\
\hline Yes & $13.7(1.2)$ & $11.8(1.3)$ & $17.0(8.3)^{*}$ \\
\hline No & $24.3(1.9)$ & $26.6(1.8)$ & $33.5(5.4)$ \\
\hline \multicolumn{4}{|l|}{ Health status } \\
\hline Excellent to good & $17.3(1.3)$ & $17.6(1.4)$ & $27.0(4.7)$ \\
\hline
\end{tabular}

$\begin{array}{lll}\text { Fair to poor } \quad 29.2 & 32.1(2.9) \quad 33.7(9.6)\end{array}$

Ever taken prednisone daily

$\begin{array}{llll}\text { Yes } & 14.5(2.4) & 25.4(4.1) & 35.5(12.1)^{*} \\ \text { No } & 19.9(1.4) & 19.7(1.3) & 28.0(5.0)\end{array}$

Calcium taken prednisone daily

$\begin{array}{llll}\text { Yes } & 11.5(1.0) & 11.5(1.7) & 22.5(5.1) \\ \text { No } & 23.5(1.7) & 24.3(1.5) & 31.2(5.5)\end{array}$

Vitamin D intake $\geq 600 \mathrm{Ul} / \mathrm{d}$

$\begin{array}{llll}\text { Yes } & 6.2(1.5) & 4.9(1.5)^{*} & 10.2(6.5)^{*} \\ \text { No } & 23.0(1.6) & 24.1(1.5) & 33.0(5.0)\end{array}$

* Relative standard error $>30 \%$ (estimate may be unreliable)

\section{P627}

\section{A FUNCTIONAL SNP IN THE 3'UTR OF FGF2 GENE INCREASES OSTEOPOROSIS RISK THROUGH MODIFYING MIRNA BINDING IN HAN CHINESE POPULATION}

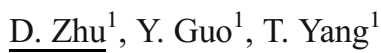

${ }^{1}$ Key Laboratory of Biomedical Information Engineering of Ministry of Education, School of Life Science and Technology, Xi'an Jiaotong University, Xi'an, China

Background: Basic fibroblast growth factor $(F G F 2)$ is an important modulator of bone growth and differentiation. Disruption of the $F G F 2$ gene could result in significantly decreased bone mass and bone formation. Previous studies have identified $F G F 2$ as a susceptibility gene for osteoporosis in white populations. Evaluating the genetic associations in Han Chinese Population is necessary and valuable. Meanwhile, discovering the unknown function of the genetic association is extremely important to offer new targets for research into the etiology of osteoporosis.

Aim: This study aimed to investigate whether the single nucleotide polymorphisms (SNPs) in FGF2 are associated with osteoporosis in Han Chinese population, and explore the potential function of the associated SNPs.

Methods: We performed association analysis to investigate the relationship between SNPs in the $F G F 2$ gene and BMD in Han Chinese population including a discovery sample of 1300 subjects and a replication sample of 1039 subjects. Bioinformatics approach was used to predict the miRNA binding to the SNP. Functional studies, such as cell transfection, dual luciferase reporter assays, qRT-PCR and Western Blot were used in this study to explore the potential function of the associated SNPs. Results: Association analyses in the discovery sample and replication study successfully validated one SNP rs 1048201 (c. ${ }^{* 757 C}>\mathrm{T}$ ) located in the 3'UTR of FGF2 associated with spine BMD (PT was localized to a miR-196a-3p seed binding region in the 3'UTR of $F G F 2$. In HEK293T cells co-transfected with FGF2 3'UTR luciferase reporter vector and a miR-196a-3p mimic, we observed that luciferase activity was significantly lower in the presence of the c.*757C 
(wild type) allele than in the presence of the c.*757 T allele (mut type), and the mutation (c.* $757 \mathrm{C}>\mathrm{T}$ ) was shown to interfere with miR-196a-3p targeting. Furthermore we found the miR-196a-3p mimic led to a moderate decrease in FGF2 mRNA and protein expression, which was completely reversed by a miR-196a-3p inhibitor. Conclusions: Our study identified that the SNP (rs1048201 C/T) variation was highly associated with an increased risk of osteoporosis through affecting binding of miR-196a-3p in the 3'UTR of FGF2 in Han Chinese population.

Acknowledgement: This work was supported by the National Natural Science Foundation of China (31371278, 31471188, 81573241, 31511140285); China Postdoctoral Science Foundation (2015 M570819); the Natural Science Basic Research Program Shaanxi Province (2015JQ3089), and the Fundamental Research Funds for the Central Universities (2012jdgz11).

\section{P628}

\section{PREDICTION OF HIP FRACTURES IN}

AUSTRALIAN MEN USING FRAX SCORES ADJUSTED WITH TRABECULAR BONE SCORE

K. L. Holloway ${ }^{1}$, M. Mohebbi ${ }^{1}$, D. Hans ${ }^{2}$ S. L. Brennan-

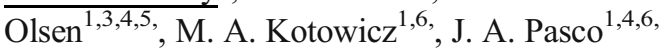

${ }^{1}$ School of Medicine, Deakin University, Melbourne, Australia, ${ }^{2}$ Center of Bone Diseases, Bone \& Joint Department, Lausanne University Hospital, Lausanne, Switzerland, ${ }^{3}$ The Institute for Health and Ageing, Australian Catholic University, Melbourne, Australia, ${ }^{4}$ Department of Medicine, The University of Melbourne, Melbourne, Australia, ${ }^{5}$ Australian Institute for Musculoskeletal Science, The University of Melbourne, Melbourne, Australia, 6Barwon Health, Geelong, Australia

Objectives: FRAX 10-year probability of fracture can be calculated with and without adjusting for trabecular bone score (TBS). Several studies have shown that TBS can improve FRAX assessments in some populations. This study aimed to determine if TBS adjusted FRAX score was better at predicting hip fractures in Australian men than the unadjusted score.

Material and Methods: In total, 597 men aged 40-90yr participated. Incident hip fractures $(\mathrm{n}=13)$ were ascertained using radiological reports. Median follow-up time was 9.5 years (IQR7.5-11.4yr). Area under receiver operating characteristic (AUROC) curves were determined for adjusted and unadjusted scores. Sensitivity, specificity, predictive values and ROC areas were also calculated for both scores using a cut-point of $\geq 3 \%$.

Results: Sensitivity was higher in the adjusted score (76.9\%) than the unadjusted score $(53.8 \%)$, with a slight decrease in specificity; $79.3 \%$ and $83.9 \%$ for the adjusted and unadjusted scores, respectively. Both adjusted and unadjusted scores were good predictors of men who did not fracture (negative predictive value: $99.4 \% \& 98.8 \%$, respectively), although the positive predictive values were low $(7.6 \% \& 6.9 \%)$. The ROC area was higher in the adjusted score $(0.781)$ than the unadjusted score (0.689). When considering adjusted and unadjusted FRAX as continuous scores AUROC was 0.839 0.842 , respectively, but Chi squared test showed no significant difference $(\mathrm{p}=0.39)$.

Conclusions: Unadjusted and TBS-adjusted FRAX scores both predicted hip fractures in Australian men with good accuracy. Diagnostics indexes for a cut-off point of $\geq 3 \%$ showed that the adjusted score performed better than the unadjusted score. However there was no difference in AUROCs.

Disclosures: Study supported by the National Health and Medical Research Council Australia, the Geelong Regional Medical Foundation, Arthritis Foundation of Australia, and Perpetual Trustees. SLB-O and KLH are supported by Alfred Deakin Postdoctoral Research Fellowships.

\section{P629}

THE HEAD SHOULD BE EXCLUDED FROM DXA TOTAL BODY LEAN MASS MEASUREMENTS D. Krueger $^{1}$, E. Fidler ${ }^{1}$, J. Libber ${ }^{1}$, B. Buehring ${ }^{1}$, N. Binkley ${ }^{1}$ ${ }^{1}$ Osteoporosis Clinical Research Program, University of Wisconsin, Madison, United States

Objective: DXA measured whole body lean mass is sometimes used as a surrogate for muscle mass in research studies and athletic performance settings. However, it must be appreciated that DXA lean mass is not limited to muscle and indeed is largely a measurement of water. Up to $\sim 10 \%$ of DXA-measured whole body lean mass is located in the head, a region that contains little skeletal muscle. This study evaluated the proportion of lean mass contained in the head region on DXA whole body scans.

Methods: DXA whole body scans were initially evaluated in 112 men and women age $\geq 70$ yrs (mean age $80.6 \pm 6.0$ ). To explore potential age relationships, the $\%$ head lean mass was calculated in a younger cohort $(n=610)$ comprised of a random sample from a mid-life US aging study and a group of university athletes. This cohort was arbitrarily divided as age 19 to $<40$ and $\geq 40$ to 70 years.

Results: Head lean mass in the oldest group was $7.3 \%(1.0)$ [mean (SD)] ranging from 4.7-9.2\%. Linear regression analysis found the $\%$ lean mass in the head to be negatively correlated with whole body lean mass (i.e., as lean mass declines, the $\%$ located in the head increases $), \mathrm{r}^{2}=0.72(p<0.0001)$. In the young and middle-aged groups mean head lean mass was $5.6 \%$ and $6.3 \%$ respectively. ANOVA revealed a difference between all age groups $(p<0.0001)$. Additionally, in the entire cohort, linear regression demonstrated a negative correlation of $\%$ head lean mass with whole body lean mass, $\mathrm{r}^{2}=0.75$ $(p<0.0001)$, similar to the observation in older adults. 
Conclusion: The head can account for up to $10 \%$ of whole body lean mass in individuals' age $\geq 70$ years. Exclusion of head lean mass is indicated when total body DXA is used as a surrogate for muscle mass. The higher proportion of head lean mass among older adults in those with lower whole body lean mass may imply head lean mass is better retained while lean mass elsewhere declines to a greater degree with aging. Further longitudinal study is required to determine whether lean mass is better retained in the head with aging.

\section{P630}

DXA ERRORS ARE COMMON AND POTENTIALLY AFFECT CLINICAL DECISION MAKING: IMPROVEMENT IS IMPERATIVE

N. Binkley $^{1}$, D. Krueger ${ }^{1}$, K. Hansen ${ }^{2}$, J. Libber ${ }^{1}$, E. Fidler ${ }^{1}$, E. Shives ${ }^{1}$, B. Buehring ${ }^{1}$

${ }^{1}$ Osteoporosis Clinical Research Program, University of Wisconsin, Madison, United States, ${ }^{2}$ Medicine, Division of Rheumatology, University of Wisconsin, Madison, United States

Objective: DXA is used to diagnose osteoporosis, assess fracture risk and monitor therapy. High quality DXA requires excellence in acquisition, analysis and interpretation. A need for excellence is under-appreciated; it may be perceived that acquiring and reporting DXA data is simple and easily automated. Based on our clinical experience, we hypothesized that DXA errors are common. This study evaluated technical and interpretation error rates in a US clinic and assessed the possibility that these may affect clinical decision making.

Methods: DXA scans on new patient referrals to our osteoporosis clinic were anonymously reviewed by three ISCD certified technologists and three ISCD certified physicians $(n=3)$. The technologists applied performance standards from the IOF/ISCD Osteoporosis Essentials course to determine if positioning, acquisition and analysis were ideal. The physicians assessed whether the interpretation complied with ISCD reporting recommendations, determined error prevalence and whether errors were major (those providing inaccurate information potentially causing incorrect patient care decisions). All assessments were performed individually and subsequently adjudicated to reach consensus.

Results: We report 100 patients; not all had both spine and hip scans. Technical errors were identified in $93 \%$ of patients being present in $60 \%$ of spine and $95 \%$ of hip scans. In 70 patients with prior scans, technical differences at baseline vs. follow-up were present in $94 \%$. The physicians concluded that these technical errors could potentially impact care in $18 \%$ of patients. Interpretation review $(n=50)$ revealed errors in $86 \%$ overall and major in $40 \%$. Half of major errors were of incorrect BMD change; an additional $20 \%$ were incorrect fracture identification on VFA.
Conclusion: DXA technical and interpretation errors are extremely common. These errors could adversely affect patient care leading to either excessive or inadequate lab evaluation and over- or under-utilization of medications. Improvement is imperative; interventions to provide high quality DXA scans and reports are needed.

\section{P631}

BONE ULTRASTRUCTURE AND ARCHITECTURE; THE ROLE OF BONE QUALITY IN FRACTURE PREDICTION

C. Greenwood ${ }^{1}$, K. Rogers ${ }^{1}$, A. Dicken ${ }^{2}$, P. Evans ${ }^{2}$, J. Clement $^{3}$, I. Lyburn ${ }^{4}$, R. Martin ${ }^{5}$, P. Zioupos ${ }^{1}$, N. Stone ${ }^{6}$

${ }^{1}$ CFI, Cranfield University, Swindon, United Kingdom, ${ }^{2}$ The Imaging Science Group, Nottingham Trent University, Nottingham, United Kingdom, ${ }^{3}$ Melbourne Dental School, University of Melbourne, Melbourne, Australia, ${ }^{4}$ Cobalt Health, Cheltenham, Cheltenham, United Kingdom, ${ }^{5}$ Social and Community Medicine, Bristol University, Bristol, United Kingdom, ${ }^{6}$ Physics and Astronomy, Exeter University, Exeter, United Kingdom

Objective(s): The risk of osteoporotic fracture is determined collectively by bone mass, architecture and physico-chemistry of the mineral composite building blocks. Thus DXA scans alone inevitably fail to fully discriminate individuals who will suffer a fragility fracture. We have been examining bone at both ultrastructure and microarchitectural levels to provide a detailed material view of bone and therefore produce a more comprehensive model of osteoporotic fracture risk.

Material and Methods: 37 femoral heads were collected from osteoporotic patients who had suffered trauma fractures at the femoral neck. A further 83 nonfracture femoral head specimens from a similar ethnocultural population were also collected as a control group. Bone architectural features were quantified using calibrated microCT (average voxel dimension $20 \mathrm{~mm}$ ) and physico-chemistry determined using X-ray diffraction and FTIR.

Results: For females of the fracture group, the trabecular architecture (e.g., morphology, density and orientation) was significantly different to that of the non-fracture group. Similar differences were demonstrated within the male cohort with the exception of trabecular number and tissue mineral density. This may suggest for the fracture group, differences in remodelling between males and females. Physico-chemical characterisation indicated significant differences in apatite crystal chemistry and microstructure between fracture and non-fracture groups. Combining ultrastructure with architectural 
properties enabled a new model for the mineral of osteoporotic bone. Different architectures were correlated with mineral chemistry and crystallite lattice order correlated to tissue mineral density.

Conclusion(s): We have been able to produce a new perspective of osteoporotic tissue simultaneously at several scale lengths and used this to produce a new predictive risk fracture model. An important clinical implication is that a different model is required for males and females.

Acknowledgments: This programme of work is funded by an EPSRC research grant (EP/ K020196: Point- of-Care High Accuracy Fracture Risk Prediction).

\section{P632}

\section{THE LEVEL OF 25(OH)D AND ALVEOLAR BONE LOSS IN PATIENTS WITH CHRONIC} PERIODONTITIS

V. Novoshytskyy $^{1}$, I. Mazur ${ }^{2}$

${ }^{1}$ Shupyk National Medical Academy of Postgraduate Education, Kyiv, Ukraine, ${ }^{2}$ Department of Dentistry PL Shupyk National Medical Academy of Postgraduate Education, Kyiv, Ukraine

Introduction: Chronic periodontitis (CHP) is one of the most common dental diseases. Clinical manifestations are bleeding, periodontal pockets, clinical attachment loss, alveolar bone loss (ABL) and teeth loss. The relationship between serum concentration of $25(\mathrm{OH}) \mathrm{D}$ and $\mathrm{CHP}$ was shown in many studies.

Aim: To investigate serum concentration of $25(\mathrm{OH}) \mathrm{D}$ in patients with CHP. To investigate relationship between serum concentration of 25(OH)D and level of ABL in mandible in patients with CHP.

Methods: The study involved 47 patients with CHP aged 23-65 years. All patients were divided into 3 groups. First group was with normal level of vitamin D (75-375 nmol/ 1), second group was with vitamin D insufficiency $(50-74,99 \mathrm{nmol} / \mathrm{l})$ and third was with vitamin D deficiency $(<50 \mathrm{nmol} / 1)$. To determine serum concentration of $25(\mathrm{OH}) \mathrm{D}$ electrochemiluminescent method was used with Elecsys 2010 analyzer (Roche Diagnostics, Germany).

Results: Normal level of vitamin D observed in $23.4 \%$ (11 patients), vitamin D insufficiency in $46.8 \%$ (22 patients) and Vitamin D deficiency in $29.8 \%$ (14 patients). The level of ABL was $3.13 \pm 1.46 \mathrm{~mm}(\mathrm{M} \pm \mathrm{SD})$, $3.24 \pm 1.54 \mathrm{~mm}$ and $4.0 \pm 2.21 \mathrm{~mm}$ in the first, second and third group. respectively. Correlation between groups is not significant $(p>0.05)$.

Conclusions: Vitamin D deficiency and insufficiency are prevails in patients with CHP. There is a tendency the lower level of the serum concentration of $25(\mathrm{OH}) \mathrm{D}$, the greater alveolar bone loss.

\section{P633}

\section{OSTEOARTHRITIS IN GENERAL PRACTICE: FROM UNIVERSITY EDUCATION TO PERSONALIZED MEDICINE}

M. Panchovska ${ }^{1}$, N. Nikolov ${ }^{2}$, L. Despotova-Toleva ${ }^{3}$, M. Krastanova $^{4}$

${ }^{1}$ Department of Internal Medicine, Medical University Plovdiv, Plovdiv, Bulgaria, ${ }^{2}$ Department of Internal Medicine, Medical University Pleven, Pleven, Bulgaria, ${ }^{3}$ Department of Health Management, Health Economy and General Practice, Medical University Plovdiv, Plovdiv, Bulgaria, ${ }^{4}$ Physical and Rehabilitation Medical Clinic, UMBAL "Dr. Georgi Stranski”, Pleven, Pleven, Bulgaria

Introduction: Osteoarthritis (OA) is the most common rheumatic disease which affects about $20 \%$ of the world population. Patients with OA occupy a significant part of the visits in the office of the general practitioner. Both pharmacological and nonpharmacological treatment of $\mathrm{OA}$ is used and regulated by guidelines according to the localization of OA. In recent years personalized medicine convincingly enters the medical practice, displaying individual approach to each patient. Specific biomarkers are investigated with respect to diagnosis, treatment and prognosis of the diseases, incl. OA University education in rheumatology should be continued and developed also after graduation, according to the latest achievements of science.

Aim: To evaluate the training of Bulgarian general practitioners (GPs) in the field of osteoarthritis - the most common rheumatic disease in general practice.

Material and methods: Anonymous questionnaire based survey was conduct, involving 35 GPs with a total number of patients 39,349 over age of 18 years. From OA suffer 7837 patients. The length of service of GPs vary from 8.1 to 35.2 years. Seven GPs have in addition to their general practice diploma an acknowledged specialty in internal diseases and one of them in neurology. Alternative and descriptive analysis was used for elaborating statistics.

Results and discussion: Eleven from 35 GPs (31.42\%) declare willingness to upgrade their training in rheumatology. Regarding patients with OA-23 of the 35 $\operatorname{GPs}(65.71 \%)$ consulted their patients with orthopedist in the frame of the first three visits. Thirty one (31) of 35 GPs $(88.57 \%)$ defined the management of pain syndrome as a main goal of treatment. Long-term treatment with chondroprotectors declare as necessary 27 of 35 GPs $(77.14 \%)$. Consultations with a rheumatologist in the frame of the first three visits scheduled 8 of 35 GPs 
$(22.85 \%)$ and by the end of the first year - 12 GPs (34.28\%). For consultation with physiotherapist asked 29 of the 35 GPs $(82.85 \%)$. On personalized medicine in OA can comment 5 of 35 respondents $(14.28 \%)$ and information about biomarkers in OA have two of them $(5.71 \%)$. The survey results show that GPs treat OA in close collaboration first with orthopaedist and physiotherapists and consultations with rheumatologists are thereafter. The main goal of treatment in general practice is pain management and the long-term treatment and prognosis is carried by orthopaedists. The future of personalized medicine in OA remains remote in the minds of general practitioners.

Conclusions: The results from the survey has shown that training in rheumatology should be expanded and upgraded. Competence both in traditional and personalized medicine is needed for the general practice. Individual approach to each patient defining him as unique is needed, including the most common rheumatic disease - osteoarthritis.

\section{P634}

\section{FIBROMYALGIA AND EXPRESSION OF SICCA} SYMPTOMS: IS THIS SJÖGREN'S SYNDROME? M. Panchovska $^{1}$, N. Nikolov ${ }^{2}$, L. Despotova-Toleva ${ }^{3}$

${ }^{1}$ Department of Internal Medicine, Medical University Plovdiv, Plovdiv, Bulgaria, ${ }^{2}$ Department of Internal Medicine, Medical University Pleven, Pleven, Bulgaria, ${ }^{3}$ Department of Health Management, Health Economy and General Practice, Medical University Plovdiv, Plovdiv, Bulgaria

Introduction: Fibromyalgia is a disease that occurs with chronic widespread muscle pain, change in sleep, irritable bowel, cognitive disorders. Fibromyalgia could accompany various rheumatic and nonrheumatic diseases. Fibromyalgia treatment is focused on normalization of altered levels of neurotransmitters -serotonin, dopamine, noradrenaline, adrenaline. Different groups of drugs antidepressants, anticonvulsants, antihypnotics, analgesics are offered. Part of the patients with fibromyalgia exhibit persistent sicca - symptoms xerostomia and xerophthalmia.

Aim: To evaluate the symptoms of xerophthalmia and xerostomia in patients with fibromyalgia who are treated with tricyclic antidepressants - amitrriptyline, selective inhibitors of serotonin reuptake - citalopram and selective antagonist and an inhibitor of serotonin reuptaketrazodone.

Material and methods: In the study are included 27 women diagnosed with fibromyalgia accordingly to the criteria of the American College of Rheumatology, 1990 Other rheumatic diseases are excluded previously to the study. The average age of patients was $47.9 \pm 5.7$ years. The mean duration of disease was $3.5 \pm 1.9$ years. Patients were treated with amitriptyline, citalopram, trazodone in a different order prior and during the study. Xerophthalmia and xerostomia symptoms persist of 2.1 \pm 0.8 years. Patients underwent investigations included in American-European consensus criteria for Sjögren's Syndrome. Alternative and descriptive analysis were used for the statistical analysis of the results.

Results and discussion: The studies in women with fibromyalgia treated with antidepressants and expression of sicca symptoms - xerostomia and xerophthalmia have shown: xerostomia - in 17 of 27 patients(62.96\%) and xerophthalmia - in 11 of 27 patients(40.74\%), positive Schirmer's test in 5 from 27 patients(18.51\%), reduced accumulation of the radiomarker in salivary glands in 2 of 27 patients $(7.40 \%)$, diagnostic focus score at biopsy of small salivary glands - one patient, positive antibodies to $\mathrm{Ro}(\mathrm{SS}-\mathrm{A})$ antigen in 2 from 27 patients(7.40\%), positive antibodies to $\mathrm{La}(\mathrm{SS}-\mathrm{B})$ antigen in 1 of 27 patients(3.70\%). From the entire group only 1 of the patients fulfill the diagnostic criteria for primary Sjogren's syndrome $-3.70 \%$, which does not exceed the rate in the general population. Xerostomia and/or xerophthalmia in patients with fibromyalgia, under therapy with antidepressants does not correlate with the results of invasive, non-invasive and immunology tests for primary Sjögren's syndrome. Reports of some authors of various oral symptoms in patients with fibromyalgia support the broad differential diagnosis of the disease

Conclusions: The manifestation of newly emerged xerostomia and xerophthalmia in patients with fibromyalgia, under treatment with different groups of antidepressants remains drug dependent as determined without objectified data for accompanying Sjogren syndrome.

\section{P635 \\ RELATIONSHIP BETWEEN SUPPRESSION OF BONE TURNOVER MARKERS AND FUTURE INCREASES IN BONE MINERAL DENSITY IN RISEDRONATE TREATMENT}

T. M. Mawatari ${ }^{1}$, R. M. Muraoka ${ }^{2}$, Y. I. Iwamoto ${ }^{3}$

${ }^{1}$ Department of Orthopaedic Surgery, Hamanomachi Hospital, Fukuoka, Japan, ${ }^{2}$ Ajinomoto Pharmaceuticals Co., Ltd., Tokyo, Japan, ${ }^{3}$ Department of Orthopaedic Surgery, Kyushu University, Fukuoka, Japan

Objective: While bone turnover markers (BTMs) are useful to monitor response and adherence to osteoporosis 
treatment, the relationship between risedronate-related suppression of BTMs and future increase in BMD has not been fully established yet.

Material and Methods: Subanalysis included 788 postmenopausal women with osteoporosis included in the risedronate $75 \mathrm{mg}$ once monthly Japanese phase III trial for 12 months, whose vertebral BMD, BTMs (serum TRACP-5b, urinary CTX, and serum BAP) were measured sequentially. The subjects were divided into three subgroups according to the change in BTMs at 3 months: Group L (reduction more than minimal significant change (MSC)), Group N (change within MSC), and Group H (elevation more than MSC). Alternatively, the subjects were divided into two groups, depending on whether the BTMs at 3 months were less than upper limit of normal (ULN). Percent change in BMD at 12 months was compared among these subgroups.

Results: Vertebral BMD gain in Group L was significantly greater than that in the other groups, for all TRACP-5b, CTX and BAP analyses (TRACP-5b: Group L [ $N=652]$ $6.22 \pm 4.12 \%$, Group N [N=97] 4.39 $\pm 4.54 \%$, Group H $[N=32] 2.12 \pm 4.27 \%, p<0.0001$; CTX: Group L [ $N=642]$ $6.11 \pm 4.08 \%$, Group $\mathrm{N}[N=116] 4.98 \pm 4.97 \%$, Group H $[N=23] 2.12 \pm 4.17 \%, p<0.0001$; BAP: Group L [ $N=454]$ $6.50 \pm 4.02 \%$, Group $\mathrm{N}[N=320] 4.94 \pm 4.48 \%$, Group H $[N=7] 2.26 \pm 3.13 \%, p<0.0001)$. Baseline BTMs higher than ULN were related to a future increase in BMD.

Conclusion: BMD gain at 12 months was greater in the subgroups showing reduction more than MSC in BTMs at 3 months, while BMD gain was more than $1.6 \%$ attenuated when reduction was less than MSC. Furthermore, BMD gain at 12 months was greater when the value of serum TRACP-5b at 3 months was less than ULN, compared with cases with a value higher than ULN. These results suggest that monitoring patients with BTMs may be useful in predicting future BMD gain with risedronate treatment.

\section{P636 \\ A PROGRAM OF YOGA HAS THE POTENTIAL TO BE EFFECTIVE IN HIP OSTEOARTHRITIS \\ A. C. Bighea ${ }^{1}$, S. Patru ${ }^{1}$, I. R. Marcu ${ }^{1}$, D. Matei ${ }^{1}$, A.-M. Bumbea $^{1}$, D. V. Caimac ${ }^{1}$ \\ ${ }^{1}$ University of Medicine and Pharmacy, Craiova, Romania}

Background: Although significant attention has focused on aerobic, stretching and strengthening for reducing symptoms of OA, yoga has the potential to be effective in OA as well, because improves both flexibility and strength and could theoretically be beneficial to some musculoskeletal problems.
Aim: The study assessed the efficacy and safety of yoga treating the symptoms and disability from osteoarthritis of the hip.

Material and methods: Were included 63 patients that met ACR criteria for hip osteoarthritis, $\geq 50$ years of age, symptomatic for at least 6 months before study entry and were not currently participating in an exercise program or other physical therapy interventions during the study. Patients were required to attend a 60-min yoga program, 2 times/week, 10 weeks. They are assessed at baseline and at the completion of the study using Western Ontario and McMaster Universities Osteoarthritis Index (WOMAC), the patient global assessment and the physician global assessment by a $100-$ $\mathrm{mm}$ visual analog scale.

Results: Patients had statistically significant improvements in their levels of pain $(40.3 \%, p=.004)$ and disability $(27.6 \%$, $p=.005$ ) measured by WOMAC. The patient global assessment showed a statistically significant improvement at $30.2 \%$ $(p=.005)$ and the physician global assessment showed a $25.7 \%$ improvement but without statistical significance. No adverse events were reported.

Conclusions: A supervised 10-week course of yoga can be beneficial in reducing pain and disability in symptomatic hip OA patients.

\section{References:}

1. Bowman AJ, et al. Eur J Clin Invest 1997;27:443

2. Kolasinski SL, et al. J Altern Compl Med 2005; 11:689

\section{P637}

\section{ESTABLISHING FRAGILITY FRACTURE RISK AMONG THE MALE SPANISH POPULATION: THE FROCAT COHORT STUDY}

$\underline{\text { R. Azagra }}^{1}$, M. Zwart ${ }^{1}$, A. Aguyé ${ }^{1}$, L. Troyano ${ }^{1}$, J. C. MartínSánchez $^{2}$, N. Puchol ${ }^{1}$, M. Iglesias ${ }^{1}$, M. Teixidó ${ }^{1}$, F. Solé-

Sancho $^{1}$, F. Julià ${ }^{1}$, M. C. Yuste ${ }^{1}$

${ }^{1}$ Catalan Institute of Health (ICS), Barcelona, Spain, ${ }^{2}$ Universitat Internacional de Catalunya, Barcelona, Spain

Objective: To evaluate the associations between the main osteoporotic risk factors and the occurrence of fragility fractures in the community.

Methods: A retrospective multicenter study of men aged $\geq 40$ and $\leq 90$ years old obtained from a randomized sample of the general population (FROCAT cohort) carried out over a 10year period. Analysis of the Spanish male population, either taking into consideration isolated risk factors or based on FRAX $\geq 5 \%$ tool for major osteoporotic fracture, according to previous studies of the Spanish female population (FRIDEX and FROCAT cohorts).

Results: A total of 865 men were included. Mean age 69 years old $(59.9 \% \geq 65$ and $39.7 \%$ between $50-64)$. 
After 10 years 72 men $(5.3 \%)$ had suffered 65 osteoporotic fractures, of which $40(55.5 \%)$ were major: hip 5 (6.9\%), vertebral 7 (9.7\%), humerus 10 (13.9\%), and wrist $18(25 \%)$. Men who suffered a major osteoporotic fracture during the follow-up study, were smokers or people who had suffered previous fractures, $\geq 2$ falls in previous year, and a mean of FRAX risk for major and hip fracture. 17 subjects $(24 \%)$ with fractures and 86 $(10.9 \%)$ without fractures had a FRAX $\geq 5$ (without Tscore) for major osteoporotic fracture, respectively [RR 2.29 (95\%CI: 1.38-3.78)]. No significance in age, BMI, parental hip fracture, alcohol intake, corticosteroids use, and history of rheumatoid arthritis.

Conclusions: Our results have shown a higher risk of fracture among male in case of: smokers, prior fractures, falls in the previous year or FRAX $\geq 5 \%$ for major osteoporotic fracture. We have found no other significant associations but the low percentage of fractures among the Spanish male population could explain the results.

\section{P638}

\section{A NONRANDOMISED CLINICAL TRIAL OF PAIN RELEASE PHENOMENON ON PATELLOFEMORAL PAIN}

S.-A. Anwar $^{1}$

${ }^{1}$ Department of Physical Therapy UOS Lyallpur Campus Faisalabad, Faisalabad, Pakistan

Purpose: Patellofemoral pain (PFP) is one of the common disabling conditions in physically active individuals in late age as a result of arthritis and in young ones due to malalignment of patella. There are different conservative treatment approaches for the treatment of PFP. The purpose of this study is to find out effectiveness of Mulligan pain release phenomenon(PRP) for the treatment of PFP.

Materials and Methods: A nonrandomized clinical trial was conducted with 45 patients selected through effect size sampling technique. Equal participant division was done with random allocation into 3 treatment groups i.e. A(control group), B (compression technique group, $\mathrm{C}$ (distraction technique group). Treatment was concealed through opaque sealed envelopes. control group(A) received baseline treatment only, i.e., $10 \times 10$ quadriceps isometric contractions (10 sec hold with 10 repetitions). The patients in Compression group(B) were treated by baseline treatment and compression technique of PRP. The distraction group $(C)$ received baseline treatment and Distraction with patellofemoral glides. The outcomes were measured as pain on VAS (visual analogue scale), knee ROM with foniometer, and functional capability through LEFS (lower extremity functional scale).
Results: The comparison among groups shows that the $p$ value for knee ROM is $0.766>0.05$ (selected alpha), LEFS $0.026<0.05$ and for VAS $0.015<0.05$. The result shows that in terms of treatment groups comparison there is a significant $p$ value for LEFS and VAS as compared to ROM. So PRP is effective in decreasing pain and improving functional outcomes in terms of LEFS.

Conclusion and Recommendations: The study concluded that Mulligan Pain Release Phenomenon through patellar compression is an effective treatment in patellofemoral pain. When compared with distraction of patellofemoral joint and quadriceps isometric strengthening method there was considerable difference in difference in functional activities measured through LEFS. It is recommended that more research work is needed to find out the efficacy of this technique in a variety of patients. Long term clinical trials are recommended.

P639

ANALYSIS OF USING FRAX® WITH FRIDEX RISK THRESHOLDS IN THE APPLICATION OF DXA-SCAN IN THE SPANISH FEMALE POPULATION OVER 45 YEARS OLD

R. Azagra ${ }^{1}$, M. Zwart ${ }^{2}$, A. Aguyé ${ }^{3}$, N. Moreno ${ }^{4}$, P. Gabriel $^{4}$, J. M. Manresa ${ }^{4}$, J. C. Martín-Sánchez ${ }^{5}$, O. Hernández-Vian ${ }^{4}$, E. Gené ${ }^{6}$

${ }^{1}$ Health Center Badia del Vallés, Institut Català de la Salut, USR MN IDIAP Jordi Gol, Universidad Autónoma de Barcelona, Barcelona, Spain, ${ }^{2}$ Health Center Can Gibert del Plà, USR Girona-IDIAP Jordi Gol. Institut Català de la Salut, Girona, Spain, ${ }^{3}$ Health Center Granollers Centre, DAP Metropolitana Nord. Institut Català de la Salut, Barcelona, Spain, ${ }^{4}$ Catalan Institute of Health (ICS), Barcelona, Spain, ${ }^{5}$ Universitat Internacional de Catalunya, Barcelona, Spain, ${ }^{6}$ Emergency Department, Consorci Sanitari Parc Taulí, Universitat Autònoma de Barcelona, Barcelona, Spain

Background: Recently several publications have suggested carrying out DXA-scan tests based on the risk of fragility fracture using the FRAX tool.

Methods: The objective is to analyze the Physicians' Training Program (PTP) results following the Clinical Practice Guidelines (CPG) that includes FRAX risk thresholds adjusted for the Spanish population. A pre and post study of the training program intervention group (IG) using the CPG and the control group (CG) was merely observed. The DXA scans were analyzed in 6 different areas: $1-3$ in the IG and 4-6 in the CG. PTP took place in 2011 and was put together by a group of several medical specialists (rheumatologists, gynecologists, family physicians and chemists). The pre-PTP was 2009-10, the post-PTP was 
2012-14. CPG advise the use of DXA in cases of FRAX $\geq 5 \%$ or fragility fractures to assess the risk of major fragility fractures. Population: 1-3 areas included 107, 141 women $\geq 45$ years old. $4-6$ areas included 167,533 women $\geq 45$. Main variable: DXA/ 1000 women $\geq 45$ years old the difference percentage 2014-2011 and ratio 201411 with $95 \%$ CI.

Results: Table 1

\begin{tabular}{llllllllll}
\hline Area & 2009 & 2010 & 2011 & 2012 & 2013 & 2014 & $2014 / 2011 \%$ & $2014-2011$ Ratio & CI 95\% Ratio \\
\hline 4 & 18.6 & 30.7 & 19.1 & 20.9 & 26.3 & 20.4 & 6.5 & 1143 & $1.044-1.250$ \\
5 & 28.3 & 32.6 & 38.1 & 38.2 & 47.5 & 29.6 & -28.7 & 0.777 & $0.708-0.853$ \\
6 & no data & no data & 21.9 & 26.4 & 32.4 & 25.0 & 12.4 & 1.069 & $0.972-1.180$ \\
1 & 16.2 & 16.2 & 23.1 & 14.8 & 16.4 & 12.4 & -86.6 & 0.527 & 0.559 \\
2 & 15.0 & 20.6 & 23.2 & 12.3 & 12.7 & 12.8 & -81.3 & $0.580-0.578$ \\
3 & 19.6 & 22.1 & 25.0 & 7.7 & 7.6 & 13.2 & -89.8 & 0.536 & 0.603 \\
\hline
\end{tabular}

Conclusions: PCT as an intervention programme is aimed at family physicians, and when applied, there is a significant reduction in annual DXA requests.

\section{P640}

\section{DOES OSTEOPOROSIS AFFECT YOUNG MEN? NATIONAL OSTEOPOROSIS ASSOCIATION OF GEORGIA; TBILISI, GEORGIA}

L.-K. Kilasonia $^{1}$, N.-K. Kirvalidze ${ }^{1}$, M.-K. Kopaliani ${ }^{2}$, N.-D. Dolidze $^{3}$, L.-L. Lagvilaval ${ }^{1}$

${ }^{1}$ Tbilisi Heart and Vascular Clinic, Tbilisi, Georgia, ${ }^{2}$ Tbilisi State University, Tbilisi, Georgia, ${ }^{3}$ National Institute of Endocrinology, Tbilisi, Georgia

Introduction: The title question explains the newly emerged tendencies of osteoporosis and its prevalence. As the rates of the incidence of osteoporosis are growing the myths related to the disease are about to crush. Many years doctors believed that women are much more likely to get osteoporosis than men, but IOF declared 2014 as the year of "Men Osteoporosis" Moreover, if previously osteoporosis was attributed to the conditions of an old age, today it is most apparent, scientists focused on osteoporosis are evaluating the disease in young population; The information about peak bone mass has a prognostic opportunity for the progression of the bone mass decrease and consequent bone fragility.

Objectives: The question in the article title line: Does osteoporosis affect young men? the rationale of the question in the title lies in those risk-factors that determine development of OP are most likely uncovered in men rather than in women. Among them are: smoking, alcohol consumption, hypogonadal state. Androgens have vital role in bone formation in both: men and women. It is proved that osteoblasts have androgen receptors. Series of research support the evidence that androgen deficiency lead to retarded skeletal growth in men. Androgen deficiency is recognized as the most potent reason of bone loss in men. The aim of the study was to assess BMD in young Georgian men, and determine correlations between BMD and basic osteoporosis risk factors. 2800 young men with the age 22-50 (median age $38 \pm 4.6)$ years were examined, all patients have filled the questioner.

OP Risk-Factors in Young Georgian Males - $n=2800$ Genetic Factor - $15 \%$ (420) Spine Deformities - $35 \%$ (980) Low Bone Mass $<70$ - 10,7 \% $(299,6)$ Alcohol consumption - 8,5 \% (238) Hypo dynamic Lifestyle - $21 \%$ (588) Low Calcium Intake - $41 \%$ (1148) Sexual Potency Decrease - 39,8\% $(1114,4)$ Bone Mass was measured using DXA technique (Hologic 1000), according to $\mathrm{T}$ and $\mathrm{Z}$ criteria $\mathrm{SD}$; in particular cases lumbar spine $\mathrm{X}$-ray has been performed. Statistical analysis was made by ExcelWindows Microsoft MedicalExe Statistical Software. Confidence indicators were calculated by the appropriate formula. Results have computed normal bone mass in $55 \%$ - 2800 young men; osteopenia in $17 \%$; osteoporosis $(\mathrm{t} \leq-2,5 \mathrm{sd})$. measured in $38 \%$ of the group.

\section{Results Received:}

1. Low bone mass detected in $45 \%$ of Healthy Young Georgian Men (more than 1000 men) Therefore, the condition should be followed to avoid progression of bone loss and its complications

2. Secondary hypogonadotropic hypogonadism and Lumbar spine vertebral deformities were major OP risk -a factor is young Georgian men are: It is apparent that emerging risk-factors are continuing the list of 
worldwide recognized risk-factor; Consequently evaluation of newly-appearing risks should be spotted and closely monitored.

3. The correlation between Osteoporosis incidence and Hypogonadism is clear. It is recommended to correct hypogonadal state for the prevention of bone loss progression.

\section{P641}

\section{BONE REPAIR UNDER TYPE 2 DIABETES} MELLITUS (EXPERIMENTAL INVESTIGATION) N. Dedukh ${ }^{1}$, A. Sykal ${ }^{1}$

${ }^{1}$ Sytenko Institute of Spine and Joint Pathology, Kharkiv, Ukraine

In developed countries diabetes mellitus occupies one of the leading places in structure of diseases. It is known that during the development of diabetes bone quality decreases, increases risk of fracture and nonunion.

Objective: To study post-traumatic bone regeneration in rats with type 2 diabetes mellitus.

Material and methods: No genetic forms of type 2 diabetes was reproduced according to the model developed by Islam S., Choi H. [2007]. For this purpose, 15 white laboratory rats (female, 2 months age) were intraperitoneally administered with nicotinamid $(230 \mathrm{mg} / \mathrm{kg})$, followed by intraperitoneal administration $(65 \mathrm{mg} / \mathrm{kg})$ of streptozotocin. Defect $(2 \times 2 \mathrm{~mm})$ was reproduced using dental bor in the metaphysis of the distal femur. Bone regeneration at 3, 7 and 28 days in rats with type 2 diabetes and control group was studied by morphology methods. Histochemical topo-optical studies include an assessment of the main component of matrix bone - collagen types I and III, sulfated glycosaminoglycans.

Results: On 3rd day in animals with type 2 diabetes studies in the regenerate among cellular elements were prevailed by neutrophils, lymphocytes, and undifferentiated connective tissue cells. The density of macrophages and fibroblasts was low. Osteoblasts were absent. The density of blood vessels when compared to control animals was low, indicating a slowing of angiogenesis. On 28 th day into regenerate of the animals with type 2 diabetes fibroreticular tissue area was at $342.1 \%$ more than in the control animals with defects. The area occupied by lamellar bone tissue was reduced by $46.8 \%$. In the regenerates in animals with type 2 diabetes, type I collagen was present with different maturity and large areas of type III collagen, refraction of glycosaminoglycans was broken.

Conclusion: Slowing bone regeneration in animals with type 2 diabetes is associated with early stage - reduction of cell proliferation and differentiation, angiogenesis, in the later stages - in violation of the organic matrix of the bone - collagen and glycosaminoglycans.

\section{P642 \\ PREVALENCE OF HYPERCALCEMIA IN ADULT POPULATION OF MINSK-CITY}

E. V. Brutskaya - Stempkovskaya ${ }^{1}$, A. P. Shepelkevich ${ }^{2}$, G. M. Kostin ${ }^{3}$, E. N. Chertko ${ }^{4}$, T. V. Rozhnova ${ }^{4}$, D. B. Haikina ${ }^{3}$ ${ }^{1}$ Belarusian State Medical University, Minsk City Polyclinic N31, Minsk, Belarus, ${ }^{2}$ Belarusian State Medical University, Minsk, Belarus, ${ }^{3}$ Minsk Consulting and Diagnostic Centre, Minsk, Belarus, ${ }^{4}$ Minsk City Polyclinic N31, Minsk, Belarus

Introduction: the prevalence of hypercalcemia varies in different populations from $0.042 \%$ to $0.2 \%$.

Objective: to investigate the prevalence of hypercalcemia in the adult population of Minsk city.

Materials and Methods: were studied 1000 people, average age 54.68 \pm 16.96 ( 727 women, 273 men) from 18-96 years. Examination: total calcium, total protein, creatinine, cholesterol and triglycerides; the analysis of morbidity were made (osteoporosis, kidney stones, cholelithiasis, ulcer disease, diabetes mellitus, hypertension, coronary heart disease, cardiovascular events, cancer, fractures in history).

Results: hypercalcemia has been found in 26 people, mean age was $57.42 \pm 11.6$ years (women-18, men- 8 ). In the age group up to 20 years -6 people hypercalcemia is not revealed; in the age group of 20-29 years - 98 people hypercalcemia was found in $1 \%$, in the age group 30-39 years - 139 people, hypercalcemia $0.7 \%$; in the age group $40-49$ years - 113 people, hypercalcemia $-2.7 \%$; in the age group 50-59 years - 174 people, hypercalcemia - $4 \%$; in the age group 60-69 years - 282 people, hypercalcemia - 4.3\%; in the age group 70-79 years - 146 people, -hypercalcemia- $1.4 \%$ of patients; in the age group $80-89$ years - 39 people, hypercalcemia is not revealed; in the age group over 90 years 4 people, hypercalcemia is not revealed.

Conclusion: The prevalence of hypercalcemia in the adult population of Minsk-city was $2.6 \%$ (26: 1000). The highest incidence of hypercalcemia has been detected in the age group 50-69 years.

\section{P643}

CHANGES IN BONE MASS IN JAPANESE ELDERLY PATIENTS WITH OSTEOPOROSIS TREATED WITH ANTI-RANKL ANTIBODY FOLLOWING BONE FORMATION BY PTH FORMULATION

N. Okada ${ }^{1}$, H. Kikuchi ${ }^{2}$, Y. Itoh ${ }^{2}$, W. Shimada ${ }^{2}$, S. Soen ${ }^{3}$

${ }^{1}$ Orthopaedic Surgery, Sumoto-itsuki Hospital, Sumoto-city, Japan, ${ }^{2}$ Orthopaedic Surgery, Sakai Hospital Kinki University Faculty of Medicine, Sakai-city, Japan, 
${ }^{3}$ Orthopaedic Surgery and Rheumatology, Nara Hospital Kinki University Faculty, Nara, Japan

Objectives: This study examined changes in bone mass (young adult mean [YAM] of the lumbar spine and femur) in Japanese elderly patients with a history of bone fracture treated with anti-receptor activator of nuclear factor kappa-B ligand (RANKL) antibody (denosumab $60 \mu \mathrm{g} / 6$ months, Daiichi Sankyo Company) after bone formation by daily PTH (PTH, teriparatide $20 \mu \mathrm{g}$ /day, Eli Lilly).

Method: We retrospectively studied urinary levels of Nterminal telopeptide (NTx) and bone minerals (in the lumbar spine and femur) before and after the administration of antiRANKL antibody twice or more in 13 patients with severe osteoporosis at the Department of Orthopaedic Surgery, Imoto Isuki Hospital (women aged 79-99 y, mean $88 \mathrm{y}$; body weight of $31-52 \mathrm{~kg}$, mean $44 \mathrm{~kg}$ ), following PTH treatment (1-24 months, mean 10 months).

Results: PTH efficacy was measured in terms of procollagen type $1 \mathrm{~N}$-terminal propeptide (P1NP) level. With PTH treatment, the P1NP level increased from $44.8 \pm 19.8 \mu \mathrm{g} / \mathrm{L}$ to $117.8 \pm 56.7 \mu \mathrm{g} / \mathrm{L}$. The treatment was switched to antiRANKL antibody treatment after a P1NP level increase was confirmed in all patients. The NTx level before and after the treatment switch was $22.0 \pm 7.3 \mathrm{nmol} / \mathrm{mmol}$ creatinine (CRE) and $18.0 \pm 4.3 \mathrm{nmol} \mathrm{BCE} / \mathrm{mmol} \mathrm{CRE}$, respectively. The YAM of the lumbar spine increased in 11 of 13 patients (from 69.1 $\pm 17.6 \%$ to $72.3 \pm 16.0 \%$ ), whereas that of the femur increased only in 4 of 13 patients (from $63.3 \pm 14.5 \%$ to 60.7 $\pm 12.1 \%$ ). No patient had a serious adverse reaction or a new clinical bone fracture during the period.

Discussion: Treatment with PTH and anti-RANKL antibody most potently increases bone mineral levels (Benjamin Z Leder et al., The Lancet); however, combination therapy is not covered under health insurance in Japan. Although taking measures against osteoporosis after menopause is important, antiRANKL antibody treatment after PTH increases bone mineral levels even in elderly patients with severe osteoporosis.

\section{P644 \\ OSTEOMETRIC PARAMETERS OF RAT SPINE VERTEBRAE WITH OSTEOPOROSIS AFTER LEAD EXPOSURE}

V. Maltseva

${ }^{1}$ Sytenko Institute of Spine and Joint Pathology, Kharkiv, Ukraine

Lead is one of the main toxic elements in the environment. According to WHO, this element included in the list of 10 leading risk factors of disease in Ukraine. It's know that lead causes a negative impact on the human skeleton, but most of the studies in which it was studied, investigated the effect of high doses of lead. The effect of lead on the spine with osteoporosis and during active growth remains unclear.

Aim: To determine the features of lead exposure on osteometric parameters of vertebral bodies of the lumbar spine of rats during the period of active growth and in rats with osteoporosis.

Object and methods: The study was performed on 80 white laboratory rats (40 males and 40 females). The experiment was investigated the lead exposure in rats, which corresponding to effect of this element on the people living in the urban environment. Series 1. On males studied the effect of lead on the spine during active growth. For this purpose animals aged 1.5 months were divided into two groups - experimental and control. The experimental group received lead acetate solution (230 mg of lead per 1 liter of distilled water) with drinking water, and the control group received distilled water for 10 weeks. Series 2 . The modeling of osteoporosis was performed on the female rats (aged 6 months) by ovariectomy. After 4 months after ovariectomy rats were divided into 2 groups. The experimental group received lead acetate solution for 10 weeks, similar experimental study on male rats, and the control group received distilled water. Rats aged 4 months (Series 1) and 12.5 months (Series 2) were removed from the experiment by decapitation and fragments of lumbar spine LI-LIV were removed for the osteometric analysis. Osteometric measurements of vertebral bodies were performed using caliper. These osteometric parameters of vertebrae were measured: height, cranial width, cranial sagittal diameter, caudal width and caudal sagittal diameter.

Results: In younger animals was found that lead exposure causes a statistically significant decrease of all measured parameters of vertebrae in average: height - by $10.5 \%$, cranial width by $11.5 \%$, caudal width-by $8.5 \%$; cranial sagittal diameter - by $12.4 \%$; caudal sagittal diameter - by $15 \%$, compared with the control group. Thus lead exposure during period of active growth lead to inhibition of growth of vertebral bodies in all planes, but greater sign was showed in the sagittal plane. In the experimental group of rats with modeling osteoporosis after lead exposure was found a statistically significant reduction in the height of the vertebral bodies: LI - by $10.9 \%$, LII - by $6.9 \%$, LIII - by $3.6 \%$, and LIV - no change compared with control group (rat with osteoporosis without of lead exposure). It was not established significant differences in these parameters: cranial width, cranial sagittal diameter, caudal width and caudal sagittal diameter (in vertebral bodies LII, LIII, LIV) of vertebrae of rats with modeling osteoporosis after lead exposure, compared with control group. Showed decrease in caudal sagittal diameter by $16.2 \%$ in the LI. Reducing of all measured 
osteometric parameters after lead exposure in young rats probably due to the inhibition of growth, while reducing of the height of the vertebral bodies in the group of rats with osteoporosis probably explains the emergence compression deformation that may lead to decrease of this parameter.

Conclusion: The spine of younger rats during the period of active growth is more sensitivity to the negative impact of lead exposure compared with older rats with osteoporosis.

\section{P645}

\section{PREVALENCE OF HYPERCALCEMIA IN WOMEN OF MINSK-CITY}

E. V. Brutskaya - Stempkovskaya ${ }^{1}$, A. Shepelkevich ${ }^{2}$, G. M. Kostin $^{3}$, E. N. Chertko

${ }^{1}$ Belarusian State Medical University, Minsk City Polyclinic N31, Minsk, Belarus, ${ }^{2}$ Belarusian State Medical University, Minsk, Belarus, ${ }^{3}$ Minsk Consulting and Diagnostic Centre, Minsk, Belarus, ${ }^{4}$ Minsk City Polyclinic N31, Minsk, Belarus

Objective: To study the prevalence of hypercalcemia in the female population of Minsk city.

Materials and Methods: Continuous survey of 1000 people, average age 54.68 \pm 16.96 ( 727 women, $273 \mathrm{men}$ ) from 18-96 years. Survey: total calcium, total protein, creatinine, cholesterol and triglycerides with an analysis of morbidity.

Results: Hypercalcemia has been found in 18 women (mean age $47.25 \pm 12.64$ ). In the age group under 40-193 women, hypercalcemia is not revealed; in the age group 40-49 years - 74 women, hypercalcemia found in $1.4 \%$ of women; in the age group 50-59 y - 126 women (hypercalcemia - $4 \%$ ); in the age group 60-69 y - 191 women (hypercalcemia $-5.2 \%$ ); in the age group 70-79 y - 114 women (hypercalcemia, $1.8 \%$ ); in the age group older than $80 \mathrm{y}-30$ women, hypercalcemia was not found. There was a significant difference in the prevalence of hypercalcemia in women according to age: more common in the age group 50-69 y $(M N=362,28, p<0.0001)$.

Conclusion: The peak incidence of hypercalcemia in the female population between the ages of 50-69 y.

\section{P646}

PREVALENCE OF HYPERCALCEMIA IN THE MALE POPULATION COMPARED TO WOMEN OF MINSK-CITY

A. P. Shepelkevich $^{1}$, E. V. Brutskaya - Stempkovskaya ${ }^{2}$, G. M. Kostin ${ }^{3}$, E. N. Chertko

${ }^{1}$ Belarusian State Medical University, Minsk, Belarus,

${ }^{2}$ Belarusian State Medical University, Minsk City Polyclinic N31, Minsk, Belarus, ${ }^{3}$ Minsk Consulting and Diagnostic
Centre, Minsk, Belarus, ${ }^{4}$ Minsk City Polyclinic N31, Minsk, Belarus

Objective: To study the prevalence of hypercalcemia in the male population compared to women in Minsk.

Materials and Methods: We studded 1000 people, average age 54.68 \pm 16.96 ( 727 women, 273 men) from 18-96 years. Examination: total calcium, total protein.

Results: Hypercalcemia was found in 26 people, 18 women (mean age 47.25 \pm 12.64 ), men (mean age $61.94 \pm 11.62$ ). In the age group up to 30 years - 104 people ( 81 women, 23 men), hypercalcemia was found in $4.3 \%$ of men, it was not found in women; in the age group 30-39 years - 139 people (112 women, 27 men), hypercalcemia: in men- $4 \%$, in women $-0 \%$; in the age group 40-49 years - 113 people ( 74 women, 39 men), hypercalcemia: in men- $5.1 \%$, in women- $1.4 \%$; in the age group 50-59 years - 174 people (126 women, 48 men), hypercalcemia: men $-4.2 \%$, of women- $4 \%$; in the age group 60-69 years - 282 people (191 women, 91 men), hypercalcemia: men $2.3 \%$, in women- $5.2 \%$; in the age group older than 70 years - 189 people (144 women, 45 men) hypercalcemia: women- $1.8 \%$, men- 0 . There was a significant difference in the incidence of hypercalcemia according to gender and age: it more common in men up to 50 years compared to women $(M N=89,09, p<0.0001)$.

Conclusion: The incidence of hypercalcemia in the age group up to 50 years in men is about $4 \%$. The results of the study indicate a significantly greater frequency of hypercalcemia in men up to 50 years compared to women.

\section{P647}

PROGNOSTIC VALUE OF X-RAY DENSITOMETRY IN OSTEOPOROSIS - NATIONAL ASSOCIATION OF OSTEOPOROSIS

$\underline{\text { L.-K. Kilasonia }}^{1}$, N.-K. Kirvalidze ${ }^{1}$, M.-K. Kopaliani ${ }^{2}$, N. Dolidze $^{3}$, L.-L. Lagvilaval ${ }^{1}$

${ }^{1}$ Tbilisi Heart and Vascular Clinic, Tbilisi, Georgia, ${ }^{2}$ Tbilisi State University, Tbilisi, Georgia, ${ }^{3}$ National Institute of Endocrinology, Tbilisi, Georgia

Introduction: X-ray densitometry had revolutionized the diagnostics of osteoporosis. Majority of scientists believed that it was a diagnostic instrument close to almost ideal one capable evaluation of both: BMD and likelihood of future fractures. Experiences accumulated during the previous years have slightly shaken this belief. It is more frequently heard that DXA is not an ideal diagnostic instrument, but no better instrument has been created yet, which is also proved by our 12 years of experience. 
Materials and Methods: X-ray densitometry research evaluated 5200 patients aged 40-70. 4100 women and 1100 men. Medical History of 825 patients showed that they had bone fractures, 136 out of them were high energy (pressure), the rest of them low trauma or no-trauma fractures. BMD index was low in 2736 patients ( $T$ score $>-2.5$ ). 48 patients had fractures in earlier lives registered in their medical records.

Results: Low BMD index was identified in 2736 patients with osteoporosis only during the DXA lateral scans. It is arguable that $\mathrm{X}$-ray densitometry and BMD evaluation is the only criteria for the standards of diagnostics and the predictor of progressive bone loss and fractures.

Discussion: While analyzing the results, diagnostic problems emerged at diagnostic process were taken into account, namely spine deformation, osteoporosis, osteochondrosis that camouflage real mineral density of the bones. At the same time, each patients with existing fractures in their record filled in special questionnaires describing clinical symptoms of the disease and risk-factors influencing the likelihood of the disease. Results of the work were processed through the analysis of variance (Fisher). Reliable correlation was not identified between DXA results and fracture risk index. This last one encourages routine use of FRAX in diagnostics of osteoporosis and prognosis of fractures before morphological evaluation of bones will be readily available to use as an additional diagnostic tool.

\section{P648}

HYPOCALCEMIA IN ADULTS IN MINSK, BELARUS A. P. Shepelkevich $^{1}$, E. V. Brutskaya - Stempkovskaya ${ }^{2}$, G. M. Kostin ${ }^{3}$, E. N. Chertko ${ }^{4}$, T. V. Rozhnova ${ }^{4}$, D. B. Haikina ${ }^{3}$ ${ }^{1}$ Belarusian State Medical University, Minsk, Belarus, ${ }^{2}$ Belarusian State Medical University, Minsk City Polyclinic N31, Minsk, Belarus, ${ }^{3}$ Minsk Consulting and Diagnostic Centre, Minsk, Belarus, ${ }^{4}$ Minsk City Polyclinic N31, Minsk, Belarus

The prevalence of hypocalcemia among nonoperated on the thyroid and parathyroid glands of patients has increased significantly with the introduction of screening of calcemia.

Objective: To study the prevalence of hypocalcemia in the adult in Minsk.

Materials and Methods: We studded 1000 people from 1896 years, the average age of $54.68 \pm 16.96$ (727 women, 273 men). Examination: total calcium, total protein; the analysis of morbidity were made.

Results: Hypocalcaemia was recorded in 32 people (3.2\%), the mean age was $44.06 \pm 17.39$ years ( 25 women, men- 8 ). The true hypocalcemia was found in $1.4 \%$ (14: 1000), the hypocalcemia was associated with hypoproteinemia in $1.8 \%$. In the age group up to 20 years of hypocalcemia was not revealed; in the age group of 20-29 years - 98 people, hypocalcemia-9 \% (women), 30-39 years - 139 people, hypocalcemia-6.5\% (men $-1.5 \%$, women - $5 \%$ ); 40-49 years - 113 people, hypocalcemia- $2.7 \%$ (men- $0.9 \%$, women- $1.8 \%$ ); 50-59 years - 174 people, hypocalcemia- $1 \%$ (men- $0.5 \%$, women- $0.5 \%$ ); 60 69 y - 282 people, hypocalcemia- $1.8 \%$ (men $0.7 \%$, women $1.1 \%$ );70-79 years - 146 people, hypocalcemia - $3.4 \%$ of cases (men-1.4\%, women- $2 \%$ ); age group over 80 years 43 people, hypocalcemia - 0 . Hypocalcemia in women younger than 40 years was associated with hypoproteinemia (94\%) without symptoms; hypocalcemia in the age group 60-69 years was not associated with hypoproteinemia (100\%).

Conclusion: The results of the study indicate a significant occurrence of hypocalcemia in women up to 40 years due to hypoproteinemia. Hypocalcemia after 60 years is not associated with hypoproteinemia, and can be pathological. The prevalence of true hypocalcemia was $1.4 \%$.

\section{P649}

ASSOCIATION BETWEEN BONE MINERAL DENSITY AND NON-ALCOHOLIC FATTY LIVER DISEASE: A CROSS-SECTIONAL STUDY OF 3866 POSTMENOPAUSAL WOMEN

D.-Y. Lee ${ }^{1}$, D. Choi ${ }^{1}$, B.-K. Yoon ${ }^{1}$

${ }^{1}$ Obstetrics and Gynecology, Samsung Medical Center, Seoul, Republic of Korea

Objective: Growing evidence suggests that non-alcoholic fatty liver disease (NAFLD) may be associated with reduced BMD, but data regarding this issue is still limited. The objective of this study was to examine the association between BMD and ultrasonography-determined NAFLD in postmenopausal Korean women.

Methods: This cross-sectional study analyzed 3866 postmenopausal women aged 50-59 years who had visited a health promotion center for a routine checkup from 2009-2014. Menopause was defined as the absence of menstruation for $\geq 12$ months with an elevated ( $>20$ IU/L) serum level of follicle stimulating hormone. Women were excluded from the study if they had a history of diseases or if they were taking medications that might affect bone metabolism. BMD was measured by DXA and compared according to the presence of NAFLD.

Results: Among 3866 postmenopausal women, 621 (16.1\%) had NAFLD. Mean BMD was significantly lower in women with NAFLD compared to those without NAFLD at the 
lumbar spine $\left(1.051 \pm 0.127\right.$ vs. $1.063 \pm 0.140 \mathrm{~g} / \mathrm{cm}^{2}$; $P=0.036)$ and femur neck $(0.836 \pm 0.093$ vs. 0.858 $\left.\pm 0.106 \mathrm{~g} / \mathrm{cm}^{2} ; P<0.001\right)$. In multivariate analysis, this association remained significant after adjusting for age, BMI, Creactive protein, insulin resistance, osteocalcin, lipid profile, smoking status, alcohol intake, and physical activity.

Conclusion: This study suggests that NAFLD per se may have a negative impact on BMD in postmenopausal women. A longitudinal study is necessary.

\section{P650}

METABOLIC MILIEU REGULATES BONE TURNOVER AND ASSOCIATES WITH IMPAIRED BONE QUALITY IN OBESITY

${ }_{\text {H. Viljakainen }}^{1}$, H. Koistinen ${ }^{2}$, S. Andersson ${ }^{1}$, O. Mäkitie ${ }^{1}$

${ }^{1}$ Children's Hospital, University of Helsinki and Helsinki University Hospital, Helsinki, Finland, ${ }^{2}$ Department of Medicine, University of Helsinki and Helsinki University Hospital, Helsinki, Finland

Objectives: High adipokine concentrations, low-grade inflammation, and insulin resistance often coexist in metabolically unhealthy obese subjects. The adverse metabolic milieu has been suggested to be the main culprit for impaired bone quality and possibly for increased fracture risk. Here we have examined the associations of leptin, hs-CRP and insulin resistance with bone turnover markers (BTM) and bone outcomes in a cohort of young obese adults and their controls.

Materials and Methods: The study involved 58 obese (median BMI $42 \mathrm{~kg} / \mathrm{m}^{2}$ ) and 67 population-based control (BMI $\left.23 \mathrm{~kg} / \mathrm{m}^{2}, p<0.001\right)$ subjects (48\% males) with mean age $19.5( \pm 2.5)$ yrs. Laboratory parameters included circulating leptin, hs-CRP, fasting plasma glucose and insulin to calculate HOMA [(Glucose x Insulin)/22.5], and BTMs (=BAP, P1NP, CTX-1, and TRAC5b). pQCT-derived radial characteristics included bone mass (Mass), trabecular and cortical density (Trb/Cort Den), strength index (SSI) and periosteal circumference (PC).

Results: Leptin, hs-CRP and HOMA correlated inversely both with P1NP, CTX-1, and TRAC5b: $r$ varied between -0.37 and -0.45 with $p<0.03$ for Sqr(Leptin) and $\operatorname{Ln}(\mathrm{CRP})$, but these were weaker for $\operatorname{Ln}($ HOMA). The associations with BTMs were independent for leptin and CRP, but not for HOMA. Moderate, inverse partial correlations were observed between these and Mass, Trb Den, SSI and PC after controlling for age, sex and BMI. By comparing groups defined by median of leptin or CRP concentration or HOMA, we were able to define the strongest modifier. Bone mass differed between low and high leptin group (1.32 [1.26-1.38] vs. $1.18[1.12-1.25] \mathrm{g} / \mathrm{cm}, p=0.01)$ and a similar tendency was seen in Trb Den $(p=0.109)$, but not when using cutoff of hsCPR and HOMA.

Conclusions: In a cohort of young obese adults and their controls leptin, CRP and HOMA associated with BTMs and radial bone characteristics. Effects of leptin and CRP were independent, while HOMA appeared a secondary modifier. Of these, leptin was the strongest determinant of bone mass and trabecular density.

P651

SIMULTANEOUS OCCURRENCE OF ATYPICAL FEMORAL FRACTURES AND OSTEONECROSIS OF THE JAW IN AN OSTEOPOROTIC WOMAN TAKING ALENDRONATE: REPORT OF A SECOND CASE

W.-Y. Chiu ${ }^{1}$, J.-J. Lee ${ }^{1}$, K.-S. Tsai ${ }^{1}$

${ }^{1}$ Taiwan University Hospital, Taipei, Taiwan, Province of China

Objective: Randomized clinical trials have provided literary evidence that antiresorptive agents reduce the incidence of fragility fractures in patients with osteoporosis. Therefore, the use of these drugs has become common. However, reports of osteonecrosis of the jaw (ONJ) and atypical femoral fractures (AFF) in patients receiving bisphosphonates have raised concerns in recent years.

Material and Methods: The diagnosis of AFF and ONJ were made according to the report of a task force of the American Society for Bone and Mineral Research $(2014)^{1}$ and the definition of medication-related ONJ proposed by the American Association of Oral and Maxillofacial Surgeons in its 2014 guideline ${ }^{2}$.

Result: The patient was a 75 -year-old woman with a history of diabetes for 10 years. She took alendronate for osteoporosis with compression fracture since December 2005. The oral bisphosphonate was discontinued in October 2014. In December 2014, two months later, she presented with an incomplete fracture in the subtrochanteric region of the left femur and received intramedullary reconstruction with fulllength nails. She was treated with subcutaneous teriparatide therapy since January 2015 and received dental extraction in March. However, a non-healing wound with pain and erythema was observed in the extraction region. The patient underwent several courses of antibiotics without relief from symptoms. She was diagnosed as having ONJ in June 2015. Prior to this case, the first case of concomitant ONJ and AFF occurrence in an osteoporotic patient receiving alendronate was reported in $2013^{3}$. 
Conclusions: This case supports the association of both ONJ and AFF with long-term bisphosphonate therapy and highlights the need to reevaluate the optimal duration of antiresorptive therapy.

\section{References:}

${ }^{1}$ J Bone Miner Res. 2014;291:1

2 J Oral Maxillofac Surg. 2014;72:1938

${ }^{3}$ J Clin Endocrinol Metab. 2013;98:E723

\section{P652}

\section{MEASUREMENTS OF BONE MINERAL DENSITY AND STIFFNESS INDEX IN YOUNG SAUDI FEMALES}

L. Hammad ${ }^{1}$

${ }^{1}$ Radiological Sciences, College of Health and Rehabilitation Sciences, Princess Nourah Bint AbdulRahman University, Riyadh, Saudi Arabia

Objective: To examine the ability to use quantitative ultrasonography (QUS) densitometer for screening of osteoporosis and osteopenia by comparing QUS values obtained at the calcaneus region to BMD values measured at the spine and the neck of the femur using DXA.

Methods: QUS (in the calcaneus region) and DXA (the spine and the neck of femur respectively) measurements were performed in 101 females.

Results: The precision of the QUS parameters varied from 1.771.78 , whereas the reliability ranged from $92.2 \%-98.6 \%$. For the QUS parameters variability between subjects was greater than that within subjects. Positive correlation were found between stiffness index (SI) and $\mathrm{BMD}_{\text {spine }}$ and $\mathrm{BMD}_{\mathrm{N}, \text { femur }}(r=0.29 \&$ $r=0.25$ respectively, $P<0.05)$ and a strong positive correlation

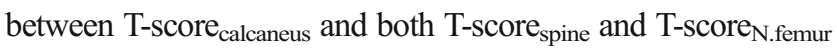
$(r=0.5 \& r=0.58$ respectively, $P<0.01)$.

Conclusions: We found that the QUS is a reliable technique to be used in combination with DXA for the investigation of osteopenia and osteoporosis.

\section{P653}

\section{NEW CARTILAGE QUALITY EVALUATION USING CYCLIC NANO INDENTATION: CASE STUDY WITH A TOOL DMOAD COMPOUND ACTIVE IN THE RAT MNX MODEL}

C. Lavet ${ }^{1}$, P. Clement-Lacroix ${ }^{2}$, D. Merciris ${ }^{2}$, S. Meurisse ${ }^{2}$, L. Lepescheux $^{2}$, V. Gaillard ${ }^{2}$, P. Pastoureau ${ }^{3}$, P. Deprez ${ }^{2}$, D.

Amantini $^{2}$, P. Ammann ${ }^{1}$

${ }^{1}$ Division of Bone Diseases, Geneva University Hospitals and Faculty of Medicine, Geneva, Switzerland, ${ }^{2}$ Galapagos SASU, Romainville, France, ${ }^{3}$ Institut de Recherches Servier, Suresnes, France
Measurement of cartilage material level properties are lacking in small osteoarthritis (OA) animal model. We evaluated a novel cyclic indentation technique in femurs from SHAM and meniscectomized (MNX) rats treated with vehicle and a disease modifying osteoarthritis drug (DMOAD). This novel target identified by Galapagos was based on its in vivo DMOAD efficacy recorded in a MNX model. DMOAD is endowed with strong anticatabolic cellular activity: IL-1b-triggered MMP13 protein induction in normal human articular chondrocytes. Therefore, the compound could positively influence the cartilage material level properties. Surgical joint destabilization was performed in 10 week-old female Lewis rats. DMOAD was then delivered by oral dosage $(20 \mathrm{mg} / \mathrm{kg} / \mathrm{d})$ until sacrifice (21 days post-surgery). Femur was used for phase-contrast micro-computed tomography allowing determination of hyaline cartilage thickness and proteoglycan (PG) content estimation of each condyle as well as trabecular and cortical subchondral bone (SB) morphometric parameters. The quality (indentation depth and Young's modulus) of each condyle of the femoral cartilage was evaluated through bioindentation. This cyclic indentation allowed for assessment of cartilage quality at different cartilage depth. Cartilage of proximal tibia was evaluated and scored using the OARSI method. As expected, analysis of MNX animals showed higher tibial OARSI score and a deterioration of the trabecular SB (BV/TV:-11\%, $p<.001)$. In MNX group, depth of indentation significantly increased and Young's modulus significantly decreased at each cartilage depth investigated in the medial condyle (respective average $+74 \%$ and $-35 \%$ ), indicating softening of the cartilage, while $\mu \mathrm{CT}$ analysis showed increased hyaline cartilage thickness $(+13 \%$, $p<.05)$. At the lateral condyle indentation depth and Young's modulus also respectively increased and decreased ( $+47 \%$ and $-41 \%)$ without morphologic alteration. DMOAD reduced OA progression in the femur and tibia and normalized femoral biomechanical and SB alterations. In medial femoral condyle, cartilage thickness remained higher in the treated group, but PG content estimation by $\mu \mathrm{CT}$ (attenuation: $-10 \%$ vs. sham, $p<.01$ ) was increased by DMOAD in both condyles and could contribute to normalization of biomechanical parameters. These results indicated that this new technique generated data on cartilage quality which were correlated to well-validated readouts. This new technique enables to differentiate $\mathrm{OA}$ and DMOAD effects. 


\section{P654}

\section{FOCAL CORTICAL THINNING}

IS ASSOCIATED WITH LOWER THIGH MUSCLE AREA IN HIP FRACTURES AND CONTROLS

\section{T. P. Rikkonen ${ }^{1}$, G. Treece ${ }^{2}$, F. Johannesdottir ${ }^{3}$, K. E. S. Poole ${ }^{3}$}

${ }^{1}$ Kuopio Musculoskeletal Research Unit, University of Eastern Finland, Kuopio, Finland, ${ }^{2}$ Engineering Division of the Department of Engineering, University of Cambridge, Cambridge, United Kingdom, ${ }^{3}$ Department of Medicine, University of Cambridge School of Clinical Medicine, Cambridge, United Kingdom

Low muscle strength and focal osteoporosis are known risk factors for hip fracture. However, associations between thigh muscle compartment size and proximal femur 3D bone mass distribution in hip fracture are less clear.

Subjects and methods: A total of 84 CT scans consisting of age matched hip fractured patients $(n=40)$ and nonhip fracture controls $(n=43)$ were analyzed (Mean age 78 years, range 61-92). Fracture cases were age matched with patients undergoing routine CT. Age, height and weight were recorded. The thigh muscle compartment was determined just below lesser trochanter using $10 \times 1 \mathrm{~mm}$ slice mean tissue values. Muscle and fat tissue separation and areal bone density (BMD) analysis were done using QCT Pro (5.1.3). Proximal femur bone mass characteristics were measured with Stradwin 5.0 software. Significant associations between muscle cross sectional area and bone parameters, including cortical mass (CM) and endocortical trabecular density (ECTD), were color mapped using SurfStat Matlab toolbox. An independent-samples $t$-test was conducted to compare the groups.

Results: A lower thigh muscle cross-sectional area $\left(\mathrm{cm}^{2}\right)$ was seen in fractures (Mean $=91.7$, standard deviation $=13.9)$ compared to controls (97.8, 12.7); $\mathrm{t}(81)=-2.1, p<0.05$. Across all patients, higher thigh muscle area was associated with more cortical mass. Most notably in upper femoral neck, which is known to associate with hip fracture (Figure). Fracture patients had lower BMI $(22.8,4.4)$ than control $(26.1,4.9)$ group; $\mathrm{t}(81)=-3.21, p<0.01$. A lower areal BMD $\left(\mathrm{g} / \mathrm{cm}^{2}\right)$ was seen in fractures $(0.624,0.162)$ vs. controls $(0.698,0.10)$ in total hip; $\mathrm{t}(81)=-2.57, p=0.01$ and in femoral neck $((0.539,0.120)$ vs. $(0.651,0.81))$, respectively; $\mathrm{t}(81)=-5.0, p<0.001$. There was no difference in the amount of intramuscular fat between the groups. Adjusting muscle and fat areas for height ${ }^{2}$, did not change the results. The association persisted after adjusting for age and volumetric bone size.
Conclusion: Femoral cortical bone distribution is associated with thigh muscle cross-sectional area, in fracture critical zones. In addition, the muscle compartment size and bone mass are significantly lower in hip fracture patients. Smaller muscle area is a modifiable risk factor suggested to contribute towards weaker bone characteristics and hip ractures.

Figure. Percentage increase in cortical mass (CM) associated with one unit increase in thigh muscle cross-sectional area $\left(\mathrm{cm}^{2}\right)$. Adjusted for age and volumetric femur size.

P655

PROTEIN MALNUTRITION AFFECTS
CARTILAGE QUALITY AND COULD
CONTRIBUTE TO OSTEOARTHRITIS
DEVELOPMENT

\section{P. Ammann ${ }^{1}$, C. Lavet ${ }^{1}$}

${ }^{1}$ Division of Bone Diseases, Geneva University Hospitals and Faculty of Medicine, Geneva, Switzerland

IGF-I is a major anabolic agent for cartilage homeostasis. Protein malnutrition results in alteration of the somatotropic axis as well as IGF-I local production and thus may contribute to osteoarthritis (OA) development. Such malnutrition could also be implicated in subchondral bone (SB) alterations. We hypothesizes that protein malnutrition could alter cartilage metabolism and subchondral bone predisposing to OA. To address this question, 9 months old rats were pair fed a normal or an isocaloric low protein diet (LP, $2.5 \%$ casein) for 2 months $(n=6 /$ group). Contrast enhanced computed tomography (Hexabrix) of distal femur allowed to assess thickness of hyaline and calcified cartilage, as well as cortical and trabecular SB micro-architecture. Since early OA is characterized by PG loss and collagen network disorganization which could alter cartilage biomechanical properties, the cartilage material level properties (indentation depth, Young's modulus and indentation energy) was evaluated using bioindentation of medial condyle. Indentation tests affects around $75 \%$ of the whole cartilage thickness were performed with accuracy lower than $9 \%$ for all investigated parameters. Systemic IGF-I was decreased in LP group $(-18 \%, p<.001)$. As expected, in LP group, SB compartment was altered: trabecular SB mass was decreased $(-10 \%, p<.01)$ as well as SB cortical plate thickness of the medial condyle $(-12 \%, p<.05)$. Despite no morphologic changes of the cartilage (thickness tended to decrease by $8 \%$ but not significantly), hyaline cartilage biomechanical properties (force, elastic modulus and working energy) were respectively decreased by -47 , 

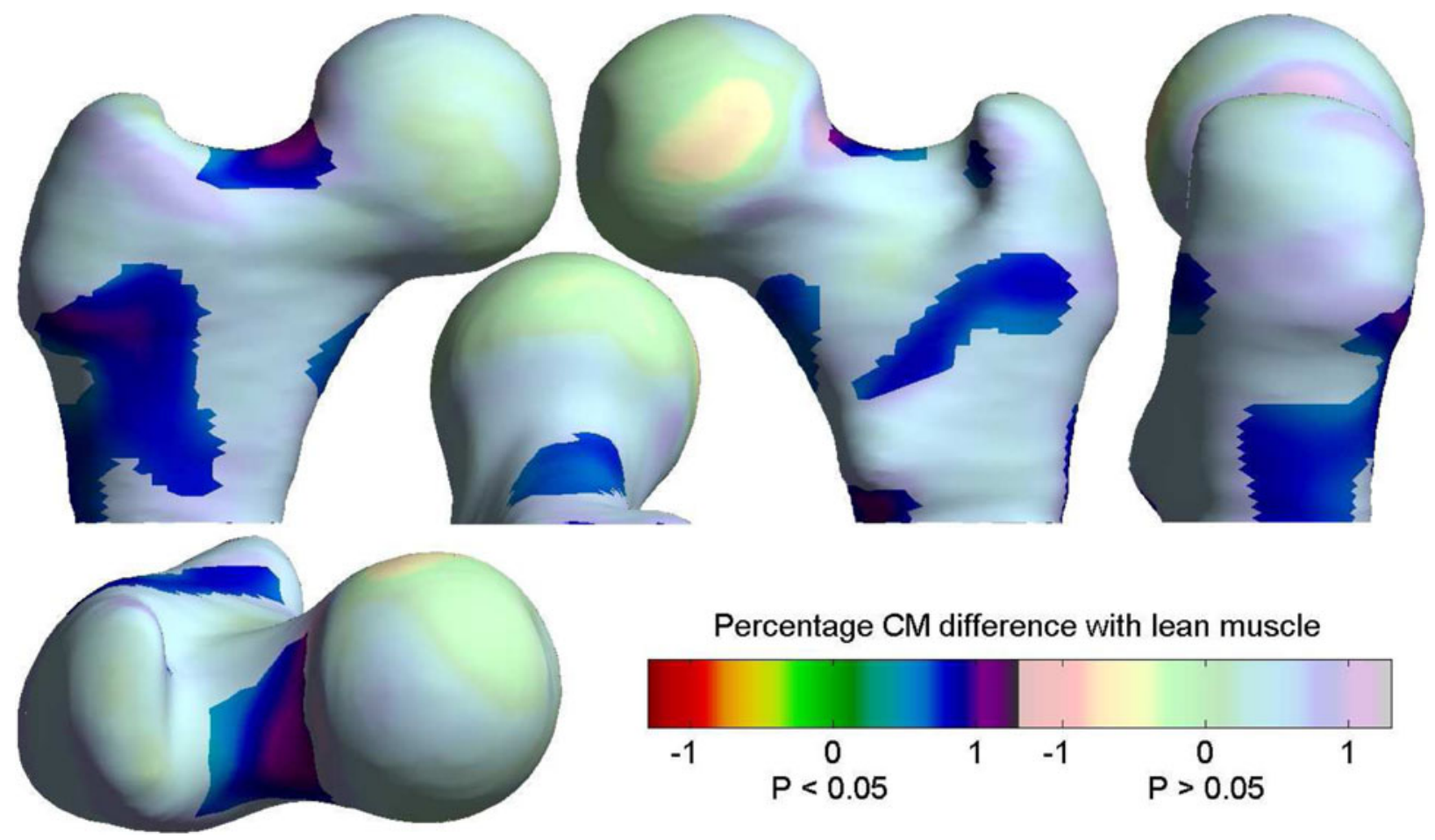

Percentage $\mathrm{CM}$ difference with lean muscle

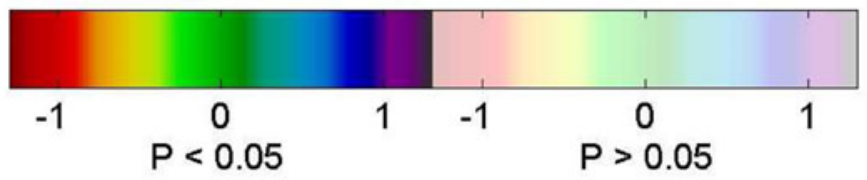

-58 and $-41 \%(p<.01)$. Similar effects on cartilage biomechanical properties were also observed in young adult rats. We identified degradation of hyaline cartilage material level properties and a SB cortical thinning as well as trabecular SB deterioration with only mild alteration of hyaline cartilage morphology in rats fed a low protein diet. Since these alterations have been described in early OA, we suggest that alteration of the somatotropic axis induced by protein malnutrition could predispose to OA.

\section{P656}

\section{EFFICACY OF HIGH INTENSITY LASER THERAPY} ON BONE MINERAL DENSITY AND FRACTURE

\section{RISK IN MALE WITH OSTEOPENIA OR}

\section{OSTEOPOROSIS: A RANDOMIZED}

\section{PLACEBO-CONTROLLED TRIAL}

M. Alayat ${ }^{1}$, E. Abd El-Kafy ${ }^{2}$, A. Thabet ${ }^{2}$, A. Abdel-Malek ${ }^{3}$, T. $\mathrm{Ali}^{4}$

${ }^{1}$ Physical Therapy Department, Faculty of Applied Medical Science, Umm Al-Qura University, Makkah, Saudi Arabia, ${ }^{2}$ Physical Therapy Department, Faculty of Applied Medical Science, Makkah, Saudi Arabia, ${ }^{3}$ Internal Medicine, Al-Qura University Medical Center, Makkah, Saudi Arabia, ${ }^{4}$ Radiology, Head of the Radiology Department, Al-Qura University Medical Center, Makkah, Saudi Arabia
Objective: to investigate the effect of high intensity laser therapy (HILT), alone or combined with exercise, on BMD and fracture risk in male with osteopenia or osteoporosis.

Methods: A total of 100 male patients with osteopenia or osteoporosis participated in the study, with a mean (SD) age, weight, and height were 53.78 (2.89) years, $80.56(7.33) \mathrm{kg}$ and $174.97(5.30) \mathrm{cm}$ respectively. They had $\mathrm{T}$-scores $\leq-1.5$. Patients were randomly assigned into four groups and treated with HILT plus exercise (HILT +EX), placebo laser plus exercise $(\mathrm{PL}+\mathrm{EX})$, HILT alone (HILT) and placebo laser (PL) in groups 1, 2, 3 and 4, respectively. Each low back and hip areas received $3000 \mathrm{~J}$ by $\operatorname{HILT}(610,710$ and $810 \mathrm{~mJ} / \mathrm{cm}^{2}$ in three successive subphases). Progressive exercise training program (treadmill, weight bearing and aerobic exercises for back, and hip) were performed for $50 \mathrm{~min}$. HILT and exercises were performed 3 times per week for 3 months. The measured outcomes were BMD (Spine $\mathrm{L}_{2}-\mathrm{L}_{4}$ and total hip), T-score of femoral neck and spine. 10-year probabilities for major osteoporotic (MO) and hip fractures (HF) were calculated using fracture risk assessment tool $\left(\right.$ FRAX $\left.^{\circledR}\right)$. Measurements were taken before and after 3 months of treatment.

Results: In the first 3 groups, BMD and T-score were significantly increased with decreased MO and HF percent with a nonsignificant difference in PL group $(p>0.05)$. HILT + EX showed a higher significant effect 
than PL +EX with a least significant effect in HILT group (effect-size $\mathrm{r}$ was $0.87,0.57$, and 0.44 for groups 1,2 and 3 , respectively).

Conclusion: HILT is an effective physical therapy modality for male patients with osteopenia or osteoporosis. HILT combined with exercise is more effective than PL with exercise or HILT alone in increasing spine and total hip BMD, T-score and in decreasing MO and HF percent after 3 months of treatment.

Acknowledgment: This project was funded by the National Plan for Science, Technology and Innovation (MAARIFAH), King Abdulaziz city for science and Technology - the Kingdom of Saudi Arabia, award number (13-MED745-10).

\section{P657}

DECLINE IN BMD TESTING : A NATIONWIDE STUDY IN FRANCE

E. Lespessailles $^{1}$, P. Gabach ${ }^{2}$, D. Buchon ${ }^{3}$, M. Douge ${ }^{2}$, J. M. Feron $^{4}$, L. Grange ${ }^{5}$, C. Leboucher ${ }^{2}$, E. Legrand ${ }^{6}$, G. A. PeyreLanquar $^{2}$, E. Ronfle ${ }^{2}$, P. Guggenbuhl ${ }^{7}$, T. Thomas ${ }^{8}$

${ }^{1}$ Rheumatology, Hospital Orleans, i3mto Laboratory, Orleans University, Orleans, France, ${ }^{2}$ CNAMTS, Paris, France, ${ }^{3}$ General Medecine Department, Limoges University, Limoges, France, ${ }^{4}$ Orthopaedic and Trauma department, Saint Antoine hospital, Paris, France, ${ }^{5}$ AFLARAssociation Francaise de Lutte Anti Rhumatismale, Rheumatology Department, grenoble, France, ${ }^{6}$ Rheumatology Department Universitary Hospital, CHU Angers, Angers, France, ${ }^{7}$ Rheumatology Department CHU Rennes, INSERM UMR 991, Rennes University, Rennes, France, ${ }^{8}$ Inserm U1059, Rheumatology Department University Hospital, CHU Saint-Etienne, Saint-Etienne, France

Background: The assessment of BMD remains crucial for the general management of osteoporosis (OP). Since 2006, French national health insurance provides conditional reimbursement for bone density testing by DXA.

Methods: We aimed to evaluate the evolution of the number of BMD testing between 2011-2013 using the sources of national health insurance information system (SNIIRAM). In addition, we sought to describe in the population of patients hospitalized for fragility fracture in 2012 the dispensation of BMD testing in the following year.

Results: The total number of BMD testing went down by $13 \%$, essentially in private activity which represents $90 \%$ of the BMD measurements in France (table).
Number of BMD testing realized in the private sector

\begin{tabular}{llll}
\hline Year & 2011 & 2012 & 2013 \\
Male & 32942 & 32883 & 32043 \\
Female & 552710 & 511665 & 474964 \\
Badly filled in & 189 & 173 & 111 \\
Total & 585841 & 544721 & 507118 \\
\hline
\end{tabular}

Source: DCIR, 2011-2013

Number of BMD testing realized in the public sector

\begin{tabular}{llll}
\hline Year & 2011 & 2012 & 2013 \\
Male & 8380 & 8648 & 9632 \\
Female & 54224 & 53677 & 54157 \\
Badly filled in & 4811 & 4269 & 518 \\
Total & 67415 & 66594 & 64307 \\
\hline
\end{tabular}

Source: French hospital national database, 2011-2013

Among patients hospitalized for a fragility fracture:

- One year before hospitalization $3 \%$ of patients have had a BMD testing

- Only $0.8 \%$ of patients had a BMD testing in the month following their fracture hospitalization. This percentage slightly increased to 6 and $8.5 \%$ at 6 and 12 months respectively. Only $25 \%$ of the patients having a BMD testing, received an anti-osteoporotic treatment within 6 months following the exam. The rate of rehospitalization was $29 \%$ in the 6 months following their stay in hospital.

Discussion: These data showed that there is a declining number of BMD testing from 2011-2013 with a $6.4 \%$ annual decrease. While the number of BMD testing plateaued in men, the downward trend of BMD testing was the most prominent in women. There is a substantial post-fracture BMD testing and treatment gap. These worrying findings underlined important undertreatment of patients with osteoporosis, especially after the fracture. Procedures that will improve postfracture coordination between healthcare professionals are needed for a better management of these patients with the objective of reducing future fracture risk.

\section{P658}

\section{IMMUNE SYSTEM AND BONE CELLS IN EARLY RHEUMATOID ARTHRITIS}

I. Buondonno ${ }^{1}$, F. Sassi ${ }^{1}$, M. Rigoni ${ }^{1}$, G. Rovera ${ }^{2}$, G. Isaia ${ }^{1}$, P. $\mathrm{D}^{\prime}$ Amelio $^{1}$

${ }^{1}$ Department of Medical Science-University of Torino, Torino, Italy, ${ }^{2}$ SSD Reumatologia-Ospedale Mauriziano, Torino, Italy

Rheumatoid arthritis (RA) is an immune-mediated disease characterized by $\mathrm{T}$ cells activation and bone erosions that severely reduces patients quality of life; however a clear role for different Thelper (Th) cells has not been established yet. This work aims to 
evaluate Th phenotypes, osteoclast (OCs) precursors cells and cytokines in peripheral blood of women affected by early RA (eRA) compared to healthy women matched for age. The study was approved by our ethical committee. We enrolled in the study 36 women affected by eRA and 31 healthy controls. To quantify OCs precursors and Th subset in peripheral blood we used flow cytometry. We measured classical OCs precursors (CD14+/ $\mathrm{CD} 11 \mathrm{~b}+/ \mathrm{VNR}+)$ and inflammatory OCs precursors (CD14+/ $\mathrm{CD} 11 \mathrm{~b}+/ \mathrm{VNR}+/ \mathrm{CD} 16+)$ and Th subset. We also measured TNF $\alpha$, IFN $\gamma$, TGF $\beta$, IL-4, IL-17, IL-23, IL-6, RANKL and OPG in the serum by ELISA. Th were significantly altered in eRA: Th17 and Th17/IFN $\gamma$ were increased, whereas Th1,Th2 and Tregs were not significantly affected. Classical OCs were reduced in $\mathrm{eR}$ patients whereas inflammatory $\mathrm{OCs}$ were increased and correlated with CRP (Rho 0.35, $p=0.039$ ). RANKL/OPG was significantly increased in eRA due to increased RANKL and directly correlated with inflammatory OCs precursors. TNF $\alpha$, TGF- $\beta$,IL-23 and IL-6 were significantly increased whereas IL17 and IFN $\gamma$ were not. The increase in inflammatory OC precursors suggests a specific role for these cells in bone erosions and possibly in systemical bone loss in RA. This is the first attempt to describe Th cells and OC precursors in early phase of RA. We showed an impairment in Th cells subtypes that may be the first pathogenetic driver in early phase of RA whereas the increase in TGF $\beta$ may be due to deregulation in T regulatory cells function. Previous paper showed conflicting results on Th subsets in RA, this is mainly due to different models used.

\section{P659}

ESTIMATION OF PREVALENCE OF BONE MINERAL DENSITY CHANGES AMONG DYSPLASTIC COXARTHROSIS PATIENTS UNDERTAKING TREATMENTS IN A SPECIALIZED HOSPITAL OF TRAUMATOLOGY AND ORTHOPEDICS

M. N. Lobanov $^{1}$, L. G. Grigoricheva ${ }^{1}$, N. A. Guseva ${ }^{1}$

${ }^{1}$ Federal State Budgetary Institution "Federal Center of Traumatology, Orthopedics and Endoprosthesis Replacement" of the Ministry of Health of the Russian Federation, Barnaul, Russian Federation

Due to disorder of joints biomechanics, dysplastic coxarthrosis leads to the development of secondary arthrosis, which causes both functional restructuring of the bone, and changes in BMD. Objective: To assess the prevalence of BMD changes in patients with dysplastic coxarthrosis undergoing treatment in the FCTOER in 2015.

Materials and Methods: The analysis of BMD was carried out in 344 patients with dysplastic coxarthrosis between the ages of $22-78$, their average age being $54.6 \pm 12.4$ (248 of them were women - $72 \%$; 96 of them were men - $28 \%$ ), their age gradation being as follows: under 45 years old - $11.4 \%$, from 45 to $65-36 \%$, over $65-52.6 \%$. The patients underwent DXA of the lumbar spine and proximal femur by Excell XR-46 (Norland, USA) with evaluation of T-criterion according to the standards of WHO.

Results: Among the patients of the group under study, the frequency of BMD reduction was $73.7 \%-253$ people ( $23 \%$ of them with osteoporosis - 79 people, $50.7 \%$ with osteopenia - 174 people). Drop of indicating values to the level of osteoporosis was revealed among women in 62 people (that is $25 \%$ ) and among men - in 17 people (that is $18 \%$ ). Drop of indicating values to the level of osteopenia was revealed among women in 130 people (52.4\%), among men in 44 people $(46 \%)$ which is higher than the average population indicators in the Russian Federation ( $43.3 \%$ for women, $44.1 \%$ for men). The proportion of patients with normal BMD indicators was $26.3 \%$, that is 91 people $(22.6 \%$ among women - 56 people, $36.4 \%$ among men - 35 people). In compliance with the age criterion the prevalence of osteoporosis was as follows: no reported cases were diagnosed at the age under $45,13.6 \%$ - at the age from 45 to $65,31.2 \%$ - at the age over 65 ; the prevalence of osteopenia was $42.8 \%$ at the age under $45,54.5 \%$ - at the age from 45 to $65,50.0 \%$ - at the age over 65 .

Conclusions: The results are indicative of a high frequency of reduced BMD in patients with dysplastic coxarthrosis. It determines the relevance of formation of vulnerable groups for preventive pharmacotherapy among patients over 45 years old with a view to decelerate BMD decline and risks of fractures, both before surgery and in the postoperative period.

\section{P660 \\ FREQUENCY OF LOW BONE MINERAL DENSITY IN SPONDYLOARTHROPATHY PRESENTING AT A TERTIARY CARE HOSPITAL}

L. Nazir ${ }^{1}$

${ }^{1}$ Liaquat National Hospital and Medical College, Karachi, Pakistan

Objective: To determine the frequency and risk factors of low BMD in patients with spondyloarthropathies.

Methods: The cross-sectional study was conducted at the Rheumatology Department of a tertiary care hospital in Karachi between June and November 2014, and comprised spondyloarthritis patients whose BMD of lumbar spine and hips was measured using DXA scan. Variables like disease duration, diagnosis, human leukocyte antigen subtype B27, erythrocyte sedimentation rate, C-reactive protein, Bath ankylosing spondylitis 
disease activity Index, Bath ankylosing spondylitis functional index, Bath ankylosing spondylitis metrology index were measured along with outcomes, differentiating between osteopenia and/or osteoporosis. SPSS 21 was used for statistical analysis

Results: Of the 25 patients in the study, 16(64\%) were males, 19(76\%) had predominant axial involvement, and $20(80 \%)$ had duration of disease less than 10 years. Low BMD at the spine and hip was found in $18(72 \%)$. Osteopenia was present in $9(36 \%)$ at hip and $8(32 \%)$ in spine, while osteoporosis was seen in $5(20 \%)$ at hip and $9(36 \%)$ in the spine. No significant association was found between BMD and all the other parameters measured ( $p>0.05$ each).

Conclusion: Majority of spondyloarthritis patients had decreased BMD which could be observed in early stages of the disease.

\section{P661}

THE PREVALENCE OF MUSCLE MASS IMPAIRMENT AMONG A GROUP WITH FRACTURE AFTER 50 YEARS OF AGE

B. S. E. Peters $^{1}$, E. B. Curatella ${ }^{1}$, N. A. G. França ${ }^{1}$, M. M. S. Lima ${ }^{1}$, P. C. Santos ${ }^{1}$, L. A. Martini ${ }^{1}$

${ }^{1}$ Nutrition Department, School of Public Health, University of São Paulo, São Paulo, Brazil

Objective: Investigate in a Brazilian sample the prevalence of muscle mass impairment among those who have experienced fractures after 50 years of age.

Material and Methods: The study was conducted with 161 old adults ( $\geq 50$ years old) of both sexes enrolled in the cross-sectional study Health Survey - Sao Paulo 2014. The prevalence of fractures it was self-reported by applying a standardized questionnaire. Body weight $(\mathrm{kg})$ and height $(\mathrm{m})$ were measured, and BMI $\left(\mathrm{kg} / \mathrm{m}^{2}\right)$ was calculated and classified according WHO/OPAS. Appendicular lean mass (ALM) was assessed by total body DXA scan (Lunar iDXA Advance). Grip strength was measured by a handheld dynamometer (Jamar) and the usual gait speed was defined as the length of the walking course $(4 \mathrm{~m})$ divided by the time to conclude it at their usual pace. All the classifications were conducted according the EWGSOP cutoffs. Descriptive analysis was performed using SPSS software v17.0 and results are presented in mean (standard deviation) or percentage $(\%)$.

Results: The sample was 64.02 (7.59) years old, predominantly female $(62.7 \%)$, and mostly overweight $(54.4 \%)$ with mean BMI of $28.51(5.88) \mathrm{kg} / \mathrm{m}^{2}$. The prevalence of fractures after age 50 was $14.3 \%$ among the sample and it was higher in women than man (56.5\% vs. $44.5 \%)$. Despite only $13 \%$ of who had fractures showed low ALM, the impairment of muscle function was quite evident. Low handgrip strength and slow gait speed was observed in $81.8 \%$ and $57.1 \%$ of the fractured group, respectively. Thus, the injury of muscle function, either by impaired strength or gait speed, was prevalent among $81.8 \%$ of those with fractures after 50 years old.

Conclusion: The prevalence of muscle strength and gait speed impairment was elevated among those who suffered fractures after age 50, suggesting the importance of maintaining muscle function to prevent fractures.

\section{P662}

INCREASED PLEKHO1 WITHIN OSTEOBLAST SUPPRESSES BMP SIGNALING TO INHIBIT BONE FORMATION DURING AGING

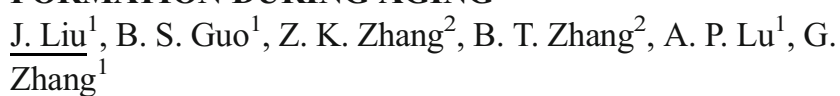

${ }^{1}$ Hong Kong Baptist University, Hong Kong, Hong Kong, ${ }^{2}$ The Chinese University of Hong Kong, Hong Kong, Hong Kong

Emerging evidences indicate that the dysregulation of protein ubiquitination plays a crucial role in agingassociated diseases. BMP signaling pathway is one of the most important signaling pathways responsible for osteoblastic bone formation, which could be disrupted by the ubiquitination and subsequent proteasomal degradation of Smad1/5, the key molecules for BMP signaling transduction. However, whether the dysregulation of Smad1/5 ubiquitination and disrupted BMP signaling pathway also responsible for the age-related bone formation reduction is still underexplored. In this study, after screening the mRNA expression of a series of previously reported ubiquitination-related molecules involved in Smad1/5 ubiquitination, we found an agerelated increase in the expression of Pleckstrin homology domain-containing family $\mathrm{O}$ member 1 (PLEKHO1) in bone specimens from elder patients with fractures and in osteoblasts from aging rodents, which was associated with the elevated ubiquitination of Smad1/5, reduced BMP signaling (phosphorylation of Smad1/5) and bone formation during aging. PLEKHO 1 was previously identified as a ubiquitination-related molecule that could specifically target the linker region between the WW domains of Smurf1 to promote the ubiquitination of Smad1/5 ( Lu 
et al. Nat Cell Biol 2008). However, we found no significant difference in the intraosseous Smurfl expression during aging. By genetic approach using osteoblast-specific Plekhol knockout/knock-in and Smadl knock-in strategy, we show that Plekhol ablation within osteoblast could attenuate the age-related bone formation reduction, whereas Plekhol overexpression within osteoblast could accelerate the age-related bone formation reduction, which could be rescued after overexpressing Smadl within osteoblast. By pharmacological approach using Plekhol siRNA encapsulated within an osteoblast-targeted delivery system, we show that silencing PLEKHO1 within osteoblast could enhance BMP signaling and promote bone formation in aging rodents. Taken together, it suggests that the increased PLEKHO1 could suppress BMP signaling to inhibit bone formation during aging, indicating the translational potential of therapeutic silencing PLEKHO1 within osteoblasts as a novel bone anabolic strategy for reversing established osteoporosis during aging.

\section{P663}

TWO-FOLD REGIONAL VARIATION IN INITIATION OF ANTI-OSTEOPOROSIS MEDICATION AFTER HIP FRACTURE IN THE UK

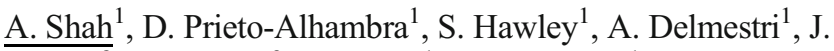
$\overline{\text { Lippett }}^{2}$, C. Cooper ${ }^{3}$, A. Judge ${ }^{1}$, M. K. Javaid ${ }^{1}$

${ }^{1}$ Nuffield Department of Orthopaedics, Rheumatology and Musculoskeletal Sciences, University of Oxford, Oxford, United Kingdom, ${ }^{2}$ Royal Berkshire NHS Foundation Trust, Reading, United Kingdom, ${ }^{3}$ MRC Epidemiology Resource Centre, University of Southampton, Southampton General Hospital, Southampton, United Kingdom

Objective: Describe UK regional variation in prescription of anti-osteoporosis drug therapy before and after a primary hip fracture during 1999-2013.

Materials and Methods: We used primary care data (Clinical Practice Research Datalink) to identify patients with a hip fracture and primary-care prescriptions of any anti-osteoporosis drugs (bisphosphonates, strontium, denosumab, oestrogen therapy, SERMS, teriparatide) prior to primary hip fracture and up to five years after. Regional variations in prescribing before and after generic oral bisphosphonates were analysed. Multivariable logistic regression models were adjusted for gender, age and indices of deprivation.

Results: 13,069 patients (mean age 82 years, $76 \%$ female) diagnosed with a primary hip fracture during
1999-2013 were identified. $11 \%$ had any prescription in the six months prior to primary hip fracture with no significant regional variation. In the $0-4$ months following a hip fracture $5 \%$ of patients were prescribed an anti-osteoporosis drug in 1999, which increased to $51 \%$ in 2011 and decreased to $39 \%$ in 2013. $15 \%$ of patients remained on treatment by 60 months. Independent predictors of treatment initiation included men $(O R=0.42$ 95\%CI: 0.36-0.49), increasing BMI $(O R=0.98$ 95\%CI: $0.97-1.00)$ and region $(O R=1.28$ $95 \%$ CI: $0.88-1.87$ North East vs. $O R=0.5795 \% \mathrm{CI}$ : $0.77-1.14$ South West). Regional differences in prescribing persisted over the 5-year follow-up. If all patients were treated at the rate of the highest performing region, then nationally 3,214 additional hip fracture patients would be initiated on therapy every year.

Conclusion: Significant regional differences exist in post-hip fracture prescribing of anti-osteoporosis drugs despite adjustment for potential confounders at the patient level. While a significant increase was observed after 2005, the rate of treatment initiation was still low. Further work examining differences in health care provision may inform strategies to improve in secondary fracture prevention after hip fracture.

\section{P664}

PHYSICAL PERFORMANCE VARIABLES AND BONE PARAMETERS IN A GROUP OF OVERWEIGHT MEN

C. El Khoury $^{1}$, A. Pinti ${ }^{2}$, H. Toumi ${ }^{2}$, G. El Khoury ${ }^{1}$, A. Alwan $^{1}$, R. El Hage ${ }^{1}$

${ }^{1}$ Department of Physical Education, Fass, University of Balamand, Kelhat El Kurah, Lebanon, ${ }^{2}$ I3MTO-EA 4708, Université d'Orléans, Orléans, France

Aim: To explore the relation between physical performance variables and bone parameters (BMD, hip geometry and trabecular bone score) in a group of overweight men.

Methods: 54 overweight $\left(\mathrm{BMI}>25 \mathrm{~kg} / \mathrm{m}^{2}\right)$ young men whose ages range between 18-30 years participated in this study. Weight and height were measured, and BMI was calculated. Body composition was evaluated by DXA. DXA measurements were performed at the whole body (WB), lumbar spine $\left(\mathrm{L}_{1}-\mathrm{L}_{4}\right)$, total hip (TH) and femoral neck (FN). FN cross-sectional area (CSA), FN section modulus $(\mathrm{Z})$ and lumbar spine trabecular bone score were also evaluated by DXA. $\mathrm{VO}_{2}$ max was determined by direct measurement while exercising on a bicycle ergometer (Siemens-Elema RE 820; Rodby 
Elektronik AB, Enhorna, Sweden). Maximal strength and maximum power of the lower limbs were evaluated using validated tests.

Results: Lean mass was the best positive determinant of WB BMC, FN CSA and FN Z. $\mathrm{VO}_{2} \max (1 / \mathrm{mn})$ was the best positive determinant of WB BMD, TH BMD and FN BMD. $\mathrm{VO}_{2} \max (\mathrm{ml} / \mathrm{mn} / \mathrm{kg})$ was the best positive determinant of TBS. Maximal strength of the lower limbs was the best positive determinant of L1-L4 BMD.

Conclusion: This study suggests that maximizing $\mathrm{VO}_{2}$ max, lean mass and maximal strength of the lower limbs positively affects bone parameters in overweight men.

\section{P665}

NEUTRALIZING ANTIBODIES AGAINST BONE MORPHOGENETIC PROTEIN 7 IN FRACTURE PATIENTS

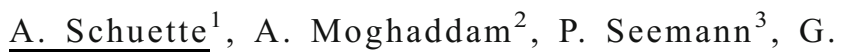
Schmidmaier $^{2}$, L. Schomburg ${ }^{1}$

${ }^{1}$ Institute for Experimental Endocrinology, CharitéUniversitätsmedizin Berlin, Berlin, Germany, ${ }^{2}$ Department of Orthopedics and Trauma Surgery, Heidelberg University Hospital, Heidelberg, Germany, ${ }^{3}$ Berlin-Brandenburg Center for Regenerative Therapies, Charité-Universitätsmedizin Berlin, Berlin, Germany

Bone is a tissue with a high regenerative potential. However, some fractures fail to regenerate spontaneously leading to delayed healing or non-union. The reasons for these complications are manifold and mostly unclear. Disturbed bone healing can be surgically enhanced by a combination of various means including the application of recombinant human growth factors like bone morphogenetic protein 7 (BMP 7). In this study we analyzed fracture patients that were treated or not with BMP7 and determined the presence of anti-BMP7 antibodies (BMP7-Ab) in their sera. To this end, we developed a detection assay for the measurement of BMP7-Ab. Sera from 265 fracture patients were analyzed at two time points, around the operational procedure and four weeks later. In some of the fracture patients we demonstrated that BMP7-Ab is present, and with a higher incidence in those treated with rhBMP7. Furthermore, BMP7-Ab were transiently increased and returned to nondetectable levels over time. In a cell-based BMP7 reporter assay a neutralizing effect of BMP7-ab isolated from patient sera was demonstrated. Our data indicate that endogenous antagonizing BMP7-Ab may transiently arise upon BMP7 treatment in some patients potentially affecting the healing process.

\section{P666}

ROLE OF PATIENT COMPLIANCE IN TREATMENT OF OSTEOPOROSIS

F. Gojkovic $^{1}$, J. Elez ${ }^{1}$, J. Vasic ${ }^{1}$, V. Culafic-Vojinovic ${ }^{2}$

${ }^{1}$ Rheumatology, Railway Healthcare Center, Belgrade, Serbia, ${ }^{2}$ Euromedic, Belgrade, Serbia

Outpatient pharmacotherapy of chronic disease relies on drug self administration et home. Due to this fact, success of achieving the desired clinical outcome depends on the patient compliance as much as on the efficacy itself. The problem of poor compliance was recognised globally and estimate as an essential factor of obtaining the suboptimal treatment results and unnecessary wasting of limited financial sources for the health care sector. During 24 months of treatment we followed compliance of bisphosphonates treatment of osteoporosis on 1700 patients. The groups were divided by the age and way of administered therapy weekly, monthly, on three months or once in a year. The statistical analysis shows that the best compliance is demonstrated in younger group of patients age from 50-59 years and it is $88.8 \%$ after 24 months of treatment. The lowest compliance was provided in the eldest group more than 80 years and it is $44.2 \%$.

Conclusion: It is statistically obvious that the patient's compliance is the lowest in the group of the eldest patients. Other groups have the very similar patients compliance, which is quite well compliance according to the published data. We wished to present the pharmacoeconomic dimension of patient compliance issue in order to intensify efforts on its improvement in local health care conditions.

\section{P667}

BONE MINERAL DENSITY IN WOMEN WITH PARKINSON'S DISEASE

V. Povoroznyuk ${ }^{1}$, M. Bystrytska ${ }^{1}$, I. Karaban ${ }^{2}$, N. Karasevych $^{2}$

${ }^{1}$ Department of Clinical Physiology and Pathology of Locomotor Apparatus, D.F. Chebotarev Institute of Gerontology NAMS Ukraine, Kyiv, Ukraine, ${ }^{2}$ Parkinson's Department, D.F. Chebotarev Institute of Gerontology NAMS Ukraine, Kyiv, Ukraine

Aim: To define the BMD in patients, with Parkinson's disease.

Methods: We examined 12 women with Parkinson's disease and 12 healthy women of appropriate age (average age: -63.6 \pm 6.25 vs. $62,2 \pm 6.83$ years, $p=0.5$ ). The duration of 
Parkinson's disease was at list 5 years. All patients received levodopa.

Results: BMD of women with Parkinson's disease was significantly lower compared with BMD of women of control group on the level of total body (T-score $=-1.86 \pm 1.32$ vs. -0.71 $\pm 1.48, p<0.05, \mathrm{Z}$-score $=-0.35 \pm 0.93$ vs. $0.51 \pm 1.05$, $p<0.05$ ), lumbar spine (T-score $=-1.56 \pm 1.22$ vs. $0.10 \pm 1.63$, $p<0.05, \mathrm{Z}$-score $=-0.66 \pm 0.87$ vs. $0.72 \pm 1.53, p<0.05)$ and at the distal forearm $(\mathrm{T}$-score $=-1.87 \pm 1.32$ vs. $0.71 \pm 1.47$, $p<0.05, \mathrm{Z}$-score $=-0.51 \pm 1.05$ vs. $0.38 \pm 1.22, p<0.05)$. Hip BMD was not different from control group. It is important to note that one woman with Parkinson's disease has two hip endoprostheses after femoral neck fractures. Conclusion: BMD in women with Parkinson's disease is significantly lower than in healthy women of the same age.

\section{P668}

\section{STATINS: ARE THEY WONDER DRUGS IN FRAC-} TURE RISK?

$\underline{\text { Z. Maghbooli }}^{1}$, A. Hossein-Nezhad ${ }^{1}$, B. Larijani ${ }^{1}$, S. M. Eshaghi $^{2}$, M. Ramezani ${ }^{2}$, E. Taheri ${ }^{1}$

${ }^{1}$ Osteoporosis Research Center, Endocrinology and Metabolism Clinical Sciences Institute, Tehran University of Medical Sciences, Tehran, Islamic Republic of Iran, ${ }^{2}$ Osteoporosis Research Center, Endocrinology and Metabolism Clinical Sciences Institute, Tehran University of Medical Sciences, National Elites Foundation, Tehran, Islamic Republic of Iran

Background: There is uncertainty about the role of statins on risk of osteoporotic fracture. The effect of statins on fracture has important clinical implication, since statins are frequently prescribed with lifelong exposure. This study aims to compare risk of fracture between statin users and nonusers among hyperlipidemic patients.

Methods: DXA results of the total hip and lumbar spine and clinical data were obtained prospectively during 2010-2014 from patients that referred to an outpatient bone clinic affiliated to osteoporosis research center of Tehran University. The study subjects were 9406 postmenopausal women older than 60 years of age. Chronic illness like auto immune disorders, cancers, neurological diseases and any conditions that under treatment with corticosteroids were excluded. Also, fractures due to high-energy injuries such as traffic accidents were excluded.

Results: Of the 9406 postmenopausal women, 1084 women had clinical and laboratory available data associated with hyperlipidemia (mean age 68.4 $\pm 7.2 \mathrm{SD}$ years, BMI $28.2 \pm 4.5 \mathrm{~kg} / \mathrm{m}^{2}$ ). Among patients with hyperlipidemia, $33.7 \%, 44.7 \%$ had history of type 2 diabetes and hypertension, respectively. Patients with hyperlipidemia were classified based on using statin drugs to statin user and nonuser. Totally 633 women used any kind of statins and 446 women did not use hypolipidemic agents. Compared with the nonusers, the statin users had higher spine and hip BMD but not statistically significant. Generally, the risk of any osteoporotic fractures was significantly higher in the statin users $(\mathrm{P}$-value $=0.04, O R=1.67,95 \% \mathrm{CI}: 1,2.2 .8)$. Related to type of fracture, there were not any significant differences in history of hip and spine fractures between statin users and nonusers, while risk of wrist fracture was higher in the statin users (P-value $=0.03$, $O R=3.02$, 95\%CI: $(1.1,9.05)$. Our findings were similar even after adjusting for history of diabetes. Also, the combined use of anti-hypertensive agents and a statin has showed an increase risk of fracture. In a logistic regression model, there was a week significant association between use of statins and history of osteoporotic fracture independent of age, BMI, type 2 diabetes and hypertension.

Conclusion: Current evidence does not support an effect of statins in preventing fracture risk. Our findings show that using statins may increase the risk of fracture. Therefore, statin drugs should be used cautiously in the elderly people who are at risk for fractures.

\section{P669}

A TEN-MONTH'S REPORT ON PARTICIPATION IN AN OSTEOPOROSIS INFO WEBSITE

U. Akarirmak $^{1}$, N. Eskiyurt ${ }^{2}$, S. Tuzun ${ }^{1}$, M. Sarıdogan ${ }^{1}$

${ }^{1}$ Istanbul University, Cerrahpasa Medical Faculty, Physical Medicine and Rehabilitation Department, Istanbul, Turkey, ${ }^{2}$ Istanbul University, Istanbul Medical Faculty, Physical Medicine and Rehabilitation Department, Istanbul, Turkey

Objective: Beginning in October 2014 the IOF Concept for Male Osteoporosis pointed out a need to raise awareness on osteoporosis also in the male population.

Methods: A website was prepared by the Turkish Osteoporosis Society www.erkekosteoporozu.com (www. maleosteoporosis.com) including the IOF One-Minute-Risk Test for men, information on osteoporosis and importance of lifestyle. We analysed the results of the participants who visited the website between March 05-December 31, 2015.

Results: A total of 22,002 visits were reported with 10 , 762 one time and 1762 multiple visits, mean duration was 01.01 minutes. $59.9 \%$ of visitors were female, $40.1 \%$ male. According to age groups the most frequent visitor group (820) was 25-34 years old (28\%) while only $7 \%$ were $65+.18 \%$ were $35-44,16 \%$ 
were $18-24,14 \%$ were $45-55,15 \%$ were $55-64$ years old. Over $60 \%$ were living in the 3 biggest cities İstanbul, Ankara and İzmir. Desktop usage was reported in $69 \%$, mobile phone $25 \%$ and tablet in $5 \%$. At least one osteoporosis risk factor was detected in 7.398 of 11.170 females, while 3.772 reported none. At least one osteoporosis risk factor was detected in 1.935 of 2.770 males, while only 3.835 reported none.

Conclusion: Preventive strategies for female as well as male healthy population is of great importance. The primary aim is to establish a lifelong approach by addressing nutrition, vitamin $\mathrm{D}$, physical activity and avoiding smoking in males. Therefore it is crucial to detect risk factors in different cohorts of the population, which were high in this study and target them in order to establish powerful preventive strategies for osteoporosis.

\section{P670}

THE ROLE OF VITAMIN D AND EXERCISES IN CORRECTION OF AGE-RELATED SKELETAL MUSCLE CHANGES IN POSTMENOPAUSAL WOMEN

V. Povoroznyuk $^{1}$, N. Dzerovych ${ }^{1}$, R. Povoroznyuk ${ }^{1}$

${ }^{1}$ Department of Clinical Physiology and Pathology of Locomotor Apparatus, D.F. Chebotarev Institute of Gerontology NAMS Ukraine, Kyiv, Ukraine

\begin{abstract}
Aim: To evaluate the role of vitamin D and exercises in correction of age-related skeletal muscle changes in postmenopausal women.
\end{abstract}

Materials and methods: 38 postmenopausal women aged 53-82 years (mean age $-67.00 \pm 7.08 \mathrm{yrs}$; mean height - $160.31 \pm 6.83 \mathrm{~cm}$; mean weight $-63.25 \pm 8.59 \mathrm{~kg}$, BMI $-24.62 \pm 3.09 \mathrm{~kg} / \mathrm{m}^{2}$ ) were examined. All subjects were free of systemic disorders (endocrine, renal, hepatic etc.) and did not take any medications known to affect skeletal and muscle metabolism. The women were divided into the following groups: A - control group $(n=10), \mathrm{B}-$ women who took an individually-targeted vitamin $\mathrm{D}$ therapy $(n=11), \mathrm{C}-$ women who took an individually-targeted vitamin D therapy and OTAGO Exercise Programme (http://www.hfwcny.org/Tools/ BroadCaster/Upload/Project13/Docs/Otago_Exercise Programme.pdf) during 12 months. The assessment of the examined women was conducted every 3 months at the medical center. We used the following questionnaires: SARC-F, IADL-questionnaire, frailty scale, Desmond fall risk questionnaire. For evaluation of skeletal muscle function and strength, we assessed the usual gait speed and used hand dynamometry. 25(OH)D total and iPTH levels were measured by electrochemiluminescent method i.e., Elecsys 2010 analytical system (Roche Diagnostics, Germany) and test-systems cobas. The lean mass was measured by the DXA method (Prodigy, GEHC Lunar, Madison, WI, USA). "Statistika 6.0" (C) StatSoft, Inc. was used for the data processing purposes.

Results: At the baseline, the groups of examined women did not differ in their age, anthropometric characteristics, 25(OH)D values, data of skeletal muscle mass, strength and function. In women of the control group, the mean 25(OH)D level significantly increased after 9 months of observation ( 9 months $-p=0.03$ ) purportedly due to the seasonal factors. In women of 2nd and 3rd groups, the 25(OH)D level significantly increased after $3,6,9$ and 12 months of observations (2nd group: 3 months $-\mathrm{p}=0.009,6$ months $p=0.007,9$ months $-\mathrm{p}=0.005,12$ months $\mathrm{p}=0.003$; 3rd group: 3 months $-\mathrm{p}<0.001,6$ months $-\mathrm{p}<0.001,9$ months $-\mathrm{p}<0.001,12$ months $\mathrm{p}<0.001)$. The data of SARC-F, IADL-questionnaires did not change during 12 months of observation in women of 1 st and 2 nd groups; however, in the $3 \mathrm{rd}$ group the SARC-F data significantly decreased after 12 months $(p=0,02)$ while the IADL data - significantly increased after $9(p=0.04)$ and 12 months $(p=0.05)$. The data of frailty scale and Desmond fall risk questionnaire did not differ in all groups during 12 months. The muscle strength significantly increased after 9 months $(p=0.01)$ in women of 3rd group while in women of 1 st and 2nd group this parameter did not change. The usual gait speed and lean mass assessed by DXA did not change in all groups during 12 months. The fall frequency in women of 1 st group significantly increased after 12 months, in women of 2 nd group it did not change while in women of 3rd group the fall frequency significantly decreased.

Conclusion: Using individually-targeted vitamin D therapy and OTAGO Exercise Programme during 12 months significantly improves daily activity, muscle strength and decreases the fall frequency in postmenopausal women.

\section{P671}

DETERMINANTS OF QUANTITATIVE ULTRASONOMETRY VARIABLES IN A GROUP OF YOUNG FRENCH ADULTS

$\underline{\text { E. Zakhem }}^{1}$, D. Theunynck ${ }^{2}$, G. Zunquin ${ }^{2}$, T. Pezé ${ }^{2}$, R. Hurdiel $^{2}$, R. El Hage ${ }^{1}$

${ }^{1}$ Department of Physical Education, Fass, University of Balamand, Kelhat El Kurah, Lebanon, ${ }^{2}$ Departement STAPS, URePSSS-EA4110/EA448, ULCO, Dunkerque, France 
Aim: To define the determinants of quantitative ultrasonometry (QUS) variables of the calcaneus in a group of young French adults.

Methods: 535 young adults (342 women and 193 men) whose ages range between 18-30 years participated in this study. Weight, height, fat mass, hip circumference and waist circumference were measured, and BMI was calculated. Physical performance variables were evaluated using standardized tests. Physical activity level, daily calcium intake and sleep quality were evaluated using validated questionnaires. Broadband ultrasound attenuation (BUA) of the calcaneus was measured using a new ultrasonometry device «Pegasus Smart Medlink ${ }^{\circledR} 》$.

Results: In women, weight $(r=0.30 ; p<0.001)$, fat mass $(r=0.20 ; p<0.01)$, BMI $(r=0.26 ; p<0.001)$, hip circumference $(r=0.23 ; p<0.01)$ and waist circumference $(r=0.19 ; p<0.01)$ were positively correlated to BUA. In men, none of the measured variables was significantly correlated to BUA.

Conclusion: This study suggests that, in young women, morphological characteristics such as body weight, fat mass, BMI, hip circumference and hip circumference are positive determinants of broadband ultrasound attenuation of the calcaneus.
P672

FRAX ${ }^{\circledR}$ - THE RELATIONSHIP BETWEEN WEB-BASED AND PAPER-BASED SCORES

G. T. Clifford $^{1}$, M. McKenna-Barry ${ }^{1}$, S. Van der Kamp ${ }^{1}$, C. Hurson $^{1}$, M. McKenna ${ }^{1}$

${ }^{1}$ St. Vincent's University Hospital, Dublin, Ireland

Background: The National Osteoporosis Guidelines Group (NOGG, 2014) recommends that fracture risk is assessed in postmenopausal women and men aged 50 years or more using FRAX ${ }^{1}$. FRAX is a webbased algorithm, incorporating clinical risk factors to determine the 10-year probability of a major osteoporotic fracture (MOF) as a percentage, and is specific to Ireland. In the absence of computer access, NOGG recommends a paper-based FRAX algorithm based on UK data. The aim of this study was to determine the relationship between the FRAX $\mathrm{WEB}_{\mathrm{B}}$ with the FRAX $X_{\text {PAPER }}$.

Methodology: A convenience sample of 150 subjects with a fracture was selected from six Orthopaedic Trauma Clinics. Patients were excluded if they had a diagnosis of osteoporosis $(n=37)$, had no clinical risk factors for osteoporosis $(n=52)$, or a history of fragility fracture if female $(n=14)$.
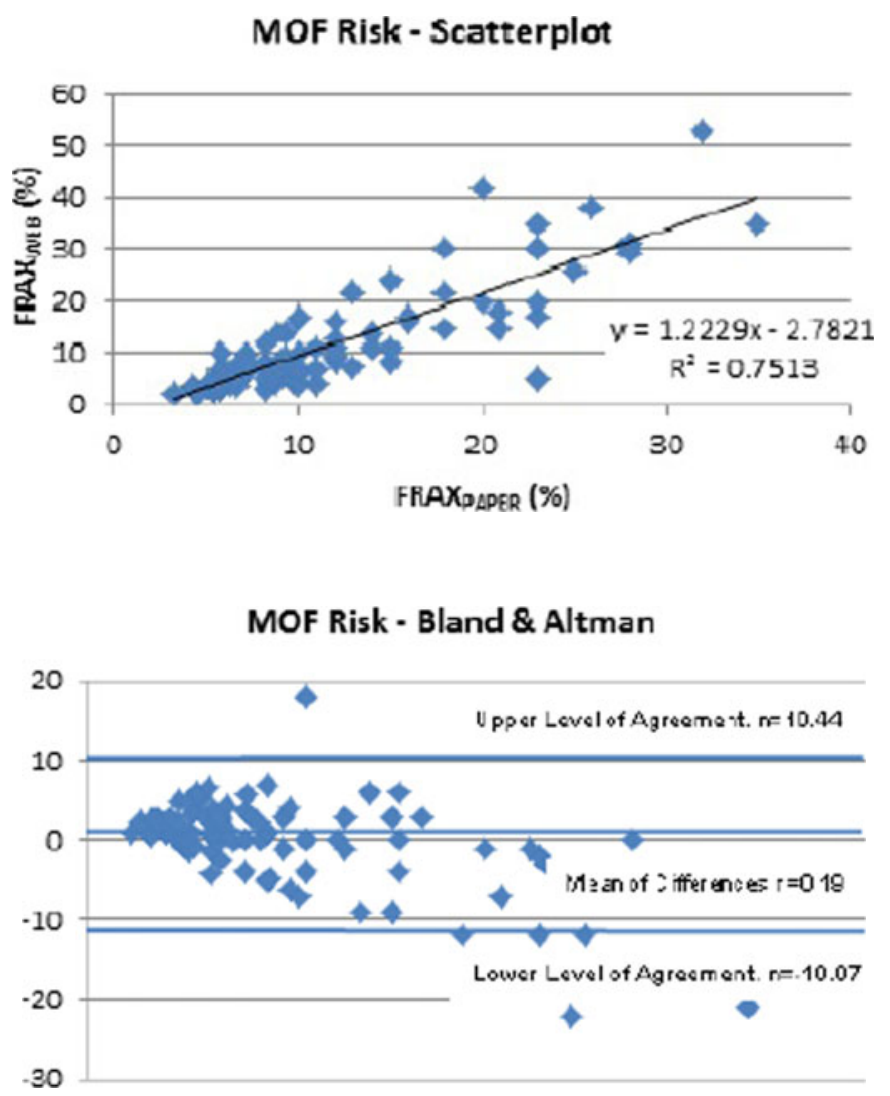
Results: Of the 101 subjects included, FRAX ${ }_{\text {WEB }}$ median score was $7.4 \%$ (IQR $10.4 \%$ ) and FRAX PAPER $_{\text {median score }}$ was $9.5 \%$ (IQR $7.95 \%$ ). Both FRAX WEB $_{\text {and FRAX }}$ PAPER identified 17 subjects as at risk of osteoporosis ( $\geq 20 \%$ ). Data was normally distributed. A strong correlation was established between both scores [Pearson's $r=0.87$, Student's $t$-test (Paired, two tail) $p=0.72]$.

Implications: The FRAX $\mathrm{WEB}_{\mathrm{W}}$ and the FRAX $\mathrm{PAPER}_{\mathrm{P}}$ corre-

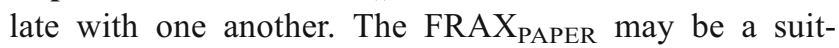
able alternative in absence of computer access however, 52 subjects who would normally be included in FRAX $_{\text {WEB }}$ could not be included in FRAX PAPER $_{\text {as }}$ they had no clinical risk factors. This may underestimate the risk of osteoporosis in this population. A FRAX $\mathrm{X}_{\mathrm{PAPER}}$ using Irish data $^{2}$ should be developed and validated in an Irish population.

\section{References:}

${ }^{1}$ Guideline for the diagnosis and management of osteoporosis in postmenopausal women and men from the age of 50 years in the UK (2014). National Osteoporosis Guideline Group

${ }^{2}$ Svedbom A, et al. Arch Osteoporos 2013;8:137.

\section{P673}

RELATIONSHIP BETWEEN VITAMIN D DEFICIENCY, FRAGILITY FRACTURES AND CARDIOVASCULAR RISK

P. J. Cossío-Jimenez ${ }^{1}$, S. M. Rojas ${ }^{1}$, T. L. Rodríguez-Araya ${ }^{1}$, E. Chamizo-Carmona ${ }^{1}$

${ }^{1}$ Reumatología, Hospital de Mérida, Mérida, Spain

Introduction: Vitamin D deficiency is highly prevalence, especially in adults and elderly population. There is controversy about its relationship with increased cardiovascular risk, osteoporosis and bone fractures.

Objective: To describe the vitamin D levels in a population of southwest Spain evaluated with suspected osteoporosis in a Bone Metabolism Unit and its relationship to other diseases.

Patients and Methods: We selected the patients attended in the Bone Metabolism Unit of the Hospital of Mérida between June 2013 and June 2014 that have at least one determination of 25-OH-cholecalciferol, bone densitometry and complete clinical history.

Results: We included 171 patients between $34-75$ years (mean 64.5, SD 11.75), 11 men and 160 postmenopausal women. The detected levels of 25-OH-cholecalciferol were normal $(>30 \mathrm{ng} / \mathrm{ml})$ in 75 and deficient in 96 patients. Deficiency was severe $(<10 \mathrm{ng} / \mathrm{ml})$ in 11 , moderate $(10-20 \mathrm{ng} / \mathrm{ml})$ in 48 and mild (between $20-30 \mathrm{ng} / \mathrm{ml}$ ) in 37 patients. We did not find any correlation (Pearson coefficient) between the levels of 25-OH-cholecalciferol and age $(-0.17)$, lumbar spine T-score $(0.1)$ and hip T-score
$(-0.004)$, major and hip fracture risk using FRAX without BMD (0.13 for both) and with BMD ( -0.017 and -0.063 , respectively). Neither did we find any association between levels of 25-OH-cholecalciferol and the existence of comorbidities and cardiovascular risk. Patients with severe deficiency $(<10 \mathrm{ng} / \mathrm{ml})$ of $25-\mathrm{OH}-$ cholecalciferol had higher rates of presence of cardiovascular risk factors (RR 5.87, 95\%CI 1.16, 1.87) and bone fractures (OR 3.76 95\%CI 1.75, 13.18).

Conclusions: Severe deficiency of 25-OH-cholecalciferol (levels below $10 \mathrm{ng} / \mathrm{ml}$ ) was significantly associated with the presence of bone fractures, irrespective of BMD values and FRAX risk, and was also more frequent in patients who had at least one cardiovascular risk factor. No association between vitamin D deficiency and the presence of comorbidities was found.

\section{P674}

INCREASES IN PROXIMAL FEMUR BONE STRENGTH ESTIMATED USING FINITE ELEMENT MODELS FROM COMPUTED TOMOGRAPHY SCANS FOLLOWING BRIEF, HIGH IMPACT EXERCISE IN OLDER MEN

K. Brooke-Wavell ${ }^{1}$, L. Yang ${ }^{2}$, D. Blackwell ${ }^{1}$, M. Qasim ${ }^{2}$, S. J. Allison $^{1}$, W. J. Rennie ${ }^{3}$, J. P. Folland ${ }^{1}$, G. D. Summers ${ }^{4}$, R. Eastell $^{2}$

${ }^{1}$ Loughborough University, Loughborough, United Kingdom, ${ }^{2}$ University of Sheffield, Sheffield, United Kingdom, ${ }^{3}$ University Hospitals Leicester, Leicester, United Kingdom, ${ }^{4}$ Derby Teaching Hospitals, Derby, United Kingdom

This study examined the influence of brief daily hopping exercises on bone strength estimates from finite element models that take into account bone distribution, based upon computed tomography (CT) and DXA scans. We analysed proximal femur DXA and CT scans of exercise and control legs, at baseline and after a year of unilateral hopping exercises, in 33 men aged 65-80y. From DXA scans, estimates of strength and stiffness in walk and fall configurations were calculated. From CT scans, estimates of strength in 2 walk ( -3 and $18^{0}$ anteroposterior rotation; both $0^{\circ}$ mediolateral rotation) and 4 fall configurations (anteroposterior and mediolateral rotation of 0,$0 ; 0,30$; 30,0 and $30,30^{\circ}$ respectively) were calculated. A repeated measure ANOVA was used to detect significant effects of time (pre vs post exercise), leg (exercise vs control) and their interaction. Mean strength based on CT scans increased by 6-9\% in the exercise leg and $1-7 \%$ in the control leg. Effects of time were significant in all the fall configurations $(0.001<\mathrm{P}<0.012)$ but not walk configurations $(0.083<\mathrm{P}<0.091)$. Responses did not differ significantly between legs $(P>0.158)$. The minimum strength 
was most often in the $30,30^{\circ}$ fall configuration. This minimum strength increased over time $(p=0.002)$ from 3300 (125) to $3514(176) \mathrm{N}$ in the exercise leg and from 3265 (136) to $3470(170) \mathrm{N}$ in the control leg but did not differ significantly between legs. There were no statistically significant effects on DXA based bone strength estimates. $\mathrm{CT}$ based estimates of proximal femur bone strength in fall configurations increased following brief hopping exercises in older men. Increases in the control leg as well as the exercise leg could represent cross-education effects or increased loading to the control leg, either during the exercise or as a consequence of neuromuscular adaptation. Exercise could reduce the risk of hip fracture following a fall.

\section{P675 \\ HYPOVITAMINOSIS D AND ITS CORRECTION IN PATIENTS WITH PSORIASIS}

O. Samakhavets ${ }^{1}$, E. Rudenka ${ }^{2}$, A. Buhlova ${ }^{3}$

${ }^{1}$ City Hospital, Minsk, Belarus, ${ }^{2}$ Belarusian State Medical University, Minsk, Belarus, ${ }^{3}$ Belarusian Medical Academy of Postgraduate Training, Minsk, Belarus

\begin{abstract}
Aim: To investigate the prevalence of hypovitaminosis D in patients with psoriatic arthritis (PsA) and cutaneous manifestations of psoriasis (CP), and to evaluate the effect of the differentiated therapeutic effects of cholecalciferol on serum level of 25(OH)D.
\end{abstract}

Materials and methods: 103 patients with psoriasis were examined. PsA was diagnosed in accordance with the diagnostic criteria CASPAR (2006) in 66 patients: 34 women (mean age $45.9 \pm 9.11$ ) and 32 men (mean age $50.2 \pm 12.71$ ). 22 patients (14 women (mean age $40.5 \pm 12.1$ ) and 8 men (mean age 46.7 $\pm 11.0)$ ) had CP. Control group consisted of 59 healthy volunteers (31 women and 28 men) who were comparable to the study group by age, sex and BMI. Exclusion criteria were the presence of chronic diseases or intake of medicines that affect bone remodeling processes, including glucocorticosteroids. Severity of the CP was determined using PASI score. Laboratory examination included evaluation of serum total vitamin $\mathrm{D}$ by electrochemiluminescence protein binding assay (Cobas e411 analyzer, Roche Diagnostic, Germany), erythrocyte sedimentation rate (ESR) and level of C-reactive protein (CRP). Statistical processing of the data was carried out using the programs of MS Office Excel 2010 and STATISTICA 8.0 (StatSoft, USA). Due to the predominance of the data corresponding to the abnormal distribution the calculation was performed using nonparametric statistical methods.

Results: Median of 25(OH)D in patients with PsA was 17.85 $[7.03,26.87] \mathrm{ng} / \mathrm{ml}$, vitamin D deficiency was detected in 37 patients $(56 \%)$, insufficiency - in $18(28 \%), 11$ individuals $(16 \%)$ had normal showings of $25(\mathrm{OH}) \mathrm{D}$. In patients with $\mathrm{CP}$ median of serum vitamin D was not significantly different from patients with PsA and was $20.05[16.81 ; 25.60] \mathrm{ng} / \mathrm{ml}$, $p=0.39$. Deficiency of $25(\mathrm{OH}) \mathrm{D}$ was observed in 10 patients with CP (45\%), insufficiency - in 9 (41\%), and only in 3 patients (14\%) level of vitamin D was sufficient. Individuals in the control group had median 25(OH)D level 20.56 [14.43; $27.71] \mathrm{ng} / \mathrm{ml}$, deficiency was observed in 28 (46\%), insufficiency - in $16(28 \%)$, in 15 patients ( $26 \%$ ) levels of vitamin D were within normal limits. Correlation analysis did not reveal any significant relationship between age, sex of patients, indicators of disease activity (ESR, CRP), PASI and level of $25(\mathrm{OH}) \mathrm{D}$ in the studied sample. For correction of hypovitaminosis D the examined patients with PsA and CP were divided into two groups according to the level of serum 25(OH)D. Group $1(n=47)$ had vitamin D deficiency and group $2(n=27)$ - insufficiency of serum vitamin $\mathrm{D}$, median of $25(\mathrm{OH}) \mathrm{D}$ in group 1 and group 2 was 11.00 [5.51, 16.94] $\mathrm{ng} / \mathrm{ml}$ and $25.26[21.92,27.98] \mathrm{ng} / \mathrm{ml}$, respectively. Patients with deficiency of vitamin $\mathrm{D}$ have been assigned cholecalciferol in a daily dose of $2000 \mathrm{IU}$. Control study of $25(\mathrm{OH}) \mathrm{D}$ levels after 3 months noted positive dynamics of $25(\mathrm{OH}) \mathrm{D}$ $33.99[32.11,38.11] \mathrm{ng} / \mathrm{ml}, p<0,001$. Patients with vitamin $\mathrm{D}$ insufficiency were prescribed with cholecalciferol $800 \mathrm{IU} /$ day. Control evaluation of serum 25(OH)D after 3 month revealed increasing of its content $-47.02[41.63 ; 49.48] \mathrm{ng} / \mathrm{ml}$, $\mathrm{p}<0.001$.

Conclusion: In the surveyed sample, hypovitaminosis D is a widespread phenomenon observed in $86 \%$ patients with $\mathrm{CP}$, $84 \%$ patients with PsA, and in $74 \%$ individuals of the control group. Correction of vitamin D deficiency and insufficiency with fat-soluble form of vitamin D in doses $800 \mathrm{IU} /$ day and $2000 \mathrm{IU} /$ day, respectively, leads to a normalization of serum $25(\mathrm{OH}) \mathrm{D}$ in 3 months.

\section{P676}

RESPONSE TO DENOSUMAB IN PATIENTS ATTENDING A SPECIALIST BONE CLINIC

R. Lannon $^{1}$, A. Farrelly ${ }^{1}$, J. Mahon ${ }^{1}$, G. Steen ${ }^{1}$, K. McCarroll $^{1}$, J. B. Walsh ${ }^{1}$

${ }^{1}$ Bone Health Unit, St James's Hospital, Dublin, Ireland

Objective: Denosumab has been available for the treatment of severe osteoporosis since October 2010. It is an antiresorptive agent which has been shown to reduce fracture risk and increase BMD. It is indicated in postmenopausal women with osteoporosis at high risk of fracture and has been shown to reduce the incidence of vertebral, non-vertebral and hip fractures.

Method: Using an electronic database we reviewed records of subjects who were prescribed denosumab at our bone health clinic. 310 patients who had been commenced on denosumab between October 2010 and January 2014. Of these, 210 patients had a repeat DXA between the date they commenced 
denosumab and Jan 2016. We recorded the change in T-score in the AP spine and the total hip in these patients.

Results: Looking at the 210 follow-up DXAs available; mean age was $74 \pm 9.6$ years and mean time to follow-up DXA was $2.12 \pm 1.04$ years. 189 subjects had spinal results and 180 had hip results. Reasons for non-availability of spine or hip scores include vertebrae not suitable for analysis due to fracture or degenerative disease or bilateral hip replacements respectively. Mean T-scores at baseline in spine and total hip were -2.96 and -2.38 , respectively. Spinal T-scores improved in 155 subjects with an average relative improvement in T-score of $0.60 \mathrm{SD}$ $( \pm 0.37)$. In 32 subjects T-score deteriorated an average of $0.40 \mathrm{SD}( \pm 0.39)$. T-score in the AP spine remained unchanged in 10 subjects. Hip T-scores improved in 120 subjects with an average relative improvement in T-score of $0.30( \pm 0.24)$. In 35 subjects T-score deteriorated an average of $0.49 \mathrm{SD}( \pm 0.84)$. Tscore in the Hip remained unchanged in 32 subjects. Overall Tscores improved by $15 \%$ at the spine and $9 \%$ at the total hip. Conclusion: These results show that in 210 patients on denosumab with follow-up DXA attending a tertiary clinical setting there is an improvement in T-score in the AP spine in $73.8 \%$ of patients and in the hip in $57.14 \%$ of patients. Reasons for lack of response will require further analysis of factors such as duration of treatment, previous treatment as well as bone turnover markers and vitamin D and fracture history.

\section{P677}

PREDICTING HIP FRACTURE TYPE OF ELDERLY ASIAN PATIENTS WITH LOW-ENERGY FALL BY VOLUMETRIC BMD AND FEMORAL MORPHOLOGY FROM QCT

Y.-B. Su${ }^{1}$, X.-G. Cheng ${ }^{1}$, L. Wang ${ }^{1}$, Y.-M. Ma ${ }^{1}$

${ }^{1}$ Department of Radiology, Beijing Jishuitan Hospital, Beijing, China

Introduction: Femoral neck fractures and trochanteric fractures are two major types of hip fractures. The BMD changing or hip strength analysis (HSA) of the two type fractures may be different. No research had focused on a large sample of Asian people. This study investigated the differences in BMD and morphologic features of the two type hip fractures of elderly Asian people from QCT.

Methods: 279 elderly Chinese patients with hip fractures due to low-energy fall were included (mean age, 74 years old; femoral neck fracture, $n=235$; trochanteric fracture, $n=144$ ). Each patient underwent QCT scan within $48 \mathrm{~h}$ after fracture. The femoral neck region was divided into four quadrants: inferioranterior (IA), superior-anterior (SA), superior-posterior (SP), and inferior-posterior (IP). Cortical thickness, cortical mass fraction, cortical BMD and trabecular BMD measurement were made at each quadrant. With HSA, measurements of the mineralized bone surface cross-sectional area (CSA), the cross- sectional moment of inertia (CSMI), the section modulus (Z), the buckling ratio $(\mathrm{BR})$ were obtained. Linear regression equation was applied to correct coefficients as age, sex and BMI.

Results: Patients with trochanteric fractures showed higher cortical thickness, cortical mass fraction, and cortical BMD at SA quadrant than patients with femoral neck fractures $(P<0.01 ; P=0.01 ; P<0.01)$. But, patients with trochanteric fractures had lower trabecular BMD at the SP, IP and IA quadrants $(P=0.02 ; P<0.01 ; P=0.03)$. And, no significant differences were found in the parameters of HSA between the two groups.

Conclusions: Severer trabecular osteoporosis is seen in patients with trochanteric fractures than in patients with femoral neck fractures. However, the patients with trochanteric fractures had shown higher cortical thickness, cortical BMD, and cortical mass fraction at femoral neck SA quadrant, which may be more important to predict hip fracture type.

\section{P678}

EFFECTS OF TWO CONSECUTIVE EXERCISE PROGRAMS ON SARCOPENIC INDICES AND BONE MINERAL DENSITY IN COMMUNITYDWELLING ELDERLY WITH HIGH FRAGILITY FRACTURE RISKS

C.-B. Chang ${ }^{1}$, D.-C. Chan ${ }^{2}$, D.-S. Han ${ }^{3}$, J.-S. Hwang ${ }^{4}$, C.-H.

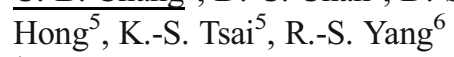

${ }^{1}$ Department of Internal Medicine, National Taiwan University Hospital, Chu-Tung branch, Hsinchu County, Taiwan, Province of China, ${ }^{2}$ Superintendent's Office, National Taiwan University Hospital, Chu-Tung Branch, Hsinchu County, Taiwan, Province of China, ${ }^{3}$ Department of Medical Affair, National Taiwan University Hospital Beihu Branch, Taipei, Taiwan, Province of China, ${ }^{4}$ Department of Internal Medicine, Chang Gung Memorial Hospital, Taoyuan, Taiwan, Province of China, ${ }^{5}$ Department of Geriatrics and Gerontology, National Taiwan University Hospital, Taipei, Taiwan, Province of China, ${ }^{6}$ Department of Orthopedics, National Taiwan University Hospital, Taipei, Taiwan, Province of China

Background/Purpose: Long-term exercise may improve sarcopenia and osteoporosis among elderly. However, whether relatively short-term consecutive campaign for 12 weeks would reach similar effects remain uncertain. We aim to examine the effects of two consecutive exercise interventions on sarcopenia and BMD in community-dwelling older adults with high fragility fracture risks.

Methods: 110 and 109 subjects with high risk for fragility fractures were separately enrolled in 2014 and 2015. Among them, 69 overlapping participants were the main focus of analysis. Each year, participants took exercise course which included core strengthening and resistance training for at least once per week 
for 12 weeks. The two separate training programs were spaced by one year interval. Assessments were done at baseline and 12weeks each year (four time points in total). Major outcomes included walking speed, chair stand test, timed up-and-go test, appendicular skeletal muscle mass index (ASMI), hand and leg muscle strength, and BMD. Comparisons were made between baseline and all other three assessments. Paired-t tests were used to compare the differences before and after interventions. Under all circumstances, $p<0.05$ was considered to be statistically significant.

Results: Mean age was $74.6 \pm 6.3$ years. In general, most sarcopenia indices (walking speed, timed up-and-go test, chair stand test, leg extension, leg curl and leg press) were improved after initial set of 12-weeks of intervention, and returned to 2014 baseline when no longer in the active exercise program. However, taking another 12-weeks boost intervention exercise would reach similar improvements. Nevertheless, walking speed at 2015 baseline was still better as compared to 2014 baseline $(1.32 \pm 0.21 \mathrm{~m} / \mathrm{s}$ vs. $1.21 \pm 0.26 \mathrm{~m} / \mathrm{s}, p<0.05)$. ASMI remained stable at 4 time points. BMD has not improved until the last two assessments indicating late effects of the exercise intervention from 2014.

Discussions: When prolonged exercise intervention is not feasible, a short-term and consecutive exercise training campaign for 12 weeks might be efficacious in improving sarcopenic indices. However, improvements of BMD may last longer even after stopping the exercise trainings for certain periods.

Conclusions: Short-term consecutive exercise interventions have beneficial effects on sarcopenic indices and BMD for elderly.

\section{P679 \\ PROMOTION OF BISPHOSPHONATE RELATED OSTEONECROSIS OF THE JAW BY MANDIBULAR TOOTH EXTRACTION IN RAT: INFLUENCE ON BONE VASCULARIZATION AND MINERA- LIZATION \\ J.-D. Kün-Darbois $^{1}$, H. Libouban ${ }^{1}$, F. PascarettiI-Grizon ${ }^{1}$, D. Chappard \\ ${ }^{1}$ GEROM, University of Angers, Angers, France}

Objectives: Bisphosphonate-related osteonecrosis of the jaws (BRONJ) is a major adverse event due to bisphosphonates (BPs). The pathogenesis of BRONJ is not fully explained and seems multifactorial. An antiangiogenic effect of some BPs (e.g., zoledronic acid-ZA) on jaw bone vascularization has been advocated. The aims of the present study were to induce BRONJ in the rat, to analyze the alveolar mandibular vascularization and to assess the quality of the bone by evaluating the mineralization degree of the bone matrix.

Material and Methods: 38 adult male rats were randomized into 2 groups: ZA, $n=27$ and control (CTRL) $n=11$. Rats of the ZA group received a weekly i.v. injection of ZA $(100 \mu \mathrm{g} /$ $\mathrm{kg}$ ) during 10 weeks. Rats of the CTRL group were similarly injected with equivalent volume of saline solution. After 6 weeks, right mandibular extraction of the 3 molars was performed in all rats. Rats were sacrificed 14 weeks after the first ZA injection. MicroCT was used to study bone lesions. Vascularization analysis was performed after intravascular injection of a radio-opaque material and $3 \mathrm{D}$ analysis by microCT. Raman microspectroscopy was performed on the alveolar bone of the molar area to evaluate bone mineralization.

Results: $55 \%$ of the ZA treated rats presented a bone exposure at extraction sites. MicroCT analysis revealed numerous signs of BRONJ at the right molar area in the ZA group (cortical erosion, periosteal reaction and bone sequestration). None of this signs were found at the left hemimandible in the ZA group and in both hemimandibles of the CTRL group. Vascular density of the molar area was found reduced at the right side in the ZA group due to tooth extraction but was not suppressed. Raman analysis showed significantly increased mineral to amide-I (band 960/1666 $\mathrm{cm}^{-1} ; p=0.031$ ) and mineral to amide II (band 960/1545 $\mathrm{cm}^{-1} ; p=0.021$ ) ratios in alveolar bone after impregnation with ZA.

Conclusion: In a rat model of BRONJ with mandibular tooth extraction, microCT evidenced signs of osteonecrosis. However, vascularization was not suppressed by ZA but reduced due to tooth extraction. Raman spectroscopy evidenced an increased mineralization of the alveolar bone after prolonged treatment with ZA.

\section{P680}

\section{PRELIMINARY COMPARISON BETWEEN NORMATIVE SPINE TBS DATA FOR MOROCCAN MEN AND WOMEN}

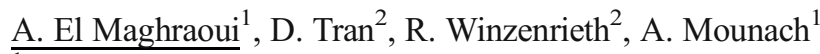

${ }^{1}$ Rheumatology Department, Military Hospital Mohammed V, Rabat, Morocco, ${ }^{2}$ R \& D Department, Med-Imaps, Bordeaux, France

Introduction: Trabecular bone score is an index reflecting the bone microarchitecture of the spine. Interpretation of this score for clinical use requires comparing TBS value to agerelated reference data based on healthy population. The aim of this study was to investigate the age related changes of the lumbar vertebrae microarchitecture assessed by TBS in healthy Moroccan men and women.

Methods: Healthy men and women from 20 y.o. and up were recruited. Inclusion criteria were age from 30-90 y.o., BMI from $15-37 \mathrm{~kg} / \mathrm{m}^{2}$ and BMD Z-score at L1-L1 within $2 \mathrm{SD}$. Exclusion criteria were previous low energy fracture at any site, traumatic fracture at spine, at femur, any treatment and/or, any illness that would be expected to impact bony metabolism (except HRT, calcium/Vit D), previous spinal surgery (such as orthopedic implant, laminectomy, vertebroplasty), a scoliosis at lumbar spine higher than $20^{\circ}$, more than two nonassessable lumbar vertebrae, 
early menopause or surgical menopause. BMD was evaluated at spine (L1-L4) of DXA scans assessed by Prodigy DXA device (GE-LUNAR, Madison). TBS was computed based on the DXA scan with TBS iNsight software (Med-Imaps, France). Agerelated curves for TBS were created using Excel solver and multiple regressions. The generalized reduced gradient approach that was used is a nonlinear optimization algorithm. The end point of the optimization was to minimize the sum of the square errors between data and predicted values.

Results: 103 men aged from 30-79 years and 383 women from 30-80 years were included in the study. TBS and BMD values at L1-L4 were poorly correlated with BMI (re- spectively $r=-0.07$ and 0.23 in men and $r=0.05$ and 0.16 in women). TBS was poorly correlated with weight $(r=-0.15$ in men and 0.04 in women) and height $(-0.19$ in men and -0.03 in women) whereas higher correlations were obtained for BMD. BMD vs. weight and BMD vs. height were respectively $r=0.29$ and 0.19 for men and $r=0.30$ and $r=0.30$ for women).TBS decreased with aging in both men and women. Between 30-80 y.o., TBS in men decreased of $14 \%(-0.9$ SD) and $17 \%(-1.5 \mathrm{SD})$ in women at L1-L4. Compared to European data results are consistent but slightly higher since linear declines of $13.5 \%$ and $16.7 \%$ have been observed between 40 and 90 for men and women, respectively.

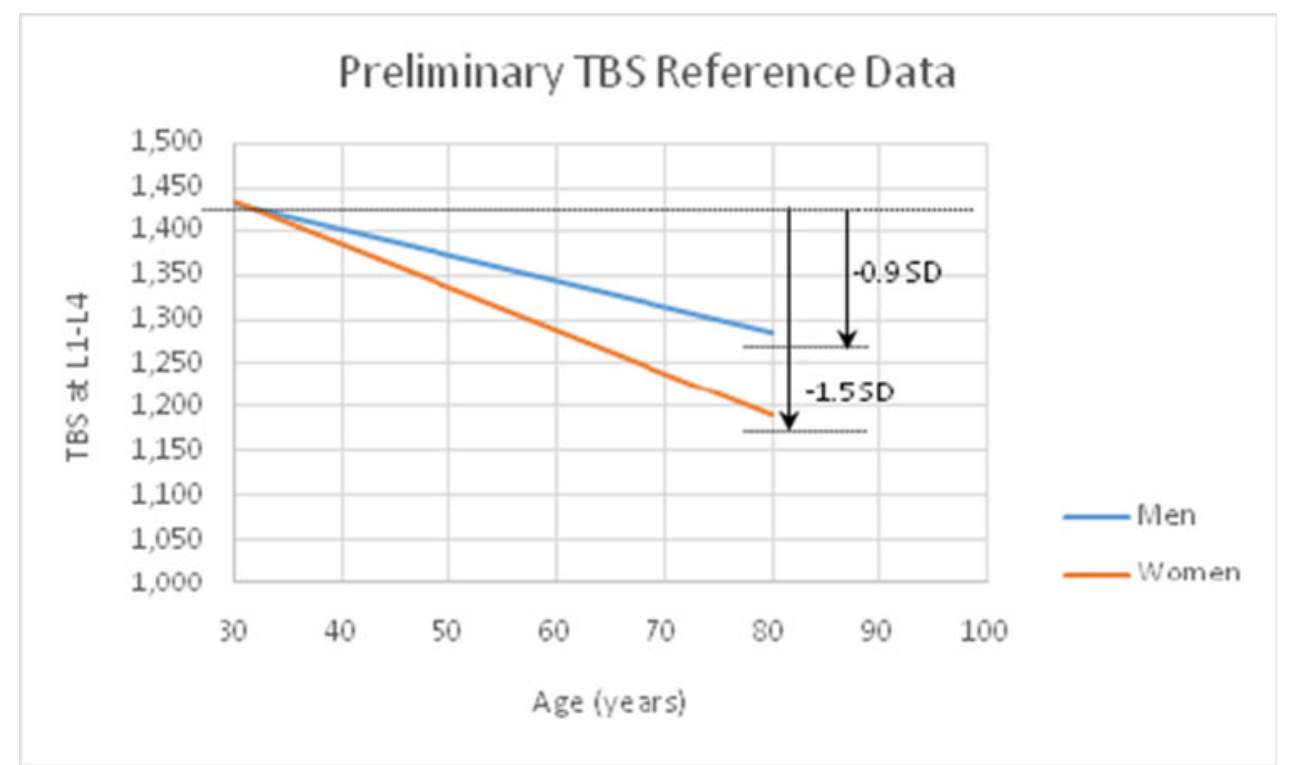

Figure 1. TBS decrease related to age in Moroccans men and women.

Conclusion: Results were consistent with previous published data for European men and women. A TBS decrease with aging was observed in both men and women. As expected, the decrease was greater for women than for men. These data are the first step to define Moroccan reference data.

\section{P681}

DIFFERENCES BETWEEN CLINICAL AND DENSITOMETRIC RISK FACTORS IN PATIENTS WITH AND WITHOUT FRAGILITY FRACTURES IN A BONE METABOLISM UNIT (BMU)

$\underline{\text { S. M. Rojas }}^{1}$, P. J. Cossío-Jimenez ${ }^{1}$, T. L. Rodríguez-Araya ${ }^{1}$, J. J. Aznar-Sánchez ${ }^{1}$, R. Veroz-Jimenez ${ }^{1}$, E. ChamizoCarmona $^{1}$

${ }^{1}$ Reumatología, Hospital de Mérida, Mérida, Spain
Introduction: Identification of fracture risk factors, BMD and FRAX application have helped to decrease the variability of the diagnosis and treatment in patients with osteoporosis (OP). More investigation is needed to identify the differences between patients that are susceptible to fractures.

Aim: Identify the differences in BMD and fracture risk factors (FRAX) between patients with and without fragility fractures. Patients and methods: Patients attended a high resolution consultation of OP in the BMU of the Rheumatology Unit in the Hospital of Mérida, from July 2013 to June 2014, collecting information about fracture risk factors, lumbar BMD (L2-L4) and hip BMD, radiology of dorsolumbar spine and vitamin D. FRAX with and without BMD was calculated in all patients.

Results: We studied 355 patients who had all the information needed. 81 patients presented clinical or morphometric fractures, with a mean age of $69.28 \pm 2,88$ years, slightly higher than the 274 patients without fractures $(62.61 \pm 1.31)$. The 
differences in the rates of densitometric OP between fractured and non-fractured patients were $23.4 \%$ and $21.1 \%$ in lumbar spine and $18.5 \%$ and $10.9 \%$ in hip with an OR $1.85(0.94$ $3.64)$ and $\mathrm{Ji}^{2} 3.24(p=0.07)$. The rate of fractured patients with Major osteoporotic fracture $>10 \%$ and hip fracture $>3 \%$ in FRAX were $12.34 \%$ and $22.22 \%$. When BMD is added to the calculation the rates increased to $16.04 \%$ and $23.45 \%$, respectively. In non fractured patients, the rate of major fracture of $20.43 \%$ and hip fracture of $25.18 \%$ decreased when BMDwas added to the calculation of FRAX to $14.96 \%$ and $21.89 \%$, respectively. The rate of patients with levels of vitamin $\mathrm{D}<20 \mathrm{ng} / \mathrm{ml}$ was $19.75 \%$ in fractured patients and $16.05 \%$ in non fractured patients.

Conclusions: The Mean age in fractured patients was higher than in non fractured patients, in accordance to other studies in the literature that age is an important risk factor for fractures.

\section{P682}

EPIDEMIOLOGICAL FEATURES OF PERIPHERAL FRAGILITY FRACTURES IN REPUBLIC OF MOLDOVA: REGIONAL RESULTS FROM THE MULTICENTRIC STUDY EVA

V. Cazac ${ }^{1}$, L. Groppa ${ }^{1}$, I. Radu ${ }^{1}$, A. Tigulea ${ }^{1}$, E. Deseatnicova $^{1}$, C. Toia ${ }^{1}$, E. Chiriacova ${ }^{1}$

${ }^{1}$ Laboratory of Rheumatology, Chisinau, Republic of Moldova

Objectives: To determine the incidence of peripheral fragility fractures in two regions of Republic of Moldova

Material and methods: The study was conducted starting with January 2013 until June 2014. The overall population of 2 regions was studied $-5.86 \%$ of the total population of the Republic. In the first (retrospective) part, cases with fragility fractures of the proximal humerus, distal forearm, proximal femur and distal third of the calf bones were collected. In the second (prospective) part, only the fragility fractures of the proximal third of the femur were collected, including femoral neck fractures and trochanteric fractures. Only the fractures in patients $>40$ years old were collected. The data gathering was performed using medical documentation of primary healthcare institutions, as well as from specialized healthcare institutions, hospital departments. When calculating epidemiological indexes, data offered by the National Bureau of Statistics of Republic of Moldova was used. The population data was updated for each year of study.

Results: In the retrospective part, 1517 cases (1000 women and 517 men) were collected, of which 197 of proximal humerus, 494 of distal forearm, 340 of proximal femur and 486 of distal third of the calf bones. In the prospective part, 249 cases (155 women and 94 men) of hip fractures were collected, during the years 2013 and the first half of 2014. The average incidence was 881.3 (2011) and 854.2 (2012) per 100000 population older than 40 years, varying from 281.6 up to 1884.5 , based on age group. An increased incidence during the cold months and months of intense agricultural work was registered. Additionally, a greater prevalence of forearm and calf bones fractures was noted in age groups $<70$ years old, whereas hip fractures were prevalent in older age groups.

Conclusion: The incidence of peripheral fragility fractures depends on age, sex and period of year.

Acknowledgements: We thank Prof. John A. Kanis for offered attention and guidance.

\section{P683}

THE ORTHOGERIATRIC MANAGEMENT: AN INSEPARABLE DUO TO FOSTER APPROPRIATE CARE

M. Baroni ${ }^{1}$, G. Pioli ${ }^{2}$, V. Boccardi ${ }^{1}$, V. Prenni ${ }^{1}$, E. Zengarini ${ }^{1}$, M. Conestabile della Staffa $^{1}$, P. Mecocci ${ }^{1}$, C. Ruggiero ${ }^{1}$

${ }^{1}$ Section of Geriatrics, Department of Medicine, University of Perugia, Perugia, Italy, ${ }^{2}$ Neuromotor Diseases Department, ASMN-IRCCS, Reggio Emilia, Italy

Objectives: Compare the effectiveness of giving the patients with acute hip fracture comprehensive geriatric care in a dedicated orthogeriatric ward vs. the usual orthopaedic care.

Material and Methods: patients with hip fractures aged 65 years or older, who were able to walk outdoors before their fracture, will be randomly assigned to the orthogeriatric unit or orthopaedic unit of our hospital, to achieve the required sample of 150 patients. Randomisation will be achieved via a webbased, computer generated, block method with unknown block size. The primary aim of the study is to estimate the effect of orthogeriatric comanagement on the prescription of appropriate antifracture therapy at hospital discharge. The secondary aim of the study is to evaluate adherence to treatments, mobility and functional independence at 6 and 12 months from surgery.

Results: We will expect the orthogeriatric comanagement of hip fracture patients to improve the appropriate prescription of antifracture therapy, the therapeutic adherence to treatments, thus a lower risk of falls and fragility refracture.

Conclusions: The treatment of older patients with hip fractures organized as orthogeriatric care may improve the appropriateness of anti-fracture drug prescription, with improved adherence.

\section{P684}

EFFECT OF MONTHLY INTRAVENOUS IBANDRONATE INJECTIONS ON BONE MINERAL DENSITY IN JAPANESE OSTEOPOROSIS PATIENTS

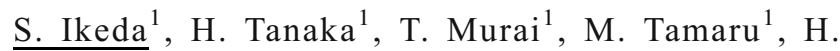
Matsumoto $^{2}$, N. Okimoto ${ }^{3}$

${ }^{1}$ Ken-Ai Memorial Hospital, Onga, Japan, ${ }^{2}$ Sanzai Hospital, Miyazaki, Japan, ${ }^{3}$ Okimoto Clinic, Kure, Japan 
Objectives: Ibandronate was marketed for the first time as monthly intravenous agent injections in Japan in August, 2013. Monthly intravenous ibandronate injections $(1 \mathrm{mg} /$ month) lead to the increase of BMD. However, there are few reports about the effect of monthly intravenous ibandronate injections in increase of BMD between nontreated patients and switch patients from other agents. The purposes of this study were to investigate the effects of increase of BMD the effect of monthly intravenous ibandronate injections in increase of BMD between nontreated patients and switch patients from other agents in Japanese osteoporosis patients.

Material and Methods: Study patients were allocated to either the new treatment (NT) group (non- treated patient at starting this study: $n=37$ ) or the Switch (SW) group (previously treated patient with other agents at starting this study: $n=23$ ). We measured the BMD values of lumbar spine (L2-4), femoral neck, and total hip, at baseline, 6, 12, and 18 months after the start of this study. We evaluated the $\%$ change of $\mathrm{BMD}$ and the number of increased BMD patients at each time points between two groups.

Results: Patients' mean age was 74.3 years in NT group and 77.0 years in SW group. The\% change of BMD of lumbar spine in NT group was significantly increased $6 \mathrm{M}$ later in comparison with baseline, and was significantly increased $12 \mathrm{M}$ later in comparison with SW group. The $\%$ change of BMD of femoral neck in NT group was significantly increased $12 \mathrm{M}$ later in comparison with baseline. The $\%$ change of BMD of total hip in NT group was significantly increased $6 \mathrm{M}$ later in comparison with baseline. The number of increased BMD patients of lumbar spine at each time points, in NT group was $83.8,88.0$, and $84.6 \%$, and in SW group was $65.2,64.7$, and $58 \%$. The number of increased BMD patients of femoral neck at each time points, in NT group was $50.0,76.0$, and $76.9 \%$, and in SW group was 66.7 , 50.0 , and $50.0 \%$. The number of increased BMD patients of total hip at each time points, in NT group was 68.6, 79.2, and $76.9 \%$, and in SW group was 50.0, 50.0, and $66.7 \%$.

Conclusions: In the effect of monthly intravenous ibandronate injections in increase of BMD values, new treatment patients were superior to switch patients from other agents.

\section{P685}

POSITIVE EFFECT OF PARENTERAL IBANDRONATE IN POSTMENOPAUSAL OSTEOPOROSIS $\mathrm{S}_{\text {. Mulic }}{ }^{1}$, A. Hajdarovic ${ }^{2}$, D. Antic ${ }^{3}$, E. Tulumovic ${ }^{4}$

${ }^{1}$ University Clinical Center Tuzla, Rheumatology Department, Tuzla, Bosnia and Herzegovina, ${ }^{2}$ University Clinial Center Tuzla, Tuzla, Bosnia and Herzegovina, ${ }^{3}$ UCC Tuzla, Rheumatology Department, Tuzla, Bosnia and Herzegovina, ${ }^{4}$ Rheumatology Department, Tuzla, Bosnia and Herzegovina
Introduction: Osteoporosis is caused by the cumulative effect of bone resorption in excess of bone formation. Bisphosphonates inhibit bone resorption with relatively few side effects. As a result, they are widely used for the prevention and treatment of osteoporosis. Ibandronate in oral or parenteral form is approved for the treatment and prevention of postmenopausal osteoporosis.

Objective: To determine the relationship between BMI, its values, T-score values and duration of menopause in postmenopausal patients before and after two years treatment with parenteral ibandronate.

Methods: 25 female patients with diagnosed postmenopausal osteoporosis were treated with parenteral ibandronate $(3 \mathrm{mg}$ i.v. every three months) for at least two years and monitored in our hospital.

Results: The average age of our patients was 61.13 years with average duration of menopause 15.24 years. The average BMI was $26.7 \pm 3.2 \mathrm{~kg} / \mathrm{m}^{2}$ before the therapy. After the two years therapy the increase of BMI values was recorded both on spine for $8.7 \%$ and hip $4 \%$, as well as T-score value for spine and hip 0.6 and 0.4 , respectively. Better results were noticed in patient with longer menopausal status ( $>10$ years) and with no history of fractures.

Conclusion: Application of parenteral ibandronate led to increase of BMI and T-score both on spine and hip in postmenopausal patient.

\section{P686}

ASSOCIATION OF LOW VITAMIN D3 LEVEL IN OSTEOPENIC AND OSTEOPOROTIC PATIENTS IN A TERTIARY LEVEL HOSPITAL OF BANGLADESH $\underline{\text { S. Anwaruzzaman }}^{1}$, M. R. Bhuiyan ${ }^{1}$, N. Sakib ${ }^{2}$, M. I. Majumder $^{3}$

${ }^{1}$ Department of Orthopaedic Surgery, Comilla Medical College Hospital, Comilla, Bangladesh, ${ }^{2}$ BRAC University, Dhaka, Bangladesh, ${ }^{3}$ Comilla Medical College, Comilla, Bangladesh.

Osteoporosis is consequence of ageing process mostly affecting postmenopausal women and also an important noncommunicable disease. The osteoporosis accounts for higher fracture risk, mortality and morbidity. Vitamin D metabolism and deficiency is closely related with osteoporosis pathophysiology. Vitamin D insufficiency or deficiency is associated with secondary hyperparathyroidism causing increased bone turn over and bone loss which increase fracture risk. Now a new term osteoporomalacia or poromalacia is meant for the condition where osteoporosis and osteomalacia both are present simultaneously. Aim of this study was to ascertain the association of osteopenia and osteoporosis with low vitamin D3 level deficiency $(<10 \mathrm{ng} / \mathrm{ml})$, Insufficiency $(10-30 \mathrm{ng} / \mathrm{ml})$ and 
sufficiency $(30-100 \mathrm{ng} / \mathrm{ml})$ in outdoor and indoor patients of Comilla Medical College Hospital. 456 osteopenic and osteoporotic patients included in the study. They were evaluated for BMD with DXA by using Lunar Prodigy Advance DXA System (Analysis version:13.60) by GE healthcare USA. Vitamin D3 estimation with enzyme linked fluorescence assay (EFLA) using Biomerieux, Mini Vidas (France). Data analysis was performed using statistical program Stata Version 12.0 (StataCorp, College Station, Texas). Total sample size was 469. Mean age of the patients was 60.81 years with SD of 11.21 years. Among the participants, 456 were females $(97 \%)$ and 13 were males (3\%). Mean BMD was -2.78 with SD 1.02. Minimum was -7.3 and maximum -1. Mean level of D3 was $23.31 \mathrm{ng}$ with SD $11.33 \mathrm{ng}$. Minimum was 2.5 and maximum 98. Analysis on BMD over D3 level shows that vitamin D3 level tends to be between 15-30 ng when the BMD is between -1 to -4 in the study. The study showed lower vitamin D3 level, mostly insufficiency in osteoporotic and osteopenic patients.

\section{P687}

ATYPICAL FEMORAL FRACTURES ASSOCIATED WITH SHORT-TERM EXPOSURE TO DENOSUMAB M. Chandran ${ }^{1}$, D. Tay ${ }^{1}$

${ }^{1}$ Osteoporosis and Bone Metabolism Unit, Singapore General Hospital, Singapore, Singapore

Introduction: Two patients who had atypical femur fractures after therapy with the RANKL inhibitor denosumab (DMB) for less than a year and a half are presented.

Method: Case Study

Case report: Patient 1 was a 72 year old Chinese female with a history of Myasthenia Gravis for which she was on long-term intermittent prednisolone therapy. She presented with lower extremity pain after a fall from standing height. X-ray showed right short oblique femoral fracture with thickened lateral cortex and periosteal breaking with no contralateral involvement. Osteoporosis had been diagnosed 15 years ago. Though she had taken alendronate for her osteoporosis, she had stopped therapy 8 years prior to the current presentation. She had started DMB injections 1.5 years ago. She had been given 3 doses of DMB 6 months apart before she developed the AFF 3 months after the last dose. Patient 2 was a 61 year old Indian female who presented with pain in her lower extremities after a fall from standing height. X-rays showed thickening of both femoral cortices with suspicious lucency noted in the left femur. Subsequent MRI of both hips documented bilateral insufficiency fractures in the lateral cortices at the midshaft of both left and right femora and diffuse thickening of the left femur lateral cortex. She had received 2 doses of DMB the last dose of which was 4 months prior to the fracture. Prior to DMB initiation, she had been on Strontium Ranelate for 6 months. Before Strontium usage, she had taken 2 years of Alendronate and prior to that she had been on 4 years of the selective estrogen receptor modulator, raloxifene.

Conclusion: Theoretically DMB should not cause persistent suppression of bone turnover unless there is long-term cumulative exposure. The occurrence of AFF in our 2 patients with very short and low cumulative exposure to DMB suggests that a different pathophysiological mechanism may at least partly be responsible for the occurrence of these rare fractures in the setting of DMB use. Whether it is due to growth of microcracks due to disruption of targeted remodeling by DMB can be speculated upon

\section{P688}

CORTICAL AND TRABECULAR BONE ANALYSIS OF PROFESSIONAL DANCERS USING 3D-DXA: A CASE CONTROL STUDY

T. A. Amorim ${ }^{1}$, L. H. Humbert ${ }^{2}$, R. F. Fonolla ${ }^{2}$, A. D. F. Flouris $^{3}$, G. D. M. Metsios ${ }^{4}$, J. M. Maia ${ }^{1}$, M. W. Wyon ${ }^{4}$, Y. K. Koutedakis ${ }^{3}$

${ }^{1}$ University of Porto, Porto, Portugal, ${ }^{2}$ Musculoskeletal Unit, Galgo Medical S.L., Barcelona, Spain, ${ }^{3}$ University of Thessaly, Trikala, Greece, ${ }^{4}$ University of Wolverhampton, Walsall, United Kingdom

Objective: To assess bone health parameters in professional dancers and control group using 3D-DXA.

Material and Methods: The total of 41 professional dancers (29 women, 12 men) together with 41 (29 women, 12 men) age- and height-matched controls volunteered. DXA scans were conducted for all participants using a Lunar DPX scanner (GE Healthcare, Madison, WI). The 3D-DXA software (Galgo Medical, Barcelona, Spain) was used to analyse the cortical and trabecular bone in both groups. This software (3D-DXA) registers a 3D appearance model of the femoral shape and density onto the DXA projection to obtain a $3 \mathrm{D}$ subject-specific model of the femur of the patient and quantify the volumetric BMD (vBMD), volume (for trabecular and cortical regions) and cortical thickness distribution.

Results: Student's t-test revealed no statistically significant differences between dancers and controls in terms of age (36.2 \pm 9.0 vs. $38.0 \pm 9.7$ years, $p=0.38)$ and height (167.6 \pm 7.7 vs. $165.0 \pm 9.3 \mathrm{~cm}, p=0.18$ ). However, the mean weight of dancers was found to be significantly lower than that of controls $(55.7 \pm 10.0$ vs. $62.9 \pm 10.8 \mathrm{~kg}, p=0.002)$. Furthermore, vBMD of dancers was on average higher compared to controls: $+8 \%\left(+29 \mathrm{mg} / \mathrm{cm}^{3}\right)$ for integral bone $(p=0.04),+14 \%$ for trabecular bone $(p=0.02)$, and $+1 \%$ 
for cortical bone $(p=0.04)$. Similarly, the femoral cortex of dancers was thicker: $+0.1 \mathrm{~mm}(+8 \%, p=0.01)$. No statistically significant difference was found for the volume of the whole proximal femur $(p=022)$ and trabecular compartment $(p=0.34)$, but the volume of the cortex was found to be significantly higher $\left(+1.3 \mathrm{~cm}^{3}\right.$ or $\left.+10 \%\right)$ in dancers.

Conclusion: Professional dancers were found to have a thicker cortex and a higher vBMD for both trabecular and cortical compartments compared to matched controls.

\section{P689}

CHANGES IN BONE MINERAL DENSITY IN PATIENTS WITH TYPE 2 DIABETES TREATED WITH LOBEGLITAZONE, A NOVEL THIAZOLIDINEDIONE, OVER 52 WEEKS: A MULTICENTER, RANDOMIZED, DOUBLE-BLIND, PLACEBO CONTROLLED TRIAL

K. M. Kim ${ }^{1}$, S. Lim ${ }^{1}$, H. C. Jang ${ }^{1}$

${ }^{1}$ Department of Internal Medicine, Seoul National University College of Medicine and Seoul National University Bundang Hospital, Seongnam, Republic of Korea

Thiazolidinediones (TZDs), a class of anti-diabetic agents, promote insulin sensitivity through activation of peroxisome proliferator activated receptor gamma. However, long term usage of TZDs is known to increase risk of fracture with inhibitory effects on osteoblasts differentiation while promoting adipogenesis, which acts a limitation of TZD usages. The aim of this study was to examine the effects of lobeglitazone, a novel TZD, on changes in BMD over 52-weeks of treatment compared to placebo in Korean patients with type 2 diabetes. In this multicenter, randomized, double-blind, placebo controlled study, 170 patients aged 34-76 years were randomly assigned with $2: 1$ ratio to lobeglitazone $0.5 \mathrm{mg}(n=112)$ or matching placebo $(n=58)$ orally once daily. BMD was assessed using DXA in each center at week 26 and 52. Significant bone loss was defined as a percentage changes in BMD of greater than $-3.5 \%$. The primary end point was the changes of BMD in femur neck and total hip areas from baseline to the end of the treatment. The secondary endpoint was percentage of subjects who showed significant bone losses at these skeletal sites. Lobeglitazone treatment did not show any significant decreases of BMD in femur neck or total hip over 52 weeks compared to the placebo group. Moreover, percentages of subjects who provided significant bone loss did not differ between two groups at both skeletal sites. In conclusion, treatment of lobeglitazone $0.5 \mathrm{mg}$ showed a neutral effect on changes in BMD over 52 weeks at femur neck and total hip without any serious side effects.

\section{P690}

IMPACT OF FRACTURE RISK ASSESSMENT TOOLS ON PROPORTIONS OF WOMEN WITHOUT PRIOR FRACTURE IDENTIFIED AT RISK AND TREATED - LESSONS FROM THE SIGN GUIDANCE E. McCloskey ${ }^{1}$, J. E. Compston ${ }^{2}$, C. Cooper ${ }^{3}$, N. C. Harvey ${ }^{3}$, H. Johansson $^{1}$, A. Oden ${ }^{1}$, J. A. Kanis ${ }^{1}$

${ }^{1}$ Centre for Metabolic Bone Diseases, University of Sheffield Medical School, Sheffield, United Kingdom, ${ }^{2}$ Department of Medicine, University of Cambridge School of Clinical Medicine, Cambridge, United Kingdom, ${ }^{3} \mathrm{MRC}$ Lifecourse Epidemiology Unit, University of Southampton, Southampton, United Kingdom

Recent guidance from the Scottish Intercollegiate Guideline Network (SIGN) proposes that women aged 50 years and older with a $\geq 10 \%$ risk of fracture, in the absence of a prior vertebral or hip fracture, should have treatment if a subsequent BMD scan shows T-score osteoporosis. We have compared the impact of the use of FRAX or QFracture on the proportion of women, without a prior fracture, that would be identified as needing a BMD scan and subsequent therapy. The number of women in Scotland with a fracture risk of $10 \%$ or more were estimated using the distributions of QFracture incidence and FRAX probabilities in an analysis of the THIN database[1] adjusted for the age demography of women in Scotland. In a total population of nearly 1 million women, 275,600 (28\%) women without prior fracture would be identified as having a 10-year FRAX probability of $10 \%$ or more (Table); the proportion increased from $2 \%$ at ages $50-59$ years to $58 \%$ at ages $80-89$ years. In contrast, only $2.7 \%$ of women would be identified by QFracture (ranging from $0 \%$ at younger ages to $16 \%$ at 90-99 years). The number of women subsequently eligible for treatment would be 81,700 (8.2\%) with FRAX, rising from $0.2 \%$ of the population at 50-59 years to $32 \%$ at $90-99 \mathrm{y}$; the proportions were much smaller with QFracture with only $12,300(1.2 \%)$ identified for treatment, ranging from $0 \%$ of the population at 50-59 years to $10 \%$ at $90-99 \mathrm{y}$. We conclude that the use of the QFracture algorithm results in a marked reduction in the treated population, reflecting well-demonstrated deficiencies in the calibration of QFracture for fractures other than hip fracture. The use of QFracture will result in little or no impact on the overall incidence of fractures in women without a prior fracture compared to the use of FRAX.

Hippisley-Cox $\mathrm{J}$ and Coupland C. Validation of QFracture compared with FRAX. Analysis prepared for NICE 2011. http://www.qfracture.org/Validation-ofQFracture-vs-FRAX-for-NICE-2011.pdf accessed 15 May 2015, 2011. 
Number of women in Scotland having FRAX probablility (major osteoporotic fracture) and QFracture incidence for major osteoporotic fracture (osteoporotic fracture) above the limit of $10 \%$ and having osteoporosis (BMD FN $\leq-2.5 \mathrm{SD}$ )

\begin{tabular}{|c|c|c|c|c|c|}
\hline \multirow[t]{2}{*}{$\begin{array}{l}\text { Age } \\
\text { interval } \\
\text { (years) }\end{array}$} & \multirow[t]{2}{*}{$\begin{array}{l}\text { Estimated } \\
\text { female } \\
\text { population }\end{array}$} & \multicolumn{2}{|c|}{$\begin{array}{l}\text { Number of women } \\
\text { above } 10 \% \\
\text { threshold with no } \\
\text { previous fracture }\end{array}$} & \multicolumn{2}{|c|}{$\begin{array}{l}\text { Number of women } \\
\text { above } 10 \% \\
\text { threshold with } \\
\text { no previous } \\
\text { fracture and } \\
\text { osteoporosis }\end{array}$} \\
\hline & & FRAX & QFracture & FRAX & QFractur \\
\hline $50-59$ & 343,800 & 6,900 & 0 & 800 & 0 \\
\hline $60-69$ & 290,600 & 60,700 & 600 & 10,000 & 100 \\
\hline $70-79$ & 207,000 & 119,200 & 5,600 & 31,400 & 2,000 \\
\hline $80-89$ & 119,800 & 71,500 & 15,300 & 29,400 & 7,100 \\
\hline \multirow[t]{2}{*}{ 90-99 } & 31,300 & 17,300 & 5,000 & 10,100 & 3,100 \\
\hline & 992,500 & 275,600 & 26,500 & 81,700 & 12,300 \\
\hline
\end{tabular}

P691

THE EFFECT OF HORMONAL CONTRACEPTION AND PHYSICAL ACTIVITY ON BODY COMPOSITION AMONG HEALTHY FINNISH YOUNG FEMALES

J. Sirola $^{1}$, J. Suuronen ${ }^{1}$, S. Sjöblom ${ }^{1}$, M. Tuppurainen ${ }^{1}$, R. Honkanen $^{1}$, T. Rikkonen ${ }^{1}$, H. Kröger ${ }^{1}$

${ }^{1}$ Kuopio Musculoskeletal Research Unit, University of Eastern Finland, Kuopio, Finland

Purpose: To determine if hormonal contraception or physical activity exert an effect on body composition.

Methods: 400 Finnish women (aged 20-40) were measured with DXA, hand-held dynamometer and knee extension bench between 2011-2014. Inclusion criteria for the study were: no chronic diseases or permanent medication. Lifestyle factors were assessed with inquiries parallel with the measurements and included: alcohol consumption, pregnancies, hormonal contraception, smoking, self-rated health and physical activity. Body composition variables (lean and fat mass) were measured with DXA. Effects of hormonal contraception and physical activity on body composition and muscle strength were investigated with AN(C)OVA.

Results: Women who used combination of progestogen + estrogen had lower appendicular skeletal mass (ASM), relative skeletal muscle index (RSMI), total lean mass (TLM) $(p<0.01)$ and grip strength $(\mathrm{GS})(p<0.001)$. After adjustment ASM, skeletal muscle index (SMI), GS $(p<0.05)$, RSMI and TLM $(p<0.01)$ prevailed significantly lower and fat- $\%$ higher $(p<0.05)$ compared to women not using hormonal contraception. In univariate model, women who used a low dose $(15 \mu \mathrm{g} \leq$ estrogen $\geq 20 \mu \mathrm{g})$ had lower ASM, RSMI, TLM $(p<0.01)$, BMI $(p<0.05)$ and GS $(p<0.001)$ compared to women who did not use estrogen. Women who used a standard dose $(25 \mu \mathrm{g} \leq$ estrogen $\geq 35 \mu \mathrm{g})$ had also lower ASM, RSMI, GS, quadriceps strength (QS) $(p<0.01)$ and TLM $(p<0.05)$ in comparison "No use"-group. There was no difference in SMI and Fat- $\%$ between groups according to use of estrogen in ANOVA. After adjustment for multiple covariates, users of low dose estrogen had lower ASM, RSMI, SMI, GS, TLM and higher fat- $\%(p<0.05)$ compared to women not using estrogen. Standard dose users had lower RSMI $(p<0.05)$ and QS $(p<0.01)$ in comparison. Women who exercised 2-3 times per week had higher ASM, RSMI $(p<0.01)$, SMI, GS, QS $(p<0.05)$ and TLM $(p<0.001)$ and lower fat- $\%(p<0.05)$ compared to physically least active group. Adjustment for multiple covariates did not diminish the differences and higher ASM, RSMI, TLM $(p<0.01)$, SMI, GS, QS $(p<0.05)$ and lower fat- $\%(p<0.05)$ remained significant after adjustments. Four to six times per week exercising women had higher ASM, RSMI, SMI, QS, TLM $(p<0.001)$ and GS $(p<0.01)$. Furthermore, they were less obese (fat-\% $p<0.001$ ). Adjustment did not alter the differences. Women exercising daily had significantly higher ASM, RSMI, SMI, TLM $(p<0.001)$, QS $(p<0.01)$ and lower fat- $\%(p<0.001)$. After adjustments, QS had higher statistical significance $(p<0.001)$; other variables remained as significant as in univariate model. Conclusions: Use of estrogen has significantly negative effect on muscle mass and strength. Exercise increases muscle mass and strength and lowers fat mass significantly.

\section{P692}

\section{CORRELATIONS BETWEEN FUNCTIONAL STATUS AND QUALITY OF LIFE IN PATIENTS WITH HIP OSTEOARTHRITIS}

I.-R. Marcu $^{1}$, S. Patru ${ }^{1}$, A. C. Bighea ${ }^{1}$

${ }^{1}$ University of Medicine and Pharmacy, Craiova, Romania

Studies concerning the quality of life in patients with hip osteoarthritis proved the impact of pain on daily activities and on their social and professional life. The purpose of this study was to evaluate the efficacy of complex rehabilitation treatment and its influence on functional status and quality of life for patients with hip osteoarthritis. This prospective, controlled, observational, randomized study included 141 patients with hip osteoarthritis, $54 \%$ women, mean age $61,52 \pm 11,63$ years, randomized function the treatment in three lots: a control lot with medication (57 patients), a control lot with kinetotherapy (42 patients) and the studied lot with complex physical and kinetic treatment (42 patients). The patients were evaluated clinical and 
functional at the beginning of the study and after 3, 6 and 12 months. The efficacy of complex physical and kinetic treatment was emphasized by the statistic significant improvement $(p<0.05)$ of mean values obtained for the indicators of functional status and quality of life, Liquesce functional index and SF-36 score (Short Form Health Survey) and also for articular mobility and muscular strength. There was a moderate correlation (0.7) between the functional improvement (Lequesne functional index) and the quality of life increase (SF-36). The improvement of the studied clinical and functional parameters obtained for the patients with hip osteoarthritis that followed a complex physical and kinetic rehabilitation program had a significant impact on their quality of life.

\section{P693}

GENDER-SPECIFIC ASSOCIATIONS BETWEEN MUSCLE AND BONE LOSSES IN LONGITUDINAL CHANGES OF MUSCLE MASS AND STRENGTH, AND BONE MASS

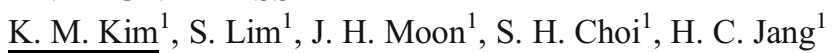

${ }^{1}$ Department of Internal Medicine, Seoul National University

College of Medicine and Seoul National University Bundang

Hospital, Seongnam, Republic of Korea

Decreases in muscle and bone mass are representative features of body compositional changes with aging, and crosstalk between these two tissues has been suggested. The aims of this study were to investigate the longitudinal changes in muscle and bone with aging in an elderly population, and to clarify the muscle-bone relationships. We conducted a community-based prospective study as a part of the Korean Longitudinal Study on Health and Aging. A total of 172 men and 165 women aged $\geq 65$ years (mean, 70.9 years) were included who had participated in body composition analysis at baseline and 5year follow-up evaluation. Body composition parameters and BMD were assessed by DXA. Appendicular skeletal muscle mass (ASM) was defined as the sum of lean mass of the arms and legs. In the associations between muscle and bone loss, the rate of BMD loss in total hip was significantly associated with baseline legs muscle mass and ASM, but not with the rate of these muscle losses in men ( $r=0.205$ for baseline leg muscle mass and $r=0.195$ for baseline ASM, $p<0.05$ respective). However, in women, it was significantly correlated with the rate of muscle loss, but not with baseline values ( $r=0.196$ for percentage changes in ASM and $r=0.169$ for percentage changes in leg muscle mass, $p<0.05$ respectively). On the other hand, the rates of decrease in leg muscle strength showed positive associations with loss in total hip BMD in both men and women $(r=0.170$ for men and $r=0.246$ for women, $p<0.05$ respectively). In conclusion, the muscle- bone interactions might differ according to gender in a longitudinal aspect.

\section{P694}

\section{LOW PEAK BONE MASS IN BOTH GENDERS BORN WITH VERY LOW BIRTH WEIGHT: A COHORT STUDY}

C. Balasuriya $^{1}$, M. P. Mosti ${ }^{2}$, K. A. I. Evensen ${ }^{3}$, A. M. Brubakk $^{4}$, M. S. Indredavik ${ }^{5}$, A. K. Stunes ${ }^{2}$, U. Syversen ${ }^{2}$

${ }^{1}$ Department of Endocrinology, St.Olav's University Hospital, Trondheim, Norway, ${ }^{2}$ Department of Cancer Research and Molecular Medicine, Norwegian University of Science and Technology, Trondheim, Norway, ${ }^{3}$ Department of Laboratory Medicine, Children's and Women's Health, St.Olav's University Hospital, Trondheim, Norway, ${ }^{4}$ Department of Laboratory Medicine, Children's and Women's Health, St.Olavs University Hospital, Trondheim, Norway, ${ }^{5}$ Child and Adolescent Psychiatry, St. Olav's University Hospital, Trondheim, Norway

Growing evidence suggests that low birth weight may lead to subnormal peak bone mass, which implies an increased risk for osteoporosis later in life. The last trimester of pregnancy is crucial for skeletal development.

Objectives: We wanted to evaluate the influence of low birth weight on BMD and bone mineral content (BMC) in young adults born preterm with very low birth weight (VLBW) and small for gestational age (SGA) at term.

Material and methods: Altogether 186 subjects (females 95, males 91) 26-28 years of age were included. Of these, 52 were born preterm with VLBW $(<1500 \mathrm{~g})$, $56 \mathrm{SGA}$ born at term $(<10$ th percentile) and 75 controls born at term with normal birth weight $(>10$ th percentile). Height and weight were recorded, and a questionnaire addressing previous fractures, smoking, physical activity, calcium and vitamin D intake were filled in. BMC and BMD at spine, hip and whole body and trabecular bone score (TBS) were measured by DXA. P1NP, CTX, sclerostin and dkk1 were analyzed in serum.

Results: The VLBW and SGA groups were significantly shorter. The VLBW group was more physically inactive and reported higher calcium intake. No differences were observed in previous fractures, smoking and vitamin D intake. The VLBW group exhibited significantly lower BMC and BMD at most sites measured. Femoral neck BMD was $6.7 \%$ lower in VLBW. The significant differences in femoral neck persisted, after adjustment for adult body weight, height, physical activity and calcium intake. The SGA group displayed lower BMC at spine and lower whole body Z-score. 
No significant differences were observed in TBS or bone markers, except for significantly higher levels of $\mathrm{dkk} 1$ in the VLBW group compared to controls.

Conclusion: Young adults born preterm with VLBW studied close to the age of peak bone mass displayed significantly lower BMC and BMD than term-born controls. Those born SGA at term also exhibited lower BMC. This may imply increase future fracture risk.

\section{P695}

INCREASED GENERAL BONE LOSS AND CARDIOVASCULAR RISK FACTORS IN PATIENTS WITH EROSIVE HAND OSTEOARTHRITIS

O. Ruzickova $^{1}$, O. Sleglova ${ }^{1}$, L. Senolt ${ }^{1}$, K. Pavelka ${ }^{1}$

${ }^{1}$ Institute of Research in Rheumatology, Prague, Czech Republic

Background: Hand osteoarthritis (OA) is a progressive and heterogeneous group of rheumatic disorders with its more severe erosive subset, which is associated with more pain and disability. Several studies investigated differences in $\mathrm{BMD}$ and bone mineral content between erosive and nonerosive hand $\mathrm{OA}^{1}$. Furthermore, symptomatic hand $\mathrm{OA}$ has been recently demonstrated to associate with an increased risk of coronary heart disease events ${ }^{2}$.

Objectives: To compare BMD and metabolic cardiovascular risk factors between patients with erosive and non-erosive hand OA in a cross-sectional study.

Methods: Patients with symptomatic disease fulfilling the American College of Rheumatology (ACR) criteria for hand $\mathrm{OA}$ were included in this study. Erosive hand OA was defined by at least one erosive interphalangeal joint. All patients underwent clinical assessments of joint swelling and radiographs of both hands. DXA examination of lumbar spine, total femur and femur neck was performed. Metabolic risk factors (BMI, hypertension, diabetes and dyslipidaemia) and smoking habits were collected.

Results: Altogether, 134 patients (13 male) with symptomatic nodal hand OA were included in this study between April 2012 and January 2015. Mean (SD) age of all patients was 66.0 (8.2) years and disease duration was 8.54 (7.14) years. Out of these patients, 72 had erosive hand OA. Baseline population characteristics did not differ between both groups. Osteoporosis (T-score $<-2.5$ SD) was diagnosed in $12.5 \%(9 / 72)$ of patients with erosive hand $\mathrm{OA}$ and in $8.06 \%(5 / 62)$ of patients with non-erosive hand $\mathrm{OA}$. Although BMD did not differ between the groups, T-scores of lumbar spine $(-0.46$ vs. $-1.04 \mathrm{SD}$, $p<0.001)$, total femur $(-0.36$ vs. $-1.20 \mathrm{SD}, p<0.001)$ and femur neck (-0.92 vs. $-1.20 \mathrm{SD}, p<0.01)$ were significantly lower in patients with erosive compared with non-erosive disease. Furthermore, there were more patients with dyslipidaemia $(p<0.05)$ and arterial hypertension $(p<0.05)$ among patients with erosive compared with non-erosive hand $\mathrm{OA}$. On the other side, there were less patients with diabetes mellitus $(p<0.05)$ among patients with erosive compared with non-erosive hand OA.

Conclusion: Based on our results we suggest that patients with erosive hand OA are at higher risk of general bone loss and increased prevalence of cardiovascular risk factors such as arterial hypertension and dyslipidaemia.

Acknowledgement: This work was supported by the project MHCR No. 023728.

\section{References:}

1. Haugen IK, et al. Ann Rheum Dis 2015;74:74.

2. Ramonda R, et al. Clin Rheumatol 2014;33:11.

\section{P696}

RELATIONSHIP BETWEEN VITAMIN D AND TRABECULAR BONE SCORE IN ELDERLY

$\underline{\text { V. Alekna }}^{1}$, M. Tamulaitiene ${ }^{1}$, A. Mastaviciute ${ }^{1}$, V. Strazdiene $^{2}$

${ }^{1}$ Faculty of Medicine, Vilnius University, Vilnius, Lithuania, ${ }^{2}$ National Osteoporosis Center, Vilnius, Lithuania

Objective: To analyze the associations between vitamin D level and trabecular bone score in elderly persons.

Materials and methods: This was a cross-sectional study on persons aged 60 years and more. The subjects with conditions known to affect bone metabolism or taking any medications known to affect bone metabolism, or taking vitamin D were excluded from this study. Serum levels of 25-hydroxyvitamin D were measured by automated immunoassay (Cobas E411, Roche Diagnostics). Lumbar spine $\left(\mathrm{L}_{1}-\mathrm{L}_{4}\right)$ trabecular bone score was measured using iDXA (GE Lunar, USA). Correlations were determined using Spearman correlation coefficient.

Results: A total of 187 individuals 91 (48.7 \%) men (from 60-95 years) and $97(51.3 \%)$ women (from 60-89 years) were included in this study. The results of this analysis showed that vitamin D was statistically significantly positively associated with lumbar spine $\left(\mathrm{L}_{1}-\mathrm{L}_{4}\right)$ TBS $(r=0.22, p=0.003)$, analyzing men and women together. The same correlations between vitamin $D$ and lumbar spine TBS were detected in men $(r=0.23, p=0.032)$ and women $(r=0.21, p=0.041)$ groups, separately. The linear relationship between vitamin D and TBS were confirmed. 
Conclusion: This study showed that vitamin D level is positively associated with trabecular bone score in elderly men and women.

\section{P697}

CHONDROITIN AND GLUCOSAMINE IN OSTEOARTHRITIS, MEDICATIONS AND SUPPLEMENTSCURRENT ISSUES AND IMPLICATIONS IN THE SAFETY APPLICATION

J. Woron ${ }^{1,3,4}$, B. Batko ${ }^{2}$, J. Schramm ${ }^{5}$

${ }^{1}$ Chair of Pharmacology, Department of Clinical Pharmacology, Jagiellonian University College of Medicine, Krakow, Poland, ${ }^{2}$ Department of Rheumatology, Dietl Hospital, Krakow, Poland, ${ }^{3}$ University Hospital in Krakow, Krakow, Poland, ${ }^{4}$ Regional Centre for Adverse Drug Reaction Monitoring and Investigation in Krakow, Krakow, Poland, ${ }^{5}$ J. Dietl Specialist Hospital in Krakow, Center of Rheumatology, Immunology and Rehabilitation of Malopolska Voivodship Jagiellonian University College of Medicine, Krakow, Poland

One group of drugs used to treat osteoarthritis are symptomatic slow acting drugs for osteoarthritis (SYSADOA). The most commonly used drugs in this class in Polish conditions include glucosamine and chondroitin. Unfortunately, on the Polish market are also available as dietary supplements containing glucosamine and chondroitin, which are mistaken for drugs by patients and doctors. Centers for supervision of the pharmacovigilance each year receive a report on the complications that occurred in patients receiving medicines. In the period from 1 January to 31 December 2015 Regional Centre for Adverse Drug Reaction Monitoring and Investigation in Krakow has received 77 reports of adverse side effects that occurred after the adoption of preparations containing in its composition of glucosamine and chondroitin. With the most common side effects experienced rash $(n=46)$, disorders of the gastrointestinal, such as nausea, vomiting, and diarrhea ( $n$ $=16)$, abnormal levels of glucose $(n=4)$, headache $(n=3)$, pruritus $(n=3)$, liver toxicity associated with cholestasis $(n=$ $3)$, arthralgia $(n=2)$. The severity of adverse reactions resulted in the need for hospitalization in 18 cases, and as many as 66 cases it was necessary to apply drug treatment side effects. After a thorough analysis, we found that as many as 75 cases of side effects were caused by the preparations which were dietary supplements and not drugs. It is worth noting that, in relation to dietary supplements there is no research on the efficacy and safety. Both EULAR and ACR do not recommend their use in osteoarthritis. Dietary supplements can not be an alternative to drugs containing in its composition of glucosamine and chondroitin.

\section{P698}

CLUSTERIN: A NEW MARKER OF SARCOPENIAOSTEOPOROSIS CONNECTION?

F. Mastrangeli ${ }^{1}$, C. Tarquini ${ }^{1}$, A. Orlandi ${ }^{2}$, G. Novelli ${ }^{2}, \mathrm{~S}$. Pucci $^{2}$, U. Tarantino ${ }^{3}$

${ }^{1}$ Medical Biotechology and Translational Medicine, University of Rome Tor Vergata, Rome, Italy, ${ }^{2}$ Department of Biomedicine and Prevention, University of Rome Tor Vergata, Rome, Italy, ${ }^{3}$ Department of Orthopedics and Traumatology, "Tor Vergata" University of Rome, Rome, Italy

Targeting the metabolic pathways leading to osteoporosis is a hot topic for drug discovery.Recent studies show that Clusterin (CLU) interferes with the most important signaling pathways involved in osteoporosis, such as $\mathrm{Ca}^{2+}$ metabolism. CLU is a heterodimeric glycoprotein functionally involved in signaling pathways that regulate inflammation and cell death. CLU has recently been demonstrated to be under the control of IL6. In osteoarthritis (OA) and degenerative joint disease CLU has been implicated in inflammatory and apoptotic process. In the present study we sought to assess the expression and the role of CLU (mRNA and protein) in OP-OA condition and characterize its function in influencing the decrease of muscle mass, the increase of fiber senescence and the raise of calcium deposition.

Methods: Quantitative RT-PCR, immunohistochemistry, and western blot were used to specify and quantify the expression of CLU, IL-6 and the level of H4 acetyl histone in skeletal muscle biopsies of OP and OA patients. Skeletal muscle cells were isolated and induced with CLU to assess the effects on cellular proliferation and differentiation.

Results: A strong overexpression and accumulation of CLU in degenerated fibers was observed in OP as compared to OA. IL-6 and acetyl histone expression were strongly increased in the fibers degenerated in OP patients. The levels of CLU closely correlated with the levels of acetyl histone and IL-6 in OP. Conversely CLU was barely detectable in OA, IL-6 was uniformly expressed while no Histone acetylation was noted. The treatment of the isolated ex vivo muscle cells of OA patients with CLU showed no effect on proliferation in the short incubation time, but strongly accelerated cells aging inducing noticeable morphological changes

Conclusion: The preliminary study showed that CLU affects the histone acetylation pattern suggesting a potential role in epigenetic regulation of muscle cell differentiation and senescence in OA. A better understanding of the role and expression of clusterin in the onset and development of the OP-OA conditions could allow to consider this protein as a new marker for evaluating the muscular alterations in this degenerative diseases proposing it as a new target for therapeutic strategy in the osteoporotic disease. 


\section{P699}

\section{THE STUDY OF DENTAL MODIFICATIONS IN CERTAIN ENDOCRINE DISORDERS}

S. A. Preda ${ }^{1}$, M. Bistriceanu ${ }^{2}$, I. Bistriceanu ${ }^{3}$, D. M. Albulescu $^{4}$, C. Constantin ${ }^{4}$, O. M. Marioara ${ }^{5}$, A.

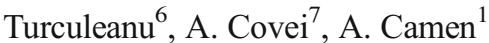

${ }^{1}$ Faculty of Dentistry, University of Medicine and Pharmacy, Craiova, Romania, ${ }^{2}$ Department of Endocrinology, University of Medicine and Pharmacy, Craiova, Romania, ${ }^{3}$ Department of Endocrinology, Emergency Hospital, Craiova, Romania, ${ }^{4}$ Department of Radiology, University of Medicine and Pharmacy, Craiova, Romania, ${ }^{5}$ University of Medicine and Pharmacy, Craiova, Romania, ${ }^{6}$ Biochemical Department, University of Medicine and Pharmacy, Craiova, Romania, ${ }^{7}$ Endocrinology Department, Philanthropy Hospital, Craiova, Romania

Introduction: Excess or deficient secretion of endocrine glands, hormone transport and differentiated tissues responsiveness are important factors that should be evaluated clinically and biologically to determine with certainty the hormonal participation quota in the induction of anomalies dentomaxillary.

Methods: The study was performed on 15 patients with: acromegaly (2), pituitary dwarfism (1), myxedema (congenital-1 and acquired autoimmune-4), Turner syndrome (5) and Klinefelter syndrome (2) Positive diagnosis of endocrine diseases was based on clinical examination and laboratory investigations Physical examination of the oral-maxillofacial dental's region, allowed us to highlight the cases included in the study - changes in the shape and volume of the oral cavity - changes in dental gums, tongue and lips.

Results: Malformations of mouth such as the type of raised palate ("the warhead") or jaw weakness have been reported in cases of Turner syndrome.The small volume of the oral cavity, was met in cases of pituitary dwarfism .However, the large volume of the oral cavity was encountered in both cases with acromegaly. Altering the rate of appearance of teeth was visible in the case of congenital myxedema, which definitive dentition was incomplete, irregular and coexists with elements of the first dentition. Microdentism is characteristic to pituitary dwarfism. The vicious implantation of teeth (dental malposition) was observed at both pituitary dwarfism and at 3 of the cases with Turner syndrome. In acromegaly, through the hypertrophy of the jaw and, especially, of the mandible, interdentally spaces become enlarged due to appearance of and diastema. Changes were noted at both gums case with adult acquired myxedema and in acromegaly. Changes of the tongue have been observed in acromegaly as well as in myxedema. Changes of the lips are also present in the pathology of pituitary (acromegaly) and myxedema.

\section{Conclusions:}

1. The activity of endocrine system begins in different moments of postnatal odontogenesis having the role to set up the genetic program, growth and development of the body and therefore the dental apparatus as an integral part.

2. The dental cavity hormonal control receptor is key to deciphering the pathogenetic mechanisms of the dental device changes at some endocrine disorders.

\section{P700}

\section{CORRELATION BETWEEN BONE DENSITY, FAT MASS AND LEAN MASS IN POSTMENOPAUSAL} WOMEN IN OLTENIA COUNTY

S. A. Preda ${ }^{1}$, M. Bistriceanu ${ }^{2}$, I. Bistriceanu ${ }^{3}$, D. M. Albulescu $^{4}$, C. Constantin ${ }^{4}$, O. M. Marioara ${ }^{5}$, A.

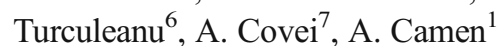

${ }^{1}$ Faculty of Dentistry, University of Medicine and Pharmacy, Craiova, Romania, ${ }^{2}$ Department of Endocrinology, University of Medicine and Pharmacy, Craiova, Romania, ${ }^{3}$ Department of Endocrinology, Emergency Hospital, Craiova, Romania, ${ }^{4}$ Department of Radiology, University of Medicine and Pharmacy, Craiova, Romania, ${ }^{5}$ University of Medicine and Pharmacy, Craiova, Romania, ${ }^{6}$ Biochemical Department, University of Medicine and Pharmacy, Craiova, Romania, ${ }^{7}$ Endocrinology Department, Philanthropy Hospital, Craiova, Romania

Introduction: Bone density is the best indicator of the risk of fractures in osteoporosis, but this variable is closely related anthropometric fat free mass and fat mass. This paper proposes an analysis of anthropometric variables correlated with BMD in postmenopausal women, the Oltenia region. Along with obesity, osteoporosis is a major health problem worldwide, given the consequences of the disease, namely increased bone fragility and an increased risk of fractures.

Method: Lot studied comprises of 183 women from urban, aged between 43 and 78 years. They excluded subjects with diseases that have the effect of osteoporosis: malabsorption, hyperthyroidism, hyperparathyroidism, renal failure, chronic arthritis, etc. Anthropometric variables studied include height and weight; measurements were performed using a stadiometer or a scale, all rules for proper anthropometric measurements. BMD was measured at the lumbar spine and whole body, using DXA 90. Medix fat mass and fat free mass resulting from scanning the whole body were also obtained BMI values. 
Discussions: The analysis of data obtained retains elevated correlation coefficient between weight and BMI, and study group is enclosed in preobesity positive correlations between BMI and BMD exists, but with very low. We note further and positive correlations between BMD spine fat mass and fat free mass, but significant values of correlation coefficients observed the relationship between BMD and total fat mass, fat free mass, respectively. In this study, higher values of correlation coefficients can be found if fat free mass. In the study group we considered only the values measured BMD in the spine and total values of BMD. The backbone is regarded as one of the places of choice for measuring BMD, especially when monitoring therapy response; hip measurements are indicated in the prediction of hip fractures However, the two anthropometric variables, fat free mass and fat mass are predictors for bone density.

Conclusion: This study shows the importance of fat free mass and fat mass in determining BMD in postmenopausal women, in the Oltenia region.

\section{P701}

\section{TISSUE BIOMARKERS OF OSTEOARTHRITIC} OSTEOCHONDRAL DEGENERATION

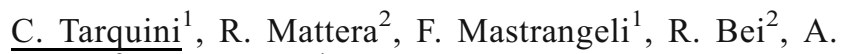

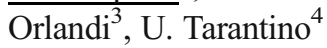

${ }^{1}$ Medical Biotechology and Translational Medicine, University of Rome Tor Vergata, Rome, Italy, ${ }^{2}$ Department of Clinical Sciences and Translational Medicine, University of Rome Tor Vergata, Rome, Italy, ${ }^{3}$ Department of Biomedicine and Prevention, University of Rome Tor Vergata, Rome, Italy, ${ }^{4}$ Department of Orthopedics and Traumatology, "Tor Vergata" University of Rome, "Policlinico Tor Vergata" Foundation, Rome, Italy

Background / Objective: Osteoarthritis (OA) is a slowly progressing chronic joint disease, with a high incidence in the aging population. Articular cartilage, subchondral bone degeneration and remodeling of adjacent bone are major features of OA. Transglutaminases (TG) play a significant role in OA alterations of extracellular matrix, whereas Osteocalcin $(\mathrm{OCN})$ and Sclerostin (SOST) influence bone mass but their real contribution in OA are not clarified. Osteopontin (OPN) is a phosphorylated acidic glycoprotein which seems to be involved in pathogenesis of OA promoting the degeneration of articular cartilage. The study of specific biomarkers would be beneficial to confirm the clinical diagnosis of OA and to predict its progression.

Material and Methods: Immunohistochemical investigation of TG2, OCN, OPN and SOST expression was performed on formalin-fixed, paraffin-embedded femoral heads of patients $(n=60)$ underwent hip arthroplasty for primary OA or osteoporotic fracture of the neck of femur (OP). Histomorphometric evaluation of bone trabecular remodeling and western blot analysis of chondrocyte subpopulations were also performed. Results: TG2 expression was increased in OA chondrocytes and extracellular matrix and almost absent in OP femoral cartilage. OCN levels were increased in OA osteocytes/ osteoblasts of trabecular bone in both men and women, while its expression was reduced in OA chondrocytes. We also observed an increased OPN expression in OA osteocytes/ osteoblasts of trabecular bone of male patients. OA biomarker changes well correlated with bone remodeling histomorphometry. No significant differences were found for SOST expression both in cartilage and bone. Blot analysis of cultured OA chondrocytes confirmed in vivo data.

Conclusions: Our study demonstrated changes of TG2, OCN and OPN expression in OA cartilage and bone tissues and correlated with OA trabecular remodeling. Besides to be additional markers for osteochondral degeneration, study of biomarkers can address new therapeutic interventions aimed to contrast the progression of $\mathrm{OA}$.

P702

SYNOVIAL FLUID ANALYSIS FROM OSTEOARTHRITIS PATIENTS REVEALS A NEGATIVE CORRELATION BETWEEN TUMOUR NECROSIS FACTOR- $\alpha$ AND ALKALINE PHOSPHATASE

$\underline{\text { S. Deshpande }}^{1}$, P. Kulkarni ${ }^{2}$, A. Mahajan ${ }^{1}$, S. Koppikar ${ }^{3}$, A. Harsulkar $^{3}$

${ }^{1}$ Department of Orthopedics, Bharati Hospital, Bharati Vidyapeeth University, Pune, India, ${ }^{2}$ Cell and Molecular Biology, Interactive Research School for Health Affairs, Bharati Vidyapeeth University, Pune, India, ${ }^{3}$ Cell and Molecular Biology, Interactive Research School for Health Affairs, Bharati Vidyapeeth University, Pune, India

Background: Loss of proteoglycan and low-grade inflammation comprise the hallmark of osteoarthritis (OA) pathology. These two processes are well studied in single and partially understood in connection with OA. However, complex interplay of both the pathologies, which is yet unexplained, has been targeted in the present communication. Synovial fluid (SF), a biological communicator between cartilage and synovium, conducts biochemical signals transmitted by both tissues; thus selected as a tool to achieve the objectives of our study. A crosstalk between inflammatory and cartilage remodelling pathways in the form of alkaline phosphatase (ALP), an established marker of chondrogenesis and tumour necrosis factor- $\alpha$ (TNF- $\alpha)$ has been discussed here.

Materials and methods: We obtained $50 \mathrm{SF}$ samples from 43 OA patients (few samples were bilateral) using arthrocentesis. ALP was estimated using diagnostic kit procured from Erba Mannheim, Germany on spectrophotometer. On the other side, $28 \mathrm{SF}$ samples were measured for their TNF- $\alpha$ levels 
using ELISA kit from Abnova, Walnut, CA on ELISA platereader. All the enrolled OA patients were radiographically graded for the disease severity, using Kellgren-Lawrence (KL) scale.

Results: Elevated ALP was observed throughout OA SFs when compared to control (SF from meniscal tear patient); however remained non-significant on statistical scale except in terminal OA (KL grade IV) due to variation. KL-IV SFs showed a substantially raised ALP levels than control $(p=0.0130)$. Marginally significant elevation of TNF- $\alpha$ was noted in all samples $(p=0.0900)$ when compared to control. On more specific account, end-stage OA patients (KL-IV) revealed a dramatic increase in $\mathrm{TNF}-\alpha(p=0.0501)$. Interestingly, a negative correlation was revealed between ALP activity and TNF- $\alpha$. When higher ALP levels in mild (KL-I) and moderate OA (KL-II and KL-III) were suggestive of well-maintained chondrogenesis; the process was seen to be affected in severe OA as denoted by dropped ALP activity. On the contrary, KL-I, II and III were associated mild inflammation which reached a peak at terminal stage of the disease, indicated by a steady rise in TNF- $\alpha$.

Conclusion: Here, elevated TNF- $\alpha$ in OA SFs were suggestive of a strong association of inflammation. Recent research reports usefulness of anti-TNF- $\alpha$ therapy in OA through amelioration of structural damage, arresting cartilage-loss and improving clinical symptoms. These studies were consisted of OA animal models and a few human clinical case reports. On the other side, it is well understood that enhanced bone remodelling is closely linked with OA pathology where ALP plays a pivotal role via bone as well as cartilage mineralization. High ALP levels in OA SFs, in the present experiment, supported increase in bone re-absorption and formation process. At the interface of inflammation and cartilage degeneration pathways, a direct inhibition of ALP by TNF- $\alpha$ was revealed through a negative correlation between TNF- $\alpha$ and ALP activity. Indeed, this cross-talk will increase our comprehension about OA pathology and demands evaluation of anti-TNF- $\alpha$ therapy as a better alternative. Nevertheless, increased ALP levels in OA were suggested to be associated with abnormal osteoblast formation ultimately results in osteoid matrix which is undermineralized; thus needs a further meticulous investigation.

\section{P703}

OCCULT METABOLIC BONE DISEASE IN ALCOHOLIC AND TROPICAL CHRONIC PANCREATITIS K. Hari $^{1}$, M. Manrai ${ }^{1}$, A. Sood ${ }^{1}$

${ }^{1}$ Command Hospital, Panchkula, India

Objective: Chronic pancreatitis (CP) has multisystemic morbidity due to associated exocrine and endocrine deficiency. Alcohol and tropical calcific varieties comprise the two common etiologies of $\mathrm{CP}$ in our population. We investigated the presence of occult metabolic bone disease (MBD) including osteopenia, osteoporosis and osteomalacia in patients with CP.

Material and Methods: In this cross-sectional, observational study, we included serial patients of CP presented to our hospital. The study was conducted for six month period from June to December 2015. The study population consists of $51 \mathrm{pa}-$ tients $(12 \mathrm{~F} ; 39 \mathrm{M})$ in different stages of $\mathrm{CP}$ and are grouped as alcoholic (Group 1; $N=30$ ) and tropical CP (Group 2; $N=21$ ). We determined serum calcium, phosphorus, alkaline phosphatase, 25-hydroxyvitamin D and intact PTH levels. BMD was measured by DXA in the lumbar spine (L1-L4) and in the proximal femur. The results were analyzed by appropriate statistical methods.

Results: The study participants had a mean age of $34.5 \pm 5.4 \mathrm{yr}$ and duration of CP $1.1 \pm 1.7 \mathrm{yr}$. Metabolic bone disease is seen in $34(67 \%)$ out of 51 patients. The prevalence of Osteopenia (23) is the most common disease followed by osteomalacia (7) and osteoporosis (4). MBD is seen more in the tropical CP (18/ $21)$ than alcoholic $(16 / 30) \mathrm{CP}(P=0.0188)$. Osteopenia and osteoporosis were observed more frequently in tropical $\mathrm{CP}$, whereas osteomalacia showed equal distribution.

Conclusion: Occult MBD is seen in two thirds of patients with $\mathrm{CP}$ and is more common in tropical CP than alcoholic $\mathrm{CP}$. The disease is mostly silent and mandates active screening in all susceptible individuals.

\section{P704}

NERVE GROWTH FACTOR/BRAIN-DERIVED NEUROTROPHIC FACTOR - OSTEOCALCIN AND OXYTOCIN GENE INTERACTION IN BRAIN, BONE, FAT STORES AND REPRODUCTIVE ORGANS OF ADULT MICE

C. Camerino $^{1}$, R. Caloiero ${ }^{1}$, E. Conte ${ }^{1}$, D. Tricarico ${ }^{1}$

${ }^{1}$ University of Bari, Bari, Italy

Objective: That gonadal failure favors the appearance of osteoporosis while obesity seems to protect from osteoporosis support linking between bone, energy, and reproduction. The bone-derived osteocalcin (Ost), the neurotrophins BDNF/NGF and oxytocin(Oxt) have effects on energy metabolism, bone mass, reproduction and brain functions suggesting a coordinated regulation.

Materials and Methods: BDNF/NGF-Oxt-Ost interactions was investigated by RT-PCR measuring mRNA levels of NGF, BDNF, Oxt, Ost and their receptors p75NTR/NTRK1, TRKb, Oxtr and Gprc6a in brain, bone, WAT/BAT and reproductive organs, of 3 months old female and male mice using brain and bone as positive controls, respectively.

Results: NGF and p75NTR expression is $50 \%$ higher in BAT than brain and are downregulated in WAT and bone in both 
genders. Ost and Gprc6a are upregulated in bone and brain, downregulated in BAT/WAT. BDNF and TRKb expression in bone is higher than brain, but lower in BAT/WAT; TRKb is downregulated in bone and up-regulated in adipose tissue. NGF is upregulated in ovaries/uterus, but downregulated in testes. p75NTR is respectively $300 \%, 100 \%$ and $50 \%$ higher in testis, ovaries and uterus than brain. NTRK1 is downregulated in all tissues. Gprc6a is expressed in testes, not in ovaries and uterus. BDNF and TRKb are downregulated in reproductive organs. Oxt is expressed in brain and in bone in either gender while Oxtr in ovaries, in fat and bone. Upregulation of NGF and related-receptors in fat is consistent with NGF as an energy regulator. Inverse correlation of NGF and BDNF in fat and bone shows these exerting opposite effects on leptin with BDNF regulating bone.

Conclusions: The upregulation of p75NTR in testes matches Gprc6a expression and may be responsible for higher LH in Ost $-1-$ mice. The pattern of expression of these molecules show a similar trend with Ost/NGF/Oxt/BDNF genes highly expressed in brain of male and female mice, while their receptors were expressed in reproductive organs showing a gender expression profile. This is consistent with the fact that these molecules have limited or no access through the blood brain barrier and adds evidences that the signalling of bone metabolism and fertility are released from CNS to act on peripheral tissues.

\section{P705}

\section{STRUCTURAL AND FUNCTIONAL DEFORMITIES} T. Petrusic $^{1}$

${ }^{1}$ Health Centre, Nis, Serbia

Introduction: The modern way of life full of passive position, and overflowing, and improper diet, expansion of computer technology, prolonged television watching, leading to hypokinesia, which increasingly affects children of preschool age favoring the emergence of posture disorders in preschoolers. Poor posture follows the protrusion sagging shoulders, relaxing the muscles of the anterior abdominal wall, the burden on the spinal column and creates the possibility of damage. Kyphosis is a spinal deformity where there is a curvature of the spine in the sagittal plane, usually in the thoracic spine. Statistics indicate a high percentage of children with flat feet, with a tendency to decrease. Therefore it is necessary as soon as possible to begin work on prevention at the stage of the plastic period of child development.

Objective: Early diagnosis, prevention and treatment of physical deformities in the posture of preschool children.

Methods: Clinical study, which included 2258 children aged 6 years examined in the period from September to December 2015. Of this number of girls was 1200, and the boys 1058 . Specialist examinations we examined the posture of children in the normal course of systematic reviews in the department of Physical Medicine and Rehabilitation Health Center in Nis. Results: The incidence posture of preschool children after physiotherapy which included assessment of posture, examination of the spine and lower extremities and distribution data are presented as percentage of the total number of children. Poor postural keeping children had a total of 592 (26.22\%), boys $270(11.96 \%)$ and 322 girls (14.26\%). Kyphosis 87 (3.85\%), 41 boys (1.81\%), 46 girls (2.04\%). Flatfeet 1160 $(51.37 \%)$, boys $627(27.77 \%)$ and 533 girls (23.60\%).

Conclusion: Poor postural posture of preschool children is the most important problem, while the percentage of kyphosis less pronounced, but far more serious problem. Flat feet are at this age often unjustly neglected deformity, but later on can lead to serious problems in posture and to functional limitations in physical activities. It is necessary in this age timely diagnosis, implement prevention and correction of deformity skeletons.

P706

HEALTH MEASURES IN BRITISH AND SPANISH CARE HOME RESIDENTS: AN INTERNATIONAL COMPARATIVE STUDY

A. I. Rodríguez-Rejón ${ }^{1,2}$, M. Edwards ${ }^{2}$, C. Parsons ${ }^{2}$, R.

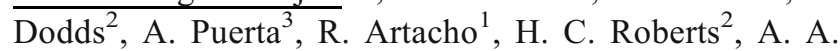
Sayer $^{2}$, C. Cooper ${ }^{2}$, M. D. Ruiz-López ${ }^{1,4}$

${ }^{1}$ Department of Nutrition and Food Science, Faculty of Pharmacy, University of Granada, Granada, Spain, ${ }^{2}$ MRC Lifecourse Epidemiology Unit, University of Southampton, Southampton, United Kingdom, ${ }^{3}$ Geriatric Physician, Granada, Spain, ${ }^{4}$ Institute of Nutrition and Food Technology, University of Granada, Granada, Spain

Objective: To compare measures of anthropometry, cognition, nutrition, and physical functioning in institutionalized people in the UK and Spain.

Material and Methods: This study was carried out in residents aged 70 years and older who are living in 5 British and 2 Spanish care homes. Demographic data were recorded; anthropometric data were measured and used to calculate BMI. Validated questionnaires were administered to assess physical function (Barthel Score), cognitive function (Mini Mental State Examination and Pfeiffer test) and nutritional status (Malnutrition Universal Screening Tool (MUST) and Mini Nutritional Assessment (MNA)). Maximum grip strength was measured using a handheld dynamometer (Jamar and Takei).

Results: The sample included 100 British residents (65 women and 35 men) and 116 Spanish residents ( 85 women and 31 men). Data was compared by sex and country. Age and weight were similar in both settings. British women were taller than Spanish women $(p<0.001)$, had a lower BMI $(p=0.004)$, similar Barthel score and a numerically lower grip strength $(p=0.054)$. British men were taller than Spanish men 
$(p<0.001)$, had a lower BMI $(p<0.001)$, lower Barthel score $(p<0.001)$ and a significantly lower grip strength $(p=0.015)$. The majority of women had moderate or severe cognitive impairment $(60 \%$ of the British and $62 \%$ of the Spanish female). In men, $77 \%$ of the British sample showed moderate or severe cognitive impairment, while $64 \%$ of the Spanish sample showed mild or no impairment. The risk of malnutrition was low in the $74 \%$ of British women and $83 \%$ of men. However, only the $26 \%$ of Spanish women and $58 \%$ of men have low risk.

Conclusion: We found interesting differences between the residents of Spanish and British care homes. Further research is required to better understand these differences and how they may affect the physical functioning of institutionalized people in both countries.

\section{P707}

EFFECTS OF REHABILITATION TREATMENT ON QUALITY OF LIFE IN PATIENTS WITH HAND OSTEOARTHRITIS

I.-R. Marcu $^{1}$, S. Patru ${ }^{1}$, A. C. Bighea ${ }^{1}$

${ }^{1}$ University of Medicine and Pharmacy, Craiova, Romania

Introduction: Hand osteoarthritis (HOA) is one of the most prevalent musculoskeletal diseases in an adult population with bad consequences on hand function and health related quality of life. Clinical guidelines for HOA management include non-pharmacological approaches like physical modalities and kinesitherapy. The main purpose of the management of these patients is to ensure a normal and independent living and an appropriated quality of life. In this context we considered it appropriate to evaluate the effectiveness of evolution of the score Short Form 36 Health Survey Questionnaire (SF-36).

Material and Method: The study included 94 patients, $64 \%$ women, divided into three groups comparable in gender, residence, duration of the disease, age: two control lots with medication (32 patients), respectively with kinesitherapy (26 subjects) and a test lot with physical therapy and exercises (36 patients). Average age of the patients was $65.3 \pm 6.21$ years, mean duration of HOA $15.5 \pm 8.75$. The patients are assessed at baseline, at 4 weeks and after 8 weeks, at the completion of the study, using the parameters: hand joint pain $(100 \mathrm{~mm}$ VAS), a hand function test with short administration time, the Moberg Picking-Up Test (MPUT) and to describe health status, the Short Form 36.

Results: SF-36 dynamics during the 8 weeks reveals a positive change especially in the physical and kinetic therapy group. Based on SF-36 indicator we calculated the minimum number of patients to be treated for 8 weeks to prevent decreasing the quality of life in at least one of them: 2 in the lot with physical and kinetic therapy and 3 in the lot with kinesitherapy. Moberg Picking-Up Test (MPUT) influence on SF-36 improvement is well above $50 \%$; there is a correlation between indicators estimated as moderately high $(0.8)$.

Conclusion: The results show the contribution of complex physical and kinetic therapy to increasing the quality of life for patients with hand osteoarthritis.

\section{P708}

\section{THE DEFORMATION OF VERTEBRAE IN PATIENTS WITH RHEUMATOID ARTHRITIS (RA)}

E. Petrova $^{1}$, I. Dydykina ${ }^{1}$, A. Smirnov ${ }^{1}$, M. Podvorotova ${ }^{1}$, P.

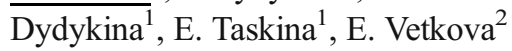

${ }^{1}$ Nasonova Research Institute of Rheumatology, Moscow, Russian Federation, ${ }^{2}$ I.M. Sechenov First Moscow State Medical University, Moscow, Russian Federation

Objective: To identify frequency of DV in RA and obtain comparative description of suffering from RA patients with/without DV.

Material and methods: The present study recruits 164 women, fulfilling ACR 1987 criteria of RA, aged from 20 to 65 y.o. Average age is $47.9 \pm 12.9$, duration of RA is $12.1 \pm 9.3$ years. $99(60.4 \%)$ of participants have RF in their serum samples, 92(56.1\%) have erosions in joints of hand and/or feet, and 3 or 4 radiographic stages, respectively. All patients were conducted radiographies of thoracic and lumbar spine with Genant's semi-quantitative assessment, and also radiographic estimate of hands and feet joints by Sharp/van der Heijde scoring (SHS) was performed. BMD was measured in 3 sites (lumbar spine (LS), femoral neck (FN) of left leg and dominant distal forearm (DF)) of all patients by Hologic «Discovery A». Data were analyzed using Statistica 6.0 StatSoft Inc.

Results: $16(10 \%)$ participants reported about back pain, DV of grade 3 by Genant's assessment was found of 10(6\%), grade $2-18(11 \%), 1-8(5 \%)$ of patients. Then all patients were shared in 2 groups: with/without DV, 36(22 \%) and $128(78 \%)$, respectively. Average age of gr. 1 is $54.3 \pm 10.8$ y.o., gr. $2-46.1 \pm 12.9$ y.o. $(\mathrm{p}<0.05)$, duration of RA -16.8 \pm 11.0 and $10.7 \pm 8.4$ years., respectively, $(\mathrm{p}<0.05)$. Groups were comparable in parameters of disease activity (DAS 28), amount of RF-positive subjects, aspects of treatment approaches (biological agents and DMARD). Average duration of receiving oral glucocorticoids (GK) in gr. 1 was $95.4 \pm 92.5$ vs. $50.9 \pm 47.8$ months in gr. $2(p<0.05)$. BMD in gr. 1 was less in each of 3 sites, than in gr. $2,(p<0.05)$. Osteoporosis according BMD was obtained in 29(82.9\%) patients of gr.1 and $62(50.8 \%)$ in gr.2, $(\mathrm{p}<0.05)$. Nonvertebral fractures were found in $61 \%$ vs. $28 \%$ patients, respectively, $(\mathrm{p}<0.05)$. The 
erosions score was $62.4 \pm 63.3$ vs. $27.5 \pm 38.8$ in groups 1 and 2 , $(\mathrm{p}<0.05)$, the joint space narrowing score was $104.0 \pm 39.8$ vs. $80.8 \pm 39.4,(\mathrm{p}<0.05)$, the total score SHS $-167.2 \pm 92.6$ vs. $108.2 \pm 72.3$ in group 1 and 2 , respectively, $(\mathrm{p}<0.05)$.

Conclusion: $22 \%$ of patients with RA have DV, interesting, that more than half of them don't feel back pain. Contributing factors of DV are age, duration of RA, supplement of GK. In patients with DV BMD in 3 studied sites of skeleton were less, and score of SHS were more, than in patients without DV.

\section{P709}

PROSPECTIVE 16-YEAR EPIDEMIOLOGICAL STUDY OF HIP AND DISTAL FOREARM FRACTURES IN MOSCOW REGION

I. Kryukova $^{1}$, A. Dreval ${ }^{1}$, L. Martchenkova ${ }^{2}$, M. Gerasimenko $^{2}$

${ }^{1}$ Moscow Region Research and Clinical Institute, Moscow, Russian Federation, ${ }^{2}$ Russian Scientific Center of Medical Rehabilitation and Balneology of Ministry of Health of the Russian Federation, Moscow, Russian Federation

The purpose of the study was comparison analysis of incidence of hip and distal forearm fractures for two five-year periods (1998-2002 vs. 2009-2013) in Moscow Region.

Methods: The study was performed among the population aged 50 years and older in Kolomensky area which is located in the South-East of Moscow Region and has total population about 190000 people. All low trauma hip and distal forearm fractures which occurred in men and women aged 50 years and older were registered from the medical documentation of traumatology service of Kolomensky area during the periods 1998-2002 and 2009-2013 years.

Results: The average incidence of hip fracture in women in 2009-2013 was slightly lower but not statistically significant than in $1998-2002-184.5$ vs. $201.1 / 100000$ respectively $(p>0.05)$. The rate of distal forearm fractures in women in 2009-2013 was $1458.5 / 100000$ vs. 1094.4/ 100000 in 1998-2002 $(p>0.05)$. Despite the lack of significant difference, the average incidence of hip fractures in 2009-2013 in men reached 170.8/100 000 and had a clear tendency to increase compared with 120.6/100 000 in 1998-2002. There was a trend to a significant increase of distal forearm fracture rate in men from 276.1/100 000 in 1998-2002 to $1520.8 / 100000$ in 2009-2013 $(p<0.05)$. Conclusions: A prospective 16-year epidemiological study of incidence of hip and distal forearm fracture in Moscow Region showed no any significant changes in fracture rate from study period 1998-2002 to 2009-2013 in women aged 50 years and older but the significant increase in distal forearm fracture incidence in men of similar age.

\section{P710}

HISTOMORPHOMETRIC STUDY IN OSTEOPOROTIC PATIENTS WITH NEW FRACTURES AFTER LONG TERM ANTI-OSTEOPOROTIC TREATMENT

$\underline{\text { B. Batteux }}^{1}$, A. Sejourné ${ }^{1}$, M. Cohen-Solal ${ }^{2}$, V. Goëb ${ }^{1}$, P. Fardellone $^{1}$

${ }^{1}$ Service de Rhumatologie CHU Amiens Nord, Amiens, France, ${ }^{2}$ Service de Rhumatologie CHU Lariboisière, Paris, France

Introduction: New fractures can occur after prolonged treatment despite adequate adhesion. These recurrent fractures occur after several years of antiresorptive treatments: bisphosphonates (BP), raloxifene (RLX) or mixed effects agents: strontium ranelate (SR). The choice of a new treatment should consider the histological mechanism underlying the new fracture: persistence of hyperresorption due to poor adhesion or bioavailability, or even low bone remodeling due to a prolonged exposure to treatment. The only reliable way to find out the underlying mechanism of this new fracture is to perform a bone biopsy (BB) to explore the dynamics of remodeling pattern. The objective of this study was to describe the histomorphometric profile of bone from osteoporotic patients with new fragility fractures after prolonged exposure to anti-resorptive agents.

Patients and methods: 8 patients were included, of which 4 treated exclusively with oral BP, whether sequentially with BP (oral or intravenous), and teriparatide and / or RLX.

Results: Histomorphometric results reveal various profiles. Five patients, treated with BP for more than 10 years, presented a decreased or very low remodeling status with osteoblastic surfaces varying from 0 to $0.99 \%$ (normal values of $4 \pm 2 \%$ ), a large range of osteoclasts of 0.11 to $0.77 / \mathrm{mm}^{2}$ (normal values of $0.45 \pm 0.05 / \mathrm{mm}^{2}$ ) and an always very thin cortical bone. Two patients presented persistent bone hyper- resorption after receiving BP orally for more than 10 years or RLX, with osteoblastic surfaces varying from 0 to $2.55 \%$, an osteoclast number ranging from 1.61 to $162 / \mathrm{mm}^{2}$ and a very thin cortical bone. Finally, one patient had a low formation rate with preserved resorption activity after treatment with BP for 5 years and teriparatide for 18 months with an osteoblastic surface of $0.74 \%$, a number of osteoclasts $0.88 / \mathrm{mm}^{2}$ and a resorbed cortical bone.

Conclusion: These results suggest that new fractures can be occurred because of a very thin cortical in patients after a long exposure to classical anti-resorptive treatments suggesting a lack of effect of BP on the cortical bone. Our study shows the interest of the BB after double labeling for histomorphometric analysis of bone in osteoporotic patients treated for many years and having a recurrent fracture to identify the underlying mechanism of bone fragility. 


\section{P711}

FREQUENCY OF EXTRASKELETAL ABNORMALITIES IN BELARUSIAN CHILDREN WITH OSTEOGENESIS IMPERFECTA

\section{A. Pachkaila ${ }^{1}$, E. Rudenka ${ }^{2}$, V. Zhernosek ${ }^{3}$}

${ }^{1}$ Belarusian Medical Academy of Postgraduate Education, Minsk, Belarus, ${ }^{2}$ Belarusian State Medical University, Minsk, Belarus, ${ }^{3}$ Belarusian Medical Academy of Postgraduate Education, Minsk, Belarus

Objective: To evaluate the frequency of extraskeletal abnormalities in Belarusian children with osteogenesis imperfecta (OI).

Methods: 32 Belarusian boys (19) and girls (13) aged 018 years with OI were included in study; they were examined by a pediatrician, ophthalmologist, dentist, geneticist, endocrinologist, hearing care professional (audiometry); TSH and free thyroxin, insulin, glucose were assessed; ultrasound evaluation of the heart, abdominal organs and the thyroid gland was carried out. In accordance with the D. Silence classification 24/32 patients had OI type I, 7/32 - type III, 1/32 - type IV. Mild OI was detected in $5 / 32$ patients, moderate - in $18 / 32$, severe - in 9/32.

Results: Short stature of different severity was observed in 11/ 32 patients, mostly - in patients with type III. In 5/32 patients, obesity was revealed (in 2 of them - complicated by the insulin resistance). Type III patients had the typical facial pattern (triangular face and etc.). Dentinogenesis imperfecta identified in 9/28 patients (4 infants had no teeth); the majority of these children had severe OI. During the audiological screening in 1 infant bilateral sensorineural hearing loss with delay of speech development was revealed; the remaining patients had normal hearing. Blue (gray) sclera detected in 25/32 patients. Ultrasound examination showed no signs of nephrocalcinosis in examined patients; 3 patients had mitral valve prolapse, in 5 patients valve dysfunction (regurgitation) was found; cysts and thyroid hyperplasia detected in 2 patients. Subclinical hypothyroidism was diagnosed in 4 children. Myopia/hyperopia/astigmatism detected in 13/32 patients. Signs of joint hypermobility were detected in half of the patients. It was difficult to identify the real incidence of muscle weakness due to the possible impact of pamidronic acid and other factors.

Conclusions: Prompt detection and correction of extraskeletal abnormalities helps verification of the diagnosis in children with OI.
P712

CATHEPSIN K AS A NEW BIOMARKER OF OSTEOCLAST ACTIVITY IN PATIENTS WITH ENDOGENOUS CUSHING'S SYNDROME

T. A. Grebennikova $^{1}$, T. T. Tsoriev ${ }^{1}$, A. V. Iljin ${ }^{2}$, L. Y. Rozhinskaya ${ }^{1}$, Z. E. Belaya ${ }^{1}$

${ }^{1}$ Neuroendocrinology and bone diseases, the National Research Centre for Endocrinology, Moscow, Russian Federation, ${ }^{2}$ Biochemistry and Hormonal Assays, Endocrinology Research Centre, Moscow, Russian Federation

An increase in the number of osteoclasts and sites of lacunar resorption has been seen in experimental studies in glucocorticoid-induced osteoporosis (GIO). Cathepsin $\mathrm{K}$, released in the circulation, may be a new specific marker of osteoclast activity.

Objectives: To evaluate serum cathepsin $\mathrm{K}$ levels in patients with endogenous Cushing's syndrome (CS).

Material and Methods: Fasting serum samples were taken from consecutive patients with biochemically confirmed active CS and healthy volunteers matched by age, sex and BMI. Twenty-four hours urine free cortisol (24hUFC) was measured by an immunochemiluminescence assay on a Vitros ECi (60-413 nmol/24 h). Osteocalcin (OC) and carboxyterminal crosslinked telopeptide of type I collagen (CTx) were assayed by electrochemiluminescence assay (ECLIA) Cobas e601 Roche. Serum cathepsin K was measured by commercially available immunoassay.

Results: Thirty patients with CS: 20 females and 10 males, Me (Q25-Q75) 33 (24-45) years old, BMI $29.9(24.6-35.0) \mathrm{kg} / \mathrm{m} 2$ and 30 healthy volunteers matched by sex, age $(p=0.21)$ and BMI $(p=0.27)$ were enrolled. Subjects with CS (19 had lowtraumatic fractures) had 24hUFC - 1343 (604-3675) $\mathrm{nmol} / 24 \mathrm{~h}$, suppressed OC $-6.5(5.2-11.7) \mathrm{ng} / \mathrm{ml}$ vs. 16.5 (13.4-21.8)ng/ml $p<0.001$; no difference in CTx levels $0.41(0.26-0.75)$ vs. $0.37(0.30-0.43) p=0.985$. Nevertheless serum cathepsin $\mathrm{K}$ levels were increased in CS $6.67(5.77-8.02) \mathrm{pmol} / 1$ vs. healthy subjects 5.2 (4.48-6.24) $\mathrm{pmol} / 1 p=0.042$. Significant correlation was found between $24 \mathrm{hUFC}$ and serum cathepsin $\mathrm{K}$ levels $\rho-0.36 p=0.05$.

Conclusions: Cathepsin $\mathrm{K}$ is increased in patients with CS and might be considered as a new marker of osteoclast activity and target molecule for intervention in patients with GIO. 


\section{P713}

VITAMIN D DEFICIENCY AND LONG MEDICAL HISTORY OF A SKULL BONE-FORMING TUMOUR M. Carsote $^{1}$, A. Goldstein ${ }^{2}$, A. Valea ${ }^{3}$, S. E. Albu ${ }^{4}$, A. Dumitrascu $^{5}$, A. Ghemigian ${ }^{1}$

${ }^{1}$ C. Davila University of Medicine and Pharmacy \& C.I.Parhon National Institute of Endocrinology, Bucharest, Romania, ${ }^{2}$ C.I. Parhon National Institute of Endocrinology, Bucharest, Romania, ${ }^{3}$ I. Hatieganu University of Medicine and Pharmacy \& Clinical County Hospital, Cluj-Napoca, Romania, ${ }^{4}$ C.Davila University of Medicine and Pharmacy \& University Emergency Hospital, Bucharest, Romania, ${ }^{5}$ C.I.Parhon National Institute of Endocrinology, Bucharest, Romania

Introduction: Bone- forming tumors (BFT) have distinct radiological and imagery features. However, the specific type (as osteoma, osteoblastoma, endoostoma, etc.) is reflected by pathological report after surgery or neurosurgery. The first step of diagnosis is provided in most of cases by selfpalpation since the masses are progressively growing in a symptomatic way manner, local pain may be associated, together with anatomic disturbances as impaired hearing or vision. Osteoma may develop on bones (especially skull, facial bones, etc.) and within soft tissues.

Objective: We introduce an adult symptomatic female with a long history of BFT who refused any surgical approach.

Material and Method: Bone turnover markers (BTM) and parameters as well as skull imagery is used to characterize the condition.

Case data: A 42-year old female is admitted for a checkup related to a previous diagnosis of a small goiter with normal thyroid function. She complains of intermittent nonspecific bone and muscle pain. She has regular menses and the medical family history is negative. Clinical exam was within normal limits except for a small left frontal lump (around $1.5 \mathrm{~cm}$ ) which, based on anamnesis, progressively grew for almost 10 years. No trauma, local pain or dysfunction is described. Thyroid ultrasound, function and autoimmunity were not specific for any new pathology. The bone parameters revealed total / ionic serum calcium of $8.84 / 3.8 \mathrm{mg} / \mathrm{dL}(\mathrm{N}: 8.5-$ $10.2 \mathrm{mg} / \mathrm{dL}$, respective $3.9-3.9 \mathrm{mg} / \mathrm{dL}$ ), serum phosphorus of $2.88 \mathrm{ng} / \mathrm{mL}$ (N:2.5-4.5); BTM: total circulating alkaline phosphatase of $36.72 \mathrm{U} / \mathrm{L}$ (N:35-129), serum osteocalcin of $120.8 \mathrm{ng} / \mathrm{mL}(\mathrm{N}: 11-43)$, serum CrossLaps of $1.45 \mathrm{ng} / \mathrm{mL}(\mathrm{N}$ : 0.137-0.573); bone hormones: 25-hydroxyvitamin D of $10.9 \mathrm{ng} / \mathrm{mL}(\mathrm{N}: 30-100 \mathrm{ng} / \mathrm{mL})$, intact serum PTH of $54.01 \mathrm{pg} / \mathrm{mL}(\mathrm{N}: 15-65 \mathrm{pg} / \mathrm{mL})$. Contrast computed tomography found a hypo-dense (compare to normal skull structure), well shaped, a bone mass of oval form at the level of left frontal area (of 2.67 by 0.96 by $1.82 \mathrm{~cm}$ ). No cerebral edema was detected. Whole body bone scintigram with $99 \mathrm{~m}$ Technetium - HDP (740 MBq) revealed an intense, homogenous, well shaped uptake of the tracer with bone tropism at the same level as CT scan did. No other lesion was detected. Daily oral vitamin D supplements were offered to the patient who is going to be clinically followed-up since she refused any further investigations, including surgery.

Conclusion: Despite the lack of pathological confirmation, the bone imagery and biochemical investigations are highly suggestive for a bone- forming tumor, probably a begin type sine the slow clinical evolution is positive for more than a decade. In the absence of tumor remove, local deformity and pain is eventually expected. The association with vitamin $\mathrm{D}$ deficiency is most probably incidental and more likely to be correlated with the mild muscle and skeleton accuses.

\section{P714}

CORRELATION BETWEEN THE LEVELS OF 25(OH) D AND BONE TURNOVERS AMONG POSTMENOPAUSAL OSTEOPOROSIS WOMEN WITH NEPHROLITHIASIS

K. Vasilenka $^{1}$, E. Rudenka ${ }^{2}$, H. Babak ${ }^{3}$

${ }^{1}$ Minsk City Center of Osteoporosis, Minsk, Belarus, ${ }^{2}$ Belarusian State Medical University, Minsk, Belarus, ${ }^{3}$ Clinical Hospital, Minsk, Belarus

Objective: An important regulator of calcium-phosphorus metabolism, including kidney, is vitamin D. Disturbance in calciumphosphorus metabolism can cause the formation of nephrolithiasis, wherein the most forms of kidney stones form with calcium. Also vitamin $\mathrm{D}$ is requisite for building bone. The aim of this study was to analyze the serum levels of $25(\mathrm{OH}) \mathrm{D}$ and bone turnovers(Total P1NP, osteocalcin and $\beta$-CrossLaps) with and without nephrolithiasis among postmenopausal osteoporosis women.

Material and Methods: 103 women with postmenopausal osteoporosis were examined. Median age and BMI were $64.2 \pm 3.38$ years and $22.6 \pm 3.57 \mathrm{~kg} / \mathrm{m} 2$, respectively. Exclusion criteria were the histories of any kind of proved rheumatologic or endocrine diseases, blood creatinine level of $>100 \mathrm{mmol} / \mathrm{l}$, intake of active vitamin D metabolites within 1 month prior the blood test. All the examined were divided into 2 groups according to presence of nephrolithiasis. Group I $(\mathrm{n}=58)$ had kidney stones, group II $(n=45)$ did not have a history of nephrolithiasis. Measurement of BMD at lumbar spine and femurs was performed by the 
method of DXA (Lunar Prodigy, GE, USA). Serum levels of 25(OH)D, Total P1NP, osteocalcin and $\beta$ - CrossLaps were determined using the chemiluminescent assay (analyzer - Cobas e 411). Statistical analysis was performed using the program Statistica 8.

Results: Group II indicated a significantly higher serum level of $25(\mathrm{OH}) \mathrm{D} 25.45$ [24.13:26.41] ng/ml, than group I, where serum level of $25(\mathrm{OH}) \mathrm{D}$ was 20.73 [19.28:21.84] $\mathrm{ng} / \mathrm{ml}$ $(p=0.034)$. There were no statistical differences between the groups for serum levels of osteocalcin, Total P1NP and $\beta$ CrossLaps ( $\mathrm{p}>0.05)$.

Conclusion: In both groups among women with postmenopausal osteoporosis vitamin D insufficiency was detected. Postmenopausal osteoporosis women without kidney stones demonstrated significantly higher levels of vitamin D in blood, than women with nephrolithiasis. Require more indepth study of the community of these states for their prevention and treatment.

\section{P716}

GASTROINTESTINAL EVENTS AND ASSOCIATION WITH TREATMENT SATISFACTION AMONG OSTEOPOROTIC PATIENTS: 12 MONTH OBSERVATIONS FROM THE MUSIC OS EUROPE AND CANADA STUDY

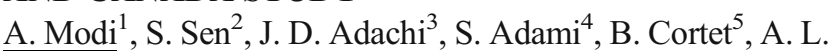

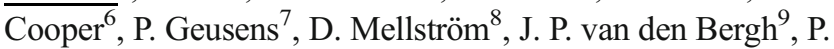
Keown ${ }^{10}$, S. Sajjan ${ }^{11}$

${ }^{1}$ Center for Observational and Real-World Evidence, Kenilworth, United States, ${ }^{2}$ Center for Observational and Real-World Evidence, Merck \& Co., Inc, Kenilworth, United States, ${ }^{3}$ Department of Medicine, St. Joseph's Hospital, McMaster University, Hamilton, Ontario, Canada, ${ }^{4}$ University of Verona, Verona, Italy, ${ }^{5}$ Department of Rheumatology, Lille University Hospital, Lille, France, ${ }^{6}$ Bridge Medical Center, Crawley, United Kingdom, ${ }^{7}$ Department of Internal Medicine, Subdivision of Rheumatology, Maastricht University Medical Centre, Maastricht, Netherlands, ${ }^{8}$ Department of Internal Medicine and Geriatrics, Gothenburg University, Göteborg, Sweden, ${ }^{9}$ Department of Internal Medicine, VieCuri Medical Center, Venlo, Netherlands, ${ }^{10}$ Syreon Corporation, Vancouver, Canada, ${ }^{11}$ Center for Observational and Real-World Evidence, Merck \& Co., Inc., Kenilworth, United States

Objective: To assess association of gastrointestinal (GI) events and treatment satisfaction at 12 months among patients treated for osteoporosis (OP).

Methods: In this analysis of the European and Canadian cohorts of the Medication Use Patterns, Treatment Satisfaction, and Inadequate Control of Osteoporosis Study (MUSIC OS), osteoporotic women $\geq 50$ years treated with OP medication were enrolled in the study during routine physician visits. Women diagnosed with Parkinson's disease, neuromuscular disease, Paget's disease, or malignant neoplasm or treated with any injectable OP medication at enrollment were excluded. Self-reported treatment satisfaction was compared between patients with and without GI events. GI events were self-assessed and included a variety of mild to moderate upper and lower GI symptoms. Developing GI events were those that were absent at baseline, but emergent at 12 months. Continuing GI events were those that were reported at both baseline and 12 months. Treatment satisfaction was measured with the Osteoporosis Patient Satisfaction Questionnaire (scale 0-100). Data were compiled at baseline and 12 months. Satisfaction scores were plotted for each time point, and the least-squares mean differences between patients with and without GI events were calculated using multivariate analyses. Results: A total of 2,959 patients with a mean age of 69.4 years were eligible for the analysis. At baseline, $68.1 \%$ of the patients reported having GI events in the past 6 months. At 12 months, $54.8 \%$ of patients reported experiencing GI events in the past 6 months. Covariate-adjusted treatment satisfaction scores were lower (i.e., worse) at each time point for patients with GI events than patients without GI events: 77.1 and 83.0, respectively, at baseline and 79.4 and 86.5, respectively, for patients with and without developing GI events at 12 months $(P<0.001$ for each comparison). The covariateadjusted least-squares mean $(95 \% \mathrm{CI})$ difference in treatment satisfaction between patients with and without continuing GI events at 12 months was $-10.59(-12.201,-8.971 ; P<0.001)$ points. The findings were similar among new and experienced users of osteoporosis therapy.

Conclusions: The presence of GI events was significantly associated with decreased treatment satisfaction in patients being treated for OP.

\section{P717}

GROWTH RATE OF MANDIBLE IN RATS OF DIFFERENT AGES AFTER EXCESSIVE PALM OIL INTAKE

$\underline{\text { K. R. Ismailova }}^{1}$, V. I. Luzin ${ }^{1}$, I. S. Prikhodchenko ${ }^{1}$, Y. P. Osipova $^{1}$

${ }^{1}$ SE Lugansk State Medical University, Lugansk, Ukraine

Objectives: To study growth and formation rates of mandible in rats after excessive palm oil (PO) intake.

Materials and methods: The experiment involved 144 rats of three ages: immature ( 1 month old), mature ( 6 month old) and senile (22 month old). All experimental animals received intragastric PO in dosage of $30 \mathrm{mg} / \mathrm{kg}$ (modeled alimentary obesity). The control animals received the same amount of saline. The animals were withdrawn from the experiment by the 1 st, the 10 th, the 30 th and the 60 th day after 6 -week of PO 
intake. The mandibles were measured according to traditional method.

Results: Excessive intake of PO resulted in inhibition of mandible growth and reduced bone mass gain rate. The alterations started manifesting from the 1st day of observation and continued growing throughout the whole experiment. Mandible length in immature animals was lower than that of the controls from the 10 th to the 60 th days by $3.55 \%, 3.60 \%$, and 3.70 respectively. Ramus height was lower in all observation terms - by $4.62 \%$, $4.61 \%, 4.95 \%$, and $5.45 \%$ respectively. Also, index of Simon (IS) was higher from the 10th up to the 60th day of observation by $3.62 \%, 3.86 \%$, and $4.19 \%$ respectively. In mature animals alterations were similar yet manifestations were observed beginning from the 10th day of observation period. IS from the 10th up to the 60th day of observation was higher than that of the controls by $3.94 \%, 3.63 \%$, and $3.67 \%$ respectively. Ramus height was lower by the 30th and the 60th days of observation by $4.03 \%$ and $4.66 \%$ respectively. In old animals alteration were registered only beginning from the 30th day of experiment. Ramus height was lower than that of the controls by the 30th and the 60 th days by $3.86 \%$ and $4.44 \%$ and IS was higher by $4.00 \%$ and $4.44 \%$ in the same terms.

Conclusions: Long-term excessive intake of $\mathrm{PO}$ results in inhibition of mandible growth and reduced bone mass gain rate. Terms and intensity of alterations depend on age of experimental animals. The earliest manifestations were observed in immature animals while in old ones same manifestations appeared later.

\section{P718}

24-MONTH TREATMENT WITH DENOSUMAB IN PATIENTS WITH OSTEOPOROSIS AND RHEUMATOID ARTHRITIS: EFFECTS ON BONE MINERAL DENSITY AND STRUCTURAL DAMAGE $\underline{\text { P. Kovalenko }^{1}}{ }^{\text {, I. Dydykina }}{ }^{1}$, A. Smirnov ${ }^{1}$, L. Alekseeva ${ }^{2}$, E. Nasonov $^{1}$

${ }^{1}$ Nasonova Research Institute of Rheumatology, Moscow, Russian Federation, ${ }^{2}$ Research Institute of Rheumatology, Moscow, Russian Federation

Objectives: To evaluate the effects of continuous treatment with denosumab in patients with rheumatoid arthritis (RA) and osteoporosis (OP).

Material and Methods: 27 postmenopausal women (mean age $58.4 \pm 7.4$ ) with RA (mean duration of RA 19.5 \pm 11.8 years) and OP received s/c denosumab $60 \mathrm{mg}$ every 6 months pro 2 years. According to X-ray 8 (29.6\%) patients had the 2nd, $8(29.6 \%)$ - the 3 rd and 11 (40.8\%) - the 4th stage of RA. 17 patients $(62.9 \%)$ continued glucocorticoids. The primary end points were the changes from baseline at 12and 24-month in BMD (by DXA at 3 sites: lumbar spine (L1L4), hip neck (HN) and distal forearm (DF) and the Sharp/van der Heijde (SVH) score of hands and feet X-rays. Statistica 6.0 was used in statistical analysis.

Results: At L1-L4 the significant increase of BMD was noted both at 12- and 24-month point in comparison with baseline: $0.789 \pm 0.079 \mathrm{~g} / \mathrm{cm}^{2}$ vs. $0.818 \pm 0.077 \mathrm{~g} / \mathrm{cm}^{2}(p<0.001)$ and $0.789 \pm 0.079 \mathrm{~g} / \mathrm{cm}^{2}$ vs. $0.836 \pm 0.078 \mathrm{~g} / \mathrm{cm}^{2}(p<0.001)$, respectively. The same was noted at DF - the increase of BMD at 12and 24-month point: $0.480 \pm 0.102 \mathrm{~g} / \mathrm{cm}^{2}$ vs. $0.493 \pm 0.095 \mathrm{~g} /$ $\mathrm{cm}^{2}(p=0.01)$ and $0.480 \pm 0.102 \mathrm{~g} / \mathrm{cm}^{2}$ vs. $0.511 \pm 0.104 \mathrm{~g} / \mathrm{cm}^{2}$ $(p<0.001)$, respectively. At HN it was noted the stabilization at both points $(p>0.05)$. The erosion and total SVH score were increased at 12 - and 24-month point $(p<0.05)$, while the narrowing score was not changed significantly at both points.

Conclusions: After 24 months of therapy with denosumab it was shown the significant increase of BMD at L1-L4 and distal part of forearm and the significant increase was observed both at the first and the following year of therapy. At $\mathrm{HN}$ it was observed the stabilization of BMD during the therapy. The erosion score and total SVH score were increased after treatment, the narrowing score did not change significantly for two years of observation.

\section{P719}

\section{GASTROINTESTINAL EVENTS AND ASSOCIATION WITH QUALITY OF LIFE AMONG OSTEOPOROSIS PATIENTS: 12 MONTH OBSERVATIONS FROM THE MUSIC OS EUROPE AND CANADA STUDY}

A. Modi ${ }^{1}$, S. Sen ${ }^{1}$, J. D. Adachi ${ }^{2}$, S. Adami ${ }^{3}$, B. Cortet ${ }^{4}$, A. L.

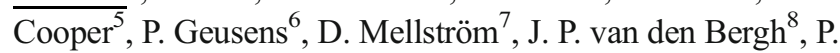
Keown ${ }^{9}$, S. Sajjan ${ }^{1}$

${ }^{1}$ Center for Observational and Real-World Evidence, Merck \& Co., Inc., Kenilworth, United States, ${ }^{2}$ Department of Medicine, St. Joseph's Hospital, McMaster University, Hamilton, Ontario, Canada, ${ }^{3}$ University of Verona, Verona, Italy, ${ }^{4}$ Department of Rheumatology, Lille University Hospital, Lille, France, ${ }^{5}$ Bridge Medical Center, Crawley, United Kingdom, ${ }^{6}$ Department of Internal Medicine, Subdivision of Rheumatology, Maastricht University Medical Centre, Maastricht, Netherlands, ${ }^{7}$ Department of Internal Medicine and Geriatrics, Gothenburg University, Göteborg, Sweden, ${ }^{8}$ Department of Internal Medicine, VieCuri Medical Center, Venlo, Netherlands, ${ }^{9}$ Syreon Corporation, Vancouver, Canada

Objective: To assess the association of gastrointestinal (GI) events with quality of life (QoL) in patients treated for osteoporosis (OP).

Material and Methods: The Medication Use Patterns, Treatment Satisfaction, and Inadequate Control of Osteoporosis Study (MUSIC OS) was a multinational, prospective study in Europe and Canada which enrolled osteoporotic women $\geq 50$ years treated with OP medication. Women 
diagnosed with Parkinson's disease, neuromuscular disease, Paget's disease, malignant neoplasm or treated with any injectable OP medication at enrollment were excluded. In this analysis, self-reported QoL was compared between patients with and without GI events. GI events were self-assessed and included a variety of mild to moderate upper (e.g., heartburn) and lower (e.g., constipation) GI symptoms. General health-related QoL was measured with the EuroQol-5D utility score (scale 0-1) and visual analog scale (scale 0-100). OPspecific QoL was measured with the Osteoporosis Assessment Questionnaire (physical function, emotional status, and back pain domains, each with scale 0-100). Data were compiled at baseline and 12 months. QoL and satisfaction scores were plotted for each time point, and the least-squares mean differences between patients with and without GI events were calculated using multivariate analyses.

Results: A total of 2,959 patients with a mean age of 69.4 years were eligible for the analysis. At baseline, $68.1 \%$ of the patients reported having GI events in the past 6 months. At 12 months, $54.8 \%$ of patients reported experiencing GI events in the past 6 months. QoL scores changed very little from baseline to 12 months. Scores at each time point were lower (i.e., worse) for patients with GI events than patients without GI events. Covariate-adjusted least-squares mean $(95 \% \mathrm{CI})$ differences between patients with and without continuing GI events at 12 months were $-0.07(-0.088,-0.053)$ for the EuroQol-5D utility score, $-7.29(-8.985,-5.603)$ for the EuroQol-5D visual analog scale, $-4.83(-6.864,-2.805)$ for physical function, $-7.38(-9.459,-5.303)$ for emotional status, and -12.27 $(-14.994,-9.549)$ for back pain. The findings were similar in subgroups of new and experienced users of OP therapy.

Conclusions: The presence of GI events was significantly associated with decreased QoL in patients being treated for OP.

\section{P720}

BONE QUALITY GENE POLYMORPHISM FREQUENCY IN BELARUSIAN POSTMENOPAUSAL WOMEN WITH OSTEOPOROSIS

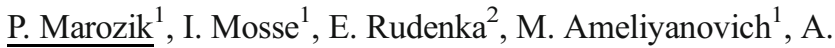
Rudenka $^{3}$, O. Samakhavets ${ }^{4}$, K. Nestsiarenka ${ }^{1}$

${ }^{1}$ Institute of Genetics \& Cytology NAS Belarus, Minsk, Belarus, ${ }^{2}$ Belarusian State Medical University, Minsk, Belarus, ${ }^{3}$ Belarusian Medical Academy of Postgraduate Training, Minsk, Belarus, ${ }^{4}$ Minsk City Clinical Hospital, Minsk, Belarus

Objective: The traditional evaluation of bone fracture risk, based on BMD measure, performed by absorption densitometry, does not always reliable, because it is not considering bone quality. Bone quality includes several aspects of bone structure, such as microarchitecture and bone matrix composition, which can be evaluated in vivo only using bone biopsy or autopsy specimens. The analysis of genes, responsible for the synthesis of bone matrix components, may help to perform osteoporotic fracture risk assessment based on evaluation of low bone quality risk. In present study, we analyzed the genetic variants of collagen type 1 ( $\alpha 1$ - and $\alpha 2$-chains), collagen type 5 and matrix metalloproteinase- 3 genes, which define bone quality, to reveal their association with postmenopausal osteoporosis (PMO) in Belarusian women.

Materials and methods: Case group included women with severe PMO (66 Belarusians, average age 58.3 \pm 6.2 years), the control group comprised postmenopausal women with the BMD T-score of $>-2.5$ and without previous osteoporotic fractures (77 Belarusians, 56.7 \pm 7.42 years). DNA was extracted from bloodspots dried on special cards (MachereyNagel, Germany). Polymorphic variants in candidate genes

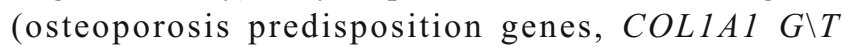
(rs1800012), COL1A2 $A \backslash G$ (rs42517), COL5A1 $C \backslash T$ (rs12722) and MMP3 $A \backslash G$ (rs679620) were determined using PCR analysis. The $\chi^{2}$ test was used to compare allele frequencies between analysed groups. The differences were considered significant at $P<0.05$.

Results: The analysis revealed statistically significant association of COL1A2 rs42517 gene polymorphisms with PMO. The risk of osteoporosis was 2.9 times higher for the bearers of $G$-allele compared to the bearers of $A$-allele $(P=0.03)$. There was also a tendency for an increase of PMO risk for T-allele bearers of COL1A1 rs1800012 gene polymorphism. It is known that these both gene polymorphisms determine increased level of homotrimeric type I collagen form with three $\alpha 1$-chains. No statistically significant association with PMO was found for COL5A1 and MMP3 gene polymorphisms.

Conclusion: The findings of this study suggest that screening of COL1A1 and COL1A2 genetic markers may help to evaluate the risk of low bone quality.

\section{P721 \\ PREVALENCE OF OSTEOPOROSIS IN AN ANKY- LOSING SPONDYLITIS COHORT}

G. E. Fitzgerald $^{1}$, D. J. Lundon ${ }^{2}$, P. Gallagher ${ }^{3}$, C. O Sullivan $^{4}$, K. O Rourke ${ }^{5}$, C. Sheehy ${ }^{6}$, F. Stafford ${ }^{7}$, C. Silke ${ }^{8}$, M. Haroon ${ }^{9}$, R. Mullan ${ }^{10}$, O. FitzGerald ${ }^{3}$, F. O Shea ${ }^{1}$

${ }^{1}$ Department of Rheumatology, St James's Hospital, Dublin, Ireland, ${ }^{2}$ School of Medicine and Medical Sciences, University College Dublin, Dublin, Ireland, ${ }^{3}$ Department of Rheumatology, St Vincent's Hospital, Dublin, Ireland, ${ }^{4}$ Department of Rheumatology, Galway University Hospital, Galway, Ireland, ${ }^{5}$ Department of Rheumatology, Midlands Regional Hospital, Tullamore, Ireland, ${ }^{6}$ Department of Rheumatology, University Hospital Waterford, Waterford, 
Ireland, ${ }^{7}$ Department of Rheumatology, Blackrock Clinic, Dublin, Ireland, ${ }^{8}$ Department of Rheumatology, Sligo General Hospital, Sligo, Ireland, ${ }^{9}$ Department of Rheumatology, Kerry General Hospital, Kerry, Ireland, ${ }^{10}$ Department of Rheumatology, Tallaght Hospital, Dublin, Ireland

Background: The Ankylosing Spondylitis Registry of Ireland (ASRI) is a web-based database established in 2013, the objectives of which are to provide descriptive epidemiological data on the ankylosing spondylitis (AS) population in Ireland and to establish a registry for potential future studies of genetics, aetiology and therapeutics. The prevalence of osteoporosis is higher in AS patients than age- and sex- matched controls, with potential for vertebral fractures increased. However, the true prevalence is unknown. There is no data on the prevalence of osteoporosis in an Irish cohort.

Objective: To determine the prevalence of low BMD in an Irish AS cohort.

Materials and methods: A standardised detailed clinical assessment was performed on each patient. Disease activity was assessed by Bath AS Disease Activity Index (BASDAI), function by the Bath AS Functional Index (BASFI) and Health Assessment Questionnaire (HAQ) and quality of life by AS Quality of Life (ASQoL). Structured interviews provided patient-reported data. Presence or absence of DXA testing and result was recorded. BMD was categorised according to the World Health Organisation criteria into normal BMD, osteopenia or osteoporosis. Statistical analysis was performed using SPSS.

Results: As of October 2015, 416 patients are enrolled in ASRI: $78.1 \%$ males, mean age 47.95 (SD 12.4), mean disease duration 20.9 years (SD 12.2), average delay to diagnosis of 8.8 years (SD 8.3). Mean BASDAI is 3.8 (SD 2.5), BASFI 3.7 (SD 2.7), HAQ 0.53 (SD 0.51) and ASQoL 6.15 (SD 5.5). DXAs have been performed in $24.75 \%(n=103)$ of the cohort, of which $39.8 \%(n=41)$ have osteopenia and $10.7 \%$ $(n=11)$ have osteoporosis. Low BMD is significantly correlated with men and advancing age. There is no association with disease activity or duration. Low BMD is more prevalent in patients treated with one or more biologics compared to those never treated. The self-reported prevalence of osteoporosis is $6.4 \%$ ( $n=27 ; 19$ males).

Conclusion: There is an elevated prevalence of low BMD in this cohort, with no association with disease severity. The majority of affected patients were unaware, as demonstrated by the low self-reported prevalence. More research and education is needed.
P722

A RANDOMIZED DOUBLE-BLIND PLACEBOCONTROLLED TRIAL OF VITAMIN D SUPPLEMENTATION IN JUVENILE-ONSET SYSTEMIC LUPUS ERYTHEMATOSUS: EFFECTS ON MICROARCHITECTURE USING HR-PQCT

J. A. Paupitz ${ }^{1}$, G. L. Lima ${ }^{1}$, N. E. Aikawa ${ }^{2}$, J. C. Alvarenga ${ }^{1}$, E. Bonfa $^{1}$, R. M. R. Pereira ${ }^{1}$

${ }^{1}$ Rheumatology Division, University of São Paulo, São Paulo, Brazil, ${ }^{2}$ Pediatric Rheumatology Unit, University of São Paulo, São Paulo, Brazil

Purpose: Vitamin D has an important effect in bone quality and vitamin D deficiency in juvenile onset systemic lupus erythematosus patients (JoSLE) may be associated with lower $\mathrm{BMD}$ at spine and whole body sites. Besides, vitamin $\mathrm{D}$ has an important immunomodulatory effect that justified its use in autoimmune diseases including JoSLE. However, there are no trials that directly addressed the boosting of serum levels of 25-hydroxyvitamin D (25OHD) in bone microarchitecture in JoSLE. The aim of this study was to evaluate the effect of vitamin D supplementation on bone microarchitecture parameters in JoSLE.

Methods: This study was a randomized double-blind placebocontrolled 24-week trial conducted in the Rheumatology Outpatient Clinic, University of São Paulo. Forty JoSLE patients (according to ACR classification criteria) up to $25 \mathrm{y}$-old were randomized (1:1) to receive oral cholecalciferol 50,000 IU/week (JoSLE-VitD, $n=20$ ) or placebo (JoSLE-PL, $n=20$ ). Before randomization a three months washout of vitamin $\mathrm{D}$ was performed for all patients. Other medications remained stable throughout the study. Serum levels of 25OHD were measured using radioimmunoassay. Disease activity was assessed using SLE Disease Activity Index (SLEDAI). Bone microarchitecture and volumetric bone density were analysed at baseline and after 24 weeks using HR-pQCT at tibia site.

Results: At baseline, groups were similar regarding, age, BMI, organ involvement, SLEDAI, glucocorticoid current dose use, immunosuppressants drugs and parameters of bone microarchitecture and volumetric bone density. The mean 25OHD serum levels at baseline were similar in JoSLE-VitD and JoSLE-PL groups $(19.1 \pm 7.5$ vs. $19.5 \pm 6.9 \mathrm{ng} / \mathrm{mL}$, $p=0.82$ ), as well the mean of total calcium serum levels ( 9.4 \pm 0.5 vs. $9.1 \pm 0.5 \mathrm{mg} / \mathrm{dL}, p=0.12$ ), respectively. After 24 weeks, the mean 25OHD serum levels was higher in the JoSLE-VitD group ( $31.3 \pm 8.6$ vs. $16.5 \pm 5.8 \mathrm{ng} / \mathrm{mL}, p<0.001)$. Concerning bone microarchitecture, an increase in trabecular number ( $\Delta$ Tb.N: $0.167 \pm 0.220$ vs. $0.031 \pm 0.190 \mathrm{~mm}, p=0.04$ ) and a decrease in trabecular separation $(\Delta \mathrm{Tb}$.Sp: $-0.044 \pm 0.050$ vs. $0.003 \pm 0.050 \mathrm{~mm}, p=0.024)$ were observed in the JoSLE-VitD group compared to JoSLE-PL at tibia site. No other differences were observed in structural parameters [trabecular (Tb.Th) and 
cortical thickness (Ct.Th)], or volumetric bone mineral densities [trabecular (Tb.BMD) and cortical (Ct.BMD)] ( $p>0.05)$.

Conclusion: This study suggests that cholecalciferol supplementation for 24 weeks is effective in improving bone microarchitecture parameters in JoSLE patients. Therefore, a therapeutic intervention may be recommended for patients with 25OHD deficiency. (Clinical Trial Registry: NCT01892748).

\section{P723}

EVALUATION OF SERUM LEVELS OF PROGRANULIN AND BONE MORPHOGENETIC PROTEIN-4 IN PATIENTS WITH OSTEOARTHRITIS S. Hira ${ }^{1}$, C. Tamam ${ }^{2}$, U. Demirpek ${ }^{3}$, M. Gem ${ }^{4}$

${ }^{1}$ Department of Biochemistry, Tatvan Military Hospital, Bitlis, Turkey, ${ }^{2}$ Department of Orthopedics and Traumatology, Tatvan Military Hospital, Bitlis, Turkey, ${ }^{3}$ Department of Clinical Microbiology, Tatvan Military Hospital, Bitlis, Turkey, ${ }^{4}$ Department of Orthopedics and Traumatology, Faculty of Medicine, Dicle University, Diyarbakir, Turkey

Osteoarthritis (OA) is a chronic, slowly progressive disease of the joints and is one of the most common causes of pain and disability in middle-aged and older people. The etiology and pathogenesis underlying this disease are poorly understood. Progranulin (PRGN), a secreted glycoprotein expressed in many cell types, has been linked to wide variety of biological processes. In recent years, increasing evidence suggests that PRGN stimulates chondrocyte proliferation and is considered an essential regulator of cartilage metabolism. Bone morphogenetic protein-4 (BMP-4), a member of transforming growth factor- $\beta$ superfamily of proteins, is involved in bone and cartilage development and induces chondrogenesis. This study aimed to investigate serum BMP-4 and PRGN levels in patients with $\mathrm{OA}$ and present a new evidence of pathogenesis OA disease. The study included 38 female osteoarthritis patients and 38 female healthy volunteers. Serum PRGN and BMP-4 concentrations were measured using enzyme-linked immunosorbent assay. We also measured BMI and erythrocyte sedimentation rate (ESR), white blood cells (WBC) and neutrophil lymphocyte ratio (NLR). Mean BMP4 levels were significantly lower in OA women compared to controls $(29.66 \pm 13.61$ vs. $72.81 \pm 44.06 \mathrm{ng} / \mathrm{mL}, p<0.001)$. Mean PRGN levels were found to be significantly lower in OA women compared to controls $(71.93 \pm 33.83$ vs. 268.33 $\pm 180.45 \mathrm{ng} / \mathrm{mL}, p<0.001)$. There were no significant differences in WBC and NLR levels between two groups ( $p=0.763$, $p=0.925$, respectively). ESR values was significantly higher in patients group than controls group $(p=0.022)$. There was a significant positive correlation between serum BMP-4 levels and serum PRGN levels in patients with OA. In conclusion,
BMP-4 and PRGN levels may play a role in the pathogenesis of $\mathrm{OA}$ and could be a useful biomarker of $\mathrm{OA}$.

\section{P724}

ESTIMATING THE BURDEN OF OSTEOPOROSISRELATED FRACTURES IN TERMS OF HEALTH-RELATED QUALITY OF LIFE, UTILITY, AND RESOURCE USE (BOOST): ASSESSMENT OF HEALTH UTILITY IN THE YEAR AFTER FRACTURE

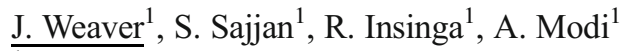

${ }^{1}$ Merck \& Co., Inc., Kenilworth, NJ, United States

Objectives: To estimate the utility of health states, just prior to an osteoporotic (OP) fracture, and during the first year post-fracture. Materials and Methods: This prospective, observational study was conducted in the Netherlands (NLD), South Korea (KOR) and Taiwan (TWN). Patients enrolled in the study were required to be female, post-menopausal, $\geq 50$ years of age, and recently diagnosed by their physician with an osteoporotic fracture (hip, clinical vertebral, wrist, humeral, other). All assessments collected data on utility using the EuroQol 5 Dimension 5 Level (EQ-5D-5 L) questionnaire. The study included a baseline visit within two weeks of OP fracture diagnosis with the EQ-5D-5 L administered retrospectively for prefracture utility, immediately after fracture and at 1, 3, 6 and 12 months post-fracture. Descriptive statistics for demographic and clinical characteristics at baseline were generated by fracture type and country. Utility was summarized by fracture type and country at baseline and each study data collection point, and EQ-5D Utility score change from baseline and ratio of cumulative 1 year post-fracture utility to pre-fracture utility was also assessed. Only fracture groups with a sample size $\geq 20$ at baseline are reported.

Results: The NLD, KOR and TWN enrolled, 143, 174 and 102 patients respectively. Mean age at enrollment ranged from the early 60 s to late 70 s, depending on fracture type and country. For all countries and fracture types, mean utility score dropped significantly immediately post-fracture, by as much as -0.46 points for patients with other fractures in KOR, to as little as -0.165 points for wrist fracture patients in NLD. Over the following year, health utility scores slowly improved (Fig. 1). The ratio of post-fracture utility (over 1 year) to pre-fracture utility was 0.751 for hip fracture (KOR), 0.789 (KOR) and 0.759 (TWN) for clinical vertebral fracture, 0.983 (NLD) and 0.808 (KOR) for wrist fracture and 0.960 (NLD) for humeral fracture. Conclusions: OP fractures substantially affect the health utility of OP patients across fracture types and countries. The long term burden of these fractures on health utility relative to prefracture values was less extensive in humeral and wrist fractures as compared to hip and vertebral fractures.

Figure 1 


\section{EQ-5D-5L}

South Korea

\begin{tabular}{|c|c|}
\hline Fracture type & PF value \\
\hline Hip & 0.719 \\
\hline CV & 0.793 \\
\hline Wrist & 0.898 \\
\hline Other & 0.911 \\
\hline
\end{tabular}

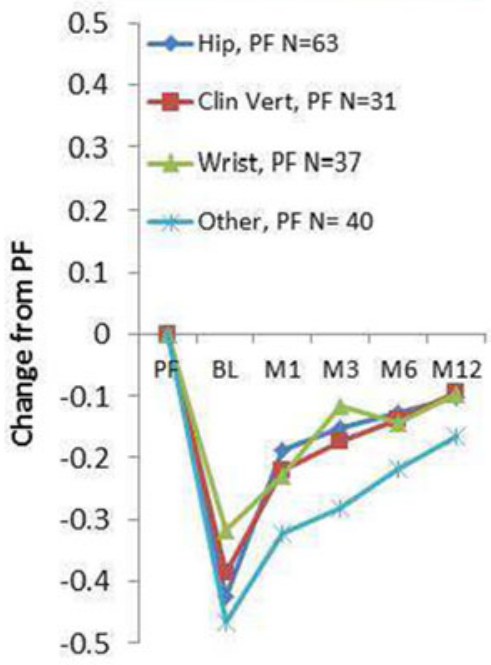

Taiwan

\begin{tabular}{|c|c|}
\hline Fracture type & PF value \\
\hline CV & 0.764 \\
\hline Other & 0.855 \\
\hline
\end{tabular}

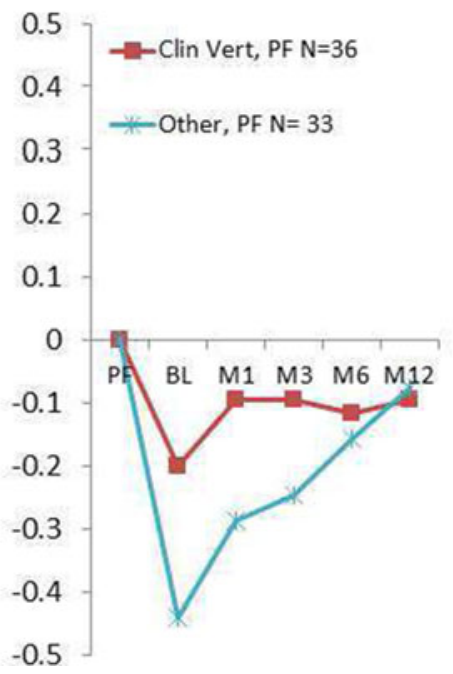

The Netherlands

\begin{tabular}{|c|c|}
\hline Fracture type & PF value \\
\hline Wrist & 0.854 \\
\hline Humeral & 0.781 \\
\hline Other & 0.848 \\
\hline
\end{tabular}

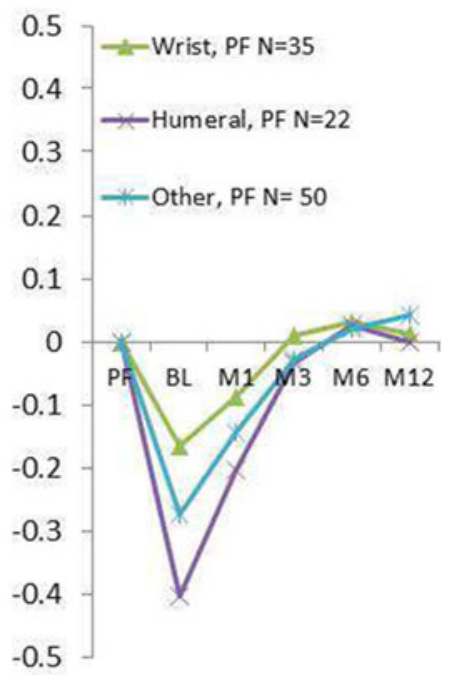

EQ-5D-5 L score change from pre-fracture in the year after fracture.

\section{P725}

PRIMARY HYPOXALURIA TYPE 1 AS A CAUSE OF SECONDARY HYPERPARATHYROIDISM WITH INCREASED BONE DENSITY

E. A. Pigarova $^{1}$, J. A. Krupinova ${ }^{1}$, L. K. Dzeranova ${ }^{1}$, L. Y. Rozhinskaya $^{1}$

${ }^{1}$ Neuroendocrinology and Bone Diseases, Endocrinology Research Centre, Moscow, Russian Federation

Objectives: Primary hypoxaluria type 1 (PH1) is a rare genetic disorder characterized by recurrent kidney and bladder calcium oxalate stones that often results in end stage renal disease (ESRD). When kidneys excretion is affected, oxalate gets deposited in tissues throughout the body negatively affecting their function. Secondary hyperparathyroidism $(\mathrm{SH})$ is common in ESRD due to low conversion of vitamin $\mathrm{D}$ to its active form, but approaches to the management of SH in patients with PH1 should be different.

Material and Methods: Clinical case presentation.

Results: A 18-yo female with unspecified cause kidney failure treated by peritoneum dialysis was diagnosed with $\mathrm{SH}$ (PTH -
$1661 \mathrm{pg} / \mathrm{ml}(<69), \mathrm{Ca}$ ion $-1.23 \mathrm{mmol} / \mathrm{l}(1.03-1.30), \mathrm{P}-$ $1.59 \mathrm{mmol} / \mathrm{l}(0.86-1.42)$, alkaline phosphatase $-787 \mathrm{U}$ $(<250)$ ). US showed all four hyperplastic parathyroid glands. Her primary complains were bone pain and leg cramps. She was started with calcium carbonate 1 tsp. day and $0.5 \mu \mathrm{g}$ of alfacalcidol with complete resolution of complains but no effect on PTH. Addition of $30 \mathrm{mg}$ cinacalcet also had no effect and was discontinued as higher dose could not be afforded. Her DXA showed an increased BMD assessed by Z-criteria $+3.1 \mathrm{SD}$ at the spine and +1.2 SD at the hip with further increase in the following two years up to $+5.0 \mathrm{SD}$ and +2.9 $\mathrm{SD}$, respectively. Later she was switched to hemodialysis and during evaluation for renal transplantation $\mathrm{PH} 1$ was suspected due to renal stone disease in relatives and AGXT gene mutation analysis confirmed compound heterozygote. Confirmation of PH1 changers the way the patient should be treated: kidney + liver transplantation and abstain from further increase in doses of calcium and alfacalcidol.

Conclusion: Diagnosis of PH1 should be a part of differential diagnosis in patients with SH due to ESRD caused by renal stone disease and high BMD as treatment approaches differ. 
P726

INVESTIGATION OF OSTEOPOROSIS IN ANKYLOSING SPONDYLITIS: EPIDEMIOLOGY, ASSESSMENT TECHNIQUES, TREATMENT - A RESEARCH PROPOSAL

G. E. Fitzgerald $^{1}$, G. Cunnane ${ }^{1}$, F. O Shea ${ }^{1}$

${ }^{1}$ Department of Rheumatology, St James's Hospital, Dublin, Ireland

Background: Ankylosing spondylitis (AS) is a chronic inflammatory rheumatic disease. It is a major cause of functional disability in young adults, with excess risk of vertebral fractures and devastating neurological outcomes. Evidence suggests the prevalence of osteoporosis is increased in AS patients, further adding to this risk. However, the true prevalence of osteoporosis in this cohort is unknown, varying from $3-47 \%$ depending on the measurement technique used. The World Health Organisation (WHO) defines osteoporosis using BMD estimation by anteroposterior (AP) DXA techniques. However, this technique can overestimate BMD in advanced AS disease due to the spinal changes that occur. Optimal evaluation techniques are yet to be ascertained. Lateral DXA scanning, quantitative ultrasound (QUS) of the calcaneus and bone turnover markers (BTM) may have a role. High-risk patients and treatment guidelines are as of yet undefined. The effect of exercise on BMD in AS has also not been evaluated.

Objectives: To establish an accurate profile of osteoporosis in AS patients, specifically to determine:

1. Epidemiology of osteoporosis

2. Accurate methods of BMD assessment

3. High-risk subgroups

4. The effect of treatment on bone turnover markers

5. The effect of an exercise intervention on BMD

Material and methods: AS patients aged 18 and over will be prospectively recruited from a database of patients attending a specialist AS clinic. Objective measures will be collected and will include anthropometric and demographic data, clinical history, medication history, disease severity (clinical and radiographic scores) and bloods (including BTM). BMD assessment will be performed (PA vs. lateral DXA, calcaneal QUS). A subset of patients will be randomised to a supervised exercise programme group or usual-treatment group, with objective measures performed pre- and post-intervention. Ethical approval will be obtained from the institution's research ethics committee. Statistical analysis will be performed using SPSS.

Conclusion: This study will expand the existing limited evidence base regarding osteoporosis epidemiology, diagnosis and management in AS patients.
P727

THE ASSOCIATION BETWEEN HYPERTENSION AND OSTEOPOROSIS AT THE RADIUS USING BMD AND T-SCORE OF THE RADIUS

I. Cotofana $^{1}$, R. I. Rosca ${ }^{1,2}$, A. Mirica ${ }^{1}$, M. Dobrescu ${ }^{1}$, M. Stoiceanu $^{1}$, C. Poiana ${ }^{1,2}$, D. L. Paun ${ }^{1,2}$

1"C.I. Parhon" National Institute of Endocrinology, Bucharest, Romania, "Carol Davila" University of Medicine and Pharmacy, Bucharest, Romania

Introduction: Hypertension and related cardiovascular diseases are reported to be associated with osteoporosis. A nutritional pathway related to dairy intake has been postulated for both diseases. Cardiovascular disease, hypertension, vascular calcification, and osteoporosis are common in elderly individuals and have previously been regarded as independent agerelated disorders. Some studies have suggested an overlap in the etiological mechanisms of these diseases - for example, bone metabolism and vascular physiology share several regulatory factors, and the process of vascular calcification in many ways resembles that of bone formation ${ }^{1}$. A high salt intake has been associated with hypertension and osteoporosis. One of the proposed pathogenetic mechanisms is an increased calcium excretion in urine. Urinary calcium loss induces a negative calcium balance that may predispose hypertensive subjects to developing greater bone $\operatorname{loss}^{2}$. A recent study from 2015 demonstrates a clear link between ischemic heart disease and cortical bone loss, and increased cortical porosity and possible fracture risk at the radius ${ }^{3}$.

Aim: We want to verify whether there is any association between hypertension and BMD of the radius in a clinical case-series of patients that presented themselves to "C.I. Parhon" - National Institute of Endocrinology in 2015 on our hospital ward.

Material \& Methods: We have included 20 patients from "C.I. Parhon" - National Institute of Endocrinology known with low T-score and BMD, osteoporosis and hypertension, and using osteodensitometry we have tested the value of BMD and T-score of the radius. We have included 10 patients from "C.I. Parhon - National Institute of Endocrinology" aged between 57 and 87.8 years (average 70.77), of female gender, known with postmenopausal osteoporosis and radius affectation. Osteoporosis presence was demonstrated by osteodensitometry examination at this level and the results showed $\mathrm{T}$ values of minimum -2.3 and maximum -4.4 (average -3.322), and BMI score of minimum 0.398 and maximum 0.543 (average 0.474 ). The subsequent cardiologic examination performed proved that 16 of 20 patients had arterial hypertension grade 2 or 3 , some of them being under antihypertensive treatment.

Conclusions: Thus we can say that there is probably a connection between arterial hypertension and 
osteoporosis with radius location. We will continue to demonstrate this link in an extensive study.

\section{References:}

1. Varenna M, et al. Calcif Tissue Int 2013;93:86

2. Caudarella R, et al. J Endocrinol Invest 2009;32

(4 Suppl): 15

3. Paccou J, et al. Osteoporos Int. 2015;26:1893

\section{P728}

DOES THE RELATIONSHIP BETWEEN BODY COMPOSITION AND BONE HEALTH VARY IN WEIGHT-BEARING AND NON-WEIGHT BEARING SITES? OBSERVATIONS FROM THE HERTFORDSHIRE COHORT STUDY

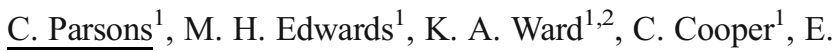
M. Dennison ${ }^{1}$

${ }^{1}$ MRC Lifecourse Epidemiology Unit, University of Southampton, Southampton, United Kingdom, ${ }^{2} \mathrm{MRC}$ Human Nutrition Research, University of Cambridge, Cambridge, United Kingdom

Objectives: BMD is related to both muscle mass and function, and adiposity. Less is understood about whether relationships are similar at both weight bearing and non-weight bearing sites. To understand this relationship further we studied the association between bone size, BMD and bone strength with muscle and fat area, as surrogates of muscle strength and adiposity respectively, in the upper and lower limbs.

Material and methods: This study was based in a subset of the Hertfordshire Cohort Study, a cohort of men and women born in 1931-9, who had undergone a pQCT scan of the radius ( $4,66 \%$ sites) and tibia ( $4 \%, 14 \%, 38 \%, 66 \%$ sites) (Stratec 2000). Bone outcomes and muscle and fat crosssectional area (CSA) were derived. Pearson's $r$ correlation coefficient was used to quantify the association between bone parameters and body composition.

Results: Data were available for 322 men and 320 women. The mean age was 69.3 (SD 2.6) years and mean BMI was 27.5 (SD 4.4). Muscle forearm CSA was positively associated with bone size and BMD in the upper limb at the $4 \%$ and $66 \%$ sites (radius total area $4 \% r=0.7,66 \%$ $r=0.7$; radius trabecular BMD $4 \% r=0.4$; cortical BMD $66 \% r=0.2$ ) and lower limbs at all 4 sites (tibia total area $4 \% r=0.6$; tibia total BMD $4 \% r=0.4$; tibia cortical BMD $14 \% r=0.4$ ). Relationships were broadly similar in both sexes. In women, a positive relationship was observed between fat CSA and both bone size and BMD in upper and lower limbs (total bone area $r=0.2$; radius total BMD $4 \% r=0.2$; tibia cortical area $14 \% r=0.2$; tibia cortical density $14 \% r=0.2$ ). No consistent relationship was found in men.
Conclusions: Muscle and fat CSA were significantly correlated with markers of bone health at both weight bearing and non-weight bearing sites, with muscle having stronger associations with bone health than fat area. However muscle and fat CSA measurements are only proxies for body composition and further study is required to understand these measurements correlate with muscle strength and adiposity.

P729

ANTIOXIDANTS AND BISPHOSPHONATES: EFFECTS IN DIFFERENT DISEASES THAT AFFECT THE BONE

$\underline{\text { J. A. Lopez-Morata }}^{1}$, M. Alcaraz ${ }^{2}$, A. Olivares ${ }^{2}$, J. D. Avilés Hernández ${ }^{3}$

${ }^{1}$ Orthogeriatrics Unit Hospital Perpetuo Socorro, Cartagena, Spain, ${ }^{2}$ University of Murcia, Murcia, Spain, ${ }^{3}$ Orthogeriatrics Unit, Hospital Virgen de la Arrixaca, Murcia, Spain

Background: Bisphosphonates are used in cancer-related hypercalcaemia, in complications of bone metastasis and in postmenopausal osteoporosis, and have often been associated with adverse complications.

Aim: To determine the protective effect of antioxidants, against growth inhibition of normal epithelial human prostatic (PNT2), transgenic adenocarcinoma of mouse prostate (TRAMP-C1) and metastatic melanoma cells (B16F10) in combined treatments with bisphosphonates and ionizing radiation (IR).

Materials and Methods: The growth inhibition on PNT2, TRAMP-C1 and B16F10 cells in the combined treatments with bisphosphonates (zoledronic acid, ibandronate and pamidronate) and IR in the presence and absence of apigenin was studied using a cell viability test.

Results: Zoledronic acid had a cytotoxic effect on PNT2, TRAMP-C1 and B16F10 cells $(p<0.001)$. However, ibandronate and pamidronate had a cytotoxic effect only on PNT2 cells $(p<0.001)$. The administration of apigenin in combined treatment with bisphosphonates and IR showed: a decrease in the cytotoxic effect on TRAMP-C1 and B16F10 cells in the treatment with ibandronate; a protective effect on normal PNT2 and melanoma cells, but not on TRAMP-C1 cells in the treatment with zoledronic acid; and provided protection only to PNT2 cells in the treatment with pamidronate. Rosmarinic acid exhibits a significant genoprotective capacity $(p<0.001)$ against X-rays with a protection factor of $58 \%$, and a dose reduction factor of 7.2. However, in metastatic B16F10 melanoma cells, rosmarinic acid acted not as a radioprotector, but as a sensitizing agent, increasing cellular death by $42 \%(p<0.001)$, with an enhancement ratio of 2.36 . Conclusion: The use of the antioxidant produced a greater decrease in the cytotoxic effect on the non tumor 
than in tumor cells when treated with bisphosphonatesalone and could be used in non tumor pathologies. However, in a combined treatment with IR, it can also provide protection to tumor cells, thus reducing the intended effect of the IR. Potentially, research into substances such as rosmarinic acid could help clarify mechanisms that provide protection on healthy normal cells, while exclusively damaging neoplastic cells, thus presenting a new strategy for patients undergoing radiotherapy for cancer.

\section{P730}

CENTRAL SENSITIZATION A LEADING MECHANISM IN CHRONIC PAIN OSTEOARTHRITIS

E. F. Turovskaia $^{1}$, L. I. Alekseeva ${ }^{1}$, E. G. Filatova ${ }^{2}$

${ }^{1}$ Department of Osteoarthritis and Osteoporosis in V.A.Nasonova Research Institute, Moscow, Russian Federation, ${ }^{2}$ Neurological Department I.M. Sechenov First Moscow State Medical University, Moscow, Russian Federation

Introduction: Traditionally pain in osteoarthritis (OA) considered being the cause of structural changes in bone, synovium, soft tissues and cartilage. But recent studies have shown some dissociations, that cannot be explained by nociceptive pain model. The level of pain doesn't correlate with structural changes in knee OA, $44 \%$ of patients continue to suffer from persistent pain after total knee transplantation and NSAID are not effective in $27 \%-61 \%$. Nociceptive mechanism cannot explain the presence of referred hyperalgesia (increase sensation to pain far from damaged joint) and other sensitive phenomena which can occur in an intact region. These data demonstrate that chronic pain(CP) in OA can also be caused by changes in central nervous system(CNS) - central sensitization(CS).

Aim: To reveal CS in patients with knee OA and demonstrate its role on pain syndrome.

Materials and methods: 89 women (45-65 age) with knee OA II-III Kellgren-Lawrence grade and CP (duration more than 3 months) and 23 women(45-65) healthy controls were included. Pain characteristics were examined by WOMAC, VAS. Structural changes were assessed by X-ray and USexamination of the knee. CS was examined by neuropathic pain scales (Paindetect and DN4), neurological examination and algometry (pressure pain thresholds(PPT) and wind-up phenomena). Emotional disturbances were assessed by Hospital Anxiety and Depression scale.

Results: Patients with chronic knee OA described neuropathic (NP) descriptors. The most common descriptors were: resembling an electric shock- $59.6 \%$, burning- $47.2 \%$, pins and needles $-46.1 \%$, numbness $-42.7 \%$ But patients did not have any neurological deficit. Examination of sensitive disorder revealed primary hyperalgesia (in the damaged knee) and also referred hyperalgesia $(\mathrm{RH})$ in the intact region(shank and even hip), in some cases allodynia(18\%) was revealed. RH is considered to be clinical features of CS. Patients were divided into two groups in accordance with presence of RH: $41.5 \%$ had RH and $58.5 \%$ did not have RH. RH did not correlate with age, duration of disease, BMI, quality of life and level of structural changes. The presence of RH associated with high levels of NP pain scales, correlated with higher pain intensity (VAS), poor WOMAC and significantly associated with higher level of depression. The presence of RH characterized by often described NP descriptors. Algometry was examined in 46 patients with OA and 23 women healthy controls. All patients had statistically lower PPT in sites near the knee and also in intact site compared with 23 healthy controls. Wind-up data showed no differences between groups. But the intensity of pain on the first stimuli and sequential 10 stimuli was statistically higher compared with controls.

Conclusion: Chronic pain OA is a complex of mechanism of pain: nociceptive pain and CS. CS plays a leading role in $40 \%$ case and cause NP phenomena and RH. Neurophysiological features of CS are low PPT and wind-up. CS can be the reason of intensive pain on VAS, low functional activity, high level of depression and doesn't correlate with structural changes. Therefore, one of the main ways of controlling pain should also target CNS mechanisms.

\section{P731}

\section{HEMATOLOGIC INDICES AND OSTEOARTHRITIS} S. Hira ${ }^{1}$, C. Tamam ${ }^{2}$

${ }^{1}$ Department of Biochemistry, Tatvan Military Hospital, Bitlis, Turkey, ${ }^{2}$ Department of Orthopedics and Traumatology, Tatvan Military Hospital, Bitlis, Turkey

Osteoarthritis (OA) is most common form of joint disease and one of the leading causes of disability and pain in elderly people worldwide. Historically, OA has been considered a noninflammatory disease, but more recently studies revealed that inflammation is a risk factor associated with both progression of cartilage destruction and signs and symptoms of disease. Mean platelet volume (MPV), red blood cell distribution width (RDW), platelet distribution width (PDW), neutrophilto-lymphocyte ratio (NLR), platelet-to-lymphocyte ratio (PLR) and RDW-to-platelet ratio (RPR) can be obtained with a basic hemogram test. These parameters have been investigated as a predictor of inflammatory process in many diseases, their roles in $\mathrm{OA}$ is unclear. The aim of the present study was to investigate the diagnostic value of routine hematological parameters on $\mathrm{OA}$ and explore their clinical significance. The study included 118 patients with osteoarthritis and 145 age and gender matched healthy individuals. Medical records, erythrocyte sedimentation rate (ESR), C-reactive protein 
(CRP), White Blood Cell (WBC) count, NLR, platelet count (PLT), PDW, RDW, RPR, PLR and MPV levels were retrospectively recorded from patient files. There were no significant differences in WBC, RDW, PLT, RPR levels between two groups (all $p>0.05$ ). NLR and PLR values were significantly higher in OA group compared to control group (all $p<0.001)$. RBC, MPV and PDW levels were significantly lower in OA than control group (all $p<0.001$ ). Patients with OA had significantly higher CRP and ESR values compared to control group ( $p=0.020$ and $p=0.011$, respectively). In addition, ESR was positively correlated with CRP and negatively correlated with RBC, MPV and PDW levels in OA patients. Also, CRP was negatively correlated with RBC and MPV levels in OA patients. Our study showed that hematological inflammatory markers might be useful parameters that can be used in patients with OA.

\section{P732}

\section{OSTEOPOROSIS RISK ASSESSMENT IN A RE-} SOURCE POOR ENVIRONMENT

N. R. Njeze ${ }^{1}$, I. E. $\mathrm{Obi}^{2}$, M. O. Ajuba ${ }^{3}$, N. C. Njeze ${ }^{4}$

${ }^{1}$ Department of Radiation Medicine, University of Nigeria Medical School, Nsukka-Enugu, Nigeria, ${ }^{2}$ Community Medicine, University of Nigeria Nsukka, Enugu Campus, Enugu, Nigeria, ${ }^{3}$ Community Medicine, ESUT College of Medicine, Parklane Enugu, Enugu, Nigeria, ${ }^{4}$ Department of Radiation Medicine, University of Nigeria Teaching Hospital, Enugu, Nigeria

Introduction/Background: Persons aged $>50$ need to be evaluated for osteoporosis risk. Few studies have assessed for osteoporosis risk among South Eastern Nigerians.

Objective: To determine and classify osteoporosis risk into mild, moderate and severe among adults in Enugu South East Nigeria.

Materials and methods: A cross-sectional descriptive study was conducted among adult respondents. An interviewer administered questionnaire was utilized by research assistants trained to administer the questionnaire. There were 15 non modifiable risk factors common to males and females, an extra 3 for the females by virtue of their disposition and 2 for males. The modifiable risk factors were 5 and common to each gender. Thus a total of 23 risk factors were assessed for females and 22 for males. The presence or absence of a risk factor was determined during the interview and coded ' 1 ' when present and ' 0 ' when absent. Those with a total score of 1-7 risk factors were classified as mild osteoporosis risk, score of 816 was classified moderate risk while a score of $>17$ meant severe risk. The parameters for osteoporosis risk were adapted from the 'one minute osteoporosis risk test' of the International Osteoporosis Foundation (IOF). This was modified.
Results: Respondents were 937 of 1000 , a response rate of $93.7 \%$. Most were aged $>56$ years. Mean age was 48.02 \pm 13.2 years. There were more females $567(60.5 \%)$, than males $370(39.5 \%)$. Majority (35.4\%) had attained managerial levels in their professions and some had retired $(18.2 \%)$. The total mean BMI was found to be $26.96 \mathrm{~kg} / \mathrm{m}^{2}, 27.51 \mathrm{~kg} / \mathrm{m}^{2}$ among females and $25.74 \mathrm{~kg} /$ $\mathrm{m}^{2}$ among males as most respondents were overweight. Among females, $83.8 \%$ were assessed to have mild osteoporosis risk, $2.1 \%$ with moderate risk. Among the males, $87.6 \%$ were assessed to have mild osteoporosis risk and $1.1 \%$ moderate risk.

Conclusion: Mild to moderate risk of osteoporosis was predominant in the study population. There is therefore need for public enlightenment, early diagnosis and treatment, to reduce the incidence of osteoporosis related fractures and its complications.

P733

ESTIMATING THE BURDEN OF OSTEOPOROSIS-RELATED FRACTURES IN TERMS OF HEALTH-RELATED QUALITY OF LIFE, UTILITY, AND RESOURCE USE (BOOST): DISEASESPECIFIC HEALTH RELATED QUALITY OF LIFE

J. Weaver $^{1}$, R. Insinga ${ }^{1}$, S. Sajjan ${ }^{1}$, A. Modi ${ }^{1}$

${ }^{1}$ Merck \& Co., Inc., Kenilworth, NJ, United States

Objectives: To estimate the disease specific quality of life (QoL) just prior to an osteoporotic (OP) fracture, and during the first year post-fracture.

Materials and Methods: This prospective, observational study was conducted in The Netherlands (NLD), South Korea (KOR) and Taiwan (TWN). Patients were required to be female, postmenopausal, $\geq 50$ years of age, and recently diagnosed by their physician with an osteoporotic fracture (hip, clinical vertebral, wrist, humeral, other). All assessments collected data on disease specific QoL using the Osteoporosis Assessment Questionnaire-short version (OPAQ-SV). Scores ranged from 0 (low QoL) to 100 (high QoL). The study included a baseline visit within two weeks of OP fracture diagnosis with a retrospective pre- fracture assessment, immediately postfracture assessment, and assessments at 1, 3,6 and 12 months post- fracture. Descriptive statistics for demographic and clinical characteristics at baseline were generated by fracture type and country. Outcomes were summarized by fracture type and country for each assessment. Mean (SD) OPAQ-SV score change from baseline was also assessed for each domain: physical functioning, emotional status and back pain.

Results: The NLD, KOR and TWN enrolled 143, 174 and 102 patients, respectively. Mean age at enrollment ranged from the early 60 s to late 70 s, depending on fracture type and country. For all countries and fracture types with a sample 
size $\geq 20$ patients, mean (SD) OPAQ-SV Physical Functioning score dropped significantly immediately post-fracture, as low as -60.5 (20.2) points for clinical vertebral fracture patients in $\mathrm{KOR}$, and returned to near pre-fracture levels over the course of a year. Mean (SD) OPAQ-SV Emotional Status score was significantly impacted by all fractures in KOR and TWN dropping by as much as -43.4 (24.4) points in clinical vertebral patients in KOR after fracture, and not recovering to prefracture levels by month 12. Patients in NLD experienced some decline in emotional status immediately after fracture but returned to near pre-fracture levels by month 6 . In all countries, mean (SD) OPAQ-SV Back pain scores were negatively affected by clinical vertebral fractures, but minimally so for other fracture types.

Conclusion: OP fractures substantially diminished the healthrelated quality of life of OP patients across countries and fracture types.

\section{P734}

RELATIONSHIPS BETWEEN RADIOGRAPHIC TIBIOFEMORAL AND PATELLOFEMORAL KNEE OSTEOARTHRITIS AND CLINICAL EXAMINATION FINDINGS IN MEN AND WOMEN FROM THE HERTFORDSHIRE COHORT STUDY

C. Parsons ${ }^{1}$, M. H. Edwards ${ }^{1}$, A. E. Litwic ${ }^{1}$, D. Jagannath ${ }^{2}$, S. van der Pas $^{3}$, C. Cooper ${ }^{1}$, E. M. Dennison ${ }^{1}$

${ }^{1}$ MRC Lifecourse Epidemiology Unit, University of Southampton, Southampton, United Kingdom, ${ }^{2}$ St Mary's Hospital, Isle of Wight, United Kingdom, ${ }^{3}$ Department of Epidemiology and Biostatistics, VU University Medical Center, Amsterdam, Netherlands

Background: A significant correlation has been previously demonstrated between radiographic and clinical diagnoses of knee osteoarthritis (OA), however the specific findings on clinical examination that relate best to a radiographic diagnosis have not been fully elicited. We explored the associations between clinical signs on knee examination and both radiological tibiofemoral (TF) and patellofemoral (PF) knee OA.

Methods: This study was based on 407 individuals from the Hertfordshire Cohort Study, born between 1931-9. Weightbearing AP radiographs were taken of both knees to assess for TF OA and lateral semi-flexed knee radiographs were obtained to assess for PF OA. Both were graded for OA severity using Kellgren and Lawrence (K\&L) grading with OA defined as a $K \& L$ grade $\geq 2$, and clinical signs were ascertained by examination. Relationships were assessed using multilevel univariate logistic regression.

Results: A total of 767 knees were included in the analyses, 237(31 \%) had TF OA and 222(29\%) had PT OA. The prevalence of each clinical examination finding were: pain on flexion $72(10 \%)$, medial $\mathrm{TF}$ tenderness
101(13\%), lateral TF tenderness $61(8 \%)$, joint effusion 32(4\%), crepitus 189(25\%), bony swelling 94(12\%), and varus deformity $167(22 \%)$. Just 8 (1\%) knees demonstrated warmth which prohibited inclusion in analysis. Tibiofemoral OA was significantly associated [OR $(95 \% \mathrm{CI}) \mathrm{p}$ value] with pain on flexion $8.4(3.0,23.9)$ $p<0.001$, medial TF tenderness 11.1(3.9,31.5) $p<0.001$, lateral $\mathrm{TF}$ tenderness $7.8(2.6,23.6) p<0.001$, joint effusion $17.3(3.6,82.0) p<0.001$, crepitus $3.7(1.8,7.8)$ $p=0.001$, and bony swelling - mild 5.2(1.6,16.8) $p=0.006$ but not the presence of varus deformity. Patellofemoral OA was only significantly associated with joint effusion $10.5(1.8,61.2) p=0.009$ and lateral tibiofemoral tenderness 3.4(1.0,11.3) $p=0.046$.

Conclusion: Clinical examination findings at the knee correlate well with TF OA, whereas PF OA is only associated with lateral TF tenderness and the presence of a joint effusion.

\section{P735}

OSTEOPOROSIS AND SARCOPENIA: WHEN TISSUE REGENERATION FAILS?

J. Baldi $^{1}$, E. Piccirilli ${ }^{1}$, M. Scimeca ${ }^{2}$, C. Rao ${ }^{1}$, E. Gasbarra ${ }^{1}$, E. Bonanno $^{3}$, U. Tarantino ${ }^{1}$

${ }^{1}$ Department of Orthopedics and Traumatology, "Tor Vergata" University of Rome, "Policlinico Tor Vergata" Foundation, Rome, Italy, ${ }^{2}$ Italian Space Agency (ASI), Spatial Biomedicine Center, Rome, Italy, ${ }^{3}$ Anatomic Pathology Section, Department of Biomedicine and Prevention, University of Rome "Tor Vergata", Rome, Italy

Introduction: Age-related bone diseases, as osteoarthritis or osteoporosis, are strongly associated with sarcopenia and specific muscle fiber atrophy. Sarcopenia is characterized by an important imbalance between synthesis and deterioration of muscle proteins and cells, with a resulting poor muscular quality. This depletion influences bone tropism and affects mobility and skeletal features during osteoporosis. The potential mechanisms involved in the reduction of skeletal muscle mass during sarcopenia converge on satellite cells, contributing to their failure in repairing damaged muscle fibers. We performed morphological and immunohistochemical studies to investigate muscle regeneration by satellite cells activity. Moreover, immunogold labeling allowed us to investigate the correlation between BMP-2 expression and the formation of stem cells syncytium.

Methods: In this study we studied vastus lateralis muscle biopsies (30 biopsies of osteoarthritic women (OA) who underwent a total hip arthroplasty and 30 biopsies from osteoporotic women (OP) with a cervical femoral fragility fracture) in order to demonstrate that in osteoporotic patients the regenerative properties of muscle stem cells are related to the same factors that could influence bone status. In particular, 
thanks to immunohistochemistry, transmission electron microscopy and immunogold labeling we investigated the role BMP-2 on CD44 positive satellite muscle cells activity. To evaluate the BMD, each patient underwent DXA scan of the lumbar spine and femoral neck; a functional evaluation of the performance status was realized in each patient through the PASE test (Physical Activity Scale for the Elderly).

Results: PASE test showed that both OA and OP patients were characterized by poor physical activity. However, in OP group patients display a lower PASE test score than OA patients. Our immunohistochemistry study reveals that osteoporotic patients have lower number of BMP-2 positive fibers than osteoarthritic group. Moreover osteoporotic patients express a lower number of CD44 positive satellite muscle cells forming syncitia as observed both with immunohistochemistry and transmission electron microscopy. The expression of BMP-2 assessed by in situ molecular characterization trough immuno-gold analysis both in the perinuclear area and in the fiber body of satellite cells syncytia suggest the strict correlation between BMP-2 expression and muscle regeneration capability.

Conclusion: Our data suggest that the poor skeletal muscle tissue regeneration capability typical of osteoporotic patients is characterized by low or absent expression of BMPs, loss of satellite cells and loss of their ability to differentiate in myotube forming cell syncitia. The finding that aged muscle tissue reduces the expression of bone-muscle crosstalk markers according to poor bone quality suggests that these two tissues are highly interconnected; physical activity should be strongly recommended in osteoporotic patients at the time of diagnosis because it could be useful to delay muscle atrophy and bone loss thanks to the biomechanical stimuli the muscle exerts on bone.

\section{P736}

PRIMARY HYPERPARATHYROIDISM AMONG ELDERLY WOMEN WITH DISTAL FOREARM FRACTURE

A. Wihlborg ${ }^{1}$, K. Bergström ${ }^{2}$, P. Gerdhem ${ }^{3}$, I. Bergström ${ }^{2}$

${ }^{1}$ Department of Clinical Science, Intervention and Technology, Karolinska Institute, Stockholm, Sweden, ${ }^{2}$ Centre for Osteoporosis, Clinic for Endocrinology, Metabolism and Diabetes, Karolinska University Hospital Huddinge, Stockholm, Sweden, ${ }^{3}$ Department of Orthopedics and Biotechnology, Karolinska Institute, Solna, Sweden

Background: Primary hyperparathyroidism (PHPT) is a common disorder among elderly women, resulting in derangement of calcium metabolism with increased bone resorption and osteoporosis. Improved diagnosis of PHPT would be beneficial as efficient treatment options are available. Distal forearm fracture is an early sign of possible osteoporosis and could therefore be an indicator of PHPT. A previous study from our group has indicated an increased rate of PHPT among women with a distal forearm fracture.

Methods: At the department of orthopaedics, Karolinska University hospital, postmenopausal women with a distal forearm fracture caused by low-energy trauma were invited to participate. No other exclusion criteria were applied. All women underwent clinical investigations at the department of endocrinology and were examined with biochemical markers for parathyroid and calcium metabolism. Increased level of PTH level with simultaneously increased serum calcium and ionized calcium was considered diagnostic for PHPT. The prevalence of PHPT was compared to population data and Chi squared test was used to determine the difference between observed and expected occurrence of PHPT among the postmenopausal women with distal forearm fracture.

Results: 166 women with a distal forearm fracture were included, mean age 65 . Of the 166 women, 12 (7.2\%) were observed with PHPT. When compared to population data with a prevalence of $2 \%$, there was a greater occurrence of PHPT among women with distal forearm fracture at a significance level of $p<0.001$.

Conclusions: The occurrence PHPT was significantly greater among elderly women with a distal forearm fracture when compared to population data, confirming previous findings. Consideration of a PTH disorder in elderly women with a distal forearm fracture could improve the diagnosis and treatment of PHPT.

\section{P737}

\section{EVALUATION OF HEMATOLOGIC PARAMETERS IN PATIENTS WITH TENDINOPATHY}

C. Tamam $^{1}$, S. Hira $^{2}$

${ }^{1}$ Department of Orthopedics and Traumatology, Tatvan Military Hospital, Bitlis, Turkey, ${ }^{2}$ Department of Biochemistry, Tatvan Military Hospital, Bitlis, Turkey

Tendinopathy is a painful condition that occurs in and around tendons in response to overuse. The role of inflammation and inflammatory mediators in the development or progression of tendinopathy have been investigated in many studies, but it is still uncertain and controversial. Mean platelet volume (MPV), red blood cell distribution width (RDW), platelet distribution width (PDW), neutrophil-to-lymphocyte ratio (NLR), platelet-to-lymphocyte ratio (PLR) and RDW-toplatelet ratio (RPR) may indicate the systemic inflammatory response associated with many diseases. In this retrospective study, we aimed to evaluate the relationship between routine hematological indices and tendinopathy. The study consisted of 65 tendinopathy patients and 77 age and gender matched healthy individuals. Age, gender, white blood cell (WBC) count, NLR, platelet count, MPV, PDW, RDW, RPR, PLR, 
erythrocyte sedimentation rate (ESR) and C-reactive protein (CRP) were retrospectively recorded from patient files. There were no significant differences in WBC, RDW, RPR and CRP levels between two groups (all $p>0.05$ ). Platelet count, NLR and PLR values of patients with tendinopathy were significantly higher than control subjects $(p=0.046$, $p=0.009, p=0.008$ respectively). MPV, RBC and PDW values were found to be significantly lower in patients group (all $p<0.001)$. Patients with tendinopathy had higher ESR values compared to control group $(p=0.046)$. Furthermore, ESR was positively correlated with CRP, PLT and PCT and negatively correlated with RDW and RPR in tendinopathy patients. Also, CRP was positively correlated with WBC and PLT and negatively correlated with RDW, MPV, PDW and RPR in tendinopathy patients. Results of our study have shown that hematologic parameters can be useful prognostic biomarkers in tendinopathy. Further large-scale prospective studies are required to confirm these findings and demonstrate the prognostic significance of these values.

\section{P738}

\section{REAL-WORLD OUTCOMES IN DAILY TERIPA- RATIDE PATIENTS IN JAPAN}

R. Burge $^{1}$, M. Sato ${ }^{2}$, T. Sugihara ${ }^{3}$

${ }^{1}$ Global Health Outcomes, Eli Lilly and Company, Indianapolis, United States, ${ }^{2}$ Health Outcomes \& Health Technology Assessment, Eli Lilly Japan K.K., Kobe, Japan, ${ }^{3}$ inVentiv Health Clinical, Princeton, United States

Objective: To estimate real-world clinical and economic outcomes for daily teriparatide (d-TPD) patients in Japan.

Material and Methods: A large database (Medical Data Vision Co., Ltd.) with medical and pharmacy claims from 121 hospitals in Japan was used. From 4/2008 - 7/2013, 1244 patients with an index prescription of d-TPD and with $6+$ months' pre- and post-index observation were identified. Administered via daily $20 \mu \mathrm{g}$ subcutaneous injection, d-TPD is indicated for individuals at high fracture risk. This study used all patients with $18+$ months of follow-up $(n=445)$. Descriptive analyses were conducted on all clinical fractures (vertebral + nonvertebral), health care resource use, and costs (Japanese Yen). D-TPD persistence was measured by consecutive prescriptions, allowing for gaps $\leq 60$ days. Adjusted analyses of lower vs. higher persistence for fracture incidence and hospital admissions (ADMs) were performed using Logistic and Poisson regression models with Log-link, controlling for cross-group differences in demographics and clinical characteristics. Adjusted cost analyses used Propensity Bin Bootstrapping, controlling for baseline characteristics.
Results: Baseline patient characteristics were: mean age 74.7 years $(\mathrm{SD}=9.0)$; $90 \%$ female; $88 \%$ aged $65+; 20 \%$ had $1+$ fracture. Incident fractures/1000 patient years for persistence $<9$ and $\geq 9$ months were 77.29 and 60.59 , respectively. Adjusted fracture risk was greater in Low persistent (1-6 months; $n=123)$ vs. High persistent patients (19-24 mo; $n=222)$ in Logistic $(O R=1.73,95 \% \mathrm{CI}=0.739,4.059)$ and Poisson models (Incidence ratio, $I R=1.538, C I=0.727$, 3.254), though statistically insignificant. Hospital ADMs and days/patient per month were 0.26 and 4.29, and 0.026 and 0.487, for Low and High persistent patients, respectively. Adjusted risk of hospital ADM was significantly greater in Low vs. High patients $(O R=1.88, C I=1.10,3.21 ; I R=1.55$, $C I=1.15,2.09)$. Adjusted inpatient costs were significantly less in High persistent patients (18,782 vs. 38,076), but pharmacy $(64,903$ vs. 23,673$)$ and outpatient costs $(20,535$ vs. 13 , 611) were greater.

Conclusions: Longer persistence to d-TPD revealed numerically lower fracture risk, and significant reductions in hospital ADMs and costs. The small sample size limited the robustness of these results however.

P739

SERUM OSTEOPONTIN AND BONE SIALOPROTEIN LEVELS IN PATIENTS WITH TENDINOPATHY

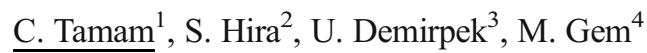

${ }^{1}$ Department of Orthopedics and Traumatology, Tatvan Military Hospital, Bitlis, Turkey, ${ }^{2}$ Department of Biochemistry, Tatvan Military Hospital, Bitlis, Turkey, ${ }^{3}$ Department of Clinical Microbiology, Tatvan Military Hospital, Bitlis, Turkey, ${ }^{4}$ Department of Orthopedics and Traumatology, Faculty of Medicine, Dicle University, Diyarbakir, Turkey

The pathogenesis of tendinopathy remains unclear. Small integrin-binding ligand N-linked glycoproteins, a family of noncollagenous proteins including osteopontin (OPN) and bone sialoprotein (BSP), were initially thought to be limited to mineralized tissue but recent studies showed that they are more widely distributed and are expressed in nonmineralized tissues. Musculoskeletal tissue cells are categorized as the same functional unit developed from the mesenchymal stem cells. This theoretical background led us to hypothesize that OPN and BSP could be involved tendinopathy pathogenesis. The aim of this study was to investigate relationship between serum OPN and BSP levels and tendinopathy disease. 39 female tendinopathy patients and 39 female healthy volunteers were recruited for this prospective observational study. Serum OPN and BSP levels were measured using enzyme-linked immunosorbent assay. We also measured BMI and erythrocyte sedimentation rate (ESR), white blood 
cells (WBC) and neutrophil lymphocyte ratio (NLR). There was no significant differences in serum BSP levels between two groups $(41.83 \pm 52.03$ vs. $53.64 \pm 53.06 \mathrm{ng} / \mathrm{mL}, p=0.276)$. There was also no significant differences in serum OPN levels between two groups $(57.37 \pm 21.61$ vs. $77.72 \pm 72.14 \mathrm{ng} / \mathrm{mL}$, $p=0.363$ respectively). There were no significant differences in WBC, NLR and ESR values between two groups ( $p=0.897, p=0.795, p=0.405$ respectively). There was no correlation between serum BSP levels and serum OPN, WBC, NLR and ESR levels in patients group. Patients with tendinopathy had a negative correlation between serum OPN levels and NLR levels. The results of this study have indicated that BSP and OPN levels are not involved in pathogenesis of tendinopathy.

\section{P740}

DIFFERENCES IN SITE SPECIFIC VOLUMETRIC BONE DENSITY MEASUREMENTS IN 65 AND 85 YEAR OLD WOMEN WITH AND WITHOUT DISTAL FOREARM FRACTURE

A. Wihlborg $^{1}$, K. Bergström ${ }^{2}$, K. Akesson ${ }^{3}$, I. Bergström ${ }^{2}$, P. Gerdhem $^{4}$

${ }^{1}$ Department of Clinical Science, Intervention and Technology, Karolinska Institute, Stockholm, Sweden, ${ }^{2}$ Centre for Osteoporosis, Clinic for Endocrinology, Metabolism and Diabetes, Karolinska University Hospital Huddinge, Stockholm, Sweden, ${ }^{3}$ Department of Orthopedics, Skane University Hospital and Clinical Sciences Malmö, Lund University, Lund, Sweden, ${ }^{4}$ Department of Orthopedics and Biotechnology, Karolinska Institute, Solna, Sweden

Background: Distal forearm fracture is one of the most common fractures associated with low energy trauma. Areal BMD assessed by DXA at the spine or hip has a poor capability of predicting distal forearm fracture. We studied volumetric BMD (vBMD) and bone area of the distal radius in women with or without a previously sustained distal forearm fracture in two different age groups.

Methods: In a case control study, 96 women with a distal forearm fracture and 107 controls with a mean (SD) age of 65 (8.6) years were recruited. From a population based study, 94 women with a distal forearm fracture and 132 women without a fracture with a mean age of $85(0.2)$ years was identified. vBMD and cross-sectional bone area was determined with peripheral quantitative computer tomography (Stratec 2000) at the $4 \%$ and the $66 \%$ level. Statistical comparisons were made with t-tests.

Results: Trabecular vBMD at $4 \%$ was lower among women with fracture than in women without fracture at age 65 and 85 $\left(125 \mathrm{vs} .166 \mathrm{~g} / \mathrm{cm}^{3}\right.$ and 108 vs. $134 \mathrm{~g} / \mathrm{cm}^{3}$, respectively, both $p<0.001)$. Corresponding figures for cross-sectional area at
$4 \%$ was 153 vs. $145 \mathrm{~mm}^{2}(p=0.030)$ at age 65 and 177 vs. $175 \mathrm{~mm}^{2}(p=0.46)$ at age 85 and for cortical vBMD at $66 \%$ 1097 vs. $1117 \mathrm{~g} / \mathrm{cm}^{3}$ at age 65 and 1000 vs. $1025 \mathrm{~g} / \mathrm{cm}^{3}$ at age 85 (both $p<0.001$ ). There were no differences in crosssectional area at $66 \%$ in either age group.

Conclusion: When compared to women without fracture, women with a history of wrist fracture had a lower trabecular bone density at the distal end of the radius, a reduced cortical bone density at the proximal end at age 65 and 85 , but a larger cross-sectional area at age 65 only.

\section{P741}

\section{ASEPTIC NECROSIS OF FEMUR IN COMBINATION WITH HYPOCORTICISM AND HYPOGONADISM IN CHILDHOOD MEDULLOBLASTOMA SURVIVOR}

E. Gubernatorova $^{1}$, M. G. Pavlova ${ }^{2}$, T. Kaznacheeva ${ }^{3}$, T. Tselovalnikova $^{1}$

${ }^{1}$ I.M. Sechenov First Moscow State Medical University, Moscow, Russian Federation, ${ }^{2}$ Endocrinology Department, I.M. Sechenov First Moscow State Medical University, Moscow, Russian Federation, ${ }^{3}$ Russian Federation Department of Reproductive Medicine and Surgery, Moscow State Medical Dental University, Moscow, Russian Federation

Introduction: Late outcomes are common among childhood cancer survivors. Craniospinal irradiation and use of antimetabolites are well known risk factors for cardiac, metabolic, endocrine and reproductive disorders. However, bone system is affected too. Mechanism of osteoporosis in this group of patients seems to be complex and include direct influence of irradiation and chemotherapy as being aggravated by endocrine disorders and low physical activity. There are only few publications concerning aseptic hip necrosis in acute leukemia survivors. There is no data available of hip necrosis in brain tumor survivors. The patient diagnosed with medulloblastoma in December 2008 year at the age of 15 years. She was treated with surgery, craniospinal irradiation at the dose of 35 Gy with direct irradiation of postcranial fossa at the dose of $54 \mathrm{~Gy}$ and chemotherapy (protocol HIT 2000). The patient developed multiple side effects of this treatment (pancytopenia, vincristine neuropathy). In December 2010 she was consulted by endocrinologist in department of endocrinology of I.M. Sechenov First Moscow State Medical University. The patient suffered from sickness, got low weight (BMI $15 \mathrm{~kg} / \mathrm{m}^{2}$ ) and low blood pressure. Insulin tolerant test was performed. There was no increase during hypoglycemia in cortisol level (baseline - $320 \mathrm{nmol} / \mathrm{l}$, hypoglycemia $303 \mathrm{nmol} / \mathrm{l})$. So far, secondary adrenal insufficiency was diagnosed. The patient was given cortisone acetate at the dose of $5 \mathrm{mg}$. During hormonal tests it was found that the patient was euthyroid (TSH $1.33 \mathrm{mMU} / \mathrm{l}$, 
T4 $19.0 \mathrm{pmol} / \mathrm{l})$. Also this patient had secondary amenorrhea due to primary hypogonadism (FSH 109.5 MIU/1, estradiol $25 \mathrm{pg} / \mathrm{ml}$, AMH $0.1 \mathrm{nmol} / \mathrm{l}$, ultrasound signs of low ovarian reserve). The patient received estrogen-gestogen replacement therapy that was well tolerated. During densitometry BMD $1.14 \mathrm{~g} / \mathrm{cm}^{2}, \mathrm{Z}$-score $(-0,3)$. We followed up the patient within 4 years with regular examination and hormonal tests. Her well-being and quality of life significantly improved and she feels well now. In December 2014 (age 23 years) the patient complained acute pain in hips. She underwent densitometry, X-ray of hips. No pathological changes were found. Pain relievers and physical exercises were recommended. There was no effect during 3 months. On the repeated hip X-ray examination bilateral aseptic hip necrosis was found. Surgery performed in August 2015 that was well tolerated by the patient. Conclusion: We performed a rare case of association of aseptic hip necrosis with secondary adrenal insufficiency, primary hypogonadism in young childhood medulloblastoma survivor

\section{P742}

VISCERAL FAT MEASURED BY DXA IN JUVENILEONSET SYSTEMIC LUPUS ERYTHEMATOSUS PATIENTS: A CORRELATION WITH DISEASE DURATION AND DAMAGE INDEX SCORE

$\underline{\text { J. A. Paupitz }}{ }^{1}$, G. L. Lima ${ }^{1}$, L. P. C. Seguro ${ }^{1}$, N. E. Aikawa ${ }^{2}$, R. M. R. Pereira ${ }^{1}$

${ }^{1}$ Rheumatology Division, University of São Paulo, São Paulo, Brazil, ${ }^{2}$ Pediatric Rheumatology Unit, University of São Paulo, São Paulo, Brazil

Introduction: Patients with juvenile onset systemic lupus erythematosus (JoSLE) are at increased risk of early atherosclerosis leading to cardiovascular disease in adult life. It has recently been shown that visceral adipose tissue (VAT) is associated with incidence metabolic risk in subclinical atherosclerosis beyond its contribution to overall adiposity. Currently, a new application of DXA has been developed to measure VAT, with strong correlation with computed tomography to quantity visceral fat. Therefore, the aim of this study was to investigate the VAT, measured by DXA, in juvenile female SLE (JoSLE) patients, compared to age-matched healthy controls and evaluate its correlation with clinical disease parameters.

Methods: We compared 56 female JoSLE patients with ageand sex- matched healthy controls. Clinical disease parameters were recorded by electronic chart review. VAT measured using DXA (Discovery, Hologic, software APEX 4.0.2).

Results: JoSLE patients used to have higher fat mass (19.26 \pm 6.60 vs. $17.86 \pm 6.22 \mathrm{~kg}, p=0.017)$ and higher percentage of fat $(32.93 \pm 6.35$ vs. $30.82 \pm 5.72 \%, p=0.004)$ than healthy controls. Also all VAT parameters, mass (280.55 \pm 132.42 vs.
$199.60 \pm 98.67 \mathrm{~g}, p<0.001)$, area $(58.19 \pm 27.47$ vs. 41.41 $\left.\pm 20.50 \mathrm{~cm}^{2}, p<0.001\right)$ and volume $(303.36 \pm 143.19 \mathrm{vs}$. $\left.215.78 \pm 106.66 \mathrm{~cm}^{3}, p<0.001\right)$ were significantly higher when compared to controls. Pearson's coefficients correlates VAT volume with disease duration $(r=0.313, p=0.019)$ and cumulative organ damage (SLICC/ACR-DI) $(r=0.300$, $p=0.025$ ).

Conclusion: This study demonstrate for the first time that VAT parameters evaluated using DXA are higher in JoSLE patients compared to healthy controls. Its correlation with disease duration and organ damage suggests that this parameter could be used to stratify cardiovascular risk in JoSLE patients.

\section{P743}

\section{THE IMPACT OF ADAPTED KINESITHERAPY PROGRAM ON DISABILITY AND BACK PAIN IN WOMEN WITH POSTMENOPAUSAL OSTEO- POROSIS}

K. Boskovic $^{1}$, D. Simić-Panić ${ }^{2}$, S. Tomasevic-Todorovic ${ }^{3}$, J. Novaković-Paro $^{4}$, A. Knežević ${ }^{2}$, M. Jeremic Knezevic ${ }^{2}$

${ }^{1}$ Clinic for Medical Rehabilitation and Physical Medicine, Clinical Center of Vojvodina, Novi Sad, Serbia, ${ }^{2}$ Medical Faculty, University of Novi Sad, Novi Sad, Serbia, ${ }^{3}$ Rheumatology, Clinic for Medical Rehabilitation, Clinical Center of Vojvodina, Faculty of Medicine, University of Novi Sad, Novi Sad, Serbia, ${ }^{4}$ Clinic of Endocrinology, Clinical Center of Vojvodina, Medical Faculty, University of Novi Sad, Novi Sad, Serbia

Objective: To determine the effect of adapted kinesitherapy program on disability and back pain in postmenopausal women with osteoporosis.

Material and methods: A total of 80 postmenopausal women with osteoporosis of the lumbar spine (mean DXA T-score of the lumbar spine $-2.63 \pm 0.631$ ) were included in this randomized prospective study. Subjects were divided into two groups: the study group ( $\mathrm{SG}, n=40 ; 59.1 \pm 7.3$ years) and the control group (CG, $n=40 ; 56.9 \pm 6.1$ years). Study and control groups were age matched. In the study group a physical activity protocol (warm up and aerobic exercises, resistance and weight exercises, coordination and balance training) was applied during 8 weeks. In the control group no exercise protocol was applied but patients were given brochures with exercises for osteoporosis. All patients were evaluated at the beginning of the treatment, at the end of applied kinesitherapy program and at 6-month follow-up after completed rehabilitation.

Results: The applied kinesitherapy program was effective vs. the control group at the end of the rehabilitation treatment and at 6-month follow-up. Disability was significantly improved (Oswestry Disability Questionnaire: $p<0.001$ at the end of rehabilitation treatment and follow-up, six minute walking test 
$p<0.001$ at the end of treatment and $p=0.005$ at follow-up). Pain was significantly reduced (Visual analogue scale: $p<0.001$ at the end of the treatment and at the follow-up).

Conclusions: Our results suggest that group kinesitherapy reduces back pain and improves functional status in women with postmenopausal osteoporosis. Furthermore such outcomes last up to 6 months after completed rehabilitation.

\section{References:}

Bošković K, et al. Osteoporos Int 2015;26:191.

Bošković K, et al. Med Pregl 2013; 66: 221.

\section{P744}

HEAVY METALS AFFECTS BONE MICROARCHITECTURE IN OSTEOPOROTIC PATIENTS

M. Scimeca ${ }^{1}$, M. Feola ${ }^{2}$, L. Romano ${ }^{3}$, C. Rao ${ }^{3}$, E. Gasbarra ${ }^{3}$, $\overline{\text { E. Bonanno }}^{1}$, U. Tarantino ${ }^{4}$

${ }^{1}$ Anatomic Pathology Section, Department of Biomedicine and Prevention, University of Rome, Rome, Italy, ${ }^{2}$ Department of Orthopedics and Traumatology, "Tor Vergata" University of Rome, "Policlinico Tor Vergata" Foundation, Rome, Italy, ${ }^{3}$ Department of Orthopedics and Traumatology, Rome, Italy, ${ }^{4}$ Department of Orthopedics and Traumatology, "Tor Vergata" University of Rome, Rome, Italy

Introduction: Bone metabolism is affected by mechanical, genetic and environmental factors. Nevertheless, the role of environmental pollution on bone metabolism and/or bone diseases is still controversial. In this context, heavy metals are the most important pollutants capable to affect bone mass. The aim of this study was to investigate whether heavy metals accumulation in bone tissues could be related to the altered bone metabolism and architecture of osteoporotic patients.

Methods: In order to investigate the influence of lead $(\mathrm{Pb})$, chromium $(\mathrm{Cr})$, cadmium $(\mathrm{Cd})$ and cobalt $(\mathrm{Co})$ accumulation on the development of OP, we analyzed 25 bone biopsies from osteoporotic patients (19 women and 6 men) and 25 bone biopsies from hip arthroplasty, as control (14 women and 11 men).

Results: Combining data obtained by BioQuant osteo software, scanning electron microscopy and energy dispersive $\mathrm{x}$ ray (EDX) microanalysis we found a prevalence of $\mathrm{Pb}, \mathrm{Cd}$ and $\mathrm{Cr}$ accumulation in osteoporotic patients. Noteworthy, in of OP group we found a significantly difference between t-score values of patients with at least 3 elements ( $\mathrm{Pb}$ included) and tscore values of patients with less 3 elements. In particular, OP patients with at least 3 elements showed a low BMD. In addition, we studied the expression of sclerostin, a protein with anti-anabolic effects on bone formation, in order to correlate heavy metal accumulation with bone metabolism. High levels of sclerostin revealed in tissues with $\mathrm{Pb}, \mathrm{Cd}$ and $\mathrm{Cr}$, candidate it as a molecular link between heavy metals accumulation and bone metabolism impairment. Indeed, sclerostin expression was higher in tissues with at least 2 heavy metals accumulated. Discussion: Our data highlighted the importance of $\mathrm{Pb}, \mathrm{Cd}$, and $\mathrm{Cr}$ in bone health. The presence of these elements is convincingly connected to the environmental pollution, as assessed by habitat conditions of the investigated patients. Indeed, they live in large urban centers in which heavy metals concentration is often upper safety threshold values. In conclusion, the identification of heavy metals into bone structure opens new perspectives about the pathogenesis of osteoporosis. In fact, heavy metals can contribute to development of osteoporosis at both cellular/molecular and epigenetic level.

\section{P745}

COGNITIVE DISORDERS AND FUNCTIONAL RECOVERY OF PATIENTS AFTER HIP FRACTURE K. Boskovic ${ }^{1}$, S. Tomasevic-Todorovic ${ }^{2}$, S. Kevic ${ }^{3}$, D. SimićPanić $^{3}$, A. Knežević ${ }^{4}$, M. Jeremic Knezevic ${ }^{5}$

${ }^{1}$ Clinic for Medical Rehabilitation and Physical Medicine, Clinical Center of Vojvodina, Novi Sad, Serbia, ${ }^{2}$ Rheumatology, Clinic for Medical Rehabilitation, Clinical Center of Vojvodina, Novi Sad, Serbia, ${ }^{3}$ Clinic for Medical Rehabilitation and Physical Medicine, Clinical Center of Vojvodina, Serbia, Novi Sad, Serbia, ${ }^{4}$ Clinic for Physical Medicine and Rehabilitation, Clinical Center of Vojvodina, Novi Sad, Serbia, ${ }^{5}$ Medical Faculty, University of Novi Sad, Novi Sad, Serbia

Objective: Annually one third of population older than 65 years suffers from consequences of falls, out of which $3-6 \%$ are hip fractures resulting in high mortality and disability rates among the elderly. Surgical procedures enable for some of the patients early mobilisation and satisfactory functional recovery. Cognitive disorders which accompany old age can interfere with the outcome of the treatment. The aim of this research was to compare levels of functional recovery of patients after surgically treated hip fracture depending on cognitive disorders.

Material and methods: 60 patients with surgically treated hip fracture were included in this research. Among participating patients two groups were singled out using Folstein test. First group included patients with lower mental abilities (12 to 23 points on Folstein test score, moderate and mild dementia), while second group included patients with intact mental abilities (24 to 30 points on Folstein test score). For assessing the presence of depressive symptomatology Cornel scale of depression in dementia was used. All patients were included in program of medical rehabilitation according to certain protocols. Functional capabilities of all patients were assessed with Functional impairment measurement test (FIM) on admission and at discharge from our institution. 
Results: The results obtained on FIM test on admission and at discharge indicate that both groups of patients improved in functional recovery.

Conclusion: Our research confirmed negative impacts of cognitive disorders on functional recovery of the patients after surgically treated hip fracture, while the impact of associated diseases was mostly simultaneous.

\section{References:}

1. Bošković K. Annals Rheum Dis 2012;71:717

\section{P746}

ASEPTIC NECROSIS OF FEMUR IN COMBINATION WITH SECONDARY AND ADRENAL INSUFFICIENCY, PRIMARY HYPOGONADISM IN CHILDHOOD MEDULLOBLASTOMA SURVIVOR

E. Gubernatorova $^{1}$, M. G. Pavlova ${ }^{2}$, T. Kaznacheeva ${ }^{3}$, T. Tselovalnikova

${ }^{1}$ I.M. Sechenov First Moscow State Medical University, Moscow, Russian Federation, ${ }^{2}$ Endocrinology Department, I.M. Sechenov First Moscow State Medical University, Moscow, Russian Federation, ${ }^{3}$ Russian Federation Department of Reproductive Medicine and Surgery, Moscow State Medical Dental University, Moscow, Russian Federation

Introduction: Late outcomes are common in childhood cancer survivors. Craniospinal irradiation and use of antimetabolites are well known risk factors for cardiac, metabolic, endocrine and reproductive disrupters. However, bone system is affected too. Mechanism of osteoporosis in this group of patients seems to be complex and include direct influence of irradiation and chemotherapy and is being aggravated by endocrine disorders and low physical activity. There are only few publications concerning aseptic hip necrosis in acute leukemia survivors. There are no data of hip necrosis in brain tumor survivors. The patient was diagnosed with medulloblastoma in December 2008 year at the age of 15 years. She was treated with surgery, craniospinal irradiation at the dose of 35 Gy with direct irradiation of post cranial fossa at the dose of $54 \mathrm{~Gy}$ and chemotherapy (protocol HIT 2000). In December 2010 she was consulted by endocrinologist in department of endocrinology of. T I.M. Sechenov First Moscow State Medical University. The patient suffered from sickness, got low weight (BMI $15 \mathrm{~kg} / \mathrm{m}^{2}$ ) and low blood pressure. Insulin tolerant test was performed. There was no increase during hypoglycemia in cortisol level (basal - $320 \mathrm{nmol} / \mathrm{l}$, hypoglycemia $303 \mathrm{nmol} / \mathrm{l}$ ). So far secondary adrenal insufficiency was diagnosed. The patient was given cortisone acetate at the dose of $5 \mathrm{mg}$. During hormonal tests it was found that the patient was euthyroid (TSH $1.33 \mathrm{mMU} / \mathrm{l}$, T4 $19.0 \mathrm{pmol} / \mathrm{l})$. Also patient had secondary amenorrhea due to primary hypogonadism (FSH 109.5 MIU/1, estradiol $25 \mathrm{pg} / \mathrm{ml}$, AMH 0,1 nmol/l, ultrasound signs of low ovarian reserve). The patient was performed estrogen-gestogen replacement therapy with good tolerance. During densitometry BMD $1.14 \mathrm{~g} / \mathrm{cm}^{2}, \mathrm{Z}$-score $(-0.3)$. We followed up the patient during 4 years with regular examination hormonal tests. Her well being and quality of life were good. In December 2014 (age 23 years) the patient complained acute pain in hips. She underwent densitometry, X-ray of hips. No pathological changes were found. She was recommended pain relief drugs and physical exercises. There was no effect during 3 months. On the repeated hip X-ray examination bilateral aseptic hip necrosis was found. Surgery was performed in August 2015 and as well tolerated by the patient.

Conclusion: We performed a rare case of association of aseptic hip necrosis with secondary adrenal insufficiency, primary hypogonadism in young childhood medulloblastoma survivor.

\section{P747}

ANALYSIS OF JOINT CHANGES IN CHILDREN WITH JUVENILE IDIOPATHIC ARTHRITIS AGAINST THE BACKGROUND OF STANDARD THERAPY

M. A. Abiodun $^{1}$, E. A. Adegoke ${ }^{1}$, N. O. Panko ${ }^{1}$

${ }^{1}$ Department of Pediatrics, V.N. Karazin Kharkiv National University, Kharkiv, Ukraine

Objective: To study the development of articular changes in patients with juvenile idiopathic arthritis (JA) against the background of standard therapy with methotrexate.

Materials and methods: The study involved 83 children within 2-18 years with JIA: oligoarthritis in $39.6 \%$, polyarticular form $37.7 \%$, systemic form $-20.8 \%$, enthesitis-related arthritis $-1.9 \%$. Changes in the joints were assessed based on the duration of the disease: $1-2$ years ( 25 patients), 3-5 years ( 23 patients), 5-10 years (35 patients). There were 51 females and 33 males. Radiologic abnormalities of joints were evaluated by Steinbrocker system. For the statistic processing of the material, Stagraphics 3.0 was used.

Results: With an increase in duration of the disease in spite of standard care, the joint tenderness according Ritchie index and joint pain according visual analogue scale were decreased, especially after 5 years of illness $(p<0.05)$. Radiologic changes in the joints were found in all patients with JIA. The frequency and stage covered the basic laws of the clinical pathological process. It was determined during the first two years, the changes that correspond to $0(27.3 \%)$ and I stage of the disease by Shteynbroker (72.7 \%), from 3 year in $45.5 \%$ of children II stage was found, from 4 th year $15.4 \%$ - III, after 6th year $14.7 \%$ people had IV stage. The main stage of deterioration of the radiological changes is the term disease for more than 5 years, when significantly the frequency of the 
III stage in comparison with the previous period is increased $(p<0.01)$ and the IV stage appeared.

Conclusion: It reflected steadily progressive destructive changes of the musculoskeletal system despite therapy with methotrexate and independent of the activity of the disease and symptom load.

\section{P748}

EFFECT OF METABOLIC SYNDROME ON PAIN AND FUNCTIONAL STATUS OF PATIENTS WITH KNEE OSTEOARTHRITIS: A CROSS SECTIONAL STUDY

A. Knežević $^{1}$, D. Simić-Panić ${ }^{2}$, K. Boskovic ${ }^{3}$, S. Tomasevic-Todorovic $^{4}$, S. Mikulić-Gutman ${ }^{2}$, M. Jeremic Knezevic $^{5}$

${ }^{1}$ Clinic for Physical Medicine and Rehabilitation, Clinical Center of Vojvodina, Novi Sad, Serbia, ${ }^{2}$ Clinic for Medical Rehabilitation and Physical Medicine, Clinical Center of Vojvodina, Serbia, Novi Sad, Serbia, ${ }^{3}$ Clinic for Medical Rehabilitation and Physical Medicine, Clinical Center of Vojvodina, Novi Sad, Serbia, ${ }^{4}$ Rheumatology, Clinic for Medical Rehabilitation, Clinical Center of Vojvodina, Novi Sad, Serbia, ${ }^{5}$ Medical Faculty, University of Novi Sad, Novi Sad, Serbia

Objectives: Osteoarthritis is the most common form of arthritis. It affects one-third of adults and tends to increase with age leading to functional impairment, and increased the risk for comorbidity. The aim of this research was to evaluate the impact of metabolic syndrome on pain intensity and functional status of patients with knee osteoarthritis.

Material and methods: A total of 70 patients with knee osteoarthritis based on American College of Rheumatology (ACR) criteria were included in this cross sectional study. They were divided in two groups based on the presence of metabolic syndrome. Diagnose of metabolic syndrome was made according to the National Cholesterol Education Program Adult Treatment Panel III criteria. The two groups were compared for pain - Visual analogue scale (VAS), and functional status - Western Ontario and McMaster Universities Osteoarthritis Index (WOMAC).

Results: The mean age was $57.14 \pm 9.12$ years, and the mean BMI was $34.67 \pm 3.27 \mathrm{~kg} / \mathrm{m}^{2}$. The prevalence of metabolic syndrome was $43.31 \%$ Patients with and without metabolic syndrome had similar osteoarthritis parameters. However patients with metabolic syndrome had higher level of pain $(p=0.007)$ and more pronounced disability $(p=0.004)$.

Conclusion: The results of this study imply that metabolic syndrome has negative effect on functional status and pain intensity of patients with osteoarthritis. Therefore appropriate treatment of this condition is crucial in management of knee osteoarthritis.

Key words: knee osteoarthritis, functional status, metabolic syndrome, pain assessment

\section{References:}

Tomašević Todorović S, et al. Osteoporos Int 2013;24:278.

\section{P749}

BENEFICIAL EFFECTS OF VITAMIN D SUPPLEMENTATION IN PATIENTS WITH TYPE 2 DIABETES MELLITUS

F. Marc $^{1}$, D. M. Farcas ${ }^{1}$, C. Moldovan ${ }^{1}$

${ }^{1}$ University of Oradea, Faculty of Medicine and Pharmacy, Oradea, Romania

Introduction: Recently, research reveals more and more the importance of vitamin D in pathology. Low blood levels have been associated with increased risk of cardiovascular diseases, cognitive impairment, asthma, cancer, diabetes mellitus, osteoporosis, sarcopenia. With aging, people become vitamin D deficient.

Objective: To evaluate the presence of vitamin D deficiency in patients with type 2 diabetes mellitus (T2DM) and if the therapy with vitamin $\mathrm{D}$ in those with deficiency may improve the glycemic control.

Material and method: Our study included 80 patients with T2DM poorly controlled admitted in the Internal Medicine Department. Their vitamin D status was evaluated by measuring 25 -hydroxyvitaminD serum levels. Values were considered: below $10 \mathrm{ng} / \mathrm{ml}$-deficit, between $10-20 \mathrm{ng} / \mathrm{ml}$-insufficiency, $30-100 \mathrm{ng} / \mathrm{ml}$-optimal. Patients found to have levels below $20 \mathrm{ng} / \mathrm{ml}$ received therapy with vitamin $\mathrm{D}$ in doses of $800 \mathrm{UI} /$ day; serum fasting glucose and HBalc (glycosylated hemoglobin) were measured at 3 and 6 months; serum vitamin D was measured after 6 months.

Results: We found vitamin D deficiency in $25 \%$ of patients and insufficiency in $42 \%$ of the patients with T2DM. Women in menopause have lower levels compared with men of the same age. Supplementation of the classic therapy (diet, oral antidiabetics, insulin therapy) with vitamin D improved glycemic control; at 3 months glucose level and HBalc decreased with $12 \%$, at 6 months with $27 \%$. Serum vitamin D increased above $20 \mathrm{ng} / \mathrm{ml}$ in $65 \%$ of the patients.

Conclusions: $67 \%$ of patients with T2DM have decreased values of vitamin $\mathrm{D}$ (deficiency and insufficiency). Adding vitamin $\mathrm{D}$ in chronic therapy of diabetes helps to have a better glycemic control and also helps in preventing cardiovascular diseases; therapy is needed for long term as these patients are aging. 


\section{P750}

VITAMIN D LEVELS AT POSTMENOPAUSAL WOMEN ACCORDING TO THE AGE AND THE BONE MINERAL DENSITY

V. K. Koevska ${ }^{1}$, E. N. Nikolic-Dimitrova ${ }^{1}$

${ }^{1}$ Institute for Physical Medicine and Rehabilitation, Skopje, R. Macedonia, Skopje, Former Yugoslav Republic of Macedonia

Objective: To establish the difference between deficiency, insufficiency and normal results of vitamin D according to patients' age and BMD at the hip and at the spine.

Material and Methods: The retrospective analyze has been done in 147 postmenopausal women who were submitted to osteodensitometric examination at Institute for Physical Medicine and Rehabilitation, Skopje, R. Macedonia between January 2015 and December 2015. All patients had their vitamin D status analyzed. Normal values of $25(\mathrm{OH}) \mathrm{D}$ were defined as levels above $30 \mathrm{ng} / \mathrm{ml}$, deficiency of vitamin D as values below $10 \mathrm{ng} / \mathrm{ml}$ and results between these two as vitamin D insufficiency. A difference was analyzed between patients with insufficient, deficient and normal levels of vitamin $\mathrm{D}$ in correlation to age and BMD at the hip and at the spine.

Results: Average age of patients was $65.7 \pm 8.33$ years. $60 \%$ of patients had insufficiency of vitamin D, $22 \%$ had deficiency and $18 \%$ had normal results. There is a statistical difference $(p<0.05)$ between these three groups of patients only regarding the spine BMD. In patients with insufficient vitamin $\mathrm{D}$, spine BMD was higher than in those with deficient vitamin D. Patients with vitamin D deficiency are older than those with insufficient and normal results.

Conclusion: Patients with lower levels of vitamin D also have lower BMD and belong to older population, which indicates the need for adequate supplementation of this vitamin.

\section{P751}

\section{ACTIVE SCREENING FOR OSTEOPOROSIS IN HEMATOLOGIC DISEASES}

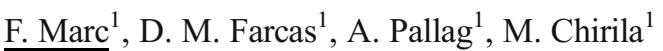

${ }^{1}$ University of Oradea, Faculty of Medicine and Pharmacy, Oradea, Romania

Objective: Patients with hematologic disorders that require long term corticotherapy are at high risk to develop osteoporosis.

Materials and Methods: Study included patients admitted in the Hematology Department over a period of 6 months in 2015; they were evaluated to see which are the diseases with the therapeutic plan that includes corticosteroids (doses and duration). They have performed DXA examination to identify the presence of osteoporosis. Life expectancy was an important factor to decide whether it is necessary or not to perform DXA (acute leukemias were excluded).
Results: Among 110 patients with chronic hematologic disorders, we found osteoporosis due to long term corticotherapy in: autoimmune hemolytic anemias (63\%), idiopathic thrombocytopenic purpura (58\%), multiple myeloma (56\%), nonHodgkin malignant lymphoma (43\%) and chronic lymphatic leukemia (32\%). Due to the fact that the first 2 diseases have a higher life expectancy than the last 2 , the prevalence of osteoporosis is higher. Patients with multiple myeloma have vertebral fractures more often than patients with other diseases. The presence of osteoporosis correlates with the number of cycles of chemotherapy (with high doses of corticosteroids) that each patient had.

Conclusion: Active screening for osteoporosis in patients with hematological diseases that use long term corticotherapy is very important, because treating osteoporosis may prevent fragility fractures and worsening of the quality of life. We found the highest prevalence of osteoporosis in patients with autoimmune hemolytic anemia.

\section{P752}

THE ROLE OF VITAMIN D IN SECONDARY PREVENTION OF FRAGILITY FRACTURES

A. Bañuelos Diaz ${ }^{1}$, F. J. Nistal Rodríguez ${ }^{1}$, R. Escudero Marcos $^{1}$, E. Cebrian Rodriguez ${ }^{1}$, I. Aguado Maestro ${ }^{1}$, A. Quintanilla Garcia $^{1}$, J. Lopez Sanchez ${ }^{1}$, M. F. Garcia Alonso ${ }^{1}$ ${ }^{1}$ Hospital Universitario Rio Hortega, Valladolid, Spain

Objectives: Analyze the results obtained after developing a protocol to treat patients with fragility fracture. This protocol sets to establish standard treatment guidelines, to follow in these patients.

Material and methods: A retrospective analysis of patients included in the protocol with hip fracture between June 2014 and February 2015, is performed. This protocol consists to collect patient data at hospital admission (functionality, previous treatment, previous fractures), determine serum levels of calcium and vitamin $\mathrm{D}$, and X-ray of dorsal and lumbar column is performed. At the time of leaving hospital, treatment with calcium and vitamin D (600 mg / 1000 IU every $24 \mathrm{~h}$ ) is established. At three months, new serum levels determination is performed, and incidents are registered.

Results: The protocol was applied in 118 fractures. The mean age was 85.5 years. $31.36 \%$ of patients had previous fragility fracture, $13.5 \%$ being hip fracture. $13.5 \%$ had previous treatment for osteoporosis. The average initial calcium was $8.33 \mathrm{mg} / \mathrm{dl}$. Average Vitamin D was $12.67 \mathrm{ng} / \mathrm{ml}(3-50)$, being low in $98.31 \%$ of patients, and this deficit was severe $(<10 \mathrm{ng} / \mathrm{ml})$ in $41.53 \%$. The spine X-ray was performed in $55 \%$ of patients, appearing unknown compression fractures in $51 \% .17$ patients died within the first 3 months. $63 \%$ of remaining patients underwent treatment. The average calcium 
was $9.04 \mathrm{mg} / \mathrm{dl}$ and average vitamin D was $27.11 \mathrm{ng} / \mathrm{ml}$; deficit persisted in $61.29 \%$, and severe in $4.84 \%$. 4 patients suffered new fractures during the follow-up.

Conclusions: Most patients with hip fragility fracture have vitamin D deficiency; this must be corrected to reduce the risk of new fractures. Treatment with standard doses of calcium and vitamin D could be enough in many cases; however, in some cases higher doses may be necessary to achieve correct serum levels, so that we modified the daily doses of calcium and vitamin D to $1200 \mathrm{mg} / 2000$ UI.

\section{P753}

APPLICATION OF FRAX IN ISRAEL: VALIDATION AND IMPACT ASSESSMENT USING REAL-WORLD MEDICAL DATABASES

I. Goldshtein ${ }^{1}$, M. Leshno ${ }^{2}$, S. Ish-Shalom ${ }^{3}$, Y. Gerber ${ }^{2}$

${ }^{1}$ Tel Aviv University, Maccabi Healthcare Services, Tel Aviv, Israel, ${ }^{2} \mathrm{Tel}$ Aviv University, Tel Aviv, Israel, ${ }^{3}$ Elisha Hospital, Haifa, Israel

Objective: To evaluate the discriminative performance of the recently constructed FRAX model for Israel, and assess the potential impact of its implementation, using real-world data.

Materials and Methods: A retrospective cohort study was conducted utilizing the computerized central database of a large Israeli health fund (both insurer and provider of healthcare services). Patients with electronically recorded BMD measured at age 50 or above at the year 2006 or earlier were eligible. First available BMD test defined the index date. Patients with less than 10 years follow-up after index (for reasons other than death) were excluded. A major osteoporotic fracture (MOF) was determined by clinical diagnoses or specific procedures of hip, spine, Colles' or humerus fractures occurring at age $50+$ for women or $60+$ for men, excluding motor vehicle accidents. FRAX scores at index were obtained using the web-calculator for a random sample of patients, and compared to actual fractures observed during the following 10 years. Area under receiver operating characteristic curve (AUC) was computed as a measure of discriminative strength. The US intervention threshold (by NOF) was applied to the sample to estimate its potential impact on detection and intervention rates.

Results: A total of 46,997 eligible patients were identified. Study sample included 6,000 patients (mean $\pm \mathrm{SD}$ age $=61 \pm 8$ years), of whom $95 \%$ women, and $50 \%$ treated for osteoporosis for at least $3 \mathrm{y}$. MOF and hip fracture events were observed for $21 \%$ and $3 \%$ of the sample population, respectively. Median (IQR) 10-year FRAX scores were $6.4 \%(4.4-9.8 \%)$ and $1.4 \%(0.7-$ $2.9 \%$ ) for any MOF and hip fracture, respectively. A total of
$15 \%$ were considered osteoporotic by BMD alone, and additional $11 \%$ met NOF's threshold (exceeded $3 \%$ hip fracture risk score or $20 \%$ MOF risk score) based on FRAX score, increasing intervention rate by $71 \%$. The observed AUC for the FRAX model was 0.63 (95\%CI: $0.61-0.65)$ and 0.77 (95\%CI: 0.72 0.82 ) for any MOF and hip fracture, respectively.

Conclusions: Among this Israeli cohort of middle-aged community dwelling patients with measured BMD, the FRAX calculator exhibited reasonable predictive value for hip fracture incidence. Adopting FRAX-based thresholds in addition to BMD would largely impact detection and intervention rates.

\section{P754}

TOPICAL NONIVAMIDE/NICOBOXIL EFFECTIVELY AND SAFELY REDUCES ACUTE UNSPECIFIC LOW BACK PAIN: A RANDOMISED, CONTROLLED TRIAL

$\underline{\text { Z. Blahova }}^{1}$, C. Holm ${ }^{1}$, T. Weiser ${ }^{2}$, E. Richter ${ }^{2}$, M. Trampisch $^{2}$, E. S. Akarachkova ${ }^{3}$

${ }^{1}$ Boehringer Ingelheim RCV GmbH \& Co KG, Vienna, Austria, ${ }^{2}$ Boehringer Ingelheim Pharma GmbH \& Co. KG, Ingelheim am Rhein, Germany, ${ }^{3}$ I.M. Sechenov First Moscow State Medical University, Moscow, Russian Federation

Objective: Low back pain affects many patients and has a high socioeconomical impact. Effective and early intervention might reduce the risk of chronification. Topical capsaicinoids have been used for decades to treat musculoskeletal pain. This study investigated the effects of the fixed dose combination (FDC) of nonivamide (a capsaicinoid) and nicoboxil (a nicotinic acid ester) cream for the treatment of acute low back pain.

Material and Methods: This phase III randomised, doubleblind, placebo-controlled, multinational, multi- centre trial investigated efficacy, safety and tolerability of topical nicoboxil $1.08 \%$ /nonivamide $0.17 \%$ for treatment of acute non-specific low back pain with the endpoints: pain intensity (PI) difference between pre-dose baseline and 8 hours after first application as well as at end of treatment, mobility score, efficacy score.

Results: Patients $(n=138), 21-65$ years of age were treated for up to 4 days with FDC or placebo cream. Baseline PI was 6.8 (on a $0-10$ point numerical rating scale). After 8 hours, pain was more reduced with the FDC (mean [95\%CI]: 2.824 [2.384, $3.264]$ points) than with placebo (mean $[95 \% \mathrm{CI}]: 0.975$ [0.546, 1.404] points), $p<0.0001$. At the end of treatment, the FDC provided more pronounced mean pain reduction (mean [95\% CI]: $5.132[4.581,5.683]$ points) compared to placebo (mean [95\%CI]: 2.174 [1.635, 2.712] points), $p<0.0001$. Mobility on Day 1 was in favour of the FDC: $35 \%$ of patients rated improvement of mobility as "good" or "very good", 
compared to $13 \%$ of placebo-treated patients $(p<0.0001)$. At the end of treatment, patients rated efficacy significantly higher: $87 \%$ treated with the FDC assessed efficacy as "good" or "very good", compared to $30 \%$ in the placebo arm $(p<0.0001)$. Both treatments were tolerated well. No treatment-related serious adverse events were reported.

Conclusion: Nicoboxil/nonivamide cream is an effective, well tolerated medication for the treatment of acute nonspecific low back pain. The study suggests that its effect size is higher when compared to the pooled effect size of systemic medications, including prescription-only analgesics and muscle-relaxants (see e.g. Keller et al. Eur Spine J 2007;16:1776).

Disclosure: Sponsor: Boehringer Ingelheim ClinicalTrials.gov No: NCT02300311

\section{P755}

THE PROGNOSTIC VALUE OF DIFFERENT COMBINATIONS OF T-SCORE AND Z-SCORE OF SPINE AND HIP DENSITOMETRY IN PREDICTING FRACTURE RISK IN POSTMENOPAUSAL WOMEN P. Borowy $^{1}$, E. Czerwinski ${ }^{2}$

${ }^{1}$ Krakow Medical Centre, Krakow, Poland, ${ }^{2}$ Metabolic Bone Diseases, Jagiellonian University College of Medicine, Krakow, Poland

Introduction: In osteoporosis the focus of the diagnosis is to predict fractures. Densitometry remains the main criterion for diagnosis; however, it has been observed that there is a great need to assess the risk of fracture resulting from BMD decrease. The aim of the study was to look for optimal $\mathrm{T}$ and Z-score combination in predicting the risk of osteoporotic fractures.

Material and methods: 1284 women aged over 50 were randomly selected into the study. Between 1997-2003 these subjects were screened for osteoporosis in the process of which they underwent the densitometry and fracture risk assessment. The questionnaire collected demographic and anthropometric data as well as risk factors based on FRAX $^{\circledR}$ - parental history of proximal femur fractures, smoking status, exposure to glucocorticosteroids, presence of rheumatoid arthritis, alcohol intake, and known secondary causes of osteoporosis. A separate part of the questionnaire was devoted to fractures. The subjects underwent the spine and/or hip densitometry, in accordance to the methodology of the International Society for Clinical Densitometry. After an average of 10 years (median 10.1) the questionnaire was repeated via telephone. ROC curve (receiver operating characteristics) was applied to evaluate the prognostic value of DXA. The following T-score and Z-score combinations were analysed by means of AUC (area under curve): T(+)Z, T(*) Z, T(-)Z, T(/)Z for spine and hip.

Objective: To evaluate the prognostic value of T-score and Zscore combinations in predicting the risk of osteoporotic fractures, particularly vertebral and proximal femur fractures.
Results: None of the above mentioned combinations showed a higher prognostic value than the assessment based on BMD in forecasting osteoporotic fractures (regardless of site). The highest value was demonstrated by spine $\mathrm{T}$-score $(+) \mathrm{Z}$-score (AUC $=0.569(0.528-0.608 ; p=0.001)$. In predicting compression fractures only three combinations showed significantly higher values: spine T-score $(+) \mathrm{Z}$ - score $(\mathrm{AUC}=0.681(0.615-$ $0.745 ; p=0.001$, proximal femur T-score $(+) \mathrm{Z}$-score $(\mathrm{AUC}=0.665(0.596-0.734 ; p=0.001)$ and proximal femur Tscore $(*)$ Z-score $(\mathrm{AUC}=0.638(0.552-0.724 ; p=0.01)$. The optimal prognostic value as per proximal femur fracture risk was demonstrated by: spine T-score $(+) \mathrm{Z}$ - score $(\mathrm{AUC}=0.616$ $(0.514-0.728 ; p=0.37)$, proximal femur T-score $\left(^{*}\right) \mathrm{Z}$-score $(\mathrm{AUC}=0.654(0.547-0.762 ; p=0.05)$.

Conclusion: There is no combination of T-score and Z-score that has a higher prognostic value with regard to fracture risk prediction than a single parameter obtained from this measurement. Densitometry, based on T- and Z-scores, remains the most valuable risk assessment tool.

P756

CAMURATI-ENGELMANN DISEASE WITH SPINAL CORD COMPRESSION: CASE REPORT AND ASSESSMENT OF BONE MICROARCHITECTURE BY HR-PQCT

C. R. Uña $^{1}$,M. B. Zanchetta ${ }^{2}$, J. J. Rivero ${ }^{3}$, F. O. Rodriguez ${ }^{3}$,J. R. Zanchetta ${ }^{2}$ O. D. Messina ${ }^{1}$

${ }^{1}$ Rheumatology Section, Hospital Gral. de Agudos Dr. C. Argerich, Ciudad Autónoma de Buenos Aires, Argentina, ${ }^{2}$ Idim, Universidad del Salvador, Ciudad Autónoma de Buenos Aires, Argentina, ${ }^{3}$ Neurology Section, Hospital Gral. de Agudos Dr. C. Argerich, Ciudad Autónoma de Buenos Aires, Argentina

Camurati-Engelmann's disease (CED) is a rare autosomal dominant condition caused by different mutations in TGF $\beta 1$, that mainly lead to increase bone formation.

Aim: Describe the first patient with presumptive diagnosis of CED and spinal cord compression in which their bone microarchitecture was assessed by HR-pQCT.

Case description: A 46 year old female hospitalized with severe pain in the lower limbs and lumbar spine (LS) plus gait disorders, progressive since 26 years old. There was similar symptoms in some relatives. She had prominent forehead, decreased muscle mass, painful march and pyramidal syndrome. Plain X-rays: symmetrical thickening and sclerotic appendicular skeleton diaphyses and skull, with narrowed medullary cavity. Initial laboratory: serum calcium $(\mathrm{sCa})$ $8.64 \mathrm{mg} / \mathrm{dl}$, phosphate (sP) $2.8 \mathrm{mg} / \mathrm{dl}$, alkaline phosphatase (sAP) 137U/L, 25-OH-vitamin D 17ng/ml, iPTH 33pg/ml. Normal protein electrophoresis. MRI LS: increased lordosis, fascetaria hypertrophy. Bone scintigraphy Tc99-MDP: increased uptake in affected bones. The patient was interpreted 
as CED and treatment with an intramuscular deposit corticosteroid followed by deflazacort, vitamin D3 and losartan. The laboratory was repeated two months later: $\mathrm{sCa} 9.37 \mathrm{mg} / \mathrm{dl}, \mathrm{sP}$ $3.9 \mathrm{mg} / \mathrm{dl}$, 24h urine Ca $140.36 \mathrm{mg}$, 24hs urine P $362.2 \mathrm{mg}$, urinary $\mathrm{Na} 163 \mathrm{mEq} / 1$, urinary creatinine $81.1 \mathrm{mg} / \mathrm{dl}, 25-\mathrm{OH}-$ vitamin D $23.8 \mathrm{ng} / \mathrm{ml}$, serum CrossLaps $723 \mathrm{pg} / \mathrm{ml}$, sAP $126 \mathrm{U} /$ L, bone fraction sAP $30.8 \mathrm{U} / \mathrm{L}(24 \%)$.

HR-pQCT: Distal radius (photo1): Tb density, BV/TV and Tb number all low; Tb spacing, Tb.I/N.SD and Ct thickness all high. distal tibia (photo 2): Ct density low; Tb.I/N.SD and $\mathrm{Ct}$ thickness high. MRI cervical spine: posteromedial herniation $\mathrm{C} 3-\mathrm{C} 4$ disc and posterolateral $\mathrm{C} 4-\mathrm{C} 5$ disc, osteophytes, facet hypertrophy, narrow spinal canal and spinal cord compression, with myelomalacia $\mathrm{C} 4-\mathrm{C} 5$.

Conclusion: CED altered bone microarchitecture was assessed using HR-pQCT. The abnormal gait was not only by the CED itself; it may be secondary to a spinal cord compression.

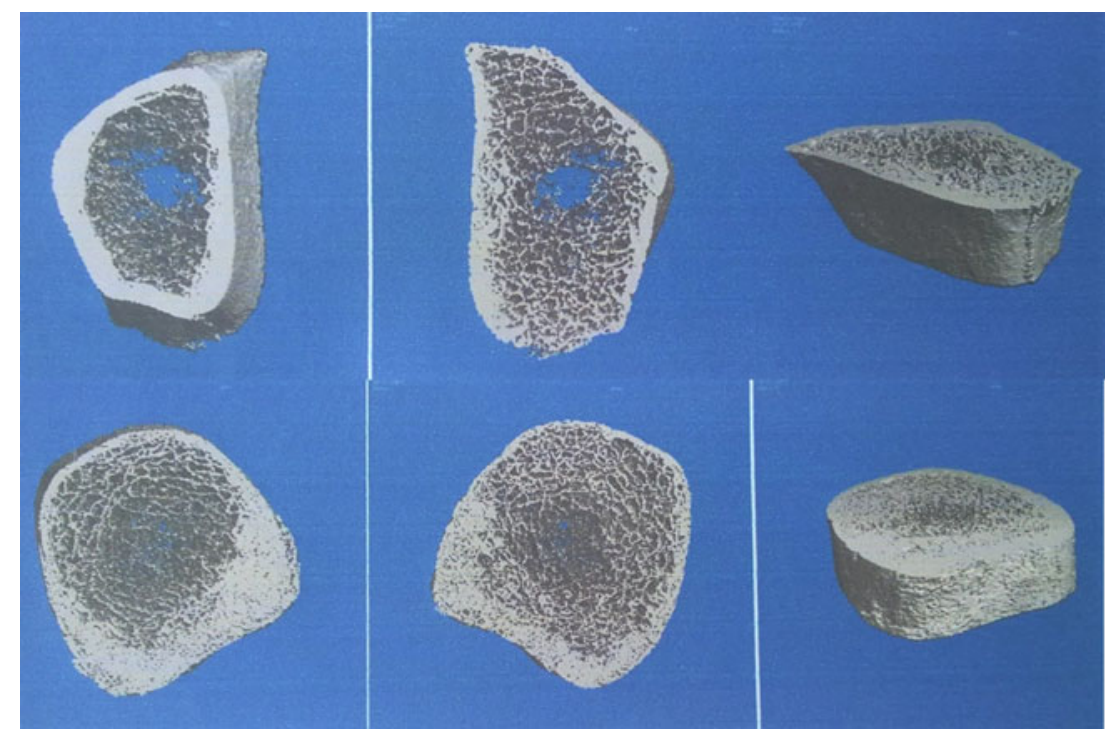

Acknowledgement: to the "Jaime Roca" Foundation (TCbaDiagnostic Center) who donated Brain and Cervical Spine MRI.

\section{P757}

\section{EVALUATION OF BONE METABOLISM IN PA-} TIENTS WITH PROLACTINOMA

F. B. Bayram $^{1}$, B. Tekin ${ }^{1}$, D. Yavuz ${ }^{2}$

${ }^{1}$ Department of Internal Medicine, Marmara University Hospital, Marmara University School of Medicine, Istanbul, Turkey, ${ }^{2}$ Department of Internal Medicine, Section of Endocrinology and Metabolism, Marmara University Hospital, Marmara University School of Medicine, Istanbul, Turkey

Objective: Hyperprolactinemia has been reported to be associated with abnormalities of bone metabolism and increased risk of vertebral fractures. The aim of this study was to evaluate the bone metabolism and vertebral fracture risk of our patients with prolactinoma in outpatient clinics.
Material and methods: In this cross-sectional study, most of which are currently or previously treated 53 hyperprolactinemic patients with PRL-secreting adenoma (32 with microadenoma and 21 with macroadenoma) and 55 healthy controls with normal PRL values and comparable gender, age and BMI with patients were included. They were evaluated for BMD by DXA at lumbar spine and femur, for vertebral fractures by a morphometric approach. Bone turn-over markers such as serum osteocalcin and c-telopeptide levels were measured. Both groups were also similar in terms of risk factors for osteopenia, such as family history, previous trauma-related fractures, alcohol ingestion, smoking and physical activity.

Results: There were no significant differences in BMD, femur neck $\mathrm{T}$ and $\mathrm{Z}$ scores, L1-4 $\mathrm{T}$ and $\mathrm{Z}$ scores between prolactinoma and control groups. However lumbar vertebrae L1-4 BMD, T and Z scores were significantly lower in macroprolactinoma group compared to microprolactinoma patients $(p=0.004, p=0.01, p=0$., $01)$. Vertebral fractures were detected in 6 patients with 
PRL-secreting adenoma $(11 \%)$ and in 1 control group (1 \%, $p=0.05)$. A significant positive correlation was observed between serum osteocalcin and c-telopeptide levels $(p=0.0001),(r=0.51)$. Also serum osteocalcin levels were significantly higher in patients with prolactinoma than control group $(p=0.03)$.

Conclusion: Contrary to previous studies; BMD measurements of prolactioma and control groups were similar in our study. This situation can be associated with that most of our patients are under treatment or were previously treated. Although treatment with dopaminergic agents have favourable results in BMD, the higher vertebral fracture rates of prolactioma patients than control group showed that; fracture risk still exists in prolactinoma patients.

\section{P758}

THE PREDICTIVE VALUE OF DENSITOMETRY OF THE SPINE AND PROXIMAL FEMUR IN PREDICTING FRACTURE IN POSTMENOPAUSAL WOMEN

P. Borowy $^{1}$, E. Czerwinski ${ }^{2}$

${ }^{1}$ Krakow Medical Centre, Krakow, Poland, ${ }^{2}$ Metabolic Bone

Diseases, Jagiellonian University, Krakow, Poland

Introduction: In osteoporosis the focus of the diagnosis is to predict fractures. Densitometry remains the main criterion for diagnosis; however, it has been observed that there is a great need to assess the risk of fracture resulting from BMD decrease.

Material and methods: 1284 women aged over 50 were randomly selected into the study. Between 1997-2003 these subjects were screened for osteoporosis in the process of which they underwent the densitometry and fracture risk assessment. The questionnaire collected demographic and anthropometric data as well as risk factors based on FRAX ${ }^{\circledR}$. A separate part of the questionnaire was devoted to fractures. The subjects underwent the spine and/or hip densitometry, in accordance to the methodology of the International Society for Clinical Densitometry. After an average of 10 years (median 10.1) the questionnaire was repeated via telephone. ROC curve was applied to evaluate the prognostic value of DXA. Relative risk (RR) of an osteoporotic fracture in different locations depending on the DXA parameters was evaluated using Cox proportional hazard model.

Objective: The aim of the study was to evaluate the prognostic value of densitometry in predicting the risk of osteoporotic fractures (RR), particularly vertebral and proximal femur fractures, with a special focus on spine and hip T-scores and Z-scores.

Results: AUC values of T-score in predicting proximal femur fractures was highest and amounted to 0.701 (vertebral fracture), 0.703 (proximal femur fracture) and 0.629 (all osteoporotic fractures). AUC values with regard to T-score in predicting fractures was 0.696 (vertebral fracture), 0.696 (proximal femur fracture) and 0.601 (all osteoporotic fractures). The highest AUC value was observed in the younger population regardless of the forecast fracture site (50-60 years old, e.g. AUC for T-score $=0.854$; in age range $>71$ AUC for T-score=0.580). The values of relative risk (RR) varied depending on site of examination and predicted fracture. It has been demonstrated that the fracture risk does not grow linearly.

Fig. 1

\begin{tabular}{|c|c|c|c|c|}
\hline \multirow{2}{*}{$\begin{array}{l}\text { BMD hip } \\
\text { (g/cm2) }\end{array}$} & \multirow[t]{2}{*}{$\mathrm{P}$} & \multirow{2}{*}{$\begin{array}{l}\text { RR of spine } \\
\text { fracture }\end{array}$} & \multicolumn{2}{|c|}{$95,0 \% \mathrm{CI}$ for $\mathrm{PR}$} \\
\hline & & & Lower Bound & Upper Bound \\
\hline$\geq 0,95$ & 0,001 & 1 & & \\
\hline 0,$85 ;<0,95$ & 0,104 & 5,561 & 0,705 & 43,897 \\
\hline 0,$75 ;<0,85$ & 0,142 & 4,607 & 0,599 & 35,443 \\
\hline 0,$65 ;<0,75$ & 0,036 & 8,571 & 1,146 & 64,076 \\
\hline$<0,65$ & 0,005 & 17,722 & 2,349 & 133,698 \\
\hline
\end{tabular}

Conclusion: The prognostic value of densitometry in predicting compression fractures and proximal femur fractures is high. Hip densitometry provides the best prediction of vertebral and proximal femur fractures. (AUC 0.703; 0.23-0.782, $p=0.000$ ). The value of densitometry in predicting peripheral fractures is low. Its sensitivity and specificity differ depending on age - they decrease with age with the highest value in the youngest group. RR increases non-linearly as BMD decreases.

\section{P759}

BIOCHEMICAL MARKERS AS PREDICTORS OF BONE HISTOMORPHOMETRIC ALTERATIONS IN PATIENTS WITH CHRONIC KIDNEY DISEASE

M. Feola $^{1}$, C. Rao ${ }^{1}$, M. Scimeca ${ }^{2}$, M. Celi $^{1}$, A. Scialdoni ${ }^{1}$, E. $\overline{\text { Gasbarra }}^{1}$, U. Tarantino ${ }^{1}$

${ }^{1}$ Department of Orthopedics and Traumatology, "Tor Vergata" University of Rome, "Policlinico Tor Vergata" Foundation, Rome, Italy, ${ }^{2}$ Anatomic Pathology Section, Department of Biomedicine and Prevention, University of Rome, Rome, Italy

Introduction: The metabolic impairment of the musculoskeletal is common in patients with kidney disease. The chronic kidney disease mineral and bone disorder (CKD-MBD) is a syndrome in which biochemical, clinical and radiographic alterations affecting the bone tissue coexist.

Objective: To evaluate the biochemical and morphostructural alterations in different states of CKD in patients with osteoarthritis or hip fracture.

Materials and Methods: We evaluated all patients undergoing hip replacement surgery from January 2012 to April 2015 for fracture of the medial femoral neck or hip osteoarthritis. For each patient blood chemistry was evaluated for the study 
of the bone metabolism and the kidney function; glomerular filtration was calculated by CKD-EPI method and the patients were divided, according to the degree of CKD, in five groups. All patients underwent the evaluation of the BMD by DXA examination and of the histomorphometric evaluation system by Bioquant of the bone sample taken from the femoral head during surgery.

Results: 272 patients (92 men and 180 women, average age $76.1)$ were included in the study. They underwent surgery for fracture $(n=144)$ or osteoarthritis $(n=128)$. The patients with grade I $(n=43)$ and II $(n=112)$ were arthritic respectively in $65 \%$ and $60 \%$, while patients with grade III $(n=99)$, IV $(n=14)$ and V $(n=4)$ were fractured respectively $69 \%, 92 \%$ and $75 \%$. PTH was lower in patients with grade I and II $(p<0.01)$, vitamin $\mathrm{D}$ in patients grade III and IV $(p<0.01)$. The BMD values were lower with the decrease in renal function both in osteoarthritic patients than in the fractured ones. Histomorphometric analysis showed a progressive decrease of the structural parameters of the bone with a bone volume significantly lower in patients with stage III-IV $\left(0.525 \mathrm{~mm}^{2}\right)$ compared with grades I-II $\left(0.63 \mathrm{~mm}^{2}, p<0.01\right)$ as well as even a reduced trabecular thickness $(13.88$ vs. 16:54, $p<0.01)$.

Conclusions: Metabolic alterations linked to CKD appear to affect bone quality already in the initial states and become striking in the later stages. The main biochemical parameters of the bone metabolism were significantly related to the degree of CKD and to the microstructural alterations.

\section{P760}

\section{EFFECT OF DENOSUMAB IN IMPROVING OR- THOSTATIC HYPOTENSION}

$\underline{\text { K. Thavarajah }}^{1}$, F. O'Sullivan ${ }^{1}$, G. O’Malley ${ }^{1}$, E. Shanahan ${ }^{1}$, D. Lyons ${ }^{1}$, M. O'Connor ${ }^{1}$, C. Peters ${ }^{1}$, A. Gabr ${ }^{1}$, N. MohdAsri $^{1}$, N. Muthalvan ${ }^{2}$

${ }^{1}$ Department of Elderly Medicine, University Hospital Limerick, Dooradoyle, Ireland, ${ }^{2}$ Department of Anaesthesia and Intensive Care, University Hospital Limerick, Dooradoyle, Ireland

Background: Denosumab is widely used for the treatment of postmenopausal women with osteoporosis at high/increased risk for fracture. It is a fully human monoclonal antibody to RANKL that blocks its binding to RANK, inhibiting the development and activity of osteoclast, decreasing bone resorption, and increasing bone density ${ }^{1}$. RANKL leads to the activation of antiapoptotic kinase and nitric oxide synthase and to nitric oxide (NO) production in endothelial cells. Moreover RANKL acts as a potent vasodilator ${ }^{2}$.

Objective: This is a pilot observational study for further longitudinal work on an interesting hypothesis. The aim is to determine if denosumab improves orthostatic hypotension $(\mathrm{OH})$ by inhibition of RANKL leading to vasoconstriction and reduction of NO production.

Methods: Data of 30 patients with head-up-tilt (HUT) test prior to denosumab treatment (T0) at 3(T3), 6(T6) and 12 months (T12) were analysed. 8 were excluded due to incomplete participation.

Results: 16 patients had $\mathrm{OH}$ prior to treatment and only two were on midodrine. Drop in systolic blood pressure varied between $20 \mathrm{mmHg}$ to $101 \mathrm{mmHg}$ and diastolic drop between $10 \mathrm{mmHg}$ to $34 \mathrm{mmHg}$ prior to treatment. Following denosumab treatment, 5 of 16 patients had completely resolved $\mathrm{OH}$. Magnitude of the blood pressure drop improved in 8 patients. 3 patients had worsened $\mathrm{OH}$; Among them 2 had Parkinson's disease and one was on antihypertensive treatment. Of 6 patients who did not have $\mathrm{OH}$ pretreatment, 5 developed $\mathrm{OH}$, in which 3 were on antihypertensive medications. 2 of 5 patients who developed $\mathrm{OH}$ at 6 months had resolution of $\mathrm{OH}$ after 12 months of treatment. Mean age of the participants was 76.09 with standard deviation (SD) of 7.38. Age range was between 65 to 88.15 people $(68.2 \%)$ had $\mathrm{OH}$ prior to treatment. T0 median $\triangle \mathrm{SBP}$ (Systolic Blood Pressure) was 27.5, minimum $\Delta$ SBP was -6 and maximum was 101, IQR of 25. 16 people had HUT at 6 months and 13 of them had OH. 4/5 people with no $\mathrm{OH}$ at $\mathrm{T} 0$ had $\mathrm{OH}$ at T6. 2/11 people with no $\mathrm{OH}$ at $\mathrm{T} 0$ do not have $\mathrm{OH}$ at T6. There was a mean reduction of $\triangle \mathrm{SBP}$ of $11.8, \mathrm{SD} 11.9$ at T6 compared to T0 ( $p$ value of 0.52 ). In those with $\mathrm{OH}$ at $\mathrm{T} 0$, there was mean reduction in $\triangle \mathrm{SBP}$ of 12.54 , SD 21.87 (p value of 0.09 ). There was no significant difference in $\triangle$ DBP (Diastolic Blood Pressure) at T6 compared to T0 ( $p$ value of 0.6). 16 people had HUT at 12 months and 11 of them had $\mathrm{OH}$. 1/4 people who did not have $\mathrm{OH}$ at $\mathrm{T} 0$ had $\mathrm{OH}$ at $\mathrm{T} 12.10 / 12$ people who had $\mathrm{OH}$ at T0 still have $\mathrm{OH}$ at T12. T12 mean $\triangle \mathrm{SBP} 16.36$, SD 20.54, T12 mean $\triangle \mathrm{DBP} 6.07$, SD 7.96. There was no significant difference in mean $\triangle \mathrm{SBP}$ between $\mathrm{T} 0$ and T12 ( $p$ value of $0 / 075$ ). There was no significant difference in mean $\triangle$ DBP between T0 and $\mathrm{t} 12$ ( $\mathrm{p}$ value of $0 / 6)$. In those who had $\mathrm{OH}$ at $\mathrm{T} 0$ there was a significant reduction in $\triangle \mathrm{SBP}$ at $\mathrm{T} 12$ compared to $\mathrm{T} 0$ ( $p$ value of 0.003 ) with mean reduction in systolic postural blood pressure drop of 17.9 and SD of 21.6

Conclusions: HUT test results after a year of denosumab treatment showed improvement in orthostatic hypotension. Deterioration of orthostatic hypotension in several patients was probably due to underlying Parkinson disease and anti-hypertensive medications. Thus, improvement in $\mathrm{OH}$ is expected in patients on longstanding denosumab treatment. 
Discussion: Freedom trial study on denosumab for prevention of fractures in postmenopausal women with osteoporosis published in New England Journal of Medicine. 2009;361(8):756-765 reported falling as an adverse event. Number of people who had falls on denosumab among was 175 (4.5) among 3886 with $p$ value of 0.02 . In this study, denosumab improves orthostatic hypotension $(\mathrm{OH})$ by inhibition of RANKL leading to vasoconstriction and by reduction of NO production. Thus, improvement in $\mathrm{OH}$ is expected to reduce the risk of falls in patients who receives denosumab treatment. Limitations of this study include small number of participants and multiple confounding factors. A further study is planned with strict protocols and also to do a HUT at 36 months in order to compare the falls risk results with freedom trial study on denosumab.

\section{References:}

1. Cummings S, et al. NEJM 2009;361:756.

2. Min J, et al. Blood 2007;109:1495.

\section{P761}

OUTCOMES OF A MULTIDISCIPLINARY APPROACH FOR THE MANAGEMENT OF HIP FRACTURES IN OLDER PATIENTS. EXPERIENCE IN A REGIONAL HOSPITAL

$\underline{\text { M. Perez Santamaria }}^{1}$, M. J. Dominguez Arevalo ${ }^{1}$, J. Manso Perez Cossio $^{1}$, M. Peraza Sanchez ${ }^{1}$

${ }^{1}$ Hospital de Laredo, Laredo, Spain

Objective: To show that outcomes of hip fractures management in older patients may be improved by implementing a multidisciplinary approach.

Material and Methods: Since 2013, our Hospital has developed guidelines for hip fracture management in older patients based on Orthogeriatrics Units. The multidisciplinary team was composed by staff from Orthopedic Surgery, Internal Medicine, Anesthesiology, Rehabilitation and Physical Therapy services, along with Social Workers, committed to assess the problem from a comprehensive and specialized point of view in order to optimize the clinical status of the patient. Our purpose was to perform surgical treatment earlier than 48 hours post-admission and to achieve an early functional recovery along with adequate socio-sanitary state at discharge. Additionally, as a secondary prevention strategy, we have implemented an osteoporosis management guideline, which gives prominence to specific medical treatment at discharge based on patient medical status. It consists of oral bisphosphonates $(65 \%)$, zolendronate $(22 \%)$ in a single intravenous dose, or PTH (13\%) in patients with previous fractures and/or high risk of hip fractures. The study was composed by 309 patients ( $79.3 \%$ women; $20.7 \%$ men). Age interval, $65-103$ years $($ mean $=84.2$ y). Hip fractures were classified as intracapsular (46.6\%) or extracapsular $(53.4 \%)$. Data were collected on admission and 6 months later.

Results: Time to surgical procedure $(67.92 \mathrm{~h}$, before guideline to $47.84 \mathrm{~h}$, after); mean inpatient stay (11.5 days to 10.4 days). Postoperative mortality ( $9.1 \%$ to $7.9 \%)$. Patient-specific functional scores: Barthel Index $>60$ (59 \% at 6 months); Holden ambulation score $>345 \%$. No cases of contralateral hip fracture were reported. Antiosteoporotic treatment adherence $61 \%$.

Conclusions: Hip fracture care demands a different approach from other trauma procedures, based on a comprehensive and multidisciplinary care of older patients. Performing surgical treatment as early as possible in optimized and stabilized patients allows to better reach functional outcomes and a shorter inpatient stay, resulting in lower health services costs and better results in patient and family satisfaction surveys. That is the reason why we consider the model implemented in our hospital as an optimal alternative to be implemented in centers of similar characteristics.

\section{P762}

\section{STANDARDIZING 25-HYDROXYVITAMIN D DATA FROM THE HUNMEN COHORT}

H. P. Bhattoa $^{1}$, E. Kulcsar-Jakab ${ }^{1}$, Z. Petho ${ }^{2}$, Z. Pap ${ }^{3}$, E.

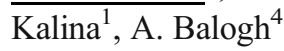

${ }^{1}$ Department of Laboratory Medicine, University of Debrecen, Debrecen, Hungary, ${ }^{2}$ Department of Rheumatology, University of Debrecen, Debrecen, Hungary, ${ }^{3}$ Department of Traumatology, University of Debrecen, Debrecen, Hungary, ${ }^{4}$ Department of Obstetrics and Gynecology, University of Debrecen, Debrecen, Hungary

Background: Development of robust healthcare policy to improve vitamin $\mathrm{D}$ sufficiency is critical and is primarily based on vitamin D data achieved from measurement of total 25hydroxyvitamin D (25OHD). Although there is abundance of data, unstandardized laboratory measurement of 25OHD has greatly hindered attempts to uniformly adapt guidelines. Lately the Vitamin D standardization Program (VDSP) has delineated protocols for standardizing existing 25OHD data from national surveys around the world.

Objective: We introduce a methodology to calibrate $25 \mathrm{OHD}$ values to the NIST reference measurement procedures using VDSP methods thereby facilitating future guidelines in Hungary. We have applied our approach to total $25 \mathrm{OHD}$ values belonging to the HunMen cohort. Briefly, the HunMen study was a local initiative to evaluate the bone health of randomly selected healthy men who confirmed to the inclusion and/or did not confirm to the exclusion criteria. Inclusion criteria were $>50$ years of age, male, ambulatory, community dwelling and 
generally regarded as healthy. Exclusion criteria were known prevalent metabolic bone disease, liver or renal disease and use of medication influencing bone metabolism. The study was approved by the ethics review board of the institution in compliance with the Declaration of Helsinki, and all subjects gave written informed consent.

Design: Vitamin D External Quality Assessment (DeQas) data on $\%$ bias from ALTM was used to correct measurement values achieved using our analytical methodology. Reagents with the same LOT number were used to measure the DeQas and the HunMen samples.

Results: All 206 samples for the HunMen cohort were evaluated using the DiaSorin platform for measuring total 25OHD. The timing of these measurements coincided with that of the October 2015 DeQas samples. Average\% bias from ALTM was $-13.5 \%$ for DeQas samples 481 to 484 , values for sample 485 was excluded since it showed an over $20 \%$ difference from the NIST assigned value. The mean total $25 \mathrm{OHD}$ value changed markedly, i.e., $72.8 \mathrm{nmol} / \mathrm{L}$ with the original HPLC methodology, $52.6 \mathrm{nmol} / \mathrm{L}$ was the observed mean using the DiaSorin platform and $59.6 \mathrm{nmol} / \mathrm{L}$ was the mean corrected value. Subsequently, the originally reported prevalence of hypovitaminosis $\mathrm{D}(<75 \mathrm{nmol} / \mathrm{L})$ for the HunMen cohort changed significantly, i.e., from $52.9 \%$ to $71.8 \%$.

Conclusion: Our simple approach towards standardizing $25 \mathrm{OHD}$ values using the DeQas survey\% bias from ALTM may encourage participating laboratories to readily correct values at primary result delivery.

\section{P763}

INFLIXIMAB INDUCTION COURSE CAN ACTIVATE BONE METABOLISM IN SEVERE PEDIATRIC CROHN'S DISEASE

T. Gabrusskaia ${ }^{1}$, M. Kostik ${ }^{1}$, M. Revnova ${ }^{1}$

${ }^{1}$ Saint-Petersburg State Pediatric Medical University, SaintPetersburg, Russian Federation

Objectives: Disturbances of bone metabolism in Crohn's disease (CD) can include osteoporosis, increased risk of lowenergy fractures and linear growth failure and have multifactorial nature: systemic inflammation, malabsorption, vitamin D deficiency, corticosteroid (CS) therapy. CS is a first line treatment for severe and moderate forms of $\mathrm{CD}$, but it can deteriorate bone mineralization and metabolism. Severe patients require treatment with infliximab (INF)- tumor necrosis factor- $\alpha$ inhibitors, which does not impact bone and possibly can improve bone status through control of the systemic inflammation. The aim of our study was to evaluate the changing of bone metabolism during the infliximab induction course.

Material and Methods: 15 children with $\mathrm{CD}$ (7 M and $8 \mathrm{~F}$ ) aged 6-17 years (median age 14.0) who failed the treatment with CS, azathioprine and 5-aminosalycilic acid were treated with infliximab induction scheme (0-2-6 weeks) in $5 \mathrm{mg} / \mathrm{kg}$ per infusion. Lumbar spine BMD (DXA), serum osteocalcin (OC), C- terminal telopeptides (CTT), 25-OHD 3 and fecal calprotectin (CP) were measured before INF. OC, CTT and $\mathrm{CP}$ were measured repeatedly before the 4 th INF infusion (week 12). We used Mann-Whitney U test, Wilcoxon test, chi-square test. Data are presented in the median and interquartile range ( $25 \% ; 75 \%)$.

Results: The initial (before INF) bone status included BMD Zscore - 1.7 SD (-2.1; -0.7), 25- $\mathrm{OHD}_{3}-17.4 \mathrm{ng} / \mathrm{ml}(16.0 ; 25.0)$. At week 12 in 13/15 patients we observed gain of OC levels from $21.1 \%$ to $3930.0 \%, \Delta=100 \%(25.5 ; 577)$ from $6.3 \mathrm{ng} / \mathrm{ml}$ $(3.3 ; 9.6)$ to $10.9 \mathrm{ng} / \mathrm{ml}(8.2 ; 32.0), p=0.005$. CTT levels increased only in $8 / 15$ patients from $1.9 \%$ to $492.9 \%, \Delta=1.9 \%$ $(-22.8 ; 65.0)$, from $1.05 \mathrm{ng} / \mathrm{ml}(0.83 ; 1.57)$ to $1.29 \mathrm{ng} / \mathrm{ml}(0.83$; 2.6), $p=0.33$ and in $7 / 15$ patients CTT levels decreased ranged $-3.3 \%$ to $-54.7 \%$. CP decreased in $12 / 15$ from $798 \mu \mathrm{g} / \mathrm{g}$ (750.0; 961.0) to $113.0 \mu \mathrm{g} / \mathrm{g}(34.0 ; 654.0), p=0.0007$. There were no differences in initial status between patients with increased and decreased CTT level at week 12. Only unchanged $\mathrm{CP}$ level was predictor of decreased CTT level during INF induction course $(O R=13.2(0.6 ; 316.7), p=0.048)$.

Conclusion: INF treatment can activate bone metabolism in pediatric CD patients. Unchanged CP level can appear as a marker of poor response to INF and bone poor outcomes. Further trials are necessary.

\section{P764}

\section{VALIDATION OF THE QFRACTURE SCALE IN} SPAIN

M. Sosa $^{1}$, N. Sosa-Cabrera ${ }^{2}$, N. Sablón ${ }^{1}$, L. Rivero ${ }^{3}$, M. J. Gómez de Tejada $^{4}$, A. I. Reyes-Domínguez ${ }^{1}$, P. Saavedra ${ }^{1}$

${ }^{1}$ Instituto Universitario de Investigaciones Biomédicas y Sanitarias, University of Las Palmas de Gran Canaria, Las Palmas de Gran Canaria, Spain, ${ }^{2}$ Centro de Salud de Tejina, La Laguna, Tenerife, Spain, ${ }^{3}$ University of Las Palmas de Gran Canaria, Las Palmas de Gran Canaria, Spain, ${ }^{4}$ Department of Medicine, University of Seville, Seville, Spain

Introduction: In the last years it has been published several clinical scales based on risk factors who estimate the risk of suffering a fracture in the next 10 years. One of these scales, Qfracture, has not been validated outside its original country, United Kingdom.

Main objective: To validate the Qfracture scale in the Spanish population. The outcome was the first fracture recorded after the determination of the Qfracture.

Material and Methods: Prospective observational study. 529 patients were included. They all had at least two clinical evaluations and were studied at least for 10 years. Qfracture was estimated at the first visit. Some patients were followed up to 
24 years. All the new fractures were recorded and verified, either with X-rays or with clinical reports.

Statistical study: Univariate analysis. The categorical variables expressed as frequencies and percentages, and you continue as averages and diversions standard when the information was following a normal distribution, and as medians and ranges interquartiles (percentiles 25-75) when the distribution was not normal. The percentages were compared using the chi-square test, the media with Student's t-test and the medians with the Wilcoxon's test for independent data. Analysis of survival. In order to explore the predictive capacity of the QFracture major, the patients were classified in agreement by the tertiles corresponding to the predictor $(<2.8 ; 2.8-7 ;>7)$. In each of these groups the curves of survival were estimated up to the appearance of the first fracture. In each of these groups the curves of survival were estimated up to the appearance of the first fracture by means of Kaplan-Meier's method. The difference between them was confirmed using the test log-rank. Likewise there was obtained the value of the statistician $\mathrm{D}$, which evaluates the capacity discriminant of the scoreboard for the fractures. In order to evaluate the capacity discriminant of the QFracture for any fracture, the patients who had a follow-up for at least 10 years they were classified as they had had or not at least a fracture in the first 10 years. For this classification it was realized a receiver operating characteristics analysis (ROC), being estimated the area under the curved correspondent ROC by means of an $95 \%$ interval. Discriminant of the QFracture was selected as ideal threshold, the value associated with the point of the curve ROC that minimized the quantity (1 sensibility $)^{2}+(1-\text { specificity })^{2}$. For this threshold there were estimated the sensibility, specificity, the predictive positive value (PPV) and the predictive negative (PNV) by means of confidence intervals to $95 \%$. A contrast of hypothesis was considered to be statistically significant when the corresponding value of $p$ was lower than $5 \%$. The information was analyzed using the program $\mathrm{R}$, version 3.1.0.

Results: Are shown in figures and tables.

Conclusion: Qfracture is now validated for its use in Spain.

\section{P765}

RELATIONSHIP BETWEEN BODY COMPOSITION, FAT MASS AND BONE MINERAL DENSITY IN ELDERLY BRAZILIANS

B. S. E. Peters ${ }^{1}$, P. C. Santos ${ }^{1}$, N. A. G. França ${ }^{1}$, M. M. S. Lima $^{1}$, E. B. Curatella ${ }^{1}$, L. A. Martini ${ }^{1}$

${ }^{1}$ Nutrition Department, School of Public Health, University of São Paulo, São Paulo, Brazil

Objective: Whereas the excess of body fat mass is an independent risk factor for osteoporosis and osteoporotic fractures, we aimed to evaluate the relationship between body fat mass (BFM) and BMD in the elderly people.

Material and Methods: This subsample of a cross-sectional population-based study evaluated the elderly population, aged 60-84 years, 78 women and 44 men, from Sao Paulo, Brazil. The body composition and BMD of the lumbar spine (L1 to L4) and femoral neck, was determined by iDXA (GE Healthcare, Madison, WI). The BMI was classified according Pan American Health Organization (2002); fat mass index (FMI, fat mass/ height) was classified according Kelly \& Wilson (2009); osteopenia and osteoporosis was classified according to WHO. Statistical analyses were performed using SPSS 20.0. The significance level was $5 \%$. Results are expressed as mean (SD).

Results: The mean weight of total sample was 74.36 $\pm 17.16 \mathrm{~kg}$. Underweight was presented in $11.5 \%$ of the subjects, normal weight in $39.3 \%$, overweight in $11.5 \%$ and obese in $37.7 \%$. In relation to FMI, $0.8 \%$ of the sample was classified as mild fat deficit, $18.9 \%$ as normal and $80.3 \%$ as excess fat. The mean of BMD L1 to L4 and BMD femoral neck were $1.136 \pm 0.216$ and $0.900 \pm 0.163 \mathrm{~g} / \mathrm{cm}^{2}$, respectively. Osteopenia and osteoporosis were presented in $31.4 \%$ and $11.6 \%$ of subjects according to BMD L1 to L4, and $55.8 \%$ and $3.3 \%$ by BMD femoral neck. BMD L1 to L4 was statistically significant lower in subjects with normal weight compared to obese $\left(1.101 \pm 0.196\right.$ vs. $1.232 \pm 0.201 \mathrm{~g} / \mathrm{cm}^{2}$; $p=0.012$ ). BMI presented significant positive correlation with BMD femoral neck $(r=0.313 ; p<0.001)$ and BMD L1 to L4 $(r=0.305 ; p=0.001)$. FMI presented significant positive correlation with BMD femoral neck $(r=0.189 ; p=0.038)$.

Conclusion: In the present study was observed that the prevalence of excess fat mass was high in our elderly population. Although controversial, similar to previous studies, the body fat mass showed a positive association with the bone mass, particularly in the body mineral density lumbar spine.

\section{P766 \\ FREQUENCY OF CERTAIN TYPES VERTEBRAL FRACTURES IN PRIMARY AND SECONDARY OSTEOPOROSIS}

V. Iriski ${ }^{1}$

${ }^{1}$ Indjija Health Center, Indjija, Serbia

Introduction: Vertebral fractures and their symptoms may be the first sign of osteoporosis. Observing changes in the vertebral bodies of the spinal column at the radiography in a patient with back pain which was not preceded by trauma is a relatively common finding, especially in the sixth (or more) years old.

Objective: We analyzed the frequency of different types of deformity in vertebral fracture in patients with osteoporosis and possible correlation of a certain type of primary or secondary OP. 
Material and Methods: The study included 103 patients: 47 with primary osteoporosis (43 women and 4 men) and 56 with secondary osteoporosis ( 38 women and $18 \mathrm{men}$ ). The causes of secondary osteoporosis were: RA-11, IDDM-12, primary biliary cirrhosis -3 , hepatitisC-2, peptic ulcer-13, COPD-5, 6 hemiplegia-6, SM polyneuropathy-2, MS-1, SEL-1. All patients were first seen in the clinic because of back pain, except for patients with RA or stroke, which are due to the primary disease before seen. In addition to physical examination, all were examined by radiography thoraco-lumbar spine in 2 directions and routine laboratory analysis.

Results: Are presented in Table 1.

\begin{tabular}{lllll}
\hline age & wedge & endplate & crush & $\sum=103$ \\
\hline $60-69$ & $\mathrm{P} / \mathrm{S} 10 / 13$ & $\mathrm{P} / \mathrm{S} / 3 / 6$ & $\mathrm{P} / \mathrm{S} 4 / 5$ & $\mathrm{P} / \mathrm{S} 17 / 24$ \\
$70-79$ & $\mathrm{P} / \mathrm{S} 8 / 5$ & $\mathrm{P} / \mathrm{S} \mathrm{6} / 6$ & $\mathrm{P} / \mathrm{S} 6 / 10$ & $\mathrm{P} / \mathrm{S} 20 / 21$ \\
$80-89$ & $\mathrm{P} / 2$ 2/1 & $\mathrm{P} / \mathrm{S} \mathrm{6} / 1$ & $\mathrm{P} / \mathrm{S} 2 / 9$ & $\mathrm{P} / \mathrm{S} 10 / 11$ \\
\hline
\end{tabular}

Patients in the seventh decade of life, the predominant type of fracture were wedge in both the primary and secondary OP. In the group in the eighth decade of life, the dominant type were wedge fractures in the primary and secondary crush fractures in OP. Biconcave (endplate) fractures are the most common type of primary OP in patients in the ninth decade of life. For peers with secondary OP, the dominant type were crush fractures.

Conclusion: These results suggest the conclusion that the type of fracture regarding with the activity of exemplary certain age but also with hormonal status that changes with aging, as well as a possible expression of certain genes in a particular age. Effect of primary disease on quality of life, bone metabolism and bone structure is essential.

\section{P767}

THE ROLE OF CORTICAL BONE IN HIP FRACTURES: FEMORAL CORTICAL INDEX AS A DIAGNOSTIC TOOL FOR BONE FRAGILITY

$\underline{\text { C. Rao }}^{1}$, M. Feola ${ }^{1}$, V. Tempesta ${ }^{1}$, M. Riccardi ${ }^{1}$, R. De Marco $^{1}$, U. Tarantino

${ }^{1}$ Department of Orthopedics and Traumatology, "Tor Vergata" University of Rome, "Policlinico Tor Vergata" Foundation, Rome, Italy

Introduction: The femoral cortical index (FCI) assesses bone stock using the ratio between the diameter of the femoral shaft and the thickness of the cortical bone calculated $10 \mathrm{~cm}$ distal to the center of the small trochanter in an AP view X-ray of the femur. Objective: Aim of our study is to evaluate a possible association among low values of FCI, risk factors, comorbidities and serum 25-hydroxyvitamin D levels and to establish the importance of FCI as a potential predictor of a new fracture.
Materials and Methods: We conducted a retrospective study on 366 consecutive patients ( 90 men and 296 women) (range 60 to 103 y) surgically treated for hip fractures from March 2013 to June 2015, after informed consent in our Orthopaedic Department and that never received any medical treatment for osteoporosis. FCI has been calculated by routine clinical radiographs of the pelvis both on fractured femur and on the opposite side. For each patient, we analyzed the presence of comorbidities (such as diabetes, hypertension, IRC, rheumatoid arthritis), osteoporosis risk factors and blood levels of vitamin $\mathrm{D}$, usually evaluated in our patients with fragility fractures.

Results: Average values of FCI were 0.42 (range $0.18-0.58$ ) at the fractured femur and 0.48 at the opposite side (range 0.25-0.66) with a statistically significant difference $(p=0.002)$. At the fractured side an average value of 0.45 was found in men, and of 0.40 in women. Patients with severe hypovitaminosis D (serum concentration $<12 \mathrm{ng} / \mathrm{ml}$ ) had a minor FCI compared to those with a moderate deficiency ( 0.41 vs. $0.46, p<0.01)$. The presence of comorbidities or osteoporosis risk factors had a different influence on the values of FCI.

Conclusions: We found a correlation among low values of FCI, clinical factors related to bone fragility and severe hypovitaminosis D in elderly patients with hip fractures. Comorbidities and risk factors have a different weight in FCI variations, while the severe hypovitaminosis has a major impact on it. As described in the literature regard the DXA limitations in elderly, FCI could be a useful tool in terms of bone fragility evaluation and fracture risk prediction.

\section{P768}

LOW BMD, STILL A MAJOR DETERMINANT OF VERTEBRAL FRACTURES IN OSTEOPOROTIC PATIENTS WITH TYPE 2 DIABETES MELLITUS?

H. Cheaito $^{1}$, S. Florea ${ }^{2}$, C. G. Barbu ${ }^{3}$

${ }^{1}$ Carol Davila University of Medicine and Pharmacy, Bucharest, Romania, ${ }^{2}$ Elias Hospital, Bucharest, Romania, ${ }^{3}$ Carol Davila University of Medicine and Pharmacy, Elias Hospital Endocrinology Department, Bucharest, Romania

Aim of the study was a retrospective analysis of the fractures prevalence among 100 osteoporotic patients related to the association with type 2 diabetes mellitus(DM).

Material and Method: A sample of 100 patients' medical records (including 50 who were suffering also from type $2 \mathrm{DM}$ ), treated for osteoporosis in our department were retrospectively analysed regarding clinical history including fragility fractures, comorbidities, metabolic pathology, BMD measurements and calcium homeostasis parameters. 
Results: Femoral neck BMD value of the type 2 DM group was not significantly different from the nonDM group (0.78 vs. $\left.0.77 \mathrm{~g} / \mathrm{cm}^{2}\right)$. The mean lumbar BMD in type $2 \mathrm{DM}$ was significantly lower than nonDM group $\left(0.72\right.$ vs. $\left.0.75 \mathrm{~g} / \mathrm{cm}^{2}, p<0.05\right)$. The nonvertebral fractures prevalence was not significantly different in the two subgroups (66\% in DM vs. $56 \%$ in nonDM group) but regarding the vertebral fractures we found a higher prevalence in the diabetic group comparing the nondiabetic group (62\% vs. $34 \%, p<0.05)$.

Conclusion: In our sample of osteoporotic patients, vertebral fractures were more frequent in the type $2 \mathrm{DM}$ group, in parallel with a lower lumbar BMD in the DM group. The similarity in femoral neck BMD in both groups was doubled by a similar prevalence of non vertebral fractures. These findings would suggest that low BMD is still a major determinant of fragility fractures when looking under a certain BMD level like in osteoporotic patients.

\section{P769}

\section{SUCCESSFUL MANAGEMENT OF ACUTE GOUTY} ARTHRITIS WITH CANACINUMAB

I. Kostoglou-Athanassiou $^{1}$, E. Pantazi ${ }^{2}$, A. Tzanavari ${ }^{3}$, O. Skioti $^{2}$, P. Athanassiou ${ }^{3}$

${ }^{1}$ Department of Endocrinology, Red Cross Hospital, Athens, Greece, ${ }^{2}$ nd Department of Endocrinology, Alexandra Hospital, Athens, Greece, ${ }^{3}$ Department of Rheumatology, St. Paul's Hospital, Thessaloniki, Greece

Objectives: Canacinumab, an anti-interleukin- $1 \beta$ monoclonal antibody, is a novel approach for the treatment of acute gouty arthritis in a targeted population of patients in whom treatment options are limited. Relative to on demand treatment with intramuscular triamcinolone acetonide on demand treatment with subcutaneous canakinumab significantly relieves pain and inflammation in acute gouty arthritis flares. The aim was to present the case of a patient with acute gouty arthritis in whom canacinumab was administered along with urate lowering therapy including febuxostat.

Materials and Methods: A patient, male, aged 43 years, was admitted due to asymmetric, migratory oligoarthritis, accompanied by fever. The patient had acute arthritis of the right knee joint causing difficulty in walking. Laboratory investigations revealed ESR $60 \mathrm{~mm} / \mathrm{h}$, CRP 9.8 (normal values $<0.5$ ), RF negative, anti-CCP antibodies negative, hyperuricemia, serum uric acid being $8.8 \mathrm{mg} / \mathrm{dl}$ and normal renal function. He was febrile, temperature $38.5{ }^{\circ} \mathrm{C}$. Aspiration of the right knee joint was performed,
$50 \mathrm{ml}$ of joint fluid were aspirated, showing 13.975 cells/ml, sugar $90 \mathrm{mg} / \mathrm{dl}$ and needle-like monosodium urate crystals.

Results: Colchicine was administered along with methylprednisolone with immediate improvement, allopurinol being added 20 days later. Despite treatment the patient had 3 episodes of acute gouty arthritis in 12 months. Canacinumab was administered. The patient improved and presented free from arthritis. As hyperuricemia persisted and liver function tests were slightly impaired, febuxostat was initiated, while allopurinol was stopped.

Conclusions: Canacinumab, an anti-interleukin- $1 \beta$ monoclonal antibody is a novel approach to treat acute gouty arthritis flares in difficult to treat patients. As inflammation appears to be a feature of both gouty arthritis and chronic hyperuricemia, canacinumab is a useful addition in the armamentarium against gouty arthritis. Febuxostat is a novel urate lowering treatment, which may be used for the management of hyperuricemia if allopurinol is not effective or has side effects.

\section{P770}

BONE MINERAL DENSITY IN MEN WITH FOREARM FRACTURE

$\underline{\text { R. F. Filipov }}^{1}$, K. M. Markovic ${ }^{1}$, S. K. Kozomara ${ }^{1}$, M. S. Stoickov $^{1}$, S. M. Mitic ${ }^{1}$

${ }^{1}$ Institute Niska Banja, Nis, Serbia

Introduction: Osteoporosis is one of the most common diseases, nowadays. Osteoporosis in men, although of significantly lower frequency, in comparison to women, is not only a growing health problem, but also a socioeconomic problem.

Objective: The objective of this written paper is to establish BMD in men who had forearm fracture.

Methods: 46 male patients were examined. Out of them, 26 were in the investigated group (IG), they were the patients who had forearm fracture, while 20 of them were without the fracture and they were in the control group (CG). Measuring of BMD was performed on the DXA Hologic apparatus, on lumbar vertebras L1-L4 and on the left hip.

Results: Average BMD value on the lumbar spine in the investigated group was $0.856 \pm 0.11 \mathrm{~g} / \mathrm{cm}^{2}$ (T-score -1.8 \pm 0.88 ), and on the hip $0.802 \pm 0.112 \mathrm{~g} / \mathrm{cm}^{2}$ (T-score -1.9 ). Average BMD value on the lumbar spine in the control group was $0.966 \pm 0.121 \mathrm{~g} / \mathrm{cm}^{2}$ (T-score $\left.-1.23 \pm 0.92\right)$ and on the hip it was $0.823 \pm 0.146 \mathrm{~g} / \mathrm{cm}^{2}$ (T-score $-1.38 \pm 0.82$ ). Statistical significant difference was not found between absolute values of BMD as on the lumbar spine $(p>0.01)$, as on the hip $(p>0.5)$, between the investigated and control group. There 
is no statistically significant difference between average Tscore values on the lumbar spine or on the hip in the investigated and control group.

Conclusion: Forearm fractures in men are not associated with the presence of osteoporosis.

\section{P771}

MEASURING MARROW ADIPOSITY AND CORTICAL POROSITY IDENTIFIES WOMEN AT RISK OF NONVERTEBRAL FRACTURES WITH GREATER SENSITIVITY

M. Osima ${ }^{1}$, R. Zebaze ${ }^{2}$, M. Lukic ${ }^{1}$, X. Wang ${ }^{2}$, A. GhasemZadeh $^{2}$, M. Bui ${ }^{3}$, J. L. Hopper ${ }^{3}$, E. F. Eriksen ${ }^{4}$, E. Seeman ${ }^{2}$, A. Bjørnerem ${ }^{5}$

${ }^{1}$ Department of Community Medicine, UiT The Arctic University of Norway, Tromsø, Norway, ${ }^{2}$ Endocrine Centre, Austin Health, University of Melbourne, Melbourne, Australia, ${ }^{3}$ Centre of Epidemiology and Biostatistics, Melbourne School of Population and Global Health, University of Melbourne, Melbourne, Australia, ${ }^{4}$ Department of Endocrinology, Morbid Obesity and Preventive Medicine, University of Oslo, Oslo, Norway, ${ }^{5}$ Department of Health and Care Sciences, UiT The Arctic University of Norway, Tromsø, Norway

Objectives: Advancing age is associated with a reduction in the volume of bone formed by the bone multicellular units that might be partly due to diversion of mesenchymal precursors towards adipocyte phenotypes rather than osteoblast lineage cells. We tested the hypothesis that marrow adiposity is associated with higher cortical porosity and lower trabecular number, and that marrow adiposity and cortical porosity were independently associated with risk of nonvertebral fracture.

Materials and methods: We measured distal tibia and distal radius marrow adiposity, cortical and trabecular morphology using HRpQCT and quantified these traits using StrAx1.0 software, and measured femoral neck (FN) areal BMD (aBMD) using DXA, for 79 women aged 40-70 years with nonvertebral fractures, and 161 age-matched controls from Melbourne, Australia. Associations were estimated using linear and logistic regression analysis adjusted for age, height, weight and FN aBMD. Thresholds of marrow adiposity and porosity $>80$ th percentile were used to determine the sensitivity and specificity of identifying women at risk of fractures.

Results: Each standard deviation (SD) increment in marrow adiposity was associated with $0.31 \mathrm{SD}$ higher cortical porosity and 0.79 SD lower trabecular number of distal tibia, respectively $(p<0.001)$. Each SD higher marrow adiposity and cortical porosity were associated with higher odds ratio for nonvertebral fracture of 1.82 (95\% confidence interval 1.17-2.84) and 1.90 (1.17-3.08), respectively, independent of FN aBMD. The sensitivity for fracture was
$36 \%$ using marrow adiposity, $35 \%$ using cortical porosity, and $52 \%$ using both, and specificity were 88,87 and $78 \%$, respectively. Results were similar for the corresponding distal radius measures.

Conclusions: Combining a measurement of marrow adiposity with cortical porosity improved identification of women with nonvertebral fractures.

\section{P772}

MODULATION OF DIFFERENT PERIPHERAL B CELL SUBPOPULATIONS IN RHEUMATOID ARTHRITIS PATIENTS DURING IL-6R INHIBITION

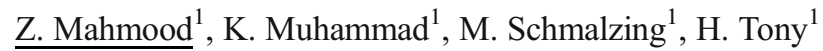
${ }^{1}$ University Hospital, Wuerzburg, Germany

Background/Purpose: Enhanced B cell activity has been proposed in the pathogenesis of rheumatoid arthritis (RA). With the advent of $\mathrm{B}$ cell targeted therapies the modulation of memory B cells seems to be a prime target. Human peripheral memory $\mathrm{B}$ cells can be distinguished by the phenotypic expression of $\mathrm{CD} 27$ and $\mathrm{IgD}$ defining three major $\mathrm{B}$ cell subpopulations: $\mathrm{CD} 27+\mathrm{IgD}+$ preswitch, $\mathrm{CD} 27+\mathrm{IgD}-$ postswitch and CD27-IgD- double negative memory B cells. We analyzed these different memory populations in RA and under IL-6R blockade.

Methods: B cells were phenotypically analyzed from RA patients at baseline, week 12 and week 24 under tocilizumab (TCZ) treatment. Memory B cell subsets were defined by $\mathrm{CD} 27$ and isotype surface expression. Mutational frequencies were analyzed by single $\mathrm{B}$ cell RT-PCR and were further analyzed by multicolor flow cytometry. B cell activation was identified by surface staining with CD95 and intracellular ki-67 staining. Mann Whitney U test was used for statistical analysis by using GraphPad Prism 5 .

Results: The Ig receptor mutational frequency was highest in class switched memory with $6.1 \pm 0.3 \%$ compared to double negative $(3.5 \pm 0.2 \%)$ and preswitch memory $(4.2 \pm 0.2 \%)$. The phenotypically analyzed isotype profile in RA patients $(n=60)$ and healthy donors $(n=20)$ revealed that the memory B cell pool was a heterogeneous mixture of $\operatorname{IgA}, \operatorname{IgG}$ and $\operatorname{IgM}$ expressing cells. The double negative B cell memory population showed a clear dominance of IgG followed by $\operatorname{IgA}$ and $\operatorname{IgM}(\sim 70 \%, 20 \%$ and $10 \%$ respectively $)$, whereas CD27+IgD- B cell had an equal distribution of $\mathrm{IgA}$ and IgG. Under IL-6R inhibition by TCZ, the isotopic profile remained stable at week 12 and 24 . Surface and intracellular staining of B cells showed a significantly higher percentage of CD95 $(p=0.01)$ and Ki-67 $(p=0.04)$ expression in RA, which was highest in post-switched memory $\mathrm{B}$ cells. 
Conclusion: Our data suggests that the three major peripheral memory B cell populations, pre-, post- switch and double negative $B$ cells harbor different numbers of mutations in their Ig receptors. They are activated in RA with enhanced CD95 and Ki-67 expression compared to healthy individuals and which can be reduced by IL-6R inhibition in vivo. The double negative B cell pool displays a significantly higher proportion of IgG bearing cells compared to postswitch B cells which is not changed by IL-6R inhibition.

\section{P773}

RECOVERY THERAPY OF PATIENTS WITH ANKYLOSING SPONDYLOARTHRITIS

O. Sarbu ${ }^{1}$, L. Rotaru ${ }^{2}$, L. Groppa ${ }^{2}$

${ }^{1}$ Laboratory of Rheumatology, Republican Clinical Hospital, Chisinau, Republic of Moldova, ${ }^{2}$ Department of Rheumatology, State Medical and Pharmaceutical University «Nicolae Testemitanu», Chisinau, Republic of Moldova

Aim: Analysis of patients with ankylosing spondyloarthritis receiving drug therapy, kinesiotherapy and other physiotherapeutic means compared to patients receiving only drug (medical) treatment.

Materials and methods: 50 patients diagnosed with ankylosing spondylitis (modified New York criteria,1984) were examined, being in a clinical remission. Study duration 20132015. All patients were examined clinically and laboratory. Patients were divided into two groups of 25 patients each. First group of patients received drug therapy with kinesiotherapy and other physiotherapeutic means, in the second group patients received only drug therapy. Therapeutic efficiency assessment was conducted using questionnaires BASDAI, BASFI, HAQ.

Results: Both groups were $80 \%$ male dominated, III and IV radiographically stage was dominant. BASDAI score diminished after treatment in first group on average 10.3 points compared to group II -8.9 points. According to BASFI questionnaire - I group patients improved with 22 points, compared with second group - 16 points. HAQ questionnaire showed improvement in I group with 9 points, compared to 5 points (II group). In I group movements in all segments of spine were obviously improved - especially menton-sternum index - $2.5 \mathrm{~cm}$ compared to $1.1 \mathrm{~cm}$ group II, tragus-acromion index decreased by $3.4 \mathrm{~cm}$ and $1.8 \mathrm{~cm}$ respectively, thoracic amplitude of movements has improved by $1.8 \mathrm{~cm}$ and $0.9 \mathrm{~cm}$ in group I and group II, Schober index decreased by $3.4 \mathrm{~cm}$ in group I and by $2.1 \mathrm{~cm}$ in group II.

Conclusions: Combination therapy with drug treatment and kinesiotherapy with other physiotherapeutic means leads to significant improvements of the life's quality in patients with ankylosing spondyloarthritis.

\section{P774}

USING THE RUDY STUDY PLATFORM TO CAPTURE QUALITY OF LIFE OF ADULTS WITH RARE DISEASES OF THE BONE

L. Zhang ${ }^{1}$, L. Watts ${ }^{1}$, A. Turner ${ }^{1}$, H. Teare ${ }^{2}$, J. Barrett ${ }^{1}$, P. Wordsworth $^{1}$, J. Kaye ${ }^{2}$, M. K. Javaid ${ }^{1}$, R. Pinedo-Villanueva ${ }^{1}$

${ }^{1}$ Nuffield Department of Orthopaedics, Rheumatology and Musculoskeletal Sciences, University of Oxford, Oxford, United Kingdom, ${ }^{2}$ Centre for Health, Law and Emerging Technologies (HeLEX), University of Oxford, Oxford, United Kingdom

Objectives: Developing novel therapies for rare diseases is an important research priority. Although current methods involve recruitment from hospital clinics, this may only include the most severely affected individuals and so overestimate the population level burden of rare diseases. Further, any new therapy will require an economic evaluation before implementation in the UK. However, there is a paucity of data on health-related quality of life measures in patients with rare diseases. We therefore compared quality of life across three rare bone diseases in adult using the EQ5D-5 L.

Method: Adults with osteogenesis imperfecta (OI), fibrous dysplasia (FD) and X-linked hypophosphataemia (XLH) were recruited via the Rare and Undiagnosed Diseases Study (RUDY), a web-based platform for patient recruitment and assessment of patient reported outcomes including the EQ5D. The EQ5D-5 L utility scores of OI participants were used to generate a costutility simulation.

Results: 82 adults completed the EQ5D-5 L questionnaire. Overall there was a wide distribution of quality of life with moderate/severe problems commonly reported in the pain and discomfort dimension (OI $60 \%$, FD $56 \%$, XLH $65 \%$ ). A cost-utility simulation showed that a hypothetical intervention which increased the health utility of the lowest utility tertile of OI patients to the mean utility level of the overall group over 10 years and costing $£ 79,000$ would be found cost-effective for the English NHS based on a $£ 30,000$ per QALY threshold.

Conclusion: These findings confirm that RUDY is recruiting patients across a range of quality of life with pain and discomfort domains most commonly affected. This is the first study to estimate the cost required to improve quality of life for adults with OI who have the lowest quality of life. A greater understanding of health-related quality of life amongst this population could help guide novel therapy developments and resource allocation. 


\section{P775}

SOME RISK FACTORS ARE MORE IMPORTANT FOR OSTEOPOROSIS THAN THE DEFICIT OF ESTROGEN AND PROGESTERONE

$\underline{\text { K. M. Markovic }}^{1}$, S. K. Kozomara ${ }^{1}$, R. F. Filipov ${ }^{1}$, M. S. Stoickov $^{1}$, S. B. Bacevic ${ }^{2}$

${ }^{1}$ Institute Niska Banja, Nis, Serbia, ${ }^{2}$ Generaly Hospital, Prokuplje, Serbia

Introduction: Osteoporosis is huge medical and socioeconomic problem. One in four women and one in eight men over age 50 suffer from osteoporosis. Fixed factors affecting the $80 \%$ of the bone density. It is possible to influence at $20 \%$ of factors, which is enough for a carefree old age. The most important is proper nutrition - an adequate intake of calcium and vitamin D. It is important everyday physical activity, eliminating the excessive consumption of alcoholic drinks, coffee and smoking. Secondary factors important for osteoporosis, diseases of thyroid and parathyroid glands, rheumatoid arthritis, some diseases of the digestive tract and neuropsychiatric diseases, drugs for depression, epilepsy and glucocorticoids.

Aim: Demonstrate that the risk factors are more important than the deficit of estrogen and progesterone for osteoporosis. Method: 80 postmenopausal women average age $62.2 \pm 3.8$ which had a menstrual cycle neat, having osteoporosis - have been analyzed in the first group. In the second group was 80 postmenopausal women average age $60.8 \pm 4.2$ - with menopause before 40 years, also having osteoporosis - menopause has occurred on average $9 \pm 2.8$ years earlier among women in this group 1. Measuring of BMD was performed on Hologic Discovery densitometer, anteroposterior scan of lumbar vertebrae L1-L4 by the method of DXA was performed. The results were interpreted according to the accepted. The obtained data were statistically analyzed with nonparametric tests, Pearson correlation test and Student t-test in the software program Microsoft Excel.

Results: Reduced physical activity in the first group had 23 women $(28.13 \%)$ and the second 3 women (4.12\%), lower BMI in the first group had 7 women $(8.33 \%)$ and the second 3 women (3.7\%), drugs and diseases that cause OP in the first group had 17 women $(21.33 \%)$, in the other 7 women $(8.36 \%)$ - these data were not statistically significant $(p<0.001)$. Low calcium intake in the first group had 44 women $(54.36 \%)$, in the other 13 women $(16 \%)$, in the first group who smoked were 52 women $(64.66 \%)$, in the other 34 women $(43.23 \%)$, decreased levels of $25(\mathrm{OH}) \mathrm{D} 3$ in the first group had most of the 47 women $(59.33 \%)$ and in the other 19 women $(23.33 \%)$ - these data were statistically significant $(p>0.001)$.

Conclusion: Inadequate intake of calcium through diet, smoking and insufficiency of $25(\mathrm{OH})$ D3 is more important for osteoporosis than the deficit of estrogen and progesterone.
P776

A NOVEL FEATURE SELECTION ALGORITHM BASED ON BONE MICROARCHITECTURE ANALYSIS TO IDENTIFY OSTEOARTHRITIS

$\underline{\text { R. Ljuhar }}^{1}$, B. Norman ${ }^{1}$, D. Ljuhar ${ }^{1}$, T. Haftner ${ }^{1}$, J. Hladuvka $^{2}$, P. Bui Thi $\mathrm{Mai}^{2}$, H. Canhão ${ }^{3}$, J. Branco ${ }^{3}$, A. M. Rodrigues $^{3}$, N. Gouveia ${ }^{3}$, S. Nehrer ${ }^{4}$, A. FahrleitnerPammer $^{5}$, H.-P. Dimai ${ }^{5}$

${ }^{1}$ Braincon Technologies, Vienna, Austria, ${ }^{2}$ VRVis Research Competence Center, Vienna, Austria, ${ }^{3}$ Faculdade de Medicina da Universidade de Lisboa, Lisboa, Portugal, ${ }^{4}$ Center for Regenerative Medicine \& Orthopedics, Danube University, Krems, Austria, ${ }^{5}$ Department of Internal Medicine, Division of Endocrinology and Metabolism, Medical University of Graz, Graz, Austria

Objective: Texture information of the subchondral bone area of $2 \mathrm{D}$ radiographs represents a promising possibility for evaluating the state of osteoarthritis $(\mathrm{OA})$ in addition to traditional clinical means such as visual and semi-quantitative assessments. Algorithms based on fractal analysis have shown to be capable of identifying differences in trabecular bone structure. However such features are likely to vary within the subchondral bone area and therefore the appropriate selection of the region of interest (ROI) plays a crucial role for the result of the analysis. Thus, a feature selection algorithm is being applied in order to determine ROIs that enable an optimum discrimination between patients with and without OA.

Methods: The study included 152 standardized knee radiographs from 66 female patients with OA, and 86 controls. Subchondral bone microarchitecture was assessed by using both fractal analysis and a Shannon Entropy (SE) algorithm at predefined regions of the proximal tibia and the distal femur. For fractal analysis the distinct parameter Bone Structure Value (BSV) was defined. The selected area of the proximal tibia involved a matrix of $3 \times 8$ ROIs, whereas a $2 \times 2$ matrix was defined for each condyle of the distal femur. SE and the BSV were calculated for each of the 32 ROIs, respectively. Based on these 64 variables, a feature selection algorithm was applied to determine the variables that showed the best discrimination power between Case and Control subjects.

Results: By combining the BSV and SE, the odds ratio increased significantly from 3.08 (95\%CI: $1.78-5.30)$ to 14.82 (95\%CI: 6.69-32.83) when using 15 features, and to 39.75 (95\%CI: 15.41-102.51) based on 10 features. By using the selected 10 features the accuracy was found to be 0.86 . This showed to be a significant improvement compared to the accuracy achieved when calculating a single mean value for the $3 \times 8$ ROIs of the proximal tibia alone ( 0.62 vs. 0.86$)$.

Conclusions: The application of a feature selection algorithm in accordance with the combination of the two texture analysis methods shows a significant improvement with respect to the discrimination power between subjects with and without OA. 
The high odds ratios confirm that reliable results can be achieved by combining the BSV and the SE. This novel algorithm for the assessment of bone microarchitecture may not only be useful in osteoarthritis subjects but also for the early prediction and assessment of other degenerative bone diseases like osteoporosis and rheumatoid arthritis.

\section{P777}

COMPARISON OF THE SCALES FRAX AND QFRACTURE IN THE SPANISH POPULATION

N. Sosa-Cabrera $^{1}$, A. I. Reyes-Domínguez ${ }^{2}$, L. Rivero ${ }^{3}$, M. J. Gómez de Tejada $^{4}$, P. Saavedra ${ }^{2}$, M. Sosa ${ }^{2}$

${ }^{1}$ Centro de Salud de Tejina, La Laguna, Tenerife, Spain,

${ }^{2}$ Instituto Universitario de Investigaciones Biomédicas y Sanitarias, University of Las Palmas de Gran Canaria, Las Palmas de Gran Canaria, Spain, ${ }^{3}$ University of Las Palmas de Gran Canaria, Las Palmas de Gran Canaria, Spain, ${ }^{4}$ Department of Medicine, University of Seville, Seville, Spain

Background: FRAX is the scale for estimating the risk of fragility fracture more used worldwide. Some other scales have also been published, as Qfracture and Garvan. Qfracture has not been validated outside its country of origin (UK) and a few studies have compared them both.

Main objective: To compare the scales Qfracture and FRAX in the Spanish population. The outcome was the first fracture recorded after the determination of both scales.

Material and Methods: Prospective observational study. 529 patients were included. They all had at least two clinical evaluations and were studied at least for 10 years. Qfracture and FRAX were estimated at the first visit. Some patients were followed up to 24 years. All the new fractures were recorded and verified, either with X-rays or with clinical reports.

\section{Statistical study:}

Univariate analysis: The categorical variables expressed as frequencies and percentages, and you continue as averages and diversions standard when the information was following a normal distribution, and as medians and ranges interquartiles (percentiles 25-75) when the distribution was not normal. The percentages were compared using the chi-square test, the media with Student's t-test and the medians with the Wilcoxon's test for independent data. Analysis of survival. In order to explore the predictive capacity of the QFracture major, the patients were classified in agreement by the tertiles corresponding to the predictor $(<2.8$; $2.8-7 ;>7)$. In each of these groups the curves of survival were estimated up to the appearance of the first fracture. In each of these groups the curves of survival were estimated up to the appearance of the first fracture by means of Kaplan- Meier's method. The difference between them was confirmed using the test log-rank. Likewise there was obtained the value of the statistician $\mathrm{D}$, which evaluates the capacity discriminant of the scoreboard for the fractures. In order to evaluate the capacity discriminant of the QFracture and FRAX for any fracture, the patients who had a follow-up for at least 10 years they were classified as they had had or not at least a fracture in the first 10 years. For this classification it was realized an analysis ROC (receiver operating characteristics), being estimated the area under the curved correspondent ROC by means of a $95 \%$ interval for both scales. Discriminant of the QFracture and FRAX were selected as ideal threshold, the value associated with the point of the curve ROC that minimized the quantity ( 1 - sensibility $) 2+(1$ - specificity $)$ 2 . For these threshold there were estimated the sensibility, specificity, the predictive positive value (PPV) and the predictive negative (PNV) by means of confidence intervals to $95 \%$. A contrast of hypothesis was considered to be statistically significant when the corresponding value of $\mathrm{p}$ was lower than $5 \%$. The information was analyzed using the program R, version 3.1.0.

Results: We found no differences with the statistics studied. Both scales seem to discriminate well. The sensibility and specificity were similar. The $\mathrm{p}$ value of the AUC was nonsignificant: $p=0.757$.

Conclusion: Qfracture and FRAX have shown similar results for calculating the 10 years risk of fragility fracture in the Spanish population.

\section{P778}

\section{COMPARISON OF DENOSUMAB VERSUS ZOLE-} DRONIC ON TBS: A HEAD TO HEAD STUDY

M. Sosa ${ }^{1}$, M. J. Gómez de Tejada ${ }^{2}$, N. Sosa-Cabrera ${ }^{3}$, E. Jodar-Gimeno $^{4}$, J. M. Quesada-Gómez ${ }^{5}$

${ }^{1}$ Instituto Universitario de Investigaciones Biomédicas y Sanitarias, University of Las Palmas de Gran Canaria, Las Palmas de Gran Canaria, Spain, ${ }^{2}$ Department of Medicine, University of Seville, Seville, Spain, ${ }^{3}$ Centro de Salud de Tejina, La Laguna, Tenerife, Spain, ${ }^{4}$ Servicio de Endocrinología, Hospital Universitario Quirón, Madrid, Spain, ${ }^{5}$ Clinical Management Unit of Endocrinology and Nutrition, Maimónides Biomedical Research Institute of Córdoba (IMIBIC), Hospital Universitario Reina Sofía RETICEF, Universidad de Córdoba, Córdoba, Spain

Background: Denosumab and zoledronic acid are the two more powerful drugs used in the treatment of the osteoporosis. Though they have many common points (pharmacological potency, parenteral administration, long duration effect) their mechanisms of action are different. We have found no one "face-to-face" study comparing the effect on the trabecular bone score (TBS) of these two drugs. 
Objective: To study the changes produced in the mineral bony metabolism in 2 groups of patients suffering from severe postmenopausal osteoporosis, which were treated either with zoledronic acid or denosumab in a random manner, and followed-up of 3 years. We have analyzed the changes in the quantity of bone (BMD), in his quality (TBS and calcaneal ultrasound - QUS) and finally the biochemical markers of bone remodeling (MRO).

Material and Methods: Observational, opened, "face to face" and longitudinal study, performed in patients suffering from severe postmenopausal osteoporosis who were assigned to group I treated with zoledronic acid, $5 \mathrm{mg}$ i.v. yearly and Group II treated with denosumab $60 \mathrm{mg}$ s.c each 6 months. We measured BMD, TBS and QUS, and also determined biochemical markers of bone remodelling. The statistical study was effected realizing the analysis of the variance with repeated measures, using the program SPSS.

Results: The patients were randomized correctly and all their basal characteristics were similar.

Conclusion: This is the first "face-to-face" study that compares denosumab with zoledronic acid to 3 years measuring BMD, TBS and calcaneus ultrasound (bone quantity and quality). We have not found any similar reference in the consulted bibliography. At the end of 3 years of the study, denosumab, produces a major increase in the quantity of bone, estimated by BMD, and also in the quality estimated by both TBS in the lumbar spine and QUS parameters.

\section{P779}

EVALUATION OF THE ACUTE EFFECTS OF WHOLE BODY VIBRATION EXERCISES IN BALANCE, FLEXIBILITY AND MUSCLE ACTIVATION OF WOMEN WITH SYSTEMIC LUPUS ERYTHEMATOSUS WITH AND WITHOUT OSTEOPOROSIS

$\underline{\text { C. Dionello }}^{1}$, E. Moreira-Marconi ${ }^{1}$, D. Morel ${ }^{1}$, D. SáCaputo $^{1}$, E. Klumb ${ }^{1}$, M. Bernardo-Filho ${ }^{1}$

${ }^{1}$ Universidade do Estado do Rio de Janeiro, Rio de Janeiro, Brazil

Objective: To evaluate the acute effects of whole body vibration exercises (WBVE) in balance, flexibility and muscle activation of women with systemic lupus erythematous (SLE) with and without osteoporosis (OP).

Methods: Following the local ethical committee designations 20 women with SLE diagnosed for more than 5 years, with ages above 40 years old, that were using corticosteroids for more than 5 years consented their participation on the study. They were divided in two groups: 10 patients had demonstrated OP in the DXA studies and 10 patients had not demonstrated OP in DXA. Patients were attended in the Rheumatology
Division of the Hospital Pedro Ernesto, in Rio de Janeiro. The 20 DXA studies were performed by the same technician and the results interpreted by the same doctor. For the entire group of SLE women it was performed 2 separate evaluations of a unique protocol, the first evaluation before the WBVE and the second evaluation within 5 minutes after the WBVE were finished. The evaluation protocol consisted of the Short Physical Performance Balance (SPPB), the timed up and go test (TUG), the finger-to-toe distance (FTT), and a electromyography (EMG) of the vastus lateralis muscle. The room was at a temperature of $21{ }^{\circ} \mathrm{C}$. The patients must have eaten $30 \mathrm{~min}$ before the exercises. WBVE were performed in a triplanar platform, the patients were stand with $130^{\circ}$ of knee flexion, holding the platform bars and being observed by a physiotherapist during the entire time of the exercise. The only set performed consisted of 15 bouts that lasted $1 \mathrm{~min}$ (frequency of $25 \mathrm{~Hz}$ and amplitude of $4 \mathrm{~mm}$; 2.54 multiples of $g$ force) with rest periods of $30 \mathrm{~s}$ between the bouts.

Results: It was demonstrated differences between the SLE with OP group and the SLE without OP group in the EMG study before and after WBVE. It was not demonstrated differences between the groups in FTT, but the first and second FTT evaluation of both groups had differences. The balance evaluation (SPPB and TUG) had no differences between the groups or moment of evaluation.

Conclusions: The WBVE are an option for physical activity for patients with SLE and OP, since it is safe and easy to perform. These patients are at increased risk of falls, due to sarcopenia and medications for the treatment of the disease. The evaluation of balance, flexibility and muscle activity is suitable and important to these patients. It was confirmed the difference of muscle activation between the groups. Flexibility evaluation was different before and after WBVE.

\section{P780}

GESTATIONAL VITAMIN D STATUS AND OFFSPRING BONE MINERAL MEASURES IN CHILDHOOD: A PRELIMINARY ANALYSIS

N. K. Hyde ${ }^{1}$, S. M. Hosking ${ }^{1}$, J. D. Wark ${ }^{2}$, K. Bennett ${ }^{1}$, A. G. Morse $^{1}$, J. A. Pasco ${ }^{1}$

${ }^{1}$ School of Medicine, Deakin University, Geelong, Australia, ${ }^{2}$ The University of Melbourne, Royal Melbourne Hospital Bone and Mineral Service, Melbourne, Australia

Objective: To determine the association between gestational vitamin $\mathrm{D}$ status and offspring bone mineral measures during childhood.

Methods: Data were collected from the Vitamin D in Pregnancy (VIP) study (2002-04); a cohort of 475 pregnant women, recruited from the Geelong Hospital in early pregnancy (gestation $12.6 \pm 2.8$ weeks). Blood samples were taken at recruitment and later pregnancy (28-32 weeks). 
Maternal serum 25-hydroxyvitamin D (25(OH)D) was measured by radioimmunoassay (Immunodiagnostic Systems). Offspring (mean age $11.0 \pm 0.46$, at time of analyses $n=168$ ) underwent an assessment of areal BMD, by DXA (GE Lunar).

Results: There was an interaction observed between sex and $25(\mathrm{OH}) \mathrm{D}$ in models predicting BMD, thus data were stratified by sex. Correlations were observed for BMD at the lumbar spine and total body less head (TBLH) with $25(\mathrm{OH}) \mathrm{D}$ levels at recruitment in boys $(r=0.21 p=0.05 ; r=0.22, p=0.05$, respectively), but not girls (both $p>0.4$ ). When adjusted for offspring height, weight and age, $25(\mathrm{OH}) \mathrm{D}$ was positively associated with BMD at the spine and TBLH in boys $(\beta 0.06 \pm 0.02, p=0.019 ; \beta 0.05 \pm 0.02, p=0.005$, respectively), but not girls (both $p>0.05$ ). All observed associations remained independent of gestational PTH concentrations, birth weight, gestation length, season, maternal age, gestational smoking, area-based socioeconomic status at recruitment and pubertal staging. $25(\mathrm{OH}) \mathrm{D}$ in later pregnancy showed no association with BMD.

Conclusions: A sexually dimorphic effect was displayed by early gestational vitamin D status; it was not associated with bone accrual in girls but was positively associated with boys. Boys born to mothers with lower vitamin D levels may be predisposed to an increased risk of osteoporosis in adulthood.

Acknowledgments: The Vitamin D in pregnancy study has received funding from the National Health and Medical Research Council, Australia and Bupa Health Foundation.

\section{P781}

CORRELATION BETWEEN FUNCTIONAL TESTS AND FRAGILITY FRACTURES IN PATIENTS WITH OSTEOPOROSIS

F. Gazoni $^{1}$, A. Santos ${ }^{1}$, J. Sarmento ${ }^{1}$, D. Martins ${ }^{2}$, B. Gusmao $^{2}$, D. Nascimento ${ }^{3}$, F. Santos ${ }^{1}$

${ }^{1}$ Sao Paulo Federal University, Sao Paulo, Brazil, ${ }^{2}$ Sao Paulo University, Sao Paulo, Brazil, ${ }^{3}$ Prevent Senior, Sao Paulo, Brazil

Objective: To check the correlation between functional tests in osteoporotic patients with the presence of previous fragility fractures.

Method: A descriptive and observational analysis of a sample of patients with osteoporosis treated in outpatient called Health of Bones in São Paulo, Brazil during the year of 2015 was carried out and evaluated the correlation between performance in the functional tests and the prevalence of fracture provided by fragility. During appointment with physician and gerontologist some functional tests were performed: running speed test (walk usual steps an overall distance of $7 \mathrm{~m}$, with $3 \mathrm{~m}$ of distraction), grip strength (handgrip), which was considered the best of 3 attempts, and calf circumference (measurement taken from the left leg, with an inelastic tape in the most protuberant part). Statistical analysis was performed with the parametric ANOVA test with a comparison of means using the variance.

Results: From 2662 patients evaluated during the year of 2015, most of them were women (93\%) with average age of 73.4 years, $30.8 \%$ of this population had history of fragility fracture. At the first visit, among those patients with fractures the handgrip average was 18.64 against 21.7 in those patients without fractures. The average walking speed was 0.72 in fractured, and 0.94 in the without fracture group, and the calf circumference was 34.13 in the group with fracture, and 35.26 in the group without fracture. In the sample studied, for all variables there was a statistically significant mean correlation between poor performance in the functional tests and the presence of previous fracture $(p<0.001)$.

Conclusion: When evaluating the presence of altered functional tests as a risk factor for fragility fractures in the population studied, it was found important correlation between these variables. The functional tests are good predictors of fragility fracture and can contribute to guide the multidisciplinary team in elaborating preventive strategies for the occurrence of new fractures.

\section{P782}

CORRELATIONS BETWEEN BONE MINERAL DENSITY, LEAN BODY MASS, HANDGRIP STRENGTH, CALF CIRCUMFERENCE AND BODY MASS INDEX IN THE OLDEST OLD COMMUNITY-DWELLING WOMEN

$\underline{\text { L. Bastos }}^{1}$, F. K. Fonte ${ }^{1}$, P. F. P. Moreira ${ }^{1}$, M. S. Cendoroglo ${ }^{1}$, F. C. Santos ${ }^{1}$

${ }^{1}$ Department of Geriatrics and Gerontology, Universidade Federal de São Paulo, São Paulo, Brazil

Background: The life expectancy is increasing and jointly there is an increase in the prevalence of osteopenia, osteoporosis and a decline in lean body mass and also on the strength of individuals who get older.

Objective: To investigate the correlation between BMD, lean body mass (LBM), calf circumference (CC), BMI and handgrip strength in oldest old women dwelling in the community.

Methods: We studied 43 women, participants of the LONGEVOS study, which is a longitudinal population study and has 139 elderly participants aged greater than or equal to 80 years old, both genders, residents in Sao Paulo, Brazil. To be eligible, participants had to be completely independent in the activities of daily, living with no severe or incapacitating illness. Body composition was measured by DXA with 
equipment Hologic QDR Discovery A series. Osteoporosis and osteopenia was classified according to the criteria of the World Health Organization, from the analysis of BMD of the lumbar spine, total hip or femoral neck T-score. Handgrip strength was assessed by a dynamometer. The dominant hand was tested and the best value of three attempts was recorded. $\mathrm{CC}$ was measured with tape, and for BMI were used weight and height.

Results: From the total of 43 oldest old women was found a high prevalence of osteoporosis (55.8\%) and osteopenia $(44.2 \%)$. The mean age of this population was 88 \pm 4.5 years old, BMI was $27 \pm 4.5 \mathrm{~kg} / \mathrm{m}^{2}$, LBM by DXA was $36.6 \pm 4.9 \mathrm{~kg}$, handgrip strength was $16 \pm 4.7 \mathrm{~kg}$, an average percentage of body fat mass of $34.1 \%$, and mean $\mathrm{CC}$ was $33.7 \pm 4.4 \mathrm{~cm}$. There were significant and positive interactions between handgrip strength with the LBM $(\mathrm{kg})$ by DXA $(r=0.36, p<0.05)$, as well as hand grip strength with $\mathrm{CC}(\mathrm{cm})(r=0.38, p<0.005)$. Also, we have found positive and significant correlations between $\mathrm{CC}$ and LBM $(r=0.71, p<0.001)$, BMI and LBM $(r=0.70$, $p<0.001)$, CC and BMI $(r=0.81, p<0.001)$. In addition, older women with osteoporosis showed significantly lower values of CC, BMI and LBM when compared with osteopenic women, $31.9 \pm 3.9 \mathrm{~cm}$ vs. $36 \pm 3.9 \mathrm{~cm}$ $(p=0.002), 25.33 \pm 3,9 \mathrm{~kg} / \mathrm{m}^{2}$ vs. $29,19 \pm 4.4 \mathrm{~kg} / \mathrm{m}^{2}$ $(p=0.005)$ and $35 \pm 4.2 \mathrm{~kg}$ vs. $41.7 \pm 3.8 \mathrm{~kg}(p=0.001)$, respectively. These data suggests that low BMD values correlate with low values of CC, LBM and BMI. Comparing osteoporotic and osteopenic women, lower values were observed in the group that had osteoporosis in relation to handgrip strength $(15 \pm 5 \mathrm{~kg}$ vs. 17.3 $\pm 3.3 \mathrm{~kg})$, percentage fat mass by DXA $(33 \pm 5.3 \mathrm{Kg}$ vs. $36.4 \pm 6 \mathrm{Kg})$ and higher results for age $(88.9 \pm 4.8$ years vs. $87 \pm 4$ years), however these differences were not statistically significant. Although the BMI is a widely used and easily applicable tool, it does not identify the body composition. With aging, it is expected a positive association between BMI and fat mass. However, in the present sample BMI was significantly correlated with the LBM, that is, the greater was the index, the greater was the muscle mass amount. Besides, the $\mathrm{CC}$ proved to be an easy and effective method in evaluation of LBM, and demonstrated correlation with muscular strength.

Conclusion: It was observed a high prevalence of osteoporosis and osteopenia in the elderly women evaluated, and BMD correlated positively with LBM, pointing to the importance of addressing the body composition in oldest old women with low BMD. BMI and CC were configured as good methods of assessing muscle mass. And along to handgrip strength they are useful methods to complement the assessment of patients with osteoporosis and osteopenia, and can be easily accomplished in the consultation.

\section{P783}

MICRODEBRIDEMENT USING RADIOFREQUENCY COBLATION FOR MANAGING REFRACTORY TENNIS ELBOW: OUR SERIES OF 22 CASES

K. K. V. Acharya ${ }^{1}$

${ }^{1}$ Arthroscopy \& Sports Injuries, Manipal University, Manipal, India

Introduction: Many treatment modalities have been prescribed for tennis elbow or lateral epicondylitis. However a limited number of cases continue to appear for follow-up with no improvement in pain relief or function and may require surgical intervention. The objective of this study was to assess the outcome of radiofrequency coblation, in a consecutive series of refractory tennis elbow patients.

Material and Methods: A retrospective review of 22 patients with lateral epicondylitis treated by microdebridement using radiofrequency coblation, between January 2010 and January 2014 was performed. There were 10 men and 12 women averaging 38 years at the time of surgery. In all patients, nonoperative management failed, and they underwent surgery at a mean of 12 months after the onset of symptoms. Under regional anaesthesia and tourniquet control, microdebridement using radiofrequency coblation was performed using Topaz microdebrider wand. Postoperatively patients mobilized their elbow, with local cryotherapy.

Results: At a mean follow-up of 12 months, pain improved from $1.5 \pm 1.3$ preoperatively to $8.1 \pm 2.4$ at follow-up $(P<.01)$. Of the patients, $7(32 \%)$ reported mild pain with strenuous activities and $1(5 \%)$ received mild benefit from the procedure. Patients required a mean of 2.8 weeks to return to regular activities and 8 weeks to return to full activity. No serious complications were identified.

Conclusions: Microdebridement using Radiofrequency coblation is a good option for refractory lateral epicondylitis. This procedure appears to be safe and effective and allows early mobilization and return to work.

\section{P784}

BONE MINERAL DENSITY OF THE FEMORAL NECK ON THE BACKGROUND OF A FOUR- YEAR RITUXIMAB THERAPY WHILE WOMEN POSTMENOPAUSAL WITH A DIAGNOSIS OF RHEUMATOID ARTHRITIS DEPENDING ON INITIAL OF DISEASE ACTIVITY

T. Raskina $^{1}$, M. Koroleva ${ }^{1}$, I. Dydykina ${ }^{2}$, V. Fanaskov ${ }^{3}$, O. Malyshenko ${ }^{1}$

${ }^{1}$ Kemerovo State Medical Academy, Kemerovo, Russian Federation, ${ }^{2}$ Nasonova Research Institute of Rheumatology, Moscow, Russian Federation, ${ }^{3}$ Regional Clinical Hospital for War Veterans, Kemerovo, Russian Federation 
Purpose: To rate the dynamics of BMD of the femoral neck on the background of a four-year rituximab (RTM) therapy while women postmenopausal with a diagnosis of rheumatoid arthritis (RA), depending on initial of disease activity.

Materials and Methods: We observed 44 postmenopausal women with documented diagnosis of RA receiving combination therapy with methotrexate (mean dose 13.18 [12.5$15.0] \mathrm{mg}$ / week) and rituximab (1000 mg intravenously twice with an interval of 14 days, the average number of courses 3.43 [3.0-4.0]). All patients were divided into two groups, depending on the degree of disease activity (index DAS28): group 1 - patients with 1 st and 2 nd degree of activity $(n=18)$, group 2 - patients with third degree of activity $(n=26)$. DXA was performed for all patients one time in the 12 months (48 months). Evaluated BMD and T-score of the proximal femur neck (densitometer «Excell» XR-46 «Norland»).

Results: It was found that all patients with RA receiving rituximab and methotrexate, regardless of initial level of activity on the background of a four-year therapy showed a significant increase in BMD of the femoral neck with respect to baseline data. Thus, in patients with the 1 st and 2 nd degree activity of the starting BMD was $0.026 \pm 0.8322 \mathrm{~g} / \mathrm{cm}^{2}$; after 48 months of therapy BMD $-0.8828 \pm 0.040 \mathrm{~g} / \mathrm{cm}^{2} \quad(p=0.038)$, T-score $-1.55 \pm 0.20 \mathrm{SD}$ and $-1.17 \pm 0.32 \mathrm{SD}$, respectively $(p=0.046)$. Similar results were obtained in the analysis of bone density values in patients with third degree of activity: baseline BMD $-0.7232 \pm 0.019 \mathrm{~g} / \mathrm{cm}^{2}$, after 48 months of therapy $-0.8035 \pm 0.024 \mathrm{~g} / \mathrm{cm}^{2}(p=0.0004)$ T-score $-2.39 \pm 0.17$ $\mathrm{SD}$ and $-1.17 \pm 0.32 \mathrm{SD}$, respectively $(p=0.001)$. Comparative analysis of BMD of the two patient groups revealed that in patients with third degree of activity BMD and T-score femoral neck baseline were significantly lower than in patients with 1 st and 2 nd degree $(p=0.0049$ and $p=0.0079)$. After 48 months of follow-BMD and T-score group 1 and group 2 did not differ significantly ( $p=0.106$ and $p=0.137$ ).

Conclusions: When the patients in the study registered the association between disease activity and the BMD. After 48 months of follow-up of all patients with RA, on the background of a combination therapy with rituximab and methotrexate showed a statistically significant increase in BMD, regardless of initial level of activity. A greater degree of RA activity corresponds to a lower baseline in BMD at the femoral neck.

\section{P785}

BONE MINERAL DENSITY AND THE RISK OF FATAL CORONARY EVENTS IN MEN WITH CORONARY HEART DISEASE

T. Raskina ${ }^{1}$, A. Voronkina ${ }^{2}$, M. Letaeva ${ }^{1}$, E. Maluta ${ }^{2}$, A. N. Kokov $^{3}$, O. Barbarash ${ }^{3}$

${ }^{1}$ Kemerovo State Medical Academy, Kemerovo, Russian Federation, ${ }^{2}$ Municipal Budget Healthcare Institution of City
Kemerovo, Kemerovo, Russian Federation, ${ }^{3}$ Federal State Budgetary Institution Research Institute for Complex Issues of Cardiovascular Diseases under the Siberian Branch of the Russian Academy of Medical Sciences, Kemerovo, Russian Federation

Purpose: To assess the degree of fatal coronary events development by coronary calcium index of the coronary arteries (CA) in dependence on BMD in men with coronary heart disease (CHD).

Materials and methods: It is examined 102 men with verified CHD at the age of $51-75$ years $[61(55 ; 65)$ y]. All patients were undergone the multislice computed tomography. Calcium index was calculated according to Agatston method. Patients with calcium index in absolute units above the 75th percentile of the corresponding age were attributed to the high risk of fatal coronary events. Method of DXA was used for determining MBD and T-criterion at the level of the lumbar spine and the femoral neck. According to T-criterion men were divided into 3 groups: I - 33 (32.4\%) with osteoporosis (T- criteria $<-2.5)$, II $-48(47,0 \%)$ with osteopenia (T-criteria between -1 and -2.5$)$ and III $-21(20.6 \%)$ with normal BMD (T-criteria $\geq-1$ ).

Results: The absolute majority of patients included to the study ( $63.7 \%$ of men) had the high risk of fatal coronary events. The distribution of men with CHD at high risk of fatal coronary events development in dependence on the T-criterion are presented as follows: in men group with osteoporosis 23 (69.7\%) patients had the high risk of fatal coronary events, at 10 patients (33.3\%) there was no such a risk, in men with osteopenia the high risk development of fatal coronary events was registered at $35(72.9 \%)$ when there is no risk at13 $(27.1 \%), p=0.9483$. Unlike groups with osteopenic syndrome at patients with normal BMD high risk of coronary events was detected significantly less frequently - at 7 (33.3 \%) patients while no risk at 14 (66.7 \%), I vs. III $p=0.0231$, II vs. III $p=0.0061$.

Conclusions: Low BMD is associated with the high risk of fatal coronary events that allows to consider the osteopenic syndrome as a possible prognostic factor of coronary complications development in men with coronary artery disease.

\section{P786}

CORONARY ARTERY CALCIFICATION AND BONE MINERAL DENSITY IN MEN WITH CORONARY HEART DISEASE

T. Raskina ${ }^{1}$, A. Voronkina ${ }^{2}$, M. Letaeva ${ }^{1}$, E. Maluta ${ }^{2}$, A. N.

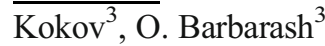

${ }^{1}$ Kemerovo State Medical Academy, Kemerovo, Russian Federation, ${ }^{2}$ Municipal Budget Healthcare Institution of City Kemerovo, Kemerovo, Russian Federation, ${ }^{3}$ Federal State 
Budgetary Institution Research Institute for Complex Issues of Cardiovascular Diseases under the Siberian Branch of the Russian Academy of Medical Sciences, Kemerovo, Russian Federation

Purpose: To investigate the relation between BMD and calcification of the coronary arteries (CA) in men with coronary heart disease (CHD).

Materials and methods: It was examined 102 men with verified CHD at the age of 51-75 years $[61(55 ; 65) \mathrm{y}]$. BMD and T-score L1-L4 and the femoral neck were determined by DXA. According to T-criterion the men were divided into 3 groups: I - $33(32.4 \%)$ with osteoporosis (OP, T-score $<-2.5)$, II $-48(47.0 \%)$ with osteopenia (OPe, T-criterion between -1 and -2.5$)$ and III - $21(20.6 \%)$ with normal BMD (T-criterion $\geq-1)$. All patients were undergone the quantification of CA calcification using multislice computed tomography. Calcium index was calculated according to Agatston method and evaluated the degree of calcification: 0 no, 1-10 - 11-100 - average, 101-400 - moderate, over 400 - expressed.

Results: Expressed CA calcification was detected at $57.8 \%$ of men, moderate - at $25.5 \%$, average - at $6.9 \%$, minimum at $2.0 \%$, no calcification - at $7.8 \%$. In the group with OP the majority of patients had the expressed CA calcification $(69.7 \%)$, at $15.1 \%$ there was revealed the moderate calcification, at $6.1 \%$ average, at $3.0 \%$ - minimum, at $6.1 \%$ cases the calcification was not detected. In patients with $\mathrm{OPe}$ at $60.4 \%$ there was recorded the expressed CA, at $33.3 \%$ - moderate, and at $4.2 \%$ - average, at $2.1 \%$ - minimum. There were no patients without calcification $\mathrm{CA}$ in this group. In the group with normal BMD at $33.3 \%$ of patients there are registered the expressed CA calcification, at $23.8 \%$ - moderate calcification, at $14.3 \%$ - average, at $28.6 \%$ the calcification was not determined. Minimal CA calcification in this group of patients has not been detected. In selected by $\mathrm{T}$ criterion groups of patients there were prevailed the patients with expressed CA calcification. The prevalence of expressed CA calcification was significantly lower in the group of patients with normal BMD compared with groups with osteopenic syndrome $(p<0.05)$. The absence of CA calcification is registered significantly more often in patients with normal BMD compared with groups with low BMD $(p<0.05)$. It is noted the inverse correlation between the degree of CA calcification by Agatston scale and T-criterion of the femoral neck $(r=-0.25 ; p=0.0136)$, T-criterion L1-L4 ( $r=$ to $-0.20 ; p=0.0484)$, the femoral neck BMD $(r=-0.23 ; p=0.0220), \mathrm{BMD}, \mathrm{L} 1-\mathrm{L} 4 \quad(r=-0.19$; $p=0.0464)$.
Conclusions: Low BMD is associated with expressed CA calcification which confirms the relationship between the deposition in the vascular wall calcifications and osteopenia syndrome in men with coronary artery disease.

\section{P787}

MATERNAL HEALTH LITERACY AND CHILDHOOD BONE MINERAL DENSITY OF OFFSPRING

S. M. Hosking ${ }^{1}$, R. Buchbinder ${ }^{2}$, S. L. Brennan-Olsen ${ }^{1}$, N. K. Hyde $^{1}$, L. J. Williams ${ }^{1}$, J. A. Pasco ${ }^{1}$

${ }^{1}$ School of Medicine, Deakin University, Geelong, Australia, ${ }^{2}$ Department of Epidemiology and Preventive Medicine, School of Public Health and Preventive Medicine, Monash University, Melbourne, Australia

Objective: Lower maternal health literacy (HL) is suggested to have a negative impact on a wide range of child health outcomes; however, its role in child bone health is currently unknown. We explored associations between maternal HL and child BMD.

Material and Methods: Data were collected at the $10 \mathrm{yr}$ follow-up of mother-child pairs enrolled in the Vitamin D in Pregnancy study. Maternal HL was ascertained using the Health Literacy Questionnaire, a multi-dimensional HL measurement tool. Child areal BMD was measured at lumbar spine and total body less head (TBLH), aged 10-12 yr ( $11.04 \pm 0.48$ yr) using DXA (Lunar DPX-L). Developmental stage was determined using the 5-stage Tanner scale. Hierarchical cluster analysis was used to group participants with similar HL profiles into 4 clusters, ranging from highest HL (cluster 1) to lowest HL (cluster 4). Using regression analyses we assessed relationships between maternal HL and child BMD, holding the mid HL cluster 3 as referent. 155 mother-child pairs had complete measures for maternal HL and child BMD.

Results: Cluster 1 was associated with higher child TBLH BMD after adjustment for weight and developmental stage ( $\beta \quad 0.048$ 95\% CI $0.012,0.084)$; results were sustained after adjustment for age and sex; however after adjustment for height only a trend remained ( $\beta 0.03295 \% \mathrm{CI}-0.003,0.069$ ). A trend was observed for BMD at the spine (cluster 1 vs. cluster $3 p=0.108$ ); this was not sustained after further adjustment for age. Cluster 4 also showed higher child BMD for TBLH ( $\beta$ $0.04395 \%$ CI $0.014,0.072$ ) with a trend observed for low maternal HL and spine BMD $(p=0.151)$.

Conclusion: Both the highest and the lowest maternal HL clusters showed an association with higher child BMD for TBLH and spine when holding mid HL cluster 3 as referent. These data suggest that mothers from both ends of the HL spectrum may have unique barriers and enablers that may influence child bone health. 


\section{P788}

\section{MUTATIONAL SPECTRUM OF TYPE I COLLAGEN} GENES IN CHINESE OI PATIENTS

Y. Lu ${ }^{1}$, X. Ren ${ }^{2}$, Y. Wang ${ }^{3}$, X. Shi ${ }^{1}$, J. Han ${ }^{1}$

${ }^{1}$ Shandong Academy of Medical Sciences, Jinan, China, ${ }^{2}$ The People's Hospital of Wuqing District, Tianjin, China, ${ }^{3}$ Shandong Provincial Hospital, Jnan, China

Aims: Osteogenesis imperfecta (OI) is a group of inheritable connective tissue diseases characterized by frequent fractures and reduced bone mass. Blue sclerae, dentinogenesis imperfecta, hearing and sight impairment, muscle weakness and joint laxity are frequently existed in OI patients. OI genetic heterogeneity is obvious and autosomal dominant form is the primary inheritance pattern, which is caused by mutations of COL1A1 or COL1A2 genes that encode the proa1 and proa2 chains of type I procollagen. There is little report about the mutation spectrum of OI patients in China. The study aims to investigate the epidemiology of OI in China.

Methods: Under the approval of the Shandong Academy of Medical Sciences Review Board, peripheral bloods were collected after the informed consents signed by all patients attended. Blood genomic DNAs were extracted for PCR amplification. PCR products covering the coding region and exon-intron boundaries of COL1A1 and COL1A2 gene were sequenced by traditional Sanger sequencing. Genetic variations were analyzed by Mutation Surveyor software 4.0, OI mutation and HGVD databases. Mutations were further confirmed by normal control samples from healthy population. In silico prediction was conducted to analyse their effects on protein structure by Polyphen, Align GVGD, SIFT and Rescue-ESE softwares.

Results: A total of 61 heterozygous mutations were identified in type I collagen genes with 30 mutation sites were novel. Equal mutation incidence of COL1A1 and COL1A2 genes was identified. In 30 COL1A1 related mutations, 24 heterogeneous missense mutations and two new frameshift nonsense and splice site mutations were discovered in respectively. In 31 COL1A2 related mutations, 29 were heterozygous missense mutations and two were splice site variation. Glycine substitutions were the primary type for missense mutation and their detection rate in the COL1A1 and COL1A2 gene was $70.00 \%$ and $83.87 \%$, respectively. The percentage of 40 was substituted by serine in COL1A1 and 45 in COL1A2 gene. Substitutions of glycine by cysteine was the second common mutation type in COL1A2 gene $(n=6,19.35 \%)$. Alanine for glycine substitutions detected only in the COL1A1 gene with $16.67 \%$ prevalence. Substitutions of glycine by arginine, asparagine, valine, glutamine and tryptophan was rare but existed in either $\alpha 1$ or $\alpha 2$ chain. Two new recurrent mutations of c. $1913 \mathrm{G}>\mathrm{C}$ in COL1A1 gene and c.3061G $>$ T in COL1A2 gene was detected in two and three unrelated OI patients, respectively.

Conclusions: The study depicts the mutational spectrum of type I collagen genes in the large number of Chinese OI patients. The identification of mutations will be useful for clinical diagnosis, genetic counseling and prenatal diagnosis. It discovers the epidemiology of OI in China and provides the data for phenotype-genotype correlations.

\section{P789}

VITAMIN D LEVELS AND THE PRESENCE OF VERTEBRAL FRACTURES IN POSTMENOPAUSAL WOMEN IN A LOCALITY OF THE COLOMBIAN CARIBBEAN REGION

$\underline{\text { J. Jaller }}^{1}$, J. Jaller Char ${ }^{2}$, P. Camargo ${ }^{3}$, R. Vargas ${ }^{4}$

${ }^{1}$ Universidad Metropolitana, Centro de Reumatología y Ortopedia, Barranquilla, Colombia, ${ }^{2}$ Universidad Central de la Florida, Orlando, United States, ${ }^{3}$ Medicina Interna, Universidad Metropolitana, Barranquilla, Colombia, ${ }^{4}$ Universidad Metropolitana, Barranquilla, Colombia

Objective: To determine the relation between vitamin D levels and the presence of vertebral fractures in postmenopausal women in a locality of the Colombian Caribbean region.

Materials and Methods: A descriptive, cross-sectional study. There were involved 319 postmenopausal women, from 6 nursing homes in Barranquilla city, with ages over 59 years old, ability to walk with independence, without metabolic disease or cancer, and belonging to the general health system. To determine vertebral fractures, morphometric assessment was performed using radiographic study and Genant's classification method for fractures (mild, moderate and severe). The levels of vitamin D (total 25-OH vitamin D metabolite) were taken by puncture of peripheral blood, the sample was analyzed by immunoassay chemiluminescence (CLIA) liaison 25OH Vit D total assay Ref 310600, using Diasorin Iiaison analyzer.

Results: The overall prevalence of fractures in the group of study was $17.9 \%$ (43.9\% severe, $35.1 \%$ moderate and $21 \%$ mild). The average of vitamin $\mathrm{D}$ was $24.1 \mathrm{ng} / \mathrm{ml}(\mathrm{SD} \pm 7.83)$. Statistical association between age and the presence of vertebral fractures $(p<0.05)$ was found, as well as between the levels of vitamin D and these fractures $(p<0.05)$. 
Conclusion: significant relation between low vitamin D levels and the presence of vertebral fractures was obtained, which can be influenced by age.

\section{P790}

EFFECT OF RITUXIMAB ON QUALITY OF LIFE WITH RHEUMATOID ARTHRITIS WOMEN TO BASELINE BONE MINERAL DENSITY

T. Raskina $^{1}$, M. Koroleva ${ }^{1}$, I. Dydykina ${ }^{2}$, M. Letaeva ${ }^{1}$

${ }^{1}$ Kemerovo State Medical Academy, Kemerovo, Russian

Federation, ${ }^{2}$ Nasonova Research Institute of Rheumatology,

Moscow, Russian Federation

Objective: On the results of a prospective four-year observation to evaluate the effect of rituximab on the quality of life with rheumatoid arthritis (RA) women with different BMD to baseline.

Material and Methods: We observed 44 postmenopausal women with documented diagnosis of RA (criteria ACR, 1987 ) with the combined therapy of methotrexate (mean dose of 13.18 [12.5-15.0] mg / week) and rituximab to standard scheme (1000 mg intravenously in the 1st and 15 th days, the course - 2 infusion, the average number of courses -3.43 [3.0-4.0]). All patients were randomized into two groups according to the level of BMD and T-score: patients with osteopenia (group 1, $n=18$ ) and with osteoporosis (group 2, $n=26$ ). To assess BMD was performed DXA. To assess life quality questionnaires EQ-5D and HAQ were used. All patients completed questionnaires on the baseline and then every 12 months to 4 years follow-up.

Results: When calculating a quantitative index for health questionnaire EQ-5D established that baseline in patients with osteoporosis health index indicator was lower than in patients with osteopenia $-0.26 \pm 0.03$ and $0.34 \pm 0.03(p=0.043)$. After 4 years of therapy there was a statistically significant increase in the index in both groups of patients relatively baseline data $-0.52 \pm 0.03$ and $0.55 \pm 0.04 \quad(p<0.0001$ and $p<0.001$, respectively). Similar relationships observed in the analysis of the dynamics of the index HAQ. Baseline in patient's osteopenia HAQ index was $1.84 \pm 0.09$, in patients with osteoporosis $-1.83 \pm 0.13(p=0.89)$, which corresponds to moderate functional impairment in patients of both groups. During therapy HAQ index was significantly decreased in both groups (HAQ group $1-1.24 \pm 0.11$, HAQ group $2-$ $1.36 \pm 0.14, p=0.0003$ and $p=0.038$ )

Conclusion: According to the results of a four-year observation noted a positive effect of rituximab on the quality of life of patients with osteopenia and with osteoporosis.
P791

USE OF TERIPARATIDE FOR TREATING DELAYED UNION AND NONUNION OF FRACTURES: REPORT OF 12 CASES

A. $\mathrm{Kar}^{1}$

${ }^{1}$ Department of Orthopaedics, Apollo Gleneagles Hospitals, Kolkata, India

Introduction: Fracture healing is a slow process. Majority fractures heal, but a few of them are notorious for delayed union and nonunion. The treatment ranges from ultrasound therapy, bone marrow injection and the more painful bone grafting procedure. Teriparatide injection has been used "off the shelf" for delayed union and nonunion of fractures with encouraging results. Even animal studies have been encouraging.

Material and Methods: A total of 12 cases of delayed union and nonunion were treated with a dose of $20 \mu \mathrm{g} /$ day for six months. Intertrochanteric fracture femur -4 cases, proximal humerus -4 cases, distal tibial fractures -2 cases, radial shaft -1 case, proximal tibia -1 case. The patients underwent radiological and clinical evaluation for union every 6 weeks till the end of six months.

Results: All hip fractures united. Two of the proximal humerus cases united. None of the distal tibial fractures united. They had to be treated with bone marrow injections. The radial shaft fracture had to be re operated with plating and bone grafting. The proximal tibial fracture also did not unite and the patient refused further treatment. 8 out of 12 cases $(66 \%)$ united.

Conclusion: Teriparatide injection shows promise in treatment of delayed and nonunions. Most of these patients had to be treated earlier with some form of invasive treatment or surgery. It is also cost effective as it is cheaper than invasive procedures. More randomized controlled trials are required before its use becomes widespread.

\section{P792}

EFFECT OF METHOTREXATE THERAPY IN RHEUMATOID ARTHRITIS ON SERUM RANKL, OPG AND DKK1

V. Dhir ${ }^{1}$, N. Pandurangan ${ }^{1}$, K. V. Mahesh ${ }^{1}$, N. Gupta ${ }^{1}$, A. $\overline{\text { Sharma }}^{1}$, S. K. Sharma ${ }^{1}$

${ }^{1}$ Department of Internal Medicine, PGIMER Chandigarh, Chandigarh, India

Objective: To assess the effect of methotrexate therapy in rheumatoid arthritis on serum RANKL, OPG and DKK1 levels. 
Methods: Prospective study that included patients of RA (fulfilling 1987 ACR), aged 18-65 years with active disease (Disease activity score 28 joints, 3 variable, DAS28$3 \mathrm{v}>3.2$ ) and not on methotrexate. Oral MTX was started at $15 \mathrm{mg} /$ week and increased to $25 \mathrm{mg} /$ week at 8 weeks and continued till 24 weeks. Serum was stored at $-80 \mathrm{C}$ at baseline and 24 weeks and commercial ELISA used to assay levels of sRANKL, OPG and DKK-1. Paired t-test used for analysis.

Results: We included 51 patients (F:M=45:6)with rheumatoid arthritis, mean age $42.7( \pm 11.4)$ years with mean disease duration $1.8( \pm 1.5)$ years. RF+ in $59 \%$ and anti $\mathrm{CCP}+$ in $79 \%$. Over 24 weeks, the mean DAS 28 3v declined from $6.1( \pm 0.9)$ to $4.8( \pm 1.0)$ and HAQ from $1.4( \pm 0.6)$ to $0.7( \pm 0.5)$. There was significant increase in DKK1 $(96.3 \pm 80.1,211.3$ $\pm 236.8, p=0.002)$ and decrease in OPG (2879.7 \pm 1038.0 , $2059.8 \pm 532.2, p<0.001)$ with treatment, however, no change in sRANKL. There was no correlation between sRANKL, OPG or DKK1 with DAS28_3v

Conclusion: There were changes contrary to expected, in terms of increase in DKK-1 (inhibitory to bone formation) and decrease in OPG (inhibitory to bone resorption). There may be a mismatch between circulating and tissue levels of these molecules.

\section{P793}

ASSOCIATIONS BETWEEN REGULAR PHYSICAL ACTIVITY AND EXCEEDING INTERVENTION THRESHOLDS FOR FRACTURE USING FRAX CALCULATED WITH TRABECULAR BONE SCORES

$\underline{\text { S. L. Brennan-Olsen }}{ }^{1}$, K. L. Holloway ${ }^{1}$, W. D. Leslie ${ }^{2}$, M. A. Kotowicz $^{1}$, J. A. Pasco ${ }^{1}$

${ }^{1}$ School of Medicine, Deakin University, Geelong, Australia, ${ }^{2}$ Department of Medicine, University of Manitoba, Winnipeg, Canada

Objective: Ten-year fracture probability estimated using FRAX $^{\circledR}$ now optionally includes spine trabecular bone score (TBS). Different types and levels of physical activity may impact on bone strength and trabecular structure, thus we examined whether regular physical activity in men was associated with exceeding intervention thresholds for fracture probability as indicated by FRAX, with the inclusion of TBS. Methods: We collected clinical data, including FRAX variables, in 545 men (40-90 yr) from the Geelong Osteoporosis Study. Femoral neck BMD was measured by DXA (Prodigy, GE Lunar); TBS was derived retrospectively. Self-reported occupational or home-based activity (sitting; standing; walking; heavy lifting) was categorized as always/often (regular) vs. sometimes/never (referent). FRAX scores including BMD were computed, followed by TBS adjustment. Using logistic regression we investigated associations between regular physical activity and FRAX scores exceeding intervention thresholds: $\geq 3 \%$ for hip fracture and $\geq 20 \%$ for major osteoporotic fracture (MOF).

Results: Independent of regular standing, walking and lifting, regular sitting increased the age-adjusted odds of exceeding hip fracture intervention thresholds $(O R=1.78,95 \%$ CI 1.05-3.04). We observed a protective trend in heavy lifting to lower odds of exceeding hip fracture intervention thresholds $(O R=0.60,95 \% \mathrm{CI}$ $0.32-1.15$, respectively). No further associations were observed for hip fracture, and none were observed for MOF intervention thresholds. Whilst significant in models, age did not explain associations.

Conclusions: Men with sedentary occupations/homebased activities were more likely to exceed hip fracture intervention thresholds; regular walking and heavy lifting may provide protection, thus suggesting implications for the promotion of preventive health behaviour.

\section{P794}

CHANGES IN KINEMATICS OF LOWER EXTREMITIES IN OSTEOARTHRITIS PATIENT WEARING KNEE BRACES

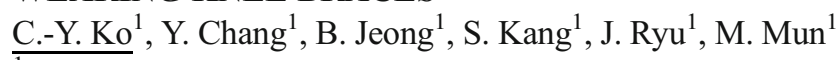
${ }^{1}$ Korea Orthopedics \& Rehabilitation Engineering Center, Incheon, Republic of Korea

Objective: The aim of this study was to evaluate lowerextremities kinematics in an OA patient wearing knee braces. Material and Methods: In this study, 7 male healthy adults as control group and $1 \mathrm{OA}$ patient were involved. They wore 3 knee brace, Neo (Ossur, USA), CTI (Ossur, USA), Patella (Ottobock, Germany), in their right knee. Then they walked along a $10 \mathrm{~m}$ long walkway and 3D kinematics of the hip, the knee and the ankle was evaluated by motion analysis system (Motion Analysis, USA).

Results: There were differences in joint kinematics between wearing knee braces and no brace. In the ankle, less internal rotation and adduction and more dorsiflexion and less plantarflexion were shown in the OA patient wearing knee braces, while more internal rotation and adduction, and less dorsiflexion and more plantflexion were shown in the healthy 
adults wearing knee braces. In the knee, less external rotation, and adduction and more flexion were shown in the OA patient wearing knee braces, while more external rotation and less abduction and flexion in the knee was shown in the healthy adults wearing knee braces. In the hip, more external rotation and adduction were shown in the OA patient and healthy adults wearing knee braces. Less extension in the OA patients was shown, while more extension and less flexion in healthy adults were shown.

Conclusion: These results indicated that lower extremities kinematics can be altered by wearing knee brace. Furthermore, the effects of knee brace were differences between healthy adults and OA patient.

Acknowledgments: This research project was supported by the Sports Promotion Fund of Seoul Olympic Sports Promotion Foundation from Ministry of Culture, Sports and Tourism.

\section{P795}

PREVALENCE OF OSTEOPOROSIS AMONG WOMEN LIVING IN DIFFERENT GEOGRAPHIC REGIONS OF UZBEKISTAN

L. S. Abboskhujaeva $^{1}$, S. I. Ismailov ${ }^{1}$, N. M. Alikhanova ${ }^{1}$, G. I. Allayarova $^{1}$, M. M. Shakirova ${ }^{1}$

${ }^{1}$ Republican Specialized Scientific-Practical Medical Centre of Endocrinology, Tashkent, Uzbekistan

Our epidemiological survey is the first step in studying of prevalence and risk factors of postmenopausal osteoporosis (PMO) in the Republic of Uzbekistan, aiming at development of early preventive and therapeutic measures to reduce osteoporosis-associated fractures.

Methods: We screened 1594 postmenopausal female residents of Tashkent, Namangan, and Qarshi - the cities with the largest populations in Uzbekistan, aged from 50-80. The duration of postmenopausal period was $\geq 1$ year.

Results: According to our data, the prevalence of osteoporosis in different regions of Uzbekistan varies widely $(23.1 \%, 33.5 \%$ and $51.1 \%$ in Qarshi, Tashkent and Namangan, respectively). The prevalence of osteoporosis increases with age from $25.6 \%$ (in age group 50-59 years) to $51.3 \%$ (in age group $>70$ ) in Tashkent, from 16.5 to $66.7 \%$ in the same age groups in Qarshi and from 44.0 to $80.0 \%$ in Namangan. In Namangan were significantly more women with body mass $<57 \mathrm{~kg}$ than in Tashkent (OR 2.44; 95\%CI 1.72-3.46; $P<0.00001$ ), but there was no difference with Qarshi (OR 1.09; 95\%CI 0.69-1.70; $P=0.80$ ). We found that the number of women doing physical exercises in Namangan $(36.1 \%)$ was significantly lower comparing to Tashkent $(65.5 \%$; OR $0.30 ; 95 \% \mathrm{CI}$
$0.23-0.38 ; P<0.0001)$ and Qarshi $(81.0 \%$; OR 0.13 ; $95 \%$ CI $0.09-0.20 ; P<0.0001)$. Women living in Namangan $(6.0 \%)$ had significantly more fractures in history comparing to women living in Tashkent $(1.7 \%$; OR 3.79; 95\%CI 2.0-7.18; $P<0.0001)$ and Qarshi (1.9 \%; OR 3.40; 95\%CI 1.17-9.89; $P=0.03$ ).

Conclusion: Our research shows that osteoporosis is widely spread among women above 50 living in three densely populated cities of Uzbekistan (Tashkent, Qarshi and Namangan). Osteoporosis prevalence among Namangan (51.1\%) female residents is higher comparing to women living in Tashkent (33.5\%) and Qarshi (23.1\%).

\section{P796}

ADVANCED GLYCATION ENDPRODUCTS INDUCES INFLAMMAGING IN OSTEOGENIC SAOS-2 CELLS $\underline{\text { S. Ashe }}^{1}$, D. Nayak ${ }^{1}$, P. R. Rauta ${ }^{1}$, M. Kumari ${ }^{1}$, B. Nayak ${ }^{1}$ ${ }^{1}$ Life Science National Institute of Technology, Rourkela, India

Objective: Advanced glycation endproducts (AGE) are formed in long-lived matrix proteins by a nonenzymatic reaction with sugar and have been implicated in the development of diabetic complications. In this work we aim to assess the involvement of AGE and its receptor (RAGE) in the reactive oxygen species production and cell death via NFKB activation.

Materials and Methods: Glucose-derived AGEs were synthesized and characterized by UV and fluorescence studies. The effect of glycation on the change in structure of BSA was done by circular dichroism. Saos-2 cells were treated with AGEs and cytotoxicity was evaluated by cell proliferation assay (MTT Assay). Extent of DNA damage was also checked by DNA fragmentation assay chromatin condensation assay. Further the decrease in colony forming ability of the Saos-2 cells was determined by the clonogenic assay. Reactive oxygen species (ROS) produced was quantified by detecting the fluorescence intensity of the oxidation-sensitive dye $2^{\prime}, 7^{\prime}$ dichlorofluorescin diacetate. Real time PCR was performed to check the mRNA levels of RAGE, NFKB, RANK, IL-1, IL-6, OPG and OPN to determine the pathway by which AGE mediated cell death and ROS production occurs.

Results: Cells treated with AGEs showed a reduced cell proliferation with an increase in DNA damage. The amount of ROS produced was also high as compared to the control cells. With the presence of AGE mRNA expression of RAGE, NFKB, RANK, IL-1 and IL-6 increased indicating increase in production of inflammatory factors.

Conclusion: Through this work we want to suggest AGEs as a potential risk factor. Understanding the pathophysiology of 
AGEs in bone biology would be paramount to identifying new targets and developing personalized therapy for osteoporosis thus needing future studies.

\section{P797}

FREQUENCY AND RISK FACTORS OF DXA DISCORDANCE BETWEEN HIP AND SPINE

L. Nazir $^{1}$, T. Perveen Umer ${ }^{1}$, S. Rehman Arain ${ }^{1}$, T. Rasool ${ }^{1}$

${ }^{1}$ Liaquat National Hospital and Medical College, Karachi, Pakistan

Objective: To assess the frequency and risk factors for T-score discordance between spine and hip measurement sites of patients coming to Rheumatology clinic at a tertiary care hospital of Karachi.

Methods: Demographic data, anthropometric measurements and risk factors for osteoporosis were collected through questionnaire based personal interviews. DXA was performed on L1-L4 lumbar spine and total hip for all cases. Minor discordance was defined when the difference between 2 sites was not more than 1 World Health Organization diagnostic class. Major discordance was present when 1 site is osteoporotic and other is normal.

Result: This is a prospective ongoing study with a calculated sample size of 595. Data of 137 patients is analyzed till now and presented in this abstract. The frequency of concordance was 55 (40.1\%), minor discordance was 66 $(48.2 \%)$ and major discordance was $16(11.7 \%)$ between spine and hip measurement sites. There is no significant association found between the discordance and risk factors like the gender, smoking, type of toilet use, BMI, previous history of fracture, family history of osteoporosis and parent's history of fracture.

Conclusion: On the basis of current findings, we concluded that there is no significant association between osteoporosis risk factors with DXA discordance between hip and spine.

\section{P798}

RELATIONSHIP BETWEEN SECONDHAND SMOKING EXPOSURE, DETERMINED BY URINARY COTININE LEVEL, AND BONE HEALTH

H.-J. Kim ${ }^{1}$, J.-H. Moon ${ }^{2}$, M.-H. Kong ${ }^{2}$, J.-S. Huh ${ }^{3}$

${ }^{1}$ Family Medicine, Jeju National Universitiy \& Hospital, Jeju, Republic of Korea, ${ }^{2}$ Jeju National University Hospital, Jeju, Republic of Korea, ${ }^{3}$ Jeju National University \& Hospital, Jeju, Republic of Korea

Objective: We evaluated the relationship between secondhand smoke (SHS) inhalation, as verified by urinary cotinine levels, and bone health.
Material and Methods: BMD data was analyzed from the Korean National Health and Nutrition Examination Survey (2008 - 2010). Men aged 50 years or older were included, while current smokers (urinary cotinine levels higher than $500 \mathrm{ng} / \mathrm{mL}$ ) were excluded. Exposure to SHS was determined using a $50 \mathrm{ng} / \mathrm{mL}$ urinary cotinine threshold.

Results: The estimated prevalence of SHS exposure in our cohort was $13.9 \%$. After adjusting for age and BMI, T-scores at total femur $(P<0.001)$, femoral neck $(P<0.001)$, and lumbar spine $(P=0.004)$ were lower in SHS exposure vs. nonexposure groups. Impaired bone health (osteopenia or osteoporosis) at femoral neck or lumbar spine was evident in $61.7 \%$ and $48.6 \%$ of SHS exposure and nonexposure cases, respectively $(P=0.004)$. Moreover, after adjusting for age, BMI, and health habits, the odds ratio for impaired bone health in the SHS exposure group was 1.89 (95\%CI: 1.31 - 2.74).

Conclusions: Our findings suggest that SHS exposure, determined by urinary cotinine levels, is negatively associated with BMD, and is a leading cause of impaired bone health in Korean men.

\section{P799}

EMPIRICAL EVALUATION OF THE RISK AND PREVENTION OF UNSTABLE COMPONENT OF THE KNEE ENDOPROSTHESIS

$\underline{\text { A. V. Alabut }}^{1}$, V. D. Sikilinda ${ }^{1}$, I. L. Filonov ${ }^{1}$, A. I. Gorbatenko $^{1}$

${ }^{1}$ Rostov State Medical University, Department of Traumatology and Orthopedics, Rostov on Don, Russian Federation

The arthroplasty of a knee joint with an osteoporosis background is accompanied by a risk development of endoprosthesis loosening.

Aim: Comparison of the data from osteodensitometry and bone strength in patients undergoing knee replacement.

Methods: 50 patients underwent densitometry in the preoperative period. After surgery, the strength of the resected fragments of the femur and tibia were examined.

Results: A classification was developed for bone strength. Patients with the worst bone strength were classified in class 1. Pathological bone breakdown occurred for excess weights of not more than 3 times. The bone strength in this group was less than $240 \mathrm{~kg}$ of force $(\mathrm{KgF})$. Patients with a bone strength of 241-400 KgF were set in class $2,401-480 \mathrm{KgF}$ to class 3, more than $480 \mathrm{KgF}$ to class 4 . There were more than 3 times more women in the classes 1 and 2 , in classes 3 and 4 more men. The average ages by class were as follows: $1-63.93$ years, $2-$ 61.45 years, $3-64.11$ years, $4-62.56$ years. According to the data from the densitometry and bone strength of the femur in classes 1 and 2, osteoporosis and osteopenia were present. Classes 3 and 4 had normal indicators. The average weights of 
the patients for each class was as follows: classes 1 and $2-$ $83.76 \mathrm{~kg}$, classes 3 and 4 - about $10 \mathrm{~kg}$ more. The average BMI for each class was as follows: Class $1-30.05$, class $2-31.11$, class $3-31.48$, class $4-30.63$.

Conclusions: It was concluded that a decrease in the bone strength depends on an increase in age, a decrease in weight and BMI. A gender dependence was found.

\section{P800}

EVALUATION OF THE RISK AND PREVENTION OF COMPLICATIONS OF TOTAL HIP ARTHROPLASTY $\underline{\text { A. V. Alabut }}^{1}$, V. D. Sikilinda ${ }^{1}$, D. S. Chuyko ${ }^{1}$, A. I. Gorbatenko ${ }^{1}$

${ }^{1}$ Rostov State Medical University, Department of Traumatology and Orthopedics, Rostov on Don, Russian Federation

Osteoporosis is currently one of the most common diseases with tremendous socioeconomic and medical significance. Fourth place among all causes of disability and death occupy fractures of the femur in osteoporosis. According to Aubrey Blumsohn(2013) osteoporosis is 2.7 times more common in women older age group, which is associated with the onset of menopause. In our study involved 32 patients of the senior age group orthopedics and trauma. Department of the clinic of Rostov state medical university. Inclusion criteria: bilateral coxarthrosis 3: before surgery, after performing unilateral hip replacement, and, after bilateral hip replacement with cement variant of the prosthesis. All patient was performed a study of bone resorption markers and bone formation, osteodensitometry of the lumbar spine and the hips before surgery, and at 3, 6 and 12 months after total arthroplasty of the first hip joint. The aim was to determine the optimal amount perioperative management of female patients of the senior age group with bilateral coxarthrosis 3 . In 28 patients 3 months after surgery on the first hip joint showed a reduction in mineral density of bone mass of the lumbar spine, a decrease in $\mathrm{T}$ criterion of 0.8 . In 4 patients remained unchanged (within 0.2). In 27 patients, the decrease of BMD in the zone of localization of the operated hip joint, the T criterion decreased on average by 2.1. The tendency to loss of BMD in the following year was observed in $100 \%$ of patients. In 17 women with the worst indicators of osteodensitometry, the increase in bone resorption (b-cross laps test) history of diagnosed early menopause. Further x-ray observation during the year of instability of the components of the implant was not observed in $100 \%$ of women. It remains an undeniable fact that effects of menopause on the rate of development of osteoporosis in women with bilateral coxarthrosis. Treatment selection should be based on data osteodensitometry studies, biochemical markers of bone metabolism. Need extended preoperative preparation and dynamic observation of the patient after the implantation with the aim of preventing the development of instability of endoprosthesis components.

\section{P801}

CORRELATES OF OSTEOPOROTIC FRACTURES AMONG TYPE 2 DIABETIC PATIENTS

$\underline{\text { I. Goldshtein }^{1}}$, J.-M. Chandler ${ }^{2}$, A. DePapp ${ }^{2}$, S. Ish-Shalom ${ }^{3}$, G. Chodick $^{1}$, A.-M. Nguyen ${ }^{2}$

${ }^{1}$ Epidemiology and Database Research Department, Maccabi Healthcare Services, Tel Aviv, Israel, ${ }^{2}$ Center for Observational and Real-World Evidence, Merck \& Co., Inc., Kenilworth, United States, ${ }^{3}$ Elisha Hospital, Haifa, Israel

Objective: To identify diabetes-specific predictors of an osteoporotic fracture.

Materials and Methods: This study utilized the computerized database of a large insurer and provider of healthcare services in Israel. The first phase included a cross sectional analysis to assess the current prevalence of fracture history among osteoporosis (OP) patients with and without type 2 diabetes (T2DM). Subsequently, a subcohort of osteoporotic T2DM patients with no fracture at OP diagnosis (index date), was retrospectively analyzed to identify correlates of incident fractures among diabetic OP patients, using time to event (Cox's proportional hazard) analysis.

Results: A total of 97,454 OP patients were identified of whom $18 \%$ were diabetic. The prevalence of major osteoporotic fractures (hip, spine, Colle's or humerus) was significantly higher among T2DM (42\%) compared to DM-free patients (31\%) across all fracture subtypes (age and sex adjusted standardized ratio $=1.30, P<0.001$ ), and particularly increased in hip fractures ( $8.8 \%$ vs. $5.8 \%$ ). Diabetic OP patients were more likely to be older (mean current age $74.3 y$ vs. $69.1 \mathrm{y})$ and males $(20.8 \%$ vs. $14.4 \%, \mathrm{p}<0.001)$ as compared with DM-free ones. Screening rates for BMD were similar in both groups $(79.3 \%$ vs. $79.9 \%)$, and median most recent t-scores were significantly $(\mathrm{P}<0.001)$ higher in both total-hip (-1.3 vs. -1.6$)$ and vertebrae (-1.4 vs. -1.8$)$ yet relatively similar in femur neck $(-1.8$ vs. $-1.9, \mathrm{p}<0.001)$ for T2DM patients vs. DM-free. In a multivariable model among T2DM patients with osteoporosis, fracture risk was significantly associated with neuropathy ( $H R=1.22,95 \% \mathrm{CI}: 1.05-1.42)$, history of hypoglycemic events $(H R=1.21,1.07-1.37)$, cardiovascular disease $(H R=1.25,1.14-1.38)$, chronic kidney disease, and prolonged intake of thiazolidinedione $(12+$ months vs. $<12$ months: $H R=1.34,1.04-1.73$ ).

Conclusions: This large population-based study quantifies the unique risk factors for fracture among diabetic patients, and confirms that patients with diabetes are at increased risk for major osteoporotic fractures and that the presence of microand macrovascular disease appears to further increase the risk. This study was supported by MSD, as part of a real-world evidence research center. 


\section{P802}

\section{CALCIUM SUPPLEMENT AND CAROTID INTIMA THICKNESS IN POSTMENOPAUSAL WOMEN WITH OSTEOPOROSIS}

D. Anghel $^{1}$, M. L. Ciobica ${ }^{1}$, M. M. Negru ${ }^{2}$, N. C. Anghel ${ }^{3}$, S.

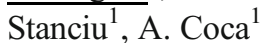

${ }^{1}$ Central Military Hospital Bucharest, Bucharest, Romania, ${ }^{2}$ Clinical Hospital "Sfanta Maria", Bucharest, Romania,

${ }^{3}$ Clinical Hospital "Constantin Angelescu", Bucharest, Romania

Background: Calcium/vitamin D supplement are widely used for the treatment of osteoporosis. Vascular calcification is a risk factor for cardiovascular disease. Calcium supplements can influence the vascular calcification and atherosclerosis.

Objectives: To evaluate the relationship between influence of calcium /vitamin D supplement use in post menopause women with osteoporosis and IMT of common carotid artery and carotid atherosclerotic plaque.

Methods: In this study were consecutively enrolled 69 women with osteoporosis and calcium/vitamin D supplement and 30 women with normal BMD. Mean age was $51.2 \pm 5.1$. OP screening has been performed by DXA scanning of the lumbar spine and hip. Mean common carotid IMT as a marker of atherosclerosis and atherosclerotic plaques were assessed by carotid ultrasonography (IMT -abnormal $>0.8 \mathrm{~mm}$ or calcified plaques). Patients were evaluated for independent cardiovascular risk factors: hypertension, diabetes, dyslipidemia.

Results: 34 patients in osteoporosis group had increased IMT or calcification plaques compared to 14 of nonOP women. OP women had 8.2 times increased IMT $(p=0.003)$ compared to non OP women. The odds of increased IMT and developed atherosclerosis are increased with $19 \%$ per year after menopause $(p<0.008)$. Comparison of cardiovascular risk factors between 2 groups reveals no significant differences.

Conclusion: Calcium/vitamin D supplement is not associated with significant increase of IMT or calcified plaque. Our results provide further evidence for a relationship between calcium/vitamin D in osteoporosis women and the atherosclerotic risk for cardiovascular disease.

\section{P803}

BMP2/4 ARE KEY REGULATORS OF ADULT SKELETAL HOMEOSTASIS AND PREREQUISITE FOR BONE ANABOLIC ACTION OF PTH

M. P. Khan ${ }^{1}$, K. Khan ${ }^{1}$, P. S. Yadav ${ }^{2}$, A. K. Singh ${ }^{3}$, A. $\mathrm{Nag}^{2}$, A. Bandyopadhyay ${ }^{2}$, N. Chattopadhyay ${ }^{1}$

${ }^{1}$ Division of Endocrinology, CSIR-Central Drug Research Institute, Lucknow, India, ${ }^{2}$ Department of Biological
Sciences and Bioengineering, Indian Institute of Technology, Kanpur, India, ${ }^{3}$ Division of Biochemistry CSIR-Central Drug Research Institute, Lucknow, India

Simultaneous ablation of Bmp2 and Bmp4 during embryonic development resulted in absolute abrogation of osteogenesis and severely malformed bones. Interestingly, although depletion of Bmp2 alone does not upset embryonic skeletal development, these mice grow up to suffer from osteopenia, frequent fractures and reduced fracture healing in adult life. Thus suggesting that BMP $2 / 4$ are indispensable for bone development. However, the role of these two BMPs in maintaining the homeostasis of adult bone is unknown. We used a tamoxifen inducible system to conditionally and simultaneous knockout Bmp2 and Bmp4 genes in adult mice $(\mathrm{KO})$ and compared these with the age matched mice having intact Bmp2 and Bmp4 expression $(\mathrm{Wt})$. Bones were evaluated by $\mu \mathrm{CT}$ to determine volumetric BMD and microstructure, bone strength tester to vertebral compression strength, histomorphometry to bone remodelling and serum levels of carboxyterminal collagen crosslinks, tartrateresistant acid phosphatase and N-terminal propeptide of type I procollagen to bone turnover. Drill-hole was made in femur metaphysis and bone regeneration in the site was measured by calcein binding and $\mu \mathrm{CT}$. Mice were either sham operated (ovary intact) or ovariectomized (OVX), and treated with PTH, 17 $\beta$ estradiol (E2) or vehicle. KO mice displayed trabecular bone loss, diminished osteoid formation and biomechanical strength compared with Wt. Both osteoblast and osteoclast functions were impaired in $\mathrm{KO}$ mice. Bone histomorphometry and serum parameters established a low turnover bone loss in $\mathrm{KO}$ mice. Bone regeneration at the fracture site in $\mathrm{KO}$ mice was lower than Wt. PTH and E2 both resulted in skeletal preservation in Wt-OVX, whereas in $\mathrm{KO}$ OVX, E2 but not PTH was effective suggesting that the skeletal action of PTH required Bmp2/4 ligands but E2 did not. To determine cellular effects of Bmp2/4, bone marrow stromal cell cultures (BMSCs) were used where KO mice BMSCs showed attenuated mineralization compared with Wt. In osteoblasts, PTH but not E2 stimulated Bmp2/4 synthesis and secretion leading to increased Smad1/5 phosphorylation. Taken together, we conclude that $\mathrm{Bmp} 2 / 4$ are indispensable for maintaining adult skeletal homeostasis and anabolic action of PTH. In future, it would be interesting to determine whether Bmp2/4 mediates the suppression effect of PTH, sclerostin secretion from osteocytes and induction effect of this hormone on bone lining cells. 


\section{P804}

THE RELATIONSHIP BETWEEN BONE PARAMETERS AND LOW SKELETAL MUSCLE MASS IN POSTMENOPAUSAL WOMEN

A. Wongsuttilert $^{1}$, K. Chaiklongkit ${ }^{2}$, W. Jaidee ${ }^{3}$

${ }^{1}$ Faculty of Medicine, Burapha University, Chonburi, Thailand, ${ }^{2}$ Faculty of Sport Science, Burapha University, Chonburi, Thailand, ${ }^{3}$ Faculty of Public Health, Burapha University, Chonburi, Thailand

Objective: We evaluated the association between the components of bone mass density and skeletal muscle mass in postmenopausal women.

Material and Methods: 356 postmenopausal women aged 5195 years were examined. BMD and body composition were assessed by DXA. The relative skeletal muscle index (RSMI) was calculated as appendicular muscle mass $(\mathrm{kg}) /$ square of height (m). The study sample was divided into two categories according to the Asian Working Group for Sarcopenia (AWGS): normal and low muscle mass $\left(\mathrm{RSMI}<5.4 \mathrm{~kg} / \mathrm{m}^{2}\right)^{1}$. Linear regression was used to determine the associations between parameters of bone mass density and skeletal muscle mass.

Results: The prevalence of low muscle mass was $23.3 \%$. Osteoporosis was higher in women with low muscle mass than in the normal group (69.7\% and $44.3 \%$, respectively). They had significantly lower BMD at distal one-third radius, femoral neck and total hip. Similarly, the most of them had lower adiposity (96.1\%). In the multiple regression analysis, lean mass of arms, after adjustments to BMI and RSMI, was able to predict BMD of distal one-third radius $\left(\mathrm{R}^{2}=24 \%\right)$. However, lean mass of legs adjusted to BMI and RSMI was unable to predict femoral neck BMD $\left(\mathrm{R}^{2}=11 \%\right)$ and total hip $\operatorname{BMD}\left(\mathrm{R}^{2}=17 \%\right)$.

Conclusions: In postmenopausal women, low skeletal muscle mass is strongly associated with osteoporosis. Lean mass of the upper limbs is associated to their BMD but lean mass of the lower limbs is not. This discordant result is a key question for future research.

References:

1. Chen LK, et al. J Am Med Dir Assoc 2014;15:95.

\section{P805}

CONSEQUENCES OF FUNCTIONAL AND MENTAL LIMITATIONS IN STILL INDEPENDENT ELDERLY M. Farago ${ }^{1}$, S. Pop ${ }^{1}$, F. Cioara ${ }^{1}$

${ }^{1}$ University of Oradea, Faculty of Medicine and Pharmacy, Oradea, Romania

Background: The state of health in older people is of vital importance. Becoming mature, the bodies start to lose muscle strength and mass, through bone loss the skeleton's mechanical strength reduces, the needed time to repair injured tissues become longer. With increasing age there is a decline in function. The aim of this study was to assess the elderly fitness capacity in correlation with quality of life in order to identify priority needs and interventions for individuals.

Methods: The selected subjects $(n=29)$, mostly women $(n=19)$, mean age 72.3 years, medium educated, hospital outpatients, agreed to complete "The American Alliance for Health, Physical Education, Recreation \& Dance (AAHPERD) Functional Fitness Test", "Mini-Cog Test" for mental state examination, and a 6 items "Quality of Life Test" (mood, walk, housework, human relationships, sleep, availability for social activities). The AAHPERD Functional Fitness Test items designed for low fitness elderly who are not yet frail were: Ponderal Index, Sit and Reach Test, Agility Test, Soda Pop Test, 800 Meter Walking Test. Prior to anterior testing, a detailed clinical examination including muscle-skeletal pain, joint range of motion and walk was performed. For testing counter-indications, concurrent illnesses were taken into account.

Results: All patients had mild to medium muscle-skeletal pain (back and lower limb joints). Walk was possible in $93.1 \%$ patients without assistive device. AAHPERD Test results: $55.97 \%$ higher level of body fat; weak to medium for flexibility test (Sit and Reach Test); medium to good for hand coordination test (Soda Pop Test); medium for agility test; and weak results in 19 patients $(65.51 \%)$ for 800 Meter Test and 3 patients had to stop the test due to higher heart rate. The mostly affected items in quality of life were: mood, sleep, human relationships, and availability for social activities.

Conclusions: In the presence of muscle-skeletal pain, of good cognitive function and coordination, the study group presents a low level of agility and aerobic fitness that reflects in the mostly affected items of quality of life. The arising needs for such individuals are muscle-skeletal and cardiovascular reeducation in conjunction with efforts to increase the level of socialization and maintain the cognitive function. Arise needs for prophylactic programs and investments for elderly in order to keep them mental and functional healthy and independent.

\section{P806}

IDENTIFICATION OF NEUROPATHIC PAIN COMPONENT IN PATIENTS OF VARIOUS AGE WITH KNEE OSTEOARTHRITIS

\section{Povoroznyuk ${ }^{1}$, U. Pryimych ${ }^{1}$}

${ }^{1}$ Department of Clinical Physiology and Pathology of Locomotor Apparatus, D.F. Chebotarev Institute of Gerontology NAMS Ukraine, Kyiv, Ukraine

Introduction: Osteoarthritis-induced pain is a result of nociceptor stimulation, associated with local tissue damage and inflammation. Resent data suggest the presence of neuropathic pain symptoms in patients with osteoarthritis. 
The aim of this study was to estimate the structure of pain syndrome, reveal the presence of neuropathic pain (NP) component, symptoms and signs of NP in patients suffering from knee osteoarthritis.

Material and Methods: We examined 44 patients with knee osteoarthritis of the II-III stages by the KellgrenLawrence scale aged 47-85 years (average age 66.1 \pm 1.5 years). Patients were divided according to age into next groups: 47-60, 61-74, 75-85 years. To assess the NP component, we used screening scales painDETECT, LANSS, DN4 questionnaires. To assess intensity of pain, visual analogue scale (VAS) was used. Besides WOMAC and EuroQol-5D questionnaire were applied. For statistical analysis of results, ANOVA, correlation and regression analysis, chi-square $\left(\chi^{2}\right)$ were applied.

Results: $72.7 \%$ of patients with knee osteoarthritis examined by painDETECT were unlikely to have the NP component, $22.7 \%$ might possibly, $4.6 \%$ probably. LANSS scale: $25 \%$ were probably to have NP. DN4 scale: $31.2 \%$ probably had NP. Moderate to significant correlations were found between intensity of pain by VAS data and neuropathic pain scales (painDETECT, LANSS, DN4) data $(p<0.05)$. It was established than higher results of screening by painDETECT and DN4 positively correlate with a disturbance of physical function tested by WOMAC $(p<0.05)$. PainDETECT data have moderate to significant correlations with EuroQol$5 \mathrm{D}$ questionnaire $(p<0.01)$. Verbal descriptors as pins and needles, tingling, numbness and allodynia, pain from light touch which are revealed by 3 screening scales can significantly contribute to the likely neuropathic component in patients with knee osteoarthritis $(p<0.05)$. Burning pain $(p<0.01)$, pins and needles $(p<0.05)$ can be associated with a more severe pain in patients with knee osteoarthritis.

Conclusion: Thus, in patients with osteoarthritis the pain syndrome may reveal NP features. Identification of these would promote a targeted treatment strategy.

\section{P807 \\ NEUROPATHIC PAIN COMPONENT IN PATIENTS WITH OSTEOPOROSIS AND LOW BACK PAIN}

V. Povoroznyuk $^{1}$, T. Shinkarenko ${ }^{1}$, U. Pryimych ${ }^{1}$

${ }^{1}$ Department of Clinical Physiology and Pathology of Locomotor Apparatus, D.F. Chebotarev Institute of Gerontology NAMS Ukraine, Kyiv, Ukraine

Introduction: Neuropathic pain caused by the musculoskeletal diseases has recently been the focus of numerous studies. The aim of this study was to estimate the structure of pain syndrome and reveal the presence of neuropathic pain component in patients suffering from the osteoporosis and low back pain.
Material and Methods: We've examined 107 patients aged 45-89 years (average age 68.1 \pm 1.2 years). Patients were divided into 2 groups: A - patients with osteoporosis $(n=49), \mathrm{B}$ - patients with low back pain $(n=58)$. And according to age into next groups: $45-60,61-74,75-89$ years. To assess the NP component, we used painDETECT, LANSS, DN4 questionnaires. To assess intensity of pain, visual analogue scale (VAS) was used. Patients completed Oswestry and RollandMorris Disability Questionnaires. For statistical analysis of results, ANOVA, correlation and regression analysis were applied.

Results: Regression analysis shows correlation between the questionnaires: LANSS and painDETECT $(r=0.74$, $p<0.001)$, DN4 and painDETECT $(r=0.8, p<0.001)$. It was found correlation between the visual analogue scale (VAS) and screening scales of neuropathic pain: painDETECT and VAS $(r=0.4 ; p<0.001)$. LANSS and VAS $(r=0.3 p<0.001)$, DN4 and VAS $(r=0.3$; $p<0.001) .79 .6 \% \%$ of patients with osteoporosis examined by painDETECT were unlikely to have the NP component, $14.3 \%$ might possibly, $6.1 \%$ - probably. LANSS scale: $14.3 \%$ were probably to have NP. DN4 scale: $24.5 \%$ probably had NP. $58.7 \%$ of patients with low back pain examined by painDETECT were unlikely to have NP, $24.1 \%$ might possibly, $17.2 \%$ - probably. LANSS scale: $24.1 \%$ were probably to have NP. DN4 scale: $44.8 \%$ had probably NP. In patients with low back pain it was found significant correlation between intensity of pain measured by VAS and Oswestry Disability Index $(r=0.7, p<0.001)$; between VAS and Rolland-Morris Disability Questionnaire $(r=0.6$, $p<0.001)$. Significant correlations were found between Oswestry Disability Index and painDETECT screening scale data $(r=0.4, p<0.05)$.

Conclusion: In patients with osteoporosis and low back pain the pain syndrome may include NP features. Identification of these would promote a treatment strategy targeted at the NP.

\section{P808}

LOWER LIMB OSTEOARTHRITIS DOES NOT IMPEDE PHYSICAL ACTIVITY AT LOW IMPACTS: THE HERTFORDSHIRE COHORT STUDY

C. L. $\operatorname{Moss}^{1}$, M. H. Edwards ${ }^{1}$, K. A. Jameson ${ }^{1}$, K. J.

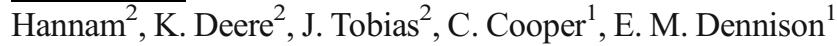
${ }^{1}$ MRC Lifecourse Epidemiology Unit, Southampton, United Kingdom, ${ }^{2}$ University of Bristol, Bristol, United Kingdom

Background: Osteoarthritis (OA) is a common disease, affecting 7.3 million people over the age of 45, in England. The disease can negatively impact levels of physical activity, although current advice promotes staying active in 
preventing joint degeneration. We report physical activity levels in a cohort of older adults where OA status is defined. Methods: The study population comprised 69 men and 45 women from the Hertfordshire Cohort Study. Participants had data on osteoarthritis, at knee and hip sites, collected from interviews and clinical examination. GCDC tri-axial accelerometers, worn on the hip, were used to measure physical activity. The accelerometers were programmed to record positive accelerations, expressed in $\mathrm{g}$ units, these results were categorised into three bands $(\geq 0.5 \mathrm{~g}-<1.0 \mathrm{~g},>-1.0 \mathrm{~g}$ $<1.5 \mathrm{~g}$ and $\geq 1.5 \mathrm{~g}$ ) selected from the distribution of positive Y-axis peak accelerations.

Results: The mean (SD) age was 75.3 (2.6) years in men and 75.4 (2.6) years in women. The mean (SD) BMI $(\mathrm{kg} / \mathrm{m} 2)$ in men was 27.3 (4.3) and 27.3 (3.8) in women. Self-report osteoarthritis data was available on all participants; 87 (76.3\%) reported no knee or hip OA, $16.7 \%$ reported knee OA only, $5.3 \%$ hip OA only and $1.8 \%$ of participants reported to suffer both. 110 participants underwent examination and $86.4 \%$ were found to have no clinical knee or hip OA. Twelve participants $(10.9 \%)$ had clinical knee OA only, $2.7 \%$ clinical hip OA only and no participants suffered both hip and knee osteoarthritis. Osteoarthritis status was used as explanatory variables for the frequency of Y-peaks across the different bandings of counts. No associations were found between osteoarthritis status and levels of physical activity, before and after adjustment for age and BMI. This was true across selfreported and clinical definitions of $\mathrm{OA}$ and in all three bandings of accelerometry counts.

Conclusions: In this small sample we observed no association between lower limb OA and low impact physical activity levels in a cohort of older adults.

\section{P809 \\ BONE MINERAL DENSITY IN A COHORT OF EARLY RHEUMATOID ARTHRITIS PATIENTS \\ $\underline{\text { A. L. Barbulescu }}^{1}$, F. A. Vreju ${ }^{1}$, C. Criveanu ${ }^{1}$, C. Mitran ${ }^{1}$, P. L. Ciurea ${ }^{1}$ \\ ${ }^{1}$ University of Medicine and Pharmacy, Craiova, Romania}

Objectives: To assess the BMD in a cohort of patients with early rheumatoid arthritis and establish the possible correlations with synovial inflammation and disease activity score.

Material and Methods: We enrolled 29 patients diagnosed with early RA, according to ACR-EULAR 2010 classification criteria. The presence of synovitis, by ultrasonography, was assessed both in grey scale (GSUS) and power-Doppler (PDUS), according to OMERACT-EULAR consent. BMD measurement of the femoral neck, spine and non-dominant wrist was performed using DXA.

Results: Our cohort included 29 consecutive patients, diagnosed with early rheumatoid arthritis, with a duration of the symptoms under 1 year, $25(86.20 \%)$ women and 4 men, with a mean age of $48.97 \pm 9.72$ years. RF was present in $72.41 \%$ of the cases (21) and anti-CCP antibodies in 58.62\% (17 cases). Disease activity registered a mean value of 5.11 \pm 0.61 and the disability index (HAQ) had a mean value of $1.31 \pm 0.53$. Assessment of synovial inflammatory activity, using both grey scale and PDUS, has shown active synovitis in all patients with a mean number of joints with active synovitis of 5; evaluating the grade, using PDUS, we found grade I in all patients, II in 14 and grade 3 in 3 patients. Using DXA we found the presence of osteoporosis in 11 patients, osteopenia in 15 and a normal BMD for 3 patients. Disease activity correlated significant with both wrist and lumbar spine BMD, with a Pearson correlation coefficient of $-0.598, p=0.0006$ for wrist BMD/GSUS; -0.5917 , $p=0.0007$ for wrist BMD/PDUS; $r=-0.453$ for lumbar spine $\mathrm{BMD} / \mathrm{GSUS}$ and $r=-0.428$ for wrist BMD/PDUS. There was a significant negative correlation between wrist and lumbar spine BMD and active synovitis $(r=-0.487, p=0.020$ for wrist BMD and $r=-0.402, p=0.02$ for lumbar spine BMD); also, we noticed a moderate negative correlation between ACPA antibodies and wrist BMD.

Conclusions: our results show the presence of low BMD in early RA patients and a significant correlation between BMD and active synovitis, disease activity score and ACPA.

\section{P810}

A COMPARATIVE STUDY OF THE EFFECTS OF LATERAL WEDGE INSOLE SHOE MODIFICATION ON THE PATIENTS WITH KNEE OSTEOARTHRITIS T. I. Khan ${ }^{1}$, B. Ahmed ${ }^{2}$

${ }^{1}$ Rajshahi Medical College Hospital, Rajshahi, Bangladesh,

${ }^{2}$ Department of Physical Medicine Bangabandhu Sheikh Mujib Medical University, Dhaka, Bangladesh

Introduction: Knee osteoarthritis (OA) is the common form of disability in comparatively elderly patient. Lateral wedge shoe insole is an easy, simple and cost effective approach which can be applied as an adjunct to pharmacotherapy to treat the patients with OA knee.

Objective: To evaluate the effectiveness of using lateral wedge shoe insole on pain and physical functioning in patients with OA knee.

Method: A randomized prospective study was carried out in the department of Physical Medicine and Rehabilitation, Bangabandhu Sheikh Mujib Medical University(BSMMU), Dhaka, Bangladesh from January, 2012 to March,2012. Patients with OA knee by American College of Rheumatology Criteria were selected. Two intervention groups were compared. In patients of Group-A (32 patients) were treated with Tab.aceclofenac $(100 \mathrm{mg})$ bid, Cap.omeprazole $(20 \mathrm{mg})$ and daily instructions of activities of daily living(ADLs), 
isometric quadriceps muscle strengthening exercise. Patients of Group-B were given lateral wedge shoe insole along with above treatment. The change between two weeks post intervention and baseline WOMAC (Western Ontario and Mc Master Universities) subscale and scores were calculated. There were 4 visits and in each visit, patients were assessed for pain, stiffness and physical function by WOMAC index.

Result: A total 65 patients with OA knee were included in this study. The mean age was $56.5 \pm 10$ years. Male to female ratio 1.32:1. Comparison of mean pretreatment and 8th week post treatment WOMAC physical function subscale score in Group A $(4.9 \pm 1.2$ vs. $2.4 \pm 0.8)$ showed significant improvement and in Group B $(4.3 \pm 1.2$ vs. $1.9 \pm 0.5)$ which also shows significant improvement more than group A. The result was compared and student t-test was done to see the level of significance. Method was found significant after treatment $(p<0.05)$.

Conclusion: Effects of use of lateral wedge shoe insole in patients with knee OA is beneficial.

\section{P811}

OSTEONECROSIS OF THE JAW IN PATIENTS WITH OSTEOPOROSIS RECEIVING ORAL BISPHOSPHONATES

C. Dalai ${ }^{1}$, G. Ciavoi ${ }^{1}$, R. Suciu ${ }^{1}$, F. Cioara ${ }^{1}$, N. Pascalau ${ }^{1}$

${ }^{1}$ University of Oradea, Faculty of Medicine and Pharmacy, Oradea, Romania

Objectives: Determining the incidence and risk factors for the occurrence of osteonecrosis of the jaw in patients with osteoporosis treated with oral bisphosphonates.

Material and Methods: We have studied a number of 60 patients diagnosed with osteoporosis and treated with oral bisphosphonates (alendronate and risedronate). Of the $60 \mathrm{pa}-$ tients taken into study, 15 patients have presented osteonecrosis of mandible and 6 patients have experienced jaw osteonecrosis. Osteonecrosis diagnosis was based on panoramic imaging made during routine dental assessments, or for different interventions.

Results: The average of women was 61.5 years. Average duration of treatment with oral bisphosphonates was 3 years old. $76.19 \%$ of patients with osteonecrosis of mandible had a history of alveolar-dental surgery procedures and $42.85 \%$ were smokers.

Conclusions: Before initiating treatment with oral bisphosphonates it would be good to do a dental checkup and a rigorous amamnesis relating to the procedures for the dento-alveolar surgery suffered by patients in history. Better collaboration between practitioners and those who prescribe the treatment for osteoporosis would be desirable, and last but not least the avoidance of smoking, a proper dental hygiene and dental checkup at regular intervals.

\section{P812}

LIGAMENTUM TERES RUPTURE IN PATIENTS WITH FEMOROACETABULAR IMPINGEMENT

$\underline{\text { R. Santos-Pereira }}^{1}$, C. Aleixo ${ }^{1}$, F. Lima-Santos ${ }^{1}$, M. Frias ${ }^{1}$, T. Pinheiro-Torres $^{1}$, D. Sá ${ }^{1}$, A. Sarmento ${ }^{1}$

${ }^{1}$ Centro Hospitalar Vila Nova de Gaia / Espinho, Vila Nova de Gaia, Portugal

Objectives: The importance of the ligamentum teres in hip biomechanics and function has been a subject of debate and controversy for decades. In recent years, the exponential increase and application of hip arthroscopy has allowed for an increased reported incidence of ligamentum teres ruptures (diagnosed in up to $9 \%$ of hip arthroscopies). This has fueled a renewed interest in the ligamentum teres as a source of intra-articular hip pain. Although there are only a few well designed studies regarding the subject, surgical debridement has emerged in recent years as the gold standard for the treatment of symptomatic ligamentum teres ruptures, as the role of ligament reconstruction is still not well defined. The aim of the present study is to evaluate the role of concomitant treatment of partial ligamentum teres ruptures with debridement in patients treated with hip arthroscopy for femoroacetabular impingement (FAI)

Methods: All 54 patients diagnosed with mixed type FAI and labral tears treated arthroscopically in our institution between January 2012 and June 2014 were identified and included. The arthroscopic procedure consisted of femur osteochondroplasty, acetabular rim trimming and labral refixation with PEEK anchors in all patients. In addition, arthroscopic ligamentum teres debridement was performed in the patients in whom ligamentum teres ruptures where identified in during central compartment visualization (9 patients). All arthroscopic procedures were performed by the same two senior surgeons and all patients followed the same postoperative rehabilitation protocol and were evaluated with Visual analogue scale (VAS), and Modified Harris Hip Score (mHHS) at 3, 6 and 12 months postoperatively. There were no significant differences between the two groups regarding age, gender or pre-operative cartilage damage.

Results: Regarding the whole study population, the mean post-operative VAS and mHHs showed statistically significant improvement within all periods evaluated $(p<0.05)$. Patients treated for ligamentum teres ruptures as well as FAI showed significantly better outcomes (mean difference in VAS 6.4 points and mHHS 52.17 points) when compared to those treated for isolated FAI (mean difference in VAS 5.4 points and mHHS 43.67 points).

Conclusions: Many prior studies have shown that arthroscopic treatment of mixed type FAI produces consistently good results. In these patients, ligamentum teres ruptures may be an associated cause of hip pain and should be thoroughly 
evaluated during hip arthroscopy because surgical debridement seems to be related with better medium term outcomes.

\section{P813}

EVALUATION OF LOW-ENERGY FOREARM FRACTURES OF CHILDREN WITH BONE MINERAL DENSITY A PROSPECTIVE STUDY

B.-B. $\mathrm{Cakmak}^{1}$, E. $\mathrm{Ercin}^{2}$, C. Kural ${ }^{2}$, M. C. Avkan' ${ }^{2}$, K.-M. $\mathrm{Ceren}^{2}$, S.-Y. Kazanc1 ${ }^{3}$, S. Hatipoglu ${ }^{3}$, M. Vural ${ }^{1}$, B. Kural ${ }^{3}$, F. Y. Aydin ${ }^{1}$

${ }^{1}$ Department of Physical Medicine and Rehabilitation, Bakirköy Dr. Sadi Konuk Training and Research Hospital, Istanbul, Turkey, ${ }^{2}$ Department of Orthopedics and Traumatology, Bakirköy Dr. Sadi Konuk Training and Research Hospital, Istanbul, Turkey, ${ }^{3}$ Department of Adolescent Medicine, Pediatrics, Bakirköy Dr. Sadi Konuk Training and Research Hospital, Istanbul, Turkey

Objective: To evaluate the risk factors associated with lowenergy trauma related (child falls from a height less than his length) forearm fractures of children. BMD, children's diet, the frequency of falls and osteoporosis in the family studied as risk factors.

Patients and Method: The study conducted in Bakirkoy Dr. Sadi Konuk Training and Research Hospital between November 2014 and January 2016, 107 patients age between 7-14 (mean age: $11.03 \pm 2.28$ ) with low-energy related forearm fractures who admitted to the Orthopedics and Traumatology Clinic included to the study. Healthy 51 patients who admitted to the Pediatrics Clinic used as a control group. In both groups, a total of 158 patients $(17.7 \%(n=28)$ boy, $82.3 \%(n=130)$ girl) were evaluated by the same physician of Physical Therapy and Rehabilitation Clinic. A standard questionnaire with queries; gestational age, maternal smoking during pregnancy, medication usage, intermarriage, the presence of breastfeeding, consumption of calcium rich diet, protein and sodium-rich diet, sedentary life, frequency of falling, were used in the evaluation. DXA used to determine osteoporosis and BMD (Lunar Prodigy 2012, GE Medical Systems, Wisconsin USA). Z-score below -2.0 were signed as 'low bone density for chronologic age' or 'low bone density for this age'. Ethical approval obtained before the study with number: 2014/261. Demographic characteristics of the patients in the study group and the control group were similar $(p>0.05)$. The frequency of falling significantly differed between groups $(p=0.031)$. There was no significant difference between other evaluated factors $(p>0.05)$. Other characteristics did not differ significantly $(p>0.05)$. There was no statistically significant difference between study and control groups regarding DXA Z-, BMD, BMC scores and percentages $(p>0.05)$.
Conclusion: BMD values of patients who admitted with lowenergy related forearm fractures are in expected ranges. Patients who had frequent falling problems should be monitored closely for the risk of forearm fracture. Balance exercises might help in preventing falls in this age.

\section{P814}

QUALITY OF OSTEOPOROSIS CARE IN AN IRANIAN POPULATION

P. Khashayar ${ }^{1}$, M. Ebrahimi ${ }^{2}$, H. Ebrahimi ${ }^{3}$, M. Raji ${ }^{2}$, M.

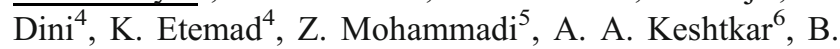
Larijani $^{7}$

${ }^{1}$ Orc/EMRI/TUMS-CMST/UGENT, Tehran, Islamic Republic of Iran, ${ }^{2}$ Orc/EMRI/TUMS, Tehran, Islamic Republic of Iran, ${ }^{3} \mathrm{Orc} /$ EMRI/TUMS, Shahroud, Islamic Republic of Iran, ${ }^{4} \mathrm{MoHME}$, Tehran, Islamic Republic of Iran, ${ }^{5}$ Ddri/TUMS, Tehran, Islamic Republic of Iran, ${ }^{6} \mathrm{Tums}$, Tehran, Islamic Republic of Iran, ${ }^{7}$ Endocrinology and Metabolism Research Center, Dr Shariati Hospital, Tehran University of Medical Sciences, Tehran, Islamic Republic of Iran

Background: While osteoporosis is becoming a health concern in Iran, the quality of care provided to osteoporotic patients has never been assessed in our country. The present study was therefore conducted to measure the quality of care provided to osteoporosis patients in outpatient clinics to identify the osteoporosis care gap.

Methods: In the present cross-sectional study, a questionnaire was filled out for all the patients diagnosed with osteoporosis or osteoporotic fracture, visiting outpatient osteoporosis clinics in two Iranian cities.

The questionnaire was composed of seven main parts:

- Demographic Information (age, gender, educational status, living place)

- Bone health-related lifestyle habits (diet (calcium and vitamin D intake), physical activity, sun exposure, smoking, medication history)

- Osteoporosis risk factors (menopause, corticosteroid use, personal and family history of related disease (hyperparathyroidism, hypogonadism), history of osteoporosis/ osteoporotic fracture in first degree relatives, personal history of fragility fracture)

- Knowledge on osteoporosis (related complications, risk factors, protective factors)

- BMD status (BMD values at femoral neck and lumbar spine)

- Quality of care (Interval of BMD measurements, osteoporosis diagnosis criteria, prescribed supplements, recommended diet and other lifestyle changes, prescribed medications, follow-up visits, etc.)

Results: 232 osteoporotic patients and 252 individuals with fractures were studied. Females accounted for $86.2 \%$ and $61.9 \%$ of 
each group, respectively. Mean age of the individuals in the two groups was $63.6 \pm 15.4$ years. In the osteoporosis group, the initial diagnosis was made based on BMD reports in 61 (26.3\%) patients. The diagnosis was made by a specialist in only $64(27.5 \%)$ patients. The initial diagnosis of the specialist was compatible with the BMD results in $104(55.9 \%)$ of the studied individuals (Cohen's kappa coefficient $=0.14$ ). The sensitivity and specificity of the specialist's diagnosis (with BMD being the gold standard) was 32.7 (CI95\%: 23.5-42.9) and 81.8 (CI95\%: 72.2-89.2), correspondingly. From among the 64 patients diagnosed by a specialist, $50(78.1 \%)$ reported a good compliance with treatment. As for those confirmed to have osteoporosis based on BMD results, about $30(85.7 \%)$ had good treatment compliance. In the osteoporotic fracture group, only 168 (66.6\%) were evaluated for osteoporosis. In 64 of them, however, the evaluation was performed upon the patient's request.

Conclusion: Only $27 \%$ of the Iranians seem to have the chance of getting appropriate osteoporosis care. From among them $21 \%$ have a good compliance for treatment. At the same time, only $16 \%$ of patients with fractures were evaluated for osteoporosis.

\section{P815}

\section{TREAT-TO-TARGET IN OSTEOPOROSIS. SPANISH EXPERT CONSENSUS}

E. Jódar $^{1}$, E. Casado ${ }^{2}$, X. Nogués ${ }^{3}$, J.-M. Nolla ${ }^{4}$, M. MuñozTorres $^{5}$, J.-M. Quesada-Gómez ${ }^{6}$, L. Canals ${ }^{7}$, M. Balcells ${ }^{7}$, L. Lizán $^{8}$

${ }^{1}$ University Hospital Quirón, Madrid, Spain, ${ }^{2}$ University Hospital Parc Taulí, Sabadell, Spain, ${ }^{3}$ Mar Institute of Medical Research (IMIM), Barcelona, Spain, ${ }^{4}$ University Hospital Bellvitge, L'Hospitalet de Llobregat, Spain, ${ }^{5}$ University Hospital San Cecilio, Granada, Spain, ${ }^{6}$ University Hospital Reina Sofía, IMIBIC, RETICEF, Córdoba, Spain, ${ }^{7}$ Amgen, Barcelona, Spain, ${ }^{8}$ Outcomes'10, University Jaume I, Castellón, Spain

Objective: To reach a Spanish expert consensus on the treatto-target (T2T) strategy in osteoporosis.

Material and methods: A scientific committee comprised of 6 experts in the field of osteoporosis (3 endocrinologists, 2 rheumatologists and 1 medical internist) led the project. A 2-round Delphi was completed. The 24 items included in the 1 st round questionnaire assessed the experts' wish (W) and prognosis (P) for each item to occur in 5-year time, in a 7-point Likert scale $(1=$ entirely disagree; 7 =entirely agree). The 2 nd round included all items without previous consensus. Consensus was established if at least $75 \%$ of the participants reached agreement (5-7) or disagreement (1-3).

Results: 112 experts completed the 1 st round (67.5\%) and 106 $(94.6 \%)$ the 2 nd round. Most of experts (60\%) were rheumatologists with a mean of 21.3 years (SD: 8.5 ) of clinical experience. Consensus was achieved on $70 \%$ of the items raised. There was a consensus (W: $96.4 \%$; P: $82.1 \%$ ) on establishing a T2T strategy in osteoporosis in order to have a well defined objective, improve the monitoring of patients and follow an established therapeutic algorithm. Experts agreed that the main therapeutic objectives were the absence of new fractures (W: $99.1 \%$; P: $97.3 \%$ ) and a significant gain in BMD (W: $91.9 \%$; P: $91.1 \%$ ). In regards to the monitoring of treated patients, consensus was achieved that all locations proposed [lumbar spine (W: $96.4 \%$; P: $96.4 \%$ ), femoral neck (W: $99.1 \%$; P: $98.2 \%$ ), and total hip (W: $92.0 \%$; P: $92.0 \%$ )], were suitable for measuring BMD changes. Experts also agreed in considering "treatment failure" when a significant BMD gain is not achieved in 2 (W: $81.3 \%$; P: $82.1 \%$ ) or 3 years (W: $77.7 \%$; P: $75.9 \%$ ) or when a new fracture occur during the first 2 (W: $92.0 \%$; P: $92.0 \%$ ) or 3 years (W: $90.2 \%$; P: $88.4 \%$ ) of treatment.

Conclusion: A T2T strategy in osteoporosis can be implemented in Spain, as therapeutic objectives, patient follow-up scheme and treatment failure criteria have been defined by an expert consensus.

Disclosures: Study was funded by Amgen.

\section{P816}

A CROSS-SECTIONAL CASE-CONTROL STUDY OF SCLEROSTIN SERUM LEVELS IN PATIENTS WITH SYSTEMIC AUTOIMMUNE DISEASES

C. Fernández-Roldán $^{1}$, F. Genre ${ }^{2}$, M. Correa-Rodriguez ${ }^{3}$, B. Rueda-Medina $^{3}$, R. López-Mejías ${ }^{2}$, B. Ubilla ${ }^{2}$, V. Mijares ${ }^{2}$, D. Sánchez-Cano $^{1}$, C. López-Robles ${ }^{1}$, J.-L. Callejas-Rubio ${ }^{1}$, R. Ríos-Fernández ${ }^{1}$, M. Expósito-Ruiz ${ }^{4}$, M.-A. González-Gay ${ }^{2}$, N. Ortego-Centeno ${ }^{1}$

${ }^{1}$ Unidad de Enfermedades Autoinmunes y Sistémicas, Hospital Universitario San Cecilio, Granada, Spain, ${ }^{2}$ Laboratorio de Epidemiología Genética y Arteriosclerosis en Enfermedades Inflamatorias Sistémicas, IDIVAL, Santander, Spain, ${ }^{3}$ Department of Nursing, University of Granada, Granada, Spain, ${ }^{4}$ FIBAO, (Fundación Pública Andaluza para la Investigación Biosanitaria de Andalucía Oriental - Alejandro Otero), Granada, Spain

Introduction: Systemic autoimmune diseases (SAD) are associated with lower bone mass and an increased risk of fractures. Although sclerostin plays a main role in the regulation of bone metabolism by blocking the Wnt signaling pathway in osteoblasts, little is known about circulating sclerostin levels in patients with SAD. Therefore, we aimed to investigate circulating sclerostin concentrations in systemic lupus erythematosus (SLE), systemic sclerosis (SSc) and Crohn's disease (CD) patients, and to analyze the factors associated with sclerostin concentrations.

Methods: We have performed a descriptive cross-sectional case control study, which included 66 patients who had been diagnosed with SLE $(n=38), \operatorname{SSc}(n=8)$ or CD $(n=20)$, and 20 healthy controls from the Andalusian Public Health's System's Biobank whose mean age was 44 years. Sclerostin 
serum levels were determined by a commercial enzymelinked immunoassay (sclerostin ELISA).

Results: The mean values of the sclerostin $(95 \% \mathrm{CI})$ were: 35.36 $\mathrm{pmol} / \mathrm{L}(12-101)$ in patients and $33.92 \mathrm{pmol} / \mathrm{L}(2.31-100)$ in control subjects. The mean sclerostin value was $36.4 \mathrm{pmol} / \mathrm{L}$ (22.1-48.5) in SLE patients, $26.7 \mathrm{pmol} / \mathrm{L}(17.3-36.3)$ in CD patients and $51.8 \mathrm{pmol} / \mathrm{L}(26.5-77.1)$ in SSc patients $(p=0.001)$. Serum sclerostin levels were positively correlated with age $(p<0.001)$, BMI $(p=0.01)$ and lumbar spine Z-score $(p=0.001)$, and negatively with creatinine clearance $(p=0.001)$. Glucocorticoid treatment did not affect sclerostin levels.

Conclusions: We have demonstrated that in different SAD sclerostin levels seem to have a heterogeneous pattern. Serum sclerostin levels in SLE and SSc are very similar to that observed in healthy controls. The CD group had significantly lower values compared with systemic sclerosis patients. In addition, in SAD the factors associated with sclerostin levels seem to be the same than in healthy subjects.

\section{P817}

PARTIAL GLUTEUS TENDON RUPTURE - RESULTS FOLLOWING ENDOSCOPIC TREATMENT

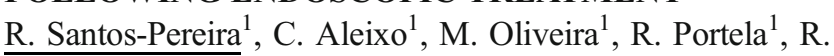
Grazina $^{1}$, M. Bernardes ${ }^{1}$, D. Sá ${ }^{1}$, A. Sarmento ${ }^{1}$

${ }^{1}$ Centro Hospitalar Vila Nova de Gaia / Espinho, Vila Nova de Gaia, Portugal

Objectives: Greater trochanteric pain syndrome (GTPS) is clinically defined as greater trochanter pain with mechanical characteristics. GTPS is a common pathology that affects mostly female patients. Most cases can be managed with conservative treatment but refractory cases may be candidates for operative treatment. With technological progress and advances in hip arthroscopy technique, favorable results have been described with extracapsular endoscopic procedures. In recent years, gluteus medius and/or minimus tendon tears have been increasingly identified as the underlying pathology behind some of these GTPS cases. The aim of the present study is to evaluate whether arthroscopic treatment of abductor tendon ruptures can improve the outcomes in patients with GTPS.

Methods: All patients diagnosed with GTPS and treated arthroscopically in our institution between January 2012 and June 2014 were identified. Patients with concomitant femoroacetabular impingement, advanced hip osteoarthritis (defined as Tonnis grade $>2$ ) or other intra-articular hip pathology were excluded, leaving 17 patients (all females) that were included in the present study. All arthroscopic procedures were performed by the same two senior surgeons. Patients were divided in two groups. Group A (6 patients) consisted of patients with has partial gluteus tendon ruptures confirmed by MRI. These were treated with all endoscopic trochanteric bursectomy and tendon suture and fixation in the greater trochanter with PEEK anchors. Patients with no identifiable gluteus tendon rupture on MRI (11 patients) were assigned to group B and treated with endoscopic trochanteric bursectomy alone. All patients followed the same postoperative rehabilitation protocol and were evaluated with Visual analogue scale (VAS), and Modified Harris Hip Score (mHHS) at 3, 6 and 12 months postoperatively.

Results: VAS improved since preoperative period to last follow-up (mean difference 6.5 points in Group A and 5.45 points in Group B) with statistically significant results within all periods evaluated $(p<0.05)$. The mHHS showed a statistically significant increase with respect to the preoperative data and final follow-up $(p<0.05)$ in both groups - mean increase 46.83 points in Group A and 38.81 points in Group B. No major complications occurred in the present study.

Conclusions: Arthroscopic treatment of refractory GTPS yields excellent results with a low complication rate. Although the small sample size in this study did not allow for the direct comparison between the two groups, patients treated specifically for abductor tendon ruptures showed a tendency towards better outcomes despite worse pre-operative functional scores. These results seem very encouraging and further studies with prospective design and larges sample sizes are warranted to better define the value of abductor tendon repair in patients with GTPS.

\section{P818}

AWARENESS OF OSTEOPOROSIS AMONG IRANIAN FEMALE HEAD OF HOUSEHOLD

${ }_{\text {P. Khashayar }}^{1,2}$, A. A. Keshtkar ${ }^{3}$, A. Ziaee ${ }^{1}$, B. Larijani ${ }^{4}$

${ }^{1}$ Qazvin University Of Medical Sciences, Qazvin, Islamic Republic of Iran, ${ }^{2}$ Orc/EMRI/TUMS-CMST/UGENT, Tehran, Islamic Republic of Iran, ${ }^{3}$ Tums, Tehran, Islamic Republic of Iran, ${ }^{4}$ Endocrinology and Metabolism Research Center, Dr Shariati Hospital, Tehran University of Medical Sciences, Tehran, Islamic Republic of Iran

Background: The prevalence of osteoporosis is increasing in many parts of the world, including Iran. The disease imposes a heavy socioeconomic burden on the society. Many studies have reported that the quality of osteoporosis care highly depends on the knowledge and attitude of patients and healthcare providers of osteoporosis, patients' socioeconomic status and access to BMD and clinics. The present study is the first such research assessing the awareness of osteoporosis among Iranian female head of households, who are considered as the most important member of the family in determining the lifestyle habits, mainly diet, of other members.

Methods: The cross-sectional study was conducted on Iranian female head of households participating in the third phase of the population-based Iranian Multicenteric Osteoporosis 
Study (IMOS) in Arak and Sanandaj. These women were the wife of the head of the household or the female household head in the families who were selected for IMOS based on one - stage cluster sampling technique. They were asked to fill out a questionnaire on their knowledge of osteoporosis, its complications, risk factors, and protective factors. Another questionnaire comprising of several sections, demographic information (age, gender, level of education, and marital and socioeconomic status), reproductive factors (only in women), lifestyle habits (sun exposure, exercising, smoking and alcohol abuse habits), and family and personal medical history and drug use (with focus on diseases and medications affecting bone metabolism) were also completed for all of them.

Results: 1125 women with mean age of $43.7 \pm 12.5$ years were included. 32(2.8\%) of them were diagnosed with osteoporosis prior to the study, while our results showed that 111(11.7\%) were affected. About half of these women (48.5\%) had never heard of osteoporosis. From those who knew the disease, $63.2 \%$ named fracture as the major complication of the disease but only $31.8 \%$ were aware of the importance of hip fracture. Only $34 \%$ considered osteoporosis as a disease that could result in death. They were all capable of naming at least one of the risk and protective factors of the disease. The socioeconomic state and previous diagnosis of osteoporosis significantly influenced the response to all of these questions.

Conclusion: To improve bone health and the quality of care for osteoporotic patients, more effort is needed regarding public education with focus on women.

\section{P819}

\section{SENSITIVITY AND SPECIFICITY OF OSTEO- POROSIS DIAGNOSTICS AT PRIMARY HEALTH- CARE WITH BINDEX}

J. P. Karjalainen $^{1}$, O. Riekkinen ${ }^{1}$, J. T. Schousboe ${ }^{2}$, H. Kröger $^{3}$

${ }^{1}$ Bone Index Finland Ltd., Kuopio, Finland, ${ }^{2}$ HealthPartners Institute, Minneapolis, United States, ${ }^{3}$ Kuopio Musculoskeletal Research Unit, University of Eastern Finland, Kuopio, Finland

Objective: An ultrasound based device (Bindex $\left.{ }^{\circledR}\right)$ has been recently introduced to address the challenges in osteoporosis (OP) diagnostics at primary care (1). Bindex measures cortical thickness and determines parameter called density index (DI). Thresholds for DI in osteoporosis assessment have been determined in Finnish Caucasian population $(n=448)$ along the International Society of Clinical Densitometry (ISCD) guidelines (2). In this study, the sensitivity and specificity are assessed in data set combining three independent trials.

Material and Methods: A total of 1830 Caucasian females were enrolled for the study (age 67.2 \pm 8.9 years) for two clinical trials in Finland and one in the United States. Subjects were measured with DXA for BMD at hip and femoral neck.
The DI was determined based on cortical thickness measurements at proximal tibia alone $\left(\mathrm{DI}_{1}\right)$, and with thickness information included from distal tibia and radius $\left(\mathrm{DI}_{3}\right)$. Subjects were diagnosed with OP when T-score at either location was at or below -2.5 (NHANES III). A subgroup of 1344 subjects was formed in which the subjects with T-score between -2.1 and -2.9 were removed due to the precision error based uncertainty of osteoporosis/healthy status (3).

Results: Osteoporosis was diagnosed in 374 subjects (Tscore $\leq-2.5$ ) and 163 subgroup subjects (T- score $\leq-2.9$ ). The sensitivity and specificity of $\mathrm{DI}_{1}$ and $\mathrm{DI}_{3}$ in the total population of 1830 ranged from $82-87 \%$. Following the guidelines of the ISCD, approximately $70 \%$ of the subjects could have been classified as healthy or osteoporotic by ultrasound measurement and $30 \%$ would have needed DXA for diagnostic verification. In the subgroup analysis the DI sensitivity ranged from $90-93 \%$ and specificity was $87 \%$.

Conclusions: The suggested thresholds for the DI were tested in a large population and performance of the technique seems consistent when data from several studies were combined. The ultrasound based Bindex measurement for osteoporosis detection suggests good performance for OP detection at primary care level.

\section{References:}

1. Karjalainen et al. Osteoporos Int 2015

2. Hans et al. J Clin Densitom 2008

3. Kiebzak et al. J Clin Densitom 2007

\section{Disclosures:}

Karjalainen, Kröger, Riekkinen, stock ownership in Bone Index Finland Ltd.

\section{P820}

RARE CASE REPORT OF AN OPEN IRREDUCIBLE INTERPHALANGEAL JOINT DISLOCATION OF THE HALLUX IN A FOOTBALL PLAYER

R. Santos-Pereira $^{1}$, A. Costa ${ }^{1}$, C. Aleixo ${ }^{1}$, A. Sarmento ${ }^{1}$

${ }^{1}$ Centro Hospitalar Vila Nova de Gaia / Espinho, Vila Nova de Gaia, Portugal

Objectives: Great toe dislocations usually occur at the metatarsophalangeal joint owing to its greater mobility and longer lever arm. Because of its inherent stability, hallux interphalangeal (IP) joint dislocation is a rare disorder. There are only a few cases of IP joint dislocation reported in the literature and most are dorsal dislocations resulting from hyperextension injuries. Closed reduction should be attempted but is often unsuccessful because of interposition of the volar plate or sesamoids within the joint space. We present the case of a 18 year old male athlete who sustained an one irreducible hallux IP joint dislocation while playing football.

Methods: The patient presented to our emergency department minutes after sustaining the injury. He was in moderate pain and 
unable to explain the exact mechanism of injury. Physical examination showed an elongated great toe, fixed in a hyperextended position and decreased range of motion. The medial condylar surface of the proximal phalanx was protruding through a $2 \mathrm{~cm}$ volar wound. Attempts at closed reduction were unsuccessful as the joint immediately springed back to its elongated hyperextended position upon release of traction. The patient was taken to the operating room and the wound was cleaned, extended and debrided. Thorough inspection of the IP joint revealed the volar plate with its sesamoid bone over the proximal phalangeal head. The interposed structure was moved in the plantar direction while the distal phalanx was under traction. After relocation of the volar plate, the joint was stable, with full range of motion. The volar plate was repaired with interrupted absorbable suture and a Kirschner wire (K-wire) was placed from the distal phalanx through the proximal phalanx under $\mathrm{C}$-arm guidance to immobilize the joint and provide stability for the volar plate during healing. Following surgery the patient was given a rocker bottom shoe and the wire was removed 4 weeks after the injury.

Results: The postoperative course was unremarkable. The patient was allowed full unrestrained sporting activity by the 12th postoperative week. At the final clinical evaluation the patient was pain-free with symmetrical range of motion compared to the contralateral hallux and was very satisfied with de treatment outcome. Conclusions: Although irreducible dislocations of the IP joint of the great toe are very rare, they can be the result of even minor sports-related trauma. Prompt treatment with open reduction and K-wire fixation can be very successful and a good outcome is to be expected.

\section{P821}

LONGITUDINAL CHANGES OF BONE MINERAL DENSITY AND BONE TURNOVER MARKERS IN PATIENTS WITH INDOLENT SYSTEMIC MASTOCYTOSIS WITHOUT OSTEOPOROSIS.

$\underline{\text { C. Caimmi }}{ }^{1}$, M. Rossini ${ }^{1}$, A. Artuso ${ }^{2}$, G. Tripi ${ }^{1}$, O. Viapiana ${ }^{1}$, R. Zanotti ${ }^{2}$, D. Gatti ${ }^{1}$, S. Adami ${ }^{1}$

${ }^{1}$ Rheumatology Unit, University of Verona, Verona, Italy,

${ }^{2}$ Hematology Unit, University of Verona, Verona, Italy

Background: Systemic mastocytosis has been long identified as a potential cause of osteoporosis; nevertheless, prevalence, characteristics and pathogenesis of osteoporotic manifestations are still not well defined. In particular, data regarding the longitudinal variation of BMD in patients with indolent systemic mastocytosis (ISM) are missing.

Methods: We studied BMD variation at lumbar spine and proximal hip after 30 months ( \pm 6 months) follow-up in a large cohort of patients (83) affected by ISM without osteoporosis, in supplementation with vitamin D and/or calcium when needed. We also analyzed the correlation between variation of BMD, serum tryptase levels and bone turnover markers (bone alkaline phosphatase and Ctelopeptide of type I collagen, CTX)

Results: $64 \%$ of our population was male, the mean age was $52.1( \pm 11.5)$ years. Vitamin D insufficiency (serum levels of 25$\mathrm{OH}$-vitamin D lower than $75 \mathrm{nmol} / \mathrm{L}$ ) were found in more than $70 \%$ of patients. After a follow-up of $30 \pm 6$ months with only vitamin D (5000-7500 IU weekly) or calcium (500 mg/day) supplementation when needed, we observed $2.1 \%$ increase of BMD at lumbar spine, with no significant changes at hip. At the end of follow-up almost $60 \%$ of patients showed vitamin D serum levels still lower than recommended, despite vitamin D supplementation. Reduction of BMD after follow-up significantly correlated with high CTX serum levels at baseline.

Conclusions: In patients with ISM without osteoporosis frequent $(<2$ years) BMD evaluation are not justified, except in presence of elevated bone turnover markers. Vitamin D supplementation is frequently needed and should be given at higher doses than those usually recommended in general population.

P822

LOW-COST AND EFFICIENT FINITE ELEMENT ANALYSIS OF BONE FOR HIGH-RESOLUTION PERIPHERAL QUANTITATIVE COMPUTED TOMOGRAPHY DATA

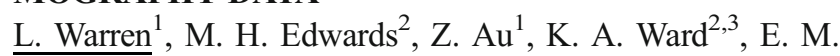
Dennison $^{2}$, C. Cooper ${ }^{2}$, P. Schneider ${ }^{1}$

${ }^{1}$ Faculty of Engineering and the Environment, University of Southampton, Southampton, United Kingdom, ${ }^{2}$ MRC Lifecourse Epidemiology Unit, University of Southampton, Southampton, United Kingdom, ${ }^{3}$ MRC Human Nutrition Research, Elsie Widdowson Laboratory, Cambridge, United Kingdom

Objectives: FEA of HRpQCT data is often performed using proprietary software and hardware of CT manufacturers, which can be expensive and time consuming. The study's objectives were to cross-validate FE outcomes from a widely available commercial FE solver vs. a proprietary FE solver and to explore in silico mechanical differences of individuals with and without low trauma fractures using low-cost and efficient FEA.

Materials and Methods: We used HR-pQCT distal radius data from an XtremeCT system (Scanco Medical) from the Hertfordshire Cohort Study (Syddall et al., Int J Epidemiol 2005;34:1234). FEAs were carried out on a VMS and on a high-performance computing (HPC) Linux node using SCANCO FE software and Ansys Mechanical ${ }^{\mathrm{TM}}$, respectively. Average von Mises equivalent strain and stress were compared using Bland-Altman plots and paired t-tests performed for differences in cortical and trabecular bone between fractures vs. age-matched controls.

Results: Mechanical outcomes from 44 distal radii (strain: Scanco $0.646 \pm 0.095 \%$ and Ansys $0.634 \pm 0.095 \%$, stress: 
Scanco 36.9 \pm 7.5 MPa, Ansys 36.1 $\pm 7.4 \mathrm{MPa}$ ) did not show any proportional errors, with a small consistent bias of $1.9 \%$ and $2.0 \%$ for strain and stress between the methods, respectively. While strain and stress (Ansys) in cortical bone for 47 agematched distal radii pairs was not significantly different between the two groups (strain: difference $0.007 \%$, stress: difference $0.3 \mathrm{MPa}$ ), there was evidence that trabecular bone plays a more important role in this cohort for maintaining bone strength in controls vs. fracture cases (strain: difference $0.041 \%$ with $p=0.06$, stress: difference $2.2 \mathrm{Mpa}$ with $p=0.04$ ).

Conclusions: We showed that FEA of HR-pQCT bone data can be performed reliably, at low cost, and efficiently using commercially FE software and rather cheap HPC systems, widely available today. In this study, the FE processing time was reduced by a factor of 20, which can be optimised further by running several FEA jobs in parallel on a HPC cluster, which would decrease the processing time for our test study (94 individuals) from 24 days to below 1 hour for a small HPC cluster (32 nodes). Low cost and efficient FE approaches will be beneficial for large HR-pQCT studies and for future developments towards clinical use of HRpQCT for diagnostic purposes in terms of fracture prediction.

\section{P823}

HPLC-MS-MS 25OHVITAMIND LEVELS ARE ASSOCIATED WITH PROGNOSIS MARKERS OF HEART FAILURE

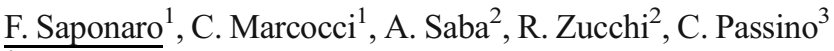
${ }^{1}$ Endocrinology 2 Unit of University of Pisa, Pisa, Italy, ${ }^{2}$ Department of Biochemistry, Pisa, Italy, ${ }^{3}$ Cardiology Unit of Fondazione Toscana Gabriele Monasterio, Pisa, Italy

Introduction and Aims: Heart failure (HF) is a health problem with poor prognosis, despite many treatments available. Vitamin D is the prehormone of the active calcitriol (1, $25(\mathrm{OH}) 2 \mathrm{D} 3)$. It is involved in bone homeostasis, but recent studies suggest extra skeletal functions, including pleiotropic effects on cardiovascular system and a relationship between low levels of $25 \mathrm{OH}-\mathrm{D}$ and worse HF prognosis. The aims of the study were: 1) to define $25 \mathrm{OH}-\mathrm{D}$ levels in the HF population, 2) to correlate $25 \mathrm{OH}-\mathrm{D}$ levels and HF outcome markers (biochemical and instrumental evaluation) in the hypothesis vitamin D represents a potential "modifiable" factor risk in HF.

Materials and Methods: We performed a retrospective study on 261 consecutive HF patients (NHYA 1-3), collecting clinical, biochemical and instrumental data (echocardiography and cardiopulmonary exercise test - CPET). We retrieved stored blood samples collected at the baseline and developed a fast isotope dilution mass spectrometry coupled with liquid chromatography (HPLC-MS-MS) method for accurate measurement of $25 \mathrm{OH}-\mathrm{D}$ levels.
Results: Patients were 47 females and 214 males (ratio $\mathrm{M}: \mathrm{F}=4: 1$ ), with a mean age of $65 \pm 12$ years and mean BMI of $28 \pm 14$. They had stable HF disease in prevalent NYHA 2 class. Mean EF (ejection fraction) was $33 \pm 8 \%$; patients had mild kidney failure (creatinine $1.12 \pm 0.3 \mathrm{mg} / \mathrm{dl}$ ) and they were normocalcemic and normo-PTH. Levels of $250 H v i t a m i n D$ ranged $2-45 \mathrm{ng} / \mathrm{ml}$, with mean of $17 \pm 9 \mathrm{ng} / \mathrm{ml} .25 \%(n=65)$ patients had vitamin D deficiency $(<10 \mathrm{ng} / \mathrm{ml}), 62 \%(n=161)$ had vitamin insufficiency (between 10-30 ng/ml) and $13 \%$ $(n=35)$ had vitamin $>30 \mathrm{ng} / \mathrm{ml}$, without any supplementation. The linear regression analysis showed that $25 \mathrm{OH}-\mathrm{D}$ levels were positively correlated with CPET parameters and negatively with mortality Mecki score and this relation was even stronger in patient with vitamin D insufficiency

Conclusion: Our study revealed a strong association between variables from CPET, a well recognized valuable tool for $\mathrm{HF}$ prognosis and 25OH-D levels, detected with a new and accurate method HPLC-MS-MS.

\section{P824}

RELATIONSHIP BETWEEN VITAMIN D METABOLITES AND MARKERS OF BONE METABOLISM IN RENAL DYSFUNCTION PATIENTS: A CROSSSECTIONAL STUDY

H. Majid $^{1}$, L. Jafri ${ }^{1}$, S. Khawaja ${ }^{1}$, A. H. Khan ${ }^{1}$

${ }^{1}$ Department of Pathology and Laboratory Medicine, Aga Khan University, Karachi, Pakistan

Background: Not many studies have studied the relationship of $1,25(\mathrm{OH})_{2} \mathrm{D}$, with calcium, phosphorus and $25(\mathrm{OH}) \mathrm{D}$ in subjects with renal dysfunction. To compare the relationship between $25(\mathrm{OH}) \mathrm{D}, 1,25(\mathrm{OH})_{2} \mathrm{D}$ with other markers of bone metabolism in healthy and subjects with renal dysfunction.

Methods: A cross-sectional study was conducted at section of Chemical Pathology, Aga Khan University during the period of Feb2015 to June 2015. 22 healthy and 18 renal dysfunction patients with high serum creatinine $(>1.3 \mathrm{mg} / \mathrm{dl})$ were included. Serum samples were collected for markers of bone metabolism calcium, phosphorus, creatinine, alkaline phosphatase and vitamin D metabolites; 25OHD and 1,25OHD. Serum calcium, phosphorus, creatinine, alkaline phosphatase were analyzed on ADVIA 1800, while 25OHD and 1,25OHD analyzed on liasionXL by electrochemiluminescence immunoassay. The calculated parameters included calcium phosphorus product and estimated PTH using formula proposed by Harvey et al. (1). Means of vitamin $\mathrm{D}$ metabolites and markers of bone metabolism were compared between the two groups by t-test and association was assessed by spearmen's correlation.

Results: The mean age of study participants was $46 \pm 20$ years with majority being females $(67 \%)$. For all subjects, 1, $25(\mathrm{OH})_{2} \mathrm{D}$ levels were found to correlate significantly with creatinine (Spearman $\mathrm{r}-0.53, p<0.001$ ), 25OHD (Spearman 
r $0.59 p<0.001$ ), calcium (Spearman r 0.44, $p<0.0001$ ) and estimated PTH (Spearman $\mathrm{r}-0.63, p<0.001$ ). No significant correlation was found between $1,25(\mathrm{OH})_{2} \mathrm{D}$ levels, Alkaline phosphatase and calcium phosphate product. On comparison of healthy and subjects with renal dysfunction, mean comparison showed that $1,25(\mathrm{OH})_{2} \mathrm{D}$ and calcium were significantly lower, while creatinine, phosphorus, alkaline phosphatase and estimated PTH were significantly higher in patients with renal dysfunction. In patients with renal dysfunction on correlating $1,25(\mathrm{OH})_{2} \mathrm{D}$ with other parameters only $25 \mathrm{OHD}$ was significantly correlated. While in healthy group only creatinine was significantly correlated with $1,25(\mathrm{OH})_{2} \mathrm{D}$. Out of 18 subjects $12 \mathrm{had}<20 \mathrm{ng} / \mathrm{ml} 25 \mathrm{OHD}$, of these 10 subjects had low 1 , $25(\mathrm{OH})_{2} \mathrm{D}$ levels, whereas all healthy subjects had both vitamin $\mathrm{D}$ metabolites in normal ranges.

Conclusion: Circulating $1,25(\mathrm{OH})_{2} \mathrm{D}$ was significantly lower in patients but levels of 25(OH)D were not significantly lower in renal dysfunction patients, indicating that simply replacing the substrate is not enough until one knows the status of 1 , $25(\mathrm{OH})_{2} \mathrm{D}$ in these patients, necessitating the measurement of $1,25(\mathrm{OH})_{2} \mathrm{D}$ in renal dysfunction patients for maintaining calcium and phosphorus homeostasis.

\section{Reference:}

1. Harvey A, et al. Endocr Pract 2011;18:124.

\section{P825}

ASSESSMENT OF BONE MINERAL DENSITY AND TRABECULAR BONE SCORE IN CHRONIC DIALYSIS PATIENTS

M. Yavropoulou $^{1}$, V. Vaios ${ }^{2}$, I. Chryssogonidis ${ }^{3}$, M. Pikilidou $^{4}$, M. Sachinidou ${ }^{3}$, K. Makris ${ }^{5}$, S. Tournis ${ }^{5}$, M. Daniilidis $^{4}$, V. Liakopoulos ${ }^{2}$

${ }^{1}$ Division of Endocrinology and Metabolism, 1st Department of Internal Medicine, AHEPA Univ. Hospital, Aristotle University of Thessaloniki, Thessaloniki, Greece, ${ }^{2}$ Nephrology Division, 1st Department of Internal Medicine, AHEPA Univ. Hospital, Aristotle University of Thessaloniki, Thessaloniki, Greece, ${ }^{3}$ Radiology Unit AHEPA Univ. Hospital, Aristotle University of Thessaloniki, Thessaloniki, Greece, ${ }^{4} 1$ st Department of Internal Medicine, AHEPA Univ. Hospital, Aristotle University of Thessaloniki, Thessaloniki, Greece, ${ }^{5}$ Laboratory of Research of Musculoskeletal System "Th. Garofalidis", Medical School, University of Athens, KAT Hospital, Athens, Greece

Patients with end stage renal disease (ESRD) on maintenance hemodialysis (HD) exhibit osteoporosis and increased fracture risk. In these patients bone loss predominantly affects cortical bone. However, histomorphometric analysis has shown that trabecular bone loss also contribute to bone fragility in ESRD patients. Since, bone biopsies are difficult to perform in everyday clinical practice, trabecular bone score (TBS) has been recently proposed as an indirect measure of bone microarchitecture. In this study we investigated TBS in ESRD patients on HD.

Patients and Methods: 45 patients with ESRD on HD, mean age 60 years, and 55 healthy individuals matched for age and gender were enrolled in the study. All participants had a BMD measurement by DXA scan at the lumbar spine (LS), femoral neck (FN) and ultra distal radius of the left arm (UD). In all patients and controls TBS was evaluated in the same regions as those used for LS using TBS iNsight. Serum fetuin and plasma FGF-23 (C-terminal) were also measured.

Results: Patients with ESRD on HD demonstrated significant lower BMD values at all skeletal sites compared to controls (LS $0.979 \pm 0.223 \mathrm{~g} / \mathrm{cm}^{2}$ vs. $1.939 \pm 0.1846 \mathrm{~g} / \mathrm{cm}^{2}, p<0.001$; FN $0.751 \pm 0.153 \mathrm{~g} / \mathrm{cm}^{2}$ vs. $1.024 \pm 0.143 \mathrm{~g} / \mathrm{cm}^{2}, p<0.001$; UR, $0.359 \pm 0.11 \mathrm{~g} / \mathrm{cm}^{2}$ vs. $0.6 \pm 0.2 \mathrm{~g} / \mathrm{cm}^{2}, p<0.001$, respectively). TBS and serum fetuin levels were also significantly reduced in ESRD patients compared to healthy controls (TBS, $1.17 \pm 0.16$ vs. $1.31 \pm 0.13, p<0.001$; fetuin, $0.742 \pm 0.19 \mathrm{~g} / \mathrm{L}$ vs. $0.889 \pm 0.2, \mathrm{~g} / \mathrm{L}, p=0.001$, respectively). Plasma FGF-23 levels were significantly increased in ESRD patients compared to controls $(1027.8 \pm 556.7 \mathrm{RU} / \mathrm{ml}$ vs. $104.36 \pm 67.4$ $\mathrm{RU} / \mathrm{ml}, p<0.001$, respectively). TBS was significantly associated with BMD of the ultra radius $(r=0.377, p<0.001)$, with serum fetuin levels $(r=0.249, p=0.023)$, and with plasma FGF-23 levels $(r=-0.549, p<0.001)$.

Conclusion: TBS appears to indirectly reflect an alteration of trabecular bone microarchitecture in patients with ESRD on $\mathrm{HD}$ and is strongly associated with cortical bone loss, as well as with circulating markers related with bone mineral disease in these patients such as fetuin and FGF-23.

\section{P826}

INFLUENCE OF THE DISEASE MODIFYING OSTEOARTHRITIS DRUG DIACEREIN ON CALCIUM HOMEOSTASIS OF CHONDROSARCOMA CELLS

B. Steinecker-Frohnwieser ${ }^{1}$, A. Mann ${ }^{2}$, H. G. Kress ${ }^{2}$, W. Kullich $^{3}$, L. Weigl ${ }^{2}$

${ }^{1}$ Ludwig Boltzmann Department for Rehabilitation of Internal Diseases, Cluster for Arthritis and Rehabilitation, Groebming, Austria, ${ }^{2}$ Department of Special Anaesthesia and Pain Therapy, Medical University Vienna, Vienna, Austria, ${ }^{3}$ Ludwig Boltzmann Department for Rehabilitation of Internal Diseases, Cluster for Arthritis and Rehabilitation, Saalfelden, Austria

Objective: To investigate the modulation of intracellular calcium $\left[\mathrm{Ca}^{2+}\right]_{\mathrm{i}}$ signaling in Cal-78 chondrosarcoma cells as a possible mechanism of action for diacerein, a disease modifying osteoarthritis drug (DMOAD).

Material and Methods: Changes in $\left[\mathrm{Ca}^{2+}\right]_{\mathrm{i}}$ were measured by the calcium imaging technique in Fura-2 
loaded cells pretreated with diacerein. Histamine, a mediator of inflammation, was applied in different concentrations to provoke calcium signaling. As a consequence, ATP production (ATP Assay Kit, Abcam) as well as the calcineurin (Calcineurin Phosphatase Activity Assay, Abcam) and calpain activity (Calpain Activity Assay, Abcam) of Cal-78 cells was measured. Cal-78 cells were transfected with a functional NFATGFP construct (Poteser et al., 2011) to monitor the NFAT translocation triggered by histamine application under diacerein and control conditions.

Results: Cal-78 cells pretreated with diacerein showed a reduction in histamine induced calcium peaks as a response to different concentrations of histamine. Interestingly, the levels for resting calcium and the time constant $\tau$ for calcium removal were increased. A change in the production of ATP was not observed though a diacerein induced regulation of calcineurin and calpain activity was detected. Further observations showed a decline in the translocation efficiency of the Nuclear Factor of activated T-cells (NFAT) into the nucleus when cells were pretreated with diacerein.

Conclusion: Our results demonstrate that diacerein modulates intracellular calcium signaling in chondrosarcoma cells. The observed down regulation of histamine induced $\left[\mathrm{Ca}^{2+}\right]_{\mathrm{i} \text {,peak }}$, by diacerein obviously influences NFAT activity, an important transcription factor involved in cell cycle regulation (Roderick \& Cook, 2008). Furthermore, the up regulation in resting $\mathrm{Ca}^{2+}$ points out a modulation of the rate between influx and efflux of $\mathrm{Ca}^{2+}$ with the $\mathrm{Na}^{+}-\mathrm{Ca}^{2+}$ exchanger as a possible target. Moreover, the presented effects on $\mathrm{Ca}^{2+}$ homeostasis followed by changes in NFAT activity might explain the inhibitory action of diacerein on cell growth and cell viability of Cal-78 cells (Lohberger et al., 2015).

References:

Poteser et al., PNAS 2011;108:10556

Roderick \& Cook, Nature Reviews Cancer 2008;8:361

Lohberger et al., BMC Cancer 2015;15:1

\section{P827}

\section{REHABILITATION OF PATIENTS WITH GOUT}

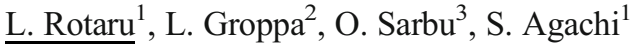

${ }^{1}$ Department of Rheumatology, State Medical and Pharmaceutical University «Nicolae Testemitanu», Chisinau, Republic of Moldova, ${ }^{2}$ Laboratory of Rheumatology, Chisinau, Republic of Moldova, ${ }^{3}$ Republican Clinical Hospital, Chisinau, Republic of Moldova
Objectives: The purpose of the present study consists in the analysis of the results of the use of rehabilitation procedures by the application of hydrocortisone ultrasound concomitantly with the treatment with pharmacological products.

Methods: 110 patients with gout were examined. 83 of them were diagnosed with chronic gout, and 27 with acute gout. The patients were monitored during the years of 2013-2015. The time persistence of gout in these patients was between 1 and 37 years. All patients were examined (instrumental and laboratory methods). The patients had been divided into 2 groups - 1st group - 55 patients (50\%) were administered medications along with a local treatment of the swollen joints with the ultrasound with hydrocortisone ointment, the 2nd group - 55 patients $(50 \%)$ used only drug treatment without the use of the ultrasound. The treatment of the patients with gout was carried out within the hospital, which allowed the dosage adjustments of the medications for the treatment of gout depending on the hyperuricemia figures.

Results: $102(92.7 \%)$ patients presented MTF I joint damage; tarsus - 65 (59\%) patients; knees - 57 (51.8\%) patients; distal interphalangeal - 54 (49 \%) patients, elbow - 38 (34.5\%) patients. In the 1st group - the inflammatory reaction of the joints of $13(24 \%)$ patients decreased during the first two day of treatment, 19 (35\%) patients - on the third day of treatment, $11(20 \%)$ patients - on the 4th day of treatment and $12(21 \%)$ patients - on the 5th day of treatment; in the 2nd group - the inflammation in the joints decreased, but belatedly, namely on the 2nd day of treatment - 8 (15\%) patients, on the 3rd day $14(25 \%)$ patients, the 4 th day $-18(33 \%)$ patients and on the 5 th day of treatment - $15(27 \%)$ patients.

Conclusion: The early rehabilitation of affected joints helps to reduce more effectively the articular inflammatory process, the pain syndrome and it delays the progression of the underlying pathology, and improves the quality of life of patients with gout.

\section{P828}

ASSESSING THE RISK OF OSTEOPOROTIC FRACTURE IN HEALTHY SUBJECTS: AN OBSERVATIONAL STUDY IN ITALIAN COMMUNITY PHARMACIES

G. Gandolini ${ }^{1}$, A. Di $\mathrm{Chio}^{2}$, M. Alemanni ${ }^{2}$, C. Giua ${ }^{2}$, E. Keber $^{2}$

${ }^{1}$ Rheumatology and Bone Metabolic Unit IRCCS Don C. Gnocchi Foundation, Milan, Italy, ${ }^{2}$ Italian Society of Clinical Pharmacy - SIFAC, Cagliari, Italy 
Objectives: An observational study was conducted in community pharmacies in order to assess the feasibility of a general population screening and to quantify the risk of fracture between genders and different age groups.

Methods: 6 community pharmacies participated in the study. All subjects with at least 55 years old who have agreed to participate and who did not present previous related fracture risk diseases, completed a questionnaire that allowed to assess the fracture risk at 10 years by the FRAX algorithm. Data are presented as median (range interquartile).

Results: 799 patients (540 women) participated in the study. The overall fracture risk was $7 \%$, and hip fracture risk of $2 \%$ with an overall fracture risk in women more than men $(P<0.0001)$ and increased with increasing age: $4 \%$ in subjects aged $55-64,8 \%$ in subjects aged $65-74$ and $14 \%$ in subjects aged 75 or more years $(P<0.0001)$. The risk of hip fracture increases with age and is significantly higher in women.

Conclusions: The study showed that pharmacies can be a valuable Presidium for a quick and economic fracture risk assessment in the population and that a considerable percentage of alleged healthy individuals has a non-negligible fracture risk. Systematic screening in Italian pharmacies may allow early detection of at risk individuals, permitting a cost savings associated with diagnostic and therapeutic osteoporotic fractures.

\begin{tabular}{|c|c|c|c|c|c|c|c|}
\hline \multirow[b]{2}{*}{ Mice group } & \multicolumn{3}{|c|}{ Parameter - soft X-RAYS $\{=12 /$ group $\}$} & \multicolumn{4}{|c|}{ Parameter $-\mu \mathrm{CT}\{\mathrm{n}=6 /$ group $\}$} \\
\hline & $\begin{array}{l}\text { Total and relative } \\
\text { () number of right } \\
\text { humeral(H) }+ \\
\text { femoral(F) fractures }\end{array}$ & $\begin{array}{l}\text { Midshaft outer } \\
\text { diameter } \\
\text { (MOD, mm) }\end{array}$ & $\begin{array}{l}\text { Ct. Th / } \\
\quad \text { MOD (\%) }\end{array}$ & $\begin{array}{l}\text { Cancellous BMD } \\
(\mathrm{mg} / \mathrm{cm} 3)\end{array}$ & $\begin{array}{l}\text { Cancellous Bone } \\
\text { volume density } \\
\text { (BV/TV, \%) }\end{array}$ & $\begin{array}{l}\text { Cancellous trabecular } \\
\text { number }(1 / \mathrm{mm})\end{array}$ & $\begin{array}{l}\text { Cortical fissure } \\
\text { mineral } \\
(\mathrm{TMD}, \mathrm{mg} / \mathrm{cm} 3)\end{array}$ \\
\hline WT & $\begin{array}{l}\text { H: } 0(0) \\
\text { F: } 0(0)\end{array}$ & $1,21 \pm 0,11$ & $18,5 \pm 1,8$ & $731 \pm 61,8$ & $6,09 \pm 0,50$ & $1,71 \pm 0,09$ & $1438,0 \pm 13,0$ \\
\hline Oim(-/-) & $\begin{array}{l}\mathrm{H}: 11(0,85) \\
\mathrm{F}: 3(0,23)\end{array}$ & $1,18 \pm 0,07$ & $11,2 \pm 1,5^{* *}$ & $139,4 \pm 141,5^{* *}$ & $179 \pm 0,89^{* *}$ & $0,43 \pm 0,25^{* *}$ & $1411,7 \pm 70,2$ \\
\hline CatK(-/-) & $\begin{array}{l}\text { H: } 0(0) \\
\text { F: } 0(0)\end{array}$ & $1,18 \pm 0,07$ & $27,5 \pm 2,06^{* *}$ & $3488,3 \pm 1366,9^{* *}$ & $27,46 \pm 12,57^{* *}$ & $4,73 \pm 1,40^{* *}$ & $1280,0 \pm 26,0^{* *}$ \\
\hline $\mathrm{O}$ im(-/-)CatK(-/-) & $\begin{array}{l}\mathrm{H}: 0(0) \\
\mathrm{F}: 0(0)\end{array}$ & $1,06 \pm 0,05+$ & $19,1 \pm 1,5++$ & $777,2 \pm 237,8$ & $6,13 \pm 1,57$ & $1,86 \pm 0,40++$ & $1436,7 \pm 16,3$ \\
\hline
\end{tabular}

Data are mean $\pm \mathrm{SD} ; * * P<0.001$ vs. WT; $++P<0.001$ vs. $\operatorname{Oim}(-/-) ;+P<0.01$ vs. $\operatorname{Oim}(-/-)$

\section{P829}

CATHEPSIN K KNOCKOUT IMPROVES BONE MASS AND SUPPRESSES LONG BONE FRACTURES IN THE OSTEOGENESIS IMPERFECTA MOUSE MODEL (OIM)

T. Roels $^{1}$, J.-P. Devogelaer ${ }^{2}$, L. T. Duong ${ }^{3}$, M. Cardinal $^{1}$, C. Behets ${ }^{1}$, D. H. Manicourt ${ }^{2}$

${ }^{1}$ Morphology, IREC, Université Catholique de Louvain, Bruxelles, Belgium, ${ }^{2}$ Department of Rheumatology, Université Catholique de Louvain, Cliniques Universitaires St Luc, Bruxelles, Belgium, ${ }^{3}$ Bone Biology, Merck Research Laboratories, West Point, PA, United States

Background and Purpose: Osteogenesis imperfecta (OI) is a severe genetic condition that results in poor bone quality and high rates of bone fractures. As preclinical studies consistently demonstrate that cathepsin $\mathrm{K}$ (CatK) inhibition increases bone mass and strength in postmenopausal osteoporosis, we hypothesized that CatK knockout (KO) might improve the bone mass and strength of long bones in OI mice [Oim( $(-/-)]$, a model of severe human OI with spontaneous fractures.

Experimental approach: As inhibitors of human CatK do not work in the mouse, we bred Oim(-l-) with CatK $\mathrm{KO}[\mathrm{CatK}(-)$ $-)]$ mice to get $\mathrm{Oim}(-/-) \mathrm{CatK}(-1-)$ mice. Mice were sacrificed at 13 weeks of age. We analyzed 12 wild/wild (WT) control mice, $12 \mathrm{Oim}(-/-)$ mice, $12 \mathrm{CatK}(-/-)$ mice and $12 \mathrm{Oim}(-/$ $-)$ CatK $(-/-)$ mice. Soft X-rays identified limb bone fractures. Midshaft outer diameter and cortical thickness (Ct. Th) were measured on the digitized radiographic image. Quantitative $\mu \mathrm{CT}$ measured femurs at metaphysis and midshaft.

Results: Soft X-ray data and $\mu \mathrm{CT}$ data of the right femur are given in table below. 
Conclusion: CatK knock-out in Oim improves cancellous BMD and cortical thickness in the femur, but does not increase the midshaft outer diameter or cortical TMD. These data show that CatK knockout in the Oim does not increase bone quantity but improves different bone parameters contributing to the absence of fractures.

\section{P830}

BONE MINERAL DENSITY AMONG SAUDI MEN R. Al-Raddadi ${ }^{1}$, W. Hashem ${ }^{2}$, N. Kabli ${ }^{1}$, F. Hakim ${ }^{3}$, H. $\mathrm{Kabli}^{4}$, R. Hakim ${ }^{5}$, M. Ardawi ${ }^{1}$

${ }^{1}$ Center of Excellence for Osteoporosis Research, Jeddah, Saudi Arabia, ${ }^{2}$ King Saud Medical City, MOH, Riyadh, Saudi Arabia, ${ }^{3}$ Family Medicine, Ministry of National Guard, Jeddah, Saudi Arabia, ${ }^{4}$ King Fahad Hospital, Jeddah, Saudi Arabia, ${ }^{5}$ King Abdulaziz University, Jeddah, Saudi Arabia

Introduction: Osteoporosis is a major public health problem common with aging in both sexes, but is often ignored in men. Measurement of bone density allows early detection of osteoporosis and prevent fractures. Effort to maximize the peak bone mass have important significances in decrease prevalence of osteoporosis and future fracture risk.

Objectives: To assess the BMD among Saudi men and to compare the peak BMD with the published peak BMD from other countries in the region.

Methodology: A sample of 330 men with no previous history of bone diseases or diseases affecting bone health was selected from the primary health care centers in Jeddah city. The BMD for the selected sample was measured for lumbar spine and proximal femur and compared to BMD of other countries in the region. Correlation coefficient was used to identify the association between BMD, age and BMI. One sample t-test was used to assess the difference between peak BMD of Saudi men and others.

Results: The mean age was 47.4, SD 12.9. The mean (SD) of the peak spine BMD was $1.13(0.14)$ with $17 \%$ prevalence of osteoporosis. The mean (SD) hip BMD 1.09 (0.14) and prevalence of osteoporosis $3.3 \%$. The peak BMD Spin and Hip of Saudi men were significantly negatively correlated with age and positively correlated with BMI. BMD spin but not hip was significantly associated with presence of any fracture. Spin BMD of Saudi men was significantly lower than Lebanese, Moroccan and Iranian but higher than Omanis. However, Hip BMD significantly lower than Moroccan, Iranian but higher than Lebanese and Omanis.

In conclusion: The peak BMD of Saudi men have a significant difference in comparing to men in other countries of the region. A preventive measure should target Saudi Men to maximize their peak BMD.
P831

HOW DOES FALL HISTORY PREDICT FUTURE FALLS?

N. Afrin ${ }^{1}$, R. Honkanen ${ }^{1}$, H. Koivumaa-Honkanen ${ }^{2}$, T. Rikkonen $^{1}$, J. Sirola ${ }^{1}$, H. Kröger ${ }^{1}$

${ }^{1}$ Kuopio Musculoskeletal Research Unit, University of Eastern Finland, Kuopio, Finland, ${ }^{2}$ Department of Psychiatry, Kuopio University Hospital, Kuopio, Finland

Objective: To assess how does falling history predict future falls in postmenopausal women before old age.

Introduction: Falls are a major health problem in the elderly. The purpose of this study was to evaluate if falling history predicts future falls. Our special interest was to compare fall prediction in occasional and frequent fallers.

Methods: This is a prospective cohort study. Falls were asked in OSTPRE (Kuopio Osteoporosis Risk Factor and Prevention Study) enquiries in 1994 and 1999. A total of 7940 women (study population) responded to fall questions in both 1994 and 1999 postal enquiries. Falls in 1994 were predictor variables, while falls in 1999 were outcome variables. Women reporting one fall during the last 12 months were classified as occasional fallers, while women reporting $2+$ falls were classified as frequent fallers. Odds ratios (OR) were computed with logistic regression.

Results: Women were 52-61 years old at baseline in 1994. Falling history in general predicted future falls with an OR of $2.68(p<0.0001)$. History of frequent falls predicted strongly slip falls $(5.42, p<0.001)$ and nonslip falls $(O R=4.95, p<0.000)$. Frequent slip fall history showed a strong and high prediction for future slip falls $(O R=8.47, p<0.001)$, similarly, history of frequent nonslip falls predicted future nonslip falls $(O R=7.93, p<0.001)$. History of occasional falls did not predict future falls $(O R=1.60)(\mathrm{ns})$, slip falls $(O R=1.68)(\mathrm{ns})$ or nonslip falls $(O R=1.59)(\mathrm{ns})$.

Conclusions: History of frequent falls is a better predictor for future fall risk than history of occasional falls.

\section{P832}

VITAMIN D SUPPLEMENTATION IN PATIENTS WITH AUTOIMMUNE DISEASES TREATED WITH GLUCOCORTICOIDS: INFLUENCE OF THE SEASON IN 25(OH)D CONCENTRATION

M. Ortego-Jurado ${ }^{1}$, M. Correa-Rodriguez ${ }^{2}$, B. RuedaMedina $^{2}$, J.-L. Callejas-Rubio ${ }^{3}$, R. Ríos-Fernández ${ }^{4}, \mathrm{~N}$. Ortego-Centeno ${ }^{4}$

${ }^{1}$ Unidad de Medicina Familiar y Comunitaria, Palma de Mallorca, Spain, ${ }^{2}$ Department of Nursing, University of Granada, Granada, Spain, ${ }^{3}$ Unidad de Enfermedades Autoinmunes, Hospital Clinico San Cecilio, Granada, Spain, 
${ }^{4}$ Unidad de Enfermedades Autoinmunes y Sistémicas, Hospital Universitario San Cecilio, Granada, Spain

Introduction: Glucocorticoids (GCs) are the crucial therapy in many autoimmune and inflammatory diseases. However, their beneficial effects are associated frequently with unwanted effects such as glucocorticoid-induced osteoporosis (GIO). Clinical practice guidelines have shown the importance of preventing bone mass loss and reduce the incidence of fractures associated with GC therapy including calcium and vitamin D supplementation. We aimed to analyze if oral vitamin D supplementation (calcidiol or cholecalciferol) helps to reach adequate $25(\mathrm{OH}) \mathrm{D}$ levels in patients with autoimmune diseases chronically treated with low doses of GCs.

Methodology: A prospective observational study was conducted in 147 Caucasian patients with autoimmune diseases undergoing GCs therapy (age 56.06 $\pm 16.11 ; 30$ men; 49 preand 68 postmenopausal women) followed up at the Outpatient Clinic of the Systemic Autoimmune Diseases Unit at San Cecilio Hospital in Granada (Spain). 86 patients received cholecalciferol (800 IU/day), 49 patients calcidiol (10.640 IU/ 4 weeks) and twelve received no supplements. At the time of inclusion in the study, the patients were already on calcidiol or cholecalciferol for at least three months. $25(\mathrm{OH}) \mathrm{D}$ assessment was done using the IDS-iSYS25OHD kit.

Results: Patients treated with calcidiol showed higher $25(\mathrm{OH}) \mathrm{D}$ annual mean than those treated with cholecalciferol or those who did not receive vitamin D3 supplements $(25(\mathrm{OH}) \mathrm{D}$ mean levels with calcidiol $35 \mathrm{ng} / \mathrm{ml}$, cholecalciferol $27.1 \mathrm{ng} / \mathrm{ml}$ and with no supplement 24.7; $p=0.002$ ). Interestingly, when considering the season of the year we observed statistically significant differences in the $25(\mathrm{OH}) \mathrm{D}$ levels only in the fall winter period (25(OH)D mean values with calcidiol $35.6 \mathrm{ng} / \mathrm{ml}$, cholecalciferol $24.6 \mathrm{ng} / \mathrm{ml}$ and with no supplement 22.8 ; $p<0.0001)$.

Conclusions: Calcidiol supplementation improves $25(\mathrm{OH}) \mathrm{D}$ levels than cholecalciferol in patients with SAD during fallwinter period and may constitute an affordable source of vita$\min \mathrm{D}$ to prevent GIO.

\section{P833}

EVALUATION OF BONE MINERAL DENSITY IN PEDIATRIC PATIENTS AND ASSOCIATION WITH CLINICAL AND DEMOGRAPHICAL VARIABLES

J. Öncü ${ }^{1}$, B. Kuran ${ }^{1}$, F. Yilmaz ${ }^{1}$, B. Doğu ${ }^{1}, H$. Bertan ${ }^{1}$, C. Mert $^{1}$, Z. Duman ${ }^{1}$

${ }^{1}$ Şişli Etfal Hospital, Istanbul, Turkey

Objectives: Osteoporosis is traditionally regarded as a disease of elderly women. However, this bone disorder is also increasingly recognised in the paediatric setting. In particular, patients, including young children, with other chronic diseases are at risk of developing bone fragility. Many children with neurological disorder (e.g., cerebral palsy, spina bifida, etc.) have diminished BMD and a propensity to fracture with minimal trauma. The aim of this study was to identify variables which are routinely assessed as part of standard clinical care and that might be correlated with low BMD.

Material and Methods: 37 participants (ages 7 years 3 month to 16 years 1 month; mean age 11 years 9 months, SD 4 years 3 months) with moderate to severe functional disability (evaluated with pediatric functional independence score $=$ WeeFIM $<5$ ) were assessed in detail. This included gathering clinical data (Gross Motor Function Scale, WeeFIM), taking anthropometric measures of weight, height and BMI, as well as DXA measures of lumbar and femoral BMD.

Results: 20 participants were cerebral palsy and 17 were spina bifida. 22 were ambulatory with assistance (Gross Motor Function Classification System [GMFCS] level III) and 14 were capable of little or no ambulation even with assistance (GMFCS level IV and V for cerebral palsy, WeeFIM score: 13). Declining BMD of spinal (L1-4 scores) was correlated with increasing age and greater severity of functional disability (correlated both with GMFCS and WeeFIM). Lower femoral BMD scores have been found in patients with spina bifida rather than cerebral palsy $(p<0.05)$. We could not find any correlation with low BMD scores with demographical factors. Conclusion: Our results showed that low BMD scores were correlated best with functional disability level. Since a child possessing low BMD can exhibit a significant proneness to fractures; early diagnosis and treatment for paediatric osteoporosis very important in this patient group.

\section{P834}

AN OPTIMIZED ECHOGRAPHIC APPROACH FOR OSTEOPOROSIS DIAGNOSIS ON FEMORAL NECK

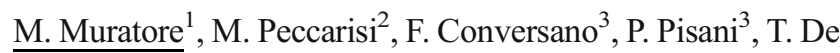
Marco $^{2}$, R. Franchini ${ }^{3}$, E. Casciaro ${ }^{3}$, A. Grimaldi ${ }^{1}$, D.

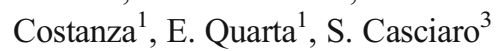

${ }^{1}$ O.U. of Rheumatology, "Galateo" Hospital, San Cesario di Lecce, ASL-LE, Lecce, Italy, ${ }^{2}$ Echolight srl, Lecce, Italy, ${ }^{3}$ National Research Council, Institute of Clinical Physiology, Lecce, Italy

Objective: To assess the clinical effectiveness of an advanced quantitative echographic method for osteoporosis diagnosis on femoral neck.

Materials and Methods: 273 female patients (55-80 years) underwent both a femoral DXA and an echographic scan of the proximal femur. Data analysis algorithm was based on the closely integrated processing of B-mode images and corresponding "raw" unfiltered signals, performing a series of combined spectral and statistical elaborations ("echosound approach" [1]). A novel 
dedicated signal compensation tool was also employed, to better take into account the physiological differences related to the variable distance between the femoral neck interface and the probe, and the subsequent frequency-dependent attenuation due to the soft tissues. The diagnostic agreement between DXA, assumed as the gold standard reference, and the proposed echographic method was assessed by accuracy calculation, Cohen's $k$, Pearson correlation coefficient $(r)$, root mean square error (RMSE), and Bland-Altman analysis.

Results: The overall agreement with DXA patient classification was $88.3 \%(k=0.794, p<0.0001)$. A further confirmation of the good diagnostic performance was also provided by the significant correlation found between the echographically estimated values of BMD and the corresponding DXA-measured ones $(r=0.81$, $p<0.001)$, and by the low residual error $\left(R M S E=0.053 \mathrm{~g} / \mathrm{cm}^{2}\right)$. The average difference between paired BMD measurements was $-0.002 \pm 0.105 \mathrm{~g} / \mathrm{cm}^{2}$ (bias $\pm 2 \mathrm{SD}$ ).

Conclusions: The adopted signal compensation method resulted in an unprecedented diagnostic agreement between DXA and echographic osteoporosis diagnoses on the femoral neck, supporting the clinical translation of the employed echosound approach.

\section{References:}

1. Casciaro et al., Clin Cases Min Bone Metab 2015;12:142.

Acknowledgements: Work partially funded by FESR PO Apulia Region 2007-2013 - Action 1.2.4 (grant n. 3Q5AX31: ECHOLIGHT Project)

\section{P835}

PRELIMINARY DATA FROM OSTEOPOROSIS STUDY IN LUNG TRANSPLANTATION PATIENTS IN VALL D'HEBRON HOSPITAL

M. Barceló ${ }^{1}$, S. Farietta ${ }^{1}$, M. López ${ }^{1}$

${ }^{1}$ Hospital Vall d'Hebron, Barcelona, Spain

Background: Osteoporosis (OP) is a known complication in lung transplantation patients that may represent a worsening of quality of life and reduced lung capacity secondary to a fracture.

Objectives: Determine the prevalence of osteoporosis in a group of lung transplantation patients at our hospital, before and after surgery and the degree of bone mass loss after transplantation.

Methods: 179 adults lung transplantation recipients between 1997-2014, that were evaluated for OP in Rheumatology Outpatient Clinic and who had available densitometry (BMD) lumbar and femoral (BMD) (Lunar Prodigy DXA System) prior and post transplantation were included. Demographics and risk factors for osteoporosis were collected. Patients were divided into 4 groups according to lung pathology. These were: COPD, diffuse interstitial disease (ILD), Cystic Fibrosis (CF) / Bronchiectasis (BE) and lymphangioleiomyomatosis (LAM). The dose of corticosteroids prior to transplantation was stratified in high cumulative doses $(>5 \mathrm{mg} / \mathrm{d}$ for $>3$ months or $>3$ sets/year) or low if they did not meet these conditions. Post-transplant cumulative corticosteroid dose and the other immunosuppressive drugs were calculated from the transplant date to the BMD realization. The presence of post-transplant fractures was collected. For the assessment of change in BMD in each group, a comparison of pre and posttransplant BMD means was performed.

Results: 179 patients, 109 men (age $10.4 \pm 51.25$ ) and 70 women (age 50.6 \pm 11.2 ), $65 \%$ postmenopausal, were included. $57 \%$ were smokers with a mean of $32.41 \pm 24.7$ packs/year, $5 \%$ consumed alcohol, $28 \%$ exhibited osteopenia; 22 (12.3\%) patients were underweight (BMI < 19.99); $22.9 \%$ reported low calcium intake ( $<499 \mathrm{mg})$ and $101(56.4 \%)$ required high doses of corticosteroids prior to transplantation. The prevalence of osteoporosis before transplantation was $37.4 \%$; of these, 41 patients $(61.2 \%)$ received treatment for osteoporosis. 17 patients had a previous fracture, 14 vertebral and 3 not vertebral. The distribution of patients according to disease was: 65 COPD patients (36.3\%), ILD 86 (48 \%), FQ/BE 19 patients (10.6\%) and 9 with LAM. The average time of the post-transplant BMD realization was 12.43 months (1-96). Evolutive results of BMD, the BMD change, the cumulative dose of corticosteroids and presence of fractures are shown in Table 1

\begin{tabular}{|c|c|c|c|c|}
\hline & $\operatorname{COPD}(n=65)$ & $\operatorname{ILD}(n=86)$ & $\mathrm{FQ} / \mathrm{BE}(n=19)$ & $\operatorname{LAM}(n=9)$ \\
\hline OP pre-transplant & $38(58,5 \%)$ & $19(22,1 \%)$ & $9(47,4 \%)$ & $1(11,1 \%)$ \\
\hline OP post- transplant & $31(47,7 \%)$ & $25(29,1 \%)$ & $10(52,6 \%)$ & $3(33,3)$ \\
\hline $\begin{array}{l}\text { BMD post-transplant (mean) } \\
\text { - L2-L4 } \\
\text { - CF } \\
\text { - FT }\end{array}$ & $\begin{array}{l}0,984 \pm 0,149 \\
0,782 \pm 0,112 \\
0,812 \pm 0,153\end{array}$ & $\begin{array}{l}1,071 \pm 0,179 \\
0,842 \pm 0,130 \\
0,884 \pm 0,149\end{array}$ & $\begin{array}{l}0,931 \pm 0,156 \\
0,762 \pm 0,095 \\
0,782 \pm 0,104\end{array}$ & $\begin{array}{l}1,021 \pm 0,127 \\
0,813 \pm 0,117 \\
0,847 \pm 0,122\end{array}$ \\
\hline $\begin{array}{l}\text { \% change BMD } \\
- \text { L2-L4 } \\
- \text { CF } \\
\text { - FT }\end{array}$ & $\begin{array}{l}+4,5^{*} \\
+0,9 \\
+0,8\end{array}$ & $\begin{array}{l}-1,2 \\
-4,2^{*} \\
-4,7^{*}\end{array}$ & $\begin{array}{l}-3,2 \\
-4,3^{*} \\
-3,1\end{array}$ & $\begin{array}{l}-3,6 \\
-4,7 * \\
-5,5^{*}\end{array}$ \\
\hline $\begin{array}{l}\text { Cumulative dose GC post-TX (gr) } \\
*_{p}<0.05\end{array}$ & $0,58 \pm 0,94$ & $0,78 \pm 1,33$ & $0,288 \pm 0,11$ & $0,27 \pm 0,08$ \\
\hline
\end{tabular}


Conclusions: OP prevalence was $37.4 \%$ prior to transplantation and $38.5 \%$ posteriorly. A higher overall percentage of patients with pre and post-transplantation OP was evidenced in the COPD and CF/BE groups, probably due to the use of corticosteroids in COPD and the risk of osteopenia in CF. A loss of BMD at all localizations and in all the groups, some with statistical significance, was found except for the COPD group. The cumulative dose of corticosteroids after transplantation was higher in the ILD group followed by the COPD group. These differences could be explained by the greater number of patients in these groups and greater proportion of patients with acute rejection, entailing an increase of the cumulative dose of drug. More fractures occurred in the groups who received a higher cumulative dose of corticosteroids.

\section{P836}

VERTEBRAL FRACTURE RISK: ALENDRONATE TREATMENT ENHANCES ANTERIOR MICROSTRUCTURE IN OSTEOPOROTIC VERTEBRAE

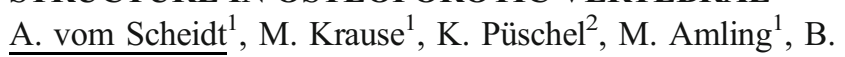
Busse $^{1}$

${ }^{1}$ Department of Osteology and Biomechanics, University Medical Center Hamburg-Eppendorf, Hamburg, Germany, ${ }^{2}$ Institute of Forensic Medicine, University Medical Center Hamburg-Eppendorf, Hamburg, Germany

Objective: Our aim was to investigate whether anti-resorptive therapy with alendronate would result in different regional variations of structural indices in the vertebral body to elucidate why the efficacy of bisphosphonate therapy is higher for the prevention of vertebral fractures compared to other fractures.
Material and Methods: We investigated the microstructure of L3 vertebrae from 14 women (mean age 83 years) with high-resolution peripheral quantitative CT $(82 \mu \mathrm{m})$. Further, we scanned cylindrical samples from the anterior and posterior vertebrae with micro-computed tomography at $3.5 \mu \mathrm{m}$ resolution and determined their mechanical competence with uniaxial compression testing.

Results: The CT results showed a significantly higher average bone volume to tissue volume ratio (BV/TV), T-Score, and trabecular thickness for the alendronate treated group compared to the osteoporotic control group. Regression analysis showed a high influence of BV/TV on the maximum force for both groups. While our results for the regional structural indices in the osteoporosis group agree well with epidemiological data, we found specific deviations in regional structures for the alendronate group. The osteoporosis group had a significantly lower BV/TV (Fig. 1 A) and trabecular number in the anterior regions compared to the posterior ones. In contrast, the alendronate group did not exhibit these regional differences (Fig. 1 B). Further, trabecular thickness was higher for the anterior cylinders from the alendronate group than those from the osteoporosis group were.

Conclusions: Alendronate therapy influences the regional variation (anterior vs. posterior) of vertebral BV/TV in comparison to treatment naïve controls. Contrary to osteoporotic bone, this study shows a uniform distribution of bone volume in anterior and posterior regions for alendronate treated bone. This uniform distribution might make the elderly vertebrae less susceptible to wedge fractures. Therefore, our study provides insight in the higher fracture risk reduction in vertebrae compared with other fracture sites.

Figure 1:
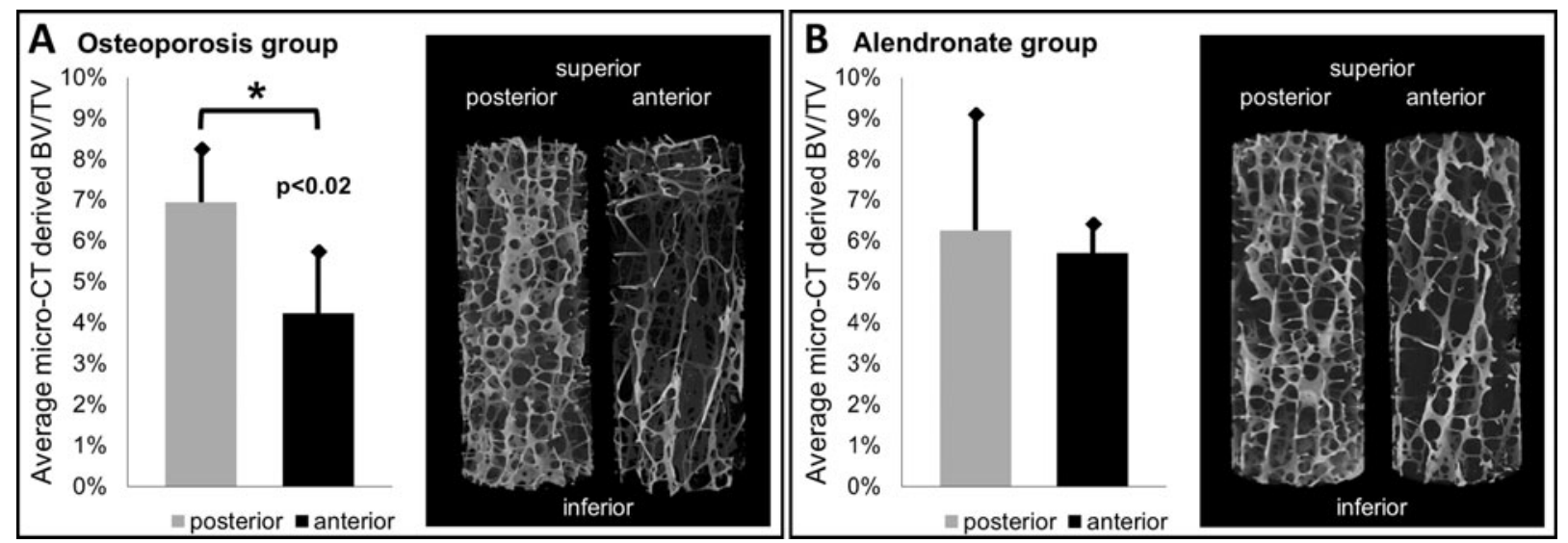


\section{P837}

\section{EFFECT OF DENOSUMAB ON CREATININE CLEARANCE}

F. Yilmaz $^{1}$, J. Öncü ${ }^{1}$, B. Doğu ${ }^{1}$, C. Mert ${ }^{1}$, C. Erçalık ${ }^{1}$, B. Kuran $^{1}$

${ }^{1}$ Şişli Etfal Hospital, Istanbul, Turkey

Objective: It is known that while administering denosumab, no dose adjustment is necessary in patients with renal impairment. The aim of this study was to investigate the effect of the drug on renal function, PTH level and quality of life (QOL) in a period of 6 months.

Material and Methods: 36 registered osteoporotic patients were included in the study. Creatinine clearance (GFR) and PTH levels were recorded before, 1 and 6 months after the treatment. QOL was assessed before and 6 months after the treatment. Renal impairment was classified as normal (GFR $>90 \mathrm{ml} / \mathrm{min}$ ), mild (GFR 60-89 $\mathrm{ml} / \mathrm{min}$ ), moderate (GFR 30-59 ml/min), severe (GFR 15-29) and very severe $($ GFR $<15)$.

Results: Initial GFRs of 36 patients ( 35 female, 1 male) was $19.4 \%$ (n: 7) normal, mildly impaired in $36.1 \%$ (n: 13), moderately impaired in $41.7 \%$ (n: 15) and severely impaired in $2.8 \%$ (n: 1). Four patients were lost at follow-up. At the $1 \mathrm{st}$ follow-up month the GFR s of 32 patients were $20 \%, 36 \%$, $40 \%$ and $4 \%$ respectively. At the 6th follow-up month, the values were $3.4 \%, 41.4 \%, 48.3 \%$ and $6.9 \%$, respectively. At the 1st and 6th months of follow-up 15 patients had moderate, 7 had mild and 1 had severe renal impairment. Renal functions of three patients were normal at the treatment period. Four patients who had normal renal functions before treatment were mildly impaired and two patients who were mildly impaired had moderately impaired renal functions 6 months of treatment. PTH levels which were normal before treatment were elevated in the 1st month after treatment in $24 \%$ and in the 6th month of treatment in $27 \%$ of the patients. Quality of life scores were similar at baseline and at sixth months.

Conclusion: In this short period (6 months) of follow-up, we have observed that denosumab has mildly affected the renal functions of the patients. The study should be extended to a longer follow-up period with more patients.

\section{P838}

\section{FOLLOW-UP DATA OF PATIENTS WITH OSTEO-} POROSIS RECEIVING ZOLEDRONIC ACID

F. Yilmaz ${ }^{1}$, B. Kuran ${ }^{1}$, B. Doğu ${ }^{1}$, J. Öncü ${ }^{1}$, Z. Duman ${ }^{1}$, C. Erçalık ${ }^{1}$

${ }^{1}$ Şişli Etfal Hospital, Istanbul, Turkey

Objective: In this study, we aimed to follow changes in BMD and quality of life and their correlation with fractures in patients with osteoporosis receiving zoledronic acid treatment.
Materials and Method: Patients followed in our outpatient clinic who were begun on zoledronic acid treatment were enrolled. The presence of femur and/or vertebra fracture, annual BMD measurements, and quality of life at the latest follow-up visit were recorded.

Results: Among 108 patients (F/M: 97/11) who were begun on zoledronic acid treatment, 34 patients were lost to followup. Female patients had a higher rate of non-attendance in follow-up visits (F: $33 \%$, M: $18 \%$ ). Eleven (10.19\%) patients had femoral fracture, $42(38.89 \%)$ had vertebral fracture, and 4 had both femoral and vertebral fracture. Fifty-nine $(54.63 \%)$ patients had no fracture. Patients with femoral fracture had a significantly lower pre-treatment BMD value compared to those who had no femoral fracture. However, femoral neck BMD values of the patients with femoral fracture were found to have increased and the significant difference between the two groups disappeared at 1 year after zoledronic acid treatment. On the other hand, no significant difference was found between pretreatment lumbar and femoral BMD values of the patients with vertebral fracture. However, there was a significant decrease in the lumbar BMD values of patients with vertebral fracture than patients without at 1 and 2 years after the treatment.

Conclusion: Although zoledronic acid treatment was associated with a greater increase in femoral neck BMD values in patients with femoral fracture, no similar increase in lumbar BMD values was observed in patients with vertebral fracture. It was also noteworthy that $31.5 \%$ of patients who were begun on zoledronic acid treatment did not attend follow-up visits, and the majority of patients who were lost to followup were women.

\section{P839}

TRANSIENT OSTEOPOROSIS OF THE HIP IN PREGNANCY OR EARLY AVASCULAR NECROSIS AS OBSERVED BY MAGNETIC RESONANCE IMAGING

$\underline{\text { S. Pavlov-Dolijanovic }}^{1}$, N. Vujasinovic Stupar ${ }^{1}$, R. Milenkovic $^{2}$, V. Koletic ${ }^{3}$

${ }^{1}$ Institute of Rheumatology, School of Medicine University of Belgrade, Belgrade, Serbia, ${ }^{2}$ Institute of Rheumatology, Belgrade, Serbia, ${ }^{3}$ Centre for Hyperbaric Medicine, Belgrade, Serbia

Transient osteoporosis of the hip (TOH) is a rare clinical condition with usually an unknown aetiology and which typically develops in middle-age men, or in women in the third trimester of their pregnancy. MRI plays an important role in the diagnosis and shows a pattern of bone marrow oedema. A 34-year-old female patient presented with severe right hip pain last month of her first pregnancy and got worse after delivery. Her history was unremarkable. MRI of the pelvis 
showed significant bone marrow oedema in the right hip with low T-1 and high T-2 signals. A diagnosis of TOH was made with a differential diagnosis of early avascular necrosis (AVN). She did not have any risk factors for AVN. BMD in the right hip was $0.498 \mathrm{~g} / \mathrm{cm}^{2}$, Z-score -3.0 ; in the left hip was $0.570 \mathrm{~g} / \mathrm{cm}^{2}, \mathrm{Z}$-score -2.4. Spine BMD was $0.884 \mathrm{~g} / \mathrm{cm}^{2}, \mathrm{Z}$ score -1.4 . All laboratory parameters, including vitamin $\mathrm{D}$, were in the normal range. Patient received vitamin D supplementation (18 $666 \mathrm{IU} /$ week) and of 45 sessions of hyperbaric oxygen (HBO) at 2.2 ATA, $70 \mathrm{~min}$ each, in a multiplace chamber. Over the course of HBO treatment, her pain was gradually relived and she became asymptomatic. MRI of the hips 7 months after onset of symptoms showed normal signal intensity, BMD in the right hip was $0.553 \mathrm{~g} / \mathrm{cm}^{2}, \mathrm{Z}$ - score -2.5 ; in the left hip was $0.585 \mathrm{~g} / \mathrm{cm}^{2}, \mathrm{Z}$-score -2.2 . Spine BMD was $0.888 \mathrm{~g} / \mathrm{cm}^{2}, Z$-score -1.4 . We concluded that the resolution without subchondral collapse of the femoral heads suggests that the original diagnosis was $\mathrm{TOH}$ right side. Diagnosis of TOH may only be achieved retrospectively as the natural course of the disease ensues and, therefore, invasive surgical treatment should be postponed unless significant stress fractures occur.

\section{P840}

\section{THEORETICAL INVESTIGATION OF OSTEOPO-} ROTIC LUMBAR VERTEBRA L1 UNDER STATIC COMPRESSION LOAD

$\underline{\text { O. Ardatov }}^{1}$, V. Alekna ${ }^{2}$, A. Maknickas ${ }^{3}$, M. Tamulaitiene ${ }^{2}$, R. Kacianauskas ${ }^{3}$

${ }^{1}$ Department of Biomechanics, Vilnius Gediminas Technical University, Vilnius, Lithuania, ${ }^{2}$ Faculty of Medicine, Vilnius University, Vilnius, Lithuania, ${ }^{3}$ Institute of Mechanics, Vilnius Gediminas Technical University, Vilnius, Lithuania O. Ardatov $^{1}$, V. Alekna ${ }^{2}$, A. Maknickas ${ }^{3}$, M. Tamulaitiene ${ }^{2}$, R. Kacianauskas ${ }^{3}$

${ }^{1}$ Department of Biomechanics, Vilnius Gediminas Technical University, Vilnius, Lithuania, ${ }^{2}$ Faculty of Medicine, Vilnius University, Vilnius, Lithuania, ${ }^{3}$ Institute of Mechanics, Vilnius Gediminas Technical University, Vilnius, Lithuania

Objective: To investigate the influence of degenerative tubercular aging behaviour on the load carrying capacity of human lumbar vertebra $\mathrm{L}_{1}$. The static failure load basing on the von Mises stress criterion is chosen to predict the risk of failure.

Materials and Methods: The new trabecular bone geometry model of perpendicular arranged cylindrical lumens was proposed. Both trabecular and surrounding compact bone was modelled as transversally orthotropic elastoplastic material. The intervertebral disks were assumed isotropic and perfectly elastic. Osteoporotic influence for the vertebra is characterised by density depending decrease of elasticity modulus of trabecular bone, increasing diameters of intertrabecular lumens and thickening of cortical shell. The 3D finite element (FE) method of the inhomogeneous vertebra body by applying volume elements was developed, and the nonlinear static analysis was performed to define its mechanical state behaviour model under the action of compression load.

Results: Numerical FE results allow discovering contribution of aging to the failure load which is predicted by the von Mises strength criterion. The contribution is demonstrated for various combinations of degradation parameters: apparent density and different load values. Stress distribution on cortical shell of the model is shown that the highest von Mises stresses occurred in the middle of the cortical shell on the front and on posterior sides of the model. The value of stress was about $200 \%$ higher for model with low mineral density $\left(0.1 \mathrm{~g} / \mathrm{cm}^{3}\right)$, than for normal $\left(0.35 \mathrm{~g} / \mathrm{cm}^{3}\right)$ under $0.75 \mathrm{MPa}$ load by shell thickness $0.4 \mathrm{~mm}$. Obtained results were compared with calculation on the similar model by continuum cancellous bone instead of trabeculated tissues.

Conclusion: The parametric study illustrates the nature of failure load due to aging bone degradation where thickening of cortical shell prevails over degradation of trabecular bone properties.

\section{P841 \\ VITAMIN D LEVELS IN PHYSICAL MEDICINE AND REHABILITATION INPATIENTS}

$\underline{\text { F. Yilmaz }}^{1}$, B. Doğu ${ }^{1}$, C. Mert ${ }^{1}$, J. Öncü ${ }^{1}$, B. Kuran ${ }^{1}$, C. Erçalık $^{1}$

${ }^{1}$ Şişli Etfal Hospital, Istanbul, Turkey

Aim: Although Turkey is rich in sunlight, 25 -hydroxyvitamin $\mathrm{D}$ deficiency is a frequent problem in our patients. This study aims to detect the vitamin D levels of inpatients in the physical medicine and rehabilitation (PM\&R) department.

Material and Method: Medical records of 410 inpatients hospitalised between January- December 2014 were reviewed. 273 patients whose vitamin D levels have been detected were included in the study. Patients were stratified according to their ages and average vitamin $\mathrm{D}$ values were calculated at each decade. Patient was vitamin D deficient if the level was $<20 \mathrm{ng} / \mathrm{ml}$, insufficient if the level was 21 $29 \mathrm{ng} / \mathrm{ml}$ and normal if the level was $>30 \mathrm{ng} / \mathrm{ml}$.

Results: Mean age of the 273 patients (F/M: 197/76) was $59.07 \pm 16.14$ years. Average vitamin D level was 13.83 $\pm 12.43 \mathrm{ng} / \mathrm{dl}$. Vitamin D level was similar in two genders and it was deficient in every decade. As a total, 218 patients (80\%) had deficient, 30 (11\%) patient had insufficient and 25 (9\%) patients had normal levels of vitamin D. While 43 patients had secondary hyperparathyroidism, none of them had primary hyperparathyroidism. Vitamin D level was observed 
to decrease as the decades increased. Most (22\%) of the deficiency was observed to be at the 7 th and 8 th decades.

Conclusion: Vitamin D deficiency was observed in inpatients of the PM\&R department at every age. We suggest that vitamin D level should be analysed and replaced whenever necessary.

\section{P842}

\section{PREVALENCE OF OA AMONG HOSPITALIZED PATIENTS AND DISEASE BURDEN}

Y. M. Soe ${ }^{1}$

${ }^{1}$ Rheumatology Department, 500-bedded Specialties Hospital, Yangon, Myanmar

Aim: To determine the prevalence of osteoarthritis among the hospitalized patients for other medical diseases and to identify the risk factors and functional status of OA patients.

Methods: The study will be performed in Rheumatology Department of 500-bedded Specialty Hospital and Medical units of Yangon General Hospital from March 2016 to March 2017 after getting the Ethical approval from University of Medicine, Yangon. All newly admitted patients aged 16 years and above will be surveyed. Diagnosis of knee OA will be reached according to the examination results based on ARA diagnostic criteria for knee OA by two rheumatology advanced trainees. Demographic data (age, sex, residence, education, occupation, marital status), their functional status by Myanmar Quality of Life index (MQoL), WOMAC score, self-administered Comorbidity Questionnaire(SACQ), consultation history for OA, treatment taken will be asked by using the structured questionnaire. Height, weight, BMI, waisthip circumference and knee examination will be done. Knee X-rays will be reviewed by consultant radiologist. The data will be analyzed by using statistical software SPSS.

Conclusion: Osteoarthritis is one of the most common forms of bone and joint diseases. The burden of osteoarthritis is neglected in least developing countries like our country. This study is very first study of OA from one and only Rheumatology department of Myanmar and aims to find out its prevalence, disease burden and patients perception and to reduce its impact.

\section{P843}

A REDUCTION IN KIDNEY FUNCTION IS ASSOCIATED WITH BMD AND BONE LOSS IN ELDERLY WOMEN

$\underline{\text { K. Akesson }}{ }^{1}$, L. Malmgren ${ }^{1}$, F. E. McGuigan ${ }^{1}$, A. Christensson $^{2}$

${ }^{1}$ Clinical and Molecular Osteoporosis Research Unit, Dept of Clinical Sciences Malmo, Lund University, Malmo, Sweden,

${ }^{2}$ Internal Medicine Research Unit, Dept of Clinical Sciences Malmo, Lund University, Malmo, Sweden

Objective: Chronic kidney disease (CKD) is associated with BMD and bone loss, but longitudinal studies in elderly women are scarce. We investigated association between estimated kidney function (eGFR) and BMD; specifically, how BMD and bone loss differ with CKD stage, in the population based OPRA cohort of women $(n=1044)$ all aged 75 at baseline and followed for 10 years.

Methods: Plasma cystatin $\mathrm{C}(\mathrm{CysC})$ was measured at age $75(n=981), 80(n=685)$ and $85(n=365)$. eGFR was calculated using the CKD-EPI equation. BMD was measured by DXA and biochemical bone markers assayed. Multilinear regression adjusted for weight, smoking and serum vitamin D was performed for eGFR. Women were also stratified by CKD stage (12, $3 \mathrm{~A}$ or $3 \mathrm{~B}-5$ ) to determine association with BMD and bone loss.

Results: Kidney function was associated with femoral neck (FN) BMD at age 75 and $80 \quad(p=0.028$; $p=0.001)$. FN-BMD did not differ with CKD stage, but women with stage $3 \mathrm{~A}$ or $3 \mathrm{~B}-5$ had higher total body (TB) BMD $(p<0.05$ at all ages). Stage $3 \mathrm{~A}$ or 3B-5 lost more FN-BMD between age 75-80 compared to stage $1-2(p=0.016$ and $p=0.007)$ and stage 3B-5 lost more TB-BMD $(p=0.030)$. Women in CKD stage 3B-5 also had higher PTH, phosphate, osteocalcin and ALP levels at baseline (all $p<0.001$ ). There was no association between kidney function and BMD at age 85 and bone loss $80-85 y$ was not significant.

Conclusion: Kidney function estimated by cystatin $\mathrm{C}$ is associated with femoral neck BMD in elderly women. Women with the worst kidney function have a higher rate of bone loss. The association between kidney function, BMD and bone loss is absent in women $>80$ years, suggesting that in the very elderly, the general decline in health outweighs the kidney specific decline with increasing age. 


\section{P844}

CHRONIC HYPOPHOSPHATEMIC OSTEOMALACIA MIMICKING OSTEOPOROSIS-CASES PRESENTATION

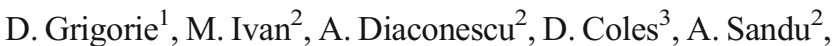
M. Grigorie ${ }^{4}$, A. Sucaliuc ${ }^{1}$

${ }^{1}$ National Institute of Endocrinology, Carol Davila University of Medicine, Bucharest, Romania, ${ }^{2}$ National Institute of Endocrinology, Bucharest, Romania, ${ }^{3}$ Medicine, Bucharest, Romania, ${ }^{4}$ National Institute of Geriatrics, Bucharest, Romania

We report on seven patients (two women and five men, aged 49-69 yrs) referred to our bone department for the evaluation of very low BMD by DXA: mean lumbar spine $\mathrm{BMD}=0.675 \mathrm{~g} / \mathrm{cm}^{2}$, mean $\mathrm{T}$ - score $=-4.5 \mathrm{SD}$ and mean $\mathrm{Z}$ score $=-2.6 \mathrm{SD}$.

Clinical findings at presentation: diffuse bone pain (all patients), multiple fractures (5 patients), muscular weakness ( 5 patients). The patients did not have any history of malabsorption, short stature, or limb deformity; one male patient had spine entesopathy and almost normal BMD. Four patients received antiresorptive therapy for "osteoporosis" before presentation. Four patients had pseudofractures on X-rays. The blood chemistries showed moderate-severe hypophosphatemia in all patients (range $0,9-2 \mathrm{mg} / \mathrm{dl}$, mean $1.44 \mathrm{mg} / \mathrm{dl}$ ), normal calcium and high levels of alkaline phosphatase (range 161-747 U/1). The serum 25OHD was very low in two patients $(6.26 \mathrm{ng} / \mathrm{ml} ; 7.41 \mathrm{ng} / \mathrm{ml})$ accompanied by secondary hyperparathyroidism, and normal in the others (mean value: $24.44 \mathrm{ng} / \mathrm{ml}$ ). Five patients had low TmP/GFR $(1-1.8 \mathrm{mg} / \mathrm{dl})$ suggesting an excessive renal loss of phosphate; one patient had acquired Fanconi syndrome. Serum FGF-23 measurements were not available. All patients had high bone remodeling by serum $\beta$ CrossLaps (mean $1.37 \mathrm{ng} / \mathrm{ml}$, range $0.61-3.31 \mathrm{ng} / \mathrm{ml}$ ), and total alkaline phosphatase; serum osteocalcin (mean $22.6 \mathrm{ng} / \mathrm{ml}$; range 15.8-32.7 ng/ml) was normal. The five patients, with apparent primary renal loss, were treated with phosphates salts ( $2 \mathrm{~g} /$ day), responded with spectacular improvement of clinical status, moderate increase in serum Pi and huge increases in BMD at both spine (about $20 \%$ at one year) and hip. One patient suspected of TIO had initial negative imaging studies; after three years focal neurologic symptoms led to removal of a cerebral hemangiopericytoma.

Conclusion: Acquired hypophosphatemic osteomalacia is sometimes discovered as low bone density on DXA scanning and incurs an appropriate differential diagnosis.

\section{P845}

ATYPICAL FEMORAL FRACTURES: CASE SERIES AND PROPOSAL OF AN ALGORITHM FOR MANAGEMENT

G. Toro ${ }^{1}$, A. de Sire ${ }^{1}$, A. Moretti ${ }^{1}$, C. Ojeda-Thies ${ }^{2}$, P. CabaDoussoux ${ }^{2}$, G. Martinez-Diaz Guera ${ }^{3}$, G. Iolascon ${ }^{1}$

${ }^{1}$ Department of Medical and Surgical Specialties and Dentistry, Second University of Naples, Naples, Italy, ${ }^{2}$ Trauma Unit, Department of Orthopaedic Surgery and Traumatology, Hospital Universitario 12 de Octubre, Madrid, Spain, ${ }^{3}$ Metabolic Bone Disease Unit, Department of Endocrinology, Hospital Universitario 12 de Octubre, Madrid, Spain

Objective: To purpose a practical tool for approaching atypical femoral fractures (AFF) considering the current evidences and experience of a multidisciplinary study group.

Material and Methods: We examined a series of clinical cases with a diagnosis of AFF according with 2013 ASBMR criteria. After a consensus of our study group, we purposed an algorithm in order to improve the knowledge in terms of early diagnosis and conservative and surgical treatment.

Results: From the analysis of 30 clinical cases of AFF (28 female and 2 male, mean aged $76.06 \pm 6.56$ years) our group debated on 4 main topics regarding the AFF: diagnosis, evaluation of bone turnover, treatment of the fractured femur, and management of the contralateral femur. These clinical issues represented the 4 steps of our proposed algorithm.

Figure 1. AFF complicated by an intraoperative fracture and subsequent infection. A) Pre-operative X-ray showing an incomplete AFF in a very bowed femur; B) Reduction and synthesis of the intraoperative fracture using a locking plate; C) External fixation after removal of locking plate because of the development of an infection.

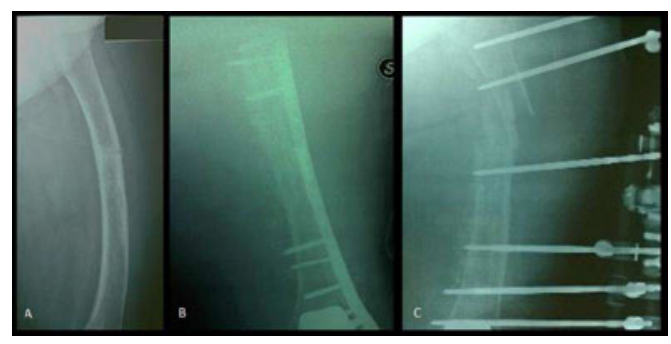

Conclusions: A multidisciplinary approach is mandatory in order to face up the AFF. Based on our clinical experience, we proposed a practical guide for diagnosis and management of AFF. However, there is a lack of adequate evidences on treatment of AFF and further studies are needed. 


\section{P846}

THE RISK OF VERTEBRAL FRACTURES IS ASSOCIATED WITH THYROID AUTOIMMUNITY IN EUTHYROID POSTMENOPAUSAL WOMEN

E. Armeni ${ }^{1}$, X. Papacharalambous ${ }^{2}$, P. Pliatsika ${ }^{1}$, D. Rizos ${ }^{3}$, G. Kaparos $^{3}$, A. Augoulea ${ }^{1}$, A. Alexandrou ${ }^{1}$, M. Flokatoula ${ }^{1}$, M. Creatsa ${ }^{1}$, C. Panoulis ${ }^{1}$, N. Triantafyllou ${ }^{2}$, I. Lambrinoudaki ${ }^{1}$

${ }^{1}$ 2nd Department of Obstetrics and Gynecology, National and Kapodistrian University of Athens, Aretaieio Hospital, Athens, Greece, ${ }^{2} 1$ st Department of Neurology, National and Kapodistrian University of Athens, Aiginiteio Hospital, Athens, Greece, ${ }^{3}$ Hormonal and Biochemical Laboratory, National and Kapodistrian University of Athens, Aretaieio Hospital, Athens, Greece

Objectives: To investigate the impact of thyroid autoimmunity on the development of vertebral fractures (VFs) in a sample of euthyroid postmenopausal women.

Methods: This cross-sectional study included a total of 335 euthyroid postmenopausal women. The euthyroid status was defined as serum levels of thyroid-stimulating hormone (TSH) within the laboratory reference range $(0.4-4.5 \mathrm{mIU} / \mathrm{ml})$. Quantitative procedures were implemented to identify the presence of VFs, using spine lateral radiographs. We compared levels of anti-thyroglobulin (anti-TG) and thyroid peroxidase antibodies (anti-TPO), according to the presence of VFs. Levels of thyroid antibodies were defined as "positive" or "negative" if the value of the antibody was higher or lower than 5 -fold the upper normal limit, respectively.

Results: Women with positive anti-TG and anti-TPO antibodies exhibited a significantly higher frequency of VFs compared to women with positive anti-TG or anti-TPO antibodies or women with negative antibodies $(26.3 \%$ vs. $5.3 \%$ vs. $5.9 \%$, chi-square p-val$\mathrm{ue}=0.003)$. Multivariate analysis showed that the development of VFs was independently predicted by: i) positive anti-TG antibodies $(O R=3.308$, p- val$\mathrm{ue}=0.026$ ), or by ii) positive anti-TG and anti-TPO antibodies $(O R=6.328$, p-value $=0.009)$; all models adjusted for age, menopausal age and insulin resistance. The results remained the same even after an additional adjustment for levels of $\ln$-transformed TSH.

Conclusion: According to our findings, the presence of anti-thyroid antibodies associated with the development of VFs in euthyroid, postmenopausal women, independently of age, menopausal age and insulin resistance. Although indicative, these findings may have implications on the evaluation of thyroid profile with respect to osteoporotic fracture risk. Further studies are necessary to elucidate the significance of our findings.
P847

CALCIUM INTAKE, ABDOMINAL FAT, BONE MICROARCHITECTURE AND BONE MINERAL DENSITY IN SPANISH MEN WITH HYPERTENSION

J. M. Moran $^{1}$, O. Leal-Hernandez ${ }^{1}$, M. L. Canal-Macias ${ }^{1}$, J. Lavado-Garcia $^{1}$, R. Roncero-Martin ${ }^{1}$, I. Aliaga ${ }^{1}$, J. D. Pedrera-Zamorano ${ }^{1}$

${ }^{1}$ Metabolic Bone Diseases Research Group, University of Extremadura, Caceres, Spain

Background: An adequate intake of calcium is only not limited to avoid the risk of osteoporosis and its benefits in long term bone health, but also it has been linked to protection against various major diseases, such as hypertension.

Aim: The primary aim of this study was to determine crosssectional association between calcium intake, abdominal fat and treatment of hypertension that could lead to any detrimental effect on bone microarchitecture evaluated by pQCT and BMD evaluated by DXA in male adults.

Methods: A Spanish cohort of male subjects participated in the study. In total 130 participants (age 67.07 \pm 7.61 years (mean $\pm \mathrm{SD}$ ); range 51-80) were analyzed. From the studied sample a total of $49(37.7 \%)$ patients were undergoing treatment for hypertension while 81 healthy volunteers matched for age $(P=0.053)$ and calcium intake $(P=0.901)$ were used as a control group. Volumetric BMD (vBMD) and structural parameters of the trabecular and cortical bone were assessed by $\mathrm{pQCT}$ of the distal radius while BMD was assessed by DXA in the femoral neck (FN), femoral trochanter (FT), Ward's Triangle (WT), L2, L3, L4 and total spine (L2-L4). Abdominal fat was measured by electric bioimpedance (BIA). Calcium intake was analyzed by a 7-day questionnaire.

Results: In the hypertension group the $31.8 \%$ of the patients have a calcium intake under the recommendations for Spanish adult population. Similarly the $35.6 \%$ of the participants in the control group have a calcium intake under the recommendations for Spanish adult population. Overall no significant differences were found between controls and treatment group in neither the bone microarchitecture nor the BMD. After further adjustment for the calcium intake, based in the recommendations for the Spanish population $(>800 \mathrm{mg} /$ day $)$, no differences in neither the bone microarchitecture nor the BMD where found. Based on the BMD similar figures were found in the diagnosis of osteopenia/osteoporosis between groups with the $61.7 \%$ of the control group and the $63.3 \%$ of the treatment group classified as normal based on the WHO criteria for T- score. A total 3 participants of the control group were diagnosed as osteoporotic $(n=3)$ while none of the hypertension group had a T-score under -2.5 in neither the FN nor the spine. In calcium intake/abdominal fat-adjusted models, 
pQCT revealed higher cortical density in HTA patients (adjusted cortical vBMD $558.07 \pm 16.11 \mathrm{~mm}^{3}$ ) than in the control group (adjusted cortical vBMD 511.71 $\left.\pm 14.56 \mathrm{~mm}^{3}\right)(P=0.045)$.

Conclusion: We have not found any detrimental effect in neither the bone microarchitecture nor the BMD in Spanish males undergoing treatment for hypertension. Due to the frequent coexistence of hypertension and osteoporosis, further investigations are needed to correctly understand the effects of long- term antihypertensive therapy on development, worsening, or improvement of osteoporosis in men.

\section{P848}

BONE-SPECIFIC ALKALINE PHOSPHATASE (BAP) AND CARBOXYTERMINAL TELOPEPTIDE OF TYPE I COLLAGEN (CTX) AS SERUM MARKERS OF BONE METASTASES IN POSTMENOPAUSAL WOMEN WITH COLORECTAL AND BREAST CANCER

R. Tozzoli ${ }^{1}$, A. Del Conte ${ }^{2}$, G. B. Chiara ${ }^{3}$, S. M. M. Basso ${ }^{3}$

${ }^{1}$ Clinical Pathology Laboratory, Department of Laboratory Medicine, S. Maria degli Angeli Hospital, Pordenone, Italy, ${ }^{2}$ Medical Oncology, S. Maria degli Angeli Hospital, Pordenone, Italy, ${ }^{3}$ Surgery 1, S. Maria degli Angeli Hospital, Pordenone, Italy

Background: Colorectal cancer (CRC) and breast cancer (BC) are common malignancy, and the bone is one of the possible sites of distant metastases from both $\mathrm{CRC}$ and $\mathrm{BC}$. Cancer cells invading the bone lead to the degradation of mineral matrix involving various cytokines, including IL- $1 \beta$, IL-3, IL- $6, \mathrm{TNF} \alpha$, prostaglandins and chemokines. Normal and malignant bone marrow cells interact together with this network of cytokines, ultimately leading to osteoclast activation adjacent to the cancer cells. Monitoring bone metabolism using various serum markers can be useful in early detection of bone metastases (BMs). The aim of this study was to investigate the usefulness of bone formation marker bone-specific alkaline phosphatase (BAP) and bone resorption marker carboxyterminal telopeptide of type I collagen (CTX) in postmenopausal women with BMs from CRC and BC.

Patients and Methods: Data were analyzed retrospectively from a prospective protocol enrolling 46 consecutive postmenopausal women (median age 61 years, range 51-70 years) with stage II-III CRC or BC. During follow-up, 21 patients developed BMs (cases), while 25 age- $(59.6 \pm 6.7$ vs. $61.0 \pm 5.5$ years, $p=0.44)$ and stage-matched (at the time of diagnosis of cancer) patients (controls) did not show BMs on ${ }^{18}$ F-FDG-
PET/CT. All patients underwent BAP and CTX measurement periodically.

Results: Cases had a significantly increased level of both BAP $(29.1 \pm 3.1$ vs. $22.3 \pm 2.8 \mathrm{ng} / \mathrm{mL}$, $p=0.0001)$ and CTX $(953.1 \pm 9.7$ vs. $767.5 \pm 5.6 \mathrm{pg} /$ $\mathrm{mL}, p=0.0001$ ) with respect to controls. An inverse correlation between age and CTX was found among cases $(R=-0.85, p<0.0001)$ and controls $(R=-0.58$, $p=0.002)$, while there was no correlation between age and BAP in both groups $(R=-033, p=0.14$ vs. $R=-0.10, p=0.62$ ). According to a cutoff value of $24 \mathrm{ng} / \mathrm{mL}$ (BAP) and $830 \mathrm{pg} / \mathrm{mL}$ (CTX), the sensitivity and specificity were $76.2 \%$ vs. $71.4 \%(p=0.73)$ and $84.0 \%$ vs. $88.0 \%(p=0.99)$, respectively. Both diagnostic accuracy $(80.4 \%)$ and pretest probability (prevalence: $45.6 \%$ ) were the same. The other results are shown in the Table below.

\begin{tabular}{lll}
\hline PARAMETER & BAP & CTX \\
\hline Sensitivity & $76.2 \%$ & $71.4 \%$ \\
Specificity & $84.0 \%$ & $88.0 \%$ \\
Positive predictive value & $80.0 \%$ & $83.3 \%$ \\
Negative predictive value & $80.7 \%$ & $78.6 \%$ \\
Diagnostic predictive value & $80.4 \%$ & $80.4 \%$ \\
Pre-test probability (prevalence) & $45.6 \%$ & $45.6 \%$ \\
Likelihood ratio for positive test result & 4.76 & 5.95 \\
Likelihood ratio for negative test result & 0.28 & 0.32 \\
Pre-test odds & 0.84 & 0.84 \\
Post-test odds & 4 & 5 \\
True-positive/false-negative ratio & 3.2 & 2.5 \\
\hline
\end{tabular}

Conclusions: In postmenopausal women with CRC or BC, the usefulness of BAP and CTX measurement is limited in early diagnosis of BMs.

\section{P849}

METABOLIC COMPENSATION AND BONE QUALITY IN TYPE 2 DIABETES

J. Payer $^{1}$, P. Jackuliak ${ }^{1}$, M. Kuzma ${ }^{1}$, Z. Killinger ${ }^{1}$

${ }^{1}$ Comenius University, Faculty of Medicine, 5th Department of Internal Medicine, University Hospital, Bratislava, Slovakia

Introduction: Patients with both types of diabetes are known to have an increased risk of fractures. While in type 1 diabetes the major reason is low bone mass, patients with type 2 diabetes are at an increased risk despite increased BMD and this is caused by inferior quality of bone. The association between glycemic compensation and diabetic complications or comorbidities is well known. Various trials observed that poor 
glycemic control is associated with higher all types of fracture risk.

Objective: To determine the role of metabolic compensation measured by $\mathrm{A} 1 \mathrm{c}$ haemoglobin $(\mathrm{HbA} 1 \mathrm{c})$ on bone quality measured by trabecular bone score (TBS) and also on BMD in T2DM patients.

Patients and methods: A cross-sectional trial in 56 women patients with T2DM and 30 controls without DM. Mean age of the patients was $51.2 \pm 6.1$ years. The mean duration of diabetes was $10.3 \pm 5.8$ yrs. All patients were treated only by oral antidiabetic drugs (metformin and DPP-4), not by insulin. The BMD at lumbar spine (LS), femoral neck (FN) were measured at baseline and after 1 year of treatment by DXA (Hologic). TBS was performed by TBS Insight software (Medimaps, France) from LS DXA scans.

Results: Diabetes was associated with higher BMD than the control group $\left(1.008 \pm 0.175 \mathrm{~g} / \mathrm{cm}^{2}\right.$ vs. 0.961 $\left.\pm 0.176 \mathrm{~g} / \mathrm{cm}^{2}, p=0.05\right)$. The LS-TBS was lower in T2DM than in control group $(1.172 \pm 0.120$ vs. 1.304 $\pm 0.018, p<0.001)$. HbA1c was an important determinant effecting BMD $(r=-0.30, p<0.05)$ and TBS $(r=-0.35, p=0.01)$. But the cutoff was $8.5 \%$ for BMD and $7.9 \%$, respectively.

Conclusion: Our data confirm that poor glycemic control based on an $\mathrm{HbAlc}$ is an important determinant for BMD as a marker of bone quantity and also TBS, a marker of bone quality. It seems that the cutoff levels of $\mathrm{HbA} 1 \mathrm{c}$ are others for $\mathrm{BMD}$ respectively TBS. Reliable HbA1c cutoffs need to be determined in larger prospective studies. Limitations of are trial are, that there can be other factors affecting the correlations like duration of diabetes, treatment modalities, other diabetic complications.

\section{P850}

EFFECTS OF BIOLOGIC DRUGS ON BONE TRABECULAR SCORE AND BONE MINERAL DENSITY IN RHEUMATOID ARTHRITIS PATIENTS Z. Killinger $^{1}$, L. Gajdarova ${ }^{1}$, M. Kuzma ${ }^{1}$, P. Jackuliak ${ }^{1}$, J. Payer $^{1}$

${ }^{1}$ Comenius University, Faculty of Medicine, 5th Department of Internal Medicine, University Hospital, Bratislava, Slovakia

Introduction: Patients with rheumatoid arthritis (RA) have a greater risk of osteoporosis (OP) and fracture than the general population. The most important factor involved in the pathogenesis is the inflammation. Thus, inhibition of TNF may influence activity of RA as well as BMD. Decreased BMD not entirely explains the fracture risk in RA patients. Past few years, trabecular bone score (TBS) is promising method for assessment of bone quality and risk of fracture.
Objective: To compare the effects of biological disease-modifying antirheumatic drugs (bDMARDs) to conventional synthetic DMARDs (methotrexate) on BMD and TBS in patients suffering from active RA. Patients and methods: A 12-month prospective trial was performed in 105 patients with active RA. The cohort was divided into two groups: group 1 ( $n=84$, mean age 54 yrs) treated with bDMARDs (anti- TNF and IL- 6 blockers) and group $2(n=21$, mean age 53 years) treated with nonbiological DMARDs (methotrexate). There was no significant difference in baseline characteristics between both groups observed. The mean daily dose of glucocorticoids (GC) was $6.2 \mathrm{mg}$ vs. $6.6 \mathrm{mg}$ between group 1 vs. 2 (NS), respectively. Patients with bisphosphonates or other antiporotic treatment were not included. All patients were administered with calcium $(1000 \mathrm{mg})$ and vitamin D (800 IU). The BMD at lumbar spine (LS), femoral neck (FN) were measured at baseline and after 1 year of treatment by DXA (Hologic). TBS was performed by TBS Insight software (Medimaps, France) from LS DXA scans.

Results: We found in patients treated with biologics a significant increase in lumbar spine BMD (0.987 g/ $\mathrm{cm}^{2}$ vs. $\left.0.994 \mathrm{~g} / \mathrm{cm}^{2}, p=0.008\right)$. FN BMD remained unchanged (NS). In group 2, significant decrease in LS BMD $\left(0.993 \mathrm{~g} / \mathrm{cm}^{2}\right.$ vs.. $\left.0.989 \mathrm{~g} / \mathrm{cm}^{2}, p<0.001\right)$ and at FN BMD $\left(0.784 \mathrm{~g} / \mathrm{cm}^{2}\right.$ vs.. $0.782 \mathrm{~g} / \mathrm{cm}^{2}$, $p=0.016)$ was observed. A significant increase of TBS $(+1.5 \%, p=0.009)$ was observed in group 1 , exclusively.

Conclusion: BMD and TBS were significantly improved in patients treated with biological agents, whereas in control group BMD decreased and TBS remained unchanged. Thus, biologics improve not only the BMD but also may have a beneficial effect on bone quality represented by TBS.

\section{P851}

RELATIONSHIP BETWEEN OSTEOPOROSIS AND BREAST CANCER IN POSTMENOPAUSAL WOMEN WHO UNDERWENT HORMONE REPLACEMENT THERAPY: A MULTIVARIATE ANALYSIS USING A LOGISTIC REGRESSION MODEL

M. Ermani ${ }^{1}$, V. Camozzi ${ }^{2}$, G. B. Chiara ${ }^{3}$, S. M. M. Basso ${ }^{3}$

${ }^{1}$ University of Padua, School of Medicine, Department of Neurosciences, Padova, Italy, ${ }^{2}$ University of Padua, School of Medicine, Department of Medicine, Padova, Italy, ${ }^{3}$ Surgery 1, S. Maria degli Angeli Hospital, Pordenone, Italy 
Background: BMD represents an indicator of lifetime cumulative risk to endogenous estrogen exposure, and in both preand postmenopausal patients, there is a strict correlation between estrogen exposure and risk of breast cancer (BC). Thus, osteoporotic women have usually a reduced risk of developing BC. The aim of this study was to investigate whether the presence of osteoporosis should be considered an independent risk factor $(\mathrm{RF})$ of $\mathrm{BC}$, and its correlation with other RFs, including age at menarche and menopause and the use of hormone replacement therapy.

Patients and Methods: The medical records of 132 postmenopausal patients (age $60.1 \pm 6.5$ years) with BC (cases) and 146 age-matched $(58.8 \pm 6.1 \mathrm{y} ; p=0.065)$ postmenopausal women (controls) who have undergone annual mammographic screening to exclude the presence of subclinical BC were retrospectively reviewed. In all patients, standard lumbar spine BMD measurement by DXA for excluding osteoporosis had been performed.
Results: No significant differences were found ( $p=\mathrm{NS}$ ) between cases and controls with regard to the most part of classical risk factors for $\mathrm{BC}$, including number of pregnancies, months of breastfeeding, the use of oral contraceptives, and history of benign breast diseases. The interval between menarche and menopause (MMI) was (cases vs. controls) $37.4 \pm 1.9$ vs. 36.9 \pm 1.7 years $(p=0.021)$. Estrogen replacement therapy has been most widely used in patients with BC than in controls (44.7 \% vs. $32.9 \%, p=0.043$ ). Overall, osteoporosis (LT-score below -2SD) was found in $33 /$ $278(11.9 \%$ ) patients: $7.8 \%$ (cases) and $15.7 \%$ (controls; $p=0.03$, respectively (sensitivity $=15.7 \%$, specificity $92.4 \%, \mathrm{PPV}=69.7 \%, \mathrm{NPV}=49.8 \%$, diagnostic accuracy $=52.1 \%$ ). Multivariate analysis using a logistic regression model showed that only MMI and osteoporosis were independent factors related to $\mathrm{BC}$, and the area under the receiver operating characteristic (ROC) curve (AUC) was 0.62 (95\%CI: 0.58-0.67).

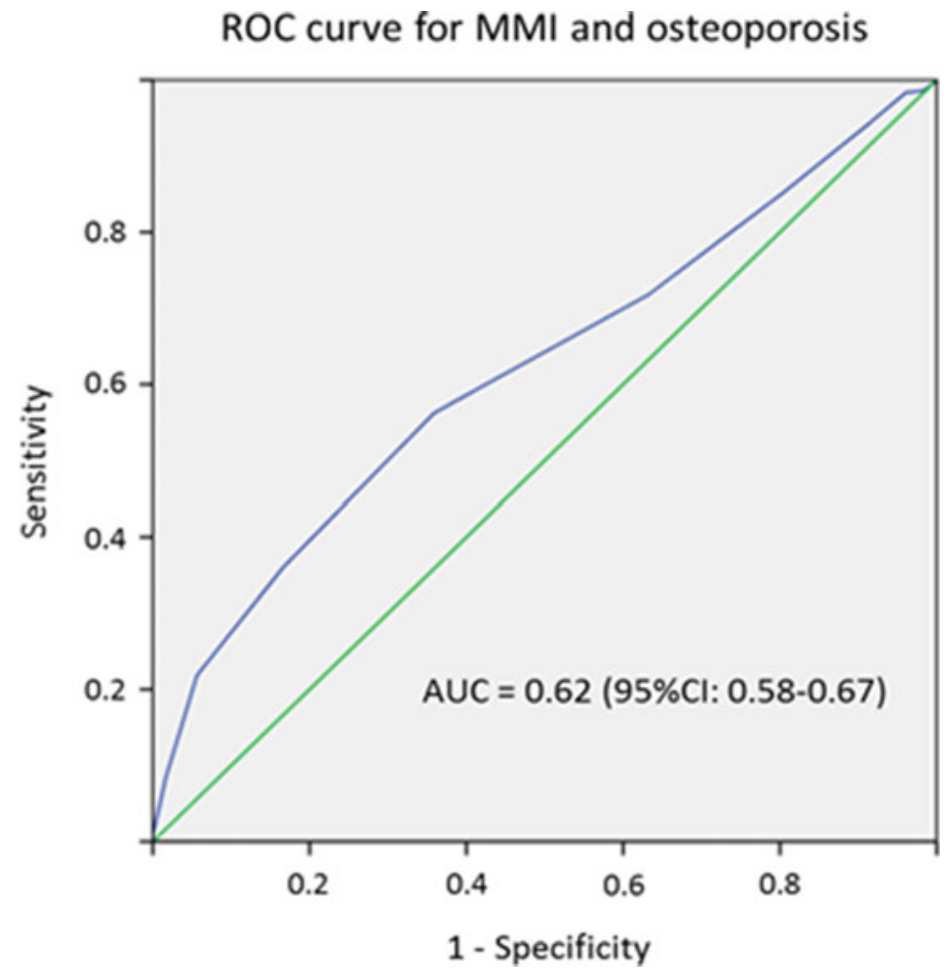

Conclusions: In our study population, there is a direct relationship between both $\mathrm{BC}$ and $\mathrm{MMI}$ and between $\mathrm{BC}$ and osteoporosis, being these RFs independent. Thus, postmenopausal women with normal BMD should require a more careful evaluation in terms of mammographic screening, according to the presence of other RFs. 


\section{P852}

MUTATIONS IN ACVR1 THAT DRIVE LESS COMMON CASES OF FIBRODYSPLASIA OSSIFICANS PROGRESSIVA PERCEIVE ACTIVIN A AS AN AGONIST

V. Idone $^{1}$, D. M. Alessi Wolken ${ }^{1}$, H. J. Kim ${ }^{1}$, J. McClain ${ }^{2}$, R. A. Corpina ${ }^{1}$, C. J. Schoenherr ${ }^{1}$, G. Della Gatta ${ }^{3}$, A. J. Murphy $^{4}$, S. J. Hatsell ${ }^{1}$, A. N. Economides ${ }^{1}$

${ }^{1}$ Skeletal Diseases TFA, Regeneron Pharmaceuticals Inc., Tarrytown, United States, ${ }^{2}$ Genome Engineering Technologies/ Regeneron Pharmaceuticals Inc., Tarrytown, United States, ${ }^{3}$ Functional Modeling, Regeneron Genetics Center, Tarrytown, United States, ${ }^{4}$ Target Discovery, Regeneron Pharmaceuticals Inc., Tarrytown, United States

Objectives: Fibrodysplasia ossificans progressiva (FOP) is a rare autosomal dominant disorder characterized by episodic, progressive and cumulative heterotopic ossification (HO) of connective tissue resulting in debilitating immobility. We recently showed that a normally antagonistic ligand - Activin A - activates Smad1/5/8 signaling through the most common mutant receptor, ACVR $1^{\text {R206H }}$. In this study we explored whether the more rare ACVR1 mutations identified in FOP also signal to Smad1/5/8 in response to Activin A.

Methods: Stable HEK293 lines overexpressing FOPcausing ACVR1 mutations and harboring a BMP responsive element (BRE) luciferase reporter to measure ligand-stimulated Smad1/5/8 signaling were used to assess the responsiveness of the FOP mutant receptors to Activin A.

Results: Mirroring what has been observed for the most common FOP variant of ACVR1 (ACVR1[R206H]) ${ }^{1}$, all FOP-associated ACVR1 variants, respond to Activin A by triggering Smad 1/5/8 signaling, while wild-type ACVR1 does not. All of the FOP variants tested either completely or partially activated the BRE reporter when stimulated with Activin A.

Conclusions: As an extension of our previous work, we found that Activin A stimulates Smad1/5/8 signaling in all of the ACVR1 mutations identified in FOP. Thus, the same therapeutic approach may apply to patients without the ACVR $1^{\mathrm{R} 206 \mathrm{H}}$ genotype, and indicates that the molecular mechanism of action of this altered property is likely shared among the majority of FOP-associated variants of ACVR1. In addition, these mutations cluster to one surface suggesting that they alter binding of a common intracellular regulator of Smad signaling; however, the exact molecular mechanism of action by which FOP-associated AVCR1 variants respond to Activin A remains to be elucidated.

\section{References:}

1. Hatsell SJ, et al. Science Translational Medicine. v.7(303). 2015
Disclosures: All the authors except for Giusy Della Gatta are employees of Regeneron Pharmaceuticals, Inc. Giusy Della Gatta is an employee of the Regeneron Genetics Center.

\section{P853}

RISK FACTORS FOR DEVELOPING OSTEOPOROSIS IN MEN

$\underline{\text { D. Grujoska Veta }}{ }^{1}$, M. Foteva ${ }^{1}$, Z. Bozinovski ${ }^{1}$, M. Samardziski ${ }^{1}$, A. Andonovski ${ }^{1}$, S. Stojkoska ${ }^{1}$

${ }^{1}$ University Clinic for Orthopaedic Surgery, Skopje, Former Yugoslav Republic of Macedonia

Osteoporosis is clinically silent disease until fractures occur, so risk factors for osteoporosis and the disease itself need to be identified and proper treatment suggested in order decreasing the morbidity as well as mortality associated with osteoporotic fractures.

Aim: The aim of the study was to present risk factors for developing osteoporosis in men through cases that were diagnosed at the university clinic for orthopaedic surgery - Skopje in the period of January 2012 to December 2013.

Material and Methods: A total of 3960 patients (2795 female, 165 male) underwent diagnostic clinical and radiological examination for BMD, at the university clinic for orthopaedic surgery-Skopje, in the period of January 2012 to December 2013. BMD was assessed with DXA with Hologic equipment. Examination was performed on the lumbar spine and hips. BMD was recorded as $\mathrm{g} / \mathrm{cm}^{2}$, as T-scores (SD from mean BMD of young adults) and Z-scores (SD from mean BMD of age-andsex matched controls). Data of laboratory examinations, previous and current diseases, surgical procedures, everyday habits, tobacco and alcohol use were noted.

Results: Osteoporosis was diagnosed in 1757 patients and osteopenia in 1708 patients. Results showed normal BMD findings in 495 patients. Osteoporosis was diagnosed in 27 men and osteopenia in $14 \mathrm{men}$. Average age was 60 years (18-81). Risk factors for male decreased BMD were: Crohn disease (4), Marfan syndrome (1), family history of osteoporosis (2), chronic gastritis and duodenal ulcers (7), orchiectomy (2), hyperparathyroidism (1), Multiple myeloma (1), dementia (1), malnutrition (4), excess alcohol consumption (4), excessive tobacco use (4), low body weight (2), previous vertebral fracture (3), neurological disorders (4), rheumatic diseases (3).

Conclusion: Findings of decreased BMD in younger men might suggest screening men earlier than 60 years of age, despite the fact that fracture rate increases rapidly after 70 years of age. 


\section{P854}

LOOKING FOR ANSWER: BREAST-FEEDING AND PARITY CONSIDER AS RISK OR PROTECTIVE FACTOR ON OSTEOPOROSIS

K. Mirzaei $^{1}$, L. Khorrami-Nezhad ${ }^{2}$, Z. Maghbooli ${ }^{2}$, S. Moradi $^{2}$, A. Hossein-Nezhad ${ }^{2}$

${ }^{1}$ Department of Community Nutrition, School of Nutritional Sciences and Dietetics, Tehran University of Medical Sciences (TUMS), Tehran, Islamic Republic of Iran, ${ }^{2}$ Osteoporosis Research Center, Endocrinology and Metabolism Clinical Sciences Institute, Tehran University of Medical Sciences, Tehran, Islamic Republic of Iran

Objective: In the present study we investigated the correlation of breast-feeding (BF) total time and number of child on BMD in menopause women. This study was conducted to know these are risk factor or have protective effect on postmenopausal osteoporosis.

Material and Method: We performed cross-sectional study including 235 postmenopausal women with the mean age of 57.8 , who were divided based on normal and low BMD (T-score $<-1)$. BMD was measured by DXA of the lumbar (L2-L4) and total hip. Associations between background characteristics and BMD were analyzed.

Results: One-way ANOVA showed a strong significance difference between hip T-score and lumbar Z-score with increasing $\mathrm{BF}$ duration $(p=0.068, p=0.033$, respectively). There was negative correlation between lumbar $\mathrm{BMD}$ and $\mathrm{BF}$ duration. It was observed that a significance difference existed between women with more than 3 parity with BMD and T-score reduction at lumbar site ( $p=0.086, p=0.040$, respectively). In linear model, after adjustment for age and BMI, there were significance difference between parity and hip T-score $(p=0.005$, CI: $-0.186,-0.036)$. There was no correlation between abortion and BMD.

Conclusion: Long term BF more than 60 months could be a risk factor for postmenopausal osteoporosis in both sites especially in lumbar. However an increase in number of parity could be causes the back pain in menopause women with effect of on lumbar. Maybe extended BF because of higher calcium turn over in this period had undesirable effect on BMD. On the other hand in developing countries, a large number of parity associated with lower quality of life and nutritional status.

Acknowledgements: The authors acknowledge Tehran University of Medical Sciences for the financial support for the study additionally, we acknowledge the osteoporosis research center, Tehran, Iran for allowing us to conduct the comparative cross sectional study.
P855

STUDY OF THE MICROALBUMINURIA IN RHEUMATOID ARTHRITIS AND ITS CORRELATION WITH DISEASE ACTIVITY

A. Mittal $^{1}$

${ }^{1}$ University of Delhi, New Delhi, India

Objective: In rheumatoid arthritis (RA), CRP and ESR are nearly always elevated and reflect the disease activity. But the role of microalbuminuria with disease activity in RA is not clear. We aimed to study the association of microalbuminuria with RA and its correlation with disease activity.

Material \& Methods: Sixty patients of RA satisfying 2010 ACR/EULAR classification criteria without renal involvement were enrolled in study. The patients were subjected to detailed clinical history and examination with special emphasis on evaluation for swollen and tender joint count. Chi Square/Fisher Exact test was used to find the association of clinical factors between patients with and without the presence of microalbuminuria. Student- $t$ test was used to find the significance of investigation parameters between two groups of patients and $p$ value of less than 0.05 was considered statistically significant.

Results: Mean age of microalbuminuria positive patient was $42.14 \pm 11.03$ vs. $43.33 \pm 11.50$ in microalbuminuria negative group. Mean ESR, Mean CRP and Mean RA factor values were $83.71,49.14$ and 200.05, respectively, in microalbuminuria positive patients that was significantly higher as compared to microalbuminuria negative group. Severity of disease correlated with both CRP and Microalbuminuria.

Conclusions: The renal involvement in RA is with both glomerular and tubular damage. Presence of microalbuminuria indicates severe disease activity and long standing RA. Microalbuminuria is a sensitive indicator of early subclinical renal dysfunction and drug induced renal damage in RA. Methods for microalbuminuria detection should routinely be used in all RA patients to detect renal involvement in initial phase to devise the most appropriate treatment.

\section{P856}

COMPLEX PILON FRACTURE MANAGEMENT IN ELDERLY, OSTEOPOROTIC WOMAN

$\underline{\text { R. Ene }}{ }^{1}$, Z. A. Panti ${ }^{1}$, M. Nica ${ }^{1}$, M. Pleniceanu ${ }^{1}$, M. Cirstoiu ${ }^{2}$, C. Cirstoiu ${ }^{1}$

${ }^{1}$ Orthopedics and Trauma, Emergency University Hospital Bucharest, Bucharest, Romania, ${ }^{2}$ Obstetrics and Gynecology, Emergency University Hospital Bucharest, Bucharest, Romania

Objectives: Tibial pilon fractures and unstable ankle fractures have a challenging surgical management in the general 
population, yielding predictably fair outcomes. The situation changes when it comes to treatment of such injuries in the osteoporotic, usually elderly patients. There is a debate about the surgical management in this age group because of a higher risk of various complications. Failure of fixation is one of these feared complications and is generally accepted to be associated with low BMD. This paper underlines the importance of the bone density for good results after surgical fixation of such demanding fracture patterns.

Material and Methods: We present the case of a 66 year old female with severe osteoporosis diagnosed with left tibial pilon and left lateral malleolus fracture after low energy trauma. Surgical fixation with an L shaped plate, malleolar screw and Kirschner wire was performed.

Results: At 3 months postoperatively the patient presents in the emergency department with unremitting pain and edema of the left ankle. The radiological examination confirms the failure of the osteosynthesis with varus deformation and revision surgery is considered. Due to high fragility of the osteoporotic bone we decided to perform closed reduction and use supplementary transcalcanean fixation with Kirschner wires instead of full revision.

Conclusion: Even for familiar injuries like ankle and tibial plafond fractures surgeons are hesitant to recommend surgery as a treatment option in the geriatric population, with low BMD. This happens because of the high risks associated with the precarious biomechanical environment provided by the osteoporotic bone. Although surgical approaches and fixation methods used are similar to the principles used in the surgical treatment of younger patients, special attention and some modifications have to command the primary management or revision after this pathology in this patient population.

\section{P857}

IS THERE ANY ASSOCIATION BETWEEN BODY FITNESS WITH DISCORDANCE OR CONCORDANCE OF BONE?

L. Khorrami-Nezhad $^{1}$, K. Mirzaei ${ }^{2}$, Z. Maghbooli ${ }^{1}$, S. Moradi $^{1}$, A. Hossein-Nezhad ${ }^{1}$

${ }^{1}$ Osteoporosis Research Center, Endocrinology and Metabolism Clinical Sciences Institute, Tehran University of Medical Sciences, Tehran, Islamic Republic of Iran, ${ }^{2}$ Department of Community Nutrition, School of Nutritional Sciences and Dietetics, Tehran University of Medical Sciences (TUMS), Tehran, Islamic Republic of Iran

Objective: There is a contradiction to relationship between weight or BMI on bone. To clarify this inconsistency we have used the discordance concept. Our aim was to assess the prevalence of T-score discordance and its association with body fitness in postmenopausal women.
Material and Method: A total of 235 obese subject, $17<\mathrm{BMI}<41$; with the mean age of 57.8 ; were included in the current cross-sectional study. We assessed the body composition with body composition analyzer and DXA was used for measurement of BMD in hip and lumbar. We divided bone dis or concordance status to 6 categories for compared to BMI, fat mass, weight, height. These groups were included: bone concordance (both normal, both osteopenia, both osteoporosis in two measured sites), Bone discordance (normal-osteopenia, osteopenia-osteoporosis, normal- osteoporosis in two measured sites).

Results: After categorized to bone discordance (41.2\%) and bone concordance $(52.8 \%$ ), we found negative relationship between hip circumferences (HP) and discordance, so with increasing discordance specially major type, HP were decreased $(p=0.013)$. Major discordance was seen in women with older age $(p=.001)$.There were positive relationship between discordance (normal-osteopenia) and obesity, fat mass, both arm fat $(p=0.011, p=0.028, p$ (right arm $)=0.028, p($ left arm $)=0.014$, respectively). By regression model in subject with osteoporosis, at least in one site appear had less weight (p (both site) $<0.001 ; O R=0.933, \mathrm{p}$ (one site) $=0.065$; $O R=0.961)$.

Conclusion: After grouping we saw the positive relationships between minor discordance and BMI, fat mass. Discordance will increase with age. Participants who were underweight were also at risk for osteoporosis in one or two sites. Discordance could be a reason for height reduction in elderly. Acknowledgements: The authors acknowledge Tehran University of Medical Sciences for the financial support for the study additionally, we acknowledge the osteoporosis research center, Tehran, Iran for allowing us to conduct the comparative cross sectional study.

\section{P858}

MINERALS INTAKE MAY PREDICT THE BONE DISCORDANCE BETWEEN TWO TYPES OF BONE TISSUE: LUMBAR (L2-L4) AND TOTAL HIP

K. Mirzaei ${ }^{1}$, L. Khorrami-Nezhad ${ }^{2}$, Z. Maghbooli ${ }^{2}$, S. A. Keshavarz $^{3}$, A. Hossein-Nezhad ${ }^{2}$

${ }^{1}$ Department of Community Nutrition, School of Nutritional Sciences and Dietetics, Tehran University of Medical Sciences (TUMS), Tehran, Islamic Republic of Iran, ${ }^{2}$ Osteoporosis Research Center, Endocrinology and Metabolism Clinical Sciences Institute, Tehran University of Medical Sciences, Tehran, Islamic Republic of Iran, ${ }^{3}$ Department of Clinical Nutrition, School of Nutritional Sciences and Dietetics, Tehran University of Medical Sciences (TUMS), Tehran, Islamic Republic of Iran

Objectives: Minerals intake is effective on BMD But they have different effects on trabecular and cortical bones and 
need to be evaluated. To determine the effect of minerals intake on the bone discordance between the total hip and lumbar (L2-L4) in women 22-50 years old, secondly due to bone discordance, which minerals are needed to prevent the progression to osteoporosis and fractures.

Material and Method: A total of 325 obese subject, $\mathrm{BMI} \geq 30$; age, 22-50; were included in the current crosssectional study. We assessed the body composition with the use of body composition analyzer and BMD measurements by DXA of the lumbar (L2-L4) and total hip. Dietary data were collected through a 3-day food record. Statistical analyses were performed using SPSS and N4 software.

Results: There was significant difference in age, BMI, fat mass and weight between groups $(p<0.0001)$. By correlation we found significance correlation between calcium intake and hip BMD $(P=0.03)$ and between fluoride intake and lumbar $\operatorname{BMD}(P=0.05)$, potassium intake with hip T-score $(P=0.01)$, manganese and selenium intake with hip Z-score (p-value $=0.04$, p-value $=0.03$, respectively).

Conclusion: We found there was a strong relationship between minerals intake and effect of that on cortical and trabecular bone. There were significant correlation between potassium, phosphorus with hip as a cortical bone and selenium with lumbar. An interesting result was deficiency of calcium intake was more effective in hip than the lumbar but the lack of fluoride has the reverse effect.

Acknowledgements: The authors acknowledge Tehran University of Medical Sciences for the financial support for the study additionally, we acknowledge the osteoporosis research center, Tehran, Iran for allowing us to conduct the comparative cross sectional study.

\section{P859}

\section{IS DIETARY ACID LOAD REVEALS FACTS ABOUT BONE LOSS IN WOMEN?}

L. Khorrami-Nezhad $^{1}$, K. Mirzaei ${ }^{2}$, Z. Maghbooli ${ }^{1}$, S. A. Keshavarz $^{3}$, A. Hossein-Nezhad ${ }^{1}$

${ }^{1}$ Osteoporosis Research Center, Endocrinology and Metabolism Clinical Sciences Institute, Tehran University of Medical Sciences, Tehran, Islamic Republic of Iran, ${ }^{2}$ Department of Community Nutrition, School of Nutritional Sciences and Dietetics, Tehran University of Medical Sciences (TUMS), Tehran, Islamic Republic of Iran, ${ }^{3}$ Department of Clinical Nutrition, School of Nutritional Sciences and Dietetics, Tehran University of Medical Sciences (TUMS), Tehran, Islamic Republic of Iran

Objective: Bone loss is a critical concern for elderly and influenced by several factors especially dietary intake. The objective of this cross-sectional study was to determine effect of dietary acid load (DAL) on BMD in hip and lumbar sites by measuring potential renal acid load (PRAL).
Material and Method: A total of 285 obese subjects, $\mathrm{BMI}=35.3$; with the mean age of 39.7; to evaluate the effect dietary acid load on BMD. Dietary intake to calculated DAL was estimated from 3-day food records by PRAL algorithm. Anthropometry was measured. We assessed the body composition with the use of body composition analyzer and DXA was used to assess BMD in hip and lumbar spine.

Results: By correlation analysis, PRAL had significant negative correlation with T-score $(r=-0.329, P=0.007)$ and Zscore $(r=-0.335, P=0.005)$ in hip site. Subjects in our study divided into two groups by PRAL (low PRAL \& high PRAL). We found women who were with low PRAL had higher fat mass, FBS and also T-score, Z-score and BMD in hip site $(P<0.05)$ but in subject with high PRAL were seen lower level on fat-free mass.

Conclusion: DAL associated with hip BMD that means obese women who take a diet with high acid load were at risk to bone loss specially in this area. This relationship was not observed for lumbar site. Another interesting finding was subject with higher PRAL had more free-fat mass and lower fat mass and FBS.

Acknowledgements: The authors acknowledge Tehran University of Medical Sciences for the financial support for the study; additionally, we acknowledge the osteoporosis research center, Tehran, Iran for allowing us to conduct the comparative cross sectional study.

\section{P860}

RELIABILITY AND VALIDITY OF THE BINDEX® ULTRASOUND BONE DENSITOMETER

M. Behrens ${ }^{1}$, J. Karjalainen ${ }^{2}$, S. Felser ${ }^{1}$, A. Mau-Moeller ${ }^{3}$, M. Weippert ${ }^{1}$, J. Pollex ${ }^{1}$, R. Skripitz ${ }^{3}$, P. Herlyn ${ }^{4}$, D.-C. Fischer ${ }^{5}$, S. Bruhn ${ }^{1}$, H.-C. Schober ${ }^{6}$, V. Zschorlich ${ }^{1}$, T. Mittlmeier ${ }^{7}$

${ }^{1}$ Institute of Sport Science, University of Rostock, Rostock, Germany, ${ }^{2}$ Department of Applied Physics, University of Eastern Finland, Kuopio, Finland, ${ }^{3}$ Department of Orthopaedics, Rostock University Medical Center, Rostock, Germany, ${ }^{4}$ Department of Traumatology, Hand- and Reconstructive Surgery, Rostock, Germany, ${ }^{5}$ Department of Pediatrics, Rostock University Medical Center, Rostock, Germany, ${ }^{6}$ Department of Internal Medicine, Klinikum Südstadt, Rostock, Germany, ${ }^{7}$ Department of Traumatology, Hand- and Reconstructive Surgery, Rostock University Medical Center, Rostock, Germany

Objectives: The purpose of this study was to analyze (I) the relative and absolute intra- and inter-session reliability of the Bindex ${ }^{\circledR}$ quantitative ultrasound (QUS) device and (II) the relationship between the data provided by Bindex ${ }^{\circledR}$-QUS and the BMD measured by DXA at different skeletal sites.

Materials and Methods: 28 young and healthy subjects (19 males, 9 females, age: $25.0 \pm 3.6 \mathrm{yrs}$, weight: $74.1 \pm 11.2 \mathrm{~kg}$, 
height: $176 \pm 8 \mathrm{~cm}$ ) with no history of injuries were invited to participate in this study. All test persons underwent two measurement sessions with the Bindex ${ }^{\circledR}$ device with one week inbetween. In both sessions, the thickness of cortical bone at the distal radius as well as at the distal and proximal tibia was measured twice in order to analyze intra- and inter-session reliability. A density index was calculated for all measurements, which is based on the QUS results in combination with subject characteristics. Participants were also investigated using DXA regarding their BMD of the distal radius, distal and proximal tibia, lumbar spine and femoral neck. Relative (intraclass correlation coefficient (ICC)) and absolute reliability (coefficient of variation $(\mathrm{CV})$ ) of the Bindex ${ }^{\circledR}$ QUS were analyzed. Pearson product-moment correlation and linear regression were applied to investigate the association between the parameters provided by DXA and the parameters calculated by the Bindex ${ }^{\circledR}$ device.

Results: Data show a very good relative and absolute intra( $\mathrm{ICC}=0.977, \mathrm{CV}=1.5 \%$ ) and inter-session reliability ( $\mathrm{ICC}=0.978, \mathrm{CV}=1.4 \%$ ) for the density index. The intrasession ICCs and CVs for the distal radius as well as distal and proximal tibia are $\geq 0.889$ and $\leq 5.5 \%$. The inter-session ICCs for the distal tibia and distal radius are $\geq 0.892$ while the values for the proximal tibia are less reliable $(\mathrm{ICC}=0.713)$. The absolute inter-session reliability (CVs) is below $7.4 \%$. The highest positive correlations were found between cortical thickness and BMD for the distal radius and distal tibia $(\mathrm{r} \geq 0.71)$.

Conclusions: Data indicate that the Bindex ${ }^{\circledR}$-QUS parameters are repeatable within and between measurement sessions. Furthermore, the measurements reflect the BMD at specific skeletal sites. Bindex ${ }^{\circledR}$-QUS might be a useful tool for the measurement of skeletal impairments and adaptations.

\section{P861 \\ REVISION SURGERY OF TOTAL KNEE JOINT REPLACEMENT IN AN ELDERLY, LOW BONE MINERAL DENSITY PATIENT

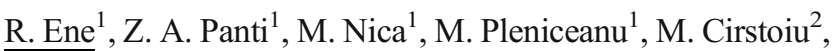 C. Cirstoiu $^{1}$ \\ ${ }^{1}$ Orthopedics and Trauma, Emergency University Hospital Bucharest, Bucharest, Romania, ${ }^{2}$ Obstetrics and Gynecology, Emergency University Hospital Bucharest, Bucharest, Romania}

Objectives: The life expectancy of total knee prosthesis depends on several elements. One of the most important elements for a long term outcome is the patient's BMD. One of the major cause of revision surgery is the loosening of the prosthetic components, which represents a major surgical challenge, and these interventions often has to be done in special orthopedic departments. The purpose of this case report is to study the latest research papers, and to highlight the importance and the challenging character of revision surgery in patients with low BMD.

Material and method: We present a case of a 62 year old female patient who was known with an untreated osteopenia in the past and right knee joint osteoarthritis, who underwent in 2012 to total knee joint replacement at the Orthopedic Department at the University Emergency Hospital of Bucharest.

Results: The short term results after the primary intervention were good. In 2015 the patient presents at our department with swelling, pain and a varus deformity of the right knee. After routine X-ray and laboratory examination we removed the tibial component which was the cause of the varus deformity. We performed the implantation of a new tibial component with a stem for a greater stability of the component.

Conclusions: One of the major causes of knee joint prosthesis failure is the aseptic loosening. It is well known that BMD is has a major influence on the implant life expectancy. The lowest the BMD is, the higher the stress-shielding is. These biological properties increase bone resorption around the implant. Association of anti-osteoporotic drugs showed a better long term results in patients with total knee joint replacement with a decrease of aseptic loosening of the implants.

\section{P862}

\section{LASER ACUPUNCTURE IN TREATMENT OF GONARTHROSIS}

N. Krstic $^{1}$, Z. Railic ${ }^{1}$

${ }^{1}$ Clinic for Physical Medicine and Rehabilitation, Clinical Center Serbia, Belgrade, Serbia

Introduction: Gonarthrosis is chronical, degenerative progressive disease. Treatment consists of medical therapy (NSAIL, analgesics) combined with physical therapy. Laser therapy and laser acupuncture are safe, painless procedures performed in short time aimed at relieving pain and increasing amplitude of joint movement. The purpose of the study is to compare therapy with laser application on painful areas of the affected ankles with laser application on acupunctural points on pain relief and ankle flexion amplitude in patients with gonarthrosis.

Materials and Methods: 36 patients (26 women and 10 men) aged 64-76 (mean age 70 years) were included and prospectively followed during gonarthrosis the treatment at Clinic for Physical Medicine and Rehabilitation, Clinical Center of Serbia, Belgrade. Diagnosis was made on ACR criteria lasted from 4-20 years (average12 years) Pain was measured by VAS scale (1-100 mm) before and after therapy. Ankle movement was measured with ankle arthrometer in degrees before and after completion of therapy. Patients were randomly assigned in two groups: First group with 20 patients, 14 
women and 6 men, aged 66-72 years and second group with 16 patients ( 12 women and 4 men) aged 64-76 years. Both groups were comparable in age, gender distribution and gonarthrosis duration. Laser used in this study was Mediclaser 637 Electronic design, valve length of $780 \mathrm{Nm}$, and power of 70-mW. First group of patients was treated with Laser beam applied on painful areas with $70 \mathrm{~mW}$, frequency of $2500 \mathrm{~Hz}$ in $60 \mathrm{~s}$ and energy absorption of $2.1 \mathrm{~J} / \mathrm{cm}^{2} 3$ times a week, in 10 consecutive doses. Patients in second group were treated with Laser applied on acupunctural points, i.e., F41,VU40, VF34, H8, G34, G35, PE31, PE32, with frequency of $70 \mathrm{~Hz}$, power of $40 \mathrm{~mW}, 0.6 \mathrm{~J} / \mathrm{cm}^{2}$ energy absorption in $30 \mathrm{~s}, 3$ times a week with 10 consecutive applications. Evaluation of treatment in both groups are performed before and after the end of treatment and statistically analyzed in Windows SPSS 16 program.

\section{Results:}

1. Analyzing the VAS scale data we found in group I significant decrease in pain before and after therapy (74.5-29.0) with 45.5 points. In group II, VAS score declined from 78.13 to 19.38 , with 58.7 points improvement. It estimated high statistical significance in both groups, Wilcoxon test, $p<0,001$.

2. In measuring ankle movement amplitude, mean flexion angle before therapy for the first group was $82.5^{\circ}$ and $120.5^{\circ}$ after treatment with $38.0^{\circ}$ improvement. In a second group the amplitude was $80.0^{\circ}$ before therapy and $123.13^{\circ}$ after treatment with $53.0^{\circ}$ improvement. High statistical significance was achieved also in improvement of ankle flexion amplitude in both groups (Wilcoxon test, $p<0.001$ ).

3. Significantly better pain relief and increased knee flexion was detected in group II - laser acupuncture application (Mann-Whitney $p<0.05$ ).

Conclusions: Analysis clearly shows positive impact of Laser therapy in pain relief, and ankle movement amplitude, with better results of laser applications on acupunctural points during treatment.

\section{P863 \\ COMPARATIVE STUDY OF OSTEOPOROSIS PROGRESSION AT PATIENTS WITH RHEU- MATOID ARTHRITIS IN REMISSION TREATMENT WITH METHOTREXATE VERSUS BIOLOGICAL AGENTS \\ A.-M. Bumbea ${ }^{1}$, S. Patru ${ }^{1}$, A. C. Bighea ${ }^{1}$, R. M. Trăistaru ${ }^{1}$, D. Matei $^{1}$, A. E. Musetescu ${ }^{1}$, I. R. Marcu ${ }^{1}$, B. S. Bumbea ${ }^{2}$, D. V. Caimac $^{1}$ \\ ${ }^{1}$ University of Medicine and Pharmacy, Craiova, Romania, ${ }^{2}$ County Emergency Hospital, Orthopedic Department, Craiova, Romania}

Background: It is known that patients with rheumatoid arthritis have frequently associated osteoporosis, both within the context of the disease and due to the treatment with corticosteroids.

Objective: We evaluated the progression of osteoporosis at patients with rheumatoid arthritis and osteoporosis in therapy with bisphosphonates, vitamin D3 and $500 \mathrm{mg}$ calcium daily in two groups of patients; one group in remissive treatment with methotrexate vs. the other group in treatment with biological agents. For this evaluation we have developed a retrospective study within a 4-year period of time, 2011-2015.

Method and Methodology: We have analyzed a number of 64 patients split in two homogeneous groups, each with 32 patients; all the patients were diagnosed with osteoporosis, T-score in between -2.5 and $-3.8 \mathrm{SD}$. The A Group received a treatment with $20 \mathrm{mg}$ of methotrexate weekly and nonsteroidal anti-inflammatory or steroids drugs for a short period. The B Group has received a treatment with biological agents: infliximab $3 \mathrm{mg} / \mathrm{kg}$ in infusion at 2 months or etanercept $50 \mathrm{mg}$ weekly or adalimumab $40 \mathrm{mg}$ once at two weeks. Both groups have received bisphosphonates in therapeutic doses being evaluated for osteoporosis. The median patient age was of 54.3 of which only 41 patients were at the beginning of the postmenopausal study. There was evaluated the level of the disease activity by the 28 DAS score. We also mention that at 14 patients treated with methotrexate there was necessary corticosteroids therapy in doses of $16 \mathrm{mg}$ per day with a diminishing level of $8 \mathrm{mg}$ on a period of 2 up to 8 months; meanwhile there was necessary the pulse therapy with metilprednisolone $1 \mathrm{mg} / \mathrm{kg}$ at 5 patients for a period of 3 days. Patients in biological therapy did not need corticosteroids therapy. We evaluated a witness group of 20 patients with rheumatoid arthritis and osteopenia who received only the supplement with D3 and $500 \mathrm{mg}$ of calcium daily.

Results: The A group of study presented the maintenance of median of T-score at $-3.3 \mathrm{SD}$, and DAS 28 median was lowered from 3.8 to 3.1. The B Group of study presented the diminishing of the level of osteoporosis from a $\mathrm{T}$ score from -3.8 to 3.0 ; the median of 28 DAS was reduced from 5.4 to 3.1. These levels showed osteopenia in the witness group a T-score from -1.7 to -2.2 with a passing to osteoporosis at 8 patients who needed to receive bisphosphonates, and the DAS 28 score presented a raise from 2.8 la 3.5 .

Conclusions: The diminishing level of osteoporosis with $0.2 \mathrm{SD}$ per year at the group of patients in therapy with biological agents as compared to the group treated with methotrexate proves the fact that despite the beginning of the first group with lower values it obtained better results in both reducing the level of disease activity and the pathogenic immune inflammatory context. The 
witness group of patients with osteopenia proves the role played by the patients' age in the beginning of menopause at 8 patients as well as the presence of the immune inflammatory factor in generating osteoporosis to patients with rheumatoid arthritis. In this context we can conclude that the biological therapy which reduce the activity of disease for low level, determine a good evolution to prevent or slow increase or maintaining osteoporosis score.

\section{P864}

BONE MASS AT THE CALCANEUS AMONG YOUNG MALE PRACTICING A HIGH IMPACT-LOADING SPORTS

E. Zakhem $^{1}$, G. Zunquin ${ }^{2}$, D. Theunynck ${ }^{2}$, T. Pezé ${ }^{2}$, R. El Hage $^{1}$

${ }^{1}$ Department of Physical Education, Fass, University of Balamand, Kelhat El Kurah, Lebanon, ${ }^{2}$ Departement STAPS, URePSSS-EA4110/EA448, ULCO, Dunkerque, France

\begin{abstract}
Aim: The aim of the present study was to compare the broadband ultrasound attenuation (BUA) among young male student participants in four distinctly different high impactloading sports (HI-LS).
\end{abstract}

Methods: 62 Lebanese students from University of Balamand mean age 21 years (18-29) participated in this study. 48 students practice four distinctly HI-LS with the following university teams: rugby (18), football (13), volleyball (9) and basketball (8), and 14 sedentary subjects. All university teams practice at least three times a week with duration of each session being approximately $2 \mathrm{~h}$. The sedentary subjects practice less than two hours per week. Body weight and height were measured, and BMI was calculated. Broadband ultrasound attenuation (BUA), the speed of sound (SOS) and the stiffness index (SI) are measured at a fixed region in the midcalcaneus, by using a new ultrasonometry device "Pegasus Smart Medlink ${ }^{\circledR}$ ".

Results: A significant difference at the BUA was found in the HI-LS between volleyball players and basketball players $(P=0.024)$. However a different but not significant at the BUA was found in the HI-LS between volleyball players and rugby players $(P=0.064)$, volleyball and football players $(P=0.052)$. The BUA was significantly different between HI-LS and sedentary subjects $(P<0.05)$. The SOS was significantly different between volleyball players, basketball players and sedentary subjects $(P<0.05)$. The SI was also significantly different between HI-LS and sedentary subjects.

Conclusion: In the present study we observed that the values of BUA, SOS and SI are significantly higher in the four distinctly different HI-LS (rugby, volleyball, basketball and football) than among sedentary group. However a significant and nonsignificant difference at the BUA was found between the four distinctly different HI-LS. This is the first study that determines the difference between several kinds of HI-LS at the BUA.

\section{P865}

\section{VERTEBRAL BODY AUGMENTATION RELIABLY REDUCES MORBIDITY IN OSTEOPOROTIC SPINE} FRACTURES

K. S. Oh ${ }^{1}$, M. Malik ${ }^{1}$

${ }^{1}$ Island Hospital Spine Centre, Georgetown, Penang, Malaysia

Aims: The morbidity of osteoporotic vertebral fractures continues to plague us as the ageing of population sets in across many communities globally. Treatment options have ranged from simple immobilization to vertebroplasty with acrylate to instrumentation of the spine. We looked at the results of our procedure which stents the vertebral body from within before augmenting with acrylate.

Methods: 60 patients with painful and symptomatic osteoporotic fractures were treated in 2012 and followed through for 36 months thereafter. All were female, postmenopausal and radiographically osteoporotic with at least moderate degrees of Genant-type fractures of the thoracic and lumbar spines. 42 received an imageguided percutaneous, transpedicular procedure that used a trocar to deliver a collapsed mesh into the corpus of the vertebra concerned, before expanding the mesh hydraulically within the body of the vertebra. 18 had the procedure as part of a more extensive open decompressive and instrumented approach. All patients were treated by the same surgeon. We studied the efficacy of pain relief and the chronology of activity resumption. Results: All patients experienced pain relief within $48 \mathrm{~h}$ after the procedure. All percutaneous cases had speedy recovery within $24 \mathrm{~h}$. In the open cases, 4 patients had residual radiculopathy pain that lasted for 48 h. 59 patients resumed pre-morbid physical activity while the sole exception suffered persistent post-traumatic lower motor neuron damage that took 12 weeks to improve. Six cases in the open category showed cement leaks, compared to one in the percutaneous group. All leaks but one were asymptomatic and inconsequential right up till 36 months of follow-up. The sole exception suffered pulmonary embolism but recovered after 3 days of intensive care support. Five patients with percutaneous treatment suffered adjacent segment fractures subsequently.

Conclusions: Vertebral body stenting has effectively relieved the symptoms of pain and radiographically 
restored stability in our patients. This procedure has been executed safely and without major complications, with the patients exhibiting continuity of symptom relief and spine stability at 36 months of follow-up. We are expanding this study into a wider pool of patients to achieve better statistical significance.

\section{P866 \\ SIGNIFICANCE OF LEVEL OF LACTATE DEHYD- ROGENASE AND LACTIC ACID IN SYNOVIAL FLUID IN DIFFERENTIATING BETWEEN NON-INFLAMMATORY, INFLAMMATORY AND SEPTIC ARTHRITIS \\ T. Umer $^{1}$ \\ ${ }^{1}$ Liaquat National Hospital and Medical College, Karachi, Pakistan}

Objective: To determine the significance of elevated LDH and Lactic acid levels in synovial fluid. To determine the significance of elevated synovial fluid LDH and Lactic acid levels in differentiating between Inflammatory arthritis, infectious arthritis and non inflammatory arthritis.

Study rationale: Tissue hypoxia is closely associated with arthritis pathogenesis. In many types of arthropathy, the synovial fluid (SF) oxygen tension and glucose levels often are low and associated with lactic acidosis which, unless joints are insensitive to the lack of fuel and oxygen, must contribute to joint damage. Many a times a physician is faced with the problem of a swollen joint, arthrocentesis of which may show a modestly elevated White cell count which may be seen with both septic or an inflammatory arthritis or a mildly elevated synovial fluid WBC count which may be seen in both inflammatory of noninflammatory arthritis. This dilemma becomes a diagnostic night mare if blood and synovial fluid cultures are negative. Similarly patients known to have gouty arthritis when present with acute swollen joint can be suffering from acute gouty arthritis or septic arthritis or both. Several studies have shown that elevated lactate and LDH levels in the synovial fluid corresponds with the bacterial culture outcome suggesting its role in the early diagnosis of septic arthritis and differentiating it from nonseptic causes ${ }^{1-3}$.

Material and Methods: Lactate and LDH will be determined in synovial fluid of all selected patients using L-Lactate PAP (4-amino-antipyrine) colorimetric and DGKC methods, respectively. Samples will be collected at rheumatology unit of our tertiary care centre.

\section{References:}

1. Alam JM, et al. Middle-East J Sci Res 2015;23:1664

2. Alam JM, et al. IJBBS 2015; 4:53

3. Lenski M, Scherer MA. Clin Biochem 2014;47:49.
P867

OSTEOPOROSIS AND A LONG TERM MEDICAL HISTORY OF NON-TREATED PROLACTIONOMA ON A PATIENT WITH TRANSFRONTAL PITUITARY SURGERY AND PANHYPOPITUITARISM

A. Geleriu $^{1}$, M. Carsote ${ }^{2}$, D. Hortopan ${ }^{3}$, A. M. Dobrin-Tripac ${ }^{1}$

${ }^{1}$ Lujerului Polyclinic, Queen Mary Healthcare Private Network, Bucharest, Romania, ${ }^{2} \mathrm{C}$. Davila University of Medicine and Pharmacy \& C.I. Parhon National Institute of Endocrinology, Bucharest, Romania, ${ }^{3}$ Gral Medical Center, Bucharest, Romania

Introduction: Large pituitary tumors cause bone damage by gonad insufficiency, high prolactin levels, and over-treated central hypothyroidism and secondary adrenal insufficiency. Overall long term endocrine follow-up is required despite a good clinical outcome.

Objective: We present an adult female with a long medical and surgical history of large pituitary prolactin producing mass.

Material and Method: We asses bone turnover markers (BTM) and calcium metabolism, and pituitary profile (based on both imagery and hormonal panel).

Case data: A 65-year-old female was diagnosed 14 years ago with a large pituitary mass based on hypopituitary symptoms (secondary amenorrhea since the age of 38). Trans-frontal surgery was performed and she was offered daily prednisone and levothyroxine (LT4) replacement therapy. 6 years ago vertebral fractures were found and therapy with weekly oral alendronate (for one year), then weekly oral risedronate (for one year), then monthly oral ibandronate (for 4 years) was given. On admission, the patient presented with diffuse bone and muscle pain and mild high blood pressure. Low FSH levels of $0.38 \mathrm{mUL} / \mathrm{mL}$ confirmed the central component of menopause, low T3 levels (of $52 \mathrm{ng} / \mathrm{mL}, \mathrm{N}$ : 80-200) indicated an under-treatment with LT4. Despite the absence of galactorrhea, high levels of prolactin of $408 \mathrm{ng} / \mathrm{mL}(\mathrm{N}<20)$ were consistent with the diagnosis of prolactinoma. An excess of prednisone replacement was suspected and tapering the doses improved the blood pressure profile. Residual bilateral eye field's defects were found. CT scan revealed an intrapituitary mass of 1.83 by $1.14 \mathrm{~cm}$ with another two suprasellar masses, one next to the optic chiasm of 1.71 by 1.11 by $2.17 \mathrm{~cm}$ (on the right side of Willis polygon) and one of 1.11 by $1.63 \mathrm{~cm}$ (on the left). Distal radius BMD-DXA (lumbar and hip noninterpretable data because of vertebral fractures and bilateral coxarthrosis) was $0.532 \mathrm{~g} / \mathrm{cm}^{2}$, T-score of $-2.5 \mathrm{SD}$, and Z-score of $-1.1 \mathrm{SD}$. 25-hydroxyvitamin D of $13.5 \mathrm{ng} / \mathrm{mL}$ (N: 30-100), intact serum PTH of $24.77 \mathrm{ng} / \mathrm{mL}$ (N:15-65), bone resorption marker serum CrossLaps of $0.16 \mathrm{ng} / \mathrm{mL}(\mathrm{N}: 0.226-1.008)$, and bone formation marker osteocalcin of $2.28 \mathrm{ng} / \mathrm{mL}$ (N: 15-65). Therapy with cabergoline (twice a week with progressive doses), higher 
LT4 doses and lower prednisone dose in association with vitamin D and calcium supplements and IV zolendronic acid was recommended.

Conclusion: Endocrine tumors-related osteoporosis underline anomalies of estrogens-FSH axis, prolactin, thyroid and adrenal function and a major component of bone loss is caused by the iatrogenic element due to insufficient (as dopamine agonists) or excess (as glucocorticoids) administration of hormones.

\section{P868}

THE RELATION BETWEEN COMORBIDITIES AND BONE MINERAL DENSITY IN POSTMENOPAUSAL OSTEOPOROSIS

B. Kuran ${ }^{1}$, C. Mert ${ }^{2}$, F. Yilmaz 2 , J. Önc̈̈ ${ }^{2}$, G. Durlanik ${ }^{2}$, B.

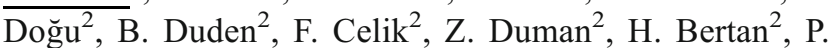
Bulut $^{2}$, M. Unal ${ }^{3}$, C. Erçalık ${ }^{4}$, H. Elik ${ }^{2}$, N. Șen ${ }^{2}$, G. Çelebi ${ }^{2}$, A. Üșen ${ }^{2}$

${ }^{1}$ Şişli Etfal Hospital, Istanbul Yeni Yüzyıl University, Istanbul, Turkey, ${ }^{2}$ Şişli Etfal Hospital, Istanbul, Turkey, ${ }^{3}$ IIstanbul Yenı Yuzyıl University, Faculty of Health Sciences, Istanbul, Turkey, ${ }^{4}$ Arel University, Istanbul, Turkey

Objective: The burden of osteoporosis is one of the major health concerns which in fact can be reduced with medical treatments and physical activity. The disability related with osteoporosis increases with the number of comorbidities. The aim of this study was to investigate the relation between BMD and comorbidities in postmenopausal osteoporotic (PMO) women.

Material and Methods: 300 postmenopausal women attending the outpatient osteoporosis polyclinic in our department were included in this observational, cross-sectional study. All the patients were screened by Cumulative Illness Rating Scale (CIRS) which provides medical information on 14 body systems. Severity of the medical problems in CIRS is defined in 5 categories (between 0-4). The scale gives both a total score (CI Total) and a severity index (CI) of the categories.

Results: Patients included in the study were between $42-90$ ages (mean $64.4 \pm 10$ ), had a mean BMI of $27.1 \pm 4.3 \mathrm{~kg} / \mathrm{m}^{2}$ $\left(16-42 \mathrm{~kg} / \mathrm{m}^{2}\right)$. The mean lumbar and femoral BMD and tscores were $0.900 \mathrm{~g} / \mathrm{cm}^{2}(0.48-1.99), 0.7 \mathrm{~g} / \mathrm{cm}^{2}$ (0.18-1.45), -2.7 (t-lumbar-5.9-3.6), -2.1 (t-fneck -6.2-0.9), respectively. CIRS-T average was $3.3 \pm 2.3(0-10)$. CI score average was $1.5 \pm 0.9(0-4)$. There was significant $(p<0.050)$ correlation between femoral BMD, femoral t-score, age, BMI and CI score. Lumbar BMD was significantly correlated only with BMI.

Conclusion: We conclude that in PMO women, not the number of comorbid conditions but the severity of the system involvement is associated with femoral bone density. As the severity increases femoral bone density decreases.

\section{P869}

SOLUBLE MILK PROTEIN SUPPLEMENTATION ASSOCIATED WITH MODERATE PHYSICAL ACTIVITY IMPROVES MUSCLE FORCE AND LOCOMOTION IN AGED RATS

F. Lara-Villoslada ${ }^{1}$, A. Lafoux ${ }^{2}$, C. Baudry ${ }^{3}$, P. le Ruyet ${ }^{3}$, C. Huchet $^{2}$

${ }^{1}$ Lactalis Research and Development, Granada, Spain, ${ }^{2}$ INSERM Institut du Thorax, Therassay, Université de Nantes, Nantes, France, ${ }^{3}$ Lactalis Research and Development, Retiers, France

Context and Objectives: Loss of muscle mass and function, called sarcopenia, occurs with aging. Adequate protein intake and physical activity are considered as two crucial interventions to maintain both muscle mass and functionality in elderly subjects. The aim of this study was to investigate the impact of a protein supplementation with different milk proteins, associated with a moderate exercise, on muscle and locomotor functions in aged rats.

Methods: We investigated the effect of 8-week supplementation with different milk proteins, such as caseins (CAS), whey (WH) or soluble milk proteins (PRO), on muscle force and gait analysis in trained aged rats Wistar RjHan (17 month-old, $n=8$ /group). Animals followed moderate intensity protocol training with running exercises on treadmill at maximum speed $10 \mathrm{~m} / \mathrm{min}$, during maximum $30 \mathrm{~min} /$ day, 5 days/week for 8 weeks. Just after treadmill exercise, a bolus of $0.85 \mathrm{~g}$ of CAS, WH or PRO was given in order to increase by $20 \%$ the protein content of the diet. Muscle force was evaluated using the grip test (Bioseb) and locomotor activity was measured in an open-field (Actimeter, Bioseb). Furthermore, an extensive gait analysis was performed using the Gait-Lab system (GaitLab, View Point) which is a highly sensitive tool recording the rat's footprints on a glass plate to assess gait and locomotion. Results: After 8 weeks, grip strength (expressed in $\mathrm{g} / \mathrm{mg}$ of muscle mass) was significantly higher in rats from the PRO group than in CAS and WH groups. Also, locomotor parameters measured in the open-filed, such as distance travelled, activity time and maximum speed, were higher in group PRO than in groups CAS and $\mathrm{WH}$, without any difference between CAS and $\mathrm{WH}$ groups. Gait analysis using the Gait Lab system showed a training-induced increase in voluntary gait speed after 8 weeks in the 3 groups, which was significantly higher in the PRO group. Also, a training-induced increase in stride frequency was observed and was higher in the PRO group.

Conclusion: In aged rats, a supplementation with soluble milk proteins after a moderate exercise is an effective way to improve muscle force and locomotor activity. These results suggest that soluble milk proteins 
consumed just after a moderate exercise could be more beneficial than whey. Therefore, soluble milk proteins could be considered as an interesting nutritional strategy in combination with physical activity to counteract the effect of sarcopenia in the elderly.

\section{P870}

\section{OSTEOPOROSIS AND FRACTURE RISK IN PRIMARY HYPERPARATHYROIDISM}

L. Y. Rozhinskaya ${ }^{1}$, N. G. Mokrysheva ${ }^{2}$, A. K. Lipatenkova ${ }^{2}$, S. S. Mirnaya ${ }^{2}$

${ }^{1}$ Neuroendocrinology and Bone Diseases, Endocrinology Research Centre, Moscow, Russian Federation, ${ }^{2}$ Department of Hyperparathyroidism, Endocrinology Research Centre, Moscow, Russian Federation

Introduction: Primary hyperparathyroidism (PHPT) results in increased bone turnover, resulting in BMD reduction and a predisposition towards fractures.

Objective: To evaluate BMD and fracture risk in patients with PHPT.

Methods: 365 (328 women and 37 men) patients with PHPT were investigated, healthy controls included 66 individuals. BMD was estimated with DXA at three sites: in lumbar vertebral bodies and in femoral neck consisting mainly of the trabecular bone, in $1 / 3$ distal part of the radius composed predominantly of the cortical bone.

Results: Osteoporosis (OP) in at least one region was diagnosed in $64 \%$ and osteopenia in $27 \%$ patients. $36 \%$ of patients had fragility fractures, $63 \%$ of them were multiple fractures. The relative risk of developing osteoporosis in young patients with PHPT compared with the healthy control increased several times: by 9 times for men [95\%: CI 1.4; 58], $p=0.0001]$, by 2.6 for women [ $95 \%$ CI: $0.7,10], p=0.03$. Relative risk of developing osteoporosis in elderly group is smaller. The OP risk in male group raised by 3 times in cortical bone, in trabecular bone it was comparable to the control group $(p<0.05)$. Among postmenopausal the risk of osteoporosis elevated by 2.6 times in the radius [CI $95 \% 1.4,4.8](p<0.01)$. The relative risk of lowtraumatic fractures in elderly patients with PHPT increased by 1.5 times in men (18\% vs. $13 \%)$ and almost by 2 times in postmenopausal woman (41\% vs. $24 \%)$. In young patients the risk of fractures increased many times with the predominance of their occurrence in trabecular bones. The risk of hip fracture is $6 \%$ for patients with PHPT, which is significantly higher than in elderly population.

Conclusions: Our work shows that osteoporosis and fracture risks are significant higher in patients with PHPT comparing to healthy individuals matched by sex and age.
P871

A CASE REPORT OF TRADITIONAL CHINESE
MEDICINE IN TREATING OSTEOSARCOPENIA P. C. $\mathrm{Wu}^{1}$

${ }^{1}$ Division of Chinese Internal Medicine, Taipei Tzu Chi Hospital, Buddhist Tzu Chi Medical Foundation, New Taipei City, Taiwan, Province of China

Objective(s): Osteosarcopenia show a high prevalence in aging individuals and incur a high risk for falls, fractures, and institutionalization. There still no medicines are approved in treating osteopenia/osteoporosis and sarcopenia currently. However, some supplementing and blood-quickening stasis-transforming formula in traditional Chinese medicine could treat osteosarcopenia. We will therefore present a case with osteosarcopenia getting effective report by using this treatment. Material and Methods: The case to be reported here involved a 89 year old man who was left femoral neck fracture treated with bipolar hemiarthroplasty in December 06, 2014. Due to severe pain, general weakness, depressed mood, and weight loss, he visited our traditional Chinese medicine clinic and received supplementing and blood-quickening stasis-transforming formula $5 \mathrm{~g}$ three times a day since December 23, 2014. The concept of this treatment is that appetite could be increased, and skeletal muscle could increase mass, quality and strength by adjusting the meridian system through traditional Chinese medicine formula.

Results: After one week of receiving traditional Chinese medicine treatment, the follow-up result of the patient revealed that the mood and appetite changed for the better, the pain improvement and the patient feel more power to stand up and walking. After one month of receiving supplementing and blood- quickening stasis-transforming formula, the patient can walk for 30 min without pain or low gait speed. Besides, no obvious side effect was found after these three months of treatment.

Conclusion: Through the positive report in this case, we suggest the supplementing and blood- quickening stasistransforming formula in traditional Chinese medicine may be a safer and more effective treatment for aging with osteosarcopenia.

\section{P872}

IS IT NECESSARY TO SUPPLEMENT WITH CALCIUM AND VITAMIN D MENOPAUSAL WOMEN?

C. Prado Martinez ${ }^{1}$, M. Díaz Curiel ${ }^{2}$, D. Navarro Despaigne ${ }^{3}$ ${ }^{1}$ Departamento Biología, Facultad de Ciencias, Universidad Autónoma de Madrid, Madrid, Spain, ${ }^{2}$ Departamento Enfermedades Metabólicas Oseas Fundación Jiménez Díaz, Madrid, Spain, ${ }^{3}$ Instituto Endocrinología, Universidad de Ciencias Médicas de La Habana, Havana, Cuba 
Introduction: For years the treatment with calcium, bound or not, vitamin D, was used as an alternative for the prevention and treatment of osteoporosis. However, as reported the Food and Nutrition Board and Health Canada committee, the dietary recommendations are applied for the general healthy population. We need information on criteria and values of dietary $\mathrm{Ca}$ intake in different population and age group indicating the need or the potential risk of supplementation. Therefore, today the usefulness of therapeutic supplementation when considering that its use could increase cardiovascular morbidity and mortality, kidney stones and gastrointestinal disorders is questioned. Although the optimal intake has not been established in premenopausal women or men, a daily amount of between $800-1000 \mathrm{mg}$ of $\mathrm{Ca}$ it is suggested, and not more than $2000 \mathrm{mg}$ of total calcium at any age.

Objective: Identify the habitual content of calcium and vitamin $\mathrm{D}$ in the diet, and related it with bone status in climacteric and postmenopausal Spanish women.

Subjects and methods: Cross-sectional study involving 803 women living in Madrid aged between 43-80 years who came to the Department of Metabolic Bone Diseases, University Hospital "Fundación Jiménez Díaz". In each patient nutritional status and body composition was identified by anthropometry and bio impedance. BMD by DXA densitometry at the lumbar spine (L2-L4). The group is subdivided into younger and older than 55 years, age at which all surveyed women had undergone menopause. In $15 \%$ of women $(n=126)$, was made a weekly intake control of consumption of different food groups. Also a quantitative evaluation of the ingested during the last 48 hours was performed.to analyze the contribution of macro and micronutrients in the diet. Less than $800 \mathrm{mg} /$ day was considered low calcium diets. Normality for nutritional variables is corroborated by the logarithmic correction and test of Shapiro - Wilks, outliers were removed resulting in a sample of $N=124$ for these variables. Student's $t$-test was used to identify differences between groups $(p<0.05)$ was considered statistical difference).

Results: In the overall group according to BMI classification, $3 \%$ of women had low weight, $42 \%$ normal weight, $38 \%$ overweight and $17 \%$ obesity. Taking into account the age groups mean of BMI was 26.97 \pm 4.28 , and $25.09 \pm 4.05$ for those under and over 55 years, respectively. Calcium intake in each of the categories of age was $944.62 \pm 416.30$ and 893.15 \pm 334.60 . $(P>0.05)$. Dietary intake of vitamin D in old and young was $6.78 \pm 9.09 \mathrm{mg} /$ day and $4.56 \pm 7.26 \mu \mathrm{g} /$ day $(p<0.05)$. Regarding daily intake of vitamin $\mathrm{D}$ according to BMI, our results shows that in obese women, it was below the recommendations. Regarding the consumption of $\mathrm{Ca}$ in diet, the $18.05 \%$ of the women not cover the recommendations. This percentage is only $11.7 \%$ in old women, as in the case of vitamin $\mathrm{D}$, intakes are more balanced in older than 55 years. Value of T-score at the lumbar spine in the total population was $-2.36 \pm 1.16$ while in women with a dietary calcium intake of $<800 \mathrm{mg}$ the value of T-score at the same location was $-2.60 \pm 1.21$. Is important to remark the loss in height in our women and the progression on this fact, In women $<55$ years was $-2.18 \pm 1.56 \mathrm{~cm}$, bur in over 55 years loss was $-5.17 \pm 1.49 \mathrm{~cm}$.

Conclusions: Must be identified in each case(subject and population)the type of action before the nutritional supplement of calcium and vitamin D. Negative impact on BMD values were found in women whose diets with less of $800 \mathrm{mg} \mathrm{Ca}$. The deficit of vitamin D in obese women could be related to a massive consumption of skim and need a confirmative study. Apparently postmenopausal women $>55$ years, with bigger clinical control, have greater concern for the bone healthy eating diet.

\section{P873}

SYNOVIAL FLUID FROM KNEE OSTEOARTHRITIS PATIENTS TRIGGERS EXPRESSION OF MATRIXMETALLOPROTEASES AND VASCULAR ENDOTHELIAL GROWTH FACTOR GENES IN SYNOVIOCYTES EXPERIMENTAL CELL MODEL S. Deshpande $^{1}$, S. Koppikar ${ }^{2}$, P. Kulkarni ${ }^{2}$, A. Harsulkar ${ }^{2}$ ${ }^{1}$ Department of Orthopedics, Bharati Hospital, Bharati Vidyapeeth University, Pune, India, ${ }^{2}$ Cell and Molecular Biology, Interactive Research School for Health Affairs, Bharati Vidyapeeth University, Pune, India

Objectives: Synovial fluid (SF) is the biological snapshot of biochemical signals released from the cartilage and synovium. We have published data on pro-inflammatory factors accumulated in SF obtained from knee osteoarthritis (OA) patients of increasing severity of the disease. By using the cultured rabbit synoviocytes as the cell model we envisage to demonstrate expression of genes that can influence cartilage remodelling as well as synovitis, both being the key pathologies in OA progression.

Methods: The SF from 10 knee OA patients were collected either at the time of total knee replacement or arthrocentesis. The fluid was analyzed for NO (nitric oxide), nitrate-nitrite $(\mathrm{NN})$, glycosaminoglycan (GAG) and IL-1 $\beta$. Cultured HIG82 (rabbit synoviocytes) cell line was challenged with these SFs to assess expression of MMP1, MMP 13 and VEGF1 genes using quantitative real time PCR.

Results: Patients divided into early OA (KL grade $1, N=3$ ), moderate OA (KL grade II /III, $N=4$ ) and advanced OA (KL grade IV, $N=3$ ). The Synovial assessment of IL- $1 \beta$ in early OA group and moderate OA group was higher (range 47.27 $2442.4 \mathrm{pg} / \mathrm{ml}$ ) as compared to grade IV (range 22.27$23.18 \mathrm{pg} / \mathrm{ml}$ ). The GAG levels in early OA group and moderate OA (range 93.75-789.28 $\mu \mathrm{g} / \mathrm{ml}$ ) higher than the grade IV OA group (range 392.50-448.33 $\mu \mathrm{g} / \mathrm{ml}$ ). The NO and NN showed the similar trend with higher values in early OA group 
and moderate OA. In cell challenge experiment, the MMP1 and MMP13 mRNA expression was reduced in OA-grade dependent manner after 48 and $72 \mathrm{~h}$. The VEGF1 expression showed enhanced levels by SFs from early OA patients, which reduced further in moderate and advanced conditions. Conclusion: Increased IL-1 $\beta$, NO and NN suggest increased inflammatory modulators accumulating in SF in association with the disease severity. These mediators can further show a pronounced impact on expression of MMPs and VEGF1 genes. To our knowledge; this is the first experimental evidence to show that the patient's fluid upregulates MMP1, MMP13 and VEGF1 in SF challenged synoviocytes as compared to IL-1 $\beta$ used as a control. Further there is a KL-grade dependent reduction in the gene expression. The MMPs represent the catalytic processes leading to cartilage degeneration that correlates with increased GAG levels, while VEGF1 triggers angiogenesis, which promotes synovial hypertrophy and synovitis. This data suggest accumulation of factors in SF that can expounds the disease symptoms and pathology.

\section{P874}

POLYMORPHISM GENES OF OSTEOPOROSIS AMONG INDIGENOUS PEOPLE OF TRANSBAIKALIAN REGION OF RUSSIAN AND BURYAT NATIONALITIES

$\underline{S}$. Verkhoturova $^{1}$, S. Tsarenok ${ }^{2}$, V. Gorbunov ${ }^{2}, \mathrm{~N}$. Strambovskaya $^{2}$, T. Aksenova ${ }^{2}$

${ }^{1}$ Russia Railway Hospital, Chita, Russian Federation, ${ }^{2}$ Chita State Medical Academy, Chita, Russian Federation

According to many surveys, different ethnic groups have a genetic predisposition to certain diseases.

Objective: To examine the frequency of genotypes of polymorphic locus of vitamin D receptor gene (VDR) Bsm1 c.IVS7G $>$ A and the lactase gene LCT $13910 \mathrm{~T}>\mathrm{C}$ in healthy peoples and patients who have osteoporosis (OP) among indigenous people of Trans-Baikalian region of Russian and Buryat nationalities. Materials and methods: We have examined 86 women with OP: 40 Russian and 46 Buryat aged 50 80 years, 103 healthy women were included in a control group. The material for the molecular genetic analysis of the DNA samples was extracted from peripheral venous blood. The point of mutations in the VDR Bsm 1 c.IVS7G $>$ A gene and the lactase (LCT) $-13910 \mathrm{~T}>\mathrm{C}$. were chosen for investigation. The diagnosis of OP was estimated according to "Russian clinical guidelines for osteoporosis," 2012. The program Statistica 10.0 was used for data processing. The relative risk (RR) and odds ratio (OR) were calculated to assess the association of the allele genotype. The law of Hardy Weinberg was used to determine the frequency of allelic variants of genes.
Results: The prevalence of genotypes of polymorphic loci prevalence Bsm1 c.IVS7G $>$ A vitamin D receptor gene and $-13910 \mathrm{~T}>\mathrm{C}$ lactase gene in a group of healthy peoples and patients who have OP correspond to Hardy-Weinberg law. The calculation of the relative risk revealed a positive association of genotype AA VDR gene polymorphism Bsm1 c.IVS7G $>$ A with the development of osteoporosis among the representatives of the Buryat nationality (OR 2.2, confidence interval (CI) $[1.3,3.6])$. The A allele is associated with lower BMD in the case of insufficient intake of calcium from food. This allele has a positive effect on bone in case of adequate calcium intake from food. In our previous works we have estimated that the Patients in both groups were insufficient calcium intake. The patients of Russian nationality had no association of genotype AA VDR gene polymorphism Bsm1 c.IVS7G $>$ A with the development of osteoporosis (OR 0.9, CI $[0.5 ; 1.5]$ ). During the calculating of the relative risk association of allele $\mathrm{C}$ gene polymorphism LCT $-13910 \mathrm{~T}>\mathrm{C}$ positive relationship have been identified in both groups (OR 0.7, CI $[0.4 ; 1.1]$ ).

Conclusion: Representatives of Buryat nationality with inadequate calcium intake from food have an increased risk of developing osteoporosis if they have AA genotype VDR gene polymorphism Bsm1 c.IVS7G $>$ A. This association was not found in patients of Russian nationality.

\section{P875}

BONE MINERAL DENSITY IN PATIENTS WITH AROMATASE INHIBITORS COMPARED TO POSTMENOPAUSAL OSTEOPOROSIS

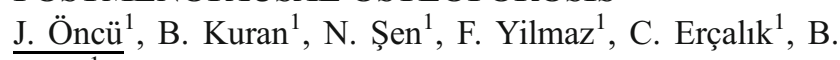
$\overline{D o g ̆ u}^{1}$

${ }^{1}$ Şişli Etfal Hospital, Istanbul, Turkey

Objectives: Osteoporotic effects are one of the known side effects of aromatase inhibitors that are used in the treatment of breast cancer. In our study we compared the BMD values of breast cancer patients using aromatase inhibitors with postmenopausal osteoporosis patients.

Material and Methods: A total of 24 patients having breast cancer and using aromatase inhibitors were included in the study. 40 postmenopausal osteoporosis patients without any malignancies were enrolled as the control group. Comparison of DXA results of the groups (femoral neck, total femur, spinal BMD (L1-4)) was the primary outcome.

Results: The mean age of the patient group was $56 \pm 11$ years (43-69) and the mean disease duration was 6 months (1-12 mos). There were significant differences between the two groups regarding spinal (L1-4) and femoral neck BMD values. Low BMD was more prevalent in patients on aromatase inhibitors compared to the other group $(p=0,045)$. There weren't any reported fracture in any patient in neither of the 
groups. Serum calcium, phosphate, alkaline phosphatase, PTH and urinary calcium and phosphate levels were normal in both groups.

Conclusion: Patients with breast cancer must be further investigated with regards to BMD and the clinician should keep in mind that these patients are prone to osteoporotic fractures. These patients are advised to be more closely monitored by physicians compared to the normal population.

\section{P876}

VITAMIN D STATUS AND ITS ASSOCIATION WITH PTH CONCENTRATIONS IN IN A REPRESENTATIVE POPULATION LIVING IN RIYADH SAUDI ARABIA

N. M. Al-Daghri ${ }^{1}$

${ }^{1}$ Biochemistry Department, College of Science, King Saud University, Riyadh, Saudi Arabia

There is a lack of large scale studies on vitamin D status and its relationship to PTH in adults living in Riyadh Saudi Arabia. The objectives were to determine vitamin $\mathrm{D}$ statues in adults living in Riyadh and to investigate the relationship of $25(\mathrm{OH}) \mathrm{D}$ with parathyroid function in order to determine the threshold for plasma $25(\mathrm{OH}) \mathrm{D}$ above which there is no further suppression of PTH. This cross-sectional study involved 373 men and 720 women aged $50.7 \pm 13.9$ years who randomly sampled from Riyadh. Serum concentrations of $25(\mathrm{OH}) \mathrm{D}, \mathrm{PTH}$, calcium and albumin were measured. Prevalence of vitamin D insufficiency (25-50 nmol/) was $57 \%$ in males and $42 \%$ in females. The prevalence of vitamin D deficiency $(<25 \mathrm{nmol} / \mathrm{L})$ was $72 \%$ in males and $27.5 \%$ in females. With increasing serum $25(\mathrm{OH}) \mathrm{D}$ concentrations mean PTH gradually decreased in both sexes $(p<0.001)$. There was an inverse relationship between the serum $25(\mathrm{OH}) \mathrm{D}$ and PTH concentrations in both genders, but no threshold of 25(OH)D at which PTH plateau was observed.

\section{P877 \\ EPIDEMIOLOGIC STUDY OF HYPERCALCEMIA IN A RHEUMATOLOGY CENTER: SYMPTOMS, PREVALENCE, ETIOLOGY AND TREATMENT \\ K. Baccouche ${ }^{1}$, M. Bouzaoueche ${ }^{1}$, N. Elamri ${ }^{1}$, S. Belghali ${ }^{1}$, Z. Alaya $^{1}$, H. Zeglaoui ${ }^{1}$, E. Bouajina ${ }^{1}$ \\ ${ }^{1}$ Rheumatology, Farhat Hached Hospital, Sousse, Tunisia}

Introduction: Hypercalcemia is a common metabolic complication in medicine can be life threatening. It is defined by a higher than normal level of calcium in the blood, usually more than $2.6 \mathrm{mmol} / \mathrm{l}$ of blood. The objective of this study is to identify the different symptoms arising therefrom, the different responsible etiologies, and different means for its therapeutic management.
Methods: retrospective study focused on 74 patients with hypercalcemia who were hospitalized in rheumatology between 1998-2013.

Results: 74 patients, with average age of $64.13 \pm 11.5$ years. We found a female predominance $(63.1 \%)$. The discovery of hypercalcemia was coincidental in $64.5 \%$ of cases following a systemic dosage. The clinical signs of hypercalcaemia were dominated by osteoarticular manifestations ( $58 \%$ ), followed by general signs $(25.8 \%)$, digestive symptoms $(6.4 \%)$, neurological signs (3.22\%) and renal signs (3.22\%). In our study, $64.5 \%$ of patients had mild hypercalcemia, $25,8 \%$ had moderate hypercalcemia and $9.7 \%$ had severe hypercalcemia. The various etiologies were primary hyperparathyroidism $(38.7 \%)$, multiple myeloma (12.9\%), bone metastases (25.8\%), lymphoma (6.5\%), sarcoidosis (3.2\%), chronic renal failure $(6.5 \%)$. No etiology was found in $6.5 \%$ of cases. The therapeutic management consisted of two main components: the symptomatic and etiological treatment. Symptomatic treatment was based on hyperhydration (48.3\%), bisphosphonate (16.1\%), calcitonin (12.9\%) and corticosteroid for only one patient. No patients received extra renal purification. The etiological treatment was medical and / or surgical according to different etiologies.

Conclusion: Hypercalcemia is still a common and potentially serious metabolic complication hence the importance of practicing systematic dosage, to know its different clinical manifestations and its main etiologies in order to have a rapid and effective therapeutic management.

\section{P878}

HYALURONIC ACID IMPROVES CARTILAGE MATERIAL PROPERTIES

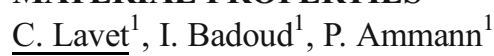

${ }^{1}$ Division of Bone Diseases, Geneva University Hospitals and Faculty of Medicine, Geneva, Switzerland

Viscosupplementation with hyaluronic acid is known to influence parameters of articular lubrication and cellular metabolism in patients suffering from osteoarthritis. An influence on articular cartilage micromechanical properties could be one of the mechanisms of action of this intra-articular treatment, recognized for its protective and long lasting effects. Indeed the main function of cartilage (shock absorption and force transmission) is influenced by its biomechanical characteristics and physicochemical interventions could bring a benefit to cartilage biomechanics. To verify this hypothesis, distal femurs were harvested from three 11-month-old rats. Bioindentations were performed at the level of the medial condyle before and after an ex-vivo overnight incubation in $1 \%$ nonchemically modified medium molecular weight HA solution (Ostenil ${ }^{\circledR}$ ). The material level properties (indentation depth and Young's modulus) of the distal femur were 
evaluated using bio-indentation with Bioindenter equipment (CSM Instrument, Switzerland). Cyclic indentation was defined as a set of 5 successive cyclic indentations at $200 \mu \mathrm{m}$ distant locations with a quadratic load increment (from 0.05-8 $\mathrm{mN}$ ) resulting in 15 successive indentations at the same location. Elastic modulus (MPa) and depth of indentation $(\mu \mathrm{m})$ were assessed for each indentation (load applied), to obtain biomechanical material level properties at different cartilage depths. The incubation with Ostenil ${ }^{\circledR}$ induced a decrease in indentation depth in the range of forces influencing the whole cartilage thickness by $30-35 \%(p<0.05)$ together with an increment of modulus by $22-25 \%(p<0.01)$. This explorative study could represent a proof-of-concept demonstrating a physicochemical effect of HA on cartilage material level properties. After meniscectomy, depth of indentation increased while elastic modulus decreased. As a result, a HA preparation such as Ostenil ${ }^{\circledR}$ could potentially counteract deleterious effects of meniscectomy. This effect on cartilage material properties could represent a major mechanism of action of viscosupplementation.

\section{P879}

PITFALLS IN PREVIOUS DUAL-ENERGY X-RAY ABSORPTIOMETRY (DXA) SCANS: ADHERENCE TO INTERNATIONAL SOCIETY FOR CLINICAL DENSITOMETRY (ISCD) 2013 GUIDELINE

L. M. Sconfienza $^{1}$, C. Messina ${ }^{2}$, M. Petrini' ${ }^{2}$, C. Monaco ${ }^{2}$, F. M. Ulivieri ${ }^{3}$, F. Sardanelli ${ }^{1}$

${ }^{1}$ Dipartimento di Science Biomediche per la Salute, University of Milan, Milan, Italy, ${ }^{2}$ Radiology, University of Milan, Milan, Italy, ${ }^{3}$ Bone Metabolic Unit, Medicina Nucleare, Fondazione IRCCS Ca' Granda Ospedale Maggiore, Milan, Italy

Objective: Due to the ageing of population, osteoporosis is becoming an emerging medical and socioeconomic threat: hip and vertebral fractures are associated with increased mortality. This disease is commonly evaluated through a quantitative assessment of BMD, DXA being most widely used technique. In fact, compared to other techniques, DXA has a higher reproducibility and is capable to detect very small BMD variations. DXA rests on numerical data. The acquired image is not aimed at visual diagnosis, even though image evaluation is fundamental to obtain proper BMD values. For this reason, is mandatory to strictly apply the instructions provided by densitometer manufacturers in the user's manual as well as the ISCD official positions. Neglecting these correctness criteria may result in erroneous data interpretation and equivocal reports. Our aim was to assess rate and type of errors in DXA examinations, evaluating a consecutive series of DXA scans of patients scanned elsewhere and later presenting to our institution for a follow-up DXA scan.
Methods and Materials: We prospectively evaluated 2476 patients who presented at our institution to perform DXA. We excluded patients without previous DXA and those who performed the previous examination at our institution. A radiologist with 4 years of experience in DXA re-evaluated previous DXA of 498 patients ( 38 males, 447 females; mean age $68 \pm 9$ years), according to ISCD 2013 guidelines. Errors were classified in four categories: patient- positioning (PP), dataanalysis (DA), artifacts, demographics.

Results: Of 2476 patients, 1198 had no previous DXA, while 793 had a previous DXA performed in our institution. The remaining $485(20 \%)$ patients entered the study (38 males and 447 females; mean age \pm SD $68 \pm 9$ years). Previous DXA examinations were performed at a total of 37 centers. Of 485 examinations, 451 (93\%) had at least one error for a total of 558 errors distributed as follows: $441(79 \%)$ were DA, 66 (12\%) PP, 39 (7\%) artifacts, and $12(2 \%)$ demographics. Regarding the lumbar spine, the most frequent error concerned the inclusion or exclusion of vertebrae (136/295, $46 \%)$; regarding the femur, it was a poor definition of the analysis box (79/263, $30 \%)$.

Conclusion: More than $90 \%$ of DXA presented at least one error, mainly of DA. ISCD guidelines are very poorly adopted. Errors in acquisition and/or analysis of DXA examinations may result in an inaccurate BMD measurement, with potential relevant implication on patients' management (diagnosis, treatment and follow-up). Strategies to prevent these errors include continuous radiographers' training and permanent monitoring of examinations quality. If radiologists want to accept the future challenge of quantitative imaging should not lose today the clinical task of good clinical practice in DXA.

\section{P880}

THE EFFECT OF BODY MASS INDEX ON TRABECULAR BONE SCORE AND BONE MINERAL DENSITY

C. Messina $^{1}$, C. Monaco ${ }^{1}$, A. Poloni ${ }^{1}$, F. M. Ulivieri ${ }^{2}$, F. Sardanelli $^{3}$, L. M. Sconfienza ${ }^{3}$

${ }^{1}$ Radiology, University of Milan, Milan, Italy, ${ }^{2}$ Bone Metabolic Unit, Medicina Nucleare, Fondazione IRCCS Ca' Granda Ospedale Maggiore, Milan, Italy, ${ }^{3}$ Dipartimento di Science Biomediche per la Salute, University of Milan, Milan, Italy

Objective: Trabecular bone score (TBS) measured on lumbar spine DXA provides an indirect index of trabecular microarchitecture. According to proponents, TBS is adjusted to the patient's BMI. In obese patient, BMD is usually increased due to image noise related to soft tissue superimposition. Our aim was to investigate the effect of BMI on BMD and TBS. 
Materials and Methods: After ethics committee approval, we retrospectively reviewed the last 197 DXA examinations performed at our institution. For each patient, data on TBS, BMI, and BMD were registered. An experienced reader evaluated all examinations. TBS and BMD were automatically averaged from L1 to L4. T-score was categorized according the WHO criteria. The presence of osteoarthrosis was determined when a difference of more than a $1.0 \mathrm{~T}$-score was found between two adjacent vertebrae, in agreement to the International Society for Clinical Densitometry guidelines. Correlation was estimated using the Pearson coefficient and multivariate regression using TBS as dependent variable. Data were presented as mean $\pm \mathrm{SD}$.

Results: T-score diagnosis was normal in 54 patients $(27.4 \%)$, osteopenia in 95 (48.2\%), and osteoporosis in 48 (24.4\%). Overall, BMI was $26 \pm 5 \mathrm{~kg} / \mathrm{m}^{2}$; BMD 0.877 $\pm 0.153 \mathrm{~g} / \mathrm{cm}^{2}$; TBS $1.224 \pm 0.117 \mathrm{~mm}^{-1}$. Osteoarthrosis was evident in $55(28 \%)$ patients. Bivariate correlation analysis between TBS and BMI was significant $(r=-0.396, P<.001)$; this correlation was higher in patients with $\mathrm{BMI} \geq 30 \mathrm{~kg} / \mathrm{m}^{2}$ $(r=-0.501, P=.015)$ than in those with BMI $<30 \mathrm{~kg} / \mathrm{m}^{2}$ $(r=-0.207, P=.006)$. The correlation between TBS and BMD was: $r=0.313(P<.001)$, overall; $r=0.431(P=.040)$, in patients with $\mathrm{BMI} \geq 30 \mathrm{~kg} / \mathrm{m}^{2} ; r=0.408(P<.001)$, in patients with $\mathrm{BMI}<30 \mathrm{~kg} / \mathrm{m}^{2}$. Multivariate regression analysis confirmed that BMI and BMD are independently associated to TBS $(P<.001)$, both overall and only in patients without osteoarthrosis.

Conclusion: The higher the BMI the lower the TBS. BMI and BMD were independent predictors of TBS. TBS evaluation may be impaired in patients with high BMI, especially when $\mathrm{BMI} \geq 30 \mathrm{~kg} / \mathrm{m}^{2}$.

\section{P881}

HEARD OF IT BUT REALLY NOT SURE: KNOWLEDGE AND AWARENESS ABOUT OSTEOPOROSIS AMONG URBAN MALAYSIAN

S. A. Mokhtar $^{1}$, S. N. Aqilah ${ }^{1}$, M. A. Sharkawi ${ }^{2}$, S. M. Zulfarina $^{2}$, K. Y. Chin ${ }^{3}$, A. S. Nazrun ${ }^{2}$, M. Isa ${ }^{2}$

${ }^{1}$ Department of Orthopaedics and Traumatology, University Kebangsaan Malaysia, Kuala Lumpur, Malaysia, ${ }^{2}$ Department of Pharmacology, Faculty of Medicine, University Kebangsaan Malaysia, Kuala Lumpur, Malaysia, ${ }^{3}$ Department of Pharmacology, University Kebangsaan Malaysia Medical Centre, Kuala Lumpur, Malaysia

Objectives: The study was to investigate the awareness and knowledge regarding osteoporosis among patients and accompanying persons attending orthopaedics clinic at a tertiary level university hospital in an urban setting of Kuala Lumpur, Malaysia.
Methods: Knowledge and awareness regarding osteoporosis among 368 participants (male and female) aged $\geq 20$ years old, was assessed using the osteoporosis questionnaire (OPQ), a validated questionnaire. The questionnaire contains 20 items encompassing knowledge in four areas: i) general knowledge, ii) risk factors, iii) treatment, and iv) consequences. MannWhitney test was used to determine the differences in the mean scores between socio-demographic variables.

Results: The mean total OPQ scores was $1.7(\mathrm{SD} \pm 3.08$; range -5 to 9 ; maximum possible score 20 ). We found a significant difference in the total OPQ scores between subjects with and without family history of osteoporosis and also with the subjects who have high education level $(p<0.05)$. However, there is no significant difference $(P>0.05)$ in osteoporosis knowledge in the subjects with respect to age group $(<50 \mathrm{y} \&>50 \mathrm{y})$, gender (male and female), ethnicity (Malay, Chinese and Indian) and menopausal status (pre menopause and postmenopausal). The main sources of osteoporosis knowledge were magazine/newspaper (45.9\%), doctors (30.4), television/radio (22.8\%) and friends (18.2\%).

Conclusion: Overall levels of knowledge and awareness about osteoporosis were low among all participants, especially those with no family members with osteoporosis and lower education level. Strategies to increase the awareness of osteoporosis among the public should be implemented. Printed media (e.g., magazine and newspaper) has been shown to be the applicable and efficient source.

\section{P882}

BONE, NEUROENDOCRINE AND ADRENAL PROFILE IN PREMENOPAUSAL MAMMARY CANCER AND ADRENAL CORTIOCOADENOMA

M. Carsote $^{1}$, A. Dumitrascu ${ }^{2}$, A. Valea ${ }^{3}$, D. Terzea ${ }^{4}$, S. E.

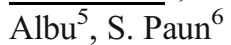

${ }^{1}$ C. Davila University of Medicine and Pharmacy \& C.I. Parhon National Institute of Endocrinology, Bucharest, Romania, ${ }^{2}$ C.I. Parhon National Institute of Endocrinology, Bucharest, Romania, ${ }^{3}$ I. Hatieganu University of Medicine and Pharmacy \& Clinical County Hospital, Cluj-Napoca, Romania, ${ }^{4}$ C.I.Parhon National Institute of Endocrinology \& Monza Oncoteam, Bucharest, Romania, ${ }^{5}$ C.Davila University of Medicine and Pharmacy \& University Emergency Hospital, Bucharest, Romania, ${ }^{6} \mathrm{C}$.Davila University of Medicine and Pharmacy \& Floreasca Emergency Hospital, Bucharest, Romania

Introduction: Skeletal neuroendocrinology with clinical applications is nicely expressed in patients with adrenal tumors or breast cancer when neuroendocrine markers as circulating serotonin (CSer) may also express potential bone anomalies. A carcinoid component might be seen in soma types of mammary neoplasia while the same monoamine (CSer) interfere 
with the bone formation in a negative mechanism (via peripheral CSer with intestinal origin and plates secretion) and a positive one (via central CSer by passing the leptin and sympathetic pathways.

Objective: We introduced a female (within her fifth decade of life) diagnosed with adrenal tumor and hypovitaminosis D while evaluation for a newly discovered mammary cancer.

Material and Method: The bone assessments included DXA; 25-hydroxyvitamin D (25-OH D; $\mathrm{N}: \geq 30 \mathrm{ng} / \mathrm{mL})$, osteocalcin (OC, N: 11-42 pg/mL), P1NP (N: 15-74 ng/mL), CrossLaps $(\mathrm{N}: 0.137-0.537 \mathrm{ng} / \mathrm{mL})$; intact serum PTH $(\mathrm{N}: 15-65 \mathrm{pg} /$ $\mathrm{mL}$ ). Computed tomography (CT) and the neuroendocrine/ adrenal markers as CSer (in $\mathrm{ng} / \mathrm{mL}$ ); chromogranin A (CA, $\mathrm{N}: 20-125 \mathrm{ng} / \mathrm{mL}$ ), baseline and cortisol ACTH (N: 3-66 pg/ $\mathrm{mL}$ ) were tested.

Case data: A Caucasian female is diagnosed with a unilateral breast nodule. She has menarche at 12, one birth and regular menses. She previously has been suspected for carcinoid syndrome due to repetitive hot flashes but no neuroendocrine marker assay could confirm it. Her family medical history for breast/ovarian cancer is irrelevant. Surgery is performed and an Invasive Ductal Carcinoma is confirmed (+ve ER of $80 \%$ ) CT scan found an adrenal tumor of $6.04 \mathrm{~cm}$. The adrenal was nonsecretor: CA of $36.5 \mathrm{ng} / \mathrm{mL}$; ACTH of $8.23 \mathrm{pg} /$ $\mathrm{mL}$, cortisol suppression after DXM. After unilateral mastectomy several surgical approaches have been done: bilateral anexectomy, laparoscopic adrenalectomy and all found benign features. 25-OHD was found low (of $16.4 \mathrm{ng} / \mathrm{mL}$ ) with normal PTH and DXA-BMD for age and bone turnover markers as following: CL of $0.29 \mathrm{ng} / \mathrm{mL}$, OC of $16.58 \mathrm{ng} /$ $\mathrm{mL}, \mathrm{P} 1 \mathrm{NP}$ of $48.52 \mathrm{pg} / \mathrm{mL}$. CSer profile was stationary through surgeries: before adrenal remove of $120 \mathrm{ng} / \mathrm{mL}$ $(\mathrm{N}: 80-450 \mathrm{ng} / \mathrm{mL}$ ) and after of $129 \mathrm{ng} / \mathrm{mL}$. Therapy with daily tamoxifen and vitamin D supplements were started.

Conclusion: Bone parameters are essential to be assessed when starting therapy for breast cancer in patients especially if adrenal tumors are presented. The link between breast and adrenal masses may be due to common genetic background or due to a complex evaluation (in the context of newly discovered malignancy). This work has been partially supported by project no. 33878/11.11.2014.

\section{P883}

MODERN ASPECTS OF TREATMENT OF OSTEOPOROSIS IN POSTMENOPAUSAL AGE IN DEVELOPING COUNTRIES

\section{N. Iobashvili ${ }^{1}, M$. Giorgadze ${ }^{2}$}

${ }^{1}$ Aversi clinic, Tbilisi, Georgia, ${ }^{2}$ Tbilisi State Medical University, Tbilisi, Georgia

Osteoporosis - an implicit epidemic of our times - takes the fourth place in noncommunicable diseases spreading rate (after cardiovascular diseases, cancer and diabetes mellitus) and maintains its relevance not only due to the complications associated with it, but also because of the fact that the elderly population of the Earth is steadily increasing. Osteoporosis becomes medico-social problem due to the high rates of its spreading, disease-associated disability and mortality and the costs related to its treatment and rehabilitation. According to WHO data, every third woman and every fifth man over 50 suffers from osteoporosis - that is over 200 million people around the world. Such an accurate data is not available in Georgia, but the numbers are impressive. Density studies confirm that every third woman over 40 and every fifth man over 50 suffer from osteoporosis. This data is determined by many different factors, among which we would like to highlight: genetic factors (Asian or Caucasian race), hormone range, inadequate nutrition, sedentary lifestyle, long-term glucocorticoid intake, intense tobacco and alcohol consumption, low level of public awareness (superstitions of the hormone replacement therapy). Unfortunately, number of undiagnosed cases in the world is 50 out of a hundred; In Georgia, the number of undiagnosed cases is much larger.

Aim and purpose of the research was to observe the patients' quality of life, BMD and pain relieving remedies during the antiosteoporosis treatment period and evaluation of proper, adequate treatment course.

Testing group consisted of 138 women (age ranged from 40 72). 107 of them were diagnosed osteoporosis. 91 women were of post-menstrual age (48-65) and the remaining 31 were going through menopause period and were not diagnosed osteoporosis. In most cases of benign uterine pathologies radical (surgical) treatment, and not conservative methods, is given preferences and, therefore, post menopause age is relevantly young. Unfortunately, this is common problem not only in Georgia, but also in a whole Caucasian region. The research was based on the participating patients' medical history, lifestyle, bone density data and bone metabolism laboratory examination results. Each patient went through the determination of calcium and non-organic phosphor levels in peripheral blood; as about bone formation markers - alkalinephosphates activity and level of osteocalcin in blood serum were examined. Osteoporosis treatment is aimed at maintaining the bone mass and bone loss prevention, prevention of fractures, pain intensity reduction, improvement of the patient's general condition, increasing of physical activity level, and enhancement of quality of life. Nowadays, the gold standard for the treatment of osteoporosis in Georgia is the usage of bisphosphonates. The group is an analogue of the natural pyrophosphates. They "organize" the bone matrix and inhibit bone resorption by restraining osteoclast activity. Strontium ranelate is also widely use, which is characterized by dual effect: it stimulates bone formation and, at the same time, inhibits bone resorption. 91 women suffering from osteoporosis were distributed into two groups: the first group (57 
women) was prescribed to take alendronate, while the second group (34 women) was given strontium ranelate. Both groups were prescribed periodical hormonal treatment according to the each patient's health sate. Evaluation of the treatment effects was conducted the third, sixth and 12th months. After 12-months alendronate treatment, vertebral pain eliminated significantly. 18-months alendronate treatment resulted into increase of bone density - femur: +5.62 (3.02-10.6)\% $(<0.001)$ and spine: $+10.02(д+6.47 ;+13.95)$. In the cases of strontium ranelate therapy, annual growth of the bone mass reached average $+6.51(+2.72$ to +12.55$)$, though the risk of thrombosis was much higher. Since Georgia is a developing country, it is important to consider many aspects - such as age, occupation, social and economic states of a woman in menopausal period, when deciding if which medicine to prescribe to the certain patient. It is essential to take into account patient's annual income before recommending a drug that should be taken in for a prolonged period. Financial aspect serves as a precondition to the fact that on Georgian market, alendronates sales figures are 3.5 times higher than those of strontium ranelate. Pharmaceutical companies carry out distribution of informational booklets in order raise public awareness in the field of healthcare. Thus, osteoporosis is a medico-social problem. In order to diagnose and treat osteoporosis timely, it is crucial to raise people's awareness and imply adequate and opportune treatment using modern antiosteoporosis therapy.

\section{P884}

THE ROLL OF BOSWELLIA IN TREATMENT OF SECONDARY KNEE OSTEOARTHRITIS AFTER STROKE

$\underline{\text { A. M. Bumbea }}^{1}$, S. Patru ${ }^{1}$, B. S. Bumbea ${ }^{2}$, A. C. Bighea ${ }^{1}$, D. Matei $^{1}$, I. R. Marcu ${ }^{1}$

${ }^{1}$ University of Medicine and Pharmacy, Craiova, Romania,

${ }^{2}$ County Emergency Hospital, Orthopedic Department, Craiova, Romania

Objectives: To provide the importance of Boswellia ingestion in patients with secondary osteoarthritis due to hemiparesis after stroke.

Material and Method: Our study included 54 patients with hemiparesis after ischemic or hemorrhagic stroke. The onset of stroke were one year ago, and patient developed standing and walking with cane. All the patients included in study had had secondary knee osteoarthritis with radiological sings, osteophytes and narrow of joint space. The symptoms of osteoarthritis occurred after stroke and after standing and walking in the hemiparetic lower limb. The median age was 65.7. We split the patients in two groups, one group, A-27 patients, received a combination of rehabilitation therapy which included electrotherapy and kinetic program for hemiparesis and group B-27 patients received the same rehabilitation program and extra medication with Boswellia $400 \mathrm{mg}$ daily for six months. We evaluated the patients initially and after six months. The evaluation scales were: VAS (Visual analogue scale), WOMAC (Western Ontario McMaster Universities Arthritis Index), 6MWD (6 Minute Walking Distance Test).

Results: The group A started from 70.5 score on VAS evaluation and reached to 52.3, comparison with group B which start from 75.0 and decrease to 38.9 , and the WOMAC scale decreased significant in group B with $29.51 \%$. The 6MWD had a major improvement in group B with $50 \mathrm{~m}$, comparison with group A where the distance was maintaining at the same level. Conclusions: Immobility and pain can affect the quality of life and determine the patient with stroke to avoid rehabilitation program due to knee osteoarthritis. The patients with stroke have a restriction of nonsteroidal drugs, and Boswellia could be an alternative way to treat the osteoarthritis in this category of patients and the results show as an improvement of mobility and decrease of pain in group which ingest $400 \mathrm{mg}$ Boswellia daily for six months.

\section{P885}

CHANGING TRENDS IN THE INCIDENCE OF HIP AND OTHER OSTEOPOROTIC TYPE FRACTURES IN IRELAND

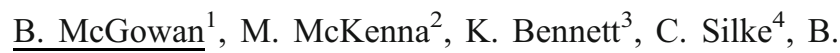
Whelan $^{1}$

${ }^{1}$ The North Western Rheumatology Unit, Our Lady's Hospital, Manorhamilton, Co Leitrim, Ireland, '2 St. Vincent's University Hospital, Dublin, Ireland, ${ }^{3}$ Pharmacology and Therapeutics, Trinity Centre for Health Sciences, St James's Hospital, Dublin, Ireland, ${ }^{4}$ Department of Rheumatology, Sligo General Hospital, Sligo, Ireland

Summary: In contrast to the results of previously published studies on trends in fracture incidence in Ireland (2) the present study identified a stabilising of the trends in the number of hospitalisations for osteoporotic-type fractures in Ireland since 2010 with the exception of hospitalisations for hip fractures in females which showed a minimal increase of $0.2 \%$. The age standardised rates in both women and men also fell with the exception of men aged 85 years and over which showed a modest increase of $1.8 \%$. The declining trends may be partly explained by the specific measures taken in recent years in falls prevention in at risk groups and the heightened awareness of osteoporosis in general in Ireland.

Objectives: The aims of the study were to carry out a trend analyses of all hospitalisations for osteoporotic-type fractures in males and females aged 50 years and over in Ireland between 2010 and 2014. A second objective was to project the number of hip fractures in the Republic of Ireland expected by 2046 based on the 2014 incidence data. 
Methods: Age- and gender-specific trends in the absolute numbers and direct age-standardised rates of hospitalisations for all osteoporotic-type fractures in men and women $\geq 50$ years were analysed along with the associated length of stay using the Hospital In-Patient Enquiry system database (1). Future projections of absolute numbers of hip fractures in years 2021, 2031, 2041 and 2046 were computed based on the 2014 incidence rates applied to the projected populations.

Results: Between 2010 and 2014, the absolute numbers of all osteoporotic-type fractures decreased by $0.4 \%$ in females and by $3.9 \%$ in males while the absolute numbers of hip fractures increased by $0.2 \%$ in women but decreased by $15 \%$ in males. The age-standardised rates for hip fractures decreased in all age groups in both females and males with the exception of males 85 years and older who showed a $1.8 \%$ increase $(p=0.534)$. The mean length of stay (LOS) for hip fractures decreased by $3 \%$ in females and increased by $3 \%$ in males while the mean LOS for all osteoporotic type fractures decreased by $9 \%$ and $12 \%$ in women and men respectively. Assuming stable age-standardised incidence rates from 2014 over the next 30 years, the number of hospitalisations for hip fractures is projected to increase 3 fold from 4,301 in 2014 to 12,708 in 2046.

Conclusion: The trend analyses identified a stabilising of the incidence of osteoporotic type fractures in Ireland between 2010-2014 and a decrease of $15 \%$ in the incidence of hip fractures in males in contrast to a previously published study of earlier trends.

\section{References:}

1. https://www.healthatlasireland.ie/f1live accessed January 2016

2. McGowan B, et al. Osteoporos Int 2013;24:849

Acknowledgments: We would like to thank Health Atlas Ireland for providing access to the HIPE data on which the study was based.

\section{P886}

THE RELATION BETWEEN THE CHRONIC PAIN AND BODY MASS INDEX IN KNEE OSTEOARTHRITIS PATIENTS

D. Matei $^{1}$, S. Patru ${ }^{1}$, R. M. Trăistaru ${ }^{1}$, A.-M. Bumbea ${ }^{1}$, D. V. Caimac $^{1}$, A. C. Bighea ${ }^{1}$

${ }^{1}$ University of Medicine and Pharmacy, Craiova, Romania

Knee osteoarthritis (OA) is a long-term disease that affects especially aged persons and the medical management is not always effective in reducing pain or improving the mobility.

Patients and Method: The patients with knee OA (n:21) were involved in this study. The diagnosis of OA was based on the criteria of American College of Rheumatology (ACR).
Demographic data, disease duration (years), BMI were noted. The pain was evaluated using visual analogue scale $(100 \mathrm{~mm})$ and also the results of HAQ were noted. All the patients had several types of treatment: medication was the most common treatment (nearly $3 / 4$ of the participants did not express any treatment preference), physiotherapy, kinetotherapy.

Results: Using linear regression equation, the correlation between the pain variable was found to be satisfactory for obese patients $\left(R=0.685\right.$ and $\left.\mathrm{R}^{2}=0.512\right)$; a statistically significant correlation and predictivity were obtained for obese patients coming from rural areas $\left(R=0.599, \mathrm{R}^{2}=0.463\right)$ and also in men patients. Analyzing the relation between pain and the HAQ results, we noticed that for obese patients is a statistically significant correlation $\left(\mathrm{R}^{2}=0.790\right)$.

Conclusions: As we expected, the pain represents an important factor for limiting the knee function and it was even more intense as the BMI is greater. It is therefore necessary that patients with knee osteoarthritis to be educated in order to maintain optimum body weight.

\section{P887}

VITAMIN D LEVEL AND BONE VARIABLES IN A GROUP OF OVERWEIGHT MEN

$\underline{\text { A. Alwan }}^{1}$, G. El Khoury ${ }^{1}$, H. Zouhal ${ }^{2}$, C. El Khoury ${ }^{1}$, R. El Hage $^{1}$

${ }^{1}$ Department of Physical Education, Fass, University of Balamand, Kelhat El Kurah, Lebanon, ${ }^{2}$ STAPS, University of Rennes 2, Rennes, France

Aim: To explore the relationship between serum vitamin D and bone variables (bone mineral content, BMD, hip bone geometry and trabecular bone score) in a group of overweight men.

Methods: 82 overweight men (BMI $>25 \mathrm{~kg} / \mathrm{m}^{2}$ ) aged from 18-30 years participated in this study. Weight and height were measured, and BMI was calculated. Body composition and bone variables were measured by DXA. Daily calcium intake and physical activity level were evaluated by validated questionnaires. Serum vitamin D was measured.

Results: Vitamin D was negatively correlated to fat mass $(r=-0.26 ; p<0.05)$ and positively correlated to physical activity level $(r=0.35 ; p<0.01)$. Vitamin $\mathrm{D}$ was not significantly correlated to bone variables. Lean mass was positively correlated to WB BMC $(r=0.72 ; p<0.001)$, WB BMD $(r=0.42$; $p<0.001)$, L1-L4 BMD $(r=0.32 ; p<0.01)$, TH BMD $(r=0.55 ; p<0.001)$, FN BMD $(r=0.37 ; p<0.001)$, FN CSA $(r=0.53 ; p<0.001)$ and FN Z $(r=0.55 ; p<0.001)$.

Conclusion: This study suggests that, in overweight men, vitamin $\mathrm{D}$ is not a determinant of bone variables while lean mass is a positive predictor of these variables. 


\section{P888}

\section{A RETROSPECTIVE STUDY OF THE SECOND HIP FRACTURE}

R. G. Crilly ${ }^{1}$, I. Gutmanis ${ }^{2}$

${ }^{1}$ University of Western Ontario, London Ontario, Canada,

${ }^{2}$ Parkwood Institute, London Ontario, Canada

There is evidence to suggest the intertrochanteric (IT) hip fracture is more associated with osteoporosis than the subcapital (SC) fracture. However which type is more likely to recur, whether the second hip fracture is concordant with the first and the time between are largely unexplored questions.

Methods: Hip fracture patients admitted to a rehabilitation unit were studied retrospectively using the 10 year long electronic medical record to see if this was their second hip fracture, when the first had occurred and if the types were concordant.

Results: 343 patients were studied. In $42(12 \%)$ a previous hip fracture had occurred. Overall in $76 \%$ the second fracture was concordant. Concordance was greater when the first fracture was an SC fracture ( $82 \%$ ) than when it had been an IT fracture $(70 \%)(\mathrm{NS})$. In $52 \%$ the first fracture had been subcapital and in $48 \%$ intertrochanteric, which is the same proportion in those with the first fracture. The average age of patients at the time of their first fracture, and the gender distribution was not different from patients presenting with their first hip fracture. In 17 cases (40\%) the second fracture was the IT fracture, $14(82 \%)$ being preceded by an IT fracture and $3(18 \%)$ by a SC fracture. On average there was 46.5 months, range 5-180 months, between fractures and this did not differ significantly with respect to the type of the first fracture. Overall $26.2 \%$ recurred within 6 months, $40.5 \%$ within a year, $28.6 \%$ in $1-5$ years and in $28.6 \%$ over 5 years.

Conclusion: In this retrospective study, $12 \%$ had had a previous hip fracture with a high degree of concordance especially in the SC fracture patient. If the IT fracture is the preventable fracture the data suggest those presenting with an IT fracture should be treated, whereas those with a first SC fracture may have less to gain. The distance apart of the two fractures may be sufficiently short or long to affect preventability with osteoporosis medication.

\section{P889}

\section{DIFFUSE BONY SCLEROSIS: AN EPIDEMIOLOGIC} RETROSPECTIVE STUDY

K. Baccouche ${ }^{1}$, D. Amri ${ }^{1}$, N. Elamri ${ }^{1}$, S. Belghali ${ }^{1}$, Z. Alaya ${ }^{1}$, H. Zeglaoui ${ }^{1}$, E. Bouajina ${ }^{1}$

${ }^{1}$ Rheumatology, Farhat Hached Hospital, Sousse, Tunisia

Introduction: Sclerotic bone lesions are common, but there are diverse groups of tumors and non- tumorous lesions.
Although plain radiograph and computed tomography can reveal important characteristics of these lesions, diagnosis is often challenging for radiologists. Aim of this study is to determine the different etiologies of generalised increased bone density in patients how were hospitalized in our rheumatology department.

Methods: Retrospective, descriptive study including all patients admitted to our rheumatology department, with diffuse bony sclerosis.

Results: In total 17 patients, with an average age of 57 years with a sex ratio of 1 . Seven patients had risk factors such as the notion of well water consumption. The chief complaint was back pain in $43 \%$ of cases, diffuse bone pain in $25 \%$ of cases, pain in the hips or pelvis in $25 \%$ of cases. The pain was mechanical type for 7 patients, inflammatory for 4 patients and mixed for 6 patients. Biologically, 2 patients had a disturbance of serum levels of calcium and phosphorus with an inflammatory syndrome in 5 patients. All patients had evidence of diffuse sclerosis in standard radiography. An MRI, required in 3 patients, has shown spinal and bone hypertrophy. A bone biopsy was performed in one patient. The different etiologies were fluorosis in 5 patients, renal bone disease in 6 patients, bone metastases in 2 patients, bone infarcts related to sickle cell disease in one patient and no etiology was found in 2 patients. The etiological treatment was medical and / or surgical according to different etiologies.

Conclusion: This study highlights that a diffuse osteosclerosis can be unnoticed and we must first think of a probably malignant progressive disease.

\section{P890}

FACTORS DETERMINING CLINICAL OUTCOME OF LONG-TERM USE OF BISPHOSPHONATES IN MCCUNE-ALBRIGHT SYNDROME AND POLYOSTOTIC FIBROUS DYSPLASIA

N. M. Appelman-Dijkstra ${ }^{1}$, B. C. J. Majoor ${ }^{2}$, M. Fiocco ${ }^{3}$, M. A. J. van de Sande $^{2}$, P. D. S. Dijkstra ${ }^{2}$, N. A. T. Hamdy ${ }^{1}$

${ }^{1}$ Department of Medicine, Division Endocrinology and Center for Bone Quality, Leiden University Medical Center, Leiden, Netherlands, ${ }^{2}$ Department of Orthopedic Surgery and Center for Bone Quality, Leiden University Medical Center, Leiden, Netherlands, ${ }^{3}$ Department of Medical Statistics \& Bioinformatics and Mathematical Institute, Leiden University, Leiden, Netherlands

Objective: McCune-Albright syndrome (MAS) is a rare bone dysplasia characterized by fibrous dysplasia (FD), café-au-lait patches and endocrinopathies. It has been suggested that patients with FD respond favorably to treatment with bisphosphonates by a decrease in bone pain and fracture rate and arrest of progression of bony lesions. Data are scarce and factors that may influence clinical outcome are not known. 
Material and Methods: We studied the clinical and biochemical response to treatment with amino-bisphosphonates in eleven patients with MAS ( 9 female) and 31 patients with polyostotic FD (PFD). Prognostic factors for treatment outcome were evaluated in both groups.

Results: MAS patients had a younger age at diagnosis $(p=0.001)$, worse fracture history $(p=0.021)$, higher alkaline phosphatase concentrations (ALP) $(p=0.005)$ and higher skeletal burden scores $(p=0.000)$ than PFD patients. MAS patients had also higher FGF-23 levels $(p=0.008)$ and higher prevalence of hypophosphatemia $(p=0.013)$. All MAS patients had precocious puberty and five (46\%) GH excess combined with severe craniofacial FD. Four MAS patients $(36 \%)$ had a complete clinical and biochemical response to bisphosphonates and 7 a partial response, of whom 4 with GH excess, compared to a complete response in 25 PFD patients $(81 \%)$ and a partial one in $6(p=0.015)$. In the whole group, levels of FGF-23 were correlated with skeletal burden $(p<0.001)$ but FGF-23 levels did not correlate with treatment response. High skeletal burden was a significant risk factor for a moderate response $(p<0.01)$. Time to achieve a response was significantly shorter in PFD patients $(p=0.002)$ and there was no further improvement in either group after 5 years of treatment. Treatment was well tolerated, and none of the patients developed an atypical fracture or osteonecrosis of the jaw despite the high cumulative doses and long term use of bisphosphonates.

Conclusion: Our data suggest a beneficial clinical and biochemical response to bisphosphonates in PFD also in the presence of endocrinopathies in MAS. MAS patients require, however, higher cumulative doses and longer-term treatment to achieve a similar response as PFD patients, and their response is often incomplete. Factors influencing clinical outcome include high skeletal burden in both PFD and MAS and additional GH excess in MAS.

Disclosures: BCJ Majoor and PDS Dijkstra received a research grant from the Bontius Foundation.

\section{P891}

IS LOW SPINAL BONE DENSITY IN WOMEN WITHOUT SPINAL FRACTURES AN ARTIFACT OR A PRECURSOR TO FUTURE SPINAL FRACTURES

R. G. Crilly $^{1}$, E. Ballantyne ${ }^{1}$, J. Sennet ${ }^{1}$

${ }^{1}$ University of Western Ontario, London Ontario, Canada

Introduction: It is common to see postmenopausal women with low spinal BMD without spinal fractures. Whether these patients are in the early stages of osteoporosis, or whether the low BMD is an artifact is unknown. We hypothesized that low BMD may be an artifact as BMD is an areal density, influenced by vertebral body shape. Wider vertebrae have a greater surface area and may be calculated to have a lower BMD than vertebrae which are deeper and narrower, but of similar mass and strength.

Methods: Postmenopausal women attending the osteoporosis clinic with a BMD T-score $\leq-3.0$ at L2-L4 site, with and without spinal fractures, were studied. Patient height, weight, age, bone mineral content (BMC), vertebral area, BMD for the spine, and BMD at 3 standard hip sites - total, neck and intertrochanteric - were collected. Lateral thoracic and lumbar radiographs were assessed for compression fractures.

Results: Of 112 women assessed, 39 patients had vertebral fractures. The patients did not differ in terms of vertebral morphometry, refuting the primary hypothesis. Fracture patients were significantly older $(71.3 \pm 10.6$ vs. $64.2 \pm 8.5, p<0.001)$ and had small but significantly greater reductions in BMD at all sites. After multiple-regression analysis, only age $(p=0.001)$ and spine T-score $(p=0.014)$ remained significant. Discussion: Low BMD in patients without fractures is not the result of differences in vertebral body shape. These patients may represent an earlier phase in the development of osteoporosis, and may end up with vertebral fractures whether due to the passage of time (risk exposure), or due to a progressive drop in BMD. A longitudinal study could assess this, but for now these patients should be considered at risk and treated accordingly.

\section{P892}

MUSCLE WASTING INDUCED BY BOTULINUM TOXIN IN MICE MEASURED BY MICROCOMPUTED TOMOGRAPHY (MICROCT)

$\underline{\text { H. Libouban }}^{1}$, N. Minier ${ }^{1}$, C. Guintard ${ }^{2}$, E. Aguado ${ }^{2}$, D. Chappard $^{1}$

${ }^{1}$ GEROM, University of Angers, Angers, France, ${ }^{2}$ UPSP BBTO CEx/ONIRIS, Nantes, France

Objective: Muscle and bone mass are highly correlated and muscle impose large load on bone. Long term immobilization or prolonged exposure to microgravity induce a severe muscle wasting and bone loss. Muscle wasting that accompanied bone loss has been poorly investigated mainly due to the lack of appropriate analytical method to quantify muscle loss by microCT. The objective of the study is to assess muscle wasting in a mouse disuse model using a specific contrast agent for optimal use of microCT.

Material and Methods: 21 female mice were spread into 7 groups. At day 0, 18 mice received Botulinum toxin (BTX) injection in the quadriceps to induce paralysis of the right hind limb; the left contralateral side was used as control. Mice were sacrificed at 7, 14, 21, 28, 56 and 90 days post-BTX (3 mice/group). The 56 and 90 day periods correspond to the recovery of disuse because of reversible effect of BTX. The remaining group was not injected and sacrificed at day 0 . Hind limbs were immersed in a contrast agent solution containing 
$\mathrm{HgCl}_{2}$ for muscle visualization in $3 \mathrm{D}$ by microCT allowing a morphometric analysis. Three parameters were calculated for the gastrocnemius, quadriceps femoris and plantaris muscles: area $\left(\mathrm{mm}^{2}\right)$ occupied by each muscle in 2D section, circularity and aspect ratio. 2 muscles were carefully dissected and weighed (gastrocnemius and quadriceps femoris).

Results: The area of gastrocnemius and of quadriceps were significantly lower in the paralyzed limb from 7 days; the decrease was maximum at resp. 21 days ( $-46.9 \%)$ and 28 days $(-47.9 \%)$. No difference in geometric form parameters were found between the paralyzed and non-paralyzed limb. No modifications were observed for the plantaris muscle which was not influence by BTX injection. Similar results were obtained with the anatomical method. Significant correlations were obtained between area and weight for the gastrocnemius and quadriceps muscles $(r=0.782, p<0.001)$.

Conclusions: The use of a specific contrast agent opens new perspective to better understand musculoskeletal relationships.

\section{P893 \\ ENDOTOXIN AND BONE TURNOVER MARKERS IN POSTMENOPAUSAL SAUDIS WITH AND WITHOUT OSTEOPOROSIS}

N. J. Aljohani ${ }^{1}$, N. M. Al-Daghri ${ }^{2}$, I. Aziz ${ }^{2}$, S. Yakout ${ }^{2}$, Y. AlSaleh $^{3}$, O. S. Al-Attas ${ }^{2}$, M. S. Alokail ${ }^{2}$

${ }^{1}$ King Saud bin Abdulaziz University for Health Sciences, Riyadh, Saudi Arabia, ${ }^{2}$ Biochemistry Department, College of Science, King Saud University, Riyadh, Saudi Arabia, ${ }^{3}$ King Abdulaziz Medical City, College of Medicine, King Saud Bin Abdulaziz University for Health Sciences, Riyadh, Saudi Arabia

Bacterial Lipopolysaccharide (LPS) also known as endotoxin which represents the outer cell wall membrane of gram-negative bacteria has been implicated as the major bacterial bone-resorbing factor. Recently, the most prevalent form of clinically significant osteopenia and osteoporosis involves periodontitis and otitis media by Gramnegative bacteria. The aim of the study is to evaluate circulating endotoxin levels and to study the association amongst endotoxin and bone turnover markers in a cohort of Saudi postmenopausal women with or without osteoporosis. We determined the levels of endotoxin, bone turnover markers, 25-OH vitamin D total and corrected calcium in 100 Saudi postmenopausal women with osteoporosis and 100 women without osteoporosis were taken under the supervision of qualified physicians in the primary care centers in Riyadh. Serum endotoxin, NTx, osteocalcin, PTH, 25-OH vitamin D total and calcium were analyzed. Serum NTX and PTH levels in patients with osteoporosis were significant higher than controls.
Serum endotoxin was significantly and positively associated with calcium in all subject and controls. Endotoxin was positively associated with NTX in both groups but not with osteocalcin, PTH or 25-OH vitamin D. Findings of the present study implicate a role for endotoxinmediated inflammation in patients with osteoporosis.

\section{P894}

EFFECTS OF TERIPARATIDE ON BONE METABOLISM OF PATIENTS WITH RHEUMATOID ARTHRITIS AND NON RHEUMATOID ARTHRITIS

D. Kida ${ }^{1}$, T. Fujito ${ }^{1}$, H. Kanda ${ }^{1}$, N. Takahashi ${ }^{1}$

${ }^{1}$ Orhopedic Surgery and Rheumatology, Nagoya Medical Center, Nagoya, Japan

Objectives: To evaluate change in bone quality in patients at high risk of fracture by quantification of several bone biochemical markers and BMD, before and after teriparatide (TPD) treatment.

Methods: 85 female patients with rheumatoid arthritis (RA; mean age $70.8 \pm 8.0 \mathrm{yrs}$, and 69 female patients with non-RA (N-RA; mean age 69.9 \pm 10.9 ) were assessed. Levels of serum osteocalcin (OC), serum undercarboxylated osteocalcin (ucOC), serum bone specific alkaline phosphatase (BAP), procollagen type I N-terminal propeptide (PINP) serum tartrate resistant acid phosphatase isoform $5 b$ (TRAP-5b), urinary N-telopeptide of type I collagen/creatinine (NTx), serum calcium (Ca), serum phosphorus $(\mathrm{P})$, vitamin $\mathrm{D}$ metabolites 1,25-dihydroxyvitamin D3 (1,25D3), intact PTH (iPTH), calcitonin (CT) and BMD at the lumbar spine (LS) and total hip (TH) were measured at baseline and 24 months after TPD by subcutaneous injection ( $20 \mu \mathrm{g}$ once a day). Statistical analysis also performed.

Results: Levels of biochemical markers before and after TPD are summarized (Table 1). Significant increasing was found in levels of $\mathrm{PPTH}$ and CT in both RA and N-RA groups. Significant differences between RA group and N-RA group were not found before and after treatment. TPD administration significantly stimulated bone formation and bone resorption and increased BMD after 24 months in both RA and N-RA groups.

Conclusions: It is possible that TPD administration might adjust the calcium related hormone dynamics, to stabilize the calcium concentration.

Table 1a. Levels of biochemical markers before TPD administration

\begin{tabular}{llll}
\hline & $\begin{array}{c}\text { RA Group } \\
(n=85)\end{array}$ & $\begin{array}{c}\text { N-RA Group } \\
(n=69)\end{array}$ & $\begin{array}{c}\text { P-Value } \\
(\text { RA vs N-RA) }\end{array}$ \\
\hline iPTH $(\mathrm{ng} / \mathrm{mL})$ & $52.1+/-0.46^{*}$ & $21.27+/-16.90^{*}$ & NS \\
$\mathrm{CT}(\mathrm{pg} / \mathrm{mL})$ & $30.7+/-12.2$ & $30.6+/-13.5$ & $\mathrm{NS}$ \\
$\mathrm{VitD}(\mathrm{mg} / \mathrm{mL})$ & $62.0+/-28.3$ & $66.7+/-27.4$ & $\mathrm{NS}$ \\
$\mathrm{Ca}(\mathrm{mg} / \mathrm{dL})$ & $9.76+/-0.41$ & $9.87+/-0.41$ & $\mathrm{NS}$ \\
\hline
\end{tabular}


Table 1b. Levels of biochemical markers after TPD administration

\begin{tabular}{llll}
\hline & $\begin{array}{c}\text { RA Group } \\
(n=85)\end{array}$ & $\begin{array}{c}\text { N-RA Group } \\
(n=69)\end{array}$ & $\begin{array}{c}\text { P-Value } \\
\text { (RA vs N-RA) }\end{array}$ \\
\hline iPTH $(\mathrm{ng} / \mathrm{mL})$ & $9.64+/-0.46^{*}$ & $21.27+/-16.90^{*}$ & NS \\
$\mathrm{CT}(\mathrm{pg} / \mathrm{mL})$ & $32.9+/-12.5^{*}$ & $32.9+/-12.1 *$ & $\mathrm{NS}$ \\
$\mathrm{VitD}(\mathrm{mg} / \mathrm{mL})$ & $69.9+/-24.7$ & $77.4+/-89.4$ & $\mathrm{NS}$ \\
$\mathrm{Ca}(\mathrm{mg} / \mathrm{dL})$ & $9.64+/--0.46^{* *}$ & $9.66+/-0.43^{* *}$ & NS \\
\hline
\end{tabular}

Two-sided P-value for the comparison between RA Group and N-RA Group are detrmined by Mann-Whitney test. Two-sided P-value for the comparison between baseline and 24 months in each group are determined by Wilcoxon signed rank test.

$*: P<0.05, * *: P<0.01, * * *: P<0.001$ vs before.

\section{P895}

\section{THE EFFECT OF GENETIC VARIANTS OF VITAMIN} D PATHWAY ON THE VARIANCE OF SERUM PTH LEVEL

B. Szili ${ }^{1}$, B. Bakos ${ }^{1}$, B. Szabó ${ }^{1}$, P. Horváth ${ }^{1}$, A. G. Tabák ${ }^{1}$, E. $\overline{\text { Toldy }^{2}}$, P. Lakatos ${ }^{1}$, I. Takács ${ }^{1} 1$ st Department of Internal Medicine, Semmelweis University, Budapest, Hungary, ${ }^{2}$ Department of Diagnostics, University of Pécs, Faculty of Health Science, Pécs, Hungary

Objective: As there are scarce data available on the genetic factors that influence serum PTH, calcium and phosphate levels, we aimed to evaluate determinants of PTH levels.

Material and Methods: In a representative cross-sectional study of the Hungarian adult population we measured PTH, calcium, phosphate, total 25-hydroxyvitamin D (t-25OHD), vitamin D-binding protein (DBP) and albumin levels. Bioactive (b25OHD) and free (f-25OHD) vitamin D concentrations have been calculated. Blood samples were taken at the end of winter. We have determined factors that affect vitamin $\mathrm{D}$ level: vitamin D intake, solarium use, and travel below the 40th latitude. Patients affected by the abovementioned factors have been excluded from our study. We genotyped 29 SNPs of VDR, GC, CYP2R1, CYP24A1 and NADSYN1 genes in the model population. Pearson's correlation, two-way ANOVA with Bonferroni post hoc test and univariate linear regression were calculated.

Results: Serum PTH level correlated with b-25OHD, f-25OHD, creatinine, BMI and age. Interestingly, no correlation was found with $\mathrm{t}-25 \mathrm{OHD}$, calcium or phosphate levels in the model population. Only three out of the examined 29 SNPs (rs1993116, rs10500804, rs11023374 [CYP2R1]) showed a significant effect on PTH levels. The three SNPs and the five factors that previously correlated with PTH were added to a linear regression model as co-variates. Creatinine and f-25OHD levels, and BMI and rs10500804 SNP status have shown no significant effect on
PTH levels, and have been excluded from the analysis. The analysis conducted with the remaining three factors showed that the two SNPs are responsible for $3 \%$ of the variance of PTH levels, while age and b-25OHD are responsible for a $9 \%$ and a $5 \%$ variance, respectively.

Conclusion: Genetic variances of CYP2R1 (vitamin D 25hydroxylase encoding gene) might be a factor of the variance of PTH levels at the end of winter.

\section{P896}

\section{UNDERREPORTING OF VERTEBRAL FRACTURES} IN AN OLDER IRISH POPULATION

O. Hannigan ${ }^{1}$, P. Claffey ${ }^{1}$, N. Fallon ${ }^{1}$, E. Laird ${ }^{2}$, J. Mahon ${ }^{3}$, B. McCarthy $^{3}$, J. B. Walsh ${ }^{3}$, K. McCarroll ${ }^{1}$, M. Casey ${ }^{1}$, R. Lannon $^{1}$

${ }^{1}$ Department of Gerontology, St James Hospital, Dublin, Ireland, ${ }^{2}$ Institute of Molecular Medicine, Trinity College, Dublin, Ireland, ${ }^{3}$ Mercers Institute for Research and Aging, St James Hospital, Dublin, Ireland

Objectives: Vertebral fractures are often asymptomatic and undiagnosed and are associated with an increased risk of future fracture at one year $(20 \%)$ and a significant increase in mortality ${ }^{1}$. Vertebral Fracture Assessment (VFA) is a modality which can be used to identify fractures on DXA that are often otherwise missed. VFA is not used as standard in all centres and it has not been clearly established which patient groups should be targeted. Data from a large cross sectional study was used to investigate the role of VFA in older adults with osteopenia/osteoporosis.

Materials and Methods: Study subjects were participants of the TUDA (Trinity, Ulster, Dept of Agriculture) study and were community dwelling Irish adults aged over 60 with a diagnosis of osteopenia/osteoporosis (using standard WHO criteria). We explored the prevalence of vertebral fractures (both self-reported and by VFA) in the overall study population. We also investigated for predictors of vertebral fracture using age, gender, BMI, IADL (Instrumental of Activities of Daily Living), TUG (Timed Up and Go), alcohol intake, smoking, eGFR and 25(OH)D as dependent variables.

Results: Data were available for 1203 subjects. Mean age 72.84 $( \pm 7.55)$, MMSE $27.5( \pm 2.2)$ and TUG $9.9( \pm 5.0)$ s. The overall prevalence of vertebral fractures identified on VFA was $17 \%$ compared to $13 \%$ that were self-reported. In regression analysis, the only positive predictor of vertebral fracture(s) was age $(\beta=0.038, p=0.016)$ whereas the only negative predictor was $\operatorname{IADL}(\beta=-0.113, p=0.000)$. The majority $(81.9 \%)$ of patients with fractures on VFA were unaware of them.

Conclusions: We identified a $30 \%$ greater prevalence of vertebral fractures using VFA vs. patient self-report. This cohort represents patients typically seen in clinical practice. It highlights the importance of VFA as an adjunct to clinical 
assessment which could alter patient management and improve outcomes. In particular, the presence of a vertebral fracture in those with osteopaenic T-scores puts patients in a much higher risk category that helps guide optimal treatment. Findings suggest VFA should be utilised especially in those who are older and have worse functional status.

\section{References:}

- Francis RM, et al. QJM Feb 2004

\section{P897}

WILL SUPPLEMENTATION WITH LOW-DOSE OF CALCIUM PREVENT THE BONE CHANGES INDUCED BY OESTROGENS DEFICIENCY WITH SAFETY IN RATS?

$\underline{\text { A. Agripino }}^{1}$, C. Mello-Sampayo ${ }^{2}$, D. Stilwell ${ }^{3}$, M. Vaz ${ }^{4}$, A. Fernandes $^{5}$, B. Silva-Lima ${ }^{2}$, H. Santos ${ }^{6}$, H. Canhão ${ }^{7}$, M. C. Marques $^{2}$

${ }^{1}$ FCT U.Nova, Lisboa, Portugal, ${ }^{2}$ Pharmacological Scs Department, FF.ULisboa, Lisboa, Portugal, ${ }^{3}$ Clinica Veterinária de Colares, Sintra, Portugal, ${ }^{4}$ IST.ULisboa, Lisboa, Portugal, ${ }^{5}$ UBiA, FCT U.Nova, Almada, Portugal, ${ }^{6}$ Hospital de Sta Maria, CHLN, Lisboa, Portugal, ${ }^{7}$ Faculdade de Medicina da Universidade de Lisboa, Lisboa, Portugal

Introduction: Calcium supplementation in adult humans in the recommended doses (1-1.2 g) still generates controversy for safety reasons. Aiming to circumvent the risks, the effectiveness of a low dose of calcium supplement in preventing the bone changes associated with estrogen deficiency as well as its safety was studied in an animal model.

Methods: Female Wistar rats $(n=18)$ randomized into 3 groups: sham, ovariectomized (OVX), OVX supplemented $(\mathrm{OVX}+\mathrm{S})$ with oyster-shells $(105 \mathrm{mg} / \mathrm{kg})$ and Guar gum $(43 \mathrm{mg} / \mathrm{kg}$ ) for 53 -days (human equivalent dose of $420 \mathrm{mg} /$ day calcium and $414.5 \mathrm{mg} /$ day Guar). At D60 were evaluated: body-weight, aorta and kidney histology and calcium deposits (von Kossa staining), femoral calcium and urinary calcium (atomic absorption); femur biomechanics and tibia cortical bone (SEM); and serum biomarkers levels. Kruskal-Wallis test $(p<0.05)$ were applied.

Results: The body weight of supplemented and nonsupplemented animals increased by $22 \%$ and $33 \%$ with no change in glycaemia and lipid profile. Histological examination of the aorta and kidney revealed no deposition of calcium. Bone remodeling increased $(p<0.05)$ in the OVX and
$\mathrm{OVX}+\mathrm{S}$. The urine calcium increased in the $\mathrm{OVX}(p<0.05)$ and $\mathrm{OVX}+\mathrm{S}$. The supplement mitigated the hypocalcaemia $(p<0.01)$, the femoral calcium reduction $(p<0.05)$ and the tibia cortical thickness while increased the stiffness $(p<0.05)$, yield stress and ultimate stress $(p<0.05)$ of the femurs compared to OVX.

Conclusion: The supplementation with low doses of calcium, provided by oyster shell and Guar gum supplement, attenuated the body-weight gain, the calciuria, the hypocalcaemia, the reduction of the tibia cortical thickness and the femoral demineralization, giving greater femoral stiffness and toughness with no apparent risk of vascular and kidney calcium deposits formation.

\section{P898}

RISK OF OSTEOPOROSIS IN TYPE 2 DIABETES MELLITUS PATIENTS

$\underline{\text { C. F. Palici }}{ }^{1}$, C. Criveanu ${ }^{2}$, A. E. Musetescu ${ }^{2}$, F. A. Vreju ${ }^{2}$, P. Ciurea $^{2}$

${ }^{1}$ Emergency County Hospital Craiova, Craiova, Romania, ${ }^{2}$ University of Medicine and Pharmacy of Craiova, Craiova, Romania

Objectives: The aim of the study was to identify the risk of type 2 diabetes mellitus with oral antidiabetic drugs and insulin treated patients to develop osteoporosis.

Material and Method: We analyzed 44 postmenopausal female patients with type 2 diabetes mellitus, without bisphosphonates drugs or vitamin $\mathrm{D}$ and calcium supplements, 32 with oral antidiabetic drugs and 12 insulin treated, to which we assessed BMD by performing DXA at lumbar spine and femoral neck.

Results: The mean age of patients was 57.5 years, ranging from 40-75 years with mean duration of type 2 diabetes of 10.5 years. In patients with oral antidiabetic drugs we recorded a T-score at femoral neck between -1.3 and -2.7 and a T-score at lumbar spine between -1.6 and -3.1 , while insulin-treated patients had an higher T-score in both on lumbar spine and femoral neck, between -1 and -1.9 , respectively 0.8 and -1.3 . In 3 insulin-dependent patients who initially were treated with oral antidiabetics, we found a lower BMD at the lumbar spine compared to the femoral neck. 
Conclusion: Patients with type 2 diabetes usually have an increase body mass and, in fact, may be relatively protected from osteoporosis, that has been associated with an increased bone mass in both normal and diabetic individuals, but oral antidiabetic drugs seem to influence BMD, these patients requiring vitamin $\mathrm{D}$ and calcium supplements.

\section{P899}

SUN EXPOSURE, SKIN COLOR AND VITAMIN D STATUS IN ARAB CHILDREN AND ADULTS

Y. Al-Saleh $^{1}$, N. Al-Daghri ${ }^{2}$, N. Khan ${ }^{2}$, S. Sabico ${ }^{2}$, N. Aljohani $^{3}$, H. Alfawaz ${ }^{4}$, M. Alsulaimani ${ }^{2}$, A. Al-Othman ${ }^{2}$, M. Alokail ${ }^{2}$

${ }^{1}$ King Abdulaziz Medical City, College of Medicine, King Saud Bin Abdulaziz University for Health Sciences, Riyadh, Saudi Arabia, ${ }^{2}$ Biochemistry Department, College of Science, King Saud University, Riyadh, Saudi Arabia, ${ }^{3}$ King Saud bin Abdulaziz University for Health Sciences, Riyadh, Saudi Arabia, ${ }^{4}$ Food and Science Nutrition, College of Food Science and Agriculture, King Saud University, Riyadh, Saudi Arabia

Accumulating evidence suggests an increased prevalence of vitamin D deficiency in the Middle East and North African countries. Sunlight has long been recognized as a major provider of vitamin $\mathrm{D}$ and lighter skin color has been associated with better vitamin D status. In this context, we aimed to determine whether 25-hydroxyvitamin D [25-(OH)D] concentrations are related to skin color, sun exposure and gender among healthy Saudi children and adults. A total of 808 Saudi children (age $=14.6 \pm 0.04$ years) and 561 (age $=31.4 \pm 0.3$ years) adults of both genders were included in this study. Levels of sun exposure and skin color were determined using a standard questionnaire. Anthropometry and serum 25-(OH)D levels were analyzed. On the basis of duration of sun exposure $(<20 \mathrm{~min}$ vs. $>20 \mathrm{~min})$, a significantly lower level of $25-(\mathrm{OH}) \mathrm{D}(40.9 \pm 1.2$ vs. $35.5 \pm 1.8 \mathrm{nmol} / 1 ; p<0.019)$ was demonstrated in dark skinned boys with exposure time less than 20 min than those exposed longer than $20 \mathrm{~min}$. Given the same age group, gender and skin color, circulating levels of 25-(OH)D in an Arabic cohort was significantly lower only in dark skinned boys having lesser sun exposure.

\section{P900}

A CLINICAL STUDY TO EXAMINE THRESHOLDS OF JOINT SPACE WIDTH AND JOINT SPACE AREA FOR IDENTIFICATION OF KNEE OSTEOARTHRITIS

R. Ljuhar $^{1}$, B. Norman ${ }^{1}$, D. Ljuhar ${ }^{1}$, T. Haftner ${ }^{1}$, J. Hladuvka $^{2}$, P. Bui Thi $\mathrm{Mai}^{2}$, H. Canhão ${ }^{3}$, J. Branco ${ }^{3}$, A. M. Rodrigues $^{3}$, N. Gouveia ${ }^{3}$, S. Nehrer ${ }^{4}$, A. FahrleitnerPammer $^{5}$, H.-P. Dimai ${ }^{5}$

${ }^{1}$ Braincon Technologies, Vienna, Austria, ${ }^{2}$ VRVis Research Competence Center, Vienna, Austria, ${ }^{3}$ Faculdade de Medicina da Universidade de Lisboa, Lisboa, Portugal, ${ }^{4}$ Center for Regenerative Medicine \& Orthopedics, Danube University, Krems, Austria, ${ }^{5}$ Department of Internal Medicine, Division of Endocrinology and Metabolism, Medical University of Graz, Graz, Austria

Objective: Osteoarthritis (OA) is a degenerative, slowly developing joint disease and characterized by pain and functional disability. Although clinical indications of OA can vary among different definitions there is a general agreement that the disease is associated with cartilage narrowing and the development of osteophytes and sclerosis within the subchondral bone. However, there is no general consensus about the threshold below which the joint space width (JSW) and the respective joint space area (JSA) can be certain indicators for the state of OA. Therefore this study evaluates these limits to reveal quantitative information about indicators of OA.

Methods: The study included 226 standardized knee radiographs from 101 female patients with OA, and 125 controls. All images were acquired in PA direction and standardized positions. The minimum JSW and JSA were calculated by using the i3a software. 3 physicians assessed the 2D radiographs by using the Kellgren \& Lawrence Score and assigned the images to either a Case or Control group. A knee was assigned to the Case group, if at least two physicians assessed it as being affected by OA. The JSW was defined as the vertical distance from the inferior femur condyle to the superior tibia condyle on both the medial and lateral compartment, obtained from 4 distinct points from each side. By building a spline curve between the points of each condyle, the upper and lower boundaries of the JSA are defined for each side, whereas the outer points define the horizontal boundaries. For the JSW and JSA, only the minimum value of each variable was taken into account.

Results: Considering the minimum JSW, an odds ratio of 5.63 (CI: 3.17 - 9.99) with an accuracy of $70.35 \%$ and a sensitivity 
of $70.30 \%$ can be obtained. Every subject that has a minimum JSW below $3.4 \mathrm{~mm}$ belongs to the Case group. With respect to the minimum JSA, the odds ratio is 3.60 with an accuracy of $65.49 \%$ and a sensitivity of $65.35 \%$. Results also show that every subject with a minimum JSA below $50 \mathrm{~mm}^{2}$ is being considered to have OA.

Conclusion: Based on this study it can be concluded that a JSW below $3.4 \mathrm{~mm}$ and a JSA below $50 \mathrm{~mm}^{2}$ at the knee joint are strong indicators for OA. Thus, for clinical assessments it is suggested to consider these threshold values for diagnostic purposes. In further studies, symptomatic knee OA should be incorporated to verify whether minimum JSWs and JSAs can also be linked to symptomatic knee pain.

\section{P901}

EXERCISE PROMOTES BONE REJUVENATION: STUDY IN BIOCHEMICAL BONE MARKER AND CIRCULATED STEM CELL

\section{Tinduh}

${ }^{1}$ Physical Medicine and Rehabilitation School of Medicine Airlangga University, Dr. Soetomo General Hospital, Surabaya, Indonesia

Introduction/Background: Biochemical bone markers reflect the activities of bone remodeling. C- telopeptide (CTx) is marker for bone resorption by osteoclast and P1NP is marker for bone formation by osteoblast. Estrogen has role in bone quality regulation especially in women, start from stem cells until mature cells. This study was aimed to reveal the effect of exercise in bone rejuvenation in postmenopausal women. It was hypothesized that there were different profiles of stem cell and bone mature cell activities in premenopausal, postmenopausal and exercised postmenopausal women.

Material and Methods: This pretest-posttest randomized study design was enrolled 18 postmenopausal women with comparison group of 38 premenopausal women. All subjects were underwent the blood sample examination for serum CTx, P1NP, IL-6 and PTH and evaluation of circulated stem cells in peripheral blood mononuclear cell with flow cytometry (percentages of CD34- cells, CD105+CD49d- cells, CD105+CD49d + cells, CD49d + cells, CD117-CD116+ cells, CD117-CD184+ cells). Postmenopausal women group was evaluated before and after play speed walking exercise with increasing cadence $25 \%$ from initial cadence, 3 times a week for 3 weeks. Premenopausal women group was not treated.

Results: There were significant different $(p=0.000)$ between premenopausal women and pre-exercised postmenopausal women in CTx, P1NP, IL-6, percentage of CD105+ CD49d + cells, CD49d + cells and CD117-CD116+ cells. Also there were significant different $(p=0.000-0.002)$ between pre-exercised postmenopausal women and post- exercised postmenopausal women in IL-6, percentages of CD34- cells, CD49d+cells and CD117-CD116+ cells. Significant different between premenopausal women and post- exercised postmenopausal women were on CTx $(p=0.000), \mathrm{P} 1 \mathrm{NP}(p=0.000)$, percentages of CD34- cells $(p=0.004), \mathrm{CD} 105+\mathrm{CD} 49 \mathrm{~d}+$ cells $(p=0.001), \mathrm{CD} 49 \mathrm{~d}+$ cells $(p=0.000)$ and CD117-CD116 + cells $(p=0.020)$. But there were no significant different between premenopausal women and post-exercised postmenopausal women in IL-6 $(p=0.094)$. PTH and percentages of CD117-CD184+ cells were no difference.

Conclusion: PTH and percentages of CD117-CD184+ cells were same for pre- and postmenopausal women. Exercise could improve profile of IL-6, percentages of CD49d+cells and CD117-CD116+ cells.

\section{P902}

OBSTRUCTIVE SLEEP APNEA AS A RISK FACTOR FOR OSTEOPENIA AND OSTEOPOROSIS IN THE MALE POPULATION

C. Liguori ${ }^{1}$, F. Placidi ${ }^{2}$, N. B. Mercuri ${ }^{2}$, E. Piccirilli ${ }^{2}$, F. Izzi $^{2}$, U. Tarantino $^{4}$

${ }^{1}$ Department of Systems Medicine, University Hospital of Rome "Tor Vergata", Rome, Italy, ${ }^{2}$ Department of Systems Medicine, University Hospital of Rome, Rome, Italy, ${ }^{3}$ Department of Orthopedics and Traumatology, Rome, Italy, ${ }^{4}$ Department of Orthopedics and Traumatology, "Tor Vergata" University of Rome, Rome, Italy

Obstructive sleep apnea (OSA) is a sleep disorder featured by recurrent apnea events leading to hypoxia, hypercapnia and sleep disruption. OSA represents a growing health problem mainly affecting men; in fact, its prevalence in the adult male population is between 4 and $24 \%$. It was recently demonstrated that OSA may lead to a deficient vitamin $\mathrm{D}$ status inducing a secondary hyperparathyroidism, which may produce the demineralization of the skeleton and the reduction of the BMD. Therefore, the aim of the present study was to evaluate bone homeostasis in a large cohort of male OSA patients compared to male controls matched for age, BMI and physical activity, also investigating and correlating the measured BMD to both polygraphic parameters, Epworth Sleepiness Scale (ESS) scores and serum biomarkers levels, such as vitamin D, PTH, calcium, fibrinogen and C-reactive protein (CRP). 240 consecutive severe OSA patients were screened from October 2014 to March 2015; 148 patients were excluded and 92 patients were included in the study. The control population consisted of 50 male subjects with age, BMI an physical activity matched with OSA patients. Lower vitamin D, higher PTH and increased 
fibrinogen and CRP serum levels were found in OSA patients with respect to controls $(p<0.0001, p<0.05$, $p<0.001, p<0.001)$. We found the significant reduction of BMD in all the regions analysed of both lumbar spine and femur in OSA patients with respect to controls. Moreover, $\chi$-square analysis showed that T-scores consistent with osteopenia/osteoporosis were more frequent in OSA patients compared to controls at all regions of lumbar spine and at femur neck, upper femur neck, and Ward triangle. The correlation test documented significant correlations between BMD and oxygen saturation parameters, BMI, ESS scores and vitamin D levels. Therefore, this study documented that male OSA patients are affected by reduced BMD in lumbar spine and femur, thus suffering more frequently from osteopenia and osteoporosis in those regions with respect to age, BMI and physical activity matched male controls. Since BMD reduction in several lumbar and femur segments significantly correlated with the alteration of night oxygen saturation indices, hypoxia seems to be the main candidate in reducing BMD in male severe OSA patients. In conclusion, this report proposes the clinical potential of performing DXA in male patients, since OSA could be a detrimental factor on BMD leading to osteopenia and osteoporosis and thus giving susceptibility to bone fractures.

\section{P903}

\section{ARE THE CRACKS STARTING TO APPEAR IN BISPHOSPHONATE TREATMENT FOR OSTEO- POROSIS?}

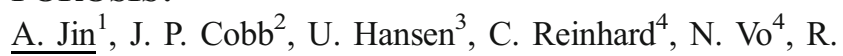

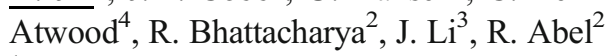

${ }^{1}$ Mechanical Engineering, London, United Kingdom, ${ }^{2}$ Faculty of Medicine, Imperial College, London, United Kingdom, ${ }^{3}$ Mechanical Engineering, Imperial College, London, United Kingdom, ${ }^{4}$ Diamond Light Source, Didcot, United Kingdom

Animal and human studies have reported that bisphosphonate (BP) is associated with over-suppression of remodelling and accumulation of microcracks, but the effect (if any) on trabecular bone strength remains unclear. Our aim was to determine whether increased microdamage associated with BP was offset by improvements in trabecular bone volume and microstructure so that there was no loss of compressive strength. Trabecular bone from hip fracture patients treated with BP $(n=10)$ was compared to naïve fractured $(n=14)$ and non-fractured controls $(n=6)$. Samples were synchrotron scanned for structural analysis, then mechanically tested in compression. BP therapy did not appear to confer any mechanical advantage in compressive strength to the bone of patients suffering hip fractures. BP therapy was associated with a significantly higher microcrack density than untreated controls, but there was no discernible improvement in bone volume or microstructure. The increase in microcrack density and reduction in bone strength may be related.

\section{P904}

RELATIONSHIP BETWEEN BONE SPECIFIC SURROGATES AND BONE MINERAL DENSITY WITH HIGH CIRCULATING CYSTATIN C IN ELDERLY MALES WITH CHRONIC HEART FAILURE

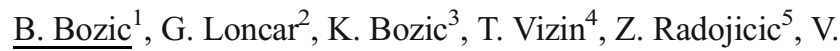
Popovic $^{6}$, J. Kos ${ }^{4}$

${ }^{1}$ University of Belgrade, Faculty of Biology, Institute for Medical Research, Military Medical Academy, Belgrade, Serbia, ${ }^{2}$ Cardiology Department, Clinical Medical Center Zvezdara, Belgrade, Serbia, ${ }^{3}$ Clinic for Rheumatology and Clinical Immunology, Military Medical Academy, Belgrade, Serbia, ${ }^{4}$ Faculty for Pharmacy, University of Ljubljana, Ljubljana, Slovenia, ${ }^{5}$ Institute for Statistics, Faculty of Organizational Sciences, University of Belgrade, Belgrade, Serbia, ${ }^{6}$ Faculty for Medicine, University of Belgrade, Belgrade, Serbia

Background: The aim of the present study was to investigate the association of bone mass and bone specific surrogates with cystatin $\mathrm{C}$ in elderly males with chronic heart failure (CHF). Methods: Sixty-eight males (mean age $68 \pm 7$ years) with stable mild to moderate CHF and 20 age- and BMI-matched healthy individuals underwent cardiovascular, BMD, and body composition assessment. Biochemical assessment included bone specific surrogates (osteocalcin (OC), $\beta$ CrossLaps ( $\beta$-CTx), osteoprotegerin (OPG), RANKL, PTH, 25-hydroxyvitamin D (25(OH)D), NT-pro-BNP, and cystatin C (Cys C).

Results: Hip, total hip and lumbar spine BMD were significantly lower in CHF patients compared to healthy controls. On the other hand, waist, waist/hip ratio, BMI, fat and lean mass were similar between studied groups. Serum Cys C was significantly increased in CHF patients compared to healthy controls. A significant positive association was found between $\mathrm{OC}$ and Cys $\mathrm{C}$ levels in CHF males, and $\mathrm{OC}$ and $\beta-\mathrm{CTx}$ increased in increasing Cys $\mathrm{C}$ tertiles. In multivariate regression analysis, OC and smoking were a significant determinant of Cys $\mathrm{C}$ in CHF patients. Level of Cys C was found to be positively associated with an increased risk of mortality in elderly CHF males.

Conclusions: Serum osteocalcin is an independent predictor of Cystatin $\mathrm{C}$ level in elderly males with mild to moderate 
CHF. Higher Cys $\mathrm{C}$ level showed a negative relation to survival and bone loss in elderly CHF patients. Further research is needed to confirm the potential for Cys $\mathrm{C}$ and bone surrogates in the crosstalk among heart, bone and energy metabolism in CHF patients.

\section{P906}

\section{METABOLIC MARKERS IN SAUDI ADULTS WITH} AND WITHOUT OSTEOPENIA AND OSTEOPOROSIS

$\underline{\text { S. Sabico }}^{1}$, N. Al-Daghri ${ }^{1}$, Y. Al-Saleh ${ }^{2}$, S. Yakout ${ }^{1}$, N. J. Aljohani $^{3}$, M. S. Alokail ${ }^{1}$

${ }^{1}$ Biochemistry Department, College of Science, King Saud University, Riyadh, Saudi Arabia, ${ }^{2}$ King Abdulaziz Medical City, College of Medicine, King Saud Bin Abdulaziz University for Health Sciences, Riyadh, Saudi Arabia, ${ }^{3}$ King Saud bin Abdulaziz University for Health Sciences, Riyadh, Saudi Arabia

Background: Accelerated bone loss has been observed in patients with inflammatory disorders. However, the impact of cytokines in the etiology of osteoporosis and osteopenia needs further investigation. Differences in the expression of inflammatory markers could provide new insights in identifying patients at early risk of skeletal diseases as well as support the development of cytokine-blocking therapies to reduce bone loss. The aim of the study was to determine differences in the pro-inflammatory cytokines and in Saudi adults with and without osteoporosis and osteopenia.

Methods: We studied 590 Saudi adult subjects taken from the Saudi Osteoporosis Registry $\mathrm{d}=$ Database; 121 with osteoporosis (age 58.27.2; BMI 30.2 \pm 5.5 ), 253 with osteopenia (age 56.5 \pm 7.5 ; BMI $32.1 \pm 5.6$ ) and 216 normal (without osteoporosis and osteopenia) (age 55.6 \pm 7.3 ; BMI $32.8 \pm 5.4$ ). Anthropometric was taken along with fasted blood samples to measure lipid profile, resistin, IL-6, osteoprotegerin, osteocalcin, RANKL and $25(\mathrm{OH}) \mathrm{D}$ using commercially available kits.

Results: Age- and BMI adjusted results showed that the normal group had the lowest HDL-cholesterol and $25(\mathrm{OH}) \mathrm{D}$ levels than those with osteoporosis and osteopenia (p-values 0.002 and 0.028 , respectively).

Resistin and IL-6 levels were highest in the osteoporosis group (p-values $<0.001$, respectively). Osteoprotegerin, osteocalcin and RANKL were also highest in the osteoporosis group ( $p$-values $<0.001$, respectively).

Conclusion: The present study showed that some of inflammatory factors such resistin and IL-6 play important role in bone metabolism. It suggests that these inflammatory factors may influence bone metabolism in distinct mechanism.

\section{P907}

DIAGNOSTIC PERFORMANCE OF MUSCULOSKELETAL ULTRASOUND IN HEEL PAIN

A. E. Musetescu ${ }^{1}$, C. Criveanu ${ }^{2}$, F. A. Vreju ${ }^{1}$, A.-M. Bumbea $^{1}$, P. L. Ciurea ${ }^{1}$, A. Rosu ${ }^{1}$

${ }^{1}$ University of Medicine and Pharmacy, Craiova, Romania,

${ }^{2}$ University of Medicine and Pharmacy of Craiova, Craiova, Romania

Objective: The objective of the study is to discriminate between mechanical and inflammatory causes of heel pain by using musculoskeletal ultrasound. Heel pain is a common condition in clinical practice with differential diagnosis between degenerative conditions and inflammatory disease being quite challenging. Plantar fasciae, Achilles tendon and retrocalcaneal bursa are involved in either degenerative processes or in inflammatory disease and musculoskeletal ultrasound may be a valuable tool in discriminating the morphological background of the heel pain.

Material and methods: 50 patients were included in the study who presented for heel pain in the Rheumatology department. Patients with recent trauma or local treatment with corticosteroids were excluded. Complete physical examination and blood analysis were performed for positive diagnosis. Ultrasound was performed by a trained rheumatologist unaware of the clinical data using an Esaote MyLabSix equipment with a multifrequency linear probe. The following structures were assessed bilaterally: plantar fasciae, Achilles tendon and retrocalcaneal bursa while pathological findings were recorded: tendon/fasciae thickness, homogeneity, calcifications, erosions, Doppler signal, entesophytes, and bursitis.

Results: Patient distribution showed 27 patients with inflammatory disease and 23 with degenerative underlying pathology. Ultrasound examination revealed a significant statistical difference only in the presence of erosions, greater in inflammatory disease group while no statistical differences were found regarding erosion number and dimension. The frequency of enthesis Doppler signal detected was also in favor of inflammatory conditions. Tendon and fasciae thickness as well as the presence of entesophytes, calcifications, and the presence of bursitis and loss of homogeneity did not show statistically significant differences between the two study groups.

Conclusions: Musculoskeletal ultrasound is a valuable tool in detecting the presence of pathological structural lesions such as erosions or enthesopathy in heel pain but unable to discriminate between the degenerative vs. inflammatory underlying condition and even the presence of erosions and hypervascularisation do not exclude a degenerative cause of enthesitis. 


\section{P908}

CORRELATIONS BETWEEN BONE MINERAL DENSITY AND SUBCLINICAL ATHEROSCLEROSIS IN A COHORT OF SYSTEMIC LUPUS ERYTHEMATOSUS PATIENTS

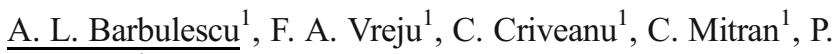
L. Ciurea $^{1}$

${ }^{1}$ University of Medicine and Pharmacy, Craiova, Romania

Objective: To determine BMD in a cohort of 22 systemic lupus erythematosus (SLE) patients, compared to controls, and establish the possible correlations with subclinical atherosclerosis, evaluated by carotid intima-media thickness.

Material and Methods: We enrolled a cohort of 22 SLE patients, 20 women and 2 men, with a mean age of $43.5+12$ years, and 18 sex and age matched controls; BMD was determined by DXA, in lumbar spine, total hip and wrist, and carotid intima media thickness (cIMT) by B-mode ultrasound.

Results: The medium cIMT in SLE patients was 0.63 $\pm 0.33 \mathrm{~mm}$, statistically significant different compared to the value calculated for controls $(0.41 \pm 0.22 \mathrm{~mm}, p=0.002)$; using DXA, we identified osteoporosis in 7 patients $(31.81 \%)$, osteopenia in $11(50 \%)$ and normal BMD in $4(18.18 \%)$. Regarding the control group, osteoporosis was identified in 4 cases (22.22 \%), osteopenia in $5(27.27 \%)$ and normal BMD in $9(50 \%)$. We noticed a moderate, negative correlation, between cIMT and T-score, evaluated in all 3 sites $(r=-0.68$, $p=0.001$ for lumbar spine; $r=-0.64, p=0.003$ for total hip and $r=-0.72, p<0.0001$ for wrist). Analysing the risk of a higher cIMT depending on the presence of osteoporosis, revealed a RR of 3.57. Compared to controls, in SLE patients, the presence of osteoporosis associated a RR of 6.22 for developing a cIMT over 9; also, in the study group, T-score $<-2.5$ predicted a cIMT over $0.9 \mathrm{~mm}$ with a specificity of $85.71 \%$ and a sensibility of $62.50 \%$.

Conclusion: The results show a direct correlation between BMD and cIMT in SLE patients and a higher risk of subclinical atherosclerosis compared to controls; therefore diagnosis of osteoporosis in these patients should extend the evaluation to cardiovascular risk assessment.

\section{P909}

TWO PREVIOUSLY UNINVESTIGATED SNPS ARE RESPONSIBLE FOR HALF OF THE GENETIC INFLUENCE ON 25OH-VITAMIN-D LEVELS IN A POPULATION FREE OF CONFOUNDING ENVIRONMENTAL FACTORS

B. Bakos ${ }^{1}$, P. Horváth ${ }^{2}$, B. Szili², B. Szabó ${ }^{2}$, P. Lakatos ${ }^{2}$, A. G. Tabák $^{2}$, E. Toldy ${ }^{3}$, I. Takács ${ }^{2}$

${ }^{1} 1$ st Department of Internal Medicine, Semmelweis University, Budapest, Hungary, ${ }^{2} 1$ st Department of Medicine, Semmelweis University, Budapest, Hungary,
${ }^{3}$ Department of Diagnostics, University of Pécs, Kaposvár, Hungary

Purpose: Data regarding genetic factors that affect serum 25hydroxyvitamin-D levels are scarce and oftentimes conflicting. Methods: We measured total 25-hydroxyvitamin-D (t25OHD), vitamin D-binding protein (DPB) and albumin levels at the end of winter in a probability stratified sample of 669 Hungarian adult men and women. Bioactive (b25OHD) and free (f-25OHD) vitamin D concentrations were calculated.

Subjects with a history of confounding factors on 25OHD level (vitamin D substitution, solarium use, travel over 40th latitude below poles, etc.) were excluded. Measured t-25OHD and DBP levels were hence only affected by endogenous vitamin D metabolism. For SNP selection of vitamin D receptor (VDR), DBP (GC), 1,25-dihydroxyvitamin D3 24hydroxylase (CYP24A1) and glutamine-dependent NAD synthetase (NADSYN1) we used publicly available online databases.

Results: We found five SNP polymorphisms that significantly affect t-25OHD (GC: rs222054, rs7041; CYP24A1: rs2762939, rs2762941; NADSYN1: rs7935125), from which only rs7935125 affected f-25OHD. The only SNP affecting b25OHD was rs2209314 (CYP24A1).

According to our univariate general linear model rs7935125, rs7041, rs222054, and rs2762941 were found to be responsible for $11 \%$ of the variance of total $25-\mathrm{OH}$-vitamin- $\mathrm{D}_{3}$ levels. The two previously unpublished SNPs; rs7935125 (NADSYN1) and rs2762941 (CYP24A1) were responsible for $6 \%$ of the variance of $\mathrm{t} 25 \mathrm{OHD}$ levels, more than half of the genetic effect.

Conclusions: In the present study we registered multiple SNP polymorphisms affecting total-, free- and bioactive-25-OHvitamin-D3 and DBP levels in a representative Hungarian population that is free of environmental factors affecting vitamin D levels. We also report two previously unpublished SNP-s which seem to be responsible for a substantial part of the genetic effect.

\section{P910}

DRUG HOLIDAY IN OSTEOPOROSIS: HOW LONG SHOULD IT LAST?

$\underline{\text { I. Kostoglou-Athanassiou }}^{1}$, E. Pantazi ${ }^{2}$, O. Skioti $^{2}$, A. Tzanavari $^{3}$, T. Banti ${ }^{3}$, P. Athanassiou ${ }^{3}$

${ }^{1}$ Department of Endocrinology, Red Cross Hospital, Athens, Greece, ${ }^{2}$ 2nd Department of Endocrinology, Alexandra Hospital, Athens, Greece, ${ }^{3}$ Department of Rheumatology, St. Paul's Hospital, Thessaloniki, Greece

Objectives: Osteoporosis is a chronic and dangerous disease, as it may be silent and may express itself with a sudden catastrophic event, such as a fracture. Treatment of osteoporosis 
demands the use of potent pharmaceutical agents, which have long lasting effects on the skeleton. In particular, bisphosphonates, such as ibandronate, may be used. It has become evident that chronic treatment with anti-osteoporotic agents may reverse the course of the disease, patients having normal BMD or only osteopenia following treatment. In such a group of patients treatment may be interrupted and patients followed up. The interruption of pharmaceutical treatment in osteoporosis is called drug holiday. The aim was to describe drug holiday in a group of patients with osteoporosis, having received treatment with ibandronate.

Materials and Methods: Within a group of 120 patients (108 female, 12 male) with osteoporosis, 20 patients (female) were receiving ibandronate $150 \mathrm{mg}$ once monthly and calcium with cholecalciferol for a period of 1.8-3 years. These patients had osteoporosis on ibandronate initiation, T-score ranging from -2.5 to -3.4

Results: On reevaluation after treatment with ibandronate $150 \mathrm{mg}$ once monthly and calcium with cholecalciferol, BMD was measured, osteopenia was observed in 12 female patients and normal BMD was observed in 8 . Ibandronate was stopped, while treatment with calcium and cholecalciferol was continued. Patients were reevaluated after 1 and 2 years. Within this group 11 patients continued to have osteopenia and treatment with calcium and cholecalciferol was continued, while 1 patient was found to have osteoporosis and he required the addition of another antiosteoporotic medication. Within the group of 8 patients with normal BMD 6 continued to have normal BMD and 2 were found to have osteopenia.

Conclusions: Treatment with antiosteoporotic agents, especially bisphosphonates appears to have beneficial and longlasting effects in some patients, enabling treatment interruption and absence of recurrence. Drug holiday seems to be possible in a significant group of osteoporosis patients, being associated with absence of recurrence. It appears that osteoporosis treatment should be individualized, as genetic factors may be associated with very good response in some of our osteoporosis patients.

\section{P911}

RELATIONSHIP BETWEEN SEX HORMONE LEVELS, BONE MINERAL DENSITY AND BONE TURNOVER MARKERS IN HEALTHY MOROCCAN MEN: A CROSS-SECTIONAL STUDY

$\underline{\text { Z. Ouzzif }}^{1}$, A. Benghabrite ${ }^{2}$, A. El Maghraoui ${ }^{3}$, L. Chabraoui $^{4}$, A. El Maataoui ${ }^{5}$

${ }^{1}$ Biochemistry, Military Hospital Mohammed V, Rabat, Morocco, ${ }^{2}$ Ministry of Health, Rabat, Morocco, ${ }^{3}$ Rheumatology, Military Hospital Mohammed V, Rabat, Morocco, ${ }^{4}$ Biochemistry Department at Ibn Sina Hospital, Rabat, Morocco, ${ }^{5}$ Ibn Zohr University, Faculty of Medicine and Pharmacy, Casablanca, Morocco
Introduction: Gonadal steroid hormones play a crucial role during skeletal growth and maturation in both men and women. The aim of this study is to evaluate the relationship of sex hormone levels, BMD and biochemical markers of bone turnover in healthy Moroccan men.

Methods: 142 Moroccan men who had no previous diagnosis of osteoporosis were enrolled prospectively in this cross-sectional study between December 2009 and August 2010. Also, subjects were excluded from the study if they had conditions affecting bone metabolism. Different biochemical parameters were assayed: Testosterone, estradiol, sex hormone binding globulin, osteocalcin, vitamin D, CrossLaps, intact PTH and alkaline phosphatase. DXA was used to measure the BMD $\left(\mathrm{g} / \mathrm{cm}^{2}\right)$.

Results: In this study, among the 142 Moroccan men, 29 $(20.1 \%)$ had densitometry osteoporosis and the prevalence of vitamin D insufficiency was $94 \%$. No correlation was found between estradiol, testosterone and BMD but we found significant differences in the levels of estradiol between patients with osteoporosis, osteopenia and normal patients. BMD at the lumbar spine was negatively correlated to hormone-binding globulin and positively correlated to free androgen index, free estrogen index and the BMI. BMD at the total hip was positively correlated to free androgen index, BMI and negatively correlated to sex hormone binding globulin, alkaline phosphatase, intact PTH, osteocalcin, CrossLaps and age.

Conclusion: Our study showed that increasing age, intact PTH and alkaline phosphatase levels and decreasing BMI were the most important independent factors associated to the presence of a low BMD at the total hip. Increasing BMI and free androgen index level were the most important independent factors associated to the presence of a low BMD at the lumbar spine. The combination of variable that best predicted the male osteoporosis is age, BMI, alkaline phosphatase and cigarette smoking.

Acknowledgments: The study was funded by grants from Roche Laboratories.

\section{P912}

DXA ERROR INFLUENCE ON THE ASSESSMENT OF A NOVEL ECHOGRAPHIC APPROACH TO OSTEOPOROSIS DIAGNOSIS ON THE SPINE

$\underline{\text { F. Conversano }}^{1}$, S. Casciaro ${ }^{1}$, A. Greco ${ }^{2}$, P. Pisani ${ }^{1}$, M. Di

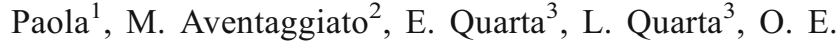
Casilli $^{3}$, M. Muratore ${ }^{3}$

${ }^{1}$ National Research Council, Institute of Clinical Physiology, Lecce, Italy, ${ }^{2}$ Echolight srl, Lecce, Italy, ${ }^{3}$ O.U. of Rheumatology, "Galateo" Hospital, San Cesario di Lecce, ASL-LE, Lecce, Italy 
Objective: To assess the actual performance of Osteoporosis Score (OS), a recently introduced ultrasound (US) parameter for osteoporosis diagnosis on lumbar spine.

Materials and Methods: In a previous study, we directly assumed the outcome of DXA investigations as the gold standard reference to assess the OS diagnostic performance in a population of 384 patients aged in 45-65 years, obtaining an overall accuracy of $84.6 \%$ in patient classification (osteoporotic, osteopenic, or healthy) coupled with a good correlation between DXA- measured BMD and corresponding OSderived values $(r=0.72, p<0.0001)$ [1]. In the present work, we performed a retrospective check of the 384 DXA reports, excluding all those cases presenting a typical DXA error as identified by recent literature (e.g., patient positioning, presence of artefacts, etc.) [2] and reassessing the actual diagnostic accuracy of our US approach by considering only those patients having a reliable DXA report.

Results: 159 patients out of the initial 384 (41.4\%) were excluded because of clear DXA pitfalls. The overall agreement between DXA and OS-based diagnoses on the remaining 225 patients was $93.5 \%(r=0.81, p<0.0001)$, without appreciable variations as a function of patient age, showing that undetected DXA errors had resulted in a significant underestimation of OS accuracy in our previous study.

Conclusions: The actual potential of the OS-based approach for osteoporosis diagnosis was previously underestimated. Furthermore, the adopted method for DXA report analysis could be used to reassess the performance of different US methods that assumed routine DXA investigations as the gold standard reference.

\section{References:}

1. Casciaro et al., Proc 2015 I.E. Int Symp Med Meas Appl (MeMeA), pp. 250-254.

2. Messina et al., Eur Radiol 2015;25:1504.

Acknowledgements: Work partially funded by FESR PO Apulia Region 2007-2013 - Action 1.2.4 (grant n. 3Q5AX31: ECHOLIGHT Project).

\section{P913}

\section{BONE VARIABLES IN SEDENTARY OVERWEIGHT} MEN AND ACTIVE OVERWEIGHT MEN

G. El Khoury $^{1}$, H. Zouhal ${ }^{2}$, C. El Khoury ${ }^{1}$, A. Alwan ${ }^{1}$, R. El Hage $^{1}$

${ }^{1}$ Department of Physical Education, Fass, University of Balamand, Kelhat El Kurah, Lebanon, ${ }^{2}$ STAPS, University of Rennes 2, Rennes, France

Aim: To compare bone variables in sedentary overweight men and active overweight men.

Methods: 20 sedentary overweight men and 20 active overweight men participated in this study. Weight and height were measured, and BMI was calculated. Body composition and bone variables (BMC, BMD and geometric indices of hip bone strength) were measured by DXA. Physical activity level, daily calcium intake (DCI), daily protein intake (DPI) and sleep duration were measured by validated questionnaires.

Results: Age, BMI, lean mass, height, DCI and DPI were not significantly different between the two groups. WB BMC, WB BMD, L1-L4 BMD, FN BMD and femoral neck crosssectional area were significantly higher in active overweight men compared to sedentary overweight men.

Conclusion: This study suggests that physical activity can positively affect BMD and bone geometry in overweight men.

\section{P914}

MANAGEMENT OF KNEE OSTEOARTHRITIS WITH LOCAL CAPSAICIN APPLICATION

$\underline{\text { P. Athanassiou }}^{1}$, A. Tzanavari ${ }^{1}$, I. Kostoglou-Athanassiou ${ }^{2}$, A. Georgiadis $^{3}$, I. Siafaka ${ }^{4}$

${ }^{1}$ Department of Rheumatology, St. Paul's Hospital, Thessaloniki, Greece, ${ }^{2}$ Department of Endocrinology, Red Cross Hospital, Athens, Greece, ${ }^{3}$ Lito Hospital, Athens, Greece, ${ }^{4}$ Department of Anesthesiology, University of Athens, Athens, Greece

Objectives: Knee osteoarthritis is a chronic and debilitating disease of unknown etiology. Management is particularly challenging, although nowadays the development of novel methods for the treatment of knee osteoarthritis, such as cell-based cartilage repair strategies is underway. The disease is characterized by chronic pain. The treatment of chronic pain is difficult and creates many problems as chronic treatment with NSAIDs is accompanied by severe and often dangerous side effects. The aim was to study the effect of local capsaicin application on pain in knee osteoarthritis.

Materials and Methods: In 55 patients with knee osteoarthritis local capsaicin $8 \%$ was applied. Applications were performed in the areas of knee, the lumbar spine, the shoulders and elsewhere, as needed. All patients had chronic knee osteoarthritis, 6 patients having also rheumatoid arthritis and 1 patient also psoriatic arthritis. All patients were on systemic treatment for osteoarthritis, taking chondroitin sulfate, diacerein, paracetamol and NSAIDs. In 11 of the patients intra-articular hyaluronic acid injection had also been performed. Before and 1 month after the application of local capsaicin $8 \%$ pain intensity was assessed using the DN4 questionnaire and the VAS scale. A group of 55 patients with knee osteoarthritis receiving a placebo application served as controls.

Results: As assessed by the DN4 questionnaire pain intensity was 4.1 (minimum 1, maximum 8 ), 14/23 of the patients having $>4$. Pain intensity as assessed by the VAS scale was $68(\min 40, \max 100)$. After the 
application of local capsaicin pain intensity improved by $49.9 \%$. Capsaicin appeared to be safe without systemic toxicity. In some of the patients mild application site burning was observed.

Conclusions: Local capsaicin application appears to be effective for the treatment of pain in the management of knee osteoarthritis. Local capsaicin seems to be a useful addition in the armamentarium of physicians for the management of knee osteoarthritis.

\section{P915}

ACCUMULATED QUALITY OF LIFE LOSS 36 MONTHS AFTER VERTEBRAL AND DISTAL FOREARM FRACTURE: RESULTS FROM ICUROS IN LITHUANIA

M. Tamulaitiene ${ }^{1}$, V. Alekna ${ }^{1}$, F. Borgström ${ }^{2}$, V. Sinkeviciene $^{1}$, A. Svedbom ${ }^{3}$, R. Stukas ${ }^{1}$, J. A. Kanis ${ }^{4}$

${ }^{1}$ Faculty of Medicine, Vilnius University, Vilnius, Lithuania,

${ }^{2}$ Karolinska Institutet, Stockholm, Sweden, ${ }^{3}$ Mapi, Stockholm, Sweden, ${ }^{4}$ Centre for Metabolic Bone Diseases, University of Sheffield Medical School, Sheffield, United Kingdom

Objective: To evaluate the changes regarding the quality of life (QoL) based on the EQ-5D during the 36 months after fracture based on the patients enrolled in the International Costs and Utilities Related to Osteoporotic fractures Study (ICUROS) in Lithuania.

Material and Methods: Patients aged 50 years and older, with vertebral fracture (VFx) or distal forearm fracture (FFx) enrolled and observed for 18 months were further interviewed at 24 and 36 months after the fracture. For the estimation of the loss in QoL associated with a fracture over 36 months, it was assumed that the patient would have remained at their reported pre-fracture level of QoL had the fracture not occurred. The accumulated QoL loss in the 36-month follow-up after fracture was calculated as the area under the curve using the trapezoid method.

Results: In total, 256 persons were included in this study: 65 subjects with VFx (51 women and 14 men) and 191 subjects with FFx (179 women and 12 men). The age did not differ significantly between the fracture types $(p=0.342)$. Our results showed that mean loss in QoL at 4 months was $0.33(95 \%$ CI $0.31-0.35)$ and at 36 months $0.13(95 \% \mathrm{CI} 0.12-0.15), p<0.001$ in patients with FFx. In VFx the mean loss was $0.41(95 \% \mathrm{CI}$ $0.36-0.45)$ at 4 months and $0.32(95 \%$ CI $0.28-0.36)$ at 36 months after fracture $(p<0.001)$.

Conclusion: The findings of this study showed that utility loss after both vertebral and forearm fracture persists on average for several years.

\section{P916}

VITAMIN D STATUS IN HEALTHY MOROCCAN MEN AND WOMEN AGED 50 YEARS AND OLDER: A CROSS SECTIONAL STUDY

A. El Maataoui ${ }^{1}$, A. Biaz ${ }^{2}$, S. El Machtani ${ }^{2}$, S. Bouhsain ${ }^{2}$, A. $\mathrm{Dami}^{2}$, A. El Maghraoui ${ }^{3}$, Z. Ouzzif ${ }^{2}$

${ }^{1} \mathrm{Ibn}$ Zohr University, Faculty of Medicine and Pharmacy, Casablanca, Morocco, ${ }^{2}$ Biochemistry, Military Hospital Mohammed V, Rabat, Morocco, ${ }^{3}$ Rheumatology, Military Hospital Mohammed V, Rabat, Morocco

Objectives: Vitamin D $(25(\mathrm{OH}) \mathrm{D})$ plays an important role in bone and calcium metabolism. The main purpose of this study was to evaluate the vitamin D status of a healthy Moroccan men and women aged 50 years and older.

Methods: This was a cross-sectional study conducted from December 2009 to August 2010 in men over 50 years old, and from October 2008 to November 2009 in postmenopausal women. A total of 186 Moroccan postmenopausal women and 68 men, who had no previous diagnosis of osteoporosis were recruited prospectively.

Results: The prevalence of vitamin D deficiency in men and women was $4.4 \%$ and $8.6 \%$, respectively, and the prevalence of vitamin D insufficiency in men and women was $85.2 \%$ and $77.4 \%$, respectively. In men and women, no correlation was found between iPTH and 25(OH)D $(r=0.056)$.

Conclusion: Despite a sunny environment, hypovitaminosis $\mathrm{D}$ is widespread in Moroccan men over 50 years and postmenopausal women.

\section{P917}

ADVANCING MATERNAL AGE AT CHILDBIRTH IS ASSOCIATED WITH LESS FAVOURABLE TRABECULAR BONE MINERAL DENSITY AND TIBIAL CORTICAL BONE GEOMETRY IN YOUNG ADULT MALE OFFSPRING

C. Verroken ${ }^{1}$, H. Zmierczak ${ }^{1}$, S. Goemaere ${ }^{1}$, J.-M. Kaufman ${ }^{1}$, B. Lapauw ${ }^{1}$

${ }^{1}$ Unit for Osteoporosis and Metabolic Bone Diseases, Ghent University Hospital, Ghent, Belgium

Objective: Advancing maternal age at childbirth has been associated with a higher risk of pregnancy complications and with adverse short-term and long-term offspring health outcomes, but little is known about the effects of increasing maternal age on offspring bone acquisition. We aimed to investigate associations of maternal age at childbirth with offspring bone parameters in healthy men at the age of peak bone mass.

Material and Methods: We used cross-sectional data from 689 subjects participating in a population- based sibling pair study including healthy men aged $25-45$ years. Data include 
maternal age at childbirth, offspring birth weight, adult weight and height, and offspring DXA and pQCT-derived areal and volumetric bone parameters and cortical bone geometry. Cross-sectional associations were investigated using linear mixed-effects modeling with adjustment for offspring age, height and weight.

Results: Maternal age at childbirth was $27.0 \pm 4.7$ years and correlated inversely with areal BMD (aBMD) and bone mineral content (BMC) at the lumbar spine $(\beta=-0.09, p=0.011$ and $\beta=-0.01, p=0.017$ ), as well as with trabecular volumetric BMD (vBMD) at the distal radius $(\beta=-0.10, p=0.014)$. No associations of maternal age were found with aBMD or $\mathrm{BMC}$ at the total hip or femoral neck, nor with cortical $\mathrm{VBMD}$ at the proximal radius or tibia. However, increasing maternal age was associated with smaller cortical thickness $(\beta=-0.13, p=0.002)$, cortical area $(\beta=-0.08, p=0.024)$, and cortical over total bone area ratio $(\beta=-0.11, p=0.010)$ as well as with larger endosteal circumference $(\beta=0.10, p=0.010)$ at the tibia. No associations of maternal age with cortical bone geometry were observed at the radius.

Conclusions: Advancing maternal age at childbirth might adversely affect the acquisition of peak bone mass and geometry in male offspring, with in particular less favorable trabecular BMD and tibial cortical bone geometry. Whether this results from altered placental function or from epigenetic changes due to lifestyle factors remains to be established.

\section{P918}

CORRELATIONS BETWEEN QUALITY OF LIFE AND PSYCHOLOGICAL WELL-BEING IN WOMEN WITH OSTEOPOROSIS COMPARING TO MEN WITH OSTEOPOROSIS

D. M. Farcas $^{1}$, F. Marc ${ }^{2}$, C. Suteu ${ }^{2}$

${ }^{1}$ Physical Rehabilitation Department, University of Oradea, Faculty of Medicine and Pharmacy, Oradea, Romania, ${ }^{2}$ University of Oradea, Faculty of Medicine and Pharmacy, Oradea, Romania

Aim: To assess quality of life, anxiety and stress in patients with osteoporosis.

Material and method: Our study consisted in patients with osteoporosis, both women and men. We divided the patients from our study in two groups. The first group consisted of 25 women, second group consisted of $25 \mathrm{men}$. We recruited all the patients from ambulatory system Bihor county, Romania. The mean age in the group of women was 65.28 \pm 3.31 and in the group of men was of $68.43 \pm 2.49$. Educational levels were similar in both groups. The inclusion criteria were: fulfilling the WHO criteria for osteoporosis, complying with the principles of medical ethics. The exclusion criteria were: severe diseases, noncompliance. The subjects from both groups were measured for osteoporosis by DXA method. We assessed all the patients for quality of life with Qualeffo 41 Questionnaire, with Hamilton Anxiety Rating Scale for anxiety, and with Levenstein Stress Perceived Questionnaire for stress.

Results: We found out low quality of life in both groups, but lower in the group of women with osteoporosis then in the group of men with osteoporosis. The mean anxiety value was higher in the group of women with osteoporosis than in the group of men with osteoporosis, and the mean stress values were almost similar in both groups. We found out also that there were correlations between quality of life and psychological factors investigated.

Conclusion: Osteoporosis has an impact on patient's well-being and quality of life, both women and men. Even if the psychological well-being was a little neglected in patients with osteoporosis, it seems that it plays an important role in their quality of life and general well-being. Further studies should be continued in this field, to elaborate new strategies in coping with patients with osteoporosis, as a chronic disease.

\section{P919}

ASSOCIATION OF PHYSICAL FITNESS WITH BODY MASS INDEX IN ANDALUSIAN PERIMENOPAUSAL WOMEN: THE FLAMENCO PROJECT

M. Borges-Cosic $^{1}$, A. Andrade ${ }^{2}$, F. J. Gallos ${ }^{3}$, P. AcostaManzano $^{1}$, I. Coll-Risco ${ }^{2}$, M. Delgado ${ }^{1}$, P. Casas-Reyes ${ }^{3}$, V. A. Aparicio ${ }^{4}$

${ }^{1}$ Department of Physical Education and Sports, Faculty of Sport Sciences, University of Granada, Granada, Spain, ${ }^{2}$ Department of Physiology, Faculty of Pharmacy, Faculty of Sport Sciences, and Institute of Nutrition and Food Technology, University of Granada, Granada, Spain, ${ }^{3}$ Zaidín Sur Healthcare Centre and Department of Medicine, Faculty of Medicine, University of Granada, Granada, Spain, ${ }^{4}$ Department of Physiology, Faculty of Pharmacy, Faculty of Sport Sciences, and Institute of Nutrition and Food Technology, University of Granada and Department of Physical Education and Sports, Faculty of Sport Sciences, University of Granada, Granada, Spain

Objectives: To assess the association of different components of physical fitness with BMI in perimenopausal women.

Material and Methods: The sample for the present analyses comprised 194 women (age range 45-60 years) from Andalusia, Spain. Participants' physical fitness status was assessed with the Senior Fitness Test battery (additionally including handgrip test). Bone mass density was measured using DXA device (Hologic Discovery QDR, Nasdaq: HOLX). 
Results: Linear regression analysis assessing the association of physical fitness with BMD showed that cardiorespiratory fitness, flexibility and motor agility were not significantly associated with BMD after adjusting for the age-adjusted and multivariate model (all, $P>0.05$ ). Handgrip muscle strength was significantly associated with BMD $(\beta=0.212, P=0.005$ for the age adjusted and multivariate model).

Conclusions: The findings of the present study indicate that muscle strength is positively associated with BMD in perimenopausal women. This suggests that muscle strength might be an important marker of bone health in perimenopausal women.

Table 1. Linear regression analysis assessing the association of physical fitness with bone mineral density in perimenopausal women

\begin{tabular}{|c|c|c|c|c|c|c|c|c|}
\hline \multirow[b]{3}{*}{ Physical fitness } & \multicolumn{8}{|c|}{ Bone Mineral Density $\left(\mathrm{g} / \mathrm{cm}^{2}\right)$} \\
\hline & \multicolumn{4}{|c|}{ Age-adjusted } & \multicolumn{4}{|c|}{ Multivariate adjusted $^{\mathrm{a}}$} \\
\hline & $\beta$ & $\mathrm{B}$ & SE & $\mathrm{P}$ & $\beta$ & $\mathrm{B}$ & SE & $\mathrm{P}$ \\
\hline Bruce test $\left(\mathrm{VO}_{2} \max \right)$ & -03072 & -0.001 & 0.001 & 0.314 & -0.001 & 0.001 & 0.001 & 0.245 \\
\hline Handgrip strength (kg) & 0.192 & 0.005 & 0.002 & 0.010 & 0.212 & 0.002 & 0.002 & 0.005 \\
\hline Back-scratch $(\mathrm{cm})$ & -0.107 & -0.001 & 0.001 & 0.142 & -0.059 & 0.001 & 0.001 & 0.449 \\
\hline Sit-and-reach (cm) & -0.104 & -0.001 & 0.001 & 0.133 & -0.115 & 0.001 & 0.001 & 0.102 \\
\hline Timed up-and-go (s)\# & 0.052 & 0.008 & 0.012 & 0.493 & 0.004 & 0.012 & 0.012 & 0.960 \\
\hline
\end{tabular}

SE: Standard Error; $\beta$, standardized regression coefficint; B, nonstandardized regression coeffiecient; \#Lower scores indicate better performance; ${ }^{a}$ Model adjusted for age, educational status, marital status, smoking status, regular menstruation and hormonal theraphy; Model additionally adjusted for body mass index

\section{P920}

\section{OSTEOPOROSIS IN MEN WITH PROSTATE} CANCER

N. Tskhovrebashvili ${ }^{1}$, M. Zodelava ${ }^{1}$, N. Tatishvili ${ }^{2}$, T. Mamaladze $^{1}$, M. Tskhovrebashvili ${ }^{3}$

${ }^{1}$ Department of Osteoporosis, Ltd “Unimedi Kakheti”, Caraps Medline, Tbilisi, Georgia, ${ }^{2}$ Department of Rheumatology, Tbilisi Central Hospital, Tbilisi, Georgia, ${ }^{3}$ Center of Endocrinology, Metabology and Dietology "Enmedic", Tbilisi, Georgia

Objective: Prostate cancer is the most common visceral malignancy in men. Osteoporosis is an important complication of androgen deprivation therapy (ADT) for prostate cancer. ADT either by bilateral orchiectomies or treatment with a gonadotropin-releasing hormone agonist $(\mathrm{GnRH})$ decreases BMD and increases the risk of fracture. The aim of the present study was to determine BMD in men with PC, to consider for them adjuvant therapy after successful treatment for PC with the following BMD monitoring.

Material and Methods: We have studied 33 men with PC aged 35-60 years. All patients were divided into three groups. I group $(n=21)$ men with PC with bilateral orchiectomy treating with $(\mathrm{ADT})$ Casodex $50 \mathrm{mg}$ daily within 10 month and the II group - the same I group of men who were examined on osteoporosis and were receiving ibandronate $3 \mathrm{mg}$ solution for intravenous injection every three month during following1 year and the III group $(n=12)$ men with PC with bilateral orchiectomy treating with Casodex $50 \mathrm{mg}$ daily within 2 years without osteoporosis monitoring and treatment for improvement BMD. BMD was measured at three sites (distal radius, midshaft tibia and proximal phalanx) using the ultrasound bone sonometer (Sunlight, Omnisense). Results were interpreted according to the criteria adopted by the WHO by T-score.

Results: The mean data for T-score in the I group of patients with PC was: distal radius $2.1 \pm 0.02$; midshaft of the tibia $-2.0 \pm 0,05$; proximal phalanx $-2.3 \pm 0,11$; in the II group Tscore: $-1.6 \pm 0,09 ;-1.5 \pm 0.15 ;-1.4 \pm 0.11$; in the III group Tscore: $-2.9 \pm 0.18 ;-2,7 \pm 0.13 ;-3.1 \pm 0.04$ at the same measurement sites, respectively.

Conclusions: In patients with PC receiving Casodex was shown a high rate of decrease of BMD, who were not treated for osteoporosis. Early intervention is warranted to prevent skeletal morbidity in patients with nonmetastatic prostate cancer receiving ADT. So, it is important that BMD must be determined in men with PC, appropriately monitored and, when necessary, treated with bisphosphonates, calcium and vitamin D supplements, avoid smoking, reduce alcohol intake and increase level of physical activity. 


\section{P921}

IS BONE MICROARCHITECTURE OF OESTROGEN DEFICIENT RATS AFFECTED BY PERMANENT HYPERGLYCEMIA?

A. Agripino $^{1}$, C. de Mello-Sampayo ${ }^{2}$, D. Stilwell ${ }^{3}$, B. Vidal ${ }^{4}$, M. Vaz ${ }^{5}$, B. Silva-Lima ${ }^{2}$, H. Canhão ${ }^{6}$, M. C. Marques ${ }^{2}$

${ }^{1}$ FCT U.Nova, Lisboa, Portugal, ${ }^{2}$ Pharmacological Scs Department, FF.ULisboa, Lisboa, Portugal, ${ }^{3}$ Clinica Veterinária de Colares, Sintra, Portugal, ${ }^{4}$ fRheumatology Research Unit, Instituto de Medicina Molecular, Faculdade de Medicina da Universidade de Lisboa, Lisboa, Portugal, ${ }^{5}$ IST.ULisboa, Lisboa, Portugal, ${ }^{6}$ Faculdade de Medicina da Universidade de Lisboa, Lisboa, Portugal

Objectives: Osteoporosis is an increasing prevalent bone disease that mainly affects postmenopausal women. In diabetic postmenopausal women it is observed an increase in BMD, but there is some controversy about the effects of diabetes on the bone ultrastructure and bone strength, being pointed in some studies increase in its fragility despite BMD. The aim of this study was to evaluate the effect of chronic hyperglycaemia in the bone turnover, morphology and mechanics of female Wistar rats in the presence and absence of E2, induced by ovariectomy.

Material and Methods: Animals $(n=24)$ divided into 4 groups: healthy control (sham); ovariectomized (OVX); hyperglycaemic induced per single dose $(40 \mathrm{mg} / \mathrm{kg}$, ip) of streptozotocin (STZ); hyperglycaemic + ovariectomized (STZ+OVX). Glucose, triglycerides, cholesterol, calcium, E2, CTX, and PINP were estimated by standard methods on blood samples taken 60 days after ovariectomy. The body weight, microarchitecture of the vertebral bone (histomorphometry), biomechanical properties of the femur (bending tests), cortical thickness and ultrastructure (scanning electron microscopy SEM) of the tibia were also evaluated.

Results: There was a significant increase in bone formation (PINP) in both hyperglycaemic groups (STZ and $\mathrm{STZ}+\mathrm{OVX}$ ) with increased PINP/CTX ratio in hyperglycaemic animals and a similar trend observed in STZ + OVX group. The histomorphometric studies (L4) were consistent with these results. The cortical thickness of the tibia from both hyperglycaemic groups was not affected, while that of OVX animals decreased. The biomechanical studies have revealed a more resistant bone in STZ + OVX compared to OVX animals in line with SEM revealed morphology of less microcracks, resorptive areas and lacunae/osteocytes.

Conclusions: chronic hyperglycaemia, through increased type I collagen formation, modulates bone microarchitecture into less fragile structures, even in the absence of oestrogens.
P922

EPIPHYSEAL BONE, SUBCHONDRAL BONE PLATE AND EPIPHYSEAL TRABECULAR BONE IN SURGICALLY AND CHEMICALLY INDUCED RAT MODELS OF OSTEOARTHRITIS

J. P. Rissanen $^{1}$, J. Morko ${ }^{1}$, Z. Peng ${ }^{1}$, J. Vääräniemi ${ }^{1}$, K. M. Fagerlund $^{1}$, J. Bernoulli ${ }^{1}$, J. M. Halleen ${ }^{1}$

${ }^{1}$ Pharmatest Services Ltd, Turku, Finland

Objective: Several experimental animal models have been developed for human osteoarthritis (OA). Recently, the histopathology initiative of Osteoarthritis Research Society International (OARSI) presented recommendations for histological OA assessment in various species. In rats, the recommendations focus on articular cartilage, synovium, joint capsule and growth plate. When studying treatment effects on osteoarthritic bone, the histological OA assessment of rat samples should be completed with the analysis of epiphyseal bone, subchondral bone plate and epiphyseal trabecular bone. Materials and Methods: Unilateral OA was induced in knee joints of male Lewis rats at 3 months of age using the following chemical and surgical OA models: intraarticular monoiodoacetate (MIA, $1 \mathrm{mg}$ ), medial meniscal tear and medial collateral ligament transection (MMT+ MCLT), anterior cruciate ligament transection and partial medial meniscectomy (ACLT + pMMx), and ACLT. Body weight, static weight bearing and static mechanical allodynia were followed during the in-life phase of the study. Histological OA assessment was performed according to the OARSI recommendations followed by the analysis of epiphyseal, subchondral and trabecular bone in medial tibial plateau.

Results: Knee joint discomfort was observed in operated hind limbs during the first week, and knee joint pain in operated and MIA-injected hind limbs during the first week and at the end of the in-life phase. Knee joint pain was associated with cartilage degeneration down to intermediate layer and mild synovial inflammation in the rat MIA model, with cartilage loss down to deep layer, osteophytes and mild synovial inflammation in the rat ACLT model, and with marked cartilage loss down to tidemark, large osteophytes and synovial inflammation in the rat MMT + MCLT and ACLT + pMMx models. These OA changes were observed together with decreased amount of epiphyseal, subchondral and/or trabecular bone in the rat MIA and ACLT models and with increased amount of epiphyseal, subchondral and/or trabecular bone in the rat MMT + MCLT and ACLT + pMMx models.

Conclusion: The amount of epiphyseal, subchondral and/ or trabecular bone decreased in the rat OA models exhibiting mild to moderate OA changes and increased 
in the rat $\mathrm{OA}$ models exhibiting moderate to severe OA changes.

\section{P923}

THE EFFECTS OF A POTENT AND SELECTIVE PARTIAL ESTROGEN RECEPTOR MODULATOR (PERD) ON BONE IN INTACT FEMALE RATS

J. P. Rissanen $^{1}$, A. Schmitz ${ }^{2}$, J. Morko ${ }^{1}$, Z. Peng ${ }^{1}$, K. M. Fagerlund $^{1}$, Y. Konkol ${ }^{1}$, M. I. Suominen ${ }^{1}$, J. Bernoulli ${ }^{1}$, J. M. Halleen ${ }^{1}$, A. Wagenfeld ${ }^{2}$

${ }^{1}$ Pharmatest Services Ltd, Turku, Finland, ${ }^{2}$ Bayer Pharma AG, Berlin, Germany

Objective: Selective estrogen receptor modulators (SERMs) are compounds that bind to estrogen receptors (ERs) and agonize or antagonize estrogen action in different tissue types. Due to a broad spectrum of physiological and pathological processes contributed by ERs, SERMs provide a potential therapeutic benefit for a variety of diseases. In this study, we characterized the effects of a novel partial estrogen receptor destabilizer ( $\mathrm{pERD}$ ) on bone under physiological estrogen levels in intact female rats.

Materials and Methods: Treatment of female Sprague Dawley rats was started at 3 months of age and continued once a day for 8 weeks. Intact rats were treated with pERD at 3,10 and $30 \mathrm{mg} / \mathrm{kg} / \mathrm{d}$ p.o., fulvestrant (FUL) at $3 \mathrm{mg} / \mathrm{kg} / \mathrm{d}$ s.c., raloxifene (RAL) at $1 \mathrm{mg} / \mathrm{kg} / \mathrm{d}$ p.o., tamoxifen (TAM) at $10 \mathrm{mg} / \mathrm{kg} / \mathrm{d}$ p.o. or with vehicle. FUL was used as a complete antagonist, whereas RAL and TAM were used as reference SERMs exhibiting agonist activity in bone. In addition, ovariectomized (OVX) rats treated with vehicle were included in the study for demonstrating estrogen deficiency. Treatment effects on bone were studied by peripheral quantitative computed tomography and measuring serum levels of bone turnover biomarkers.

Results: In metaphyseal trabecular bone, treatment with pERD at $3 \mathrm{mg} / \mathrm{kg} / \mathrm{d}$ decreased BMD and bone mineral content slightly. These pERD effects were only minor when compared with the effects of OVX and FUL. In diaphyseal cortical bone, treatment with pERD at $3 \mathrm{mg} / \mathrm{kg} / \mathrm{d}$ decreased cortical bone area and thickness slightly. These pERD effects were opposite to the effects of OVX and similar with the effects of RAL. Treatment with pERD at 10 and $30 \mathrm{mg} / \mathrm{kg} / \mathrm{d}$ did not affect metaphyseal trabecular bone and diaphyseal cortical bone significantly. In circulation, treatment with pERD decreased serum levels of N-terminal mid-fragment of osteocalcin at $30 \mathrm{mg} / \mathrm{kg} / \mathrm{d}$ and serum activity of tartrate-resistant acid phosphatase isoform $5 b$ at 3,10 and $30 \mathrm{mg} / \mathrm{kg} / \mathrm{d}$.

Conclusion: The effects of oral pERD treatment indicate a minor antagonist activity in trabecular bone and an agonist activity in cortical bone at $3 \mathrm{mg} / \mathrm{kg} / \mathrm{d}$. Subsequently, this study presents $\mathrm{pERD}$ as a novel mixed partial antagonist and agonist in bone at $3 \mathrm{mg} / \mathrm{kg} / \mathrm{d}$, and as a novel SERM without ER activities in bone at $10-30 \mathrm{mg} / \mathrm{kg} / \mathrm{d}$ in young adult female rats.

\section{P924}

THE REGISTER OF PATIENTS WITH HIP FRACTURES IN YAROSLAVL, RUSSIAN FEDERATION: THE PRELIMINARY ASSESSMENT

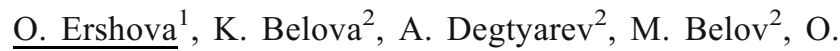
Ganert $^{2}$, M. Romanova ${ }^{2}$, I. Abissova ${ }^{2}$

${ }^{1}$ Yaroslavl State Medical Academy, Yaroslavl, Russian Federation, ${ }^{2}$ Yaroslavl Emergensy Care Hospital, Yaroslavl, Russian Federation

Introduction: Hip fractures are the most severe complications of osteoporosis, characterized by a high mortality rate.

Aim: To establish a register of patients with hip fractures; to assess the major indicators of trauma care during the hospital stay.

Materials and methods: An automated system called REALITY of processing patient medical information was created in the clinical emergency care hospital n.a. N.V.Solovyov. This program was established under the target project of the Russian Association of osteoporosis. The system includes information about the residents of Yaroslavl city who suffered by hip fracture from 01.01 .2013 to 16.04 .2015 . Totally the register included the data on 1748 patients (487 (27.86\%) men and 1261 (72.13\%) women). The average age was 74.84 years (in men it was $68.17 \pm 11.29$ years, in women $77.41 \pm 10.40$ years, $p=0.003)$. At the age $75-89$ years were $912(52.17 \%)$ patients. 39 (2.23\%) people received outpatient medical care, the others were hospitalized. The average length of hospital stay was 8.6 days. $1504(88.52 \%)$ patients were operated on. The average age of the non-operated patients was $77.14 \pm 11.77$ years, among the operated it was $74.47 \pm 11.33$ years, $p=0.0006$. Surgical treatment was performed on the first day after admission to the hospital in $22.87 \%$, on the second in $26.05 \%$ and the third $17.93 \%$, after three days received surgery $33.13 \%$ of patients. 47 $(2.77 \%)$ patients died during hospitalization. The lethality rate before operative treatment was $0.88 \%$ (among 
them $7(46.66 \%)$ patients died within 1-2 days of hospital stay due to a critical condition on admission), the mortality rate after operative treatment was low and amounted to $1.8 \%$.

Conclusion: We created the register of the patients with hip fracture, which can be used as an effective system of registration of all cases of hip fractures and to obtain objective information on medical care and outcomes by these patients.

\section{P925}

STANDARDIZED HISTOLOGICAL ASSESSMENT OF LATE-PHASE MELOXICAM EFFECT IN IODOACETATE-INDUCED RAT OSTEOARTHRITIS E. Nagy $^{1}$, E. Vajda ${ }^{2}$, D.-L. Muntean ${ }^{2}$, C. Vari ${ }^{3}$, E. Horvath ${ }^{4}$ ${ }^{1}$ Dept. of Pharmaceutical Biochemistry, University of Medicine and Pharmacy, Targu-Mures, Romania, ${ }^{2}$ Dept. of Drug Analysis, University of Medicine and Pharmacy Targu-Mures, Targu-Mures, Romania, ${ }^{3}$ Dept. of Pharmacology, University of Medicine and Pharmacy TarguMures, Targu-Mures, Romania, ${ }^{4}$ Dept. of Pathology, University of Medicine and Pharmacy Targu-Mures, TarguMures, Romania

Objective: To compare the late phase histologic effects of low-dose vs. high-dose meloxicam treatment in monoiodoacetate (MIA) induced osteoarthritis of the knee, applying the Osteoarthritis Research Society International (OARSI) Cartilage Histopathology Assessment System (OOCHAS) and the OARSI histopathology initiative recommended methodology.

Materials and methods: 10 Wistar rats served as controls and were injected with isotonic solution, while osteoarthritis was triggered in 34 animals with an intra-articular monodose of MIA. The osteoarthritic group was subdivided and treated between weeks 3-7 as follows: 11 animals received vehicle, 12 low-dose and 11 high-dose meloxicam (mxc) p.o. daily. At the end of the 11th week, the animals were sacrificed, the knee joints being submitted to histological investigation. Haematoxylin-eosin and periodic acid Schiff stainings were performed, and the lesions were evaluated in conformity with the OOCHAS and the OARSI histopathology initiative recommendations.

Results: The cartilage degeneration score and the total cartilage degeneration width were significantly lower in both MXC treated groups, while the OOCHAS grade was lower only in the high-dose MXC group than in the placebo receiving animals. At the level of deep cartilage and subchondral bone, only the high- dose meloxicam treatment generated a protective effect.

Conclusion: both low- and high-dose meloxicam exert chondroprotection in the late-stage of MIA- induced rat osteoarthritis of the knee. High-doses are effective also in reduction of the deep cartilage and subchondral bone lesions, that may be beneficial in arresting the disease progression.

Acknowledgments: This work was partly supported by the Sectorial Operational Programme Human Resources Development, financed from the European Social Fund and by the Romanian Government under the contract number POSDR 80641.

P926

DELETION OF THE C-TERMINAL DOMAIN OF CONNEXIN 43 RESULTS IN ALTERATIONS IN NORMAL CHONDROCYTE PHENOTYPE AND CARTILAGE FORMATION

R. Gago-Fuentes $^{1}$

${ }^{1}$ CellCOM Research Group, Institute for Biomedical Research of A Coruña (INIBIC), A Coruña, Spain

Abstract: Recent results have demonstrated the involvement of connexin $43(\mathrm{Cx} 43)$ in the pathogenesis of degenerative skeletal and joint disorders such as osteoporosis and osteoarthritis. Connexins are proteins responsible of the gap junction (GJ) intercellular communication (GJIC), which ability to synchronize groups of cells for coordinated electrical, mechanical, metabolic and chemical communication make these proteins essential for tissue function. $\mathrm{Cx} 43$ the most abundant GJ protein expressed in chondrocytes and bone cells. We have used genetically modified murine models to characterize, for the first time, the role of the C-terminal domain (CTD) of $\mathrm{Cx} 43$ on cartilage development and function.

Methods: Mouse genotyping was achieved by PCR using DNA extracted from ear tissue and further tested by western blotting using whole cell lysate from primary chondrocytes. Mice knee joints were fixed and analyzed by immunohistochemical and staining techniques in order to study the articular cartilage morphology, structure, and the rates of cell proliferation in tissue. Chondrocytes were isolation and were kept in primary culture. Immunofluorescence techniques against osteopontin, fibronectin, collagen type II, $\mathrm{Cx} 43$ and Ki67 to study cellular phenotypes. Cellular proliferation in culture was determined by measuring the Ki67 positive cells and by using an automated cell counter. GJIC was tested by scrape load assays. 
Results: Using primary chondrocytes from CTD-deficient mice exhibited changes in their phenotype together with a dramatically reduced GJIC; CTD-deficient chondrocytes have increased rates of cell proliferation and significantly reduced expression of collagen type II. The staining techniques demonstrated that the CTD-deficient animals contain significantly lower levels of proteoglycans. CTD-deficient mice showed a significantly shorter body as compared with the control mice without detectable loss in fat mass.

Conclusions: The results illustrate that a complete isolation of Cx43 from its CTD have a negative impact on chondrocyte phenotype, cartilage structure and growth leading to a significant reduced body size. The data further suggest that CTDdependent channel gating is involved in preserving normal cartilage ECM by altering cellular proliferation and gene expression. Our study in mice suggests that previous findings and alterations described for $\mathrm{Cx} 43$ in skeletal or joint tissue may be due to alterations in the CTD more than full-length Cx43, thereby opening new avenues to the study and understanding of the pathophysiological mechanisms that will provide new insights in order to treat bone and cartilage diseases such as OA.

\section{P927}

GREEK ORTHODOX FASTING: EXPLORING ITS EFFECTS ON METABOLISM AND VITAMIN D EQUILIBRIUM IN MOUNT ATHOS MONKS

S. Karras ${ }^{1}$, A. Persynaki ${ }^{1}$, A. Petrozci ${ }^{2}$, E. Barkans ${ }^{2}$, H. Mulrooney $^{2}$, M. Kypraiou ${ }^{1}$, T. Tzotzas ${ }^{1}$, K. Kotsa ${ }^{1}$, E. Rapti $^{1}$, M. Grammatiki ${ }^{1}$, D. Naughton ${ }^{2}$

${ }^{1}$ First Department of Internal Medicine, Division of Endocrinology and Metabolism, AHEPA Hospital, Thessaloniki, Greece, ${ }^{2}$ Kingston University London, School of Life Sciences, Pharmacy and Chemistry, Penrhyn Road, Kingston upon Thames, Surrey, KT1 2EE, United Kingdom, London, United Kingdom

Objectives: The aim of this study was to examine the effect of an integral part of the Mediterranean diet, the orthodox fasting (OF), as it is practiced by the Orthodox monks from Mount Athos. We especially emphasized on the vitamin D equilibrium of the participants, due to their nutritional and sartorial habits in a sunny Mediterranean region of Northern Greece.

Materials and methods: In a relatively large sample of Athonian monks (AM, $n=70$ ), we aimed to report the micro- and macronutrient and calorie intake between fasting days (FD) and non-fasting days (NFD). We also assessed the health status in terms of body anthropometry and biochemical markers considering age and physical activity, with a special discourse on PTH and vitamin D homeostasis.

Results: In this sample of Athonian monks (AM, $n=70$, mean age $38.8 \pm 9.69$ years), nutrient intake was significantly different ( $p>.001)$ between FD and NFD for fat, protein, saturated fat, cholesterol, fibre, minerals and most vitamins; and was especially low for energy intake (1362 \pm 84 vs. 1561 $\pm 81 \mathrm{kcal}, p>.001)$ mainly as carbohydrates $(159 \pm 22 \mathrm{vs}$. $294 \pm 23 \mathrm{~g}, p>.0001)$. Anthropometric measures, including BMI and body weight, were independent of age and level of physical activity. The averaged results for all markers fell within normal ranges with the exception of elevated PTH (115.54 \pm 51.09$)$, INS $(4.64 \pm 3.19)$ and extremely low concentrations of $25 \mathrm{OHD}(8.89 \pm 6.26)$.

Conclusions: This report presents, for the first time, results on dietary intake and health markers between the Athonian monks, using dietary data, anthropometric measurement and blood test results embracing age and levels of physical activity. The key outcomes are (i) the markedly low levels of dietary energy, carbohydrate and vitamin $\mathrm{D}$, and significant differences between intake on FD and NFD, (ii) anthropometric measures within the healthy range and (iii) blood results revealing secondary hyperparathyroidism as a result of profound hypovitaminosis $\mathrm{D}$. These results suggest that $\mathrm{OF}$ fasting as practiced by AM, demonstrates a beneficial metabolic profile, but due to sartorial habits results in profound hypovitaminosis D Further work should be directed at the potential impact of these results on bone microarchitecture in this specific population.

\section{P928}

ORGANIZATION OF THE SYSTEM OF SECONDARY FRACTURE PREVENTION IN THE YAROSLAVL CITY, RUSSIAN FEDERATION

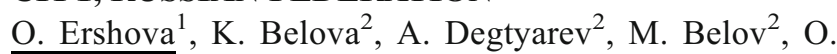
Ganert $^{2}$, M. Romanova ${ }^{2}$, I. Abissova ${ }^{2}$

${ }^{1}$ Yaroslavl State Medical Academy, Yaroslavl, Russian Federation, ${ }^{2}$ Yaroslavl Emergensy Care Hospital, Yaroslavl, Russian Federation

Introduction: To reduce the incidence of secondary osteoporotic fractures, the IOF initiated "Capture the Fracture" Campaign, and in 2012 the Russian Association on Osteoporosis project launched PROMETHEUS ("Creation of the system for prevent re-fractures in patients with osteoporosis").

Aim: To established the center of secondary fracture prevention in Yaroslavl, Russian Federation. Materials and methods: The organization of the working of the center has begun from 01.01.2014. It is constructed with dedicated nurse-coordinator, and the consultations of the specialist are carried out on the basis of osteoporosis center, which is a part of the clinical emergency care hospital n.a. N.V.Solovyov.

Results: Identification of the patients with osteoporotic fractures is carried out by the nurse-coordinator using the logs of registration from the accident ward. The coordinator carries out an evaluation of the subsequent fractures risk (includes the 
assessment of a trauma level, risk factors, FRAX calculation) and the risk of falling. In the emergency department the patient is completely examined. The coordinator provides advice to the patient's by the specialist of osteoporosis center and DXA. The physician diagnoses secondary causes osteoporosis, initiate treatment in accordance with relevant Russian guidelines, give recommendations on modification of risk factors, diet, physical activities, prevention of falling, defined the plan for further supervision. In 6 and 12 months the nurse-coordinator carries out the telephone contact with the patient.

Conclusion: In the Yaroslavl the Center of secondary fracture prevention was organized, it based on the work of a dedicated nurse-coordinator. In August 2015 it was evaluated and recognized the silver level standard of the Capture programme in patients with hip fractures.

\section{P929 \\ BONE BIOLOGY AND BONE METABOLISM AFTER THR \\ T. Minasov ${ }^{1}$ \\ ${ }^{1}$ Department of Orthopaedic Surgery and Traumatology, Novi Sad, Serbia}

Objective: Hip arthroplasty associated with bone alteration and activate local and systemic inflammatory response which can affects the systemic mineral homeostasis.

Material and Methods: The results of 72 patients' activity with impaired bone metabolism after total hip arthroplasty had been analyzed. Functional activity, life quality and X- ray monitoring including whole body DXA and $\mathrm{pQCT}$ had been researched.

Results: Functional activity by HHS scale a year after arthroplasty was 82.9 points $\pm 12.4 \mathrm{SD}$, which was significantly higher than comparison group - 75.6 $\pm 8.2 \mathrm{SD} .(p<0.01)$, and significance of differences persisted for up to 18 months. Life quality parameters showed a positive trend during the rehabilitation period in both groups. However, significant benefits had been observed in main group after 3 months after the arthroplasty, in particular the parameters of physical, emotional and social component of quality of life. Patients comparison group noted a slow recovery of the vital and mental component of quality of life, which was associated with both the operated limb function, and subjective general health and low values of these parameters are marked even 6 months after arthroplasty. X-ray monitoring in 6 months showed the formation of areas of enlightenment mainly in 1 and 7 Gruen zones. At 12 and 18 months similar changes was in 2 and 6 Gruen zones. Whole body DXA after 6 months showed a significant decrease in BMD in all segments of the skeleton. The most intensive changes occurred in the thoracic spine and operated limb ranged from -3.39 to $-6.22 \%$, which was significantly higher than main group of patients $(p<0.05)$.
pQCT in distal tibia shown decreasing of volumetric bone density in compeering group and negative dynamic was significantly higher than main group of patient.

Discussion: Thus we can conclude that antiresorptive therapy increase functional activity, life quality and stabilize bone metabolism in postoperative period after THR.

\section{P930}

GLUCOCORTICOID-ASSOCIATED SDI: A POTENTIAL INSTRUMENT FOR THE ASSESSMENT OF GLUCOCORTICOID-INDUCED DAMAGE IN SYSTEMIC LUPUS ERYTHEMATOSUS

S. Mittal ${ }^{1}$

${ }^{1}$ Safdarjung Hospital, New Delhi, India

Objective: In systemic lupus erythematosus (SLE), organ damage occurs in response to autoimmune- induced inflammation but also glucocorticoid therapy. Inflammatory activity and damage are measured with validated instruments, but the glucocorticoid-induced organ damage is not routinely quantified. We evaluated whether a modified damage index could be utilised to assess glucocorticoid-induced damage in SLE.

Material and Methods: SLE patients $(\mathrm{ACR} \geq 4)$ were studied. Disease activity (SLEDAI-2 k) and treatment details were recorded at each visit and organ damage measured annually (SLICC-SDI). Four domains of the SDI that relate directly to glucocorticoid use (osteoporosis, avascular necrosis, diabetes mellitus and cataracts) were combined to form the glucocorticoid-associated SDI (GC-SDI).

Correspondingly, all other domains of the SDI comprised the non-GC-SDI.

Results: 184 patients were included; 138 received glucocorticoids during the study period. Baseline SDI was not significantly different in the subsets exposed to glucocorticoids, however final SDI was significantly worse in glucocorticoid-exposed patients $(p=0.02)$. There was a strong correlation between time-adjusted mean SLEDAI (AMS) and cumulative prednisolone usage $(p<0.0001)$. Total SDI was significantly correlated with AMS, $r=0.258, p=0.0004$ ) and with cumulative prednisolone dose $(r=0.269, p=0.0002)$, indicating that damage accrual is associated with both disease activity and exposure to prednisolone. GC-SDI was not significantly associated with disease activity (AMS) in either all patients $(r=0.075, p=0.31)$ or in glucocorticoid exposed patients $(r=0.038, p=0.65)$. In glucocorticoid users, cumulative prednisolone exposure was correlated with change in GC-SDI $(r=0.214, p=0.012)$ and with final GC-SDI $(r=0.16, p=0.063)$. The non-GC-SDI was significantly associated with both AMS $(r=0.267$, $p=0.0002)$ and cumulative prednisolone use $(r=0.243$, $p=0.0009)$ in all patients and in glucocorticoid users. 
Conclusions: A novel instrument, GC-SDI, associates with glucocorticoid use in SLE. Validation with further longitudinal data and multivariate analysis is justified.

\section{P931}

THE EFFECTS OF A POTENT AND SELECTIVE PARTIAL ESTROGEN RECEPTOR MODULATOR (PERD) ON BONE IN OVARIECTOMIZED RATS

J. P. Rissanen ${ }^{1}$, C. Möller ${ }^{2}$, J. Morko ${ }^{1}$, Z. Peng ${ }^{1}$, J. Vääräniemi $^{1}$, K. M. Fagerlund ${ }^{1}$, T. A. Suutari ${ }^{1}$, J. Bernoulli ${ }^{1}$, A. Wagenfeld ${ }^{2}$, A. Schmitz ${ }^{2}$, J. M. Halleen ${ }^{1}$

${ }^{1}$ Pharmatest Services Ltd, Turku, Finland, ${ }^{2}$ Bayer Pharma AG, Berlin, Germany

Objective: Selective estrogen receptor modulators (SERMs) are compounds that bind to estrogen receptors (ERs) and agonize or antagonize estrogen action in different tissue types. Due to a broad spectrum of physiological and pathological processes contributed by ERs, SERMs provide a potential therapeutic benefit for a variety of diseases. In this study, we characterized the effects of a novel partial estrogen receptor destabilizer (pERD) on bone under estrogen deficiency in ovariectomized (OVX) rats.

Materials and Methods: Female Sprague Dawley rats were OVX or SHAM operated at 3 months of age. Treatment of rats was started at one day after the operations and continued once a day for 8 weeks. OVX rats were treated with pERD at 1,3 or $10 \mathrm{mg} / \mathrm{kg} / \mathrm{d}$ p.o., $17 \beta$-estradiol (E2) at $4 \mu \mathrm{g} / \mathrm{kg} / \mathrm{d}$ s.c., raloxifene (RAL) at $1 \mathrm{mg} / \mathrm{kg} / \mathrm{d}$ p.o. or with vehicle, and SHAMoperated rats were treated with vehicle. E2 was used as a complete agonist and RAL as a reference SERM exhibiting agonist activity in bone. Treatment effects on bone were studied by peripheral quantitative computed tomography, bone histomorphometry and measuring serum levels of bone turnover biomarkers.

Results: In metaphyseal trabecular bone, treatment with pERD at 1,3 and $10 \mathrm{mg} / \mathrm{kg} / \mathrm{d}$ prevented an OVXinduced reduction in BMD, bone mineral content (BMC), bone volume fraction and trabecular number. The effects of pERD were associated with a prevention of OVX-induced increase in osteoclastogenesis (inclucing N.Oc/B.Pm and Oc.S/BS) and bone turnover and formation (including MS/BS, MAR, BFR/BS and BFR/BV). In diaphyseal cortical bone, treatment with pERD at 1 and $3 \mathrm{mg} / \mathrm{kg} / \mathrm{d}$ prevented an OVX-induced increase in cortical BMC, cortical bone area and periosteal perimeter. In circulation, treatment with $\mathrm{pERD}$ at 1 , 3 or $10 \mathrm{mg} / \mathrm{kg} / \mathrm{d}$ prevented an OVX-induced increase in serum levels of crosslinked telopeptides of type I collagen, N-terminal mid-fragment of osteocalcin, and procollagen type I N-terminal propeptide.

Conclusion: The effects of oral pERD treatment were similar with the effects of E2 and RAL indicating a boneprotective agonist activity for pERD in trabecular bone at $1-10 \mathrm{mg} / \mathrm{kg} / \mathrm{d}$ and in cortical bone at $1-3 \mathrm{mg} / \mathrm{kg} / \mathrm{d}$. The maximal efficacy of pERD was lower than the efficacy of E2 presenting pERD as a novel partial agonist in bone in young adult OVX rats.

\section{P932}

\section{EFFECT OF PROTEIN DIET ON THE MECHANICAL PROPERTIES OF BONE TISSUE IN OVARIECTO-} MIZED RATS

$\underline{\text { R. C. Shimano }}^{1}$, G. R. Yanagihara ${ }^{1}$, A. P. Macedo ${ }^{1}$, B. B. Brancaleon $^{1}$, A. C. Shimano ${ }^{1}$, J. P. M. Issa ${ }^{2}$

${ }^{1}$ Department of Biomechanics, Medicine and Rehabilitation of the Locomotor System, Ribeirão Preto Medical School, University of São Paulo, Ribeirão Preto, Brazil, ${ }^{2}$ Department of Morphology, Physiology and Basic Pathology, School of Dentistry of Ribeirão Preto, University of São Paulo, Ribeirao Preto, Brazil

Introduction: Appropriate nutrition is important for bone mass maintenance and is an important tool for fracture prevention in people with osteoporosis. Osteoporosis is a metabolic disease characterized by bone mass and strength losses. Many variables may interfere in this situation; however, focus is given to women after menopause due to estrogen declination. To simulate an osteopenic condition in experimental study, ovariectomy, a surgical procedure that involves ovaries removing, has been used in the current literature.

Materials and Methods: Forty Wistar rats were used, body mass 150-180 g (juvenile stage), in total of four groups $(n=10)$ : OVX, ovariectomized and standard diet; OVXP, ovariectomized and protein diet (40\% casein); SHAM, sham surgery and standard diet; SHAMP, sham surgery and protein diet. All groups were euthanized after 12 weeks for femurs remotion, and these samples were submitted to mechanical tests, stiffness and maximum strength. Data were evaluated for normal distribution (Shapiro-Wilk), and analysis of variance were done using the general linear model, Bonferroni test $(p<0.05)$.

Results: For flexocompression test, the stiffness, the OVX group was lower when compared to SHAM group $(p=0.008)$ and OVXP group was less than the SHAMP group $(p=0.007)$. Already at maximum strength, the OVX group was lower than the SHAM $(p=0.013)$. 
In conclusion, based on this experimental model and according to the limits of this study, only the ovariectomy had negative influence of the mechanical properties of bone tissue, and high protein diet amount was not able to sensitize the bone tissue.

\section{P933}

\section{ASSESSMENT THE EFFECT OF AGING AND} MUSCULOSKELETAL DISORDERS ON ADL AND IADL IN OLD PERSONS: ARTICLES REVIEW

\section{G. Niketeghad $^{1}$}

${ }^{1}$ Osteoporosis Research Center, Endocrinology and Metabolism Clinical Sciences Institute, Tehran University of Medical Sciences, Tehran, Islamic Republic of Iran

Background: Approximately $20 \%$ of the persons aged 70 years or older report difficulty with performing essential ADL and IADL. It makes a serious decline in functional health and increases the risk of outpatient care and hospitalization. Articles show the most common problem that impacts and causes dependency in elderly people is functional disability.

Method: Systematic search was performed in Google Scholar, PubMed and Science Direct from 1995 to 2015, articles were reviewed by one reviewer who also assessed quality of the included studies.

Results: Articles show that elderly with weight loss, slower gait speed, lower grip strength, lower physical activity, lower exercise, poor balance, lower extremity function and musculoskeletal disorders have higher risk of disability in ADL and IADL in future. Slow gait and low physical activity because of aging and musculoskeletal disorders have the highest effect on elderly people. Impairment of IADL function occurs prior to that of ADL. Differences in incidence of IADL disabilities can be because of cultures. Bathing was found to be the most difficulty for elderly to perform. Dressing, transferring and toileting are very frequent of dependency.

Discussion: There is an urgent need to develop effective interventions that may increase the years of life spent without disability and dependency. According to articles suggest an exercise program may significantly reduce incidence of ADL disability in older persons with chronic disorders such as osteoarthritis, osteoporosis, according to the results ADL and IADL independency have favorable results such as decreased depressed mood and fear of falling, increased self-care and quality of life and finally this can increase life expectancy. Highest independency rate had the lowest female proportion and married rate, this suggest social factors such as gender and marital status associated with functional disability in elderly so study of this problem separately in female and men is necessary. It is essential that we know about special problems that influence on ADL and IADL disabilities such as knee osteoarthritis or osteoporosis of lower extremity joints.

\section{P934}

HOW THE LOCAL INHIBITOR FACTORS OF BONE FORMATION BEHAVE FOR OSTEOPOROTIC HIP FRACTURES

A. Sepici Dincel ${ }^{1}$, V. E. Dincel ${ }^{2}$

${ }^{1}$ Department of Medical Biochemistry, Gazi University, Ankara, Turkey, ${ }^{2}$ Clinics of Orthopedics and Traumatology, Ministry of Health, Ankara Training and Research Hospital, Ankara, Turkey

Objectives: Today, the most important topic is the quality of life after fractures. Beta-catenin signal can be activated at fracture sites, highly expressed during fracture repair, especially in the early stage of healing and controls the differentiation of mesenchymal cells into osteoblasts. However dickkopf 1 (DKK1) forms a complex with Lrp5/6, disrupts the Wnt signaling pathway, prevent the differentiation of osteoprogenitor cells and blocked bone formation at the injury site. In our study we aimed to evaluate the relationship between the inhibitor factor dickkopf 1 and Wnt-beta catenin pathway for osteoporotic hip fractures in order to speculate on the prognosis of patients.

Methods: The total of the study group were included 62 patients with osteoporotic hip fracture; 38 of those (Group 1; 23 female/15 male, mean age 77.52 \pm 6.74 years) had intertrochanteric fractures of hip and 24 of them (Group 2; 18 female/6 male, mean age $75.16 \pm 6.89$ years) had collum femoris fractures. All fractures were due to low energy trauma, simple falls. None of the patients had neoplastic pathology of bone, long-term corticosteroid usage, bone metabolism disease or arthritis and any other metabolic disease. BMD measurements were done with Lunar DXA. Human serum beta-catenin levels were determined both by western blot and ELISA (R\&D Systems, pg/mL) and dickkopf 1 values were determined by ELISA (Cusabio kit, pg/ $\mathrm{mL})$.

Results: Neck, trochanter, wards and total BMD values were in agreement for osteoporosis and no significant differences between the two groups for all BMD values. The mean and standard error values for DKK1 in Group $1(1489.84 \pm 131.27)$ were significantly increased $(p<0.05)$ compared to Group $2(1032.71 \pm 139.35)$ and beta-catenin levels in Group $1(230.67 \pm 13.18)$ and in 
Group $2(235.91 \pm 20.980)$ were not different. All groups had significantly different compared to control group.

Conclusion: Wnt-beta catenin signal transduction pathway is a bone remodelling regulator which some of the cytokines and BMPs mediate their effects on bone formation and resorption. The increased inhibitor factor DKK1 in intertrochanteric fractures could be related with the severity and diagnosis of patients. Inhibition of DKK 1 enhanced the healing process, resulting in mechanically stronger bone at the fracture site.

\section{P935}

NOVEL LONG TERM SUBSTANCE DELIVERY METHOD IN PRECLINICAL OSTEOPOROSIS STUDIES

J. P. Rissanen $^{1}$, S. T. Ruohonen ${ }^{1}$, H. J. Jukarainen ${ }^{1}$

${ }^{1}$ Preclinapps Ltd, Turku, Finland

Objective: Chronic dosing of compounds often has a pivotal role in osteoporosis preclinical studies as the study length can be several months or even over a year. To avoid challenges with traditional injections, we have developed a polymerbased substance delivery system called MedRod ${ }^{\mathrm{TM}}$. In this study, we characterized the in vitro and in vivo release of 17b-estradiol (E2) from MedRod ${ }^{\mathrm{TM}}$ system up to 8 weeks.

Materials and Methods: E2 was dispersed in the polydimethylsiloxane matrix and two types of silicone membrane covered rods (OD $3 \mathrm{~mm}$ ) were produced: $20 \mathrm{~mm}$ (11 $\mathrm{mg}$ E2) and $10 \mathrm{~mm}$ (5.5 mg E2). The release of E2 was studied for 8 weeks first in dissolution in vitro test and then in animal studies with aged intact and ovariectomized (OVX) female Sprague Dawley rats. ELISA E2 immunoassay was used to quantify E2 levels from the dissolution or serum samples. The macroscopic evaluation of foreign body reaction was conducted during necropsy, and sensitive tissues for E2 metabolisms, such as uterine, liver and bones were harvested and weighed. BMD was measured from tibias by pQCT.

Results: In both, in vitro and in vivo studies, the release profiles of E2 were stable for 8 weeks. In the in vivo experiments, E2 levels were elevated, and the difference between the two doses remained accurately two-fold throughout the study. The increase in uterine weights correlated significantly with the elevated E2 levels. In metaphyseal trabecular bone, treatment with E2 prevented an OVX-induced reduction in BMD and bone mineral content confirming the anabolic effect of the hormone treatment. Liver weights were slightly elevated after hormone treatment in response to increased metabolisms, but during necropsy, no evidence of toxicity was observed. In addition, no irritation or inflammation was found from implant surrounding tissue, neither did any of the animals scratch the implanted site, thus proofing comfortable and safe delivery system.
Conclusion: The MedRod ${ }^{\mathrm{TM}}$ system enables long-term and stable 17b-estradiol delivery in preclinical osteoporosis studies. Benefits also include decreased handling stress for the animals and decreased variability which follows closely the 3R principle (Replacement, Reduction, and Refinement).

\section{P936}

CORRELATIONS BETWEEN QUALITY OF LIFE AND SOME PSYCHOLOGICAL ASPECTS IN PATIENTS WITH UPPER LIMB OSTEOARTHRITIS C. Suteu ${ }^{1}$, D. M. Farcas ${ }^{2}$

${ }^{1}$ University of Oradea Faculty of Medicine and Pharmacy, Oradea, Romania, ${ }^{2}$ University of Oradea, Faculty of Medicine and Pharmacy, Oradea, Romania

Aim: To assess quality of life and it's correlations with some psychological aspects in patients with upper limb osteoarthritis.

Material and method: We studied 104 patients with upper limb osteoarthritis. They were divided into two groups, first group consisted in 52 patients which underwent a physical rehabilitation program three times per week for 12 months and the second subgroup of 52 sedentary patients. All the patients were assessed with Short Form 36 for quality of life, with Hamilton Anxiety Rating Scale for anxiety and with Rosenberg self-esteem scale for self-esteem. We assessed all the patients at baseline, at six months and at twelve months. Results: In the group of patients who underwent rehabilitation program quality of life, anxiety mean value and self-esteem mean value improved at 6 months and the improvement in quality of life was greater at 12 months than in the second group of sedentary patients which showed no improvement at all. In our study we found also correlations between quality of life, anxiety and self-esteem.

Conclusion: It seems that a healthy lifestyle that includes a regular physical exercise programme has a beneficial effect on well-being and on quality of life in patients with chronic upper limb osteoarthritis.

\section{P937}

PREDICTORS FOR FUNCTIONAL STATUS IN RHEUMATOID ARTHRITIS PATIENTS

D. Matei ${ }^{1}$, S. Patru ${ }^{1}$, R. M. Trăistaru ${ }^{1}$, A.-M. Bumbea ${ }^{1}$, D. V. Caimac $^{1}$, A. C. Bighea ${ }^{1}$

${ }^{1}$ University of Medicine and Pharmacy, Craiova, Romania

Objectives: To determine the predictive values of different factors for functional disability in patients with rheumatoid arthritis. 
Material and Methods: We observed 53 patients with rheumatoid arthritis during $1 \mathrm{y}$; the mean age was 47.91 and mean disease duration: 53.8 months. The patients were laboratory (complete blood count, CRP, anti CCP and RF) and clinically (morning stiffness and extra-articular manifestations) evaluated. The functional status was assessed using HAQ. The results were analyzed with the medical statistics programme SPSS.

Results: The most common clinical manifestations were morning stiffness $(100 \%)$; extra-articular manifestations were represented by anemia (46.8\%), and thrombocytosis $(18.9 \%)$. The functional capacity assessed by HAQ was significantly correlated with age of onset ( $r$ 0.524), the extra-articular manifestations ( $\mathrm{r}$ 0.517) and rheumatoid factors value ( $\mathrm{r}$ 0.313 ), respectively.

Conclusion: There are many factors involved in the evolution of functional status in patients with rheumatoid arthritis. Permanent monitoring of all clinical and biological parameters may prevent all the disabilities founded in the disease evolution.

\section{P938}

\section{THE PLACE OF KINETOTHERAPY IN HIP} ARTHROPLASTY

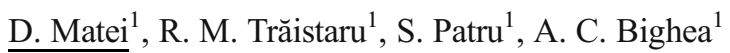

${ }^{1}$ University of Medicine and Pharmacy, Craiova, Romania

The aim of this study was to determine whether the kinetic exercises and massage is useful for patients with hip replacement for osteoarthritis.

Material and method: A number of patients with recent hip arthroplasty for osteoarthritis (n:31) were included in this study. Demographic data, the level of pain, hip osteoarthritis duration (y) were noted. We compared physiotherapy exercises group of patients (n:16) vs. standard care group (n: 15) looking for the hip range of motion (ROM) and muscle strength and quality of life (SF-36).

Result: Compared to the usual care group, the patients with kinetotherapy program showed a better correlation between the clinical and functional variables, with a superior predictability for ROM and muscle strength in connection to pain $\left(R=0.712\right.$ and $\left.R^{2}=0.545\right)$; moreover, the early rehabilitation patients group showed a significant correlation with almost $60 \%$ (58.6) predictability for improving the quality of life.

Conclusion: It is obvious the benefit of rehabilitation treatment both of clinical and functional parameters and it is expected that the improvement of these parameters to increase the patients " life quality.
P939

POSSIBLE ETIOLOGIES AND DIAGNOSTIC DIFFICULTIES IN TRANSIENT OSTEOPOROSIS OF THE HIP: CASE REPORT

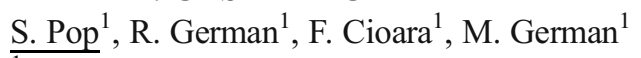

${ }^{1}$ University of Oradea, Faculty of Medicine and Pharmacy, Oradea, Romania

When a young adult female patient accuses spontaneous hip pain, the most common causes considered are osteoarthritis, tendonitis, bursitis, endometriosis or lumbarsacral spine pathology. But sometimes the patient history, clinical, biological and radiographic examinations not confirm these pathologies, causing difficulty in establishing certain diagnosis. We present the case of a female patient, aged 39 years, presenting with spontaneous left hip pain during active mobilization. Anamnesis stands out 12 weeks pregnancy that stopped evolving two weeks ago, mitral valve prolapse, lower limbs venous insufficiency, multiple sports injuries in adolescence, smoking about 11 years. The case is interpreted as impingement syndrome. MRI examination show femoral neck edema interpreted as avascular osteonecrosis of the femoral head. Patient refused surgical treatment, and after 4 weeks evolution became unable to load on left lower limb and with increased left hip pain. MRI examination show the extension of femoral neck edema but with femoral head integrity and liquid in minimum quantity, aspects that raise suspicion of an infectious osteitis, considering the recent abortion, but biological samples not confirm this diagnosis. We initiated treatment with NSAIDs and unloading the left hip joint. After 2 weeks the MRI examination show worsening evolution, with extending edema and osteoporosis, interpreted as transient osteoporosis of the hip, therefore was initiated therapy with ibandronic acid intravenously. Edema remission was obtained after five months. In the hip pathology in young adult we can face with difficulties of diagnosis, transient osteoporosis being a relatively rare disease, with several possible etiologies, but under diagnosed and often confused with avascular necrosis of the femoral head.

\section{P940}

THE INCIDENCE OF RISK FACTORS IN PATIENTS WITH OSTEOPOROSIS

R. Suciu $^{1}$, F. Cioara ${ }^{1}$, A. Pallag ${ }^{1}$, S. Bungau ${ }^{1}$, D. Zaha ${ }^{1}$

${ }^{1}$ University of Oradea, Faculty of Medicine and Pharmacy, Oradea, Romania

Objectives: To determine the incidence of risk factors which lead to osteoporosis in women in the age group 51-90 years. 
Materials and methods: We took in the study a number of 30 patients with diagnosis of osteoporosis from Rehabilitation Clinical Hospital Baile Felix during March to October 2015 to whom questionnaires were administered with predefined questions relating to existing risk factors in the patients history.

Results: Subjects included in the study had an average weight of $65.72 \mathrm{~kg}$, an average height of $161.8 \mathrm{~cm}$ and an average BMI of $24.08 \mathrm{~kg} / \mathrm{m}^{2}$. As regards physiological personal background to the 30 patients included in the study, showed an average age of menarche of 12.76 years and average age of menopause installation of 45.82 years. Average duration of menopause was 15.67 years. Of the risk factors linked to lifestyle $60 \%$ were low in calcium nutrition as well as vitamin $\mathrm{D}, 40 \%$ were smokers and $66.66 \%$ had a sedentary life. Women which smoke have a lower BMD, an increased rate of bone loss and an age of menopause 2 years less compared to nonsmoker. Using FRAX algorithm, we discovered an increased risk for both major fracture osteoporotic and hip-level fracture, with a risk of occurrence of an osteoporotic fracture in the next 10 years of $10.948 .16 \%$, i.e. $\pm 4.73 \pm 6.066$ at hip level. Conclusion: Osteoporosis is an important disease because of the severity they manifest and because of the length, irreparably damaging the life of those who are ill. Covers a large segment of the population (one in five men and one in three women over 50 years) and can be held in check by means of prevention applied in different periods of life.

\section{P941 \\ -643C > T RANKL GENE POLYMORPHISM BUT NOT -693 G $>$ C IS ASSOCIATED WITH OSTEOPOROSIS AND BONE MINERAL DENSITY IN TUNISIAN POST- MENOPAUSAL WOMEN}

R. Sassi ${ }^{1}$, S. Sahli ${ }^{2}$, S. Sellami ${ }^{2}$, A. Benammar ElGaaied ${ }^{1}$

${ }^{1}$ Genetics, Immunology and Human Pathologies Laboratory, Faculty of Mathematical, Physical and Natural Sciences of Tunis, Tunis EL Manar University, Tunis, Tunisia, ${ }^{2}$ Osteoporosis and Arthritis Laboratory, Rabta Hospital, Faculty of Medicine of Tunis, Tunis EL Manar University, Tunis, Tunisia

Background: RANKL gene has been associated to osteoporosis susceptibility in candidate gene association studies then confirmed by GWAS as true susceptibility locus regulating BMD. SNPs identified in the promotor and 5'UTR region of the RANKL gene have shown an association with BMD and also correlated to low trauma fractures. In the current study, we have investigated the association between the RANKL gene $-693 \mathrm{G}>\mathrm{C}$ and $-643 \mathrm{C}>\mathrm{T}$ polymorphisms and BMD in a population of postmenopausal Tunisian women.
Methods: A case control study including 566 Tunisian postmenopausal women, who consecutively visited the Rabta and Charles Nicolles Hospitals of Tunis, was carried out. The BMD was measured for the lumbar spine (L2-L4) and femoral neck (dual femur). RANKL polymorphism analysis was performed by PCR-RFLP technique and verified by direct sequencing.

Results: We have detected a significant difference in lumbar spine and hip BMD for $-643 C>T$ genotypes. For $-693 G>C$ genotypes a significant difference was detected only in hip BMD. The TT genotype of $-643 C>T$ polymorphism is associated with osteoporosis risk $(p=0.01 ; O R=2.15)$ in Tunisian postmenopausal women but not with low impact fracture incidence.

Conclusion: We have demonstrated the association of the $-643 \mathrm{C}>\mathrm{T}$ polymorphism with BMD variation and osteoporosis risk in postmenopausal Tunisian women. This result confirms the important role of RANKL in bone metabolism and osteoporosis risk. More investigation are needed to evaluate the interaction of RANKL gene polymorphisms with other genetic factors like OPG and VDR polymorphisms and environmental factors like physical activity.

\section{P942}

ANTI-CYCLIC CITRULLINATED PEPTIDE (ANTI-CCP) ANTIBODY AS A MARKER OF EROSIVE ARTHRITIS IN PATIENTS WITH LUPUS NEPHRITIS

D. Monova ${ }^{1}$, S. Monov ${ }^{2}$, E. Peneva ${ }^{1}$

${ }^{1}$ Department of Internal Diseases, Medical Institute, Sofia, Bulgaria, ${ }^{2}$ Department of Rheumatology, Medical University, University Hospital, Sofia, Bulgaria

Introduction: Systemic lupus erythematosus (SLE) is an autoimmune and inflammatory disease characterized by a variety of symptoms, including arthropathy. The clinical presentation of joint involvement varies, ranging from arthralgia without erosions or deformity to an erosive arthropathy and severe functional disability, similar to that of rheumatoid arthritis (RA). Rhupus was characterised as the association of SLE and chronic inflammatory arthritis with erosion and positive rheumatoid factor. The aim of this study was to describe the association of anti-CCP antibodies in patients with lupus nephritis and articular damage and determine whether anti-CCP antibody is a useful marker of erosive disease in SLE patients presenting with arthritis.

Methods: Retrospective medical record review of a 174 lupus nephritis patients. The initial SLE presentation in all patients was arthralgia without erosions or deformity to an erosive arthropathy. All patients had recent $\mathrm{x}$-ray 
films of the hands that were evaluated according to Sharp's method. Lupus nephritis patients had a mean $7.26 \pm 0.73$ American College of Rheumatology (ACR) criteria for SLE. The DerSimonian-Laird random effects model was used to calculate pooled sensitivity and specificity of anti-CCP antibody for erosive arthritis in lupus nephritis patients.

Results: Erosive arthritis was strongly associated with the presence of anti-CCP antibodies. Anti-CCP antibodies in patients with lupus nephritis may be a marker of a more severe joint disease. Anti-CCP antibodies were detected in 58 out of $174(33.33 \%)$ patients, 31 of whom had a positive rheumatoid factor. Patients with lupus deforming arthropathy were more frequently positive for rheumatoid factor than patients with nondeforming arthritis $(p<0.05)$.

Conclusion: Anti-CCP antibodies do not associate with lupus arthropathy, whether deforming or non deforming. Our findings suggest that anti-CCP antibody is a highly specific marker for erosive arthritis in SLE.

\section{P943}

ASSOCIATION BETWEEN BODY COMPOSITION AND BONE MINERAL DENSITY IN ANDALUSIAN PERIMENOPAUSAL WOMEN: THE FLAMENCO PROJECT

$\underline{\text { I. Coll-Risco }}^{1}$, P. Ruiz-Cabello ${ }^{1}$, M. Borges-Cosic ${ }^{2}$, P. AcostaManzano $^{2}$, A. Soriano-Maldonado ${ }^{2}$, J. J. Martín-Martín ${ }^{3}$, M. D. Quesada ${ }^{4}$, V. A. Aparicio ${ }^{5}$

${ }^{1}$ Department of Physiology, Faculty of Pharmacy, Faculty of Sport Sciences, and Institute of Nutrition and Food Technology, University of Granada, Granada, Spain, ${ }^{2}$ Department of Physical Education and Sports, Faculty of Sport Sciences, University of Granada, Granada, Spain,
${ }^{3}$ Department of Applied Economics, Faculty of Economics, University of Granada, Granada, Spain, ${ }^{4}$ Zaidín Sur Healthcare Centre and Department of Medicine, Faculty of Medicine, University of Granada, Granada, Spain, ${ }^{5}$ Department of Physiology, Faculty of Pharmacy, Faculty of Sport Sciences, and Institute of Nutrition and Food Technology, University of Granada and Department of Physical Education and Sports, Faculty of Sport Sciences, University of Granada, Granada, Spain

Objectives: To assess the association of different components of body composition with BMD in perimenopausal women.

Material and Methods: The sample for the present analyses comprised 194 women (age range 45-60 years). Fat and lean mass and bone mass density were measured using DXA device (Hologic Discovery QDR, Nasdaq: HOLX). Weight and height were used to calculate BMI $\left(\mathrm{kg} / \mathrm{m}^{2}\right)$.

Results: The results showed that body weight $(\beta=0.244$, $P=0.001)$, BMI $(\beta=0.180, P=0.011)$ and lean mass $((\beta=0.379, P<0.001)$ were positively associated with BMD. Lean mass was independently associated with BMD $(\beta=0.392, P<0.001)$ and explained $20 \%$ of the variability in $\operatorname{BMD}(P<0.001)$.

Conclusions: The findings of the present study indicate that lean mass is consistently associated with BMD in perimenopausal women. On the other hand, body fat mass did not show a significant association with BMD in this population. Furthermore body lean mass showed an independent association with BMD, explaining $\sim 20 \%$ of the variability in BMD. Further research is needed to determine whether increasing lean mass might lead to improvements in BMD in this critical period of women's life.

Table 1. Linear regression analysis assessing the association of body composition with bone mineral density in perimenopausal women.

\begin{tabular}{|c|c|c|c|c|c|c|c|c|}
\hline \multirow[b]{3}{*}{ Body composition } & \multicolumn{8}{|c|}{ Bone Mineral Density $\left(\mathrm{g} / \mathrm{cm}^{2}\right)$} \\
\hline & \multicolumn{4}{|c|}{ Age-adjusted } & \multicolumn{4}{|c|}{ Multivariate adjusted $^{\mathrm{a}}$} \\
\hline & $\beta$ & $\mathrm{B}$ & SE & $\mathrm{P}$ & $\beta$ & $\mathrm{B}$ & $\mathrm{SE}$ & $\mathrm{P}$ \\
\hline Weight $(\mathrm{kg})$ & 0.205 & 0.002 & 0.001 & 0.003 & 0.244 & 0.002 & 0.001 & 0.001 \\
\hline Height (m) & 0.064 & 0.001 & 0.001 & 0.366 & 0.069 & 0.001 & 0.001 & 0337 \\
\hline Body mass index $(\mathrm{kg})$ & 0.145 & 0.003 & 0.001 & 0.036 & 0.180 & 0.004 & 0.002 & 0.011 \\
\hline Fat mass $(\%)$ & -0.060 & -0.001 & 0.001 & 0.390 & -0.042 & -0.001 & 0.001 & 0.560 \\
\hline Lean mass (kg) & 0.344 & 0.007 & 0.001 & $<0.001$ & 0.379 & 0.008 & 0.003 & $<0.001$ \\
\hline
\end{tabular}

SE: Standards Error; $\beta$, standardized regression coefficient; B, nonstandardized regression coefficient; \#Lower scores indicate better performance; ${ }^{a}$ Model adjusted for age, educational status, marital status, smoking status, regular menstruation and hormonal therapy; ${ }^{*}$ Model additionally adjusted for body mass index 


\section{P944}

FEMORAL GEOMETRY AND FRAX INDICES AS INDEPENDENT RISK FACTORS FOR HIP FRACTURES IN UKRAINIAN PATIENTS

N. Grygorieva ${ }^{1}$, O. Zubach ${ }^{1}$

${ }^{1}$ Department of Clinical Physiology and Pathology of Locomotor Apparatus, D.F. Chebotarev Institute of Gerontology NAMS Ukraine, Kyiv, Ukraine

Current evidence suggests that BMD score is crucial but not the only criterion to determine fracture risk. Osteoporosis and fractures risk factors studies collected a large evidence base to define the contribution of each different factor in osteoporotic fractures. According to the literature data, some parameters of the femur (length of axis, length of femoral neck) are independent predictors of hip fractures, but such studies among Ukrainian patients are absent. Although FRAX is an important instrument for measuring the hip fracture risk, right now there are few studies in Ukrainian patients using FRAX-model. From NOF guidelines FRAX scores 3 or more for hip fractures is the important criteria for treatment initiation.

The purpose of this research was to study FRAX indices and some geometric parameters of the upper third of the hip and in patients with intra- and extra-articular fractures of the femur. Material and Methods: 94 patients aged 50-89 years (median age $70.98 \pm 0.99$ years) were examined, 74 of whom (37 women and $37 \mathrm{men}$ ) were hospitalized with intra- and extraarticular hip fractures. We calculated 10-y risk for hip osteoporotic fractures using FRAX algorithm for Austrian model without BMD determination (before and after hip fracture). Assessment of geometry parameters of the femur was performed on the contralateral limb in relation to fracture.

Results: In patients aged 60-69 and 70-79 years the indices of FRAX-hip before fracture were significantly higher than the limits which are used in NOF guidelines for treatment initiation. In patients with hip fractures indices of FRAX-hip were 7.7 and 12.4 before and after hip fractures accordingly in all groups (1.4 and 3.4 in age group 60-69 years, 6.5 and 11.2 in age group 70-79 years and 17.0 and 24.5 in age group 8089). Although the significant effect of age on femoral geometry parameters in men and women with intra- and extraarticular fractures was established, but this effect was not present in patients without fractures. In men with intra-articular hip fractures the indices of the length of hip axis, length of femoral neck, intertrochanteric distance, basis of the head and head diameter were significant lower in comparison with indices of patients without fractures. In men with extra-articular hip fractures the indices of length of hip axis, intertrochanteric distance, basis of the head and head diameter were also significant lower in comparison with indices of patients without fractures. We did not find the significant differences of hip geometry parameters in women depending on the hip fractures.
Conclusion: Femoral geometry and FRAX indices are independent risk factors for hip fractures in Ukrainian patients. Identified differences should be considered for both planning surgery after hip fracture and for predicting the risk of hip fracture in older age patients.

\section{P945}

\section{EPIDEMIOLOGY OF LOWER LIMB FRACTURES IN UKRAINIAN POPULATION}

N. Grygorieva ${ }^{1}$, R. Vlasenko ${ }^{1}$, V. Povoroznyuk ${ }^{1}$

${ }^{1}$ Department of Clinical Physiology and Pathology of Locomotor Apparatus, D.F. Chebotarev Institute of Gerontology NAMS Ukraine, Kyiv, Ukraine

Lower limb fractures (LLF) account for approximately one third of all fractures and may result in substantial mortality and morbidity. Age, osteoporosis, road collision, obesity and different diseases (osteoarthritis, Parkinsonism, cataract, dementia etc.) are the risk factors of LLF. Fractures are a considerable public health burden but information on their epidemiology in Ukraine is limited.

Material and Methods: We identified 665 subjects from 76765 citizens, living in Vinnitsa region, who had a first time (incident) diagnosis of LLF recorded in the regional Hospital database from 1.01.2011 to 31.12.2011.

Results: Frequency the LLF of was $42.4 \%$ from the total fractures in all patients and $44.4 \%$ from the total fractures in patient aged 50 years and older. The most common anatomic site of LLF was the tibia and/or fibula $(48.9 \%$ of all incident lower limb fractures), followed by the hip (29.5\%), and the tarsal/ metatarsal bones $(21.6 \%)$. Incidence of fracture in patient 50 years old was 519.8 per 10000 patient for all LLF, 212.3 per 10000 patient for tibia and/or fibula fractures and 226.9 per 10000 patient for hip fracture. Lower limb fractures were more common among males than among females in the younger age groups (up to 39 years old). Among subjects 50 years and older the incidence of LLF was higher in women than in men, and the difference increased with increasing age. Incidence of the tibia and/or fibula fractures was 340.7 per 10000 patient in the age group 60-69 years old, 44.9 per 10000 patient in age group 70-79 years old, and 102.4 per 10000 patient in age group 80-89 years old.

Conclusion: Our study provided the new information about the epidemiology of lower limb fractures in Ukrainian population according the age. This information is important for planning of the prevention and treatment strategy in patients of different ages. 


\section{P946}

GENETIC RISK FACTORS OF OSTEOPOROSIS IN UKRAINIAN POSTMENOPAUSAL WOMEN

N. Grygorieva ${ }^{1}$, V. Povoroznyuk ${ }^{1}$, N. Balatska ${ }^{1}$, A. Kaliada ${ }^{1}$

${ }^{1}$ Department of Clinical Physiology and Pathology of

Locomotor Apparatus, D.F. Chebotarev Institute of

Gerontology NAMS Ukraine, Kyiv, Ukraine

Determination of molecular genetic causes of osteoporosis is an actual problem. There are several approaches to assess the contribution of a candidate gene in the pathogenesis of osteoporosis. One of them consists in determining the correlation between allelic polymorphism and candidate factors which cause the disease, which in comparison estimates allele frequencies of candidate genes in osteoporosis patients with individuals not having the disease and preserving normal BMD. The aim of the work was to determine the allele frequency of genes - regulators of bone metabolism in patients with osteoporosis in Ukrainian population, and to assess the contribution of different polymorphisms in the risk of developing the disease.

Material and Methods: DNA extraction was performed using the phenol-chloroform method from whole blood. Using PCR followed by restriction digestion and visualization of the reaction products in polyacrylamide gel have been studied 180 patients with osteoporosis and 160 healthy people of the same age.

Results: We have found association of polymorphism 60890 $\mathrm{A} / \mathrm{G}$ of vitamin D receptor gene $(O R=3.2(95 \%$ CI 2.2-4.6)) and $-234 \mathrm{~T} / \mathrm{G}$ polymorphism of collagen type 1 gene $(O R=2.8$ (95\% CI 2.1-4.1)) with the risk of osteoporosis developing. We have not found association of polymorphism $-764 \mathrm{~T} / \mathrm{G}$ of estrogen receptor gene $(O R=1.2(95 \% \mathrm{CI} 0.6-2.3))$.

Conclusion: Knowing association between pathogenetic alleles, candidate genes and osteoporosis in Ukrainian population will allow use genetic testing to identify predisposition to the disease. The results of this study are important for a more rational organization of the prevention and treatment of the illness in the early stages of disease development.

\section{P947}

\section{EVOLVING ROLE OF OUTPATIENT PHARMACISTS} IN OSTEOPOROSIS CLINICAL OUTCOMES

H. D. Al-Dqour ${ }^{1}$

${ }^{1}$ Hikaya Center for Civil Society Development, Amman, Jordan

Background: Osteoporosis is a clinical problem with social, psychological, and economic burdens. Improving patient disease management and quality of life (QOL) is critical to obtain a satisfactory medical outcome. Pharmacists play a key role in many healthcare systems by helping patients manage chronic diseases, as their role in patient care have expanded over the last three decades, from the traditional tasks of dispensing medications to working with other health professionals and the public to ensure the therapy goal been achieved. The objective is to examine the effect of outpatient pharmacists' roles on patient and health professional outcomes.

Method: A questionnaire was mailed to a sample of outpatient pharmacists, focusing on the impact of pharmacist-provided services in osteoporosis management settings.

Results: The data supports the evolving role of outpatient pharmacists in patient counseling, therapeutic management, and providing health professional education with the goal of improving patients' clinical outcomes. The data suggests that educational outreach visits may impact physician prescribing patterns.

Conclusion: Data suggest the potential role for pharmacists to help reduce gaps in osteoporosis management through improved identification of high-risk patients. More research is needed to examine pharmacists' interventions on osteoporosis treatment adherence.

\section{P948}

ASSOCIATION BETWEEN METABOLIC SYNDROME AND BONE MINERAL DENSITY: THE IMOS STUDY

$\underline{\text { P. Khashayar }}{ }^{1}$, F. Razi ${ }^{2}$, E. Nasli Esfehani ${ }^{3}$, A. A. Keshtkar ${ }^{4}$, B. Larijani ${ }^{5}$

${ }^{1}$ Osteoporosis Research Center, Endocrinology and Metabolism Clinical Sciences Institute, Tehran University of Medical Sciences - Centre for Microsystems Technology, University of Gent, Tehran, Islamic Republic of Iran, ${ }^{2}$ Endocrinology and Metabolism Research Center, Endocrinology and Metabolism Clinical Sciences Institute, Tehran University of Medical Sciences, Tehran, Islamic Republic of Iran, ${ }^{3}$ Diabetes Research Center, Endocrinology and Metabolism Research Institute, Tehran, Islamic Republic of Iran, ${ }^{4}$ Tums, Tehran, Islamic Republic of Iran, ${ }^{5}$ Endocrinology and Metabolism Research Center, Dr Shariati Hospital, Tehran University of Medical Sciences, Tehran, Islamic Republic of Iran

Background: Metabolic syndrome (MS) is a cluster of conditions linked to increased risk of several conditions, particularly cardiovascular diseases, stroke and diabetes. The association between MS and bone health remains unclear as each of the MS components are believed to affect bone differently. This study was conducted to assess the relationship between MS and BMD in a representative Iranian population.

Methods: We conducted a cross-sectional analysis of osteoporosis status and BMD values for subjects with and without the metabolic syndrome who participated in the third Phase of Iranian Multicenteric Osteoporosis Study (IMOS). IMOS is a population-based study conducted in 2012 on the adults from 
two main Iranian cities, Arak and Sanandaj. A questionnaire on demographic information and bone health- related risk factors was filled out for all the patients and they underwent BMD measurement.

Results: Among 1791 adults who underwent BMD measurements, 533(29.8 \%) were diagnosed with MS. There was no significant difference in BMD value of femoral neck in individuals with and without MS. As for lumbar spine, however, women with MS were reported to have significantly lower BMD values $\left(0.39 \pm 0.47\right.$ vs. $\left.0.51 \pm 0.48 \mathrm{~g} / \mathrm{cm}^{2} ; p<0.001\right)$. When stratified by BMI, adjusted BMD values at both studied sites were reported to be lower in women with MS.

Conclusions: In IMOS, the metabolic syndrome is associated with reduced BMD values in women.

\section{P949}

\section{HIP FRACTURE AND CHRONIC ALCOHOLISM}

C. Criveanu ${ }^{1}$, A. E. Musetescu ${ }^{1}$, F. A. Vreju ${ }^{1}$, A. L.

Barbulescu $^{1}$, A. Rosu ${ }^{1}$, P. Ciurea ${ }^{1}$

${ }^{1}$ University of Medicine and Pharmacy, Craiova, Romania

Objectives: To evaluate the incidence of hip fracture in male patients with chronic alcohol consumption and to correlate with BMI and BMD on femoral neck.

Material and method: We assessed 25 male patients with chronic alcohol consumption without history of bisphosphonates, calcium and vitamin $\mathrm{D}$; patients with liver and endocrinology diseases were not included in the study. BMD was evaluated in all patients by using DXA.

Results: The 25 patients' mean age was 52.5 years, ranging from 40-65 years, were active alcohol drinkers, 9 of them (36\%) with an average alcohol consumption of $25 \mathrm{~g}$ alcohol/day, the remaining 16 patients $(64 \%)$ had an average of $40 \mathrm{~g}$ alcohol per day. The mean BMI was $26.35 \mathrm{~kg} / \mathrm{m}^{2}$. In the study group were 6 underweight patients, 12 overweight and 7 obese patients. The 6 underweight patients ( $24 \%$ ) had a daily consumption of $25 \mathrm{~g}$ alcohol with a T-score between $-1,3$ and $-3,2$, the 12 (48 \%) overweigh patients with BMI between $27-29.99 \mathrm{~kg} / \mathrm{m}^{2}$, from which $8(66.6 \%)$ had a daily consumption of less than $40 \mathrm{~g}$ alcohol per day and a T-score between -1 and -2.3 , while the remaining 4 had a daily consumption of more than $40 \mathrm{~g}$ alcohol with a T-score between -1.5 and -2.8 . The 7 (28\%) obese patients that had a BMI between 34 and $35.7 \mathrm{~kg} / \mathrm{m} 2$ and a daily consumption of $40 \mathrm{~g}$ alcohol/day had a T-score between 0.8 and -1.7 . Four patients $(66.6 \%)$ from the 6 underweight, with a daily consumption of $25 \mathrm{~g}$ of ethanol, had femoral neck fracture, while from the others 19 patients, that had a BMI between 27 and $35.7 \mathrm{~kg} / \mathrm{m}^{2}$, only 4 (21.05\%) had femoral neck fracture.

Conclusion: This study showed that underweight patients with chronic alcoholism had a higher risk of hip fracture for having a lower BMD, while overweight and obese patients had a reduce risk due to the protective effect of estrogen.

\section{P950}

IMPORTANCE OF TENS -TRANSCUTANEOUS ELECTRICAL NERVE STIMULATION THERAPY IN ACUTE LOW BACK PAIN

S. Szasz ${ }^{1}$ H. V. Popoviciu ${ }^{1}$, E. G. Papp ${ }^{2}$, C. Popa $^{2}$, E. S. Ianosi $^{2}$, G. Moldovan ${ }^{2}$

${ }^{1}$ Rheumatology Department, University of Medicine and Pharmacy of Tirgu Mures, Tirgu Mures, Romania, ${ }^{2}$ University of Medicine and Pharmacy of Tirgu Mures, Tirgu Mures, Romania

Introduction: Low back pain represents a very common pathology in current practice, it is a multifactorial disease, which affects quality of life and it also have an important role in personal and socio- professional life of patients. Treatment involves postural rest, reduce overloading on lumbar spine and appropriate rehabilitation and medication therapy.

Material and Method: We have included in this study 60 patients enrolled during March-December 2015, which were diagnosed with acute low back pain. Patients were divided in two groups A and B, each including 30 patients. All patients received antalgic and anti-inflammatory treatment. For patients from group B we added a home based TENS therapy with portable devices, applied four times per day for $20 \mathrm{~min}$ for ten days, after training them in our office. Patient evaluation was done at baseline and after 10 days of treatment. We used as outcome measures VAS scale and Schober scale.

Results: On patients from group B who received electrotherapy using portable TENS devices we observed improving on pain level and disability.

Conclusion: TENS represents a very reliable, easy to apply therapy with low cost involvement and few adverse effects and it shows to be efficient in improving acute low back pain as home base therapy.

\section{P951}

INTERACTION OF BONE MINERAL DENSITY AND ADIPOKINES IN ADVANCED CHRONIC OBSTRUCTIVE PULMONARY DISEASE

E. Kochetkova $^{1}$, L. Ugay ${ }^{1}$, Y. Maistrovskaia ${ }^{1}$

${ }^{1}$ Central Scientific Research Laboratory, Pacific State Medical University, Vladivostok, Russian Federation

Introduction: Body composition is an important prognostic factor in COPD patients with osteoporosis. Nutritional depletion is associated with an elevated secretion of adipocytokines which promote systemic inflammation. Moreover, adiponectin, leptin, resistin, chemerin, omentin and visfatin may play an important role in osteoimmunology. However, their association with BMD in COPD is unclear.

Aim: To evaluate body composition and the adipocytokines profile in advanced COPD in relation with bone status. 
Methods: Body and bone composition examination were performed by densitometry in 48 male patients with endstage COPD and 36 healthy males. Serum concentrations of adipokines (adiponectin, leptin, resistin, visfatin, omentin-1 and chemerin) were determined by immunoenzymatic methods. Results: In COPD males we observed significantly reduced fat mass, lean mass, bone mineral content and BMD both lumbar and femur T-score compared with healthy. Increased serum adiponectin, omentin and visfatin correlated negatively with body weight and fat mass index. Omentin and visfatin were related to fat free mass $(r=-0.45, p=0.001$ and $r=-0.48, p=0.006)$ and lean mass $(r=-0.42, p<0.0001$ and $r=-0.46, p=0.001)$. Decreased leptin level was positively correlated with weight $(r=0.46, p=0.0009)$ and fat mass index $(r=0.29, p=0.042)$, while chemerin had direct association with fat mass index $(r=0.30, p=0.038)$ and leg lean mass $(r=0.46, p=0.0009)$. No correlations were found between resistin and bone composition. Leptin and chemerin showed a direct correlation with femur and lumbar T-score while omentin-1 and visfatin had negative relation with both sites. Adiponectin and resistin correlated inversely with femur neck only. Using backward selection multivariable regression, serum levels of decreased leptin and increased visfatin remained significantly associated with reduced BMD in male COPD patients.

Conclusion: Nutritional depletion is related with an alteration in the adipokines profile, resulting in a decrease in BMD in advanced COPD. It provides a novel physiologically concept that may shed light on the etiology of osteoporosis and help to identify new therapeutic targets.

Acknowledgments: This study was supported by grants from Russian Science Foundation (No. 14-33-00009).

\section{P952}

BRAIN-DERIVED NEUROTROPHIC FACTOR (BDNF) IN NORMAL HEALTHY AND HEMODIALYZED POPULATIONS

E. Cavalier ${ }^{1}$, I. Carlisi ${ }^{1}$, S. Kovacs ${ }^{1}$, F. Watar ${ }^{1}$, C. Beaudart ${ }^{2}$, $\overline{\text { F. Buckinx }}^{3}$, O. Bruyère ${ }^{3}$, J.-Y. Reginster ${ }^{4}$

${ }^{1}$ Clinical Chemistry, University of Liège, CHU de Liège, Liège, Belgium, ${ }^{2}$ Department of Public Health, University of Liège, Liège, Belgium, ${ }^{3}$ Department of Public Health, Epidemiology and Health Economics, University of Liège, Liège, Belgium, ${ }^{4}$ Centre Académique de Recherche et d'Expérimentation en Santé SPRL (CARES SPRL), Liège, Belgium

Introduction: BDNF is a member of the neurotrophin family, also produced in the skeletal muscle, that acts as a muscle promoter. It is indeed involved in development and differentiation of myoblasts and muscle fibers as well as in the regulation of motoneuron survival [1]. Exercise has an important influence on BDNF levels and the well-known anti- inflammatory effects of exercise may be linked to the activation of the BDNF/TrkBK pathway. As it plays a role in fat oxidation, in immunity and in inflammation regulation but also maintains the integrity of the trophic interactions between motor neurons and muscle fibers, BDNF could play a role in sarcopenia. In this study, we aimed to establish the reference range in a normal healthy population and compared the values obtained with a population of hemodialyzed patients.

Material and methods: We used the BDNF Quantikine ELISA Kit from R\&D. The Company claims a CV $<10 \%$ and a recovery not different from $100 \%$. The range of values observed in an apparently healthy population ( $n=33$ ) was $6.1-42.6 \mathrm{ng} / \mathrm{mL}$, but no reference range was provided. We established the reference range in a healthy population of 62 young individuals $(27$ males, 35 females, $29.0 \pm 5.6$ yo) and compared them to the levels observed in a population of 10 hemodialyzed (HD) patients.

Results: The mean BDNF level observed in the healthy population was $25.2 \pm 7.3 \mathrm{ng} / \mathrm{mL}$. There was no gender difference. Hence, the reference range calculated in the healthy population was $10.0-39.3 \mathrm{ng} / \mathrm{mL}$. The mean level observed in the HD patients was significantly $(p=0.0007)$ lower and was calculated at $17.4 \pm 4.1 \mathrm{ng} / \mathrm{mL}$.

Conclusions: In this study, we established the reference range of BDNF, a promising marker of sarcopenia and compared the results obtained in a young and healthy population with a HD one. The values observed in our healthy population are compatible with the range proposed by the manufacturer on a smaller subset of individuals. Our results show that BDNF was decreased in HD patients. Zoladz et al. have shown that a single HD session decreased BDNF levels [2]. Since this decrease was accompanied by elevated isoprostanes and decreased plasma total antioxidant capacity the authors suggested that the cause could be the enhanced oxidative stress induced by hemodialysis. Our findings confirm that chronic HD patients, that are a model of oxidative stress, present lower levels of BDNF. If the sensibility of BDNF to oxidative stress is confirmed, this will increase the potential interest of BDNF in sarcopenia.

1. Kalinkovich A, Livshits G. Ageing Res Rev 2015;22:58.

2. Zoladz JA, et al. Neurochem Res 2012;37:2715.

\section{P953}

EFFICACY AND SAFETY OF HIGH-DOSE ORAL VITAMIN D SUPPLEMENTATION: A PILOT STUDY

A. A. Petrushkina $^{1}$, E. A. Pigarova ${ }^{1}$, T. S. Tarasova ${ }^{1}$, L. Y. Rozhinskaya $^{1}$

${ }^{1}$ Neuroendocrinology and Bone Diseases, Endocrinology

Research Centre, Moscow, Russian Federation

Objectives: Vitamin D deficiency is a widespread pathologic condition with skeletal and nonskeletal consequences. Low dose daily vitamin $\mathrm{D}$ supplementation often fails to improve vitamin D status due to poor compliance or deep deficit takes 
months to achieve sufficiency. Treatment with high doses is a compelling alternative, but negative outcomes such as hypercalcemia should be avoided.

Material and Methods: 24 apparently healthy volunteers were included in the study: Group $1(11 / 24)$ with blood serum levels of $25(\mathrm{OH}) \mathrm{D}<10 \mathrm{ng} / \mathrm{mL}$ and Group $2(13 / 24)$ with $25(\mathrm{OH}) \mathrm{D}$ levels $10-20 \mathrm{ng} / \mathrm{mL}$. All patients received orally single dose of 200000 IU of cholecalciferol oil solution. Assessments were made at baseline, $3 \mathrm{~h} ; 3,7$ and 28 days after intake: 25(OH)D total (Diasorin, Liason), PTH (ELECSYS, Roche), $\mathrm{Ca}_{\text {tot }}, \mathrm{Ca}_{\text {ion }}, \mathrm{P}$, creatinine blood serum levels and $\mathrm{Ca}$, creatinine in first void urine (Abbott Architect, Abbott.)

Results: By 28th day 10 (41.6\%) and 21 (87.5\%) from 24 participants achieved $25(\mathrm{OH}) \mathrm{D}$ blood serum level $>30 \mathrm{ng} / \mathrm{mL}$ and $>20 \mathrm{ng} / \mathrm{mL}$, respectively. On the $3 \mathrm{~d}$ day we observed an increase of $25(\mathrm{OH}) \mathrm{D}>20 \mathrm{ng} / \mathrm{mL}$ in $72.7 \%$ in Group 1, on the 7 th day - increase higher than $30 \mathrm{ng} / \mathrm{mL}$ was seen in $36.4 \%$ in Group 1 and $76.9 \%$ in Group 2. Mean level of PTH decreased significantly by 28 th day in both groups, $\mathrm{Ca}_{\text {tot }}, \mathrm{Ca}_{\text {ion }}, \mathrm{P}$ blood serum levels andurine $\mathrm{Ca}$-creatinine ratio were similar to the baseline. There were no cases of hypercalcuria and nephrolithiasis, but we observed one case of hypercalcemia (max $\mathrm{Ca}_{\mathrm{tot}}=2.65 \mathrm{mmol} / \mathrm{L}$ ) considered as transient.

Conclusion: High dose cholecalciferol therapy for vitamin D deficiency appears to be safe and effective in young patients but further studies are needed to assess the safety at long term and in other patient populations.

\section{P954}

TWO ATYPICAL FRACTURES ON THE SAME FEMUR IN PATIENT TREATED LONG-TERM IBANDRONATE? CASE REPORT

\author{
L. Franekova $^{1}$ \\ ${ }^{1}$ Internal Clinic - Central Military Hospital - The Military \\ University Hospital Prague, Prague, Czech Republic
}

Introduction: Long-term continuous use of bisphosphonate in the therapy of osteoporosis is associated with a low risk of the occurrence of an atypical subtrochanteric and diaphyseal femoral fracture. These fractures may occur bilaterally in one patient. The case report is presented here of a patient in whom we retroactively assessed the suffered right side diaphyseal femoral fracture as an atypical femur fracture complicating 8-y usage of ibandronate. 4 months later, a subtrochanteric fracture of the same femur occurred.

Case: 62-y-old female patient with postmenopausal osteoporosis treated for 10 years with ibandronate was sent for osteologic care in our hospital. The patient had already suffered several fractures with minimal trauma - of the left olecranon, right ankle, right femoral shaft and subsequently subtrochanteric right femur fracture. Old compression fractures 12th thoracic and first lumbar vertebrae and healed fractures of both rami of the right os pubis of uncertain age were discovered by X-ray examination. For localisation of the right femur fractures that is unusual for osteoporosis, the $\mathrm{X}$ ray findings were retroactively reviewed. The diaphyseal femoral fracture fulfilled the definition of an atypical femoral fracture, which occurred after 8 years of using ibandronate. The fracture was treated operatively with intramedullary nail. The patient continued using ibandronate. After 4 months, the patient fell at home and suffered a right subtrochanteric fracture; at the point of the femoral fracture, the osteosynthesis also broke; at the time, the right diaphyseal fracture was already healed.

Conclusion: To assess the atypical femoral fracture as a side effect of bisphosphonate therapy, we use the American Society for Bone and Mineral Research (ASBMR) Case Definition. In the case of the first diaphyseal femoral fracture in the female patient, these criteria were fulfilled. During continued therapy using ibandronate, a low energy fracture of the same femur occurred after 4 months. It is not clear as to how the subtrochanteric femur fracture should be assessed, if the osteosynthesis fails at this point. The exclusionary criteria for atypical femur fracture according ASBMR is a periprosthetic fracture, which was not the case here.

\section{P955 \\ CONCOMITANT BILATERAL HIP TRANSIENT OSTEOPOROSIS IN PREGNANCY: A CASE PRESENTATION}

A. Bitzidis $^{1}$

${ }^{1}$ Orthopaedic Department, Saint Loukas Private Hospital, ThessalonikiI, Greece

Hip transient osteoporosis developed during pregnancy is an uncommon self- limited musculoskeletal disorder with an unknown aetiology. We would like to present a rare case of concomitant transient osteoporosis in bilateral hips in a Caucasian 38 year old female, secundipara in her 32nd week of gestation. She was referred to the Orthopaedic team by her treating Obs-Gyn team of doctors complaining of progressive bilateral groin and proximal femoral pain for the last 2 weeks. On presentation she was unable to bear weight on both lower limbs even with the use of 2 crutches, moving around with a wheel chair. She described her hip pain as continuous, severe (9/10 VAS) even at rest with progressive loss of hip active and passive ROM. From her past medical history she was diagnosed with sympysis pubic dysfunction syndrome in her first pregnancy 3 years before. No other pathologies were reported. On examination she had very limited active and passive ROM in both hips and bilateral knee effusions with 0-100 active ROM. Rest of physical examination was normal in the context of a pregnancy in the 3rd trimester. A MRI of bilateral hip and proximal femur showed gross osteopenia in both femoral 
heads extended to the peritrochanteric area. These images were consistent with concomitant transient osteoporosis in bilateral hips. In the next 4 weeks the pain became worse associated with very limited mobility and an elective birth was organized in her 36 gestational week. After the birth there was a gradual remission of the symptoms. The pain management included only calcium and vit D supplementation as she refused to take any antiresorptive or anabolic antiosteoporotic agent due to her decision of child breast feeding. In 12 weeks after birth, she was able to fully weight bear with a mild left hip pain (3/10 VAS). We present this case as it is unique in 2 different ways. Firstly, it is the first reported case of hip transient osteoporosis during pregnancy presented in the same time and not as a unilateral complicated at a later stage. Secondly, the antiosteoporotic management was successful only with calcium and vitamin D supplementation, proving a point about hip transient osteoporosis' self-limited characteristic.

\section{P956}

EFFECTS OF KINETIC TREATMENT APPLIED TO THE PATIENTS WITH ACUTE MYOCARDIAL INFARCTION IN PHASE I

$\underline{\text { F. Cioara }}^{1}$, M. Rus ${ }^{1}$, L. Lazar ${ }^{1}$, C. Nistor Cseppento ${ }^{1}$, A.

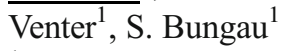

${ }^{1}$ University of Oradea, Faculty of Medicine and Pharmacy, Oradea, Romania

Objective: To investigate the quality of life of patients with acute myocardial infarction (AMI) in phase I (hospital) after a physical therapy program.

Material and Methods: We studied a number of 15 patients (8 women and $7 \mathrm{men}$ ) with acute myocardial infarction, hospitalized on the cardiology ward of Emergency County Hospital Oradea, in the period from January to September 2015. As the evaluation methods we used: questionnaire of quality of life, stress testing using treadmill (Bruce protocol), Borg scale.

Results: $73.33 \%$ of patients are sedentary, $80 \%$ had hypertension, dyslipidemia was present in $33.33 \%$ of patients, $53.33 \%$ are smokers, $26.66 \%$ are obese, $53.33 \%$ of patients had accused social and professional stress. In terms of assessing quality of life, patients have had in the first 24$48 \mathrm{~h}$ postinfarct acute myocardial a severe impaired quality of life (around 56), and acute myocardial infarction 14 days before discharge moderate impairment of quality of life (around 11). Patients had a tolerance to effort between 0:46 (the weakest), 7: 59 (the best) at the end of phase I recovery (the hospital), tolerance to effort, evidenced by a submaximal test effort carried out with $24-48 \mathrm{~h}$ prior to discharge. After applying the Borg scale during the testing effort with the treadmill (Bruce protocol), it was observed that five patients have reached Level 1 (barely noticeable pain), 4 patients have achieved Level 2 (persistent pain), 5 patients have achieved Level 3 (intense pain that stopped the effort) and 1 patient has achieved level 4 (intolerable pain).

Conclusions: In this study, all patients were admitted to hospital in the Department of Cardiology during phase I of recovery (14 days) after acute myocardial infarction, this contributing significantly to chest pain relief through medication and cessation of the action coronarien risk factors. The decrease in the incidence and recurrence of this type of pathology, it is very important that the self education of the patient by adopting a healthy lifestyle, active, primarily by eliminating the risk factors that lead to coronary accident (sedentary, obesity, smoking, social and professional stress) both at home (family support, avoid bickering, avoid negative emotions or intensely positive) and at work (adjusting working hours, the rate, the intensity of the effort or if necessary change the workplace according to functional capacity).

P957

SSOCIATION OF CARDIOMETABOLIC MARKERS AND THE METABOLIC SYNDROME WITH BONE MINERAL DENSITY IN PERIMENOPAUSAL WOMEN: THE FLAMENCO PROJECT

$\underline{\text { P. Acosta-Manzano }}^{1}$, A. R. Peces-Rama ${ }^{1}$, M. Borges-Cosic ${ }^{1}$, P. Ruiz-Cabello ${ }^{2}$, M. Puerto del $\mathrm{Amo}^{3}$, E. Rojas ${ }^{4}$, I. CollRisco $^{5}$, V. A. Aparicio 5

${ }^{1}$ Department of Physical Education and Sports, Faculty of Sport Sciences, University of Granada, Granada, Spain, ${ }^{2}$ Department of Physiology, Faculty of Pharmacy, Faculty of Sport Sciences, and Institute of Nutrition and Food Technology, University of Granada, Granada, Spain, ${ }^{3}$ Department of Applied Economics, Faculty of Economics, University of Granada, Granada, Spain, ${ }^{4}$ Zaidín Sur Healthcare Centre and Department of Medicine, Faculty of Medicine, University of Granada, Granada, Spain, ${ }^{5}$ Department of Physiology, Faculty of Pharmacy, Faculty of Sport Sciences, and Institute of Nutrition and Food Technology, University of Granada and Department of Physical Education and Sports, Faculty of Sport Sciences, University of Granada, Granada, Spain

Objectives: To examine the association of cardiometabolic markers and the metabolic syndrome with BMD in perimenopausal women.

Material and Methods: This cross-sectional study comprised 197 women (age range 45-60 years) from Granada (Southeast Spain). Waist circumference was assessed at the middle point between the ribs and the iliac crest, with the participant standing. A blood pressure monitor was used to assess systolic and diastolic blood pressure and resting heart rate when women were 
seated in a proper relaxed state at rest. Venous blood samples after all night fasting were collected. Plasma alkaline phosphatase, C-reactive protein, HDL-cholesterol, triglycerides and glucose concentrations were estimated using an autoanalyzer.

Results: Systolic blood pressure was independently associated with BMD in perimenopausal women after adjusting for age $(p<0.05)$, whereas the rest of cardiometabolic markers $\operatorname{did}$ not $(p>0.05)$. None of the cardiometabolic markers under study (vascular, lipid, glycemic and inflammatory markers) was independently associated with BMD after controlling for age, educational status, marital status, smoking status, regular menstruation, hormonal therapy and BMI (all $p>0.05$ for the multivariate model). Perimenopausal women with metabolic syndrome did not show higher BMD than those without metabolic syndrome $(p>0.05)$.

Conclusions: Neither the cardiometabolic markers nor the presence of metabolic syndrome was associated with BMD in this population.

\section{P958}

PECULIARITIES OF DISEASE ACTIVITY IN PATIENTS WITH RHEUMATOID ARTHRITIS AND VITAMIN D DEFICIENCY

V. Povoroznyuk $^{1}$, N. Balatska ${ }^{1}$, O. Synenky ${ }^{2}$

${ }^{1}$ Department of Clinical Physiology and Pathology of Locomotor Apparatus, D.F. Chebotarev Institute of Gerontology NAMS Ukraine, Kyiv, Ukraine, ${ }^{2}$ Lviv Regional Clinical Hospital, Lviv, Ukraine

Introduction: Rheumatoid arthritis is an autoimmune disease that results in chronic inflammation predominantly of the flexible joints. Vitamin D deficiency can have association with the pathogenesis of the rheumatoid arthritis. The aim of the study was to evaluate the association between 25(OH)D level and disease activity in patients with rheumatoid arthritis.

Materials and methods: The study included 61 patients with rheumatoid arthritis, $73.8 \%$ were women, 70.4 patients were younger 60 years old. Subjects suffering from liver and kidney insufficiency and those who had received vitamin D in the previous 3 months have been excluded. Disease activity was assessed by DAS-28 score, joint pain degree, morning stiffness time and laboratory measures including $\mathrm{Hb}$ and ESR. The level of $25(\mathrm{OH}) \mathrm{D}_{\text {total }}$ was evaluated by electrochemiluminescence method (Elecsys 2010, Roche). Vitamin D deficiency was defined as a $25(\mathrm{OH}) \mathrm{D}$ below $20 \mathrm{ng} / \mathrm{ml}$, and vitamin D insufficiency as $25(\mathrm{OH}) \mathrm{D}$ of $21-$ $29 \mathrm{ng} / \mathrm{ml}$.

Results: In patients with rheumatoid arthritis the frequency of vitamin D insufficiency and deficiency was 32.8 and $55.7 \%$ accordingly. $14.7 \%$ subjects with rheumatoid arthritis had severe vitamin D deficiency. $25(\mathrm{OH}) \mathrm{D}$ was associated with morning stiffness $(r=-0.35 ; p=0.04)$, ESR level $(r=-0.36$; $p=0.05), \mathrm{DAS}-28(r=-0.24 ; p=0.05), \mathrm{Hb}(r=0.27$; $p=0.01)$.

Summary: Vitamin D deficiency can be an important factor in worsening of disease activity in patients with rheumatoid arthritis.

\section{P959}

VITAMIN D VARIATIONS IN SUMMER/WINTER: PRELIMINARY RESULTS OF A PILOT STUDY IN PORTUGUESE YOUNG ADULTS

A. P. Barbosa ${ }^{1}$, C. Godinho ${ }^{2}$, D. Madureira ${ }^{3}$, I. Rodrigues ${ }^{2}$, E. S. Leite $^{4}$, O. Shapovalova ${ }^{4}$, L. Galaio ${ }^{4}$, M. R. Mascarenhas ${ }^{1}$

${ }^{1}$ Endocrinology, Diabetes and Metabolism Department, Santa Maria Hospital - CHLN, EPE and Endocrinology and Metabolic Diseases, Faculty of Medicine of Lisbon University, Osteoporosis and Metabolic Bone Diseases Task force, Portuguese Society of Endocrinology, Diabetes and Metabolism, Lisboa, Portugal, ${ }^{2}$ Clinical Pathology Department, Curry Cabral Hospital - CHLC, EPE Endocrinology Laboratory Task force, Portuguese Society of Endocrinology, Diabetes and Metabolism, Lisboa, Portugal, ${ }^{3}$ Clinical Pathology Department, Portuguese Oncology Institute Endocrinology Laboratory Task force, Portuguese Society of Endocrinology, Diabetes and Metabolism, Lisboa, Portugal, ${ }^{4}$ Occupational Health Department- CHLN, EPE, Lisboa, Portugal

Several studies done in different countries showed inadequate levels (deficient and insufficient) of vitamin D. Besides the classical skeletal consequences, such a "pandemia" has also been associated to an increased risk of severe diseases namely cardiovascular and cancer. The diagnosis is made by the measurements of total plasma 25-hydroxyvitamin D [25(OH)D], which measures the concentrations of both vitamin D2 and D3. In Portugal the studies about vitamin D levels in different aged populations are scarce.

Objectives: To study the variation type, in summer and winter, of the vitamin D levels in Portuguese young adults.

Material and Methods: In 267 healthy women and men, aged 18-35 years, fasting blood was taken to measure $25(\mathrm{OH}) \mathrm{D}$, iPTH and several biochemical and hormonal parameters, in summer 2014 and in the winter after. The hormones were analyzed by Liaison technology.

Adequate statistical tests were used to describe the summer / winter groups and their differences. Statistical significance was considered for $P<0.05$.

Results: The mean $( \pm \mathrm{SD}) 25(\mathrm{OH}) \mathrm{D}$ and iPTH blood concentrations of the groups are in Table 1. 
Table 1. Means $( \pm \mathrm{SD})$ of the blood concentrations of 25(OH)D and PTHi

\begin{tabular}{lll}
\hline Groups & $25(\mathrm{OH}) \mathrm{D} n g / m l$ & iPTH $p g / m l$ \\
\hline Summer & $27.0( \pm 9.3)$ & $12.3( \pm 4.7)$ \\
Winter & $15.4( \pm 5.2)$ & $13.0( \pm 5.2)$ \\
$P$ & 0.001 & 0.3 \\
\hline
\end{tabular}

Significant relations between the blood collections during the summer vs. those collected during winter, for both $25(\mathrm{OH}) \mathrm{D}$ $(r=0.683 ; P=0.001)$ and iPTH $(r=0.467 ; P=0.001)$.

Conclusions: In this group of young healthy adults we found significant variations summer/winter of both $25(\mathrm{OH}) \mathrm{D}$ and iPTH plasma levels, as already described for populations of other countries. Moreover, the means of $25(\mathrm{OH}) \mathrm{D}$ are relatively low, suggesting that there are already levels of deficiency/ insufficiency of vitamin D in many young adults; the clinical significance of such a "pandemic" needs urgent clarification.

\section{P960}

EFFICACY OF INTRAARTICULAR INFILTRATION OF HYALURONIC ACID IN PATIENTS WITH SECONDARY OSTEOARTHRITIS INDUCED BY RHEUMATOID ARTHRITIS AND INFLUENCE OF THIS TREATMENT ON QUALITY OF LIFE OF PATIENTS

O. Bujor $^{1}$, L. Groppa ${ }^{1}$, S. Aghachi ${ }^{1}$, R. Usatii ${ }^{1}$

${ }^{1}$ Republican Clinical Hospital, Chisinau, Republic of Moldova

Introduction: Rheumatoid arthritis is a systemic inflammatory condition, its main manifestation is symmetric, persistent arthritis of synovial joints. Because of its chronicity rheumatoid arthritis can induce secondary osteoarthritis, a pathology that consist in degradation of joint cartilage, alteration of joint function, with installation of great disability, and decrease of quality of live. We propose to realize a study in this domain. Objectives: Appreciation of efficacy of intraarticular infiltration of hyaluronic acid in patients with secondary osteoarthritis induced by rheumatoid arthritis and influence of this treatment on quality of life of patients.

Methods: In the study 50 patients were included with secondary osteoarthritis induced by rheumatoid arthritis, radiologic stage II. Inclusion criteria:

- Age of patients between 40-60 y

- Patient with rheumatoid arthritis with moderate disease activity (DAS 28 3.2-5.1), with secondary osteoarthritis radiologic stage II

- Disease duration until $10 \mathrm{y}$

- Treatment duration with DMARDs until 5 y

- Patient agreement in collaboration, 1 year Patients were divided into II groups.
I group includes 25 patient whom was administrated 2 intraarticular infiltration with hyaluronic acid $60 \mathrm{mg}-3 \mathrm{ml}, 1$ infiltration per month during 2 months, II group without intraarticular infiltration of hyaluronic acid. Patients from both groups had administrated methotrexate and nonsterioidal antiinflamatory drugs (NSAIDS).

During the study on patients were performed clinical examinations, laboratory tests and radiological examinations. The intensity of pain was appreciated with VAS and quality of live with HAQ questionnaire.

Results: The median pain on rest before treatment in the 1st group of patient was $4.9 \pm 0.92$, in the 2nd group $5.0 \pm 0.65$. The pain during movement in the1 st group of patient was $6.04 \pm 0.71$, in the II nd group $6.05 \pm 0.78$. For the 1st group of patient intraarticular infiltration with hyaluronic acid decrease pain with 2.5 $(p<0.001)$ points on rest, and on movement with $2.3(p<0.001)$ points, in the second group the pain was improved with 0.9 $(p<0.001)$ points, in movement with $0.5(p<0.001)$. The residual pain reported by patient after treatment was in average $28.33 \% \pm 9.69$ in the 1 st group, and $85.32 \% \pm 3.97$ for the 2 nd group. Among 25 patients from 1st group total HAQ score between 0 and 1 point had 21 patients, between 1 and 2 had 3 patients and between 2 and 3 had 1 patient. In the 2nd group HAQ score between 0 and 1 point had 5 patients, between 1 and 2 had 5 patients, and between 2 and 3 had 15 patients.

Conclusions: The patients from 1st group, whom was administrated intraarticular infiltration with hyaluronic acid in combination with methotrexate and NSAIDS, reported the smallest residual pain, opposite the patient from 2 nd group treated with methotrexate and NSAIDS, had worse results. According to our study, the most affected quality of live was reported in the 2nd group of patients treated with methotrexate and NSAIDS, better quality of live had the patients from 1st group treated with intraarticular infiltration with hyaluronic acid in combination with methotrexate and NSAIDS. That permitted us to conclude that intraarticular infiltration with hyaluronic acid is a good treatment option for patients with secondary osteoarthritis.

\section{References:}

1. Altman RD, et al. Arthritis Rheum 2000; 43:1905e15

2. Osteoarthritis. National clinical guideline for care and management in adults. The National Collaborating Centre for Chronic Conditions for NHS, NICE 2008, London.

3. Carli P, et al. Rev Med Interne 2009;30:1067

\section{P961}

METHODS OF PREVENTION IN OSTEOPOROSIS

${ }_{\text {R. Suciu }}{ }^{1}$, F. Cioara ${ }^{1}$, E. Szarka ${ }^{1}$, M. L. Cevei ${ }^{1}$, M. Tit ${ }^{1}$

${ }^{1}$ University of Oradea, Faculty of Medicine and Pharmacy, Oradea, Romania

Objectives: revention is the most effective and inexpensive in the management of osteoporosis. Epidemiologic evidence suggests that lifestyle factors, such as exercise, calcium intake, 
and tobacco consumption, have effects on bone density. This study tracked the frequency of the causes that gave rise to the appearance of osteoporosis in patients admitted for rehabilitation treatment after accidents with fractures.

Materials and methods: Have been assessed a number of 30 patients with diagnosis of fracture from Rehabilitation Hospital Felix, who showed osteoporosis in DXA examination. Patients were investigated during hospitalization: anamnesis, medical examination and questionnaire relating to lifestyle and history of the patients.

Results: As a result of statistical processing of survey data taken from related investigations carried out, has been highlighted: $60 \%$ of patients had a grade of overweight and were smokers, $33.33 \%$ had high blood pressure, $6.66 \%$ were diabetics, $6.66 \%$ hyperthyroidism, $6.66 \%$ had in history a form of neoplasm with chemotherapy treatment. Answers relating to measures for the reduction and prevention of osteoporosis have been applied by the patients surveyed were: $40 \%$ healthy diet avoiding hyperprotein diets and avoiding rich in phosphates and vitamin $\mathrm{D}$ and calcium intake and rigorously monitoring the glycemic levels, $40 \%$ change in lifestyle (giving up smoking, excess alcohol, coffee), $30 \%$ adoption active lifestyle.

Conclusion: Osteoporosis is influenced by the structure of each individual, but also by poor diet or lack of physical activity. Women, especially young ones, should be aware of in advance of the risk of osteoporosis and to act to slow the evolution of the disease and possible complications that may occur with the progression of osteoporosis. These data indicate that lifestyle factors, such as tobacco consumption, calcium intake, and physical activity, may influence bone density in the elderly. Modification of these lifestyle factors may influence osteoporotic fracture risk in elderly men and women.

\section{P962}

NUCLEAR EXPRESSION OF VITAMIN D RECEPTOR AS A MOLECULAR LINK OF OSTEOPOROSIS-SARCOPENIA CONNECTION

E. Piccirilli $^{1}$, M. Scimeca ${ }^{2}$, L. Romano ${ }^{1}$, E. Bonanno ${ }^{2}$, F. M. Cannari $^{1}$, E. Gasbarra ${ }^{1}$, U. Tarantino ${ }^{3}$

${ }^{1}$ Department of Orthopedics and Traumatology, Rome, Italy,

${ }^{2}$ Anatomic Pathology Section, Department of Biomedicine and Prevention, University of Rome, Rome, Italy, ${ }^{3}$ Department of Orthopedics and Traumatology, "Tor Vergata” University of Rome, Rome, Italy

Introduction: Bone and muscle are tissue in close relationship and emerging evidence suggests that Vitamin D (Vit.D) may play a direct role in both muscle and bone homeostasis. Nevertheless, precise molecular mechanisms by which vitamin D affects the homeostasis of muscle and bone tissues are still unclear. The main aim of this study was investigated the role of Vit.D and their receptor (VdR) in regulation of muscle homeostasis of osteoporotic and osteoarthritis patients.

Methods: For this study, we analyzed 60 muscle biopsies of vastus lateralis in total: 30 biopsies of osteoporotic patients and 30 biopsies of osteoarthritic women, as control (OA/ CTRL). Clinical report included anamnestic data, serum concentration of vitamin D and PTH and DXA. Muscle atrophy, VDR, BMP-2 and myostatin expression were evaluated by immunohistochemical reaction.

Results: The morphometric analysis of muscle fibers in OP patients showed more than $48.00 \%$ of atrophic fibers with prevalence of type II fibers. As concern VdR, we note a significantly different expression of activated VdR (nuclear) in OA/CTRL muscle tissues respect to OP group. Conversely, no different was observed for cytoplasmic expressions of VdR (OP: 213.94 127.51 ; OA: $193.90 \pm 107.08)$. Surprisingly, we note that in muscle tissue of OP patients the absence of nuclear VdR was associated with atrophy of type II fibers and absence of BMP-2 expression. Unlike, the number of myostatinpositive fibers in OP patients $(42.85 \pm 59.52)$ was significantly higher compared to OA group (17.21 \pm 17.66$)$.

Discussion: Our results demonstrated that activation of $\mathrm{VdR}$, and their translocation into nucleus, is related to onset of sarcopenia. Indeed, we note low level of nuclear VdR in muscle samples characterized by high percent of atrophic fibers. Moreover, the evidence of relationship between type II fibers atrophy and impairment of VdR activation in OP patients, suggest a molecular correlation between Vit.D signaling and muscle regeneration. In conclusion, the identification of early and late mechanisms by which Vit.D affects muscle homeostasis could provide a rationale for the establishment of a clinical correlation between Vit.D levels and muscle health. In future, we will expect to identify patient's molecular profiles able to predict the clinical response to Vit.D supplementation.

\section{P963}

ASSESSMENT OF RISK FACTORS FOR OSTEOPOROSIS IN YOUNG ADULTS BY USING A DIGITAL INTERFACE

M. Copotoiu ${ }^{1}$, M. Hulub ${ }^{2}$, H. V. Popoviciu ${ }^{3}$, J. Cristina ${ }^{2}$, A. Palaghie $^{2}$, C. Bogatan ${ }^{2}$, M. Susca ${ }^{1}$, L. Bordean ${ }^{1}$

${ }^{1}$ University of Medicine and Pharmacy of Targu Mures, Department of Rheumatology, Targu Mures, Romania, ${ }^{2}$ Emergency County Hospital of Targu Mures, Targu Mures, Romania, ${ }^{3}$ Rheumatology Department, University of Medicine and Pharmacy of Tirgu Mures, Tirgu Mures, Romania

Objective: The main aim of our pilot study was to identify the association of risk factors for osteoporosis (OP) 
in a young population by using a dedicated digital platform.

Material and method: We created a website and uploaded two gender discriminated questionnaires (15 items for women and 14 items for men) in order to start the assessment of risk factors for osteoporosis in young adults. The items were focused on known data concerning risk for osteoporosis. The following variables were registered: family history, fractures, secondary microtrauma fractures, steroids intake, coffee use, smoking, alcohol intake, diet (e.g., vegan), milk (vegetal or not), use of birth control pills, low levels of testosterone, menstrual irregularities, physical exercise routine, sun exposure and the BMI. We used GraphPad Prism 6.0 for statistic correlations.

Results: 119 subjects ( 87 women and 32 men) with a mean age of $29.10 \pm 6.84$ years old, logged in during a two-week period. The majority of the subjects ( $82 \%$, 98 out of 119 individuals) were coffee consumers. A positive correlation was observed between the smokers and the coffee consumption (p: 0.0172, r: 0.2181). Lack of sun exposure was associated with coffee drinkers (p: 0.0387, r: -0.1898 ). Sedentarism was recorded in $74 \%$ of the subjects ( 88 out of 119). A strong correlation was noted between physical exercise and diet enhanced with nutritional supplements (p: 0.005, r: 0.3126). The environment might play a role in the onset of osteoporosis. A link between the registered cases of osteoporosis in the family history of subjects living in less sun exposure zones was obtained ( $\mathrm{p}: 0.0431, \mathrm{r}:-0.1858$ ).

Conclusion: Chronic coffee consumption, sedentarism and lack of sun exposure are the main evidenced risk variables for the development of osteoporosis in our young adult subjects.

\section{References:}

1. Hernlund E, et al. Arch Osteoporos 2013; 8:136

2. Svedbom A, et al. Arch Osteoporos 2013;8:137

3. Ferrari S, et al., Osteoporos Int 2012;23:2735

\section{P964 \\ MICROARCHITECTURE OF BONE TISSUE AND BLOOD VITAMIN D LEVELS IN MEN}

M. R. Mascarenhas ${ }^{1}$, A. P. Barbosa ${ }^{1}$, N. Duarte $^{2}$, A.

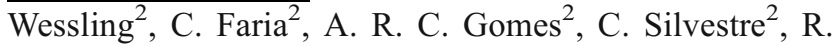
Paixão $^{2}$, D. O. Barbosa ${ }^{2}$, J. Poupino ${ }^{2}$, A. S. Osório ${ }^{2}$, V. Gomes $^{2}$, F. Sampaio ${ }^{2}$, J. Monteiro ${ }^{2}$, D. H. Hans ${ }^{3}$, M. Bicho ${ }^{4}$ ${ }^{1}$ Endocrinology, Diabetes and Metabolism Department, Santa Maria University Hospital - CHLN, EPE and Endocrinology and Metabolic Diseases, Faculty of Medicine of Lisbon University; Osteoporosis and Metabolic Bone Diseases Task force, Portuguese Society of Endocrinology, Diabetes and Metabolism,
Lisboa, Portugal, ${ }^{2}$ Fracturary Osteoporosis Outpatient Clinic - Endocrinology, Diabetes and Metabolism Department, Santa University Maria Hospital-CHLN, EPE, Lisboa, Portugal, ${ }^{3}$ Center of Bone Diseases, Lausanne University Hospital Switzerland, Lausanne, Switzerland, ${ }^{4}$ Endocrinology and Metabolic Diseases, Faculty Medicine of Lisbon, Lisboa, Portugal

Blood vitamin D concentrations and BMD decline with ageing. Inadequate vitamin D levels may be associated to an increased falls number and osteoporotic fractures among elderly people. DXA and clinical fracture risk factors do not provide total info on bone strength. The TBS (trabecular bone score) can estimate the bone microarchitectural texture or bone quality; together, TBS and DXA may evidence the bone strength. Nevertheless, the influence of vitamin D on the microarchitecture of bone tissue by TBS is almost unknown.

Objectives: To evaluate the influence of the blood vitamin D levels on the BMD and TBS at the L1-L4 in normal men.

Material and Methods: The BMD $\left(\mathrm{g} / \mathrm{cm}^{2}\right)$, the BMC $(\mathrm{g})$ and TBS (derived from each DXA scan) at the lumbar spine, the blood 25(OH)D (ng/ml), the iPTH and the osteocalcin concentrations were evaluated in a group of 56 normal men [mean age $55.7( \pm 12.7) \mathrm{y}$ ]. These otherwise normal men were divided in the vitamin D sufficiency, insufficiency and deficiency groups (Endocrine Society Guidelines). BMI was also calculated $\left(\mathrm{kg} / \mathrm{m}^{2}\right)$. Adequate statistical tests were used (statistical significance $P<0.05$ ).

Results: The mean $( \pm \mathrm{SD}) \mathrm{BMD}, \mathrm{BMC}$ and TBS are shown in Table 1.

Table 1. The mean $( \pm \mathrm{SD}) \mathrm{BMC}, \mathrm{BMD}$ and TBS of the three groups.

\begin{tabular}{lllll}
\hline $\begin{array}{l}\text { Groups } \\
\mathrm{L}_{1}-\mathrm{L}_{4}\end{array}$ & $\begin{array}{l}\text { Sufficiency } \\
n=8(14.3 \%)\end{array}$ & $\begin{array}{l}\text { Insufficiency } \\
n=17(30.4 \%)\end{array}$ & $\begin{array}{l}\text { Deficiency } \\
n=31(55.3 \%)\end{array}$ & $\mathrm{P}$ \\
\hline $\begin{array}{c}\text { BMD } g / \\
\mathrm{cm}^{2}\end{array}$ & $0.990( \pm 0.1)$ & $1.059( \pm 0.1)$ & $1.040( \pm 0.1)$ & $N S$ \\
$\mathrm{BMC} g$ & $69.6( \pm 16.5)$ & $70.3( \pm 14.2)$ & $66.0( \pm 10.1)$ & $N S$ \\
TBS & $1.403( \pm 0.09)^{*}$ & $1.331( \pm 0.10)$ & $1.299( \pm 0.12)^{*}$ & $* P<0.05$ \\
\hline
\end{tabular}

Conclusions: Blood vitamin D3 levels may play an important role on the bone quality by TBS, as men with vitamin D deficiency may have worse bone quality development. Further studies are needed on a larger cohort and it might be worth to investigate also men with osteomalacia. 


\section{P965}

COMMUNITY VS. SELF-MANAGEMENT OF OSTEOARTHRITIS: WHAT DOES THE EVIDENCE TELL US?

S. A. Ali ${ }^{1}$, K. Kokorelias ${ }^{1}$, M. Kloseck ${ }^{1}$

${ }^{1}$ Western University, London, Canada

Objectives: Self-management programs for chronic conditions have received considerable attention. A review of self-management education programs for osteoarthritis $(\mathrm{OA})$ found minimal evidence of benefit. We propose an alternative approach, the community-management of chronic musculoskeletal conditions. Our model includes both education and ongoing peer support to implement lifestyle changes to ameliorate daily management of OA. Here we review the literature for evidence to support such a model.

Material and Methods: A literature review (1990-2015) examined community-based management strategies for arthritis. We considered literature specific to ongoing and community-based OA education and mentorship. Results are presented as a narrative synthesis. We retrieved 814 studies and of these, 35 met the inclusion criteria.

Results: Traditional self-management models for arthritis have not worked. Most arthritis self-management models fail to deliver disease-specific strategies (grouping OA with other arthritis subtypes), and only $12 \%$ offer social support over time for implementing lifestyle changes ${ }^{1}$. Components of our proposed community-management model have been reported in the literature. Regarding education, community-based interventions were shown to improve medication adherence and have significant effects on psychological status of OA patients. No study exclusively explored peer support as an OA community-management strategy.

Conclusion: These findings provide justification to explore a community-management model for $\mathrm{OA}$ in which education is reinforced with peer support, so that disease-management strategies are implemented more successfully and sustainably.

References: ${ }^{1}$ Kroon FP, et al. Cochrane Database Syst Rev 1, CD008963 (2014)

\section{P966 \\ ZOLEDRONATE INFUSION IN OSTEOPOROTIC PATIENTS PROMOTES OSTEOGENIC DIFFE- RENTIATION OF MESENCHYMAL STEM CELLS COMPARTMENT}

F. del Forno $^{1}$, L. Dalle Carbonare ${ }^{1}$

${ }^{1}$ Department of Medicine, University of Verona, Verona, Italy
Objective: Zoledronate is a powerful treatment for fragility fractures. While its action on osteoclasts has been investigated, lately some studies suggested an effect also on the osteoblast-lineage. This study was aimed to evaluate the possible positive effect of zoledronate on the osteogenic differentiation in an ex vivo human model.

Methods: We selected 22 postmenopausal women with densitometric diagnosis of osteoporosis, never treated for fracture prevention. We supplied all patients with cholecalciferol 1000 $\mathrm{UI} / \mathrm{d}$ and administered zoledronate $5 \mathrm{mg}$ i.v. At the baseline and 12 months after the infusion of zoledronate, all patients underwent to densitometric evaluation at the lumbar spine and hip. At the baseline, 3 and 12 months after the infusion, we evaluated bone turnover markers (CTX and bALP) and we analyzed the differentiation of the osteoblast lineage in mesenchymal stem cells (MSCs) obtained from the peripheral blood of patients. The cells were evaluated by CFU-F assay and cultured in medium with osteogenic stimulating factors, to analyze the expression of the transcription factor $R U N X 2$ and the bone-related genes SPARC, SPP1, COLIA1 after 3, 7 and $14 \mathrm{~d}$ of differentiation.

Results: BMD after the administration of zoledronate was significantly increased in all patients. Bone turnover markers were significantly reduced 3 and 12 months after the infusion. The pool of circulating MSCs, evaluated by CFU-F assay, was significantly reduced after infusion of zoledronate. Furthermore, we observed an increased expression of RUNX2, SPP1, SPARC and COLIA1 after the treatment.

Conclusion: The gain in BMD observed after the administration of zoledronate associated to the increased expression of genes involved in osteogenic lineage suggests a possible anabolic activity of zoledronate. According to these results, the reduced pool of circulating MSCs might reflect a possible commitment of these cells toward the osteogenic lineage induced

\section{P967}

PREGNANCY AND LACTATION ASSOCIATED OSTEOPOROSIS: SLOVENIAN EXPERIENCE IN NINE CASES

$\underline{\text { A. Sabati Rajic }}{ }^{1}$, T. Kocjan ${ }^{1}$, M. Jensterle Sever ${ }^{1}$, J. Prezelj ${ }^{1}$, M. Pfeifer ${ }^{1}$

${ }^{1}$ University Medical Center Ljubljana, Ljubljana, Slovenia

Pregnancy and lactation associated osteoporosis is a rare disorder, occurring usually in late pregnancy and the early postpartum period. Most commonly are multiple fragility fractures diagnosed in the vertebral bodies. The prevalence, etiology and pathogenesis remains unknown. There are also no clear treatment guidelines. We report 9 cases of pregnancy and lactation associated osteoporosis we diagnosed and treated from the year 2006. The mean age of the patients at symptom onset 
was 33 years. The main complaint in our patients was severe pain in the lower back. In the majority of cases the pain occurred in the third pregnancy trimester. The vertebral fractures were diagnosed by MRI. The mean number of the vertebral fractures was 4 (range from 1-8). Most often we diagnosed fractures in the lower thoracic and in the first two lumbar vertebras. After exclusion of the other possible causes of the osteoporosis they all were treated with teriparatide in dose $20 \mu \mathrm{g}$ subcutaneous daily during 18-24 months. They were also treated with calcium supplementation $1000 \mathrm{mg}$ daily and cholecalciferol $1000 \mathrm{IU}$ daily. In one of our patients we diagnosed celiac disease. BMD was measured using DXA at the beginning, after 12 months and at the end of treatment. In the first year of treatment BMD measured on the lumbar spine was increased by $6.9 \%$ and $31.2 \%$, respectively. The pain was completely relieved in few months in all patients. BMD measured on the lumbar spine at the end of the treatment was at total increased by $33 \%$. After the completed teriparatide treatment two of the patients have had subsequent pregnancy without any complication or new osteoporotic fracture. There are many pharmacological therapy modalities that were used in pregnancy or lactation related osteoporosis. Bisphosphonates are effective, but there are some concerns that they accumulate in bone and may expose fetus in subsequent pregnancies. Our experience showed that teriparatide is a candidate drug for optimal and safe therapy modality. Although vertebral fractures are rare complication in expecting mothers, they should always be considered in the presence of acute pain in the peripregnancy period.

\section{P968}

EFFECT OF EXERCISE ON BALANCE AND FUNCTIONAL MOBILITY IN POSTMENOPAUSAL WOMEN WITH VERTEBRAL FRACTURES

$\underline{\text { L. Evstigneeva }}^{1}$, E. Negodaeva ${ }^{2}$, E. Kozhemykina ${ }^{1}$, A. Belkin $^{1}$

${ }^{1}$ Sverdlovsk Regional Clinical Hospital \#1, Ekaterinburg, Russian Federation, ${ }^{2}$ Urals State Medical University, Ekaterinburg, Russian Federation

Background: In accordance with population studies conducted in Russia, the prevalence of morphometric fractures for patients aged 50 years and older is high and ranges from 7.2-12 \% in men and 7-16\% in women. Osteoporotic vertebral fractures lead to lower quality of life and disability. Patients with osteoporotic fractures have to stick to specially developed exercise such as spinal stability exercise and postural corrections. The objective of our study was to evaluate the effectiveness of a structured physical exercise intervention on functional mobility and balance in patients with osteoporotic vertebral fractures.
Materials and methods: The study participants were women aged 50 years and older with postmenopausal osteoporosis and at least one radiographically confirmed vertebral fracture and chronic back pain. Exclusion criteria were vertebral and nonvertebral fracture within past 6 months, secondary osteoporosis, osteomalacia, inflammatory diseases of joints and spine, diffuse connective tissue diseases, history of malignancies in the last 5 years, significant functional changes in the cardiovascular, respiratory, gastrointestinal, or nervous system, renal disease, and corticosteroid intake during the last year. 135 women were invited to participate, and 78 subjects accepted the invitation. Patients were randomly assigned to the exercise and control groups in a 1:1 ratio. Patients in the exercise group performed a specially designed program of physical exercises in the exercise therapy gym twice a week under the instructor's supervision during 12 months. The duration of a session was $40 \mathrm{~min}$. Patients in the control group were asked to maintain their current physical activity. For assessment of balance, functional mobility and its dynamics, stabilometry was used. To assess physical function and balance possibly reflecting disorders of the musculoskeletal system three tests were chosen: 1) 'Weight bearing/Squat' test to maintain static balance is performed to quantify the weight held by each leg; 2) 'Sit-to-Stand' test provides an estimate of the functional mobility while a patient is lifting from a sitting position without using his hands, with the following parameters to be evaluated: weight transfer and left/right weight symmetry; 3) Test 'Tandem Walk and Sway' is performed as follows: a patient is asked to walk on a straight line moving the toe to the heel, to stop moving on command and to keep balance for $5 \mathrm{~s}$. Oscillations of the center of gravity for $5 \mathrm{~s}$ after the command to stop motion (degrees per second) are assessed. Functional mobility was also assessed with the test 'Timed Up and Go'. When performing this test a patient is asked to stand up from a chair, walk three meters, then go back and sit down. The test was evaluated twice measuring the time in seconds. The best test result was included in the analysis.

Results: We recruited 78 participants with at least one osteoporotic vertebral fracture and chronic back pain (40 in the exercise group and 38 controls), mean age 69.2 \pm 7.7 years. Demographic, clinical characteristics and study parameters (stabilometry, test 'Timed Up and Go') did not differ between groups at baseline. Both 'Weight bearing/Squat' test with extended legs and 'Sit to Stand: left/right weight symmetry' test reflecting weight distribution to each leg showed change neither in the exercise group nor in the controls. In opposite, the time of performing the 'Sit to Stand: weight transfer' test and 'Tandem Walk and Sway' test demonstrated statistically significant between-group difference with statistically significant worsening in the control group and statistically nonsignificant improvement in the exercise group. Average change in mean during 12 months for the 'Sit to Stand: weight transfer' test was $-0.24(95 \% \mathrm{CI}-1.12,0.64)$ in the exercise group, 0.43 
$(95 \%$ CI $0.13,0.73)$ in the control group, $p=0.01$. Average change in mean for the 'Tandem Walk and Sway' test was $-0.08(95 \%-1.62,1.47)$ in the exercise group, $1.72(95 \%$ $0.15,3.30)$ in the control group, $p=0.029$. Values in the 'Timed Up and Go' test at 12 months were not different between the exercise group and the control group. However, there was a significant improvement in the exercise group with a decrease in test performance time from $12.0 \pm 3.8-11.4 \pm 3.3 \mathrm{~s}(\mathrm{p}=0.02)$ while performance time remained unchanged in the control group $(11.0 \pm 3.3$ and $11.3 \pm 2.9 \mathrm{~s}$, respectively). Based on changes in mean values from baseline to the 12 months evaluation, we found a significant difference in change in mean between the intervention and the control group. The exercise group had better results than the control $(p=0.02)$.

Conclusion: The study demonstrated the effectiveness of a structured physical exercise program in improvement of functional mobility and balance in postmenopausal women with osteoporotic vertebral fractures.

\section{P969}

VITAMIN D LEVELS IN CANTON SARAJEVO OSTEOPOROSIS PATIENTS

S. Saric $^{1}$, R. Alimanovic-Alagic ${ }^{2}$

${ }^{1}$ Public Medical Care Institution of Canton Sarajevo, Sarajevo, Bosnia and Herzegovina, ${ }^{2}$ University of Sarajevo Clinical Center, Sarajevo, Bosnia and Herzegovina

Introduction: Vitamin D plays a very important role in the metabolism bone tissue. Its main role is to maintain a constant concentration of calcium in blood, which is achieved by increase of calcium absorption from small intestine as well as by mobilisation of calcium from bones and reduction of renal excretion of calcium. Vitamin D stimulates synthesis of osteocalcin, binding calcium in the bone matrix.

Objective: establish vitamin D levels in patients with osteoporosis in Sarajevo Canton.

Method: The research included 118 patients with DXA diagnosed osteoporosis treated in CBR-Saraj Polje in the period from October 1, 2014 to October 1, 2015. All patients were measured total concentration of vitamin $\mathrm{D}$, total calcium in blood and BMI.

Results: The above mentioned group consisted of $98.3 \%$ (116) white women and $1.7 \%$ (2) white men with 61.87 average age ranging from 38-84 years. Lumbar spine average T-score level equaled to -2.8 SD ranging from -2.2 to -5.9 SD. Proximal femur average T-score level equaled to $-2.33 \mathrm{SD}$ ranging from -1.4 to $-4.8 \mathrm{SD}$. Average vitamin D level equaled to $17.33 \mu \mathrm{g} / \mathrm{ml}$ ranging from $3 \mu \mathrm{g} / \mathrm{ml}$ to $65.9 \mu \mathrm{g} / \mathrm{ml}$. Vitamin D within referential levels was found in $11.8 \%$ (14) patients ranging from 30.4 $45.5 \mu \mathrm{g} / \mathrm{ml}$, with the average of $34.5 \mu \mathrm{g} / \mathrm{ml}$. Insufficient vitamin $\mathrm{D}$ levels were found in $87.28 \%$ (103) patients, with the average of $14.53 \mu \mathrm{g} / \mathrm{ml}$ ranging from $3 \mu \mathrm{g} / \mathrm{ml}$ to $29.4 \mu \mathrm{g} / \mathrm{ml}$. Vitamin D above referential levels, that is $65.9 \mu \mathrm{g} / \mathrm{ml}$, was found in $0.87 \%$ (1) patients. Average level of total calcium in blood in the group equaled to $2.37 \mathrm{mmol} / \mathrm{L}$ ranging from $2.04-2.81 \mathrm{mmol} / \mathrm{L}$. Referential calcium levels in blood were found in $97.5 \%$ (115) patients, with $2.4 \mathrm{mmol} / \mathrm{L}$ average levels ranging from 2.16 $2.57 \mathrm{mmol} / \mathrm{L}$. Insufficient calcium levels were found in $1.7 \%$ (2) patients, with $2.05 \mathrm{mmol} / \mathrm{L}$ average levels ranging from 2.04-2.07 mmol/L. Hypercalcemia was found in $0.87 \%$ (1) patients, with the level of $2.81 \mathrm{mmol} / \mathrm{L}$. Average BMI value amounted to $23.8 \mathrm{~kg} / \mathrm{m}^{2}$ ranging from $18-39.5 \mathrm{~kg} / \mathrm{m}^{2}$.

Conclusion: The research showed that the patients with osteoporosis exhibit highly insufficient vitamin D levels. Therapy induced increase of vitamin D level has to be exclusively adjusted to individual cases under strict medical follow-up.

References:

1. National Osteoporosis Foundation. Clinician,s Guide to Prevention and Treatment of Osteoporosis. Washington, DC: National Osteoporosis Foundation; 2014.

2. International Osteoporosis Foundation and National Osteoporosis Foundation. European guidance for the diagnosis and management of osteoporosis in postmenopausal women. Published online:19.October2012.International Osteoporosis Foundation and National Osteoporosis Foundation, 2012.

3. Black JA, Sandison R, Reid M.D. Osteoporosis the facts. Oxford University Press Inc., NewYork 2009.

\section{P970}

BAZEDOXIFENE 20MG RETAINS TRABECULAR AND CORTICAL BONE MINERAL MASS, CORTICAL GEOMETRY AND DERIVED STRENGTH AFTER 12 MONTHS OF TREATMENT IN POSTMENOPAUSAL WOMEN WITH OSTEOPOROSIS: A SINGLE-ARM, PHASE IV, TIBIA PQCT STUDY

K. D. Stathopoulos ${ }^{1}$, C. Kosmidis ${ }^{2}$, A. Mavrogenis ${ }^{3}$, E. Atsali $^{3}$, I. Bournazos ${ }^{3}$, P. J. Papagelopoulos ${ }^{3}$, G. Skarantavos ${ }^{3}$ ${ }^{1}$ Bone Metabolic Unit, 1st Department of Orthopaedics, University of Athens, "Attikon" Athens University General Hospital, Athens, Greece, ${ }^{2}$ Ika Athens, Athens, Greece, ${ }^{3}$ Bone Metabolic Unit, 1st Department of Orthopaedics, University of Athens, Athens, Greece

Objective: This phase 4 single-arm prospective study evaluated the effects of bazedoxifene (BZA) on trabecular and cortical bone mineral mass (BMC) and volumetric BMD as well as bone geometry and derived strength in postmenopausal women with osteoporosis assessed with pQCT of the tibia.

Methods: Subjects were Caucasian postmenopausal women with osteoporosis who were either treatment-naive or had previously discontinued oral bisphosphonates within less than a year of treatment and at least 6 months prior to enrolment. All study subjects received BZA $20 \mathrm{mg}$ daily as well as calcium and 
vitamin D supplements (1000 mg and 800 IU cholecalciferol, respectively). pQCT of the tibia was performed at baseline and 12 months after treatment. For each measurement, 3 radiological slices were obtained for each individual at the $4 \%$ (trabecular bone), $14 \%$ (subcortical and cortical bone) and $38 \%$ (cortical bone) of tibia length sites, respectively. Primary endpoint was percent change in trabecular BMC $(\mathrm{mg})$ and volumetric BMD $\left(\mathrm{mg} / \mathrm{cm}^{3}\right)$ at 12 months. Secondary endpoints included percent change in cortical BMC and volumetric BMD, cortical crosssectional area $\left(\mathrm{CSA}, \mathrm{mm}^{2}\right)$, cortical thickness $(\mathrm{mm})$ and strength-strain index (SSI, mm.) after 12 months of treatment.

Results: At 12 months, trabecular BMC and vBMD were shown to increase by $+1.52 \%$ and $+1.08 \%$, respectively, but these results were not statistically significant. Cortical BMC, vBMD, CSA, mean cortical thickness and SSI did not present significant differences after therapy. No serious adverse events were reported. Conclusions: BZA retained trabecular and cortical BMC and volumetric BMD as well as derived strength at 12 months as assessed with pQCT of the tibia, and was generally safe and well tolerated.

Disclosures: The study was sponsored (research grant) from Pfizer.

\section{P971}

\section{THE EFFECT OF INTRA-ARTICULAR INJECTION} OF HYALURONIC ACID ON OA: SYMPTOMS RELIEF VS. INTRA-ARTICULAR NSAID

G. Golubev $^{1}$

${ }^{1}$ Chair of Trauma and Orthopedic Surgery, Rostov State Medical University, Rostov-na-Donu, Russian Federation

Objectives: The recommendations to use intra-articular injection of hyaluronic acid (HA) to relief OA symptoms are contradictive. European guidelines recommend HA as symptomatic remedy, but the AAOS recently published data against using HA.

Material and Methods: Open randomized prospective cohort study was undertaken to assess the effectiveness and safety of intra-articular injection HA $(2.5 \mathrm{ml} 1 \%$ sodium hyaluronate solution) and compare it against NSAID intra-articular injections (lornoxicam $8 \mathrm{mg}+$ lidocaine $0.25 \% 5 \mathrm{ml}$ ). 40 patients (14 men, 26 women, the mean age $54 \pm 8$ years) with second (18 persons) and third ( 22 persons) stages of AO by Ahlback were randomly distributed to research (HA) and control (Lornoxicam) groups (GrR and GrC below). All patients answered inclusion/exclusion criteria: bilateral process, no previous arthroscopic or open surgical procedures to knee, no corticosteroid intraarticular injections during previous 6 months, no signs of acute thrombosis or varicose disease. Remedies injected into upper knee compartment once a week during 5 sequential weeks. Manifestations of OA were registered by Goldberg score (Gs) and visual analogous pain score (VAPS), clinical investigation performed according to "2000 IKDC knee Examination Form, surgeons part". The stage of OA was classified radiographically by Ahlback score. Routine blood and liver function tests performed before treatment, and monthly till the end of research. Contacts with 3 patients lost.

Results: On inclusion there were no Gs and VAPS difference between groups and 2nd and 3rd stages of AO (57.33 \pm 9.64 and 49.36 $\pm 9.38 ; 5$ and $5.5 \mathrm{~mm}$ accordingly). At GrR knee functional score increased gradually and average Gs was $88.00 \pm 7.50$ after 4 months. At GrC Gs increased rapidly and in 1 month after start of treatment reached $69.64 \pm 11.38$ then decreased gradually to base values at the end of followup. VAPS values had statistically significant differences between $\mathrm{GrC}(3.5 \pm 0.25 \mathrm{~mm})$ and $\mathrm{GrR}(2.2 \pm 0.4 \mathrm{~mm})$. No statistical differences between blood and liver tests were registered at start and end periods. The symptomatic improvement remained during 16 weeks since the completion of the course of treatment in 17 of 19 patients from GrR. Systemic reactions and complications were not registered during research.

Conclusions: This study allows to assert that HA has evident middle follow-up symptomatic effect leading to the reduction of pain intensity and the improvement of the functional characteristics of knee joints. The duration of symptomatic effects of intra-articular injections of HA exceeds length of NSAID effects.

\section{P972}

ATYPICAL FRACTURES IN PATIENTS WITH OSTEOPOROSIS

A. Wawrzyniak $^{1}$, M. Marcinkowska ${ }^{1}$, J. Dytfeld $^{1}$

${ }^{1}$ Department of Family Medicine University of Medical Science Poznan, Poznan, Poland

Introduction: Subtrochanteric/atypical fractures are rare, but serious complication of antiresorptive therapy in osteoporosis. The retrospective analysis of clinical data of patients who underwent this type of fracture might help to identify the ones that are most vulnerable.

Material and methods: In a large group of 875 females, postmenopausal, Caucasian, noninstitutionalized patients of osteoporosis outpatient clinic in Poznań, the history of vertebral, nonvertebral and hip fractures was analyzed. In the latter group, atypical femur fractures were distinguished. Particularly age of patient while fractured, type and length of previous osteoporosis treatment, secondary causes of osteoporosis, vitamin D status and BMD (DXA).

Results: Among 875 women, 247 underwent vertebral fractures, and 389 nonvertebral. Within nonvertebral fractures there were 33 hip fractures and 7 femoral subtrochanteric fractures (atypical). On hospital discharge cards none of hip fractures were identified as atypical ones, but as fractures of femoral shaft. 


\begin{tabular}{|c|c|c|c|c|c|c|c|}
\hline Patient's number & 1 & 2 & 3 & 4 & 5 & 6 & 7 \\
\hline Age of fracture & 66 & 77 & 71 & 74 & 85 & 59 & 58 \\
\hline $\begin{array}{l}\text { Initial indication to } \\
\text { treatment }\end{array}$ & $\begin{array}{l}\text { Wrist fracture } \\
\text { low bone mass }\end{array}$ & DXA & Risk factors & DXA & Risk factors & No treatment & No treatment \\
\hline Length of therapy (yrs) & 12 & 15 & 10 & 3 & 5 & 0 & 0 \\
\hline Type of drugs & $\begin{array}{l}\text { BS-10 } \\
\text { SR - } 3\end{array}$ & $\begin{array}{l}\text { BS-10 } \\
\text { SR - } 3\end{array}$ & BS-10 & BS - 3 & SR - 5 & No treatment & No treatment \\
\hline $\begin{array}{l}\text { Type of secondary } \\
\text { osteoporosis }\end{array}$ & PPIs & & $\begin{array}{l}\text { GKS } \\
\text { IPP } \\
\text { RA }\end{array}$ & & RA & $\begin{array}{l}\text { Smoking Alcohol abuse } \\
\text { PPIs No dairy diet }\end{array}$ & PPIs \\
\hline $25 \mathrm{OHD} \mathrm{ng} / \mathrm{ml}$ & 28 & 29 & 26 & 29 & 20 & 43 & 24 \\
\hline BMD L1L4 g/cm2 & 0,850 & 0,854 & 1,119 & 0,784 & 0,881 & 0,953 & 0,823 \\
\hline BMD Hip g/cm2 & 0,932 & 0,745 & 0,853 & 0,820 & 0,826 & 0,753 & - \\
\hline
\end{tabular}

Abbreviation

BS- bisphosphonate

SR- strontium ranelate

RA- rheumatoid arthritis

\section{Conclusions:}

1. Patients with subtrochanteric femoral fractures are not the same: two of them were not diagnosed with osteoporosis and had no antiresorptive treatment.

2. The risk of atypical fracture possibly increases with duration of antiosteoporotic therapy and accumulation of risk factors such as chronic inflammatory diseases - rheumatoid arthritis, use of PPIs, glucocorticosteroids.

3. 2 from 3 patients with atypical fractures, treated with antiresorptive drugs for more than 10 years had initially no indication to take them (had not had history of any osteoporotic fracture, only low bone mass).

4. The decision on osteoporosis treatment should be systematically verified.

\section{P973}

\section{ROLE OF PHYSICAL EXERCISE IN PATIENTS WITH KNEE OSTEOARTHRITIS}

I.-R. Marcu ${ }^{1}$, S. Patru ${ }^{1}$, A. C. Bighea ${ }^{1}$

${ }^{1}$ University of Medicine and Pharmacy, Craiova, Romania

Introduction: Knee osteoarthritis evolution is slow, but progressive, increasing degradation of the joint, worsening instability, with permanent pain and reduced walk perimeter.

Purpose: To examine the effects of a 3 weeks kinetic program on pain and the functional status of patients with knee osteoarthritis.

Method: We conducted an observational, prospective, randomized study on a sample of 80 patients with knee osteoarthritis. The patients were randomly assigned to a control group (40 patients) who received electrotherapy, physical therapy and massage (group 1) and a study group (40 patients) whose therapeutic program also included daily physical exercise (group 2). Evaluation of patients was done on day1 and after 3 weeks of rehabilitation treatment. The clinical and functional parameters assessed were: pain on a visual analogue scale (100 mm VAS), physical impairments (muscular strength, static disorders and mobility of knee joint) and disabilities (Tinetti Gait Scale, ADL 24, D'Aubigné Scale and movement capacity).

Results: The scores for functional parameters improved: pain$43.5 \%$ (group 2) and $32.9 \%$ (group 1) $(p=0.000054)$; physical impairments: muscular strength- $9.7 \%$ (group 2), without improving by group 1, static disorders- $24.6 \%$ (group 2) and $13.6 \%$ (group 1), mobility: $34.8 \%$ (group2) and $23.2 \%$ (group 1); disabilities: Tinetti Gait Scale- $36.3 \%$ (group 2) and $22.1 \%$ (group 1), ADL- $53.6 \%$ (group 2) and $40.2 \%$ (group 1), D’Aubigné Scale- $34.7 \%$ (group 2) and $25.9 \%$ (group 1), movement capacity- $45.7 \%$ (group 2 ) and $32.7 \%$ (group 1). The results were statistic significant $(p<0.05)$.

Discussion and Conclusions: Improvement of pain, physical impairments and disabilities for the study group certifies the efficacy of the rehabilitation program including physical exercise for the patients suffering from knee osteoarthritis and motivates the continuation of the study on a longer period of time and on a larger number of patients.

\section{P974}

VITAMIN D REPLETION IN OSTEOPOROSIS RELATED TO IDIOPATHIC HYPERCALCIURIA

\section{S. L. Calvisi ${ }^{1}$, M. Meloni ${ }^{2}$}

${ }^{1}$ Unità Operativa Medicina Interna, Nuoro, Italy, ${ }^{2}$ Unità Operativa Neurochirurgia, Gravedona, Italy 
Idiopathic hypercalciuria $(\mathrm{IH})$ is defined as an excess urine calcium excretion more than $0.1 \mathrm{mmol} / \mathrm{kg} / 24 \mathrm{~h}$ without an apparent underlying etiology. It recognizes three pathogenetic mechanisms: increased intestinal absorption of calcium, defective reabsorption of calcium by the renal tubule, increased bone resorption due to an overexpression of the vitamin D (VD) receptors, increased tissue VD response and deficiencies in renal tubule enzymes. Patients might have either elevated or normal serum levels of VD. We reported the case of a 32 years old woman with osteoporosis and associated multiple bone fractures affected by $\mathrm{IH}$, with low levels of VD and normal levels of PTH. Despite the reluctance to prescribe VD, several studies have demonstrated that giving VD is effective and safe, therefore, VD supplementation may lead to a positive calcium balance and be beneficial for maintaining bone health. We decided to start therapy with ergocalciferol, hydrochlorothiazide, bisphosphonates and dietary restrictions for 8 weeks. The outcome is the change in urinary calcium excretion that will be evaluated at the end of the treatment.

\section{P975}

RETROSPECTIVE RADIOGRAPHIC EVALUATION OF SCREENING AND MANAGEMENT OF DEVELOPMENTAL DYSPLASIA OF THE HIP IN A DISTRICT GENERAL HOSPITAL

T. Amuthalingam $1^{1}$,U. Ramkumar ${ }^{1}$,A. Amini ${ }^{1}$

${ }^{1}$ The Princess Alexandra Hospital, Harlow, United Kingdom

Objective: We hoped to revalidate the need to screen for developmental dysplasia of the hip in newborns and further investigations for at risk groups. We evaluated the use of appropriate radiological methods in diagnosing and managing the condition as well the prompt initiation of treatment.

Methods: A retrospective, longitudinal study was undertaken of 26 patients who were referred to the senior clinician in the year 2012 of the 1363 births in the catchment area, Harlow. Screening was conducted by radiographers and patients with abnormal findings referred to the clinician. Ultrasounds of the hips were reported using Graf type by calculating the alpha angle and/or percentage bone coverage. Data was collected from radiological imaging records and clinical letters.

Results: Of the 26 patients, $77 \%$ presented with bilateral dysplasia in contrary to literature and $84 \%$ of the patients were female in line with literature (Wilkinson, 1992). Screening ultrasonography reported a Graf type II in $41 \%$ and type III in 38\% of the hip. A relatively short period of 10 weeks was spent in Pavlik harness on average. 4 ultrasounds and 2 radiographs per patient were required on average during the course of management.

Conclusions: Pavlik harness treatment was sufficient in managing $98 \%$ of the patients due to early diagnosis of developmental dysplasia of the hip by the screening programme. 19.1 newborns per 1000 had at least one sonographically dysplastic hip. We recommended that ultrasounds of the hip are reported using Graf classifications and not percentage bone coverage to improve outcomes and quality of care.

Reference: Wilkinson J. Clin Orthop Relat Res 1992;281:75.

\section{P976}

EVALUATION OF THE OSTEOGENIC POTENTIAL OF HUMAN MESENCHYMAL STEM CELLS (MSC) FROM DIFFERENT NICHES: MSC-DERIVED ORGANOID-LIKE MICROSPHERES AS A MODEL FOR TISSUE ENGINEERING AND BONE DISEASE E. M. Haddouti ${ }^{1}$, F. A. Schildberg ${ }^{2}$, C. Hilgers ${ }^{1}$, W. Masson ${ }^{1}$, R. Pflugmacher ${ }^{1}$, C. Burger ${ }^{1}$, D. C. Wirtz ${ }^{1}$, W. Götz ${ }^{3}$, A. Limmer $^{1}$, S. Gravius ${ }^{1}$, T. M. Randau ${ }^{1}$

${ }^{1}$ Department of Orthopedics and Trauma Surgery, University Clinic Bonn, Bonn, Germany, ${ }^{2}$ Department of Microbiology and Immunobiology, Harvard Medical School, Boston, United States, ${ }^{3}$ Department of Orthodontics, Oral Biology Laboratory, University Clinic Bonn, Bonn, Germany

Objectives: Bone remodeling is regulated by MSC-derived osteoblasts forming bone and resorbing osteoclasts. The aim of the current study was to isolate, characterize, compare MSCs from different niches from osteoporotic and healthy patients. The osteogenic potential was investigated using bone organoid-like generated via 3D cultures.

Methods: MSCs were harvested from vertebral bodies, adipose tissue and femoral head. MSCs were characterized via their differentiation towards three lineages, surface marker expression, proliferation and immunomodulatory capacity. The calcification process was evaluated by measuring the optical density and free phosphate ions $(\mathrm{Pi})$ during the osteogenic differentiation. MSC-derived bone organoid-like microspheres were generated on agarose.

Results: All investigated MSCs showed typical fibroblast-like morphology, expressed MSCs surface marker, differentiated towards adipo-chondro-osteogenic lineages confirmed via specific stainings. Proliferation and calcification showed comparable tendencies for all analyzed MSCs. Amounts of Pi measured were clearly elevated in osteogenic induced MSCs compared to controls. Vertebral body-derived MSCs from osteoporotic and healthy patients showed immunomodulatory capacity by suppressing lymphocytes proliferation. All MSCs were able to self-organize and form microspheres on agarose. At the end of the induction period cryosections were generated from the MSC-derived bone organoid-like microspheres and the mineralization was conformed via specific staining.

Conclusion: MSCs from osteoporotic patients showed similar characteristics investigated like calcification, proliferation and no impairment was detected when compared to MSCs from healthy patients. Nevertheless, MSCs could provide a useful tool for further studies to investigate their contribution in the pathogenesis of 
osteoporosis. Furthermore, MSCs from osteoporotic patients could be possibly used in cell-based therapy approaches. MSCderived bone organoid-like can serve as an in-vitro model to investigate bone related diseases like infection, drug testing and toxicology as well as individual and regeneration medicine. Our established model can be extended by taking other cells orchestrating bone remodeling into consideration like osteoclasts.

\section{P977}

FROM OSTEOPOROSIS TO OSTEOARTHRITIS: APPLYING LESSONS LEARNED FOR A NEW COMMUNITY MANAGEMENT MODEL FOR MUSCULOSKELETAL DISEASES

S. A. Ali ${ }^{1}$, M. Kloseck ${ }^{1}$, R. G. Crilly ${ }^{1}$, K. Walsh ${ }^{1}$

${ }^{1}$ Western University, London, Canada

Objectives: There is good evidence to suggest that modification of lifestyle factors can reduce the burden of osteoarthritis (OA). The challenge lies in conveying this information down the research pipeline to the patient. Despite consistent clinical practice guideline recommendations for patient education, there is a large gap in bringing information to community settings. Facilitating, and most importantly, sustaining behavior change, is essential. A recent osteoporosis study ${ }^{1}(n=105$; mean age $=80.5 \pm 6.9 \mathrm{SD} ; 89 \%$ female) showed that a community peer-led program could lead to positive behavior change, even with no change in knowledge. This is in contrast to self-management models which have been inconsistent in their effect. We are now exploring the value of a community management model for OA.

Material and Methods: Our OA model consists of 4 phases: 1) a qualitative phase with patient and physician interviews to identify issues including information access, care gaps, resource availability, and access to specialists in order to inform the following phases, 2) design of a care program which could be run by patients and other lay people to encourage and provide on-going support for evidence-based behavior change; including peer-based education and peer-mentoring as shown to work in our osteoporosis study, 3) design and perform a RCT of the model to demonstrate efficacy and cost-effectiveness, and 4) if successful work with The Arthritis Society to implement this program nationally.

Results: Phase 1 is now in progress, with promising preliminary results.

Conclusion: Although in the early stages of development we anticipate this community approach will make a difference to the care of OA patients in an inexpensive, effective manner.

References: ${ }^{1}$ Kloseck M, Fitzsimons D, Speechley M, Crilly RG. Improving the treatment and diagnosis of osteoporosis using a senior-friendly peer-led community education model: a randomized controlled trial. Trials (under review).

\section{P978}

DYSMOBILITY SYNDROME IS ASSOCIATED WITH FALLS AND FACTURES IN OLDER CHILEAN PEOPLE

H. Sanchez ${ }^{1}$, L. Lera ${ }^{1}$, B. Angel ${ }^{1}$, C. Marquez ${ }^{1}$, C. Albala ${ }^{1}$

${ }^{1}$ INTA, University of Chile, Santiago, Chile

Background: Impaired mobility, fall and fractures are among the main causes of dependence, institutionalisation and death in older people.

Objective: To determine the frequency dysmobility syndrome and its association with falls and fractures in older Chilean people. Methods: Follow-up of ALEXANDROS cohorts designed to study disability associated with obesity in community dwelling people 60 years and older ( $\min 60$ years, max 99 years) living in Santiago, Chile. At baseline 1006 people (68.3\% women) from 2372 participating subjects had DXA scan and the measurements needed for the identification of dysmobility syndrome: slow walk speed $(<0.8 \mathrm{~m} / \mathrm{s})$, weak handgrip strength $(\leq$ p25 older Chilean people) balance problems, low BMD $(<$ $-1 \mathrm{SD}$ WHO standard), low skeletal muscle mass ( $\leq$ p20 older Chilean people) and high body fat ( $\geq \mathrm{p} 60$ older Chilean people). Dysmobility was defined as having at least three of the conditions. Results: Prevalence of dysmobility at baseline was $32.6 \%$, higher in women than in men (37.0 vs. $23.2 \%$ ). The frequency of dysmobility increased with increasing age $(60-69 \mathrm{y}=27.9 \%$; $70-79 y=44.2 \% ; \geq 80=51.1 \%, p<0.0001$ ) Falls were more frequent in people with dysmobility compared with people without dysmobility ( $39.8 \%$ vs. $25.1 \%, p<0.0001)$, respectively. Similar situation was observed for fractures $($ dysmobility $=39.9 \%$, nondysmobility $=31.4 \%, p<0.0001)$; After age, sex, BMI and diabetes adjusted regression analysis, the association of dysmobility with falls remained significant $(O R=1.71 ; 95 \%$ CI $1.23-2.37)$. Dysmobility was associated with fractures (crude $O R=1.45 ; 95 \%$ CI 1.03-2.04, $p=0.03$ ). After adjusting by sex, age, BMI and diabetes the association increased $(O R=1.91 ; 95 \% \mathrm{CI} 1.31-2.77)$

Conclusion: Dysmobility was associated with increased risk of falls and fractures in older Chilean people.

Acknowledgements: Fondecyt Grant 1130947

\section{P979}

TRABECULAR BONE SCORE IN COMPARISON TO BONE MINERAL DENSITY IN DISCRIMINATION BETWEEN FEMALE POSTMENOPAUSAL PATIENTS WITH AND WITHOUT OSTEOPOROTIC FRACTURES

M. Ignaszak-Szczepaniak ${ }^{1}$, M. Michalak ${ }^{2}$

${ }^{1}$ Laboratory of Metabolic Bone Diseases, Department of Family Medicine, University of Medical Sciences, Poznan, Poland, ${ }^{2}$ Department of Computer Science and Statistics, University of Medical Sciences, Poznan, Poland 
Objectives: (1) to assess whether TBS discriminates between patients with and those without previous osteoporotic fractures $(\mathrm{OFs})$, particularly women with a $\mathrm{T}$-score $>-2.5 \mathrm{SD}$, as well as (2) to determine the usefulness of TBS in fracture risk assessment in the population of Polish postmenopausal women.

Material and methods: Retrospective, case-control study of 490 postmenopausal women diagnosed for osteoporosis. Fracture status was reviewed in medical records and vertebral fractures (VFs) were identified by VFA for each subject (only $\mathrm{VFs}>\mathrm{I}$ by Genant were included). Spine DXA images performed for all subjects were reanalyzed using TBS iNsight $\mathrm{v}$. 2.1 to calculate individual TBS value. Prevalence of osteoporotic fractures (VFs, hip, forearm, shoulder, rib) were analyzed in particular TBS tertiles: lowest $(\mathrm{L})$, middle $(\mathrm{M})$ and highest $(\mathrm{H})$ as well as in BMD T-score categories. Comparison between fractured and nonfractured women depending on BMD Tscore and TBS tertiles were assessed by chi-square. ORs and 95\% CIs were calculated with Statistica 10.

Results: Complete datasets of 439 women (mean age 71.7 years, mean BMI $30.2 \mathrm{~kg} / \mathrm{m} 2$ ) of 490 patients were available. We identified $184 \mathrm{OFs}$, including116VFs and 55 forearm, 5 hip, 5 shoulder, 3 rib fractures. Mean TBS value in fractured women (FW) was 1.13 vs. 1.20 for nonfractured $(p<0.001)$. Number of OFs increased with a decrease of TBS value irrespective of T-score. $71 \%$ OFs was observed in the lowest tertile, tertile $\mathrm{M}$ was found in every third patient $(26 \%)$ with OFs and only in $2.7 \%$ of FW TBS score was high. $37 \%$ of OFs occurred in the nonosteoporotic zone. TBS value in $69 \%$ of them was low and in $94 \%<1.350$. Women whose TBS was low had 3.6 higher risk of OFs as compared to highest tertile ( $p=0.0014,95 \% \mathrm{CI}: 1.573-8.156)$ and 2.05 higher when compared $\mathrm{L}$ to $\mathrm{M}(p=0.0008,95 \%$ CI $1.618-4.489)$. The association was weaker when compared $\mathrm{M}$ and $\mathrm{H}$ tertiles (OR-2.46, $p=0.027$ ). Highest TBS score was associated with low probability of OFs also in women with $\mathrm{T}$-score $<-2.5 \mathrm{SD}$. Conclusions: Low TBS score is related to 3.6-fold higher probability of any site osteoporotic fracture, irrespective of BMD. TBS score improves identification of patients with OFs whose BMD is in nonosteoporotic range. We suggest the inclusion of TBS value in fracture risk assessment of Polish women for better identification of women prone to low energy fractures.

\section{P980}

25(OH)2D DEFICIENCY AND POOR SLEEP QUALITY DURING FIRST TRIMESTER IN PREGNANT SAUDI WOMEN

S. AL-Musharaf ${ }^{1}$, P. McTernan ${ }^{1}$, P. Sarvanan ${ }^{1}$, N. M. AlDaghri $^{2}$, A. Al-Ajlan ${ }^{3}$

${ }^{1}$ University of Warwick, Coventry, United Kingdom, ${ }^{2}$ Biochemistry Department, College of Science, King Saud University, Riyadh, Saudi Arabia, ${ }^{3}$ King Saud University, Riyadh, Saudi Arabia
Objectives: To measure vitamin D levels and sleep quality in first trimester pregnant Saudi women.

Material and Methods: This was a cross-sectional study that collected biochemical and anthropometric data on randomly selected Saudi pregnant women during their first antenatal checkup (8-16 weeks), in Riyadh, KSA $(n=610) .216$ patients were simultaneously assessed for their quality of sleep using Pittsburgh Sleep Questionnaire (PSQI).

Results: Serum mean of $25(\mathrm{OH})_{2} \mathrm{D}$ was $29.21 \pm 16.27 \mathrm{nmol} / \mathrm{l}$. $49.5 \%$ of the pregnant women were deficient $(<25 \mathrm{nmol} / \mathrm{l})(302 /$ $610)$. The mean duration of sleep per night was $6.36 \pm 2.73 \mathrm{~h}$ and mean sleep latency was $26.12 \pm 22.79 \mathrm{~min}$ in the deficient group compared to $31.13 \pm 27.61 \mathrm{~min}$ in the nondeficient group. The mean total PSQI score was $8.03 \pm 4$. $79.4 \%(117 / 216)$ of the women had poor quality sleep. Women with $25(\mathrm{OH})_{2} \mathrm{D}$ deficiency had significantly higher PSQI score than the women with sufficient $25(\mathrm{OH})_{2} \mathrm{D}(8.81 \pm 3.74$ vs. $7.64 \pm 4.10 ; p<0.05)$. Additionally, day dysfunction was significantly higher (2.11 \pm 1.29 vs. $1.72 \pm 1.42 ; p<0.01)$ and sleep latency was significantly lesser in the deficient compared to sufficient group (26.12 \pm 22.79 vs. $31.13 \pm 27.61 \mathrm{~min} ; p<0.01)$. However, sleep disturbance components were significantly higher in the sufficient group compared to the deficient. There were no differences between the $25(\mathrm{OH})_{2} \mathrm{D}$ deficient group and the sufficient group with regards to sleep duration, habitual sleep efficiency, sleep quality and sleep medications ( $p>0.05$ ). Additionally, no significant differences were observed between poor or good sleep groups with regards to age, waist to hip ratio, BMI and \%fat.

Conclusion: This novel study in pregnant Saudi women shows that high prevalence of $25(\mathrm{OH})_{2} \mathrm{D}$ deficiency in early pregnancy and is associated with higher total PSQI score which indicates poor sleep quality. Women with $25(\mathrm{OH})_{2} \mathrm{D}$ deficiency have poor sleep quality, higher daytime dysfunction and took less time to fall asleep compared with the sufficient group. Based on current literature this represents the first study that has assessed the relationship between sleep and $25(\mathrm{OH})_{2} \mathrm{D}$ in early pregnancy. Further studies should be undertaken to analyze the relationship over time between vitamin D and sleep quality as well as how vitamin D supplementation may improve sleep during pregnancy.

\section{P981}

BILATERAL QUADRICEPS TENDON RUPTURE IN A HEALTHY MAN SECONDARY TO A FALL DURING A NONPROFESSIONAL TENNIS MATCH

$\underline{\text { C. Aleixo }}^{1}$, R. Santos-Pereira ${ }^{1}$, F. Lima-Santos ${ }^{1}$, A. Costa $^{1}$, J.

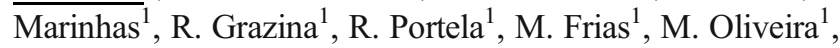
R. Freitas ${ }^{1}$

${ }^{1}$ Centro Hospitalar Vila Nova de Gaia / Espinho, Vila Nova de Gaia, Portugal

Objectives: Ruptures of the quadriceps tendon are uncommon 
and usually occur in patients older than 50 years. Bilateral ruptures are rare and highly correlated with systemic disease. In younger individuals, bilateral simultaneous quadriceps ruptures has been associated with anabolic steroid use, but more frequently with underlying comorbid medical conditions such as hyperparathyroidism, chronic renal failure, obesity and gout that predispose the patients to tendon rupture.

Methods: We present a case report of a 38 years old male patient with no significant past medical history presented to our emergency room with a bilateral thigh pain after a fall during a nonprofessional tennis match. He was unable to walk. He had no equimosis, hematoma or swelling. Physical examination showed the patient to be unable to actively extend his legs and had bilateral suprapatellar gaps. The X-ray of both knees did not show signs of acute fracture. Ultrasonography was performed and showed a complete bilateral rupture of quadriceps tendon near its insertion site on the patella. His haematological and metabolic profiles were all within normal limits. There was no history of use of anabolic steroids, previous local steroid injections, or tendinitis. Surgical repair of tendon was performed with a Krackow type suture trough drill holes in the patella and the patient was immobilized with knee in extension for 4 weeks. Following that period he received physiotherapy to regain strength and range of motion.

Results: The patient was followed up during 6 months. He was allowed full weight-bearing by the 8th postoperative week and returned to playing sports at 4 months after the initial injury. At the last clinical evaluation the patient was asymptomatic with symmetrical bilateral quadriceps strength, normal range of motion and no extensor lag. He was able to play tennis at a casual level and was very satisfied with the outcome.

Conclusion: Bilateral quadriceps tendon rupture in a healthy individual is an extremely rare occurrence and a high index of suspicion is necessary to diagnose this lesions. Complete quadriceps tendon rupture is a disabling injury and to obtain the best results, early diagnosis and surgical repair is mandatory.

\section{P982}

LOSS OF HEIGHT AS THE CLINICAL INDICATOR OF PREVALENT VERTEBRAL FRACTURE: ANALYSIS OF VERTEBRAL FRACTURES BY VFA VS. HEIGHT MEASUREMENT IN WOMEN OVER $50 \mathrm{Y}$

M. Ignaszak-Szczepaniak ${ }^{1}$, M. Michalak ${ }^{2}$, M. Padzik ${ }^{3}$

${ }^{1}$ Laboratory of Metabolic Bone Diseases, Department of Family Medicine, University of Medical Sciences, Poznan, Poland, ${ }^{2}$ Department of Computer Science and Statistics, University of Medical Sciences, Poznan, Poland, ${ }^{3}$ Family Medicine Department, University of Medical Sciences, Poznan, Poland

Objective: To determine which height loss threshold: at least $3 \mathrm{~cm}$ or $4 \mathrm{~cm}$ is better clinical indicator of prevalent vertebral fracture(s) (VFs) in postmenopausal women.
Material and methods: Retrospective analysis of 411 postmenopausal women aged 50-85y (mean 71.7) screened for osteoporosis. Clinical data included the maximal height in youth determined from personal identity card. PE was performed including current height measurements. The presence and number of VFs were assessed by VFA on DXA device (Lunar Prodigy, GE). Statistical analyzes were performed using Statistica 10 (StatSoft Inc.)

Results: 110 VFs were detected. Loss of height ranged from $1-13 \mathrm{~cm}$ (mean $4.2 \mathrm{~cm}$ ). Two analyses were performed. First for the threshold of $3 \mathrm{~cm}$. Loss $\geq 3 \mathrm{~cm}$ was detected in $50 \%$ of subjects. In this group, the VFs were detected in 93 patients (45\%), but in remaining 113 there was no fracture despite height loss (in 3 cases $>9 \mathrm{~cm}$, all aged $>80 \mathrm{y}$ ). On the other hand, among those who fractured min. 1vertebra, patients with loss $\geq 3 \mathrm{~cm}$ accounted for $84.5 \%$ (93/110 VFs, sensitivity 0.85 ). In $37 \%$ of patients there were no fractures, despite the height reduction (specificity 0.63 ). Second analysis was performed for $4 \mathrm{~cm}$. Loss of height $\geq 4 \mathrm{~cm}$ concerned 165 women, while VFs were confirmed in $51.5 \%$ of them. 85 out of 110 VFs occurred in patients with decreased $\geq 4 \mathrm{~cm}$ (sensitivity 0.77). 80 women who reported no loss $\geq 4 \mathrm{~cm}$ had no VFs in VFA (specificity 0.73 ). ORs for the criteria $\geq 3$ and $\geq 4 \mathrm{~cm}$ were respectively: 9.10 vs. 9.34 ; CI: $[5,16-16]$ vs. [5,16-15.7]; $p=0.00001$. Among the patients who had height loss $<3$ or $<4 \mathrm{~cm}$, the fracture was detected respectively in only $8.3 \%$ vs. $10 \%$; the negative predictive value was higher for the threshold $3 \mathrm{~cm}$. The degree of height loss did not correlate with the number of fractured vertebrae $(p=0.06)$.

Conclusions: Both thresholds of height loss are comparable for VF detection and associated with 9- fold higher probability of prior vertebral fracture. VF was confirmed in every second women both for $3 \mathrm{~cm}$ and for $4 \mathrm{~cm}$ of height loss, however the criterion of $3 \mathrm{~cm}$ has better sensitivity. Loss of height up to $3 \mathrm{~cm}$ excludes VF in $92 \%$ of women $>50$ years.

\section{P983}

\section{TERIPARATIDE TREATMENT IN PELVIC FRACTURE}

M. Stanciu ${ }^{1}$, F.-L. Popa ${ }^{2}$

${ }^{1}$ Department of Endocrinology, Lucian Blaga University Sibiu, Sibiu, Romania, ${ }^{2}$ Department of Medical Rehabilitation, Lucian Blaga University Sibiu, Sibiu, Romania

Pelvic insufficiency fractures are common in female patients with osteoporosis and mostly occurred by trivial injury or slip down. As elderly population increases, the incidence of pelvic insufficiency fractures tends to be also increased rapidly. We present a 83 years old female with left hip pain that was occurred after a fall. Fractures in left pubic ramus were observed on simple radiograph at the admission and fractures in sacrum left side were found in CT scan. In BMD examination utilizing 
DXA (Prodigy Advance ${ }^{\mathrm{TM}}$, GE-Lunar Corp., Madison, WI, USA), osteoporosis was diagnosed with T-score -5.1 (L1-4), but the patient did not have history of diagnosis and treatments in osteoporosis before the injury. We recommended $20 \mu \mathrm{g}$ of teriparatide (1-34 PTH) subcutaneously once a day in order to promote fracture healing as well as treat osteoporosis. Teriparatide was administered for 20 weeks. Bed rest and medication for pain relief was combined at the same time. The patient was able to move using a wheelchair a week after the injury and walked using a walker $15 \mathrm{~d}$ after the injury. Then the patient was able to walk herself without pain 8 weeks after the injury. On simple radiograph a month after the injury, callus formation was observed in left pubic ramus and then it was even definitely denser on 4 months follow-up x-ray.

\section{P984}

DEVELOPMENT AND VALIDATION OF A MODIFIED MALE OSTEOPOROSIS SELF-ASSESSMENT TOOL FOR TAIWAN (MOSTAI)

$\underline{\text { D.-H. Liu }}^{1}$, T.-T. Cheng ${ }^{2}$, F.-M. Su${ }^{3}$, J.-F. Chen ${ }^{3}$, S.-F. Yu ${ }^{3}$, W.-C. Chiu ${ }^{3}$, C.-Y. $\mathrm{Hsu}^{3}$, C.-H. Ko ${ }^{3}$, C.-C. Tsai ${ }^{4}$

${ }^{1}$ Department of Physical Medicine and Rehabilitation, Taipei Tzu Chi Hospital, Buddhist Tzu Chi Medical Foundation, New Taipei City, Taiwan, Province of China, ${ }^{2}$ Section of Rheumatology, Allergy and Immunology, Chang Gung Memorial Hospital - Kaohsiung Medical Center, Kaohsiung, Taiwan, Province of China, ${ }^{3}$ Division of Rheumatology, Allergy, and Immunology, Department of Internal Medicine, Kaohsiung Chang Gung Memorial Hospital, Kaohsiung, Taiwan, Province of China, ${ }^{4}$ Department of Obstetrics and Gynecology, Kaohsiung Chang Gung Memorial Hospital, Kaohsiung, Taiwan, Province of China

Purpose: To develop a MOSTAi and validate it by comparing with Osteoporosis Self-assessment Tool for Asians (OSTA) and the National Osteoporosis Foundation recommendations in 2013 (NOF 2013) for BMD testing in Taiwanese men.

Methods: A bus, equipped with DXA and conducted by Taiwan Osteoporosis Association, serving for countrywide BMD testing in Taiwanese, was available between 20082011. All participants must complete a questionnaire regarding risk factors of osteoporotic fracture in FRAX ${ }^{\circledR}$ tool before BMD testing. Followed the original OSTA model of Koh et al., we used the database to analyze potential risk factors and develop a risk index via multiple variable regression analysis and item reduction. Then, we used these index values to set up a simple algorithm (namely MOSTAi) for identifying those men who need BMD testing. Moreover, we validated MOSTAi and compared it with the OSTA and NOF 2013 by the receiver operating characteristic (ROC) curve analysis and analyzed their sensitivity/specificity.
Results: A total of 2290 Taiwanese men ( $\geq 50$ years) were enrolled in this survey. Through the random computerized number generator assigning these men to the development $(n=1145)$ and validation $(n=1145)$ cohorts, we used the split-sample method to develop and validate a MOSTAi index to identify Taiwanese men at increased risk for osteoporosis. In the development cohort, the index score was derived by age and body weight of the participants according to weighted odds of each risk factor and the selected index score was set at "11". In the validation cohort, the AUC for MOSTAi, OSTA and NOF 2013 were 0.706 (95 \% CI: $0.664-0.748, p<0.001), 0.697(95 \%$ CI: $0.657-$ $0.738, p<0.001)$ and $0.593(95 \%$ CI: $0.552-0.634, P<0.001)$, respectively. The sensitivity, specificity and accuracy of MOSTAi (cutoff value $=11$ ) and NOF 2013 to identify osteoporosis were $63.0 \%, 71.4 \%, 70.1 \%$ and $86.2 \%, 32.4 \%, 41.3 \%$, respectively. Conclusions: MOSTAi could be a more suitable model to identify Taiwanese men with osteoporosis than the original OSTA model. In comparison with NOF 2013, MOSTAi may be an easier and better tool for referral to BMD testing by DXA in Taiwanese men.

\section{P985}

MEDIAL COLLATERAL LIGAMENT BURSITIS IN A PATIENT WITH KNEE OSTEOARTHRITIS

H. Nur $^{1}$, A. Aytekin ${ }^{1}$, E. Gilgil ${ }^{1}$

${ }^{1}$ Department of Physical Medicine and Rehabilitation, Akdeniz University, Faculty of Medicine, Antalya, Turkey

Introduction: Medial collateral ligament (MCL) bursitis has been described as a distended and inflamed bursa often with fine septations between the superficial and deep portions of the MCL. It is a rare but important cause of medial knee pain and should be differentiated from other common conditions making medial knee pain like meniscal tears.

Case: A 65-y-old woman with knee osteoarthritis (OA) was admitted to our outpatient clinic with complaints of severe medial knee pain and swelling in her left knee. She did not have a traumatic injury and was treated elsewhere with nonsteroidal anti-inflammatory drugs with limited benefit. After a clinical examination slight decrease of knee range of motion, and a painful swelling and tenderness at the medial joint line were detected. The pain was exacerbated by valgus stress test. Laboratory tests were within normal range. Narrowing of the medial joint space, osteophyte formation, and soft tissue swelling were seen on plain radiographs. The MRI finding was a distended bursa with internal septations between the superficial and deep portions of the MCL. After a diagnosis of MCL bursitis an ultrasound-guided injection in the bursa was applied. There was a significant improvement of pain intensity and functional limitations in the follow-up examination, and a repeated MRI scan showed marked improvement and approximation of the deep and superficial MCL. 
Conclusion: Medial knee pain is widely seen and there are many possible etiologies such as medial meniscus tears, MCL tears, stress fractures, articular cartilage injury, and bursitis. MCL bursitis which is a rare condition must be considered for the differential diagnosis of medial knee pain in patients with knee OA. Isolated medial joint line pain reproduced by palpation and valgus stress is typical. The combination of clinical symptoms and imaging findings are important in confirming the diagnosis. Corticosteroid injection is an effective treatment of MCL bursitis.

\section{P986}

AN OPPORTUNITY TO BREAK THE FRAGILITY FRACTURE CYCLE: 10 MONTHS OF A FRACTURE LIAISON SERVICE

D. Rosa-Gonçalves $^{1}$, R. Vieira ${ }^{1}$, G. Terroso ${ }^{1}$, R. Matos $^{2}$, R.

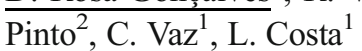

${ }^{1}$ Rheumatology Department, Centro Hospitalar São João, Oporto, Portugal, ${ }^{2}$ Orthopaedic Surgery and Traumatology Department, Centro Hospitalar São João, Oporto, Portugal

Introduction: A Geriatric Trauma Unit (GTU) was created in our hospital to optimize the acute treatment of patients with fragility fractures. The rheumatology department assists in subsequent evaluation of these.

Aim: To report the results of the evaluation after 10 months of a fracture liaison service (FLS).

Patients and Methods: All patients over 65 years old admitted to the GTU with a fragility hip fracture were identified on a daily basis. The protocol was implemented on March 2015 and we describe all patients included until December 2015. After a first evaluation during the hospital admission, patients are referred to the outpatient clinic for characterization. Investigations included were DXA (GE-Lunar), FRAX including DXA and laboratory blood tests. A descriptive study was performed.

Results: 208 cases of fragility hip fracture in patients above 65 years were identified, $78.9 \%$ women. Mean age (sd) of 83 years old (7.6). The most frequently affected segment was the trochanteric region $(n=109,52.4 \%)$, followed by the femoral neck $(n=75,36.1 \%)$. Most patients $(68.8 \%)$ underwent surgical intervention within the first $48 \mathrm{~h}$. Regarding risk factors for osteoporosis (OP), there was a high prevalence of selective serotonin reuptake inhibitor use $(n=40,20.1 \%)$ as well as antiepileptics $(n=10,5 \%)$. Only $13.5 \%(n=28)$ of the patients had past or current use of bisphosphonates (BP) and only $5.8 \%$ of those reporting previous fragility fracture were ever treated for OP. The prevalence of previous fragility fracture was $25.3 \%$ $(n=47)$, most commonly hip fracture $(n=19,10.2 \%)$. Prior to fracture, $62.5 \%$ of patients were independent for daily activities. However, within 3 months after the fracture, only $32 \%$ of these regain their autonomy. We have recorded 22 deaths. Full assessment of bone health was conducted in 44 patients. In $34 \%$ (15/
44) of the cases, the densitometric criteria for OP were not met and $20(45.5 \%)$ had radiographic signs of vertebral fragility fracture. Using the FRAX toll, the absolute risk of major osteoporotic fracture was higher than $20 \%$ in $8(18 \%)$ patients and the absolute risk of hip fracture was higher than $3 \%$ in 30 (68 \%) patients. Regarding treatment, 35 (84\%) started antiosteoporotic treatment (33 BP and 2 denosumab).

Conclusion: Fracture Liaison Services are key pieces in secondary fracture prevention and related complications.

\section{P987}

NUTRITIONAL MEASUREMENTS AS PREDICTOR'S OF BONE HEALTH IN OLDER IRISH ADULTS ( $>60$ YRS)

M. Casey ${ }^{1}$, C. Cunningham ${ }^{1}$, E. Laird ${ }^{2}, \mathrm{H} . \mathrm{McNulty}^{3}, \mathrm{~K}$.

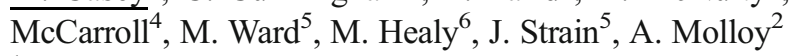

${ }^{1}$ Department of Medicine for the Elderly, St James Hospital, Dublin, Ireland, ${ }^{2}$ Institute of Molecular Medicine, Trinity College, Dublin, Ireland, ${ }^{3}$ Northern Ireland Centre for Food and Health (NICHE), University of Ulster, Colraine, Ireland, ${ }^{4}$ Department of Gerontology, St James Hospital, Dublin, Ireland, ${ }^{5}$ Northern Ireland Centre for Food and Health (NICHE), University of Ulster, Coleraine, Ireland, ${ }^{6}$ Department of Biochemistry, St James Hospital, Dublin, Ireland

Background and Aims: Osteoporosis in older subjects has a multifactorial aetiology. Nutritional factors contributing to this disease should be ameliorated where possible in conjunction with (if necessary) pharmacological therapies. Indeed the response to pharmacological therapies will be suboptimal if there are nutritional deficiencies. The aim of this study was to investigate the associations of nutritional abnormalities with measures of BMD with potential fracture risk.

Methods: This was an observational study conducted in a subset of community dwelling older participants ( $n$ 2983) from the Trinity, Ulster and Department of Agriculture (TUDA) cohort study, who had had DXA measures. Participants provided blood samples for serum albumin calcium, phosphate, glucose, vitamin D, PTH, serum Cholesterol HDL, LDL and renal function. A food frequency questionnaire was administered and BMD was measured by DXA at the lumbar spine, femoral neck and total hip.

Results: The cohort population was $67 \%$ Female (n 2008), with a mean age of the 70.8 y $\mathrm{SD} \pm 6.97$. Approximately $34.5 \%$ of participants were identified as osteopenic and $42.9 \%$ with osteoporosis being more prevalent in females $(30.8 \%)$ compared to males $(22.7 \% ; P<0.0001)$. In the total cohort, daily red meat intake was found to correlate positively with vertebral BMD $(r=+0.051 ; P=0.014)$. Serum cholesterol concentration was negatively correlated with femoral neck $(r=-0.149)$; total hip $(r=-0.184)$ and vertebral BMD 
$(r=-0.236)(P<0.0001)$. In a linear regression analysis, serum albumin was a positive predictor of total hip $(P<0.0001)$, femoral neck $(P=0.002)$ and vertebral BMD $(P=0.018)$, while serum cholesterol was a negative predictor of total hip $(P=0.006)$, femoral neck $(P=0.003)$ and vertebral BMD $(P=0.026)$ after adjustment for covariates (after age, BMI, smoking, alcohol consumption, GFR, phosphate, vitamin $\mathrm{D} /$ calcium supplement use and red meat).

Conclusion: This study has identified several nutritional risk factors for fracture, some of which are well recognized namely protein intake (as reflected in serum albumen and red meat intake) and others such as cholesterol are not known to have such a clear association with bone disease and may indeed be an indirect marker of another causative agent. However it underlines the importance of a broad biochemical screening of these patients and adjustment of these risk factors where possible.

\section{P988}

\section{OSTEOPOIKILOSIS: A CASE REPORT}

H. Nur ${ }^{1}$, T. Tuncer ${ }^{1}$

${ }^{1}$ Department of Physical Medicine and Rehabilitation, Akdeniz University, Faculty of Medicine, Antalya, Turkey

Objective: Osteopoikilosis is a rare asymptomatic bone dysplasia characterized by defective endochondral bone formation. Inheritance is autosomal dominant but sporadic forms are also present. Prevalence is approximately $1 / 50.000$, incidence is similar in both sex, and it can be seen at any age. Symmetric, numerous, well defined circular or ovoid sclerotic bone lesions are generally distributed in the epiphyses and metaphyses of the long tubular bones, carpal and tarsal bones, pelvis and scapulae. Case: A 26-y-old woman was admitted to outpatient clinic with complaints of low back and leg pain for the past 5 months. There was no history of trauma, systemic disease, joint pain, morning stiffness, and fever. Paravertebral muscle spasm, slightly limited and painful lumbar spine flexion, and a positive straight leg raise test were detected after a musculoskeletal examination. Multiple, symmetric, well defined sclerotic lesions were identified in femoral head, acetabulum, symphysis pubis, and ischium on lumbosacral plain radiographs. Similar lesions were also seen on shoulders, elbows, knees, hands and feet after radiologic examination. Laboratory tests were within normal range. Radionuclide bone scintigraphy was normal. A lumbosacral MRI was also performed and a herniated disc was detected between L4-L5. With these clinical and radiological findings, we concluded the diagnosis as osteopoikilosis and lumbar disc herniation. Management with nonsteroidal antiinflammatory drugs and physical therapy resulted in improvement of back and leg pain.

Conclusion: Osteopoikilosis is typically asymptomatic and most reported cases are identified during the evaluation of unrelated problems. It must be differentiated from other sclerosing dysplasias and sclerotic metastases. Multiple, circular or ovoid, well defined, symmetric, periarticular sclerotic bone lesions are characteristic radiological features of osteopoikilosis. When these lesions are found incidentally in radiological studies, osteopoikilosis should be considered for differential diagnosis to avoid misdiagnosis.

\section{P989}

NEUROFIBROMATOSIS TYPE 1 (VON RECKLINGHAUSEN DISEASE) AND OSTEOPOROSIS

I. Kostoglou-Athanassiou $^{1}$, I. Karaitianos ${ }^{2}$, C. Tsomidou ${ }^{3}$, P. Athanassiou $^{4}$

${ }^{1}$ Department of Endocrinology, Red Cross Hospital, Athens, Greece, ${ }^{2}$ Department of Surgery, St. Savvas Hospital, Athens, Greece, ${ }^{3}$ Department of Internal Medicine, Hippocrates Hospital, Pireaus, Greece, ${ }^{4}$ Department of Rheumatology, St. Paul's Hospital, Thessaloniki, Greece

Objectives: Neurofibromatosis type 1 or von Recklinghausen disease is a systemic hereditary disease characterized by disorders regarding the skin, neural and skeletal systems. Osteoporosis is one of the skeletal manifestations of neurofibromatosis type 1, being associated with increased fracture risk. Neurofibromatosis type 1 is associated with a propensity for the development of cancer, breast cancer in particular being associated with neurofibromatosis type 1 . The aim was to present the case of a patient with neurofibromatosis type 1 who developed breast and thyroid cancer and was diagnosed with osteoporosis.

Materials and Methods: A female patient, aged 47, with neurofibromatosis type 1 developed breast cancer at the age of 45 and had surgery and radiotherapy. The patient had a mother with neurofibromatosis type 1, who had breast cancer and had died from metastatic generalized breast cancer. A year later the patient presented with a nodule in the thyroid gland. Fine needle aspiration biopsy was performed and revealed the presence of papillary thyroid cancer. Near total thyroidectomy was performed and histology was positive for a papillary thyroid cancer. The patient complained for diffuse musculoskeletal pain, $25(\mathrm{OH}) \mathrm{D}_{3}$ levels and BMD were measured. $25(\mathrm{OH}) \mathrm{D}_{3}$ levels were $20 \mathrm{ng} / \mathrm{ml}$, Tscore was -2.9 .

Results: Ibandronate was administered at a dose of $150 \mathrm{mg}$ once monthly along with calcium and vitamin D orally. A year later BMD was measured, T-score being -2.7 , compatible with improvement in BMD.

Conclusions: Neurofibromatosis type 1 is a systemic hereditary disease. Recently, osteoporosis has been described as one of the features of the disease. The case of a patient has been described with neurofibromatosis type 1 and osteoporosis who improved after treatment with risedronate. The patient described herein was also given bisphosphonates with a good therapeutic response. 


\section{References:}

Benlidayi IC, et al. Acta Clin Belg 2015;70:130.

Kühnisch J, et al. PLoS One 2014;9:e86115.

\section{P990}

OSTEOPOROSIS IN MULTIPLE SCLEROSIS: SHOULD WE WORRY?

O.-M. Sirbu ${ }^{1}$, A. Sandu ${ }^{2}$, C. A. Sirbu ${ }^{3}$

${ }^{1}$ Bagdasar-Arseni Clinical Emergency Hospital, Bucharest, Romania, ${ }^{2}$ National Institute of Endocrinology, Bucharest, Romania, ${ }^{3}$ Central Military Hospital Bucharest, Bucharest, Romania

Background: Many neurological conditions are at high risk for bone fractures due to osteoporosis. People with multiple sclerosis (MS) have low bone density even at the onset of the disease, the role of the inflammatory processes being incriminated for both. Methods: We present a case series of 21 MS diagnosed patients and our findings regarding the risk factors for osteoporosis considering the lifestyle, medications, heredity.

Results: We found cumulative effects of various factors like age, Caucasian race, female, cigarette smoking, vitamin D deficiency, lack of physical activity and the use of medications such as glucocorticoids. The effect of different MS disease-modifying therapies on bone health was studied.

Conclusions: MS is a cause of secondary osteoporosis and an algorithm for diagnosis and monitoring of it should be considered. As well, the nursing processes of such patients should be managed properly.

\section{P991}

\section{ANTISYNTHETASE OR MECHANIC'S HANDS SYNDROME}

P. Athanassiou $^{1}$, A. Spyridis ${ }^{1}$, M. Kostopoulos ${ }^{1}$

${ }^{1}$ Department of Rheumatology, St. Paul's Hospital, Thessaloniki, Greece

Objectives: Antisynthetase syndrome is an inflammatory myopathy frequently associated with pulmonary manifestations, especially interstitial lung disease, and uncommonly pulmonary hypertension. In the context of a suggestive clinical and radiological picture, positive anti-RNA synthetase antibodies confirm the diagnosis. Anti-Jo1, anti-PL7, and anti-PL12 antibodies are the more commonly encountered. The presence of a number of extra-thoracic manifestations in association with pulmonary disease may suggest the diagnosis. The aim was to describe the case of a patient with antisynthetase or mechanic's hands syndrome.

Materials and Methods: A female patient, aged 37, presented with painful edema of upper extremities and thickened skin of tips and margins of fingers resembling a mechanic's hands. She had increased serum creatine kinase levels. She exhibited Raynaud's phenomenon and had arthritis of the wrists and knees. The patient did not have muscle weakness. Muscle biopsy was negative for vasculitis. Laboratory investigations revealed positive anti-Jo-1 and anti-Ro/SSA antibodies, while anti-La/SSB, anti-scl70 and rheumatoid factor were negative. A high resolution $\mathrm{CT}$ of the chest revealed ground glass appearance in the lower segments of both lungs, while spirometry showed restrictive lung disease. The diagnosis of antisynthetase or mechanic's hands syndrome was made.

Results: Due to lung involvement pulse methylprednisolone i.v. was administered followed by pulse cyclophosphamide i.v. The patient improved. Azathioprine along corticosteroids orally with calcium and vitamin $\mathrm{D}$ were administered. The patient is now stable.

Conclusions: Antisynthetase syndrome is characterized by the presence of interstitial lung disease, inflammatory myopathy, joint disease, Raynaud's phenomenon and characteristic skin lesions of the hands known as "mechanic's hands" associated with the presence of serum antibodies to RNA synthetases. In the case described herein immunosuppressive treatment resulted in improvement of disease manifestations. In the context of antisynthetase syndrome mechanic's hands may be considered a marker of visceral involvement.

References: Nahas O, et al. Ann Dermatol Venereo 2015;142:189.

\section{P992}

CORRELATION OF RADIOGRAPHIC EVIDENCE OF KNEE OSTEOARTHRITIS WITH THE CLINICO-DEMOGRAPHIC PROFILE AND MEASURES OF PAIN AND DISABILITY IN FILIPINO PATIENTS $\mathrm{J}_{\text {. De Jesus }}{ }^{1}$, A. Villarubin ${ }^{2}$, J. P. Lorenzo ${ }^{2}$

${ }^{1}$ Makati Medical Center, Makati City, Philippines, ${ }^{2}$ Rheumatology/Makati Medical Center, Makati City, Philippines

Background: Osteoarthritis $(\mathrm{OA})$ is the most common chronic joint disease locally and worldwide, and the most common joint associated with disability and functional impairment is the knee. Studies have found conflicting results with regards to the association of knee pain and radiographic severity.

Objectives: 1) To describe the demographic and clinical profile of Filipino patients with radiographic knee OA seen in the outpatient Rheumatology clinics of Makati Medical Center; and 2) To determine the presence or absence of correlation between patient characteristics, disability scores, pain, and radiographic severity of knee OA.

Methods: A single-center cross-sectional analytical study on adult Filipino patients $\geq 18$ years old, with symptomatic and radiologically confirmed knee OA seen in Rheumatology clinics of Makati Medical Center were included. Bilateral standing 
antero-posterior and lateral knee x-rays were reviewed and classified as KL grade 1, 2, 3, or 4 by a single radiologist.

Results: 43 out of 50 patients seen were enrolled. Subjects were predominantly female $(83.7 \%)$, with a mean age of $61.53 \mathrm{y}$ \pm 9.65 , and a mean BMI of $28.47 \mathrm{~kg} / \mathrm{m}^{2} \pm 6.9$. The HAQ-DI scores positively correlated with the VAS $(p=0.004)$, and KL grade $(p=0.003)$. History of smoking and alcohol intake, and presence of varus deformity were seen in patients with KL 3 $(p<0.05)$, while KL 1 and 2 had no knee deformity $(p=0.039)$. Bony enlargement was significantly associated with HAQ-DI and moderate to severe KL grade $(p<0.05)$. Obesity was significantly associated with VAS $(p=0.045)$, but no significant correlation was seen between knee pain and KL grade.

Conclusions: In the knee OA patients studied, higher HAQ-DI scores, indicative of physical function and disability, were directly proportional to pain and radiologic severity. Patient characteristics associated with pain and disability were BMI and bony enlargement. Smoking and alcohol intake were associated with moderate to severe radiographic OA. The VAS pain scores did not correlate significantly with radiographic findings.

\section{P993}

RELATIONSHIP BETWEEN BONE MINERAL DENSITY, PTH, 25(OH)D AND IGF-1 SERUM LEVELS IN ELDERLY MEN WITH OSTEOPOROSIS: RESULTS FROM A PRELIMINARY STUDY

$\underline{\text { F. Lumachi }}^{1}$, V. Camozzi ${ }^{2}$, R. Tozzoli ${ }^{3}$, G. B. Chiara ${ }^{4}$, S. M. M. Basso ${ }^{4}$

${ }^{1}$ University of Padua, School of Medicine, Department of Surgery, Oncology \& Gastroenterology (DiSCOG), Padova, Italy, ${ }^{2}$ University of Padua, School of Medicine, Department of Medicine, Padova, Italy, ${ }^{3}$ Clinical Pathology Laboratory, Department of Laboratory Medicine, S. Maria degli Angeli Hospital, Pordenone, Italy, ${ }^{4}$ Surgery 1, S. Maria degli Angeli Hospital, Pordenone, Italy
Background: Osteoporosis is relatively rare among men, but represents one of the leading causes of morbidity and mortality among elderly men. The precocious recognition of patients at increased risk of fracture is still a challenge for physicians. In this preliminary study we evaluated the relationship between BMD, PTH, 25-hydroxyvitamin D [25(OH)D] and IGF-1 serum levels in a group of osteoporotic men $(\geq 65$ years old).

Patients and Methods: A group of 15 elderly (median age 68 years, range 65-74 years) men with confirmed osteoporosis (LS T-score below $-2 \mathrm{SD}$ ) underwent PTH, 25(OH)D and IGF-1 serum levels measurement. As previously reported, PTH was analyzed by an immunometric assay (Intact PTH Bridge; Adaltis, Bologna, Italy) with a detection limit $10 \mathrm{pg} / \mathrm{mL}$ and inter- and intra-assay CV of 4 and $3.5 \%$, respectively. $25(\mathrm{OH}) \mathrm{D}$ was determined by a radioimmunoassay $(25(\mathrm{OH}) \mathrm{D}$ 125I RIA kit; DiaSorin, Stillwater, MN, USA); detection limit $5 \mathrm{nmol} / \mathrm{L}$ and inter- and intra-assay CV of $9 \%$. Serum IGF-I was measured by a immunoradiometric method (IGF-1 kit; Immunotech, Marseille, France) with detection limit $10 \mathrm{ng} / \mathrm{mL}$ and inter- and intra-assay CV of 11 and $4 \%$, respectively. The evaluation of lumbar spine (LS) BMD was obtained with DXA in all patients.

Results: The results were the following: LS$\mathrm{BMD}=0.777 \pm 0.060 \mathrm{~g} / \mathrm{cm} 2, \mathrm{PTH}=75.2 \pm 8.3 \mathrm{pg} / \mathrm{mL}$, $25(\mathrm{OH}) \mathrm{D}=365.1 \pm 134.4 \mathrm{nmol} / \mathrm{L}, \quad \mathrm{IGF}-1=201.3$ $\pm 55.3 \mathrm{ng} / \mathrm{mL}$. No correlation was found between age and LS- BMD $(R=-0.034, p=0.91)$, PTH $(R=0.276$, $p=0.31), 25(\mathrm{OH}) \mathrm{D} \quad(R=-0.235, p=0.40), \mathrm{IGF}-1$ $(R=0.219, p=0.43)$. No correlation between $25(\mathrm{OH}) \mathrm{D}$ and $\operatorname{BMD}(R=0.278, p=0.32), \mathrm{PTH} \quad(R=0.036$, $p=0.88)$, IGF-1 $(R=0.032, p=0.91)$, and between IGF-1 and PTH $(R=0.292, p=0.29)$. There was e significant inverse relationship between BMD and both PTH $(R=-0.511, p=0.047)$ and IGF-1 $(R=-0.662$, $p=0.007)$.

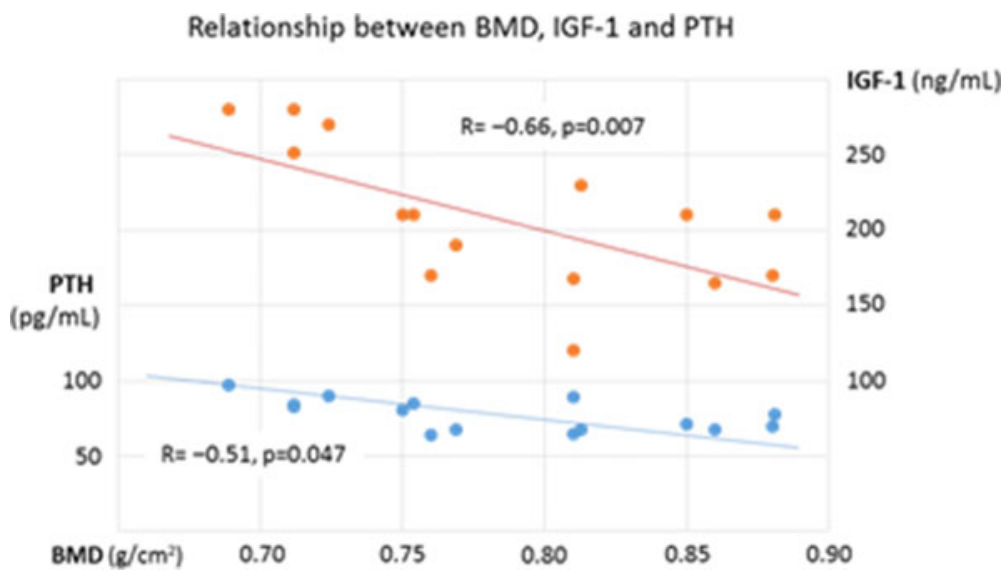


Conclusions: In this group of elderly osteoporotic men, LSBMD seems to be independent from the age and circulating $25(\mathrm{OH}) \mathrm{D}$, suggesting the need of a preventive therapy (e.g., bisphosphonates) to treat bone loss.

References: Lumachi F, et al. In Vivo 2013; 27: 415.

\section{P994}

RELATIONSHIP OF THYROID AND SEX HORMONES WITH OSTEOARTHRITIS IN POSTMENOPAUSAL INDIAN WOMEN

D. Sur ${ }^{1}$, R. Chakravorty ${ }^{1}$

${ }^{1}$ Obstetrics and Gynecology Outpatient Department MAGS Medical \& Research Center, Kolkata, India

Introduction: Osteoarthritis (OA) is the most frequent joint disease encountered in the clinical practice and is the most common cause of movement disability in the elderly. Females are found to have more severe OA; it increases in prevalence and incidence after menopause. OA affects all articular tissues and finally leads to joint failure. Many experimental and clinical studies suggest that loss of hormone especially estrogen at the time of menopause increases a woman's risk of getting OA and use of HRT did seem to be associated with not only relieving of symptoms but also reduced rate of progression of $\mathrm{OA}$.

Objective: The aim of this case control study was to investigate whether the thyroid and sex hormones are associated with OA in postmenopausal Indian women.

Methods: 125 patients suffering from OA and 82 control subjects, all aged 45-65 years, were included in this study. Thyroid and sex hormones were measured in the serum by ELISA and chemiluminescent immunoassay technique.

Results: Mean serum estrogen levels in the OA and control samples were $29.53 \pm 3.27$ and $49.21 \pm 2.18(p<0.0001)$, which were significantly lower in OA patients compared with controls. Also these patients show significant change in thyroid hormones and progesterone hormone levels when compared with control subjects.

Conclusion: The findings suggest that estrogen deficiency after menopause may contribute to develop OA in postmenopausal women. Estrogen can render help in such patients but with the current level of evidence, it cannot be recommended as a first-line treatment.

\section{P995}

ERDHEIM-CHESTER DISEASE: AN EXTREMELY RARE FORM OF HISTIOCYTOSIS WITH MULTIPLE BONE INVOLVEMENT

P. Athanassiou $^{1}$, T. Banti ${ }^{1}$

${ }^{1}$ Department of Rheumatology, St. Paul's Hospital, Thessaloniki, Greece
Objectives: Erdheim-Chester disease is an extremely rare form of non-Langerhans cell histiocytosis. Patients present with a wide variety of non-specific symptoms and signs that can occur in the disease process. The aim was to describe the case of a patient with a rare constellation of symptoms and multiple bone involvement, in whom the diagnosis of Erdheim-Chester disease was made.

Materials and Methods: A patient, male, aged 77 years, presented with dyspnea on exertion, fatigue, edema of the lower extremities and diffuse bone pain. He had a history of coronary artery disease, diabetes mellitus since 10 years and multiple recurring episodes of pleural effusion. On admission he was found to have bilateral pleural effusion and a diagnostic and evacuating paracentesis was performed. The fluid was an effusion; cytology performed was negative for the presence of cells. A CT scan of the chest and abdomen performed revealed the presence of abnormal tissue in the areas of the aortic arch, the pericardium, the left pleura, renal hilus and around the kidneys leading to the appearance of "hairy kidneys". Bone radiography revealed osteosclerotic bones in the upper and lower extremities. Osteosclerotic foci were also found in the metacarpal and metatarsal bones and calcification of the aortic arch was observed. A kidney biopsy performed revealed areas of fibrosis and infiltration of renal fatty tissue from histiocytes with foamy cytoplasm. CD68(+)CD1a(-) histiocytes were observed leading to the diagnosis of Erdheim-Chester disease.

Results: Methylprednisolone was administered orally at an initial dose of $32 \mathrm{mg}$ daily with subsequent tapering. The patient improved and is now stable.

Conclusions: The case of a patient with Erdheim-Chester disease is described. Erdheim-Chester disease is a rare, nonLangerhans histiocytosis, characterized by infiltration of tissues by foamy $\mathrm{CD} 68(+) \mathrm{CD} 1 \mathrm{a}(-)$ histiocytes. Interferon- $\alpha$ seems to be the best initial treatment for Erdheim-Chester disease, although tocilizumab, anakinra and infliximab are being tested. At the moment there is no definite cure for the disease.

References: Caglar E, et al. Spine J 2015;Pii:S15299430(15)01635-6.

\section{P996}

OSTEOPOROSIS IN A YOUNG MALE PATIENT WITH ANKYLOSING SPONDYLITIS AND BEHCET'S DISEASE

P. Athanassiou ${ }^{1}$, A. Spyridis ${ }^{1}$, A. Tzanavari ${ }^{1}$, M. Kostopoulos $^{1}$, I. Kostoglou-Athanassiou ${ }^{2}$

${ }^{1}$ Department of Rheumatology, St. Paul's Hospital, Thessaloniki, Greece, ${ }^{2}$ Department of Endocrinology, Red Cross Hospital, Athens, Greece

Objectives: Ankylosing spondylitis is a systemic inflammatory disease. Behcet's disease is a systemic inflammatory disease of unknown etiology, its pathogenesis possibly being 
related to an infectious agent. The coexistence of ankylosing spondylitis with Behcet's disease is extremely rare. It has become apparent that ankylosing spondylitis is related to the pathogenesis and development of osteoporosis. The aim was to describe the case of a young male patient with ankylosing spondylitis and Behcet's disease who developed osteoporosis. Materials and Methods: A patient, male, aged 42 years, developed at a young age recurrent oral and genital ulcers, anterior uveitis, arthritis and gastrointestinal symptoms. The diagnosis of Behcet's disease was made; corticosteroids were administered locally and orally for the treatment of uveitis resulting in disease remission. The patient had low back pain and arthralgias of the wrists and lower extremities since young adulthood. The diagnosis of ankylosing spondylitis with positive HLA-B27 was made. Initially, NSAIDs and corticosteroids were administered, while subsequently infliximab for a period lasting 2 years with very good response. Recently, he had a disease flare of ankylosing spondylitis, with limited mobility of the spine, sclerosis of the sacroiliac joints, ESR $50 \mathrm{~mm} / \mathrm{h}$, CRP $3.7 \mathrm{mg} / \mathrm{dl}$ (normal values $<0.5 \mathrm{mg} / \mathrm{dl}$ ) and a BASDAI of 6.4 .

Results: Infliximab was administered. BMD was measured and osteoporosis was diagnosed, $\mathrm{T}$-score being -2.7 . He was treated for osteoporosis with alendronate, along with calcium and vitamin D.

Conclusions: The coexistence of Behcet's disease with ankylosing spondylitis is extremely rare. In the patient described the coexistence of the above named disorders and the respective treatment caused the development of osteoporosis at a young age.

\section{P997}

THE IMPACT OF A CORE STRENGTHENING EXERCISE PROGRAM ON OSTEOPOROTIC WOMEN'S SELF-CARE MANAGEMENT TO IMPROVED HEALTH OUTCOMES AND QUALITY OF LIFE

\author{
A. Varahrami Vigeh ${ }^{1}$ \\ ${ }^{1}$ University of Western Ontario, London, Canada
}

Osteoporosis is a major health threat. Self-care management, monitoring and maintenance may have the most potential for improving osteoporotic women's health-related quality of life. This study focuses on how having women with osteoporosis (WwOP) participate in a core strengthening exercise program will help them in their daily activities. In particular, helping them overcome their fear of falling and breaking bones which tends to limit their capacity to enjoy their lives. Exercise programs for $\mathrm{WwOP}$ normally focus on limb strengthening and balance. However, osteoporosis is not just limited to their limbs. By not helping them strengthen their total body WwOP are not provided with the means to maintain their capacity to be both physically and psychologically active in their desired lives.
Objectives:

- To improve health outcomes by involving WwOP actively in their self-care management, monitoring and maintenance of their mobility and normal daily activities

- To test the impact of a core (instead of lower extremity) strengthening exercise program on osteoporotic women's confidence, fear of falls and fractures

Conceptual Framework: This study is to highlight the role of WwOP and their confidence and willingness to take action related to their capacity to manage, monitor, and maintain their health based on their level of activation (beliefs) about having an active role in one's health decisions and the extent of engagement in health behaviors (actions). Their level of activation will influence their capacity to manage, to monitor, and to maintain their health and their confidence in being able to carry out their daily activities and to perceive an improvement in their quality of life.

Outcomes: Findings from this study may reduce demand for health services, resulting in reduction of physical injuries and psychological impacts.

\section{P998}

BODY COMPOSITION USING DUAL-ENERGY X-RAY ABSORPTIOMETRY IN WOMEN OF REPRODUCTIVE AGE

N. Dal Ava ${ }^{1}$, C. Stein ${ }^{1}$, L. Bahamondes ${ }^{1}$, I. Monteiro ${ }^{1}$

${ }^{1}$ Department of Obstetrics \& Gynecology/State University of Campinas, Campinas, Brazil

Introduction: Quantifying and determining the components of body composition (BC) represent an important step in evaluating clinical and nutritional disorders. Notwithstanding, studies have yet to be conducted in Brazil to define reference values of fat mass (FM) and lean mass (LM) for the evaluation of BC using DXA.

Objectives: To evaluate body weight and the percentage of FM and LM in women of 20-50 years of age.

Methods: A cross-sectional study in which body weight, BMI, FM (kg), LM (kg),\%FM, \%LM, fat mass index (FMI) and fat-free mass index (FFMI) were evaluated in 639 healthy women stratified according to age group (20-25, 26-30, 31$35,36-40,41-45$ and $46-50$ years).

Results: Mean weight $( \pm \mathrm{SD})$ was $66.9 \pm 12.3 \mathrm{~kg}$, BMI was $26.2 \pm 4.6 \mathrm{~kg} / \mathrm{m} 2$ and $\% \mathrm{FM}$ was $41.6 \pm 7.4$. The women in the oldest age group (46-50 years) had an additional $8.0 \mathrm{~kg}$ in weight $(p<0.001)$ and $4.1 \mathrm{~kg} / \mathrm{m}^{2}$ in BMI $(p<0.001)$ compared to the youngest women, those in the 20-25-y age group. The highest mean values for weight, BMI, FM, LM and \%FM were found in the 46-50-y age group in which FM was $>3 \mathrm{~kg}$ $(p<0.001)$ and LM was $1.7 \mathrm{~kg}(p<0.005)$. There was no statistically significant difference between any of the age groups in relation to percentage FM $(p=0.063)$ or to 
percentage LM $(p=0.051)$. Mean FMI was $10.5 \pm 3.5$ and mean FFMI was $14.2 \pm 1.6$.

Conclusions: The population evaluated was overweight, with weight increasing as a function of age. Although the percentages of fat and lean mass remained unchanged, there was an increase in both fat and lean mass in women up to 50 years of age.

\section{P999}

INTRAPELVIC CEPHALIC SCREW MIGRATION OF AN INTRAMEDULLARY NAIL IN PATIENT WITH SEVERE OSTEOPOROSIS WHO SUFFERED HIP FRACTURE

$\underline{\text { R. Cañadillas Rueda }}^{1}$, N. Domingo Montesinos ${ }^{1}$, M. Natividad Pedreño $^{1}$

${ }^{1}$ Servicio de Cirugía Ortopédica y Traumatología, Complejo Hospitalario de Jaén, Jaén, Spain

Introduction: Hip fractures, distal radius fractures and vertebral fractures are the most common fragility fractures in the elderly directly related to the condition of osteoporosis. For its high morbidity and mortality, produced great social and economic impact. It is estimated that the incidence is approximately $250,000 /$ year in USA, and Spain are seeing a peak frequency, for the improved quality of life, increased life expectancy and improved the sanitary conditions of the population. It is estimated that in Spain the incidence is about 60,000 hip fractures/year over 60 years, with a mean age of 82 years for women and 79 men. Also, associated with an increase of cardiovascular, respiratory, renal, neurological comorbilities, and the direct suffering of others directly related to the elderly osteoporosis fractures. In osteoporotic hip fractures in the elderly, we distinguish two groups. Intracapsular, (more common in women) with important biological impact on the viability of the femoral head to be interrupted vascularization, which is usually treated hip arthroplasty (total or partial), and (more common in men) extracapsular fractures, biomechanics with important implications for the march to settle in a region with important anatomical muscle attachments on which depends the mobility of the thigh and hip, the most representative group are the intertrochanteric fractures and which is usually treated osteosynthesis.

Case: We present the case of a patient of 84 years that is brought to the emergency department of our hospital, after suffering accidental trauma left hip after falling own height. It is diagnosed with unstable intertrochanteric fracture type 31-A2 according to the classification system of the AO. Urgent surgery is scheduled, achieving complete fracture reduction and intramedullary nail fixation with $180 \mathrm{~mm}$, with dynamic cephalic screw $130^{\circ}$ and distal dynamic screw, under fluoroscopy and postoperative radiographic proper control. The patient ambulation and early rehabilitation begins without incident. A month later, the patient wanders with support walker, and referred pain in thigh and hip operated with correct radiological control. Three months referred pain is still present and is accented intermittently. In the radiograph bone healing is not yet appreciated. At 6 months the patient suffers own height fall spontaneously while wandering, which is brought to the emergency room. Radiography is performed, in which intertrochanteric fracture nonunion and CUT IN is appreciated: Removal of the intramedullary nail system, intrapelvic proximal migration of the cephalic screw. Massive rupture of the femoral head and the acetabulum by drilling it with guts and commitment intrapelvic neurovascular structures. It involved performing emergency removal of osteosynthesis material (intramedullary nail), sealing the acetabular defect and left partially cemented hip arthroplasty. Postoperative control is satisfactory. The patient ambulation and early rehabilitation begins with good results, with adequate pain control and support walker. At 6 months the patient is well, wanders with the support of a cane, no pain and

Discussion: Osteoporosis prevalence increases significantly with age and is the most important risk factor for the condition of fractures "fragility" to occur at low energy trauma in relation to poor bone quality severely osteoporotic bone, which treatment conditions:

1. Quality decreases bone implant fixation

2. Problems consolidation

3. Causes greater comminution of the posteromedial cortex (they are more unstable fractures)

4. There are more refractures index

Conclusion: The condition of a hip fracture at low energy trauma, implant failure, nonunion, removal of osteosynthesis system, and the destruction of the femoral head and acetabulum are feared complications and catastrophic consequences attributable to poor bone quality osteoporotic patient. It should establish a medical, rehabilitation and behavioral treatment for primary/secondary prevention of osteoporotic fractures in the population at risk.

\section{$\mathbf{P 1 0 0 0}$}

BILATERAL ATYPICAL FEMORAL FRACTURE PATIENT AGED 44 WITH EARLY MENOPAUSE AND SEVERE OSTEOPOROSIS TREATED WITH PROLONGED BISPHOSPHONATES

R. Cañadillas Rueda ${ }^{1}$, N. Domingo Montesinos ${ }^{1}, M$. Natividad Pedreño ${ }^{1}$

${ }^{1}$ Servicio de Cirugía Ortopédica y Traumatología, Complejo Hospitalario de Jaén, Jaén, Spain

Introduction: The "atypical femoral fractures" are a potential complication in patients diagnosed with severe osteoporosis receiving prolonged treatment with antiresorptive (Denosumab or aminobisphosphonates). They constitute a group of atraumatic fractures or trauma associated with low energy located in the subtrochanteric region or femoral shaft. 
They are characterized by a typical pattern of fracture; Usually they are not comminuted, and in a horizontal line that starts in the outer cortical, sometimes taking a peak at the inner cortex. There may be a periosteal reaction in the outer cortical and cortical thickening. The incidence of such fractures associated with bisphosphonate treatment is very low (3.2 to 50 cases/ $100,000 /$ year), especially when compared with the number of vertebral and hip fractures is estimated to be avoided with this treatment. They are seated on bone fractures with cortical thickened, reduced size, more common in women to be risk population suffering from osteoporosis and therefore be treated with antiresorptive medullary canal. The clinic is similar to the clinic of a femur fracture traumatic, although in the months before the fracture, the patient complains of dull and diffuse pain in the anterior thigh unyielding night. The treatment is the antegrade intramedullary nailing, with intense milling, given the cortical thickening and decreasing the diameter of the spinal canal. The American Society for Bone and Mineral Research (ASBMR) in 2014 has issued revised criteria for the diagnosis of this type of fracture. They must meet at least 4 of the 5 major criteria:

1. Major criteria of ASBMR:Produced spontaneously fracture or minimal trauma, such as falling from the same height

2. The line of the fracture must be horizontal, starting at the outer cortex, although it can sometimes be slightly oblique

3. When the fracture is complete may have a "peak in the medial cortical. Incomplete fractures affect only the lateral cortex

4. Are not comminuted or with minimal comminution fractures

5. There endosteal or periosteal thickening of the lateral cortex

Minor criteria of ASBMR:

1. There is a general thickening of the femoral cortical thickness

2. Prodromal symptoms in the form of unilateral or bilateral pain as pain in the thigh or groin

3. Bilateral femoral fracture Complete or incomplete

4. Delay of consolidation

Case: We present the case of a 45 -year-old diagnosed with early menopause at age 40 , in prophylactic treatment of osteoporosis with alendronate $70 \mathrm{mg} /$ week for 5 years. It is followed by the Gynecology and good evolution. It has come to the emergency room several times afflicting pain in both thighs and flashing legs, walking, resting and at night, you have come to affect their daily activity with diagnosis of peripheral vascular disease. Casually, the patient suffers fall of his own height, and is taken to the emergency room of our hospital with pain, deformity and loss of function in right thigh where after radiographic study and application of the criteria ASMBR is diagnosed with "atypical femoral fractures" in relation to long-term use of alendronate. It is subjected to elective surgery, which is performed antegrade intramedullary nailing, milling and vigorous double encerrojado under fluoroscopy in the right distal femur. At discharge, the patient begins a program of intense rehabilitation, and begins walking with canes support. Revisions per month, 3 months and 6 months elapse without incident and with good results, to walking without assistance and independent living. She consults a year without pain involved femur, but afflicting dull pain in the left thigh with similar characteristics to that I had before suffering a fractured right femur. Rx femur showing pathological features as criteria ASMBR and lateral cortical reaction takes place, which is clinically and radiologically stage prior to fracture. TAC unicortical study showing linear image, thickened cortical side and hypertrophic is performed. It is proposed to prophylactic surgery sick, proceeding to intramedullary nailing of the left femur, undergoing complete fracture of the femur during handling in the intervention. The subsequent radiological check is OK, and proceed to discharge to start a program of intensive rehabilitation to march with independent support, which is achieved at 3 months. At one year, the patient is asymptomatic and makes normal life.

Discussion: According to the algorithms proposed by the Group of Studies and Research on Osteoporosis (GEIOS), adopted by the Andalusian Society of Orthopedics and Traumatology, before a menopausal patient at high risk for osteoporotic fracture should ensure adequate intake of calcium and Vitamin D, and starting antiresorptive treatment (bisphosphonate first place, election Alendronate). Given the poor tolerance or treatment failure, treatment should be evaluated denosumab (alternative SERMs) and to a high risk of fracture or existence of various osteoporotic vertebral fractures or greater + osteoporotic vertebral, fracture treatment would assess teriparatide. After 5 years of treatment with antiresorptive, and 2 years of treatment with bone forming, treatment should be stopped in what is known as "therapeutic holidays". After this period of rest should valorarar continuity through risk reassessment Frop clinic with FRAX Tool (Spain version).

Conclusion: The atypical femoral fractures are associated with prolonged use of antiresorptive fractures. Patients treated with these drugs should be evaluated regularly, and after completing the maximum time limitation, reassessed to evaluate drug substitution secondary prevention of osteoporotic fractures.

\section{P1001}

ATYPICAL STRESS FRACTURE IN ACETABULUM WITHOUT ANTECEDENT TRAUMA IN PATIENT DIAGNOSED OF SEVERE OSTEOPOROSIS IN PROLONGED TREATMENT WITH BISPHOSPHONATES

R. Cañadillas Rueda $^{1}$, N. Domingo Montesinos ${ }^{1}$, M. Natividad Pedreño ${ }^{1}$

${ }^{1}$ Servicio de Cirugía Ortopédica y Traumatología, Complejo Hospitalario de Jaén, Jaén, Spain 
Introduction: The "atypical fractures" are a potential complication in patients diagnosed with severe osteoporosis receiving prolonged treatment with antiresorptive (aminobisphosphonates or Denosumab). They are a group or atraumatic fractures associated with low energy trauma usually located in the subtrochanteric region or femoral shaft. They are characterized by a typical pattern of fracture; Usually they are not comminuted, and in a horizontal line that starts in the outer cortical, sometimes taking a peak at the inner cortex. There may be a periosteal reaction in the outer cortical and cortical thickening. The incidence of such fractures associated with bisphosphonate treatment is very low (3.2 to 50 cases/ $100,000 /$ year), especially when compared with the number of vertebral and hip fractures is estimated to be avoided with this treatment. They are seated on bone fractures with cortical thickened, reduced size, more common in women to be risk population suffering from osteoporosis and therefore be treated with antiresorptive medullary canal. Acute clinical fracture clinic differs from conventional traumatic fracture, although typically, in the months leading to fracture the patient reports deaf and diffuse pain in the region affects unyielding night. The American Society for Bone and Mineral Research (ASBMR) in 2014 has issued revised criteria for the diagnosis of this type of fracture. They must meet at least 4 of the 5 major criteria:

Major criteria of ASBMR:

1. Produced spontaneously fracture or minimal trauma, such as falling from the same height

2. The line of the fracture must be horizontal, starting at the outer cortex, although it can sometimes be slightly oblique

3. When the fracture is complete may have a "peak in the medial cortical. Incomplete fractures affect only the lateral cortex

4. Are not comminuted or with minimal comminution fractures

5. There endosteal or periosteal thickening of the lateral cortex

Minor criteria of ASBMR:

1. There is a general thickening of the femoral cortical thickness

2. Prodromal symptoms in the form of unilateral or bilateral pain as pain in the thigh or groin.

3. Bilateral femoral fracture Complete or incomplete

3. Delay of consolidation

Case: We present the case of a 72 year old patient with a history of DM, hypertension and dyslipidemia, hysterectomy, with two crushed vertebrae L3 and L4 without remembering history of trauma, bilateral wrist fracture and fracture of proximal humerus after fall his own height eight years before, so it required surgery. After recovery of the fracture of the humerus, the patient has been followed up by the Department of Rheumatology of our hospital, diagnosed with severe osteoporosis T-score of -3.2 , so he began treatment for osteoporosis with alendronate $10 \mathrm{mg} / 24$ hours. It is derived from trauma outpatient primary care for nonspecific hip pain with no history of trauma, coxartrosis for evaluation of surgical treatment. The patient reported having a continuous dull pain in left hip that prevented ambulation autonomy, which uses a cane. It bothers him even at rest and when it is sleeping. Suspecting coxartrosis, Rx of pelvis and bilateral hip axial request. Strengthening the background of the left acetabulum, sclerosis and thickening of the background can be seen. Projections are repeated, and linear fracture set image seen with very strong well-defined sclerotic borders and periosteal reaction, compatible image left acetabular fracture. Insisting to the patient denies traumatic history now or years, and has never had pain so far. imaging study is expanded with TAC, in which linear image shown without fracture fragment separation, large sclerosis affecting medial anterior wall of the left acetabulum with small cysts marginal thickness and thickening, suggestive of fracture stress. MRI study was requested to rule out acute nature of the injury, in which bone edema image shown in the left acetabulum with linear stroke hyposignal regarding non-displaced fracture line.

Discussion: Prolonged use of antiresorptive has been associated with increased cortical and sclerotic bone, more common in long bones such as femur. However, a stress fracture in the acetabulum is extremely rare, and there are no documented cases in recent literature. According to the criteria of the ASBMR acetabular radiological images presented they may correspond to an insufficiency fracture in a secondary hiperescleroso bone metabolic change induced by prolonged treatment with bisphosphonates. According to the algorithms proposed by the Group of Studies and Research on Osteoporosis (GEIOS), adopted by the Andalusian Society of Orthopedics and Traumatology, before a menopausal patient at high risk for osteoporotic fracture should ensure adequate intake of calcium and vitamin D, and starting antiresorptive treatment (bisphosphonate first place, election Alendronate). Given the poor tolerance or treatment failure, treatment should be evaluated denosumab (alternative SERMs) and to a high risk of fracture or existence of various osteoporotic vertebral fractures or greater + osteoporotic vertebral, fracture treatment would assess teriparatide. After 5 years of treatment with antiresorptive, and 2 years of treatment with bone forming, treatment should be stopped in what is known as "therapeutic holidays". After this period of rest should valorarar continuity through risk reassessment Frop clinic with FRAX Tool (Spain version)

Conclusion: We suggest antiresorptive withdrawal after five years, to initiate a washout. After individual assessment of the risk of new fractures, if necessary you can start treatment with teriparatide for 2 years. The monitoring of patients treated with antiresorptive must be precise and avoid the development of complications such as atypical fractures. 


\section{P1002}

CALCANEAL AND IPSILATERAL FEMUR SPONTANEOUS FRACTURES AFTER LOW ENERGY TRAUMA IN A PATIENT WITH SEVERE OSTEOPOROSIS

$\underline{\text { R. Cañadillas Rueda }}^{1}$, N. Domingo Montesinos ${ }^{1}, M$. Natividad Pedreño $^{1}$

${ }^{1}$ Servicio de Cirugía Ortopédica y Traumatología, Complejo

Hospitalario de Jaén, Jaén, Spain

Introduction: Osteoporosis is the most common metabolic bone disease, a reduction in bone mass with a parallel loss of bone mineral and matrix collagen for bone resorption rate greater than the sum, with deterioration of bone microarchitecture and decreased resistance, bone fragility and consequently increased risk of fracture (whose most common locations are: spine, hip, distal forearm and proximal humerus). Prevalence: the $6 \%$ of men and $21 \%$ of women aged between 50-84 years are classified as osteoporotic.

Bone mass reaches its maximum peak at 30-35 years, with a progressive decrease of $20-30 \%$ in men and $40-50 \%$ in women. Is increasing evidence that the origin of osteoporosis can be started at an early age if adequate bone mass at maturity is not obtained, it is at risk of premature osteoporosis. The sloughs bone remodeling and skeletal remains after completion of growth. It occurs in areas of the skeleton called basic multicellular units (BMU). Approximately $10 \%$ of the skeleton is remodeled each year. Remodeling involves the succession of bone resorption and formation, two processes in which three proteins involved coupling cascade tumor necrosis factor (TNF). Two of these proteins RANK (receptor activator of nuclear factor kappa B) and RANKL molecules are attached to the membrane of osteoclasts and osteoblasts respectively pro. The RANKL binds to RANK in the presence of M-CSF (colony stimulating mast cell factor). The glycoprotein osteoprotegerin (OPG), secreted by osteoblasts, inhibits this binding.

Among the risk factors for osteoporosis we are:

- Genetic: Caucasian skin and white hair, scoliosis, osteogenesis imperfecta, premature menopause, thin constitution

- Behavioral: Snuff, excess alcohol, inactivity, malnutrition, Caffeine, amenorrhea induced by exercise, diet rich in fiber diet rich in phosphates

- Medications: glucocorticoids, thyroid hormones, diuretics, anticonvulsants (phenytoin), benzodiazepines and antidepressants.

The three major groups of settlement of osteoporotic fragility fractures are fractures of the hip, distal radius, and spine. But because osteoporosis is a systemic disease, any bone in the body can be fractured to trauma minimum energy in an osteoporotic patient.

Case: We present the case of a patient of 86 years with a history of dyslipidemia, smoking, a hemorrhagic stroke eight years ago with full resolution and with right hemiparesis sequel, acute myocardial infarction by taking anticoagulants, FMD, hip fracture 3 years and 3 vertebral compression. The patient is not wandering, is institutionalized, it is completely dependent caregivers and assistants. Despite his trauma history, the patient is not in treatment for osteoporosis, or make secondary prevention of fractures. The patient can stand up to daily grooming. In the course of his toilet, in a given time suffers sharp pain on one foot like a pinch, by falling to the ground, aquejándose of pain in the thigh and foot. It is brought to the emergency department of our hospital, where after performing the corresponding radiological study is diagnosed with comminuted fracture of left heel and shaft fracture of the left femur (which was already having an implant after being operated on for a hip fracture). CT study expands the calcaneus, which Sanders type IV fracture is seen, with irreconstruibles joint surfaces, and opt for orthopedic treatment, scheduling surgery for femur. During admission, suffers confusional state, disorientation with decreased level of consciousness, progressive deterioration, respiratory failure requiring urgent assistance on 2 occasions and that fails to recover. CT scan in which moderate $\mathrm{SAH}$ seen is performed. treatment and monitoring guidelines is established until the patient died four days later.

Discussion: The elderly osteoporotic fractures are among the most frequent causes of admission to a Traumatology. They are seriously ill by the severity and severity of trauma, as well as presenting comorbidities. In certain circumstances, the fracture should be operated to try to get the patient's recovery, but often can not be achieved by the development of complications in the patient: direct mortality, cognitive impairment, constipation, thromboembolism, surgical wound infection, urinary tract infections, decubitus ulcers, cardiovascular diseases, disoriented patient, pressure ulcers: in $20 \%$ patients (very variable rates), dementia, malnutrition and delayed surgery. In addition, osteoporosis extremely difficult surgery: technical and mechanical failures, failure to achieve anatomic reduction, penetration of implants in joints, false roads, lack of integration of implants, nonunion, acceptance of residual deformities, osteolysis, migration of implants, refractures by effect tip of nails or screws, collapse of joint surfaces, etc.

Conclusions: Patients treated at a Trauma Service, admitted for a fracture with the aggravation of having severe osteoporosis, handled worse, have higher rates of sequels, more complicated and have more risk of repeat or subsequent events resulting from trauma after discharge, the brittle fracture at minimum energy trauma. Osteoporosis is the most prevalent metabolic bone disease, and that most conditions the successful outcome of surgery, the patient management, and subsequent events due to immobilization and prolonged stay in bed. 


\section{P1003}

\section{COMPREHENSIVE TREATMENT AND SECONDARY} PREVENTION OF FRAGILITY FRACTURES IN THE ELDERLY IN AN ORTHOGERIATRIC UNIT. MULTIDISCIPLINARY MANAGEMENT OF OSTEOPOROTIC PATIENTS PRE AND POST SURGERY. ADVANTAGES AND RESULTS.

R. Cañadillas Rueda $^{1}$, N. Domingo Montesinos ${ }^{1}, \mathrm{M}$. Natividad Pedreño $^{1}$

${ }^{1}$ Servicio de Cirugía Ortopédica y Traumatología, Complejo

Hospitalario de Jaén, Jaén, Spain

Introduction: Hip fracture and fracture of the proximal end of the femur, is the most serious complication of osteoporosis in terms of mortality, morbidity and costs. It is a common entity in the elderly population, if we consider the increased life expectancy and population growth in civilized countries, hip fracture and represents a major health challenge. Despite being surgical pathology, the complexity of patients from the medical point of view the problem worse and complications and mortality in these patients is more associated with medical comorbidity surgery. Are elderly, osteoporotic pluripatológicos in multidrug therapy, a significant percentage have a history of cognitive/ dementia deterioration, all or which favors the occurrence of medical complications during hospitalization. The incidence of Fx C in Spain to follow an upward trend, with 50,000-60,000 cases/year and it is estimated that this figure will double by 2050 . It can occur at any age, but $85-90 \%$ of cases occur in people over 65 , the average age of affected patients is 82 years, although in recent publications and talking of 84 years. The mortality rate per year is around $30 \%$. Recovering the previous functional capacity less than $50 \%$ of cases. For its prevalence, consequences and the high consumption of resources involved, the elderly hip fracture has become the focus of medical, surgical collaboration, with the paradigm of multidisciplinary team in order to offer these patients Integrated treatment. The primary goal of treatment is to get a proper consolidation of fracture after surgery, with the lowest degree of perioperative morbidity and mortality, subsequent functional recovery maximum reduce the degree of dependence that this disease generates. Beyond a better optimization of resource consumption. Thus arises the concept of unit Orthogeriatrics as the multidisciplinary team composed of internists, rehabilitation, orthopedic surgeons, anesthesiologists, nurses and nutritionists, in collaboration with primary care physicians, is responsible for the comprehensive treatment of elderly patients with hip fracture osteoporotic origin, not only from a surgical point of view, but of all medical, rehabilitation and social processes that is associated with the patient.

Objective: To assess the role of each health care unit Orthogeriatrics and various items of improvement in attendance: Internists, rehabilitation, orthopedic surgeons, anesthesiologists, nurses and nutritionists. Comparison of data obtained with the previous year and assess the results.
Material and methods: From March 2015 to March 2016, we selected all patients seen at our center, diagnostiados hip fracture and underwent surgery (approximately 320). They have been divided into groups by gender, age range, gender, type of hip fracture and surgical considerations. They are rated preadmission comorbidities, treatments, days of delay from admission to surgery and its cause (most common taking anticoagulants or antiplatelet drugs or anesthetic contraindication for medical problems), has been valued time to the high and incidents during hospitalization, such as surgical or medical complications. We evaluate the rate of intraoperative exitus, and the incidence of events that have doomed to exitus. It has valued the start of loading and improved function after surgery and in subsequent months, and has spent a questionnaire Barthel function, an analog scale of pain and a specific questionnaire of satisfaction and benefit to the patient and family.

Results: Fewer medical complications from surgery wait. Lower hospital mortality ( $7.2 \%+/-11 \%$ Prior to our center). Reduction of the average hospital stay of 13 days to 7 days. Reduction in the number of postoperative complications due to early loading and immediate restoration. Improving autonomy, pain and function, with Reducers dependency. Optimized drug prescriptions. Decrease in the number of readmissions for hip fracture. Treatment of osteoporosis at high/secondary prevention of fractures. Increased number of diagnostic and early detection of clinical problems (stroke, gastrointestinal, kidney, etc.), improvement in functional status at discharge. Better use of outpatient care levels, social workers and nurses liaison team working together orthogeriatric. Improvement in Barthel score on average 30 points, decreased pain in average 4.3 points and degree of high or very high patient satisfaction and family.

Conclusions: The implementation of Orthogeriatrics Unit in our center, is a model of multidisciplinary care to patients with fragility fractures, of which the paradigm is hip fracture the elderly. It has brought a revolution in social and healtheconomic terms and is a model of management and management of patients with spectacular for resource optimization results, quality care, systematizing the individualized assistance and personnel adequacy of personal needs. It has decreased morbidity and mortality, hospital stay and increased happiness and satisfaction of the patient and family.

\section{P1004}

CONDITION OF BONE MINERAL DENSITY IN CHILDREN WITH SYSTEMIC SCLEROSIS AND LIMITED SCLERODERMA

I. Chyzheuskaya $^{1}$

${ }^{1}$ Belarusian Medical Academy of Postgraduate Education, Minsk, Belarus

Osteoporosis is a systemic complications rheumatic diseases. The purpose of research - to assess bone mineral density in children 
suffering from various forms of scleroderma. 24 children with limited scleroderma and 11 children with systemic sclerosis were examined by dual-energy X-ray absorptiometry. In 13 children with limited scleroderma and 8 children with systemic sclerosis observed decline in bone mineral density. All of these patients received basic therapy with corticosteroids and cytotoxic drugs for more than 6 months. A direct correlation between the decrease in bone mineral density and the level of tumor necrosis factor alpha, and disease activity. The results make it possible to assume that the decrease in bone mineral density in children with scleroderma may be due to ongoing therapy and immune processes caused by the underlying disease.

\section{P1005}

BONE MINERAL DENSITY AND ASSOCIATED RISK FACTORS - IN HEALTHY INDIAN POPULATION

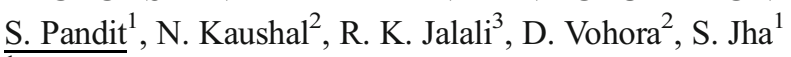
${ }^{1}$ Institute of Endocrinology, Diabetes and Matabolism, Max Healthcare Inst. Ltd, New Delhi, India, ${ }^{2}$ Pharmaceutical Medicine, Faculty of Pharmacy, Jamia Hamdard, New Delhi, India, ${ }^{3}$ Medical Affairs \& Clinical Research and Global Head Pharmacovigilance, Sun Pharmaceutical Limited, Gurgaon, India

Introduction: Osteoporosis is a global public health problem affecting more than 200 million people worldwide. Osteoporosis has clinical and public health implications because of the mortality, morbidity, and cost of medical care associated with osteoporotic fractures. The World Health Organization (WHO) has defined criteria for assessing bone status by DEXA, which are defined by the T-score, which is the number of standard deviations (SDs) by which a patient's test result exceeds (positive T-score) or falls below (negative T-score) the mean value expected in young healthy individuals. Epidemiology of osteoporosis varies based on risk factors prevalent in a particular geography e.g., dietary habits, socioeconomic pattern such as parda custom, exposure to sun, alcohol drinking, smoking habits etc. An understanding of bone mineral density pattern and associated risk factors is crucial for prevention, diagnosis of osteoporosis and management of its complications in later life. Better understanding of known and novel risk factors is expected to improve the decision taken by physician in the prevention and management of osteoporosis.

Methods: This research involves retrospective data collection, from the Preventive Health Check up Department at Max Superspeciality Hospital, Saket, New Delhi, India, over a period of 1 year (2014-15). The classification for status of BMD has been done based on WHO criteria i.e., normal BMD (T score $\geq-1$ ), osteopenia ( $\mathrm{T}$ score $<-1$ but $>-2.5$ ) and osteoporosis ( $\mathrm{T}$ score $\leq-2.5$ ). The data was collected in structured questionnaire and then recorded in Excel file. Statistical Analysis has partially been done using SPSS version 16.0.
Results: The analyzed population included $57 \%$ males; age range: $20-85$ years and $43 \%$ females; age range: 21-79 years. As per preliminary analysis, osteoporosis has been observed in $7.7,5.4,5.4,3.2$ and $4.5 \%$ subjects at lumber spine (L1-L4), femur neck (left), femur neck (right), total femur (left) and total femur (right) respectively. Osteopenia has been observed in 31.7 , $34.8,32.6,30.8$ and $28.5 \%$ subjects at lumber spine (L1-L4), femur neck (left), femur neck (right), total femur (left) and total femur (right) respectively. Prevalence of osteoporosis increased in this population as the age progressed: $<40$ years $(0 \%), \geq 40$ years $(10 \%), \geq 50$ years $(13 \%), \geq 60$ years $(16 \%)$ and $\geq 70$ years $(17 \%)$. In gender-wise analysis for left femur neck bone status, more females reported low BMD (osteoporosis + osteopenia) as compared to males (F vs. M: 48 vs. $35 \%$ ). This is to reiterate that results are preliminary only based on unpublished data.

Conclusion: Taking the aforementioned into consideration, an exploratory research has been initiated aimed to analyze osteoporosis disease in adult urban population and to identify risk factors influencing bone mineral density. Further data collection on BMD and risk factors is currently ongoing and will be presented at the Conference.

\section{P1006}

CHARACTERIZATION OF HUMAN PERIPHERAL BLOOD CD34+ HEMATOPOIETIC STEM CELLS TRANSDUCED WITH TELOMERASE GENE AND THE EFFECT TO BONE PROFILES OF SPRAGUE-DAWLEY RATS OSTEOPOROSIS MODEL

$\underline{\text { S. Basalamah }}^{1}$, E. Hutagalung ${ }^{2}$, I. H. Dilogo ${ }^{2}$, D. Suhendro ${ }^{3}$, M. Hidayat $^{4}$, A. Boediono ${ }^{5}$, A. A. Yusuf ${ }^{6}$, J. Pandelaki $^{7}$, S. Bardosono $^{8}$

${ }^{1}$ Doctoral Program, Fakultas Kedokteran Universitas Indonesia - Rumah Sakit Cipto Mangunkusumo, Jawa Barat, Indonesia, ${ }^{2}$ Department of Orthopaedic and Traumatology, Fakultas Kedokteran Universitas Indonesia - Rumah Sakit Cipto Mangunkusumo, Jawa Barat, Indonesia, ${ }^{3}$ Department of Internal Medicine, Fakultas Kedokteran Universitas Indonesia - Rumah Sakit Cipto Mangunkusumo, Jawa Barat, Indonesia, ${ }^{4}$ Department of Surgery, Universitas Brawijaya, Jawa Timur, Indonesia, ${ }^{5}$ Department of Anatomy, Physiology, and Pharmacology, Institut Pertanian Bogor, Jawa Barat, Indonesia, ${ }^{6}$ Department of Histology, Fakultas Kedokteran Universitas Indonesia - Rumah Sakit Cipto Mangunkusumo, Jawa Barat, Indonesia, ${ }^{7}$ Department of Radiology, Fakultas Kedokteran Universitas Indonesia Rumah Sakit Cipto Mangunkusumo, Jawa Barat, Indonesia, ${ }^{8}$ Department of Nutrition, Fakultas Kedokteran Universitas 
Indonesia - Rumah Sakit Cipto Mangunkusumo, Jawa Barat, Indonesia

Background: The role of osteoblasts decreased in osteoporosis. Stem cells are required as an alternative to long-term therapy of osteoporosis. Human peripheral blood CD34+ hematopoietic stem cells can transdifferentiate into osteoblast and have antiosteoclast activity. Telomerase gene transduction on CD34+ stem cells is expected to enhance the osteogenic potential to improve bone profiles of sprague dawley rats osteoporosis model.

Objective: To prove the osteogenic potential of human peripheral blood CD34+ hematopoietic stem cells into osteoblasts have better result after telomerase gene transduction.

Methods: The preparation studies consisting of the making of osteoporosis model, stem cell isolation, transduction, rejection study, and in vitro study. The main study using a control group, nontelomerized CD34+ stem cells, and telomerized CD34+ stem cells. Analysis was performed to evaluate the radiological profile, histomorphometrical profile, and biomarkers of osteocalcin and TRAP.

Results: CD34+ stem cell transdifferentiate into osteoblast, but not into osteoclast. There were improvements in osteoporosis bone profiles in all treatments. Telomerase did not give significant differences.

Conclusion: This study still cannot prove that the CD34+ stem cells with telomerase better in improving bone profile compared with that without telomerase.
P1007

\section{AGE-STANDARDIZED QUALITY OF LIFE AFTER HIP OR KNEE ARTHROPLASTY}

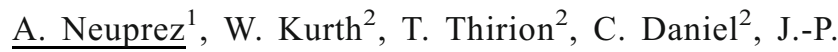
Huskin $^{2}$, P. Gillet ${ }^{2}$, O. Bruyère ${ }^{1}$, J.-Y. Reginster ${ }^{1}$

${ }^{1}$ Department of Public Health, Epidemiology and Health Economics, Liège, Belgium, ${ }^{2}$ Orthopaedic Surgery CHU, Liège, Belgium

Objective: To compare quality of life benefits of total knee/hip replacement for osteoarthritis $(\mathrm{OA})$ in patients below and above the age of 70 years.

Methods: 626 consecutive patients undergoing total knee or hip arthroplasty (TKA/THA) were enrolled in this prospective study and were stratified into 2 groups based on their age ( $<$ or $>70$ years). The primary analysis compared changes between baseline and 12 months post-surgery in health related quality of life (HRQOL) using the EQ5D and EQ5D Visual analogue scale (VAS). The Western Ontario and McMaster Universities Osteoarthritis Index (WOMAC) was obtained preoperatively and 1 year postoperatively (short-term follow-up) and the values (total and subscales) were compared between groups. Paired Student T-tests were used to compare mean values of EQ5D, EQVAS, and WOMAC between the 2 groups preoperatively and 12 months post-surgery.

Results: 626 patients were enrolled the day before TKA (280) or THA (346). They were all diagnosed with primary OA following the ACR criteria. Our cohort included 270 men and 356 women aged from 26 to 86 years (mean 65.6 \pm 10.1 ) and with a body mass index (BMI) of $28.4 \pm 5.8$. We calculated the various dimensions of WOMAC (pain, stiffness, physical function), EQ5D and EQVAS.

\begin{tabular}{|c|c|c|c|c|}
\hline \multirow{2}{*}{$\begin{array}{c}\text { Time of administration } \\
\text { Age }\end{array}$} & \multicolumn{2}{|c|}{ Preoperatively } & \multicolumn{2}{|c|}{12 months post-surgery } \\
\hline & $<70$ years & $\geq 70$ years & $<70$ years & $\geq 70$ years \\
\hline Variable & $n=402$ & $\mathrm{n}=\mathbf{2 2 4}$ & $n=362$ & $\mathrm{n}=187$ \\
\hline \multirow[t]{2}{*}{ BMI } & $28.8 \pm 5.2$ & $\begin{array}{l}27.5 \pm 4.6 \\
.05\end{array}$ & & \\
\hline & \multicolumn{4}{|c|}{$p<0.05$} \\
\hline \multirow[t]{2}{*}{ EQ5D (0-1) } & \multicolumn{2}{|c|}{$p=0.09$} & \multicolumn{2}{|c|}{$p=0.49$} \\
\hline & \multicolumn{4}{|c|}{$p<0.05$} \\
\hline EQVAS $(0-100)$ & \multicolumn{2}{|c|}{$p=0.32$} & \multicolumn{2}{|c|}{$p=0.88$} \\
\hline WOMAC & \multicolumn{4}{|c|}{$p<0.05$} \\
\hline Pain (0-20) & \multicolumn{2}{|c|}{$p=0.06$} & \multicolumn{2}{|c|}{$p=0.58$} \\
\hline Stiffness (0-8) & \multicolumn{2}{|c|}{$p=0.21$} & \multicolumn{2}{|c|}{$p=0.70$} \\
\hline Physical function (0-68) & \multicolumn{2}{|c|}{$p=0.9$} & \multicolumn{2}{|c|}{$p=0.57$} \\
\hline TOTAL (0-96) & $53.7 \pm 16.4$ & $\begin{array}{l}53 \pm 18.3 \\
.64\end{array}$ & $24.2 \pm 21.6$ & $\begin{array}{l}24.7 \pm 19.2 \\
0.79\end{array}$ \\
\hline
\end{tabular}


Conclusion: We concluded that OA patients undergoing total joint replacement in a University setting, in Belgium, experience a significant improvement of health and QOL 12 months after surgery. 12 month after the operation, the EQ5D, the EQVAS and all components of the WOMAC scores show a statistically significant improvement compared to pre-surgical status. Patients 70 years and older have similar improvements in HRQOL and OA symptoms compared with patients younger than 70 years. The only observed difference relates to the BMI. Therefore, changes in quality of life support TKA/THA as a treatment option for elderly patients at advanced stage knee/hip osteoarthritis.

\section{P1008}

HIP FRACTURE INCIDENCE IN JAPAN: ESTIMATES OF NEW PATIENTS IN 2012 AND 25-YEAR TRENDS

$\underline{\text { H. Orimo }}^{1}$, Y. Yaegashi ${ }^{2}$, T. Hosoi ${ }^{1}$, Y. Fukushima ${ }^{3}$, T.

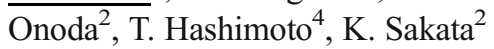

${ }^{1}$ Kenkoin Clinic, 6-7-4, Ginza, Chuo-ku, Tokyo, Japan, ${ }^{2}$ Department of Hygiene and Preventive Medicine, School of Medicine, Iwate Medical University, 2-1-1, Nishitokuta, Yahaba-cho, Shiwa-gun, Iwate, Japan, ${ }^{3}$ Ministry of Health, Labour and Welfare, 1-2-2, Kasumigaseki, Chiyoda-ku, Tokyo, Japan, ${ }^{4}$ Department of Public Health, Wakayama Medical University, 811-1, Kimii-dera, Wakayama City, Wakayama, Japan

Objectives: The objectives of this study were to estimate the number of hip fracture patients in 2012, to investigate the trends in incidence during a 25 -year period from 1987 to 2012, and to determine the regional differences in Japan.

Material and Methods: We estimated the number of hip fracture patients in 2012 in Japan and investigated the trends in incidence during a 25-year period from 1987 to 2012. The data for new hip fracture patients were collected through a nationwide mail-in survey based on hospitals and clinics throughout Japan. Hip fracture incidences by sex and age and standardized incidence ratios by 12 regions were calculated. These data are summarized with the survey previously reported ${ }^{1}$.

Results: The estimated numbers of new hip fracture patients in 2012 were 175,700 in total ( $95 \%$ CI 170,300-181,100), 37, $600(36,600-38,600)$ for men and 138,100 (134,300-141, $900)$ for women. The incidence rates in both men and women aged 70-79 years were the lowest in the 20-year period from 1992 to 2012. The incidence was higher in western areas of Japan than that in eastern areas in both men and women; however, the difference in the incidence of hip fracture between western and eastern areas is becoming smaller.
Conclusions: Despite the increasing number of new patients, the incidence of hip fracture in both men and women aged 60 79 years showed the trend to decline. The exact reasons for this are unknown, but various drugs for improving bone mineral density or preventing hip fracture might have influenced the results. A decrease in the differences in nutrient intake levels might explain some of the change in regional differences in Japan.

\section{References:}

1. Orimo H, Yaegashi Y et al., Hip fracture incidence in Japan: estimates of new patients in 2007 and 20-year trends, Arch Osteoporosis 4;71-77, 2009

Acknowledgement: This study was supported by Japan Osteoporosis Foundation and Japan Osteoporosis Society. We wish to express our sincere thanks to all hospitals and clinics which participated in this survey.

\section{P1009}

THE DEVELOPMENT OF A ONE YEAR FRACTURE PREDICTION TOOL FOR ABSOLUTE HIP FRACTURE RISK IN THE FRAIL ELDERLY

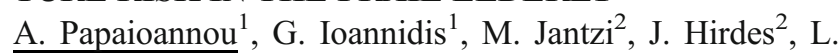
Giangregorio $^{3}$, M.-L. van der Horst ${ }^{4}$, J. D. Adachi ${ }^{2}$

${ }^{1}$ Medicine, McMaster University, GERAS Centre, Hamilton, Canada, ${ }^{2}$ Health Studies and Gerontology, University of Waterloo, Waterloo, Canada, ${ }^{3}$ Kinesiology, University of Waterloo, Waterloo, Canada, ${ }^{4}$ Medicine, McMaster University, Hamilton, Canada

Objectives:The purpose of our study was to develop and validate a 1-year hip fracture risk prediction tool for longtermcare (LTC) residents.

Materials and Methods:Using the Resident Assessment Instrument - Minimum Data Set 2.0 (RAI-MDS) all residents with a LTC admission assessment from Ontario, Canada from April, 1, 2006 to March, 31, 2010 were eligible for study inclusion $(\mathrm{N}=47,556)$. Residents were excluded from the analysis if they had a high likelihood of not surviving the one year follow-up period (i.e. receiving hospice or respite care). All potential risk factors variables for tool development including traditional risk factors such as prior fractures and fall, age, gender, height and weight, and LTC specific risk factors such as walking ability, wandering status, transfer status and cognitive function were extracted from the RAI-MDS. The RAIMDS data were then linked to the Discharge Abstract Database and National Ambulatory Care Reporting System to collect additional data on the residents' new hip fracture status. Using the combine dataset $(\mathrm{N}=29$, 848 ), decision tree analyses were conducted utilizing a derivation $(\mathrm{N}=22,386)$ and validation $(\mathrm{N}=7462)$ dataset to determine the final hip fracture prediction tool. The c- 
statistic was calculated for both the derivation and validation datasets to determine the decision tree's performance. Results:Of the 29,848 residents included, 19706 (66\%) were women and 13708 (46\%) were over the age of 85 years. A total of $959(3.2 \%)$ residents developed a new hip fracture. The final tool had 8 categories of absolute hip fracture risk (Table). A c-statistic of 0.67 and 0.69 was calculated for the derivation and validation datasets, respectively.

Conclusion:Our hip fracture prediction tool is effective at both predicting and discriminating the frail elderly at risk for a future hip fracture. The tool may be beneficial as a Clinical Assessment Protocol (CAP) for standardized fracture risk assessment in both LTC and home care settings.Categories of absolute hip fracture risk

\begin{tabular}{ll}
\hline Risk level & Incidence of hip fracture (1 year) \\
\hline 0 & 0.6 \\
1 & 1.8 \\
2 & 2.5 \\
3 & 3.1 \\
4 & 5.0 \\
5 & 6.8 \\
6 & 7.8 \\
7 & 12.6 \\
\hline
\end{tabular}

Acknowledgement:The study was supported by Amgen Inc.

Disclosures:AP- Grants/Research support: Amgen, Eli Lilly, Merck; Speaker's Bureau: Amgen, Eli Lilly, Merck; Consulting Fees: Amgen, Eli Lilly, Merck. LG- Grants/ Research support: Amgen; Consulting Fees: Eli Lilly. JDGrants/ Research support: Amgen, Eli Lilly, Merck; Speaker's Bureau: Actavis, Amgen, Eli Lilly, Merck; Consulting Fees: AgNovos, Amgen, Eli Lilly, Merck. GI, MJ, JH, M-L VH- None.

\section{P1010 \\ HYPOVITAMINOSIS D IN A YEAR-ROUND SUNNY AREA: OUTDOORS AND INDOORS LIFESTYLE PERSPECTIVES}

H. M. Al Attia ${ }^{1}$

${ }^{1}$ Department of Internal Medicine and Rheumatology, Universal Hospital, Abu Dhabi, United Arab Emirates

Background: Abu Dhabi, the capital city of UAE enjoys sunny blue skies throughout the year with an averagetemperature of $34.46 \mathrm{C}$. The temperature between May-September is usually as high as 39.3$40.4 \mathrm{C}$. Generally, the residents do not prefer to expose themselves to sunlight to avoid the intense heat. This is an attempt to have more understanding of the relationship between the outdoors and indoors lifestyle in patients with hypovitaminosis $\mathrm{D}(<26 \mathrm{ng} / \mathrm{ml})$ in $\mathrm{Abu}$ Dhabi.

Methods: Data of patients (different socioeconomic classes) without vitamin D supplementation underwent 25 (OH) D3 assay along with assays for $\mathrm{Ca}$ and PTH as well are presented. The patients were asked to classify their daily activity (from morning to 3 or $5 \mathrm{PM}$ ) as being predominantly (outdoors, indoors or both). Patients with chronic renal failure or on corticosteroids treatment were not included here.

Results:By collecting 150 patients information it became obvious that the majority 119 (79.5\%) were of predominantly indoors lifestyle ( $\mathrm{G} 1$ ) vs. 12 only of outdoors lifestyle $(8 \%)(\mathrm{G} 2)$ and 19 of both $(12.5 \%)$ (G3) together $\mathrm{P}=0.0001$. Individuals from various Arab states formed the bulk (76.5\%) of patients. Interestingly, the mean of $25(\mathrm{OH}) \mathrm{D}(17.5 \pm 5.64$ in $\mathrm{G} 1$ vs. $16.3 \pm 6.42$ in $\mathrm{G} 2, \mathrm{P}=0.48,17.5 \pm 5.64$ in $\mathrm{G} 1$ vs. $17.6 \pm 7.92$ in $\mathrm{G} 3, \mathrm{P}=0.99 \& 16 . \pm 6.42$ in $\mathrm{G} 2$ vs. $17.6 \pm 7.92 \mathrm{ng} / \mathrm{ml}$ in $\mathrm{G} 3 \mathrm{P}=0.65)$, Ca, age, and prevalence of secondary hyperparathyroidism (SHPTH) and vitamin $\mathrm{D}$ deficiency were not different between the groups, $\mathrm{Ps}=\mathrm{NS}$. Likewise, when the same data of $\mathrm{G} 1$ were compared to those of G2 \& G3 collectively, $\mathrm{Ps}=\mathrm{NS}$. Gender data showed no difference in $\mathrm{G} 1(\mathrm{~F}=66$ vs. $\mathrm{M}=53), \mathrm{P}=0.119$, but with higher tendency to male predominance in $\mathrm{G} 3(\mathrm{M}=13$ vs. $\mathrm{F}=6)$, $\mathrm{P}=0.05$ and total absence of females in $\mathrm{G} 2$ (12vs.0) $\mathrm{P}=0.0001$.

Conclusions: Despite the fact that the majority of individuals tends to be indoors and away from excessive heat, surprisingly, the contribution of such finding does not seem to have an impact on the hypovitaminosis D status in this area. Studying the skin type scale of these individuals may lead to more understanding of this issue.

\section{P1011}

PRE-SARCOPENIA, SARCOPENIA, CACHEXIA AND OSTEOPOROSIS IN PATIENTS WITH ANKYLOSING SPONDYLITIS

A. El Maghraoui ${ }^{1}$, F. E. Bertin ${ }^{1}$, S. Sadni ${ }^{1}$, A. Majjad ${ }^{1}$, T. Hamza $^{1}$, A. Mounach ${ }^{1}$

${ }^{1}$ Rheumatology Department, Military Hospital Mohammed V, Rabat, Morocco

Objective: To determine the prevalence and risk factors of pre-sarcopenia, sarcopenia and cachexia in a group of patients with AS and analyze its relationship with bone loss and symptomatic and severity parameters of the disease.

Methods: 67 consecutive male patients with AS (mean age of $40.9 \pm 11.0$ years) and 67 healthy controls were studied. Body composition and scans of the lumbar 
spine and proximal femur were obtained using DXA. The fat-free mass index (FFMI; fat-free mass divided by height squared) and the percent fat mass (\%FM) were calculated. Pre-sarcopenia was defined by low skeletal muscle mass $\left(\mathrm{SMI}<7.25 \mathrm{~kg} / \mathrm{m}^{2}\right)$.

Sarcopenia was defined by the combined presence of the two following criteria: $\mathrm{SMI}<7.25 \mathrm{~kg} / \mathrm{m}^{2}$ and a low muscle strength (handgrip strength $<30 \mathrm{~kg}$ ) or a low muscle performance (timed get-up-and-go test $>10 \mathrm{~s}$ ). Cachexia was defined by a $\mathrm{BMI}<20 \mathrm{~kg} / \mathrm{m}^{2}$ plus 3 from the 5 following parameters: anorexia, fatigue, handgrip strength $<30 \mathrm{~kg}, \mathrm{CRP}>5 \mathrm{mg} / \mathrm{l}, \mathrm{SMI}<7.25 \mathrm{~kg} / \mathrm{m}^{2}$.

Results: Prevalence of pre-sarcopenia, sarcopenia, cachexia, and osteoporosis were 50.4, 34.3, 11.9, and 16.0, respectively. Patients had a mean $3 \mathrm{~kg}$ significant decrease in FFM and a 1 $\mathrm{kg} / \mathrm{m}^{2}$ decrease in appendicular mass vs. healthy controls. Presarcopenia, sarcopenia and cachexia were significantly associated to higher BASDAI levels and lumbar spine and hip bone loss.

Conclusion: Our study showed that men with AS had a statistically significant reduction in total and appendicular lean mass that is related to higher disease activity and significantly associated to bone loss.

\section{P1012}

\section{REFRACTURE RATE IN FLS IN RIO DE JANEIRO}

B. Stolnicki ${ }^{1}$, R. Galdino ${ }^{1}$, R. Melo ${ }^{1}$, A. Libman ${ }^{1}$, H. Maleh ${ }^{1}$

${ }^{1}$ Prevrefrat, Rio de Janeiro, Brazil

Prevrefrat is a FLS, already active 5 years in Rio de Janeiro, Brazil. We provide services in a federal hospital and two HMO. We assessed the ability of our FLS to directly reduce re-fracture rate in patients with a history of fragility fractures. In this period were evaluated 553 patients, men and women above 50 years of age with a minimal trauma fracture. All patients were subjected to $\mathrm{X}$-rays of the dorsal and lumbar spine, DXA scans, blood tests (calcium, 25OHVit D, PTH and creatinine clearance). All patients were supplemented with calcium and vitamin D treated with annual infusion of zoledronic acid $5 \mathrm{mg}$ or semestral subcutaneous injection of denosumab $60 \mathrm{mg}$. The option for the mentioned drug was in order to facilitate adherence to treatment. Note that these scenarios the incident and prevalent cost of an osteoporotic fracture is on average $\$ 20,000$, justifying the cost effectiveness of these drugs. 26 fractures were found; six of them occurred before the first six months of treatment. We describe the profile of these patients for age, sex, initial fractures, new fractures, clinical risk factors, BMD values, vitamin D levels and refracture time related to treatment.

\section{P1013}

EFFECT OF MINODRONATE IN PATIENTS WITH LOW BONE MINERAL DENSITY GAIN WHILE USING OTHER BISPHOSPHONATES

H. Hagino ${ }^{1}$, K. Kita ${ }^{2}$, J. Takada ${ }^{3}$, N. Yamagata ${ }^{4}$, M. Ishii ${ }^{5}$

${ }^{1}$ Tottori University, Yonago, Japan, ${ }^{2}$ Kita Orthopedic Clinic, Kobe, Japan, ${ }^{3}$ Kitago Orthopedic Clinic, Sapporo, Japan, ${ }^{4}$ Yamagata Orthopedic Clinic, Yonago, Japan, ${ }^{5}$ Yoro Orthopedic Clinic, Yoro Cho, Japan

Objective: Minodronate, a third-generation bisphosphonate with an imidazopyridine ring side chain, is currently marketed in Japan for the treatment of osteoporosis. The aim of this study was to evaluate the effect of minodronate on BMD in patients who had low rates of BMD gain while using other bisphosphonates.

Methods: The study included postmenopausal women with primary osteoporosis who had less than 3\% increase in BMD over more than 2 years of alendronate or risedronate therapy. All patients changed from alendronate or risedronate to minodronate $1 \mathrm{mg} /$ day (daily administration) or $50 \mathrm{mg} / \mathrm{month}$ (monthly administration). BMD at the lumbar spine (L-BMD) and femoral neck (FN-BMD) was measured at baseline, 6 months, and 12 months, and urinary NTX and serum TRACP-5b were measured at 3 months, 6 months, and 12 months after the change from alendronate or risedronate to minodronate.

Results: Forty-seven women with a mean age of 71.7 years (range, 55-90) were enrolled in this study. Mean annual change in BMD before the switch over 2 years was $-0.55 \%$ and $-0.71 \%$ in L-BMD and FN-BMD, respectively. There were 32 patients in the study for 6 months and 20 patients for 12 months. Mean change in L-BMD was $1.2 \%$ at 6 months and $2.3 \%$ at 12 months. There was a significant difference in L-BMD between baseline and 12 months. Mean change in FN-BMD was $1.1 \%$ at 6 months and $2.1 \%$ at 12 months; however, these changes were not statistically significant from baseline. NTX and TRACP-5b decreased at 3 months after the change to minodronate. There was a significant negative correlation between change in TRACP-5b and L-BMD and FN$\mathrm{BMD}$, respectively.

Conclusion: Minodronate has the potential to increase BMD in patients with low BMD gain while using other bisphosphonates.

\section{P1014}

THE ASSOCIATIONS BETWEEN FEAR OF FALLING, FUNCTIONAL ABILITY AND MUSCLE MASS IN THE ELDERLY

A. Mastaviciute ${ }^{1}$, A. Versalovic ${ }^{1}$, I. TamulaityteMorozoviene $^{1}$, M. Tamulaitiene ${ }^{1}$, V. Alekna ${ }^{1}$

${ }^{1}$ Faculty of Medicine, Vilnius University, Vilnius, Lithuania 
Objective: To analyze the associations between fear of falling, functional ability and low muscle mass in older persons.

Materials and methods: The inclusion criteria for this crosssectional study were: age 65 years and more, absence of diseases of musculoskeletal or nervous system and any other conditions resulting in restricted mobility. Fear of falling was evaluated using short version of the Falls Efficacy ScaleInternational (Short FES-I). Functional ability was evaluated using the Instrumental Activities of Daily Living (IADL) scale. Muscle mass was evaluated by SARC-F scale, and score $\geq 4$ was used as an indicator of low muscle mass. Statistical analysis was carried out using SPSS version 18.0 for Windows. Correlations were assessed using Spearman correlation coefficient.

Results: A total of 41 community dwelling subjects of mean age $77.12 \pm 8.38$ years were included in this study ( 13 men and 28 women). It was found that fear of falling level was low in nine patients $(22 \%)$, moderate - in $12(29.3 \%)$ patients and high - in 20 (48.7\%) patients. Only one (2.4\%) patient had poor functional ability, $19(46.4 \%)$ - moderate functional ability and $21(51.2 \%)$ patients were functionally independent. Twenty nine (70.7\%) patients had low muscle mass evaluated by SARC-F scale. It was found that fear of falling was positively associated with low muscle mass $(\mathrm{r}=0.89, \mathrm{p}=0.01)$. Negative association between functional ability and low muscle mass $(\mathrm{r}=-0.61, \mathrm{p}=0.02)$ was revealed.

Conclusion: This study showed that in older community dwelling persons the higher level of fear of falling and poorer functional ability was associated with lower muscle mass assessed by SARC-F scale.

\section{P1015}

\section{GENDER DIFFERENCES IN PATIENT INVOLVE- MENT IN OSTEOPOROSIS CARE}

A. I. Gasparik $^{1}$, D. M. Farcas ${ }^{2}$, M. L. Cevei ${ }^{2}$

${ }^{1}$ University of Medicine and Pharmacy, Tirgu Mures, Romania, 2University of Oradea, Faculty of Medicine and Pharmacy, Oradea, Romania

Involving the patient in decision making process is especially important in osteoporosis, where compliance and persistence with therapy are suboptimal and of particular importance.

Objective: To explore possible gender related differences in patient-involvement during the consultation process of osteoporotic patients.

Methods: We observed 120 patients (68 female, 52 male) to assess communication willingness and information-need of males and females. We compared the number of questions formulated and the average duration of discussion during the encounter.
Results: Statistically significant differences were observed between the two genders in the number of formulated questions $(\mathrm{p}<0.0001)$ and duration of discussions $(p=0.03)$. The probability to formulate more than two questions is more than 7 times higher in female compared to men.

Conclusions: Male patients with osteoporosis require more attention to explore and address their concerns and expectations.

\section{P1016}

ASSOCIATION BETWEEN LEAN MASS AND DIETARY PROTEIN INTAKE IN POSTMENOPAUSAL WOMEN

V. Povoroznyuk $^{1}$, N. Dzerovych ${ }^{1}$, R. Povoroznyuk ${ }^{1}$

${ }^{1}$ Department of Clinical Physiology and Pathology of Locomotor Apparatus, D.F. Chebotarev Institute of Gerontology NAMS Ukraine, Kyiv, Ukraine

Introduction: The skeletal muscle is a key component of the body composition, and it is highly correlated with physical activity. There are many factors leading to age-related muscle mass loss. Recent studies attest to a strong connection of dietary peculiarities and the body composition of elderly people. In this context, protein with its prominent dietary status gains an especial standing as far as the older population's health is concerned.

The aim of the study: was to evaluate the appendicular lean mass depending on the dietary protein intake in the Ukrainian postmenopausal women.

Materials and methods: The study involved 63 women aged 52-89 years, who, depending on their ages, were divided into groups: 52-59 years $(\mathrm{n}=9), 60-69$ years $(n=26), 70-79$ years $(n=21), 80-89$ years $(n=7)$. To assess the dietary habits of women, we used the three-day sampling method and SEC «Viria» software. Lean mass was evaluated using a DXA (Prodigy, GE). We also calculated appendicular lean mass index (ALMI) by the formula: ALMI=lean mass of upper and lower extremities $(\mathrm{kg}) /$ height $\left(\mathrm{m}^{2}\right)$

Results: Examination of patients' dietary habits showed an age-related decrease. Women of 80-89 years consuming less than $1.0 \mathrm{~g}$ of protein per $1 \mathrm{~kg}$ of body weight accounted for more than a half of their group (57.1\%), which is significantly different from the parameters established in women of 52-59 years $(22.2 \%)$. For the purpose of quartile analysis, women were divided into 4 groups depending on their ALMI values: Q1 - ALMI $=5.20-5.84 \mathrm{~kg} / \mathrm{m}^{2} \quad(\mathrm{n}=15), \mathrm{Q} 2-$ $\mathrm{ALMI}=5.85-6.25 \mathrm{~kg} / \mathrm{m}^{2} \quad(\mathrm{n}=17), \mathrm{Q} 3-\mathrm{ALMI}=6.26-6.56$ $\mathrm{kg} / \mathrm{m}^{2} \quad(\mathrm{n}=16), \mathrm{Q} 4-\mathrm{ALMI}=6.57-7.65 \mathrm{~kg} / \mathrm{m}^{2} \quad(\mathrm{n}=15)$. Women with the lowest ALMI values consume the lowest amounts of dietary protein $(\mathrm{F}=3.67 ; \mathrm{p}=0.02)$. 
Significant correlations among dietary protein, nonessential, essential amino acids and ALMI values $(r=0.40$, $\mathrm{t}=3.44, \mathrm{p}=0.001 ; \mathrm{r}=0.39, \mathrm{t}=3.30, \mathrm{p}=0.002 ; \mathrm{r}=0.35$, $\mathrm{t}=2.91, \mathrm{p}=0.005$; accordingly) were determined.

Conclusion: Further studies are needed to elaborate a set of recommendations aimed at correction of nutritional habits observed in older women of different countries.

\section{P1017}

CHANGES IN QUALITY OF LIFE AFTER FRAGILITY HIP FRACTURES, THAILAND

T. Sangkomkamhang ${ }^{1}$, U. Sangkomkamhang ${ }^{2}$

${ }^{1}$ Department of Orthopedic, Khon Kaen Regional Hospital and Academic Center, KhonKaen, Thailand, 2Department of Obstetrics and Gynecology, Khon Kaen Regional Hospital and Academic Center, Khon Kaen, Thailand

Fragility hip fractures are common and lead to prolong pain and suffering, disability, complication, increased risk of death. The burden of hip fractures is expected to grow considerably during next years due to population aging.

Objective: to describe the health-related quality of life (HRQoL) and the determinants in patients sustaining a fragile hip fracture in Thailand.

Methods: Data from review chart 2011-2013 was gathered. Patients had to be diagnosed with a fragile hip fracture and more than 50 years old. HRQoL was prospectively collected 12 months after fracture using the EQ-5D methods. The accumulated HRQoL loss in the first year after fracture was calculated using the trapezoid method. Multivariate regression analysis was conducted to identify determinants of HRQoL reductions.

Results: 316 patients were evaluated. Mean $( \pm$ SD) age was $76 \pm 8$ years. $73 \%$ were women. $12 \%$ of the patients had a prior fracture in last 5 years; $32 \%$ of patients can walks without restrictions before fracture and $85 \%$ were classified into the low level of income category. Mean $(95 \% \mathrm{CI})$ utility value before fracture was $0.68(0.52-$ $0.75)$. Utility dropped to $0.01(0.01-0.03)$ immediately after injury and then improved to $0.43(0.39-0.50)$ and $0.50(0.45-0.61)$ at month 6 and 12 after fracture, respectively. The accumulated utility loss over the first year was $0.33(0.31-0.37)$. HRQoL before fragile hip fractures, comorbidity, preambulatory status and age of patients were the most relevant characteristics associated with the accumulated utility loss.

Conclusion: Fragility hip fractures had impaired HRQoL and leading to increase morbidity and mortality after fracture. The mean utility after 1 year of follow-up was lower than before injury.

\section{P1018}

THE PREVALENCE OF UNEXPLAINED HBM AMONGST A DXA POPULATION IN AN OSTEOPOROSIS REFERENCE CENTRE FROM ROMANIA D. Grigorie $^{1}$, A. Sucaliuc ${ }^{1}$

${ }^{1}$ National Institute of Endocrinology, Carol Davila University of Medicine, Bucharest, Romania

Aim: Unexplained high bone mass (HBM) is a rare condition and the mechanisms responsible are yet to be identified. We aimed to determine the prevalence of unexplained HBM amongst a DXA population.

Methods and Results: Our DXA databases comprise 16.352 individuals, scanned in the last 15 years, including osteoporosis patients referred for evaluation and treatment and screened patients for osteoporosis. All DXA images were visually inspected by us, and those with identifiable explanations for a high BMD values were excluded. As there is no consensus definition of HBM, we defined HBM using different thresholds for DXA Z-score at lumbar spine and/or hip. DXA databases were searched for individuals with BMD Z-score $\geq+3$ $\mathrm{SD}$ at least at a single lumbar spine vertebra or any hip region. With these criteria we identified 323 patients (mean age 61.7 years, range 21-85, mean BMI $27.4 \mathrm{~kg} / \mathrm{m}^{2}$, mean LS Z-score 2.6 SD, mean total hip Z-score 1.9 SD) with a prevalence of 1 , $97 \%$. In this group we refined criteria using 2 cutoffs: 1) Zscore $\geq+3 \mathrm{SD}$ at any of the following regions L1, L1 to L4, femoral neck, total hip, or the sum L1 plus total hip $\geq+3.2 \mathrm{SD}$; this identified 132 patients and a prevalence of $0.81 \%$; 2) Zscore $\geq+2.5 \mathrm{SD}$ at any of the following regions L1, L1 to L4, femoral neck, total hip or L1 plus total hip $\geq+2.5 \mathrm{SD}$; this refined search identified 207 patients with a prevalence of $1.27 \%$.

Conclusion: We found a high prevalence of HBM amongst our DXA databases using different cutoffs.

\section{P1019}

CHANGES IN HAND BONE DENSITOMETRY AFTER DISTAL RADIUS FRACTURE AND THEIR ASSOCIATIONS WITH CLINICAL PARAMETERS

J. Öncü ${ }^{1}$, A. Üșen ${ }^{1}$, B. Kuran ${ }^{1}$, K. Alptekin ${ }^{2}$

${ }^{1}$ Department of PM\&R, Şişli Etfal Teaching Hospital, Istanbul, Turkey, 2Department of Physiotherapy, Bahçeşehir University, Istanbul, Turkey

Purpose: This study was undertaken to evaluate hand bone mineral density (BMD) in patients with distal radius fracture, and to correlate with clinical parameters.

Subjects: Forty-nine patients and 45 healthy subjects were included.

Methods: All patients underwent BMD of fractured and nonfractured hand by DEXA at baseline and 52th week. The 
severity of CRPS-I was graded on the basis of shoulder hand syndrome score (SHSS). Clinical tests were pain severity (VAS), SHSS, range of motion of the wrist, dynamometric hand grip strength (Jamar Dynamometer) and Disability Arm Shoulder Hand (DASH) score.

Results: BMD of fractured hand was significantly lower than non-fractured hand in patient group both at baseline and 52th week. In females BMD of fractured site at both visits were significantly lower than males and controls. In males BMD of fractured site only at the 2nd visit was significantly lower than that of controls. Compared with baseline; fractured hand BMD was decreased by $0,8 \%$; non-fractured hand by $0,3 \%$ after oneyear. The bone density reduction was correlated with SHSS, DASH score most significantly.

Conclusion: Our results show significant reduction of hand BMD in patients with distal radius fracture during one year. The reduction in BMD correlates with the severity of SHSS and DASH score.

\section{P1020}

CLINICAL FEATURES OF PATIENTS WITH OSTEOPOROSIS TREATED IN A PRIMARY CARE CENTER

R. Sánchez-de las Matas $^{1}$, G. Fernández-García ${ }^{1}$

${ }^{1}$ Hospital Universitario de Puerto Real, Puerto Real, Spain

Introduction: The prevalence of osteoporosis increases with age. It is estimated that $30-40 \%$ of people over 60 have osteoporosis. The number of patients treated with any drugs has increased. The role of General Practitioners in identifying patients at risk, diagnosis, treatment and follow-up is essential. Objective: To find out the clinical and demographic features of patients in treatment for osteoporosis, look for risk factors for osteoporosis and bone fractures, and assess whether treatment is indicated.

Material and methods: Descriptive study of a series of cases, 217 patients with a treatment for osteoporosis for one year. Demographic variables, risk factors, risk of falls and information related to the prescription and treatment. To assess the type of treatment we will take as a reference the recommendations of the main guidelines.

Results: Most of the patients were women with an average of 66.1 years. $39.3 \%$ had a history of fracture and in $16.1 \%$ there is a fracture in some member of the family $73.1 \%$ of the stories have no reference to bone densitometry. The existence of risk factors is not reflected in a high percentage of medical records. $51.8 \%$ met criteria for initiating treatment. $44 \%$ of the treatments are initiated in primary care.

Discussion: Nearly $50 \%$ of treatments are not well diagnosed and a high percentage of our clinical histories do not record risk factors correctly. We should improve our clinical histories, as we are responsible for identifying, studying, evaluating, treating and controlling the progress of osteoporosis.

\section{P1021}

IMPACT OF OSTEOPOROSIS ON THE POPULATION

R. Sánchez-de las Matas ${ }^{1}$, G. Fernández-García ${ }^{1}$

${ }^{1}$ Hospital Universitario de Puerto Real, Puerto Real, Spain

Introduction: This disease affects $35 \%$ of Spanish women over 50 years, a percentage that rises to $52 \%$ for the over 70 years. The incidence of Hip fractures in patients older than 50 is 1.3 to 1.9 cases per 1000 inhabitants per year. In most patients with osteoporosis incidence of new vertebral fractures the 3-4 years is 3-4 \%. The direct costs of this disease in Europe totaled 4,800 million euros in 2000 only hospital expenses.

Objective: With this work is to determine the influence of osteoporosis on population health and expense accounts in Spain and other countries.

Material and methods: Literature Review.

Results: For the aforementioned reasons it is evident that it is a disease that needs for its prevention more education and awareness of the population for the benefit a better quality of life, less expenses health and higher productivity will result to benefit the health and economy community.

Discussion: Osteoporosis affects a large part of the world's population over 50 years. Its complications on the one hand reduces the quality of life on the other side carries a high cost. Is you should add that throughout the world there is a tendency to increase in population over 60 years and the growth in life expectancy leading to an increase in population will age advanced with a consequent increase of osteoporosis and associated fractures.

\section{P1022}

OSTEOPOROSIS AND HIP FRACTURE: RISK FACTOR'S

R. Sánchez-de las Matas ${ }^{1}$, G. Fernández-García ${ }^{1}$

${ }^{1}$ Hospital Universitario de Puerto Real, Puerto Real, Spain

Introduction: Osteoporosis is a serious problem for public health due to the strong socio- economic impact generated and the burden on the health system. It causes significant morbidity and mortality and high costs associated with their care. Hip fracture is one of the most serious complications of osteoporosis.

Objective: To determine the association between risk factors and hip fracture in hospitalized patients. To evaluate the influence of those risk factors associated with low bone mass and/or trauma. Explore the existence of protective factors. 
Material and methods: Observational, case-control study of 188 patients ( 75 hip fractures and 133 controls). The variables studied were: consumption of snuff, coffee, alcohol, sun exposure, physical activity, intake of calcium, phosphorus and protein, body mass index.

Results: The average age of the study population was 80.6 years. The female/male ratio was $3: 1$. The average age of fractured women was 82.5 years and 79.7 years for males. Physical activity was low, consumption of alcohol and snuff scarce and reduced sun exposure. The proportion of patients with BMI below $23 \mathrm{~kg} / \mathrm{m}^{2}$ was higher in cases than in controls. When analyzed data on calcium intake, significant differences between the group of fractured patients and the control group were found, with lower values in cases.

Discussion: Excessive consumption of snuff and alcohol, low weight and low BMI associated with hip fractures. Previous falls and have been repeated frequently in fractured patients than in controls.

\section{P1023}

\section{OSTEOPOROSIS ASSOCIATED INDINAVIR IN HIV PATIENT: REPORT OF A CASE}

R. Sánchez-de las Matas $^{1}$, G. Fernández-García ${ }^{1}$

${ }^{1}$ Hospital Universitario de Puerto Real, Puerto Real, Spain

Introduction: Following the development of effective therapies against HIV, we have faced a number of adverse effects associated with prolonged use of these drugs: peripheral neuropathy, pancreatitis, blood disorders etc... It has been reported significant bone loss associated with the use of protease inhibitors as part of antiretroviral therapy.

Objective: A case of osteoporosis associated with indinavir is presented.

Material and methods: 45-year-old male, HIV carrier started antiretroviral therapy (ART): zidovudine 600 $\mathrm{mg} /$ day, lamivudine $300 \mathrm{mg} /$ day and indinavir 2,400 $\mathrm{mg} /$ day. At 22 months he reported mild hip pain that was managed with NSAIDs initially going down but becoming constant from the 28 months of treatment. The 33 month ART a bone densitometry is performed which concluded that there was osteoporosis of the lumbar spine and severe osteopenia left hip.

Results: The patient develops a secondary osteoporosis indinavir sulfate. Management consisted of alendronate, calcium and vitamin D along with suspension of indinavir and starting efavirenz. After 16 months of treatment an increase in bone mineral density of $11 \%$ on average was observed. Fractures or adverse effects or drug interactions weren't found.
Discussion: The association of HIV infection and bone loss is not new. It was thought that the TAR could promote bone remodeling and avert osteoporosis; however, cases have been reported related to ART patients and especially those that include indinavir sulfate osteoporosis, detected $28.4 \%$ incidence of osteopenia and 9.5 to $21 \%$ of severe osteoporosis.

\section{P1024}

OSTEOPOROSIS IN THE ELDERLY: IDENTIFICATION OF RISK FACTORS FOR PREVENTION R. Sánchez-de las Matas ${ }^{1}$, G. Fernández-García ${ }^{1}$

${ }^{1}$ Hospital Universitario de Puerto Real, Puerto Real, Spain

Introduction: Osteoporosis is an up growing problem. During the last years of the past century, Westem Countries have experienced a progressive aging on its population. Due to this change, degenerative and involutive origin illnesses have increased notoriously, among these we can find Osteoporosis.

Objective: This review main objectives are: updating concepts about the disease, identifying potential risk factors and most important steps to take to prevent and face this growing entity.

Material and methods: To carry out this work he used the MEDLINE database through PUBMED search engine and the search SECYT. They were selected articles from magazines Clinical Geriatrics; Spanish magazine Geriatrics and Gerontology; Practice Guidelines Geriatric Clinic and continuing education program for osteoporosis.

Results: Among the risk factors are: female, Caucasian, postmenopausal and steroid treatment. A change in lifestyle, avoiding smoking and alcohol, a proper diet and physical activity are considered key strategies in preventing this disease. Drug therapy has proven effective in preventing bone loss. Hormone replacement therapy with estrogen in postmenopausal women is of choice to prevent osteoporotic fractures. Calcitonin and bisphosphonates is reserved for groups most at risk.

Discussion: The phenomenon of bone loss is a process considered practically irreversible, the most efficient method to stop these skeletal changes appear to be early and effective prevention. They could establish effective strategies, low cost and with minimal side effects, such as public health priorities considering between these improving calcium intake and physical activity. 